Date Submitted: $3 / 6 / 07$

Originator: L.M. Dittmer

Phone: $\quad 372-9664$
WASTE SITE RECLASSIFICATION FORM

Operable Unit(s): $\quad$ 100-BC-1

Waste Site Code: $\quad 1607-\mathrm{B} 2$

Type of Reclassification Action:

Closed Out $\square \cdot$ Interim Closed Out $₫$ No Action $\square$

RCRA Postclosure $\square \quad$ Rejected $\square$ Consolidated $\square$

This form documents agreement among parties listed authorizing classification of the subject unit as Closed Out, Interim Closed Out, No Action, RCRA Postclosure, Rejected, or Consolidated. This form also authorizes backfill of the waste management unit, if appropriate, for Closed Out and Interim Closed Out units. Final removal from the NPL of No Action and Closed Out waste management units will occur at a future date.

Description of current waste site condition:

The 1607-B2 waste site is a former septic system associated with various 100-B facilities, including the 105-B, 108-B, 115-B/C, and 185/190-B buildings. The site was evaluated based on confirmatory results for feeder lines within the 100-B-14:2 subsite and determined to require remediation. During remediation, the site was administratively divided into two subsites: the drain field (1607-B2:1) and the collection main, septic tank, and effluent piping (1607-B2:2). Remedial actions and verification sampling at both subsites have been performed in accordance with remedial action objectives and goals established.by the Interim Action Record of Decision for the 100-BC-1, 100-BC-2, 100-DR-1, 100-DR-2, 100-FR-1, 100-FR-2, 100-HR-1, 100-HR-2, 100-KR-1, 100KR-2, 100-IU-2, 100-IU-6, and 200-CW-3 Operable Units, Hanford Site, Benton County, Washington (Remaining Sites ROD), U.S. Environmental Protection Agency, Region 10, Seattle, Washington. The selected action involved (1) evaluating the site using available process information and confirmatory sample data, (2) remediating the site, (3) demonstrating through verification sampling that cleanup goals have been met, and (4) proposing the site for reclassification as interim closed out.

Basis for reclassification:

The 1607-B2 waste site has been remediated to meet the remedial action objectives specified in the Remaining Sites ROD. The results of verification sampling demonstrated that residual contaminant concentrations do not preclude any future uses (as bounded by the rural-residential scenario) and allow for unrestricted use of shallow zone soils (i.e.; surface to $4.6 \mathrm{~m}$ [15 ft] deep). The results also showed that residual contaminant concentrations are protective of groundwater and the Columbia River. This site does not have a deep zone; therefore, no deep zone institutional controls are required. The basis for reclassification is described in detail in the Remaining Sites Verification Package for the 1607-B2 Septic System and 100-B-14:2 Sanitary Sewer System (attached

Waste Site Controls:

Engineered Controls: Yes $\square$ No $\square \quad$ Institutional Controls: Yes $\square$ No $\square \quad$ O\&M requirements: Yes $\square$ No $\square$ If any of the Waste Site Controls are checked Yes specify control requirements including reference to the Record of Decision, TSD Closure Letter, or other relevant documents.

K. D. Bazzell

DOE Federal Project Director (printed)

NA

Ecology Project Manager (printed)

D. A. Faulk

EPA Project Manager (printed)
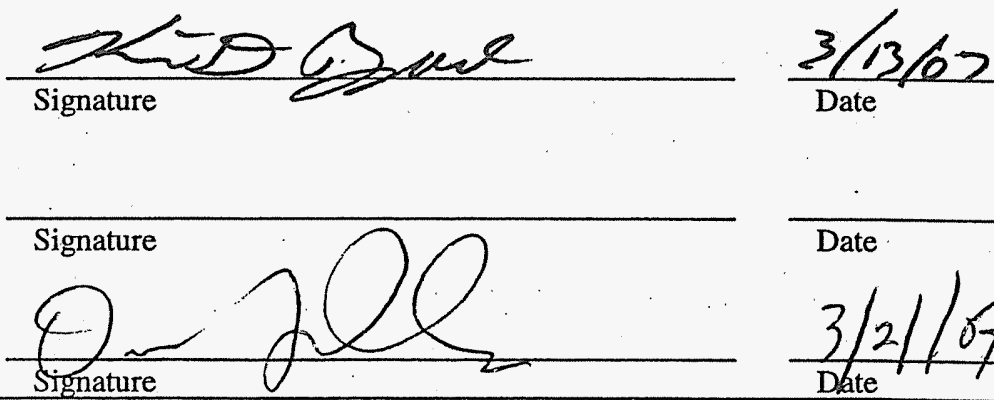
REMAINING SITES VERIFICATION PACKAGE FOR THE 1607-B2 SEPTIC SYSTEM AND 100-B-14:2 SANITARY SEWER SYSTEM Attachment to Waste Site Reclassification Form 2006-055 and 2004-006 March 2007 


\section{REMAINING SITES VERIFICATION PACKAGE FOR THE 1607-B2 SEPTIC SYSTEM AND THE 100-B-14:2 SANITARY SEWER SYSTEM}

\section{EXECUTIVE SUMMARY}

The 1607-B2 waste site was the primary septic system for 100-B facilities, formerly providing service to the $105-\mathrm{B}, 108-\mathrm{B}, 115-\mathrm{B} / \mathrm{C}, 185 / 190-\mathrm{B}$, and various $1700-\mathrm{B}$-series buildings. The $100-\mathrm{B}-14$ waste site encompasses pre-reactor underground process and sanitary pipelines and sewers, and has been divided into subsites for decision-making purposes based on functional use and geographical location. This remaining sites verification package addresses the 1607-B2 septic system and the 100-B-14:2 subsite, which encompasses the sanitary sewer feeder lines that formerly discharged to the 1607-B2 septic system, as well as the sanitary sewer formerly discharging to the 1607-B7 septic system. The 1607-B7 septic system has been separately remediated and closure documented (BHI 2003a). Portions of the 100-B-14:2 subsite were previously given redundant subsite identification numbers as follows: 100-B-14:8 and 100-B-14:9. These designations have been cancelled to resolve the redundancy.

The sites (100-B-14:2 and 1607-B2) were evaluated during September/October 2003 and June 2005 using confirmatory sampling efforts to make a decision whether remedial action would be required. Focused samples were collected from manholes, pipelines exposed by other remedial activities, and underlying soils. Multiple chemical and radiological contaminants were detected above action levels within the feeder pipelines, and remedial action was determined to be necessary for all of the pipelines, except the feeder lines formerly associated with the 1704-B, 1707-BA, 1713-B, 1717-B, 1719-B, and 1722-B facilities (designated as area 3 of the 100-B-14:2 subsite).

Site remediation consisted of the removal of the sewer piping, septic tank, and drain field, as well as adjacent, potentially-contaminated soils for disposal at the Environmental Restoration Disposal Facility. Overburden material presumed to be below cleanup levels (BCL) was stockpiled on site for use in backfill. Remedial actions were performed so as to not preclude any future uses (as bounded by the rural-residential scenario) and to allow unrestricted use of shallow zone soils (i.e., surface to $4.6 \mathrm{~m}[15 \mathrm{ft}]$ deep).

Following site remediation, verification sampling of BCL material and soil within the remediation footprints was conducted from August 2005 to July 2006. The results indicated that the waste removal action achieved compliance with the remedial action objectives for the 100-B-14:2 and 1607-B2 waste sites. A summary of the cleanup evaluation for the soil results against the applicable criteria is presented in Table ES-1. The results of the verification sampling are used to make reclassification decisions for the 100-B-14 and 1607-B2 waste sites in accordance with the TPA-MP-14 (DOE-RL 1998) procedure.

In accordance with this evaluation, the verification sampling results support a reclassification of these sites to interim closed out. The current site conditions achieve the remedial action objectives and the corresponding remedial action goals established in the Remedial Design 
Report/Remedial Action Work Plan for the 100 Area (DOE-RL 2005b) and the Interim Action Record of Decision for the 100-BC-1, 100-BC-2, 100-DR-1, 100-DR-2, 100-FR-1, 100-FR-2, 100-HR-1, 100-HR-2, 100-KR-1, 100-KR-2, 100-IU-2, 100-IU-6, and 200-CW-3 Operable Units, Hanford Site, Benton County, Washington (Remaining Sites ROD) (EPA 1999). The results of verification sampling show that residual contaminant concentrations do not preclude any future uses (as bounded by the rural-residential scenario) and allow for unrestricted use of shallow zone

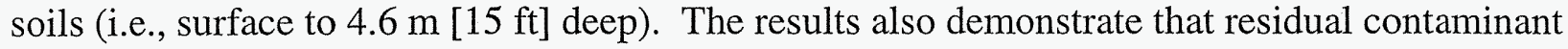
concentrations are protective of groundwater and the Columbia River. These sites do not have a deep zone component; therefore, no deep zone institutional controls are required.

Soil cleanup levels were established in the Remaining Sites ROD (EPA 1999) based on a limited ecological risk assessment. Although not required by the Remaining Sites ROD, a comparison to ecological risk screening levels has been made for the site contaminants of concern, contaminants of potential concern, and other constituents. Ecological screening levels were exceeded for arsenic, barium, boron, cadmium, chromium (total), copper, lead, mercury, nickel, vanadium, zinc, 4,4'-DDD/DDE/DDT, dieldrin, pentachlorophenol, and polychlorinated biphenyls. Exceedance of screening values does not necessarily indicate the existence of risk to ecological receptors. The majority of the exceedances are for constituents confined to the interior of residual pipelines within 100-B-14:2 (area 3). Concentrations of these constituents were quantified below screening levels and/or background levels in soils at other areas of the 100-B-14:2/1607-B2 site. All exceedances at the 100-B-14:2 and 1607-B2 sites will be evaluated in the context of additional lines of evidence for ecological effects following a baseline risk assessment for the river corridor portion of the Hanford Site, which includes a more complete quantitative ecological risk assessment. That baseline risk assessment will be used to support the final closeout decision for the 100-B-14 and 1607-B2 waste sites. 
Table ES-1. Summary of Remedial Action Goals for the 1 Waste Sites. (2 Pages)

\begin{tabular}{|c|c|c|}
\hline $\begin{array}{l}\text { Regulatory } \\
\text { Requirement }\end{array}$ & Remedial Action Goals & Resul \\
\hline $\begin{array}{l}\text { Direct Exposure - } \\
\text { Radionuclides }\end{array}$ & $\begin{array}{l}\text { Attain } 15-\mathrm{mrem} / \mathrm{yr} \text { dose rate above } \\
\text { background over } 1,000 \text { years. }\end{array}$ & $\begin{array}{l}\text { Only cesium-137, trit } \\
\text { strontium-90 were de } \\
\text { samples, at activities } \\
\text { below the direct expo } \\
\text { equivalence lookup v: }\end{array}$ \\
\hline $\begin{array}{l}\text { Direct Exposure - } \\
\text { Nonradionuclides }\end{array}$ & $\begin{array}{l}\text { Attain individual COC/COPC } \\
\text { RAGs. }\end{array}$ & $\begin{array}{l}\text { Aroclor- } 1248 \text { and die } \\
\text { detected within the 1( } \\
\text { (area 3) pipelines abo } \\
\text { exposure RAGs. Bas } \\
\text { specific risk assessme } \\
\text { residual concentratior } \\
\text { aroclor-1248 and diel } \\
\text { RAOs for direct expo } \\
\text { pyrene and dibenzo(a } \\
\text { were detected within } \\
\text { (area } 4 \text { ) remediation } 1 \\
\text { BCL stockpiles abovt } \\
\text { RAGs. Residual PAF } \\
\text { within area } 4 \text { were de } \\
\text { the result of asphalt c } \\
\text { contamination. Asph } \\
\text { used for structural an } \\
\text { purposes is excluded } \\
\text { consideration as a da1 } \\
\text { listed as an inert solic } \\
\text { not present a signific: } \\
\text { this waste site. All o1 } \\
\text { COPCs were not qua] } \\
\text { direct exposure RAG }\end{array}$ \\
\hline \multirow[t]{4}{*}{$\begin{array}{l}\text { Risk Requirements - } \\
\text { Nonradionuclides }\end{array}$} & $\begin{array}{l}\text { Attain a hazard quotient of }<1 \text { for } \\
\text { all individual noncarcinogens. }\end{array}$ & $\begin{array}{l}\text { All individual hazard } \\
\text { than } 1 .\end{array}$ \\
\hline & $\begin{array}{l}\text { Attain a cumulative hazard } \\
\text { quotient of }<1 \text { for noncarcinogens. }\end{array}$ & $\begin{array}{l}\text { The cumulative hazai } \\
\text { each area is less than }\end{array}$ \\
\hline & $\begin{array}{l}\text { Attain an excess cancer risk of } \\
<1 \times 10^{-6} \text { for individual } \\
\text { carcinogens. }\end{array}$ & $\begin{array}{l}\text { All individual excess } \\
\text { values are less than } 1\end{array}$ \\
\hline & $\begin{array}{l}\text { Attain a cumulative excess cancer } \\
\text { risk of }<1 \times 10^{-5} \text { for carcinogens. }\end{array}$ & $\begin{array}{l}\text { The total excess canc } \\
\text { each area is less than }\end{array}$ \\
\hline
\end{tabular}


Table ES-1. Summary of Remedial Action Goals for the 1607-B2 and 100-B-14:2 Waste Sites. (2 Pages)

\begin{tabular}{|c|c|c|c|}
\hline $\begin{array}{l}\text { Regulatory } \\
\text { Requirement }\end{array}$ & Remedial Action Goals & Results & $\begin{array}{l}\text { Remedial Action } \\
\text { Objectives } \\
\text { Attained? }\end{array}$ \\
\hline \multirow{4}{*}{$\begin{array}{l}\text { Groundwater/River } \\
\text { Protection - } \\
\text { Radionuclides }\end{array}$} & $\begin{array}{l}\text { Attain single-COPC groundwater } \\
\text { and river protection RAGs. }\end{array}$ & \multirow{2}{*}{$\begin{array}{l}\text { Only cesium-137, tritium, and } \\
\text { strontium- } 90 \text { were detected in closure } \\
\text { samples, at activities significantly } \\
\text { below the lookup values for protection } \\
\text { of groundwater and the Columbia } \\
\text { River. }\end{array}$} & \multirow{3}{*}{ Yes } \\
\hline & $\begin{array}{l}\text { Attain national primary drinking } \\
\text { water standards: }{ }^{a} 4 \mathrm{mrem} / \mathrm{yr} \\
\text { (beta/gamma) dose rate to target } \\
\text { receptor/organs. }\end{array}$ & & \\
\hline & $\begin{array}{l}\text { Meet drinking water standards for } \\
\text { alpha emitters: the most stringent } \\
\text { of } 15 \mathrm{pCi} / \mathrm{L} \text { MCL or } 1 / 25 \text { th of the } \\
\text { derived concentration guides from } \\
\text { DOE Order } 5400.5^{\text {b }}\end{array}$ & $\begin{array}{l}\text { No alpha-emitting radionuclides were } \\
\text { detected in verification samples. }\end{array}$ & \\
\hline & $\begin{array}{l}\text { Meet total uranium standard of } \\
30 \mu \mathrm{g} / \mathrm{L}(21.2 \mathrm{pCi} / \mathrm{L})^{\mathrm{c}}\end{array}$ & $\begin{array}{l}\text { Uranium was not identified as a site } \\
\text { COC/COPC. }\end{array}$ & N/A \\
\hline $\begin{array}{l}\text { Groundwater/River } \\
\text { Protection - } \\
\text { Nonradionuclides }\end{array}$ & $\begin{array}{l}\text { Attain individual nonradionuclide } \\
\text { groundwater and river cleanup } \\
\text { requirements. }\end{array}$ & $\begin{array}{l}\text { Residual concentrations of multiple } \\
\text { organic and inorganic contaminants } \\
\text { are above soil RAGs for groundwater } \\
\text { and/or river protection. However, } \\
\text { these contaminants are not predicted } \\
\text { to reach groundwater (and, therefore, } \\
\text { the Columbia River) within } \\
1,000 \text { years. Therefore, the residual } \\
\text { concentrations achieve the RAOs for } \\
\text { groundwater and river protection. }\end{array}$ & Yes \\
\hline
\end{tabular}

a "National Primary Drinking Water Regulations" (40 Code of Federal Regulations 141).

b Radiation Protection of the Public and the Environment (DOE Order 5400.5).

c Based on the isotopic distribution of uranium in the 100 Areas, the $30 \mu \mathrm{g} / \mathrm{L} \mathrm{MCL}$ corresponds to $21.2 \mathrm{pCi} / \mathrm{L}$. Concentration-toactivity calculations are documented in Calculation of Total Uranium Activity Corresponding to a Maximum Contaminant Level for Total Uranium of 30 Micrograms per Liter in Groundwater (BHI 2001).

$\mathrm{BCL}$ = below cleanup levels

$\mathrm{COC}=$ contaminant of concern

$\mathrm{COPC}=$ contaminant of potential concern

$\mathrm{MCL}=$ maximum contaminant level

N/A $=$ not applicable

$\mathrm{PAH}=$ polycyclic aromatic hydrocarbon

$\mathrm{RAG}=$ remedial action goal

$\mathrm{RAO}=$ remedial action objective 


\section{REMAINING SITES VERIFICATION PACKAGE FOR THE 1607-B2 SEPTIC SYSTEM AND THE 100-B-14:2 SANITARY SEWER SYSTEM}

\section{STATEMENT OF PROTECTIVENESS}

This report demonstrates that the 1607-B2 and 100-B-14:2 waste sites meet the objectives for interim closure as established in the Remedial Design Report/Remedial Action Work Plan for the 100 Area (RDR/RAWP) (DOE-RL 2005b) and the Interim Action Record of Decision for the 100-BC-1, 100-BC-2, 100-DR-1, 100-DR-2, 100-FR-1, 100-FR-2, 100-HR-1, 100-HR-2, 100-KR-1, 100-KR-2, 100-IU-2, 100-IU-6, and 200-CW-3 Operable Units, Hanford Site, Benton County, Washington (Remaining Sites ROD) (EPA 1999). Portions of the pipelines within the 100-B-14:2 subsite have also been identified as the 100-B-14:8 and 100-B-14:9 subsites; the latter two subsite designations have been administratively cancelled to resolve the redundancy. The results of verification sampling show that residual contaminant concentrations do not preclude any future uses (as bounded by the rural-residential scenario) and allow for unrestricted use of shallow zone soils (i.e., surface to $4.6 \mathrm{~m}$ [15 ft] deep). The results also demonstrate that residual contaminant concentrations are protective of groundwater and the Columbia River. This site does not have a deep zone component; therefore, no deep zone institutional controls are required.

Soil cleanup levels were established in the Remaining Sites ROD (EPA 1999) based on a limited ecological risk assessment. Although not required by the Remaining Sites ROD, a comparison to ecological risk screening levels has been made for the site contaminants of concern (COCs), contaminants of potential concern (COPCs), and other constituents. Ecological screening levels were exceeded for arsenic, barium, boron, cadmium, chromium (total), copper, lead, mercury, nickel, vanadium, zinc, 4,4'-DDD/DDE/DDT, dieldrin, pentachlorophenol, and polychlorinated biphenyls. Exceedance of screening values does not necessarily indicate the existence of risk to ecological receptors. The majority of the exceedances are for constituents confined to the residual pipelines within 100-B-14:2 (area 3). Concentrations of these constituents were quantified below screening levels and/or background levels in soils at other areas of the 100-B14:2/1607-B2 site. All exceedances at the 100-B-14:2 and 1607-B2 sites will be evaluated in the context of additional lines of evidence for ecological effects following a baseline risk assessment for the river corridor portion of the Hanford Site, which includes a more complete quantitative ecological risk assessment. That baseline risk assessment will be used to support the final closeout decision for the 100-B-14 and 1607-B2 waste sites.

\section{GENERAL SITE INFORMATION AND BACKGROUND}

The 1607-B2 and 100-B-14:2 waste sites encompass the vitrified clay sanitary sewers, septic tank, and drain field that formerly serviced the 105-B Reactor Building, the 185/190-B Pumphouse, the 108-B Chemical Pumphouse and Tritium Separation Facility, the 115-B/C Gas Recirculation Facility, and miscellaneous 1700-series support buildings. The 100-B-14:2 subsite is also inclusive of the sanitary sewer collection line for the 1607-B7 septic system, formerly servicing the 183-B Filter Plant Pumphouse. 
According to the Waste Information Data System (WIDS) (WCH 2006e), the 1607-B2 septic system included a large-capacity $(59,620 \mathrm{~L}$ [15,750 gal]) septic tank and drain field located north of the 105-B Reactor Building. The system was initially designed to support waste loading from

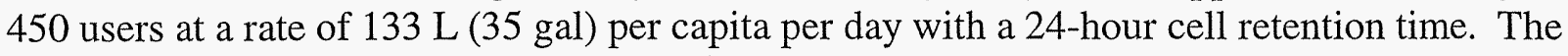
1607-B2 waste site has been administratively divided into two subsites for the purposes of verification sampling: the drain field is designated as the 1607-B2:1 subsite, and the collection main, septic tank, and discharge line to the drain field are collectively designated as the 1607-B2:2 subsite. Both of these subsites are addressed within this remaining sites verification package.

The 100-B-14 waste site, in its entirety, encompasses the underground process pipelines and process and sanitary sewers associated with the 100-B Area pre-reactor cooling water treatment facilities. For confirmatory sampling efforts, the 100-B-14 site was administratively divided into seven subsites for decision-making purposes based on the use of the pipelines (e.g., sanitary versus process sewers), expected sources of contamination, potential differing remedial action determinations, and geographical location. Two additional subsites were, subsequently, created to encompass pipelines thought to have been excluded from the initial delineation, but which had actually been included within the 100-B-14:2 subsite; these subsites were administratively cancelled to resolve the redundancy. The nine subsites, including those cancelled, are as follows:

- 100-B-14:1 Main process sewer collection pipeline

- 100-B-14:2 Sanitary sewer pipelines

- 100-B-14:3 West process sewer feeder lines (182-B and 183-B)

- 100-B-14:4 190-B/105-B cooling water tunnel pipelines

- 100-B-14:5 Sodium dichromate and sodium silicate pipelines

- 100-B-14:6 184-B Powerhouse pipelines

- 100-B-14:7 185-B/190-B sump and process sewer pipelines

- 100-B-14:8 190-B sanitary sewer pipelines (cancelled)

- 100-B-14:9 1607-B7 sanitary sewer pipelines (cancelled).

The 100-B-14:2 subsite was further divided into five service areas based on the facilities serviced, as follows:

- 100-B-14:2 (area 1)

108-B and 1703-B sanitary sewer pipelines, including sanitary sewer pipelines discovered during previous remedial activites (100-B-21 waste site, pipelines DS-100BC-012 and DS-100BC-034)

- 100-B-14:2 (area 2) 1607-B7 sanitary sewer pipelines (variously identified as the 100-B-14:9 subsite)

- 100-B-14:2 (area 3) Miscellaneous 1700-series buildings sanitary sewer pipelines

- 100-B-14:2 (area 4) 190-B Pumphouse sanitary sewer pipelines (variously identified as the 100-B-14:8 subsite)

- $100-B-14: 2$ (area 5) 115-B/C Gas Recirculation Facility sanitary sewer pipelines. 
This remaining sites verification package addresses only the sanitary sewers included within the 100-B-14:2 subsite and the 1607-B2 septic system.

Administrative boundaries for the 1607-B2 and 100-B-14:2 waste sites are shown in Figure 1.

\section{CONFIRMATORY EVALUATION AND SAMPLING ACTIVITIES}

The 100-B-14:2 subsite was evaluated during September and October 2003 using confirmatory sampling efforts to make a decision whether remedial action would be required at the subsite, with the exception of areas 1 and 3. Sanitary sewer pipelines associated with area 1 were partially removed during the remediation of the underlying 100-B-8 cooling water effluent pipelines (BHI 2004b), and field radiological surveys of exposed portions of the remaining pipelines detected sufficient radiological activity to warrant remedial action without further investigation. Evaluation of area 3 was deferred until June 2005 due to the presence of office trailers associated with 100-B/C Area remediation activities.

Based on the results of confirmatory sampling and previous investigations, it was decided that remedial action was necessary at areas 1,2, 4, and 5 (BHI 2003c and Feist 2004) and that no further action was required at area 3 . A decision was also made that remediation was necessary at the 1607-B2 waste site without further investigation based on the confirmatory sampling results for the influent feeder pipelines.

The following subsections provide additional discussion of the information used to develop the confirmatory sampling design. The results of confirmatory sampling are also summarized to provide support for the no action determination for area 3 and development of the remedial action strategies and verification sample designs for the remaining pipelines.

\section{Contaminants of Potential Concern for Confirmatory Sampling}

The COPCs for confirmatory sampling at the 100-B-14:2 subsite were identified based on existing analytical data and process knowledge of the facilities serviced by the sewer system. The COPC list included metals, polychlorinated biphenyls (PCBs), semivolatile organic compounds (SVOCs), hexavalent chromium, and chlorinated pesticides. Additionally, tritium was identified as a COPC for area 1, because those pipelines formerly provided service to the 108-B Tritium Separation Facility; and tritium and carbon-14 were identified as COPCs for area 5, because those pipelines formerly provided service to the 115-B Gas Recirculation Facility (BHI 2003c).

\section{Confirmatory Sample Design}

Historical information, site construction drawings, process knowledge, and site visit observations were used to develop a confirmatory sampling design with focused sampling in consideration of access and the likely worst case for potential residual contamination, as described below. 
Figure 1. Location of the 1607-B2 and 100-B-14:2 Waste Sites.

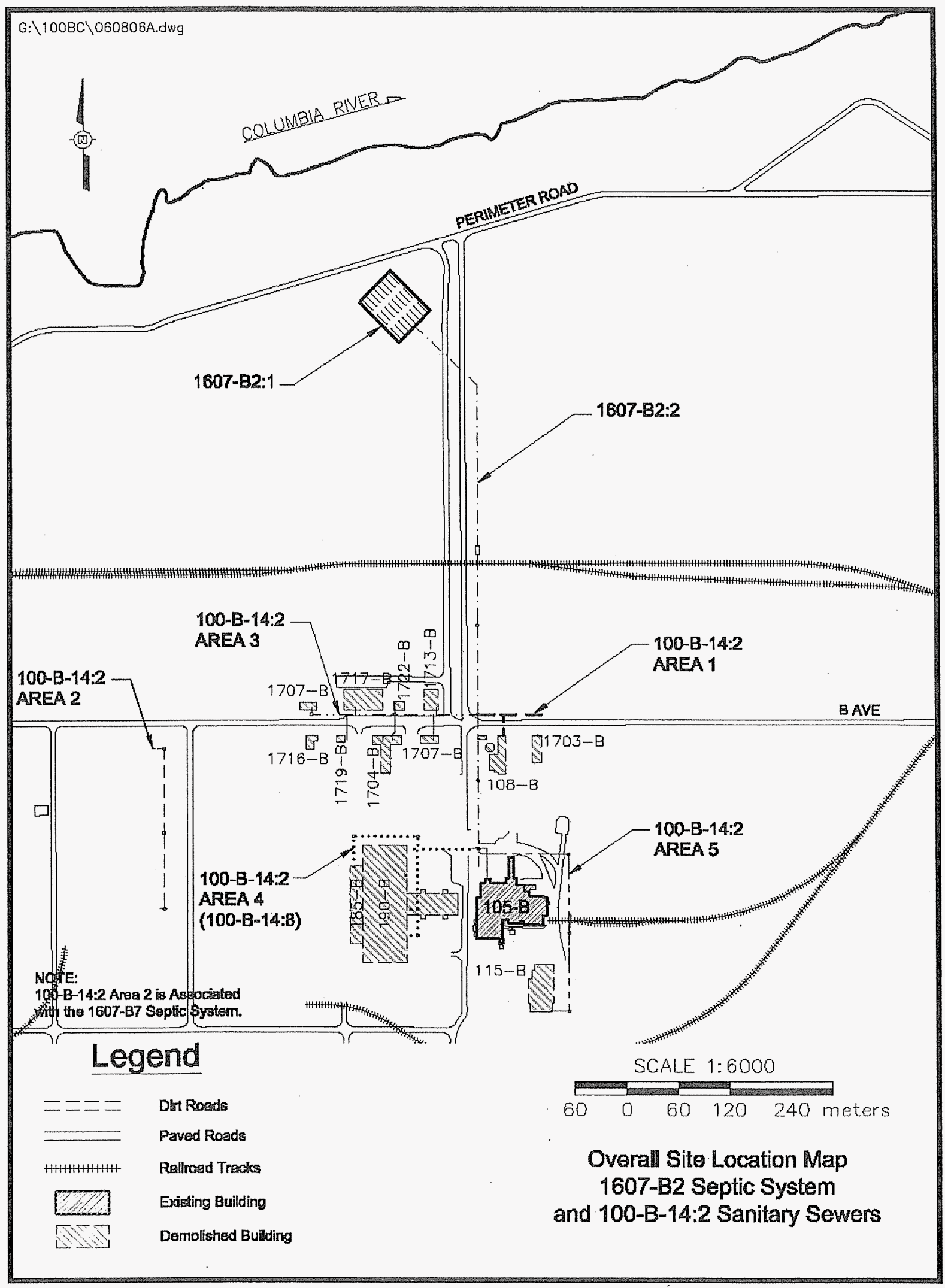


100-B-14:2 (Area 2), 1607-B7 Sanitary Sewer Pipeline

The confirmatory sample design for the 1607-B7 sanitary sewer pipelines identified two manholes (A1 and A2) and the exposed end of the piping at the boundary of the 1607-B7 septic system remediation footprint (A3/A4) as sampling locations. No accessible sediment was identified at location A1, and an alternate location approximately $50 \mathrm{~m}$ (160 ft) south was selected. The remaining samples were collected at the locations identified in the sample design. Sampling locations are shown in Figure 2; a summary of the samples collected and laboratory analyses performed is provided in Table 1 .

100-B-14:2 (Area 3), Miscellaneous 1700-Series Buildings Sanitary Sewer Pipelines

Confirmatory sampling for area 3 of the 100-B-14:2 subsite consisted of one sample of pipe sediment collected directly upstream (west) of the former discharge to the 1607-B2:2 pipeline (B1), and one sample from underlying soil at that location (B2). Sampling locations are shown in Figure 2; a summary of the samples collected and laboratory analyses performed is provided in Table 1.

100-B-14:2 (Area 4), 190-B Pumphouse Sanitary Sewer Pipelines

Confirmatory sampling for the 190-B Pumphouse sanitary sewers consisted of samples of sewer contents and underlying soils at two manholes (A9/A10 and A11/A12). Sampling locations are shown in Figure 2; a summary of the samples collected and laboratory analyses performed is provided in Table 1.

100-B-14:2 (Area 5), 115-B/C Gas Recirculation Facility Sanitary Sewer Pipelines

The confirmatory sample design for the $115-\mathrm{B} / \mathrm{C}$ Gas Recirculation Facility sanitary sewer pipelines called for collection of samples from sewer contents and underlying soils at a manhole (A13/A14) and at a pipeline location exposed by remediation of the underlying 100-B-8:1 pipeline (A15/A16). A sample was collected from within the manhole, but soils underlying the pipeline were sampled approximately $13.5 \mathrm{~m}(44 \mathrm{ft})$ west of the manhole due to access considerations. The exposed section of pipeline identified by the sampling design was inaccessible; therefore, samples were collected at the exposed pipeline at the opposite side of the 100-B-8:1 pipeline excavation (approximately $50 \mathrm{~m}$ [164 ft] north of the planned location. Sampling locations are shown in Figure 2; a summary of the samples collected and laboratory analyses performed is provided in Table 1 . 
Figure 2. Confirmatory Sampling Locations at the 100-B-14:2 Subsite.

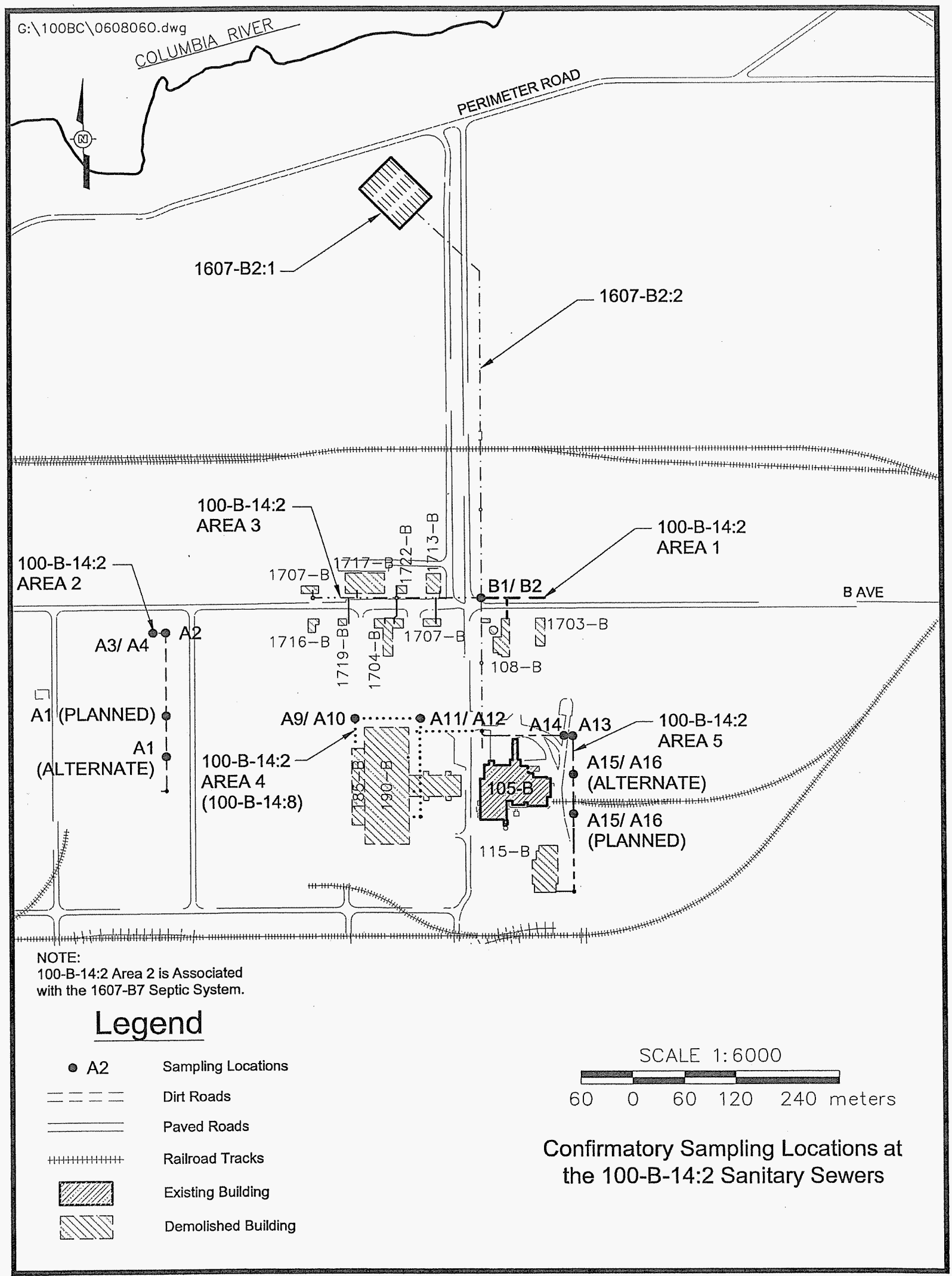


Table 1. Confirmatory Sample Summary for the 100-B-14:2 Subsite. (3 Pages)

\begin{tabular}{|c|c|c|c|c|c|}
\hline $\begin{array}{l}\text { Sample } \\
\text { Location }\end{array}$ & Sample Media & $\begin{array}{l}\text { Sample } \\
\text { Number }\end{array}$ & $\begin{array}{l}\text { Coordinate } \\
\text { Locations }\end{array}$ & $\begin{array}{l}\text { Depth } \\
\text { (Field estimate, } \\
\text { bgs) }\end{array}$ & Sample Analyses \\
\hline \multicolumn{6}{|c|}{ 100-B-14:2 Area 2} \\
\hline \multirow[t]{2}{*}{$\begin{array}{c}\mathrm{Al} \\
\left(\text { alternate }^{\mathrm{a}}\right)\end{array}$} & \multirow[t]{2}{*}{$\begin{array}{l}\text { Sediment in } \\
\text { manhole }\end{array}$} & J00Y63 & \multirow[t]{2}{*}{$\begin{array}{l}\text { N } 144551.1 \\
\text { E } 564887.4\end{array}$} & \multirow[t]{2}{*}{$2.4 \mathrm{~m}(8 \mathrm{ft})$} & $\begin{array}{l}\text { ICP metals, mercury, PCB, } \\
\text { pesticide, SVOA, GEA, gross } \\
\text { alpha, and gross beta }\end{array}$ \\
\hline & & J00Y67 & & & Hexavalent chromium \\
\hline \multirow[t]{2}{*}{$\mathrm{A} 2$} & \multirow{2}{*}{$\begin{array}{l}\text { Sediment and } \\
\text { debris in } \\
\text { manhole }\end{array}$} & J00Y64 & \multirow[t]{2}{*}{$\begin{array}{l}\text { N } 144699.0 \\
\text { E } 564887.1\end{array}$} & \multirow[t]{2}{*}{$2.4 \mathrm{~m}(8 \mathrm{ft})$} & $\begin{array}{l}\text { ICP metals, mercury, PCB, } \\
\text { pesticide, SVOA, GEA, gross } \\
\text { alpha, and gross beta }\end{array}$ \\
\hline & & J00Y68 & & & Hexavalent chromium \\
\hline \multirow[t]{2}{*}{ A3 } & \multirow[t]{2}{*}{ Pipe debris } & J00Y65 & \multirow[t]{2}{*}{$\begin{array}{l}\text { N } 144699.2 \\
\text { E } 564855.7\end{array}$} & \multirow[t]{2}{*}{$2.4 \mathrm{~m}(8 \mathrm{ft})$} & $\begin{array}{l}\text { ICP metals, mercury, PCB, } \\
\text { pesticide, SVOA, GEA, gross } \\
\text { alpha, and gross beta }\end{array}$ \\
\hline & & J00Y69 & & & Hexavalent chromium \\
\hline A4 & $\begin{array}{l}\text { Soil underlying } \\
\text { pipeline }\end{array}$ & J00Y70 & $\begin{array}{l}\text { N } 144699.2 \\
\text { E } 564855.7\end{array}$ & $2.4 \mathrm{~m}(8 \mathrm{ft})$ & $\begin{array}{l}\text { ICP metals, mercury, } \\
\text { hexavalent chromium, PCB, } \\
\text { pesticide, SVOA, GEA, gross } \\
\text { alpha, and gross beta }\end{array}$ \\
\hline $\begin{array}{l}\text { Duplicate of } \\
\text { J00Y70 }\end{array}$ & $\begin{array}{l}\text { Soil underlying } \\
\text { pipeline }\end{array}$ & J00Y71 & $\begin{array}{l}\text { N } 144699.2 \\
\text { E } 564855.7\end{array}$ & $2.4 \mathrm{~m}(8 \mathrm{ft})$ & $\begin{array}{l}\text { ICP metals, mercury, } \\
\text { hexavalent chromium, PCB, } \\
\text { pesticide, SVOA, GEA, gross } \\
\text { alpha, and gross beta }\end{array}$ \\
\hline $\begin{array}{l}\text { Equipment } \\
\text { blank }\end{array}$ & Silica sand & J00Y72 & N/A & N/A & $\begin{array}{l}\text { ICP metals, mercury, } \\
\text { hexavalent chromium, PCB, } \\
\text { pesticide, and SVOA }\end{array}$ \\
\hline \multicolumn{6}{|c|}{ 100-B-14:2 Area 3} \\
\hline \multirow[t]{2}{*}{ B1 } & \multirow[t]{2}{*}{$\begin{array}{l}\text { Sediment in } \\
\text { pipeline }\end{array}$} & J037M8 & \multirow[t]{2}{*}{$\begin{array}{l}\text { N } 144739.8 \\
\quad \text { E } 565255.4\end{array}$} & \multirow[t]{2}{*}{$3.4 \mathrm{~m}(11 \mathrm{ft})$} & $\begin{array}{l}\text { ICP metals, mercury, PCB, } \\
\text { pesticide, herbicide, SVOA, } \\
\text { GEA, gross alpha, gross beta, } \\
\text { ICP metals (TCLP), mercury } \\
\text { (TCLP), PCB (TCLP), and } \\
\text { SVOA (TCLP) }\end{array}$ \\
\hline & & J037M7 & & & Hexavalent chromium \\
\hline B2 & $\begin{array}{l}\text { Soil underlying } \\
\text { pipeline }\end{array}$ & J037M6 & $\begin{array}{l}\text { N } 144739.8 \\
\text { E } 565255.4\end{array}$ & $3.7 \mathrm{~m}(12 \mathrm{ft})$ & $\begin{array}{l}\text { ICP metals, mercury, } \\
\text { hexavalent chromium, PCB, } \\
\text { pesticide, herbicide, SVOA, } \\
\text { GEA, gross alpha, gross beta, } \\
\text { ICP metals (TCLP), mercury } \\
\text { (TCLP), PCB (TCLP), and } \\
\text { SVOA (TCLP) }\end{array}$ \\
\hline
\end{tabular}


Table 1. Confirmatory Sample Summary for the 100-B-14:2 Subsite. (3 Pages)

\begin{tabular}{|c|c|c|c|c|c|}
\hline $\begin{array}{l}\text { Sample } \\
\text { Location }\end{array}$ & Sample Media & $\begin{array}{l}\text { Sample } \\
\text { Number }\end{array}$ & $\begin{array}{l}\text { Coordinate } \\
\text { Locations }\end{array}$ & $\begin{array}{l}\text { Depth } \\
\text { (Field estimate, } \\
\text { bgs) }\end{array}$ & Sample Analyses \\
\hline \multicolumn{6}{|c|}{ 100-B-14:2 Area 4} \\
\hline \multirow[t]{2}{*}{ A9 } & \multirow{2}{*}{$\begin{array}{l}\text { Sediment and } \\
\text { debris in } \\
\text { manhole }\end{array}$} & J00Y74 & \multirow[t]{2}{*}{$\begin{array}{l}\text { N } 565108.3 \\
\text { E } 144595.9\end{array}$} & \multirow[t]{2}{*}{ Not recorded } & $\begin{array}{l}\text { ICP metals, mercury, PCB, } \\
\text { pesticide, SVOA, GEA, gross } \\
\text { alpha, and gross beta }\end{array}$ \\
\hline & & $\mathrm{J} 00 \mathrm{Y7} 76$ & & & Hexavalent chromium \\
\hline \multirow[b]{2}{*}{ A10 } & \multirow{2}{*}{$\begin{array}{l}\text { Soil underlying } \\
\text { manhole }\end{array}$} & J00YV2 & \multirow{2}{*}{$\begin{array}{l}\text { N } 565108.3 \\
\text { E } 144595.9\end{array}$} & \multirow[b]{2}{*}{ Not recorded } & $\begin{array}{l}\text { GEA, gross alpha, and gross } \\
\text { beta }\end{array}$ \\
\hline & & J00YV6 & & & $\begin{array}{l}\text { ICP metals, mercury, } \\
\text { hexavalent chromium, and } \\
\text { SVOA }\end{array}$ \\
\hline \multirow[t]{2}{*}{ A11 } & \multirow[t]{2}{*}{$\begin{array}{l}\text { Sediment and } \\
\text { debris in } \\
\text { manhole }\end{array}$} & J00Y75 & \multirow[t]{2}{*}{$\begin{array}{l}\text { N } 565186.0 \\
\text { E } 144596.2\end{array}$} & \multirow[t]{2}{*}{ Not recorded } & $\begin{array}{l}\text { ICP metals, mercury, PCB, } \\
\text { pesticide, SVOA, GEA, gross } \\
\text { alpha, and gross beta }\end{array}$ \\
\hline & & J00Y77 & & & Hexavalent chromium \\
\hline \multirow[b]{2}{*}{ A12 } & \multirow{2}{*}{$\begin{array}{l}\text { Soil underlying } \\
\text { manhole }\end{array}$} & J00YV1 & \multirow{2}{*}{$\begin{array}{l}\text { N } 565186.0 \\
\text { E } 144596.2\end{array}$} & \multirow[b]{2}{*}{ Not recorded } & $\begin{array}{l}\text { GEA, gross alpha, and gross } \\
\text { beta }\end{array}$ \\
\hline & & J00YV5 & & & $\begin{array}{l}\text { ICP metals, mercury, } \\
\text { hexavalent chromium, and } \\
\text { SVOA }\end{array}$ \\
\hline $\begin{array}{l}\text { Duplicate of } \\
\text { J00YV2 }\end{array}$ & $\begin{array}{l}\text { Sediment and } \\
\text { debris in } \\
\text { manhole }\end{array}$ & J00YV3 & $\begin{array}{l}\text { N } 565108.3 \\
\text { E } 144595.9\end{array}$ & Not recorded & $\begin{array}{l}\text { GEA, gross alpha, and gross } \\
\text { beta }\end{array}$ \\
\hline $\begin{array}{l}\text { Duplicate of } \\
\text { J00YV6 }\end{array}$ & $\begin{array}{l}\text { Sediment and } \\
\text { debris in } \\
\text { manhole }\end{array}$ & J00YV7 & $\begin{array}{l}\text { N } 565108.3 \\
\text { E } 144595.9\end{array}$ & Not recorded & $\begin{array}{l}\text { ICP metals, mercury, } \\
\text { hexavalent chromium, and } \\
\text { SVOA }\end{array}$ \\
\hline $\begin{array}{l}\text { Equipment } \\
\text { blank }\end{array}$ & Silica sand & J00YV8 & N/A & $\mathrm{N} / \mathrm{A}$ & $\begin{array}{l}\text { ICP metals, mercury, } \\
\text { hèxavalent chromium, and } \\
\text { SVOA }\end{array}$ \\
\hline \multicolumn{6}{|c|}{ 100-B-14:2 Area 5} \\
\hline \multirow[t]{2}{*}{ A13 } & \multirow[t]{2}{*}{$\begin{array}{l}\text { Sediment in } \\
\text { manhole }\end{array}$} & J00Y79 & \multirow[t]{2}{*}{$\begin{array}{l}\text { N } 144574.8 \\
\text { E } 565361.0\end{array}$} & \multirow[t]{2}{*}{$3.7 \mathrm{~m}(12 \mathrm{ft})$} & $\begin{array}{l}\text { PCB, pesticide, SVOA, ICP } \\
\text { metals (TCLP), mercury } \\
\text { (TCLP), GEA, gross alpha, } \\
\text { gross beta, tritium, and } \\
\text { carbon-14 }\end{array}$ \\
\hline & & J00Y81 & & & Hexavalent chromium \\
\hline A14 & $\begin{array}{l}\text { Soil underlying } \\
\text { pipeline }\end{array}$ & J00Y83 & $\begin{array}{l}\text { N } 144574.8 \\
\text { E } 565347.3\end{array}$ & $3.7 \mathrm{~m}(12 \mathrm{ft})$ & $\begin{array}{l}\text { ICP metals, mercury, } \\
\text { hexavalent chromium, PCB, } \\
\text { pesticide, SVOA, GEA, gross } \\
\text { alpha, gross beta, tritium, and } \\
\text { carbon-14 }\end{array}$ \\
\hline
\end{tabular}


Table 1. Confirmatory Sample Summary for the 100-B-14:2 Subsite. (3 Pages)

\begin{tabular}{|c|c|c|c|c|c|}
\hline $\begin{array}{c}\text { Sample } \\
\text { Location }\end{array}$ & Sample Media & $\begin{array}{l}\text { Sample } \\
\text { Number }\end{array}$ & $\begin{array}{l}\text { Coordinate } \\
\text { Locations }\end{array}$ & $\begin{array}{c}\text { Depth } \\
\text { (Field estimate, } \\
\text { bgs) }\end{array}$ & Sample Analyses \\
\hline \multirow[t]{2}{*}{$\begin{array}{c}\mathrm{A} 15 \\
\text { (alternate }\end{array}$} & \multirow[t]{2}{*}{ Pipe debris } & J00Y80 & \multirow[t]{2}{*}{$\begin{array}{l}\text { N } 144528.7 \\
\text { E } 565361.6\end{array}$} & \multirow[t]{2}{*}{$3.7 \mathrm{~m}(12 \mathrm{ft})$} & $\begin{array}{l}\text { PCB, pesticide, SVOA, ICP } \\
\text { metals (TCLP), mercury } \\
\text { (TCLP), GEA, gross alpha, } \\
\text { gross beta, tritium, and } \\
\text { carbon-14 }\end{array}$ \\
\hline & & J00Y82 & & & Hexavalent chromium \\
\hline $\begin{array}{c}\mathrm{A} 16 \\
\text { (alternate }^{\mathrm{b}} \text { ) }\end{array}$ & $\begin{array}{l}\text { Soil underlying } \\
\text { pipeline }\end{array}$ & J00Y84 & $\begin{array}{l}\text { N } 144528.7 \\
\text { E } 565361.6\end{array}$ & $3.7 \mathrm{~m}(12 \mathrm{ft})$ & $\begin{array}{l}\text { ICP metals, mercury, } \\
\text { hexavalent chromium, PCB, } \\
\text { pesticide, SVOA, GEA, gross } \\
\text { alpha, gross beta, tritium, } \\
\text { carbon-14, and total beta } \\
\text { radiostrontium }\end{array}$ \\
\hline $\begin{array}{l}\text { Duplicate of } \\
\text { J00Y84 }\end{array}$ & $\begin{array}{l}\text { Soil underlying } \\
\text { pipeline }\end{array}$ & J00Y85 & $\begin{array}{l}\text { N } 144528.7 \\
\text { E } 565361.6\end{array}$ & $3.7 \mathrm{~m}(12 \mathrm{ft})$ & $\begin{array}{l}\text { ICP metals, mercury, } \\
\text { hexavalent chromium, PCB, } \\
\text { pesticide, SVOA, GEA, gross } \\
\text { alpha, gross beta, tritium, } \\
\text { carbon-14, and total beta } \\
\text { radiostrontium }\end{array}$ \\
\hline $\begin{array}{l}\text { Equipment } \\
\text { blank }\end{array}$ & Silica sand & J00YF5 & N/A & N/A & $\begin{array}{l}\text { ICP metals, mercury, PCB, } \\
\text { pesticide, herbicide and } \\
\text { SVOA }\end{array}$ \\
\hline
\end{tabular}

Sources: Remaining Sites Field Sampling, Logbook EL-1578-1 (BHI 2003b) and 100BC Remaining Pipeline and Sewers Sampling and Field Services, Logbook EL-1585-2 (BHI 2005b)

a No sample material was present at the planned location; alternate location sampled.

b Planned location was inaccessible; alternate location sampled.

bgs = below ground surface

GEA = gamma energy analysis

ICP $=$ inductively coupled plasma

N/A = not applicable

$\mathrm{PCB}=$ polychlorinated biphenyl

SVOA = semivolatile organic analysis

TCLP $=$ toxicity characteristic leaching procedure

\section{Confirmatory Sample Results}

Confirmatory samples were analyzed using analytical methods approved by the U.S. Environmental Protection Agency (EPA) (DOE-RL 2005a), and the results were compared to the cleanup criteria specified in the RDR/RAWP (DOE-RL 2005b). The laboratory results were stored in the Environmental Restoration (ENRE) project-specific database prior to being provided to the Hanford Environmental Information System (HEIS) and are provided in Appendix A as well as summarized below.

\section{0-B-14:2 (Area 2), 1607-B7 Sanitary Sewer Pipeline}

Aroclor-1254 was detected in the sample of manhole sediments at a concentration ( $49 \mathrm{mg} / \mathrm{kg}$ ) exceeding the direct exposure remedial action goal (RAG). Based on this exceedance, it was 
determined that remedial action was necessary at the site (Feist 2004), with aroclor-1254 retained as a COC. Multiple metals (including hexavalent chromium), polycyclic aromatic hydrocarbons (PAHs), and chlorinated pesticides were also detected above RAGs in the sample of manhole sediments and retained as site COCs/COPCs.

\section{0-B-14:2 (Area 3), Miscellaneous 1700-Series Buildings Sanitary Sewer Pipelines}

A comparison of the maximum concentrations of detected analytes in area 3 of the 100-B-14:2 subsite and the site RAGs is summarized in Table 2 (due to the large number of comparison tables in this document, all comparison table footnotes are provided at the end of the document body to reduce redundancy). Contaminants that were not detected by laboratory analysis are excluded from Table 2. Potassium-40, radium-226, radium-228, thorium-228, and thorium-232 were detected in samples but are not considered in Table 2 because these isotopes are not related to the operational history of the site and were all detected at levels below statistical background activities. (Based on an assumption of secular equilibrium, the background activities for radium228 and thorium-228 are equal to the statistical background activity of $1.32 \mathrm{pCi} / \mathrm{g}$ for thorium-232 [DOE-RL 1996]). Calculated cleanup levels are not presented in the Cleanup Levels and Risk Calculations (CLARC) Database (Ecology 2005) under WAC 173-340-740(3) for aluminum, calcium, iron, magnesium, phosphate, potassium, silicon, sodium, and zirconium (results for total phosphorus are attributed to phosphorus in phosphate). These analytes are also essential nutrients and can be eliminated from evaluation as human health concerns per EPA guidance (EPA 1989). Therefore, these constituents are not considered COPCs. The laboratoryreported confirmatory data results for all constituents are provided in Appendix A (Table A-2).

Residual concentrations of aroclor-1248 and dieldrin were detected slightly above their respective direct exposure RAGs in the pipe sediment sample. However, because of the small footprint of the 100-B-14:2 (area 3) pipelines, site-specific risk assessments were performed using Hanford Site Risk Assessment Methodology (DOE-RL 1995), incorporating area and occupancy correction factors used in RESRAD software (ANL 2005). Based on these assessments (Appendix B), residual concentrations of aroclor-1248 and dieldrin individually pose excess carcinogenic risks less than $1 \times 10^{-6}$, and thus satisfy RAOs. All other site COPCs were detected below direct exposure RAGs.

\section{Protection of Groundwater and/or the Columbia River}

A contaminant depth/soil-partitioning coefficient $\left(\mathrm{K}_{\mathrm{d}}\right)$ value model has been developed to predict if the concentrations of contaminants in soil that exceed cleanup levels for groundwater or river protection are protective of groundwater and the river at a site. The 100 Area Analogous Sites RESRAD Calculations calculation brief (included in this RSVP as Appendix D) predicts whether or not contaminants in 100 Area soils are expected to migrate to groundwater within a 1,000 year time frame based on their $\mathrm{K}_{d}$ value and the vertical distance to groundwater. The contaminant $\mathrm{depth} / \mathrm{K}_{\mathrm{d}}$ value model assumes that uncontaminated soil exists in the vadose zone between the bottom of the waste site and groundwater. The assumption of an uncontaminated zone beneath the waste site is reasonable based on analogous site data that includes test pits and boreholes completed at several operable units in the 100 Area, including the 100-B/C Area. The test pit and/or borehole data show that contaminant concentrations that are below direct exposure 
Table 2. Comparison of Maximum Detected Contaminant Concentrations to Action Levels for the 100-B-14:2 (Area 3) Confirmatory Sampling Event." (3 Pages)

\begin{tabular}{|c|c|c|c|c|c|c|c|c|}
\hline \multirow[b]{2}{*}{ COPC } & \multirow[b]{2}{*}{$\begin{array}{c}\text { Pipe } \\
\text { Sediment } \\
\text { Analytical } \\
\text { Result } \\
(\text { pCi/g) }\end{array}$} & \multirow[b]{2}{*}{$\begin{array}{l}\text { Underlying } \\
\text { Soil } \\
\text { Analytical } \\
\text { Result } \\
\text { (pCi/g) }\end{array}$} & \multicolumn{3}{|c|}{ Generic Site Lookup Values ${ }^{\mathrm{a}}$ (pCi/g) } & \multirow[b]{2}{*}{$\begin{array}{l}\text { Does the Pipe } \\
\text { Sediment } \\
\text { Result Exceed } \\
\text { Lookup } \\
\text { Values? }\end{array}$} & \multirow[b]{2}{*}{$\begin{array}{l}\text { Does the Pipe } \\
\text { Sediment } \\
\text { Result Pass } \\
\text { Site-Specific } \\
\text { Modeling? }\end{array}$} & \multirow{2}{*}{$\begin{array}{l}\text { Does the } \\
\text { Underlying } \\
\text { Soil Result } \\
\text { Exceed } \\
\text { Loolup } \\
\text { Values? }\end{array}$} \\
\hline & & & $\begin{array}{l}\text { Shallow } \\
\text { Zone } \\
\text { Lookup } \\
\text { Value }^{b}\end{array}$ & $\begin{array}{c}\text { Groundwater } \\
\text { Protection } \\
\text { Lookup } \\
\text { Value }\end{array}$ & $\begin{array}{c}\text { River } \\
\text { Protection } \\
\text { Lookup } \\
\text { Value }\end{array}$ & & & \\
\hline Cesium-137 & 0.177 & ND & 6.2 & $1,465^{\mathrm{c}}$ & $1,465^{\mathrm{c}}$ & No & -- & - \\
\hline \multirow[b]{2}{*}{ COPC } & \multirow{2}{*}{$\begin{array}{c}\text { Pipe } \\
\text { Sediment } \\
\text { Analytical } \\
\text { Result } \\
(\mathrm{mg} / \mathrm{kg})\end{array}$} & \multirow{2}{*}{$\begin{array}{l}\text { Underlying } \\
\text { Soil } \\
\text { Analytical } \\
\text { Result } \\
(\mathrm{mg} / \mathrm{kg})\end{array}$} & \multicolumn{3}{|c|}{ Remedial Action Goals ${ }^{\mathrm{a}}$ (mg/kg) } & \multirow[b]{2}{*}{$\begin{array}{l}\text { Does the Pipe } \\
\text { Sediment } \\
\text { Result Exceed } \\
\text { RAGs? }\end{array}$} & \multirow[b]{2}{*}{$\begin{array}{l}\text { Does the Pipe } \\
\text { Sediment } \\
\text { Result Pass } \\
\text { Site-Specific } \\
\text { Modeling? }\end{array}$} & \multirow[b]{2}{*}{$\begin{array}{l}\text { Does the } \\
\text { Underlying } \\
\text { Soil Result } \\
\text { Exceed RAGs? }\end{array}$} \\
\hline & & & $\begin{array}{c}\text { Direct } \\
\text { Exposure }\end{array}$ & $\begin{array}{l}\text { Soil Cleanup } \\
\text { Level for } \\
\text { Groundwater } \\
\text { Protection }\end{array}$ & $\begin{array}{l}\text { Soil Cleanup } \\
\text { Level for } \\
\text { River } \\
\text { Protection }\end{array}$ & & & \\
\hline Arsenic & 7.7 & $4.1(<\mathrm{BG})$ & 20 & 20 & 20 & No & -- & No \\
\hline Barium & 998 & $73.1(<B G)$ & $5,600^{\mathrm{d}}$ & $132^{\mathrm{e}, \mathrm{f}}$ & $224^{\mathrm{g}}$ & Yes & $\mathrm{Yes}^{\mathrm{h}}$ & No \\
\hline Beryllium & $0.81(<\mathrm{BG})$ & $0.58(<\mathrm{BG})$ & $10.4^{\mathrm{i}}$ & $1.51^{\mathrm{f}}$ & $1.51^{\mathrm{f}}$ & No & -- & No \\
\hline Boron $^{j}$ & 4.4 & 3.0 & 16,000 & 320 & $--{ }^{k}$ & No & - & No \\
\hline Cadmium $^{1}$ & 1.5 & $0.24(<\mathrm{BG})$ & 13.9 & $0.81^{f}$ & $0.81^{\text {f }}$ & Yes & Yes $^{h}$ & No \\
\hline Chromium (total) & 67.8 & $9.4(<B G)$ & $80,000^{\mathrm{d}}$ & $18.5^{\mathrm{f}}$ & $18.5^{\mathrm{f}}$ & Yes & Yes $^{\mathrm{h}}$ & No \\
\hline Chromium (hexavalent) & 0.25 & ND & 2.1 & $4.8^{\mathrm{m}}$ & 2 & No & -- & -- \\
\hline Cobalt & $5.3(<\mathrm{BG})$ & $7.9(<B G)$ & 1,600 & 32 & $--^{k}$ & No & -- & No \\
\hline Copper & 102 & $16.5(<\mathrm{BG})$ & 2,960 & 59.2 & $22.0^{\mathrm{f}}$ & Yes & $\mathrm{Yes}^{\mathrm{h}}$ & No \\
\hline Lead & 279 & $8.9(<\mathrm{BG})$ & 353 & $10.2^{\mathrm{f}}$ & $10.2^{f}$ & Yes & Yes $^{\mathrm{h}}$ & No \\
\hline Lithium & $6.1(<\mathrm{BG})$ & $6.4(<\mathrm{BG})$ & 1,600 & $33.5^{f}$ & $--\mathrm{k}$ & No & -- & No \\
\hline Manganese & $166(<\mathrm{BG})$ & $347(<\mathrm{BG})$ & 11,200 & $512^{f}$ & $512^{f}$ & No & - & No \\
\hline Mercury & 7.2 & $0.04(<\mathrm{BG})$ & 24 & $0.33^{\mathrm{f}}$ & $0.33^{\mathrm{f}}$ & Yes & Yes $^{h}$ & No \\
\hline Molybdenum ${ }^{j}$ & 1.7 & ND & 400 & 8 & $-{ }_{-}^{k}$ & No & -- & -- \\
\hline Nickel & 34.0 & $12.5(<\mathrm{BG})$ & 1,600 & $19.1^{\mathrm{f}}$ & 27.4 & Yes & $\mathrm{Yes}^{\mathrm{h}}$ & $\mathrm{No}$ \\
\hline Silver & 2.8 & ND & 400 & 8 & $0.73^{f}$ & Yes & Yes $^{\mathrm{h}}$ & - \\
\hline Strontium $^{\mathrm{j}}$ & 40.4 & 34.2 & 48,000 & 960 & $-{ }_{-}^{k}$ & No & -- & No \\
\hline
\end{tabular}




\begin{tabular}{|c|c|c|c|c|c|c|c|c|}
\hline \multirow[b]{2}{*}{ COPC } & \multirow[b]{2}{*}{$\begin{array}{c}\text { Pipe } \\
\text { Sediment } \\
\text { Analytical } \\
\text { Result } \\
(\mathbf{m g} / \mathrm{kg})\end{array}$} & \multirow{2}{*}{$\begin{array}{l}\text { Underlying } \\
\text { Soil } \\
\text { Analytical } \\
\text { Result } \\
(\mathrm{mg} / \mathrm{kg})\end{array}$} & \multicolumn{3}{|c|}{ Remedial Action Goals ${ }^{\mathrm{a}}$ (mg/kg) } & \multirow[b]{2}{*}{$\begin{array}{l}\text { Does the Pipe } \\
\text { Sediment } \\
\text { Result Exceed } \\
\text { RAGs? }\end{array}$} & \multirow[b]{2}{*}{$\begin{array}{l}\text { Does the Pipe } \\
\text { Sediment } \\
\text { Result Pass } \\
\text { Site-Specific } \\
\text { Modeling? }\end{array}$} & \multirow[b]{2}{*}{$\begin{array}{l}\text { Does the } \\
\text { Underlying } \\
\text { Soil Result } \\
\text { Exceed RAGs? }\end{array}$} \\
\hline & & & $\begin{array}{c}\text { Direct } \\
\text { Exposure }\end{array}$ & $\begin{array}{c}\text { Soil Cleanup } \\
\text { Level for } \\
\text { Groundwater } \\
\text { Protection } \\
\end{array}$ & $\begin{array}{l}\text { Soil Cleanup } \\
\text { Level for } \\
\text { River } \\
\text { Protection } \\
\end{array}$ & & & \\
\hline $\operatorname{Tin}^{j}$ & 13.5 & ND & 48,000 & 960 & $--^{\mathrm{k}}$ & No & -- & -- \\
\hline Titanium & $1,080(<\mathrm{BG})$ & $1,450(<\mathrm{BG})$ & $320,000^{\mathrm{n}}$ & $6,400^{n}$ & $-{ }^{k}$ & No & - & No \\
\hline Vanadium & $40.9(<\mathrm{BG})$ & $47.8(<B G)$ & 560 & $85.1^{\mathrm{f}}$ & $-{ }^{k}$ & No & -- & No \\
\hline Zinc & 223 & $50.8(<\mathrm{BG})$ & 24,000 & 480 & $67.8^{\mathrm{f}}$ & Yes & Yes $^{\mathrm{h}}$ & No \\
\hline Aroclor-1248 & 1.1 & ND & 0.5 & $0.017^{\circ}$ & $0.017^{\circ}$ & Yes & Yes $^{\mathrm{h}, \mathrm{p}}$ & -- \\
\hline Aroclor-1260 & 0.28 & ND & 0.5 & $0.017^{\circ}$ & $0.017^{\circ}$ & Yes & Yes $^{\mathrm{h}}$ & -- \\
\hline beta-BHC & 0.0040 & ND & 0.556 & 0.00486 & 0.00554 & No & -- & -- \\
\hline 4,4'-DDD & 0.023 & $\mathrm{ND}$ & 4.17 & 0.0365 & $0.005^{\circ}$ & Yes & Yes $^{\mathrm{h}}$ & - \\
\hline 4,4'-DDE & 0.037 & ND & 2.94 & 0.0257 & $0.005^{\circ}$ & Yes & Yes $^{\mathrm{h}}$ & -- \\
\hline 4,4'-DDT & 0.031 & $\mathrm{ND}$ & 2.94 & 0.0257 & $0.005^{\circ}$ & Yes & Yes $^{\mathrm{h}}$ & -- \\
\hline Dieldrin & 0.084 & ND & 0.0625 & $0.003^{\circ}$ & $0.003^{\circ}$ & Yes. & Yes & -- \\
\hline Endosulfan I & 0.0046 & ND & 480 & 9.6 & 0.186 & No & -- & -- \\
\hline Endosulfan sulfate & 0.0036 & ND & 480 & 9.6 & 0.186 & No & -- & -- \\
\hline Endrin aldehyde & 0.011 & ND & 24 & 0.2 & 0.039 & No & -- & - \\
\hline gamma-Chlordane & 0.022 & ND & $2.86^{\mathrm{q}}$ & $0.025^{q}$ & $0.0165^{\circ}$ & Yes & Yes $^{\mathrm{h}}$ & - \\
\hline Methoxychlor & 0.032 & ND & 400 & 4 & 1.67 & No & -- & - \\
\hline Benzo(a)anthracene & 0.21 & ND & $1.37^{\mathrm{r}}$ & $0.33^{\circ}$ & $0.33^{\circ}$ & No & -- & - \\
\hline Benzo(a)pyrene & 0.18 & ND & $0.33^{\circ}$ & $0.33^{\circ}$ & $0.33^{\circ}$ & No & - & -- \\
\hline Benzo(b)fluoranthene & 0.18 & ND & $1.37^{\mathrm{r}}$ & $0.33^{\circ}$ & $0.33^{\circ}$ & No & -- & -- \\
\hline Benzo(g,h,i)perylene ${ }^{\mathrm{s}}$ & 0.13 & $\mathrm{ND}$ & 2,400 & 48 & 192 & No & -- & -- \\
\hline Benzo(k)fluoranthene & 0.18 & $\mathrm{ND}$ & $13.7^{\mathrm{r}}$ & $0.33^{\circ}$ & $0.33^{\circ}$ & No & -- & -- \\
\hline bis(2-Ethylhexyl)phthalate & 1.1 & 0.30 & 71.4 & 0.625 & 0.36 & Yes & Yes $^{\mathrm{h}}$ & No \\
\hline
\end{tabular}


Table 2. Comparison of Maximum Detected Contaminant Concentrations to Action Levels for the 100-B-14:2 (Area 3) Confirmatory Sampling Event.* (3 Pages)

\begin{tabular}{|c|c|c|c|c|c|c|c|c|}
\hline \multirow[b]{2}{*}{ COPC } & \multirow{2}{*}{$\begin{array}{c}\text { Pipe } \\
\text { Sediment } \\
\text { Analytical } \\
\text { Result } \\
(\mathrm{mg} / \mathrm{kg})\end{array}$} & \multirow{2}{*}{$\begin{array}{l}\text { Underlying } \\
\text { Soil } \\
\text { Analytical } \\
\text { Result } \\
(\mathrm{mg} / \mathrm{kg})\end{array}$} & \multicolumn{3}{|c|}{ Remedial Action Goals ${ }^{\mathrm{a}}$ (mg/kg) } & \multirow{2}{*}{$\begin{array}{l}\text { Does the Pipe } \\
\text { Sediment } \\
\text { Result Exceed } \\
\text { RAGs? }\end{array}$} & \multirow{2}{*}{$\begin{array}{l}\text { Does the Pipe } \\
\text { Sediment } \\
\text { Result Pass } \\
\text { Site-Specific } \\
\text { Modeling? }\end{array}$} & \multirow{2}{*}{$\begin{array}{c}\text { Does the } \\
\text { Underlying } \\
\text { Soil Result } \\
\text { Exceed RAGs? }\end{array}$} \\
\hline & & & $\begin{array}{c}\text { Direct } \\
\text { Exposure }\end{array}$ & $\begin{array}{l}\text { Soil Cleanup } \\
\text { Level for } \\
\text { Groundwater } \\
\text { Protection }\end{array}$ & $\begin{array}{l}\text { Soil Cleanup } \\
\text { Level for } \\
\text { River } \\
\text { Protection }\end{array}$ & & & \\
\hline Butylbenzylphthalate & 0.42 & ND & 16,000 & 320 & 250 & No & - & -- \\
\hline Chrysene & 0.24 & ND & $137^{\mathrm{r}}$ & $1.2^{\mathrm{r}}$ & $0.33^{\circ}$ & No & -- & -- \\
\hline Di-n-butylphthalate & 21 & $\mathrm{ND}$ & 8,000 & 160 & 540 & No & -- & -- \\
\hline Fluoranthene & 0.38 & $\mathrm{ND}$ & 3,200 & 64 & 18 & No & - & - \\
\hline Indeno(1,2,3-cd)pyrene & 0.12 & ND & 1.37 & $0.33^{\circ}$ & $0.33^{\circ}$ & No & -- & -- \\
\hline Phenanthrene $^{s}$ & 0.19 & ND & 24,000 & 240 & 1,920 & No & -- & -- \\
\hline Pyrene & 0.37 & $\mathrm{ND}$ & 2,400 & 48 & 192 & No & -- & -- \\
\hline
\end{tabular}

* All Table 2 notes and acronyms are provided at the end of the document body. 
cleanup levels decrease to background concentrations within less than $3 \mathrm{~m} \mathrm{(10} \mathrm{ft)} \mathrm{below} \mathrm{the}$ elevation at which the contamination occurs. For the 100-B/C Area, references BHI 1999, DOE-RL 1992, and DOE-RL 1994 discuss the thickness of the uncontaminated vadose zone beneath remediated waste sites.

To apply the contaminant depth/$/ \mathrm{K}_{d}$ value model, the thickness of the vadose zone beneath a remediated waste site must be at least $3 \mathrm{~m}(10 \mathrm{ft})$, per the penultimate sentence in the previous paragraph. Table 3 a shows the minimum $\mathrm{K}_{\mathrm{d}}$ values from Appendix $\mathrm{D}$ at which contaminants are not predicted to migrate to groundwater within 1,000 years at uncontaminated vadose zone thicknesses from $0 \mathrm{~m}$ to $25 \mathrm{~m}$. Table $3 \mathrm{~b}$ shows the $\mathrm{K}_{\mathrm{d}}$ values of contaminants in this RSVP that present groundwater protection concerns.

Table 3a. Minimum Contaminant $\mathrm{K}_{\mathbf{d}}$ Values Protective of Groundwater.

\begin{tabular}{|c|c|}
\hline $\begin{array}{c}\text { Uncontaminated Zone } \\
\text { Thickness } \\
(\mathbf{m})\end{array}$ & $\begin{array}{c}\mathbf{K}_{\mathbf{d}} \text { Value Protective } \\
\text { of Groundwater } \\
(\mathbf{m L} / \mathbf{g})\end{array}$ \\
\hline 0 & 80 \\
\hline 2 & 40 \\
\hline 2 & 27 \\
\hline 3 & 20 \\
\hline 4 & 16 \\
\hline 5 & 14 \\
\hline 6 & 12 \\
\hline 7 & 10 \\
\hline 8 & 9 \\
\hline 9 & 8 \\
\hline 10 & 8 \\
\hline 11 & 7 \\
\hline 12 & 7 \\
\hline 16 & 6 \\
\hline 18 & 6 \\
\hline 20 & 4 \\
\hline 22 & 4 \\
\hline 25 & 3 \\
\hline
\end{tabular}

$\mathrm{K}_{\mathrm{d}}=$ Distribution coefficient
Table $3 b . \mathbb{K}_{d}$ Values for Selected Contaminants.

\begin{tabular}{|l|c|}
\hline \multicolumn{1}{|c|}{ Contaminant } & $\begin{array}{c}\text { Contaminant } \\
\mathbf{K}_{\mathbf{d}} \text { Value } \\
(\mathbf{m L} / \mathbf{g})\end{array}$ \\
\hline Barium & 25 \\
\hline Cadmium & 30 \\
\hline Chromium, Total & 200 \\
\hline Copper & 22 \\
\hline Lead & 30 \\
\hline Manganese & 50 \\
\hline Mercury & 30 \\
\hline Nickel & 30 \\
\hline Silver & 90 \\
\hline Zinc & 30 \\
\hline Aldrin & 48.7 \\
\hline Aroclor-1248 & 43.9 \\
\hline Aroclor-1254 & 75.6 \\
\hline Aroclor-1260 & 530 \\
\hline BEHP & 110 \\
\hline DDD, 4,4'- & 45.8 \\
\hline DDE, 4,4'- & 86.4 \\
\hline DDT, 4,4'- & 678 \\
\hline Dieldrin & 25.6 \\
\hline Endrin aldehyde & 10.8 \\
\hline Gamma-chlordane & 51.3 \\
\hline BEHP = bis(2-ethylhexyl)phthalate \\
\hline
\end{tabular}

Barium, cadmium, chromium (total), copper, lead, mercury, nickel, silver, zinc, aroclor-1248, aroclor-1260, bis(2-ethylhexyl)phthalate, 4,4'-DDD, 4,4'-DDE, 4,4'-DDT, dieldrin, endrin aldehyde, and gamma-chlordane were detected above soil RAGs for protection of groundwater and/or the Columbia River in the pipe sediment sample. Based on the analytical results for the underlying soil sample, elevated concentrations of these COPCs are confined to the pipeline. Further, based on the contaminant depth/ $\mathrm{K}_{d}$ value model discussed above and the $\mathrm{K}_{\mathrm{d}}$ values for these constituents (the lowest of which is $10.8 \mathrm{~mL} / \mathrm{g}$ for endrin aldehyde), none are expected to 


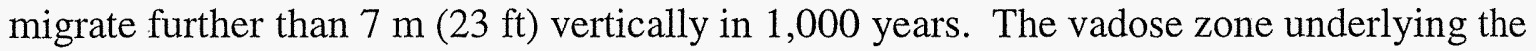
100-B-14:2 (area 3) pipelines is approximately 18-m (59-ft)-thick; therefore, residual concentrations of these constituents are protective of groundwater. The only pathway for contamination to reach the Columbia River is via groundwater migration, so these contaminant concentrations are also protective of river water.

Nonradionuclide risk requirements include an individual hazard quotient of $<1.0$, a cumulative hazard quotient of $<1.0$, an individual contaminant carcinogenic risk of $<1 \times 10^{-6}$, and a cumulative carcinogenic risk of $<1 \times 10^{-5}$. For area 3 of the 100-B-14:2 subsite, these risk values were not calculated for constituents that were either not detected or were detected at concentrations below Hanford Site or Washington State background values. All individual hazard quotients were less than 1.0, and all individual excess carcinogenic risk values were less than $1 \times 10^{-6}$ (Appendix B). The cumulative hazard quotient for those noncarcinogenic constituents above background or detection levels is $6.0 \times 10^{-1}$, and the cumulative excess carcinogenic risk value for these constituents is $2.8 \times 10^{-6}$. Therefore, nonradionuclide risk requirements are met.

When using a statistical sampling approach, a RAG requirement for nonradionuclides is the WAC 173-340-740(7)(e) three-part test. However, this test is not applicable to this focused sampling approach because maximum detected concentration data are used as the compliance basis.

\section{0-B-14:2 (Area 4), 190-B Pumphouse Sanitary Sewer Pipelines}

Aroclor-1254 was detected in one sample of manhole sediments at a concentration $(2.6 \mathrm{mg} / \mathrm{kg})$ exceeding the direct exposure RAG. Based on this exceedance, it was determined that remedial action was necessary at the site (Feist 2004), with aroclor-1254 retained as a COC. Multiple metals (including hexavalent chromium), PAHs, and chlorinated pesticides were also detected above RAGs in the sample of manhole sediments and retained as site COCs/COPCs.

\section{0-B-14:2 (Area 5), 115-B/C Gas Recirculation Facility Sanitary Sewer Pipelines}

Cesium-137, cobalt-60, europium-152, europium-154, and strontium-90 were detected in soil samples at activities cumulatively exceeding the direct exposure RAG for beta-/gamma-emitters. Based on this exceedance, it was determined that remedial action was necessary at the site (Feist 2004), with these radionuclides retained as COCs. Hexavalent chromium, aroclor-1260, and chlorinated pesticides were also detected above RAGs in samples of pipeline material and retained as site COCs/COPCs. Further, metals analyses for pipeline material samples were mistakenly performed by the toxicity characteristic leaching procedure rather than totals procedures; therefore, these metals were retained as site COPCs.

\section{REMEDIAL ACTION SUMMARY}

Remediation of the 100-B-14:2 and 1607-B2 waste sites was performed in stages from January 2005 through June 2006 as part of the 100-B/C Area Remaining Pipes and Sewers remediation. 
In general, remediation of pipelines consisted of the excavation and stockpiling (on-site) of overlying soils presumed to contain no residual contamination above cleanup levels (referred to as "below cleanup levels" [BCL]), removal of soils around and approximately $0.3 \mathrm{~m}(1 \mathrm{ft})$ below the pipelines, and disposal of the pipeline and suspect potentially contaminated soil at the Environmental Restoration Disposal Facility. Stockpiled BCL material was used in site backfill operations following verification that the soil did not contain site COCs/COPCs at levels exceeding cleanup criteria.

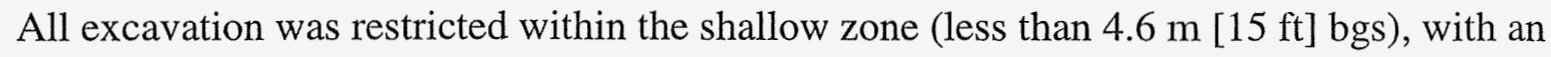

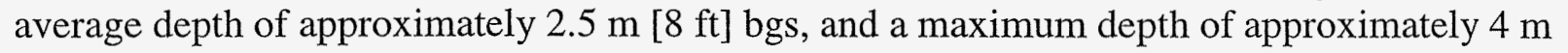
[13 ft] bgs at the septic tank. Excavation at the northern portion of the site continued to greater depth due to concurrent remediation of the underlying 100-B-14:1 process sewer. Due to colocation and concurrent remediation of differing functional pipeline groups, excavated and disposed material quantities could not be explicitly separated for the 1607-B2 and 100-B-14:2 sites. A pre-excavation topographic survey of the sites is shown in Figure 3; post-excavation civil surveys are shown in Figures 4 through 9.

The eastern portion of the 100-B-14:2 (area 1) pipelines was previously removed with remediation of the underlying 100-B-8:1 reactor cooling water effluent lines (BHI 2004b) and was not further considered in 100-B-14:2 remedial activities or verification sampling. The southernmost portion of the 100-B-14:2 (area 2) pipeline, at the former 183-B Filter Plant pumphouse, is coincident with several other pipelines in the area. During excavation, suspect hexavalent chromium staining was identified in soils in the area. The staining is presumably related to the 100-B-28 sodium dichromate transfer line, and remediation and subsequent closure of footprint soils was deferred until planned remediation of that site. The southernmost portion of the 1607-B2 collection line, formerly servicing the 105-B Reactor Building, was not removed, in agreement with the U.S. Environmental Protection Agency (WCH 2005) due to its proximity to the reactor building. This portion of the pipeline will be addressed with the 118-B-8, 105-B Reactor waste site.

In-process samples of soil and waste materials were collected during site remediation as needed to support waste characterization and evaluation of the waste profile for disposal of excavated material and to guide remediation efforts. The analytical results for these samples are provided in Appendix A. Photographs taken during remedial activities are provided in Appendix C.

\section{VERIFICATION SAMPLING ACTIVITIES}

Verification sampling was performed separately for the 100-B-14:2 (area 2), 100-B-14:2 (area 4), 100-B-14:2 (area 5), and 1607-B2:1 excavations and associated BCL material from August 2005 to July 2006. The 100-B-14:2 (area 1) and 1607-B2:2 excavations were combined into one decision unit for the purposes of verification sampling, due to the small footprint of the 100-B-14:2 (area 1) pipelines, and sampled in June 2006. Verification sampling was performed to collect data to make a decision whether the remedial action objectives had been reached. Based on statistical evaluation of the resulting data, the residual contaminant concentrations meet the cleanup criteria specified in the RDR/RAWP (DOE-RL 2005b) and the Remaining Sites ROD (EPA 1999). The following 
Figure 3. Pre-Excavation Topographic Survey of the 100-B-14:2 and 1607-B2 Waste Site Vicinity.

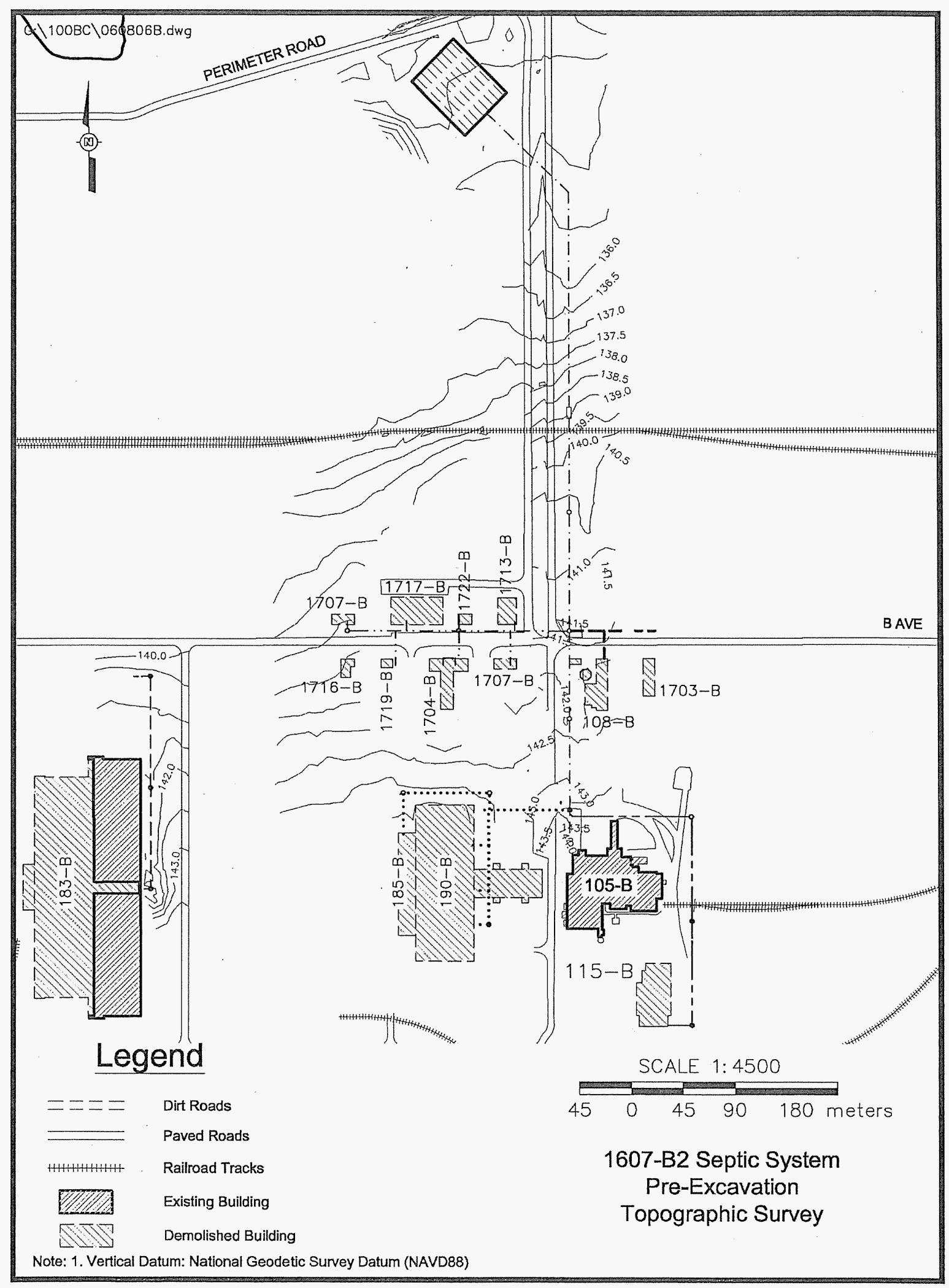


Figure 4. Post-Excavation Civil Survey of the 100-B-14:2 (Area 2) Subsite.

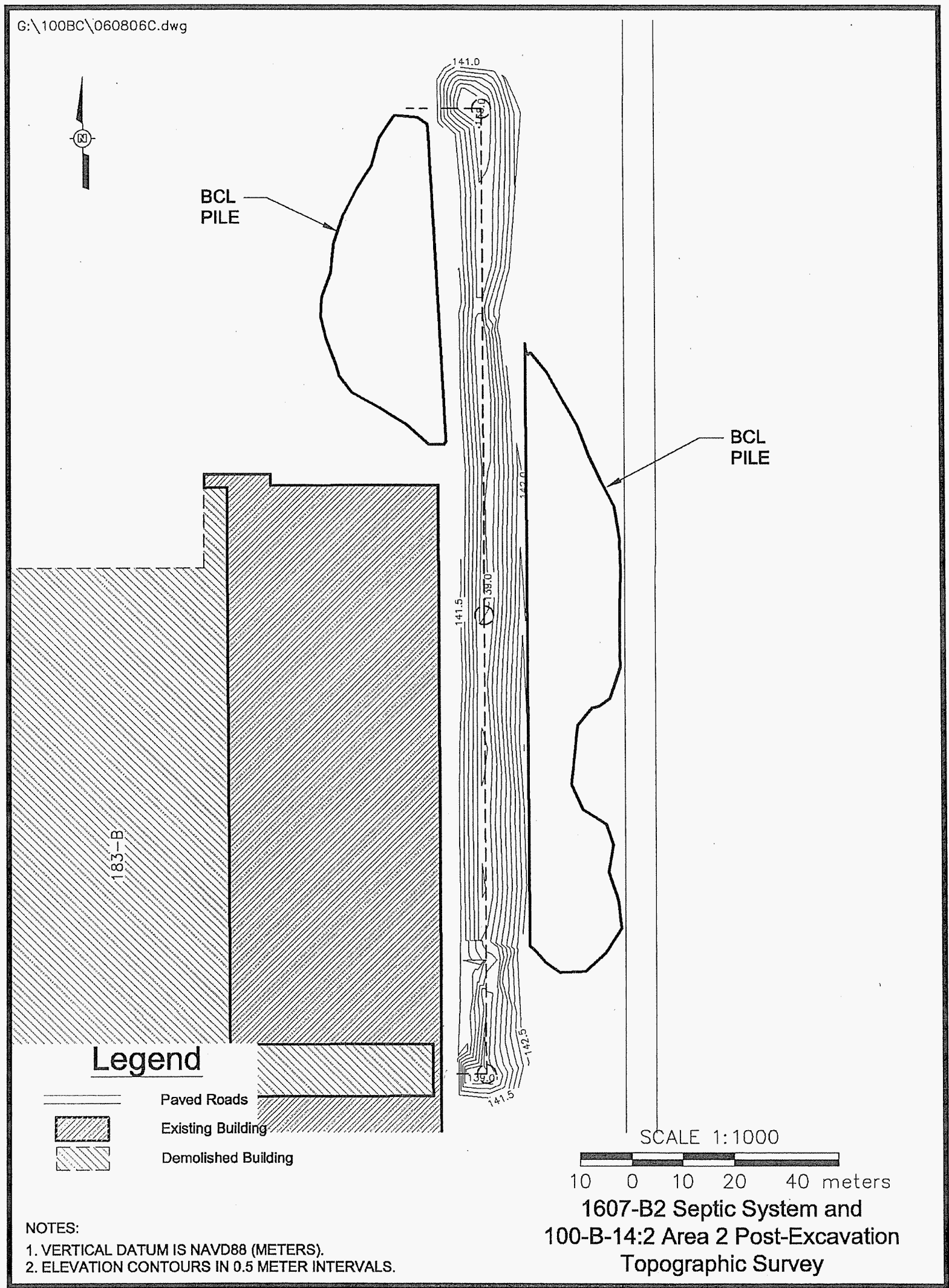


Figure 5. Post-Excavation Civil Survey of the 100-B-14:2 (Area 4) Subsite.

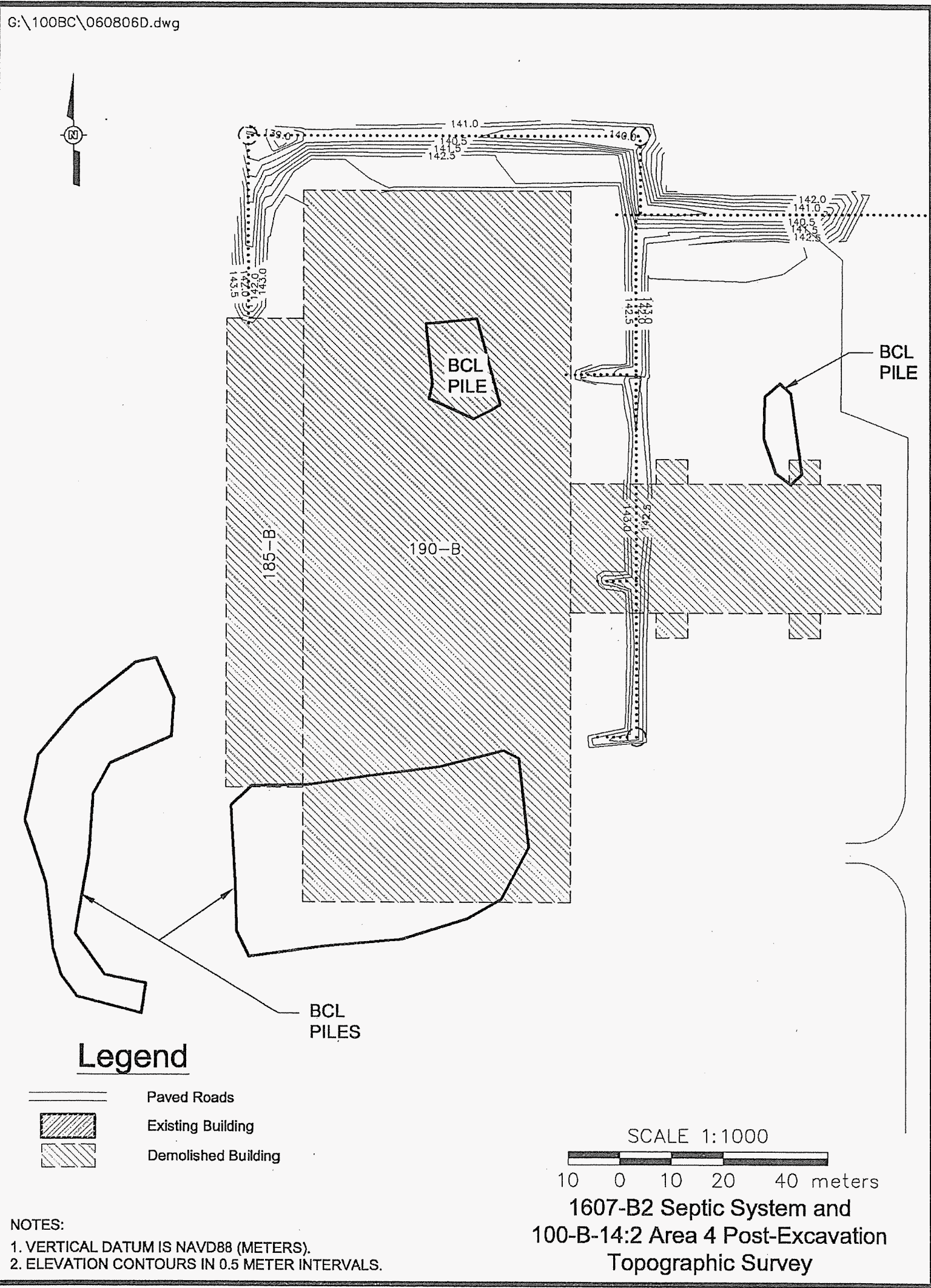


Figure 6. Post-Excavation Civil Survey of the 100-B-14:2 (Area 5) Subsite.

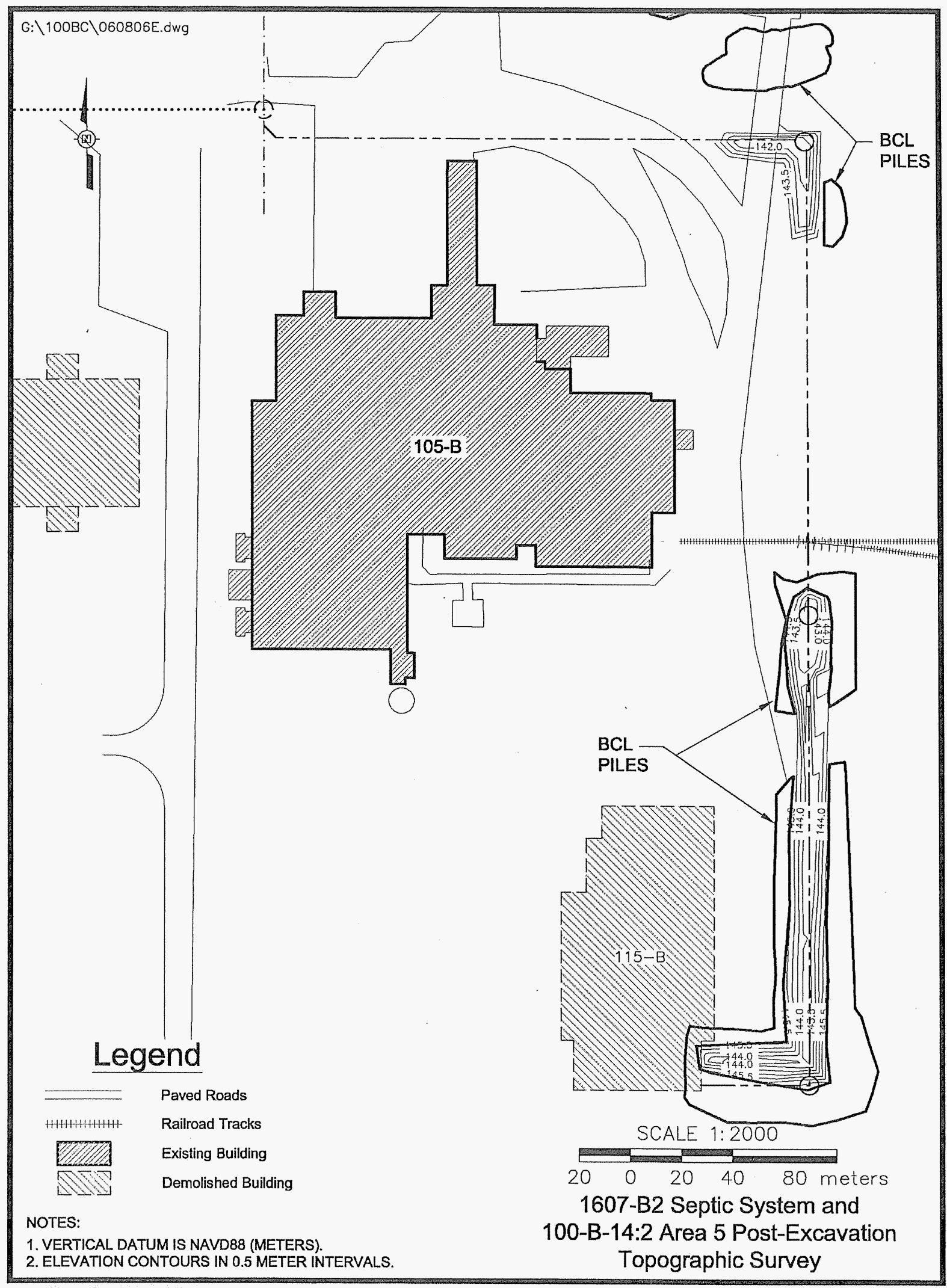


Figure 7. Post-Excavation Civil Survey of the Southern 1607-B2:2 Subsite and 100-B-14:2 (Area 1) Subsite.

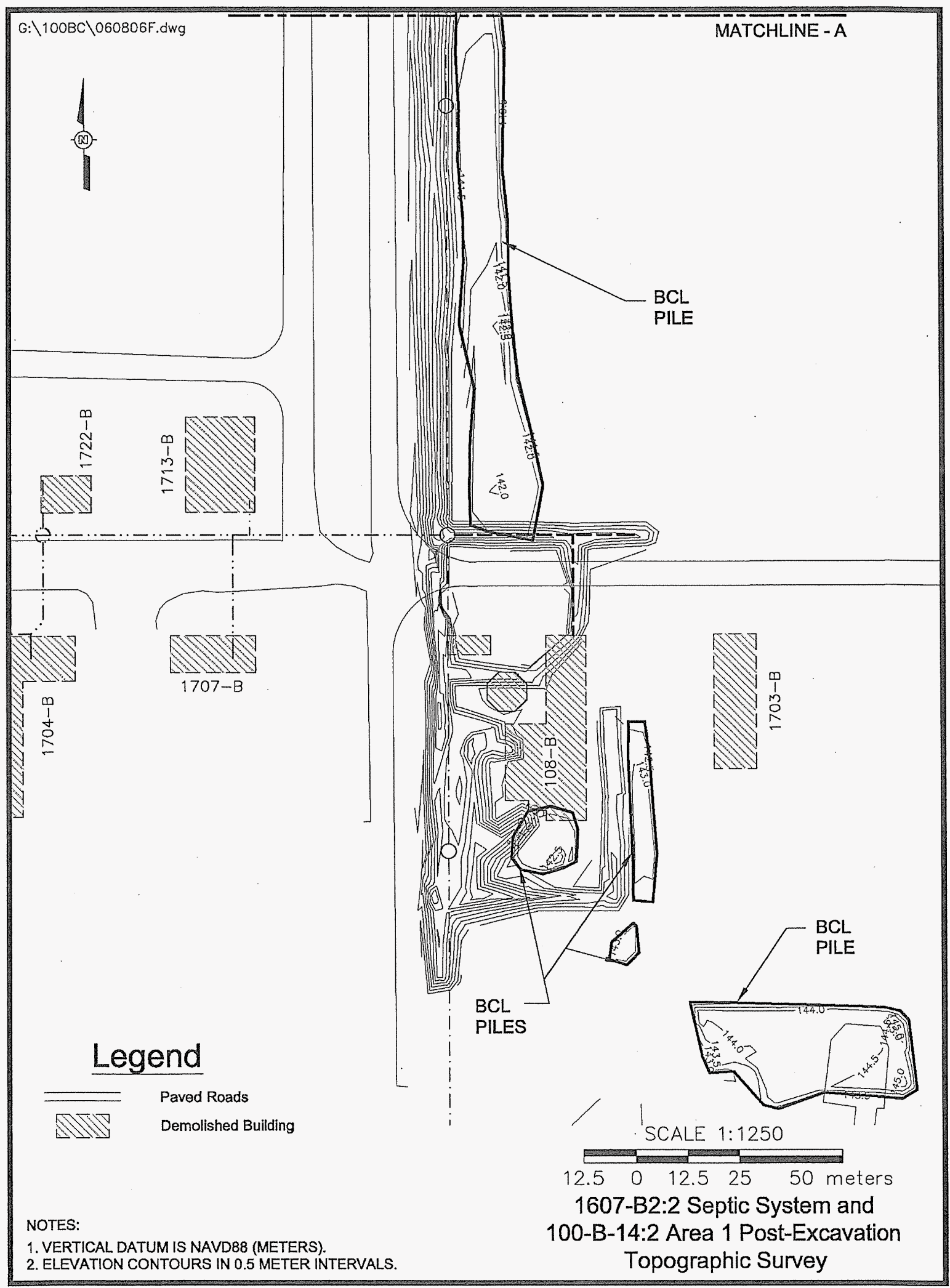


Figure 8. Post-Excavation Civil Survey of the Northern 1607-B2:2 Subsite.

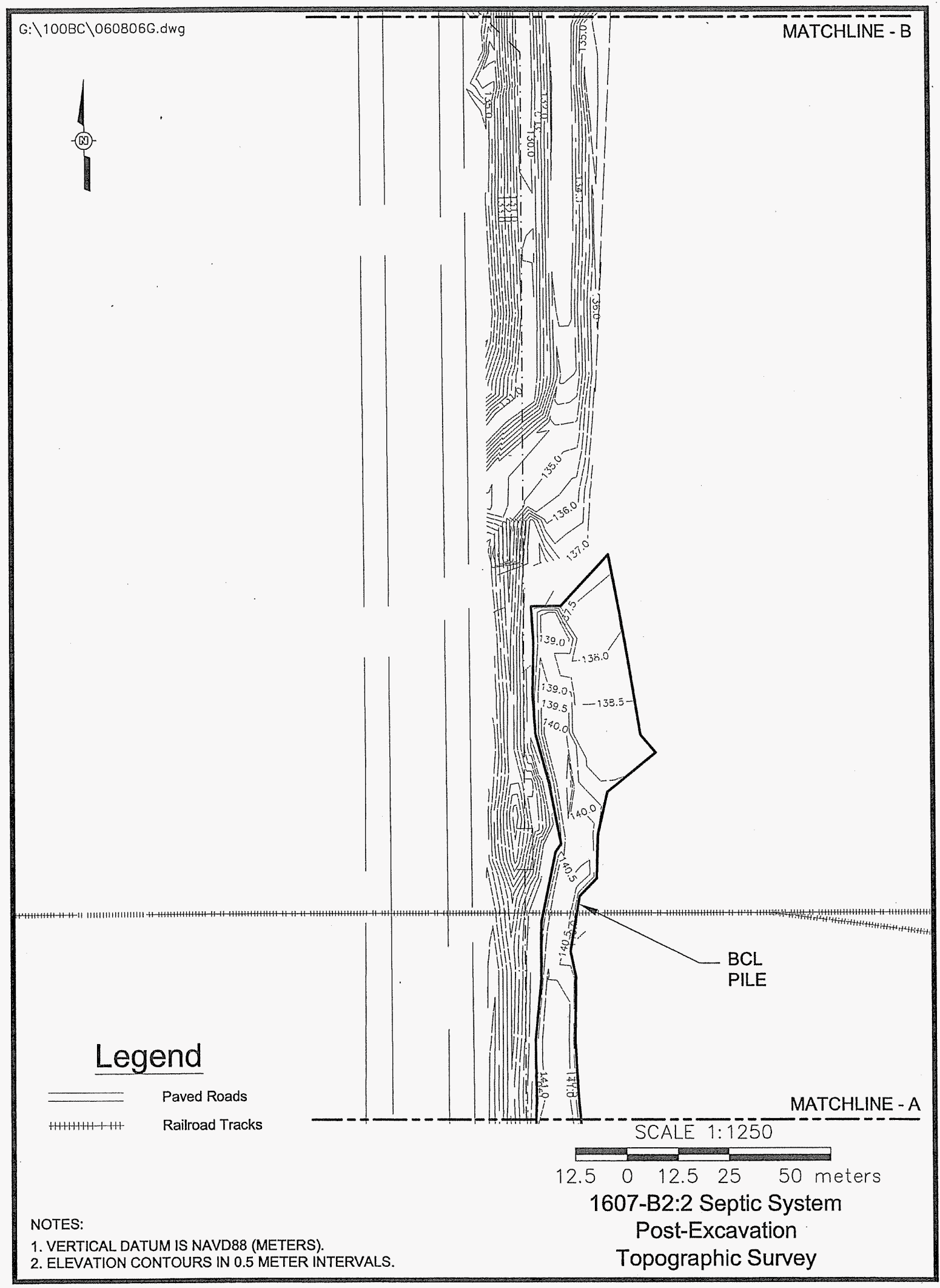


Figure 9. Post-Excavation Civil Survey of the 1607-B2:1 Subsite.

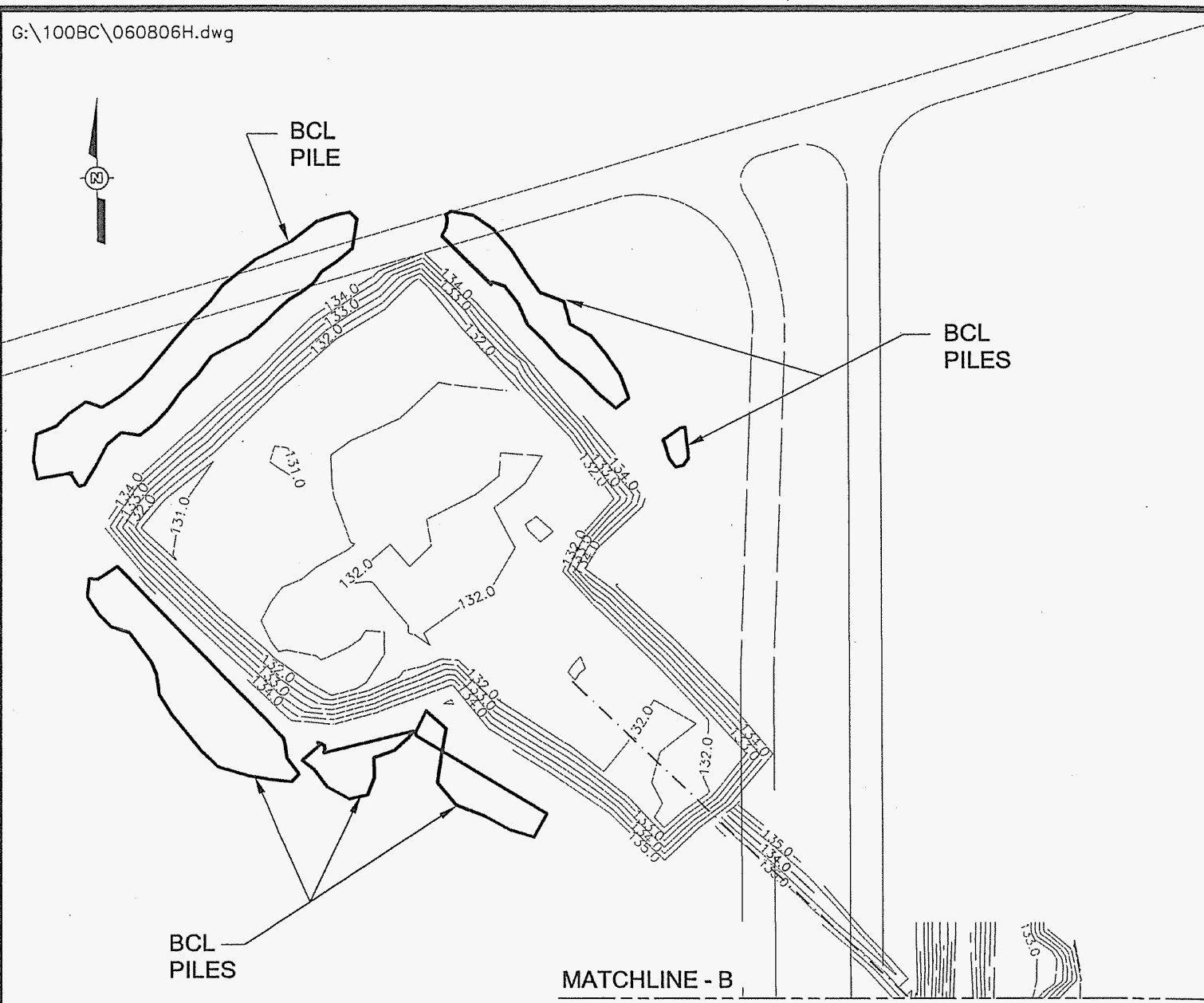

\section{Legend}

Paved Roads

SCALE 1:1250

$\begin{array}{lllll}12.5 & 0 & 12.5 & 25 & 50 \text { meters }\end{array}$ 1607-B2:2 and 1607-B2:1 Septic

NOTES:

1. VERTICAL DATUM IS NAVD88 (METERS).

2. ELEVATION CONTOURS IN 0.5 METER INTERVALS.

System Post-Excavation

Topographic Survey 
subsections provide additional discussion of the information used to develop the verification sampling design. The results of verification sampling are also summarized to support interim closure of the 100-B-14:2 and 1607-B2 waste sites.

\section{Contaminants of Concern and Contaminants of Potential Concern}

The results of confirmatory sampling, waste characterization sampling, and field-screening were used to determine the COCs and COPCs for verification sampling. The analyses performed for verification samples from each area, listed in Table 4, are inclusive of the constituents that were detected above direct exposure RAGs or dose-equivalence lookup values and/or above RAGs for the protection of groundwater and the Columbia River. These analyses are also inclusive of those methods with significant data quality deficiencies in the confirmatory data set.

Table 4. Analyses Performed on Verification Samples for Site Contaminants of Concern and Contaminants of Potential Concern.

\begin{tabular}{|c|c|c|c|c|c|}
\hline \multirow[b]{2}{*}{ Analysis } & \multicolumn{5}{|c|}{ Subsite } \\
\hline & $\begin{array}{c}\text { 100-B-14:2 } \\
\text { Area } 2\end{array}$ & $\begin{array}{c}\text { 100-B-14:2 } \\
\text { Area } 4\end{array}$ & $\begin{array}{c}\text { 100-B-14:2 } \\
\text { Area } 5\end{array}$ & 1607-B2:1 & $\begin{array}{c}\text { 1607-B2:2 } \\
\text { and } \\
\text { 100-B-14:2 } \\
\text { Area } 1\end{array}$ \\
\hline Gamma energy analysis & - & - & - & & 0 \\
\hline $\begin{array}{l}\text { Gross alpha proportional } \\
\text { counting }\end{array}$ & - & & ○ & & \\
\hline $\begin{array}{l}\text { Gross beta proportional } \\
\text { counting }\end{array}$ & 0 & & - & & \\
\hline $\begin{array}{l}\text { Total beta radiostrontium } \\
\text { (strontium-90) }\end{array}$ & & & $0^{a}$ & & - \\
\hline $\begin{array}{l}\text { Tritium by liquid } \\
\text { scintillation counting }\end{array}$ & & & 0 & & - \\
\hline $\begin{array}{l}\text { ICP metals } \\
\text { EPA Method } 6010\end{array}$ & - & - & - & - & ○ \\
\hline $\begin{array}{l}\text { Mercury } \\
\text { EPA Method } 7471\end{array}$ & - & - & - & 0 & - \\
\hline $\begin{array}{l}\text { Hexavalent chromium } \\
\text { EPA Method } 7196\end{array}$ & - & - & - & - & - \\
\hline $\begin{array}{l}\text { PCB } \\
\text { EPA Method } 8082\end{array}$ & - & - & - & - & - \\
\hline $\begin{array}{l}\text { Pesticide } \\
\text { EPA Method } 8081\end{array}$ & - & - & - & - & 0 \\
\hline $\begin{array}{l}\text { Herbicide } \\
\text { EPA Method } 8151\end{array}$ & & & & $0^{a}$ & \\
\hline $\begin{array}{l}\text { SVOA } \\
\text { EPA Method } 8270\end{array}$ & - & 0 & $\theta$ & - & - \\
\hline $\begin{array}{l}\text { VOA } \\
\text { EPA Method } 8260\end{array}$ & ' & & & $0^{a}$ & \\
\hline
\end{tabular}

${ }^{a}$ Extra analysis inadvertently requested at the analytical laboratory.

$\mathrm{EPA}=$ U.S. Environmental Protection Agency

ICP = inductively coupled plasma

$\mathrm{PCB}=$ polychlorinated biphenyl

SVOA = semivolatile organic analysis

VOA = volatile organic analysis 


\section{Verification Sample Design}

Statistical sampling was performed for the 100-B-14:2 and 1607-B2 remediation footprints because the spatial distribution of potential residual soil contamination over the study area was uncertain. The decision rule for demonstrating compliance with the cleanup criteria requires comparison of the true population mean of $\mathrm{COCs} / \mathrm{COPCs}$, as estimated by the $95 \%$ upper confidence limit on the sample mean, with the cleanup level. The Washington State Department of Ecology publication Guidance on Sampling and Data Analysis Methods (Ecology 1995) recommends that systematic sampling with sample locations distributed over the entire study area be used. Therefore, sampling locations were distributed over the entire remediation footprint on an area-by-area basis using random-start systematic grids in an effort to determine the potential residual presence of contamination.

Visual Sample Plan ${ }^{1}$ (VSP) was used as a tool to develop the statistical sampling design for the 100-B-14:2 and 1607-B2 waste sites. The remediation footprints (Figures 4 through 9) were delineated in VSP and used as the basis for location of systematic grid for verification soil sample collection.

Ten soil verification sample locations were identified for each of the 100-B-14:2 areas 2, 4, and 5 remediation footprints; 11 soil verification sample locations were identified for the 1607-B2:1 drain field remediation footprint; 14 soil verification sample locations were identified for the 1607-B2:2 and 100-B-14:2 area 1 remediation footprints. Additional details concerning the use of VSP to develop the statistical sampling designs are provided in the subsite-specific verification sampling work instructions (BHI 2005d, WCH 2006c, 2006d, 2006e).

Verification samples were also collected from stockpiled suspect BCL soils associated with the 100-B-14:2 and 1607-B2 excavations. Because this material is not believed to have received discharges from the gravity-flow sanitary sewer system, statistical sampling designs were not warranted, and professional judgment was used to develop the sampling approach. Sampling consisted of the collection of 25 to 30 aliquots of soil distributed across the surface of each BCL pile or group of smaller BCL piles and combining into one sample per pile or pile group for laboratory analysis (BHI 2005d; WCH 2006c, 2006d, 2006e).

Figures 10 through 15 show maps of the statistical sample locations that were selected for verification sampling; coordinates for each statistical sample are provided in Table 5. The soil sample locations were surveyed and staked prior to sample collection (BHI 2005c, WCH 2006a, 2006b). All sampling was performed in accordance with ENV-1, Environmental Monitoring \& Management, to fulfill the requirements of the 100 Area Remedial Action Sampling and Analysis Plan (SAP) (DOE-RL 2005a). One soil sample was collected at each location by collecting 25

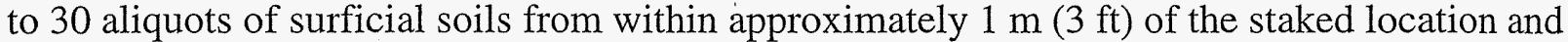
combining the aliquots into 1 sample. Field quality control (QC) samples consisted of one field duplicate sample and one equipment blank per area or subsite; field duplicate sample locations are identified in Table 5. All samples were submitted for full protocol laboratory analysis.

\footnotetext{
${ }^{1}$ Visual Sample Plan is a site map-based user-interface program that may be downloaded at http://dqo.pnl.gov.
} 
Figure 10. Statistical Verification Sampling Locations at the 100-B-14:2 (Area 2) Subsite.

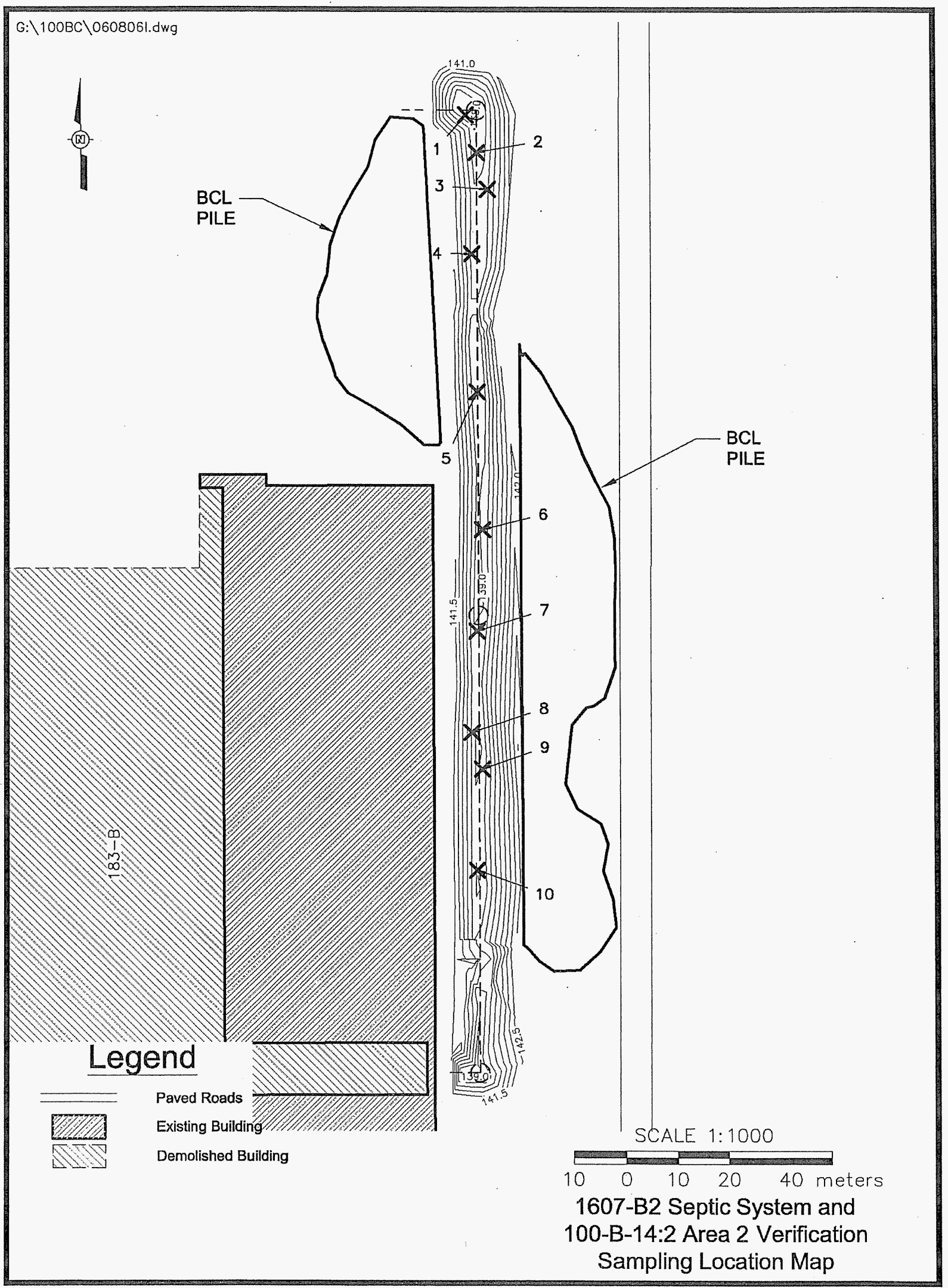


Figure 11. Statistical Verification Sampling Locations at the 100-B-14:2 (Area 4) Subsite.

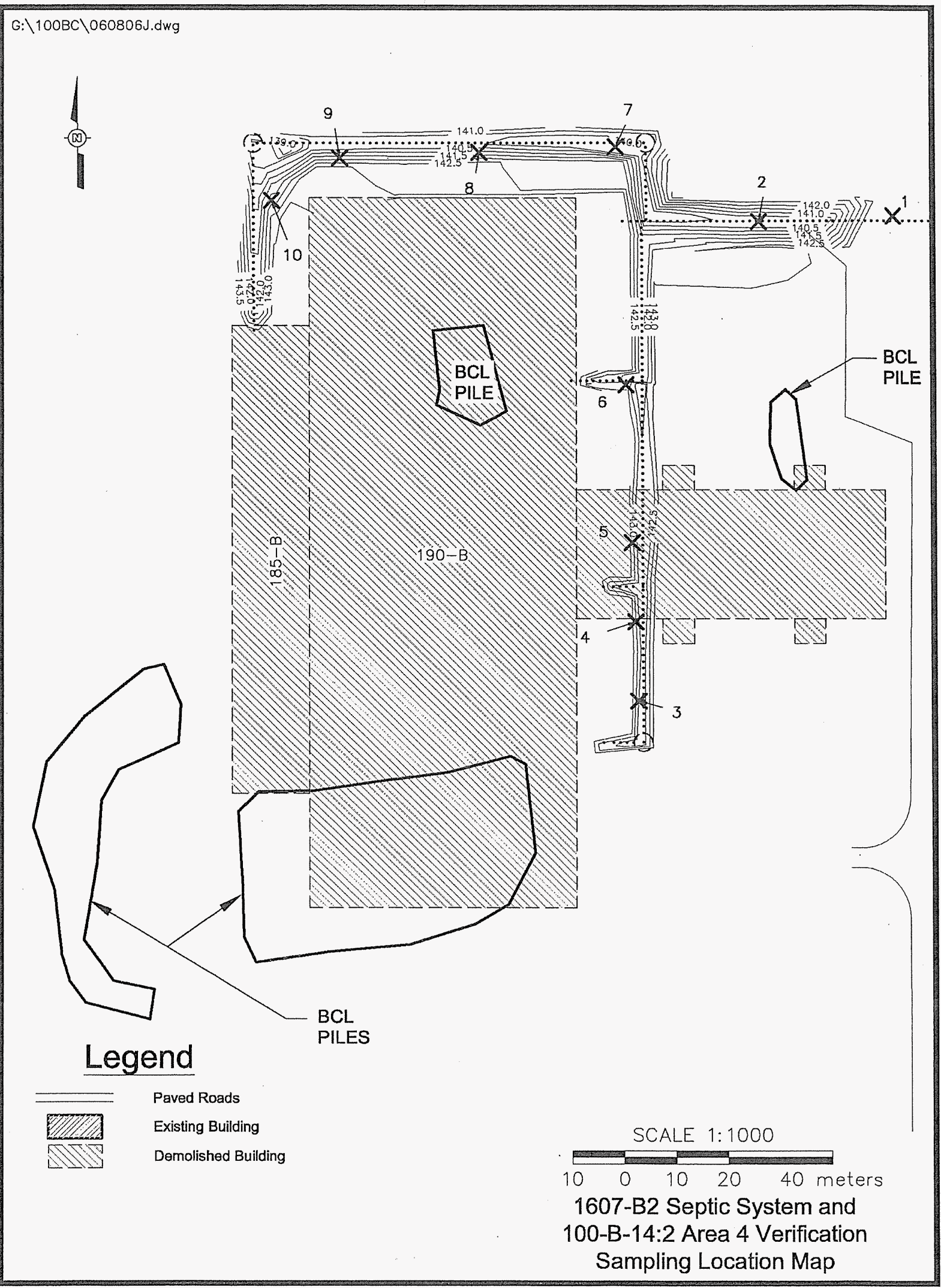


Figure 12. Statistical Verification Sampling Locations at the 100-B-14:2 (Area 5) Subsite.

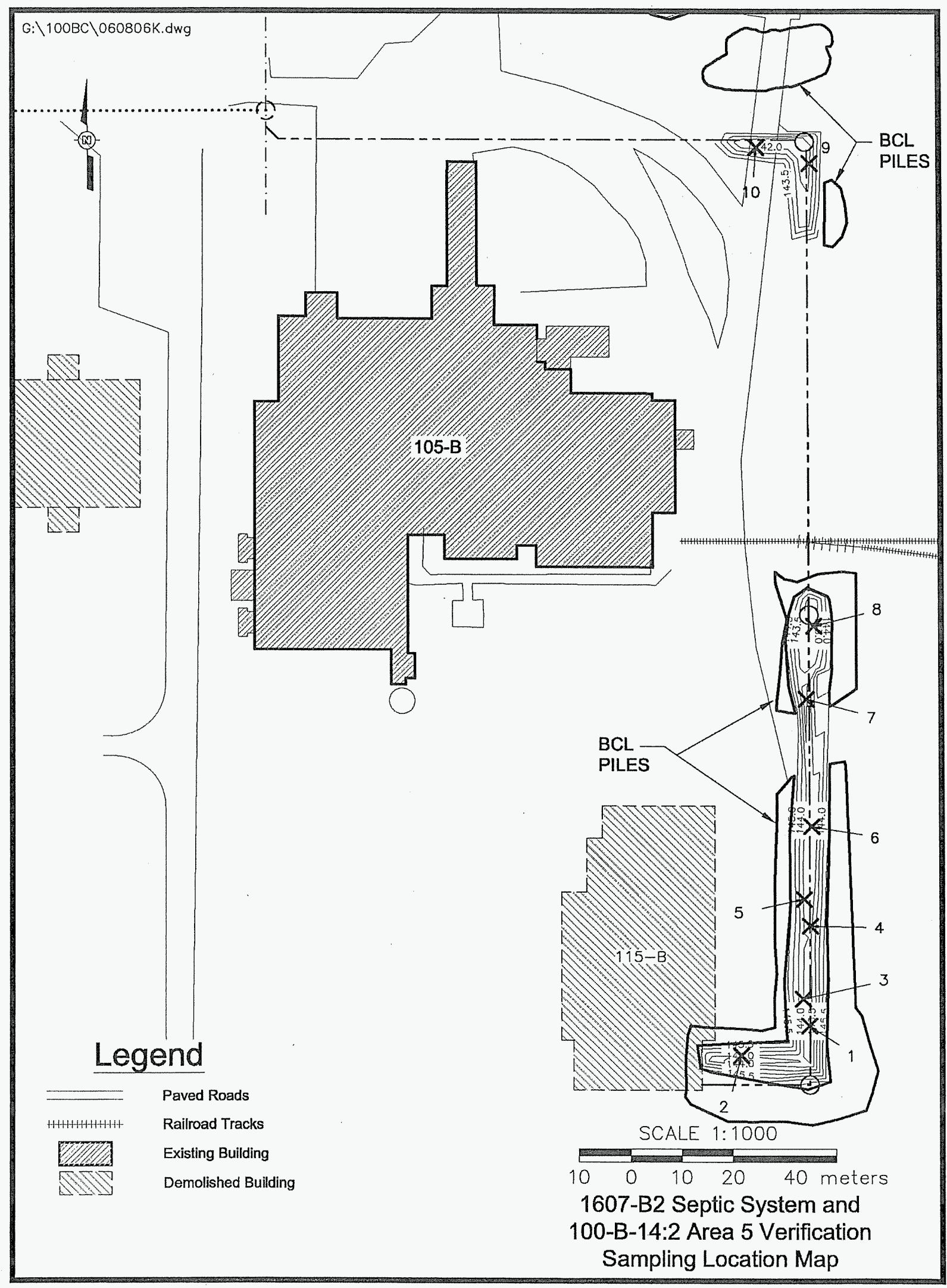


Figure 13. Statistical Verification Sampling Locations at the Southern 1607-B2:2 Subsite and 100-BB-14:2 (Area 1) Subsite.

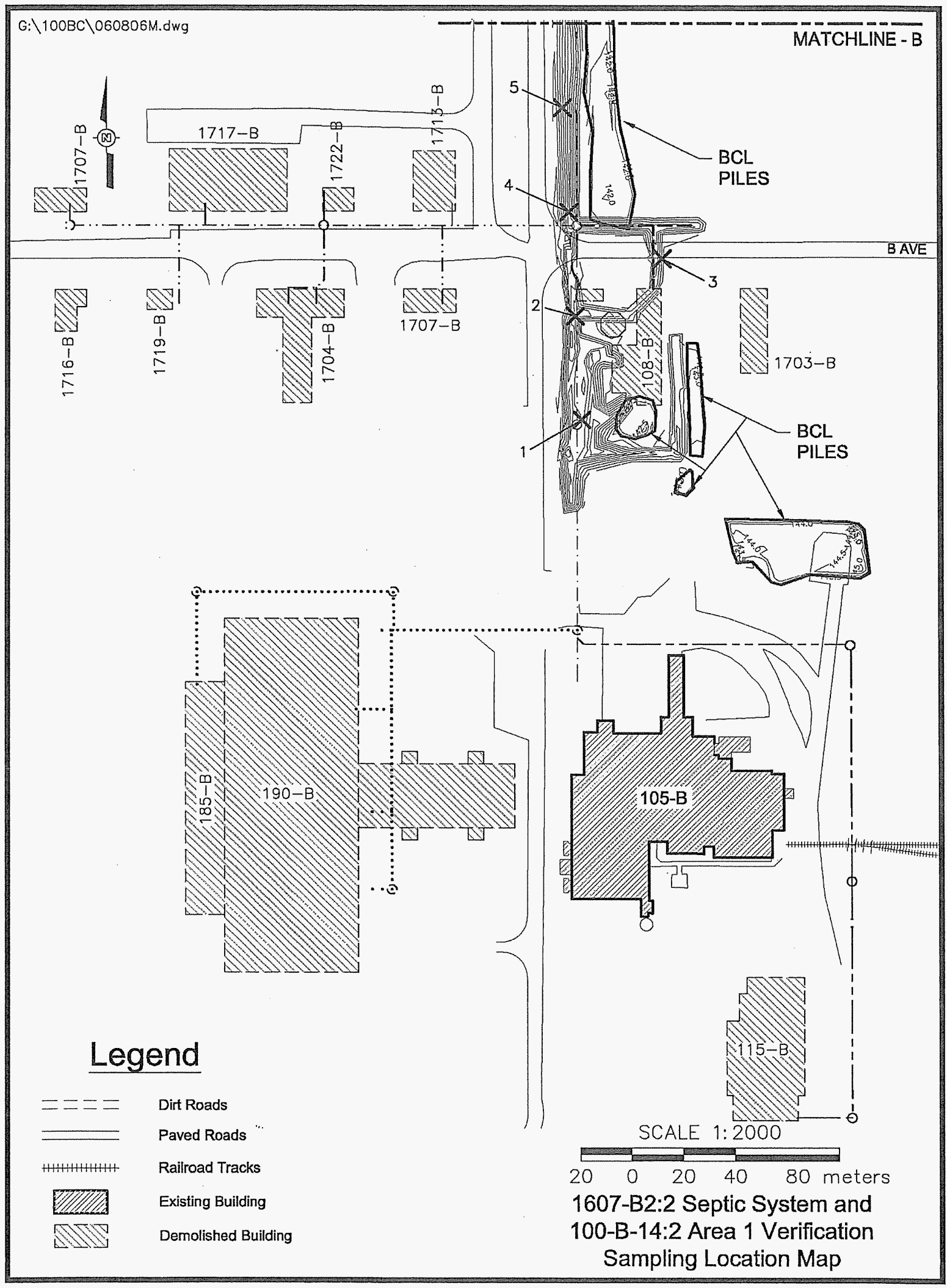


Figure 14. Statistical Verification Sampling Locations at the Northern 1607-B2:2 Subsite.

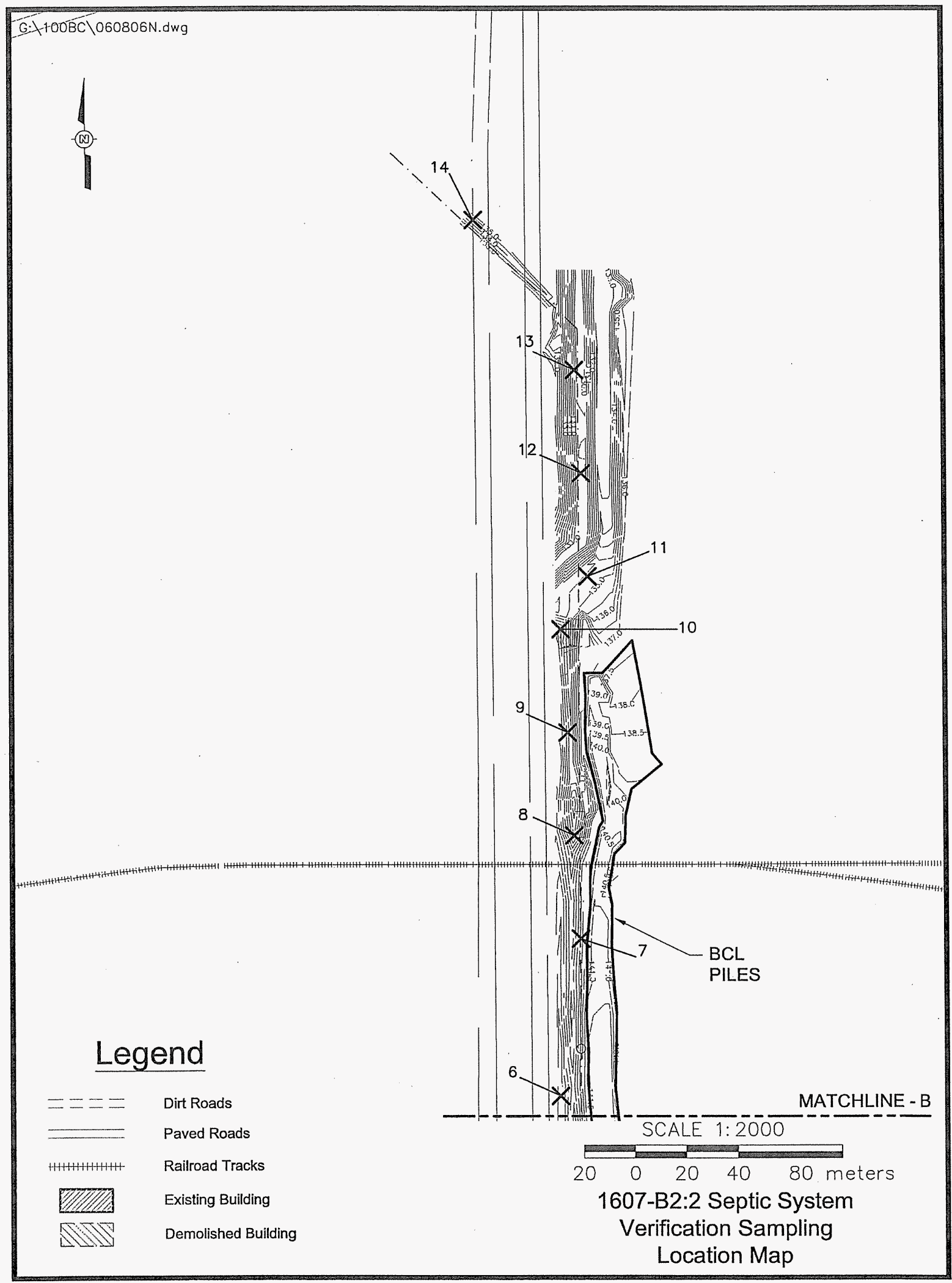


Figure 15. Statistical Verification Sampling Locations at the 1607-B2:1 Subsite.

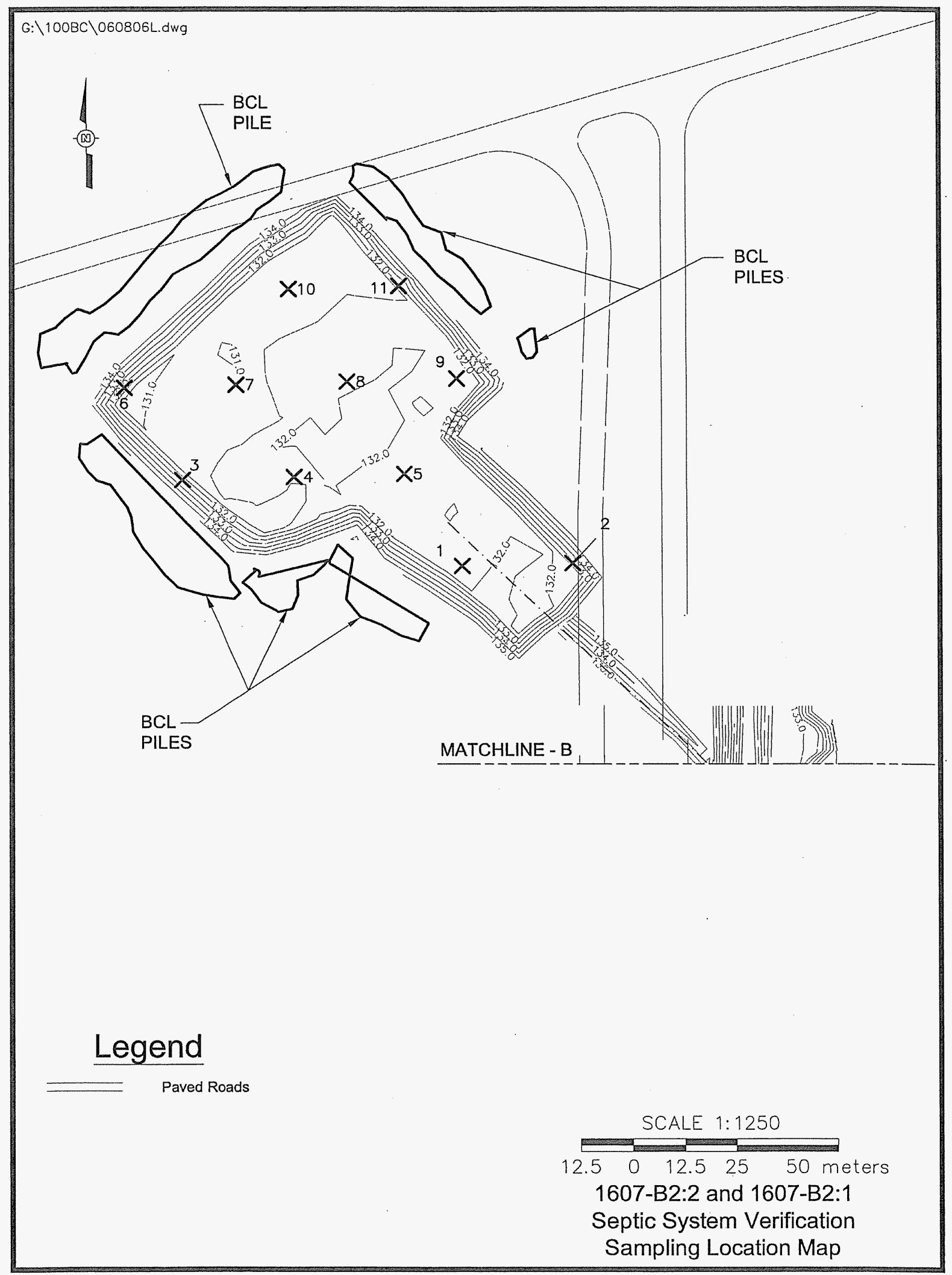


Table 5. 100-B-14:2 and 1607-B2 Verification Sample Location Coordinates. (4 Pages)

\begin{tabular}{|c|c|c|}
\hline Sample Location & Sample Number & $\begin{array}{c}\text { Washington State Plane } \\
\text { Coordinates }\end{array}$ \\
\hline \multicolumn{3}{|c|}{ 100-B-14:2 Area 2} \\
\hline 1 & $\mathrm{~J} 11 \mathrm{~K} 85$ & $\begin{array}{l}\text { N } 144698.1 \\
\text { E } 564885.0\end{array}$ \\
\hline $2^{\mathrm{a}}$ & $\mathrm{J} 11 \mathrm{~K} 86 / \mathrm{J} 11 \mathrm{~K} 95^{\mathrm{a}}$ & $\begin{array}{l}\text { N } 144690.8 \\
\text { E } 564887.1\end{array}$ \\
\hline 3 & $\mathrm{~J} 11 \mathrm{~K} 87$ & $\begin{array}{l}\text { N } 144683.6 \\
\text { E } 564889.2\end{array}$ \\
\hline 4 & $\mathrm{~J} 11 \mathrm{~K} 88$ & $\begin{array}{l}\text { N } 144671.0 \\
\text { E } 564886.1\end{array}$ \\
\hline 5 & J11K89 & $\begin{array}{l}\text { N } 144643.9 \\
\text { E } 564887.1\end{array}$ \\
\hline 6 & $\mathrm{~J} 11 \mathrm{~K} 90$ & $\begin{array}{l}\text { N } 144616.8 \\
\text { E } 564888.1\end{array}$ \\
\hline 7 & J11K91 & $\begin{array}{l}\text { N } 144596.9 \\
\text { E } 564887.0\end{array}$ \\
\hline 8 & $\mathrm{~J} 11 \mathrm{~K} 92$ & $\begin{array}{l}\text { N } 144577.0 \\
\text { E } 564886.0\end{array}$ \\
\hline 9 & $\mathrm{~J} 11 \mathrm{~K} 93$ & $\begin{array}{l}\text { N } 144569.8 \\
\text { E } 564888.0\end{array}$ \\
\hline 10 & J11K94 & $\begin{array}{l}\text { N } 144549.9 \\
\text { E } 564887.0\end{array}$ \\
\hline East BCL stockpile & $\mathrm{J} 11 \mathrm{KD} 1$ & $\mathrm{~N} / \mathrm{A}^{\mathrm{b}}$ \\
\hline West BCL stockpile & $\mathrm{J} 11 \mathrm{KD} 2$ & $\mathrm{~N} / \mathrm{A}^{\mathrm{b}}$ \\
\hline Equipment blank & J11K96 & N/A \\
\hline \multicolumn{3}{|c|}{ 100-B-14:2 Area 4} \\
\hline 1 & $\mathrm{~J} 12 \mathrm{R} 02$ & $\begin{array}{l}\text { N } 144581.6 \\
\text { E } 565232.8\end{array}$ \\
\hline 2 & $\mathrm{~J} 12 \mathrm{R} 03$ & $\begin{array}{l}\text { N } 144580.5 \\
\text { E } 565206.0\end{array}$ \\
\hline 3 & J12R04 & $\begin{array}{l}\text { N } 144486.5 \\
\text { E } 565182.8\end{array}$ \\
\hline 4 & J12R05 & $\begin{array}{l}\text { N } 144502.0 \\
\text { E } 565182.2\end{array}$ \\
\hline 5 & J12R06 & $\begin{array}{l}\text { N } 144517.5 \\
\text { E } 565181.6\end{array}$ \\
\hline 6 & $\mathrm{~J} 12 \mathrm{R} 07$ & $\begin{array}{l}\text { N } 144548.5 \\
\text { E } 565180.4\end{array}$ \\
\hline 7 & J12R08 & $\begin{array}{l}\text { N } 144595.0 \\
\text { E } 565178.5\end{array}$ \\
\hline
\end{tabular}


Table 5. 100-B-14:2 and 1607-B2 Verification Sample Location Coordinates. (4 Pages)

\begin{tabular}{|c|c|c|}
\hline Sample Location & Sample Number & $\begin{array}{c}\text { Washington State Plane } \\
\text { Coordinates }\end{array}$ \\
\hline $8^{a}$ & $\mathrm{~J} 12 \mathrm{R} 09 / \mathrm{J} 12 \mathrm{R} 14^{\mathrm{a}}$ & $\begin{array}{l}\text { N } 144593.9 \\
\text { E } 565151.7\end{array}$ \\
\hline 9 & $\mathrm{~J} 12 \mathrm{R} 10$ & $\begin{array}{l}\text { N } 144592.8 \\
\text { E } 565124.8\end{array}$ \\
\hline 10 & J12R11 & $\begin{array}{l}\text { N } 144584.5 \\
\text { E } 565111.7\end{array}$ \\
\hline East BCL stockpile & $\mathrm{J} 12 \mathrm{R} 12$ & $N / A^{b}$ \\
\hline West BCL stockpile & J12R13 & $\mathrm{N} / \mathrm{A}^{\mathrm{b}}$ \\
\hline Equipment blank & J12R15 & N/A \\
\hline \multicolumn{3}{|c|}{ 100-B-14:2 Area 5} \\
\hline 1 & $\mathrm{~J} 11 \mathrm{~KB} 3$ & $\begin{array}{l}\text { N } 144400.6 \\
\text { E } 565361.4\end{array}$ \\
\hline 2 & $\mathrm{~J} 11 \mathrm{~KB} 4$ & $\begin{array}{l}\text { N } 144394.7 \\
\text { E } 565348.2\end{array}$ \\
\hline 3 & J11KB5 & $\begin{array}{l}\text { N } 144405.9 \\
\text { E } 565360.2\end{array}$ \\
\hline $4^{a}$ & $\mathrm{~J} 11 \mathrm{~KB} 6 / \mathrm{J} 11 \mathrm{KC} 3^{\mathrm{a}}$ & $\begin{array}{l}\text { N } 144420.3 \\
\text { E } 565361.6\end{array}$ \\
\hline 5 & $\mathrm{~J} 11 \mathrm{~KB} 7$ & $\begin{array}{l}\text { N } 144425.6 \\
\text { E } 565360.4\end{array}$ \\
\hline 6 & $\mathrm{~J} 11 \mathrm{~KB} 8$ & $\begin{array}{l}\text { N } 144440.0 \\
\text { E } 565361.9\end{array}$ \\
\hline 7 & $\mathrm{~J} 11 \mathrm{~KB} 9$ & $\begin{array}{l}\text { N } 144465.1 \\
\text { E } 565360.9\end{array}$ \\
\hline 8 & $\mathrm{~J} 11 \mathrm{KC} 0$ & $\begin{array}{l}\text { N } 144479.5 \\
\text { E } 565362.4\end{array}$ \\
\hline 9 & $\mathrm{~J} 11 \mathrm{KC} 1$ & $\begin{array}{l}\text { N } 144570.6 \\
\text { E } 565361.9\end{array}$ \\
\hline 10 & $\mathrm{~J} 11 \mathrm{KC} 2$ & $\begin{array}{l}\text { N } 144573.7 \\
\text { E } 565351.4\end{array}$ \\
\hline $\begin{array}{l}\text { South BCL stockpile } \\
\text { (south) }\end{array}$ & $\mathrm{J} 11 \mathrm{KD} 3$ & $N / A^{b}$ \\
\hline $\begin{array}{l}\text { South BCL stockpile } \\
\text { (north) }\end{array}$ & $\mathrm{J} 11 \mathrm{KD} 4$ & $N / A^{b}$ \\
\hline Central BCL stockpile & J11KD5 & $N^{\prime} / A^{b}$ \\
\hline North BCL stockpile & $\mathrm{J} 11 \mathrm{KD} 6$ & $\mathrm{~N} / \mathrm{A}^{\mathrm{b}}$ \\
\hline Equipment blank & $\mathrm{J} 11 \mathrm{KC} 4$ & N/A \\
\hline
\end{tabular}


Table 5. 100-B-14:2 and 1607-B2 Verification Sample Location Coordinates. (4 Pages)

\begin{tabular}{|c|c|c|}
\hline Sample Location & Sample Number & $\begin{array}{c}\text { Washington State Plane } \\
\text { Coordinates }\end{array}$ \\
\hline \multicolumn{3}{|c|}{$1607-B 2: 1$} \\
\hline 1 & J03VB8 & $\begin{array}{l}\text { N } 145187.4 \\
\text { E } 565186.9\end{array}$ \\
\hline $2^{\mathrm{a}}$ & J03VB9/J03VD2 ${ }^{\mathrm{a}}$ & $\begin{array}{l}\text { N } 145188.1 \\
\text { E } 565213.8\end{array}$ \\
\hline 3 & J03VC0 & $\begin{array}{l}\text { N } 145208.8 \\
\text { E } 565119\end{array}$ \\
\hline 4 & J03VC1 & $\begin{array}{l}\text { N } 145209.5 \\
\text { E } 565145.9\end{array}$ \\
\hline 5 & $\mathrm{~J} 03 \mathrm{VC} 2$ & $\begin{array}{l}\text { N } 145210.3 \\
\text { E } 565172.8\end{array}$ \\
\hline 6 & J03VC3 & $\begin{array}{l}\text { N } 145231.7 \\
\text { E } 565104.9\end{array}$ \\
\hline 7 & J03VC4 & $\begin{array}{l}\text { N } 145232.4 \\
\text { E } 565131.8\end{array}$ \\
\hline 8 & J03VC5 & $\begin{array}{l}\text { N } 145233.2 \\
\text { E } 565158.7\end{array}$ \\
\hline 9 & J03VC6 & $\begin{array}{l}\text { N } 145234 \\
\text { E } 565185.6\end{array}$ \\
\hline 10 & J03VC7 & $\begin{array}{l}\text { N } 145256.1 \\
\text { E } 565144.6\end{array}$ \\
\hline 11 & J03VC8 & $\begin{array}{l}\text { N } 145256.9 \\
\text { E } 565171.5\end{array}$ \\
\hline $\begin{array}{l}\text { Southwest overburden } \\
\text { stockpile }\end{array}$ & J03VC9 & $\mathrm{N} / \mathrm{A}^{\mathrm{b}}$ \\
\hline $\begin{array}{l}\text { Northwest overburden } \\
\text { stockpile }\end{array}$ & J03VD0 & $\mathrm{N} / \mathrm{A}^{\mathrm{b}}$ \\
\hline $\begin{array}{c}\text { Northeast overburden } \\
\text { stockpile }\end{array}$ & J03VD1 & $\mathrm{N} / \mathrm{A}^{\mathrm{b}}$ \\
\hline Equipment blank & J03WW6 & N/A \\
\hline \multicolumn{3}{|c|}{$1607-B 2: 2$} \\
\hline 1 & $\mathrm{~J} 12 \mathrm{NX} 9$ & $\begin{array}{l}\text { N } 144663.7 \\
\text { E } 565257.3\end{array}$ \\
\hline 2 & J12NY0 & $\begin{array}{l}\text { N } 144704.4 \\
\text { E } 565254.8\end{array}$ \\
\hline 3 & $\mathrm{~J} 12 \mathrm{NY} 1$ & $\begin{array}{l}\text { N } 144726.9 \\
\text { E } 565288.9\end{array}$ \\
\hline 4 & $\mathrm{~J} 12 \mathrm{NY} 2$ & $\begin{array}{l}\text { N } 144745.1 \\
\text { E } 565252.4\end{array}$ \\
\hline
\end{tabular}


Table 5. 100-B-14:2 and 1607-B2

Verification Sample Location Coordinates. (4 Pages)

\begin{tabular}{|c|c|c|}
\hline Sample Location & Sample Number & $\begin{array}{c}\text { Washington State Plane } \\
\text { Coordinates }\end{array}$ \\
\hline $5^{\mathrm{a}}$ & $\mathrm{J} 12 \mathrm{NY} 3 / \mathrm{J} 12 \mathrm{NY} 4^{\mathrm{a}}$ & $\begin{array}{l}\text { N } 144785.8 \\
\text { E } 565250.0\end{array}$ \\
\hline 6 & J12NY5 & $\begin{array}{l}\text { N } 144826.6 \\
\text { E } 565247.5\end{array}$ \\
\hline 7 & J12NY6 & $\begin{array}{l}\text { N } 144888.4 \\
\text { E } 565255.6\end{array}$ \\
\hline 8 & J12NY7 & $\begin{array}{l}\text { N } 144929.1 \\
\text { E } 565253.2\end{array}$ \\
\hline 9 & J12NY8 & $\begin{array}{l}\text { N } 144969.8 \\
\text { E } 565250.8\end{array}$ \\
\hline 10 & J12NY9 & $\begin{array}{l}\text { N } 145010.6 \\
\text { E } 565248.3\end{array}$ \\
\hline 11 & J12PW5 & $\begin{array}{l}\text { N } 145031.6 \\
\text { E } 565258.9\end{array}$ \\
\hline 12 & J12PW6 & $\begin{array}{l}\text { N } 145072.4 \\
\text { E } 565256.5\end{array}$ \\
\hline 13 & J12PW7 & $\begin{array}{l}\text { N } 145113.1 \\
\text { E } 565254.0\end{array}$ \\
\hline 14 & J12PW8 & $\begin{array}{l}\text { N } 145172.1 \\
\text { E } 565215.1\end{array}$ \\
\hline $\begin{array}{l}\text { North BCL stockpile } \\
\text { (north) }\end{array}$ & $\mathrm{J} 12 \mathrm{NX} 4$ & $N / A^{b}$ \\
\hline $\begin{array}{l}\text { North BCL stockpile } \\
\text { (middle) }\end{array}$ & $\mathrm{J} 12 \mathrm{NX} 5$ & $N / A^{b}$ \\
\hline $\begin{array}{l}\text { North BCL stockpile } \\
\text { (south) }\end{array}$ & J12NX6 & $N / A^{b}$ \\
\hline $\begin{array}{l}\text { Southeast BCL } \\
\text { stockpile }\end{array}$ & $\mathrm{J} 12 \mathrm{NX} 7$ & $N / A^{b}$ \\
\hline $\begin{array}{l}\text { Small south BCL } \\
\text { stockpiles }\end{array}$ & $\mathrm{J} 12 \mathrm{NX} 8$ & $N / A^{b}$ \\
\hline Equipment blank & J12P04 & N/A \\
\hline
\end{tabular}

Source: Remaining Pipeline and Sewers Sampling and Field Services, Logbooks EL-1585-3, EL-1585-5, and EL-1585-6 (BHI 2005c, WCH 2006a, 2006b).

a A field duplicate sample was collected at sample location 7.

b Sampling of the BCL stockpiles consisted of collecting aliquots across the stockpile surface(s) and combining into one sample per pile or pile group for analysis.

$\mathrm{BCL}=$ below cleanup levels

N/A = not applicable 


\section{Verification Sampling Results}

Verification samples were analyzed using U.S. Environmental Protection Agency-approved analytical methods. The $95 \%$ upper confidence limits on the true population means for residual concentrations of COCs and COPCs were calculated for the remediation footprints as specified by the RDR/RAWP (DOE-RL 2005b), with calculations provided in Appendix B. When a nonradionuclide COC or COPC was detected in fewer than $50 \%$ of the verification samples collected for each decision unit, the maximum detected value was used for comparison to RAGs. If no detections for a given COC/COPC were reported a given data set, then no statistical evaluation or calculations were performed for that COC/COPC for the associated decision unit. Evaluation of the verification data from the BCL stockpiles was performed by direct comparison of the maximum sample results for each COC/COPC against cleanup criteria for each sampling area.

Comparisons of the statistical and maximum results for COCs and COPCs and the site RAGs for the remediation footprints and BCL stockpiles are summarized in Tables 6 a through $7 \mathrm{~b}$ and $9 \mathrm{a}$ through $11 \mathrm{~b}$ (due to the large number of comparison tables in this document, all comparison table footnotes are provided at the end of the document body to reduce redundancy). Contaminants that were not detected by laboratory analysis are excluded from these tables. Potassium-40, radium-226, radium-228, thorium-228, and thorium-232 were detected in samples but are not considered within these tables because these isotopes are not related to the operational history of the sanitary sewer system. Calculated cleanup levels for aluminum, calcium, iron, magnesium, phosphate, potassium, silicon, sodium, and zirconium are not presented in the RDR/RAWP (DOE-RL 2005b). Parameters to calculate cleanup levels for these constituents are not presented in the Cleanup Levels and Risk Calculations (CLARC) Database (Ecology 2005) under WAC 173-340-740(3) or other reference databases (results for total phosphorus are attributed to phosphorus in phosphate). These analytes are also essential nutrients and can be eliminated from evaluation as human health concerns per EPA guidance (EPA 1989). Therefore, these constituents are not considered COPCs and are not included in the tables. The laboratoryreported data results for all constituents are stored in the ENRE project-specific database prior to providing to HEIS and are presented within the statistical calculations in Appendix B.

Table 6a. Comparison of Statistical Contaminant Concentrations to Action Levels for the 100-B-14:2 (Area 2) Remediation Footprint Verification Sampling Event." (2 Pages)

\begin{tabular}{|c|c|c|c|c|c|c|}
\hline \multirow[b]{2}{*}{$\mathrm{COC} / \mathrm{COPC}$} & \multirow[b]{2}{*}{$\begin{array}{l}\text { Analytical } \\
\text { Result } \\
(\mathrm{pCi} / \mathrm{g})\end{array}$} & \multicolumn{3}{|c|}{ Generic Site Lookup Values $^{\text {a }}$ (pCi/g) } & \multirow[b]{2}{*}{$\begin{array}{l}\text { Does the } \\
\text { Result } \\
\text { Exceed } \\
\text { Lookup } \\
\text { Values? }\end{array}$} & \multirow[b]{2}{*}{$\begin{array}{l}\text { Does the } \\
\text { Result Pass } \\
\text { RESRAD } \\
\text { Modeling? }\end{array}$} \\
\hline & & $\begin{array}{l}\text { Shallow } \\
\text { Zone } \\
\text { Lookup } \\
\text { Value }^{\text {b }}\end{array}$ & $\begin{array}{c}\text { Groundwater } \\
\text { Protection } \\
\text { Lookup } \\
\text { Value }\end{array}$ & $\begin{array}{c}\text { River } \\
\text { Protection } \\
\text { Lookup } \\
\text { Value } \\
\end{array}$ & & \\
\hline Strontium-90 & 0.311 & 4.5 & $27.6^{c}$ & $27.6^{\mathrm{c}}$ & No & -- \\
\hline \multirow[b]{2}{*}{$\mathrm{COC} / \mathrm{COPC}$} & \multirow[b]{2}{*}{$\begin{array}{c}\text { Statistical } \\
\text { Result } \\
(\mathbf{m g} / \mathrm{kg})\end{array}$} & \multicolumn{3}{|c|}{ Remedial Action Goals ${ }^{\mathrm{a}}$ (mg/kg) } & Dees the & Does the \\
\hline & & $\begin{array}{c}\text { Direct } \\
\text { Exposure }\end{array}$ & $\begin{array}{l}\text { Soil Cleanup } \\
\text { Level for } \\
\text { Groundwater } \\
\text { Protection }\end{array}$ & $\begin{array}{c}\text { Soil } \\
\text { Cleanup } \\
\text { Level for } \\
\text { River } \\
\text { Protection }\end{array}$ & $\begin{array}{c}\text { Statistical } \\
\text { Data Set } \\
\text { Exceed } \\
\text { RAGs? }\end{array}$ & $\begin{array}{l}\text { Statistical } \\
\text { Result Pass } \\
\text { RESRAD } \\
\text { Modeling? }\end{array}$ \\
\hline Arsenic & $3.4(<\mathrm{BG})$ & 20 & 20 & 20 & No & -. \\
\hline
\end{tabular}


Table 6a. Comparison of Statistical Contaminant Concentrations to Action Levels for the 100-B-14:2 (Area 2) Remediation Footprint Verification Sampling Event." (2 Pages)

\begin{tabular}{|c|c|c|c|c|c|c|}
\hline \multirow[b]{2}{*}{$\mathrm{COC} / \mathrm{COPC}$} & \multirow[b]{2}{*}{$\begin{array}{l}\text { Statistical } \\
\text { Result } \\
(\mathrm{mg} / \mathrm{kg})\end{array}$} & \multicolumn{3}{|c|}{ Remedial Action Goals ${ }^{\mathrm{a}}$ (mg/kg) } & \multirow[b]{2}{*}{$\begin{array}{c}\text { Does the } \\
\text { Statistical } \\
\text { Data Set } \\
\text { Exceed } \\
\text { RAGs? }\end{array}$} & \multirow[b]{2}{*}{$\begin{array}{l}\text { Does the } \\
\text { Statistical } \\
\text { Result Pass } \\
\text { RESRAD } \\
\text { Modeling? }\end{array}$} \\
\hline & & $\begin{array}{c}\text { Direct } \\
\text { Exposure }\end{array}$ & $\begin{array}{l}\text { Soil Cleanup } \\
\text { Level for } \\
\text { Groundwater } \\
\text { Protection }\end{array}$ & $\begin{array}{c}\text { Soil } \\
\text { Cleanup } \\
\text { Level for } \\
\text { River } \\
\text { Protection }\end{array}$ & & \\
\hline Barium & 163 & $5,600^{\mathrm{d}}$ & $132^{\mathrm{e}, \mathrm{f}}$ & $224^{\mathrm{g}}$ & Yes & Yes $^{\mathrm{h}}$ \\
\hline Beryllium & $0.38(<\mathrm{BG})$ & $10.4^{\mathrm{i}}$ & $1.51^{\mathrm{f}}$ & $1.51^{\mathrm{f}}$ & No & -- \\
\hline Boron $^{j}$ & 4.5 & 16,000 & 320 & $--^{\mathrm{k}}$ & No & -- \\
\hline Cadmium $^{1}$ & $0.14(<\mathrm{BG})$ & 13.9 & $0.81^{\mathrm{f}}$ & $0.81^{\mathrm{f}}$ & No & - \\
\hline Chromium (total) & $9.0(<\mathrm{BG})$ & $80,000^{d}$ & $18.5^{\mathrm{f}}$ & $18.5^{\mathrm{f}}$ & No & -- \\
\hline Chromium (hexavalent) & 0.26 & 2.1 & $4.8^{\mathrm{m}}$ & 2 & No & -- \\
\hline Cobalt & $8.3(<\mathrm{BG})$ & 1,600 & 32 & $--^{k}$ & No & -- \\
\hline Copper & $17.7(<\mathrm{BG})$ & 2,960 & 59.2 & $22.0^{\mathrm{f}}$ & No & - \\
\hline Lead & $5.8(<\mathrm{BG})$ & 353 & $10.2^{\mathrm{f}}$ & $10.2^{\mathrm{f}}$ & No & -- \\
\hline Lithium & $7.4(<\mathrm{BG})$ & 1,600 & $33.5^{\mathrm{f}}$ & $--^{k}$ & No & -- \\
\hline Manganese & $349(<\mathrm{BG})$ & 11,200 & $512^{\mathrm{f}}$ & $512^{f}$ & No & -- \\
\hline Mercury & $0.05(<\mathrm{BG})$ & 24 & $0.33^{\mathrm{f}}$ & $0.33^{\mathrm{f}}$ & No & -- \\
\hline Molybdenum $^{\mathrm{j}}$ & 0.7 & 400 & 8 & $--^{k}$ & No & - \\
\hline Nickel & $11.5(<\mathrm{BG})$ & 1,600 & $19.1^{\mathrm{f}}$ & 27.4 & No & - \\
\hline Strontium $^{\mathrm{j}}$ & 57 & 48,000 & 960 & $--^{k}$ & No & -- \\
\hline Titanium & $1,637(<\mathrm{BG})$ & $320,000^{n}$ & $6,400^{\mathrm{n}}$ & $--^{k}$ & No & - \\
\hline Vanadium & $48.6(<\mathrm{BG})$ & 560 & $85.1^{\mathrm{f}}$ & $-{ }^{\mathrm{k}}$ & No & -- \\
\hline Zinc & $44.8(<\mathrm{BG})$ & 24,000 & 480 & $67.8^{f}$ & No & -- \\
\hline beta-BHC & 0.00060 & 0.556 & 0.00486 & 0.00554 & No & -- \\
\hline Endosulfan sulfate & 0.00053 & 480 & 9.6 & 0.186 & No & - \\
\hline 2-Methylnaphthalene & 0.034 & 320 & 3.2 & $--^{k}$ & No & -- \\
\hline Benzo(a)pyrene & 0.021 & $0.33^{\circ}$ & $0.33^{\circ}$ & $0.33^{\circ}$ & No & -- \\
\hline Benzo(b)fluoranthene & 0.023 & $1.37^{\mathrm{r}}$ & $0.33^{\circ}$ & $0.33^{\circ}$ & No & -- \\
\hline Benzo(k)fluoranthene & 0.022 & $13.7^{\mathrm{r}}$ & $0.33^{\circ}$ & $0.33^{\circ}$ & No & -- \\
\hline Chrysene & 0.022 & $137^{\mathrm{r}}$ & $1.2^{\mathrm{r}}$ & $0.33^{\circ}$ & No & -- \\
\hline Naphthalene & 0.024 & 1,600 & 16 & 988 & No & -- \\
\hline Phenanthrene $^{\mathrm{s}}$ & 0.024 & 24,000 & 240 & 1,920 & No & -- \\
\hline
\end{tabular}

* All Table 6 a notes and acronyms are provided at the end of the document body. 
Table 6b. Comparison of Statistical Contaminant Concentrations to Action Levels for the 100-B-14:2 (Area 2) BCL Stockpiles Verification Sampling Event."

\begin{tabular}{|c|c|c|c|c|c|c|}
\hline \multirow[b]{2}{*}{$\mathrm{COC} / \mathrm{COPC}$} & \multirow[b]{2}{*}{$\begin{array}{c}\text { Maximum } \\
\text { Result } \\
(\mathbf{m g} / \mathrm{kg})\end{array}$} & \multicolumn{3}{|c|}{ Remedial Action Goals ${ }^{\mathrm{a}}$ (mg/kg) } & \multirow[b]{2}{*}{$\begin{array}{l}\text { Does the } \\
\text { Maximum } \\
\text { Result } \\
\text { Exceed } \\
\text { RAGs? }\end{array}$} & \multirow[b]{2}{*}{$\begin{array}{l}\text { Does the } \\
\text { Maximum } \\
\text { Result Pass } \\
\text { RESRAD } \\
\text { Modeling? }\end{array}$} \\
\hline & & $\begin{array}{c}\text { Direct } \\
\text { Exposure }\end{array}$ & $\begin{array}{l}\text { Soil Cleanup } \\
\text { Level for } \\
\text { Groundwater } \\
\text { Protection }\end{array}$ & $\begin{array}{l}\text { Soil } \\
\text { Cleanup } \\
\text { Level for } \\
\text { River } \\
\text { Protection }\end{array}$ & & \\
\hline Arsenic & $2.9(<\mathrm{BG})$ & 20 & 20 & 20 & No & -- \\
\hline Barium & 141 & $5,600^{\mathrm{d}}$ & $132^{\mathrm{e}, \mathrm{f}}$ & $224^{\mathrm{g}}$ & Yes & Yes $^{\mathrm{h}}$ \\
\hline Beryllium & $0.34(<\mathrm{BG})$ & $10.4^{\mathrm{i}}$ & $1.51^{\mathrm{f}}$ & $1.51^{\mathrm{f}}$ & No & -- \\
\hline Boron $^{\mathrm{j}}$ & 3.6 & 16,000 & 320 & $--^{k}$ & No & -- \\
\hline Cadmium $^{1}$ & $0.21(<\mathrm{BG})$ & 13.9 & $0.81^{\mathrm{f}}$ & $0.81^{\mathrm{f}}$ & No & -- \\
\hline Chromium (total) & $8.9(<\mathrm{BG})$ & $80,000^{d}$ & $18.5^{\mathrm{f}}$ & $18.5^{\mathrm{f}}$ & No & -- \\
\hline Chromium (hexavalent) & 0.28 & 2.1 & $4.8^{\mathrm{m}}$ & 2 & No & - \\
\hline Cobalt & $9.2(<\mathrm{BG})$ & 1,600 & 32 & $--^{k}$ & No & - \\
\hline Copper & $17.6(<\mathrm{BG})$ & 2,960 & 59.2 & $22.0^{\mathrm{f}}$ & No & - \\
\hline Lead & $6.0(<\mathrm{BG})$ & 353 & $10.2^{\mathrm{f}}$ & $10.2^{\mathrm{f}}$ & No & - \\
\hline Lithium & $6.7(<\mathrm{BG})$ & 1,600 & $33.5^{\mathrm{f}}$ & $-{ }^{k}$ & No & - \\
\hline Manganese & $351(<\mathrm{BG})$ & 11,200 & $512^{\mathrm{f}}$ & $512^{\mathrm{f}}$ & No & -- \\
\hline Mercury & $0.08(<\mathrm{BG})$ & 24 & $0.33^{\mathrm{f}}$ & $0.33^{\mathrm{f}}$ & No & - \\
\hline Molybdenum $^{\mathrm{j}}$ & 0.51 & 400 & 8 & $-{ }^{k}$ & No & - \\
\hline Nickel & $11.1(<\mathrm{BG})$ & 1,600 & $19.1^{\mathrm{f}}$ & 27.4 & No & -- \\
\hline Strontium ${ }^{\mathrm{j}}$ & 57.1 & 48,000 & 960 & $--^{k}$ & No & -- \\
\hline Titanium & $1,490(<\mathrm{BG})$ & $320,000^{n}$ & $6,400^{\mathrm{n}}$ & $--^{k}$ & No & -- \\
\hline Vanadium & $47.2(<\mathrm{BG})$ & 560 & $85.1^{\mathrm{f}}$ & $--^{k}$ & No & -- \\
\hline Zinc & $43.6(<\mathrm{BG})$ & 24,000 & 480 & $67.8^{f}$ & No & -- \\
\hline beta-BHC & 0.0043 & 0.556 & 0.00486 & 0.00554 & No & -. \\
\hline
\end{tabular}

* All Table 6 b notes and acronyms are provided at the end of the document body.

100-B-14:2 (Area 2), 1607-B7 Sanitary Sewer Pipeline Data Evaluation

Residual concentrations of barium in the remediation footprint and BCL stockpiles were determined to exceed soil RAGs for the protection of groundwater at area 2 of the 100-B-14:2 subsite. Based on the $\mathrm{K}_{\mathrm{d}}$ value for barium $(25 \mathrm{~mL} / \mathrm{g})$ and the discussion of the contaminant depth/K $\mathrm{K}_{\mathrm{d}}$ value model presented within the confirmatory sampling discussion, above, and the 100 Area Analogous Sites RESRAD Calculations calculation brief (Appendix D), this constituent is not expected to migrate further than $3 \mathrm{~m}(10 \mathrm{ft})$ vertically in 1,000 years. The vadose zone underlying the 100-B-14:2 (area 2) excavation is approximately 18-m (59-ft)-thick; therefore, residual concentrations of barium are protective of groundwater.

The individual gross alpha and beta analytical results were below background activity levels for all samples, with the exception of the statistical sample collected at location 7 (J11K91), which 
had a slightly elevated gross beta reading (28.8 pCi/g). Accordingly, per the work instruction (WCH 2006c), radiostrontium-specific analysis was performed for this sample only. This result was used to quantify residual strontium-90 for the entire remediation footprint (as reported in Table 6a) without further statistical evaluation, and is likely conservative, as the gross beta measurements for all other statistical samples were below background.

Nonradionuclide risk requirements include an individual hazard quotient of less than 1.0, a cumulative hazard quotient of less than 1.0, an individual contaminant carcinogenic risk of less than $1 \times 10^{-6}$, and a cumulative carcinogenic risk of less than $1 \times 10^{-5}$. For area 2 of the 100-B-14:2 subsite, these risk values were conservatively calculated using the higher of the remediation footprint statistical value and the BCL material maximum value for each constituent. These risk values were not calculated for constituents that were either not detected or were detected at concentrations below Hanford Site or Washington State background values. All individual hazard quotients were less than 1.0, and all individual excess carcinogenic risk values were less than $1 \times 10^{-6}$ (Appendix B). The cumulative hazard quotient for those noncarcinogenic constituents above background or detection levels is $3.4 \times 10^{-2}$, and the cumulative excess carcinogenic risk value for these constituents is $3.1 \times 10^{-7}$. Therefore, nonradionuclide risk requirements are met.

When using a statistical sampling approach, a RAG requirement for nonradionuclides is the WAC 173-340-740(7)(e) three-part test. The application of the three-part test for the 100-B-14:2 (area 2) remediation footprint is included in the site-specific statistical calculations (Appendix B). The three-part test is not applicable to the BCL stockpile results, since direct evaluation of the maximum detected sampling results was used as the compliance basis. All residual COC/COPC concentrations for the 100-B-14:2 (area 2) remediation footprint pass the three-part test in comparison to direct exposure RAGs. Residual concentrations of barium fail the three-part test in comparison to soil RAGs for the protection of groundwater. However, as described above, barium is not predicted to reach groundwater within 1,000 years. Residual concentrations are, therefore, protective of groundwater.

Table 7a. Comparison of Statistical Contaminant Concentrations to Action Levels for the 100-B-14:2 (Area 4) Remediation Footprint Verification Sampling Event." (3 Pages)

\begin{tabular}{|c|c|c|c|c|c|c|}
\hline \multirow[b]{2}{*}{ COC/COPC } & \multirow[b]{2}{*}{$\begin{array}{c}\text { Statistical } \\
\text { Result } \\
(\mathrm{mg} / \mathrm{kg})\end{array}$} & \multicolumn{3}{|c|}{ Remedial Action Goals ${ }^{\mathrm{a}}$ (mg/kg) } & \multirow[b]{2}{*}{$\begin{array}{c}\text { Does the } \\
\text { Statistical } \\
\text { Data Set } \\
\text { Exceed } \\
\text { RAGs? }\end{array}$} & \multirow[b]{2}{*}{$\begin{array}{l}\text { Does the } \\
\text { Statistical } \\
\text { Result Pass } \\
\text { RESRAD } \\
\text { Modeling? }\end{array}$} \\
\hline & & $\begin{array}{c}\text { Direct } \\
\text { Exposure }\end{array}$ & $\begin{array}{l}\text { Soil Cleanup } \\
\text { Level for } \\
\text { Groundwater } \\
\text { Protection }\end{array}$ & $\begin{array}{c}\text { Soil } \\
\text { Cleanup } \\
\text { Level for } \\
\text { River } \\
\text { Protection }\end{array}$ & & \\
\hline Antimony $^{1}$ & $0.64(<\mathrm{BG})$ & 32 & $5^{\mathrm{f}}$ & $5^{f}$ & No & -- \\
\hline Arsenic & $4.2(<\mathrm{BG})$ & 20 & 20 & 20 & No & -- \\
\hline Barium & $74(<\mathrm{BG})$ & $5,600^{\mathrm{d}}$ & $132^{\mathrm{e}, \mathrm{f}}$ & $224^{g}$ & No & -- \\
\hline Beryllium & $0.50(<\mathrm{BG})$ & $10.4^{\mathrm{i}}$ & $1.51^{\mathrm{f}}$ & $1.51^{\mathrm{f}}$ & No & -- \\
\hline Boron $^{j}$ & 3.6 & 16,000 & 320 & $--^{k}$ & No & -- \\
\hline Cadmium $^{1}$ & $0.18(<\mathrm{BG})$ & 13.9 & $0.81^{\mathrm{f}}$ & $0.81^{\mathrm{f}}$ & No & - \\
\hline
\end{tabular}


Table 7a. Comparison of Statistical Contaminant Concentrations to Action Levels for the 100-B-14:2 (Area 4) Remediation Footprint Verification Sampling Event. ${ }^{*}$ (3 Pages)

\begin{tabular}{|c|c|c|c|c|c|c|}
\hline \multirow[b]{2}{*}{$\mathrm{COC} / \mathrm{COPC}$} & \multirow[b]{2}{*}{$\begin{array}{c}\text { Statistical } \\
\text { Result } \\
(\mathbf{m g} / \mathrm{kg})\end{array}$} & \multicolumn{3}{|c|}{ Remedial Action Goals ${ }^{\text {a }}$ (mg/kg) } & \multirow[b]{2}{*}{$\begin{array}{c}\text { Does the } \\
\text { Statistical } \\
\text { Data Set } \\
\text { Exceed } \\
\text { RAGs? }\end{array}$} & \multirow[b]{2}{*}{$\begin{array}{l}\text { Does the } \\
\text { Statistical } \\
\text { Result Pass } \\
\text { RESRAD } \\
\text { Modeling? }\end{array}$} \\
\hline & & $\begin{array}{c}\text { Direct } \\
\text { Exposure }\end{array}$ & $\begin{array}{l}\text { Soil Cleanup } \\
\text { Level for } \\
\text { Groundwater } \\
\text { Protection }\end{array}$ & $\begin{array}{c}\text { Soil } \\
\text { Cleanup } \\
\text { Level for } \\
\text { River } \\
\text { Protection }\end{array}$ & & \\
\hline Chromium (total) & $11.2(<\mathrm{BG})$ & $80,000^{\mathrm{d}}$ & $18.5^{\mathrm{f}}$ & $18.5^{\mathrm{f}}$ & No & -- \\
\hline Chromium (hexavalent) & 0.74 & 2.1 & $4.8^{\mathrm{m}}$ & 2 & No & - \\
\hline Cobalt & $8.4(<\mathrm{BG})$ & 1,600 & 32 & $--^{k}$ & No & -- \\
\hline Copper & 23.8 & 2,960 & 59.2 & $22.0^{\mathrm{f}}$ & Yes & Yes $^{\mathrm{h}}$ \\
\hline Lead & 27.1 & 353 & $10.2^{\mathrm{f}}$ & $10.2^{\mathrm{f}}$ & Yes & Yes $^{\mathrm{h}}$ \\
\hline Lithium & $6.8(<\mathrm{BG})$ & 1,600 & $33.5^{\mathrm{f}}$ & $--^{k}$ & No & -- \\
\hline Manganese & $343(<\mathrm{BG})$ & 11,200 & $512^{\mathrm{f}}$ & $512^{\mathrm{f}}$ & No & -- \\
\hline Mercury & 0.47 & 24 & $0.33^{\mathrm{f}}$ & $0.33^{\mathrm{f}}$ & Yes & Yes $^{\mathrm{h}}$ \\
\hline Molybdenum $^{\mathrm{j}}$ & 0.8 & 400 & 8 & $--^{k}$ & No & -- \\
\hline Nickel & $15.8(<\mathrm{BG})$ & 1,600 & $19.1^{\mathrm{f}}$ & 27.4 & No & -- \\
\hline Strontium $^{\mathrm{j}}$ & 36.2 & 48,000 & 960 & $--^{k}$ & No & -- \\
\hline $\operatorname{Tin}^{j}$ & 1.1 & 48,000 & 960 & $-{ }^{k}$ & No & -- \\
\hline Titanium & $1,411(<\mathrm{BG})$ & $320,000^{\mathrm{n}}$ & $6,400^{n}$ & $-{ }^{k}$ & No & -- \\
\hline Vanadium & $45.6(<\mathrm{BG})$ & 560 & $85.1^{\mathrm{f}}$ & $-{ }^{k}$ & No & -- \\
\hline Zinc & 82 & 24,000 & 480 & $67.8^{\mathrm{f}}$ & Yes & Yes $^{\mathrm{h}}$ \\
\hline Aroclor-1254 & 0.050 & 0.5 & $0.017^{\circ}$ & $0.017^{\circ}$ & Yes & Yes $^{\mathrm{h}}$ \\
\hline Aroclor-1260 & 0.0053 & 0.5 & $0.017^{\circ}$ & $0.017^{\circ}$ & No & -- \\
\hline Aldrin & 0.0024 & 0.0588 & $0.00165^{\circ}$ & $0.00165^{\circ}$ & Yes & Yes $^{\mathrm{h}}$ \\
\hline 4,4'-DDD & 0.0021 & 4.17 & 0.0365 & $0.005^{\circ}$ & No & -- \\
\hline 4,4'-DDE & 0.0076 & 2.94 & 0.0257 & $0.005^{\circ}$ & Yes & Yes $^{\mathrm{h}}$ \\
\hline 4,4'-DDT & 0.0067 & 2.94 & 0.0257 & $0.005^{\circ}$ & Yes & Yes $^{\mathrm{h}}$ \\
\hline Dieldrin & 0.0036 & 0.0625 & $0.003^{\circ}$ & $0.003^{\circ}$ & Yes & Yes $^{h}$ \\
\hline Endosulfan sulfate & 0.0033 & 480 & 9.6 & 0.186 & No & -- \\
\hline Endrin aldehyde & 0.0016 & 24 & 0.2 & 0.039 & No & - \\
\hline gamma-Chlordane & 0.0013 & $2.86^{\mathrm{q}}$ & $0.025^{\mathrm{q}}$ & $0.0165^{\circ}$ & No & -- \\
\hline Acenaphthene & 0.21 & 4,800 & 96 & 129 & No & -- \\
\hline Anthracene & 0.39 & 24,000 & 240 & 1,920 & No & -- \\
\hline Benzo(a)anthracene & 1.2 & $1.37^{\mathrm{r}}$ & $0.33^{\circ}$ & $0.33^{\circ}$ & $\mathrm{No}^{\mathrm{t}}$ & - \\
\hline Benzo(a)pyrene & 1.1 & $0.33^{\circ}$ & $0.33^{\circ}$ & $0.33^{\circ}$ & $\mathrm{No}^{\mathrm{t}}$ & -- \\
\hline Benzo(b)fluoranthene & 0.8 & $1.37^{\mathrm{r}}$ & $0.33^{\circ}$ & $0.33^{\circ}$ & $\mathrm{No}^{\mathrm{t}}$ & $\cdots$ \\
\hline Benzo(g,h,i)perylene & 0.8 & 2,400 & 48 & 192 & No & -- \\
\hline Benzo(k)fluoranthene & 0.9 & $13.7^{\mathrm{r}}$ & $0.33^{\circ}$ & $0.33^{\circ}$ & $\mathrm{No}^{\mathrm{t}}$ & -- \\
\hline Carbazole & 0.25 & 50 & 0.438 & $--^{k}$ & No & -- \\
\hline
\end{tabular}


Table 7a. Comparison of Statistical Contaminant Concentrations to Action Levels for the 100-B-14:2 (Area 4) Remediation Footprint Verification Sampling Event." (3 Pages)

\begin{tabular}{|c|c|c|c|c|c|c|}
\hline \multirow[b]{2}{*}{$\mathrm{COC} / \mathrm{COPC}$} & \multirow[b]{2}{*}{$\begin{array}{c}\text { Statistical } \\
\text { Result } \\
(\mathrm{mg} / \mathrm{kg})\end{array}$} & \multicolumn{3}{|c|}{ Remedial Action Goals ${ }^{\mathrm{a}}$ (mg/kg) } & \multirow[b]{2}{*}{$\begin{array}{c}\text { Does the } \\
\text { Statistical } \\
\text { Data Set } \\
\text { Exceed } \\
\text { RAGs? }\end{array}$} & \multirow[b]{2}{*}{$\begin{array}{l}\text { Does the } \\
\text { Statistical } \\
\text { Result Pass } \\
\text { RESRAD } \\
\text { Modeling? }\end{array}$} \\
\hline & & $\begin{array}{c}\text { Direct } \\
\text { Exposure }\end{array}$ & $\begin{array}{l}\text { Soil Cleanup } \\
\text { Level for } \\
\text { Groundwater } \\
\text { Protection }\end{array}$ & $\begin{array}{c}\text { Soil } \\
\text { Cleanup } \\
\text { Level for } \\
\text { River } \\
\text { Protection }\end{array}$ & & \\
\hline Chrysene & 1.1 & $137^{\mathrm{r}}$ & $1.2^{\mathrm{r}}$ & $0.33^{\circ}$ & $\mathrm{No}^{\mathrm{t}}$ & -- \\
\hline Di-n-butylphthalate & 0.030 & 8,000 & 160 & 540 & No & -- \\
\hline Di-n-octylphthalate & 0.095 & 1,600 & 32 & $--^{k}$ & No & -- \\
\hline Dibenz(a,h)anthracene & 0.66 & $0.33^{\circ}$ & $0.33^{\circ}$ & $0.33^{\circ}$ & $\mathrm{No}^{\mathrm{t}}$ & -- \\
\hline Dibenzofuran & 0.082 & 160 & 3.2 & $-{ }^{k}$ & No & -- \\
\hline Fluoranthene & 10.4 & 3,200 & 64 & 18 & No & -- \\
\hline Fluorene & 0.13 & 3,200 & 64 & 260 & No & -- \\
\hline Indeno(1,2,3-cd)pyrene & 0.9 & 1.37 & $0.33^{\circ}$ & $0.33^{\circ}$ & $\mathrm{No}^{\mathrm{t}}$ & -- \\
\hline Naphthalene & 0.055 & 1,600 & 16 & 988 & No & -- \\
\hline Pentachlorophenol & 1.9 & 8.33 & $0.33^{\circ}$ & $0.33^{\circ}$ & $\mathrm{No}^{\mathrm{u}}$ & -- \\
\hline Phenanthrene $^{\mathrm{s}}$ & 3.0 & 24,000 & 240 & 1,920 & No & -- \\
\hline Pyrene & 9.1 & 2,400 & 48 & 192 & No & -- \\
\hline
\end{tabular}

* All Table 7a notes and acronyms are provided at the end of the document body.

Table 7b. Comparison of Statistical Contaminant Concentrations to Action Levels for the 100-B-14:2 (Area 4) BCL Stockpiles Verification Sampling Event." (2 Pages)

\begin{tabular}{|c|c|c|c|c|c|c|}
\hline \multirow[b]{2}{*}{$\mathrm{COC} / \mathrm{COPC}$} & \multirow[b]{2}{*}{$\begin{array}{c}\text { Maximum } \\
\text { Result } \\
(\mathrm{mg} / \mathrm{kg})\end{array}$} & \multicolumn{3}{|c|}{ Remedial Action Goals ${ }^{\mathbf{a}}$ (mg/kg) } & \multirow[b]{2}{*}{$\begin{array}{l}\text { Does the } \\
\text { Maximum } \\
\text { Result } \\
\text { Exceed } \\
\text { RAGs? }\end{array}$} & \multirow[b]{2}{*}{$\begin{array}{l}\text { Does the } \\
\text { Maximum } \\
\text { Result Pass } \\
\text { RESRAD } \\
\text { Modeling? }\end{array}$} \\
\hline & & $\begin{array}{c}\text { Direct } \\
\text { Exposure }\end{array}$ & $\begin{array}{l}\text { Soil Cleanup } \\
\text { Level for } \\
\text { Groundwater } \\
\text { Protection }\end{array}$ & $\begin{array}{c}\text { Soil } \\
\text { Cleanup } \\
\text { Level for } \\
\text { River } \\
\text { Protection }\end{array}$ & & \\
\hline Arsenic & $3.8(<\mathrm{BG})$ & 20 & 20 & 20 & No & -- \\
\hline Barium & $72.1(<\mathrm{BG})$ & $5,600^{\mathrm{d}}$ & $132^{\mathrm{e}, \mathrm{f}}$ & $224^{g}$ & No & -- \\
\hline Beryllium & $0.55(<\mathrm{BG})$ & $10.4^{\mathrm{i}}$ & $1.51^{\mathrm{f}}$ & $1.51^{\mathrm{f}}$ & No & -- \\
\hline Cadmium $^{1}$ & $0.10(<\mathrm{BG})$ & 13.9 & $0.81^{\mathrm{f}}$ & $0.81^{\mathrm{f}}$ & No & -- \\
\hline Chromium (total) & $10.7(<\mathrm{BG})$ & $80,000^{\mathrm{d}}$ & $18.5^{\mathrm{f}}$ & $18.5^{\mathrm{f}}$ & No & -- \\
\hline Chromium (hexavalent) & 0.98 & 2.1 & $4.8^{\mathrm{m}}$ & 2 & No & -- \\
\hline Cobalt & $7.7(<\mathrm{BG})$ & 1,600 & 32 & $-{ }^{\mathrm{k}}$ & No & -- \\
\hline Copper & $18.1(<\mathrm{BG})$ & 2,960 & 59.2 & $22.0^{\mathrm{f}}$ & No & -- \\
\hline Lead & 17.0 & 353 & $10.2^{\mathrm{f}}$ & $10.2^{\mathrm{f}}$ & Yes & Yes $^{\mathrm{h}}$ \\
\hline Lithium & $7.0(<\mathrm{BG})$ & 1,600 & $33.5^{\mathrm{f}}$ & $--^{k}$ & No & -- \\
\hline Manganese & $335(<\mathrm{BG})$ & 11,200 & $512^{f}$ & $512^{\mathrm{f}}$ & No & -- \\
\hline
\end{tabular}


Table 7b. Comparison of Statistical Contaminant Concentrations to Action Levels for the 100-B-14:2 (Area 4) BCL Stockpiles Verification Sampling Event." (2 Pages)

\begin{tabular}{|c|c|c|c|c|c|c|}
\hline \multirow[b]{2}{*}{$\mathrm{COC} / \mathrm{COPC}$} & \multirow[b]{2}{*}{$\begin{array}{c}\text { Maximum } \\
\text { Result } \\
(\mathbf{m g} / \mathrm{kg})\end{array}$} & \multicolumn{3}{|c|}{ Remedial Action Goals ${ }^{\mathrm{a}}(\mathrm{mg} / \mathrm{kg}$ ) } & \multirow[b]{2}{*}{$\begin{array}{l}\text { Does the } \\
\text { Maximum } \\
\text { Result } \\
\text { Exceed } \\
\text { RAGs? }\end{array}$} & \multirow[b]{2}{*}{$\begin{array}{l}\text { Does the } \\
\text { Maximum } \\
\text { Result Pass } \\
\text { RESRAD } \\
\text { Modeling? }\end{array}$} \\
\hline & & $\begin{array}{c}\text { Direct } \\
\text { Exposure }\end{array}$ & $\begin{array}{l}\text { Soil Cleanup } \\
\text { Level for } \\
\text { Groundwater } \\
\text { Protection }\end{array}$ & $\begin{array}{c}\text { Soil } \\
\text { Cleanup } \\
\text { Level for } \\
\text { River } \\
\text { Protection } \\
\end{array}$ & & \\
\hline Mercury & 0.47 & 24 & $0.33^{\mathrm{f}}$ & $0.33^{\mathrm{f}}$ & Yes & $\mathrm{Yes}^{\mathrm{h}}$ \\
\hline Molybdenum $^{\mathrm{j}}$ & 0.60 & 400 & 8 & $-{ }^{\mathrm{k}}$ & No & -- \\
\hline Nickel & $12.2(<\mathrm{BG})$ & 1,600 & $19.1^{\mathrm{f}}$ & 27.4 & No & -- \\
\hline Strontium ${ }^{\mathrm{j}}$ & 31.1 & 48,000 & 960 & $--^{k}$ & No & -- \\
\hline Titanium & $1,480(<\mathrm{BG})$ & $320,000^{n}$ & $6,400^{\mathrm{n}}$ & $-{ }^{\mathrm{k}}$ & No & - \\
\hline Vanadium & $47.4(<\mathrm{BG})$ & 560 & $85.1^{\mathrm{f}}$ & $--^{k}$ & No & -- \\
\hline Zinc & $48.8(<\mathrm{BG})$ & 24,000 & 480 & $67.8^{\mathrm{f}}$ & No & - \\
\hline Aroclor-1254 & 0.015 & 0.5 & $0.017^{\circ}$ & $0.017^{\circ}$ & No & - \\
\hline alpha-BHC & 0.0020 & 0.159 & $0.00165^{\circ}$ & $0.00165^{\circ}$ & No & -- \\
\hline Dieldrin & 0.00070 & 0.0625 & $0.003^{\circ}$ & $0.003^{\circ}$ & Yes & Yes $^{\mathrm{h}}$ \\
\hline Anthracene & 0.39 & 24,000 & 240 & 1,920 & No & -- \\
\hline Benzo(a)anthracene & 1.6 & $1.37^{\mathrm{r}}$ & $0.33^{\circ}$ & $0.33^{\circ}$ & $\mathrm{No}^{\mathrm{t}}$ & - \\
\hline Benzo(a)pyrene & 1.5 & $0.33^{\circ}$ & $0.33^{\circ}$ & $0.33^{\circ}$ & $\mathrm{No}^{\mathrm{t}}$ & - \\
\hline Benzo(b)fluoranthene & 1.3 & $1.37^{\mathrm{r}}$ & $0.33^{\circ}$ & $0.33^{\circ}$ & $\mathrm{No}^{\mathrm{t}}$ & - \\
\hline Benzo(g,h,i)perylene ${ }^{\mathrm{s}}$ & 1.2 & 2,400 & 48 & 192 & No & -- \\
\hline Benzo(k)fluoranthene & 1.3 & $13.7^{\mathrm{r}}$ & $0.33^{\circ}$ & $0.33^{\circ}$ & $\mathrm{No}^{\mathrm{t}}$ & -- \\
\hline Chrysene & 0.26 & $137^{\mathrm{r}}$ & $1.2^{\mathrm{r}}$ & $0.33^{\circ}$ & No & -- \\
\hline Fluoranthene & 0.44 & 3,200 & 64 & 18 & No & - \\
\hline Indeno(1,2,3-cd)pyrene & 0.087 & 1.37 & $0.33^{\circ}$ & $0.33^{\circ}$ & No & -- \\
\hline Phenanthrene $^{\mathrm{s}}$ & 0.42 & 24,000 & 240 & 1,920 & No & - \\
\hline Pyrene & 0.71 & 2,400 & 48 & 192 & No & -- \\
\hline
\end{tabular}

* All Table $7 \mathrm{~b}$ notes and acronyms are provided at the end of the document body.

100-B-14:2 (Area 4), 190-B Pumphouse Sanitary Sewer Pipelines Data Evaluation

Residual concentrations of copper, lead, mercury, zinc, aroclor-1254, Aldrin, 4,4'-DDE, $4,4^{\prime}$-DDT, and dieldrin in the remediation footprint and concentrations of lead, mercury, and dieldrin in the BCL stockpiles were determined to exceed soil RAGs for the protection of groundwater and/or the river at area 4 of the 100-B-14:2 subsite. Based on the $\mathrm{K}_{\mathrm{d}}$ values for these contaminants $(>22 \mathrm{~mL} / \mathrm{g})$ and the discussion of the contaminant depth/ $\mathrm{K}_{\mathrm{d}}$ value model presented within the confirmatory sampling discussion, above, and the 100 Area Analogous Sites RESRAD Calculations calculation brief (Appendix D), none are expected to migrate further than $3 \mathrm{~m}$ (10 ft) vertically in 1,000 years. The vadose zone underlying the 100-B-14:2 (area 4) excavation is approximately $18-\mathrm{m}$ (59-ft)-thick; therefore, residual concentrations of these 
constituents are protective of groundwater. The only pathway for contamination to reach the Columbia River is via groundwater migration, so these contaminant concentrations are also protective of river water.

Multiple PAHs were also detected above soil RAGs within the remediation footprint and BCL stockpiles at the site. However, the sanitary sewers associated with the 185-B/190-B Building were formerly overlain by asphalt paving and residual fragments of the paving were visible within the site excavation. Asphaltic fragments within the verification samples would result in elevated detections of PAHs. A comparison of the statistical PAH concentrations in the verification data set to a known asphalt sample (Table 8) shows a good correlation, as indicated by the Ratio Column. Asphalt that has been used for structural and construction purposes is excluded from consideration as a dangerous waste in WAC 173-303-071(3)(e), is listed as an inert waste in WAC 173-350-990(2)(b), and does not present a significant risk to human health or the environment.

Table 8. Comparison of 100-B-14:2 (Area 4) Statistical Results to a Known Asphalt Sample. (1 Pages)

\begin{tabular}{|l|c|c|c|}
\hline \multicolumn{1}{|c|}{ Analyte } & $\begin{array}{c}\text { Asphalt Sample } \\
\text { Result } \\
\text { (mg/kg) }\end{array}$ & $\begin{array}{c}\text { Statistical } \\
\text { Verification } \\
\text { Result } \\
\text { (mg/kg) }\end{array}$ & $\begin{array}{c}\text { Ratio } \\
\text { (x 10 }\end{array}$ \\
\hline 2-Methylnaphthalene & 394 & ND & -- \\
\hline Acenaphthene & 1,783 & 0.21 & 1.18 \\
\hline Anthracene & 3,699 & 0.39 & 1.05 \\
\hline Benzo(a)anthracene & 5,792 & 1.2 & 2.07 \\
\hline Benzo(a)pyrene & 5,533 & 1.1 & 1.99 \\
\hline Benzo(b)fluoranthene & 4,619 & 0.8 & 1.73 \\
\hline Benzo(g,h,i)perylene & 2,839 & 0.8 & 2.82 \\
\hline Benzo(k)fluoranthene & 4,527 & 0.9 & 1.99 \\
\hline Carbazole & 2,049 & 0.25 & 1.22 \\
\hline Chrysene & 5,580 & 1.1 & 1.97 \\
\hline Dibenzo(a,h)anthracene & 1,531 & 0.66 & 4.31 \\
\hline Dibenzofuran & 1,135 & 0.082 & 0.72 \\
\hline Fluoranthene & 10,665 & 10.4 & 9.75 \\
\hline Fluorene & 1,756 & 0.13 & 0.74 \\
\hline Indeno(1,2,3-cd) pyrene & 2,751 & 0.9 & 3.27 \\
\hline Naphthalene & 1,917 & 0.055 & 0.29 \\
\hline Phenanthrene & 10,975 & 3.0 & 2.73 \\
\hline Pyrene & 10,205 & 9.1 & 8.92 \\
\hline
\end{tabular}

$--=$ not applicable

$\mathrm{ND}=$ not detected 
Nonradionuclide risk requirements include an individual hazard quotient of $<1.0$, a cumulative hazard quotient of $<1.0$, an individual contaminant carcinogenic risk of $<1 \times 10^{-6}$, and a cumulative carcinogenic risk of $<1 \times 10^{-5}$. For area 4 of the 100-B-14:2 subsite, these risk values were conservatively calculated using the higher of the remediation footprint statistical value and the BCL material maximum value for each constituent. These risk values were not calculated for constituents that were not detected, were detected at concentrations below Hanford Site or Washington State background values, or were the result of asphalt cross-contamination of the sample matrices. All individual hazard quotients were $<1.0$, and all individual excess carcinogenic risk values were $<1 \times 10^{-6}$ (Appendix B). The cumulative hazard quotient for those noncarcinogenic constituents above background or detection levels is $7.3 \times 10^{-2}$, and the cumulative excess carcinogenic risk value for these constituents is $9.2 \times 10^{-7}$. Therefore, nonradionuclide risk requirements are met.

When using a statistical sampling approach, a RAG requirement for nonradionuclides is the WAC 173-340-740(7)(e) three-part test. The application of the three-part test for the 100-B-14:2 (area 4) remediation footprint is included in the site-specific statistical calculations (Appendix B). The three-part test is not applicable to the BCL stockpile results since direct evaluation of the maximum detected sampling results was used as the compliance basis. Copper, lead, zinc, aroclor-1254, and multiple PAHs failed the three-part test in comparison to the most restrictive RAGs. However, as described above, residual concentrations of these contaminants have either been demonstrated to be protective of groundwater and the Columbia River or the result of asphalt cross-contamination.

Table 9a. Comparison of Statistical Contaminant Concentrations to Action Levels for the 100-B-14:2 (Area 5) Remediation Footprint Verification Sampling Event." (2 Pages)

\begin{tabular}{|c|c|c|c|c|c|c|}
\hline \multirow[b]{2}{*}{$\mathrm{COC} / \mathrm{COPC}$} & \multirow[b]{2}{*}{$\begin{array}{l}\text { Statistical } \\
\text { Result } \\
(\mathbf{p C i} / \mathrm{g})\end{array}$} & \multicolumn{3}{|c|}{ Generic Site Lookup Values ${ }^{\mathrm{a}}(\mathrm{pCi} / \mathrm{g})$} & \multirow{2}{*}{$\begin{array}{c}\text { Does the } \\
\text { Statistical } \\
\text { Result } \\
\text { Exceed } \\
\text { Lookup } \\
\text { Values? }\end{array}$} & \multirow[b]{2}{*}{$\begin{array}{l}\text { Does the } \\
\text { Statistical } \\
\text { Result Pass } \\
\text { RESRAD } \\
\text { Modeling? }\end{array}$} \\
\hline & & $\begin{array}{l}\text { Shallow } \\
\text { Zone } \\
\text { Lookup } \\
\text { Value }^{b}\end{array}$ & $\begin{array}{c}\text { Groundwater } \\
\text { Protection } \\
\text { Lookup } \\
\text { Value }\end{array}$ & $\begin{array}{c}\text { River } \\
\text { Protection } \\
\text { Lookup } \\
\text { Value }\end{array}$ & & \\
\hline Cesium-137 & 0.048 & 6.2 & $1,465^{\mathrm{c}}$ & $1,465^{\mathrm{c}}$ & No & -- \\
\hline \multirow[b]{2}{*}{$\mathrm{COC} / \mathrm{COPC}$} & \multirow[b]{2}{*}{$\begin{array}{c}\text { Statistical } \\
\text { Result } \\
(\mathbf{m g} / \mathrm{kg})\end{array}$} & \multicolumn{3}{|c|}{ Remedial Action Goals ${ }^{\text {a }}$ (mg/kg) } & \multirow[b]{2}{*}{$\begin{array}{l}\text { Does the } \\
\text { Statistical } \\
\text { Data Set } \\
\text { Exceed } \\
\text { RAGs? }\end{array}$} & \multirow[b]{2}{*}{$\begin{array}{l}\text { Does the } \\
\text { Statistical } \\
\text { Result Pass } \\
\text { RESRAD } \\
\text { Modeling? }\end{array}$} \\
\hline & & $\begin{array}{c}\text { Direct } \\
\text { Exposure }\end{array}$ & $\begin{array}{l}\text { Soil Cleanup } \\
\text { Level for } \\
\text { Groundwater } \\
\text { Protection }\end{array}$ & $\begin{array}{c}\text { Soil } \\
\text { Cleanup } \\
\text { Level for } \\
\text { River } \\
\text { Protection } \\
\end{array}$ & & \\
\hline Arsenic & $3.8(<\mathrm{BG})$ & 20 & 20 & 20 & No & -- \\
\hline Barium & $60.4(<\mathrm{BG})$ & $5,600^{d}$ & $132^{\mathrm{e}, \mathrm{f}}$ & $224^{\mathrm{g}}$ & No & -- \\
\hline Beryllium & $0.33(<\mathrm{BG})$ & $10.4^{\mathrm{i}}$ & $1.51^{\mathrm{f}}$ & $1.51^{\mathrm{f}}$ & No & -- \\
\hline Boron $^{j}$ & 1.7 & 16,000 & 320 & $-{ }^{k}$ & No & -- \\
\hline Cadmium $^{1}$ & $0.16(<\mathrm{BG})$ & 13.9 & $0.81^{\mathrm{f}}$ & $0.81^{\mathrm{f}}$ & No & -- \\
\hline Chromium (total) & $7.7(<\mathrm{BG})$ & $80,000^{d}$ & $18.5^{\mathrm{f}}$ & $18.5^{\mathrm{f}}$ & No & - \\
\hline
\end{tabular}


Table 9a. Comparison of Statistical Contaminant Concentrations to Action Levels for the 100-B-14:2 (Area 5) Remediation Footprint Verification Sampling Event." (2 Pages)

\begin{tabular}{|c|c|c|c|c|c|c|}
\hline \multirow[b]{2}{*}{$\mathrm{COC} / \mathrm{COPC}$} & \multirow[b]{2}{*}{$\begin{array}{c}\text { Statistical } \\
\text { Result } \\
\text { (mg/kg) }\end{array}$} & \multicolumn{3}{|c|}{ Remedial Action Goals ${ }^{\text {a }}$ (mg/kg) } & \multirow[b]{2}{*}{$\begin{array}{l}\text { Does the } \\
\text { Statistical } \\
\text { Data Set } \\
\text { Exceed } \\
\text { RAGs? }\end{array}$} & \multirow[b]{2}{*}{$\begin{array}{l}\text { Does the } \\
\text { Statistical } \\
\text { Result Pass } \\
\text { RESRAD } \\
\text { Modeling? }\end{array}$} \\
\hline & & $\begin{array}{c}\text { Direct } \\
\text { Exposure }\end{array}$ & $\begin{array}{l}\text { Soil Cleanup } \\
\text { Level for } \\
\text { Groundwater } \\
\text { Protection }\end{array}$ & $\begin{array}{c}\text { Soil } \\
\text { Cleanup } \\
\text { Level for } \\
\text { River } \\
\text { Protection }\end{array}$ & & \\
\hline Chromium (hexavalent) & 0.37 & 2.1 & $4.8^{\mathrm{m}}$ & 2 & No & -- \\
\hline Cobalt & $7.9(<\mathrm{BG})$ & 1,600 & 32 & $--^{k}$ & No & - \\
\hline Copper & $17.6(<\mathrm{BG})$ & 2,960 & 59.2 & $22.0^{f}$ & No & -- \\
\hline Lead & $7.0(<\mathrm{BG})$ & 353 & $10.2^{f}$ & $10.2^{\mathrm{f}}$ & No & -- \\
\hline Lithium & $7.6(<\mathrm{BG})$ & 1,600 & $33.5^{f}$ & $--^{k}$ & No & -- \\
\hline Manganese & $338(<\mathrm{BG})$ & 11,200 & $512^{\mathrm{f}}$ & $512^{f}$ & No & -- \\
\hline Mercury & $0.1(<\mathrm{BG})$ & 24 & $0: 33^{\mathrm{f}}$ & $0.33^{f}$ & No & -- \\
\hline Molybdenum $^{\mathrm{j}}$ & 0.52 & 400 & 8 & $--^{k}$ & No & -- \\
\hline Nickel & $10.9(<\mathrm{BG})$ & 1,600 & $19.1^{\mathrm{f}}$ & 27.4 & No & -- \\
\hline Strontium & 33.0 & 48,000 & 960 & $--^{k}$ & No & -- \\
\hline $\operatorname{Tin}^{\mathrm{j}}$ & 1.3 & 48,000 & 960 & $--^{k}$ & No & -- \\
\hline Titanium & $1,490(<\mathrm{BG})$ & $320,000^{\mathrm{n}}$ & $6,400^{n}$ & $--^{k}$ & No & -- \\
\hline Vanadium & $44.6(<\mathrm{BG})$ & 560 & $85.1^{\mathrm{f}}$ & $-{ }^{k}$ & No & -- \\
\hline Zinc & $39.8(<\mathrm{BG})$ & 24,000 & 480 & $67.8^{f}$ & No & -- \\
\hline Aroclor-1254 & 0.011 & 0.5 & $0.017^{\circ}$ & $0.017^{\circ}$ & No & -- \\
\hline beta-BHC & 0.00062 & 0.556 & 0.00486 & 0.00554 & No & -- \\
\hline Endrin & 0.0013 & 24 & 0.2 & 0.039 & No & - \\
\hline Methoxychlor & 0.049 & 400 & 4 & 1.67 & No & -- \\
\hline bis(2-Ethylhexyl)phthalate & 0.036 & 71.4 & 0.625 & 0.36 & No & -- \\
\hline Di-n-butylphthalate & 0.021 & 8,000 & 160 & 540 & No & -- \\
\hline Pyrene & 0.020 & 2,400 & 48 & 192 & No & -- \\
\hline
\end{tabular}

* All Table 9a notes and acronyms are provided at the end of the document body.

Table 9b. Comparison of Statistical Contaminant Concentrations to Action Levels for the 100-B-14:2 (Area 5) BCL Stockpiles Verification Sampling Event." (2 Pages)

\begin{tabular}{|c|c|c|c|c|c|c|}
\hline \multirow[b]{2}{*}{$\mathrm{COC} / \mathrm{COPC}$} & \multirow[b]{2}{*}{$\begin{array}{c}\text { Maximum } \\
\text { Result } \\
\text { (pCi/g) }\end{array}$} & \multicolumn{3}{|c|}{ Generic Site Lookup Values ${ }^{a}(\mathrm{pCi} / \mathrm{g})$} & \multirow{2}{*}{$\begin{array}{l}\text { Does the } \\
\text { Maximum } \\
\text { Result } \\
\text { Exceed } \\
\text { Lookup } \\
\text { Values? }\end{array}$} & \multirow[b]{2}{*}{$\begin{array}{l}\text { Does the } \\
\text { Maximum } \\
\text { Result Pass } \\
\text { RESRAD } \\
\text { Modeling? }\end{array}$} \\
\hline & & $\begin{array}{l}\text { Shallow } \\
\text { Zone } \\
\text { Lookup } \\
\text { Value }^{b}\end{array}$ & $\begin{array}{c}\text { Groundwater } \\
\text { Protection } \\
\text { Lookup } \\
\text { Value }\end{array}$ & $\begin{array}{c}\text { River } \\
\text { Protection } \\
\text { Lookup } \\
\text { Value }\end{array}$ & & \\
\hline Tritium & 0.296 & $459^{c}$ & $12.6^{\mathrm{c}}$ & $12.6^{\mathrm{c}}$ & No & $\ldots$ \\
\hline
\end{tabular}


Table 9b. Comparison of Statistical Contaminant Concentrations to Action Levels for the 100-B-14:2 (Area 5) BCL Stockpiles Verification Sampling Event." (2 Pages)

\begin{tabular}{|c|c|c|c|c|c|c|}
\hline \multirow[b]{2}{*}{$\mathrm{COC} / \mathrm{COPC}$} & \multirow[b]{2}{*}{$\begin{array}{c}\text { Maximum } \\
\text { Result } \\
(\mathrm{mg} / \mathrm{kg})\end{array}$} & \multicolumn{3}{|c|}{ Remedial Action Goals ${ }^{\mathrm{a}}$ (mg/kg) } & \multirow[b]{2}{*}{$\begin{array}{c}\text { Does the } \\
\text { Maximum } \\
\text { Result } \\
\text { Exceed } \\
\text { RAGs? }\end{array}$} & \multirow[b]{2}{*}{$\begin{array}{l}\text { Does the } \\
\text { Maximum } \\
\text { Result Pass } \\
\text { RESRAD } \\
\text { Modeling? }\end{array}$} \\
\hline & & $\begin{array}{c}\text { Direct } \\
\text { Exposure }\end{array}$ & $\begin{array}{l}\text { Soil Cleanup } \\
\text { Level for } \\
\text { Groundwater } \\
\text { Protection }\end{array}$ & $\begin{array}{c}\text { Soil } \\
\text { Cleanup } \\
\text { Level for } \\
\text { River } \\
\text { Protection }\end{array}$ & & \\
\hline Arsenic & $2.6(<B G)$ & 20 & 20 & 20 & No & -- \\
\hline Barium & $75.0(<\mathrm{BG})$ & $5,600^{d}$ & $132^{\mathrm{e}, \mathrm{f}}$ & $224^{g}$ & No & - \\
\hline Beryllium & $0.32(<\mathrm{BG})$ & $10.4^{\mathrm{i}}$ & $1.51^{\mathrm{f}}$ & $1.51^{\mathrm{f}}$ & No & -- \\
\hline Boron $^{\mathrm{j}}$ & 3.3 & 16,000 & 320 & $-{ }^{k}$ & No & - \\
\hline Cadmium $^{1}$ & $0.14(<\mathrm{BG})$ & 13.9 & $0.81^{f}$ & $0.81^{\mathrm{f}}$ & No & -- \\
\hline Chromium (total) & $12.7(<\mathrm{BG})$ & $80,000^{d}$ & $18.5^{\mathrm{f}}$ & $18.5^{\mathrm{f}}$ & No & -- \\
\hline Chromium (hexavalent) & 0.65 & 2.1 & $4.8^{\mathrm{m}}$ & 2 & No & -- \\
\hline Cobalt & $7.4(<B G)$ & 1,600 & 32 & $--^{k}$ & No & -- \\
\hline Copper & $15.8(<\mathrm{BG})$ & 2,960 & 59.2 & $22.0^{\mathrm{f}}$ & No & -- \\
\hline Lead & 26.1 & 353 & $10.2^{\mathrm{f}}$ & $10.2^{\mathrm{f}}$ & Yes & $\mathrm{Yes}^{\mathrm{v}}$ \\
\hline Lithium & $5.9(<\mathrm{BG})$ & 1,600 & $33.5^{\mathrm{f}}$ & $-{ }^{k}$ & No & -- \\
\hline Manganese & $324(<B G)$ & 11,200 & $512^{f}$ & $512^{f}$ & No & - \\
\hline Mercury & $0.1(<\mathrm{BG})$ & 24 & $0.33^{\mathrm{f}}$ & $0.33^{\mathrm{f}}$ & No & - \\
\hline Molybdenum $^{\mathrm{j}}$ & 0.50 & 400 & 8 & $--^{k}$ & No & -- \\
\hline Nickel & $9.7(<\mathrm{BG})$ & 1,600 & $19.1^{\mathrm{f}}$ & 27.4 & No & -- \\
\hline Strontium ${ }^{\mathrm{j}}$ & 33.1 & 48,000 & 960 & $-{ }^{k}$ & No & -- \\
\hline $\operatorname{Tin}^{j}$ & 1.3 & 48,000 & 960 & $--^{k}$ & No & -- \\
\hline Titanium & $1,250(<\mathrm{BG})$ & $320,000^{\mathrm{n}}$ & $6,400^{n}$ & $--^{k}$ & No & - \\
\hline Vanadium & $42.0(<\mathrm{BG})$ & 560 & $85.1^{\mathrm{f}}$ & $--^{k}$ & No & -- \\
\hline Zinc & $41.3(<\mathrm{BG})$ & 24,000 & 480 & $67.8^{\mathrm{f}}$ & No & -- \\
\hline bis(2-Ethylhexyl)phthalate & 0.027 & 71.4 & 0.625 & 0.36 & No & -- \\
\hline Chrysene & 0.021 & $137^{\mathrm{r}}$ & $1.2^{\mathrm{r}}$ & $0.33^{\circ}$ & No & -- \\
\hline Fluoranthene & 0.019 & 3,200 & 64 & 18 & No & - \\
\hline Pyrene & 0.019 & 2,400 & 48 & 192 & No & -- \\
\hline
\end{tabular}

* All Table $9 \mathrm{~b}$ notes and acronyms are provided at the end of the document body.

\section{0-B-14:2 (Area 5), 115-B/C Gas Recirculation Facility Sanitary Sewer Pipelines}

The residual concentration of lead in the BCL stockpiles was determined to exceed soil RAGs for the protection of groundwater and the Columbia River at area 5 of the 100-B-14:2 subsite. Based on the $K_{d}$ value for lead $(30 \mathrm{~mL} / \mathrm{g})$ and the discussion of the contaminant depth/K $\mathrm{K}_{\mathrm{d}}$ value model presented within the confirmatory sampling discussion, above, and the 100 Area Analogous Sites RESRAD Calculations calculation brief (Appendix D), this constituent is not 
expected to migrate further than $3 \mathrm{~m}(10 \mathrm{ft})$ vertically in 1,000 years. The vadose zone underlying the 100-B-14:2 (area 5) excavation is approximately 22-m (72-ft)-thick; therefore, residual concentrations of lead are protective of groundwater. No COCs/COPCs were quantified above RAGs within the remediation footprint.

The individual gross alpha and gross beta analytical results were below background activity levels for all samples. Additionally, radiostrontium analysis was inadvertently requested for samples collected from the remediation footprint, and no beta-strontium was quantitated above minimum detectable activity levels.

Nonradionuclide risk requirements include an individual hazard quotient of less than 1.0 , a cumulative hazard quotient of less than 1.0, an individual contaminant carcinogenic risk of less than $1 \times 10^{-6}$, and a cumulative carcinogenic risk of less than $1 \times 10^{-5}$. For area 5 of the 100-B-14:2 subsite, these risk values were conservatively calculated using the higher of the remediation footprint statistical value and the BCL material maximum value for each constituent. These risk values were not calculated for constituents that were either not detected or were detected at concentrations below Hanford Site or Washington State background values. All individual hazard quotients were less than 1.0, and all individual excess carcinogenic risk values were less than $1 \times 10^{-6}$ (Appendix B). The cumulative hazard quotient for those noncarcinogenic constituents above background or detection levels is $1.2 \times 10^{-2}$, and the cumulative excess carcinogenic risk value for these constituents is $3.3 \times 10^{-7}$. Therefore, nonradionuclide risk requirements are met.

When using a statistical sampling approach, a RAG requirement for nonradionuclides is the WAC 173-340-740(7)(e) three-part test. The application of the three-part test for the 100-B-14:2 (area 2) remediation footprint is included in the site-specific statistical calculations (Appendix B). The three-part test is not applicable to the BCL stockpile results since direct evaluation of the maximum detected sampling results was used as the compliance basis. All residual COC/COPC concentrations for the 100-B-14:2 (area 5) remediation footprint pass the three-part test in comparison to the most restrictive RAG.

Table 10a. Comparison of Statistical Contaminant Concentrations to Action Levels for the 1607-B2:1 Remediation Footprint Verification Sampling Event.* (2 Pages)

\begin{tabular}{|c|c|c|c|c|c|c|}
\hline \multirow[b]{2}{*}{$\mathrm{COC} / \mathrm{COPC}$} & \multirow[b]{2}{*}{$\begin{array}{c}\text { Statistical } \\
\text { Result } \\
\text { (mg/kg) }\end{array}$} & \multicolumn{3}{|c|}{ Remedial Action Goals ${ }^{\mathrm{a}}$ (mg/kg) } & \multirow[b]{2}{*}{$\begin{array}{c}\text { Does the } \\
\text { Statistical } \\
\text { Data Set } \\
\text { Exceed } \\
\text { RAGs? }\end{array}$} & \multirow[b]{2}{*}{$\begin{array}{l}\text { Does the } \\
\text { Statistical } \\
\text { Result Pass } \\
\text { RESRAD } \\
\text { Modeling? }\end{array}$} \\
\hline & & $\begin{array}{c}\text { Direct } \\
\text { Exposure }\end{array}$ & $\begin{array}{l}\text { Soil Cleanup } \\
\text { Level for } \\
\text { Groundwater } \\
\text { Protection }\end{array}$ & $\begin{array}{c}\text { Soil } \\
\text { Cleanup } \\
\text { Level for } \\
\text { River } \\
\text { Protection }\end{array}$ & & \\
\hline Antimony & $0.58(<\mathrm{BG})$ & 32 & $5^{\mathrm{f}}$ & $5^{\mathrm{f}}$ & No & -- \\
\hline Arsenic & 7.2 & 20 & 20 & 20 & No & -- \\
\hline Barium & 258 & $5,600^{d}$ & $132^{e, f}$ & $224^{\mathrm{g}}$ & Yes & Yes $^{w}$ \\
\hline Beryllium & $0.8(<\mathrm{BG})$ & $10.4^{\mathrm{i}}$ & $1.51^{\mathrm{f}}$ & $1.51^{\mathrm{f}}$ & No & -- \\
\hline Boron $^{j}$ & 8.3 & 16,000 & 320 & $--^{k}$ & No & -- \\
\hline
\end{tabular}


Table 10a. Comparison of Statistical Contaminant Concentrations to Action Levels for the 1607-B2:1 Remediation Footprint Verification Sampling Event." (2 Pages)

\begin{tabular}{|c|c|c|c|c|c|c|}
\hline \multirow[b]{2}{*}{$\mathrm{COC} / \mathrm{COPC}$} & \multirow[b]{2}{*}{$\begin{array}{c}\text { Statistical } \\
\text { Result } \\
(\mathrm{mg} / \mathrm{kg})\end{array}$} & \multicolumn{3}{|c|}{ Remedial Action Goals ${ }^{\mathrm{a}}$ (mg/kg) } & \multirow[b]{2}{*}{$\begin{array}{c}\text { Does the } \\
\text { Statistical } \\
\text { Data Set } \\
\text { Exceed } \\
\text { RAGs? }\end{array}$} & \multirow[b]{2}{*}{$\begin{array}{l}\text { Does the } \\
\text { Statistical } \\
\text { Result Pass } \\
\text { RESRAD } \\
\text { Modeling? }\end{array}$} \\
\hline & & $\begin{array}{c}\text { Direct } \\
\text { Exposure }\end{array}$ & $\begin{array}{l}\text { Soil Cleanup } \\
\text { Level for } \\
\text { Groundwater } \\
\text { Protection }\end{array}$ & $\begin{array}{c}\text { Soil } \\
\text { Cleanup } \\
\text { Level for } \\
\text { River } \\
\text { Protection }\end{array}$ & & \\
\hline Cadmium $^{1}$ & $0.13(<\mathrm{BG})$ & 13.9 & $0.81^{\mathrm{f}}$ & $0.81^{\mathrm{f}}$ & No & -- \\
\hline Chromium (total) & 19.8 & $80,000^{d}$ & $18.5^{\mathrm{f}}$ & $18.5^{\mathrm{f}}$ & Yes & Yes $^{w}$ \\
\hline Chromium (hexavalent) & 0.33 & 2.1 & $4.8^{\mathrm{m}}$ & 2 & No & -- \\
\hline Cobalt & $12.9(<\mathrm{BG})$ & 1,600 & 32 & $-{ }^{k}$ & No & -- \\
\hline Copper & 29.6 & 2,960 & 59.2 & $22.0^{\mathrm{f}}$ & Yes & Yes $^{\mathrm{w}}$ \\
\hline Lead & $10.2(<\mathrm{BG})$ & 353 & $10.2^{\mathrm{f}}$ & $10.2^{\mathrm{f}}$ & $\operatorname{Yes}^{\mathrm{x}}$ & Yes $^{\mathrm{w}}$ \\
\hline Manganese & 588 & 11,200 & $512^{\mathrm{f}}$ & $512^{\mathrm{f}}$ & Yes & Yes $^{w}$ \\
\hline Mercury & $0.02(<\mathrm{BG})$ & 24 & $0.33^{\mathrm{f}}$ & $0.33^{\mathrm{f}}$ & No & -- \\
\hline Molybdenum $^{\mathrm{j}}$ & 1.2 & 400 & 8 & $-{ }^{k}$ & No & -- \\
\hline Nickel & 21.2 & 1,600 & $19.1^{\mathrm{f}}$ & 27.4 & Yes & Yes $^{w}$ \\
\hline Silver & $0.20(<\mathrm{BG})$ & 400 & 8 & $0.73^{\mathrm{f}}$ & No & - \\
\hline Vanadium & $54.9(<\mathrm{BG})$ & 560 & $85.1^{\mathrm{f}}$ & $--^{k}$ & No & -- \\
\hline Zinc & 69 & 24,000 & 480 & $67.8^{\mathrm{f}}$ & Yes & Yes $^{w}$ \\
\hline 4,4'-DDD & 0.0017 & 4.17 & 0.0365 & $0.005^{\circ}$ & No & -- \\
\hline Dieldrin & 0.0017 & 0.0625 & $0.003^{\circ}$ & $0.003^{\circ}$ & No & -- \\
\hline Endrin aldehyde & 0.0022 & 24 & 0.2 & 0.039 & No & - \\
\hline $2,4-\mathrm{D}$ & 0.11 & 800 & 7 & $-{ }^{\mathrm{k}}$ & No & -- \\
\hline $2,4-\mathrm{Db}$ & 0.25 & 640 & 12.8 & $-{ }^{k}$ & No & -- \\
\hline $2,4,5-\mathrm{T}$ & 0.041 & 800 & 16 & $--^{k}$ & No & -- \\
\hline 2,4,5-Tp (Silvex) & 0.023 & 640 & 5 & $-{ }^{k}$ & No & -- \\
\hline Dinoseb & 0.027 & 80 & 0.7 & $-{ }^{k}$ & No & -- \\
\hline 2-Methylnaphthalene & 0.15 & 320 & 3.2 & $--^{k}$ & No & - \\
\hline Di-n-butylphthalate & 0.021 & 8,000 & 160 & 540 & No & - \\
\hline Dibenzofuran & 0.034 & 160 & 3.2 & $--^{k}$ & No & -- \\
\hline Diethylphthalate & 0.026 & 64,000 & 1,280 & 4,600 & No & - \\
\hline Indeno(1,2,3-cd)pyrene & 0.024 & 1.37 & $0.33^{\circ}$ & $0.33^{\circ}$ & No & -- \\
\hline Naphthalene & 0.11 & 1,600 & 16 & 988 & No & -- \\
\hline Phenanthrene $^{\mathrm{s}}$ & 0.037 & 24,000 & 240 & 1,920 & No & -- \\
\hline Acetone & 0.010 & 72,000 & 720 & $--^{k}$ & No & - \\
\hline
\end{tabular}

* All Table 10a notes and acronyms are provided at the end of the document body. 
Table 10b. Comparison of Statistical Contaminant Concentrations to Action Levels for the 1607-B2:1 BCL Stockpiles Verification Sampling Event."

\begin{tabular}{|c|c|c|c|c|c|c|}
\hline \multirow[b]{2}{*}{ COC/COPC } & \multirow[b]{2}{*}{$\begin{array}{c}\text { Maximum } \\
\text { Result } \\
(\mathrm{mg} / \mathrm{kg})\end{array}$} & \multicolumn{3}{|c|}{ Remedial Action Goals ${ }^{\mathrm{a}}$ (mg/kg) } & \multirow[b]{2}{*}{$\begin{array}{l}\text { Does the } \\
\text { Maximum } \\
\text { Result } \\
\text { Exceed } \\
\text { RAGs? }\end{array}$} & \multirow[b]{2}{*}{$\begin{array}{l}\text { Does the } \\
\text { Maximum } \\
\text { Result Pass } \\
\text { RESRAD } \\
\text { Modeling? }\end{array}$} \\
\hline & & $\begin{array}{c}\text { Direct } \\
\text { Exposure }\end{array}$ & $\begin{array}{l}\text { Soil Cleanup } \\
\text { Level for } \\
\text { Groundwater } \\
\text { Protection }\end{array}$ & $\begin{array}{c}\text { Soil } \\
\text { Cleanup } \\
\text { Level for } \\
\text { River } \\
\text { Protection }\end{array}$ & & \\
\hline Antimony $^{1}$ & $0.49(<\mathrm{BG})$ & 32 & $5^{\mathrm{f}}$ & $5^{\mathrm{f}}$ & No & -- \\
\hline Arsenic & $5.3(<\mathrm{BG})$ & 20 & 20 & 20 & No & -- \\
\hline Barium & $101(<\mathrm{BG})$ & $5,600^{d}$ & $132^{\mathrm{e}, \mathrm{f}}$ & $224^{g}$ & No & $-\ldots$ \\
\hline Beryllium & $0.47(<\mathrm{BG})$ & $10.4^{\mathrm{i}}$ & $1.51^{\mathrm{f}}$ & $1.51^{\mathrm{f}}$ & No & -- \\
\hline Boron $^{\mathrm{j}}$ & 2.3 & 16,000 & 320 & $--^{k}$ & No & - \\
\hline Cadmium $^{1}$ & $0.13(<\mathrm{BG})$ & 13.9 & $0.81^{\mathrm{f}}$ & $0.81^{\mathrm{f}}$ & No & -- \\
\hline Chromium (total) & $14.6(<\mathrm{BG})$ & $80,000^{\mathrm{d}}$ & $18.5^{\mathrm{f}}$ & $18.5^{\mathrm{f}}$ & No & $\ldots$ \\
\hline Chromium (hexavalent) & 0.24 & 2.1 & $4.8^{\mathrm{m}}$ & 2 & No & -- \\
\hline Cobalt & $8.0(<\mathrm{BG})$ & 1,600 & 32 & $--^{k}$ & No & - \\
\hline Copper & $17.5(<\mathrm{BG})$ & 2,960 & 59.2 & $22.0^{\mathrm{f}}$ & No & -- \\
\hline Lead & $6.6(<\mathrm{BG})$ & 353 & $10.2^{\mathrm{f}}$ & $10.2^{\mathrm{f}}$ & No & -- \\
\hline Manganese & $357(<\mathrm{BG})$ & 11,200 & $512^{\mathrm{f}}$ & $512^{\mathrm{f}}$ & No & -- \\
\hline Molybdenum $^{\mathrm{j}}$ & 0.61 & 400 & 8 & $--^{k}$ & No & -- \\
\hline Nickel & $14.4(<\mathrm{BG})$ & 1,600 & $19.1^{\mathrm{f}}$ & 27.4 & No & -- \\
\hline Silver & $0.09(<\mathrm{BG})$ & 400 & 8 & $0.73^{\mathrm{f}}$ & No & -- \\
\hline Vanadium & $40.0(<\mathrm{BG})$ & 560 & $85.1^{\mathrm{f}}$ & $--^{k}$ & No & -- \\
\hline Zinc & $42.5(<\mathrm{BG})$ & 24,000 & 480 & $67.8^{\mathrm{f}}$ & No & - \\
\hline $2,4,5-\mathrm{T}$ & 0.049 & 800 & 16 & $--^{k}$ & No & -- \\
\hline Acetone & 0.009 & 72,000 & 720 & $--^{k}$ & No & -- \\
\hline
\end{tabular}

* All Table $10 \mathrm{~b}$ notes and acronyms are provided at the end of the document body.

\section{7-B2:1 Drain Field Data Evaluation}

Residual concentrations of barium, chromium (total), copper, manganese, nickel, and zinc in the remediation footprint were determined to exceed soil RAGs for the protection of groundwater and/or the Columbia River at the 1607-B2:1 subsite. Based on the $\mathrm{K}_{\mathrm{d}}$ value for these constituents $\left(>25 \mathrm{~mL} / \mathrm{g}\right.$ ) and the discussion of the contaminant depth/$/ \mathrm{K}_{\mathrm{d}}$ value model presented within the confirmatory sampling discussion, above, and the 100 Area Analogous Sites RESRAD Calculations calculation brief (Appendix D), none are expected to migrate further than $3 \mathrm{~m}$ $(10 \mathrm{ft})$ vertically in 1,000 years. The vadose zone underlying the 1607-B2:1 excavation is approximately 11-m (36-ft)-thick; therefore, residual concentrations of lead are protective of groundwater. No COCs/COPCs were quantified above RAGs within the BCL stockpiles. 
When using a statistical sampling approach, a RAG requirement for nonradionuclides is the WAC 173-340-740(7)(e) three-part test. The application of the three-part test for the 1607-B2:1 remediation footprint is included in the site-specific statistical calculations (Appendix B). The three-part test is not applicable to the BCL stockpile results since direct evaluation of the maximum detected sampling results was used as the compliance basis. All residual COC/COPC concentrations for the 1607-B2:1 remediation footprint pass the three-part test in comparison to direct exposure RAGs. In addition to the exceedances addressed above, lead fails the three-part test in comparison to soil RAGs for the protection of groundwater and the Columbia River. As with the other metals, lead $\left(\mathrm{K}_{\mathrm{d}}=30 \mathrm{~mL} / \mathrm{g}\right)$ is not predicted to migrate more than $3 \mathrm{~m}(10 \mathrm{ft})$ vertically in 1,000 years, as compared to an $11-\mathrm{m}-(36-\mathrm{ft}-)$ thick vadose zone underlying the lowest point of the 1607-B2:1 excavation. Therefore, residual concentrations of lead are also protective of groundwater and the Columbia River.

Nonradionuclide risk requirements include an individual hazard quotient of less than 1.0, a cumulative hazard quotient of less than 1.0, an individual contaminant carcinogenic risk of less than $1 \times 10^{-6}$, and a cumulative carcinogenic risk of less than $1 \times 10^{-5}$. For the 1607-B2:1 subsite, these risk values were conservatively calculated using the higher of the remediation footprint statistical value and the BCL material maximum value for each constituent. Risk values were not calculated for constituents that were either not detected or were detected at concentrations below Hanford Site or Washington State background values. All individual hazard quotients were less than 1.0, and all individual excess carcinogenic risk values were less than $1 \times 10^{-6}$ (Appendix B). The cumulative hazard quotient for those noncarcinogenic constituents above background or detection levels is $1.3 \times 10^{-1}$, and the cumulative excess carcinogenic risk value for these constituents is $2.0 \times 10^{-7}$. Therefore, nonradionuclide risk requirements are met.

Table 11a. Comparison of Statistical Contaminant Concentrations to Action Levels for the 1607-B2:2 and 100-B-14:2 (Area 1) Remediation Footprint Verification Sampling Event."

(3 Pages)

\begin{tabular}{|c|c|c|c|c|c|c|}
\hline \multirow[b]{2}{*}{$\mathrm{COC} / \mathrm{COPC}$} & \multirow[b]{2}{*}{$\begin{array}{c}\text { Statistical } \\
\text { Result } \\
(\mathrm{pCi} / \mathrm{g})\end{array}$} & \multicolumn{3}{|c|}{ Generic Site Lookup Values ${ }^{\mathrm{a}}$ (pCi/g) } & \multirow{2}{*}{$\begin{array}{c}\text { Does the } \\
\text { Statistical } \\
\text { Result } \\
\text { Exceed } \\
\text { Lookup } \\
\text { Values? }\end{array}$} & \multirow[b]{2}{*}{$\begin{array}{l}\text { Does the } \\
\text { Statistical } \\
\text { Result Pass } \\
\text { RESRAD } \\
\text { Modeling? }\end{array}$} \\
\hline & & $\begin{array}{l}\text { Shallow } \\
\text { Zone } \\
\text { Lookup } \\
\text { Value }^{b}\end{array}$ & $\begin{array}{c}\text { Groundwater } \\
\text { Protection } \\
\text { Lookup } \\
\text { Value }\end{array}$ & $\begin{array}{c}\text { River } \\
\text { Protection } \\
\text { Lookup } \\
\text { Value }\end{array}$ & & \\
\hline Cesium-137 & 0.107 & 6.2 & $1,465^{\mathrm{c}}$ & $1,465^{\mathrm{c}}$ & No & -- \\
\hline Strontium-90 & 0.181 & 4.5 & $27.6^{\mathrm{c}}$ & $27.6^{\mathrm{c}}$ & No & -- \\
\hline \multirow[b]{2}{*}{ COC/COPC } & \multirow[b]{2}{*}{$\begin{array}{c}\text { Statistical } \\
\text { Result } \\
(\mathrm{mg} / \mathrm{kg})\end{array}$} & \multicolumn{3}{|c|}{ Remedial Action Goals ${ }^{a}$ (mg/kg) } & \multirow[b]{2}{*}{$\begin{array}{l}\text { Does the } \\
\text { Statistical } \\
\text { Data Set } \\
\text { Exceed } \\
\text { RAGs? }\end{array}$} & \multirow[b]{2}{*}{$\begin{array}{l}\text { Does the } \\
\text { Statistical } \\
\text { Result Pass } \\
\text { RESRAD } \\
\text { Modeling? }\end{array}$} \\
\hline & & $\begin{array}{c}\text { Direct } \\
\text { Exposure }\end{array}$ & $\begin{array}{l}\text { Soil Cleanup } \\
\text { Level for } \\
\text { Groundwater } \\
\text { Protection }\end{array}$ & $\begin{array}{c}\text { Soil } \\
\text { Cleanup } \\
\text { Level for } \\
\text { River } \\
\text { Protection }\end{array}$ & & \\
\hline Antimony $^{1}$ & $0.51(<\mathrm{BG})$ & 32 & $5^{\mathrm{f}}$ & $5^{\mathrm{f}}$ & No & -- \\
\hline Arsenic & $4.1(<\mathrm{BG})$ & 20 & 20 & 20 & No & - \\
\hline Barium & $112(<\mathrm{BG})$ & $5,600^{\mathrm{d}}$ & $132^{\mathrm{e}, \mathrm{f}}$ & $224^{\mathrm{g}}$ & Yes $^{x}$ & Yes $^{y}$ \\
\hline
\end{tabular}


Table 11a. Comparison of Statistical Contaminant Concentrations to Action Levels for the 1607-B2:2 and 100-B-14:2 (Area 1) Remediation Footprint Verification Sampling Event."

(3 Pages)

\begin{tabular}{|c|c|c|c|c|c|c|}
\hline \multirow[b]{2}{*}{$\mathrm{COC} / \mathrm{COPC}$} & \multirow[b]{2}{*}{$\begin{array}{c}\text { Statistical } \\
\text { Result } \\
\text { (mg/kg) }\end{array}$} & \multicolumn{3}{|c|}{ Remedial Action Goals ${ }^{\mathrm{a}}$ (mg/kg) } & \multirow[b]{2}{*}{$\begin{array}{c}\text { Does the } \\
\text { Statistical } \\
\text { Data Set } \\
\text { Exceed } \\
\text { RAGs? }\end{array}$} & \multirow[b]{2}{*}{$\begin{array}{l}\text { Does the } \\
\text { Statistical } \\
\text { Result Pass } \\
\text { RESRAD } \\
\text { Modeling? }\end{array}$} \\
\hline & & $\begin{array}{c}\text { Direct } \\
\text { Exposure }\end{array}$ & $\begin{array}{l}\text { Soil Cleanup } \\
\text { Level for } \\
\text { Groundwater } \\
\text { Protection }\end{array}$ & $\begin{array}{c}\text { Soil } \\
\text { Cleanup } \\
\text { Level for } \\
\text { River } \\
\text { Protection }\end{array}$ & & \\
\hline Beryllium & $0.44(<\mathrm{BG})$ & $10.4^{\mathrm{i}}$ & $1.51^{\mathrm{f}}$ & $1.51^{\mathrm{f}}$ & No & -- \\
\hline Boron $^{j}$ & 4.7 & 16,000 & 320 & $--^{k}$ & No & -- \\
\hline Cadmium $^{1}$ & $0.43(<\mathrm{BG})$ & 13.9 & $0.81^{\mathrm{f}}$ & $0.81^{\mathrm{f}}$ & No & -- \\
\hline Chromium (total) & $10.5(<\mathrm{BG})$ & $80,000^{\mathrm{d}}$ & $18.5^{\mathrm{f}}$ & $18.5^{\mathrm{f}}$ & No & -- \\
\hline Chromium (hexavalent) & 0.35 & 2.1 & $4.8^{\mathrm{m}}$ & 2 & No & -- \\
\hline Cobalt & $8.5(<\mathrm{BG})$ & 1,600 & 32 & $-{ }^{\mathrm{k}}$ & No & -- \\
\hline Copper & 34 & 2,960 & 59.2 & $22.0^{\mathrm{f}}$ & Yes & $\mathrm{Yes}^{\mathrm{y}}$ \\
\hline Lead & $10.1(<\mathrm{BG})$ & 353 & $10.2^{\mathrm{f}}$ & $10.2^{\mathrm{f}}$ & $\operatorname{Yes}^{x}$ & $\mathrm{Yes}^{\mathrm{y}}$ \\
\hline Lithium & $8.3(<\mathrm{BG})$ & 1,600 & $33.5^{\mathrm{f}}$ & $--^{k}$ & No & -- \\
\hline Manganese & $362(<\mathrm{BG})$ & 11,200 & $512^{\mathrm{f}}$ & $512^{f}$ & No & -- \\
\hline Mercury & 0.92 & 24 & $0.33^{\mathrm{f}}$ & $0.33^{\mathrm{f}}$ & Yes & Yes $^{y}$ \\
\hline Molybdenum $^{\mathrm{j}}$ & 0.37 & 400 & 8 & $--^{k}$ & No & -- \\
\hline Nickel & $12.5(<\mathrm{BG})$ & 1,600 & $19.1^{\mathrm{f}}$ & 27.4 & No & -- \\
\hline Strontium ${ }^{\mathrm{j}}$ & 48.2 & 48,000 & 960 & $-{ }^{k}$ & No & -- \\
\hline Titanium & $1,509(<\mathrm{BG})$ & $320,000^{\mathrm{n}}$ & $6,400^{\mathrm{n}}$ & $--^{k}$ & No & -- \\
\hline Vanadium & $46.4(<\mathrm{BG})$ & 560 & $85.1^{\mathrm{f}}$ & $--^{k}$ & No & -- \\
\hline Zinc & $51.5(<\mathrm{BG})$ & 24,000 & 480 & $67.8^{\mathrm{f}}$ & No & -- \\
\hline Aroclor-1254 & 0.33 & 0.5 & $0.017^{\circ}$ & $0.017^{\circ}$ & Yes & Yes $^{y}$ \\
\hline Aroclor-1260 & 0.0067 & 0.5 & $0.017^{\circ}$ & $0.017^{\circ}$ & No & - \\
\hline alpha-Chlordane & 0.00087 & $2.86^{\mathrm{q}}$ & $0.025^{q}$ & $0.0165^{\circ}$ & No & -- \\
\hline Beta-BHC & 0.0019 & 0.556 & 0.00486 & 0.00554 & No & -- \\
\hline 4,4'-DDE & 0.018 & 2.94 & 0.0257 & $0.005^{\circ}$ & Yes & Yes $^{y}$ \\
\hline 4,4'-DDT & 0.017 & 2.94 & 0.0257 & $0.005^{\circ}$ & Yes & Yes $^{y}$ \\
\hline Endosulfan I & 0.0069 & 480 & 9.6 & 0.186 & No & -- \\
\hline Endosulfan II & 0.0034 & 480 & 9.6 & 0.186 & No & -- \\
\hline Endosulfan sulfate & 0.00050 & 480 & 9.6 & 0.186 & No & -- \\
\hline Endrin aldehyde & 0.0074 & 24 & 0.2 & 0.039 & No & -- \\
\hline Endrin ketone & 0.0011 & 24 & 0.2 & 0.039 & No & - \\
\hline gamma-Chlordane & 0.00043 & $2.86^{\mathrm{q}}$ & $0.025^{\mathrm{q}}$ & $0.0165^{\circ}$ & No & -- \\
\hline Heptachlor epoxide & 0.00060 & 0.11 & $0.002^{\circ}$ & $0.002^{\circ}$ & No & -- \\
\hline Methoxychlor & 0.015 & 400 & 4 & 1.67 & No & -- \\
\hline 2-Methylnaphthalene & 0.019 & 320 & 3.2 & $--^{k}$ & No & -. \\
\hline
\end{tabular}


Table 11a. Comparison of Statistical Contaminant Concentrations to Action Levels for the 1607-B2:2 and 100-B-14:2 (Area 1) Remediation Footprint Verification Sampling Event." (3 Pages)

\begin{tabular}{|c|c|c|c|c|c|c|}
\hline \multirow[b]{2}{*}{$\mathrm{COC} / \mathrm{COPC}$} & \multirow[b]{2}{*}{$\begin{array}{c}\text { Statistical } \\
\text { Result } \\
(\mathbf{m g} / \mathrm{kg})\end{array}$} & \multicolumn{3}{|c|}{ Remedial Action Goals ${ }^{\mathrm{a}}(\mathrm{mg} / \mathrm{kg}$ ) } & \multirow[b]{2}{*}{$\begin{array}{c}\text { Does the } \\
\text { Statistical } \\
\text { Data Set } \\
\text { Exceed } \\
\text { RAGs? }\end{array}$} & \multirow[b]{2}{*}{$\begin{array}{l}\text { Does the } \\
\text { Statistical } \\
\text { Result Pass } \\
\text { RESRAD } \\
\text { Modeling? }\end{array}$} \\
\hline & & $\begin{array}{c}\text { Direct } \\
\text { Exposure }\end{array}$ & $\begin{array}{l}\text { Soil Cleanup } \\
\text { Level for } \\
\text { Groundwater } \\
\text { Protection }\end{array}$ & $\begin{array}{c}\text { Soil } \\
\text { Cleanup } \\
\text { Level for } \\
\text { River } \\
\text { Protection }\end{array}$ & & \\
\hline Benzo(a)anthracene & 0.041 & $1.37^{\mathrm{r}}$ & $0.33^{\circ}$ & $0.33^{\circ}$ & No & - \\
\hline Benzo(a)pyrene & 0.033 & $0.33^{\circ}$ & $0.33^{\circ}$ & $0.33^{\circ}$ & No & - \\
\hline Benzo(b)fluoranthene & 0.041 & $1.37^{\mathrm{r}}$ & $0.33^{\circ}$ & $0.33^{\circ}$ & No & - \\
\hline Benzo(g,h,i)perylene ${ }^{\mathrm{s}}$ & 0.030 & 2,400 & 48 & 192 & No & - \\
\hline Benzo(k)fluoranthene & 0.035 & $13.7^{\mathrm{r}}$ & $0.33^{\circ}$ & $0.33^{\circ}$ & No & -- \\
\hline bis(2-Ethylhexyl)phthalate & 1.6 & 71.4 & 0.625 & 0.36 & Yes & Yes $^{y}$ \\
\hline Chrysene & 0.064 & $137^{\mathrm{r}}$ & $1.2^{\mathrm{r}}$ & $0.33^{\circ}$ & No & -- \\
\hline Di-n-butylphthalate & 0.07 & 8,000 & 160 & 540 & No & - \\
\hline Dibenz(a,h)anthracene & 0.022 & $0.33^{\circ}$ & $0.33^{\circ}$ & $0.33^{\circ}$ & No & -- \\
\hline Fluoranthene & 0.079 & 3,200 & 64 & 18 & No & -- \\
\hline Indeno(1,2,3-cd)pyrene & 0.028 & 1.37 & $0.33^{\circ}$ & $0.33^{\circ}$ & No & -- \\
\hline Naphthalene & 0.017 & 1,600 & 16 & 988 & No & - \\
\hline Phenanthrene $^{s}$ & 0.046 & 24,000 & 240 & 1,920 & No & -- \\
\hline Phenol & 0.017 & 24,000 & 480 & 4,200 & No & -- \\
\hline Pyrene & 0.066 & 2,400 & 48 & 192 & No & -- \\
\hline
\end{tabular}

* All Table 11a notes and acronyms are provided at the end of the document body.

Table 11b. Comparison of Statistical Contaminant Concentrations to Action Levels for the 1607-B2:2 and 100-B-14:2 (Area 1) BCL Stockpiles Verification Sampling Event." (2 Pages)

\begin{tabular}{|c|c|c|c|c|c|c|}
\hline \multirow[b]{2}{*}{$\mathrm{COC} / \mathrm{COPC}$} & \multirow[b]{2}{*}{$\begin{array}{c}\text { Maximum } \\
\text { Result } \\
\text { (pCi/g) }\end{array}$} & \multicolumn{3}{|c|}{ Generic Site Lookup Values $^{\mathrm{a}}(\mathrm{pCi} / \mathrm{g}$ ) } & \multirow{2}{*}{$\begin{array}{l}\text { Does the } \\
\text { Maximum } \\
\text { Result } \\
\text { Exceed } \\
\text { Lookup } \\
\text { Values? }\end{array}$} & \multirow[b]{2}{*}{$\begin{array}{l}\text { Does the } \\
\text { Maximum } \\
\text { Result Pass } \\
\text { RESRAD } \\
\text { Modeling? }\end{array}$} \\
\hline & & $\begin{array}{l}\text { Shallow } \\
\text { Zone } \\
\text { Lookup } \\
\text { Value }^{\mathbf{b}}\end{array}$ & $\begin{array}{c}\text { Groundwater } \\
\text { Protection } \\
\text { Lookup } \\
\text { Value }\end{array}$ & $\begin{array}{l}\text { River } \\
\text { Protection } \\
\text { Lookup } \\
\text { Value }\end{array}$ & & \\
\hline Strontium-90 & 2.10 & 4.5 & $27.6^{\mathrm{C}}$ & $27.6^{\mathrm{c}}$ & No & -- \\
\hline \multirow[b]{2}{*}{$\mathrm{COC} / \mathrm{COPC}$} & \multirow[b]{2}{*}{$\begin{array}{l}\text { Maximum } \\
\text { Result } \\
(\mathrm{mg} / \mathrm{kg})\end{array}$} & \multicolumn{3}{|c|}{ Remedial Action Goals ${ }^{\mathrm{a}}$ (mg/kg) } & \multirow[b]{2}{*}{$\begin{array}{l}\text { Does the } \\
\text { Maximum } \\
\text { Result } \\
\text { Exceed } \\
\text { RAGs? }\end{array}$} & \multirow[b]{2}{*}{$\begin{array}{l}\text { Does the } \\
\text { Maximum } \\
\text { Result Pass } \\
\text { RESRAD } \\
\text { Modeling? }\end{array}$} \\
\hline & & $\begin{array}{c}\text { Direct } \\
\text { Exposure }\end{array}$ & $\begin{array}{c}\text { Soil Cleanup } \\
\text { Level for } \\
\text { Groundwater } \\
\text { Protection }\end{array}$ & $\begin{array}{c}\text { Soil } \\
\text { Cleanup } \\
\text { Level for } \\
\text { River } \\
\text { Protection } \\
\end{array}$ & & \\
\hline Antimony $^{1}$ & $0.47(<\mathrm{BG})$ & 32 & $5^{\mathrm{f}}$ & $5^{\mathrm{f}}$ & No & -- \\
\hline Arsenic & $3.6(<\mathrm{BG})$ & 20 & 20 & 20 & No & -- \\
\hline
\end{tabular}


Table 11b. Comparison of Statistical Contaminant Concentrations to Action Levels for the 1607-B2:2 and 100-B-14:2 (Area 1) BCL Stockpiles Verification Sampling Event."

(2 Pages)

\begin{tabular}{|c|c|c|c|c|c|c|}
\hline \multirow[b]{2}{*}{$\mathrm{COC} / \mathrm{COPC}$} & \multirow[b]{2}{*}{$\begin{array}{c}\text { Maximum } \\
\text { Result } \\
(\mathrm{mg} / \mathrm{kg})\end{array}$} & \multicolumn{3}{|c|}{ Remedial Action Goals ${ }^{\mathrm{a}}$ (mg/kg) } & \multirow[b]{2}{*}{$\begin{array}{l}\text { Does the } \\
\text { Maximum } \\
\text { Result } \\
\text { Exceed } \\
\text { RAGs? }\end{array}$} & \multirow[b]{2}{*}{$\begin{array}{l}\text { Does the } \\
\text { Maximum } \\
\text { Result Pass } \\
\text { RESRAD } \\
\text { Modeling? }\end{array}$} \\
\hline & & $\begin{array}{c}\text { Direct } \\
\text { Exposure }\end{array}$ & $\begin{array}{l}\text { Soil Cleanup } \\
\text { Level for } \\
\text { Groundwater } \\
\text { Protection }\end{array}$ & $\begin{array}{c}\text { Soil } \\
\text { Cleanup } \\
\text { Level for } \\
\text { River } \\
\text { Protection }\end{array}$ & & \\
\hline Barium & $91.0(<\mathrm{BG})$ & $5,600^{\mathrm{d}}$ & $132^{\mathrm{e}, \mathrm{f}}$ & $224^{g}$ & No & -- \\
\hline Beryllium & $0.39(<\mathrm{BG})$ & $10.4^{\mathrm{i}}$ & $1.51^{\mathrm{f}}$ & $1.51^{\mathrm{f}}$ & No & -- \\
\hline Boron $^{j}$ & 2.8 & 16,000 & 320 & $--^{k}$ & No & -- \\
\hline Cadmium $^{I}$ & $0.1(<\mathrm{BG})$ & 13.9 & $0.81^{\mathrm{f}}$ & $0.81^{\mathrm{f}}$ & No & - \\
\hline Chromium (total) & $12.8(<\mathrm{BG})$ & $80,000^{d}$ & $18.5^{\mathrm{f}}$ & $18.5^{\mathrm{f}}$ & No & -- \\
\hline Chromium (hexavalent) & 0.28 & 2.1 & $4.8^{\mathrm{m}}$ & 2 & No & -- \\
\hline Cobalt & $8.2(<\mathrm{BG})$ & 1,600 & 32 & $--^{k}$ & No & -- \\
\hline Copper & $16.0(<\mathrm{BG})$ & 2,960 & 59.2 & $22.0^{\mathrm{f}}$ & No & -- \\
\hline Lead & $8.9(<\mathrm{BG})$ & 353 & $10.2^{\mathrm{f}}$ & $10.2^{\mathrm{f}}$ & No & - \\
\hline Lithium & $7.7(<\mathrm{BG})$ & 1,600 & $33.5^{\mathrm{f}}$ & $--^{k}$ & No & - \\
\hline Manganese & $340(<\mathrm{BG})$ & 11,200 & $512^{f}$ & $512^{\mathrm{f}}$ & No & -- \\
\hline Mercury & $0.14(<\mathrm{BG})$ & 24 & $0.33^{\mathrm{f}}$ & $0.33^{\mathrm{f}}$ & No & -- \\
\hline Molybdenum $^{\mathrm{j}}$ & 0.36 & 400 & 8 & $--^{k}$ & No & - \\
\hline Nickel & $12.5(<\mathrm{BG})$ & 1,600 & $19.1^{\mathrm{f}}$ & 27.4 & No & - \\
\hline Strontium $^{j}$ & 31.0 & 48,000 & 960 & $--^{k}$ & No & -- \\
\hline Titanium & $1,330(<\mathrm{BG})$ & $320,000^{n}$ & $6,400^{\mathrm{n}}$ & $--^{k}$ & No & - \\
\hline Vanadium & $46.6(<\mathrm{BG})$ & 560 & $85.1^{\mathrm{f}}$ & $--^{k}$ & No & -- \\
\hline Zinc & $49.6(<\mathrm{BG})$ & 24,000 & 480 & $67.8^{\mathrm{f}}$ & No & -- \\
\hline Aroclor-1254 & 0.0062 & 0.5 & $0.017^{\circ}$ & $0.017^{\circ}$ & No & - \\
\hline Aroclor- 1260 & 0.011 & 0.5 & $0.017^{\circ}$ & $0.017^{\circ}$ & No & -- \\
\hline 4,4'-DDE & 0.00044 & 2.94 & 0.0257 & $0.005^{\circ}$ & No & -- \\
\hline 4,4'-DDT & 0.016 & 2.94 & 0.0257 & $0.005^{\circ}$ & Yes & $\mathrm{Yes}^{y}$ \\
\hline Endrin aldehyde & 0.0022 & 24 & 0.2 & 0.039 & No & -- \\
\hline Benzo(b)fluoranthene & 0.018 & $1.37^{\mathrm{r}}$ & $0.33^{\circ}$ & $0.33^{\circ}$ & No & -- \\
\hline bis(2-Ethylhexyl)phthalate & 1.1 & 71.4 & 0.625 & 0.36 & Yes & $\mathrm{Yes}^{\mathrm{y}}$ \\
\hline Di-n-butylphthalate & 0.080 & 8,000 & 160 & 540 & No & -- \\
\hline Diethylphthalate & 0.018 & 64,000 & 1,280 & 4,600 & No & - \\
\hline Fluoranthene & 0.032 & 3,200 & 64 & 18 & No & -- \\
\hline Phenol & 0.027 & 24,000 & 480 & 4,200 & No & - \\
\hline Pyrene & 0.026 & 2,400 & 48 & 192 & No & - \\
\hline
\end{tabular}

* All Table $11 \mathrm{~b}$ notes and acronyms are provided at the end of the document body. 
1607-B2:2 Collection Main, Septic Tank, and Effluent Piping and 100-B-14:2 (Area 1), 108-B Facility Sanitary Sewers Data Evaluation

Residual concentrations of copper, mercury, aroclor-1254, 4,4'-DDE, 4,4'-DDT, and bis(2-ethylhexyl)phthalate in the remediation footprint and 4,4'-DDT and bis(2-ethylhexyl)phthalate in the BCL stockpiles were determined to exceed soil RAGs for the protection of groundwater and/or the Columbia River at the 1607-B2:2 subsite. Based on the $\mathrm{K}_{\mathrm{d}}$ value for these constituents $\left(>22 \mathrm{~mL} / \mathrm{g}\right.$ ) and the discussion of the contaminant depth/ $\mathrm{K}_{\mathrm{d}}$ value model presented within the confirmatory sampling discussion, above, and the 100 Area Analogous Sites RESRAD Calculations calculation brief (Appendix D), none are expected to migrate further than $3 \mathrm{~m}(10 \mathrm{ft})$ vertically in 1,000 years. The vadose zone underlying the 1607-

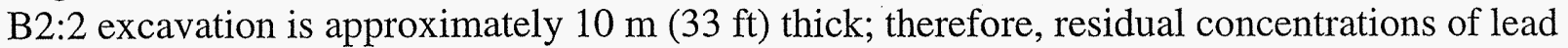
are protective of groundwater.

When using a statistical sampling approach, a RAG requirement for nonradionuclides is the WAC 173-340-740(7)(e) three-part test. The application of the three-part test for the 1607-B2:2 remediation footprint is included in the site-specific statistical calculations (Appendix B). The three-part test is not applicable to the BCL stockpile results since direct evaluation of the maximum detected sampling results was used as the compliance basis. All residual COC/COPC concentrations for the 1607-B2:2 remediation footprint pass the three-part test in comparison to direct exposure RAGs. In addition to the exceedances addressed above, barium and lead fail the three-part test in comparison to soil RAGs for the protection of groundwater and/or the Columbia River. As above, neither barium $\left(\mathrm{K}_{\mathrm{d}}=25 \mathrm{~mL} / \mathrm{g}\right)$ nor lead $\left(\mathrm{K}_{\mathrm{d}}=30 \mathrm{~mL} / \mathrm{g}\right)$ is predicted to migrate more than $3 \mathrm{~m}(10 \mathrm{ft})$ vertically in 1,000 years, as compared to an $10-\mathrm{m}$ (30-ft)- thick vadose zone underlying the lowest point of the 1607-B2:2 excavation. Therefore, residual concentrations of these metals are also protective of groundwater and the Columbia River.

Nonradionuclide risk requirements include an individual hazard quotient of less than 1.0, a cumulative hazard quotient of less than 1.0, an individual contaminant carcinogenic risk of less than $1 \times 10^{-6}$, and a cumulative carcinogenic risk of less than $1 \times 10^{-5}$. For the 1607-B2:2 subsite, these risk values were conservatively calculated using the higher of the remediation footprint statistical value and the $\mathrm{BCL}$ material maximum value for each constituent. These risk values were not calculated for constituents that were either not detected or were detected at concentrations below Hanford Site or Washington State background values. All individual hazard quotients were less than 1.0, and all individual excess carcinogenic risk values were less than $1 \times 10^{-6}$ (Appendix B). The cumulative hazard quotient for those noncarcinogenic constituents above background or detection levels is $2.6 \times 10^{-1}$, and the cumulative excess carcinogenic risk value for these constituents is $1.4 \times 10^{-6}$. Therefore, nonradionuclide risk requirements are met.

\section{DATA QUALITY ASSESSMENTS}

Individual data quality assessments (DQAs) were performed for each sampling event, as is described in the follow subsections. Each DQA was performed to compare the sampling approach and resulting analytical data with the sampling and data requirements specified in the site-specific 
work instructions. All DQAs were performed in accordance with specific data quality objectives found in the SAP (DOE-RL 2005a). The data quality requirements in the SAP are used for assessing data resulting from statistical sampling and do not specifically apply to the data sets resulting from the (confirmatory) focused sampling performed for the 100-B-14:2 subsite. However, to ensure quality data sets, the SAP data quality assurance requirements, as well as the data validation procedures for chemical and radiochemical analysis (BHI 2000a, 2000b), have been followed where appropriate.

The DQAs involve evaluation of the data to determine if they are of the right type, quality, and quantity to support the intended use (i.e., closeout decisions). A DQA completes the data life cycle (i.e., planning, implementation, and assessment) initiated by the data quality objectives process (EPA 2000). It was concluded that all interim closure data was of the right quality and quantity to support a closeout decision.

\section{0-B-14:2 (Area 2) Confirmatory Sampling Data Quality Assessment}

Because holding times for hexavalent chromium analysis in other solids (i.e., pipe sediment/debris) were exceeded by more than twice the requirement, third-party validation of confirmatory data directed that all nondetects be rejected. Nondetected hexavalent chromium results were not found in pipe sediments, but the detections should be considered estimated and potentially low-biased.

Due to detections and data quality issues in the confirmatory data set, all confirmatory analyses were retained for verification sampling following site remediation.

Verification sampling for the 100-B-14:2 (area 2) subsite was combined with that for the 100-B-14:2 (area 5) subsite, and the verification DQA for the combined sampling event is presented following the 100-B-14:2 (area 5) confirmatory DQA.

\section{0-B-14:2 (Area 5) Confirmatory Sampling Data Quality Assessment}

Because holding times for hexavalent chromium analysis in other solids (i.e., pipe sediment/debris) were exceeded by more than twice the requirement, the nondetected result in sample J00Y82 was rejected by the project, and the detection in sample J00Y81 should be considered estimated and potentially low-biased.

Samples of residual pipeline contents (J00Y79 and J00Y80) were mistakenly submitted for ICP metals analysis by toxicity characteristic leaching procedure (TCLP), rather than totals procedures; no reanalysis was performed to correct the deficiency.

Due to detections and data quality issues in the confirmatory data set, all confirmatory analyses were retained for verification sampling following site remediation.

\section{0-B-14:2 (Areas 2 and 5) Verification Sampling Data Quality Assessment}

A review of the work instruction (WCH 2006c), the field logbook (WCH 2006a), and applicable analytical data packages has been performed as part of this DQA. All samples were collected 
per the sample design. Where the sample design allowed for additional samples, if required to properly characterize the site, no additional data needs were identified at the time of field sampling.

Data from verification samples collected at the 100-B-14:2 (area 2) subsite were provided by the laboratory in sample delivery group (SDG) K0284, and from the 100-B-14:2 (area 5) subsite in SDG K0285. Both SDGs were submitted for third-party validation. No major deficiencies were found in the data and all of the data were found to be acceptable for decision-making purposes. Minor deficiencies, as well as qualifiers applied by third-party validation, will be discussed below.

\section{$\underline{\text { SDG K0284 }}$}

SDG K0284 consists of 13 field samples (J11KD1, J11KD2, and J11K85 through J11K95) collected from the 100-B-14:2 (area 2) subsite. These field samples were analyzed for SVOCs, pesticides, PCBs, ICP metals, mercury, hexavalent chromium, and by gross alpha, gross beta, and gamma spectroscopy. This SDG also includes a field equipment blank (J11K96), analyzed for SVOCs, ICP metals, and mercury. Sample J11K91 had an elevated gross beta reading and was further analyzed for total beta strontium. Sample J11K95 is a field duplicate of sample J11K86. Sample J11KD1 is from the east BCL stockpile. Sample J11KD2 is from the west BCL stockpile. The ten remaining samples were collected from statistically selected locations within the 100-B-14:2 (area 2) excavation (WCH 2006c).

The SVOC analysis matrix spike (MS) for hexachlorocyclopentadiene was above the acceptance criteria at $101 \%$ recovery (some compounds are expected to have low recoveries; recoveries around $100 \%$ are considered high for those compounds). This result may suggest a high bias in the field sample data. High-biased data is acceptable for the intended purpose of the data.

The common laboratory contaminants bis(2-ethylhexyl)phthalate and di-n-butylphthalate were found in the method blank (MB) below the contract-required quantitation limit (CRQL). All of the field samples and MBs were reported in the range of $21 \mu \mathrm{g} / \mathrm{kg}$ to $62 \mu \mathrm{g} / \mathrm{kg}$ for both phthalates. Third-party validation identified the field samples as being within a 5-fold multiplier of the MB results and reset all of the values in the field samples to the CRQLs and requalified the samples as nondetected with " $U$ " flags.

The SVOC analyte 2,4-dinitrophenol, in the laboratory duplicate, had a relative percent difference (RPD) value of $68 \%$. Therefore, third-party validation qualified all of the 2,4-dinitrophenol results as estimated with "J" flags. Estimated data is accepted for decisionmaking purposes.

In the chlorinated pesticide analysis, many of the surrogates tested above the acceptance criteria, suggesting a high bias in the sample data. However, almost all of the sample results were nondetect. A high bias in the data has no affect on nondetected analytes. In samples J11KD1 and $\mathrm{J} 11 \mathrm{~K} 93$, the analyte beta-BHC was detected. Third-party validation identified the beta-BHC results in samples J11KD1 and J11K93 as estimates and qualified those results with "J" flags. 
The laboratory control sample (LCS) in the chlorinated pesticide analysis had consistently low recoveries (13-17\%) for all analytes except toxaphene, which was not included in the LCS (see below). The MS/matrix spike duplicate (MSD) results were within criteria. Third-party validation qualified the SDG K0284 data for all chlorinated pesticide analytes, except toxaphene, as estimated with "I" flags because of the LCS results.

In the chlorinated pesticide analysis, the analyte toxaphene was not included in the MS, MSD, or LCS samples. Toxaphene is actually a mixture of compounds rather than a discrete analyte. While the overall concentration of toxaphene can be calculated using several unobstructed peaks in the chromatography, the inclusion of toxaphene in the spiking mixture would be problematic for the other pesticide analytes. The laboratory typically quantitates toxaphene but does not include toxaphene in quality assurance $(\mathrm{QA}) / \mathrm{QC}$ samples. The toxaphene data is therefore considered estimated but useable for decision-making purposes.

In the metals analysis, eight analytes were out of criteria for MS recovery. For most of these analytes, the spiking concentration was insignificant compared to the native concentration in the sample from which the MS was prepared. For these analytes, the deficiency in the MS is a reflection of the analytical variability of the native concentration rather than a measure of the recovery from the sample. To confirm quantitation, post digestion spikes (PDSs) and serial dilutions were prepared for all eight analytes with acceptable results. The analytes antimony, calcium, and phosphorus did not have mismatched spike and native concentrations in the original MS. These three analytes have been qualified by third party validation as estimates with "J" flags for all samples in SDG K0284. The original MS recoveries for antimony, calcium, and phosphorus were $46.3 \%, 137 \%$, and $55.4 \%$, respectively. Estimated, or "J"-flagged, data are considered acceptable for the intended use of the data.

The analytes boron, tin, aluminum, and lithium were reported in the $\mathrm{MB}$ at concentrations that were below the CRQLs but not less than $1 / 5^{\text {th }}$ of some of the concentrations reported in the field samples (i.e., the field sample concentrations were low enough that the MB concentration is of similar magnitude). Third-party validation has qualified the analytical data for aluminum and lithium in sample J11K96 (the equipment blank), for tin in samples J11K85, J11K93, and $\mathrm{J} 11 \mathrm{KD} 1$, and for boron in samples $\mathrm{J} 11 \mathrm{~K} 86, \mathrm{~J} 11 \mathrm{~K} 88$, J11K89, J11 K90, J11K92, J11K95, and $\mathrm{J} 11 \mathrm{~K} 96$, as estimated nondetections with "UJ" flags.

The RPDs for eight analytes (cadmium, potassium, silicon, titanium, vanadium, tin, mercury, and total uranium) in the metals analysis were above the laboratory acceptance criteria of $20 \%$. Most of these were less than the project acceptance criteria of $30 \%$. The RPDs for mercury and total uranium were $65 \%$ and $75 \%$, respectively. Elevated RPDs in environmental soil samples are generally attributed to natural heterogeneities in the soil matrix from which the sample and duplicate are prepared. However, all of the total uranium data in SDG K0284 were qualified as estimated, with "J" flags, by third-party validation.

In the gamma spectroscopy data, an elevated RPD was reported for radium-226 at 56\%. Thirdparty validation has qualified all of the radium-226 results in SDG K0284 as estimated, with "J" flags. 
An elevated gross-beta reading was reported for sample J11K91 (28.8 pCi/g). The project requested the analysis of total beta strontium on sample J11K91. There were no problems with the total beta strontium data.

\section{$\underline{\text { SDG K0286 }}$}

SDG K0286 comprises 15 field samples (J11KB3 through J11KB9, J11KC0 through J11KC3, and J11KD3 through J11KD6) collected from the 100-B-14:2 (area 5) subsite. These field samples were analyzed for SVOCs, pesticides, PCBs, ICP metals, mercury, hexavalent chromium, tritium, total beta strontium, and by gross alpha, gross beta, and gamma spectroscopy. This SDG also includes a field equipment blank (J11KC4) that was analyzed for SVOCs, ICP metals, and mercury. Sample J11C3 is a field duplicate of sample J11KC6. Four samples (J11KD3 through J11KD6) were collected from the BCL stockpiles associated with the 115-B sanitary sewer pipeline remediation. The 10 remaining samples were collected from statistically selected locations within the 100-B-14:2 (area 5) excavation (WCH 2006c).

The work instruction called for a sample to be collected from a small BCL stockpile north of the 100-B-14:2 (area 5) excavation. As described in the logbook (WCH 2006a), the northern BCL stockpile had been removed. Further investigation determined that the missing BCL stockpile was incorporated into the larger BCL stockpile associated with the nearby excavation of the 1607-B2:2 and 100-B-14:2 (area 1) subsites. The lack of a sample for the northern 100-B-14:2 (area 5) BCL stockpile has been determined to acceptable because the other BCL samples from (area 5) are all significantly below action levels and the "missing" BCL material was still evaluated as part of the 100-B-14:2/1607-B2 waste sites. Confusion caused by the relocation of the material resulted in sampling of the 100-B-14:2 (area 5) southeastern BCL stockpile twice rather than once, as called for in the work instruction (WCH 2006c).

In the SVOC analysis, the MS recovery for 1,2,4-trichlorobenzene was below the laboratory criteria (60-120\%) at 59\%. The MSD for 1,2,4-trichlorobenzene was within criteria at $79 \%$. Third-party validation qualified all of the 1,2,4-trichlorobenzene data in SDG K0286 as estimated with "J" flags.

The RPDs for eleven analytes (phenol, 1,2-dichlorobenzene, 2-methylphenol, bis(2-chloro-1methylethyl)ether, 4-methylphenol, n-nitroso-di-n-propylamine, 2-nitrophenol, 2,4-dimethylphenol, 2,4-dichlorophenol, 4,6-dinitro-2-methylphenol, and 2,4,6-trichlorophenol) were reported above the $30 \%$ acceptance criteria in the range of $30-40 \%$. The analyte 2,4-dinitrophenol was reported with an RPD of 52\%. Third-party validation has qualified all twelve of the analytes listed above, in all of the samples in SDG K0286, as estimates with "J" flags. Elevated RPDs in environmental soil samples are generally attributed to natural heterogeneities in the soil matrix from which the sample and duplicate are prepared.

The LCS in the chlorinated pesticide analysis had consistently low recoveries (9-15\%) for all analytes except toxaphene, which was not included in the LCS (see below). The MS/MSD results were within criteria. Third party validation qualified the SDG K0286 data for all chlorinated pesticide analytes, except toxaphene, as estimated with "J" flags. 
In the chlorinated pesticide analysis, the analyte toxaphene was not included in the MS, MSD, or LCS samples (see the discussion on toxaphene given for SDG K0284). All of the toxaphene data in SDG K0286 was qualified by third-party validation as estimated with "J" flags.

Surrogates in the chlorinated pesticide analysis were consistently above the acceptance criteria in the field samples. This suggests a high bias in the data. However, most of the data is listed as nondetected and a high bias has no affect on nondetected analytical data. The detected concentrations of beta-BHC in samples $\mathrm{J} 11 \mathrm{~KB} 3$ and $\mathrm{J} 11 \mathrm{KC} 3$, as well as the detected concentration of methoxychlor in sample $\mathrm{J} 11 \mathrm{~KB} 4$ were qualified as estimates with " $\mathrm{J}$ " flags by third-party validation.

Surrogates in the PCB analysis were also consistently above the acceptance criteria. This suggests a high bias in the data. However, most of the data is listed as nondetected and a high bias has no affect on nondetected analytical data. The detected concentrations of aroclor-1254 in samples $\mathrm{J} 11 \mathrm{~KB} 6, \mathrm{~J} 11 \mathrm{~KB} 9, \mathrm{~J} 11 \mathrm{KC} 0$, and $\mathrm{J} 11 \mathrm{KC} 3$ were qualified as estimates with " $\mathrm{J}$ " flags by third-party validation.

In the metals analysis, the detected concentrations of lithium, sodium, and phosphorus in sample $\mathrm{J} 11 \mathrm{KC} 4$ (the equipment blank) were low enough to be less than $1 / 5^{\text {th }}$ of the reported concentrations in the MB. All of these detections were below the CRQL. Third-party validation has qualified the lithium, sodium, and phosphorus data in sample $\mathrm{J} 11 \mathrm{KC} 4$ as estimated nondetections with "UJ" flags. Similarly, all of the detected concentrations of uranium in SDG K0286 were qualified by third-party validation as estimated nondetections with "UJ" flags.

The LCS recovery for silicon was reported below the acceptance criteria at 50.3\%. Third party validation has qualified all of the silicon results in SDG K0286 as estimated with "J" flags.

In the metals analysis, seven analytes had MS recoveries outside of acceptance criteria. For most of these analytes, the spiking concentration was insignificant compared to the native concentration in the sample from which the MS was prepared. For these analytes, the deficiency in the MS is a reflection of the analytical variability of the native concentration, rather than a measure of the recovery from the sample. To confirm quantitation, PDSs and serial dilutions were prepared for all seven analytes with acceptable results. The analytes antimony and calcium did not have mismatched spike and native concentrations in the original MS. Both of these analytes have been qualified by third-party validation as estimates with "J" flags for all samples in SDG K0286. The original MS recoveries for antimony and calcium were $59.2 \%$ and $68.6 \%$, respectively. Estimated, or "J"-flagged, data are considered acceptable for the intended use of the data.

In the hexavalent chromium data, the laboratory reported an RPD of $60.3 \%$. This calculation is misleading because the main sample $(\mathrm{J} 11 \mathrm{~KB} 3)$ was reported as nondetected at $0.22 \mathrm{mg} / \mathrm{kg} \mathrm{U}$ and the duplicate sample was reported as detected at $0.26 \mathrm{mg} / \mathrm{kg}$. It is more common not to calculate RPDs for analytes where one value is detected and one value is not detected. The actual meaning of such a calculation is unclear. Third-party validation did not assign any qualifiers to the hexavalent chromium data. 
The RPD calculated for thorium-232 was above the acceptance criteria at $39 \%$. Third-party validation qualified all of the thorium-232 data in SDG K0286 as estimated with "J" flags.

In order to calculate the number of samples needed in the statistical sampling plan, the standard deviation in the then-unknown data set had to be assumed. Examination of the verification data set shows that the assumptions in the sampling plan were valid for the design.

\section{Summary}

Limited, random, or sample matrix-specific influenced batch QC issues such as those identified above are a potential for any analysis. The number and types seen in these data sets were within expectations for the matrix types and analyses performed. The DQA review of the 100-B-14:2 areas 2 and 5 verification sampling data found the results to be accurate within the standard errors associated with the analytical methods, sampling, and sample handling. The DQA review for 100-B-14:2 areas 2 and 5 concludes that the reviewed data are of the right type, quality, and quantity to support the intended use. Detection limits, precision, accuracy, and sampling data group completeness were assessed to determine if any analytical results should be rejected as a result of QA/QC deficiencies. All analytical data were found acceptable for decision-making purposes.

\section{0-B-14:2 (Area 3) Confirmatory Sampling Data Quality Assessment}

A review of the field logbook (BHI 2005b) and applicable analytical data packages has been performed as part of this DQA. All samples were collected per agreements with the lead regulatory agency.

Three SDGs were generated by confirmatory sampling activities: W04683, H3219, and H3220. Some of the samples in these SDGs were analyzed using by TCLP for potential waste designation purposes. The TCLP data is not used for site closure and is not addressed in this DQA.

\section{$\underline{\text { SDG W04683 }}$}

SDG W04683 consists of one sample, J037M7, analyzed for hexavalent chromium. The MS and MSD were both nondetected for hexavalent chromium. The LCS had a good recovery (102\%) for hexavalent chromium. As hexavalent chromium also was not detected in the field sample, the sample matrix appears to be reactive (destructive) with hexavalent chromium. The field sample and the MS/MSD samples were prepared, extracted, and analyzed a second time (out of hold time) for sample J037M7. For the second analysis, $1 / 10^{\text {th }}$ of the sample matrix was used so that when the MS/MSD were prepared, they were effectively at 10 times greater concentration than in the previous extraction. In the second analysis, the MS/MSD did not return nondetects but did confirm that the hexavalent chromium was reacting with the sample matrix. Because the laboratory was able to run a valid standard curve with calibration verifications, and obtained a $102 \%$ result for the LCS in the first analysis, it is clear that the analytical equipment was functioning. Hexavalent chromium was added to the sample matrix in the MS/MSD pair but did not persist long enough to be analyzed for in the first analysis. Results from the second analysis confirm that the sample matrix is destructive to hexavalent chromium. Under these conditions, it 
is expected that no hexavalent chromium would exist in the field sample, which was nondetect for hexavalent chromium. The original data, which was not out of hold time, has been reported in the data set. The data is valid and useable for decision-making purposes.

\section{$\underline{\text { SDG H3219 }}$}

SDG H3219 consists of one sample, J037M6, analyzed for SVOCs, chlorinated pesticides, PCBs, herbicides, ICP metals, mercury, hexavalent chromium, and analyzed by gross alpha, gross beta, and gamma spectroscopy methods.

In the SVOC analysis for SDG H3219, there were 58 MS/MSD recoveries outside of acceptance criteria. Almost all of these deficiencies were in the MSD. The MS and LCS show good recoveries. The MSD result is certainly the result of a lab error--the analyst cites "loss during extraction" in reference to the MSD result, although a error during spiking seems more likely to have caused the entire range of analytes to be systemically reduced by a similar amount. This error is limited to the MSD and the field sample data is not affected. The data is useable for decision-making purposes.

Also in the SVOC analysis for SDG H3219, the analytes 2,6-dinitrotoluene, 4-nitroaniline, and carbazole had high recoveries in the MS. The analyte 2,6-dinitrotoluene also had a high recovery in the LCS. In each of these cases, the high recovery suggests a high bias in the sample data. None of these analytes were detected in the field sample so their results are unaffected. The data is useable for decision-making purposes.

Bis(2-ethylhexyl)phthalate and di-n-butyl phthalate were found in the MB for the SVOC analysis of SDG H3219. These analytes are common laboratory contaminants, and the results are below the CRQLs. There is no significant impact to the field sample data, relative to the RAGs. The data is useable for decision-making purposes.

In the PCB analysis for SDG H3219, the LCS recoveries for aroclor-1016 (184\%) and aroclor-1260 (147\%) were above criteria. In the herbicide analysis for SDG H3219, the MS recovery for dinoseb (164\%) was also above acceptance criteria. These high recoveries suggest a high bias in the data for these analytes. However, the field sample was nondetect for these analytes. Therefore, there is no impact on the field sample data. The data is useable for decision-making purposes.

In the metals analysis for SDG H3219, the analyte boron was detected in the MB at $0.84 \mathrm{mg} / \mathrm{kg}$. Boron was also detected in the field sample, at $3.0 \mathrm{mg} / \mathrm{kg}$. The boron result in the MB is greater than three times the instrument detection limit (IDL). The MB result is also more than $1 / 20^{\text {th }}$ of the field sample result. For both of these reasons, the laboratory is required to identify the boron results. However, the boron results are not significant with respect to the applicable RAGs and will have no impact on this site. The data is useable for decision-making purposes. 


\section{$\underline{\text { SDG H3220 }}$}

SDG H3220 consists of one sample, J037M8, analyzed for SVOCs, chlorinated pesticides, PCBs, herbicides, ICP metals, and analyzed by gross alpha, gross beta, and gamma spectroscopy methods.

In the SVOC analysis for SDG H3220, the nature of the extract forced the laboratory to dilute the samples as much as 10-fold. With this type of extract, it is expected that matrix effects will be observed. Twenty-one of the analytes had low recoveries in the MS or MSD. None of these recoveries were low enough to indicate any significant problems. There is no impact on the sample data. The data is useable for decision-making purposes.

Bis(2-ethylhexyl)phthalate and di-n-butyl phthalate were found in the MB for the SVOC analysis of SDG H3220. These analytes are common laboratory contaminants and there is no significant impact to the field sample data, relative to the RAGs. The data is useable for decision-making purposes.

The LCS in the SVOC analysis of SDG H3220 for 3-nitroanaline had a high recovery (147\%). This suggests a high bias in the data set for this analyte. However, 3-nitroanaline was not detected in the field samples. There is no impact on the field data. The data is useable for decision-making purposes.

In the chlorinated pesticide analysis for SDG H3220, five analytes were out of acceptance criteria for the MS (heptachlor epoxide 189\%, dieldrin 190\% in MS [140\% in MSD], 4-4' DDE $138 \%$, and methoxychlor $229 \%$ ). This suggests a high bias in the sample data for these analytes. Examination of the field sample data shows low-level detections for some of these analytes. The data is useable for decision-making purposes.

In the herbicide analysis for SDG H3220, the analyte dinoseb had a MS recovery of $182 \%$. This suggests a high bias in the sample data for dinoseb. However dinoseb was not detected in the field sample data. There is no impact on the sample data. The data is useable for decisionmaking purposes.

In the metals analysis for SDG H3220, the analytes boron and zirconium were detected in the $\mathrm{MB}$ at levels that were greater than the IDL and more than $1 / 20^{\text {th }}$ of the sample results for these metals. Examination of the data shows that the results are insignificant compared to the applicable RAGs. Any laboratory contamination suggested by these results has no impact on the site. The data is useable for decision-making purposes.

Samples in the SVOC analyses in SDGs H3219 and H3220 were diluted for analysis, resulting in practical quantitation limits (PQLs) elevated above RAGs for multiple nondetected analytes. Similarly, trace metals analysis was inadvertently not specified for selenium in the ICP metals analyses in these SDGs, resulting in a PQL above the soil RAG for protection of the Columbia River. However, PQLs reported by the laboratory are as much as an order of magnitude above the IDLs. Analytes that are detected below the PQLs and above the IDLs are regularly reported with " $\mathrm{J}$ " qualifiers. In the case of the nondetected analytes, it is assumed that, had the analytes 
been present below the PQLs and above RAG concentrations, they would have been detected and reported with "J" qualifiers.

Limited, random, or sample matrix-specific influenced batch quality control issues such as those identified are a potential for any analysis. The number and types seen in these data sets were within expectations for the matrix types and analyses performed.

The DQA review for the 100-B-14:2 (area 3) subsite found the results to be accurate within the standard errors associated with the methods, including sampling and sample handling. The DQA review concludes that the data are of the right type, quality, and quantity to support the intended use. Detection limits, precision, accuracy, and sampling data group completeness were assessed to determine if any analytical results should be rejected as a result of QA and QC deficiencies. All analytical data were found to be acceptable for decision-making purposes.

\section{0-B-14:2 (Area 4) Confirmatory Sampling Data Quality Assessment}

Because holding times for hexavalent chromium analysis in other solids (i.e., pipe sediment/debris) were exceeded by more than twice the requirement, third-party validation of confirmatory data directed that all nondetects be rejected. There were no nondetected hexavalent chromium results in pipe sediments, but the detections should be considered estimated and potentially low-biased.

Due to detections and data quality issues in the confirmatory data set, all confirmatory analyses were retained for verification sampling following site remediation, with the exception of gross alpha and gross beta analyses. There were no quantitations above background or significant data quality issues in these analyses.

\section{0-B-14:2 (Area 4) Verification Sampling Data Quality Assessment}

A review of the work instruction (WCH 2006d), the field logbook (WCH 2006a), and applicable analytical data packages has been performed as part of this DQA. All samples were collected per the sample design.

Data from verification samples collected at the 100-B-14:2 (area 4) subsite was provided by the laboratory in SDG K0468. This SDG was submitted for third-party validation. No major deficiencies were found in the data and all of the data was found to be acceptable for decisionmaking purposes. Minor deficiencies, as well as qualifiers applied by third-party validation, will be discussed below.

\section{$\underline{\text { SDG K0468 }}$}

SDG K0468 comprises 13 field samples (J12R02 through J12R14) collected from the 100-B14:2 (area 4) subsite. These field samples were analyzed for SVOCs, pesticides, PCBs, ICP metals, mercury, hexavalent chromium, and tested by gamma spectroscopy. Sample J12R14 is a field duplicate of sample J12R09; sample J12R12 is from the east BCL stockpile; sample J12R13 is from the west BCL stockpile. Ten samples were collected from statistically selected locations 
within the 100-B-14:2 (area 4) excavation (WCH 2006d). This SDG also includes an equipment blank, sample J12R15, analyzed for SVOCs, ICP metals, and mercury.

As discussed in the verification sampling results data evaluation section, the samples collected contained pieces of asphalt. Analytically, the asphalt presents a myriad of difficulties to the various methods which are not designed for elevated concentrations of the oils and tars in asphalt. As a result, many of the analytes in the following discussion were qualified as estimated for one or more QA/QC issues. However, none of the data have been rejected and all remain useable for decision-making purposes.

The SVOC analysis MS and MSD were prepared in separate batches. Third-party validation qualified all of the SVOC analytes in all of the samples in SDG K0468 as estimates with "J" flags because the MS/MSD pair was not prepared together.

The SVOC analysis MS for 1,2,4-trichlorobenzene was below the acceptance criteria at 59\%. Third-party validation qualified the 1,2,4-trichlorobenzene results in all SDG K0468 samples, except samples J12R03, J12R05, and J12R15, as estimated with "J" flags.

An SVOC surrogate recovery (2-fluorobiphenyl) in sample J12R02 was below acceptance criteria at $27 \%$. Third party validation qualified the related SVOC analytes in sample J12R02 as estimates with "J" flags. The analytes related to the 2-fluorobiphenyl surrogate and qualified by third-party validation are 1,3-dichlorobenzene, 1,4-dichlorobenzene, 1,2-dichlorobenzene, 1,2,4-trichlorobenzene, 2-chloronaphthalene, hexachlorobenzene, hexachloroethane, hexachlorobenzene, hexachloroethane, hexachlorobutadiene, hexachlorocyclopentadiene, 4-chloroaniline, and 3,3'-dichlorobenzidine.

The SVOC analyte and common laboratory contaminant bis(2-ethylhexyl)phthalate was found in the MB at less than the CRQL. Bis(2-ethylhexyl)phthalate was also detected in field samples J12R02 through J12R08 and sample J12R11 at similar concentrations to that found in the MB and also below the CRQL. Third-party validation raised the reporting level for bis(2-ethylhexyl)phthalate to the CRQL and qualified the results as nondetected with "U" flags for the samples in which bis(2-ethylhexyl)phthalate was detected.

The SVOC analytes and common laboratory contaminants di-n-butylphthalate and diethylphthalate were detected in the equipment blank (and some samples) at concentrations below the CRQL. This is an artifact of the sampling process and is small enough to not have any impact on the decision-making process. No qualifications were added to the data set based on this issue.

The LCS results for analytes 2-methylnaphthalene, 2,6-dinitrotoluene, and hexachlorocyclopentadiene are above the acceptance criteria at $102 \%, 111 \%$, and $115 \%$. These analytes are expected to display low recoveries, therefore, recoveries around $100 \%$ are considered high. These results may suggest a high bias in the field sample data for 2-methylnaphthalene, 2,6-dinitrotoluene, and hexachlorocyclopentadiene. High-biased data is acceptable for the intended use of the data. No qualifications were added to the data set based on this issue. 
Boron was detected in the ICP metals MB. Third-party validation qualified the boron data in all of the samples except J12R07 as nondetected estimates with "UJ" flags.

Beryllium and lithium were also detected in the ICP metals MB at low levels below the CRQL. Only sample J12R15, the equipment blank, had low enough concentrations for the concentration in the method blank to be considered significant. Third-party validation qualified the beryllium and lithium results in sample J12R15 as nondetected estimates with a "UJ" flag.

The MS recoveries for antimony and mercury were below the acceptance criteria at $56.7 \%$ and $54.5 \%$, respectively. Third-party validation qualified all of the antimony and mercury results in SDG K0468 as estimates with "J" flags.

The LCS recovery for silicon was below the acceptance criteria at 37.5\%. Third-party validation qualified all of the silicon results in SDG K0468 as estimates with "J" flags.

The RPDs calculated for molybdenum, nickel, and mercury in the laboratory duplicate were above the acceptance criteria $(+/-30 \%)$ at $35.1 \%, 32.6 \%$, and $67.5 \%$, respectively. Difficulty in producing truly homogeneous mixtures of soils is well known, and the lack of homogenous samples often results in high RPDs. No qualifications were added to the data set based on this issue.

MS recoveries in the ICP metals analysis were out of criteria for aluminum, iron, manganese, phosphorus, antimony, silicon, and titanium. Because the MS is prepared from a field sample, the initial concentrations of the analytes are not known when the MS is being prepared. When the spike concentration is small compared to the already present concentration of an analyte, the spike response can be overwhelmed by the variability in the analytical response and in the variability introduced by the heterogeneity in the sample. The standard laboratory response to this situation is to run serial dilution PDSs to confirm the quantitation of the affected analytes. All of the PDS results were within criteria, in the range of $73 \%$ to $98.7 \%$, except for iron which had a $50.9 \%$ recovery in the PDS. Iron is not a COC/COPC for the 100-B-14:2 (area 4) subsite. No qualifications were added to the data set based on these issues.

In the pesticide analysis, the analyte toxaphene was not included in the MS, MSD, or LCS samples. Toxaphene is actually a mixture of compounds rather than a discrete analyte. While the overall concentration of toxaphene can be calculated using several unobstructed peaks in the chromatography, the inclusion of toxaphene in the spiking mixture would be problematic for the other pesticide analytes. The laboratory typically quantitates toxaphene but does not include toxaphene in QA/QC samples. Third-party validation qualified all of the toxaphene data in SDG K0468 as estimated with "J" flags and, therefore, useable for decision-making purposes.

The pesticide MS had low recoveries (approximately 28\%) on all of the analytes (except toxaphene) while the MSD recoveries were all acceptable (approximately 71\%). This resulted in RPDs between the MS and MSD that were above the acceptance criteria for all of the analytes, except toxaphene. Third-party validation qualified all of the pesticide data, except toxaphene, as estimates with "J" flags due to the MS recoveries and the RPDs. 
The pesticide analysis surrogate recoveries were elevated above the acceptance criteria in samples J12R05, J12R06, J12R10, and J12R14. The elevated surrogates suggest a high bias in the sample data. Third-party validation qualified all of the detected analytical results in these samples as estimated with "J" flags.

The laboratory reported an elevated RPD for the laboratory duplicate in the hexavalent chromium analysis. The main sample and duplicate had results of $0.20 \mathrm{mg} / \mathrm{kg} \mathrm{U}$, and $0.35 \mathrm{mg} / \mathrm{kg}$, respectively. It is more common not to calculate RPD when one analysis in a pair is nondetected, and interpretation of this result is problematic. No qualifications were added to the data set based on this issue.

In the PCB analysis, patterns for aroclor-1254 and aroclor-1260 were recognized. Due to possible interferences between the two analytes, quantitation was performed using congeners common to both aroclors. This approach gives the best overall PCB concentration but is not specific between the two aroclors. The overall values have been reported as aroclor- 1254 while aroclor-1260 has been reported as nondetected. No qualifications were added to the data set based on this issue.

The gamma spectroscopy analysis of SDG K0468 had elevated RPDs for radium-226 in the laboratory duplicate, and for thorium-232 in the field duplicate. Third-party validation qualified all of the radium-226 data in SDG K0468 as estimated with "J" flags due to the 67\% RPD in the laboratory duplicate. The $36 \%$ RPD result for thorium-232 in the field duplicate did not result in any qualification of the data set.

In order to calculate the number of samples needed in the statistical sampling plan, the standard deviation in the then unknown data set had to be assumed. Examination of the verification data set shows that the assumptions in the sampling plan were valid for the design.

\section{Summary}

Limited, random, or sample matrix-specific influenced batch QC issues such as those identified are a potential for any analysis. The number and types seen in these data sets were within expectations for the matrix types and analyses performed. The DQA review of the 100-B-14:2 (area 4) verification sampling data found the results to be accurate within the standard errors associated with the analytical methods, sampling, and sample handling. The DQA review for 100-B-14:2 (area 4) verification data set concludes that the reviewed data are of the right type, quality, and quantity to support the intended use. Detection limits, precision, accuracy, and sampling data group completeness were assessed to determine if any analytical results should be rejected as a result of QA and QC deficiencies. All analytical data were found acceptable for decision-making purposes.

\section{7-B2:1 Verification Sampling Data Quality Assessment}

A review of the work instruction (BHI 2005d), the field logbook (BHI 2005c), and applicable analytical data packages has been performed as part of this DQA. All samples were collected 
per the sample design. Where the sample design allowed for additional samples, if required to properly characterize the site, no additional data needs were identified at the time of field sampling.

Data from verification samples collected at the 1607-B2:1 subsite were provided by the laboratory in SDG H3339. The analytical laboratory reprepared and reanalyzed sample J03VC1 for SVOCs within this SDG due to surrogate recovery problems. Third-party validation rejected the majority of the data from the initial analysis. SVOC data presented for this sample is based on the reanalysis. Beyond this issue, no major deficiencies were found in the data and all of the remaining data was found to be acceptable for decision-making purposes. A more complete discussion of sample J03VC1, minor deficiencies in the data, and the third-party validation data qualifiers is presented below.

\section{$\underline{\text { SDG H3339 }}$}

SDG H3339 comprises 14 field samples (J03VB8, J03VB9, and J03VC0 through J03VC9, J03VD0, and J03VD1) collected from the 1607-B2:1 subsite. The field samples were analyzed for volatile organic compounds (VOCs), SVOCs, pesticides, herbicides, PCBs, ICP metals, mercury, and hexavalent chromium. This SDG also includes a field equipment blank (J03WW6), analyzed for SVOCs, ICP metals, and mercury. Sample J03VD2 is a field duplicate of sample J03VB9. Samples J03VC9, J03VD0, and J03VD1 were collected from overburden stockpiles to the southwest, northwest, and northeast of the 1607-B2:1 excavation. The 11 remaining samples were collected from statistically selected locations within the 1607-B2:1 excavation (BHI 2005d).

In the SVOC analysis, sample J03VC1 had very poor recoveries for 4 of 5 surrogates. Thirdparty validation rejected approximately $80 \%$ of the SVOC analytes in that analysis of J03VC1. The laboratory re-extracted and reanalyzed the sample with significantly improved results. The project determined that the remaining $20 \%$ of the data from the original run of sample J03VC1 were not of significantly higher quality than the rejected data for that analysis. Therefore, none of the original SVOC data from sample J03VC1 were used for decision-making. All SVOC data presented for sample $\mathrm{J} 03 \mathrm{VC} 1$ is from the second aliquot of the J03VC1 sample that was prepared, extracted, and analyzed separately from the rest of the samples. The MS and MSD normally included with a sample batch were not included in the separate J03VC1 sample batch. Third-party validation has, therefore, qualified all of the data for sample J03VC1 with "J" flags as estimated values.

The SVOC carbazole recovery for the LCS run with the J03VC1 batch was below acceptance criteria at $46 \%$. Third-party validation qualified the carbazole result in sample J03VC1 as an estimated value with a "J" flag.

The SVOC RPDs for 3-nitroanaline, 4-nitrophenol, and 4-nitroanaline were above the acceptance criteria at $50 \%, 38 \%$, and $43 \%$, respectively. Third-party validation qualified the sample data for these three analytes as estimated in all samples except sample J03VC1. 
The common SVOC laboratory contaminant bis(2-ethylhexyl)phthalate was detected in the MB at $24 \mu \mathrm{g} / \mathrm{kg}$, and in all but one of the field samples in the range of 21 to $61 \mu \mathrm{g} / \mathrm{kg}$. Third-party validation raised the reported sample values to the CRQL $(660 \mu \mathrm{g} / \mathrm{kg})$ and requalified the samples as nondetected with " $U$ " flags for all samples except sample J03VD0, where bis(2-ethylhexyl)phthalate was not detected.

In the pesticide analysis, the MS had low recoveries for all analytes, including the surrogates, all in the range of 8 to $21 \%$. The MSD and LCS results were all acceptable in the ranges of 92 to $117 \%$ and 94 to $119 \%$, respectively. The laboratory commented that the MS may have been prepared with the wrong spike concentration. However, that would not explain the low surrogate concentrations in the MS, which are added separately. The spiked analytes and surrogates, all low in a narrow range, suggest that the liquid injector system delivered a poor injection to the analytical system. Surrogate recoveries are ideal indicators for this type of error. Fortunately, the typical symptom (low recovery on all surrogates in a narrow range) is not seen in the remaining samples.

In the pesticide analysis, the analyte toxaphene was not included in the MS, MSD, or LCS samples. Toxaphene is actually a mixture of compounds rather than a discrete analyte. While the overall concentration of toxaphene can be calculated using several unobstructed peaks in the chromatography, the inclusion of toxaphene in the spiking mixture would be problematic for the other pesticide analytes. The laboratory typically quantitates toxaphene but does not include toxaphene in QA/QC samples. Third party validation qualified all of the toxaphene data as estimated with "J" flags.

The MS result in the ICP metals analysis for antimony was below criteria at 36.7\%. Third-party validation qualified all of the antimony data as estimated with " $\mathrm{J}$ " flags.

The analyte, chromium (total), was detected in the ICP metals MB. Third-party validation requalified the chromium (total) result in sample J03WW6, the equipment blank, as an estimated nondetection with a "UJ" flag.

The MS recovery for 4-methyl-2-pentanone in the VOA analysis was above the acceptance criteria at $155 \%$. Similarly, the MS and MSD recoveries for 1,1,2,2-tetrachloroethane are above the acceptance criteria at $144 \%$ and $133 \%$, respectively. These results suggest a high bias in the field sample data for these analytes. High biased data are acceptable for decision-making purposes.

The VOA analysis MS and MSD were prepared in a separate batch from samples J03VB8, J03VC1, J03VC4, J03VC7, and J03VC8. Third-party validation has qualified all VOA results in these samples as estimated with "J" flags.

The common VOA laboratory contaminant, methylene chloride, was detected in the method blank and the field samples at concentrations below the CRQL, in the range of $2 \mu \mathrm{g} / \mathrm{kg}$ to $13 \mu \mathrm{g} / \mathrm{kg}$. Methylene chloride results for all of the samples, except J03VC5, J03VC9, and J03VC7R, were raised to the CRQL and qualified as nondetections with "U" flags by third-party 
validation. Third-party validation also qualified the results for samples J03VC5, J03VC9, and J03VC7R as nondetections with "U" flags.

Herbicide analysis was not called for in the work instruction. The chain of custody shows that the project inadvertently requested this analysis for SDG H3339.

The herbicide surrogate recovery of DCAA in sample J03VB8 was below the acceptance criteria at $27 \%$. However, the sample meets the secondary criteria of no more than one surrogate outlier per sample.

The herbicide RPDs for the analytes dicamba, dichloroprop, 2,4,5-Tp, and 2,4,5-T were above the acceptance criteria at 33\%,33\%, 32\%, and 32\%, respectively. Elevated RPDs are attributed to natural heterogeneities in the sample matrix. Third-party validation qualified the results for these analytes as estimates with "J" flags.

\section{Summary}

Limited, random, or sample matrix-specific influenced batch QC issues such as those identified are a potential for any analysis. The number and types in this data set are within expectations for the matrix types and analyses performed. With the exception of the rejected data for the original SVOC analysis of sample J03VC1, the DQA review of the 1607-B2:1 verification sampling data found the results to be accurate within the standard errors associated with the analytical methods, sampling, and sample handling. The DQA review for the 1607-B2:1 verification data set concludes that the retained data reviewed are of the right type, quality, and quantity to support the intended use. Detection limits, precision, accuracy, and sampling data group completeness were assessed to determine if any analytical results should be rejected as a result of QA/QC deficiencies. All analytical data other than the original SVOC analysis of sample J03VC1 were found to be acceptable for decision-making purposes.

\section{7-B2:2 and 100-B-14:2 (Area 1) Verification Sampling Data Quality Assessment}

Review of the work instruction (WCH 2006e), the field logbook (WCH 2006b), and applicable analytical data packages has been performed as part of this DQA. All samples were collected per the sample design.

Data from verification samples collected at the 1607-B2:2 and 100-B-14:2 (area 1) subsites were provided by the laboratory in SDGs K0454 and K0459. No major deficiencies were found in the data and all of the data was found to be acceptable for decision-making purposes. SDG K0459 was submitted for third-party validation. Minor deficiencies, as well as qualifiers applied by third-party validation, will be discussed below.

\section{$\underline{\text { SDG K0454 }}$}

This SDG consists of 16 field samples (J12NX4 through J12NX9, and J12NY0 through J12NY9) collected from the 1607-B2:2 and 100-B-14:2 (area 1) subsites. The field samples were analyzed for SVOCs, pesticides, PCBs, ICP metals, mercury, hexavalent chromium, gamma-emitting 
radionuclides, total beta radiostrontium, and tritium. This SDG also includes a field equipment blank (J12P04), analyzed for SVOCs, ICP metals, and mercury. Sample J12NY4 is a field duplicate of sample J12NY3. Samples J12NX4, J12NX5, J12NX6, J12NX7, and J12NX8 were collected from BCL stockpiles from the 1607-B2:2 and 100-B-14:2 (area 1) excavations. The remaining samples were collected from statistically selected locations within the excavations (WCH 2006e).

In the SVOC analysis of sample J12NX4, the surrogate recovery for 2,4,6-tribromophenol was above the laboratory acceptance criteria at $123 \%$. However, this is within the project acceptance criteria $(+/-30 \%)$. This was the only surrogate deficiency found out of 156 surrogates analyzed in this SDG.

The common laboratory contaminant analytes bis(2-ethylhexyl)phthalate and di-n-butylphthalate were found in the method blank at concentrations less than twice their CRQLs. The data is not significantly affected.

The original extraction of sample J12P04 had a low recovery on the internal standard, so the sample was reprepared, re-extracted, and reported. The resulting data is acceptable.

In the chlorinated pesticide analysis, 9 of 46 surrogate recoveries were above the acceptance criteria with recoveries in the range of $119 \%$ to $156 \%$. These high recoveries suggest a high bias in the sample results. High-biased data are useable for decision-making purposes.

In the chlorinated pesticide analysis, the analyte toxaphene was not included in the MS, MSD, or LCS samples. Toxaphene is actually a mixture of compounds rather than a discrete analyte. While the overall concentration of toxaphene can be calculated using several unobstructed peaks in the chromatography, the inclusion of toxaphene in the spiking mixture would be problematic for the other pesticide analytes. The laboratory typically quantitates toxaphene but does not include toxaphene in QA/QC samples. The toxaphene data is considered estimated but useable for decision-making purposes.

The laboratory reported interferences on one analytical column for the analytes endrin aldehyde (J12NX6, J12NY9), methoxychlor (J12NX9, J12NY0), endosulfan II (J12NY5), and endrin ketone (J12NY5). Results were quantitated based on the remaining column, and the data is useable for decision-making purposes.

In the PCB analysis, 9 of 44 surrogate recoveries were above the laboratory acceptance criteria with recoveries in the range of $119 \%$ to $123 \%$. These are within the project acceptance criteria. The results may suggest a high bias in the data, which is acceptable for decision-making purposes.

The PCB analysis of the LCS for aroclor- 1260 was above the acceptance criteria at $148 \%$. This result suggests a high bias in the sample data. High biased data are useable for decision-making purposes. 
A continuing calibration verification (CCV) sample returned a high bias reading of $17.6 \%$ (laboratory limit $=15.6 \%$ ) in the PCB analysis. This CCV is only associated with the MB and the LCS samples. None of the field sample data are affected. The data are useable for decisionmaking purposes.

In the ICP metals, analysis of a CCV sample returned a high bias reading for calcium and phosphorus. The affected samples were rerun. There is no issue with the final data.

The ICP metals MB result for potassium $(6.9 \mathrm{mg} / \mathrm{kg}$ ) was greater than the PQL for potassium. $\mathrm{MB}$ contamination is considered significant when it is within 20 times a sample concentration. Sample J12P04 (the equipment blank) was the only sample with a low enough concentration to be within the 20 times the $\mathrm{MB}$ concentration for potassium. While the equipment blank value for potassium may be considered estimated, none of the field verification samples are affected. The data are useable for decision-making purposes.

The LCS recovery for silicon was above the acceptance criteria (+/- 30\%) at $40.1 \%$. This may suggest a high bias in the silicon data for the field samples. However, high biased data are acceptable for decision-making purposes.

The MS recoveries for aluminum, iron, antimony, silicon, and titanium were out of acceptance criteria. Because the concentrations of these analytes are unknown when the matrix spike is prepared, the concentration of the spike is often inappropriate for the sample. The laboratory confirms quantitation of these analytes by running serial dilutions and PDSs. All of these analytes had good results in the PDSs, with recoveries in the range of $70.9 \%$ to $115.3 \%$. The data are useable for decision-making purposes.

The laboratory duplicate had a high RPD for lead at $40 \%$. Difficulty in producing truly homogeneous mixtures in soils is well-known. Heterogeneous soil samples often result in high RPDs. It is likely that more essentially inert material (e.g., larger size gravel) was present in either the sample or the duplicate. The data are useable for decision-making purposes.

The laboratory reported a high RPD for hexavalent chromium at $66.1 \%$. In this case, hexavalent chromium was not detected in the main sample $(0.20 \mathrm{mg} / \mathrm{kg} \mathrm{U})$, but was detected in the laboratory duplicate $(0.32 \mathrm{mg} / \mathrm{kg})$. Calculation of RPDs is usually not performed for sample pairs that have one nondetected value. This is because the analytical response near the detection limit is variable, which is the reason for the detection limit. The RPD is meant to gauge the laboratory performance in general, rather than the analytical device performance at its defined limit of usefulness. The field sample data are not affected by this result, and the data are useable for decision-making purposes.

\section{$\underline{\text { SDG K0459 }}$}

This SDG consists of the remaining 4 statistical samples (J12PW5, J12PW6, J12PW7, and J12PW8) collected from within the 1607-B2:2/100-B-14:2 (area 1) excavation. The field samples were analyzed for SVOCs, pesticides, PCBs, ICP metals, mercury, hexavalent 
chromium, gamma-emitting radionuclides, total beta radiostrontium, and tritium. This SDG was submitted for third-party validation.

In the SVOC analysis, the MSD recovery for 2,4-dinitrophenol was low at $18 \%$. The MS recovery was within criteria. Because of the low recovery in the MSD, the RPD calculated for 2,4-dinitrophenol in the MS/MSD pair was high at 43\%. Third-party validation qualified all of the 2,4-dinitrophenol results in SDG K0459 as estimates with "J" flags. The data remain useable for decision-making purposes.

The common laboratory contaminant bis(2-ethylhexyl)phthalate was found in the MB. Thirdparty validation increased the reported value for all samples in SDG K0459 to the CRQL and requalified these results as nondetections with " $U$ " flags. The data remain useable for decisionmaking purposes.

In the chlorinated pesticide analysis, the analyte toxaphene was not included in the MS, MSD, or LCS samples. As discussed previously, the laboratory typically quantitates toxaphene but does not include toxaphene in QA/QC samples. Third-party validation qualified all of the toxaphene data in SDG K0459 as estimated with "J" flags. The data remain useable for decision-making purposes.

The LCS recovery for silicon was above the acceptance criteria $(+/-30 \%)$ at $45.5 \%$. This may suggest a high bias in the silicon data for the field samples. However, high-biased data are acceptable for decision-making purposes.

The MS recoveries for aluminum, iron, manganese, phosphorus, antimony, silicon, and titanium were outside of acceptance criteria. Because the concentrations of these analytes are unknown when the matrix spike is prepared, the concentration of the spike is often inappropriate for the sample. The laboratory routinely confirms quantitation of these analytes by running serial dilutions and PDSs. All of these analytes had good results in the PDSs, with recoveries in the range of $80.9 \%$ to $95.7 \%$. The data are useable for decision-making purposes.

There were no issues found in the radiological data (gamma spectrum, total strontium, and tritium) for SDG K0459. However, it should be noted that the radiological data was mistakenly not included in the SDG K0459 data package submitted for third-party validation. The amount of data submitted for third-party validation is still within the criteria established in the SAP (DOE-RL 2005a). The data are useable for decision-making purposes.

In order to calculate the number of samples needed in the statistical sampling plan, the standard deviation in the then-unknown data set had to be assumed. Examination of the verification data set shows that the assumptions in the sampling plan were valid for the design.

\section{Summary}

Limited, random, or sample matrix-specific influenced batch QC issues such as those identified are a potential for any analysis. The number and types seen in this data set are within expectations for the matrix types and analyses performed. The DQA review of the 
1607-B2:2/100-B-14:2 (area 1) verification sampling data found the results to be accurate within the standard errors associated with the analytical methods, sampling, and sample handling. The DQA review of the 1607-B2:2/100-B-14:2 (area 1) verification data set concludes that the data is of the right type, quality, and quantity to support the intended use. Detection limits, precision, accuracy, and sampling data group completeness were assessed to determine if any analytical results should be rejected as a result of QA/QC deficiencies. All analytical data were found acceptable for decision-making purposes.

All analytical data are stored in the ENRE project-specific database prior to being submitted for inclusion in the HEIS database. The confirmatory sample analytical data are also summarized in Appendix A, and verification sample data are included with the calculations provided in Appendix B.

\section{SUMMARY FOR INTERIM CLOSURE}

The 100-B-14:2 and 1607-B2 waste sites have been evaluated and remediated in accordance with the Remaining Sites ROD (EPA 1999) and the RDR/RAWP (DOE-RL 2005b). Because chemical and radionuclide contaminants were detected within feeder pipelines, the site was remediated by removing piping, the septic system, and surrounding soils and transporting them to ERDF, except at 100-B-14:2 (area 3), where confirmatory sampling did not demonstrate a need for remediation. Statistical and judgmental sampling to verify the completeness of remediation was performed, and analytical results were shown to meet the cleanup objectives for direct exposure, groundwater protection, and river protection. In accordance with this evaluation, the verification sampling results support a reclassification of the 100-B-14:2 and 1607-B2 sites to interim closed out. These sites do not have a deep zone component; therefore, no deep zone institutional controls are required.

\section{ENDNOTES}

The following endnotes apply to the data result comparison tables presented in the document body (Tables 2, 6a, 6b, 7a, 7b, 9a, 9b, 10a, 10b, 11a, and 11b).

a Lookup values and RAGs obtained from the RDR/RAWP (DOE-RL 2005b) or calculated per WAC 173-340-720, 173-340-730, and 173-340-740, Method B, 1996, unless otherwise noted.

b Activity corresponding to a single-radionuclide $15 \mathrm{mrem} / \mathrm{yr}$ exposure as calculated using a generic RESRAD model (DOE-RL 2005b).

c Revised lookup value per 100 Area Radionuclide and Nonradionuclide Lookup Values for the 1995 Interim Remedial Action Record of Decision (BHI 2004a).

d Noncarcinogenic cleanup level calculated from WAC 173-340-740(3), 1996 (Method B for soils) (as presented in the 100 Area RDR/RAWP [DOE-RL 2005b]). Updated oral reference dose values (as provided in the Integrated Risk Information System [IRIS]) yield Method B direct exposure RAG values of 16,000 mg/kg and 120,000 $\mathrm{mg} / \mathrm{kg}$ for barium and chromium (total), respectively.

e Barium soil cleanup level for groundwater protection calculated from WAC 173-340-740(3)(a)(ii)(A), 1996 ("100 times rule") and WAC 173-340-720(3), 1996 (Method B for groundwater) is $112 \mathrm{mg} / \mathrm{kg}$ (as presented in the RDR/RAWP [DOE-RL 2005b]). The updated oral reference dose value (as provided in IRIS) yields a Method B groundwater cleanup criteria of $7 \mathrm{mg} / \mathrm{L}$, as compared to the more restrictive maximum contaminant level (MCL) 
of $2 \mathrm{mg} / \mathrm{L}$ (40 CFR 141). Per WAC 173-340-740(3)(a)(ii)(A), 1996 ("100 times rule"), the most restrictive updated soil cleanup level for groundwater protection would be $200 \mathrm{mg} / \mathrm{kg}$.

f Where cleanup levels are less than background, cleanup levels default to background (WAC 173-340-700[4][d]) (1996).

g Barium soil cleanup level for river protection calculated from WAC 173-340-740(3)(a)(ii)(A), 1996 ("100 times rule"), a dilution-attenuation factor of 2, and WAC 173-340-720(3), 1996 (Method B for groundwater) is $224 \mathrm{mg} / \mathrm{kg}$ (as presented in the RDR/RAWP [DOE-RL 2005b]). No surface water bioconcentration factor is available for barium and no ambient water quality criteria (AWQC) value exists separate from the previous drinking water standard; therefore no WAC 173-340-730(3), 1996 (Method B for surface waters) value can be determined.

${ }^{\mathrm{h}}$ Contaminant is not predicted to reach groundwater (and thus the Columbia River) based on discussion of the contaminant depth/ $\mathrm{K}_{\mathrm{d}}$ value model presented within the confirmatory sampling discussion, above, and the 100 Area Analogous Sites RESRAD Calculations calculation brief (Appendix D), with a groundwater table

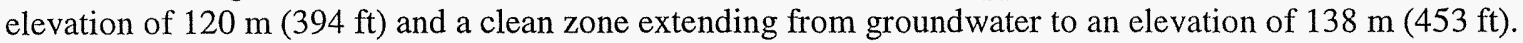

i Carcinogenic cleanup level calculated based on the inhalation exposure pathway per WAC 173-340-750[3], 1996 (Method B for air quality) and an airborne particulate mass loading rate of $0.0001 \mathrm{~g} / \mathrm{m}^{3}$ (WDOH 1997).

j No Hanford Site-specific or Washington State background value available.

${ }^{k}$ No parameters are available from the Ecology Cleanup Levels and Risk Calculations database (Ecology 2005), and no bioconcentration factor or AWQC values are available to calculate cleanup levels (WAC 173-340730(3)(a)(iii), 1996 [Method B for surface waters]).

1 Hanford Site-specific background value is not available; not evaluated during background study. Value used is from Natural Background Soil Metals Concentrations in Washington State (Ecology 1994).

${ }^{\mathrm{m}}$ Calculated cleanup level (per WAC 173-340-720(3), 1996 [Method B for groundwater] and WAC 173-340-740(3)(a)(ii)(A), 1996 ["100 times rule"]) presented is lower than that presented in the RDR/RAWP (DOE-RL 2005b), based on updated oral reference dose value (as provided in IRIS).

${ }^{\mathrm{n}}$ No cleanup levels are available from the Ecology: Cleanup Levels and Risk Calculations database (Ecology 2005); RAG values calculated using toxicity data from the Oak Ridge National Laboratory (ORNL) risk assessment database.

- Where cleanup levels are less than the required detection limit (RDL), cleanup levels default to the RDL (WAC 173-340-707[2], 1996 and DOE-RL 2005b).

$\mathrm{p}$ Based on site-specific risk assessments (Appendix B), residual pipe sediment concentrations of aroclor-1248 and dieldrin at the 100-B-14:2 subsite (area 3) satisfy RAOs for direct exposure.

${ }^{q}$ Direct exposure and groundwater protection RAG values for chlordane were mistakenly calculated based on carcinogenicity data for lindane in the RDR/RAWP (DOE-RL 2005b). Corrected values are presented in this table.

${ }^{r}$ RAG value listed in the RDR/RAWP (DOE-RL 2005b) is based on the use of benzo(a)pyrene as a surrogate. Compound-specific carcinogenic cleanup level calculated per WAC 173-340-740(3), 1996 (Method B for soils) using ORNL oral cancer potency factors.

$s$ Toxicity data for this chemical are not available. RAGs for benzo(g,h,i)perylene and phenanthrene are based on the surrogate chemicals pyrene and anthracene, respectively.

${ }^{t}$ Constituent is the result of asphalt cross-contamination of the sample matrix. Asphalt that has been used for structural and construction purposes is excluded from consideration as a dangerous waste by WAC 173-303-071(3)(e), 2004, is listed as an inert waste in WAC 173-350-990(2)(b), 2005, and does not present a significant human health risk.

"Pentachlorophenol was detected below the associated PQL $(2.5 \mathrm{mg} / \mathrm{kg})$ in one sample, and satisfies the conditions for considering cleanup criteria as achieved under the Analytical Considerations Rule (WAC 173-340-707[2], 1996).

$\checkmark$ Contaminant is not predicted to reach groundwater (and thus the Columbia River) based on discussion of the contaminant depth/ $\mathrm{K}_{\mathrm{d}}$ value model presented within the confirmatory sampling discussion, above, and the 100 Area Analogous Sites RESRAD Calculations calculation brief (Appendix D), with a groundwater table

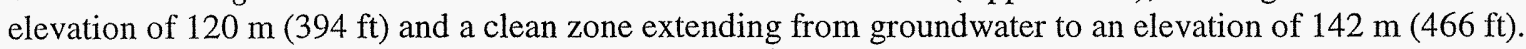

" Contaminant is not predicted to reach groundwater (and thus the Columbia River) based on discussion of the contaminant depth/ $\mathrm{K}_{\mathrm{d}}$ value model presented within the confirmatory sampling discussion, above, and the 100 Area Analogous Sites RESRAD Calculations calculation brief (Appendix D), with a groundwater table

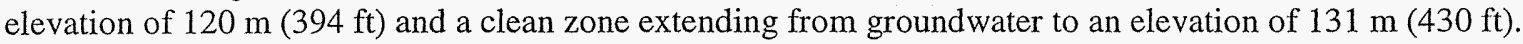


$x$ The statistical verification data set fails the second and/or third component of the WAC 173-340-740(7)(e) threepart test in comparison to soil RAGs for groundwater and/or river protection (one or more sample concentrations exceed twice the soil RAG value and/or more than $10 \%$ of the data set exceeds the soil RAG value).

y Contaminant is not predicted to reach groundwater (and thus the Columbia River) based on discussion of the contaminant depth/K $\mathrm{K}_{\mathrm{d}}$ value model presented within the confirmatory sampling discussion, above, and the 100 Area Analogous Sites RESRAD Calculations calculation brief (Appendix D), with a groundwater table

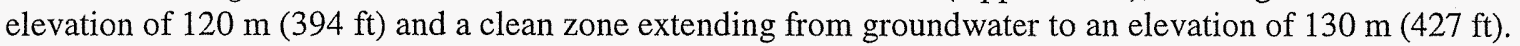

\section{Table Acronyms}

$\begin{array}{ll}\text { BCL } & =\text { not applicable } \\ \mathrm{BG} & =\text { background (obtained from DOE-RL [1996 and 2001], unless otherwise noted) } \\ \mathrm{COC} & =\text { contaminant of concern } \\ \mathrm{COPC} & =\text { contaminant of potential concern } \\ \mathrm{ND} & =\text { not detected } \\ \mathrm{RAG} & =\text { remedial action goal } \\ \text { RESRAD } & =\text { RESidual RADioactivity (dose-assessment model) }\end{array}$

\section{REFERENCES}

40 CFR 141, "National Primary Drinking Water Regulations," Code of Federal Regulations, as amended.

ANL, 2005, User's Manual for RESRAD Version 6, ANL/EAD-4, Environmental Assessment Division, Argonne National Laboratory, Argonne, Illinois.

BHI, 1999, Cleanup Verification Package for the 116-C-1 Process Effluent Trench, CVP-98-00006, Rev. 0, Bechtel Hanford, Inc., Richland, Washington.

BHI, 2000a, Data Validation Procedure for Chemical Analysis, BHI-01435, Rev. 0, Bechtel Hanford, Inc., Richland, Washington.

BHI, 2000b, Data Validation Procedure for Radiochemical Analysis, BHI-01433, Rev. 0, Bechtel Hanford, Inc., Richland, Washington.

BHI, 2001, Calculation of Total Uranium Activity Corresponding to a Maximum Contaminant Level for Total Uranium of 30 Micrograms per Liter in Groundwater, 0100X-CA-V0038, Rev. 0, Bechtel Hanford, Inc., Richland, Washington.

BHI, 2003a, Cleanup Verification Package for the 1607-B7 Septic Tank System, CVP-2003-00004, Rev. 0, Bechtel Hanford, Inc., Richland, Washington.

BHI, 2003b, Remaining Sites Field Sampling, Logbook EL-1578-1, Bechtel Hanford, Inc., Richland, Washington.

BHI, 2003c, Waste Site Evaluation for the 100-B-14:2 Sanitary Sewer (100-B-14 Process and Sanitary Sewer Underground Pipelines), Calc No. 0100B-CA-V0180, Rev. 0, Bechtel Hanford, Inc., Richland, Washington. 
BHI, 2004a, 100 Area Radionuclide and Nonradionuclide Lookup Values for the 1995 Interim Remedial Action Record of Decision, 0100X-CA-V0046, Rev. 0, Bechtel Hanford Inc., Richland, Washington.

BHI, 2004b, Cleanup Verification Package for the 100-B-8:1 and 100-C-6:1 100-B/C South Effluent Pipelines, CVP-2003-00022, Rev. 0, Bechtel Hanford, Inc., Richland, Washington.

BHI, 2005a, 100 Area Analogous Sites RESRAD Calculations, Calculation No.

0100X-CA-V0050, Rev. 0, Bechtel Hanford, Inc., Richland, Washington.

BHI, 2005b, 100BC Remaining Pipeline and Sewers Sampling and Field Services, Logbook EL-1585-2, Bechtel Hanford, Inc., Richland, Washington.

BHI, 2005c, 100BC Remaining Pipeline and Sewers Sampling and Field Services, Logbook EL-1585-3, Bechtel Hanford, Inc., Richland, Washington.

BHI, 2005d, Work Instruction for Verification Sampling of Waste Site 1607-B2:1, Septic Drain Field, Work Instruction No. 0100B-WI-G0007, Rev. 0, Bechtel Hanford, Inc., Richland, Washington.

DOE Order 5400.5, Radiation Protection of the Public and Environment, as amended, U.S. Department of Energy, Washington, D.C.

DOE-RL, 1992, Remedial Investigation Feasibility Study for the 100-BC-1 Operable Unit, DOE/RL-90-07, Rev. 0, U.S. Department of Energy, Richland Operations Office, Richland, Washington.

DOE-RL, 1994, Limited Field Investigation Report for the 100-BC-1 Operable Unit, DOE/RL-93-06, Rev. 0, U.S. Department of Energy, Richland Operations Office, Richland, Washington.

DOE-RL, 1995, Hanford Site Risk Assessment Methodology, DOE/RL-91-45, Rev. 3, U.S. Department of Energy, Richland Operations Office, Richland, Washington.

DOE-RL, 1996, Hanford Site Background: Part 2, Soil Background for Radionuclides, DOE/RL-96-12, Rev. 0, U.S. Department of Energy, Richland Operations Office, Richland, Washington.

DOE-RL, 1998, Tri-Party Agreement Handbook Management Procedures, RL-TPA-90-0001, Guideline Number TPA-MP-14, "Maintenance of the Waste Information Data System (WIDS)," U.S. Department of Energy, Richland Operations Office, Richland, Washington. 
DOE-RL, 2001, Hanford Site Background: Part 1, Soil Background for Nonradioactive Analytes, DOE/RL-92-24, Rev. 4, U.S. Department of Energy, Richland Operations Office, Richland, Washington.

DOE-RL, 2005a, 100 Area Remedial Action Sampling and Analysis Plan, DOE/RL-96-22, Rev. 4, U.S. Department of Energy, Richland Operations Office, Richland, Washington.

DOE-RL, 2005b, Remedial Design Report/Remedial Action Work Plan for the 100 Area, DOE/RL-96-17, Rev. 5, U.S. Department of Energy, Richland Operations Office, Richland, Washington.

Ecology, 1994, Natural Background Soil Metals Concentrations in Washington State, Publication No. 94-115, Washington State Department of Ecology, Olympia, Washington.

Ecology, 1995, Guidance on Sampling and Data Analysis Methods, Publication No. 94-49, Washington State Department of Ecology, Olympia, Washington.

Ecology, 2005, Cleanup Levels and Risk Calculations (CLARC) Database, Washington State Department of Ecology, Olympia, Washington, $<$ https://fortress.wa.gov/ecy/clarc/CLARCHome.aspx>.

ENV-1, Environmental Monitoring \& Management, Washington Closure Hanford, Richland, Washington.

EPA, 1989, Risk Assessment Guidance for Superfund, Volume I, Human Health Evaluation Manual (Part A), EPA/540/1-89/002, U.S. Environmental Protection Agency, Washington, D.C.

EPA, 1999, Interim Action Record of Decision for the 100-BC-1, 100-BC-2, 100-DR-1, 100-DR-2, 100-FR-1, 100-FR-2, 100-HR-1, 100-HR-2, 100-KR-1, 100-KR-2, 100-IU-2, 100-IU-6, and 200-CW-3 Operable Units, Hanford Site, Benton County, Washington, U.S. Environmental Protection Agency, Region 10, Seattle, Washington.

EPA, 2000, Guidance for Data Quality Assessment, EPA QA/G-9, QA00 Update, EPA/600/R-96/084, U.S. Environmental Protection Agency, Office of Environmental Information, Washington, D.C.

Feist, E. T., 2004, 100-B/C-1 and 100-B/C-2 Operable Units Remaining Sites for Remedial Action, CCN 112480, Interoffice Memorandum to R. A. Cárlson, dated May 28, 2004, Bechtel Hanford, Inc., Richland, Washington.

WAC 173-303, 2004, "Dangerous Waste Regulations," Washington Administrative Code.

WAC 173-340, 1996, “Model Toxics Control Act -- Cleanup,” Washington Administrative Code. 
WAC 173-350, 2005, "Solid Waste Handling Standards," Washington Administrative Code.

WCH, 2005, 100 Area / 300 Area Unit Managers' Meeting Minutes, Groundwater/Remedial Action Unit/Source Operable Units, December 8, 2005, Draft, Washington Closure Hanford, Inc., Richland, Washington.

WCH, 2006a, 100BC Remaining Pipeline and Sewers Sampling and Field Services, Logbook EL-1585-5, Washington Closure Hanford, Richland, Washington.

WCH, 2006b, 100BC Remaining Pipeline and Sewers Sampling and Field Services, Logbook EL-1585-6, Washington Closure Hanford, Richland, Washington.

WCH, 2006c, Work Instruction for Verification Sampling of the 100-B-14:2, Area 2, 1607-B7 Sanitary Sewer Pipeline and 100-B-14:2, Area 5, 115-B Sanitary Sewer Pipeline, Work Instruction No. 0100B-WI-G0012, Rev. 0, Washington Closure Hanford, Richland, Washington.

WCH, 2006d, Work Instruction for Verification Sampling of the 100-B-14:2 (Area 4)/100-B14:8, 190-B Sanitary Sewers, Work Instruction No. 0100B-WI-G0019, Rev. 0, Washington Closure Hanford, Richland, Washington.

WCH, 2006e, Work Instruction for Verification Sampling of the 1607-B2:2 Septic System and Collection Main, Work Instruction No. 0100B-WI-G0015, Rev. 0, Washington Closure Hanford, Richland, Washington.

WDOH, 1997, State of Washington Department of Health Interim Regulatory Guidance: Hanford Guidance for Radiological Cleanup, WDOH/320-015, Rev. 1, Washington Department of Health, Richland, Washington. 


\section{APPENDIX A}

\section{CONFIRMATORY SAMPLING AND WASTE CHARACTERIZATION ANAL YTICAL RESULTS}

Note: Confirmatory sample results for the 100-B-14:2 (areas 2 , 4, and 5) subsite led to a decision that remediation was necessary. Verification sampling results and calculations to support reclassification of these areas, as well as the 1607-B2 site, to interim closed out are provided in Appendix $\mathbb{B}$. 
Table A-1. 100-B-14:2 Area 2 Confirmatory Data Results. (7 Pages)

\begin{tabular}{|c|c|c|c|c|c|c|c|c|c|c|c|c|c|c|c|c|c|}
\hline \multirow{2}{*}{ Sample Location } & \multirow{2}{*}{$\begin{array}{c}\text { Sample } \\
\text { Number }\end{array}$} & \multirow{2}{*}{$\begin{array}{c}\text { Sample } \\
\text { Date }\end{array}$} & \multicolumn{3}{|c|}{ Americium-241 } & \multicolumn{3}{|c|}{ Cesium-137 } & \multicolumn{3}{|c|}{ Cobalt-60 } & \multicolumn{3}{|c|}{ Europium-152 } & \multicolumn{3}{|c|}{ Europium-154 } \\
\hline & & & $\mathrm{pCi} / \mathrm{g}$ & Q & $\mathrm{MDA}$ & $\mathrm{pCi} / \mathrm{g}$ & $Q$ & $\mathrm{MDA}$ & $\mathrm{pCi} / \mathrm{g}$ & $\mathrm{Q}$ & $\mathrm{MDA}$ & $\mathrm{pCi} / \mathrm{g}$ & $Q$ & MDA & $\mathrm{pCi} / \mathrm{g}$ & $Q$ & MDA \\
\hline A1 & J00Y63 & $9 / 16 / 03$ & 0.072 & $\mathrm{U}$ & 0.072 & 0.137 & & 0.043 & 0.037 & $\mathrm{U}$ & 0.037 & 0.084 & $\mathrm{U}$ & 0.084 & 0.12 & $\mathrm{U}$ & 0.12 \\
\hline $\mathrm{A} 2$ & J00Y64 & $9 / 16 / 03$ & 0.19 & $\mathrm{U}$ & 0.19 & 0.13 & & 0.063 & 0.051 & $\mathrm{U}$ & 0.051 & 0.12 & $\mathrm{U}$ & 0.12 & 0.17 & $\mathrm{U}$ & 0.17 \\
\hline A3 & J00Y65 & $9 / 16 / 03$ & 0.11 & $\mathrm{U}$ & 0.11 & 0.038 & $\mathrm{U}$ & 0.038 & 0.04 & $\mathrm{U}$ & 0.04 & 0.11 & $\mathrm{U}$ & 0.11 & 0.13 & $\mathrm{U}$ & 0.13 \\
\hline $\mathrm{A} 4$ & J00Y70 & $9 / 16 / 03$ & 0.092 & $\mathrm{U}$ & 0.092 & 0.042 & $\mathrm{U}$ & 0.042 & 0.045 & $\mathrm{U}$ & 0.045 & 0.12 & $\mathrm{U}$ & 0.12 & 0.14 & $\mathrm{U}$ & 0.14 \\
\hline Duplicate of J00Y70 & J00Y71 & $9 / 16 / 03$ & 0.29 & $\mathrm{U}$ & 0.29 & 0.068 & $\mathrm{U}$ & 0.068 & 0.067 & $\mathrm{U}$ & 0.067 & 0.2 & $\mathrm{U}$ & 0.2 & 0.27 & $\mathrm{U}$ & 0.27 \\
\hline
\end{tabular}

\begin{tabular}{|c|c|c|c|c|c|c|c|c|c|c|c|c|c|c|c|c|c|}
\hline \multirow{2}{*}{ Sample Location } & \multirow{2}{*}{\begin{tabular}{c|} 
Sample \\
Number
\end{tabular}} & \multirow{2}{*}{$\begin{array}{c}\text { Sample } \\
\text { Date }\end{array}$} & \multicolumn{3}{|c|}{ Europium-155 } & \multicolumn{3}{|c|}{ Gross alpha } & \multicolumn{3}{|c|}{ Gross beta } & \multicolumn{3}{|c|}{ Potassium-40 } & \multicolumn{3}{|c|}{ Radium-226 } \\
\hline & & & $\mathrm{pCi} / \mathrm{g}$ & $Q$ & MDA & $\mathrm{pCi} / \mathrm{g}$ & $\mathrm{Q}$ & MDA & $\mathrm{pCi} / \mathrm{g}$ & $\mathrm{Q}$ & MDA & $\mathrm{pCi} / \mathrm{g}$ & $Q$ & MDA & $\mathrm{pCi} / \mathrm{g}$ & $Q$ & MDA \\
\hline A1 & J00Y63 & $9 / 16 / 03$ & 0.071 & $\mathrm{U}$ & 0.071 & 12.4 & & 2.8 & 16.8 & & 7.1 & 8.4 & & 0.36 & 0.385 & & 0.068 \\
\hline $\mathrm{A} 2$ & J00Y64 & $9 / 16 / 03$ & 0.12 & $\bar{U}$ & 0.12 & 9.72 & & 4.2 & 13.1 & & 6.4 & 9.46 & & 0.42 & 0.559 & & 0.09 \\
\hline A3 & J00Y65 & $9 / 16 / 03$ & 0.12 & $\mathrm{U}$ & 0.12 & 17.2 & & 2.8 & 23.3 & & 7.1 & 10.1 & & 0.46 & 1.17 & & 0.086 \\
\hline $\mathrm{A} 4$ & J00Y70 & $9 / 16 / 03$ & 0.1 & $\mathrm{U}$ & 0.1 & 5.74 & & 3.2 & 15 & & 7.2 & 9.38 & & 0.6 & 0.39 & & 0.082 \\
\hline Duplicate of J00Y70 & J00Y71 & $9 / 16 / 03$ & 0.18 & $\mathrm{U}$ & 0.18 & 6.74 & & 3 & 16.6 & & 7.2 & 10.7 & & 0.46 & 0.492 & & 0.12 \\
\hline
\end{tabular}

\begin{tabular}{|c|c|c|c|c|c|c|c|c|c|c|c|c|c|c|c|c|c|}
\hline \multirow{2}{*}{ Sample Location } & \multirow{2}{*}{\begin{tabular}{|l|} 
Sample \\
Number
\end{tabular}} & \multirow{2}{*}{$\begin{array}{c}\text { Sample } \\
\text { Date }\end{array}$} & \multicolumn{3}{|c|}{ Radium-228 } & \multicolumn{3}{|c|}{ Thorium-228 } & \multicolumn{3}{|c|}{ Thorium-232 } & \multicolumn{3}{|c|}{ Uranium-235 } & \multicolumn{3}{|c|}{ Uranium-238 } \\
\hline & & & $\mathrm{pCi} / \mathrm{g}$ & $Q$ & MDA & $\mathrm{pCi} / \mathrm{g}$ & $Q$ & MDA & $\mathrm{pCi} / \mathrm{g}$ & $Q$ & MDA & $\mathrm{pCi} / \mathrm{g}$ & Q & MDA & $\mathrm{pCi} / \mathrm{g}$ & $Q$ & MDA \\
\hline $\mathrm{A} 1$ & J00Y63 & $9 / 16 / 03$ & 0.404 & & 0.12 & 0.357 & & 0.04 & 0.404 & & 0.12 & 0.12 & $\mathrm{U}$ & 0.12 & 4.8 & $\mathrm{U}$ & 4.8 \\
\hline $\mathrm{A} 2$ & J00Y64 & $9 / 16 / 03$ & 0.593 & & 0.21 & 0.504 & & 0.053 & 0.593 & & 0.21 & 0.17 & $\mathrm{U}$ & 0.17 & 6 & $\bar{U}$ & 6 \\
\hline$\overline{\mathrm{A} 3}$ & J00Y65 & $9 / 16 / 03$ & 1.39 & & 0.18 & 1.36 & & 0.049 & 1.39 & & 0.18 & 0.17 & $\mathrm{U}$ & 0.17 & 4.4 & $\mathrm{U}$ & 4.4 \\
\hline $\mathrm{A} 4$ & $\mathrm{~J} 00 \mathrm{Y} 70$ & $9 / 16 / 03$ & 0.565 & & 0.16 & 0.5 & & 0.055 & 0.565 & & 0.16 & 0.16 & $\bar{U}$ & 0.16 & 6.3 & $\mathrm{U}$ & 6.3 \\
\hline Duplicate of J00Y70 & J00Y71 & $9 / 16 / 03$ & 0.755 & & 0.21 & 0.487 & & 0.078 & 0.755 & & 0.21 & 0.26 & $\mathrm{U}$ & 0.26 & 8.9 & $\mathrm{U}$ & 8.9 \\
\hline
\end{tabular}

Acronyms and notes apply to all of the tables in this appendix.

Note: Data qualified with B, C, D, I, and/or J are considered acceptable values.

$\mathrm{B}=$ method blank contamination (organic constituents) $\quad \mathrm{PQL}=$ practical quantitation limit

$\mathrm{C}=$ method blank contamination (organic constituents) $\quad \mathrm{Q}=$ qualifier

$\mathrm{D}=$ diluted (organic constituents) $\quad \mathrm{R}=$ rejected

$\mathrm{I}=$ interference on one analytical column $\quad$ TCLP $=$ toxicity characteristic leaching procedure

$\mathrm{J}=$ estimate

$\mathrm{U}=$ undetected

$\mathrm{MDA}=$ minimum detectable activity 
Table A-1. 100-B-14:2 Area 2 Confirmatory Data Results. (7 Pages)

\begin{tabular}{|c|c|c|c|c|c|c|c|c|c|c|c|c|c|c|c|c|c|c|c|c|}
\hline \multirow{2}{*}{ Sample Location } & \multirow{2}{*}{$\begin{array}{l}\text { Sample } \\
\text { Number }\end{array}$} & \multirow{2}{*}{$\begin{array}{c}\text { Sample } \\
\text { Date }\end{array}$} & \multicolumn{3}{|c|}{ Aluminum } & \multicolumn{3}{|c|}{ Antimony } & \multicolumn{3}{|c|}{ Arsenic } & \multicolumn{3}{|c|}{ Barium } & \multicolumn{3}{|c|}{ Beryllium } & \multicolumn{3}{|c|}{ Boron } \\
\hline & & & $\mathrm{mg} / \mathrm{kg}$ & $Q$ & $\mathrm{PQL}$ & $\mathrm{mg} / \mathrm{kg}$ & $\mathrm{Q}$ & $\mathrm{PQL}$ & $\mathrm{mg} / \mathrm{kg}$ & $Q$ & $\mathrm{PQL}$ & $\mathrm{mg} / \mathrm{kg}$ & $Q$ & $\mathrm{PQL}$ & $\mathrm{mg} / \mathrm{kg}$ & $Q$ & PQL & $\mathrm{mg} / \mathrm{kg}$ & Q & $\mathrm{PQL}$ \\
\hline $\mathrm{A} 1$ & J00Y63 & $9 / 16 / 03$ & 6850 & & 4.8 & 8.1 & & 0.3 & 8.1 & & 0.43 & 3250 & & 0.02 & 0.14 & & 0.04 & 14 & & 0.22 \\
\hline $\mathrm{A} 2$ & J00Y64 & $9 / 16 / 03$ & 5970 & & 4.3 & 1.9 & & 0.27 & 10.1 & & 0.39 & 172 & & 0.02 & 0.3 & & 0.04 & 29.5 & & 0.2 \\
\hline $\mathrm{A} 3$ & J00Y65 & $9 / 16 / 03$ & 666 & & 4.6 & 0.29 & $\mathrm{U}$ & 0.29 & 0.88 & & 0.41 & 23.5 & & 0.02 & 0.04 & & 0.04 & 0.65 & & 0.22 \\
\hline $\mathrm{A} 4$ & J00Y70 & $9 / 16 / 03$ & 6070 & & 3.7 & 0.23 & $\mathrm{U}$ & 0.23 & 2.4 & & 0.34 & 78.5 & & 0.02 & 0.34 & & 0.03 & 3.4 & & 0.18 \\
\hline Duplicate of J00Y70 & J00Y71 & $9 / 16 / 03$ & 6710 & & 4.2 & 0.26 & $\mathrm{U}$ & 0.26 & 2.4 & & 0.38 & 115 & & 0.02 & 0.41 & & 0.04 & 10 & & 0.2 \\
\hline Equipment Blank & J00Y72 & $9 / 16 / 03$ & 55.9 & & 3.8 & 0.24 & $\bar{U}$ & 0.24 & 0.34 & $\mathrm{U}$ & 0.34 & 1.2 & & 0.02 & 0.05 & & 0.03 & 0.18 & $\mathrm{U}$ & 0.18 \\
\hline
\end{tabular}

\begin{tabular}{|c|c|c|c|c|c|c|c|c|c|c|c|c|c|c|c|c|c|c|c|c|}
\hline \multirow{2}{*}{ Sample Location } & \multirow{2}{*}{$\begin{array}{l}\text { Sample } \\
\text { Number }\end{array}$} & \multirow{2}{*}{$\begin{array}{c}\text { Sample } \\
\text { Date }\end{array}$} & \multicolumn{3}{|c|}{ Cadmium } & \multicolumn{3}{|c|}{ Calcium } & \multicolumn{3}{|c|}{ Chromium } & \multicolumn{3}{|c|}{ Cobalt } & \multicolumn{3}{|c|}{ Copper } & \multicolumn{3}{|c|}{$\begin{array}{c}\text { Hexavalent } \\
\text { Chromium }\end{array}$} \\
\hline & & & $\mathrm{mg} / \mathrm{kg}$ & $Q$ & $\mathrm{PQL}$ & $\mathrm{mg} / \mathrm{kg}$ & $Q$ & $\mathrm{PQL}$ & $\mathrm{mg} / \mathrm{kg}$ & $Q$ & $\mathrm{PQL}$ & $\mathrm{mg} / \mathrm{kg}$ & $Q$ & PQL & $\mathrm{mg} / \mathrm{kg}$ & $Q$ & PQL & $\mathrm{mg} / \mathrm{kg}$ & Q & PQL \\
\hline A1 & J00Y63 & $09 / 16 / 03$ & 8.1 & & 0.04 & 16000 & $\mathrm{C}$ & 3.2 & 60.2 & $\mathrm{C}$ & 0.1 & 8.8 & & 0.12 & 304 & & 0.12 & & & \\
\hline $\mathrm{A} 2$ & J00Y64 & $09 / 16 / 03$ & 0.76 & & 0.04 & 32700 & $\mathrm{C}$ & 2.9 & 32.7 & $\mathrm{C}$ & 0.09 & 13.1 & & 0.11 & 38.3 & & 0.11 & & & \\
\hline $\mathrm{A} 3$ & J00Y65 & $09 / 16 / 03$ & 0.04 & $\mathrm{U}$ & 0.04 & 1650 & $\mathrm{C}$ & 3 & 0.28 & $\mathrm{UC}$ & 0.1 & 0.12 & & 0.12 & 0.58 & $\mathrm{U}$ & 0.12 & & & \\
\hline $\mathrm{A} 4$ & J00Y70 & $09 / 16 / 03$ & 0.04 & & 0.03 & 4380 & $\mathrm{C}$ & 2.5 & 8 & $\mathrm{C}$ & 0.08 & 9.2 & & 0.1 & 17.4 & & 0.1 & 0.41 & $\mathrm{U}$ & 0.41 \\
\hline $\mathrm{A} 1$ & J00Y67* & $09 / 16 / 03$ & & & & & & & & & & & & & & & & 2.41 & & 0.35 \\
\hline $\mathrm{A} 2$ & J00Y68* & $09 / 16 / 03$ & & & & & & & & & & & & & & & & 1.79 & & 0.35 \\
\hline $\mathrm{A} 3$ & J00Y69* & $09 / 16 / 03$ & & & & & & & & & & & & & & & & 1.1 & & 0.35 \\
\hline Duplicate of J00Y70 & J00Y71 & $09 / 16 / 03$ & 0.04 & $\mathrm{U}$ & 0.04 & 5830 & $\mathrm{C}$ & 2.8 & 8.4 & $\mathrm{C}$ & 0.09 & 9.7 & & 0.11 & 17.8 & & 0.11 & 0.41 & $\mathrm{U}$ & 0.41 \\
\hline Equipment Blank & J00Y72 & $09 / 16 / 03$ & 0.03 & $\bar{U}$ & 0.03 & 27.4 & $\mathrm{C}$ & 2.6 & 0.17 & $\mathrm{C}$ & 0.08 & 0.1 & $\mathrm{U}$ & 0.1 & 0.1 & $\overline{\mathrm{U}}$ & 0.1 & 0.41 & $\mathrm{U}$ & 0.41 \\
\hline
\end{tabular}

*Only analyte was hexavalent chromium.

\begin{tabular}{|c|c|c|c|c|c|c|c|c|c|c|c|c|c|c|c|c|c|c|c|c|}
\hline \multirow{2}{*}{ Sample Location } & \multirow{2}{*}{$\begin{array}{c}\text { Sample } \\
\text { Number }\end{array}$} & \multirow{2}{*}{$\begin{array}{c}\text { Sample } \\
\text { Date }\end{array}$} & \multicolumn{3}{|c|}{ Iron } & \multicolumn{3}{|c|}{ Lead } & \multicolumn{3}{|c|}{ Magnesium } & \multicolumn{3}{|c|}{ Manganese } & \multicolumn{3}{|c|}{ Mercury } & \multicolumn{3}{|c|}{ Molybdenum } \\
\hline & & & $\mathrm{mg} / \mathrm{kg}$ & Q & PQL & $\mathrm{mg} / \mathrm{kg}$ & $Q$ & $\mathrm{PQL}$ & $\mathrm{mg} / \mathrm{kg}$ & $Q$ & $\mathrm{PQL}$ & $\mathrm{mg} / \mathrm{kg}$ & Q & $\mathrm{PQL}$ & $\mathrm{mg} / \mathrm{kg}$ & $Q$ & $\mathrm{PQL}$ & $\mathrm{mg} / \mathrm{kg}$ & Q & $\mathrm{PQL}$ \\
\hline A1 & J00Y63 & $9 / 16 / 03$ & 13400 & $\mathrm{C}$ & 2 & 218 & $\mathrm{C}$ & 0.19 & 2400 & & 0.71 & 203 & & 0.03 & 21.5 & & 0.4 & 4.2 & & 0.19 \\
\hline $\mathrm{A} 2$ & J00Y64 & $9 / 16 / 03$ & 14900 & $\mathrm{C}$ & 1.8 & 42.4 & $\mathrm{C}$ & 0.18 & 3930 & & 0.65 & 267 & & 0.03 & 1.2 & & 0.02 & 0.99 & & 0.18 \\
\hline $\mathrm{A} 3$ & J00Y65 & $9 / 16 / 03$ & 201 & $\mathrm{C}$ & 1.9 & 0.44 & $\mathrm{C}$ & 0.19 & 85.4 & & 0.69 & 6 & & 0.03 & 0.1 & & 0.02 & 0.19 & & 0.19 \\
\hline $\mathrm{A} 4$ & J00Y70 & $9 / 16 / 03$ & 22500 & C & 1.6 & 4.7 & $\mathrm{C}$ & 0.15 & 4030 & & 0.56 & 364 & & 0.02 & 0.02 & & 0.02 & 0.15 & & 0.15 \\
\hline Duplicate of J00Y70 & J00Y71 & $9 / 16 / 03$ & 24800 & C & 1.8 & 4.7 & $\mathrm{C}$ & 0.17 & 4530 & & 0.63 & 368 & & 0.03 & 0.02 & $\mathrm{U}$ & 0.02 & 0.3 & & 0.17 \\
\hline Equipment Blank & $\mathrm{J} 00 \mathrm{Y72}$ & $9 / 16 / 03$ & 135 & $\mathrm{C}$ & 1.6 & 0.34 & $\mathrm{C}$ & 0.15 & 8.3 & & 0.57 & 3.7 & & 0.02 & 0.01 & $\mathrm{U}$ & 0.02 & 0.15 & $\mathrm{U}$ & 0.15 \\
\hline
\end{tabular}


Table A-1. 100-B-14:2 Area 2 Confirmatory Data Results. (7 Pages)

\begin{tabular}{|c|c|c|c|c|c|c|c|c|c|c|c|c|c|c|c|c|c|c|c|c|}
\hline \multirow{2}{*}{ Sample Location } & \multirow{2}{*}{$\begin{array}{l}\text { Sample } \\
\text { Number }\end{array}$} & \multirow{2}{*}{$\begin{array}{c}\text { Sample } \\
\text { Date }\end{array}$} & \multicolumn{3}{|c|}{ Nickel } & \multicolumn{3}{|c|}{ Potassium } & \multicolumn{3}{|c|}{ Selenium } & \multicolumn{3}{|c|}{ Silicon } & \multicolumn{3}{|c|}{ Silver } & \multicolumn{3}{|c|}{ Sodium } \\
\hline & & & $\mathrm{mg} / \mathrm{kg}$ & Q & $\mathrm{PQL}$ & $\mathrm{mg} / \mathrm{kg}$ & $Q$ & $\mathrm{PQL}$ & $\mathrm{mg} / \mathrm{kg}$ & $Q$ & $\mathrm{PQL}$ & $\mathrm{mg} / \mathrm{kg}$ & $Q$ & PQL & $\mathrm{mg} / \mathrm{kg}$ & $Q$ & PQL & $\mathrm{mg} / \mathrm{kg}$ & $Q$ & PQL \\
\hline $\mathrm{A} 1$ & J00Y63 & $9 / 16 / 03$ & 20.4 & C & 0.2 & 1090 & & 2.4 & 3.4 & & 0.3 & 380 & $\mathrm{C}$ & 0.55 & 18.7 & & 0.08 & 2990 & $\mathrm{C}$ & 0.72 \\
\hline $\mathrm{A} 2$ & J00Y64 & $9 / 16 / 03$ & 12.7 & C & 0.19 & 3080 & & 2.3 & 0.59 & & 0.27 & 454 & $\mathrm{C}$ & 0.5 & 0.43 & & 0.07 & 7290 & $\mathrm{C}$ & 0.66 \\
\hline A3 & J00Y65 & $9 / 16 / 03$ & 0.42 & $\mathrm{C}$ & 0.2 & 114 & & 2.3 & 0.29 & $\mathrm{U}$ & 0.29 & 46.4 & $\mathrm{C}$ & 0.53 & 0.08 & $\mathrm{U}$ & 0.08 & 300 & $\mathrm{C}$ & 0.7 \\
\hline $\mathrm{A} 4$ & J00Y70 & $9 / 16 / 03$ & 10.1 & $\mathrm{C}$ & 0.16 & 1460 & & 1.9 & 0.23 & $\mathrm{U}$ & 0.23 & 382 & $\mathrm{C}$ & 0.43 & 0.06 & $\mathrm{U}$ & 0.06 & 302 & $\mathrm{C}$ & 0.57 \\
\hline Duplicate of J00Y70 & J00Y71 & $9 / 16 / 03$ & 11.5 & C & 0.18 & 1470 & & 3.1 & 0.26 & $\mathrm{U}$ & 0.26 & 395 & $\mathrm{C}$ & 0.48 & 0.07 & $\mathrm{U}$ & 0.07 & 333 & $\mathrm{C}$ & 0.63 \\
\hline Equipment Blank & $\mathrm{J} 00 \mathrm{Y} 72$ & $9 / 16 / 03$ & 0.16 & $\mathrm{C}$ & 0.16 & 24.6 & & 1.9 & 0.24 & $\mathrm{U}$ & 0.24 & 38.2 & $\mathrm{C}$ & 0.44 & 0.07 & $\mathrm{U}$ & 0.07 & 12 & $\mathrm{C}$ & 0.58 \\
\hline
\end{tabular}

\begin{tabular}{|c|c|c|c|c|c|c|c|c|}
\hline \multirow{2}{*}{ Sample Location } & \multirow{2}{*}{$\begin{array}{c}\text { Sample } \\
\text { Number }\end{array}$} & Sample & \multicolumn{3}{|c|}{ Vanadium } & \multicolumn{3}{|c|}{ Zinc } \\
\cline { 5 - 9 } & Dug/kg & Q & PQL & mg/kg & Q & PQL \\
\hline A1 & J00Y63 & $9 / 16 / 03$ & 94.6 & & 0.09 & 852 & 0.26 \\
\hline A2 & J00Y64 & $9 / 16 / 03$ & 61.2 & & 0.08 & 250 & 0.24 \\
\hline A3 & J00Y65 & $9 / 16 / 03$ & 0.67 & & 0.09 & 5.7 & 0.26 \\
\hline A4 & J00Y70 & $9 / 16 / 03$ & 56.6 & & 0.07 & 49.4 & 0.21 \\
\hline Duplicate of J00Y70 & J00Y71 & $9 / 16 / 03$ & 60.6 & & 0.08 & 51.9 & 0.23 \\
\hline Equipment Blank & J00Y72 & $9 / 16 / 03$ & 0.07 & & 0.07 & 0.63 & & 0.21 \\
\hline
\end{tabular}


Table A-1. 100-B-14:2 Area 2 Confirmatory Data Results. (7 Pages)

\begin{tabular}{|c|c|c|c|c|c|c|c|c|c|c|c|c|}
\hline \multirow[t]{2}{*}{ Constituents } & \multicolumn{3}{|c|}{$\begin{array}{c}\text { J00Y63 } \\
\text { Location A1 } \\
\text { Sample Date } 9 / 16 / 03\end{array}$} & \multicolumn{3}{|c|}{$\begin{array}{c}\text { J00Y64 } \\
\text { Location A2 } \\
\text { Sample Date } 9 / 16 / 03\end{array}$} & \multicolumn{3}{|c|}{$\begin{array}{c}\text { J00Y65 } \\
\text { Location A3 } \\
\text { Sample Date 9/16/03 } \\
\end{array}$} & \multicolumn{3}{|c|}{$\begin{array}{c}\text { J00Y70 } \\
\text { Location A4 } \\
\text { Sample Date } 9 / 16 / 03 \\
\end{array}$} \\
\hline & $\mu \mathrm{g} / \mathrm{kg}$ & $Q$ & PQL & $\mu \mathrm{g} / \mathrm{kg}$ & $Q$ & $\mathrm{PQL}$ & $\mu \mathrm{g} / \mathrm{kg}$ & $Q$ & PQL & $\mu \mathrm{g} / \mathrm{kg}$ & $Q$ & PQL \\
\hline \multicolumn{13}{|c|}{ Polychlorinated Biphenyls } \\
\hline Aroclor-1016 & 3000 & UD & 3000 & 13 & $\mathrm{U}$ & 13 & 13 & $\mathrm{U}$ & 13 & 14 & $\mathrm{U}$ & 14 \\
\hline Aroclor-1221 & 3000 & UD & 3000 & 13 & $\mathrm{U}$ & 13 & 13 & $\mathrm{U}$ & 13 & 14 & $\mathrm{U}$ & 14 \\
\hline Aroclor-1232 & 3000 & $\mathrm{UD}$ & 3000 & 13 & $\mathrm{U}$ & 13 & 13 & $\mathrm{U}$ & 13 & 14 & $\mathrm{U}$ & 14 \\
\hline $\begin{array}{l}\text { Aroclor- } 1242 \\
\end{array}$ & 3000 & UD & 3000 & 13 & $\mathrm{U}$ & 13 & 13 & $\mathrm{U}$ & 13 & 14 & $\mathrm{U}$ & 14 \\
\hline Aroclor-1248 & 3000 & UD & 3000 & 13 & $\mathrm{U}$ & 13 & 13 & $\mathrm{U}$ & 13 & 14 & $\mathrm{U}$ & 14 \\
\hline Aroclor-1254 & 49000 & $\mathrm{D}$ & 49000 & 70 & & 13 & 13 & $\mathrm{U}$ & 13 & 14 & $\mathrm{U}$ & 14 \\
\hline Aroclor-1260 & 3000 & UD & 3000 & 13 & $\mathrm{U}$ & 13 & 13 & $\mathrm{U}$ & 13 & 13 & $\mathrm{U}$ & 13 \\
\hline \multicolumn{13}{|c|}{ Pesticides } \\
\hline Aldrin & 19 & UD & 19 & 17 & UD & 17 & 1.7 & $\mathrm{U}$ & 1.7 & 1.7 & $\mathrm{U}$ & 1.7 \\
\hline alpha-BHC & 19 & UD & 19 & 17 & UD & 17 & 1.7 & $\mathrm{U}$ & 1.7 & 1.7 & $\mathrm{U}$ & 1.7 \\
\hline alpha-Chlordane & 19 & UD & 19 & 17 & UD & 17 & 1.7 & $\mathrm{U}$ & 1.7 & 1.7 & $\mathrm{U}$ & 1.7 \\
\hline beta-BHC & 19 & UD & 19 & 17 & UD & 17 & 1.7 & $\mathrm{U}$ & 1.7 & 1.7 & $\mathrm{U}$ & 1.7 \\
\hline delta-BHC & 19 & UD & 19 & 17 & UD & 17 & 1.7 & $\mathrm{U}$ & 1.7 & 1.7 & $\mathrm{U}$ & 1.7 \\
\hline Dichlorodiphenyldichloroethane & 81 & $\mathrm{I}$ & 37 & 34 & UD & 34 & 3.3 & $\mathrm{U}$ & 3.3 & 3.4 & $\mathrm{U}$ & 3.4 \\
\hline Dichlorodiphenyldichloroethylene & 74 & $\mathrm{I}$ & 37 & 34 & UD & 34 & 3.3 & $\mathrm{U}$ & 3.3 & 3.4 & $\mathrm{U}$ & 3.4 \\
\hline Dichlorodiphenyltrichloroethane & 4800 & $\mathrm{D}$ & 37 & 34 & UD & 34 & 3.3 & $\mathrm{U}$ & 3.3 & 3.4 & $\mathrm{U}$ & 3.4 \\
\hline Dieldrin & 37 & UD & 37 & 34 & UD & 34 & 3.3 & $\mathrm{U}$ & 3.3 & 3.4 & $\mathrm{U}$ & 3.4 \\
\hline Endosulfan I & 19 & UD & 19 & 17 & UD & 17 & 1.7 & $\mathrm{U}$ & 1.7 & 1.7 & $\mathrm{U}$ & 1.7 \\
\hline Endosulfan II & 37 & UD & 37 & 34 & UD & 34 & 3.3 & $\mathrm{U}$ & 3.3 & 3.4 & $\bar{U}$ & 3.4 \\
\hline Endosulfan sulfate & 43 & $\mathrm{I}$ & 37 & 34 & UD & 34 & 3.3 & $\mathrm{U}$ & 3.3 & 3.4 & $\mathrm{U}$ & 3.4 \\
\hline Endrin & 37 & UD & 37 & 34 & UD & 34 & 3.3 & $\mathrm{U}$ & 3.3 & 3.4 & $\mathrm{U}$ & 3.4 \\
\hline Endrin aldehyde & 37 & UD & 37 & 34 & UD & 34 & 3.3 & $\mathrm{U}$ & 3.3 & 3.4 & $\mathrm{U}$ & 3.4 \\
\hline Endrin ketone & 37 & UD & 37 & 34 & UD & 34 & 3.3 & $\mathrm{U}$ & 3.3 & 3.4 & $\mathrm{U}$ & 3.4 \\
\hline gamma-BHC (Lindane) & 19 & UD & 19 & 17 & UD & 17 & 1.7 & $\mathrm{U}$ & 1.7 & 1.7 & U & 1.7 \\
\hline gamma-Chlordane & 210 & $\mathrm{I}$ & 19 & 17 & UD & 17 & 1.7 & $\mathrm{U}$ & 1.7 & 1.7 & $\mathrm{U}$ & 1.7 \\
\hline Heptachlor & 19 & UD & 19 & 17 & UD & 17 & 1.7 & $\mathrm{U}$ & 1.7 & 1.7 & $\mathrm{U}$ & 1.7 \\
\hline Heptachlor epoxide & 19 & UD & 19 & 17 & UD & 17 & 1.7 & $\mathrm{U}$ & 1.7 & 1.7 & $\mathrm{U}$ & 1.7 \\
\hline Methoxychlor & 190 & UD & 190 & 170 & UD & 170 & 17 & $\mathrm{U}$ & 17 & 17 & $\mathrm{U}$ & 17 \\
\hline Toxaphene & 1900 & UD & 1900 & 1700 & UD & 1700 & 170 & $\mathrm{U}$ & 170 & 170 & $\mathrm{U}$ & 170 \\
\hline \multicolumn{13}{|c|}{ Semivolatile Organic Compounds } \\
\hline 1,2,4-Trichlorobenzene & 370 & $\mathrm{U}$ & 370 & 1700 & UD & 1700 & 330 & $\mathrm{U}$ & 330 & 340 & $\mathrm{U}$ & 340 \\
\hline 1,2-Dichlorobenzene & 370 & $\mathrm{U}$ & 370 & 1700 & UD & 1700 & 330 & $\mathrm{U}$ & 330 & 340 & $\mathrm{U}$ & 340 \\
\hline 1,3-Dichlorobenzene & 370 & $\mathrm{U}$ & 370 & 1700 & UD & 1700 & 330 & $\mathrm{U}$ & 330 & 340 & $\mathrm{U}$ & 340 \\
\hline 1,4-Dichlorobenzene & 370 & $\mathrm{U}$ & 370 & 1700 & UD & 1700 & 330 & $\mathrm{U}$ & 330 & 340 & $\mathrm{U}$ & 340 \\
\hline 2,4,5-Trichlorophenol & 930 & $\mathrm{U}$ & 930 & 4200 & UD & 4200 & 840 & $\mathrm{U}$ & 840 & 860 & $\mathrm{U}$ & 860 \\
\hline 2,4,6-Trichlorophenol & 370 & $\mathrm{U}$ & 370 & 1700 & UD & 1700 & 330 & $\mathrm{U}$ & 330 & 340 & $\mathrm{U}$ & 340 \\
\hline 2,4-Dichlorophenol & 370 & $\mathrm{U}$ & 370 & 1700 & UD & 1700 & 330 & $\mathrm{U}$ & 330 & 340 & $\mathrm{U}$ & 340 \\
\hline 2,4-Dimethylphenol & 370 & $\mathrm{U}$ & 370 & 1700 & UD & 1700 & 330 & $\mathrm{U}$ & 330 & 340 & $\mathrm{U}$ & 340 \\
\hline 2,4-Dinitrophenol & 930 & $\mathrm{U}$ & 930 & 4200 & UD & 4200 & 840 & $\mathrm{U}$ & 840 & 860 & $\mathrm{U}$ & 860 \\
\hline 2,4-Dinitrotoluene & 370 & $\mathrm{U}$ & 370 & 1700 & UD & 1700 & 330 & $\mathrm{U}$ & 330 & 340 & $\mathrm{U}$ & 340 \\
\hline 2,6-Dinitrotoluene & 370 & $\mathrm{U}$ & 370 & 1700 & UD & 1700 & 330 & $\mathrm{U}$ & 330 & 340 & $\mathrm{U}$ & 340 \\
\hline 2-Chloronaphthalene & 370 & $\mathrm{U}$ & 370 & 1700 & UD & 1700 & 330 & $\mathrm{U}$ & 330 & 340 & $\mathrm{U}$ & 340 \\
\hline 2-Chlorophenol & 370 & $\mathrm{U}$ & 370 & 1700 & UD & 1700 & 330 & $\mathrm{U}$ & 330 & 340 & $\mathrm{U}$ & 340 \\
\hline 2-Methylnaphthalene & 370 & $\mathrm{U}$ & 370 & 200 & JD & 1700 & 330 & $\mathrm{U}$ & 330 & 340 & $\mathrm{U}$ & 340 \\
\hline 2-Methylphenol (cresol, o-) & 370 & $\mathrm{U}$ & 370 & 1700 & UD & 1700 & 330 & $\mathrm{U}$ & 330 & 340 & $\mathrm{U}$ & 340 \\
\hline 2-Nitroaniline & 930 & $\mathrm{U}$ & 930 & 4200 & UD & 4200 & 840 & $\mathrm{U}$ & 840 & 860 & $\mathrm{U}$ & 860 \\
\hline 2-Nitrophenol & 370 & $\mathrm{U}$ & 370 & 1700 & UD & 1700 & 330 & $\mathrm{U}$ & 330 & 340 & $\mathrm{U}$ & 340 \\
\hline 3,3'-Dichlorobenzidine & 370 & $\mathrm{U}$ & 370 & 1700 & UD & 1700 & 330 & $\mathrm{U}$ & 330 & 340 & $\mathrm{U}$ & 340 \\
\hline 3-Nitroaniline & 930 & $\mathrm{U}$ & 930 & 4200 & UD & 4200 & 840 & $\mathrm{U}$ & 840 & 860 & $\mathrm{U}$ & 860 \\
\hline 4,6-Dinitro-2-methylphenol & 930 & $\mathrm{U}$ & 930 & 4200 & UD & 4200 & 840 & U & 840 & 860 & $\mathrm{U}$ & 860 \\
\hline 4-Bromophenylphenyl ether & 370 & $\mathrm{U}$ & 370 & 1700 & UD & 1700 & 330 & $\mathrm{U}$ & 330 & 340 & $\mathrm{U}$ & 340 \\
\hline 4-Chloro-3-methylphenol & 370 & $\mathrm{U}$ & 370 & 1700 & UD & 1700 & 330 & $\mathrm{U}$ & 330 & 340 & $\mathrm{U}$ & 340 \\
\hline 4-Chloroaniline & 370 & $\mathrm{U}$ & 370 & 1700 & UD & 1700 & 330 & $\mathrm{U}$ & 330 & 340 & $\mathrm{U}$ & 340 \\
\hline 4-Chlorophenylphenyl ether & 370 & $\mathrm{U}$ & 370 & 1700 & UD & 1700 & 330 & U & 330 & 340 & $U$ & 340 \\
\hline 4-Methylphenol (cresol, p-) & 370 & $\mathrm{U}$ & 370 & 1700 & UD & 1700 & 330 & $\mathrm{U}$ & 330 & 340 & $\mathrm{U}$ & 340 \\
\hline 4-Nitroaniline & 930 & $\mathrm{U}$ & 930 & 4200 & UD & 4200 & 840 & $\mathrm{U}$ & 840 & 860 & $\mathrm{U}$ & 860 \\
\hline 4-Nitrophenol & 930 & $\mathrm{U}$ & 930 & 4200 & UD & 4200 & 840 & $\mathrm{U}$ & 840 & 860 & $U$ & 860 \\
\hline
\end{tabular}


Table A-1. 100-B-14:2 Area 2 Confirmatory Data Results. (7 Pages)

\begin{tabular}{|c|c|c|c|c|c|c|c|c|c|c|c|c|}
\hline \multirow[t]{2}{*}{ Constituents } & \multicolumn{3}{|c|}{$\begin{array}{c}\text { J00Y63 } \\
\text { Location A1 } \\
\text { Sample Date } 9 / 16 / 03\end{array}$} & \multicolumn{3}{|c|}{$\begin{array}{c}\text { J00Y64 } \\
\text { Location A2 } \\
\text { Sample Date } 9 / 16 / 03\end{array}$} & \multicolumn{3}{|c|}{$\begin{array}{c}\text { J00Y65 } \\
\text { Location A3 } \\
\text { Sample Date } 9 / 16 / 03\end{array}$} & \multicolumn{3}{|c|}{$\begin{array}{c}\text { J00Y70 } \\
\text { Location A4 } \\
\text { Sample Date } 9 / 16 / 03\end{array}$} \\
\hline & $\mu \mathrm{g} / \mathrm{kg}$ & $\mathbf{Q}$ & PQL & $\mu \mathrm{g} / \mathrm{kg}$ & $\mathbf{Q}$ & PQL & $\mu \mathrm{g} / \mathrm{kg}$ & $\mathbf{Q}$ & $\overline{P Q L}$ & $\mu \mathrm{g} / \mathrm{kg}$ & $\mathbf{Q}$ & PQL \\
\hline \multicolumn{13}{|c|}{ Semivolatile Organic Compounds (continued) } \\
\hline Acenaphthene & 370 & $\mathrm{U}$ & 370 & 1700 & UD & 1700 & 330 & $\mathrm{U}$ & 330 & 340 & $\mathrm{U}$ & 340 \\
\hline Acenaphthylene & 370 & $\mathrm{U}$ & 370 & 1900 & $\mathrm{D}$ & 1700 & 330 & $\mathrm{U}$ & 330 & 340 & $\mathrm{U}$ & 340 \\
\hline Anthracene & 370 & $\mathrm{U}$ & 370 & 1200 & JD & 1700 & 330 & $\mathrm{U}$ & 330 & 340 & $\mathrm{U}$ & 340 \\
\hline Benzo(a)anthracene & 33 & $\mathrm{~J}$ & 370 & 500 & JD & 1700 & 330 & $\mathrm{U}$ & 330 & 340 & $\mathrm{U}$ & 340 \\
\hline Benzo(a)pyrene & 38 & $\mathrm{~J}$ & 370 & 1400 & JD & 1700 & 330 & $\mathrm{U}$ & 330 & 340 & $\mathrm{U}$ & 340 \\
\hline Benzo(b)fluoranthene & 370 & $\mathrm{U}$ & 370 & 830 & $\mathrm{JD}$ & 1700 & 330 & $\mathrm{U}$ & 330 & 340 & $\mathrm{U}$ & 340 \\
\hline Benzo $(\mathrm{g}, \mathrm{h}, \mathrm{i})$ perylene & 25 & $\mathrm{~J}$ & 370 & 1700 & $\mathrm{D}$ & 1700 & 330 & $\mathrm{U}$ & 330 & 340 & $\mathrm{U}$ & 340 \\
\hline Benzo(k)fluoranthene & 370 & $\mathrm{U}$ & 370 & 690 & JD & 1700 & 330 & $\mathrm{U}$ & 330 & 340 & $\mathrm{U}$ & 340 \\
\hline bis(2-Chloro-1-methylethyl)ether & 370 & $\mathrm{U}$ & 370 & 1700 & UD & 1700 & 330 & $\mathrm{U}$ & 330 & 340 & $\mathrm{U}$ & 340 \\
\hline bis(2-Chloroethoxy)methane & 370 & $\mathrm{U}$ & 370 & 1700 & UD & 1700 & 330 & $\mathrm{U}$ & 330 & 340 & $\mathrm{U}$ & 340 \\
\hline bis(2-Chloroethyl) ether & 370 & $\mathrm{U}$ & 370 & 1700 & UD & 1700 & 330 & $\mathrm{U}$ & 330 & 340 & $\mathrm{U}$ & 340 \\
\hline bis(2-Ethylhexyl) phthalàte & 69 & $\mathrm{~J}$ & 370 & 1700 & UD & 1700 & 330 & $\mathrm{U}$ & 330 & 340 & $\mathrm{U}$ & 340 \\
\hline Butylbenzylphthalate & 370 & $\mathrm{U}$ & 370 & 1700 & UD & 1700 & 330 & $\mathrm{U}$ & 330 & 340 & $\mathrm{U}$ & 340 \\
\hline Carbazole & 370 & $\mathrm{U}$ & 370 & 1700 & UD & 1700 & 330 & $\mathrm{U}$ & 330 & 340 & $\mathrm{U}$ & 340 \\
\hline Chrysene & 48 & $\mathrm{~J}$ & 370 & 830 & JD & 1700 & 330 & $\mathrm{U}$ & 330 & 340 & $\mathrm{U}$ & 340 \\
\hline Dibenz $[a, h]$ anthracene & 20 & $\mathrm{~J}$ & 370 & 1700 & UD & 1700 & 330 & $\mathrm{U}$ & 330 & 340 & $\mathrm{U}$ & 340 \\
\hline Dibenzofuran & 370 & $\mathrm{U}$ & 370 & 1700 & UD & 1700 & 330 & $\mathrm{U}$ & 330 & 340 & $\mathrm{U}$ & 340 \\
\hline Diethylphthalate & 370 & $\mathrm{U}$ & 370 & 360 & $\mathrm{JD}$ & 1700 & 330 & $\mathrm{U}$ & 330 & 340 & $\mathrm{U}$ & 340 \\
\hline Dimethyl phthalate & 370 & $\mathrm{U}$ & 370 & 130 & $\mathrm{JD}$ & 1700 & 330 & $\mathrm{U}$ & 330 & 340 & $\mathrm{U}$ & 340 \\
\hline Di-n-butylphthalate & 370 & $\mathrm{U}$ & 370 & 1700 & UD & 1700 & 330 & $\mathrm{U}$ & 330 & 340 & $\mathrm{U}$ & 340 \\
\hline Di-n-octylphthalate & 370 & $\mathrm{U}$ & 370 & 1700 & UD & 1700 & 330 & $\mathrm{U}$ & 330 & 340 & $\mathrm{U}$ & 340 \\
\hline Fluoranthene & 54 & $\mathrm{~J}$ & 370 & 520 & $\mathrm{JD}$ & 1700 & 330 & $\mathrm{U}$ & 330 & 340 & $\mathrm{U}$ & 340 \\
\hline Fluorene & 370 & $\mathrm{U}$ & 370 & 190 & $\mathrm{JD}$ & 1700 & 330 & $\mathrm{U}$ & 330 & 340 & $\mathrm{U}$ & 340 \\
\hline Hexachlorobenzene & 370 & $\mathrm{U}$ & 370 & 1700 & UD & 1700 & 330 & $\mathrm{U}$ & 330 & 340 & $\mathrm{U}$ & 340 \\
\hline Hexachlorobutadiene & 370 & $\mathrm{U}$ & 370 & 1700 & UD & 1700 & 330 & $\mathrm{U}$ & 330 & 340 & $\mathrm{U}$ & 340 \\
\hline Hexachlorocyclopentadiene & 370 & $\mathrm{U}$ & 370 & 1700 & UD & 1700 & 330 & $\mathrm{U}$ & 330 & 340 & $\mathrm{U}$ & 340 \\
\hline Hexachloroethane & 370 & $\mathrm{U}$ & 370 & 1700 & UD & 1700 & 330 & $\mathrm{U}$ & 330 & 340 & $\mathrm{U}$ & 340 \\
\hline Indeno(1,2,3-cd)pyrene & 22 & $\mathrm{~J}$ & 370 & 1200 & $\mathrm{JD}$ & 1200 & 330 & $\mathrm{U}$ & 330 & 340 & $\mathrm{U}$ & 340 \\
\hline Isophorone & 370 & $\mathrm{U}$ & 370 & 1700 & UD & 1700 & 330 & $\mathrm{U}$ & 330 & 340 & $\mathrm{U}$ & 340 \\
\hline Naphthalene & 370 & $\mathrm{U}$ & 370 & 1700 & UD & 1700 & 330 & $\mathrm{U}$ & 330 & 340 & $\mathrm{U}$ & 340 \\
\hline Nitrobenzene & 370 & $\mathrm{U}$ & 370 & 1700 & UD & 1700 & 330 & $\mathrm{U}$ & 330 & 340 & $\mathrm{U}$ & 340 \\
\hline N-Nitroso-di-n-dipropylamine & 370 & $\mathrm{U}$ & 370 & 1700 & UD & 1700 & 330 & $\mathrm{U}$ & 330 & 340 & $U$ & 340 \\
\hline N-Nitrosodiphenylamine & 370 & $\mathrm{U}$ & 370 & 1700 & UD & 1700 & 330 & $\mathrm{U}$ & 330 & 340 & $\mathrm{U}$ & 340 \\
\hline Pentachlorophenol & 930 & $\mathrm{U}$ & 930 & 4200 & UD & 4200 & 840 & $\mathrm{U}$ & 840 & 860 & $\mathrm{U}$ & 860 \\
\hline Phenanthrene & 24 & $\mathrm{~J}$ & 370 & 190 & JD & 1700 & 330 & $\mathrm{U}$ & 330 & 340 & $\mathrm{U}$ & 340 \\
\hline Phenol & 370 & $\mathrm{U}$ & 370 & 1700 & UD & 1700 & 330 & $\mathrm{U}$ & 330 & 340 & $\mathrm{U}$ & 340 \\
\hline Pyrene & 24 & $\mathrm{~J}$ & 370 & 510 & $\mathrm{JD}$ & 1700 & 330 & $\mathrm{U}$ & 330 & 340 & $\bar{U}$ & 340 \\
\hline
\end{tabular}


Table A-1. 100-B-14:2 Area 2 Confirmatory Data Results. (7 Pages)

\begin{tabular}{|c|c|c|c|c|c|c|}
\hline \multirow[t]{2}{*}{ Constituents } & \multicolumn{3}{|c|}{$\begin{array}{c}\text { J00Y71 } \\
\text { Duplicate of J00Y70 } \\
\text { Sample Date } 9 / 16 / 03 \\
\end{array}$} & \multicolumn{3}{|c|}{$\begin{array}{c}\text { J00Y'72 } \\
\text { Equipment Blank } \\
\text { Sample Date } 9 / 16 / 03 \\
\end{array}$} \\
\hline & $\mu \mathrm{g} / \mathrm{kg}$ & $\mathbf{Q}$ & $\mathrm{PQL}$ & $\mu \mathrm{g} / \mathrm{kg}$ & $\mathbf{Q}$ & PQL \\
\hline \multicolumn{7}{|c|}{ Polychlorinated Biphenyls } \\
\hline Aroclor-1016 & 14 & $\mathrm{U}$ & 14 & 13 & $\mathrm{U}$ & 13 \\
\hline Aroclor-1221 & 14 & $\mathrm{U}$ & 14 & 13 & $\mathrm{U}$ & 13 \\
\hline Aroclor-1232 & 14 & $\mathrm{U}$ & 14 & 13 & $\mathrm{U}$ & 13 \\
\hline Aroclor-1242 & 14 & $\mathrm{U}$ & 14 & 13 & $\mathrm{U}$ & 13 \\
\hline Aroclor-1248 & 14 & $\mathrm{U}$ & 14 & 13 & $\mathrm{U}$ & 13 \\
\hline Aroclor-1254 & 14 & $\mathrm{U}$ & 14 & 13 & $\mathrm{U}$ & 13 \\
\hline Aroclor-1260 & 14 & $\mathrm{U}$ & 14 & 13 & $\mathrm{U}$ & 13 \\
\hline \multicolumn{7}{|c|}{ Pesticides } \\
\hline Aldrin & 1.7 & $\mathrm{U}$ & 1.7 & 1.7 & $\mathrm{U}$ & 1.7 \\
\hline alpha-BHC & 1.7 & $\mathrm{U}$ & 1.7 & 1.7 & $\mathrm{U}$ & 1.7 \\
\hline alpha-Chlordane & 1.7 & $\mathrm{U}$ & 1.7 & 1.7 & $\mathrm{U}$ & 1.7 \\
\hline beta-BHC & 1.7 & $\mathrm{U}$ & 1.7 & 1.7 & $\mathrm{U}$ & 1.7 \\
\hline delta-BHC & 1.7 & $\mathrm{U}$ & 1.7 & 1.7 & $\mathrm{U}$ & 1.7 \\
\hline Dichlorodiphenyldichloroethane & 3.4 & $\mathrm{U}$ & 3.4 & 3.3 & $\mathrm{U}$ & 3.3 \\
\hline Dichlorodiphenyldichloroethylene & 3.4 & $\mathrm{U}$ & 3.4 & 3.3 & $\mathrm{U}$ & 3.3 \\
\hline Dichlorodiphenyltrichloroethane & 3.4 & $\mathrm{U}$ & 3.4 & 3.3 & $\mathrm{U}$ & 3.3 \\
\hline Dieldrin & 3.4 & $\mathrm{U}$ & 3.4 & 3.3 & $\mathrm{U}$ & 3.3 \\
\hline Endosulfan I & 1.7 & $\mathrm{U}$ & 1.7 & 1.7 & $\mathrm{U}$ & 1.7 \\
\hline Endosulfan II & 3.4 & $\mathrm{U}$ & 3.4 & 3.3 & $\mathrm{U}$ & 3.3 \\
\hline Endosulfan sulfate & 3.4 & $\mathrm{U}$ & 3.4 & 3.3 & $\mathrm{U}$ & 3.3 \\
\hline Endrin & 3.4 & $\mathrm{U}$ & 3.4 & 3.3 & $\mathrm{U}$ & 3.3 \\
\hline Endrin aldehyde & 3.4 & $\mathrm{U}$ & 3.4 & 3.3 & $\mathrm{U}$ & 3.3 \\
\hline Endrin ketone & 3.4 & $\mathrm{U}$ & 3.4 & 3.3 & $\mathrm{U}$ & 3.3 \\
\hline gamma-BHC (Lindane) & 1.7 & $\mathrm{U}$ & 1.7 & 1.7 & $\mathrm{U}$ & 1.7 \\
\hline gamma-Chlordane & 1.7 & $\mathrm{U}$ & 1.7 & 1.7 & $\mathrm{U}$ & 1.7 \\
\hline Heptachlor & 1.7 & $\mathrm{U}$ & 1.7 & 1.7 & $\mathrm{U}$ & 1.7 \\
\hline Heptachlor epoxide & 1.7 & $\bar{U}$ & 1.7 & 1.7 & $\mathrm{U}$ & 1.7 \\
\hline Methoxychlor & 17 & $\mathrm{U}$ & 17 & 17 & $\mathrm{U}$ & 17 \\
\hline Toxaphené & 170 & $\mathrm{U}$ & 170 & 170 & $\bar{U}$ & 170 \\
\hline \multicolumn{7}{|c|}{ Semivolatile Organic Compounds } \\
\hline 1,2,4-Trichlorobenzene & 340 & $\mathrm{U}$ & 340 & 330 & $\mathrm{U}$ & 330 \\
\hline 1,2-Dichlorobenzene & 340 & $\mathrm{U}$ & 340 & 330 & $\mathrm{U}$ & 330 \\
\hline 1,3-Dichlorobenzene & 340 & $\mathrm{U}$ & 340 & 330 & $\mathrm{U}$ & 330 \\
\hline 1,4-Dichlorobenzene & 340 & $\mathrm{U}$ & 340 & 330 & $\mathrm{U}$ & 330 \\
\hline 2,4,5-Trichlorophenol & 850 & $\mathrm{U}$ & 850 & 830 & $\mathrm{U}$ & 830 \\
\hline 2,4,6-Trichlorophenol & 340 & $\mathrm{U}$ & 340 & 330 & $\mathrm{U}$ & 330 \\
\hline 2,4-Dichlorophenol & 340 & $\mathrm{U}$ & 340 & 330 & $\mathrm{U}$ & 330 \\
\hline 2,4-Dimethylphenol & 340 & $\mathrm{U}$ & 340 & 330 & $\mathrm{U}$ & 330 \\
\hline 2,4-Dinitrophenol & 850 & $\mathrm{U}$ & 850 & 830 & $\mathrm{U}$ & 830 \\
\hline 2,4-Dinitrotoluene & 340 & $\mathrm{U}$ & 340 & 330 & $\mathrm{U}$ & 330 \\
\hline 2,6-Dinitrotoluene & 340 & $\mathrm{U}$ & 340 & 330 & $\mathrm{U}$ & 330 \\
\hline 2-Chloronaphthalene & 340 & $\mathrm{U}$ & 340 & 330 & $\mathrm{U}$ & 330 \\
\hline 2-Chlorophenol & 340 & $\mathrm{U}$ & 340 & 330 & $\mathrm{U}$ & 330 \\
\hline 2-Methylnaphthalene & 340 & $\mathrm{U}$ & 340 & 330 & $\mathrm{U}$ & 330 \\
\hline 2-Methylphenol (cresol, o-) & 340 & $\mathrm{U}$ & 340 & 330 & $\mathrm{U}$ & 330 \\
\hline 2-Nitroaniline & 850 & $\mathrm{U}$ & 850 & 830 & $U$ & 830 \\
\hline 2-Nitrophenol & 340 & $\mathrm{U}$ & 340 & 330 & $\mathrm{U}$ & 330 \\
\hline 3,3'-Dichlorobenzidine & 340 & $\mathrm{U}$ & 340 & 330 & $\mathrm{U}$ & 330 \\
\hline 3-Nitroaniline & 850 & $\mathrm{U}$ & 850 & 830 & $\mathrm{U}$ & 830 \\
\hline 4,6-Dinitro-2-methylphenol & 850 & $\mathrm{U}$ & 850 & 830 & $\mathrm{U}$ & 830 \\
\hline 4-Bromophenylphenyl ether & 340 & $\mathrm{U}$ & 340 & 330 & $\mathrm{U}$ & 330 \\
\hline 4-Chloro-3-methylphenol & 340 & $\mathrm{U}$ & 340 & 330 & $\mathrm{U}$ & 330 \\
\hline 4-Chloroaniline & 340 & $\mathrm{U}$ & 340 & 330 & $\mathrm{U}$ & 330 \\
\hline 4-Chlorophenylphenyl ether & 340 & $\mathrm{U}$ & 340 & 330 & $\mathrm{U}$ & 330 \\
\hline 4-Methylphenol (cresol, p-) & 340 & $\mathrm{U}$ & 340 & 330 & $\mathrm{U}$ & 330 \\
\hline 4-Nitroaniline & 850 & $\mathrm{U}$ & 850 & 830 & $\mathrm{U}$ & 830 \\
\hline 4-Nitrophenol & 850 & $\mathrm{U}$ & 850 & 830 & $\mathrm{U}$ & 830 \\
\hline
\end{tabular}


Table A-1. 100-B-14:2 Area 2 Confirmatory Data Results. (7 Pages)

\begin{tabular}{|c|c|c|c|c|c|c|}
\hline \multirow[t]{2}{*}{ Constituents } & \multicolumn{3}{|c|}{$\begin{array}{c}\text { J00Y71 } \\
\text { Duplicate of J00Y70 } \\
\text { Sample Date } 9 / 16 / 03\end{array}$} & \multicolumn{3}{|c|}{$\begin{array}{c}\text { J00Y72 } \\
\text { Equipment Blank } \\
\text { Sample Date } 9 / 16 / 03 \\
\end{array}$} \\
\hline & $\mu \mathrm{g} / \mathrm{kg}$ & $\mathbf{Q}$ & PQL & $\mu \mathrm{g} / \mathrm{kg}$ & $\mathbf{Q}$ & PQL \\
\hline \multicolumn{7}{|c|}{ Semivolatile Organic Compounds (continued) } \\
\hline Acenaphthene & 340 & $\mathrm{U}$ & 340 & 330 & $\mathrm{U}$ & 330 \\
\hline Acenaphthylene & 340 & $\mathrm{U}$ & 340 & 330 & $\mathrm{U}$ & 330 \\
\hline Anthracene & 340 & $\mathrm{U}$ & 340 & 330 & $\mathrm{U}$ & 330 \\
\hline Benzo(a)anthracene & 340 & $\mathrm{U}$ & 340 & 330 & $\mathrm{U}$ & 330 \\
\hline Benzo(a)pyrene & 340 & $\mathrm{U}$ & 340 & 330 & $\mathrm{U}$ & 330 \\
\hline Benzo(b)fluoranthene & 340 & $\mathrm{U}$ & 340 & 330 & $\mathrm{U}$ & 330 \\
\hline Benzo(g,h,i)perylene & 340 & $\mathrm{U}$ & 340 & 330 & $\mathrm{U}$ & 330 \\
\hline Benzo(k)fluoranthene & 340 & $\mathrm{U}$ & 340 & 330 & $\mathrm{U}$ & 330 \\
\hline bis(2-Chloro-1-methylethyl)ether & 340 & $\mathrm{U}$ & 340 & 330 & $\mathrm{U}$ & 330 \\
\hline bis(2-Chloroethoxy)methane & 340 & $\mathrm{U}$ & 340 & 330 & $\mathrm{U}$ & 330 \\
\hline bis(2-Chloroethyl) ether & 340 & $\mathrm{U}$ & 340 & 330 & $\mathrm{U}$ & 330 \\
\hline bis(2-Ethylhexyl) phthalate & 340 & $\mathrm{U}$ & 340 & 36 & $\mathrm{~J}$ & 330 \\
\hline Butylbenzylphthalate & 340 & $\mathrm{U}$ & 340 & 330 & $\mathrm{U}$ & 330 \\
\hline Carbazole & 340 & $\mathrm{U}$ & 340 & 330 & $\mathrm{U}$ & 330 \\
\hline Chrysene & 340 & $\mathrm{U}$ & 340 & 330 & $\mathrm{U}$ & 330 \\
\hline Dibenz[a,h]anthracene & 340 & $\mathrm{U}$ & 340 & 330 & $\mathrm{U}$ & 330 \\
\hline \begin{tabular}{|l} 
Dibenzofuran \\
\end{tabular} & 340 & $\mathrm{U}$ & 340 & 330 & $\mathrm{U}$ & 330 \\
\hline Diethylphthalate & 340 & $\mathrm{U}$ & 340 & 330 & $\mathrm{U}$ & 330 \\
\hline Dimethyl phthalate & 340 & $\mathrm{U}$ & 340 & 330 & $\mathrm{U}$ & 330 \\
\hline Di-n-butylphthalate & 340 & $\mathrm{U}$ & 340 & 330 & $\mathrm{U}$ & 330 \\
\hline Di-n-octylphthalate & 340 & $\mathrm{U}$ & 340 & 330 & $\mathrm{U}$ & 330 \\
\hline Fluoranthene & 340 & $\mathrm{U}$ & 340 & 330 & $\mathrm{U}$ & 330 \\
\hline Fluorene & 340 & $\mathrm{U}$ & 340 & 330 & $\mathrm{U}$ & 330 \\
\hline Hexachlorobenzene & 340 & $\mathrm{U}$ & 340 & 330 & $\mathrm{U}$ & 330 \\
\hline Hexachlorobutadiene & 340 & $\mathrm{U}$ & 340 & 330 & $\mathrm{U}$ & 330 \\
\hline Hexachlorocyclopentadiene & 340 & $\mathrm{U}$ & 340 & 330 & $\mathrm{U}$ & 330 \\
\hline Hexachloroethane & 340 & $\mathrm{U}$ & 340 & 330 & $\mathrm{U}$ & 330 \\
\hline Indeno(1,2,3-cd)pyrene & 340 & $\mathrm{U}$ & 340 & 330 & $\mathrm{U}$ & 330 \\
\hline Isophorone & 340 & $\mathrm{U}$ & 340 & 330 & $\mathrm{U}$ & 330 \\
\hline Naphthalene & 340 & $\mathrm{U}$ & 340 & 330 & $\mathrm{U}$ & 330 \\
\hline Nitrobenzene & 340 & $\mathrm{U}$ & 340 & 330 & $\mathrm{U}$ & 330 \\
\hline N-Nitroso-di-n-dipropylamine & 340 & $\mathrm{U}$ & 340 & 330 & U & 330 \\
\hline $\mathrm{N}$-Nitrosodiphenylamine & 340 & $\mathrm{U}$ & 340 & 330 & $\mathrm{U}$ & 330 \\
\hline Pentachlorophenol & 850 & $\mathrm{U}$ & 850 & 830 & $\mathrm{U}$ & 830 \\
\hline Phenanthrene & 340 & $\mathrm{U}$ & 340 & 330 & $\mathrm{U}$ & 330 \\
\hline Phenol & 340 & $\mathrm{U}$ & 340 & 330 & $\mathrm{U}$ & 330 \\
\hline Pyrene & 340 & $\mathrm{U}$ & 340 & 330 & $\mathrm{U}$ & 330 \\
\hline
\end{tabular}


Table A-2. 100-B-14:2 Area 3 Confirmatory Data Results. (4 Pages)

\begin{tabular}{|c|c|c|c|c|c|c|c|c|c|c|c|c|c|c|c|c|c|}
\hline \multirow{2}{*}{ Sample Location } & \multirow{2}{*}{$\begin{array}{l}\text { Sample } \\
\text { Number }\end{array}$} & \multirow{2}{*}{$\begin{array}{c}\text { Sample } \\
\text { Date }\end{array}$} & \multicolumn{3}{|c|}{ Americium-241 } & \multicolumn{3}{|c|}{ Cesium-137 } & \multicolumn{3}{|c|}{ Cobalt-60 } & \multicolumn{3}{|c|}{ Europium-152 } & \multicolumn{3}{|c|}{ Europium-154 } \\
\hline & & & $\mathrm{pCi} / \mathrm{g}$ & Q & MDA & $\mathrm{pCi} / \mathrm{g}$ & $\mathrm{Q}$ & MDA & $\mathrm{pCi} / \mathrm{g}$ & Q & MDA & $\mathrm{pCi} / \mathrm{g}$ & Q & MDA & $\mathrm{pCi} / \mathrm{g}$ & Q & MDA \\
\hline$\overline{\mathrm{B} 2}$ & J037M6 & $6 / 20 / 05$ & 0.12 & $\overline{\mathrm{U}}$ & 0.12 & 0.047 & U & 0.047 & 0.048 & U & 0.048 & 0.12 & $\mathrm{U}$ & 0.12 & 0.15 & Uे & 0.15 \\
\hline $\bar{B} 1$ & J037M8 & $6 / 20 / 05$ & 0.21 & $\mathrm{U}$ & 0.21 & 0.177 & & 0.055 & 0.056 & $\mathrm{U}$ & 0.056 & 0.13 & $\mathrm{U}$ & 0.13 & 0.18 & $\mathrm{U}$ & 0.18 \\
\hline
\end{tabular}

\begin{tabular}{|c|c|c|c|c|c|c|c|c|c|c|c|c|c|c|c|c|}
\hline \multirow{2}{*}{ Sample Location } & \multirow{2}{*}{$\begin{array}{c}\text { Sample } \\
\text { Number }\end{array}$} & $\begin{array}{c}\text { Sample } \\
\text { Date }\end{array}$ & \multicolumn{2}{|c|}{ Europium-155 } & \multicolumn{3}{|c|}{ Gross alpha } & \multicolumn{3}{|c|}{ Gross beta } & \multicolumn{3}{|c|}{ Potassium-40 } & \multicolumn{3}{|c|}{ Radium-226 } \\
\hline
\end{tabular}

\begin{tabular}{|c|c|c|c|c|c|c|c|c|c|c|c|c|c|c|c|c|c|}
\hline \multirow{2}{*}{ Sample Location } & \multirow{2}{*}{$\begin{array}{l}\text { Sample } \\
\text { Number }\end{array}$} & \multirow{2}{*}{$\begin{array}{c}\text { Sample } \\
\text { Date }\end{array}$} & \multicolumn{3}{|c|}{ Radium-228 } & \multicolumn{3}{|c|}{ Thorium-228 } & \multicolumn{3}{|c|}{ Thorium-232 } & \multicolumn{3}{|c|}{ Uranium-235 } & \multicolumn{3}{|c|}{ Uranium-238 } \\
\hline & & & $\mathrm{pCi} / \mathrm{g}$ & Q & $\overline{\text { MDA }}$ & $\mathrm{pCi} / \mathrm{g}$ & $\bar{Q}$ & MDA & $\mathrm{pCi} / \mathrm{g}$ & $Q$ & MDA & $\mathrm{pCi} / \mathrm{g}$ & $Q$ & $\overline{\mathrm{MDA}}$ & $\overline{\mathrm{pCi} / \mathrm{g}}$ & Q & $\overline{M D A}$ \\
\hline B2 & J037M6 & $6 / 20 / 05$ & 0.700 & & 0.19 & 0.583 & & 0.048 & 0.700 & & 0.19 & 0.16 & U & 0.16 & 5.4 & U⿺ & 5.4 \\
\hline B1 & J037M8 & $6 / 20 / 05$ & 0.694 & & 0.23 & 0.565 & & 0.063 & 0.694 & & 0.23 & 0.20 & $\mathrm{U}$ & 0.20 & 6.2 & $\bar{U}$ & 6.2 \\
\hline
\end{tabular}


Table A-2. 100-B-14:2 Area 3 Confirmatory Data Results. (4 Pages)

\begin{tabular}{|c|c|c|c|c|c|c|c|c|c|c|c|c|c|c|c|c|c|c|c|}
\hline \multirow{2}{*}{ Sample Location } & \multirow{2}{*}{$\begin{array}{l}\text { Sample } \\
\text { Number } \\
\end{array}$} & \multirow{2}{*}{$\begin{array}{c}\text { Sample } \\
\text { Date }\end{array}$} & \multicolumn{2}{|c|}{ Aluminum } & \multicolumn{3}{|c|}{ Antimony } & \multicolumn{3}{|c|}{ Arsenic } & \multicolumn{3}{|c|}{ Barium } & \multicolumn{3}{|c|}{ Beryllium } & \multicolumn{3}{|c|}{ Boron } \\
\hline & & & $\mathrm{mg} / \mathrm{kg}$ & \begin{tabular}{l|l|}
$\mathbf{Q}$ & PQL \\
\end{tabular} & $\mathrm{mg} / \mathrm{kg}$ & \begin{tabular}{l|l}
$\mathrm{Q}$ \\
\end{tabular} & $\mathrm{PQL}$ & $\mathrm{mg} / \mathrm{kg}$ & $\mathrm{Q}$ & $\mathrm{PQL}$ & $\mathrm{mg} / \mathrm{kg}$ & $\mathbf{Q}$ & PQL & $\mathrm{mg} / \mathrm{kg}$ & $Q$ & PQL & $\mathrm{mg} / \mathrm{kg}$ & $\mathrm{Q}$ & $\mathrm{PQL}$ \\
\hline B2 & J037M6 & $6 / 20 / 05$ & 6350 & 2.2 & 2.2 & $\overrightarrow{\mathrm{U}}$ & 2.2 & 4.1 & & 2.5 & 73.1 & $\mathrm{C}$ & 0.11 & 0.81 & & 0.06 & 3.0 & C & 1.3 \\
\hline$\overline{B 1}$ & J037M8 & $6 / 20 / 05$ & 5970 & 6.8 & 2.5 & $\overline{\mathrm{U}}$ & 2.5 & 7.7 & & 2.8 & 998 & $\bar{C}$ & 0.12 & 0.58 & & 0.06 & 4.4 & C & 1.4 \\
\hline
\end{tabular}

\begin{tabular}{|c|c|c|c|c|c|c|c|c|c|c|c|c|c|c|c|c|c|c|c|c|}
\hline \multirow{2}{*}{ Sample Location } & \multirow{2}{*}{$\begin{array}{l}\text { Sample } \\
\text { Number }\end{array}$} & \multirow{2}{*}{$\begin{array}{l}\text { Sample } \\
\text { Date }\end{array}$} & \multicolumn{3}{|c|}{ Cadmium } & \multicolumn{3}{|c|}{ Calcium } & \multicolumn{3}{|c|}{ Chromium } & \multicolumn{3}{|c|}{ Cobalt } & \multicolumn{3}{|c|}{ Copper } & \multicolumn{3}{|c|}{$\begin{array}{l}\text { Hexavalent } \\
\text { Chromium }\end{array}$} \\
\hline & & & $\mathrm{mg} / \mathrm{kg}$ & $Q$ & PQL & $\mathrm{mg} / \mathrm{kg}$ & $Q$ & $\mathrm{PQL}$ & $\mathrm{mg} / \mathrm{kg}$ & Q & $\mathrm{PQL}$ & $\mathrm{mg} / \mathrm{kg}$ & Q & $\mathrm{PQL}$ & $\mathrm{mg} / \mathrm{kg}$ & $\bar{Q}$ & $\overline{P Q L}$ & $\mathrm{mg} / \mathrm{kg}$ & Q & $\mathrm{PQL}$ \\
\hline B2 & J037M6 & $6 / 20 / 05$ & 0.24 & & 0.17 & 6740 & & 1.7 & 9.4 & C & 0.39 & 7.9 & & 0.5 & 16.5 & & 0.27 & 0.25 & & 0.22 \\
\hline B1 & J037M7* & $6 / 20 / 05$ & & & & & & & & & & & & & & & & 0.35 & $\bar{U}$ & 0.35 \\
\hline B1 & J037M8 & $6 / 20 / 05$ & 1.5 & & 0.19 & 2570 & & 1.9 & 67.8 & $\mathrm{C}$ & 0.44 & 5.3 & & 0.56 & 102 & & 0.3 & & & \\
\hline
\end{tabular}

*Only analyte was hexavalent chromium.

\begin{tabular}{|c|c|c|c|c|c|c|c|c|c|c|c|c|c|c|c|c|c|c|c|}
\hline \multirow{2}{*}{ Sample Location } & \multirow{2}{*}{$\begin{array}{c}\text { Sample } \\
\text { Number }\end{array}$} & \multirow{2}{*}{$\begin{array}{c}\text { Sample } \\
\text { Date }\end{array}$} & \multicolumn{2}{|c|}{ Iron } & \multicolumn{3}{|c|}{ Lead } & \multicolumn{3}{|c|}{ Lithium } & \multicolumn{3}{|c|}{ Magnesium } & \multicolumn{3}{|c|}{ Manganese } & \multicolumn{3}{|c|}{ Mercury } \\
\hline & & & $\mathrm{mg} / \mathrm{kg}$ & \begin{tabular}{|l|l|}
$\mathrm{Q}$ & $\mathrm{PQL}$ \\
\end{tabular} & $\mathrm{mg} / \mathrm{kg}$ & Q & $\mathrm{PQL}$ & $\mathrm{mg} / \mathrm{kg}$ & $Q$ & $\mathrm{PQL}$ & $\mathrm{mg} / \mathrm{kg}$ & $Q$ & PQL & $\mathrm{mg} / \mathrm{kg}$ & $Q$ & PQL & $\mathrm{mg} / \mathrm{kg}$ & $\mathrm{Q}$ & $\mathrm{PQL}$ \\
\hline B2 & J037M6 & $6 / 20 / 05$ & 20500 & 16.4 & 8.9 & & 1.4 & 6.4 & $\mathrm{C}$ & 0.11 & 4340 & & 4 & 347 & & 0.11 & 0.04 & & 0.02 \\
\hline B1 & J037M8 & $6 / 20 / 05$ & 20500 & 18.3 & 279 & & 1.6 & 6.1 & $\mathrm{C}$ & 0.12 & 3190 & & 4.4 & 166 & & 0.12 & 7.2 & & 0.14 \\
\hline
\end{tabular}

\begin{tabular}{|c|c|c|c|c|c|c|c|c|c|c|c|c|c|c|c|c|c|c|c|}
\hline \multirow{2}{*}{ Sample Location } & \multirow{2}{*}{$\begin{array}{c}\text { Sample } \\
\text { Number } \\
\end{array}$} & \multirow{2}{*}{$\begin{array}{c}\text { Sample } \\
\text { Date } \\
\end{array}$} & \multicolumn{3}{|c|}{ Molybdenum } & \multicolumn{2}{|c|}{ Nickel } & \multicolumn{3}{|c|}{ Phosphorus } & \multicolumn{3}{|c|}{ Potassium } & \multicolumn{3}{|c|}{ Selenium } & \multicolumn{3}{|c|}{$\begin{array}{l}\text { Silicon } \\
\end{array}$} \\
\hline & & & $\mathrm{mg} / \mathrm{kg}$ & Q & $\mathbf{P Q L}$ & $\mathrm{mg} / \mathrm{kg}$ & \begin{tabular}{|l|l|}
$\mathrm{Q}$ & $\mathrm{PQL}$ \\
\end{tabular} & $\mathrm{mg} / \mathrm{kg}$ & $Q$ & $\mathrm{PQL}$ & $\mathrm{mg} / \mathrm{kg}$ & $\mathbf{Q}$ & $\mathrm{PQL}$ & $\mathrm{mg} / \mathrm{kg}$ & $Q$ & $\mathrm{PQL}$ & $\mathrm{mg} / \mathrm{kg}$ & \begin{tabular}{|l|l|}
$Q$ \\
\end{tabular} & $\mathbf{P Q L}$ \\
\hline $\mathrm{B} 2$ & J037M6 & $6 / 20 / 05$ & 0.9 & $\mathrm{U}$ & 0.9 & 12.5 & 1.2 & 953 & & 1.1 & 1200 & & 50.8 & 2.7 & $\mathrm{U}$ & 2.7 & 402 & & 3.8 \\
\hline B1 & J037M8 & $6 / 20 / 05$ & 1.7 & & 1.0 & 34.0 & 1.4 & 1520 & & 1.2 & 1130 & & 56.7 & 3.1 & $\mathrm{U}$ & 3.1 & 420 & & 4.2 \\
\hline
\end{tabular}

\begin{tabular}{|c|c|c|c|c|c|c|c|c|c|c|c|c|c|c|c|c|c|c|c|c|}
\hline \multirow{2}{*}{ Sample Location } & \multirow{2}{*}{$\begin{array}{l}\text { Sample } \\
\text { Number }\end{array}$} & \multirow{2}{*}{$\begin{array}{c}\text { Sample } \\
\text { Date }\end{array}$} & \multicolumn{3}{|c|}{ Silver } & \multicolumn{3}{|c|}{ Sodium } & \multicolumn{3}{|c|}{ Strontium } & \multicolumn{3}{|c|}{ Thallium } & \multicolumn{3}{|c|}{ Tin } & \multicolumn{3}{|c|}{ Titanium } \\
\hline & & & $\mathrm{mg} / \mathrm{kg}$ & Q & $\overline{P Q L}$ & $\mathrm{mg} / \mathrm{kg}$ & Q & $\mathrm{PQL}$ & $\mathrm{mg} / \mathrm{kg}$ & $\mathrm{Q}$ & PQL & $\mathrm{mg} / \mathrm{kg}$ & $Q$ & $\mathbf{P Q L}$ & $\mathrm{mg} / \mathrm{kg}$ & Q & $\mathrm{PQL}$ & $\mathrm{mg} / \mathrm{kg}$ & $Q$ & $\overline{\mathrm{PQL}}$ \\
\hline $\mathrm{B} 2$ & J037M6 & $6 / 20 / 05$ & 0.50 & U & 0.50 & 199 & & 2.200 & 34.2 & $\mathrm{C}$ & 0.06 & 4.42 & $\mathrm{U}$ & 4.4 & 3.1 & $\overline{\mathrm{UC}}$ & 3.1 & 1450 & C & 0.17 \\
\hline$\overline{B 1}$ & J037M8 & $6 / 20 / 05$ & 2.8 & & 0.56 & 264 & & 2.400 & 40.4 & $\mathrm{C}$ & 0.06 & 4.9 & \begin{tabular}{|l}
$U$ \\
\end{tabular} & 4.9 & 13.5 & C & 3.5 & 1080 & C & 0.19 \\
\hline
\end{tabular}

\begin{tabular}{|c|c|c|c|c|c|c|c|c|c|c|c|c|c|c|}
\hline \multirow{2}{*}{ Sample Location } & \multirow{2}{*}{$\begin{array}{l}\text { Sample } \\
\text { Number }\end{array}$} & \multirow{2}{*}{$\begin{array}{c}\text { Sample } \\
\text { Date }\end{array}$} & \multicolumn{3}{|c|}{ Uranium } & \multicolumn{3}{|c|}{ Vanadium } & \multicolumn{3}{|c|}{ Zinc } & \multicolumn{3}{|c|}{ Zirconium } \\
\hline & & & $\mathrm{mg} / \mathrm{kg}$ & Q & $\overline{\mathrm{PQL}}$ & $\mathrm{mg} / \mathrm{kg}$ & $Q$ & PQL & $\mathrm{mg} / \mathrm{kg}$ & $Q$ & $\mathrm{PQL}$ & $\mathrm{mg} / \mathrm{kg}$ & $Q$ & $\mathrm{PQL}$ \\
\hline B2 & J037M6 & $6 / 20 / 05$ & 11.1 & $\mathrm{U}$ & 11.1 & 47.8 & & 0.34 & 50.8 & & 0.28 & 32.6 & $\mathrm{C}$ & 2 \\
\hline B1 & J037M8 & $6 / 20 / 05$ & 12.4 & $\mathrm{U}$ & 12.4 & 40.9 & & 0.37 & 223 & & 0.31 & 11.5 & $\mathrm{C}$ & 2.2 \\
\hline
\end{tabular}


Table A-2. 100-B-14:2 Area 3 Confirmatory Data Results. (4 Pages)

\begin{tabular}{|c|c|c|c|c|c|c|}
\hline \multirow[t]{2}{*}{ Constituents } & \multicolumn{3}{|c|}{$\begin{array}{c}\text { J037M6 } \\
\text { Location B2 } \\
\text { Sample Date } 6 / 20 / 05\end{array}$} & \multicolumn{3}{|c|}{$\begin{array}{c}\text { J037M8 } \\
\text { Location B1 } \\
\text { Sample Date } 6 / 20 / 05\end{array}$} \\
\hline & $\mu \mathrm{g} / \mathrm{kg}$ & $\mathbf{Q}$ & PQL & $\mu \mathrm{g} / \mathrm{kg}$ & $Q$ & PQL \\
\hline \multicolumn{7}{|c|}{ Polychlorinated Biphenyls } \\
\hline Aroclor-1016 & 14 & $\mathrm{U}$ & 14 & 330 & UD & 330 \\
\hline Aroclor-1221 & 14 & $\mathrm{U}$ & 14 & 330 & UD & 330 \\
\hline Aroclor -1232 & 14 & $\mathrm{U}$ & 14 & 330 & UD & 330 \\
\hline Aroclor-1242 & 14 & $\mathrm{U}$ & 14 & 330 & UD & 330 \\
\hline Aroclor-1248 & 14 & $\mathrm{U}$ & 14 & 1100 & $\mathrm{D}$ & 330 \\
\hline Aroclor-1254 & 14 & $\mathrm{U}$ & 14 & 330 & UD & 330 \\
\hline Aroclor -1260 & 14 & $U$ & 14 & 280 & JD & 330 \\
\hline \multicolumn{7}{|c|}{ Pesticides } \\
\hline Aldrin & 8.9 & UD & 8.9 & 8.3 & UD & 8.3 \\
\hline alpha-BHC & 8.9 & UD & 8.9 & 8.3 & UD & 8.3 \\
\hline alpha-Chlordane. & 8.9 & UD & 8.9 & 8.3 & UD & 8.3 \\
\hline beta-BHC & 8.9 & UD & 8.9 & 4.0 & ID & 8.3 \\
\hline delta-BHC & 8.9 & UD & 8.9 & 8.3 & UD & 8.3 \\
\hline Dichlorodiphenyldichloroethane & 18 & UD & 18 & 23 & $\mathrm{D}$ & 17 \\
\hline Dichlorodiphenyldichloroethylene & 18 & UD & 18 & 37 & $\mathrm{D}$ & 17 \\
\hline Dichlorodiphenyltrichloroethane & 18 & UD & 18 & 31 & $\mathrm{D}$ & 17 \\
\hline Dieldrin & 8.9 & UD & 8.9 & 84 & $\mathrm{D}$ & 8.3 \\
\hline Endosulfan I & 8.9 & UD & 8.9 & 4.6 & JD & 8.3 \\
\hline Endosulfan II & 18 & UD & 18 & 17 & UD & 17 \\
\hline Endosulfan sulfate & 18 & UD & 18 & 3.6 & ID & 17 \\
\hline Endrin & 18 & UD & 18 & 17 & UD & 17 \\
\hline Endrin aldehyde & 18 & UD & 18 & 11 & $\mathrm{JD}$ & 17 \\
\hline Endrin ketone & 18 & UD & 18 & 17 & UD & 17 \\
\hline gamma-BHC (Lindane) & 8.9 & UD & 8.9 & 8.3 & UD & 8.3 \\
\hline gamma-Chlordane & 8.9 & UD & 8.9 & 22 & $\mathrm{D}$ & 8.3 \\
\hline Heptachlor & 8.9 & UD & 8.9 & 8.3 & UD & 8.3 \\
\hline Heptachlor epoxide & 8.9 & UD & 8.9 & 8.3 & UD & 8.3 \\
\hline Methoxychlor & 89 & UD & 89 & 32 & JD & 83 \\
\hline Toxaphene & 890 & UD & 890 & 830 & UD & 830 \\
\hline \multicolumn{7}{|c|}{ Herbicides } \\
\hline 2,4,5-Trichlorophenoxyacetic acid & 18 & $\mathrm{U}$ & 18 & 17 & $\bar{U}$ & 17 \\
\hline 2,4-Dichlorophenoxyacetic acid & 36 & $\mathrm{U}$ & 36 & 33 & $\mathrm{U}$ & 33 \\
\hline 2-(2,4,5-Trichlorophenoxy)propionic acid & 18 & $U$ & 18 & 17 & $\mathrm{U}$ & 17 \\
\hline 2-secButyl-4,6-dinitrophenol (DNBP) & 18 & $\mathrm{U}$ & 18 & 17 & $\mathrm{U}$ & 17 \\
\hline 4-(2,4-Dichlorophenoxy)butanoic acid & 180 & $\mathrm{U}$ & 180 & 170 & $\mathrm{U}$ & 170 \\
\hline Dalapon & 180 & $\mathrm{U}$ & 180 & 170 & $\mathrm{U}$ & 170 \\
\hline Dicamba & 72 & $\mathrm{U}$ & 72 & 67 & $\mathrm{U}$ & 67 \\
\hline Dichloroprop & 180 & U & 180 & 170 & $\mathrm{U}$ & 170 \\
\hline \multicolumn{7}{|c|}{ Semivolatile Organic Compounds } \\
\hline 1,2,4-Trichlorobenzene & 1800 & UD & 1800 & 2000 & UD & 2000 \\
\hline 1,2-Dichlorobenzene & 1800 & UD & 1800 & 2000 & UD & 2000 \\
\hline 1,3-Dichlorobenzene & 1800 & UD & 1800 & 2000 & UD & 2000 \\
\hline 1,4-Dichlorobenzene & 1800 & UD & 1800 & 2000 & UD & 2000 \\
\hline 2,4,5-Trichlorophenol & 4500 & UD & 4500 & 5000 & UD & 5000 \\
\hline 2,4,6-Trichlorophenol & 1800 & UD & 1800 & 2000 & UD & 2000 \\
\hline 2,4-Dichlorophenol & 1800 & UD & 1800 & 2000 & UD & 2000 \\
\hline 2,4-Dimethylphenol & 1800 & UD & 1800 & 2000 & UD & 2000 \\
\hline 2,4-Dinitrophenol & 4500 & UD & 4500 & 5000 & UD & 5000 \\
\hline 2,4-Dinitrotoluene & 1800 & UD & 1800 & 2000 & UD & 2000 \\
\hline 2,6-Dinitrotoluene & 1800 & UD & 1800 & 2000 & UD & 2000 \\
\hline 2-Chloronaphthalene & 1800 & UD & 1800 & 2000 & UD & 2000 \\
\hline 2-Chlorophenol & 1800 & UD & 1800 & 2000 & UD & 2000 \\
\hline 2-Methylnaphthalene & 1800 & UD & 1800 & 2000 & UD & 2000 \\
\hline 2-Methylphenol (cresol, o-) & 1800 & UD & 1800 & 2000 & UD & 2000 \\
\hline 2-Nitroaniline & 4500 & UD & 4500 & 5000 & UD & 5000 \\
\hline 2-Nitrophenol & 1800 & UD & 1800 & 2000 & UD & 2000 \\
\hline 3,3'-Dichlorobenzidine & 1800 & UD & 1800 & 2000 & UD & 2000 \\
\hline \begin{tabular}{|l} 
3-Nitroaniline \\
\end{tabular} & 4500 & UD & 4500 & 5000 & UD & 5000 \\
\hline 4,6-Dinitro-2-methylphenol & 4500 & UD & 4500 & 5000 & UD & 5000 \\
\hline
\end{tabular}


Table A-2. 100-B-14:2 Area 3 Confirmatory Data Results. (4 Pages)

\begin{tabular}{|c|c|c|c|c|c|c|}
\hline \multirow[t]{2}{*}{ Constituents } & \multicolumn{3}{|c|}{$\begin{array}{c}\text { J037M6 } \\
\text { Location B2 } \\
\text { Sample Date } 6 / 20 / 05\end{array}$} & \multicolumn{3}{|c|}{$\begin{array}{c}\text { J037M8 } \\
\text { Location } \mathrm{B1} \\
\text { Sample Date 6/20/05 }\end{array}$} \\
\hline & $\mu \mathrm{g} / \mathrm{kg}$ & $Q$ & PQL & $\mu \mathrm{g} / \mathrm{kg}$ & $Q$ & PQL \\
\hline \multicolumn{7}{|c|}{$\begin{array}{l}\text { Semivolatile Organic Compounds (continued) } \\
\end{array}$} \\
\hline 4-Bromophenylphenyl ether & 1800 & UD & 1800 & 2000 & UD & 2000 \\
\hline 4-Chloro-3-methylphenol & 1800 & UD & 1800 & 2000 & UD & 2000 \\
\hline 4-Chloroaniline & 1800 & UD & 1800 & 2000 & $\overline{\mathrm{UD}}$ & 2000 \\
\hline 4-Chlorophenylphenyl ether & 1800 & UD & 1800 & 2000 & UD & 2000 \\
\hline 4-Methylphenol (cresol, p-) & 1800 & UD & 1800 & 2000 & UD & 2000 \\
\hline 4-Nitroaniline & 4500 & UD & 4500 & 5000 & UD & 5000 \\
\hline 4-Nitrophenol & 4500 & UD & 4500 & 5000 & UD & 5000 \\
\hline Acenaphthene & 1800 & UD & 1800 & 2000 & UD & 2000 \\
\hline Acenaphthylene & 1800 & UD & 1800 & 2000 & UD & 2000 \\
\hline Anthracene & 1800 & UD & 1800 & 2000 & UD & 2000 \\
\hline Benzo(a)anthracene & 1800 & UD & 1800 & 210 & JD & 2000 \\
\hline Benzo(a)pyrene & 1800 & UD & 1800 & 180 & JD & 2000 \\
\hline Benzo(b)fluoranthene & 1800 & UD & 1800 & 180 & JD & 2000 \\
\hline Benzo(g,h,i)perylene & 1800 & UD & 1800 & 130 & JD & 2000 \\
\hline Benzo(k)fluoranthene & 1800 & UD & 1800 & 180 & JD & 2000 \\
\hline bis(2-Chloro-1-methylethyl)ether & 1800 & UD & 1800 & 2000 & UD & 2000 \\
\hline bis(2-Chloroethoxy)methane & 1800 & UD & 1800 & 2000 & UD & 2000 \\
\hline bis(2-Chloroethyl) ether & 1800 & UD & 1800 & 2000 & UD & 2000 \\
\hline bis(2-Ethylhexyl) phthalate & 300 & JBD & 1800 & 1100 & $\mathrm{JBD}$ & 2000 \\
\hline Butylbenzylphthalate & 1800 & UD & 1800 & 420 & JD & 2000 \\
\hline Carbazole & 1800 & UD & 1800 & 2000 & UD & 2000 \\
\hline Chrysene & 1800 & UD & 1800 & 240 & JD & 2000 \\
\hline Di-n-butylphthalate & 1800 & UD & 1800 & 21000 & $\mathrm{BD}$ & 2000 \\
\hline Di-n-octylphthalate & 1800 & UD & 1800 & 2000 & UD & 2000 \\
\hline Dibenz[a,h]anthracene & 1800 & UD & 1800 & 2000 & UD & 2000 \\
\hline Dibenzofuran & 1800 & UD & 1800 & 2000 & UD & 2000 \\
\hline Diethylphthalate & 1800 & UD & 1800 & 2000 & UD & 2000 \\
\hline Dimethyl phthalate & 1800 & UD & 1800 & 2000 & UD & 2000 \\
\hline Fluoranthene & 1800 & UD & 1800 & 380 & JD & 2000 \\
\hline Fluorene & 1800 & UD & 1800 & 2000 & UD & 2000 \\
\hline Hexachlorobenzene & 1800 & UD & 1800 & 2000 & UD & 2000 \\
\hline Hexachlorobutadiene & 1800 & UD & 1800 & 2000 & UD & 2000 \\
\hline Hexachlorocyclopentadiene & 1800 & UD & 1800 & 2000 & UD & 2000 \\
\hline Hexachloroethane & 1800 & UD & 1800 & 2000 & UD & 2000 \\
\hline Indeno(1,2,3-cd)pyrene & 1800 & UD & 1800 & 120 & JD & 2000 \\
\hline Isophorone & 1800 & UD & 1800 & 2000 & UD & 2000 \\
\hline N-Nitroso-di-n-dipropylamine & 1800 & UD & 1800 & 2000 & UD & 2000 \\
\hline $\mathrm{N}$-Nitrosodiphenylamine & 1800 & UD & 1800 & 2000 & UD & 2000 \\
\hline Naphthalene & 1800 & UD & 1800 & 2000 & UD & 2000 \\
\hline Nitrobenzene & 1800 & UD & 1800 & 2000 & UD & 2000 \\
\hline Pentachlorophenol & 4500 & UD & 4500 & 5000 & UD & 5000 \\
\hline Phenanthrene & 1800 & UD & 1800 & 190 & JD & 2000 \\
\hline Phenol & 1800 & UD & 1800 & 2000 & UD & 2000 \\
\hline Pyrene & 1800 & UD & 1800 & 370 & JD & 2000 \\
\hline
\end{tabular}


Table A-3. 100-B-14:2 Area 3 Additional Characterization Data Results.

\begin{tabular}{|c|c|c|c|c|c|c|c|c|c|c|c|c|c|c|c|c|c|c|c|c|}
\hline \multirow{2}{*}{$\begin{array}{l}\text { Sample } \\
\text { Location }\end{array}$} & \multirow{2}{*}{$\begin{array}{c}\text { Sample } \\
\text { Number } \\
\end{array}$} & \multirow{2}{*}{$\begin{array}{c}\text { Sample } \\
\text { Date } \\
\end{array}$} & \multicolumn{3}{|c|}{ 1,4-Dichlorobenzene } & \multicolumn{3}{|c|}{ 2,4,5-Trichlorophenol } & \multicolumn{3}{|c|}{ 2,4,6-Trichlorophenol } & \multicolumn{3}{|c|}{ 2,4-Dinitrotoluene } & \multicolumn{3}{|c|}{ 2-Methylphenol } & \multicolumn{3}{|c|}{ 3+4 Methylphenol } \\
\hline & & & $\mathrm{mg} / \mathrm{L}$ & $\bar{Q}$ & $\mathrm{PQL}$ & $\mathrm{mg} / \mathrm{L}$ & $Q$ & PQL & $\mathrm{mg} / \mathrm{L}$ & $Q$ & PQL & $\mathrm{mg} / \mathrm{L}$ & $Q$ & $\begin{array}{l}\mathrm{PQL} \\
\end{array}$ & $\mathrm{mg} / \mathrm{L}$ & Q & PQL & $\mathrm{mg} / \mathrm{L}$ & Q & PQL \\
\hline B2 & J037M6 & $6 / 20 / 05$ & 0.05 & $\mathrm{U}$ & 0.05 & 0.1 & $\mathrm{U}$ & 0.12 & 0.05 & $\mathrm{U}$ & 0.05 & 0.05 & $\mathrm{U}$ & 0.05 & 0.05 & $\mathrm{U}$ & 0.05 & 0.05 & $\mathrm{U}$ & 0.05 \\
\hline B1 & J037M8 & $6 / 20 / 05$ & 0.05 & $\bar{U}$ & 0.05 & 0.12 & $\bar{U}$ & 0.12 & 0.05 & $\mathrm{U}$ & 0.05 & 0.05 & $\mathrm{U}$ & 0.05 & 0.05 & $\bar{U}$ & 0.05 & 0.05 & $\mathrm{U}$ & 0.05 \\
\hline
\end{tabular}

\begin{tabular}{|c|c|c|c|c|c|c|c|c|c|c|c|c|c|c|c|c|c|c|c|c|}
\hline \multirow{2}{*}{$\begin{array}{l}\text { Sample } \\
\text { Location }\end{array}$} & \multirow{2}{*}{$\begin{array}{l}\text { Sample } \\
\text { Number }\end{array}$} & \multirow{2}{*}{$\begin{array}{c}\text { Sample } \\
\text { Date }\end{array}$} & \multicolumn{3}{|c|}{ Hexachlorobenzene } & \multicolumn{3}{|c|}{ Hexachlorobutadiene } & \multicolumn{3}{|c|}{ Hexachloroethane } & \multicolumn{3}{|c|}{ Nitrobenzene } & \multicolumn{3}{|c|}{ Pentachlorophenol } & \multicolumn{3}{|c|}{ Pyridine } \\
\hline & & & $\mathrm{mg} / \mathrm{L}$ & $\bar{Q}$ & $\overline{\mathrm{PQL}}$ & $\mathrm{mg} / \mathrm{L}$ & $Q$ & PQL & $\mathrm{mg} / \mathrm{L}$ & Q & $\overline{\mathrm{PQL}}$ & $\mathrm{mg} / \mathrm{L}$ & Q & $\mathrm{PQL}$ & $\mathrm{mg} / \mathrm{L}$ & $Q$ & $\mathrm{PQL}$ & $\mathrm{mg} / \mathrm{L}$ & Q & $\mathrm{PQL}$ \\
\hline B2 & J037M6 & $6 / 20 / 05$ & 0.05 & $\mathrm{U}$ & 0.05 & 0.05 & $\mathrm{U}$ & 0.05 & 0.05 & $\mathrm{U}$ & 0.05 & 0.05 & $\overline{\mathrm{U}}$ & 0.05 & 0.12 & $\mathrm{U}$ & 0.12 & 0.05 & $\mathrm{U}$ & 0.05 \\
\hline B1 & J037M8 & $6 / 20 / 05$ & 0.05 & $\bar{U}$ & 0.05 & 0.05 & $\bar{U}$ & 0.05 & 0.05 & $\mathrm{U}$ & 0.05 & 0.1 & $\mathrm{U}$ & 0.05 & 0.12 & $\mathrm{U}$ & 0.12 & 0.05 & $\mathrm{U}$ & 0.05 \\
\hline
\end{tabular}

\begin{tabular}{|c|c|c|c|c|c|c|c|c|c|c|c|c|c|c|c|c|c|c|c|c|}
\hline \multirow{2}{*}{$\begin{array}{l}\text { Sample } \\
\text { Location }\end{array}$} & \multirow{2}{*}{$\begin{array}{l}\text { Sample } \\
\text { Number }\end{array}$} & \multirow{2}{*}{$\begin{array}{c}\text { Sample } \\
\text { Date }\end{array}$} & \multicolumn{3}{|c|}{ Aroclor-1016 } & \multicolumn{3}{|c|}{ Aroclor-1221 } & \multicolumn{3}{|c|}{ Aroclor -1232} & \multicolumn{3}{|c|}{ Aroclor-1242 } & \multicolumn{3}{|c|}{ Aroclor-1248 } & \multicolumn{3}{|c|}{ Aroclor-1254 } \\
\hline & & & $\mu \mathrm{g} / \mathrm{L}$ & $Q$ & PQL & $\mu \mathrm{g} / \mathrm{L}$ & $Q$ & PQL & $\mu \mathrm{g} / \mathrm{L}$ & $Q$ & $\mathrm{PQL}$ & $\mu \mathrm{g} / \mathrm{L}$ & Q & $\mathrm{PQL}$ & $\mu \mathrm{g} / \mathrm{L}$ & $Q$ & $\mathrm{PQL}$ & $\mu \mathrm{g} / \mathrm{L}$ & Q & $\mathbf{P Q L}$ \\
\hline B2 & J037M6 & $6 / 20 / 05$ & 4 & $\bar{U}$ & 4 & 4 & $\mathrm{U}$ & 4 & 4 & $\mathrm{U}$ & 4 & 4 & Uे & 4 & 4.0 & $\mathrm{U}$ & 4 & 4 & $\mathrm{U}$ & 4.00 \\
\hline B1 & J037M8 & $6 / 20 / 05$ & 10 & $\bar{U}$ & 10 & 10 & $\bar{U}$ & 10 & 10 & $\mathrm{U}$ & 10 & 10 & $\bar{U}$ & 10 & 10 & $\overline{\mathrm{U}}$ & 10 & 10 & $\bar{U}$ & 10 \\
\hline
\end{tabular}

\begin{tabular}{|c|c|c|c|c|c|}
\hline Sample & Sample & Sample & \multicolumn{3}{|c|}{ Aroclor-1260 } \\
\cline { 3 - 5 } Location & Number & Date & $\mu \mathrm{g} / \mathrm{L}$ & $\mathrm{Q}$ & PQL \\
\hline B2 & J037M6 & $6 / 20 / 05$ & 4 & $\mathrm{U}$ & 4 \\
\hline B1 & J037M8 & $6 / 20 / 05$ & 10 & $\mathrm{U}$ & 10 \\
\hline
\end{tabular}

\begin{tabular}{|c|c|c|c|c|c|c|c|c|c|c|c|c|c|c|c|c|c|c|c|c|}
\hline \multirow{2}{*}{$\begin{array}{l}\text { Sample } \\
\text { Location }\end{array}$} & \multirow{2}{*}{$\begin{array}{c}\text { Sample } \\
\text { Number } \\
\end{array}$} & \multirow{2}{*}{$\begin{array}{c}\text { Sample } \\
\text { Date }\end{array}$} & \multicolumn{3}{|c|}{ Arsenic } & \multicolumn{3}{|c|}{ Barium } & \multicolumn{3}{|c|}{ Cadmium } & \multicolumn{3}{|c|}{ Chromium } & \multicolumn{3}{|c|}{ Lead } & \multicolumn{3}{|c|}{ Mercury } \\
\hline & & & $\mu \mathrm{g} / \mathrm{L}$ & $Q$ & PQL & $\mu \mathrm{g} / \mathrm{L}$ & $\bar{Q}$ & $\mathrm{PQL}$ & $\mu g / \mathrm{L}$ & $Q$ & $\mathbf{P Q L}$ & $\mu \mathrm{g} / \mathrm{L}$ & Q & $\overline{P Q L}$ & $\mu \mathrm{g} / \mathrm{L}$ & \begin{tabular}{|l|} 
Q \\
\end{tabular} & PQL & $\mu \mathrm{g} / \mathrm{L}$ & Q & PQL \\
\hline B2 & J037M6 & $6 / 20 / 05$ & 27 & $\mathrm{U}$ & 27 & 228 & $\mathrm{C}$ & 1.200 & 1.8 & $\mathrm{U}$ & 1.8 & 4.2 & $\mathrm{U}$ & 4.2 & 15 & $\mathrm{U}$ & 15 & 0.1 & $\mathrm{U}$ & 0.1 \\
\hline B1 & J037M8 & $6 / 20 / 05$ & 27 & $\overline{\mathrm{U}}$ & 27 & 597 & $\bar{C}$ & 1.200 & 9.7 & & 1.8 & 5.5 & & 4.2 & 28.3 & & 15 & 0.1 & & 0.1 \\
\hline
\end{tabular}

\begin{tabular}{|c|c|c|c|c|c|c|c|c|}
\hline Sample & Sample & Sample & \multicolumn{3}{|c|}{ Selenium } & \multicolumn{3}{c|}{ Silver } \\
\cline { 5 - 8 } Location & Number & Date & $\mu \mathrm{g} / \mathrm{L}$ & $\mathrm{Q}$ & $\mathbf{P Q L}$ & $\mu \mathrm{g} / \mathrm{L}$ & $\mathrm{Q}$ & PQL \\
\hline B2 & J037M6 & $6 / 20 / 05$ & 29.4 & $\mathrm{U}$ & 29.4 & 5.4 & $\mathrm{UC}$ & 5.4 \\
\hline B1 & $\mathrm{J} 037 \mathrm{M} 8$ & $6 / 20 / 05$ & 29.4 & $\mathrm{U}$ & 29.4 & 5.4 & $\mathrm{U}$ & 5.4 \\
\hline
\end{tabular}


Table A-4. 100-B-14:2 Area 4 Confirmatory Data Results. (7 Pages)

\begin{tabular}{|c|c|c|c|c|c|c|c|c|c|c|c|c|c|c|c|c|c|}
\hline \multirow{2}{*}{ Sample Location } & \multirow{2}{*}{$\begin{array}{l}\text { Sample } \\
\text { Number }\end{array}$} & \multirow{2}{*}{$\begin{array}{c}\text { Sample } \\
\text { Date }\end{array}$} & \multicolumn{3}{|c|}{ Americium-241 } & \multicolumn{3}{|c|}{ Cesium-137 } & \multicolumn{3}{|c|}{ Cobalt-60 } & \multicolumn{3}{|c|}{ Europium-152 } & \multicolumn{3}{|c|}{ Europium-154 } \\
\hline & & & $\mathrm{pCi} / \mathrm{g}$ & $Q$ & MDA & $\mathrm{pCi} / \mathrm{g}$ & $Q$ & MDA & $\mathrm{pCi} / \mathrm{g}$ & $\mathrm{Q}$ & MDA & $\overline{p C i} / g$ & $\mathrm{Q}$ & MDA & $\mathrm{pCi} / \mathrm{g}$ & Q & MDA \\
\hline A9 & J00Y74 & $09 / 18 / 03$ & 0.2 & $\mathrm{U}$ & 0.2 & 0.064 & & 0.043 & 0.041 & $\mathrm{U}$ & 0.041 & 0.094 & $\mathrm{U}$ & 0.094 & 0.13 & $\mathrm{U}$ & 0.13 \\
\hline $\mathrm{A} 11$ & J00Y75 & $09 / 18 / 03$ & 0.047 & $\mathrm{U}$ & 0.047 & 0.18 & & 0.062 & 0.054 & $\mathrm{U}$ & 0.054 & 0.12 & $\mathrm{U}$ & 0.12 & 0.15 & $\mathrm{U}$ & 0.15 \\
\hline $\mathrm{A} 12$ & J00YV1 & $10 / 02 / 03$ & 0.09 & $\mathrm{U}$ & 0.09 & 0.022 & $\mathrm{U}$ & 0.022 & 0.026 & $\mathrm{U}$ & 0.026 & 0.06 & $\mathrm{U}$ & 0.06 & 0.082 & $\mathrm{U}$ & 0.082 \\
\hline A10 & J00YV2 & $10 / 02 / 03$ & 0.07 & $\mathrm{U}$ & 0.07 & 0.066 & $\mathrm{U}$ & 0.066 & 0.076 & $\mathrm{U}$ & 0.076 & 0.17 & $\mathrm{U}$ & 0.17 & 0.26 & $\mathrm{U}$ & 0.26 \\
\hline Duplicate of J00YV2 & J00YV3 & $10 / 02 / 03$ & 0.32 & $\mathrm{U}$ & 0.32 & 0.032 & $\bar{U}$ & 0.032 & 0.029 & $\mathrm{U}$ & 0.029 & 0.081 & $\mathrm{U}$ & 0.081 & 0.13 & $\mathrm{U}$ & 0.13 \\
\hline
\end{tabular}

\begin{tabular}{|c|c|c|c|c|c|c|c|c|c|c|c|c|c|c|c|c|c|}
\hline \multirow{2}{*}{ Sample Location } & \multirow{2}{*}{\begin{tabular}{c|} 
Sample \\
Number
\end{tabular}} & \multirow{2}{*}{$\begin{array}{c}\text { Sample } \\
\text { Date }\end{array}$} & \multicolumn{3}{|c|}{ Europium-155 } & \multicolumn{3}{|c|}{ Gross alpha } & \multicolumn{3}{|c|}{ Gross beta } & \multicolumn{3}{|c|}{ Potassium-40 } & \multicolumn{3}{|c|}{ Radium-226 } \\
\hline & & & $\mathrm{pCi} / \mathrm{g}$ & $Q$ & MDA & $\mathrm{pCi} / \mathrm{g}$ & $\mathrm{Q}$ & MDA & $\mathrm{pCi} / \mathrm{g}$ & $Q$ & MDA & $\mathrm{pCi} / \mathrm{g}$ & $Q$ & MDA & $\mathrm{pCi} / \mathrm{g}$ & $Q$ & MDA \\
\hline A9 & J00Y74 & $09 / 18 / 03$ & 0.099 & $\mathrm{U}$ & 0.099 & 3.73 & $\overline{\mathrm{U}}$ & 6 & 8.4 & & 7.7 & 7.11 & & 0.44 & 0.321 & & 0.077 \\
\hline A11 & J00Y75 & $09 / 18 / 03$ & 0.088 & $\mathrm{U}$ & 0.088 & 3.02 & $\mathrm{U}$ & 7.2 & 12.9 & & 6.7 & 5.3 & & 0.48 & 0.318 & & 0.1 \\
\hline $\mathrm{A} 12$ & J00YV1 & $10 / 02 / 03$ & 0.071 & $\mathrm{U}$ & 0.071 & 2.03 & $\mathrm{U}$ & 3.7 & 13.8 & & 5.6 & 12.5 & & 0.25 & 0.434 & & $\overline{0.046}$ \\
\hline A10 & J00YV2 & $10 / 02 / 03$ & 0.14 & $\overline{\mathrm{U}}$ & 0.14 & 3.38 & $\bar{U}$ & 4.7 & 14.5 & & 5.6 & 8.27 & & 0.66 & 0.299 & & 0.13 \\
\hline Duplicate of J00YV2 & J00YV3 & $10 / 02 / 03$ & 0.11 & $\overline{\mathrm{U}}$ & 0.11 & 2.34 & $\mathrm{U}$ & 4.3 & 15.5 & & 6.4 & 10.9 & & 0.46 & 0.42 & & 0.066 \\
\hline
\end{tabular}

\begin{tabular}{|c|c|c|c|c|c|c|c|c|c|c|c|c|c|c|c|c|c|}
\hline \multirow{2}{*}{ Sample Location } & \multirow{2}{*}{$\begin{array}{l}\text { Sample } \\
\text { Number }\end{array}$} & \multirow{2}{*}{$\begin{array}{c}\text { Sample } \\
\text { Date }\end{array}$} & \multicolumn{3}{|c|}{ Radium-228 } & \multicolumn{3}{|c|}{ Thorium-228 } & \multicolumn{3}{|c|}{ Thorium-232 } & \multicolumn{3}{|c|}{ Uranium-235 } & \multicolumn{3}{|c|}{ Uranium-238 } \\
\hline & & & $\mathrm{pCi} / \mathrm{g}$ & $Q$ & MDA & $\mathrm{pCi} / \mathrm{g}$ & $Q$ & MDA & $\mathrm{pCi} / \mathrm{g}$ & $Q$ & MDA & $\mathrm{pCi} / \mathrm{g}$ & $Q$ & MDA & $\mathrm{pCi} / \mathrm{g}$ & $Q$ & MDA \\
\hline A9 & J00Y74 & $09 / 18 / 03$ & 0.356 & & 0.16 & 0.334 & & 0.043 & 0.356 & & 0.16 & 0.14 & $\mathrm{U}$ & 0.14 & 4.6 & $\mathrm{U}$ & 4.6 \\
\hline A11 & J00Y75 & $09 / 18 / 03$ & 0.46 & $\mathrm{U}$ & 0.46 & 0.248 & & 0.053 & 0.46 & $\mathrm{U}$ & 0.46 & 0.16 & $\mathrm{U}$ & 0.16 & 6 & $\mathrm{U}$ & 6 \\
\hline $\mathrm{A} 12$ & J00YV1 & $10 / 02 / 03$ & 0.636 & & 0.1 & 0.641 & & 0.028 & 0.636 & & 0.1 & 0.09 & $\mathrm{U}$ & 0.09 & 3 & $\mathrm{U}$ & 3 \\
\hline $\mathrm{A} 10$ & J00YV2 & $10 / 02 / 03$ & 0.546 & & 0.31 & 0.373 & & 0.087 & 0.546 & & 0.31 & 0.24 & $\mathrm{U}$ & 0.24 & 9 & $\mathrm{U}$ & 9 \\
\hline Duplicate of J00YV2 & J00YV3 & $10 / 02 / 03$ & 0.577 & & 0.17 & 0.535 & & 0.045 & 0.577 & & 0.17 & 0.14 & $\mathrm{U}$ & 0.14 & 4.5 & $\mathrm{U}$ & 4.5 \\
\hline
\end{tabular}


Table A-4. 100-B-14:2 Area 4 Confirmatory Data Results. (7 Pages)

\begin{tabular}{|c|c|c|c|c|c|c|c|c|c|c|c|c|c|c|c|c|c|c|c|c|}
\hline \multirow{2}{*}{ Sample Location } & \multirow{2}{*}{$\begin{array}{c}\text { Sample } \\
\text { Number }\end{array}$} & \multirow{2}{*}{$\begin{array}{c}\text { Sample } \\
\text { Date }\end{array}$} & \multicolumn{3}{|c|}{ Aluminum } & \multicolumn{3}{|c|}{ Antimony } & \multicolumn{3}{|c|}{ Arsenic } & \multicolumn{3}{|c|}{ Barium } & \multicolumn{3}{|c|}{ Beryllium } & \multicolumn{3}{|c|}{ Boron } \\
\hline & & & $\mathrm{mg} / \mathrm{kg}$ & $\mathrm{Q}$ & $\mathrm{PQL}$ & $\mathrm{mg} / \mathrm{kg}$ & $Q$ & $\mathrm{PQL}$ & $\mathrm{mg} / \mathrm{kg}$ & $Q$ & $\mathrm{PQL}$ & $\mathrm{mg} / \mathrm{kg}$ & $Q$ & $\mathrm{PQL}$ & $\mathrm{mg} / \mathrm{kg}$ & $Q$ & $\mathrm{PQL}$ & $\mathrm{mg} / \mathrm{kg}$ & $Q$ & $\mathrm{PQL}$ \\
\hline A9 & J00Y74 & $09 / 18 / 03$ & 4600 & & 4.7 & 7.5 & & 0.29 & 32.9 & & 0.42 & 162 & & 0.02 & 0.07 & & 0.04 & 31.9 & & 0.22 \\
\hline A11 & J00Y75 & $09 / 18 / 03$ & 6010 & & 4.6 & 9.1 & & 0.29 & 22.2 & & 0.42 & 178 & & 0.02 & 0.12 & & 0.04 & 6.4 & & 0.22 \\
\hline A12 & J00YV5 & $10 / 02 / 03$ & 5940 & & 4.6 & 0.28 & $\mathrm{U}$ & 0.28 & 4 & & 0.41 & 51.9 & & 0.02 & 0.3 & & 0.04 & 0.92 & & 0.22 \\
\hline $\mathrm{A} 10$ & J00YV6 & $10 / 02 / 03$ & 5380 & & 4.6 & 0.29 & $\bar{U}$ & 0.29 & 2.3 & & 0.42 & 55.2 & & 0.02 & 0.32 & & 0.04 & 1.2 & & 0.22 \\
\hline Duplicate of J00YV6 & J00YV7 & $10 / 02 / 03$ & 5250 & & 4.2 & 0.26 & $\mathrm{U}$ & 0.26 & 2.3 & & 0.38 & 55.5 & & 0.02 & 0.25 & & 0.04 & 1 & & 0.2 \\
\hline Equipment blank & J00YV8 & $10 / 02 / 03$ & 53.7 & & 3.8 & 0.23 & $\mathrm{U}$ & 0.23 & 0.34 & $\mathrm{U}$ & 0.34 & 1.2 & & 0.02 & 0.03 & $\mathrm{U}$ & 0.03 & 0.18 & $\mathrm{U}$ & 0.18 \\
\hline
\end{tabular}

\begin{tabular}{|c|c|c|c|c|c|c|c|c|c|c|c|c|c|c|c|c|c|c|c|c|}
\hline \multirow[t]{2}{*}{ Sample Location } & \multirow{2}{*}{$\begin{array}{l}\text { Sample } \\
\text { Number }\end{array}$} & \multirow{2}{*}{$\begin{array}{c}\text { Sample } \\
\text { Date }\end{array}$} & \multicolumn{3}{|c|}{ Cadmium } & \multicolumn{3}{|c|}{ Calcium } & \multicolumn{3}{|c|}{ Chromium } & \multicolumn{3}{|c|}{ Cobalt } & \multicolumn{3}{|c|}{ Copper } & \multicolumn{3}{|c|}{$\begin{array}{c}\text { Hexavalent } \\
\text { Chromium }\end{array}$} \\
\hline & & & $\mathrm{mg} / \mathrm{kg}$ & $Q$ & $\mathrm{PQL}$ & $\mathrm{mg} / \mathrm{kg}$ & $Q$ & PQL & $\mathrm{mg} / \mathrm{kg}$ & $Q$ & PQL & $\mathrm{mg} / \mathrm{kg}$ & $Q$ & PQL & $\mathrm{mg} / \mathrm{kg}$ & $Q$ & $\mathrm{PQL}$ & $\mathrm{mg} / \mathrm{kg}$ & $Q$ & $\overline{\mathrm{PQL}}$ \\
\hline A9 & J00Y74 & $09 / 18 / 03$ & 3.1 & & 0.04 & 22000 & & 3.1 & 62.2 & & 0.1 & 13.8 & & 0.12 & 311 & & 0.12 & & & \\
\hline A11 & J00Y75 & $09 / 18 / 03$ & 2.6 & & 0.04 & 68600 & & 36.8 & 97.6 & & 0.1 & 10.1 & & 0.12 & 186 & & 0.12 & & & \\
\hline A9 & $\mathrm{J} 00 \mathrm{Y}^{7} 6^{*}$ & $09 / 18 / 03$ & & & & & & & & & & & & & & & & 2.52 & & 0.35 \\
\hline A11 & J00Y77* & $09 / 18 / 03$ & & & & & & & & & & & & & & & & 2.14 & & 0.35 \\
\hline $\mathrm{A} 12$ & J00YV5 & $10 / 02 / 03$ & 0.13 & & 0.04 & 9810 & & 3 & 7.1 & & 0.1 & 9.9 & & 0.12 & 18.4 & & 0.12 & 0.42 & $\mathrm{U}$ & 0.42 \\
\hline $\mathrm{A} 10$ & J00YV6 & $10 / 02 / 03$ & 0.43 & & 0.04 & 5250 & & 3.1 & 7.1 & & 0.1 & 9.9 & & 0.12 & 18.8 & & 0.12 & 0.425 & $\mathrm{U}$ & 0.425 \\
\hline Duplicate of J00YV6 & J00YV7 & $10 / 02 / 03$ & 0.43 & & 0.04 & 5550 & & 2.8 & 7.1 & & 0.09 & 8.7 & & 0.11 & 17.3 & & 0.11 & 0.42 & $U$ & 0.42 \\
\hline Equipment Blank & J00YV8 & $10 / 02 / 03$ & 0.03 & $\mathrm{U}$ & 0.03 & 18.3 & & 2.5 & 0.15 & & 0.08 & 0.1 & $\mathrm{U}$ & 0.1 & 0.1 & $\mathrm{U}$ & 0.1 & 0.4 & $\mathrm{U}$ & 0.4 \\
\hline
\end{tabular}

*Only analyte was hexavalent chromium.

\begin{tabular}{|c|c|c|c|c|c|c|c|c|c|c|c|c|c|c|c|c|c|c|c|}
\hline \multirow{2}{*}{ Sample Location } & \multirow{2}{*}{$\begin{array}{l}\text { Sample } \\
\text { Number }\end{array}$} & \multirow{2}{*}{$\begin{array}{c}\text { Sample } \\
\text { Date }\end{array}$} & \multicolumn{3}{|c|}{ Iron } & \multicolumn{3}{|c|}{ Lead } & \multicolumn{3}{|c|}{ Magnesium } & \multicolumn{3}{|c|}{ Manganese } & \multicolumn{3}{|c|}{ Mercury } & \multicolumn{2}{|c|}{ Molybdenum } \\
\hline & & & $\mathrm{mg} / \mathrm{kg}$ & 0 & POL & $\mathrm{mg} / \mathrm{kg}$ & 0 & PQL & $\mathrm{mg} / \mathrm{kg}$ & $Q$ & PQL & $\mathrm{mg} / \mathrm{kg}$ & $Q$ & PQL & $\mathrm{mg} / \mathrm{kg}$ & $\mathrm{Q}$ & PQL & $\mathrm{mg} / \mathrm{kg}$ & \begin{tabular}{|l|l}
$\mathrm{Q}$ & $\mathrm{PQL}$
\end{tabular} \\
\hline A9 & J00Y74 & $09 / 18 / 03$ & 99000 & & 23.8 & 172 & & 0.19 & 3440 & & 0.7 & 476 & & 0.03 & 8.2 & & 0.15 & 7.6 & 0.19 \\
\hline$\overline{\mathrm{A} 11}$ & J00Y75 & $09 / 18 / 03$ & 61900 & & 23.5 & 71.7 & & 0.19 & 3810 & & 0.69 & 476 & & 0.03 & 2.7 & & 0.06 & 3 & 0.19 \\
\hline $\mathrm{A} 12$ & J00YV5 & $10 / 02 / 03$ & 26000 & & 1.9 & 5.3 & & 0.19 & 5410 & & 0.68 & 378 & & 0.03 & 0.01 & $\bar{U}$ & 0.01 & 0.44 & 0.19 \\
\hline $\mathrm{A} 10$ & J00YV6 & $10 / 02 / 03$ & 27300 & & 2 & 5.1 & & 0.19 & 4920 & & 0.7 & 364 & & 0.03 & 0.21 & & 0.01 & 0.44 & 0.19 \\
\hline Duplicate of J00YV6 & J00YV7 & $10 / 02 / 03$ & 25000 & & 1.8 & 5.2 & & 0.17 & 4260 & & 0.63 & 316 & & 0.03 & 0.22 & & 0.01 & 0.43 & 0.17 \\
\hline Equipment blank & J00YV8 & $10 / 02 / 03$ & 118 & & 1.6 & 0.36 & & 0.18 & 9.2 & & 0.56 & 3.1 & & 0.03 & 0.01 & $\overline{\mathrm{U}}$ & 0.01 & 0.15 & 0.16 \\
\hline
\end{tabular}


Table A-4. 100-B-14:2 Area 4 Confirmatory Data Results. (7 Pages)

\begin{tabular}{|c|c|c|c|c|c|c|c|c|c|c|c|c|c|c|c|c|c|c|c|c|}
\hline \multirow{2}{*}{ Sample Location } & \multirow{2}{*}{$\begin{array}{l}\text { Sample } \\
\text { Number }\end{array}$} & \multirow{2}{*}{$\begin{array}{c}\text { Sample } \\
\text { Date }\end{array}$} & \multicolumn{3}{|c|}{ Nickel } & \multicolumn{3}{|c|}{ Potassium } & \multicolumn{3}{|c|}{ Selenium } & \multicolumn{3}{|c|}{ Silicon } & \multicolumn{3}{|c|}{ Silver } & \multicolumn{3}{|c|}{ Sodium } \\
\hline & & & $\mathrm{mg} / \mathrm{kg}$ & Q & $\mathrm{PQL}$ & $\mathrm{gg} / \mathrm{kg}$ & $\bar{Q}$ & $\mathrm{PQL}$ & $\mathrm{mg} / \mathrm{kg}$ & $\bar{Q}$ & $\mathrm{PQL}$ & $\mathrm{mg} / \mathrm{kg}$ & Q & $\mathrm{PQL}$ & $\mathrm{mg} / \mathrm{kg}$ & $\mathbf{Q}$ & $\mathbf{P Q L}$ & $\mathrm{mg} / \mathrm{kg}$ & Q & PQL \\
\hline A9 & J00Y74 & $09 / 18 / 03$ & 99.2 & & 0.2 & 678 & & 2.4 & 1.4 & & 0.29 & 406 & & 0.54 & 0.48 & & 0.008 & 546 & & 0.71 \\
\hline & & $9 / 18 / 03$ & 43.4 & & 0.2 & 2030 & & 2.3 & 0.37 & & 0.29 & 384 & & 0.53 & 0.65 & & 0.08 & 1030 & & 0.7 \\
\hline & & & 11 & & 0.2 & & & 2.3 & .28 & & 0.28 & 167 & & 0.53 & 0.08 & $\mathrm{U}$ & 0.08 & 234 & & 0.69 \\
\hline & J00YV6 & $10 / 02 / 03$ & 12.1 & & $\overline{0.2}$ & 888 & & 2.3 & 0.29 & $\overline{\mathrm{U}}$ & 0.29 & 144 & & 0.54 & 0.08 & & 0.08 & 237 & & 0.71 \\
\hline Duplicate of J0 & J00YV7 & $10 / 02 / 03$ & 10.8 & & 0.18 & 874 & & 2.1 & 0.26 & $\mathrm{U}$ & 0.26 & 159 & & 0.49 & 0.07 & $\mathrm{U}$ & 0.07 & 215 & & 0.64 \\
\hline Equipment blank & J00YV8 & $10 / 02 / 03$ & 0.16 & $\mathrm{U}$ & 0.16 & 20.1 & & 1.9 & 0.23 & U & 0.23 & 44.9 & & 0.44 & 0.06 & $\mathrm{U}$ & 0.06 & 4.8 & & 0.57 \\
\hline
\end{tabular}

\begin{tabular}{|c|c|c|c|c|c|c|c|c|}
\hline \multirow{2}{*}{ Sample Location } & \multirow{2}{*}{$\begin{array}{l}\text { Sample } \\
\text { Number }\end{array}$} & \multirow{2}{*}{$\begin{array}{c}\text { Sample } \\
\text { Date }\end{array}$} & \multicolumn{3}{|c|}{ Vanadium } & \multicolumn{3}{|c|}{ Zinc } \\
\hline & & & $\mathrm{mg} / \mathrm{kg}$ & $\bar{Q}$ & $\mathrm{PQL}$ & $\mathrm{mg} / \mathrm{kg}$ & $Q$ & PQL \\
\hline A9 & J00Y74 & $09 / 18 / 03$ & 34.5 & & 0.09 & 995 & & 0.26 \\
\hline A11 & Y75 & $09 / 18 / 03$ & 47 & & 0.09 & 730 & & 0.26 \\
\hline $\mathrm{A} 12$ & J00YV5 & $10 / 02 / 03$ & 60.7 & & 0.09 & 49.2 & & 0.26 \\
\hline $\mathrm{A} 10$ & J00YV6 & $10 / 02 / 03$ & 67.3 & & 0.09 & 241 & & 0.26 \\
\hline Duplicate of J00YV6 & J00YV7 & $10 / 02 / 03$ & 64.3 & & 0.08 & 249 & & 0.24 \\
\hline Equipment blank & J00YV8 & $10 / 02 / 03$ & 0.07 & & 0.07 & 0.66 & & 0.21 \\
\hline
\end{tabular}


Table A-4. 100-B-14:2 Area 4 Confirmatory Data Results. (7 Pages)

\begin{tabular}{|c|c|c|c|c|c|c|c|c|c|c|c|c|}
\hline \multirow[t]{2}{*}{ Constituents } & \multicolumn{3}{|c|}{$\begin{array}{c}\text { J00YV5 } \\
\text { Location A12 } \\
\text { Sample Date } 10 / 2 / 03\end{array}$} & \multicolumn{3}{|c|}{$\begin{array}{c}\text { J00YV6 } \\
\text { Location A10 } \\
\text { Sample Date } 10 / 2 / 03\end{array}$} & \multicolumn{3}{|c|}{$\begin{array}{c}\text { J00YV7 } \\
\text { Duplicate of J00YV6 } \\
\text { Sample Date } 10 / 2 / 03 \\
\end{array}$} & \multicolumn{3}{|c|}{$\begin{array}{c}\text { J00YV8 } \\
\text { Equipment Blank } \\
\text { Sample Date } 10 / 2 / 03 \\
\end{array}$} \\
\hline & $\mu \mathrm{g} / \mathrm{kg}$ & $Q$ & $\mathrm{PQL}$ & $\mu \mathrm{g} / \mathrm{kg}$ & $Q$ & PQL & $\mu \mathrm{g} / \mathrm{kg}$ & $\mathbf{Q}$ & PQL & $\mu \mathrm{g} / \mathrm{kg}$ & $\mathbf{Q}$ & PQL \\
\hline \multicolumn{13}{|c|}{ Semivolatile Organic Compounds } \\
\hline 1,2,4-Trichlorobenzene & 350 & $\mathrm{U}$ & 350 & 350 & $\mathrm{U}$ & 350 & 350 & $\mathrm{U}$ & 350 & 330 & $\mathrm{U}$ & 330 \\
\hline 1,2-Dichlorobenzene & 350 & $\mathrm{U}$ & 350 & 350 & $\mathrm{U}$ & 350 & 350 & $\mathrm{U}$ & 350 & 330 & $\mathrm{U}$ & 330 \\
\hline 1,3-Dichlorobenzene & 350 & $\mathrm{U}$ & 350 & 350 & $\mathrm{U}$ & 350 & 350 & $\mathrm{U}$ & 350 & 330 & $\mathrm{U}$ & 330 \\
\hline 1,4-Dichlorobenzene & 350 & $\mathrm{U}$ & 350 & 350 & $\mathrm{U}$ & 350 & 350 & $\mathrm{U}$ & 350 & 330 & $\mathrm{U}$ & 330 \\
\hline 2,4,5-Trichlorophenol & 870 & $\mathrm{U}$ & 870 & 890 & $\mathrm{U}$ & 890 & 880 & $\mathrm{U}$ & 880 & 830 & $\mathrm{U}$ & 830 \\
\hline 2,4,6-Trichlorophenol & 350 & $\mathrm{U}$ & 350 & 350 & $\mathrm{U}$ & 350 & 350 & $\mathrm{U}$ & 350 & 330 & $\mathrm{U}$ & 330 \\
\hline 2,4-Dichlorophenol & 350 & $\mathrm{U}$ & 350 & 350 & $\mathrm{U}$ & 350 & 350 & $\mathrm{U}$ & 350 & 330 & $\mathrm{U}$ & 330 \\
\hline 2,4-Dimethylphenol & 350 & $\mathrm{U}$ & 350 & 350 & $\mathrm{U}$ & 350 & 350 & $\mathrm{U}$ & 350 & 330 & $\mathrm{U}$ & 330 \\
\hline 2,4-Dinitrophenol & 870 & $\mathrm{U}$ & 870 & 890 & $\mathrm{U}$ & 890 & 880 & $\mathrm{U}$ & 880 & 830 & $\mathrm{U}$ & 830 \\
\hline 2,4-Dinitrotoluene & 350 & $\mathrm{U}$ & 350 & 350 & $\mathrm{U}$ & 350 & 350 & $\mathrm{U}$ & 350 & 330 & $\mathrm{U}$ & 330 \\
\hline 2,6-Dinitrotoluene & 350 & $\mathrm{U}$ & 350 & 350 & $\mathrm{U}$ & 350 & 350 & $\mathrm{U}$ & 350 & 330 & $\mathrm{U}$ & 330 \\
\hline 2-Chloronaphthalene & 350 & $\mathrm{U}$ & 350 & 350 & $\mathrm{U}$ & 350 & 350 & $\mathrm{U}$ & 350 & 330 & $\mathrm{U}$ & 330 \\
\hline 2-Chlorophenol & 350 & $\mathrm{U}$ & 350 & 350 & $\mathrm{U}$ & 350 & 350 & $\mathrm{U}$ & 350 & 330 & $\mathrm{U}$ & 330 \\
\hline 2-Methylnaphthalene & 350 & $\mathrm{U}$ & 350 & 350 & $\mathrm{U}$ & 350 & 350 & $\mathrm{U}$ & 350 & 330 & $\mathrm{U}$ & 330 \\
\hline 2-Methylphenol (cresol, o-) & 350 & $\mathrm{U}$ & 350 & 350 & $\mathrm{U}$ & 350 & 350 & $\mathrm{U}$ & 350 & 330 & $\mathrm{U}$ & 330 \\
\hline 2-Nitroaniline & 870 & $\mathrm{U}$ & 870 & 890 & $\mathrm{U}$ & 890 & 880 & $\mathrm{U}$ & 880 & 830 & $\mathrm{U}$ & 830 \\
\hline 2-Nitrophenol & 350 & $\mathrm{U}$ & 350 & 350 & $\mathrm{U}$ & 350 & 350 & $\mathrm{U}$ & 350 & 330 & $\mathrm{U}$ & 330 \\
\hline 3,3'-Dichlorobenzidine & 350 & $\mathrm{U}$ & 350 & 350 & $\mathrm{U}$ & 350 & 350 & $\mathrm{U}$ & 350 & 330 & $\mathrm{U}$ & 330 \\
\hline 3-Nitroaniline & 870 & $\mathrm{U}$ & 870 & 890 & $\mathrm{U}$ & 890 & 880 & $\mathrm{U}$ & 880 & 830 & $\mathrm{U}$ & 830 \\
\hline 4,6-Dinitro-2-methylphenol & 870 & $\mathrm{U}$ & 870 & 890 & $\mathrm{U}$ & 890 & 880 & $\mathrm{U}$ & 880 & 830 & $\mathrm{U}$ & 830 \\
\hline 4-Bromophenylphenyl ether & 350 & $\mathrm{U}$ & 350 & 350 & $\mathrm{U}$ & 350 & 350 & $\mathrm{U}$ & 350 & 330 & $\mathrm{U}$ & 330 \\
\hline 4-Chloro-3-methylphenol & 350 & $\mathrm{U}$ & 350 & 350 & $\mathrm{U}$ & 350 & 350 & $\mathrm{U}$ & 350 & 330 & $\mathrm{U}$ & 330 \\
\hline 4-Chloroaniline & 350 & $\mathrm{U}$ & 350 & 350 & $\mathrm{U}$ & 350 & 350 & $\mathrm{U}$ & 350 & 330 & $\mathrm{U}$ & 330 \\
\hline 4-Chlorophenylphenyl ether & 350 & $\mathrm{U}$ & 350 & 350 & $\mathrm{U}$ & 350 & 350 & $\mathrm{U}$ & 350 & 330 & $\mathrm{U}$ & 330 \\
\hline 4-Methylphenol (cresol, p-) & 350 & $\mathrm{U}$ & 350 & 350 & $\mathrm{U}$ & 350 & 350 & $\mathrm{U}$ & 350 & 330 & $\mathrm{U}$ & 330 \\
\hline 4-Nitroaniline & 870 & $\mathrm{U}$ & 870 & 890 & $\mathrm{U}$ & 890 & 880 & $\mathrm{U}$ & 880 & 830 & $\mathrm{U}$ & 830 \\
\hline 4-Nitrophenol & 870 & $\mathrm{U}$ & 870 & 890 & $\mathrm{U}$ & 890 & 880 & $\mathrm{U}$ & 880 & 830 & $\mathrm{U}$ & 830 \\
\hline Acenaphthene & 350 & $\mathrm{U}$ & 350 & 350 & $\mathrm{U}$ & 350 & 350 & $\mathrm{U}$ & 350 & 330 & $\mathrm{U}$ & 330 \\
\hline Acenaphthylene & 350 & $\mathrm{U}$ & 350 & 350 & $\mathrm{U}$ & 350 & 350 & $\mathrm{U}$ & 350 & 330 & $\mathrm{U}$ & 330 \\
\hline Anthracene & 350 & $\mathrm{U}$ & 350 & 350 & $\mathrm{U}$ & 350 & 350 & $\mathrm{U}$ & 350 & 330 & $\mathrm{U}$ & 330 \\
\hline Benzo(a)anthracene & 350 & $\mathrm{U}$ & 350 & 350 & $\mathrm{U}$ & 350 & 350 & $\mathrm{U}$ & 350 & 330 & $\mathrm{U}$ & 330 \\
\hline Benzo(a)pyrene & 350 & $\mathrm{U}$ & 350 & 350 & $\mathrm{U}$ & 350 & 350 & $\mathrm{U}$ & 350 & 330 & $\mathrm{U}$ & 330 \\
\hline Benzo(b)fluoranthene & 350 & $\mathrm{U}$ & 350 & 350 & $\mathrm{U}$ & 350 & 350 & $\mathrm{U}$ & 350 & 330 & U & 330 \\
\hline Benzo(g,h,i)perylene & 350 & $\mathrm{U}$ & 350 & 350 & $\mathrm{U}$ & 350 & 350 & $\mathrm{U}$ & 350 & 330 & $\mathrm{U}$ & 330 \\
\hline Benzo(k)fluoranthene & 350 & $\mathrm{U}$ & 350 & 350 & $\mathrm{U}$ & 350 & 350 & $\mathrm{U}$ & 350 & 330 & $\mathrm{U}$ & 330 \\
\hline bis(2-Chloro-1-methylethyl)ether & 350 & $\mathrm{U}$ & 350 & 350 & $\mathrm{U}$ & 350 & 350 & $\mathrm{U}$ & 350 & 330 & $\mathrm{U}$ & 330 \\
\hline bis(2-Chloroethoxy)methane & 350 & $\mathrm{U}$ & 350 & 350 & $\mathrm{U}$ & 350 & 350 & $\mathrm{U}$ & 350 & 330 & $\mathrm{U}$ & 330 \\
\hline bis(2-Chloroethyl) ether & 350 & $\mathrm{U}$ & 350 & 350 & $\mathrm{U}$ & 350 & 350 & $\mathrm{U}$ & 350 & 330 & $\mathrm{U}$ & 330 \\
\hline bis(2-Ethylhexyl) phthalate & 25 & $\mathrm{C}$ & 350 & 350 & $\mathrm{U}$ & 350 & 30 & $\mathrm{C}$ & 350 & 330 & $\mathrm{U}$ & 330 \\
\hline Butylbenzylphthalate & 350 & $\mathrm{U}$ & 350 & 350 & $\mathrm{U}$ & 350 & 350 & $\mathrm{U}$ & 350 & 330 & $\mathrm{U}$ & 330 \\
\hline Carbazole & 350 & $\mathrm{U}$ & 350 & 350 & $\mathrm{U}$ & 350 & 350 & $\mathrm{U}$ & 350 & 330 & $\mathrm{U}$ & 330 \\
\hline \begin{tabular}{|l} 
Chrysene \\
\end{tabular} & 350 & $\mathrm{U}$ & 350 & 350 & $\mathrm{U}$ & 350 & 350 & $\mathrm{U}$ & 350 & 330 & $\mathrm{U}$ & 330 \\
\hline Dibenz[a,h]anthracene & 350 & $\mathrm{U}$ & 350 & 350 & $\mathrm{U}$ & 350 & 350 & $\mathrm{U}$ & 350 & 330 & $\mathrm{U}$ & 330 \\
\hline Dibenzofuran & 350 & $\mathrm{U}$ & 350 & 350 & $\mathrm{U}$ & 350 & 350 & $\mathrm{U}$ & 350 & 330 & $\mathrm{U}$ & 330 \\
\hline Diethylphthalate & 350 & $\mathrm{U}$ & 350 & 350 & $\mathrm{U}$ & 350 & 350 & $\mathrm{U}$ & 350 & 330 & $\mathrm{U}$ & 330 \\
\hline Dimethyl phthalate & 350 & $\mathrm{U}$ & 350 & 350 & $\mathrm{U}$ & 350 & 350 & $\mathrm{U}$ & 350 & 330 & $\mathrm{U}$ & 330 \\
\hline Di-n-butylphthalate & 350 & $\mathrm{U}$ & 350 & 100 & $\mathrm{~J}$ & 350 & 110 & $\mathrm{~J}$ & 350 & 330 & $\mathrm{U}$ & 330 \\
\hline Di-n-octylphthalate & 350 & $\mathrm{U}$ & 350 & 350 & $\mathrm{U}$ & 350 & 350 & $\mathrm{U}$ & 350 & 330 & $\mathrm{U}$ & 330 \\
\hline
\end{tabular}


Table A-4. 100-B-14:2 Area 4 Confirmatory Data Results. (7 Pages)

\begin{tabular}{|c|c|c|c|c|c|c|c|c|c|c|c|c|}
\hline \multirow[t]{2}{*}{ Constituents } & \multicolumn{3}{|c|}{$\begin{array}{c}\text { J00YV5 } \\
\text { Location A12 } \\
\text { Sample Date } 10 / 2 / 03 \\
\end{array}$} & \multicolumn{3}{|c|}{$\begin{array}{c}\text { J00YV6 } \\
\text { Location A10 } \\
\text { Sample Date } 10 / 2 / 03 \\
\end{array}$} & \multicolumn{3}{|c|}{$\begin{array}{c}\text { J00YV7 } \\
\text { Duplicate of J00YV6 } \\
\text { Sample Date } 10 / 2 / 03\end{array}$} & \multicolumn{3}{|c|}{$\begin{array}{c}\text { J00YV8 } \\
\text { Equipment Blank } \\
\text { Sample Date 10/2/03 } \\
\end{array}$} \\
\hline & $\mu \mathrm{g} / \mathrm{kg}$ & $\mathbf{Q}$ & PQL & $\mu \mathrm{g} / \mathrm{kg}$ & $\mathbf{Q}$ & $\mathrm{PQL}$ & $\mu \mathrm{g} / \mathrm{kg}$ & Q & PQL & $\mu \mathrm{g} / \mathrm{kg}$ & Q & PQL \\
\hline \multicolumn{13}{|c|}{ Semivolatile Organic Compounds (continued) } \\
\hline Fluoranthene & 350 & $\mathrm{U}$ & 350 & 350 & $\mathrm{U}$ & 350 & 350 & $\mathrm{U}$ & 350 & 330 & $\mathrm{U}$ & 330 \\
\hline Fluorene & 350 & $\mathrm{U}$ & 350 & 350 & $\mathrm{U}$ & 350 & 350 & $\mathrm{U}$ & 350 & 330 & $\mathrm{U}$ & 330 \\
\hline Hexachlorobenzene & 350 & $\mathrm{U}$ & 350 & 350 & $\mathrm{U}$ & 350 & 350 & $\mathrm{U}$ & 350 & 330 & $\mathrm{U}$ & 330 \\
\hline Hexachlorobutadiene & 350 & $\mathrm{U}$ & 350 & 350 & $\mathrm{U}$ & 350 & 350 & $\mathrm{U}$ & 350 & 330 & $\mathrm{U}$ & 330 \\
\hline Hexachlorocyclopentadiene & 350 & $\mathrm{U}$ & 350 & 350 & $\mathrm{U}$ & 350 & 350 & $\mathrm{U}$ & 350 & 330 & $\mathrm{U}$ & 330 \\
\hline Hexachloroethane & 350 & $\mathrm{U}$ & 350 & 350 & $\mathrm{U}$ & 350 & 350 & $\mathrm{U}$ & 350 & 330 & $\mathrm{U}$ & 330 \\
\hline Indeno(1,2,3-cd)pyrene & 350 & $\mathrm{U}$ & 350 & 350 & $\mathrm{U}$ & 350 & 350 & $\mathrm{U}$ & 350 & 330 & $\mathrm{U}$ & 330 \\
\hline Isophorone & 350 & $\mathrm{U}$ & 350 & 350 & $\mathrm{U}$ & 350 & 350 & $\mathrm{U}$ & 350 & 330 & $\mathrm{U}$ & 330 \\
\hline Naphthalene & 350 & $\mathrm{U}$ & 350 & 350 & $\mathrm{U}$ & 350 & 350 & $\mathrm{U}$ & 350 & 330 & $\mathrm{U}$ & 330 \\
\hline Nitrobenzene & 350 & $\mathrm{U}$ & 350 & 350 & $\mathrm{U}$ & 350 & 350 & $\mathrm{U}$ & 350 & 330 & $\mathrm{U}$ & 330 \\
\hline N-Nitroso-di-n-dipropylamine & 350 & $\mathrm{U}$ & 350 & 350 & $\mathrm{U}$ & 350 & 350 & $\mathrm{U}$ & 350 & 330 & $\mathrm{U}$ & 330 \\
\hline N-Nitrosodiphenylamine & 350 & $\mathrm{U}$ & 350 & 350 & $\mathrm{U}$ & 350 & 350 & $\mathrm{U}$ & 350 & 330 & $\mathrm{U}$ & 330 \\
\hline Pentachlorophenol & 870 & $\mathrm{U}$ & 870 & 890 & $\mathrm{U}$ & 890 & 880 & $\mathrm{U}$ & 880 & 830 & $\mathrm{U}$ & 830 \\
\hline Phenanthrene & 350 & $\mathrm{U}$ & 350 & 350 & $\mathrm{U}$ & 350 & 350 & $\mathrm{U}$ & 350 & 330 & $\bar{U}$ & 330 \\
\hline Phenol & 350 & $\mathrm{U}$ & 350 & 350 & $\mathrm{U}$ & 350 & 350 & $\mathrm{U}$ & 350 & 330 & $\mathrm{U}$ & 330 \\
\hline Pyrene & 350 & $\mathrm{U}$ & 350 & 350 & $\mathrm{U}$ & 350 & 350 & $\mathrm{U}$ & 350 & 330 & $\mathrm{U}$ & 330 \\
\hline
\end{tabular}


Table A-4. 100-B-14:2 Area 4 Confirmatory Data Results. (7 Pages)

\begin{tabular}{|c|c|c|c|c|c|c|}
\hline \multirow[t]{2}{*}{ Constituents } & \multicolumn{3}{|c|}{$\begin{array}{c}\text { J00Y74 } \\
\text { Location A9 } \\
\text { Sample Date 9/18/03 }\end{array}$} & \multicolumn{3}{|c|}{$\begin{array}{c}\text { J00Y75 } \\
\text { Location A11 } \\
\text { Sample Date } 9 / 18 / 03\end{array}$} \\
\hline & $\mu \mathrm{g} / \mathrm{kg}$ & $Q$ & PQL & $\mu \mathrm{g} / \mathrm{kg}$ & $Q$ & PQL \\
\hline
\end{tabular}

\begin{tabular}{|l|c|c|c|c|c|c|}
\hline \multicolumn{9}{c|}{ Polychlorinated Biphenyls } & \multicolumn{3}{|c|}{} \\
\hline Aroclor-1016 & 270 & $\mathrm{U}$ & 270 & 15 & $\mathrm{U}$ & 15 \\
\hline Aroclor-1221 & 270 & $\mathrm{U}$ & 270 & 15 & $\mathrm{U}$ & 15 \\
\hline Aroclor-1232 & 270 & $\mathrm{U}$ & 270 & 15 & $\mathrm{U}$ & 15 \\
\hline Aroclor-1242 & 270 & $\mathrm{U}$ & 270 & 15 & $\mathrm{U}$ & 15 \\
\hline Aroclor-1248 & 270 & $\mathrm{U}$ & 270 & 15 & $\mathrm{U}$ & 15 \\
\hline Aroclor-1254 & 2600 & & 270 & 150 & & 15 \\
\hline Aroclor-1260 & 270 & $\mathrm{U}$ & 270 & 15 & $\mathrm{U}$ & 15 \\
\hline
\end{tabular}

\begin{tabular}{|c|c|c|c|c|c|c|}
\hline \multicolumn{7}{|c|}{ Pesticides } \\
\hline Aldrin & 17 & $\mathrm{U}$ & 17 & 18 & $\mathrm{U}$ & 18 \\
\hline alpha-BHC & 17 & $\overline{\mathrm{U}}$ & 17 & 18 & $\bar{U}$ & 18 \\
\hline alpha-Chlordane & 17 & $\bar{U}$ & 17 & 18 & $\mathrm{U}$ & 18 \\
\hline beta-BHC & 17 & $\bar{U}$ & 17 & 18 & $\mathrm{U}$ & 18 \\
\hline delta-BHC & 17 & $\overline{\mathrm{U}}$ & 17 & 18 & $\mathrm{U}$ & 18 \\
\hline Dichlorodiphenyldichloroethane & 34 & $\mathrm{U}$ & 34 & 37 & $\mathrm{U}$ & 37 \\
\hline Dichlorodiphenyldichloroethylene & 34 & $\bar{U}$ & 34 & 37 & $\mathrm{U}$ & 37 \\
\hline Dichlorodiphenyltrichloroethane & 210 & & 34 & 37 & $\mathrm{U}$ & 37 \\
\hline Dieldrin & 34 & $\bar{U}$ & 34 & 37 & $\mathrm{U}$ & 37 \\
\hline Endosulfan I & 17 & $\bar{U}$ & 17 & 18 & $\bar{U}$ & 18 \\
\hline Endosulfan II & 34 & $\bar{U}$ & 34 & 37 & $\bar{U}$ & 37 \\
\hline Endosulfan sulfate & 34 & $\mathrm{U}$ & 34 & 37 & $\mathrm{U}$ & 37 \\
\hline Endrin & 34 & $\mathrm{U}$ & 34 & 37 & $\mathrm{U}$ & 37 \\
\hline Endrin aldehyde & 34 & $\bar{U}$ & 34 & 37 & $\mathrm{U}$ & 37 \\
\hline Endrin ketone & 34 & $\bar{U}$ & 34 & 37 & $\mathrm{U}$ & 37 \\
\hline gamma-BHC(Lindane) & 17 & $\mathrm{U}$ & 17 & 18 & $\mathrm{U}$ & 18 \\
\hline gamma-Chlordane & 19 & & 17 & 18 & $\mathrm{U}$ & 18 \\
\hline Heptachlor & 17 & $\mathrm{U}$ & 17 & 18 & $\mathrm{U}$ & 18 \\
\hline Heptachlor epoxide & 17 & $\mathrm{U}$ & 17 & 18 & $\mathrm{U}$ & 18 \\
\hline Methoxychlor & 170 & $\bar{U}$ & 170 & 180 & $\mathrm{U}$ & 180 \\
\hline Toxaphene & 1700 & $\bar{U}$ & 1700 & 1800 & $\mathrm{U}$ & 1800 \\
\hline
\end{tabular}

\begin{tabular}{ll|c|c|c}
\hline Toxaphene & 1700 & $\mathrm{U}$ & 1700 \\
\hline & Semivolatile Organic Compounds
\end{tabular}

\begin{tabular}{|c|c|c|c|c|c|c|}
\hline \\
\hline 1,2,4-Trichlorobenzene & 1000 & $\mathrm{U}$ & 1000 & 730 & $\mathrm{U}$ & 730 \\
\hline 1,2-Dichlorobenzene & 1000 & $\mathrm{U}$ & 1000 & 730 & $\mathrm{U}$ & 730 \\
\hline 1,3-Dichlorobenzene & 1000 & $\mathrm{U}$ & 1000 & 730 & $\mathrm{U}$ & 730 \\
\hline 1,4-Dichlorobenzene & 1000 & $\mathrm{U}$ & 1000 & 730 & $\mathrm{U}$ & 730 \\
\hline 2,4,5-Trichlorophenol & 2600 & $\mathrm{U}$ & 2600 & 1800 & $\mathrm{U}$ & 1800 \\
\hline 2,4,6-Trichlorophenol & 1000 & $\mathrm{U}$ & 1000 & 730 & $\mathrm{U}$ & 730 \\
\hline 2,4-Dichlorophenol & 1000 & $\mathrm{U}$ & 1000 & 730 & $\mathrm{U}$ & 730 \\
\hline 2,4-Dimethylphenol & 1000 & $\mathrm{U}$ & 1000 & 730 & $\mathrm{U}$ & 730 \\
\hline 2,4-Dinitrophenol & 2600 & $\mathrm{U}$ & 2600 & 1800 & $\mathrm{U}$ & 1800 \\
\hline 2,4-Dinitrotoluene & 1000 & $\mathrm{U}$ & 1000 & 730 & $\mathrm{U}$ & 730 \\
\hline 2,6-Dinitrotoluene & 1000 & $\mathrm{U}$ & 1000 & 730 & $\mathrm{U}$ & 730 \\
\hline 2-Chloronaphthalene & 1000 & $\mathrm{U}$ & 1000 & 730 & $\mathrm{U}$ & 730 \\
\hline 2-Chlorophenol & 1000 & $\mathrm{U}$ & 1000 & 730 & $\mathrm{U}$ & 730 \\
\hline 2-Methylnaphthalene & 1000 & $\mathrm{U}$ & 1000 & 730 & $\mathrm{U}$ & 730 \\
\hline 2-Methylphenol (cresol, o-) & 1000 & $\mathrm{U}$ & 1000 & 730 & $\mathrm{U}$ & 730 \\
\hline 2-Nitroaniline & 2600 & $\mathrm{U}$ & 2600 & 1800 & $\mathrm{U}$ & 1800 \\
\hline 2-Nitrophenol & 1000 & $\mathrm{U}$ & 1000 & 730 & $\mathrm{U}$ & 730 \\
\hline 3,3'-Dichlorobenzidine & 1000 & $\mathrm{U}$ & 1000 & 730 & $\mathrm{U}$ & 730 \\
\hline 3-Nitroaniline & 2600 & $\mathrm{U}$ & 2600 & 1800 & $\mathrm{U}$ & 1800 \\
\hline 4,6-Dinitro-2-methylphenol & 2600 & $\mathrm{U}$ & 2600 & 1800 & $\mathrm{U}$ & 1800 \\
\hline 4-Bromophenylphenyl ether & 1000 & $\mathrm{U}$ & 1000 & 730 & $\mathrm{U}$ & 730 \\
\hline 4-Chloro-3-methylphenol & 1000 & $\mathrm{U}$ & 1000 & 730 & $\mathrm{U}$ & 730 \\
\hline 4-Chloroaniline & 1000 & $\mathrm{U}$ & 1000 & 730 & $\mathrm{U}$ & 730 \\
\hline 4-Chlorophenylphenyl ether & 1000 & $\mathrm{U}$ & 1000 & 730 & $\mathrm{U}$ & 730 \\
\hline 4-Methylphenol (cresol, p-) & 1000 & $\mathrm{U}$ & 1000 & 730 & $\mathrm{U}$ & 730 \\
\hline 4-Nitroaniline & 2600 & $\mathrm{U}$ & 2600 & 1800 & $\mathrm{U}$ & 1800 \\
\hline 4-Nitrophenol & 2600 & $\mathrm{U}$ & 2600 & 1800 & $\mathrm{U}$ & 1800 \\
\hline
\end{tabular}


Table A-4. 100-B-14:2 Area 4 Confirmatory Data Results. (7 Pages)

\begin{tabular}{|c|c|c|c|c|c|c|}
\hline \multirow[t]{2}{*}{ Constituents } & \multicolumn{3}{|c|}{$\begin{array}{c}\text { J00Y74 } \\
\text { Location A9 } \\
\text { Sample Date } 9 / 18 / 03\end{array}$} & \multicolumn{3}{|c|}{$\begin{array}{c}\text { J00Y75 } \\
\text { Location A11 } \\
\text { Sample Date 9/18/03 }\end{array}$} \\
\hline & $\mu \mathrm{g} / \mathrm{kg}$ & $\mathbf{Q}$ & PQL & $\mu \mathrm{g} / \mathrm{kg}$ & $\mathbf{Q}$ & PQL \\
\hline \multicolumn{7}{|c|}{ Semivolatile Organic Compounds (continued) } \\
\hline Acenaphthene & 1000 & $\mathrm{U}$ & 1000 & 120 & $\mathrm{~J}$ & 730 \\
\hline Acenaphthylene & 1000 & $\mathrm{U}$ & 1000 & 48 & $\mathrm{~J}$ & 730 \\
\hline Anthracene & 1000 & $\mathrm{U}$ & 1000 & 210 & $\mathrm{~J}$ & 730 \\
\hline Benzo(a)anthracene & 160 & $\mathrm{~J}$ & 1000 & 650 & $\mathrm{~J}$ & 730 \\
\hline Benzo(a)pyrene & 160 & $\mathrm{~J}$ & 1000 & 600 & $\mathrm{~J}$ & 730 \\
\hline Benzo(b)fluoranthene & 120 & $\mathrm{~J}$ & 1000 & 530 & $\mathrm{~J}$ & 730 \\
\hline Benzo(g,h,i)perylene & 1000 & $\mathrm{U}$ & 1000 & 380 & $\mathrm{~J}$ & 730 \\
\hline Benzo(k)fluoranthene & 150 & $\mathrm{~J}$ & 1000 & 510 & $\mathrm{~J}$ & 730 \\
\hline bis(2-Chloro-1-methylethyl)ether & 1000 & $\mathrm{U}$ & 1000 & 730 & $\mathrm{U}$ & 730 \\
\hline bis(2-Chloroethoxy)methane & 1000 & $\mathrm{U}$ & 1000 & 730 & $\mathrm{U}$ & 730 \\
\hline bis(2-Chloroethyl) ether & 1000 & $\mathrm{U}$ & 1000 & 730 & $\mathrm{U}$ & 730 \\
\hline bis(2-Ethylhexyl) phthalate & 330 & $\mathrm{~J}$ & 1000 & 160 & $\mathrm{~J}$ & 730 \\
\hline Butylbenzylphthalate & 1000 & $\mathrm{U}$ & 1000 & 730 & $\mathrm{U}$ & 730 \\
\hline Carbazole & 1000 & $\mathrm{U}$ & 1000 & 160 & $\mathrm{~J}$ & 730 \\
\hline Chrysene & 180 & $\mathrm{~J}$ & 1000 & 780 & & 730 \\
\hline Dibenz[a,h]anthracene & 1000 & $\mathrm{U}$ & 1000 & 100 & $\mathrm{~J}$ & 730 \\
\hline Dibenzofuran & 1000 & $\mathrm{U}$ & 1000 & 68 & $\mathrm{~J}$ & 730 \\
\hline Diethylphthalate & 1000 & $\mathrm{U}$ & 1000 & 730 & $\mathrm{U}$ & 730 \\
\hline Dimethyl phthalate & 1000 & $\mathrm{U}$ & 1000 & 730 & $\mathrm{U}$ & 730 \\
\hline Di-n-butylphthalate & 4200 & & 1000 & 4000 & & 730 \\
\hline Di-n-octylphthalate & 1000 & $\mathrm{U}$ & 1000 & 730 & $\mathrm{U}$ & 730 \\
\hline Fluoranthene & 230 & $\mathrm{~J}$ & 230 & 1900 & & 730 \\
\hline Fluorene & 1000 & $\bar{U}$ & 1000 & 100 & $\mathrm{~J}$ & 730 \\
\hline Hexachlorobenzene & 1000 & $\mathrm{U}$ & 1000 & 730 & $\mathrm{U}$ & 730 \\
\hline Hexachlorobutadiene & 1000 & U & 1000 & 730 & $\mathrm{U}$ & 730 \\
\hline Hexachlorocyclopentadiene & 1000 & $\mathrm{U}$ & 1000 & 730 & $\mathrm{U}$ & 730 \\
\hline Hexachloroethane & 1000 & $\mathrm{U}$ & 1000 & 730 & $\mathrm{U}$ & 730 \\
\hline Indeno(1,2,3-cd)pyrene & 58 & $\mathrm{~J}$ & 58 & 330 & $\mathrm{~J}$ & 730 \\
\hline Isophorone & 1000 & $\mathrm{U}$ & 1000 & 730 & $\mathrm{U}$ & 730 \\
\hline Naphthalene & 1000 & $\mathrm{U}$ & 1000 & 60 & $\mathrm{~J}$ & 730 \\
\hline Nitrobenzene & 1000 & $\mathrm{U}$ & 1000 & 730 & $\mathrm{U}$ & 730 \\
\hline N-Nitroso-di-n-dipropylamine & 1000 & $\mathrm{U}$ & 1000 & 730 & $\mathrm{U}$ & 730 \\
\hline N-Nitrosodiphenylamine & 1000 & $\mathrm{U}$ & 1000 & 730 & $\mathrm{U}$ & 730 \\
\hline Pentachlorophenol & 2600 & $\mathrm{U}$ & 2600 & 1800 & $\mathrm{U}$ & 1800 \\
\hline Phenanthrene & 160 & $\mathrm{~J}$ & 1000 & 1200 & & 730 \\
\hline Phenol & 1000 & $\mathrm{U}$ & 1000 & 730 & $\mathrm{U}$ & 730 \\
\hline Pyrene & 330 & $\mathrm{~J}$ & 1000 & 980 & & 730 \\
\hline
\end{tabular}


Table A-5. 100-B-14:2 Area 5 Confirmatory Data Results. (6 Pages)

\begin{tabular}{|c|c|c|c|c|c|c|c|c|c|c|c|c|c|c|c|c|c|c|c|c|}
\hline \multirow{2}{*}{ Sample Location } & \multirow{2}{*}{\begin{tabular}{|c|} 
Sample \\
Number
\end{tabular}} & \multirow{2}{*}{$\begin{array}{c}\text { Sample } \\
\text { Date }\end{array}$} & \multicolumn{3}{|c|}{ Americium-241 GEA } & \multicolumn{3}{|c|}{ Carbon-14 } & \multicolumn{3}{|c|}{ Cesium-137 } & \multicolumn{3}{|c|}{ Cobalt- 60} & \multicolumn{3}{|c|}{ Europium-152 } & \multicolumn{3}{|c|}{ Europium-154 } \\
\hline & & & $\mathrm{pCi} / \mathrm{g}$ & $Q$ & MDA & $\mathrm{pCi} / \mathrm{g}$ & $Q$ & MDA & $\mathrm{pCi} / \mathrm{g}$ & $Q$ & MDA & $\mathrm{pCi} / \mathrm{g}$ & $Q$ & MDA & $\mathrm{pCi} / \mathrm{g}$ & Q & MDA & $\mathrm{pCi} / \mathrm{g}$ & Q & MDA \\
\hline A13 & J00Y79 & $09 / 18 / 03$ & 0.15 & U & 0.15 & -0.915 & $\mathrm{U}$ & 3.4 & 0.423 & & 0.069 & 0.07 & U & 0.07 & 0.15 & $\mathrm{U}$ & 0.15 & 0.2 & $\mathrm{U}$ & 0.2 \\
\hline $\mathrm{A} 14$ & J00Y83 & $09 / 18 / 03$ & 0.12 & $\mathrm{U}$ & 0.12 & -1.02 & $\mathrm{UJ}$ & 2.8 & 0.15 & & 0.058 & 0.057 & $\mathrm{U}$ & 0.057 & 0.13 & $\mathrm{U}$ & 0.13 & 0.15 & $\mathrm{U}$ & 0.15 \\
\hline $\mathrm{A} 15$ & J00Y80 & $09 / 18 / 03$ & 0.083 & $\mathrm{U}$ & 0.083 & -0.912 & $\mathrm{U}$ & 2.7 & 0.123 & & 0.039 & 0.03 & $\mathrm{U}$ & 0.03 & 0.081 & $\mathrm{U}$ & 0.081 & 0.095 & $\mathrm{U}$ & 0.095 \\
\hline $\mathrm{A} 16$ & J00Y84 & $09 / 18 / 03$ & 0.99 & U & 0.99 & 0.365 & UJ & 2.9 & 41 & & 0.23 & 0.427 & & 0.092 & 4.4 & & 0.75 & 0.454 & & 0.33 \\
\hline Duplicate of J00Y84 & J00Y85 & $09 / 18 / 03$ & 0.46 & $\mathrm{U}$ & 0.46 & -0.035 & UJ & 2.7 & 46.8 & & 0.11 & 0.506 & & 0.05 & 5.57 & & 0.42 & 0.588 & & 0.16 \\
\hline
\end{tabular}

\begin{tabular}{|c|c|c|c|c|c|c|c|c|c|c|c|c|c|c|c|c|c|c|c|c|}
\hline \multirow{2}{*}{ Sample Location } & \multirow{2}{*}{\begin{tabular}{|c|} 
Sample \\
Number
\end{tabular}} & \multirow{2}{*}{$\begin{array}{c}\text { Sample } \\
\text { Date }\end{array}$} & \multicolumn{3}{|c|}{ Europium-155 } & \multicolumn{3}{|c|}{ Gross alpha } & \multicolumn{3}{|c|}{ Gross beta } & \multicolumn{3}{|c|}{ Potassium-40 } & \multicolumn{3}{|c|}{ Radium-226 } & \multicolumn{3}{|c|}{ Radium-228 } \\
\hline & & & $\mathrm{pCi} / \mathrm{g}$ & $Q$ & MDA & $\mathrm{pCi} / \mathrm{g}$ & $Q$ & MDA & $\mathrm{pCi} / \mathrm{g}$ & Q & MDA & $\mathrm{pCi} / \mathrm{g}$ & Q & MDA & $\mathrm{pCi} / \mathrm{g}$ & Q & MDA & $\mathrm{pCi} / \mathrm{g}$ & $Q$ & MDA \\
\hline $\mathrm{A} 13$ & J00Y79 & $09 / 18 / 03$ & 0.13 & $\mathrm{U}$ & 0.13 & 10.1 & & 3.3 & 14.1 & & 7.2 & 9.42 & & 0.79 & 0.432 & & 0.12 & 0.583 & & 0.25 \\
\hline $\mathrm{A} 14$ & J00Y83 & $09 / 18 / 03$ & 0.13 & $\mathrm{U}$ & 0.13 & 6.47 & & 2.4 & 16.5 & & 5.7 & 10.7 & & 0.51 & 0.402 & & 0.1 & 0.377 & $\mathrm{~J}$ & 0.27 \\
\hline $\mathrm{A} 15$ & J00Y80 & $09 / 18 / 03$ & 0.1 & $\mathrm{U}$ & 0.1 & 18.7 & & 3.8 & 24 & & 6.3 & 10.1 & & 0.36 & 1.04 & & 0.058 & 1.48 & & 0.12 \\
\hline A16 & J00Y84 & $09 / 18 / 03$ & 0.49 & $\mathrm{U}$ & 0.49 & 7.39 & & 4 & 91.8 & & 6.3 & 7.61 & & 0.78 & 0.43 & $\mathrm{U}$ & 0.43 & 0.611 & $\mathrm{~J}$ & 0.51 \\
\hline Duplicate of J00Y84 & J00Y85 & $09 / 18 / 03$ & 0.27 & $\mathrm{U}$ & 0.27 & 9.71 & & 3.3 & 110 & & 7.2 & 10.1 & & 0.38 & 0.365 & & 0.18 & 0.481 & $\mathrm{~J}$ & 0.26 \\
\hline
\end{tabular}

\begin{tabular}{|c|c|c|c|c|c|c|c|c|c|c|c|c|c|c|c|c|c|c|c|c|}
\hline \multirow{2}{*}{ Sample Location } & \multirow{2}{*}{$\begin{array}{c}\text { Sample } \\
\text { Number }\end{array}$} & \multirow{2}{*}{$\begin{array}{c}\text { Sample } \\
\text { Date }\end{array}$} & \multicolumn{3}{|c|}{ Thorium-228 } & \multicolumn{3}{|c|}{ Thorium-232 } & \multicolumn{3}{|c|}{$\begin{array}{c}\text { Total beta } \\
\text { Radiostrontium }\end{array}$} & \multicolumn{3}{|c|}{ Tritium } & \multicolumn{3}{|c|}{ Uranium-235 } & \multicolumn{3}{|c|}{ Uranium-238 } \\
\hline & & & $\mathrm{pCi} / \mathrm{g}$ & $\mathrm{Q}$ & MDA & $\mathrm{pCi} / \mathrm{g}$ & $Q$ & MDA & $\mathrm{pCi} / \mathrm{g}$ & $Q$ & MDA & $\mathrm{pCi} / \mathrm{g}$ & $Q$ & MDA & $\mathrm{pCi} / \mathrm{g}$ & $Q$ & MDA & $\mathrm{pCi} / \mathrm{g}$ & Q & MDA \\
\hline $\mathrm{A} 13$ & J00Y79 & $09 / 18 / 03$ & 0.407 & & 0.067 & 0.583 & & 0.25 & & & & 11.9 & & 3.1 & 0.21 & $\mathrm{U}$ & 0.21 & 7.4 & $\mathrm{U}$ & 7.4 \\
\hline $\mathrm{A} 14$ & J00Y83 & $09 / 18 / 03$ & 0.473 & & 0.059 & 0.377 & & 0.27 & & & & 0.147 & $\mathrm{U}$ & 0.24 & 0.17 & $\mathrm{U}$ & 0.17 & 6.6 & $\mathrm{U}$ & 6.6 \\
\hline $\mathrm{A} 15$ & J00Y80 & $09 / 18 / 03$ & 1.3 & & 0.039 & 1.48 & & 0.12 & & & & -0.31 & $\mathrm{U}$ & 2.3 & 0.21 & $\mathrm{U}$ & 0.21 & 3.6 & $\mathrm{U}$ & 3.6 \\
\hline $\mathrm{A} 16$ & J00Y84 & $09 / 18 / 03$ & 0.384 & & 0.3 & 0.611 & & 0.51 & 2.47 & & 0.22 & 0.046 & $\mathrm{U}$ & 0.22 & 0.65 & $\mathrm{U}$ & 0.65 & 13 & $\mathrm{U}$ & 13 \\
\hline Duplicate of J00Y84 & J00Y85 & $09 / 18 / 03$ & 0.462 & & 0.15 & 0.481 & & 0.26 & 2.66 & & 0.2 & 0.017 & $\mathrm{U}$ & 0.23 & 0.35 & $\mathrm{U}$ & 0.35 & 8.6 & $\mathrm{U}$ & 8.6 \\
\hline
\end{tabular}


Table A-5. 100-B-14:2 Area 5 Confirmatory Data Results. (6 Pages)

\begin{tabular}{|c|c|c|c|c|c|c|c|c|c|c|c|c|c|c|c|c|c|c|c|c|}
\hline \multirow{2}{*}{ Sample Location } & \multirow{2}{*}{$\begin{array}{c}\text { Sample } \\
\text { Number }\end{array}$} & \multirow{2}{*}{$\begin{array}{c}\text { Sample } \\
\text { Date }\end{array}$} & \multicolumn{3}{|c|}{ Aluminum } & \multicolumn{3}{|c|}{ Antimony } & \multicolumn{3}{|c|}{ Arsenic } & \multicolumn{3}{|c|}{ Barium } & \multicolumn{3}{|c|}{ Beryllium } & \multicolumn{3}{|c|}{ Boron } \\
\hline & & & $\mathrm{mg} / \mathrm{kg}$ & $Q$ & PQL & $\mathrm{mg} / \mathrm{kg}$ & $Q$ & $\mathrm{PQL}$ & $\mathrm{mg} / \mathrm{kg}$ & $Q$ & PQL & $\mathrm{mg} / \mathrm{kg}$ & $Q$ & $\mathrm{PQL}$ & $\mathrm{mg} / \mathrm{kg}$ & $Q$ & PQL & $\mathrm{mg} / \mathrm{kg}$ & Q & $\mathrm{PQL}$ \\
\hline A14 & $300 \mathrm{Y} 83$ & $09 / 18 / 03$ & 8250 & & 4.6 & 0.28 & $\mathrm{~J}$ & 0.28 & 4.1 & & 0.41 & 92.6 & & 0.02 & 0.64 & & 0.04 & 2.9 & & 0.22 \\
\hline A16 & J00Y84 & $09 / 18 / 03$ & 5980 & & 4.6 & 0.28 & $J$ & 0.28 & 3.2 & & 0.41 & 60.3 & & 0.02 & 0.59 & & 0.04 & 1.5 & & 0.21 \\
\hline Duplicate of J00Y84 & J00Y85 & $09 / 18 / 03$ & 6050 & & 4.7 & 0.29 & $\mathrm{~J}$ & 0.29 & 2.9 & & 0.42 & 64.9 & & 0.02 & 0.61 & & 0.04 & 1.7 & & 0.22 \\
\hline Equipment Blank & J00YF5 & $09 / 18 / 03$ & 44.1 & & 4.6 & 0.28 & $\mathrm{U}$ & 0.28 & 0.41 & $\mathrm{U}$ & 0.41 & 0.9 & & 0.02 & 0.04 & $\mathrm{U}$ & 0.04 & 0.23 & & 0.22 \\
\hline
\end{tabular}

\begin{tabular}{|c|c|c|c|c|c|c|c|c|c|c|c|c|c|c|c|c|c|c|c|c|}
\hline \multirow[t]{2}{*}{ Sample Location } & \multirow{2}{*}{$\begin{array}{l}\text { Sample } \\
\text { Number }\end{array}$} & \multirow{2}{*}{$\begin{array}{c}\text { Sample } \\
\text { Date }\end{array}$} & \multicolumn{3}{|c|}{ Cadmium } & \multicolumn{3}{|c|}{ Calcium } & \multicolumn{3}{|c|}{ Chromium } & \multicolumn{3}{|c|}{ Cobalt } & \multicolumn{3}{|c|}{ Copper } & \multicolumn{3}{|c|}{$\begin{array}{c}\text { Hexavalent } \\
\text { Chromium }\end{array}$} \\
\hline & & & $\mathrm{mg} / \mathrm{kg}$ & $Q$ & PQL & $\mathrm{mg} / \mathrm{kg}$ & $\bar{Q}$ & PQL & $\mathrm{mg} / \mathrm{kg}$ & $\bar{Q}$ & $\overline{P Q L}$ & $\mathrm{mg} / \mathrm{kg}$ & Q & PQL & $\mathrm{mg} / \mathrm{kg}$ & $\mathbf{Q}$ & PQL & $\mathrm{mg} / \mathrm{kg}$ & Q & PQL \\
\hline $\mathrm{A} 13$ & J00Y81* & $09 / 18 / 03$ & & & & & & & & & & & & & & & & 2.21 & & 0.35 \\
\hline A14 & J00Y83 & $09 / 18 / 03$ & 0.1 & & 0.04 & 8470 & $\mathrm{~J}$ & 3 & 12 & & 0.1 & 9.9 & & 0.12 & 20 & & 0.12 & 0.8 & $\mathrm{U}$ & 0.8 \\
\hline A15 & J00Y82* & $09 / 18 / 03$ & & & & & & & & & & & & & & & & 0.35 & $\mathrm{U}$ & 0.35 \\
\hline A16 & J00Y84 & $09 / 18 / 03$ & 0.05 & & 0.04 & 5840 & $\mathrm{~J}$ & 3 & 15.8 & & 0.1 & 8.5 & & 0.12 & 18.7 & & 0.12 & 0.41 & $\mathrm{U}$ & 0.41 \\
\hline Duplicate of J00Y84 & J00Y85 & $09 / 18 / 03$ & 0.04 & & 0.04 & 6280 & $\mathrm{~J}$ & 3.1 & 14 & & 0.1 & 7.9 & & 0.12 & 18.1 & & 0.12 & 0.41 & $\mathrm{U}$ & 0.41 \\
\hline Equipment Blank & J00YF5 & $09 / 18 / 03$ & 0.04 & $\mathrm{U}$ & 0.04 & 18.5 & & 3 & 1.9 & & 0.1 & 0.12 & $U$ & 0.12 & 0.28 & & 0.12 & & & \\
\hline
\end{tabular}

*Only analyte was hexavalent chromium.

\begin{tabular}{|c|c|c|c|c|c|c|c|c|c|c|c|c|c|c|c|c|c|c|c|c|}
\hline \multirow{2}{*}{ Sample Location } & \multirow{2}{*}{$\begin{array}{c}\text { Sample } \\
\text { Number }\end{array}$} & \multirow{2}{*}{$\begin{array}{c}\text { Sample } \\
\text { Date }\end{array}$} & \multicolumn{3}{|c|}{ Iron } & \multicolumn{3}{|c|}{ Lead } & \multicolumn{3}{|c|}{ Magnesium } & \multicolumn{3}{|c|}{ Manganese } & \multicolumn{3}{|c|}{ Mercury } & \multicolumn{3}{|c|}{ Molybdenum } \\
\hline & & & $\mathrm{mg} / \mathrm{kg}$ & $\mathrm{Q}$ & PQL & $\mathrm{mg} / \mathrm{kg}$ & $Q$ & $\overline{\mathrm{PQL}}$ & $\mathrm{mg} / \mathrm{kg}$ & $Q$ & PQL & $\mathrm{mg} / \mathrm{kg}$ & $\mathrm{Q}$ & PQL & $\mathrm{mg} / \mathrm{kg}$ & $Q$ & PQL & $\mathrm{mg} / \mathrm{kg}$ & $Q$ & PQL \\
\hline $\mathrm{A} 14$ & J00Y83 & $09 / 18 / 03$ & 24300 & & 1.09 & 7.1 & & 0.19 & 5280 & & 0.68 & 412 & & 0.03 & 0.02 & & 0.02 & 0.19 & U & 0.19 \\
\hline A16 & J00Y84 & $09 / 18 / 03$ & 24500 & & $1.9^{\circ}$ & 5 & & 0.19 & 4310 & & 0.68 & 330 & & 0.03 & 0.1 & & 0.02 & 0.42 & & 0.19 \\
\hline Duplicate of J00Y84 & J00Y85 & $09 / 18 / 03$ & 22700 & & 2 & 4.5 & & 0.2 & 4200 & & 0.7 & 324 & & 0.03 & 0.13 & & 0.02 & 0.25 & & 0.19 \\
\hline Equipment Blank & J00YF5 & $09 / 18 / 03$ & 151 & & 1.9 & 0.27 & & 0.19 & 6.2 & & 0.69 & 2.4 & & 0.03 & 0.01 & $\mathrm{U}$ & 0.01 & 0.19 & $\vec{U}$ & 0.19 \\
\hline
\end{tabular}

\begin{tabular}{|c|c|c|c|c|c|c|c|c|c|c|c|c|c|c|c|c|c|c|c|c|}
\hline \multirow{2}{*}{ Sample Location } & \multirow{2}{*}{$\begin{array}{l}\text { Sample } \\
\text { Number }\end{array}$} & \multirow{2}{*}{$\begin{array}{c}\text { Sample } \\
\text { Date }\end{array}$} & \multicolumn{3}{|c|}{ Nickel } & \multicolumn{3}{|c|}{ Potassium } & \multicolumn{3}{|c|}{ Selenium } & \multicolumn{3}{|c|}{ Silicon } & \multicolumn{3}{|c|}{ Silver } & \multicolumn{3}{|c|}{ Sodium } \\
\hline & & & $\mathrm{mg} / \mathrm{kg}$ & Q & PQL & $\mathrm{mg} / \mathrm{kg}$ & $Q$ & PQL & $\mathrm{mg} / \mathrm{kg}$ & $Q$ & PQL & $\mathrm{mg} / \mathrm{kg}$ & $Q$ & $\mathrm{PQL}$ & $\mathrm{mg} / \mathrm{kg}$ & $Q$ & PQL & $\mathrm{mg} / \mathrm{kg}$ & Q & $\mathrm{PQL}$ \\
\hline $\mathrm{A} 14$ & J00Y83 & $09 / 18 / 03$ & 13.8 & & 0.2 & 2000 & & 2.3 & 0.28 & $\mathrm{U}$ & 0.28 & 336 & & 0.53 & 0.08 & $\vec{U}$ & 0.08 & 466 & & 0.69 \\
\hline $\mathrm{A} 16$ & J00Y84 & $09 / 18 / 03$ & 10.8 & & 0.2 & 970 & & 2.3 & 0.28 & $\mathrm{U}$ & 0.28 & 313 & & 0.53 & 0.08 & $\mathrm{U}$ & 0.08 & 275 & & 0.69 \\
\hline Duplicate of J00Y84 & J00Y85 & $09 / 18 / 03$ & 10.3 & & 0.2 & 1010 & & 2.4 & 0.29 & $\mathrm{U}$ & 0.29 & 331 & & 0.54 & 0.08 & $\mathrm{U}$ & 0.08 & 281 & & 0.71 \\
\hline Equipment Blank & J00YF5 & $09 / 18 / 03$ & 1.1 & & 0.2 & 17.2 & & 2.3 & 0.28 & $\mathrm{U}$ & 0.28 & 39.3 & & 0.53 & 0.08 & $\mathrm{U}$ & 0.08 & 6.6 & & 0.7 \\
\hline
\end{tabular}

\begin{tabular}{|c|c|c|c|c|c|c|c|c|}
\hline \multirow{2}{*}{ Sample Location } & Sample & Sample & \multicolumn{3}{|c|}{ Vanadium } & \multicolumn{3}{|c|}{ Zinc } \\
\cline { 5 - 8 } & Number & Date & $\mathbf{m g} / \mathbf{k g}$ & $\mathbf{Q}$ & PQL & $\mathbf{m g} / \mathbf{k g}$ & $\mathbf{Q}$ & PQL \\
\hline A14 & J00Y83 & $09 / 18 / 03$ & 58 & & 0.09 & 51.2 & & 0.25 \\
\hline A16 & J00Y84 & $09 / 18 / 03$ & 51 & & 0.09 & 50 & & 0.25 \\
\hline Duplicate of J00Y84 & J00Y85 & $09 / 18 / 03$ & 55.5 & & 0.09 & 47.5 & & 0.26 \\
\hline Equipment Blank & J00YF5 & $09 / 18 / 03$ & 0.09 & & 0.09 & 0.53 & & 0.25 \\
\hline
\end{tabular}


Table A-5. 100-B-14:2 Area 5 Confirmatory Data Results. (6 Pages)

\begin{tabular}{|c|c|c|c|c|c|c|c|c|c|c|c|c|}
\hline \multirow[t]{2}{*}{ Constituents } & \multicolumn{3}{|c|}{$\begin{array}{c}\text { J00Y79 } \\
\text { Location A13 } \\
\text { Sample Date } 9 / 18 / 03 \\
\end{array}$} & \multicolumn{3}{|c|}{$\begin{array}{c}\text { J00Y83 } \\
\text { Location A14 } \\
\text { Sample Date 9/18/03 } \\
\end{array}$} & \multicolumn{3}{|c|}{$\begin{array}{c}\text { J00Y80 } \\
\text { Location A15 } \\
\text { Sample Date 9/18/03 }\end{array}$} & \multicolumn{3}{|c|}{$\begin{array}{c}\text { J00Y84 } \\
\text { Location A16 } \\
\text { Sample Date 9/18/03 } \\
\end{array}$} \\
\hline & $\mu \mathrm{g} / \mathrm{kg}$ & $\mathbf{Q}$ & PQL & $\mu \mathrm{g} / \mathrm{kg}$ & $Q$ & PQL & $\mu \mathrm{g} / \mathrm{kg}$ & $Q$ & PQL & $\mu \mathrm{g} / \mathrm{kg}$ & Q & PQL \\
\hline Aroclor-1016 & 270 & $\mathrm{U}$ & 270 & 13 & $\mathrm{U}$ & 13 & 12 & $\mathrm{U}$ & 12 & 14 & $\mathrm{U}$ & 14 \\
\hline Aroclor-1221 & 270 & $\mathrm{U}$ & 270 & 13 & $\mathrm{U}$ & 13 & 12 & $\mathrm{U}$ & 12 & 14 & $\mathrm{U}$ & 14 \\
\hline Aroclor-1232 & 270 & $\mathrm{U}$ & 270 & 13 & $\mathrm{U}$ & 13 & 12 & $\mathrm{U}$ & 12 & 14 & $\mathrm{U}$ & 14 \\
\hline Aroclor-1254 & 1200 & & 270 & 13 & $\mathrm{U}$ & 13 & 12 & $\mathrm{U}$ & 12 & 14 & $\mathrm{U}$ & 14 \\
\hline Aroclor- 1260 & 270 & $\mathrm{U}$ & 270 & 13 & $\mathrm{U}$ & 13 & 12 & $\mathrm{U}$ & 12 & 14 & $\mathrm{U}$ & 14 \\
\hline \multicolumn{13}{|c|}{ Pesticides } \\
\hline Aldrin & 86 & $\mathrm{U}$ & 86 & 17 & UJ & 17 & 17 & $\mathrm{U}$ & 17 & 17 & UJ & 17 \\
\hline alpha-BHC & 86 & $\mathrm{U}$ & 86 & 17 & UJ & 17 & 17 & $\mathrm{U}$ & 17 & 17 & $\mathrm{UJ}$ & 17 \\
\hline alpha-Chlordane & 290 & & 86 & 17 & $\mathrm{UJ}$ & 17 & 17 & $\mathrm{U}$ & 17 & 17 & UJ & 17 \\
\hline Dichlorodiphenyltrichloroethane & 140 & $\mathrm{~J}$ & 170 & 34 & $\mathrm{UJ}$ & 34 & 34 & $\mathrm{U}$ & 34 & 34 & UJ & 34 \\
\hline Dieldrin & 170 & $\mathrm{U}$ & 170 & 34 & $\mathrm{UJ}$ & 34 & 34 & $\mathrm{U}$ & 34 & 34 & UJ & 34 \\
\hline Endosulfan I & 86 & $\mathrm{U}$ & 86 & 17 & UJ & 17 & 17 & $\mathrm{U}$ & 17 & 17 & UJ & 17 \\
\hline Endosulfan II & 170 & $\mathrm{U}$ & 170 & 34 & UJ & 34 & 34 & $\mathrm{U}$ & 34 & 34 & UJ & 34 \\
\hline Endosulfan sulfate & 170 & $\mathrm{U}$ & 170 & 34 & UJ & 34 & 34 & $\mathrm{U}$ & 34 & 34 & UJ & 34 \\
\hline Endrin & 170 & $\mathrm{U}$ & 170 & 34 & UJ & 34 & 34 & $\mathrm{U}$ & 34 & 34 & $\mathrm{UJ}$ & 34 \\
\hline Endrin aldehyde & 170 & $\mathrm{U}$ & 170 & 34 & UJ & 34 & 34 & $\mathrm{U}$ & 34 & 34 & UJ & 34 \\
\hline Endrin ketone & 170 & $\mathrm{U}$ & 170 & 34 & UJ & 34 & 34 & $\mathrm{U}$ & 34 & 34 & UJ & 34 \\
\hline gamma-BHC (Lindane) & 86 & $\mathrm{U}$ & 86 & 17 & UJ & 17 & 17 & $\mathrm{U}$ & 17 & 17 & $\mathrm{UJ}$ & 17 \\
\hline gamma-Chlordane & 480 & & 86 & 17 & UJ & 17 & 17 & $\mathrm{U}$ & 17 & 17 & UJ & 17 \\
\hline Heptachlor & 86 & $\mathrm{U}$ & 86 & 17 & UJ & 17 & 17 & $\mathrm{U}$ & 17 & 17 & UJ & 17 \\
\hline Heptachlor epoxide & 86 & $\mathrm{U}$ & 86 & 17 & $\mathrm{UJ}$ & 17 & 17 & $\mathrm{U}$ & 17 & 17 & UJ & 17 \\
\hline 2,4,6-Trichlorophenol & 680 & $\mathrm{U}$ & 680 & 670 & $\mathrm{U}$ & 670 & 670 & $\mathrm{U}$ & 670 & 690 & $\mathrm{U}$ & 690 \\
\hline 2,4-Dichlorophenol & 680 & $\mathrm{U}$ & 680 & 670 & $\mathrm{U}$ & 670 & 670 & $\mathrm{U}$ & 670 & 690 & $\mathrm{U}$ & 690 \\
\hline 2,4-Dimethylphenol & 680 & $\mathrm{U}$ & 680 & 670 & $\mathrm{U}$ & 670 & 670 & $\mathrm{U}$ & 670 & 690 & $\mathrm{U}$ & 690 \\
\hline 2,4-Dinitrophenol & 1700 & $\mathrm{U}$ & 1700 & 1700 & $\mathrm{U}$ & 1700 & 1700 & $\mathrm{U}$ & 1700 & 1700 & $\mathrm{U}$ & 1700 \\
\hline 2,4-Dinitrotoluene & 680 & $\mathrm{U}$ & 680 & 670 & $\mathrm{U}$ & 670 & 670 & $\mathrm{U}$ & 670 & 690 & $\mathrm{U}$ & 690 \\
\hline 2,6-Dinitrotoluene & 680 & $\mathrm{U}$ & 680 & 670 & $\mathrm{U}$ & 670 & 670 & $\mathrm{U}$ & 670 & 690 & $\mathrm{U}$ & 690 \\
\hline 2-Chloronaphthalene & 680 & $\mathrm{U}$ & 680 & 670 & $\mathrm{U}$ & 670 & 670 & $\mathrm{U}$ & 670 & 690 & $\mathrm{U}$ & 690 \\
\hline 2-Chlorophenol & 680 & $\mathrm{U}$ & 680 & 670 & $\mathrm{U}$ & 670 & 670 & $\mathrm{U}$ & 670 & 690 & $U$ & 690 \\
\hline 2-Methylnaphthalene & 680 & $\mathrm{U}$ & 680 & 670 & $\mathrm{U}$ & 670 & 670 & $\mathrm{U}$ & 670 & 690 & $\mathrm{U}$ & 690 \\
\hline 2-Methylphenol (cresol, o-) & 680 & $\mathrm{U}$ & 680 & 670 & $\mathrm{U}$ & 670 & 670 & $\mathrm{U}$ & 670 & 690 & $\mathrm{U}$ & 690 \\
\hline 2-Nitroaniline & 1700 & $\mathrm{U}$ & 1700 & 1700 & $\mathrm{U}$ & 1700 & 1700 & $\mathrm{U}$ & 1700 & 1700 & $\mathrm{U}$ & 1700 \\
\hline 2-Nitrophenol & 680 & $\mathrm{U}$ & 680 & 670 & $\mathrm{U}$ & 670 & 670 & $\mathrm{U}$ & 670 & 690 & $\mathrm{U}$ & 690 \\
\hline 3,3'-Dichlorobenzidine & 680 & $\mathrm{U}$ & 680 & 670 & $\mathrm{U}$ & 670 & 670 & $\mathrm{U}$ & 670 & 690 & $\mathrm{U}$ & 690 \\
\hline 3-Nitroaniline & 1700 & $\mathrm{U}$ & 1700 & 1700 & $\mathrm{U}$ & 1700 & 1700 & $\mathrm{U}$ & 1700 & 1700 & $\mathrm{U}$ & 1700 \\
\hline 4,6-Dinitro-2-methylphenol & 1700 & $\mathrm{U}$ & 1700 & 1700 & $\mathrm{U}$ & 1700 & 1700 & $\mathrm{U}$ & 1700 & 1700 & $\mathrm{U}$ & 1700 \\
\hline 4-Bromophenylphenyl ether & 680 & $\mathrm{U}$ & 680 & 670 & $\mathrm{U}$ & 670 & 670 & $\mathrm{U}$ & 670 & 690 & $\mathrm{U}$ & 690 \\
\hline 4-Chloro-3-methylphenol & 680 & 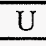 & 680 & 670 & $\mathrm{U}$ & 670 & 670 & $\mathrm{U}$ & 670 & 690 & $\mathrm{U}$ & 690 \\
\hline 4-Chloroaniline & 680 & $\mathrm{U}$ & 680 & 670 & $\mathrm{U}$ & 670 & 670 & $\mathrm{U}$ & 670 & 690 & $\mathrm{U}$ & 690 \\
\hline 4-Chlorophenylphenyl ether & 680 & $\mathrm{U}$ & 680 & 670 & $\mathrm{U}$ & 670 & 670 & $\mathrm{U}$ & 670 & 690 & $\mathrm{U}$ & 690 \\
\hline 4-Methylphenol (cresol, p-) & 680 & $\mathrm{U}$ & 680 & 670 & $\mathrm{U}$ & 670 & 670 & $\mathrm{U}$ & 670 & 690 & $\mathrm{U}$ & 690 \\
\hline 4-Nitroaniline & 1700 & $\mathrm{U}$ & 1700 & 1700 & $\mathrm{U}$ & 1700 & 1700 & $\mathrm{U}$ & 1700 & 1700 & $\mathrm{U}$ & 1700 \\
\hline 4-Nitrophenol & 1700 & $\mathrm{U}$ & 1700 & 1700 & $\mathrm{U}$ & 1700 & 1700 & $\mathrm{U}$ & 1700 & 1700 & $\mathrm{U}$ & 1700 \\
\hline
\end{tabular}


Table A-5. 100-B-14:2 Area 5 Confirmatory Data Results. (6 Pages)

\begin{tabular}{|c|c|c|c|c|c|c|c|c|c|c|c|c|}
\hline \multirow[t]{2}{*}{ Constituents } & \multicolumn{3}{|c|}{$\begin{array}{c}\text { J00Y79 } \\
\text { Location A13 } \\
\text { Sample Date } 9 / 18 / 03\end{array}$} & \multicolumn{3}{|c|}{$\begin{array}{c}\text { J00Y83 } \\
\text { Location A14 } \\
\text { Sample Date } 9 / 18 / 03\end{array}$} & \multicolumn{3}{|c|}{$\begin{array}{c}\text { J00Y80 } \\
\text { Location A15 } \\
\text { Sample Date } 9 / 18 / 03\end{array}$} & \multicolumn{3}{|c|}{$\begin{array}{c}\text { J00Y84 } \\
\text { Location A16 } \\
\text { Sample Date 9/18/03 } \\
\end{array}$} \\
\hline & $\mu \mathrm{g} / \mathrm{kg}$ & $\mathbf{Q}$ & PQL & $\mu \mathrm{g} / \mathrm{kg}$ & $\mathbf{Q}$ & PQL & $\mu \mathrm{g} / \mathrm{kg}$ & $\mathbf{Q}$ & $\mathrm{PQL}$ & $\mu \mathrm{g} / \mathrm{kg}$ & Q & PQL \\
\hline \multicolumn{13}{|c|}{ Semivolatile Organic Compounds (continued) } \\
\hline Acenaphthene & 680 & $\mathrm{U}$ & 680 & 670 & $\mathrm{U}$ & 670 & 670 & $\mathrm{U}$ & 670 & 690 & $\mathrm{U}$ & 690 \\
\hline Acenaphthylene & 680 & $\mathrm{U}$ & 680 & 670 & $\bar{U}$ & 670 & 670 & $\mathrm{U}$ & 670 & 690 & $\mathrm{U}$ & 690 \\
\hline Anthracene & 680 & $\mathrm{U}$ & 680 & 670 & $\mathrm{U}$ & 670 & 670 & $U$ & 670 & 690 & $\mathrm{U}$ & 690 \\
\hline Benzo(a)anthracene & 73 & $\mathrm{~J}$ & 680 & 670 & $\mathrm{U}$ & 670 & 670 & $\mathrm{U}$ & 670 & 110 & $\mathrm{~J}$ & 690 \\
\hline Benzo(a)pyrene & 67 & $\mathrm{~J}$ & 680 & 670 & $\mathrm{U}$ & 670 & 670 & $\mathrm{U}$ & 670 & 81 & $\mathrm{~J}$ & 690 \\
\hline Benzo(b)fluoranthene & 65 & $\mathrm{~J}$ & 680 & 670 & $\mathrm{U}$ & 670 & 670 & $\mathrm{U}$ & 670 & 92 & $\mathrm{~J}$ & 690 \\
\hline Benzo(g,h,i)perylene & 46 & $\mathrm{~J}$ & 680 & 670 & $\mathrm{U}$ & 670 & 670 & $\mathrm{U}$ & 670 & 39 & $\mathrm{~J}$ & 690 \\
\hline Benzo(k)fluoranthene & 63 & $\mathrm{~J}$ & 680 & 670 & $\mathrm{U}$ & 670 & 670 & $\mathrm{U}$ & 670 & 80 & $\mathrm{~J}$ & 690 \\
\hline bis(2-Chloro-1-methylethyl)ether & 680 & $\mathrm{U}$ & 680 & 670 & $\mathrm{U}$ & 670 & 670 & $\mathrm{U}$ & 670 & 690 & $\mathrm{U}$ & 690 \\
\hline bis(2-Chloroethoxy)methane & 680 & $\mathrm{U}$ & 680 & 670 & $\mathrm{U}$ & 670 & 670 & $\mathrm{U}$ & 670 & 690 & $\mathrm{U}$ & 690 \\
\hline bis(2-Chloroethyl) ether & 680 & $\mathrm{U}$ & 680 & 670 & $\mathrm{U}$ & 670 & 670 & $\mathrm{U}$ & 670 & 690 & $\mathrm{U}$ & 690 \\
\hline bis(2-Ethylhexyl) phthalate & 220 & $\mathrm{~J}$ & 680 & 670 & $\mathrm{U}$ & 670 & 58 & $\mathrm{~J}$ & 670 & 690 & $\mathrm{U}$ & 690 \\
\hline Butylbenzylphthalate & 680 & $\mathrm{U}$ & 680 & 670 & $\mathrm{U}$ & 670 & 670 & $\mathrm{U}$ & 670 & 690 & $\mathrm{U}$ & 690 \\
\hline Carbazole & 680 & $\mathrm{U}$ & 680 & 670 & $\mathrm{U}$ & 670 & 670 & $\mathrm{U}$ & 670 & 690 & $\mathrm{U}$ & 690 \\
\hline Chrysene & 100 & $\mathrm{~J}$ & 680 & 670 & $\mathrm{U}$ & 670 & 670 & $\mathrm{U}$ & 670 & 120 & $\mathrm{~J}$ & 690 \\
\hline Di-n-butylphthalate & 510 & $\mathrm{~J}$ & 680 & 670 & $\mathrm{U}$ & 670 & 670 & $\mathrm{U}$ & 670 & 690 & $\mathrm{U}$ & 690 \\
\hline Di-n-octylphthalate & 680 & $\mathrm{U}$ & 680 & 670 & $\mathrm{U}$ & 670 & 670 & $\mathrm{U}$ & 670 & 690 & U & 690 \\
\hline Dibenz $[a, h]$ anthracene & 680 & $\mathrm{U}$ & 680 & 670 & $\mathrm{U}$ & 670 & 670 & $\mathrm{U}$ & 670 & 690 & $\mathrm{U}$ & 690 \\
\hline Dibenzofuran & 680 & $\mathrm{U}$ & 680 & 670 & $\mathrm{U}$ & 670 & 670 & $\mathrm{U}$ & 670 & 690 & $\mathrm{U}$ & 690 \\
\hline Diethylphthalate & 680 & $\mathrm{U}$ & 680 & 670 & $\mathrm{U}$ & 670 & 670 & $\mathrm{U}$ & 670 & 690 & $\mathrm{U}$ & 690 \\
\hline Dimethyl phthalate & 680 & $\mathrm{U}$ & 680 & 670 & $\mathrm{U}$ & 670 & 670 & $\mathrm{U}$ & 670 & 690 & $\mathrm{U}$ & 690 \\
\hline Fluoranthene & 180 & $\mathrm{~J}$ & 680 & 670 & $\mathrm{U}$ & 670 & 670 & $\mathrm{U}$ & 670 & 210 & $\mathrm{~J}$ & 690 \\
\hline Fluorene & 680 & $\mathrm{U}$ & 680 & 670 & $\mathrm{U}$ & 670 & 670 & $\mathrm{U}$ & 670 & 690 & $\mathrm{U}$ & 690 \\
\hline Hexachlorobenzene & 680 & $\mathrm{U}$ & 680 & 670 & $\mathrm{U}$ & 670 & 670 & $\mathrm{U}$ & 670 & 690 & $\mathrm{U}$ & 690 \\
\hline Hexachlorobutadiene & 680 & $\mathrm{U}$ & 680 & 670 & $\mathrm{U}$ & 670 & 670 & $\mathrm{U}$ & 670 & 690 & $\mathrm{U}$ & 690 \\
\hline Hexachlorocyclopentadiene & 680 & $\mathrm{U}$ & 680 & 670 & $\mathrm{U}$ & 670 & 670 & $\mathrm{U}$ & 670 & 690 & $\mathrm{U}$ & 690 \\
\hline Hexachloroethane & 680 & $\mathrm{U}$ & 680 & 670 & $\mathrm{U}$ & 670 & 670 & $\mathrm{U}$ & 670 & 690 & $\mathrm{U}$ & 690 \\
\hline Indeno $(1,2,3$-cd)pyrene & 48 & $\mathrm{~J}$ & 680 & 670 & $\mathrm{U}$ & 670 & 670 & $\mathrm{U}$ & 670 & 35 & $\mathrm{~J}$ & 690 \\
\hline Isophorone & 680 & $\mathrm{U}$ & 680 & 670 & $\mathrm{U}$ & 670 & 670 & $\mathrm{U}$ & 670 & 690 & $\mathrm{U}$ & 690 \\
\hline N-Nitroso-di-n-dipropylamine & 680 & $\mathrm{U}$ & 680 & 670 & $\mathrm{U}$ & 670 & 670 & $\mathrm{U}$ & 670 & 690 & $\mathrm{U}$ & 690 \\
\hline N-Nitrosodiphenylamine & 680 & $\mathrm{U}$ & 680 & 670 & $\mathrm{U}$ & 670 & 670 & $\mathrm{U}$ & 670 & 690 & $\mathrm{U}$ & 690 \\
\hline Naphthalene & 680 & $\mathrm{U}$ & 680 & 670 & $\mathrm{U}$ & 670 & 670 & $\mathrm{U}$ & 670 & 690 & $\mathrm{U}$ & 690 \\
\hline Nitrobenzene & 680 & $\mathrm{U}$ & 680 & 670 & $\mathrm{U}$ & 670 & 670 & $\mathrm{U}$ & 670 & 690 & $\mathrm{U}$ & 690 \\
\hline Pentachlorophenol & 1700 & $\mathrm{U}$ & 1700 & 1700 & $\mathrm{U}$ & 1700 & 1700 & $\mathrm{U}$ & 1700 & 1700 & $\mathrm{U}$ & 1700 \\
\hline Phenanthrene & 94 & $\mathrm{~J}$ & 680 & 670 & $\mathrm{U}$ & 670 & 670 & $\mathrm{U}$ & 670 & 690 & $\mathrm{U}$ & 690 \\
\hline Phenol & 680 & $\mathrm{U}$ & 680 & 670 & $\mathrm{U}$ & 670 & 670 & $\mathrm{U}$ & 670 & 690 & $\mathrm{U}$ & 690 \\
\hline Pyrene & 110 & $\mathrm{~J}$ & 680 & 670 & $\mathrm{U}$ & 670 & 670 & $\mathrm{U}$ & 670 & 150 & $\mathrm{~J}$ & 690 \\
\hline
\end{tabular}


Table A-5. 100-B-14:2 Area 5 Confirmatory Data Results. (6 Pages)

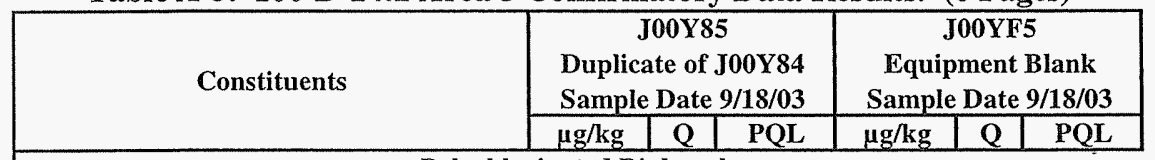

\begin{tabular}{|c|c|c|c|c|c|c|}
\hline \multicolumn{7}{|c|}{ Polychlorinated Biphenyls } \\
\hline Aroclor-1016 & 14 & $\mathrm{U}$ & 14 & 13 & $\mathrm{U}$ & 13 \\
\hline Aroclor-1221 & 14 & $\bar{U}$ & 14 & 13 & $\overline{\mathrm{U}}$ & 13 \\
\hline Aroclor-1232 & 14 & $\mathrm{U}$ & 14 & 13 & $\mathrm{U}$ & 13 \\
\hline Aroclor-1242 & 14 & $\mathrm{U}$ & 14 & 13 & $\bar{U}$ & 13 \\
\hline Aroclor-1248 & 14 & $\mathrm{U}$ & 14 & 13 & $\mathrm{U}$ & 13 \\
\hline Aroclor-1254 & 14 & $\mathrm{U}$ & 14 & 13 & $\mathrm{U}$ & 13 \\
\hline Aroclor -1260 & 16 & & 14 & 13 & $\mathrm{U}$ & 13 \\
\hline \multicolumn{7}{|c|}{ Pesticides } \\
\hline Aldrin & 17 & UJ & 17 & 1.7 & $\mathrm{U}$ & 1.7 \\
\hline alpha-BHC & 17. & $\mathrm{UJ}$ & 17 & 1.7 & $\bar{U}$ & 1.7 \\
\hline alpha-Chlordane & 17 & UJ & 17 & 1.7 & $\mathrm{U}$ & 1.7 \\
\hline beta-BHC & 17 & $\mathrm{UJ}$ & 17 & 1.7 & $\bar{U}$ & 1.7 \\
\hline delta-BHC & 17 & $\mathrm{UJ}$ & 17 & 1.7 & $\mathrm{U}$ & 1.7 \\
\hline Dichlorodiphenyldichloroethane & 34 & $\overline{\mathrm{UJ}}$ & 34 & 3.3 & $\bar{U}$ & 3.3 \\
\hline Dichlorodiphenyldichloroethylene & 34 & $\overline{\mathrm{UJ}}$ & 34 & 3.3 & $\overline{\mathrm{U}}$ & 3.3 \\
\hline Dichlorodiphenyltrichloroethane & 34 & $\overline{\mathrm{UJ}}$ & 34 & 3.3 & $\overline{\mathrm{U}}$ & 3.3 \\
\hline Dieldrin & 34 & $\overline{\mathrm{UJ}}$ & 34 & 3.3 & $\bar{U}$ & 3.3 \\
\hline Endosulfan I & 17 & UJ & 17 & 1.7 & $\mathrm{U}$ & 1.7 \\
\hline Endosulfan II & 34 & UJ & 34 & 3.3 & $\mathrm{U}$ & 3.3 \\
\hline Endosulfan sulfate & 34 & UJ & 34 & 3.3 & $\mathrm{U}$ & 3.3 \\
\hline Endrin & 34 & $\overline{\mathrm{UJ}}$ & 34 & 3.3 & $\overline{\mathrm{U}}$ & 3.3 \\
\hline Endrin aldehyde & 34 & $\overline{\mathrm{UJ}}$ & 34 & 3.3 & $\bar{U}$ & 3.3 \\
\hline Endrin ketone & 34 & UJ & 34 & 3.3 & $\overline{\mathrm{U}}$ & 3.3 \\
\hline gamma-BHC (Lindane) & 17 & UJ & 17 & 1.7 & $\overline{\mathrm{U}}$ & 1.7 \\
\hline gamma-Chlordane & 17 & UJ & 17 & 1.7 & $\bar{U}$ & 1.7 \\
\hline Heptachlor & 17 & UJ & 17 & 1.7 & $\mathrm{U}$ & 1.7 \\
\hline Heptachlor epoxide & 17 & UJ & 17 & 1.7 & $\bar{U}$ & 1.7 \\
\hline Methoxychlor & 170 & UJ & 170 & 17 & $\mathrm{U}$ & 17 \\
\hline Toxaphene & 1700 & UJ & 1700 & 170 & $\mathrm{U}$ & 170 \\
\hline \multicolumn{7}{|c|}{ Herbicides } \\
\hline 2,4,5-Trichlorophenoxyacetic acid & & & & 17 & $\overline{\mathrm{U}}$ & 17 \\
\hline 2,4-Dichlorophenoxyacetic acid & & & & 33 & $\mathrm{U}$ & 33 \\
\hline 2-(2,4,5-Trichlorophenoxy)propionic acid & & & & 17 & $\bar{U}$ & 17 \\
\hline 2-secButyl-4,6-dinitrophenol (DNBP) & & & & 17 & $\bar{U}$ & 17 \\
\hline 4-(2,4-Dichlorophenoxy)butanoic acid & & & & 170 & $\mathrm{U}$ & 170 \\
\hline Dalapon & & & & 170 & $\mathrm{U}$ & 170 \\
\hline Dicamba & & & & 67 & $\mathrm{U}$ & 67 \\
\hline Dichloroprop & & & & 170 & $\mathrm{U}$ & 170 \\
\hline
\end{tabular}

\begin{tabular}{|l|l|l|l|}
\hline & & & \\
\hline \multicolumn{3}{c}{ Semivolatile Organic Compounds }
\end{tabular}

\begin{tabular}{|l|c|c|c|c|c|c|}
\hline 1,2,4-Trichlorobenzene & 690 & $\mathrm{U}$ & 690 & 330 & $\mathrm{U}$ & 330 \\
\hline 1,2-Dichlorobenzene & 690 & $\mathrm{U}$ & 690 & 330 & $\mathrm{U}$ & 330 \\
\hline 1,3-Dichlorobenzene & 690 & $\mathrm{U}$ & 690 & 330 & $\mathrm{U}$ & 330 \\
\hline 1,4-Dichlorobenzene & 690 & $\mathrm{U}$ & 690 & 330 & $\mathrm{U}$ & 330 \\
\hline 2,4,5-Trichlorophenol & 1700 & $\mathrm{U}$ & 1700 & 830 & $\mathrm{U}$ & 830 \\
\hline 2,4,6-Trichlorophenol & 690 & $\mathrm{U}$ & 690 & 330 & $\mathrm{U}$ & 330 \\
\hline 2,4-Dichlorophenol & 690 & $\mathrm{U}$ & 690 & 330 & $\mathrm{U}$ & 330 \\
\hline 2,4-Dimethylphenol & 690 & $\mathrm{U}$ & 690 & 330 & $\mathrm{U}$ & 330 \\
\hline 2,4-Dinitrophenol & 1700 & $\mathrm{U}$ & 1700 & 830 & $\mathrm{U}$ & 830 \\
\hline 2,4-Dinitrotoluene & 690 & $\mathrm{U}$ & 690 & 330 & $\mathrm{U}$ & 330 \\
\hline 2,6-Dinitrotoluene & 690 & $\mathrm{U}$ & 690 & 330 & $\mathrm{U}$ & 330 \\
\hline 2-Chloronaphthalene & 690 & $\mathrm{U}$ & 690 & 330 & $\mathrm{U}$ & 330 \\
\hline 2-Chlorophenol & 690 & $\mathrm{U}$ & 690 & 330 & $\mathrm{U}$ & 330 \\
\hline 2-Methylnaphthalene & 690 & $\mathrm{U}$ & 690 & 330 & $\mathrm{U}$ & 330 \\
\hline 2-Methylphenol (cresol, o-) & 690 & $\mathrm{U}$ & 690 & 330 & $\mathrm{U}$ & 330 \\
\hline 2-Nitroaniline & 1700 & $\mathrm{U}$ & 1700 & 830 & $\mathrm{U}$ & 830 \\
\hline 2-Nitrophenol & 690 & $\mathrm{U}$ & 690 & 330 & $\mathrm{U}$ & 330 \\
\hline 3,3'-Dichlorobenzidine & 690 & $\mathrm{U}$ & 690 & 330 & $\mathrm{U}$ & 330 \\
\hline 3-Nitroaniline & 1700 & $\mathrm{U}$ & 1700 & 830 & $\mathrm{U}$ & 830 \\
\hline 4,6-Dinitro-2-methylphenol & 1700 & $\mathrm{U}$ & 1700 & 830 & $\mathrm{U}$ & 830 \\
\hline
\end{tabular}


Table A-5. 100-B-14:2 Area 5 Confirmatory Data Results. (6 Pages)

\begin{tabular}{|c|c|c|c|c|c|c|}
\hline \multirow[t]{2}{*}{ Constituents } & \multicolumn{3}{|c|}{$\begin{array}{c}\text { J00Y } 85 \\
\text { Duplicate of J00Y84 } \\
\text { Sample Date } 9 / 18 / 03\end{array}$} & \multicolumn{3}{|c|}{$\begin{array}{c}\text { J00YF5 } \\
\text { Equipment Blank } \\
\text { Sample Date 9/18/03 }\end{array}$} \\
\hline & $\mu \mathrm{g} / \mathrm{kg}$ & $\mathrm{Q}$ & PQL & $\mu g / \mathrm{kg}$ & $\mathbf{Q}$ & PQL \\
\hline \multicolumn{7}{|c|}{ Semivolatile Organic Compounds (continued) } \\
\hline 4-Bromophenylphenyl ether & 690 & $\mathrm{U}$ & 690 & 330 & $\mathrm{U}$ & 330 \\
\hline 4-Chloro-3-methylphenol & 690 & $\mathrm{U}$ & 690 & 330 & $\mathrm{U}$ & 330 \\
\hline 4-Chloroaniline & 690 & $\mathrm{U}$ & 690 & 330 & $\mathrm{U}$ & 330 \\
\hline 4-Chlorophenylphenyl ether & 690 & $\mathrm{U}$ & 690 & 330 & $\mathrm{U}$ & 330 \\
\hline 4-Methylphenol (cresol, p-) & 690 & $\mathrm{U}$ & 690 & 330 & $\mathrm{U}$ & 330 \\
\hline 4-Nitroaniline & 1700 & $\mathrm{U}$ & 1700 & 830 & $\mathrm{U}$ & 830 \\
\hline 4-Nitrophenol & 1700 & $\mathrm{U}$ & 1700 & 830 & $\mathrm{U}$ & 830 \\
\hline Acenaphthene & 690 & $\mathrm{U}$ & 690 & 330 & $\mathrm{U}$ & 330 \\
\hline Acenaphthylene & 690 & $\mathrm{U}$ & 690 & 330 & $\mathrm{U}$ & 330 \\
\hline Anthracene & 690 & $\mathrm{U}$ & 690 & 330 & $\mathrm{U}$ & 330 \\
\hline Benzo(a)anthracene & 690 & $\mathrm{U}$ & 690 & 330 & $\mathrm{U}$ & 330 \\
\hline Benzo(a)pyrene & 690 & $\mathrm{U}$ & 690 & 330 & $\mathrm{U}$ & 330 \\
\hline Benzo(b)fluoranthene & 690 & $\mathrm{U}$ & 690 & 330 & $\mathrm{U}$ & 330 \\
\hline Benzo(g,h,i)perylene & 690 & $\mathrm{U}$ & 690 & 330 & $\mathrm{U}$ & 330 \\
\hline Benzo(k)fluoranthene & 690 & $\mathrm{U}$ & 690 & 330 & $\mathrm{U}$ & 330 \\
\hline bis(2-Chloro-1-methylethyl)ether & 690 & $\mathrm{U}$ & 690 & 330 & $\mathrm{U}$ & 330 \\
\hline bis(2-Chloroethoxy)methane & 690 & $\mathrm{U}$ & 690 & 330 & $\mathrm{U}$ & 330 \\
\hline bis(2-Chloroethyl) ether & 690 & $\mathrm{U}$ & 690 & 330 & $\mathrm{U}$ & 330 \\
\hline bis(2-Ethylhexyl) phthalate & 690 & $\mathrm{U}$ & 690 & 330 & $\mathrm{U}$ & 330 \\
\hline Butylbenzylphthalate & 690 & $\mathrm{U}$ & 690 & 330 & $\mathrm{U}$ & 330 \\
\hline Carbazole & 690 & $\mathrm{U}$ & 690 & 330 & $\mathrm{U}$ & 330 \\
\hline Chrysene & 690 & $\mathrm{U}$ & 690 & 330 & $\mathrm{U}$ & 330 \\
\hline Di-n-butylphthalate & 690 & $\mathrm{U}$ & 690 & 29 & $\mathrm{~J}$ & 330 \\
\hline Di-n-octylphthalate & 690 & $\mathrm{U}$ & 690 & 330 & $\mathrm{U}$ & 330 \\
\hline Dibenz $[\mathrm{a}, \mathrm{h}]$ anthracene & 690 & $\mathrm{U}$ & 690 & 330 & $\mathrm{U}$ & 330 \\
\hline Dibenzofuran & 690 & $\mathrm{U}$ & 690 & 330 & $\mathrm{U}$ & 330 \\
\hline Diethylphthalate & 690 & $\mathrm{U}$ & 690 & 330 & $\mathrm{U}$ & 330 \\
\hline Dimethyl phthalate & 690 & $\mathrm{U}$ & 690 & 330 & $\mathrm{U}$ & 330 \\
\hline Fluoranthene & 47 & $\mathrm{~J}$ & 690 & 330 & $\mathrm{U}$ & 330 \\
\hline Fluorene & 690 & $\mathrm{U}$ & 690 & 330 & $\mathrm{U}$ & 330 \\
\hline Hexachlorobenzene & 690 & $\mathrm{U}$ & 690 & 330 & $\mathrm{U}$ & 330 \\
\hline Hexachlorobutadiene & 690 & $\mathrm{U}$ & 690 & 330 & $\mathrm{U}$ & 330 \\
\hline Hexachlorocyclopentadiene & 690 & $\mathrm{U}$ & 690 & 330 & $\mathrm{U}$ & 330 \\
\hline Hexachloroethane & 690 & $\mathrm{U}$ & 690 & 330 & $\mathrm{U}$ & 330 \\
\hline Indeno(1,2,3-cd)pyrene & 690 & $\mathrm{U}$ & 690 & 330 & $\mathrm{U}$ & 330 \\
\hline Isophorone & 690 & $\mathrm{U}$ & 690 & 330 & $\mathrm{U}$ & 330 \\
\hline N-Nitroso-di-n-dipropylamine & 690 & $\mathrm{U}$ & 690 & 330 & $\mathrm{U}$ & 330 \\
\hline N-Nitrosodiphenylamine & 690 & $\mathrm{U}$ & 690 & 330 & $\mathrm{U}$ & 330 \\
\hline Naphthalene & 690 & $\mathrm{U}$ & 690 & 330 & $\mathrm{U}$ & 330 \\
\hline Nitrobenzene & 690 & $\mathrm{U}$ & 690 & 330 & $\mathrm{U}$ & 330 \\
\hline Pentachlorophenol & 1700 & $\mathrm{U}$ & 1700 & 830 & $\mathrm{U}$ & 830 \\
\hline Phenanthrene & 690 & $\mathrm{U}$ & 690 & 330 & $\mathrm{U}$ & 330 \\
\hline Phenol & 690 & $\mathrm{U}$ & 690 & 330 & $\mathrm{U}$ & 330 \\
\hline Pyrene & 35 & $\mathrm{~J}$ & 690 & 330 & $\mathrm{U}$ & 330 \\
\hline
\end{tabular}


Table A-6. 100-B-14:2 Area 5 Additional Characterization Data Results.

\begin{tabular}{|c|c|c|c|c|c|c|c|c|c|c|c|c|c|c|c|c|c|}
\hline \multirow{2}{*}{ Sample Location } & \multirow{2}{*}{$\begin{array}{l}\text { Sample } \\
\text { Number }\end{array}$} & \multirow{2}{*}{$\begin{array}{c}\text { Sample } \\
\text { Date }\end{array}$} & \multicolumn{3}{|c|}{ Arsenic (TCLP) } & \multicolumn{3}{|c|}{ Barium (TCLP) } & \multicolumn{3}{|c|}{ Cadmium (TCLP) } & \multicolumn{3}{|c|}{ Chromium (TCLP) } & \multicolumn{3}{|c|}{ Lead (TCLP) } \\
\hline & & & $\mu \mathrm{g} / \mathrm{L}$ & $Q$ & $\mathrm{PQL}$ & $\mu \mathrm{g} / \mathbb{L}$ & $Q$ & $\overline{\mathrm{PQL}}$ & $\mu \mathrm{g} / \mathrm{L}$ & $\mathrm{Q}$ & $\mathrm{PQL}$ & $\mu \mathrm{g} / \mathrm{L}$ & $Q$ & $\mathrm{PQL}$ & $\mu \mathrm{g} / \mathrm{L}$ & Q & $\mathrm{PQL}$ \\
\hline $\mathrm{A} 13$ & J00Y79 & $09 / 18 / 03$ & 47.4 & $\mathrm{U}$ & 47.4 & 252 & & 2.8 & 37.4 & & 4.7 & 34.7 & & 7.9 & 39.5 & U & 39.5 \\
\hline A15 & J00Y80 & $09 / 18 / 03$ & 47.4 & $\mathrm{U}$ & 47.4 & 346 & & 2.8 & 4.7 & $\mathrm{U}$ & 4.7 & 7.9 & $\mathrm{U}$ & 7.9 & 39.5 & $\mathrm{U}$ & 39.5 \\
\hline
\end{tabular}

\begin{tabular}{|c|c|c|c|c|c|c|c|c|c|c|c|}
\hline \multirow{2}{*}{ Sample Location } & \multirow{2}{*}{$\begin{array}{c}\text { Sample } \\
\text { Number }\end{array}$} & \multirow{2}{*}{$\begin{array}{c}\text { Sample } \\
\text { Date }\end{array}$} & \multicolumn{3}{|c|}{ Mercury (TCLP) } & \multicolumn{3}{|c|}{ Selenium (TCLP) } & \multicolumn{3}{|c|}{ Silver (TCLP) } \\
\hline & & & $\mu \mathrm{g} / \mathrm{L}$ & $Q$ & $\mathrm{PQL}$ & $\mu \mathrm{g} / \mathrm{L}$ & $\bar{Q}$ & $\overline{\mathbf{P Q L}}$ & $\mu \mathrm{g} / \mathrm{L}$ & $Q$ & $\overline{\mathrm{PQL}}$ \\
\hline A13 & J00Y79 & $09 / 18 / 03$ & 0.59 & & 0.1 & 77.6 & $\mathrm{U}$ & 77.6 & 4.3 & $\mathrm{U}$ & 4.3 \\
\hline A15 & $\mathrm{J} 00 \mathrm{Y} 80$ & $09 / 18 / 03$ & 0.1 & $\mathrm{U}$ & 0.1 & 77.6 & $\mathrm{U}$ & 77.6 & 4.3 & $\mathrm{U}$ & 4.3 \\
\hline
\end{tabular}


Table A-7. 100-B-14:2 Waste Characterization and In-Process Analytical Results. (6 Pages)

\begin{tabular}{|c|c|c|c|c|c|c|c|c|c|c|c|c|c|c|c|c|c|}
\hline \multirow{2}{*}{$\begin{array}{c}\text { Sample } \\
\text { Location }\end{array}$} & \multirow{2}{*}{$\begin{array}{l}\text { Sample } \\
\text { Number }\end{array}$} & \multirow{2}{*}{$\begin{array}{l}\text { Sample } \\
\text { Date }\end{array}$} & \multicolumn{3}{|c|}{ Americium-241 } & \multicolumn{3}{|c|}{ Cesium-137 } & \multicolumn{3}{|c|}{ Cobalt-60 } & \multicolumn{3}{|c|}{ Europium-152 } & \multicolumn{3}{|c|}{ Europium-154 } \\
\hline & & & $\mathrm{pCi} / \mathrm{g}$ & \begin{tabular}{l|l} 
\\
$Q$
\end{tabular} & MDA & $\mathrm{pCi} / \mathrm{g}$ & $\bar{Q}$ & MDA & $\mathrm{pCi} / \mathrm{g}$ & $\mathrm{Q}$ & MDA & $\mathrm{pCi} / \mathrm{g}$ & $\mathbf{Q}$ & MDA & $\mathrm{pCi} / \mathrm{g}$ & Q & MDA \\
\hline Suspect BCL & J03JT7 & $6 / 30 / 05$ & 0.22 & U & 0.22 & 0.095 & $\bar{U}$ & 0.095 & 0.089 & $\overrightarrow{\mathrm{U}}$ & 0.089 & 0.22 & $\mathrm{U}$ & 0.22 & 0.26 & $\overrightarrow{\mathrm{U}}$ & 0.26 \\
\hline Suspect BCL & J03JT8 & $6 / 30 / 05$ & 0.29 & $\mathrm{U}$ & 0.29 & 0.083 & $\bar{U}$ & 0.083 & 0.082 & $\bar{U}$ & 0.082 & 0.18 & $\mathrm{U}$ & 0.18 & 0.23 & U & 0.23 \\
\hline Suspect BCL & J03JT9 & $6 / 30 / 05$ & 0.21 & $\overline{\mathrm{U}}$ & 0.21 & 0.059 & $\overline{\mathrm{U}}$ & 0.059 & 0.062 & $\overline{\mathrm{U}}$ & 0.062 & 0.21 & $\overline{\mathrm{U}}$ & 0.21 & 0.19 & $\mathrm{U}$ & 0.19 \\
\hline
\end{tabular}

\begin{tabular}{|c|c|c|c|c|c|c|c|c|c|c|c|c|c|c|c|c|c|}
\hline \multirow{2}{*}{$\begin{array}{c}\text { Sample } \\
\text { Location }\end{array}$} & \multirow{2}{*}{$\begin{array}{c}\text { Sample } \\
\text { Number }\end{array}$} & \multirow{2}{*}{$\begin{array}{c}\text { Sample } \\
\text { Date }\end{array}$} & \multicolumn{3}{|c|}{ Europium-155 } & \multicolumn{3}{|c|}{ Potassium-40 } & \multicolumn{3}{|c|}{ Radium-226 } & \multicolumn{3}{|c|}{ Radium-228 } & \multicolumn{3}{|c|}{ Thorium-228 } \\
\hline & & & $\mathrm{pCi} / \mathrm{g}$ & Q & MDA & $\mathrm{pCi} / \mathrm{g}$ & $Q$ & MDA & $\mathrm{pCi} / \mathrm{g}$ & $\mathbf{Q}$ & MDA & $\mathrm{pCi} / \mathrm{g}$ & Q & MDA & $\mathrm{pCi} / \mathrm{g}$ & Q & MDA \\
\hline Suspect BCL & J03JT7 & $6 / 30 / 05$ & 0.21 & $\mathrm{U}$ & 0.21 & 9.46 & & 1.1 & 0.402 & & 0.16 & 0.611 & & 0.36 & 0.52 & & 0.093 \\
\hline Suspect BCL & J03JT8 & $6 / 30 / 05$ & 0.19 & $\mathrm{U}$ & 0.19 & 11.6 & & 0.49 & 0.453 & & 0.14 & 0.53 & & 0.31 & 0.67 & & 0.13 \\
\hline Suspect BCL & J03JT9 & $6 / 30 / 05$ & 0.18 & $\overline{\mathrm{U}}$ & 0.18 & 4.76 & & 0.64 & 0.208 & & 0.11 & 0.328 & & 0.19 & 0.486 & & 0.091 \\
\hline
\end{tabular}

\begin{tabular}{|c|c|c|c|c|c|c|c|c|c|c|c|}
\hline \multirow{2}{*}{$\begin{array}{c}\text { Sample } \\
\text { Location }\end{array}$} & \multirow{2}{*}{$\begin{array}{c}\text { Sample } \\
\text { Number } \\
\end{array}$} & \multirow{2}{*}{$\begin{array}{c}\text { Sample } \\
\text { Date } \\
\end{array}$} & \multicolumn{3}{|c|}{ Thorium-232 } & \multicolumn{3}{|c|}{ Uranium-235 } & \multicolumn{3}{|c|}{ Uranium-238 } \\
\hline & & & $\mathrm{pCi} / \mathrm{g}$ & $Q$ & MDA & $\mathrm{pCi} / \mathrm{g}$ & $Q$ & MDA & $\mathrm{pCi} / \mathrm{g}$ & $\mathbf{Q}$ & MDA \\
\hline Suspect BCL & J03JT7 & $6 / 30 / 05$ & 0.611 & & 0.36 & 0.29 & $\mathrm{U}$ & 0.29 & 13 & $\mathrm{U}$ & 13 \\
\hline Suspect BCL & J03JT8 & $6 / 30 / 05$ & 0.53 & & 0.31 & 0.26 & $\mathrm{U}$ & 0.26 & 9.3 & $\mathrm{U}$ & 9.3 \\
\hline Suspect BCL & J03JT9 & $6 / 30 / 05$ & 0.328 & & 0.19 & 0.28 & $\bar{U}$ & 0.28 & 7.2 & $\mathrm{U}$ & 7.2 \\
\hline
\end{tabular}


Table A-7. 100-B-14:2 Waste Characterization and In-Process Analytical Results. (6 Pages)

\begin{tabular}{|c|c|c|c|c|c|c|c|c|c|c|c|c|c|c|c|c|c|c|c|c|}
\hline \multirow[b]{2}{*}{ Sample Location } & \multirow{2}{*}{$\begin{array}{l}\text { Sample } \\
\text { Number }\end{array}$} & \multirow{2}{*}{$\begin{array}{c}\text { Sample } \\
\text { Date }\end{array}$} & \multicolumn{3}{|c|}{ Aluminum } & \multicolumn{3}{|c|}{ Antimony } & \multicolumn{3}{|c|}{ Arsenic } & \multicolumn{3}{|c|}{ Barium } & \multicolumn{3}{|c|}{ Beryllium } & \multicolumn{3}{|c|}{ Boron } \\
\hline & & & $\mathrm{mg} / \mathrm{kg}$ & $\mathrm{Q}$ & PQL & $\mathrm{mg} / \mathrm{kg}$ & $\mathrm{Q}$ & PQL & $\mathrm{mg} / \mathrm{kg}$ & Q & PQL & $\mathrm{mg} / \mathrm{kg}$ & Q & PQL & $\mathrm{mg} / \mathrm{kg}$ & Q & $\mathrm{PQL}$ & $\mathrm{mg} / \mathrm{kg}$ & $Q$ & PQL \\
\hline West of B Reactor & J02MB6 & $2 / 23 / 05$ & 6410 & & 1.40 & 0.244 & $\overrightarrow{\mathrm{U}}$ & 0.244 & 6 & & 0.56 & 46.1 & C & 0.03 & 0.709 & & 0.01 & 9.5 & & 0.28 \\
\hline $\begin{array}{c}\text { Northeast of } \\
\text { B Reactor }\end{array}$ & $\mathrm{J} 032 \mathrm{X} 8$ & $4 / 20 / 05$ & 4190 & & 1.46 & 0.76 & & 0.3 & 2.2 & & 0.58 & 61.4 & $\mathrm{C}$ & 0.03 & 0.23 & & 0.01 & 29.4 & $\mathrm{C}$ & 0.29 \\
\hline West of BG trailers & $\mathrm{J} 032 \times 9$ & $4 / 20 / 05$ & 1190 & & 1.10 & 0.24 & $\mathrm{U}$ & 0.24 & 5.1 & & 0.44 & 591 & $\mathrm{C}$ & 0.02 & 0.01 & $\mathrm{U}$ & 0.01 & 18.7 & $\mathrm{C}$ & 0.22 \\
\hline West of B Reactor & J03D44 & $6 / 24 / 05$ & 3850 & & 1.12 & 0.434 & $\mathrm{U}$ & 0.434 & 3.6 & & 0.45 & 32.4 & $\mathrm{C}$ & 0.02 & 0.574 & & 0.01 & 2.4 & & 0.22 \\
\hline $\begin{array}{c}\text { Southern part of } \\
1607-\mathrm{B} 7 \text { stockpile }\end{array}$ & J03JT7 & $6 / 30 / 05$ & 6150 & & 1.01 & 2.3 & $U$ & 2.3 & 4 & & 2.41 & 143 & C & 0.12 & 0.68 & & 0.06 & 4.6 & $\mathrm{C}$ & 1.21 \\
\hline $\begin{array}{c}\text { Northern part of } \\
1607-B 7 \text { stockpile }\end{array}$ & J03JT8 & $6 / 30 / 05$ & 5480 & & 1.00 & 2.3 & $U$ & 2.3 & 4.4 & & 2.40 & 132 & $\mathrm{C}$ & 0.12 & 0.78 & & 0.06 & 3.6 & $\mathrm{C}$ & 1.20 \\
\hline $\begin{array}{c}\text { Small 1607-B7 } \\
\text { stockpile }\end{array}$ & J03JT9 & $6 / 30 / 05$ & 5250 & & 1.00 & 2.2 & $\mathrm{U}$ & 2.2 & 2.9 & & 2.40 & 91.6 & $\mathrm{C}$ & 0.12 & 0.73 & & 0.06 & 2.5 & $\mathrm{C}$ & 1.20 \\
\hline
\end{tabular}

\begin{tabular}{|c|c|c|c|c|c|c|c|c|c|c|c|c|c|c|c|c|c|c|c|}
\hline \multirow{2}{*}{ Sample Location } & \multirow{2}{*}{\begin{tabular}{c|} 
Sample \\
Number
\end{tabular}} & \multirow{2}{*}{$\begin{array}{c}\text { Sample } \\
\text { Date }\end{array}$} & \multicolumn{3}{|c|}{ Cadmium } & \multicolumn{3}{|c|}{ Calcium } & \multicolumn{3}{|c|}{ Chromium } & \multicolumn{2}{|c|}{ Cobalt } & \multicolumn{3}{|c|}{ Copper } & \multicolumn{3}{|c|}{ Hexavalent Chromium } \\
\hline & & & $\mathrm{mg} / \mathrm{kg}$ & Q & $\overline{P Q L}$ & $\mathrm{mg} / \mathrm{kg}$ & $Q$ & PQL & $\mathrm{mg} / \mathrm{kg}$ & $Q$ & PQL & $\mathrm{mg} / \mathrm{kg}$ & \begin{tabular}{l|l}
$\mathbf{Q}$ & $\mathrm{PQL}$ \\
\end{tabular} & $\mathrm{mg} / \mathrm{kg}$ & Q & PQL & $\mathrm{mg} / \mathrm{kg}$ & Q & PQL \\
\hline West of B Reactor & J02MB6 & $2 / 23 / 05$ & 0.151 & & 0.04 & 66200 & $\mathrm{C}$ & 41.90 & 7.7 & C & 0.04 & 7.2 & 0.10 & 30.2 & & 0.07 & & & \\
\hline $\begin{array}{c}\text { Northeast of } \\
\text { B Reactor }\end{array}$ & $\mathrm{J} 032 \mathrm{X} 8$ & $4 / 20 / 05$ & 0.45 & & 0.04 & 269000 & & 21.83 & 713 & C & 0.04 & 4.1 & 0.10 & 99.2 & & 0.07 & & & \\
\hline West of BG trailers & $\mathrm{J} 032 \times 9$ & $4 / 20 / 05$ & 1.7 & & 0.03 & 339000 & & 33.08 & 0.79 & $\mathrm{C}$ & 0.03 & 0.09 & 0.08 & 42.6 & & 0.06 & & & \\
\hline West of B Reactor. & J03D44 & $6 / 24 / 05$ & 0.173 & & 0.03 & 32100 & $\mathrm{C}$ & 2.79 & 5.1 & & 0.03 & 6.7 & 0.08 & 19.4 & & 0.06 & 0.223 & $\mathrm{U}$ & 0.223 \\
\hline $\begin{array}{c}\text { Southern part of } \\
1607-B 7 \text { stockpile }\end{array}$ & J03JT7 & $6 / 30 / 05$ & 0.17 & $\mathrm{U}$ & 0.17 & 8060 & $\mathrm{C}$ & 2.51 & 10.9 & $\mathrm{C}$ & 0.18 & 6.6 & 0.42 & 17.1 & & 0.05 & & & \\
\hline $\begin{array}{c}\text { Northern part of } \\
1607-B 7 \text { stockpile }\end{array}$ & J03JT8 & $6 / 30 / 05$ & 0.22 & & 0.18 & 8470 & $\mathrm{C}$ & 2.51 & 7.4 & C & 0.18 & 8.5 & 0.42 & 15.5 & & 0.05 & & & \\
\hline $\begin{array}{l}\text { Small 1607-B7 } \\
\text { stockpile }\end{array}$ & J03JT9 & $6 / 30 / 05$ & 0.22 & & 0.18 & 5690 & $\mathrm{C}$ & 2.51 & 8.2 & C & 0.18 & 7.3 & 0.42 & 15 & & 0.05 & & & \\
\hline
\end{tabular}

\begin{tabular}{|c|c|c|c|c|c|c|c|c|c|c|c|c|c|c|c|c|c|c|c|c|}
\hline \multirow{2}{*}{ Sample Location } & \multirow{2}{*}{$\begin{array}{c}\text { Sample } \\
\text { Number }\end{array}$} & \multirow{2}{*}{$\begin{array}{l}\text { Sample } \\
\text { Date }\end{array}$} & \multicolumn{3}{|c|}{ Iron } & \multicolumn{3}{|c|}{ Lead } & \multicolumn{3}{|c|}{ Lithium } & \multicolumn{3}{|c|}{ Magnesium } & \multicolumn{3}{|c|}{ Manganese } & \multicolumn{3}{|c|}{ Mercury } \\
\hline & & & $\mathrm{mg} / \mathrm{kg}$ & $Q$ & PQL & $\mathrm{mg} / \mathrm{kg}$ & $Q$ & $\mathrm{PQL}$ & $\mathrm{mg} / \mathrm{kg}$ & Q & $\mathrm{PQL}$ & $\mathrm{mg} / \mathrm{kg}$ & $Q$ & $\mathrm{PQL}$ & $\mathrm{mg} / \mathrm{kg}$ & Q & $\mathrm{PQL}$ & $\mathrm{mg} / \mathrm{kg}$ & $Q$ & $\mathrm{PQL}$ \\
\hline West of B Reactor & $\mathrm{J} 02 \mathrm{MB} 6$ & $2 / 23 / 05$ & 18100 & $\mathrm{C}$ & 1.40 & 5.6 & & 0.28 & 11.2 & $\mathrm{C}$ & 0.01 & 11600 & & 5.59 & 266 & & 0.03 & 0.129 & & 0.02 \\
\hline $\begin{array}{c}\text { Northeast of } \\
\text { B Reactor }\end{array}$ & $\mathrm{J} 032 \times 8$ & $4 / 20 / 05$ & 14600 & $\mathrm{C}$ & 1.46 & 17.8 & & 0.29 & 5.5 & C & 0.01 & 3470 & $\mathrm{C}$ & 5.82 & 226 & & 0.03 & 7.5 & & 0.1 \\
\hline West of BG trailers & $\mathrm{J} 032 \times 9$ & $4 / 20 / 05$ & 184 & $\mathrm{C}$ & 1.10 & 4.3 & & 0.22 & 0.5 & $\mathrm{C}$ & 0.01 & 5710 & $\mathrm{C}$ & 4.41 & 11.2 & & 0.02 & 0.38 & & 0.02 \\
\hline West of B Reactor & J03D44 & $6 / 24 / 05$ & 17800 & & 1.12 & 3.6 & & 0.22 & 5.1 & & 0.01 & 4170 & $\mathrm{C}$ & 4.46 & 241 & & 0.02 & 0.018 & $\mathrm{U}$ & 0.018 \\
\hline $\begin{array}{c}\text { Southern part of } \\
\text { 1607-B7 stockpile }\end{array}$ & J03JT7 & $6 / 30 / 05$ & 17200 & & 6.03 & 7 & & 1.21 & 7.5 & $\mathrm{C}$ & 0.06 & 4280 & & 4.02 & 310 & C & 0.12 & 0.02 & & 0.02 \\
\hline $\begin{array}{c}\text { Northern part of } \\
1607-B 7 \text { stockpile }\end{array}$ & J03JT8 & $6 / 30 / 05$ & 19000 & & 6.01 & 5.9 & & 1.20 & 5.8 & C & 0.06 & 4100 & & 4.01 & 358 & $\mathrm{C}$ & 0.12 & 0.16 & & 0.02 \\
\hline $\begin{array}{l}\text { Small 1607-B7 } \\
\text { stockpile }\end{array}$ & J03JT9 & $6 / 30 / 05$ & 18200 & & 6.01 & 7.3 & & 1.20 & 5.6 & C & 0.06 & 3910 & & 4.01 & 323 & C & 0.12 & 0.02 & & 0.02 \\
\hline
\end{tabular}


Table A-7. 100-B-14:2 Waste Characterization and In-Process Analytical Results. (6 Pages)

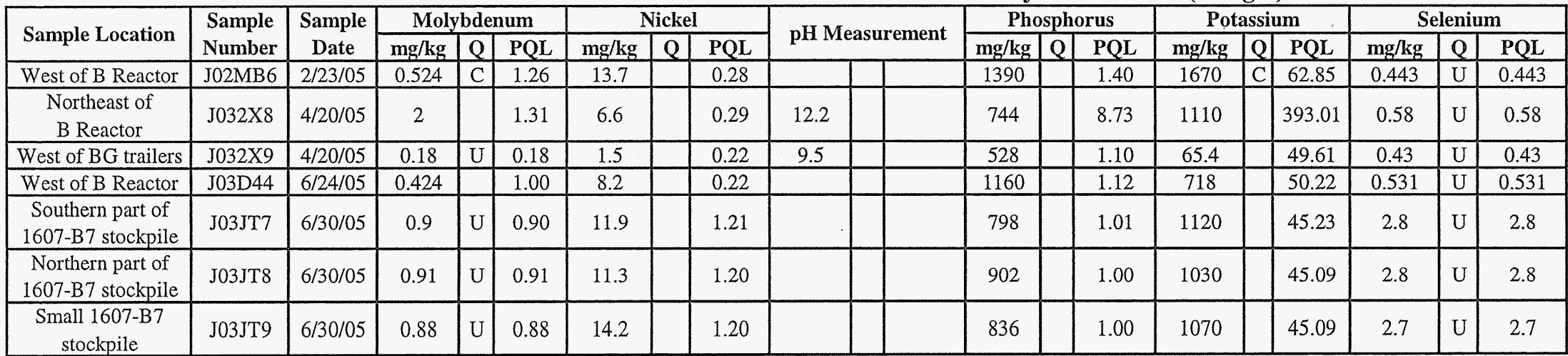

\begin{tabular}{|c|c|c|c|c|c|c|c|c|c|c|c|c|c|c|c|c|c|c|c|c|}
\hline \multirow{2}{*}{ Sample Location } & \multirow{2}{*}{$\begin{array}{l}\text { Sample } \\
\text { Number }\end{array}$} & \multirow{2}{*}{$\begin{array}{c}\text { Sample } \\
\text { Date }\end{array}$} & \multicolumn{3}{|c|}{ Silicon } & \multicolumn{3}{|c|}{ Silver } & \multicolumn{3}{|c|}{ Sodium } & \multicolumn{3}{|c|}{ Strontium } & \multicolumn{3}{|c|}{ Thallium } & \multicolumn{3}{|c|}{ Tin } \\
\hline & & & $\mathrm{mg} / \mathrm{kg}$ & $\mathbf{Q}$ & $\overline{P Q L}$ & $\mathrm{mg} / \mathrm{kg}$ & $Q$ & $\overline{P Q L}$ & $\mathrm{mg} / \mathrm{kg}$ & $Q$ & $\overline{\text { PQL }}$ & $\mathrm{mg} / \mathrm{kg}$ & Q & PQL & $\mathrm{mg} / \mathrm{kg}$ & Q & $\mathrm{PQL}$ & $\mathrm{mg} / \mathrm{kg}$ & $Q$ & $\mathrm{PQL}$ \\
\hline West of B Reactor & $\mathrm{J} 02 \mathrm{MB} 6$ & $2 / 23 / 05$ & 291 & & 0.98 & 0.055 & U & 0.055 & 564 & $\mathrm{C}$ & 2.79 & 226 & C & 0.01 & 0.72 & U & 0.72 & 1 & $\mathrm{C}$ & 0.84 \\
\hline $\begin{array}{l}\text { Northeast of } \\
\text { B Reactor }\end{array}$ & $\mathrm{J} 032 \mathrm{X} 8$ & $4 / 20 / 05$ & 922 & & 1.02 & 0.12 & & 0.05 & 1160 & $\mathrm{C}$ & 17.47 & 74.7 & $\mathrm{C}$ & 0.01 & 0.95 & $\mathrm{U}$ & 0.95 & 2 & $\mathrm{C}$ & 3.00 \\
\hline West of BG trailers & $\mathrm{J} 032 \times 9$ & $4 / 20 / 05$ & 662 & & 0.77 & 0.05 & $\mathrm{U}$ & 0.05 & 211 & $\mathrm{C}$ & 2.21 & 721 & $\mathrm{C}$ & 0.01 & 0.7 & U & 0.7 & 0.93 & $\mathrm{C}$ & 0.66 \\
\hline West of B Reactor & J03D44 & $6 / 24 / 05$ & 333 & & 0.78 & 0.098 & $\mathrm{U}$ & 0.098 & 199 & & 2.23 & 61.7 & & 0.01 & 0.856 & $\mathrm{U}$ & 0.856 & 0.661 & $\mathrm{C}$ & 0.67 \\
\hline $\begin{array}{l}\text { Southern part of } \\
1607-B 7 \text { stockpile }\end{array}$ & J03JT7 & $6 / 30 / 05$ & 414 & C & 4.22 & 0.51 & $\mathrm{U}$ & 0.51 & 186 & $\mathrm{C}$ & 2.01 & 51.3 & C & 0.06 & 4.5 & $\mathrm{U}$ & 4.5 & 3.2 & $\mathrm{UC}$ & 3.20 \\
\hline $\begin{array}{l}\text { Northern part of } \\
1607-B 7 \text { stockpile }\end{array}$ & J03JT8 & $6 / 30 / 05$ & 317 & $\mathrm{C}$ & 4.21 & 0.51 & $U$ & 0.51 & 181 & $\mathrm{C}$ & 2.00 & 54.4 & C & 0.06 & 4.5 & $\mathrm{U}$ & 4.5 & 3.2 & $\mathrm{UC}$ & 3.20 \\
\hline $\begin{array}{c}\text { Small 1607-B7 } \\
\text { stockpile }\end{array}$ & J03JT9 & $6 / 30 / 05$ & 375 & $\mathrm{C}$ & 4.21 & 0.5 & $U$ & 0.5 & 154 & $\mathrm{C}$ & 2.00 & 32.8 & C & 0.06 & 4.4 & $\mathrm{U}$ & 4.4 & 3.1 & $\mathrm{UC}$ & 3.10 \\
\hline
\end{tabular}

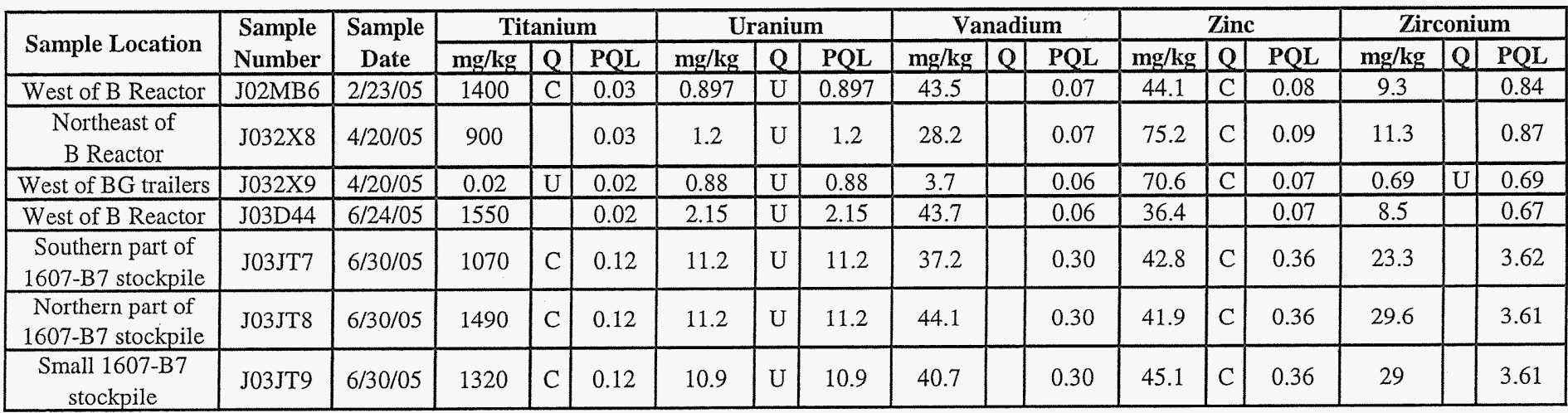

stockpile

J03JT 
Table A-7. 100-B-14:2 Waste Characterization and In-Process Analytical Results. (6 Pages)

\begin{tabular}{|c|c|c|c|c|c|c|c|c|c|c|c|c|}
\hline \multirow[t]{2}{*}{ Constituent } & \multicolumn{3}{|c|}{$\begin{array}{c}\text { J02MB6 } \\
\text { West of B Reactor } \\
\text { Sample Date } 2 / 23 / 05 \\
\end{array}$} & \multicolumn{3}{|c|}{$\begin{array}{c}\text { J032X8 } \\
\text { NE of B Reactor } \\
\text { Sample Date } 4 / 20 / 05\end{array}$} & \multicolumn{3}{|c|}{$\begin{array}{c}\text { J032X9 } \\
\text { West of BG trailers } \\
\text { Sample Date } 4 / 20 / 05\end{array}$} & \multicolumn{3}{|c|}{$\begin{array}{c}\text { J03D44 } \\
\text { West of B Reactor } \\
\text { Sample Date } 6 / 24 / 05\end{array}$} \\
\hline & $\mu \mathrm{g} / \mathrm{kg}$ & $\mathbf{Q}$ & PQL & $\mu \mathrm{g} / \mathrm{kg}$ & $Q$ & PQL & $\mu \mathrm{g} / \mathrm{kg}$ & $\mathbf{Q}$ & PQL & $\mu \mathrm{g} / \mathrm{kg}$ & $Q$ & PQL \\
\hline \multicolumn{13}{|c|}{ Semivolatile Organic Analytes } \\
\hline 1,2,4-Trichlorobenzene & 470 & $\mathrm{U}$ & 470 & 480 & $\mathrm{U}$ & 480 & 370 & $\mathrm{U}$ & 370 & 370 & $\mathrm{U}$ & 370 \\
\hline 1,2-Dichlorobenzene & 470 & $\mathrm{U}$ & 470 & 480 & $\bar{U}$ & 480 & 370 & $\mathrm{U}$ & 370 & 370 & $\mathrm{U}$ & 370 \\
\hline 1,3-Dichlorobenzene & 470 & $\mathrm{U}$ & 470 & 480 & $\mathrm{U}$ & 480 & 370 & $\mathrm{U}$ & 370 & 370 & $\mathrm{U}$ & 370 \\
\hline 1,4-Dichlorobenzene & 470 & $\mathrm{U}$ & 470 & 480 & $\mathrm{U}$ & 480 & 370 & $\mathrm{U}$ & 370 & 370 & $\mathrm{U}$ & 370 \\
\hline 2,4,5-Trichlorophenol & 1200 & $\mathrm{U}$ & 1200 & 1200 & $\mathrm{U}$ & 1200 & 920 & $\mathrm{U}$ & 920 & 930 & $\mathrm{U}$ & 930 \\
\hline 2,4,6-Trichlorophenol & 470 & $\mathrm{U}$ & 470 & 480 & $\mathrm{U}$ & 480 & 370 & $\mathrm{U}$ & 370 & 370 & $\mathrm{U}$ & 370 \\
\hline 2,4-Dichlorophenol & 470 & $\mathrm{U}$ & 470 & 480 & $\mathrm{U}$ & 480 & 370 & $\mathrm{U}$ & 370 & 370 & $\bar{U}$ & 370 \\
\hline 2,4-Dimethylphenol & 470 & $\mathrm{U}$ & 470 & 480 & $\mathrm{U}$ & 480 & 370 & $\mathrm{U}$ & 370 & 370 & $\mathrm{U}$ & 370 \\
\hline 2,4-Dinitrophenol & 1200 & $\mathrm{U}$ & 1200 & 1200 & $\mathrm{U}$ & 1200 & 920 & $\mathrm{U}$ & 920 & 930 & $\mathrm{U}$ & 930 \\
\hline 2,4-Dinitrotoluene & 470 & $\mathrm{U}$ & 470 & 480 & $\mathrm{U}$ & 480 & 370 & $\mathrm{U}$ & 370 & 370 & $\mathrm{U}$ & 370 \\
\hline 2,6-Dinitrotoluene & 470 & $\mathrm{U}$ & 470 & 480 & $\mathrm{U}$ & 480 & 370 & $\mathrm{U}$ & 370 & 370 & $\mathrm{U}$ & 370 \\
\hline 2-Chloronaphthalene & 470 & $\mathrm{U}$ & 470 & 480 & $\mathrm{U}$ & 480 & 370 & $\mathrm{U}$ & 370 & 370 & $\mathrm{U}$ & 370 \\
\hline 2-Chlorophenol & 470 & $\mathrm{U}$ & 470 & 480 & $\mathrm{U}$ & 480 & 370 & $\mathrm{U}$ & 370 & 370 & $\mathrm{U}$ & 370 \\
\hline 2-Methylnaphthalene & 470 & $\mathrm{U}$ & 470 & 480 & $\mathrm{U}$ & 480 & 370 & $\mathrm{U}$ & 370 & 370 & $\mathrm{U}$ & 370 \\
\hline 2-Methylphenol (cresol, o-) & 470 & $\mathrm{U}$ & 470 & 480 & $\mathrm{U}$ & 480 & 370 & $\mathrm{U}$ & 370 & 370 & $\mathrm{U}$ & 370 \\
\hline 2-Nitroaniline & 1200 & $\mathrm{U}$ & 1200 & $1200^{\circ}$ & $\mathrm{U}$ & 1200 & 920 & $\mathrm{U}$ & 920 & 930 & $\mathrm{U}$ & 930 \\
\hline 2-Nitrophenol & 470 & $\mathrm{U}$ & 470 & 480 & $\mathrm{U}$ & 480 & 370 & $\mathrm{U}$ & 370 & 370 & $\mathrm{U}$ & 370 \\
\hline $3+4$ Methylphenol (cresol, $m+p)$ & 470 & $\mathrm{U}$ & 470 & 480 & $\mathrm{U}$ & 480 & 370 & $\mathrm{U}$ & 370 & 370 & $\mathrm{U}$ & 370 \\
\hline 3,3'-Dichlorobenzidine & 470 & $\mathrm{U}$ & 470 & 480 & $\mathrm{U}$ & 480 & 370 & $\mathrm{U}$ & 370 & 370 & $\mathrm{U}$ & 370 \\
\hline 3-Nitroaniline & 1200 & $\mathrm{U}$ & 1200 & 1200 & $\mathrm{U}$ & 1200 & 920 & $\mathrm{U}$ & 920 & 930 & $\mathrm{U}$ & 930 \\
\hline 4,6-Dinitro-2-methylphenol & 1200 & $\mathrm{U}$ & 1200 & 1200 & $\mathrm{U}$ & 1200 & 920 & $\mathrm{U}$ & 920 & 930 & $\mathrm{U}$ & 930 \\
\hline 4-Bromophenylphenyl ether & 470 & $\mathrm{U}$ & 470 & 480 & $\mathrm{U}$ & 480 & 370 & $\mathrm{U}$ & 370 & 370 & $\mathrm{U}$ & 370 \\
\hline 4-Chloro-3-methylphenol & 470 & $\mathrm{U}$ & 470 & 480 & $\mathrm{U}$ & 480 & 370 & $\mathrm{U}$ & 370 & 370 & $\mathrm{U}$ & 370 \\
\hline 4-Chloroaniline & 470 & $\mathrm{U}$ & 470 & 480 & $\mathrm{U}$ & 480 & 370 & $\mathrm{U}$ & 370 & 370 & $\mathrm{U}$ & 370 \\
\hline 4-Chlorophenylphenyl ether & 470 & $\mathrm{U}$ & 470 & 480 & $\mathrm{U}$ & 480 & 370 & $\mathrm{U}$ & 370 & 370 & $\mathrm{U}$ & 370 \\
\hline 4-Nitroaniline & 1200 & $\mathrm{U}$ & 1200 & 1200 & $\mathrm{U}$ & 1200 & 920 & $\mathrm{U}$ & 920 & 930 & $\mathrm{U}$ & 930 \\
\hline 4-Nitrophenol & 1200 & $\mathrm{U}$ & 1200 & 1200 & $\mathrm{U}$ & 1200 & 920 & $\mathrm{U}$ & 920 & 930 & $\mathrm{U}$ & 930 \\
\hline $\begin{array}{l}\text { Acenaphthene } \\
\end{array}$ & 470 & $\mathrm{U}$ & 470 & 480 & $\mathrm{U}$ & 480 & 370 & $\mathrm{U}$ & 370 & 370 & $\mathrm{U}$ & 370 \\
\hline Acenaphthylene & 470 & $\mathrm{U}$ & 470 & 480 & $\mathrm{U}$ & 480 & 370 & $\mathrm{U}$ & 370 & 370 & $\mathrm{U}$ & 370 \\
\hline \begin{tabular}{|l} 
Anthracene \\
\end{tabular} & 470 & $\mathrm{U}$ & 470 & 480 & $\mathrm{U}$ & 480 & 370 & $\mathrm{U}$ & 370 & 370 & $\mathrm{U}$ & 370 \\
\hline Benzo(a)anthracene & 470 & $\mathrm{U}$ & 470 & 480 & $\mathrm{U}$ & 480 & 370 & $\mathrm{U}$ & 370 & 370 & $\mathrm{U}$ & 370 \\
\hline Benzo(a)pyrene & 470 & $\mathrm{U}$ & 470 & 480 & $\mathrm{U}$ & 480 & 370 & $\mathrm{U}$ & 370 & 370 & $\mathrm{U}$ & 370 \\
\hline Benzo(b)fluoranthene & 470 & $\mathrm{U}$ & 470 & 480 & $\mathrm{U}$ & 480 & 370 & $\mathrm{U}$ & 370 & 370 & $\mathrm{U}$ & 370 \\
\hline Benzo(ghi)perylene & 470 & $\mathrm{U}$ & 470 & 480 & $\mathrm{U}$ & 480 & 370 & $\mathrm{U}$ & 370 & 370 & $\mathrm{U}$ & 370 \\
\hline Benzo(k)fluoranthene & 470 & $\mathrm{U}$ & 470 & 480 & $\mathrm{U}$ & 480 & 370 & $\mathrm{U}$ & 370 & 370 & $\mathrm{U}$ & 370 \\
\hline Bis(2-chloro-1-methylethyl)ether & 470 & $\mathrm{U}$ & 470 & 480 & $\mathrm{U}$ & 480 & 370 & $\mathrm{U}$ & 370 & 370 & $\mathrm{U}$ & 370 \\
\hline Bis(2-Chloroethoxy)methane & 470 & $\mathrm{U}$ & 470 & 480 & $\mathrm{U}$ & 480 & 370 & $\mathrm{U}$ & 370 & 370 & $\mathrm{U}$ & 370 \\
\hline Bis(2-chloroethyl) ether & 470 & $\mathrm{U}$ & 470 & 480 & $\mathrm{U}$ & 480 & 370 & $\mathrm{U}$ & 370 & 370 & $\mathrm{U}$ & 370 \\
\hline Bis(2-ethylhexyl) phthalate & 107 & JBD & 470 & 80 & $\mathrm{JB}$ & 480 & 56 & $\mathrm{JB}$ & 370 & 309 & $\mathrm{JBC}$ & 370 \\
\hline Butylbenzylphthalate & 470 & $\mathrm{U}$ & 470 & 480 & $\mathrm{U}$ & 480 & 370 & $\mathrm{U}$ & 370 & 370 & $\mathrm{U}$ & 370 \\
\hline Carbazole & 470 & $\mathrm{U}$ & 470 & 480 & $\mathrm{U}$ & 480 & 370 & $\mathrm{U}$ & 370 & 370 & $\mathrm{U}$ & 370 \\
\hline Chrysene & 470 & $\mathrm{U}$ & 470 & 480 & $\mathrm{U}$ & 480 & 370 & $\mathrm{U}$ & 370 & 370 & $\mathrm{U}$ & 370 \\
\hline Di-n-butylphthalate & 47 & $\mathrm{~J}$ & 470 & 35 & $\mathrm{~J}$ & 480 & 25 & $\mathrm{~J}$ & 370 & 55 & $\mathrm{~J}$ & 370 \\
\hline Di-n-octylphthalate & 470 & $\mathrm{U}$ & 470 & 480 & $\mathrm{U}$ & 480 & 370 & $\mathrm{U}$ & 370 & 370 & $\mathrm{U}$ & 370 \\
\hline Dibenz[a,h]anthracene & 470 & $\mathrm{U}$ & 470 & 480 & $\mathrm{U}$ & 480 & 370 & $\mathrm{U}$ & 370 & 370 & $\mathrm{U}$ & 370 \\
\hline Dibenzofuran & 470 & $\mathrm{U}$ & 470 & 480 & $\mathrm{U}$ & 480 & 370 & $\mathrm{U}$ & 370 & 370 & $U$ & 370 \\
\hline
\end{tabular}


Table A-7. 100-B-14:2 Waste Characterization and In-Process Analytical Results. (6 Pages)

\begin{tabular}{|c|c|c|c|c|c|c|c|c|c|c|c|c|}
\hline \multirow[t]{2}{*}{ Constituent } & \multicolumn{3}{|c|}{$\begin{array}{c}\text { J02MB6 } \\
\text { West of B Reactor } \\
\text { Sample Date } 2 / 23 / 05\end{array}$} & \multicolumn{3}{|c|}{$\begin{array}{c}\text { J032X8 } \\
\text { NE of B Reactor } \\
\text { Sample Date 4/20/05 } \\
\end{array}$} & \multicolumn{3}{|c|}{$\begin{array}{c}\mathrm{J032X9} \\
\text { West of BG trailers } \\
\text { Sample Date } 4 / 20 / 05\end{array}$} & \multicolumn{3}{|c|}{$\begin{array}{c}\text { J03D44 } \\
\text { West of B Reactor } \\
\text { Sample Date } 6 / 24 / 05\end{array}$} \\
\hline & $\mu \mathrm{g} / \mathrm{kg}$ & $\mathbf{Q}$ & PQL & $\mu \mathrm{g} / \mathrm{kg}$ & $Q$ & $\mathrm{PQL}$ & $\mu \mathrm{g} / \mathrm{kg}$ & $Q$ & PQL & $\mu \mathrm{g} / \mathrm{kg}$ & $Q$ & PQL \\
\hline \multicolumn{13}{|c|}{ Semivolatile Organic Analytes (cont.) } \\
\hline Diethylphthalate & 470 & $\mathrm{U}$ & 470 & 480 & $\mathrm{U}$ & 480 & 370 & $\mathrm{U}$ & 370 & 19 & $\mathrm{~J}$ & 370 \\
\hline Dimethyl phthalate & 470 & $\mathrm{U}$ & 470 & 480 & $\mathrm{U}$ & 480 & 370 & $\mathrm{U}$ & 370 & 370 & $\mathrm{U}$ & 370 \\
\hline Fluoranthene & 470 & $\mathrm{U}$ & 470 & 480 & $\mathrm{U}$ & 480 & 370 & $\mathrm{U}$ & 370 & 370 & $\mathrm{U}$ & 370 \\
\hline Fluorene & 470 & $\mathrm{U}$ & 470 & 480 & $\mathrm{U}$ & 480 & 370 & $\mathrm{U}$ & 370 & 370 & $\mathrm{U}$ & 370 \\
\hline Hexachlorobenzene & 470 & $\mathrm{U}$ & 470 & 480 & $\mathrm{U}$ & 480 & 370 & $\mathrm{U}$ & 370 & 370 & $\mathrm{U}$ & 370 \\
\hline Hexachlorobutadiene & 470 & $\mathrm{U}$ & 470 & 480 & $\mathrm{U}$ & 480 & 370 & $\mathrm{U}$ & 370 & 370 & $\mathrm{U}$ & 370 \\
\hline Hexachlorocyclopentadiene & 470 & $\mathrm{U}$ & 470 & 480 & $\mathrm{U}$ & 480 & 370 & $\mathrm{U}$ & 370 & 370 & $\mathrm{U}$ & 370 \\
\hline Hexachloroethane & 470 & $\mathrm{U}$ & 470 & 480 & $\mathrm{U}$ & 480 & 370 & $\mathrm{U}$ & 370 & 370 & $\mathrm{U}$ & 370 \\
\hline Indeno(1,2,3-cd)pyrene & 470 & $\mathrm{U}$ & 470 & 480 & $\mathrm{U}$ & 480 & 370 & $\mathrm{U}$ & 370 & 370 & $\mathrm{U}$ & 370 \\
\hline Isophorone & 470 & $\mathrm{U}$ & 470 & 480 & $\mathrm{U}$ & 480 & 370 & $\mathrm{U}$ & 370 & 370 & $\mathrm{U}$ & 370 \\
\hline $\mathrm{N}$-Nitroso-di-n-dipropylamine & 470 & $\mathrm{U}$ & 470 & 480 & $\mathrm{U}$ & 480 & 370 & $\mathrm{U}$ & 370 & 370 & $\mathrm{U}$ & 370 \\
\hline N-Nitrosodiphenylamine & 470 & $\mathrm{U}$ & 470 & 480 & $\mathrm{U}$ & 480 & 370 & $\mathrm{U}$ & 370 & 370 & $\mathrm{U}$ & 370 \\
\hline Naphthalene & 470 & $\mathrm{U}$ & 470 & 480 & $\mathrm{U}$ & 480 & 370 & $\mathrm{U}$ & 370 & 370 & $\mathrm{U}$ & 370 \\
\hline Nitrobenzene & 470 & $\mathrm{U}$ & 470 & 480 & $\mathrm{U}$ & 480 & 370 & $\mathrm{U}$ & 370 & 370 & $\mathrm{U}$ & 370 \\
\hline Pentachlorophenol & 1200 & $\mathrm{U}$ & 1200 & 1200 & $\mathrm{U}$ & 1200 & 920 & $\mathrm{U}$ & 920 & 930 & $\mathrm{U}$ & 930 \\
\hline Phenanthrene & 470 & $\mathrm{U}$ & 470 & 480 & $\mathrm{U}$ & 480 & 370 & $\mathrm{U}$ & 370 & 370 & $\mathrm{U}$ & 370 \\
\hline Phenol & 470 & $\mathrm{U}$ & 470 & 480 & $\mathrm{U}$ & 480 & 370 & $\mathrm{U}$ & 370 & 29 & $\mathrm{~J}$ & 370 \\
\hline Pyrene & 470 & $\mathrm{U}$ & 470 & 480 & $\mathrm{U}$ & 480 & 370 & $\mathrm{U}$ & 370 & 370 & $\mathrm{U}$ & 370 \\
\hline
\end{tabular}


Table A-7. 100-B-14:2 Waste Characterization and In-Process Analytical Results. (6 Pages)

\begin{tabular}{|c|c|c|c|c|c|c|c|c|c|c|c|c|c|c|c|c|c|}
\hline \multirow{2}{*}{ Sample Location } & \multirow{2}{*}{$\begin{array}{l}\text { Sample } \\
\text { Number }\end{array}$} & \multirow{2}{*}{$\begin{array}{c}\text { Sample } \\
\text { Date }\end{array}$} & \multicolumn{3}{|c|}{ Arsenic (TCLP) } & \multicolumn{3}{|c|}{ Barium (TCLP) } & \multicolumn{3}{|c|}{ Cadmium (TCLP) } & \multicolumn{3}{|c|}{ Chromium (TCLP) } & \multicolumn{3}{|c|}{ Lead (TCLP) } \\
\hline & & & $\mu \mathrm{g} / \mathrm{L}$ & $Q$ & $\mathrm{PQL}$ & $\mu \mathrm{g} / \mathrm{L}$ & $Q$ & $\mathrm{PQL}$ & $\mu \mathrm{g} / \mathrm{L}$ & $Q$ & PQL & $\mu \mathrm{g} / \mathrm{L}$ & $Q$ & $\mathrm{PQL}$ & $\mu \mathrm{g} / \mathrm{L}$ & $Q$ & $\mathrm{PQL}$ \\
\hline West of B Reactor & J02MB6 & $2 / 23 / 05$ & 32.9 & & 20 & 100 & C & 12 & 2.4 & $\mathrm{U}$ & 2.4 & 2.4 & U & 2.4 & 11.4 & $\mathrm{U}$ & 11.4 \\
\hline NE of B Reactor & $\mathrm{J} 032 \times 8$ & $4 / 20 / 05$ & 17.4 & $\mathrm{U}$ & 17.4 & 1310 & & 12 & 2.4 & $\mathrm{U}$ & 2.4 & 2.4 & $\mathrm{U}$ & 2.4 & 11.4 & $\mathrm{U}$ & 11.4 \\
\hline West of BG trailers & J032X9 & $4 / 20 / 05$ & 17.4 & $\mathrm{U}$ & 17.4 & 621 & & 12 & 2.4 & $\mathrm{U}$ & 2.4 & 12300 & & 12300 & 11.4 & $\mathrm{U}$ & 11.4 \\
\hline West of B Reactor & J03D44 & $6 / 24 / 05$ & 34.9 & & 20 & 141 & $\bar{C}$ & 12 & 1.8 & UC & 1.8 & 8.5 & $\mathrm{C}$ & 2.4 & 15 & $\mathrm{U}$ & 15 \\
\hline
\end{tabular}

\begin{tabular}{|c|c|c|c|c|c|c|c|c|c|c|c|}
\hline \multirow{2}{*}{ Sample Location } & \multirow{2}{*}{$\begin{array}{c}\text { Sample } \\
\text { Number }\end{array}$} & \multirow{2}{*}{$\begin{array}{c}\text { Sample } \\
\text { Date }\end{array}$} & \multicolumn{3}{|c|}{ Mercury (TCLP) } & \multicolumn{3}{|c|}{ Selenium (TCLP) } & \multicolumn{3}{|c|}{ Silver (TCLP) } \\
\hline & & & $\mu \mathrm{g} / \mathrm{L}$ & Q & PQL & $\mu \mathrm{g} / \mathrm{L}$ & $Q$ & PQL & $\mu \mathrm{g} / \mathrm{L}$ & $Q$ & PQL \\
\hline West of B Reactor & J02MB6 & $2 / 23 / 05$ & 0.1 & $\mathrm{UC}$ & 0.1 & 24 & U & 24 & 3 & $\mathrm{U}$ & 3 \\
\hline NE of B Reactor & $\mathrm{J} 032 \times 8$ & $4 / 20 / 05$ & 0.1 & $\mathrm{U}$ & 0.1 & 24 & $\bar{U}$ & 24 & 3 & $\mathrm{U}$ & 3 \\
\hline West of BG trailers & $\mathrm{J} 032 \times 9$ & $4 / 20 / 05$ & 0.55 & & 0.1 & 24 & $\mathrm{U}$ & 24 & 3 & $\bar{U}$ & 3 \\
\hline West of B Reactor & J03D44 & $6 / 24 / 05$ & 0.1 & $\bar{U}$ & 0.1 & 29.4 & $\bar{U}$ & 29.4 & 5.4 & $\overline{\mathrm{U}}$ & 5.4 \\
\hline
\end{tabular}

\begin{tabular}{|c|c|c|c|c|c|c|c|c|c|c|c|c|c|c|c|c|c|}
\hline \multirow{2}{*}{ Sample Location } & \multirow{2}{*}{$\begin{array}{c}\text { Sample } \\
\text { Number }\end{array}$} & \multirow{2}{*}{$\begin{array}{c}\text { Sample } \\
\text { Date }\end{array}$} & \multicolumn{3}{|c|}{ 1,1-Dichloroethene* } & \multicolumn{3}{|c|}{ 1,2-Dichloroethane* } & \multicolumn{3}{|c|}{ 2-Butanone* } & \multicolumn{3}{|c|}{ Benzene* } & \multicolumn{3}{|c|}{ Carbon tetrachloride* } \\
\hline & & & $\mathrm{mg} / \mathrm{L}$ & $Q$ & PQL & $\mathrm{mg} / \mathrm{L}$ & Q & $\mathrm{PQL}$ & $\mathrm{mg} / \mathrm{L}$ & $Q$ & PQL & $\mathrm{mg} / \mathrm{L}$ & Q & PQL & $\mathrm{mg} / \mathrm{L}$ & $Q$ & PQL \\
\hline West of B I & J02MB6 & $2 / 23 / 05$ & 0.025 & UD & 0.025 & 0.025 & UD & 0.025 & 0.006 & JD & 0.025 & 0.025 & UD & 0.025 & 0.025 & UD & 0.025 \\
\hline
\end{tabular}

\begin{tabular}{|c|c|c|c|c|c|c|c|c|c|c|c|c|c|c|c|c|c|}
\hline \multirow{2}{*}{ Sample Location } & \multirow{2}{*}{$\begin{array}{l}\text { Sample } \\
\text { Number }\end{array}$} & \multirow{2}{*}{$\begin{array}{c}\text { Sample } \\
\text { Date }\end{array}$} & \multicolumn{3}{|c|}{ Chlorobenzene* } & \multicolumn{3}{|c|}{ Chloroform* } & \multicolumn{3}{|c|}{ Tetrachloroethene ${ }^{*}$} & \multicolumn{3}{|c|}{ Trichloroethene* } & \multicolumn{3}{|c|}{ Vinyl chloride* } \\
\hline & & & $\mathrm{mg} / \mathrm{L}$ & $\mathbf{Q}$ & $\mathrm{PQL}$ & $\mathrm{mg} / \mathrm{L}$ & $Q$ & $\mathrm{PQL}$ & $\mathrm{mg} / \mathrm{L}$ & $Q$ & PQL & $\mathrm{mg} / \mathrm{L}$ & $\mathbf{Q}$ & PQL & $\mathrm{mg} / \mathrm{L}$ & $Q$ & PQL \\
\hline West of B R & J02MB6 & $2 / 23 / 05$ & 0.025 & UD & 0.025 & 0.025 & UD & 0.025 & 0.025 & UD & 0.025 & 0.025 & UD & 0.025 & 0.05 & $\overline{U D}$ & 0.05 \\
\hline
\end{tabular}

\begin{tabular}{|c|c|c|c|c|c|c|c|c|c|c|c|c|c|c|c|c|c|}
\hline \multirow{2}{*}{ Sample Location } & \multirow{2}{*}{$\begin{array}{c}\text { Sample } \\
\text { Number }\end{array}$} & \multirow{2}{*}{$\begin{array}{c}\text { Sample } \\
\text { Date }\end{array}$} & \multicolumn{3}{|c|}{ 1,4-Dichlorobenzene* } & \multicolumn{3}{|c|}{ 2,4,5-Trichlorophenol* } & \multicolumn{3}{|c|}{ 2,4,6-Trichlorophenol* } & \multicolumn{3}{|c|}{ 2,4-Dinitrotoluene* } & \multicolumn{3}{|c|}{ 2-Methylphenol (cresol, o-)* } \\
\hline & & & $\mathrm{mg} / \mathrm{L}$ & $Q$ & PQL & $\mathrm{mg} / \mathrm{L}$ & $Q$ & PQL & $\mathrm{mg} / \mathrm{L}$ & $Q$ & PQL & $\mathrm{mg} / \mathrm{L}$ & $\mathbf{Q}$ & PQL & $\mathrm{mg} / \mathrm{L}$ & $Q$ & PQL \\
\hline NE of B Reactor & $\mathrm{J} 032 \times 8$ & $4 / 20 / 05$ & 0.05 & $\mathrm{U}$ & 0.05 & 0.12 & $\mathrm{U}$ & 0.12 & 0.05 & $\mathrm{U}$ & 0.05 & 0.05 & $\mathrm{U}$ & 0.05 & 0.05 & $\mathrm{U}$ & 0.05 \\
\hline West of BG trailers & $\mathrm{J} 032 \times 9$ & $4 / 20 / 05$ & 0.05 & $\mathrm{U}$ & 0.05 & 0.12 & $\mathrm{U}$ & 0.12 & 0.05 & $\mathrm{U}$ & 0.05 & 0.05 & $\overline{\mathrm{U}}$ & 0.05 & 0.05 & $\mathrm{U}$ & 0.05 \\
\hline West of B Reactor & J03D44 & $6 / 24 / 05$ & 0.05 & $\mathrm{U}$ & 0.05 & 0.12 & $\mathrm{U}$ & 0.12 & 0.05 & $\mathrm{U}$ & 0.05 & 0.05 & $\mathrm{U}$ & 0.05 & 0.05 & $\mathrm{U}$ & 0.05 \\
\hline
\end{tabular}

\begin{tabular}{|c|c|c|c|c|c|c|c|c|c|c|c|c|c|c|c|c|c|}
\hline \multirow{2}{*}{ Sample Location } & \multirow{2}{*}{$\begin{array}{l}\text { Sample } \\
\text { Number }\end{array}$} & \multirow{2}{*}{$\begin{array}{l}\text { Sample } \\
\text { Date }\end{array}$} & \multicolumn{3}{|c|}{$\begin{array}{l}\text { 3+4 Methylphenol } \\
\text { (cresol, } \mathrm{m}+\mathrm{p} \text { )* }\end{array}$} & \multicolumn{3}{|c|}{ Hexachlorobenzene* } & \multicolumn{3}{|c|}{ Hexachlorobutadiene* } & \multicolumn{3}{|c|}{ Hexachloroethane* } & \multicolumn{3}{|c|}{ Nitrobenzene* } \\
\hline & & & $\mathrm{mg} / \mathrm{L}$ & $Q$ & PQL & $\mathrm{mg} / \mathrm{L}$ & $Q$ & $\mathrm{PQL}$ & $\mathrm{mg} / \mathrm{L}$ & $\mathrm{Q}$ & $\mathrm{PQL}$ & $\mathrm{mg} / \mathrm{L}$ & $Q$ & $\mathrm{PQL}$ & $\mathrm{mg} / \mathrm{L}$ & Q & PQL \\
\hline NE of B Reactor & $\mathrm{J} 032 \mathrm{X} 8$ & $4 / 20 / 05$ & 0.05 & $\mathrm{U}$ & 0.05 & 0.05 & $\mathrm{U}$ & 0.05 & 0.05 & $\mathrm{U}$ & 0.05 & 0.05 & $\mathrm{U}$ & 0.05 & 0.05 & $\mathrm{U}$ & 0.05 \\
\hline West of BG trailers & J032X9 & $4 / 20 / 05$ & 0.05 & $\mathrm{U}$ & 0.05 & 0.05 & $U$ & 0.05 & 0.05 & $\bar{U}$ & 0.05 & 0.05 & $\bar{U}$ & 0.05 & 0.05 & $\bar{U}$ & 0.05 \\
\hline West of B Reactor & $\mathrm{J} 03 \mathrm{D} 44$ & $6 / 24 / 05$ & 0.05 & $\mathrm{U}$ & 0.05 & 0.05 & $\mathrm{U}$ & 0.05 & 0.05 & $\mathrm{U}$ & 0.05 & 0.05 & $\mathrm{U}$ & 0.05 & 0.05 & $\mathrm{U}$ & 0.05 \\
\hline
\end{tabular}

\begin{tabular}{|c|c|c|c|c|c|c|c|c|}
\hline \multirow{2}{*}{ Sample Location } & \multirow{2}{*}{$\begin{array}{c}\text { Sample } \\
\text { Number }\end{array}$} & Sample & \multicolumn{3}{|c|}{ Pentachlorophenol* } & \multicolumn{3}{|c|}{ Pyridine* $^{*}$} \\
\cline { 4 - 9 } & Numg/L & $\mathbf{Q}$ & PQL & mg/L & Q & PQL \\
\hline NE of B Reactor & J032X8 & $4 / 20 / 05$ & 0.12 & U & 0.12 & 0.05 & U & 0.05 \\
\hline West of BG trailers & J032X9 & $4 / 20 / 05$ & 0.12 & U & 0.12 & 0.05 & U & 0.05 \\
\hline West of B Reactor & J03D44 & $6 / 24 / 05$ & 0.12 & U & 0.12 & 0.05 & U & 0.05 \\
\hline
\end{tabular}


Table A-8. 1607-B2 Waste Characterization and In-Process Analytical Results. (6 Pages)

\begin{tabular}{|c|c|c|c|c|c|c|c|c|c|c|c|c|c|c|c|c|c|c|c|c|}
\hline \multirow{2}{*}{$\begin{array}{c}\text { Sample } \\
\text { Location }\end{array}$} & \multirow{2}{*}{$\begin{array}{l}\text { Sample } \\
\text { Number }\end{array}$} & \multirow{2}{*}{$\begin{array}{c}\text { Sample } \\
\text { Date }\end{array}$} & \multicolumn{3}{|c|}{ Americium-241 } & \multicolumn{3}{|c|}{ Carbon-14 } & \multicolumn{3}{|c|}{ Cesium-137 } & \multicolumn{3}{|c|}{ Cobalt-60 } & \multicolumn{3}{|c|}{ Europium-152 } & \multicolumn{3}{|c|}{ Europium-154 } \\
\hline & & & $\mathrm{pCi} / \mathrm{g}$ & $\mathrm{Q}$ & MDA & $\mathrm{pCi} / \mathrm{g}$ & $\mathbf{Q}$ & MDA & $\mathrm{pCi} / \mathrm{g}$ & \begin{tabular}{|l|l}
$\mathrm{Q}$ \\
\end{tabular} & MDA & $\mathrm{pCi} / \mathrm{g}$ & QQ & MDA & $\mathrm{pCi} / \mathrm{g}$ & $\mathrm{Q}$ & MDA & $\mathrm{pCi} / \mathrm{g}$ & $\mathrm{Q}$ & MDA \\
\hline $\begin{array}{c}\text { Contaminated } \\
\text { area }\end{array}$ & J02HF9 & $2 / 9 / 05$ & 0.22 & $U$ & 0.22 & -0.67 & $\mathrm{U}$ & 3.1 & 0.097 & U & 0.097 & 0.10 & $\mathrm{U}$ & 0.10 & 0.21 & $\mathrm{U}$ & 0.21 & 0.26 & $U$ & 0.26 \\
\hline Drainfield & $\mathrm{J} 02 \mathrm{~J} 04$ & $2 / 17 / 05$ & 0.38 & $\mathrm{U}$ & 0.38 & -0.42 & U & 3.1 & 0.07 & $\mathrm{U}$ & 0.07 & 0.072 & $\mathrm{U}$ & 0.072 & 0.19 & $\mathrm{U}$ & 0.19 & 0.24 & $\bar{U}$ & 0.24 \\
\hline Drainfield & J02MB8 & $02 / 23 / 05$ & 0.23 & $\mathrm{U}$ & 0.23 & -1.97 & $\mathrm{U}$ & 4.3 & 0.1 & $\mathrm{U}$ & 0.1 & 0.085 & $\mathrm{U}$ & 0.085 & 0.23 & $\mathrm{U}$ & 0.23 & 0.27 & $\mathrm{U}$ & 0.27 \\
\hline Drainfield & J02MF8 & $02 / 28 / 05$ & 0.22 & $\mathrm{U}$ & 0.22 & -1.66 & $\mathrm{U}$ & 2.8 & 0.083 & $\mathrm{U}$ & 0.083 & 0.091 & $\mathrm{U}$ & 0.091 & 0.23 & $\mathrm{U}$ & 0.23 & 0.32 & $\mathrm{U}$ & 0.32 \\
\hline $\begin{array}{c}\text { Pipe to } \\
\text { drainfield }\end{array}$ & J02MJ8 & $3 / 7 / 05$ & 0.30 & $\mathrm{U}$ & 0.30 & -1.28 & $\mathrm{U}$ & 4.8 & 0.12 & $\mathrm{U}$ & 0.12 & 0.14 & $\mathrm{U}$ & 0.14 & 0.26 & $\mathrm{U}$ & 0.26 & 0.32 & U & 0.32 \\
\hline Stockpiles & J03JN6 & $6 / 29 / 05$ & 0.23 & $\mathrm{U}$ & 0.23 & & & & 0.065 & U & 0.065 & 0.068 & U & 0.068 & 0.23 & $\mathrm{U}$ & 0.23 & 0.24 & $\bar{U}$ & 0.24 \\
\hline
\end{tabular}

\begin{tabular}{|c|c|c|c|c|c|c|c|c|c|c|c|c|c|c|c|c|c|}
\hline \multirow{2}{*}{$\begin{array}{c}\text { Sample } \\
\text { Location } \\
\end{array}$} & \multirow{2}{*}{$\begin{array}{c}\text { Sample } \\
\text { Number } \\
\end{array}$} & \multirow{2}{*}{$\begin{array}{c}\text { Sample } \\
\text { Date }\end{array}$} & \multicolumn{3}{|c|}{ Europium-155 } & \multicolumn{3}{|c|}{ Nickel-63 } & \multicolumn{2}{|c|}{$\begin{array}{l}\text { Potassium-40 } \\
\end{array}$} & \multicolumn{2}{|c|}{ Radium-226 } & \multicolumn{2}{|c|}{ Radium-228 } & \multicolumn{3}{|c|}{ Thorium-228 } \\
\hline & & & $\mathrm{pCi} / \mathrm{g}$ & $Q$ & MDA & $\mathrm{pCi} / \mathrm{g}$ & $\bar{Q}$ & MDA & $\mathrm{pCi} / \mathrm{g}$ & \begin{tabular}{l|l|}
$\mathrm{Q}$ & $\mathrm{MDA}$ \\
\end{tabular} & $\mathrm{pCi} / \mathrm{g}$ & \begin{tabular}{l|l|} 
Q & MDA \\
\end{tabular} & $\mathrm{pCi} / \mathrm{g}$ & \begin{tabular}{l|l|}
$\mathrm{Q}$ & $\mathrm{MDA}$ \\
\end{tabular} & $\mathrm{pCi} / \mathrm{g}$ & Q & MDA \\
\hline $\begin{array}{c}\text { Contaminated } \\
\text { area }\end{array}$ & J02HF9 & $2 / 9 / 05$ & 0.20 & $\mathrm{U}$ & 0.20 & 2.24 & $\mathrm{U}$ & 3.3 & 12.5 & 0.63 & 0.406 & 0.18 & 0.647 & 0.39 & 0.597 & & 0.08 \\
\hline Drainfield & J02J04 & $2 / 17 / 05$ & 0.2 & $\mathrm{U}$ & 0.2 & -0.67 & $\mathrm{U}$ & 3.3 & 11.1 & 0.69 & 0.538 & 0.15 & 0.77 & 0.27 & 0.756 & & 0.079 \\
\hline Drainfield & J02MB8 & $02 / 23 / 05$ & 0.21 & $\mathrm{U}$ & 0.21 & -0.79 & $\mathrm{U}$ & 4.1 & 10.8 & 0.72 & 0.514 & 0.16 & 0.615 & 0.4 & 0.692 & & 0.14 \\
\hline Drainfield & J02MF8 & $02 / 28 / 05$ & 0.2 & $\overline{\mathrm{U}}$ & 0.2 & -0.665 & $\mathrm{U}$ & 3.3 & 10.7 & 1 & 0.451 & 0.17 & 0.825 & 0.35 & 0.616 & & 0.14 \\
\hline $\begin{array}{l}\text { Pipe to } \\
\text { drainfield }\end{array}$ & J02MJ8 & $3 / 7 / 05$ & 0.27 & $\mathrm{U}$ & 0.27 & -0.553 & U & 3.3 & 13.2 & 0.20 & 0.674 & 0.16 & 0.767 & 0.30 & 0.716 & & 0.11 \\
\hline Stockpiles & J03JN6 & $6 / 29 / 05$ & 0.21 & $\mathrm{U}$ & 0.21 & & & & 6.26 & 0.51 & 0.169 & 0.14 & 0.42 & 0.42 & 0.446 & & 0.1 \\
\hline
\end{tabular}

\begin{tabular}{|c|c|c|c|c|c|c|c|c|c|c|c|c|c|c|c|c|c|}
\hline \multirow{2}{*}{$\begin{array}{c}\text { Sample } \\
\text { Location } \\
\end{array}$} & \multirow{2}{*}{\begin{tabular}{l|} 
Sample \\
Number \\
\end{tabular}} & \multirow{2}{*}{$\begin{array}{c}\text { Sample } \\
\text { Date }\end{array}$} & \multicolumn{3}{|c|}{ Thorium-232 } & \multicolumn{3}{|c|}{ Total beta Radiostrontium } & \multicolumn{3}{|c|}{ Tritium } & \multicolumn{3}{|c|}{ Uranium-235 } & \multicolumn{3}{|c|}{ Uranium-238 } \\
\hline & & & $\mathrm{pCi} / \mathrm{g}$ & Q & MDA & $\mathrm{pCi} / \mathrm{g}$ & $\mathbf{Q}$ & MDA & $\mathrm{pCi} / \mathrm{g}$ & Q & MDA & $\mathrm{pCi} / \mathrm{g}$ & Q & $\overline{\mathrm{MDA}}$ & $\mathrm{pCi} / \mathrm{g}$ & Q & $\overline{M D A}$ \\
\hline $\begin{array}{l}\text { Contaminated } \\
\text { area }\end{array}$ & J02HF9 & 2/9/05 & 0.647 & & 0.39 & 0.014 & U & 0.27 & 0.082 & $\mathrm{U}$ & 0.40 & 0.30 & $\mathrm{U}$ & 0.30 & 12 & $\mathrm{U}$ & 12 \\
\hline Drainfield & $\mathrm{J} 02 \mathrm{~J} 04$ & $2 / 17 / 05$ & 0.77 & & 0.27 & 0.02 & $\mathrm{U}$ & 027 & 2.16 & $\overline{\mathrm{U}}$ & 3.6 & 0.27 & $\mathrm{U}$ & 0.27 & 8.8 & $\mathrm{U}$ & 8.8 \\
\hline & & & & & & & $\bar{U}$ & & -0.457 & $\bar{U}$ & $\overline{5.3}$ & 0.31 & & 3 & 11 & $\mathrm{U}$ & 11 \\
\hline Drainf & J02MF8 & $02 / 28 / 05$ & 0.825 & & 0.35 & 0.002 & $\bar{U}$ & 0.22 & 2.01 & $\bar{U}$ & 3 & 0.29 & 10 & 0.29 & 10 & $\mathrm{U}$ & 10 \\
\hline $\begin{array}{c}\text { Pipe to } \\
\text { drainfield }\end{array}$ & J02MJ8 & $3 / 7 / 05$ & 0.767 & & 0.30 & -0.077 & U & 0.28 & -0.676 & U & 4.1 & 0.41 & $\mathrm{U}$ & 0.41 & 14 & $\mathrm{U}$ & 14 \\
\hline Stockpiles & J03JN6 & $6 / 29 / 05$ & 0.42 & & 0.42 & & & & & & & 0.3 & $\mathrm{U}$ & 0.3 & 7.6 & U & $\overline{7.6}$ \\
\hline
\end{tabular}


Table A-8. 1607-B2 Waste Characterization and In-Process Analytical Results. (6 Pages)

\begin{tabular}{|c|c|c|c|c|c|c|c|c|c|c|c|c|c|c|c|c|c|c|c|}
\hline \multirow{2}{*}{$\begin{array}{c}\text { Sample } \\
\text { Location }\end{array}$} & \multirow{2}{*}{$\begin{array}{l}\text { Sample } \\
\text { Number }\end{array}$} & \multirow{2}{*}{$\begin{array}{c}\text { Sample } \\
\text { Date }\end{array}$} & \multicolumn{2}{|c|}{ Aluminum } & \multicolumn{3}{|c|}{ Antimony } & \multicolumn{3}{|c|}{ Arsenic } & \multicolumn{3}{|c|}{ Barium } & \multicolumn{3}{|c|}{ Beryllium } & \multicolumn{3}{|c|}{ Boron } \\
\hline & & & $\mathrm{mg} / \mathrm{kg}$ & \begin{tabular}{l|l}
$\mathrm{Q}$ & $\mathrm{PQL}$ \\
\end{tabular} & $\mathrm{mg} / \mathrm{kg}$ & $Q$ & PQL & $\mathrm{mg} / \mathrm{kg}$ & Q & PQL & $\mathrm{mg} / \mathrm{kg}$ & $Q$ & $\mathrm{PQL}$ & $\mathrm{mg} / \mathrm{kg}$ & $Q$ & $\mathrm{PQL}$ & $\mathrm{mg} / \mathrm{kg}$ & $Q$ & PQL \\
\hline $\begin{array}{c}\text { Contaminated } \\
\text { area } \\
\end{array}$ & J02HF8 & $2 / 9 / 05$ & 7430 & 1.0 & 0.40 & & 0.25 & 2.7 & & 0.3 & 73.5 & $\mathrm{C}$ & 0.02 & 0.96 & & 0.01 & 2.1 & & 0.2 \\
\hline Equip. Blank & J02J01 & $2 / 17 / 05$ & 38.4 & 1.0 & 0.17 & $\mathrm{U}$ & 0.17 & & & & 0.8 & $\mathrm{C}$ & 0.0 & 0.01 & & 0.0 & 0.13 & $\mathrm{U}$ & 0.1 \\
\hline Drainfield & $\mathrm{J} 02 \mathrm{J02}$ & $2 / 17 / 05$ & 9800 & 1.2 & 0.24 & & 2.5 & & & & 106 & $\mathrm{C}$ & 0.0 & 0.84 & & 0.0 & 4.5 & & 0.2 \\
\hline Dup. of J02J02 & J02J03 & $2 / 17 / 05$ & 8980 & 1.2 & 0.24 & U & 0.24 & & & & 110 & $\mathrm{C}$ & 0.0 & 0.86 & & 0.0 & 2.6 & & 0.2 \\
\hline Drainfield & $\mathrm{J} 02 \mathrm{~J} 05$ & $2 / 17 / 05$ & & & 0.81 & $\mathrm{~B}$ & 0.1 & 3.8 & & 1 & 107 & $\mathrm{C}$ & 0.0 & 0.39 & $B$ & 0.0 & 11.4 & $\mathrm{BC}$ & 1.0 \\
\hline Drainfield & J02MB7 & $02 / 23 / 05$ & 10500 & 0.99 & 0.229 & $\mathrm{U}$ & 0.23 & 5.5 & & 0.31 & 131 & $\mathrm{C}$ & 0.02 & 1.1 & & 0.01 & 4.1 & & 0.18 \\
\hline Drainfield & J02MF7 & $02 / 28 / 05$ & 9930 & 0.8 & 0.57 & & 0.19 & 5.2 & & 0.25 & 112 & & 0.02 & 1.2 & & 0.009 & 2.4 & $\mathrm{C}$ & 0.15 \\
\hline $\begin{array}{c}\text { Pipe to } \\
\text { drainfield }\end{array}$ & J02MJ7 & $3 / 7 / 05$ & 9180 & 6.0 & 1.2 & $\mathrm{U}$ & 1.5 & 2.4 & & 1.8 & 97.6 & $\mathrm{C}$ & 0.12 & 0.76 & & 0.06 & 3.1 & & 1.2 \\
\hline $\begin{array}{c}\text { Rust-stained } \\
\text { soil }\end{array}$ & J03JNO & $6 / 28 / 05$ & 4940 & 2.3 & 2.3 & U & 2.3 & 2.6 & & 2.6 & 110 & $\mathrm{C}$ & 0.11 & 0.16 & & 0.06 & 1.3 & UC & 1.3 \\
\hline Stockpile & J03JN6 & $6 / 29 / 05$ & 6590 & 1.0 & 2.1 & $\mathrm{U}$ & 2.1 & 2.4 & $\mathrm{U}$ & 2.4 & 82.1 & $\mathrm{C}$ & 0.1 & 0.14 & & 0.1 & 1.2 & $\mathrm{UC}$ & 1.2 \\
\hline
\end{tabular}

\begin{tabular}{|c|c|c|c|c|c|c|c|c|c|c|c|c|c|c|c|c|c|c|c|c|}
\hline \multirow{2}{*}{$\begin{array}{l}\text { Sample } \\
\text { Location }\end{array}$} & \multirow{2}{*}{\begin{tabular}{|c|} 
Sample \\
Number
\end{tabular}} & \multirow{2}{*}{$\begin{array}{c}\text { Sample } \\
\text { Date }\end{array}$} & \multicolumn{3}{|c|}{ Cadmium } & \multicolumn{3}{|c|}{ Calcium } & \multicolumn{3}{|c|}{ Chromium } & \multicolumn{3}{|c|}{ Cobalt } & \multicolumn{3}{|c|}{ Copper } & \multicolumn{3}{|c|}{ Hexavalent Chromium } \\
\hline & & & $\mathrm{mg} / \mathrm{kg}$ & $\mathbf{Q}$ & PQL & $\mathrm{mg} / \mathrm{kg}$ & $Q$ & PQL & $\mathrm{mg} / \mathrm{kg}$ & $Q$ & PQL & $\mathrm{mg} / \mathrm{kg}$ & $Q$ & PQL & $\mathrm{mg} / \mathrm{kg}$ & $Q$ & PQL & $\mathrm{mg} / \mathrm{kg}$ & $\mathbf{Q}$ & PQL \\
\hline $\begin{array}{c}\text { Contaminated } \\
\text { area }\end{array}$ & J02HF8 & $2 / 9 / 05$ & 0.26 & & 0.04 & 4530 & $\mathrm{C}$ & 1.0 & 11.2 & C & 0.05 & 6.9 & & 0.08 & 15.8 & $\mathrm{C}$ & 0.05 & 0.23 & $\mathrm{U}$ & 0.23 \\
\hline Equip. Blank & J02J01 & $2 / 17 / 05$ & 0.03 & $\bar{U}$ & 0.0 & 17.1 & C & 2.5 & 0.03 & $\mathrm{U}$ & 0.0 & 0.06 & $\mathrm{U}$ & 0.1 & 0.04 & $\mathrm{U}$ & 0.0 & 0.2 & $\mathrm{U}$ & 0.2 \\
\hline Drainfield & $\mathrm{J} 02 \mathrm{~J} 02$ & $2 / 17 / 05$ & 0.07 & & 0.0 & 4960 & $\mathrm{C}$ & 2.9 & 15.5 & & 0.0 & 9.1 & & 0.1 & 20.8 & & 0.1 & 0.23 & $\mathrm{U}$ & 0.23 \\
\hline Dup. of J02J02 & J02J03 & $2 / 17 / 05$ & 0.04 & $\mathrm{U}$ & 0.0 & 5400 & $\mathrm{C}$ & 2.9 & 14.4 & & 0.0 & 9 & & 0.1 & 19.1 & & 0.1 & 0.32 & & 0.25 \\
\hline Drainfield & J02J05 & $2 / 17 / 05$ & 0.025 & $\overline{\mathrm{U}}$ & 0.0 & 5450 & $\mathrm{C}$ & 2.8 & 13 & & 0.0 & 12.2 & & 0.1 & 18.8 & & 0.1 & 0.35 & $\mathrm{U}$ & 0.35 \\
\hline Drainfield & J02MB7 & $02 / 23 / 05$ & 0.045 & & 0.04 & 5910 & $\mathrm{C}$ & 0.83 & 15.3 & C & 0.04 & 9.6 & & 0.07 & 24.5 & & 0.05 & 0.782 & & 0.22 \\
\hline Drainfield & J02MF7 & $02 / 28 / 05$ & 0.11 & & 0.03 & 4690 & $\mathrm{C}$ & 0.67 & 15.2 & C & 0.03 & 8.8 & & 0.06 & 22.7 & C & 0.04 & 0.43 & & 0.22 \\
\hline $\begin{array}{c}\text { Pipe to } \\
\text { drainfield }\end{array}$ & $\mathrm{J} 02 \mathrm{MJ} 7$ & $3 / 7 / 05$ & 0.22 & $\mathrm{U}$ & 0.24 & 5810 & C & 6.0 & 17.7 & C & 0.30 & 8.8 & & 0.32 & 19.8 & C & 0.30 & 0.22 & $\mathrm{U}$ & 0.22 \\
\hline $\begin{array}{c}\text { Rust-stained } \\
\text { soil }\end{array}$ & J03JN0 & $6 / 28 / 05$ & 1.7 & & 0.17 & 4270 & & 1.8 & 14.5 & C & 0.40 & 10.0 & & 0.52 & 22.6 & & 0.28 & & & \\
\hline Stockpile & J03JN6 & $6 / 29 / 05$ & 1.4 & & 0.2 & 5250 & & 2.5 & 9.9 & $\mathrm{C}$ & 0.2 & 8.5 & & 0.4 & 16.3 & & 0.1 & & & \\
\hline
\end{tabular}


Table A-8. 1607-B2 Waste Characterization and In-Process Analytical Results. (6 Pages)

\begin{tabular}{|c|c|c|c|c|c|c|c|c|c|c|c|c|c|c|c|c|c|c|c|c|}
\hline \multirow{2}{*}{$\begin{array}{c}\text { Sample } \\
\text { Location }\end{array}$} & \multirow{2}{*}{$\begin{array}{c}\text { Sample } \\
\text { Number } \\
\end{array}$} & \multirow{2}{*}{$\begin{array}{c}\text { Sample } \\
\text { Date }\end{array}$} & \multicolumn{3}{|c|}{ Iron } & \multicolumn{3}{|c|}{ Lead } & \multicolumn{3}{|c|}{ Lithium } & \multicolumn{3}{|c|}{ Magnesium } & \multicolumn{3}{|c|}{ Manganese } & \multicolumn{3}{|c|}{ Mercury } \\
\hline & & & $\mathrm{mg} / \mathrm{kg}$ & $Q$ & PQL & $\mathrm{mg} / \mathrm{kg}$ & $Q$ & PQL & $\mathrm{mg} / \mathrm{kg}$ & Q & $\mathrm{PQL}$ & $\mathrm{mg} / \mathrm{kg}$ & Q & PQL & $\mathrm{mg} / \mathrm{kg}$ & $Q$ & $\mathrm{PQL}$ & $\mathrm{mg} / \mathrm{kg}$ & $Q$ & $\mathrm{PQL}$ \\
\hline $\begin{array}{c}\text { Contaminated } \\
\text { area } \\
\end{array}$ & J02HF8 & $2 / 9 / 05$ & 21100 & C & 1.0 & 4.6 & & 0.2 & 7.6 & $\mathrm{C}$ & 0.01 & 4210 & $\mathrm{C}$ & 0.6 & 290 & & 0.02 & 0.06 & & 0.02 \\
\hline Equip. Blank & J02J01 & $2 / 17 / 05$ & 80.4 & & 1.0 & & & & 0.04 & $\mathrm{C}$ & 0.0 & 6.2 & $\mathrm{C}$ & 4.0 & 1.8 & & 0.0 & 0.01 & U & 0.01 \\
\hline Drainfield & $\mathrm{J} 02 \mathrm{~J} 02$ & $2 / 17 / 05$ & 25800 & & 1.2 & & & & 10.2 & $\mathrm{C}$ & 0.0 & 5340 & $\mathrm{C}$ & 4.6 & 421 & & 0.0 & 0.02 & $\mathrm{U}$ & 0.02 \\
\hline Dup. of J02J02 & $\mathrm{J} 02 \mathrm{~J} 03$ & $2 / 17 / 05$ & 24800 & & 1.2 & & & & 9.5 & $\mathrm{C}$ & 0.0 & 5190 & $\mathrm{C}$ & 4.6 & 402 & & 0.0 & 0.02 & U & 0.02 \\
\hline Drainfield & $\mathrm{J} 02 \mathrm{~J} 05$ & $2 / 17 / 05$ & 23500 & $\mathrm{C}$ & 1.1 & 5 & & 0.2 & 9.6 & $\mathrm{C}$ & 0.0 & 4470 & $\mathrm{C}$ & 4.5 & 320 & & 0.0 & 0.091 & $\mathrm{C}$ & 0.091 \\
\hline Drainfield & J02MB7 & $02 / 23 / 05$ & 28700 & C & 0.89 & 7.4 & & 0.20 & 12.4 & C & 0.01 & 6000 & & 0.60 & 432 & & 0.02 & 0.071 & & 0.02 \\
\hline Drainfield & J02MF7 & $02 / 28 / 05$ & 24500 & $\mathrm{C}$ & 0.72 & 6.9 & & 0.16 & 12 & & 0.009 & 5430 & & 0.49 & 368 & $\mathrm{C}$ & 0.02 & 0.04 & & 0.02 \\
\hline $\begin{array}{c}\text { Pipe to } \\
\text { drainfield }\end{array}$ & $\mathrm{J} 02 \mathrm{MJ} 7$ & $3 / 7 / 05$ & 23100 & C & 6.0 & 7.2 & & 1.2 & 10.6 & $\mathrm{C}$ & 0.06 & 5470 & C & 3.2 & 422 & & 0.12 & 0.02 & U & 0.02 \\
\hline $\begin{array}{l}\text { Rust-stained } \\
\text { soil }\end{array}$ & J03JN0 & $6 / 28 / 05$ & 23200 & & 16.8 & 7.7 & & 1.4 & 5.2 & C & 0.11 & 3810 & & 4.1 & 258 & & 0.11 & 0.43 & & 0.02 \\
\hline Stockpile & J03JN6 & $6 / 29 / 05$ & 21200 & & 6.0 & 5 & & 1.2 & 6 & $\mathrm{C}$ & 0.1 & 4350 & & 4.0 & 350 & & 0.1 & 0.01 & $\mathrm{U}$ & 0.01 \\
\hline
\end{tabular}

\begin{tabular}{|c|c|c|c|c|c|c|c|c|c|c|c|c|c|c|c|c|c|c|c|c|}
\hline \multirow{2}{*}{$\begin{array}{c}\text { Sample } \\
\text { Location }\end{array}$} & \multirow{2}{*}{$\begin{array}{l}\text { Sample } \\
\text { Number }\end{array}$} & \multirow{2}{*}{$\begin{array}{l}\text { Sample } \\
\text { Date }\end{array}$} & \multicolumn{3}{|c|}{ Molybdenum } & \multicolumn{3}{|c|}{ Nickel } & \multicolumn{3}{|c|}{ Phosphorus } & \multicolumn{3}{|c|}{ Potassium } & \multicolumn{3}{|c|}{ Selenium } & \multicolumn{3}{|c|}{ Silicon } \\
\hline & & & $\mathrm{mg} / \mathrm{kg}$ & Q & PQL & $\mathrm{mg} / \mathrm{kg}$ & $\mathrm{Q}$ & PQL & $\mathrm{mg} / \mathrm{kg}$ & Q & $\mathrm{PQL}$ & $\mathrm{mg} / \mathrm{kg}$ & $Q$ & PQL & $\mathrm{mg} / \mathrm{kg}$ & $Q$ & $\mathbf{P Q L}$ & $\mathrm{mg} / \mathrm{kg}$ & $Q$ & PQL \\
\hline $\begin{array}{c}\text { Contaminated } \\
\text { area }\end{array}$ & J02HF8 & $2 / 9 / 05$ & 0.47 & C & 0.2 & 10.8 & & 0.12 & 869 & & 1.5 & 1550 & & 1.0 & 0.48 & & 0.5 & 300 & $\mathrm{C}$ & 0.6 \\
\hline Equip. Blank & $\mathrm{J} 02 \mathrm{~J} 01$ & $2 / 17 / 05$ & 0.13 & $\mathrm{U}$ & 0.1 & 0.08 & $\mathrm{U}$ & 0.1 & 3.6 & & 1.0 & 60.9 & & 45.0 & & & & 31.5 & & 0.7 \\
\hline Drainfield & $\mathrm{J} 02 \mathrm{~J} 02$ & $2 / 17 / 05$ & 0.56 & & 1.0 & 15 & & 0.2 & 958.0 & & 1.2 & 2070 & & 52.3 & & & & 278 & & 0.8 \\
\hline Dup. of J02J02 & J02J03 & $2 / 17 / 05$ & 0.53 & & 1.0 & 14.6 & & 0.2 & 875.0 & & 1.2 & 1940 & & 51.8 & & & & 226 & & 0.8 \\
\hline Drainfield & $\mathrm{J} 02 \mathrm{~J} 05$ & $2 / 17 / 05$ & 0.8 & $\mathrm{U}$ & 0.8 & 11.9 & & 0.2 & 1150.0 & C & 1.1 & 1850 & & 50.6 & 0.34 & $\mathrm{U}$ & 0.34 & 1340 & $\mathrm{C}$ & 0.8 \\
\hline Drainfield & J02MB7 & $02 / 23 / 05$ & 0.807 & C & 0.18 & 15.7 & & 0.11 & 1050 & & 1.07 & 1900 & $\mathrm{C}$ & 0.89 & 0.417 & $\mathrm{U}$ & 0.42 & 273 & & 0.60 \\
\hline Drainfield & J02MF7 & $02 / 28 / 05$ & 0.72 & & 0.15 & 15.5 & & 0.09 & 967 & & 0.87 & 1760 & $\mathrm{C}$ & 0.72 & 0.34 & $\mathrm{U}$ & 0.34 & 121 & & 0.49 \\
\hline $\begin{array}{c}\text { Pipe to } \\
\text { drainfield }\end{array}$ & J02MJ7 & $3 / 7 / 05$ & 1.0 & & 1.2 & 18.3 & & 0.72 & 834 & & 9.0 & 1240 & $\mathrm{C}$ & 6.0 & 2.2 & $\mathrm{U}$ & 3.0 & 272 & $\mathrm{C}$ & 3.6 \\
\hline $\begin{array}{c}\text { Rust-stained } \\
\text { soil }\end{array}$ & J03JN0 & $6 / 28 / 05$ & 0.92 & $\mathrm{U}$ & 0.92 & 10.1 & & 1.3 & 1520 & & 1.1 & 790 & & 52.2 & 2.8 & U & 2.8 & 335 & & 3.9 \\
\hline Stockpile & J03JN6 & $6 / 29 / 05$ & 0.85 & UI & 0.9 & 12.3 & & 1.2 & 897.0 & & 1.0 & 1260 & & 45.3 & 2.6 & $\mathrm{U}$ & 2.6 & 298 & & 4.2 \\
\hline
\end{tabular}


Table A-8. 1607-B2 Waste Characterization and In-Process Analytical Results. (6 Pages)

\begin{tabular}{|c|c|c|c|c|c|c|c|c|c|c|c|c|c|c|c|c|c|c|c|c|}
\hline \multirow{2}{*}{$\begin{array}{c}\text { Sample } \\
\text { Location }\end{array}$} & \multirow{2}{*}{$\begin{array}{l}\text { Sample } \\
\text { Number }\end{array}$} & \multirow{2}{*}{$\begin{array}{c}\text { Sample } \\
\text { Date }\end{array}$} & \multicolumn{3}{|c|}{ Silver } & \multicolumn{3}{|c|}{ Sodium } & \multicolumn{3}{|c|}{ Strontium } & \multicolumn{3}{|c|}{ Thallium } & \multicolumn{3}{|c|}{ Tin } & \multicolumn{3}{|c|}{ Titanium } \\
\hline & & & $\mathrm{mg} / \mathrm{kg}$ & $Q$ & PQL & $\mathrm{mg} / \mathrm{kg}$ & $Q$ & $\mathrm{PQL}$ & $\mathrm{mg} / \mathrm{kg}$ & $Q$ & PQL & $\mathrm{mg} / \mathrm{kg}$ & $\bar{Q}$ & $\overline{\mathrm{PQL}}$ & $\mathrm{mg} / \mathrm{kg}$ & $Q$ & PQL & $\mathrm{mg} / \mathrm{kg}$ & Q & $\mathrm{PQL}$ \\
\hline $\begin{array}{c}\text { Contaminated } \\
\text { area }\end{array}$ & J02HF8 & $2 / 9 / 05$ & 0.05 & $\mathrm{U}$ & 0.05 & 193 & & 0.17 & 28 & $\mathrm{C}$ & 0.01 & 0.71 & U & 0.71 & 1.2 & $\mathrm{C}$ & 0.5 & 1280 & & 0.02 \\
\hline Equip. Blank & J02J01 & $2 / 17 / 05$ & 0.04 & $\mathrm{U}$ & 0.04 & 5.5 & $\mathrm{C}$ & 2.0 & 0.17 & $\mathrm{C}$ & 0.0 & 0.52 & $\bar{U}$ & 0.52 & 0.37 & $\mathrm{UC}$ & 0.4 & 1.7 & & 0.0 \\
\hline Drainfield & $\mathrm{J} 02 \mathrm{~J} 02$ & $2 / 17 / 05$ & 0.05 & $\mathrm{U}$ & 0.05 & 229 & $\mathrm{C}$ & 2.3 & 36.1 & $\mathrm{C}$ & 0.0 & 0.69 & $\bar{U}$ & 0.69 & 0.92 & $\mathrm{C}$ & 0.7 & 1510 & & 0.0 \\
\hline Dup. of J02J02 & J02J03 & $2 / 17 / 05$ & 0.05 & $\mathrm{U}$ & 0.05 & 210 & C & 2.3 & 32.7 & $\mathrm{C}$ & 0.0 & 0.71 & U & 0.71 & 0.74 & $\mathrm{C}$ & 0.7 & 1680 & & 0.0 \\
\hline Drainfield & J02J05 & $2 / 17 / 05$ & 0.65 & $\mathrm{U}$ & 0.65 & 305 & $\mathrm{BC}$ & 2.2 & 35.8 & $\mathrm{C}$ & 0.0 & 1.1 & & 0.5 & 6.2 & $\mathrm{U}$ & 6.2 & 1970 & $\mathrm{C}$ & 0.0 \\
\hline Drainfield & J02MB7 & $02 / 23 / 05$ & 0.052 & $\mathrm{U}$ & 0.06 & 282 & $\mathrm{C}$ & 0.16 & 40.6 & $\mathrm{C}$ & 0.01 & 0.678 & $\mathrm{U}$ & 0.68 & 1.2 & $\mathrm{C}$ & 0.49 & 2220 & $\mathrm{C}$ & 0.02 \\
\hline Drainfield & J02MF7 & $02 / 28 / 05$ & 0.04 & $\mathrm{U}$ & 0.05 & 257 & & 0.13 & 30.6 & & 0.009 & 0.55 & $\mathrm{U}$ & 0.55 & 1.4 & $\mathrm{C}$ & 0.4 & 1900 & & 0.02 \\
\hline $\begin{array}{c}\text { Pipe to } \\
\text { drainfield }\end{array}$ & J02MJ7 & $3 / 7 / 05$ & 0.27 & $\mathrm{U}$ & 0.27 & 213 & $\mathrm{C}$ & 1 & 32 & C & 0.06 & 3.5 & $\mathrm{U}$ & 3.5 & 2.6 & $\mathrm{UC}$ & 2.6 & 1400 & $\mathrm{C}$ & 0.12 \\
\hline $\begin{array}{l}\text { Rust-stained } \\
\text { soil }\end{array}$ & J03JN0 & $6 / 28 / 05$ & 0.52 & $\mathrm{U}$ & 0.52 & 151 & & 2.2 & 19.9 & $\mathrm{C}$ & 0.06 & 4.5 & $U$ & 4.5 & 8.9 & $\mathrm{C}$ & 3.2 & 1510 & $\mathrm{C}$ & 0.17 \\
\hline & & & 010 & $I$ & 048 & 108 & & ח & 373 & $C$ & 01 & 42 & $\bar{U}$ & 42 & 3 & $\mathrm{UC}$ & 30 & 1560 & $\mathrm{C}$ & \\
\hline
\end{tabular}

\begin{tabular}{|c|c|c|c|c|c|c|c|c|c|c|c|c|c|c|}
\hline \multirow{2}{*}{$\begin{array}{c}\text { Sample } \\
\text { Location }\end{array}$} & \multirow{2}{*}{$\begin{array}{c}\text { Sample } \\
\text { Number }\end{array}$} & \multirow{2}{*}{$\begin{array}{c}\text { Sample } \\
\text { Date }\end{array}$} & \multicolumn{3}{|c|}{ Uranium } & \multicolumn{3}{|c|}{ Vanadium } & \multicolumn{3}{|c|}{ Zinc } & \multicolumn{3}{|c|}{ Zirconium } \\
\hline & & & $\mathrm{mg} / \mathrm{kg}$ & $Q$ & PQL & $\mathrm{mg} / \mathrm{kg}$ & $\mathbf{Q}$ & PQL & $\mathrm{mg} / \mathrm{kg}$ & $\mathrm{Q}$ & PQL & $\mathrm{mg} / \mathrm{kg}$ & $Q$ & $\overline{P Q L}$ \\
\hline $\begin{array}{c}\text { Contaminated } \\
\text { area }\end{array}$ & J02HF8 & $2 / 9 / 05$ & 1.9 & & 1.00 & 46.5 & & 0.06 & 45.1 & & 0.05 & 17.7 & & 0.07 \\
\hline Equip. Blank & $\mathrm{J} 02 \mathrm{~J} 01$ & $2 / 17 / 05$ & 0.64 & $\mathrm{U}$ & 0.64 & 0.05 & $U$ & 0.1 & 0.37 & & 0.1 & 0.54 & & 0.6 \\
\hline Drainfield & $\mathrm{J} 02 \mathrm{~J} 02$ & $2 / 17 / 05$ & 0.86 & $\mathrm{U}$ & 0.86 & 59.1 & & 0.1 & 52.8 & & 0.1 & 26.3 & & 0.7 \\
\hline Dup. of J02J02 & J02J03 & $2 / 17 / 05$ & 0.89 & $\mathrm{U}$ & 0.89 & 57.7 & & 0.1 & 49.9 & & 0.1 & 26.1 & & 0.7 \\
\hline Drainfield & J02J05 & $2 / 17 / 05$ & 15.7 & $\mathrm{U}$ & 15.7 & 70 & & 0.1 & 53.3 & & 0.1 & 177 & & 0.7 \\
\hline Drainfield & J02MB7 & $02 / 23 / 05$ & 1.1 & & 0.85 & 69.4 & & 0.06 & 68.4 & $\mathrm{C}$ & 0.05 & 29.7 & & 0.07 \\
\hline Drainfield & J02MF7 & $02 / 28 / 05$ & 0.69 & $\mathrm{U}$ & 0.69 & 60.2 & & 0.05 & 68.8 & & 0.04 & 23.9 & & 0.06 \\
\hline $\begin{array}{c}\text { Pipe to } \\
\text { drainfield }\end{array}$ & J02MJ7 & $3 / 7 / 05$ & 4.4 & $\mathrm{U}$ & 4.4 & 53.3 & & 0.36 & 54.7 & $\mathrm{C}$ & 0.30 & 15.2 & & 0.42 \\
\hline $\begin{array}{c}\text { Rust-stained } \\
\text { soil }\end{array}$ & J03JN0 & $6 / 28 / 05$ & 11.4 & $\mathrm{U}$ & 11.4 & 46.5 & & 0.34 & 121 & & 0.29 & 2.1 & $\mathrm{UC}$ & 2.1 \\
\hline Stockpile & J03JN6 & $6 / 29 / 05$ & 10.5 & $\mathrm{U}$ & 10.5 & 52.9 & & 0.3 & 47.7 & & 0.4 & 5 & $\mathrm{C}$ & 3.6 \\
\hline
\end{tabular}


Table A-8. 1607-B2 Waste Characterization and In-Process Analytical Results. (6 Pages)

\begin{tabular}{|c|c|c|c|c|c|c|c|c|c|c|c|c|c|c|c|c|c|c|c|c|}
\hline \multirow{2}{*}{$\begin{array}{c}\text { Sample } \\
\text { Location }\end{array}$} & \multirow{2}{*}{$\begin{array}{c}\text { Sample } \\
\text { Number }\end{array}$} & \multirow{2}{*}{$\begin{array}{c}\text { Sample } \\
\text { Date }\end{array}$} & \multicolumn{3}{|c|}{ Arsenic (TCLP) } & \multicolumn{3}{|c|}{ Barium (TCLP) } & \multicolumn{3}{|c|}{ Cadmium (TCLP) } & \multicolumn{3}{|c|}{ Chromium (TCLP) } & \multicolumn{3}{|c|}{ Lead (TCLP) } & \multicolumn{3}{|c|}{ Mercury (TCLP) } \\
\hline & & & $\mu \mathrm{g} / \mathrm{L}$ & Q & PQL & $\mu \mathrm{g} / \mathrm{L}$ & $\mathbf{Q}$ & PQL & $\mu \mathrm{g} / \mathrm{L}$ & $\mathrm{Q}$ & $\mathrm{PQL}$ & $\mu \mathrm{g} / \mathrm{L}$ & Q & PQL & $\mu \mathrm{g} / \mathrm{L}$ & Q & $\mathrm{PQL}$ & $\mu \mathrm{g} / \mathrm{L}$ & $Q$ & $\mathrm{PQL}$ \\
\hline $\begin{array}{c}\text { Contaminated } \\
\text { area }\end{array}$ & J02HF8 & $2 / 9 / 05$ & 18.8 & & 17.4 & 230 & $\mathrm{C}$ & 1.2 & 2.4 & $\mathrm{U}$ & 2.4 & 6.7 & & 2.4 & 12.5 & & 11.4 & 0.10 & $\mathrm{U}$ & 0.10 \\
\hline Equip. Blank & $\mathrm{J} 02 \mathrm{~J} 01$ & $2 / 17 / 05$ & 17.4 & $\mathrm{U}$ & 17.4 & 44.3 & C & 12 & 2.4 & $\mathrm{U}$ & 2.4 & 2.4 & $\mathrm{U}$ & 2.4 & 11.4 & $\mathrm{U}$ & 11.4 & 0.1 & $\mathrm{U}$ & 0.1 \\
\hline Drainfield & $\mathrm{J} 02 \mathrm{~J} 02$ & $2 / 17 / 05$ & 17.4 & $\mathrm{U}$ & 17.4 & 363 & $\mathrm{C}$ & 12 & 2.4 & $\mathrm{U}$ & 2.4 & 2.4 & $\mathrm{U}$ & 2.4 & 11.4 & $\mathrm{U}$ & 11.4 & 0.1 & $\bar{U}$ & 0.1 \\
\hline Dup. of J02J02 & $\mathrm{J} 02 \mathrm{~J} 03$ & $2 / 17 / 05$ & 17.4 & $\mathrm{U}$ & 17.4 & 411 & $\mathrm{C}$ & 12 & 2.4 & $\mathrm{U}$ & 2.4 & 2.4 & $\mathrm{U}$ & 2.4 & 11.4 & $\mathrm{U}$ & 11.4 & 0.1 & $\mathrm{U}$ & 0.1 \\
\hline Drainfield & J02J05 & $2 / 17 / 05$ & 180 & $\mathrm{U}$ & 180 & 400 & $\mathrm{BC}$ & 5 & 5 & $\mathrm{U}$ & 5 & 18 & $\mathrm{U}$ & 18 & 200 & $\bar{U}$ & 200 & 0.046 & $\bar{U}$ & 0.046 \\
\hline Drainfield & J02MB7 & $02 / 23 / 05$ & 17.4 & $\mathrm{U}$ & 17.4 & 390 & $\mathrm{C}$ & 1.2 & 3.1 & & 2.4 & 19.4 & & 2.4 & 11.4 & $\mathrm{U}$ & 11.4 & 0.1 & $\mathrm{UC}$ & 0.1 \\
\hline Drainfield & J02MF7 & $02 / 28 / 05$ & 17.4 & $\mathrm{U}$ & 17.4 & 418 & C & 1.2 & 2.4 & $\mathrm{U}$ & 2.4 & 2.4 & $\mathrm{U}$ & 2.4 & 11.4 & $\mathrm{U}$ & 11.4 & 0.1 & $\mathrm{U}$ & 0.1 \\
\hline $\begin{array}{c}\text { Pipe to } \\
\text { drainfield }\end{array}$ & J02MJ7 & $3 / 7 / 05$ & 17.4 & $U$ & 17.4 & 389 & $\mathrm{C}$ & 1.2 & 2.4 & $\mathrm{U}$ & 2.4 & 5.6 & $\mathrm{C}$ & 2.4 & 11.4 & U & 11.4 & 0.10 & U & 0.10 \\
\hline
\end{tabular}

\begin{tabular}{|c|c|c|c|c|c|c|c|c|}
\hline Sample & \multirow{2}{*}{$\begin{array}{c}\text { Sample } \\
\text { Location }\end{array}$} & Sumber & Sample & \multicolumn{3}{|c|}{ Selenium (TCLP) } & \multicolumn{3}{|c|}{ Silver (TCLP) } \\
\cline { 5 - 9 } & Dag/L & Q & PQL & $\mu \mathrm{g} / \mathrm{L}$ & $\mathbf{Q}$ & PQL \\
\hline $\begin{array}{c}\text { Contaminated } \\
\text { area }\end{array}$ & $\mathrm{J} 02 \mathrm{HF} 8$ & $2 / 9 / 05$ & 24.0 & $\mathrm{U}$ & 24.0 & 3.0 & $\mathrm{U}$ & 3.0 \\
\hline Equip. Blank & $\mathrm{J} 02 \mathrm{~J} 01$ & $2 / 17 / 05$ & 24 & $\mathrm{U}$ & 24 & 3 & $\mathrm{U}$ & 3 \\
\hline Drainfield & $\mathrm{J} 02 \mathrm{~J} 02$ & $2 / 17 / 05$ & 24 & $\mathrm{U}$ & 24 & 3 & $\mathrm{U}$ & 3 \\
\hline Dup. of J02J02 & $\mathrm{J} 02 \mathrm{~J} 03$ & $2 / 17 / 05$ & 24 & $\mathrm{U}$ & 24 & 3 & $\mathrm{U}$ & 3 \\
\hline Drainfield & $\mathrm{J} 02 \mathrm{~J} 05$ & $2 / 17 / 05$ & 240 & $\mathrm{U}$ & 240 & 4.5 & $\mathrm{U}$ & 4.5 \\
\hline Drainfield & $\mathrm{J02 \textrm {MB } 7}$ & $02 / 23 / 05$ & 24 & $\mathrm{U}$ & 24 & 3 & $\mathrm{U}$ & 3 \\
\hline Drainfield & $\mathrm{J} 02 \mathrm{MF7} 7$ & $02 / 28 / 05$ & 24 & $\mathrm{U}$ & 24 & 3 & $\mathrm{UC}$ & 3 \\
\hline $\begin{array}{c}\text { Pipe to } \\
\text { drainfield }\end{array}$ & $\mathrm{J} 02 \mathrm{MJ7} 7$ & $3 / 7 / 05$ & 24.0 & $\mathrm{U}$ & 24.0 & 3.0 & $\mathrm{U}$ & 3.0 \\
\hline
\end{tabular}


Table A-8. 1607-B2 Waste Characterization and In-Process Analytical Results. (6 Pages)

\begin{tabular}{|c|c|c|c|c|c|c|c|c|c|c|c|c|c|c|c|c|c|c|c|c|}
\hline \multirow{2}{*}{$\begin{array}{l}\text { Sample } \\
\text { Location }\end{array}$} & \multirow{2}{*}{$\begin{array}{l}\text { Sample } \\
\text { Number }\end{array}$} & \multirow{2}{*}{$\begin{array}{c}\text { Sample } \\
\text { Date }\end{array}$} & \multicolumn{3}{|c|}{$\begin{array}{l}\text { 1,4-Dichlorobenzene } \\
\text { (TCLP) }\end{array}$} & \multicolumn{3}{|c|}{$\begin{array}{c}\text { 2,4,5-Trichlorophenol } \\
\text { (TCLP) }\end{array}$} & \multicolumn{3}{|c|}{ 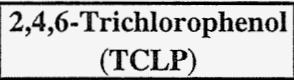 } & \multicolumn{3}{|c|}{$\begin{array}{l}\text { 2,4-Dinitrotoluene } \\
\text { (TCLP) }\end{array}$} & \multicolumn{3}{|c|}{$\begin{array}{c}\text { 2-Methylphenol } \\
\text { (cresol, o-) (TCLP) }\end{array}$} & \multicolumn{3}{|c|}{$\begin{array}{c}\text { 3+4 Methylphenol } \\
(\text { cresol, } \mathrm{m}+\mathrm{p})(\mathrm{TCLP})\end{array}$} \\
\hline & & & $\mathrm{mg} / \mathrm{L}$ & $Q$ & PQL & $\mathrm{mg} / \mathrm{L}$ & $Q$ & $\mathbf{P Q L}$ & $\mathrm{mg} / \mathrm{L}$ & $\bar{Q}$ & PQL & $\mathrm{mg} / \mathrm{L}$ & Q & $\mathrm{PQL}$ & $\mathrm{mg} / \mathrm{L}$ & $\mathrm{Q}$ & PQL & $\mathrm{mg} / \mathrm{L}$ & $Q$ & PQL \\
\hline $\begin{array}{c}\text { Contaminated } \\
\text { area }\end{array}$ & J02HF8 & $2 / 9 / 05$ & 0.050 & $\mathrm{U}$ & 0.050 & 0.12 & U & 0.12 & 0.050 & $\mathrm{U}$ & 0.050 & 0.050 & U & 0.050 & 0.050 & $U$ & 0.050 & 0.050 & U & 0.050 \\
\hline Equip. Blank & J02J01 & $2 / 17 / 05$ & 0.05 & $\bar{U}$ & 0.05 & 0.12 & $\bar{U}$ & 0.12 & 0.05 & $\mathrm{U}$ & 0.05 & 0.05 & $\bar{U}$ & 0.05 & 0.05 & $\mathrm{U}$ & 0.05 & 0.05 & $\bar{U}$ & 0.05 \\
\hline Drainfield & $\mathrm{J} 02 \mathrm{~J} 02$ & $2 / 17 / 05$ & 0.05 & $\bar{U}$ & 0.05 & 0.12 & $\bar{U}$ & 0.12 & 0.05 & $\mathrm{U}$ & 0.05 & 0.05 & $\mathrm{U}$ & 0.05 & 0.05 & $U$ & 0.05 & 0.05 & $\bar{U}$ & 0.05 \\
\hline Dup. of J02J02 & J02J03 & $2 / 17 / 05$ & 0.05 & $\bar{U}$ & 0.05 & 0.12 & $\bar{U}$ & 0.12 & 0.05 & $\bar{U}$ & 0.05 & 0.05 & $\mathrm{U}$ & 0.05 & 0.05 & $\mathrm{U}$ & 0.05 & 0.05 & $\mathrm{U}$ & 0.05 \\
\hline Drainfield & $2 \longdiv { 2 3 0 5 }$ & $2 / 17 / 05$ & 0.0046 & $\bar{U}$ & 0.0046 & 0.012 & $\bar{U}$ & $\overline{0.012}$ & 0.012 & $\overline{\mathrm{U}}$ & 0.012 & 0.02 & $\mathrm{U}$ & 0.02 & 0.0046 & $\mathrm{U}$ & 0.0046 & 0.0086 & $\bar{U}$ & 0.0086 \\
\hline Drainfield & J02MB7 & $02 / 23 / 05$ & 0.05 & $\mathrm{U}$ & 0.05 & 0.12 & $\mathrm{U}$ & 0.12 & 0.05 & $\mathrm{U}$ & 0.05 & 0.05 & $\mathrm{U}$ & 0.05 & 0.05 & $\mathrm{U}$ & 0.05 & 0.05 & U & 0.05 \\
\hline Drainfield & J02MF7 & $02 / 28 / 05$ & 0.05 & U & 0.05 & 0.12 & U & 0.12 & 0.05 & $\mathrm{U}$ & 0.05 & 0.05 & $\mathrm{U}$ & 0.05 & 0.05 & $\mathrm{U}$ & 0.05 & 0.05 & $\mathrm{U}$ & 0.05 \\
\hline $\begin{array}{l}\text { Pipe to } \\
\text { drainfield }\end{array}$ & J02MJ7 & $3 / 7 / 05$ & 0.050 & $U$ & 0.050 & 0.12 & U & 0.12 & 0.050 & $\mathrm{U}$ & 0.050 & 0.050 & $U$ & 0.050 & 0.050 & U & 0.050 & 0.050 & U & 0.050 \\
\hline
\end{tabular}

\begin{tabular}{|c|c|c|c|c|c|c|c|c|c|c|c|c|c|c|c|c|c|c|c|c|}
\hline \multirow{2}{*}{$\begin{array}{l}\text { Sample } \\
\text { Location }\end{array}$} & \multirow{2}{*}{$\begin{array}{l}\text { Sample } \\
\text { Number }\end{array}$} & \multirow{2}{*}{$\begin{array}{c}\text { Sample } \\
\text { Date }\end{array}$} & \multicolumn{3}{|c|}{$\begin{array}{l}\text { Hexachlorobenzene } \\
\text { (TCLP) }\end{array}$} & \multicolumn{3}{|c|}{$\begin{array}{l}\text { Hexachlorobutadiene } \\
\text { (TCLP) }\end{array}$} & \multicolumn{3}{|c|}{$\begin{array}{l}\text { Hexachloroethane } \\
\text { (TCLP) }\end{array}$} & \multicolumn{3}{|c|}{$\begin{array}{l}\text { Nitrobenzene } \\
\text { (TCLP) }\end{array}$} & \multicolumn{3}{|c|}{$\begin{array}{l}\text { Pentachlorophenol } \\
\text { (TCLP) }\end{array}$} & \multicolumn{3}{|c|}{ Pyridine (TCLP) } \\
\hline & & & $\mathrm{mg} / \mathrm{L}$ & $Q$ & PQL & $\mathrm{mg} / \mathrm{L}$ & $Q$ & PQL & $\mathrm{mg} / \mathrm{L}$ & $Q$ & PQL & $\mathrm{mg} / \mathrm{L}$ & Q & $\mathrm{PQL}$ & $\mathrm{mg} / \mathrm{L}$ & $Q$ & $\mathrm{PQL}$ & $\mathrm{mg} / \mathrm{L}$ & $Q$ & $\mathrm{PQL}$ \\
\hline $\begin{array}{c}\text { Contaminated } \\
\text { area }\end{array}$ & J02HF8 & $2 / 9 / 05$ & 0.050 & $\mathrm{U}$ & 0.050 & 0.050 & $\mathrm{U}$ & 0.050 & 0.050 & $\mathrm{U}$ & 0.050 & 0.050 & $\mathrm{U}$ & 0.050 & 0.12 & $U$ & 0.12 & 0.050 & $\mathrm{U}$ & 0.050 \\
\hline Equip. Blank & J02J01 & $2 / 17 / 05$ & 0.05 & $\mathrm{U}$ & 0.05 & 0.05 & $\mathrm{U}$ & 0.05 & 0.05 & $\mathrm{U}$ & 0.05 & 0.05 & U & 0.05 & 0.12 & $\mathrm{U}$ & 0.12 & 0.05 & $\mathrm{U}$ & 0.05 \\
\hline Drainfield & $\mathrm{J} 02 \mathrm{~J} 02$ & $2 / 17 / 05$ & 0.05 & $\mathrm{U}$ & 0.05 & 0.05 & $\mathrm{U}$ & 0.05 & 0.05 & $\mathrm{U}$ & 0.05 & 0.05 & $\mathrm{U}$ & 0.05 & 0.12 & $\mathrm{U}$ & 0.12 & 0.05 & $\mathrm{U}$ & 0.05 \\
\hline Dup. of J02J02 & $\mathrm{J} 02 \mathrm{~J} 03$ & $2 / 17 / 05$ & 0.05 & $\mathrm{U}$ & 0.05 & 0.05 & U & 0.05 & 0.05 & $\mathrm{U}$ & 0.05 & 0.05 & $\mathrm{U}$ & 0.05 & 0.12 & $\mathrm{U}$ & 0.12 & 0.05 & $\mathrm{U}$ & 0.05 \\
\hline Drainfield & J02J05 & $2 / 17 / 05$ & 0.0058 & $\mathrm{U}$ & 0.0058 & 0.0046 & $\bar{U}$ & 0.0046 & 0.004 & $\mathrm{U}$ & 0.004 & 0.0043 & $U$ & 0.0043 & 0.019 & $\mathrm{U}$ & 0.019 & 0.0085 & $\mathrm{U}$ & 0.0085 \\
\hline Drainfield & J02MB7 & $02 / 23 / 05$ & 0.05 & $\mathrm{U}$ & 0.05 & 0.05 & $\bar{U}$ & 0.05 & 0.05 & $\mathrm{U}$ & 0.05 & 0.05 & $\mathrm{U}$ & 0.05 & 0.12 & $\mathrm{U}$ & 0.12 & 0.05 & $\mathrm{U}$ & 0.05 \\
\hline Drainfield & J02MF7 & $02 / 28 / 05$ & 0.05 & $\mathrm{U}$ & 0.05 & 0.05 & U & 0.05 & 0.05 & $\mathrm{U}$ & 0.05 & 0.05 & $\mathrm{U}$ & 0.05 & 0.12 & $\mathrm{U}$ & 0.12 & 0.05 & $\mathrm{U}$ & 0.05 \\
\hline $\begin{array}{l}\text { Pipe to } \\
\text { drainfield }\end{array}$ & J02MJ7 & $3 / 7 / 05$ & 0.050 & $\mathrm{U}$ & 0.050 & 0.050 & $\mathrm{U}$ & 0.050 & 0.050 & $\mathrm{U}$ & 0.050 & 0.050 & $U$ & 0.050 & 0.12 & $U$ & 0.12 & 0.050 & $U$ & 0.050 \\
\hline
\end{tabular}




\section{APPENDIX B}

\section{CALCULATION BRIEFS}




\section{DISCLAIMER FOR CALCULATIONS}

The calculations that are provided in this appendix have been generated to document compliance with established cleanup levels. These calculations should be used in conjunction with other relevant documents in the administrative record.

A list of calculations provided in this appendix is as follows:

Human Health Risk Assessment for PCB's at the 100-B-14:2, Area 3, Pipeline Waste Sub-Site, Calculation No. 0100B-CA-V0266, Rev. 1, Washington Closure Hanford, Richland, Washington.

Human Health Risk Assessment for Dieldrin at the 100-B-14:2, Area 3, Pipeline Waste Sub-Site, Calculation No. 0100B-CA-V0268, Rev. 1, Washington Closure Hanford, Richland, Washington.

100-B-14:2 (Area 3) Hazard Quotient and Carcinogenic Risk Calculations, Calculation No. 0100B-CA-V0269, Rev. 1, Washington Closure Hanford, Richland, Washington.

100-B-14:2 (Areas 2 \& 5) Waste Site Cleanup Verification 95\% UCL Calculations, Calculation No. 0100B-CA-V0281, Rev. 0, Washington Closure Hanford, Richland, Washington.

100-B-14:2 (Areas 2 \& 5) Waste Site Hazard Quotient and Carcinogenic Risk Calculations, Calculation No. 0100B-CA-V0282, Rev. 1, Washington Closure Hanford, Richland, Washington.

100-B-14:2 (Area 4) Waste Site Cleanup Verification 95\% UCL Calculations, Calculation No. 0100B-CA-V0290, Rev. 0, Washington Closure Hanford, Richland, Washington.

100-B-14:2 (Area 4) Waste Site Hazard Quotient and Carcinogenic Risk Calculations, Calculation No. 0100B-CA-V0291, Rev. 1, Washington Closure Hanford, Richland, Washington.

1607-B2:1 Drain Field Cleanup Verification 95\% UCL Calculations, Calculation No. 0100B-CA-V0264, Rev. 0, Washington Closure Hanford, Richland, Washington.

1607-B2:1 Drain Field Hazard Quotient and Carcinogenic Risk Calculations, Calculation No. 0100B-CA-V0265, Rev. 1, Washington Closure Hanford, Richland, Washington.

1607-B2:2 Waste Site Cleanup Verification 95\% UCL Calculations, Calculation No. 0100B-CAV0292, Rev. 0, Washington Closure Hanford, Richland, Washington.

1607-B2:2 Drain Field Hazard Quotient and Carcinogenic Risk Calculations, Calculation No. 0100B-CA-V0293, Rev. 0, Washington Closure Hanford, Richland, Washington. 


\section{CALCULATION COVER SHEET}

Project Title 100-B/C Field Remediation

Job No. 14655

Area $100-\mathrm{B} / \mathrm{C}$ Area

Discipline Environmental

*Calc. No. 0100B-CA-V0266

Subject Human Health Risk Assessment for PCB's at the 100-B-14:2, Area 3, Pipeline Waste Sub-Site

Computer Program Excel Program No. Excel 2003

The attached calculations have been generated to document compliance with established cleanup levels.

These documents should be used in conjunction with other relevant documents in the administrative record.

Committed Calculation $\otimes \quad$ Preliminary $\square \quad$ Superseded $\square \quad$ Voided $\square$

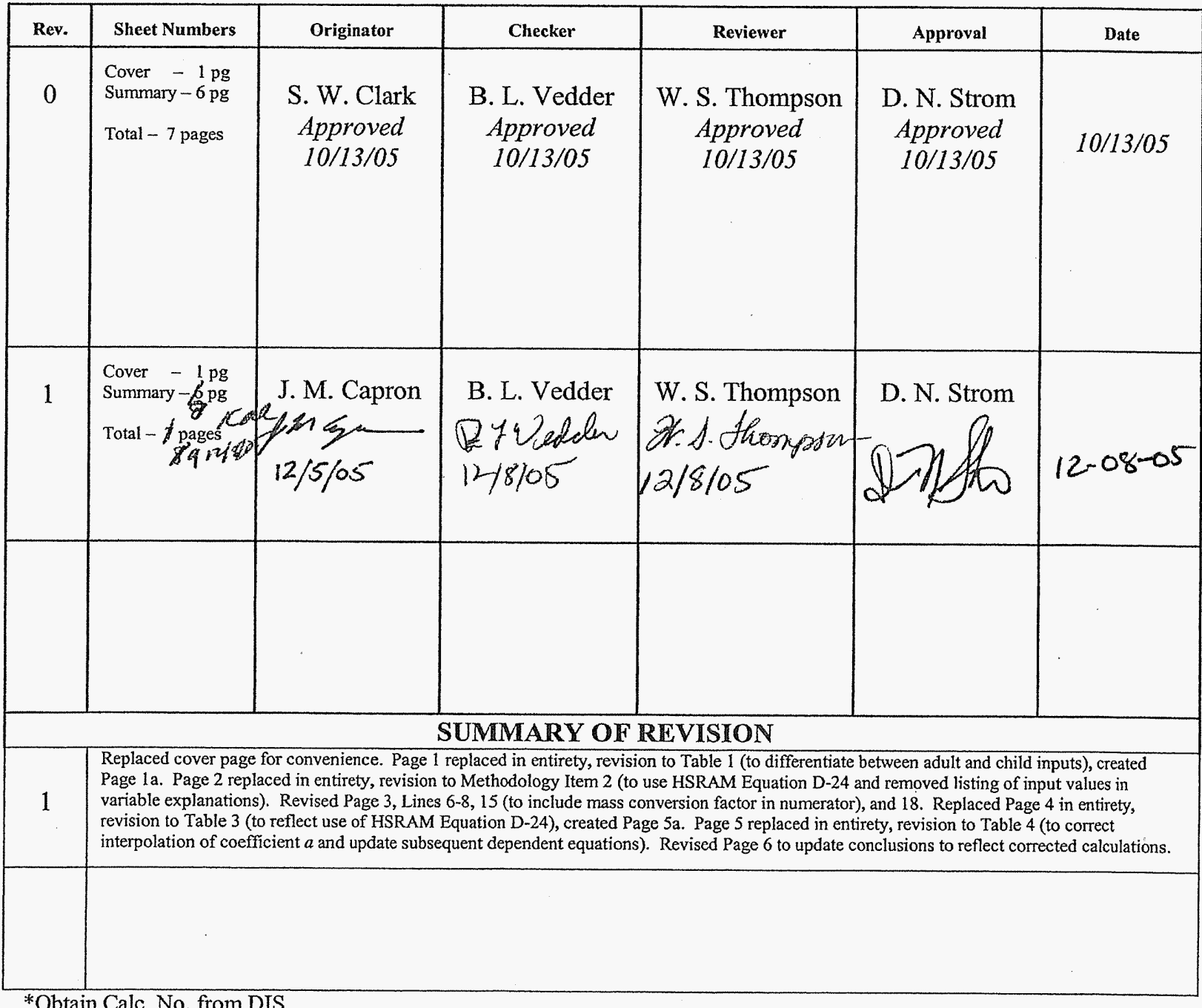

DE01-437.03 (12/09/2004) 
WII River Corridor

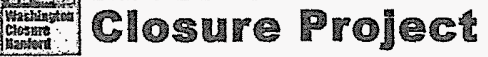

\begin{tabular}{|c|c|c|c|c|c|c|c|}
\hline Originator: & J. M. Capron Qen< & Date: & $12 / 5 / 05$ & Calc. No.: & 0100B-CA-V0266 & Rev.: & 1 \\
\hline Project: & 100-B/C Field Remediation & Job No: & 14655 & Checked: & B. L. Vedder But the & Date: & $12 / 8 / 05$ \\
\hline Subject: & \multicolumn{5}{|c|}{ Human Health Risk Assessment for PCB's at the 100-B-14:2, Area 3, Pipeline Waste Sub-Site } & \multicolumn{2}{|c|}{ Sheet No. 1 of 6} \\
\hline
\end{tabular}

\section{PURPOSE:}

Calculate the incremental cancer risk from residual concentrations of polychlorinated biphenyls (PCBs) at the 100-B-14:2, Area 3, pipeline waste sub-site.

\section{GIVEN/REFERENCES:}

1) Maximum residual concentration of PCBs from Hanford Environmental Information System (HEIS) number J037M8.

2) Remedial Design Report/Remedial Action Work Plan for the 100 Area (RDR/RAWP), DOE/RL-96-17, Rev. 5, U.S. Department of Energy, Richland Operations Office, Richland, Washington.

3) Equations for calculating contaminant intake from Appendix D of Hanford Site Risk Assessment Methodology (HSRAM), DOE/RL-91-45, Rev. 3, U.S. Department of Energy, Richland Operations Office, Richland, Washington.

4) Use of area factors and occupancy factors to account for small waste site size and actual period of occupancy in the rural-residential scenario is discussed in the User's Manual for RESRAD Version 6, ANL/EAD-4, Environmental Assessment Division, Argonne National Laboratory, Argonne, Illinois.

\section{SOLUTION:}

1) Table 1 shows the risk assessment input parameters used for this calculation.

Table 1. Risk Assessment Input Parameters.

\begin{tabular}{|l|c|c|c|}
\hline \multicolumn{2}{|c|}{ Table 1. Risk Assessment Input Parameters. } \\
\hline \multicolumn{1}{|c|}{ Parameter } & Units & Value & Citation \\
\hline Area of contaminated zone & $\mathrm{m}^{2}$ & 57.1 & Site-specific \\
\hline Exposure duration, adult & years & 24 & HSRAM \\
\hline Exposure duration, child & years & 6 & HSRAM \\
\hline Fraction of time spent indoors & unitless & 0.6 & 100 Area RDR/RAWP \\
\hline Fraction of time spent outdoors (on site) & unitless & 0.2 & 100 Area RDR/RAWP \\
\hline Soil ingestion rate, adult & $\mathrm{g} / \mathrm{yr}$ & 36.5 & HSRAM \\
\hline Soil ingestion rate, child & $\mathrm{g} / \mathrm{yr}$ & 73 & HSRAM \\
\hline Inhalation rate & $\mathrm{m} / \mathrm{yr}$ & 7,300 & 100 Area RDR/RAWP \\
\hline Mass dust loading for inhalation & $\mathrm{g} / \mathrm{m}^{3}$ & 0.0001 & 100 Area RDR/RAWP \\
\hline Wind speed & $\mathrm{m} / \mathrm{s}$ & 3.4 & 100 Area RDR/RAWP \\
\hline Body weight, adult & $\mathrm{kg}$ & 70 & HSRAM \\
\hline Body weight, child & $\mathrm{kg}$ & 16 & HSRAM \\
\hline Average lifetime & $\mathrm{yr}$ & 70 & HSRAM \\
\hline
\end{tabular}




\section{MMin River Corridor} Closure Project

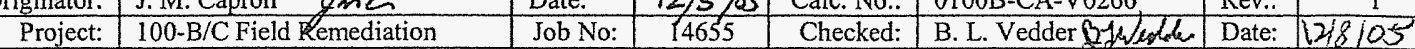

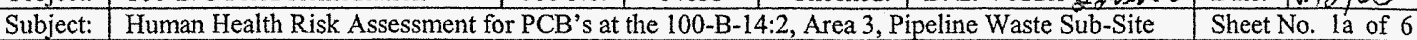

1 2) Table 2 shows the contaminant-specific risk assessment input parameters for the inhalation and soil ingestion pathways. PCBs have high distribution coefficients $(>30 \mathrm{~mL} / \mathrm{g})$ and will not move through the vadose zone in water-dependent pathways within 1,000 years. Only the inhalation and soil ingestion pathways will be affected by the PCBs. There are no 


\section{TII River Cormidor}

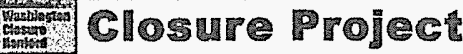

\begin{tabular}{|c|c|c|c|c|c|c|c|}
\hline Originator: & J. M. Capron $Z$ & Date: & $12 / 5 / 05$ & Calc. $\mathrm{N}$ & 0100B-CA-V0266 & Rey & 1 \\
\hline & 100-B/C Field Remediation & Job No: & 14655 & Checked: & B. L. Vedder 0146 \& & Date: & $12 / 8105$ \\
\hline Subiect: & \multicolumn{5}{|c|}{ Human Health Risk Assessment for PCB's at the 100-B-14:2, Area 3, Pipeline Waste Sub-Site } & \multicolumn{2}{|c|}{ Sheet No. 2 of 6} \\
\hline
\end{tabular}

noncarcinogenic reference doses for soil ingestion or inhalation (RfDo or RfDi) for PCBs so there is no hazard quotient calculation.

\begin{tabular}{|c|c|c|c|c|}
\hline \multirow{2}{*}{ Contaminant } & \multicolumn{2}{|c|}{ Pathway: Inhalation (Fugitive Dust) } & \multicolumn{2}{|c|}{ Pathway: Soil Ingestion } \\
\hline & $\begin{array}{c}\mathrm{RfDi}^{\mathrm{a}} \\
(\mathrm{mg} / \mathrm{kg}-\mathrm{d})\end{array}$ & $\begin{array}{c}\mathrm{CSFi}^{\mathrm{b}} \\
(\mathrm{kg}-\mathrm{d} / \mathrm{mg})\end{array}$ & $\begin{array}{c}\mathrm{RfDo}^{\mathrm{a}} \\
(\mathrm{mg} / \mathrm{kg}-\mathrm{d})\end{array}$ & $\begin{array}{c}\mathrm{CSFo}^{\mathrm{b}} \\
(\mathrm{kg}-\mathrm{d} / \mathrm{mg})\end{array}$ \\
\hline PCBs & N/A & $2.00 \mathrm{E}+00$ & N/A & $2.00 \mathrm{E}+00$ \\
\hline \multicolumn{5}{|c|}{$\begin{array}{l}\text { a RfDi or RfDo = Noncarcinogenic Reference Dose for dust inhalation or soil ingestion. Refers to chemical-specific } \\
\text { toxicity values used to evaluate noncarcinogenic effects resulting from exposures to chemicals. Obtained from the EPA } \\
\text { IRIS (Integrated Risk Information System) database or the Oak Ridge National Laboratory Risk Assessment Information } \\
\text { System database. } \\
\text { b CSFi or CSFo = Cancer Slope Factor for dust inhalation or soil ingestion. Refers to chemical-specific Cancer Slope } \\
\text { Factors used to calculate carcinogenic risk Obtained from the EPA IRIS (Integrated Risk Information System) database } \\
\text { or the Oak Ridge National Laboratory Risk Assessment Information System database. } \\
\text { N/A = Not Available }\end{array}$} \\
\hline
\end{tabular}

\section{METHODOLOGY:}

1) Incremental Cancer Risk:

The incremental cancer risk is calculated from the following general formula:

$$
\mathrm{ICR}=(\text { Daily Intake }) \mathrm{CSF}
$$

Where CSF $=$ the cancer slope factor with units of $\mathrm{kg}$ - day/mg. As applicable, the EPA provides separate values of the cancer slope factor for the inhalation and oral ingestion pathways (CSFi and CSFo, respectively).

\section{2) Daily Intake for the Soil Ingestion Pathway:}

Daily Intake for the soil ingestion pathway is calculated from the following formula from HSRAM Equation D-24, including the area factor and occupancy factor from the User's Manual for RESRAD Version 6:

$$
D I S=\frac{C \times\left[\left(\frac{S I \times E D}{B W}\right)_{\text {child }}+\left(\frac{S I \times E D}{B W}\right)_{\text {adult }}\right] \times A F S \times O F S \times 0.001(\mathrm{~kg} / \mathrm{g})}{A L \times 365(d / y r)}
$$

Where: $\mathrm{C}$ is contaminant concentration, (site-specific statistical value, $\mathrm{mg} / \mathrm{kg}$ )

SI is Soil Ingestion Rate, (g/yr)

AFS is an area factor for soil ingestion: AFS $=\mathrm{A} / 1000$ for $\mathrm{A}<1000 \mathrm{~m}^{2}$

$$
\mathrm{AFS}=1 \text { for } \mathrm{A}>1000 \mathrm{~m}^{2}
$$

$\mathrm{A}$ is the area of the contaminated zone, $\mathrm{m}^{2}$ 


\begin{tabular}{|c|c|c|c|c|c|c|c|}
\hline Originator: & J. M. Capron Y/ne & Date: & $12 / 6 / 05$ & Calc. No.: & 0100B-CA-V0266 & Rev.: & 1 \\
\hline Project: & 100-B/C Field Remediation & Job No: & 14655 & Checked: & B. L. Vedder Byluetion & Date: & $12 / 8 / 05$ \\
\hline Subject: & \multicolumn{5}{|c|}{ Human Health Risk Assessment for PCB's at the 100-B-14:2, Area 3, Pipeline Waste Sub-Site } & \multicolumn{2}{|c|}{ Sheet No. 3 of 6} \\
\hline
\end{tabular}

OFS is the occupancy factor for soils: OFS $=(\mathrm{IT})+(\mathrm{OT})$

IT is the Indoor Time Factor $(0.6)$

OT is the Outdoor Time Factor $(0.2)$

$\mathrm{OFS}=0.6+0.2=0.8$

$\mathrm{ED}$ is exposure duration ( $30 \mathrm{yr})$

$\mathrm{BW}$ is body weight $(70 \mathrm{~kg})$

AL is average lifetime (70-yr)

3) Daily Intake for the Inhalation Pathway:

Daily Intake for the inhalation pathway is calculated using the following formula from HSRAM Equation D-30, including the area factor and occupancy factor from the User's Manual for RESRAD Version 6:

$D I I=\frac{C \times I R \times M L \times E D \times A F I \times O F I \times 0.001(\mathrm{~kg} / \mathrm{g})}{B W \times A L \times 365(d / y r)}$

Where: $\mathrm{C}$ is contaminant concentration, (site-specific statistical value, $\mathrm{mg} / \mathrm{kg}$ )

IR is Inhalation Rate, $\left(7,300 \mathrm{~m}^{3} / \mathrm{yr}\right)$

ML is Mass Loading, $\left(0.0001 \mathrm{~g} / \mathrm{m}^{3}\right)$

$\mathrm{ED}$ is exposure duration ( $30 \mathrm{yr}$ )

AFI is the site specific area factor for dust inhalation calculated from formula B.4 of the User's Manual for RESRAD Version 6:

$$
A F I=\frac{a}{1+b(\sqrt{A})^{c}}
$$

In this equation, $\mathrm{A}$ is the area of the contaminated zone, $\mathrm{m}^{2}$, and $\mathrm{a}, \mathrm{b}$, and $\mathrm{c}$ are least squares regression coefficients dependent upon the average wind speed as described in Table B.2 of the User's Manual for RESRAD Version 6. Calculation results are shown in the RESULTS section of this Calculation Summary.

OFI is the occupancy factor for inhalation: $\mathrm{OFI}=(\mathrm{IT} \times \mathrm{IDF})+(\mathrm{OT})$

IT is the Indoor Time Factor (0.6)

$\mathrm{IDF}=$ Indoor dust filtration factor $(0.4)$

OT is the Outdoor Time Factor (0.2)

$\mathrm{OFI}=(0.6 \times 0.4)+0.2=0.44$

$\mathrm{BW}$ is body weight $(70 \mathrm{~kg})$

$\mathrm{AL}$ is average lifetime ( $70 \mathrm{yr})$ 
4.1. River Corridor Fin Closure Project

\begin{tabular}{r|l|l|l|l|l|l|l|l|}
\hline Project: & $100-B / C$ Field Remediation & Date: & $13 / 5 / 05$ & Calc. No.: & $0100 B-C A-V 0266$ & Rev.: & 1 \\
\hline
\end{tabular}

Subject: Human Health Risk Assessment for PCB's at the 100-B-14:2, Area 3, Pipeline Waste Sub-Site Sheet No. 4 of 6

1

2

3

4

5

\section{RESULTS:}

Calculations were performed using an Excel spreadsheet, incorporating the formulas shown in the METHODOLOGY section of this Calculation Summary.

1) Incremental Cancer Risk from the Soil Ingestion Pathway:

The following Excel spreadsheet incorporates the formulas for calculation of incremental cancer risk from PCBs in the soil ingestion pathway:

Table 3. Excel Calculation of Incremental Cancer Risk in the Soil Ingestion Pathway

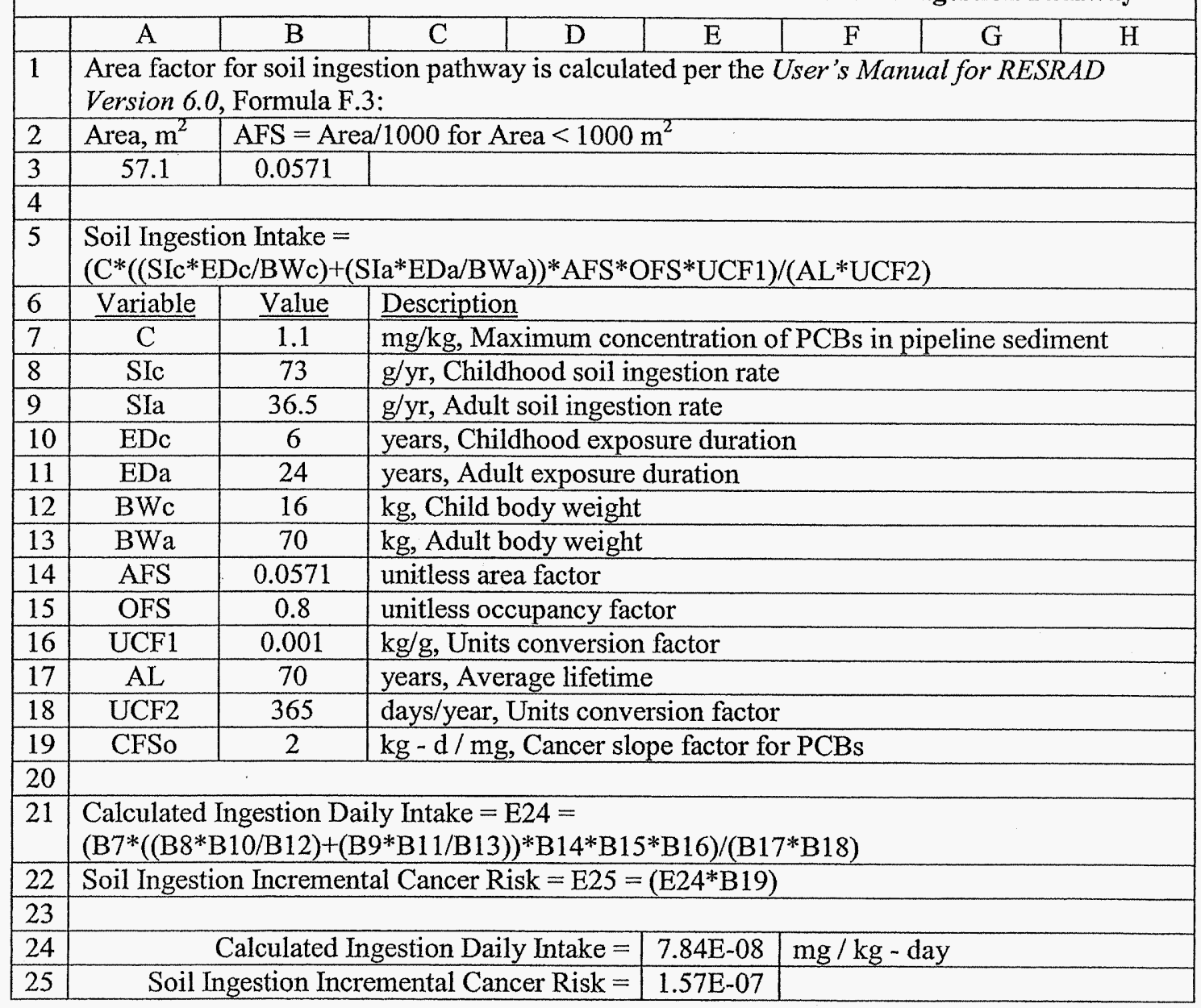




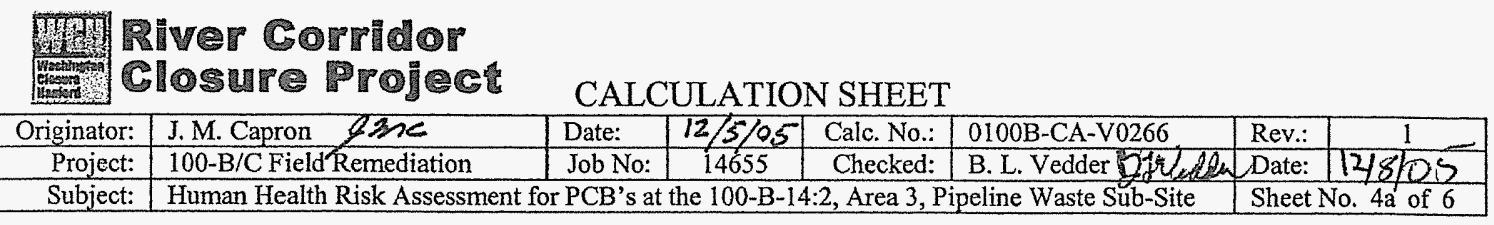

1 2) Incremental Cancer Risk from the Inhalation Pathway:

2 The following Excel spreadsheet incorporates the formulas for calculation of incremental cancer

3 risk from PCB's in the inhalation pathway: 
River Cormidor Closure Prolect

CALCULATION SHEET \begin{tabular}{|l|l|l|l|l|l|l}
\hline Date: & $12 / 5 / 65$ & Calc. No.: \\
\hline
\end{tabular}

Project: 100-B/C Field Remediation

Subject: Human Health Risk Assessment for PCB's at the 100-B-14:2, Area 3, Pipeline Waste Sub-Site

\begin{tabular}{c|c|c|} 
Rev.: & 1 \\
\hline Date: & $12 / 05$ \\
\hline
\end{tabular}

Sheet No. 5 of 6

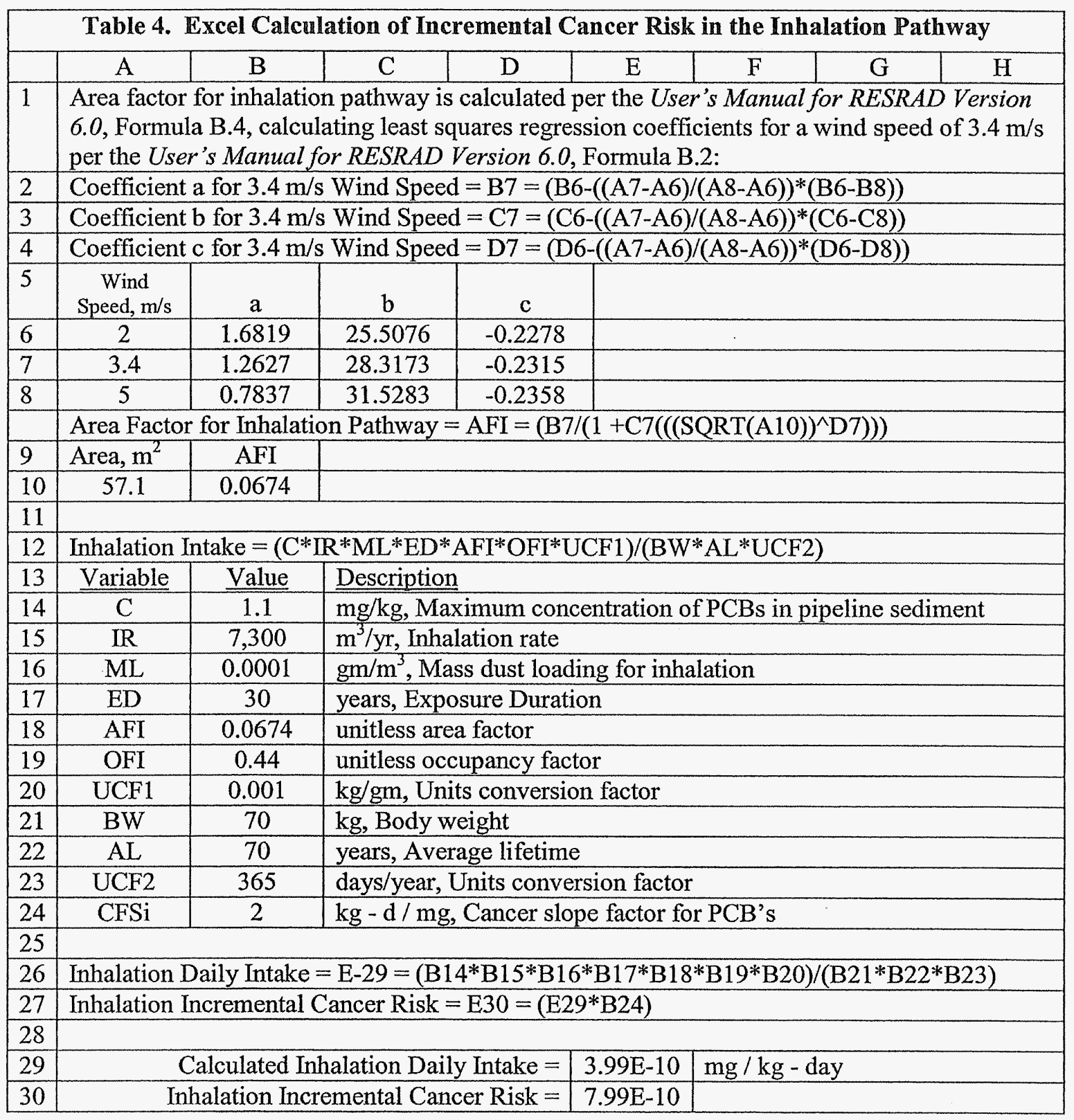

1 
MIIII River Cormidor Closure Project

\begin{tabular}{|c|c|c|c|c|c|c|}
\hline & J.1n. capron 820 & Date: & $24 / 2,05$ & Calc. No.: & $0100 \mathrm{~B}-\mathrm{CA}-\mathrm{V} 0260$ & Rev.: \\
\hline Proj & 100-B/C Field Remediation & Job No: & 14655 & Checked: & B. L. Vedder 124 lobur & Date: \\
\hline
\end{tabular}

Subject: Human Health Risk Assessment for PCB's at the 100-B-14:2, Area 3, Pipeline Waste Sub-Site Sheet No. 6 of 6

\section{CONCLUSIONS:}

3 - The incremental cancer risk due to PCB's in the soil ingestion pathway is

4

5

6

7

8

9

10

- The incremental cancer risk due to PCB's in the inhalation pathway is $7.61 \mathrm{E}-107.99 \mathrm{E}-10$.

- The total human health excess cancer risk due to PCB's at the 100-B-14:2, Area 3, Pipeline Waste Sub-Site is sum of the incremental cancer risks from the soil ingestion and inhalation pathways: $1.24 \mathrm{E}-071.58 \mathrm{E}-07$. 


\section{CALCULATION COVER SHEET}

Project Title 100-B/C Field Remediation

Job No. 14655

\section{Area $\quad 100-\mathrm{B} / \mathrm{C}$}

Disciplime Environmental *Calc. No. 0100B-CA-V0268

Subject Human Health Risk Assessment of Dieldrin at the 100-B-14:2 (Area 3) Pipeline Waste Sub-Site

Computer Program Excel Program No. Excel 2003

The attached calculations have been generated to document compliance with established cleanup levels. These documents should be used in conjunction with other relevant documents in the administrative record.

\section{Committed Calculation $\otimes \quad$ Preliminary $\square \quad$ Superseded $\square \quad$ Voided $\square$}

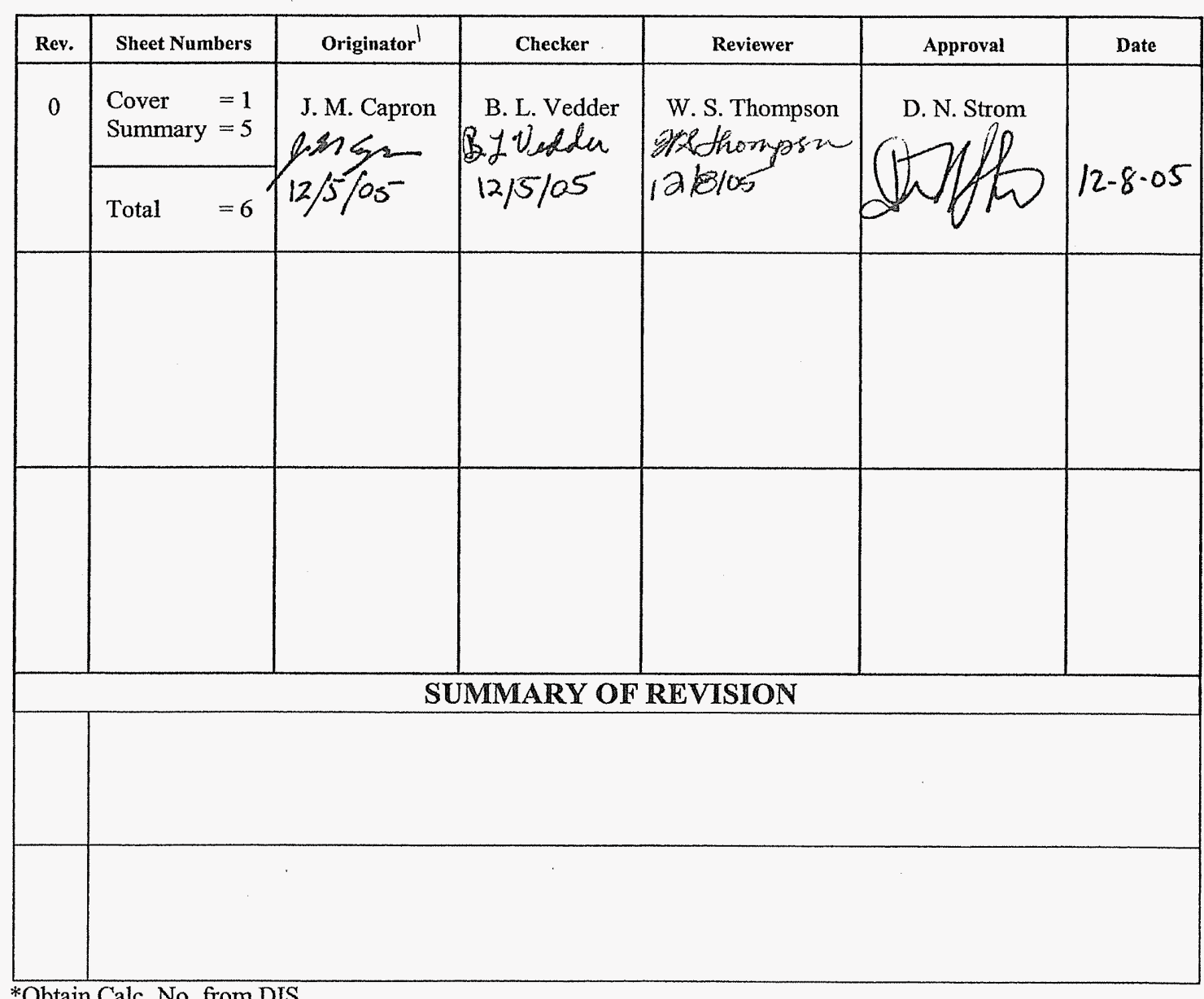

DE01-437.03 (12/09/2004) 
Tinin River Corrildor Closure Project

\begin{tabular}{|c|c|c|c|c|c|c|c|}
\hline Originator: & J. M. Capron & Date: & $12 / 5 / 05$ & Calc. No.: & 0100B-CA-V0268 & Rev.: & 0 \\
\hline Project: & 100-B/C Field/Remediation & Job No: & 14655 & Checked: & B. L. Vedder DIVILdu & Date: & $12 / 5 / 05$ \\
\hline
\end{tabular}

Subject: Human Health Risk Assessment for Dieldrin at the 100-B-14:2, Area 3, Pipeline Waste Sub-Site Sheet No. 1 of 5

1

2

3

4

5

6

7

\section{PURPOSE:}

Calculate the excess carcinogenic risk from the residual concentration of dieldrin at the 100-B-14:2 (Area 3) pipeline waste sub-site.

\section{GIVEN/REFERENCES:}

1) Analytical results for samples J037M6, J037M7, and J037M8.

2) Remedial Design Report/Remedial Action Work Plan for the 100 Area (RDR/RAWP), DOE/RL-96-17, Rev. 5, U.S. Department of Energy, Richland Operations Office, Richland, Washington.

3) Equations for calculating contaminant intake from Appendix D of Hanford Site Risk Assessment Methodology (HSRAM), DOE/RL-91-45, Rev. 3, U.S. Department of Energy, Richland Operations Office, Richland, Washington.

4) Use of area factors and occupancy factors to account for small waste site size and actual period of occupancy in the rural-residential scenario is discussed in the User's Manual for RESRAD Version 6, ANL/EAD-4, Environmental Assessment Division, Argonne National Laboratory, Argonne, Illinois.

\section{SOLUTION:}

1) Table 1 shows the risk assessment input parameters used for this calculation.

Table 1. Risk Assessment Input Parameters.

\begin{tabular}{|c|c|c|c|}
\hline Parameter & Units & Value & Citation \\
\hline Area of contaminated zone & $\mathrm{m}^{2}$ & 57.1 & Site-specific \\
\hline Exposure duration, adult & years & 24 & HSRAM \\
\hline Exposure duration, child & years & 6 & HSRAM \\
\hline Fraction of time spent indoors & unitless & 0.6 & RDR/RAWP \\
\hline Fraction of time spent outdoors (on-site) & unitless & 0.2 & RDR/RAWP \\
\hline Soil ingestion rate, adult & $\mathrm{g} / \mathrm{yr}$ & 36.5 & HSRAM \\
\hline Soil ingestion rate, child & $\mathrm{g} / \mathrm{yr}$ & 73 & HSRAM \\
\hline Inhalation rate & $\mathrm{m}^{3} / \mathrm{yr}$ & 7,300 & RDR/RAWP \\
\hline Dust mass loading for inhalation & $\mathrm{g} / \mathrm{m}^{3}$ & 0.0001 & RDR/RAWP \\
\hline Wind speed & $\mathrm{m} / \mathrm{s}$ & 3.4 & RDR/RAWP \\
\hline Body weight, adult & $\mathrm{kg}$ & 70 & HSRAM \\
\hline Body weight, child & $\mathrm{kg}$ & 16 & HSRAM \\
\hline Average lifetime & $\mathrm{yr}$ & 70 & HSRAM \\
\hline
\end{tabular}

2) Table 2 shows the contaminant-specific risk assessment input parameters for the inhalation and soil ingestion pathways. Dieldrin has a high distribution coefficient $(25.6 \mathrm{~mL} / \mathrm{g})$ and 


\section{WT: River Corridor} F- Closure Projege

\begin{tabular}{|c|c|c|c|c|c|c|c|}
\hline Originator: & J. M. Capron pane & Date: & $12 / 5 / 05$ & Calc. No.: & $0100 \mathrm{~B}-\mathrm{CA}-\mathrm{V} 0268$ & Rev.: & 0 \\
\hline Project: & 100-B/C Field Remediation & Job No: & 14655 & Checked: & B. L. Vedder 324 & Date: & $19 / 5 / 05$ \\
\hline Subject: & \multicolumn{5}{|c|}{ Human Health Risk Assessment for Dieldrin at the 100-B-14:2, Area 3, Pipeline Waste Sub-Site } & \multicolumn{2}{|c|}{ Sheet No. 2 of 5} \\
\hline
\end{tabular}

will not migrate through the vadose zone at the site in water-dependent pathways within 1,000 years. Only the inhalation and soil ingestion pathways will be affected.

Table 2. Contaminant-Specific Cancer Slope Factor Values.

\begin{tabular}{|c|c|c|}
\hline Contaminant & $\begin{array}{c}\mathrm{CSFi}^{\mathrm{a}} \\
(\mathrm{kg-d} / \mathrm{mg})\end{array}$ & $\begin{array}{c}\mathrm{CSFo}^{\mathrm{a}} \\
(\mathrm{kg}-\mathbf{d} / \mathbf{m g})\end{array}$ \\
\hline Dieldrin & 16.1 & 16 \\
\hline \multicolumn{2}{|c|}{ CSFi/CSFo = Cancer slope factor for dust inhalation or soil ingestion. } \\
Refers to the chemical-specific cancer slope factors used to calculate \\
carcinogenic risk. Obtained from the U. S. Environmental Protection \\
Agency's Integrated Risk Information System database.
\end{tabular}

\section{METHODOLOGY:}

\section{1) Excess Carcinogenic Risk:}

The incremental excess carcinogenic risk is calculated from the following general formula:

$$
\mathrm{ICR}=(\text { Daily Intake }) \times(\mathrm{CSF})
$$

Where, $\mathrm{CSF}=$ the cancer slope factor with units of $\mathrm{kg}$ - day/mg. As applicable, the

U.S. Environmental Protection Agency (EPA) provides separate values of the cancer slope factor for the inhalation and oral ingestion pathways (CSFi and CSFo, respectively).

\section{2) Daily Intake for the Soil Ingestion Pathway:}

Daily intake for the soil ingestion pathway is calculated from the following formula from HSRAM Equation D-24, including the area factor and occupancy factor from the User's Manual for RESRAD Version 6:

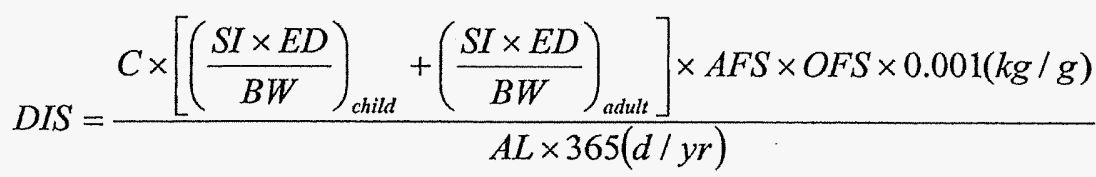

Where, $\mathrm{C}$ is contaminant concentration, (site-specific statistical value, $\mathrm{mg} / \mathrm{kg}$ )

$\mathrm{SI}$ is Soil Ingestion Rate, $(\mathrm{g} / \mathrm{yr})$

AFS is an area factor for soil ingestion: $\mathrm{AFS}=\mathrm{A} / 1000$ for $\mathrm{A}<1000 \mathrm{~m}^{2}$

$\mathrm{AFS}=1$ for $\mathrm{A}>1000 \mathrm{~m}^{2}$

$A$ is the area of the contaminated zone, $\mathrm{m}^{2}$

OFS is the occupancy factor for soils: OFS $=(\mathrm{IT})+(\mathrm{OT})$

IT is the Indoor Time Factor (0.6)

OT is the Outdoor Time Factor (0.2)

ED is exposure duration (yr)

$\mathrm{OFS}=0.6+0.2=0.8$

$\mathrm{BW}$ is body weight $(\mathrm{kg})$

$\mathrm{AL}$ is average lifetime (yr) 
1017 River Corridor Closure Project

\begin{tabular}{|c|c|c|c|c|c|c|c|}
\hline Originator: & J. M. Capron & Date: & $12 / 5 / 05$ & Calc. No.: & 0100B-CA-V0268 & Rev.: & 0 \\
\hline Project: & 100-B/C Field Remediation & Job No: & 14655 & Checked: & B. L. Vedder $(2)^{10}$ & Date: & $12 / 5 / 05$ \\
\hline Subject: & \multicolumn{5}{|c|}{ Human Health Risk Assessment for Dieldrin at the 100-B-14:2, Area 3, Pipeline Waste Sub-Site } & \multicolumn{2}{|c|}{ Sheet No. 3 of 5} \\
\hline
\end{tabular}

Daily Intake for the Inhalation Pathway:

Daily intake for the inhalation pathway is calculated using the following formula from HSRAM Equation D-30, including the area factor and occupancy factor from the User's Manual for RESRAD Version 6:

$D I I=\frac{C \times I R \times M L \times E D \times A F I \times O F I \times 0.001(\mathrm{~kg} / \mathrm{g})}{B W \times A L \times 365(d / y r)}$

Where, $\mathrm{C}$ is contaminant concentration, (site-specific statistical value, $\mathrm{mg} / \mathrm{kg}$ ) IR is Inhalation Rate, $\left(\mathrm{m}^{3} / \mathrm{yr}\right)$

ML is Mass Loading, $\left(\mathrm{g} / \mathrm{m}^{3}\right)$

ED is exposure duration (yr)

AFI is the area factor for dust inhalation calculated from formula B.4 of the User's

Manual for RESRAD Version 6:

$$
A F I=\frac{a}{1+b(\sqrt{A})^{c}}
$$

In this equation, $\mathrm{A}$ is the area of the contaminated zone, $\mathrm{m}^{2}$, and $\mathrm{a}, \mathrm{b}$, and $\mathrm{c}$ are least squares regression coefficients dependent upon the average wind speed as described in Table B.2 of the User's Manual for RESRAD Version 6. Calculation results are shown in the Results section of this Calculation Summary.

OFI is the occupancy factor for inhalation: OFI $=(\mathrm{IT} \times \mathrm{IDF})+(\mathrm{OT})$

IT is the Indoor Time Factor (0.6)

$\mathrm{IDF}=$ Indoor dust filtration factor (0.4)

OT is the Outdoor Time Factor (0.2)

$\mathrm{BW}$ is body weight $(\mathrm{kg})$

$\mathrm{OFI}=(0.6 \times 0.4)+0.2=0.44$

\section{RESULTS:}

Calculations were performed using an Excel spreadsheet, incorporating the formulae shown in the Methodology section of this Calculation Summary.

1) Excess Carcinogenic Risk from the Soil Ingestion Pathway:

Table 3 shows the results from the Excel-based calculation of excess incremental carcinogenic risk from dieldrin in the soil ingestion pathway. 
Tin: River Corridor Closure Project

\begin{tabular}{|c|c|c|c|c|c|c|c|}
\hline Originator: & J. M. Capron & Date: & $12 / 5 / 05$ & Calc. No.: & 0100B-CA-V0268 & Rev.: & 0 \\
\hline & 100-B/C Field Remediation & Job No: & 14655 & Checked: & B. L. Vedder BLIV & Date: & 015105 \\
\hline Subject: & \multicolumn{5}{|c|}{ Human Health Risk Assessment for Dieldrin at the 100-B-14:2, Area 3, Pipeline Waste Sub-Site } & \multicolumn{2}{|c|}{ Sheet No. 4 of 5} \\
\hline
\end{tabular}

Table 3. Excel Calculation of Excess Incremental Carcinogenic Risk from the Soil Ingestion Pathway.

2

3

4

5

6

7

8

9

10

11

12

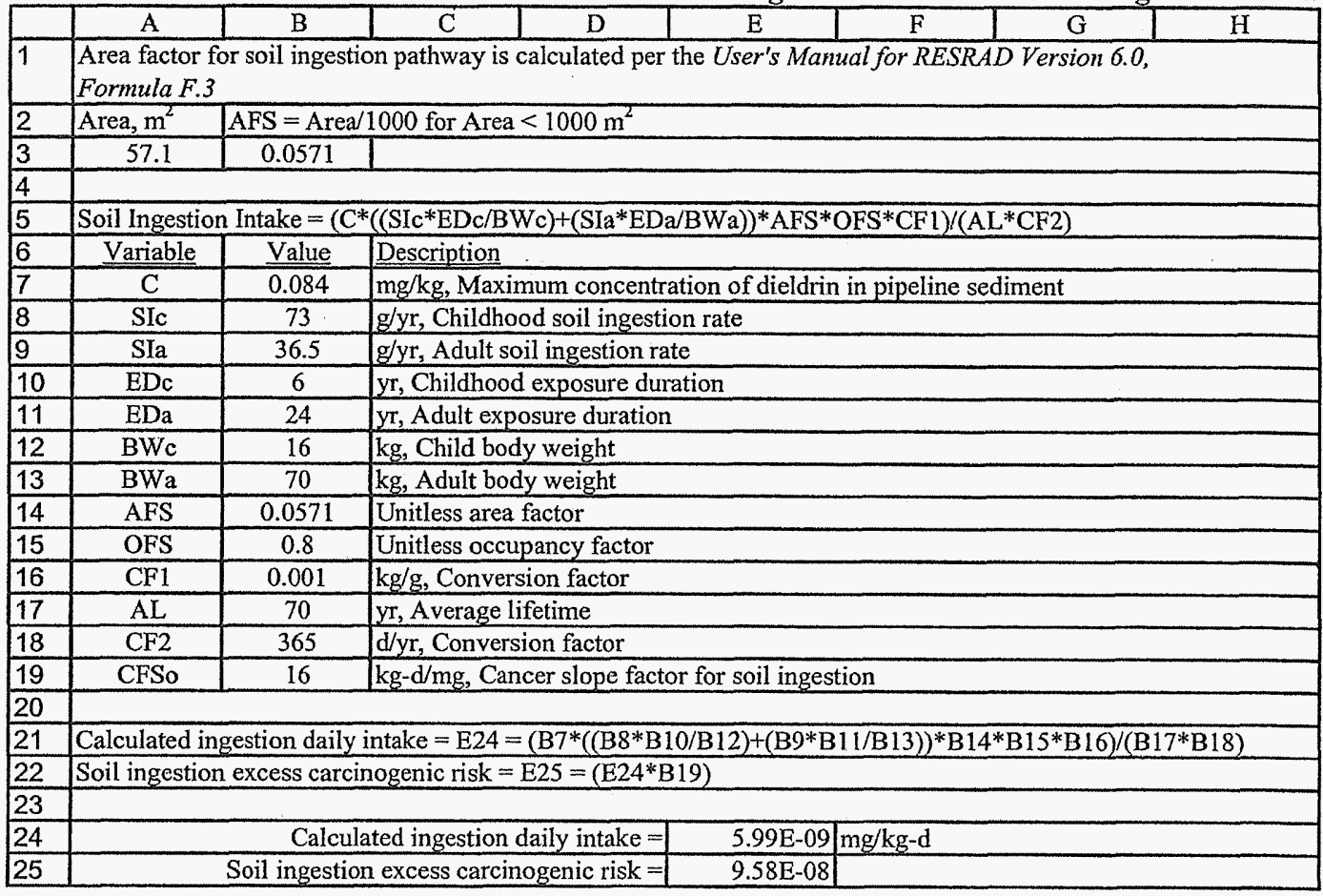

2) Incremental Cancer Risk from the Inhalation Pathway:

Table 4 shows the results from the Excel-based calculation of excess incremental carcinogenic risk from dieldrin in the dust inhalation pathway. 


\section{Mint Rer Corrildor Closere Prolecter}

CALCULATION SHEET

\begin{tabular}{|c|c|c|c|c|c|c|c|}
\hline jinator: & J.M. Capron ge & Date: & $12 / 5 / 05$ & Calc. No.: & 0100B-CA-V0268 & Rev.: & 0 \\
\hline Project: & 100-B/C Field Remediation & Job No: & 14655 & Checked: & B. L. Vedder MV & Date: & 1215105 \\
\hline
\end{tabular}

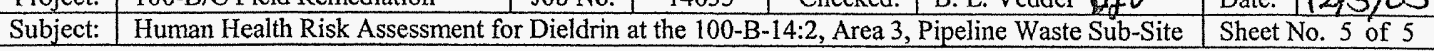

Table 4. Excel Calculation of Excess Incremental Carcinogenic Risk from the Dust Inhalation Pathway.

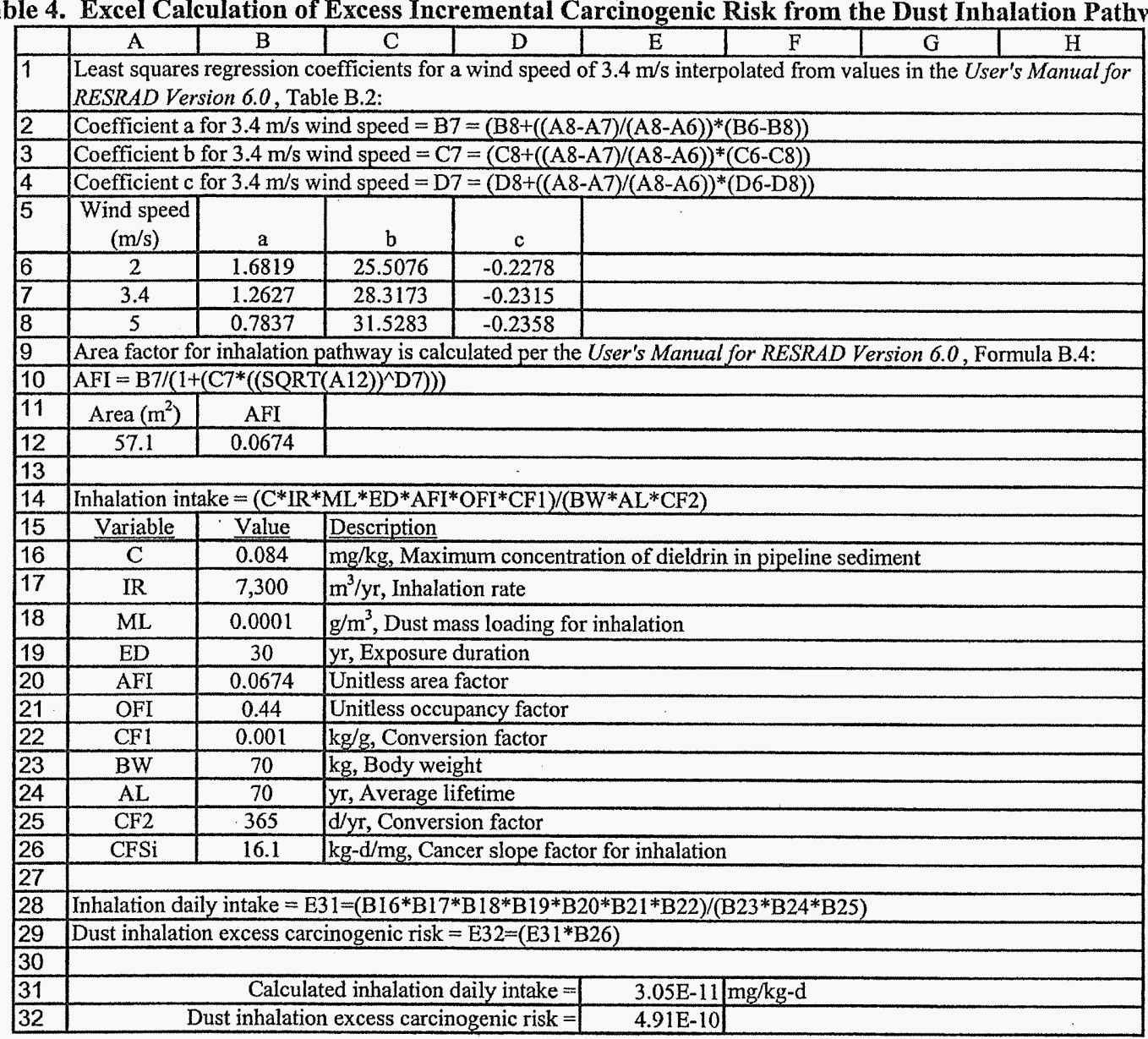

\section{CONCLUSIONS:}

- The excess carcinogenic risk due to dieldrin in the soil ingestion pathway is $9.58 \times 10^{-8}$.

- The excess carcinogenic risk due to dieldrin in the inhalation pathway is $4.91 \times 10^{-10}$.

- The total human health excess carcinogenic risk due to dieldrin at the 100-B-14:2, Area 3, pipeline waste sub-site is the sum of the excess carcinogenic risks from the soil ingestion and inhalation pathways: $9.63 \times 10^{-8}$. 


\section{CALCULATION COVER SHEET}

Project Title $100-\mathrm{B} / \mathrm{C}$ Field Remediation

Job No. 14655

Area $100-\mathrm{B} / \mathrm{C}$

Discipline Environmental

*Calc. No. O100B-CA-V0269

Subject 100-B-14:2 (Area 3) Hazard Quotient and Carcinogenic Risk Calculations

Computer Program Excel Program No. Excel 2003

The attached calculations have been generated to document compliance with established cleanup levels. These calculations should be used in conjunction with other relevant documents in the administrative record.

Committed Calculation $\otimes \quad$ Preliminary $\square \quad$ Superseded $\square \quad$ Voided

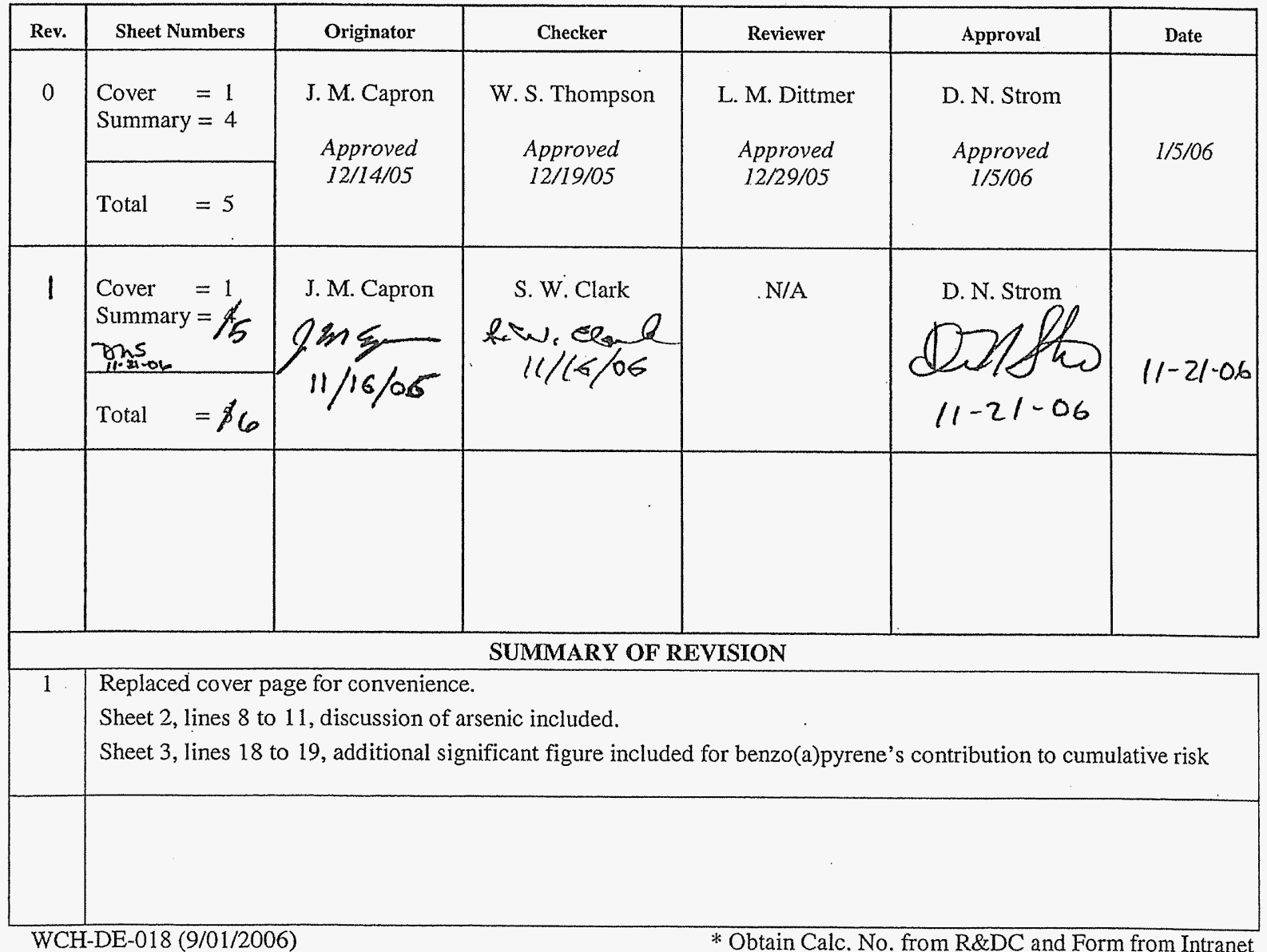


Washington Closure Hanford

CALCULATION SHEET

\begin{tabular}{|c|c|c|c|c|c|c|c|}
\hline Originator: & J.M. Capron gen & Date: & $12 / 14 / 05$ & Calc. No: & $0100 \mathrm{~B}-\mathrm{CA}-\mathrm{V} 0269$ & Rev.: & 0 \\
\hline Project: & 100-B/C Field 6 Remediation & Job No: & 14655 & Checked: & W.S. Thompson Th & Date: & 12119105 \\
\hline
\end{tabular}

\section{PURPOSE:}

Provide documentation to support the calculation of the hazard quotient (HQ) and excess carcinogenic risk values for the 100-B-14:2 (Area 3) pipelines. In accordance with the remedial action goals (RAGs) in the remedial design report/remedial action work plan (RDR/RAWP) (DOE-RL 2005), the following criteria must be met:

1) An HQ of $<1.0$ for all individual noncarcinogens

2) A cumulative $H Q$ of $<1.0$ for noncarcinogens

3) An excess cancer risk of $<1 \times 10^{-6}$ for individual carcinogens

4) A cumulative excess cancer risk of $<1 \times 10^{-5}$ for carcinogens.

\section{GIVEN/REFERENCES:}

1) Analytical results for samples J037M6, J037M7, and J037M8.

2) DOE-RL, 2005, Remedial Design Report/Remedial Action Work Plan for the 100 Areas, DOE/RL-96-17, Rev. 5, U.S. Department of Energy, Richland Operations Office, Richland, Washington.

3) WAC 173-340, "Model Toxics Control Act - Cleanup," Washington Administrative Code, 1996.

4) WCH, 2005a, Human Health Risk Assessment of Dieldrin at the 100-B-14:2 (Area 3) Pipeline Waste Sub-Site, 0100B-CA-V0268, Rev, 0, Washington Closure Hanford, Richland, Washington.

5) WCH, 2005b, Human Health Risk Assessment for PCB's at the 100-B-14:2, Area 3, Pipeline Waste Sub-Site, 0100B-CA-V0266, Rev. 1, Washington Closure Hanford, Richland, Washington.

\section{SOLUTION:}

1) Generate an HQ for each noncarcinogenic constituent detected above background or required detection limit/practical quantitation limit and compare it to the individual HQ of $<1.0$ (DOE-RL 2005).

2) Sum the HQs and compare to the cumulative HQ criterion of $<1.0$.

3) Generate an excess cancer risk value for each carcinogenic constituent detected above background or required detection limit/practical quantitation limit and compare it to the excess cancer risk of $<1 \times 10^{-6}$ (DOE-RL 2005).

4) Sum the excess cancer risk values and compare to the cumulative cancer risk criterion of $<1 \times 10^{-5}$.

\section{METHODOLOGY:}

Hazard quotient and carcinogenic risk calculations were computed using the higher of the values between underlying soil results and pipe sediment results. Of the detected analytes for the site, boron, molybdenum, strontium, and tin require the risk calculations because these analytes were detected and a Washington State or Hanford Site background value is not available. Barium, cadmium, chromium, 
Washington Closure Hanford CALCULATION SHEET

\begin{tabular}{|r|l|r|c|c|c|c|c|}
\hline Originator: & J. M. Capron 22ne & Date: & $11 / 16 / 06$ & Calc. No.: & 0100 B-CA-V0269 & Rev.: & 1 \\
\hline Project: & $100-$ B/C Field Remediation & Job No: & 14655 & Checked: & S. W. Clark \& Swe & Date: & $11 / 16 / 65$ \\
\hline Subject: & $100-$ B-14:2 (Area 3) Hazard Quotient and Carcinogenic Risk Calculations & Sheet No. 2 of 4 \\
\hline
\end{tabular}

1 copper, mercury, nickel, silver, and zinc are included because they were detected at concentrations 2 above their respective Washington State or Hanford Site background value. Lead does not have a 3 reference dose for calculation of a hazard quotient because toxic effects of lead are correlated with 4 blood-lead levels rather than exposure levels or daily intake. As a result, the maximum lead 5 concentration is reported but not included in the hazard quotient calculation. Hexavalent chromium, 6 aroclor-1248, arcolor-1260, multiple pesticides, and multiple semivolatile organic analytes (as shown in 7 Table 1) are included because they were detected by laboratory analysis and cannot be attributed to 8 natural occurrence. Arsenic was detected above the Hanford Site Background value but below the 9 WAC 173-340 Method A cleanup level. Due to the intent of Method A cleanup values and the allowance to use such values for arsenic (DOE-RL 2005), arsenic has been excluded from the Method B individual analyte and cumulative risk requirements. An example of the $\mathrm{HQ}$ and risk calculations is presented below:

1) For example, the maximum value for boron is $4.4 \mathrm{mg} / \mathrm{kg}$, divided by the noncarcinogenic $\mathrm{RAG}$ value of $16,000 \mathrm{mg} / \mathrm{kg}$ (boron is identified as a noncarcinogen in WAC 173-340-740[3]), is $2.8 \times 10^{-4}$. Comparing this value, and all other individual values, to the requirement of $<1.0$, this criterion is met.

2) After the HQ calculation is completed for the appropriate analytes, the cumulative HQ is obtained by summing the individual values. (To avoid errors due to intermediate rounding, the individual HQ values prior to rounding are used for this calculation.) The sum of the HQ values is $6.0 \times 10^{-1}$, which satisfies the requirement of $<1.0$.

3) Residual dieldrin and aroclor -1248 concentrations in pipeline sediment exceed the carcinogenic direct exposure RAG values calculated by WAC 173-340-740(3), Method B. However, these RAGs were calculated based on generic assumptions that result in highly conservative values. Site-specific risk assessments (WCH 2005a, 2005b) were therefore performed and residual concentrations of dieldrin and aroclor-1248 shown to satisfy the WAC 173-340-740(3), Method B carcinogenic requirements for human health protection from soil direct exposure.

4) To calculate the excess carcinogenic risk for all other residual contaminants, the maximum value is divided by the carcinogenic RAG value, then multiplied by $1 \times 10^{-6}$. For example, the maximum value for benzo(a)anthracene is $0.21 \mathrm{mg} / \mathrm{kg}$; divided by $1.37 \mathrm{mg} / \mathrm{kg}$ and multiplied as indicated is $1.5 \times 10^{-7}$. Comparing this value, and all other individual values, to the requirement of $<1 \times 10^{-6}$, this criterion is met.

5) After these calculations are completed for the carcinogenic analytes, the cumulative excess cancer risk is obtained by summing the individual values. (To avoid errors due to intermediate rounding, the individual excess carcinogenic risk values prior to rounding are used for this calculation.) The sum of the excess cancer risk values is $2.8 \times 10^{-6}$, which satisfies the requirement of $<1 \times 10^{-5}$.

\section{RESULTS:}

1) List individual noncarcinogens and corresponding $H Q s>1.0$ : None

2) List the cumulative noncarcinogenic $\mathrm{HQ}>1.0$ : None

3) List individual carcinogens and corresponding excess cancer risk $>1 \times 10^{-6}$ : None 
Washington Closure Hanford CALCULATION SHEET Originator: J. M. Capron Q2zec Project: $100-\mathrm{B} / \mathrm{C}$ Field Remediation \begin{tabular}{c|c|c|c|} 
Date: & $11 / 16 / 06$ & Calc. No.: & 0100B-CA-V0269 \\
\hline
\end{tabular} Job No: 14655 Checked: S. W. Clark 70 , Subject: 1 100-B-14:2 (Area 3) Hazard Quotient and Carcinogenic Risk Calculations

4) List the cumulative excess cancer risk for carcinogens $>1 \times 10^{-5}$ : None.

Table 1 shows the results of the hazard quotient and excess carcinogenic risk calculations. 
Washington Closure Hanford

CALCULATION SHEET

\begin{tabular}{r|l|r|c|c|c|}
\hline Originator: & J. M. Capron qoose & Date: & $11 / 16 / 06$ & Calc. No.: & 0100B-CA-V0269 \\
\hline Project: & 100 -B/C Field Remediation & Job No: & 14655 & Checked: & S. W. Clark
\end{tabular}

Subject: $100-$ B-14:2 (Area 3) Hazard Quotient and Carcinogenic Risk Calculations

Table 1. Hazard Quotient and Excess Carcinogenic Risk Results for the 100-B-14:2 (Area 3) Pipelines.

5

6

7

8

9

10

11

\section{Maximum}

\begin{tabular}{|c|c|c|c|c|c|}
\hline Contaminants of Potential Concern & $\begin{array}{c}\text { Maximum } \\
\text { Value }^{\mathrm{a}} \\
(\mathrm{mg} / \mathrm{kg})\end{array}$ & $\begin{array}{c}\text { Noncarcinogen } \\
\mathrm{RAG}^{\mathrm{b}} \\
(\mathrm{mg} / \mathrm{kg})\end{array}$ & $\begin{array}{c}\text { Hazard } \\
\text { Quotient }\end{array}$ & $\begin{array}{c}\text { Carcinogen } \\
\mathrm{RAG}^{\mathrm{b}} \\
(\mathrm{mg} / \mathrm{kg})\end{array}$ & Carcinogen Risk \\
\hline
\end{tabular}

Barium

Barium

Cadmium

Chromium, total

Chromium, hexavalent ${ }^{c}$

Copper

Lead

Mercury

Molybdenum

Nickel

Silver

Strontium

Tin

Zinc

\begin{tabular}{l|r}
998 & 5,600 \\
\hline 4,4 & 16,000 \\
\hline
\end{tabular}

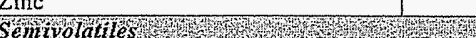

Benzo(a)anthracene

Benzo(a)pyrene ${ }^{\text {d }}$

Benzo(b)fluoranthene

Benzo(k)fluoranthene

Benzo(ghi)perylene

Bis(2-ethylhexyl) phthalate

Butylbenzylphthalate

Chrysene

Di-n-butylphthalate

Fluoranthene

Indeno(1,2,3-cd) pyrene

Phenanthrene $^{c}$

Pyrene

\begin{tabular}{l|l}
5,600 & $1.8 \mathrm{E}-0$ \\
\hline
\end{tabular}

80

$2.8 \mathrm{E}-04$

\begin{tabular}{r|r}
1.5 & 80 \\
\hline 67.8 & 120.0 \\
\hline
\end{tabular}

24000

\begin{tabular}{l|r}
0.25 & 240 \\
\hline 102 & 2,96 \\
\hline
\end{tabular}

\begin{tabular}{c|c}
279 & -- \\
\hline 7.2 & 24 \\
\hline
\end{tabular}

\begin{tabular}{l|c|c}
7.7 & 24 & $3.0 \mathrm{E}-0$ \\
\hline 1.7 & 400 & $4.3 \mathrm{E}-03$
\end{tabular}

\begin{tabular}{l|l}
1.7 & 400 \\
\hline 34.0 & 1.600
\end{tabular}

2.8

\begin{tabular}{l|r}
2.8 & 400 \\
\hline 40.4 & 48,000
\end{tabular}

\begin{tabular}{l|l}
\hline 13.5 & 48,000 \\
\hline 223 & 48,000 \\
\hline
\end{tabular}

24,000

$1.9 \mathrm{E}-02$

BHC, beta (Hexachlorocyclohexane)

Chlordane (alpha, gamma)

DDD, 4, 4'

DDE, $4,4^{\circ}$ -

DDT, 4, 4'

Dieldrin

Endosulfan (I, II, sulfate)

Endrin (and ketone, aldehyde)

Methoxychlor

\begin{tabular}{|c|c|}
\hline- & - \\
\hline- & - \\
\hline 13.9 & $1.1 \mathrm{E}-07$ \\
\hline- & - \\
\hline
\end{tabular}

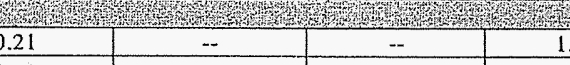

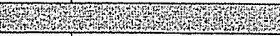

olychlonimated Biphenyls on

Aroclor-1248

Aroclor-1260

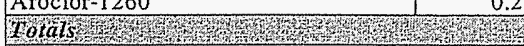

Cumulative Hazard Quotient:

Cumulative Excess Cancer Risk:

Notes:

$\mathrm{RAG}=$ remedial action goal

-. = not applicable

${ }^{2}=$ Maximum value determined by comparison of analytical results for samples J037M6, J037M7, and J037M8.

${ }^{b}=$ Value obtained from Washington Administrative Code (WAC) 173-340-740(3), Method B, 1996, unless otherwise noted.

${ }^{c}=$ Value for the carcinogen RAG calculated based on the inhalation exposure pathway (WAC) 173-340-750(3), 1996.

$\mathrm{d}=$ Individual carcinogenic risk (5.5E-07) calculated using the required detection limit $(0.33 \mathrm{mg} / \mathrm{kg})$. Contribution to cumulative carcinogenic

risk (1.3E-06) calculated based on the cleanup level $(0.137 \mathrm{mg} / \mathrm{kg}$ ) instead of the required detection limit, per WAC 173-340-740(3),

Method B, 1996.

$c=$ Toxicity data are not available for phenanthrene; RAG based on the surrogate chemical anthracene.

${ }^{r}=$ Value from site-specific risk assessment (WCH 2005a).

$s=$ Value from site-specific risk assessment (WCH 2005b). 
Washington Closure Hanford CALCULATION SHEET

\begin{tabular}{|l|l|l|l|l|l}
\hline Originator: & J. M. Capron Ganc & Date: & $12 / 14 / 05$ & Calc. No.: & 0100B-CA-V0269
\end{tabular}

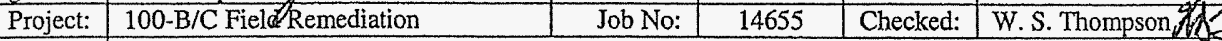

Date: 1219105

Subject: 100-B-14:2 (Area 3) Hazard Quotient and Carcinogenic Risk Calculations

Sheet No. 4 of 4

\section{CONCLUSION:}

3 This calculation demonstrates that the 100-B-14:2 (Area 3) pipelines meet the requirements for the

4 hazard quotient and excess carcinogenic risk as identified in the RDR/RAWP (DOE-RL 2005). 


\section{CALCULATION COVER SHEET}

Project Title:

Area

Discipline

Subject

Computer Program
$\frac{100-\mathrm{B} / \mathrm{C}}{100-\mathrm{B} / \mathrm{C}}$

Environmental

100-B-14:2 (Areas 2 \& 5) Waste Site Cleanup Verification 95\% UCL Calculations
Job No.

14655

The attached calculations have been generated to document compliance with established cleanup levels. These calculations should be used in conjunction with other relevant documents in the administrative record.

Committed Calculation

冈

Preliminary $\square \quad$ Superseded

Voided

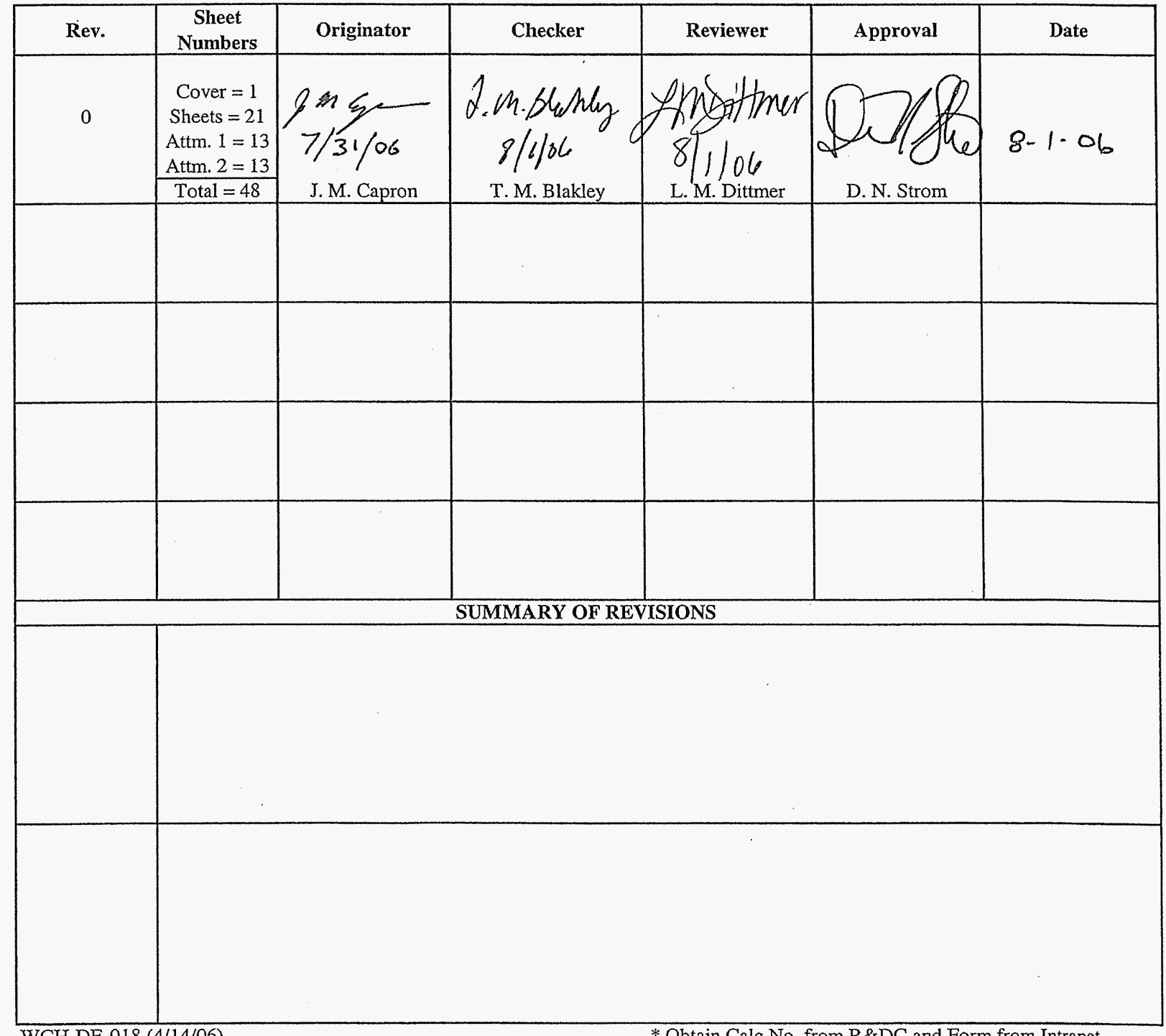

WCH-DE-018 (4/14/06)

* Obtain Calc No. from R\&DC and Form from Intranet 


\section{Washington Closure Hanford}

CALCULATION SHEET

\begin{tabular}{|c|c|c|}
\hline Originator J.M. Capron \& 20 & Calc. No. 0100B-CA-V0281 & Rev. No. \\
\hline 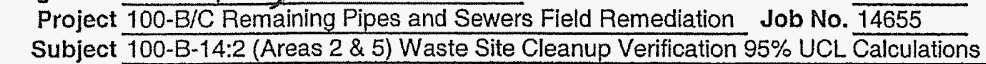 & Checked T. M. Blakley 20us & $\begin{array}{l}\text { Date } 8 / 106 \\
\text { Sheet No. } \frac{106}{101} 21\end{array}$ \\
\hline
\end{tabular}

Summary

1 Purpose:

2 Calculate the $95 \%$ upper confidence limit (UCL) values to evaluate compliance with cleanup standards for the remediation

3 footprints of Areas 2 and 5 of the 100-B-14:2 subsite. Also, perform the Washington Administrative Code (WAC) 173-340-

4 740(7)(e) 3-part test for each nonradioactive contaminant of concern (COC) and contaminant of potential concern (COPC) and

6 calculate the relative percent difference (RPD) for primary-duplicate sample pairs, as necessary.

8 Table of Contents:

Sheets 1 to 4 - Calculation Sheet Summary

10 Sheets 5 to 6 - Calculation Sheet 100-B-14:2 Area 2 Remediation Footprint Verification Data

11 Sheet 7 - Calculation Sheet 100-B-14:2 Area 2 Duplicate Analysis

12 Sheets 8 to 12 - Ecology Software (MTCAStat) Results (Area 2)

13 Sheets 13 to 15 - Calculation Sheet 100-B-14:2 Area 5 Remediation Footprint Verification Data

14 Sheet 16 - Calculation Sheet 100-B-14:2 Area 5 Duplicate Analysis

Sheets 17 to 21 - Ecology Software (MTCAStat) Results (Area 5)

17 Attachment 1 - 100-B-14:2 Area 2 Verification Sampling Results (13 sheets)

20 Given/References:

21 1) Sample Results (Attachments 1 and 2).

2 2) Background values and remedial action goals (RAGs) are taken from DOE-RL (2005b), DOE-RL (2001), Ecology (1994), and Ecology (2005).

3) DOE-RL, 2001, Hanford Site Background: Part 1, Soil Background for Nonradioactive Analytes, DOE/RL-92-24, Rev. 4, U.S. Department of Energy, Richland Operations Office, Richland, Washington.

4) DOE-RL, 2005a, 100 Area Remedial Action Sampling and Analysis Plan (SAP), DOE/RL-96-22, Rev. 4, U.S. Department of Energy, Richland Operations Office, Richland, Washington.

5) DOE-RL, 2005b, Remedial Design Report/Remedial Action Work Plan for the 100 Area (RDR/RAWP), DOE/RL-96-17, Rev. 5, U.S. Department of Energy, Richland Operations Office, Richland, Washington.

6) Ecology, 1992, Statistical Guidance for Ecology Site Managers, Publication \#92-54, Washington Department of Ecology, Olympia, Washington.

7) Ecology, 1993, Statistical Guidance for Ecology Site Managers, Supplement S-6, Analyzing Site or Background Data with Below-detection Limit or Below-PQL Values (Censored Data Sets), Publication \#92-54, Washington Department of Ecology, Olympia, Washington.

8) Ecology, 1994, Natural Background Soil Metals Concentrations in Washington State, Publication No. 94-115, Washington State Department of Ecology, Olympia, Washington.

8) Ecology, 2005, Cleanup Levels and Risk Calculations (CLARC) Database, Washington State Department of Ecology, Olympia, Washington, <https://fortress.wa.gov/ecy/clarc/CLARCHome.aspx>.

9) EPA, 1994, USEPA Contract Laboratory Program National Functional Guidelines for Inorganic Data Review, EPA 540/R-94/013. U.S. Environmental Protection Agency, Washington, D.C.

10) WAC 173-340, 1996, "Model Toxic Control Act - Cleanup," Washington Administrative Code.

Solution:

Calculation methodology is described in Ecology Pub. \#92-54 (Ecology 1992, 1993), below, and in the RDR/RAWP (DOE-RL 2005b). Use data from attached worksheets to perform the $95 \%$ UCL calculation for each analyte, the

WAC 173-340-740(7)(e) 3-part test for nonradionuclides, and the RPD calculations, as required. The hazard quotient and carcinogenic risk calculations are located in a separate calculation brief as an appendix to the Remaining Sites Verification Package (RSVP).

\section{Calculation Description:}

The subject calculations were performed on data from soil verification samples from the subject waste site. The data were entered into an EXCEL 2003 spreadsheet and calculations performed by using the built-in spreadsheet functions and/or creating formulae within the cells. The statistical evaluation of data for use in accordance with the RDR/RAWP (DOE-RL 2005b) is documented by this calculation. Duplicate RPD results are used in evaluation of data quality within the RSVP for this site. 
CALCULATION SHEET

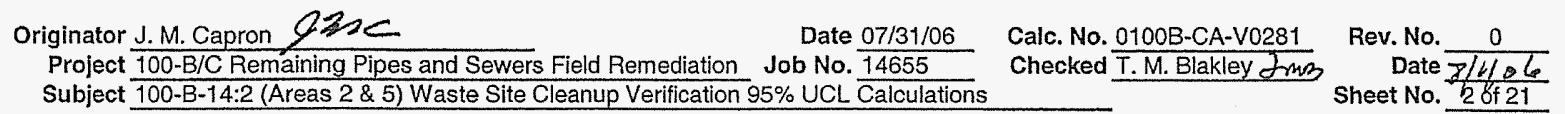

Summary (continued)

1 Methodology:

2 For nonradioactive analytes with $\leq 50 \%$ of the data below detection limits and detected radionuclide analytes (with the exception of

3 strontium-90 in Area 2), the statistical value calculated to evaluate the effectiveness of cleanup is the $95 \%$ UCL. For

4 nonradioactive analytes with $>50 \%$ of the data below detection limits, the maximum detected value for the data set is used instead

5 of the $95 \%$ UCL. The $95 \%$ UCL is not calculated for data sets with no reported detections. The evaluation of the portion of each

7 analyte's data set below detection limits was performed by direct inspection of the attached sample results, and no further

8 Calculations were performed for those data sets where $>50 \%$ of the data was below detection limits. Gross beta analysis results for statistical verification samples for Area 2 were all below background, with the exception of sample J11K91. Radiostrontium

10 analysis was requested for that sample only, and the result used as the residual strontium-90 activity for the Area 2 remediation

11 footprint. The $95 \%$ UCL values were not calculated for aluminum, calcium, iron, magnesium, phosphate, potassium, silicon,

12 sodium, and zirconium, as no cleanup values are available in Ecology (2005) under WAC 173-340-740(3), and these constituents

13 are thus not considered site COPCs (results for total phosphorus are attributed to phosphorus in phosphate).

All nonradionuclide data reported as being undetected are set to $1 / 2$ the detection limit value for calculation of the statistics (Ecology 1993). In cases where third-party validation requalified detected results as nondetected due to method blank contamination, but did not change the laboratory quantitation limit (e.g. , boron in Area 2), $1 / 2$ the reported value was used for calculation of the statistics. For radionuclide data, calculation of the statistics was done on the reported value. In cases where the laboratory does not report a value below the minimum detectable activity (MDA), half of the MDA is used in the calculation. For the statistical evaluation of duplicate sample pairs, the samples are averaged before being included in the data set, after adjustments for censored data as described above.

For nonradionuclides, the WAC 173-340 statistical guidance suggests that a test for distributional form be performed on the data and the $95 \%$ UCL calculated on the appropriate distribution using Ecology software. For nonradionuclide small data sets $(n<10)$ and all radionuclide data sets, the calculations are performed assuming nonparametric distribution, so no tests for distribution are performed. For nonradionuclide data sets of ten or greater, as for the subject site, distributional testing and calculation of the $95 \%$ UCL is done using Ecology's MTCAStat software (Ecology 1993). Due to differences in addressing censored data between the RDR/RAWP (DOE-RL 2005b) and MTCAStat coding and due to a limitation in the MTCAStat coding (no direct capability to address variable quantitation limits within a data set), substitutions for censored data are performed before software input and the resulting input set treated as uncensored.

The WAC 173-340-740(7)(e) 3-part test is performed for nonradionuclide analytes only and determines if: 1) the $95 \%$ UCL exceeds the most stringent cleanup limit for each $\mathrm{COPC} / \mathrm{COC}$,

2) greater than $10 \%$ of the raw data exceed the most stringent cleanup limit for each COPC/COC,

3) the maximum value of the raw data set exceeds two times the most stringent cleanup limit for each COPC/COC.

The WAC 173-340-740(7)(e) 3-part test is not performed for COPCs/COCs where the statistical value defaults to the maximum value in the data set. Instead, direct comparison of the maximum value against site RAGs (within the RSVP) is used as the compliance basis.

The RPD is calculated when both the primary value and the duplicate value for a given analyte are above detection limits and are greater than 5 times the target detection limit (TDL). The TDL is a laboratory detection limit pre-determined for each analytical method, listed in Table II-1 of the SAP (DOE-RL 2005a). Where direct evaluation of the attached sample data showed that a given analyte was not detected in the primary and/or duplicate sample, further evaluation of the RPD value was not performed. The RPD calculations use the following formula:

$$
\mathrm{RPD}=[|\mathrm{M}-\mathrm{S}| /((\mathrm{M}+\mathrm{S}) / 2)]^{*} 100
$$

where, $M=$ main sample value $\quad S=$ split (or duplicate) sample value

or quality assurance/quality control (QA/QC) split and duplicate RPD calculations, a value less than $30 \%$ indicates the data compare favorably. For regulatory splits, a threshold of $35 \%$ is used (EPA 1994). If the RPD is greater than $30 \%$ (or $35 \%$ for regulatory split data), further investigation regarding the usability of the data is performed. No split samples were collected for cleanup verification of the subject site. Additional discussion is provided in the data quality assessment section of the applicable RSVP, as necessary.

In addition to the statistical samples collected from the remediation footprint at the subject site, multi-aliquot samples were collected from stockpiles of overburden and other material assumed to be below cleanup levels. Statistical methodology is not applicable to non-statistical sampling, and direct evaluation of maximum detected values within these decision units will be used as the compliance basis. These maximum detected values are presented in the results summary for use in the RSVP. 
Washington Closure Hanford

CALCULATION SHEET

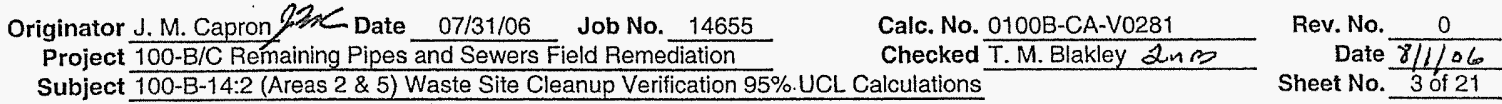

1 Summary (continued)

2 Results:

3 The results presented in the summary tables that follow are for use in risk analysis and the RSVP for this site.

5 Results Summary - Area 2 Remediation Footprint

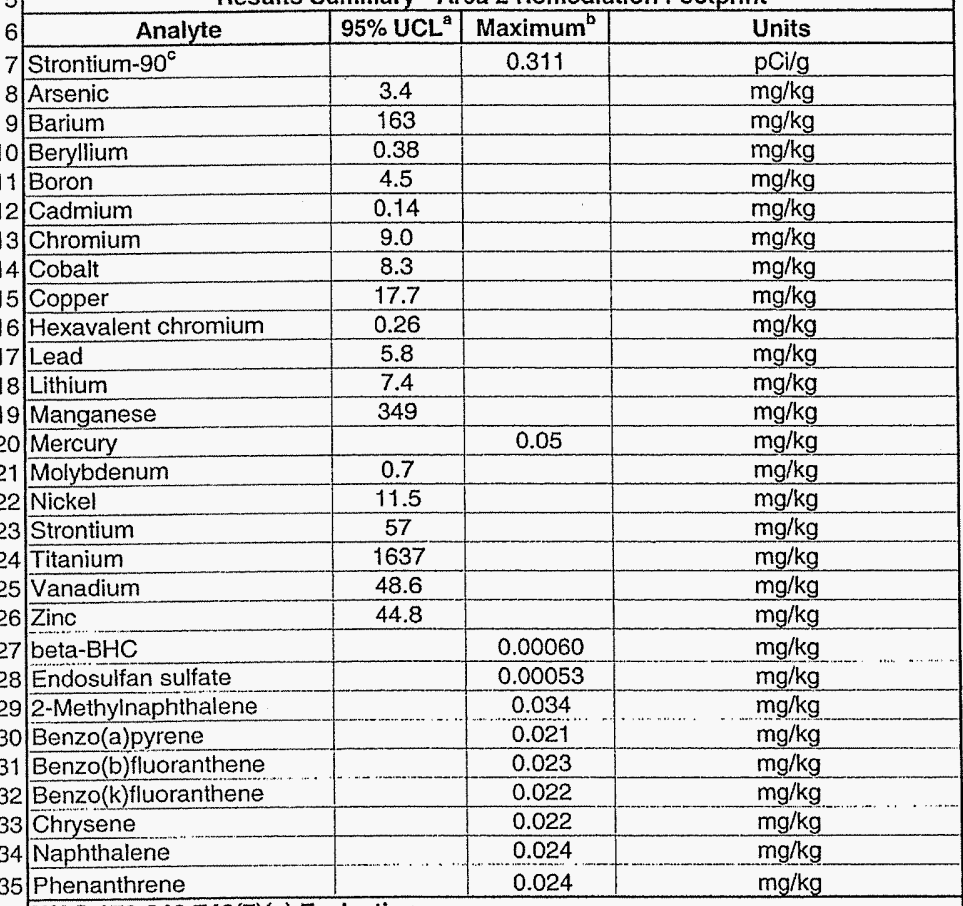

\section{WAC 173-340-740(7)(e) Evaluation}

38 WAC 173-340 3-Part Test for most stringent RAG:

39 95\% UCL > Cleanup Limit? YES

$40>10 \%$ above Cleanup Limit? NO

41 Any sample $>2 x$ Cleanup Limit? YES

Because of the "yes" answers to

the WAC 173-340 3-part test for

barium, additional evaluation of

the attainment of cleanup

criteria will be performed.

\begin{tabular}{|l|c|c|}
\hline \multicolumn{3}{|c|}{ Results Summary - Area 2 BCL Stockpiles } \\
\hline \multicolumn{1}{|c|}{ Analyte } & Maximum & Units \\
\hline Arsenic & 2.9 & $\mathrm{mg} / \mathrm{kg}$ \\
\hline Barium & 141 & $\mathrm{mg} / \mathrm{kg}$ \\
\hline Beryllium & 0.34 & $\mathrm{mg} / \mathrm{kg}$ \\
\hline Boron & 3.6 & $\mathrm{mg} / \mathrm{kg}$ \\
\hline Cadmium & 0.21 & $\mathrm{mg} / \mathrm{kg}$ \\
\hline Chromium & 8.9 & $\mathrm{mg} / \mathrm{kg}$ \\
\hline Cobalt & 9.2 & $\mathrm{mg} / \mathrm{kg}$ \\
\hline Copper & 17.6 & $\mathrm{mg} / \mathrm{kg}$ \\
\hline Hexavalent chromium & 0.28 & $\mathrm{mg} / \mathrm{kg}$ \\
\hline Lead & 6.0 & $\mathrm{mg} / \mathrm{kg}$ \\
\hline Lithium & 6.7 & $\mathrm{mg} / \mathrm{kg}$ \\
\hline Manganese & 351 & $\mathrm{mg} / \mathrm{kg}$ \\
\hline Mercury & 0.08 & $\mathrm{mg} / \mathrm{kg}$ \\
\hline Molybdenum & 0.51 & $\mathrm{mg} / \mathrm{kg}$ \\
\hline Nickel & 11.1 & $\mathrm{mg} / \mathrm{kg}$ \\
\hline Strontium & 57.1 & $\mathrm{mg} / \mathrm{kg}$ \\
\hline Titanium & 1490 & $\mathrm{mg} / \mathrm{kg}$ \\
\hline Vanadium & 47.2 & $\mathrm{mg} / \mathrm{kg}$ \\
\hline Zinc & 43.6 & $\mathrm{mg} / \mathrm{kg}$ \\
\hline beta-BHC & 0.0043 & $\mathrm{mg} / \mathrm{kg}$ \\
\hline
\end{tabular}

${ }^{\mathrm{a} V e r i f i c a t i o n ~ s a m p l i n g ~ o f ~ t h e ~ B C L ~ s t o c k p i l e s ~}$ was based on multi-aliquot, rather than statistical, sampling.

$\mathrm{BCL}=$ below cleanup levels

For nonradionuclides, where $\leq 50 \%$ of a data set is censored (below detection limits), the $95 \%$ UCL value is used for a given analyte.

$43^{\mathrm{b}}$ For nonradionuclides, where $>50 \%$ of a data set is censored, the statistical value defaults to the maximum detected value in the data set 44 (Attachment 1).

$45^{\circ}$ Strontium-90 results based on the reported value for sample $\mathrm{J11} \mathrm{K} 91$ (radiostrontium-specific analysis was not performed for other samples

46 in the data set based on evaluation of gross beta results).

$47 \mathrm{RAG}=$ remedial action goal $\quad$ WAC $=$ Washington Adminstrative Code

$48 \mathrm{UCL}=$ upper confidence level

49

\begin{tabular}{|c|c|c|c|c|}
\hline \multirow[t]{2}{*}{50} & \multicolumn{4}{|c|}{ Relative Percent Difference Results ${ }^{\mathrm{D}}$ - QAVC Analysis } \\
\hline & Analyte & $\begin{array}{l}\text { Duplicate } \\
\text { Analysis }^{\mathrm{b}}\end{array}$ & Analyte & Duplicate Analysis $^{b}$ \\
\hline 52 & Potassium-40 & $2.8 \%$ & Manganese & $3.2 \%$ \\
\hline 53 & Aluminum & $5.1 \%$ & Phosphorus & $11 \%$ \\
\hline 54 & Barium & $3.0 \%$ & Silicon & $10 \%$ \\
\hline 55 & Calcium & $13 \%$ & Strontium & $7.2 \%$ \\
\hline 56 & Chromium & $2.6 \%$ & Titanium & $7.4 \%$ \\
\hline 57 & Copper & $2.4 \%$ & Vanadium & $4.5 \%$ \\
\hline 58 & Iron & $1.7 \%$ & Zinc & $1.1 \%$ \\
\hline 59 & Magnesium & $0.90 \%$ & Zirconium & $2.6 \%$ \\
\hline
\end{tabular}

60 Relative percent difference evaluation was not required for analytes not included in this table.

$61^{\circ}$ The significance of relative percent difference values are discussed within the RSVP for the subject site.

$62 \mathrm{QA} / \mathrm{QC}=$ quality assurance/quality control

63 RSVP = remaining sites verification package 


\section{Washington Closure Hanford}

\section{CALCULATION SHEET}

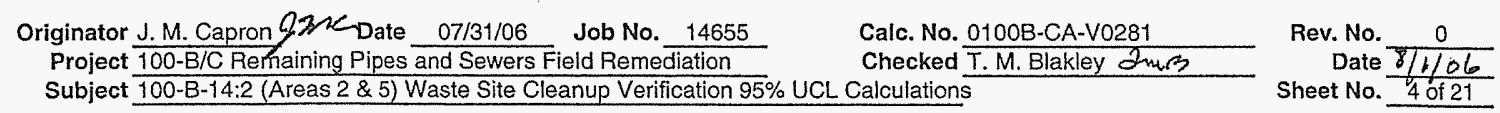

1 Summary (continued)

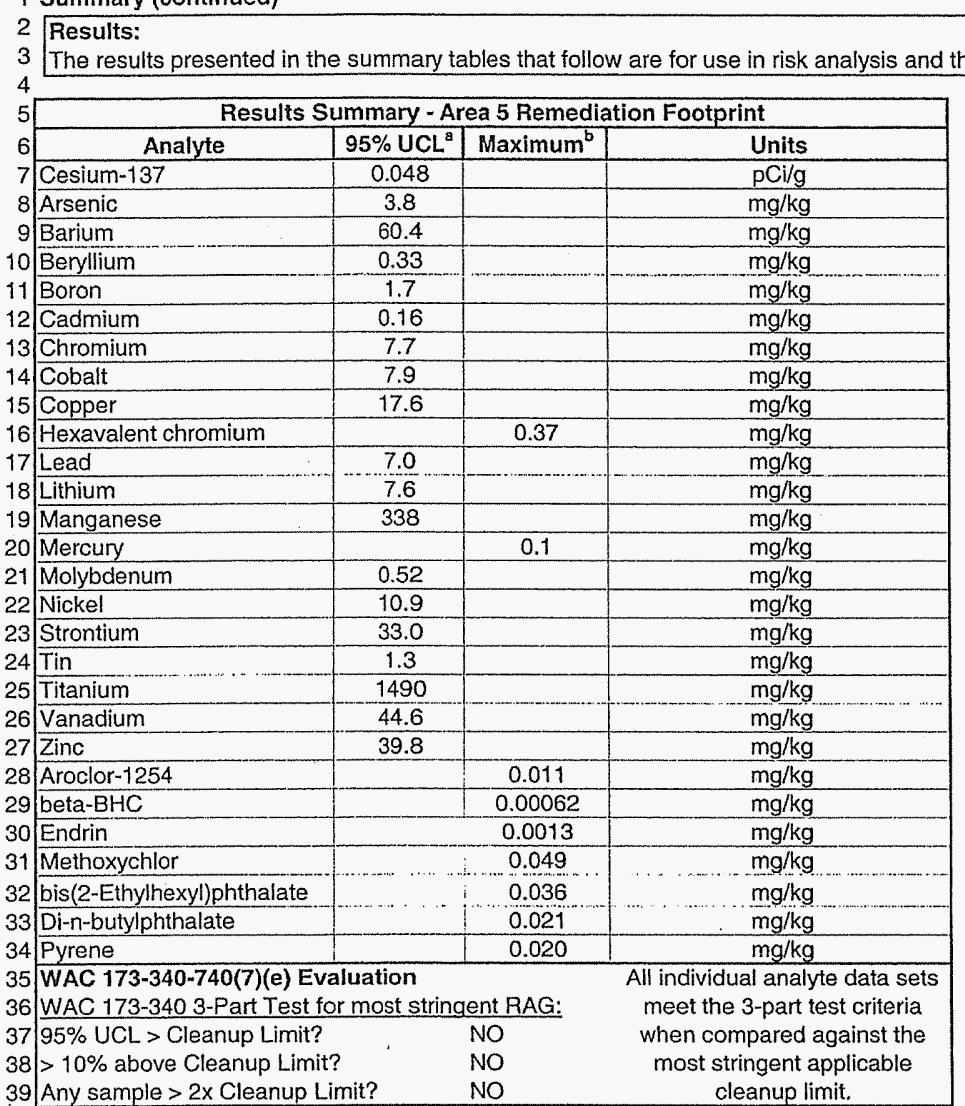

$40{ }^{2}$ For nonradionuclides, where $\leq 50 \%$ of a data set is censored (below detection limits), the $95 \%$ UCL value is used for a given analyte.

$41^{\circ}$ For nonradionuclides, where $>50 \%$ of a data set is censored, the statistical value defaults to the maximum detected value in the data set

42 (Attachment 2).

$43 \mathrm{RAG}=$ remedial action goal

$44 \mathrm{UCL}=$ upper confidence level

45 WAC $=$ Washington Adminstrative Code

46

\begin{tabular}{|c|c|}
\hline \multicolumn{2}{|c|}{ Relative Percent Difference Results ${ }^{A}$ - QAVQC Analysis } \\
\hline Analyte & Duplicate Analysis ${ }^{b}$ \\
\hline Manganese & $5.6 \%$ \\
\hline Phosphorus & $6.6 \%$ \\
\hline Silicon & $8.8 \%$ \\
\hline Strontium & $2.5 \%$ \\
\hline Titanium & $0.69 \%$ \\
\hline Vanadium & $3.5 \%$ \\
\hline Zinc & $4.3 \%$ \\
\hline Zirconium & $1.0 \%$ \\
\hline
\end{tabular}

$1.0 \%$

\begin{tabular}{|c|c|c|}
\hline \multicolumn{3}{|c|}{ Results Summary - Area 5 BCL Stockpiles } \\
\hline Analyte & Maximum ${ }^{8}$ & Units \\
\hline Tritium & 0.296 & $\mathrm{pCi} / \mathrm{g}$ \\
\hline Arsenic & 2.6 & $\mathrm{mg} / \mathrm{kg}$ \\
\hline Barium & 75.0 & $\mathrm{mg} / \mathrm{kg}$ \\
\hline Beryllium & 0.32 & $\mathrm{mg} / \mathrm{kg}$ \\
\hline Boron & 3.3 & $\mathrm{mg} / \mathrm{kg}$ \\
\hline Cadmium & 0.14 & $\mathrm{mg} / \mathrm{kg}$ \\
\hline Chromium & 12.7 & $\mathrm{mg} / \mathrm{kg}$ \\
\hline Cobalt & 7.4 & $\mathrm{mg} / \mathrm{kg}$ \\
\hline Copper & 15.8 & $\mathrm{mg} / \mathrm{kg}$ \\
\hline Hexavalent chromium & 0.65 & $\mathrm{mg} / \mathrm{kg}$ \\
\hline Lead & 26.1 & $\mathrm{mg} / \mathrm{kg}$ \\
\hline Lithium & 5.9 & $\mathrm{mg} / \mathrm{kg}$ \\
\hline Manganese & 324 & $\mathrm{mg} / \mathrm{kg}$ \\
\hline Mercury & 0.1 & $\mathrm{mg} / \mathrm{kg}$ \\
\hline Molybdenum & 0.50 & $\mathrm{mg} / \mathrm{kg}$ \\
\hline Nickel & 9.7 & $\mathrm{mg} / \mathrm{kg}$ \\
\hline Strontium & 33.1 & $\mathrm{mg} / \mathrm{kg}$ \\
\hline Tin & 1.3 & $\mathrm{mg} / \mathrm{kg}$ \\
\hline Titanium & 1250 & $\mathrm{mg} / \mathrm{kg}$ \\
\hline Vanadium & 42.0 & $\mathrm{mg} / \mathrm{kg}$ \\
\hline Zinc & 41.3 & $\mathrm{mg} / \mathrm{kg}$ \\
\hline bis(2-Ethylhexyl)phthalate & 0.027 & $\mathrm{mg} / \mathrm{kg}$ \\
\hline Chrysene & 0.021 & $\mathrm{mg} / \mathrm{kg}$ \\
\hline Fluoranthene & 0.019 & $\mathrm{mg} / \mathrm{kg}$ \\
\hline Pyrene & 0.019 & $\mathrm{mg} / \mathrm{kg}$ \\
\hline $\begin{array}{l}\text { "Verification sampling of } \\
\text { was based on multi-aliq } \\
\text { statistical, sampling. }\end{array}$ & $\begin{array}{l}\mathrm{BCL} \text { stockp } \\
\text { rather than }\end{array}$ & \\
\hline
\end{tabular}

58 'The significance of relative percent difference values are discussed within the RSVP for the subject site.

$59 \mathrm{QAVC}=$ quality assurance/quality control

60 RSVP $=$ remaining sites verification package 
Washington Closure Hanford

Originator J. M. Capron gane Project 100-B/C Renaiaining Pipes and Sewers Field Remediation
Subject 100-B-14:2 (Areas 2 2 ) Waste Site Cleanup Verification 95\% UCL Calculations
CALCULATION SHEET
Date $07 / 31 / 06$
Job No. $\frac{14655}{}$
Calc. No. $\frac{0100 B-C A-V 0281}{}$
Checked T. M. Blakley 2 me'
Rev. No. $\frac{0}{\text { Date }} \frac{0}{81606}$
Sheet No.

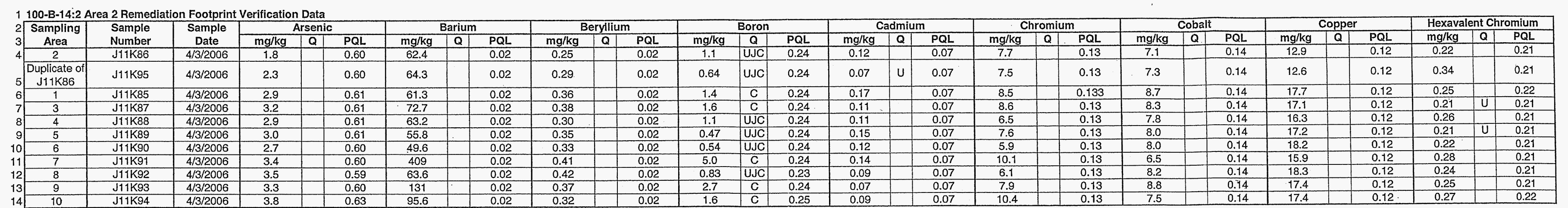

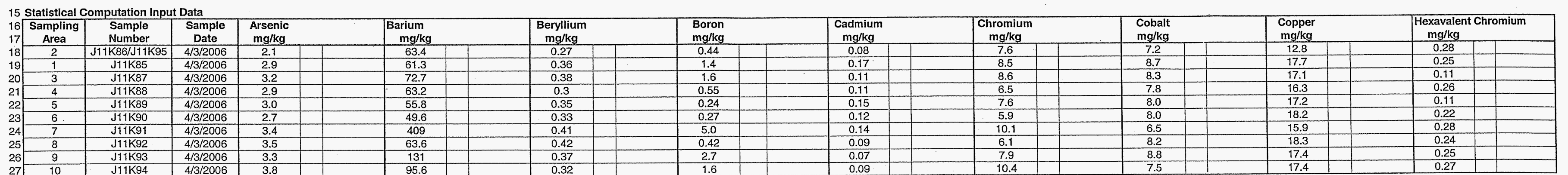

\begin{tabular}{|c|c|}
\hline 10 & J11K94 \\
\hline
\end{tabular}

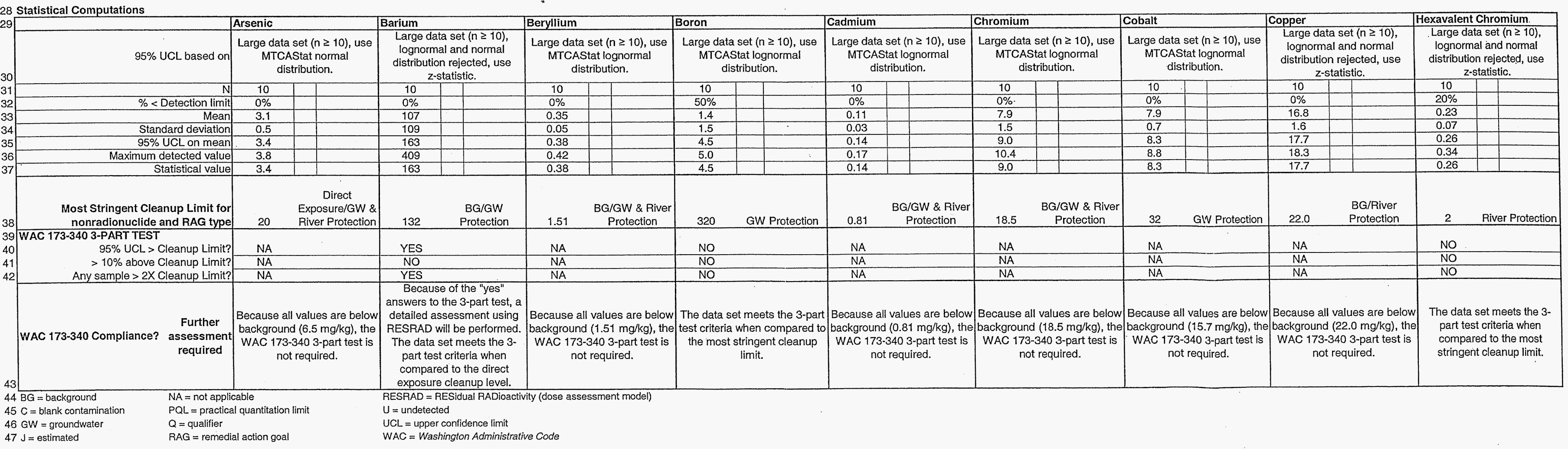


Originator J.M. Capron 9 .h C

CALCULATION SHEET

Project
Subject
100-B-B-14:2 Remaining Pipes and Sewers Field Remediation $2 \& 5$ ) Waste Site Cleanup Verification $95 \%$ UCL Calculations

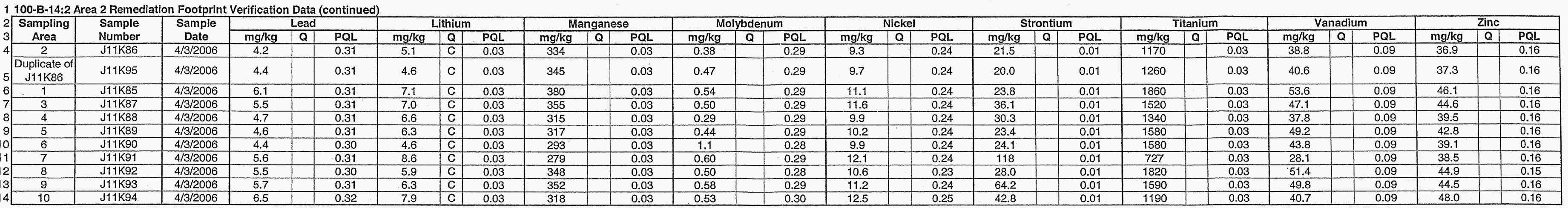

\section{Statistical Computation Input Data}

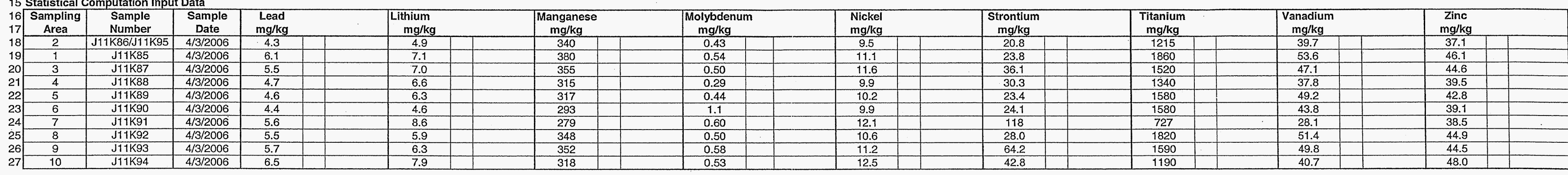

28 Statistical Computations

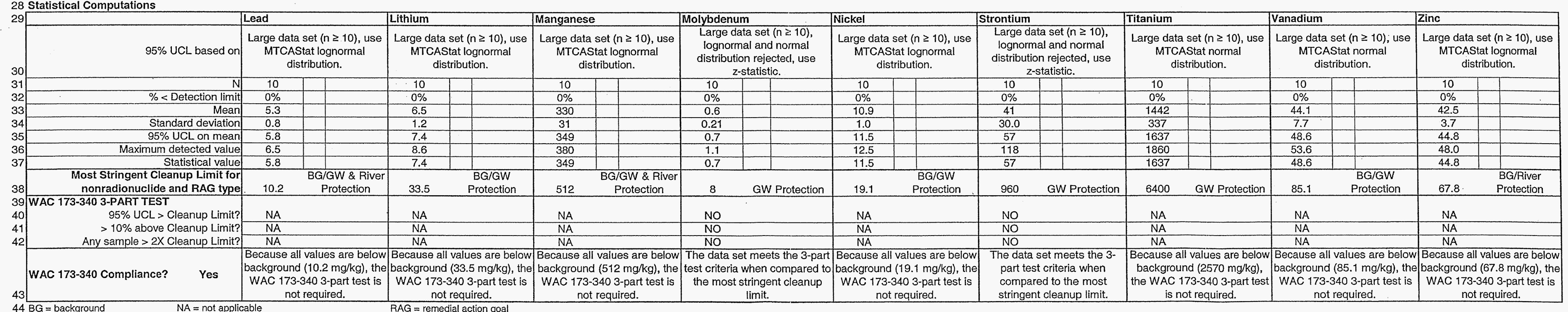

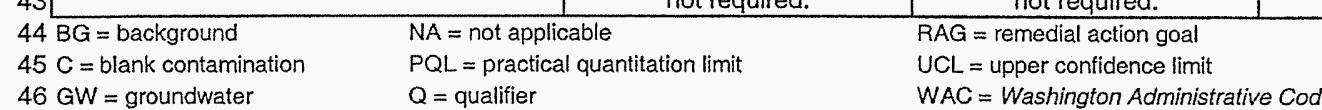


Washington Closure Hanford

Originator J.M. Capron lime

Project $100-\mathrm{B} / \mathrm{C}$ Remaining Pipes and Sewers Field Remediation

Subject 100-B-14:2 (Areas 2 25) Waste Site Cleanup Verification 95\% UCL Calculations

CALCULATION SHEET

1 100-B-14:2 Area 2 Duplicate Analysis

\begin{tabular}{|c|c|c|c|c|c|c|c|c|c|c|c|c|c|c|c|c|c|c|c|c|c|c|c|c|c|}
\hline \multirow{2}{*}{$\begin{array}{c}\text { Sampling } \\
\text { Area }\end{array}$} & \multirow{2}{*}{$\begin{array}{c}\text { Sample } \\
\text { Number } \\
\text { J11K86 }\end{array}$} & \multirow{2}{*}{$\begin{array}{c}\begin{array}{c}\text { Sample } \\
\text { Date }\end{array} \\
4 / 2 / 2006\end{array}$} & \multicolumn{3}{|c|}{ Gross Beta } & \multicolumn{3}{|c|}{ Potassium-40 } & \multicolumn{3}{|c|}{ Radium-226 } & \multicolumn{3}{|c|}{ Radium-228 } & \multicolumn{2}{|c|}{ Thorium-228 } & \multicolumn{3}{|c|}{ Thorium-232 } & \multicolumn{3}{|c|}{ Aluminum } & \multicolumn{3}{|c|}{$\begin{array}{c}\text { Arsenic } \\
\end{array}$} \\
\hline & & & $\frac{\mathrm{pCi} / \mathrm{g}}{13.4}$ & Q & $\frac{\text { MDA }}{4.0}$ & $\frac{p C i / g}{10.2}$ & $a$ & $\frac{\text { MDA }}{0.73}$ & $\frac{\mathrm{pCi} / \mathrm{g}}{0.470}$ & $\mid$\begin{tabular}{|l} 
\\
\end{tabular} & $\frac{\text { MDA }}{0.16}$ & $\frac{\mathrm{pCi} / \mathrm{g}}{0.576}$ & $Q$ & $\frac{\text { MDA }}{0.38}$ & $\frac{\mathrm{pCi} / \mathrm{g}}{0.595}$ & \begin{tabular}{l|l}
$Q$ & MDA \\
& 0.094
\end{tabular} & $\frac{\mathrm{pCi} / \mathrm{g}}{0.576}$ & Q & $\begin{array}{l}\text { MDA } \\
0.38\end{array}$ & $\frac{\mathrm{mg} / \mathrm{kg}}{5210}$ & $\mid$\begin{tabular}{|l}
$a$ \\
$C$
\end{tabular} & $\frac{P Q L}{2.3}$ & $\frac{\mathrm{mg} / \mathrm{kg}}{1.8}$ & Q & $\frac{P Q L}{0.60}$ \\
\hline $\begin{array}{l}\text { Duplicate of } \\
\mathrm{J} 11 \mathrm{~K} 86\end{array}$ & J11K95 & $4 / 3 / 2006$ & 17.6 & & 4.6 & 9.92 & & 0.67 & 0.439 & $J$ & 0.13 & 0.860 & & 0.25 & 0.597 & 0.082 & 0.860 & & 0.25 & 4950 & c & 2.3 & 2.3 & & 0.60 \\
\hline
\end{tabular}

\begin{tabular}{|c|c|c|c|c|c|c|c|c|c|}
\hline . & TDL & 15 & 0.5 & 0.1 & 0.2 & 1 & 1 & 5 & 10 \\
\hline & Both > PQL/MDA? & Yes (continue) & Yes (continue) & Yes (continue) & Yes (continue) & Yes (continue) & Yes (continue) & Yes (continue) & Yes (continue) \\
\hline $\begin{array}{l}\text { Duplicate } \\
\text { Analysis }\end{array}$ & $\begin{array}{l}\text { Both }>5 \times T D L ? \\
\text { BPD }\end{array}$ & No-Stop (acceptable) & Yes (calc RPD) & No-Stop (acceptable) & No-Stop (acceptable) & No-Stop (acceptable) & No-Stop (acceptable) & $\begin{array}{l}\text { Yes (calc RPD) } \\
5.1 \%\end{array}$ & No-Stop (acceptable) \\
\hline
\end{tabular}

\begin{tabular}{|c|c|c|c|c|c|c|c|c|c|c|c|c|c|c|c|c|c|c|c|c|c|c|c|c|c|c|c|}
\hline \multirow{3}{*}{$\begin{array}{l}12 \\
13\end{array}$} & \multirow{2}{*}{$\begin{array}{l}\text { Sampling } \\
\text { Area }\end{array}$} & \multirow{2}{*}{$\begin{array}{l}\text { Sample } \\
\text { Number }\end{array}$} & \multirow{2}{*}{ Sample Date } & \multicolumn{3}{|c|}{ Barium } & \multicolumn{3}{|c|}{ Beryllium } & \multicolumn{3}{|c|}{ Calcium } & \multicolumn{3}{|c|}{ Chromium } & \multicolumn{3}{|c|}{ Cobalt } & \multicolumn{3}{|c|}{ Copper } & \multicolumn{3}{|c|}{ Hexavalent Chromium } & \multicolumn{3}{|c|}{ Iron } \\
\hline & & & & $\mathrm{mg} / \mathrm{kg}$ & $Q_{1}$ & $\mathrm{PQL}$ & $\mathrm{mg} / \mathrm{kg}$ & Q & $\mathrm{PQL}$ & $\mathrm{mg} / \mathrm{kg}$ & Q & $\mathrm{PQL}$ & $\mathrm{mg} / \mathrm{kg}$ & Q & $\mathrm{PQL}$ & $\mathrm{mg} / \mathrm{kg}$ & Q & $\mathrm{PQL}$ & $\mathrm{mg} / \mathrm{kg}$ & Q & $\mathrm{PQL}$ & $\mathrm{mg} / \mathrm{kg}$ & Q & PQL & $\mathrm{mg} / \mathrm{kg}$ & Q & PQL \\
\hline & 2 & J11K86 & $4 / 3 / 2006$ & 62.4 & & 0.02 & 0.25 & & 0.02 & 4080 & $J$ & 2.2 & 7.7 & & 0.13 & 7.1 & & 0.14 & 12.9 & & 0.12 & 0.22 & & 0.21 & 17000 & C & 3.5 \\
\hline & $\begin{array}{l}\text { Duplicate of } \\
\mathrm{J} 11 \mathrm{~K} 86\end{array}$ & J11K95 & $4 / 3 / 2006$ & 64.3 & & 0.02 & 0.29 & & 0.02 & 3600 & $\mathrm{~J}$ & 2.2 & 7.5 & & 0.13 & 7.3 & & 0.14 & 12.6 & & 0.12 & 0.34 & & 0.21 & 17300 & c & 3.4 \\
\hline \multirow{2}{*}{\multicolumn{28}{|c|}{ 16 Analysis: }} \\
\hline & \multicolumn{3}{|c|}{ TDL } & \multicolumn{3}{|c|}{2} & \multicolumn{3}{|c|}{0.5} & \multicolumn{3}{|c|}{100} & \multicolumn{3}{|c|}{$\frac{1}{1}$} & \multicolumn{3}{|c|}{2} & \multicolumn{3}{|c|}{$\frac{1}{1}$} & \multicolumn{3}{|c|}{0.5} & \multicolumn{3}{|c|}{5} \\
\hline & $\begin{array}{l}\text { Duplicate } \\
\text { Analysis }\end{array}$ & \multicolumn{2}{|c|}{$\begin{array}{l}\text { Both }>\text { PQL? } \\
\text { Both }>5 \times \text { TDL? }\end{array}$} & \multicolumn{3}{|c|}{$\begin{array}{l}\text { Yes (continue) } \\
\text { Yes (calc RPD) }\end{array}$} & \multicolumn{3}{|c|}{$\begin{array}{l}\text { Yes (continue) } \\
\text { No-Stop (acceptable) }\end{array}$} & \multicolumn{3}{|c|}{$\begin{array}{l}\text { Yes (continue) } \\
\text { Yes (calc RPD) }\end{array}$} & \multicolumn{3}{|c|}{$\begin{array}{l}\text { Yes (continue) } \\
\text { Yes (calc RPD) }\end{array}$} & \multicolumn{3}{|c|}{$\begin{array}{l}\text { Yes (continue) } \\
\text { No-Stop (acceptable) }\end{array}$} & \multicolumn{3}{|c|}{$\begin{array}{l}\text { Yes (continue) } \\
\text { Yes (calc RPD) }\end{array}$} & \multicolumn{3}{|c|}{$\begin{array}{c}\text { Yes (continue) } \\
\text { No-Stop (acceptable) }\end{array}$} & \multicolumn{3}{|c|}{$\begin{array}{l}\text { Yes (continue) } \\
\text { Yes (calc RPD) }\end{array}$} \\
\hline
\end{tabular}

21 Sampling Sample

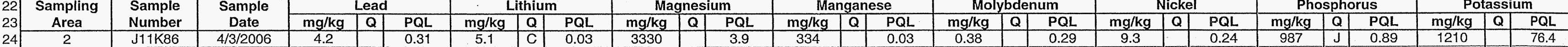

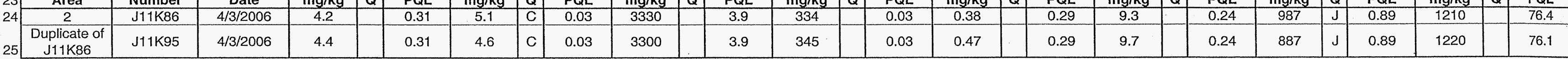

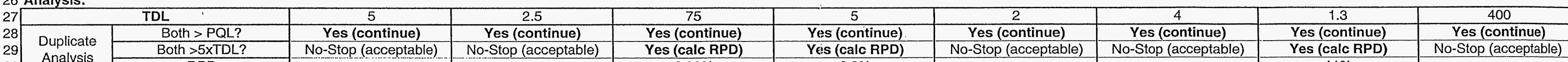

\begin{tabular}{|c|c|c|c|c|c|c|c|c|c|c|c|c|c|c|c|c|c|c|c|}
\hline & \multirow{3}{*}{\begin{tabular}{|c|}
$\begin{array}{c}\text { Sampling } \\
\text { Area }\end{array}$ \\
2
\end{tabular}} & \multirow{3}{*}{$\begin{array}{c}\text { Sample } \\
\text { Number } \\
\text { J11K86 } \\
\end{array}$} & \multirow{3}{*}{$\begin{array}{c}\begin{array}{c}\text { Sample } \\
\text { Date }\end{array} \\
4 / 3 / 2006 \\
\end{array}$} & \multicolumn{3}{|c|}{ Silicon } & \multicolumn{2}{|c|}{ Sodium } & \multicolumn{2}{|c|}{ Strontium } & \multicolumn{2}{|c|}{ Titanium } & \multicolumn{2}{|c|}{ Vanadium } & \multicolumn{2}{|r|}{ Zinc } & \multicolumn{3}{|c|}{ Zirconium } \\
\hline & & & & $\mathrm{mg} / \mathrm{kg}$ & $Q$ & $\mathrm{PQL}$ & $\mathrm{mg} / \mathrm{kg}$ & \begin{tabular}{|l|l|}
$\mathrm{Q}$ & $\mathrm{PQL}$ \\
\end{tabular} & $\mathrm{mg} / \mathrm{kg}$ & \begin{tabular}{|l|l|}
$\mathrm{Q}$ & $\mathrm{PQL}$ \\
\end{tabular} & $\mathrm{mg} / \mathrm{kg}$ & \begin{tabular}{l|l}
$Q$ & $P Q L$ \\
\end{tabular} & $\mathrm{mg} / \mathrm{kg}$ & \begin{tabular}{|l|l|}
$Q$ & $P Q L$ \\
\end{tabular} & $\mathrm{mg} / \mathrm{kg}$ & $\mathrm{PQL}$ & $\mathrm{mg} / \mathrm{kg}$ & Q & PQL \\
\hline & & & & 502 & $J$ & 2.2 & 118 & 2.5 & 21.5 & 0.01 & $1170^{\circ}$ & 0.03 & 38.8 & 0.09 & 36.9 & 0.16 & 19.2 & & 1.1 \\
\hline & $\begin{array}{l}\text { Duplicate of } \\
\text { J11 K86 }\end{array}$ & J11K95 & $4 / 3 / 2006$ & 452 & $\mathrm{~J}$ & 2.2 & 109 & 2.5 & 20.0 & 0.01 & 1260 & 0.03 & 40.6 & 0.09 & 37.3 & 0.16 & 19.7 & & 1.0 \\
\hline \multicolumn{20}{|c|}{36 Analysis: } \\
\hline \multirow{3}{*}{ 38 39} & \multicolumn{3}{|c|}{\begin{tabular}{c|c} 
& $T D L$ \\
& Both $>P Q L ?$
\end{tabular}} & & \multicolumn{2}{|r|}{50} & \multicolumn{2}{|r|}{1} & \multicolumn{2}{|c|}{0.5} & & \multicolumn{2}{|r|}{1} & \multicolumn{3}{|c|}{2.5} \\
\hline & Duplicate & \multirow{2}{*}{\multicolumn{2}{|c|}{$\begin{array}{l}\text { Both > PQL? } \\
\text { Both >5XTDL? }\end{array}$}} & \multicolumn{3}{|c|}{$\begin{array}{l}\text { Yes (continue) } \\
\text { Yes (calc RPD) }\end{array}$} & \multirow{2}{*}{\multicolumn{2}{|c|}{$\begin{array}{l}\text { Yes (continue) } \\
\text { No-Stop (acceptable) }\end{array}$}} & \multicolumn{2}{|c|}{$\frac{\text { Yes (continue) }}{\text { Yes (calc RPD) }}$} & \multicolumn{2}{|c|}{$\frac{\text { Yes (continue) }}{\text { Yes calc RPD) }}$} & \multicolumn{2}{|c|}{$\frac{\text { Yes (continue) }}{\text { Yes (calc RPD) }}$} & \multicolumn{2}{|c|}{$\begin{array}{l}\text { Yes (continue) } \\
\text { Yes (calc RPD) }\end{array}$} & \multicolumn{3}{|c|}{$\frac{\text { Yes (continue) }}{\text { Yes (calc RPD) }}$} \\
\hline & \multicolumn{2}{|c|}{ RPD } & & \multicolumn{3}{|c|}{$\frac{\text { Pes (calc RPU) }}{10 \%}$} & & & \multicolumn{2}{|c|}{$\frac{Y \text { Pes (calc RPD) }}{72 \%}$} & \multicolumn{2}{|c|}{ Yes (calc RPD) } & \multicolumn{2}{|c|}{ Yes (calco } & \multicolumn{2}{|c|}{$1.1 \%$} & & $2.6 \%$ & \\
\hline
\end{tabular}

$42 \mathrm{~J}=$ estimated

$44 \mathrm{PQL}=$ practical detectable activity

$\mathrm{RPD}=$ relative percent difference
$\mathrm{TDL}=$ = target detection limit 



\section{CALCULATION SHEET}

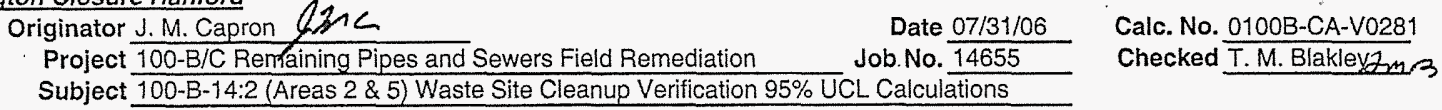

Ecology Software (MTCAStat) Results (Area 2)

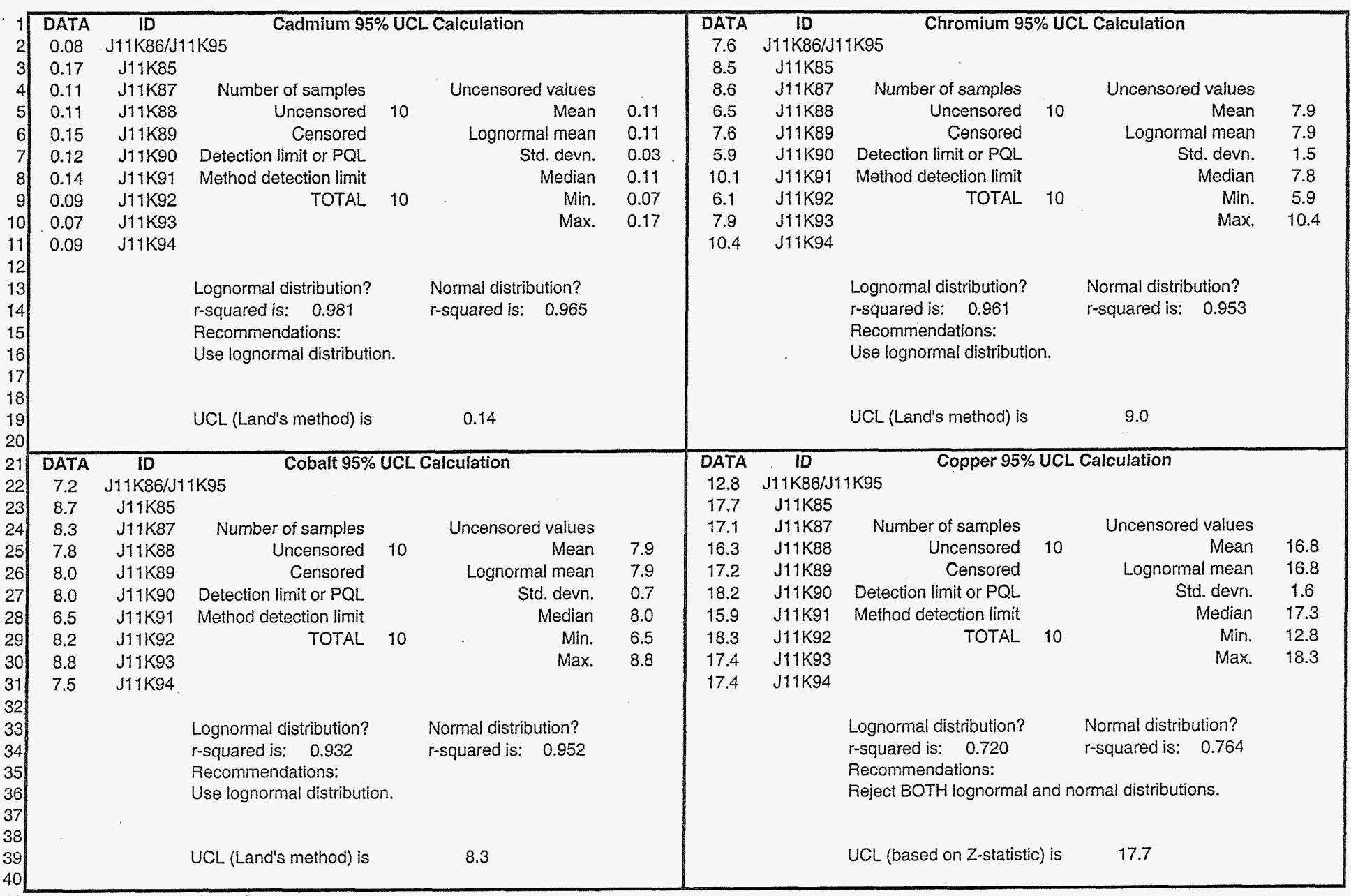


Washington Closure Hanford

CALCULATION SHEET

Originator J.M. Capron finc

Proiet $100-B / C$ Remaining Pipes and Sewers Field Remediation

Date $07 / 31 / 06$

Job No. 1465

Calc. No. 0100B-CA-V0281 Subject 100-B-14:2 (Areas 2\& 5) Waste Site Cleanup Verification 95\% UCL Calculations

Ecology Software (MTCAStat) Results (Area 2)

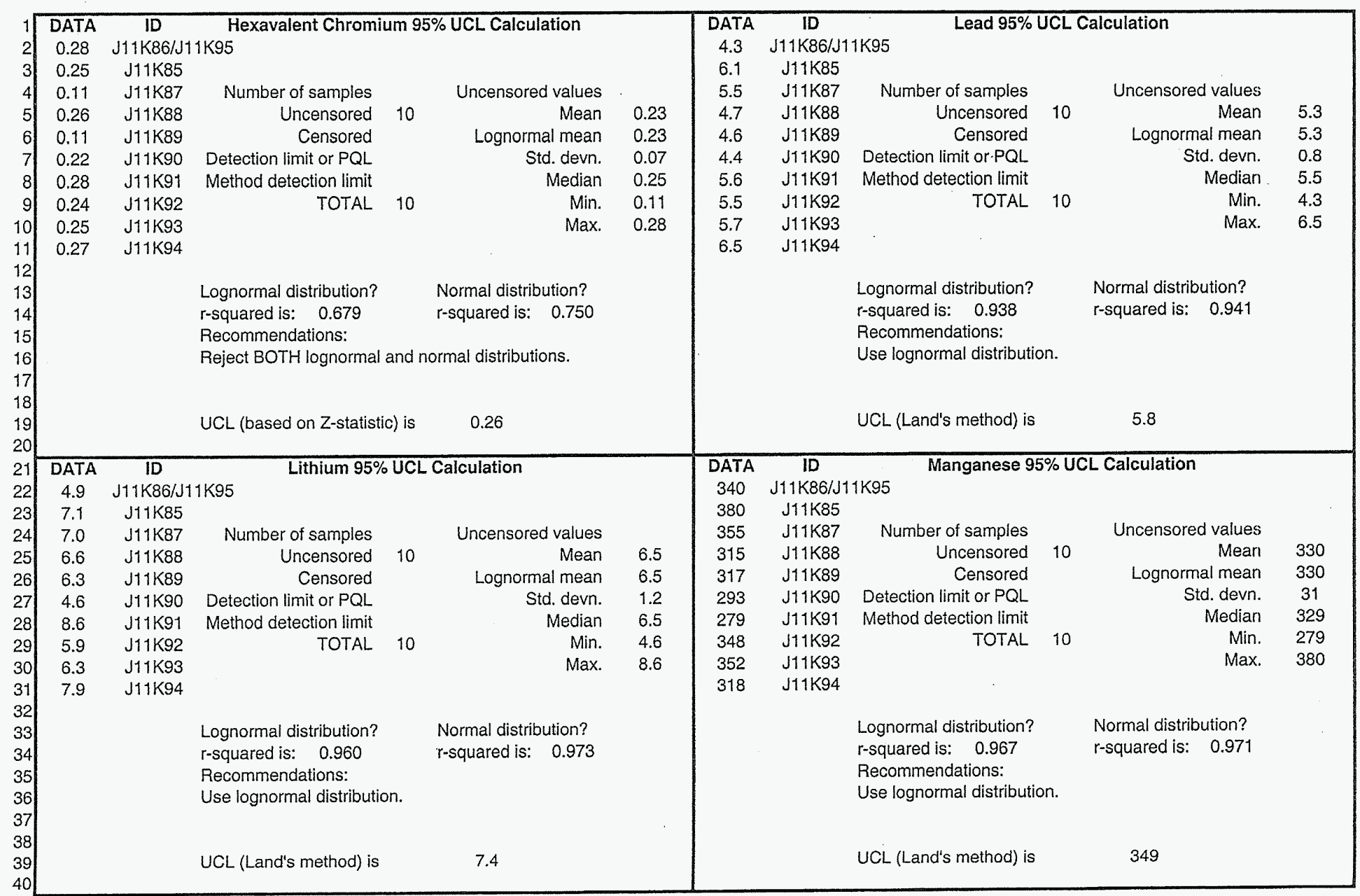


CALCULATION SHEET

Originator J M Capron Gisc

Project 100-B/C Remaining Pipes and Sewers Field Remediation

Date 07/31/06

Job No. 14655

Calc. No. 0100B-CA-V0281

Subject 100-B-14:2 (Areas 2 \& 5) Waste Site Cleanup Verification 95\% UCL Calculations

Ecology Software (MTCAStat) Results (Area 2)

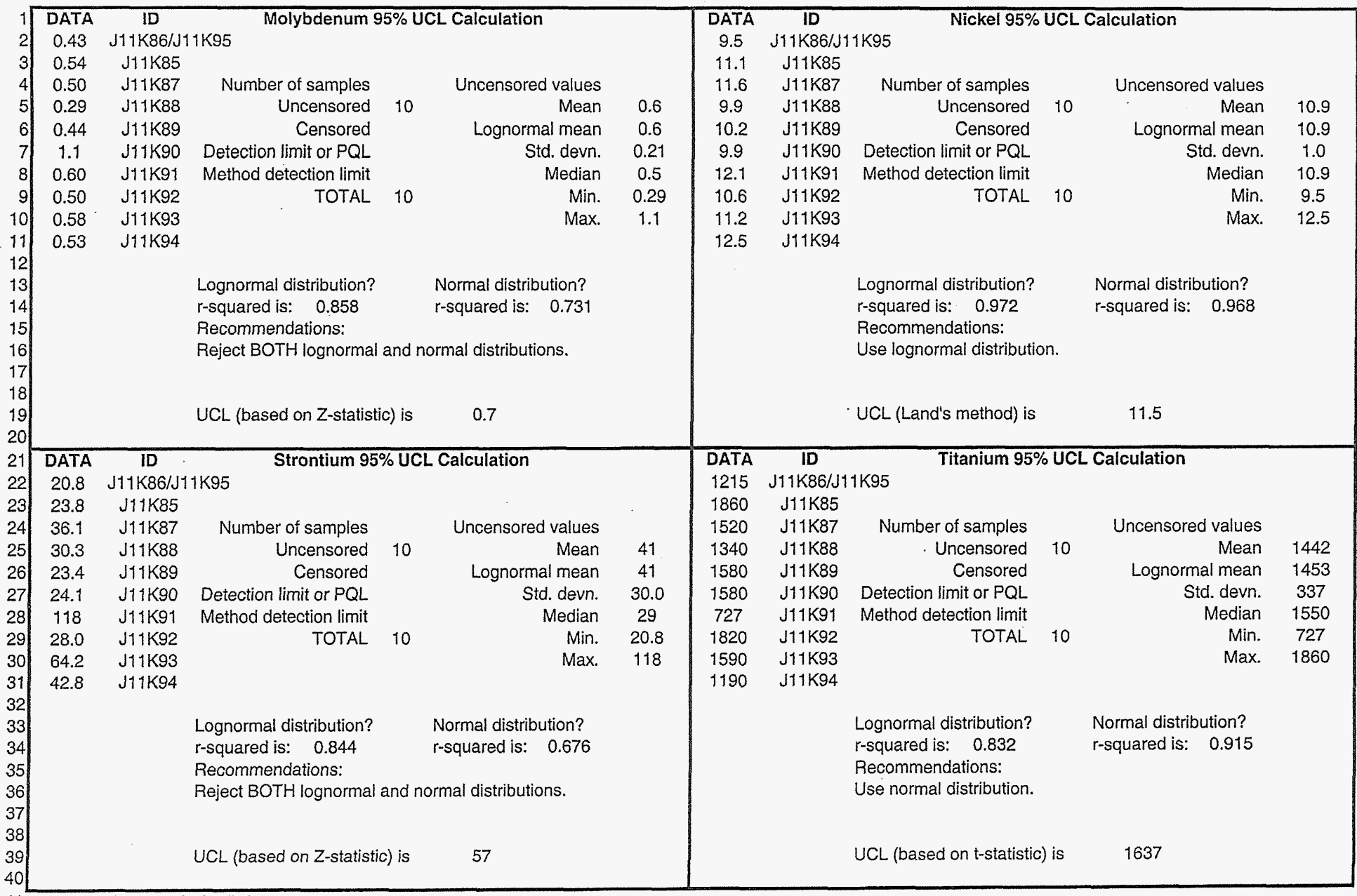


CALCULATION SHEET

\section{Washington Closure Hanford}

Originator J.M. Capron SPe

Project 100-B/C Remaining Pipes and Sewers Field Remediation

Subject 100-B-14:2 (Areas 2 \& 5) Waste Site Cleanup Verification 95\%
Date $07 / 31 / 06$ Job No. 14655

Calculation
Calc. No. 0100B-CA-V0281

Checked T.M. Blakley 2 .
Rev No.
Datej/10
Sheet No. 12 of 21

Ecology Software (MTCAStat) Results (Area 2)

\begin{tabular}{|c|c|c|c|c|c|c|c|c|c|c|c|c|}
\hline 1 & DATA & ID & Vanadium $95^{\circ}$ & $\% \mathrm{UC}$ & - Calculation & & DATA & & Zinc $95 \% \mathrm{~L}$ & $\mathrm{JCL}$ & alculation & \\
\hline 2 & 39.7 & $\mathrm{~J} 11 \mathrm{~K} 86 / \mathrm{J} 1$ & $1 \mathrm{~K} 95$ & & & & 37.1 & $\mathrm{~J} 11 \mathrm{~K} 86 / \mathrm{J} 1$ & $1 \mathrm{~K} 95$ & & & \\
\hline 3 & 53.6 & $J 11 K 85$ & & & & & 46.1 & J11K85 & & & & \\
\hline 4 & 47.1 & J11K87 & Number of samples & & Uncensored values & & 44.6 & J11K87 & Number of samples & & Uncensored values & \\
\hline 5 & 37.8 & J11K88 & Uncensored & 10 & Mean & 44.1 & 39.5 & J11K88 & Uncensored & 10 & Mean & 42.5 \\
\hline 6 & 49.2 & J11K89 & Censored & & Lognormal mean & 44.3 & 42.8 & J11K89 & Censored & & Lognormal mean & 42.5 \\
\hline 7 & 43.8 & J11K90 & Detection limit or $\mathrm{PQL}$ & & Std. devn. & 7.7 & 39.1 & J11K90 & Detection limit or $P Q L$ & & Std. devn. & 3.7 \\
\hline 8 & 28.1 & J11K91 & Method detection limit & & Median & 45.5 & 38.5 & J11K91 & Method detection limit & & Median & 43.7 \\
\hline 9 & 51.4 & J11K92 & TOTAL & 10 & Min. & 28.1 & 44.9 & J11K92 & TOTAL & 10 & Min. & 37.1 \\
\hline 10 & 49.8 & J11K93 & & & Max. & 53.6 & 44.5 & $\mathrm{J11K93}$ & & & Max. & 48.0 \\
\hline 11 & 40.7 & J11K94 & & & & & 48.0 & J11K94 & & & & \\
\hline 3 & & & Lognormal distribution? & & Normal distribution? & & & & Lognormal distribution? & & Normal distribution? & \\
\hline 4 & & & r-squared is: $\quad 0.885$ & & $r$-squared is: 0.935 & & & & r-squared is: 0.938 & & $r$-squared is: 0.942 & \\
\hline 5 & & & Recommendations: & & & & & & Recommendations: & & & \\
\hline 6 & & & Use normal distribution. & & & & & & Use lognormal distributio & & & \\
\hline 17 & & & & & & & & & & & & \\
\hline 18 & & & UCL (based on t-statistic & & 48.6 & & & & UCL (Land's method) is & & 44.8 & \\
\hline
\end{tabular}

$21 \mathrm{PQL}=$ practical quantitation limit

$22 \mathrm{UCL}=$ upper confidence limit 
Washington Closure Hanford

Originator J.M. Capron $4 \mathrm{mc}$

Project $100-B / C$ Remaining Pipes and Sewers Field Remediation Subject 100-B-14:2 (Areas 2 \& 5) Waste Site Cleanup Verification 95\% UCL Calculations

$\frac{07 / 31 / 06}{14655}$ Job No. 1465

alc. No. 0100B-CA-V0281

Ecology Software (MTCAStat) Results (Area 2)

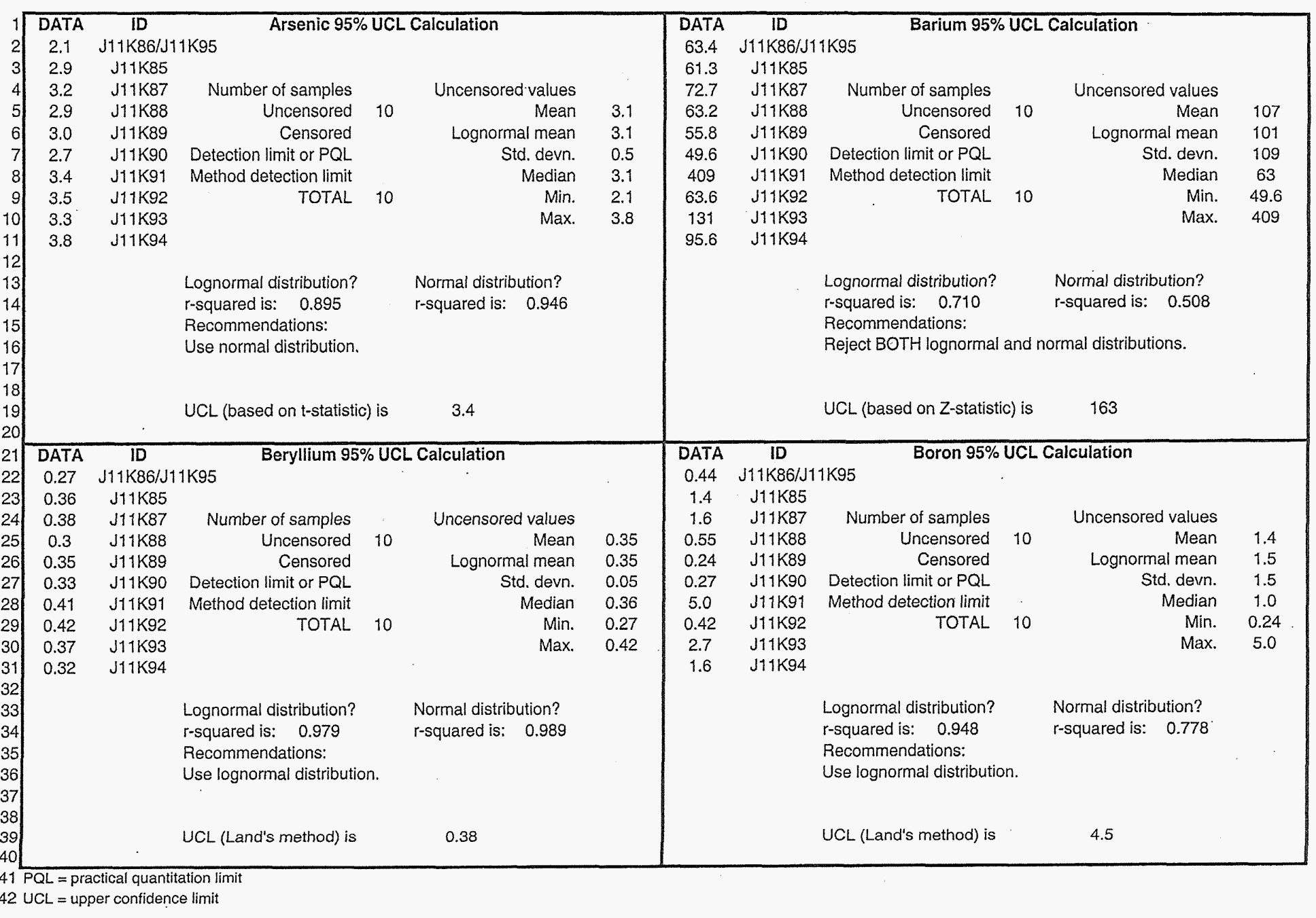

Rev. No. $\frac{0}{0}$ Date $8 / 1 / 06$ 
Originator J. M. Capron \&2arC
Project $100-B / C$ Remaining Pipes Project 100-B/C Remaining Pipes and Sewers Field Remediation
CALCULATION SHEET

Date $07 / 31 / 06$
Job No. 14655
Calc. No. $0100 B-C A-v_{0281}$

Rev. No. $\frac{0}{\text { Date }}$
Sheet No. $\frac{81106}{13 \text { of } 21}$

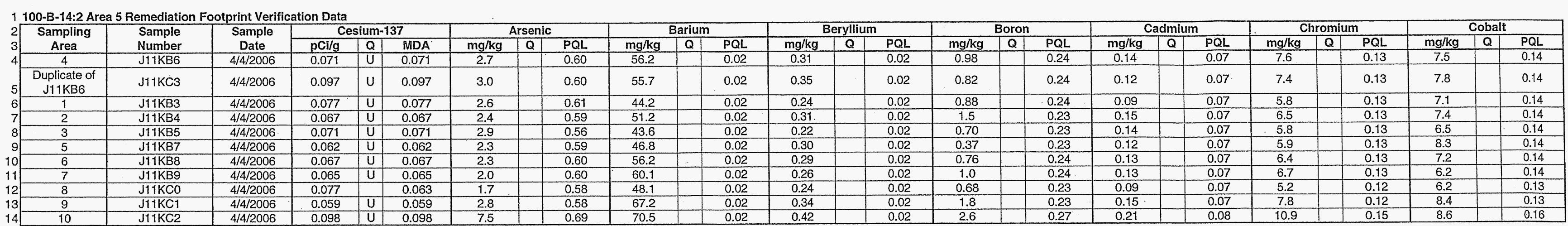

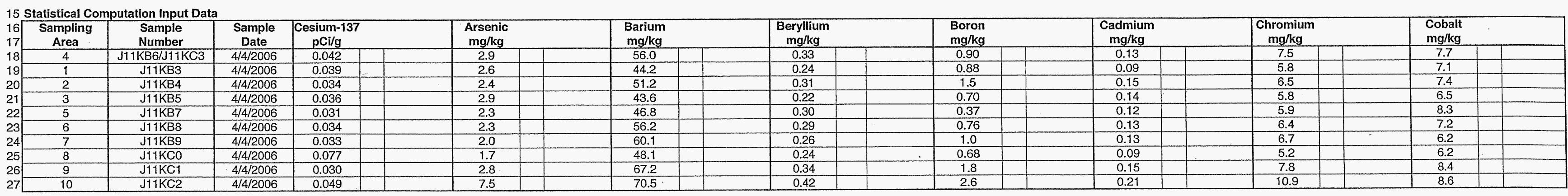

28 Statistical Computations

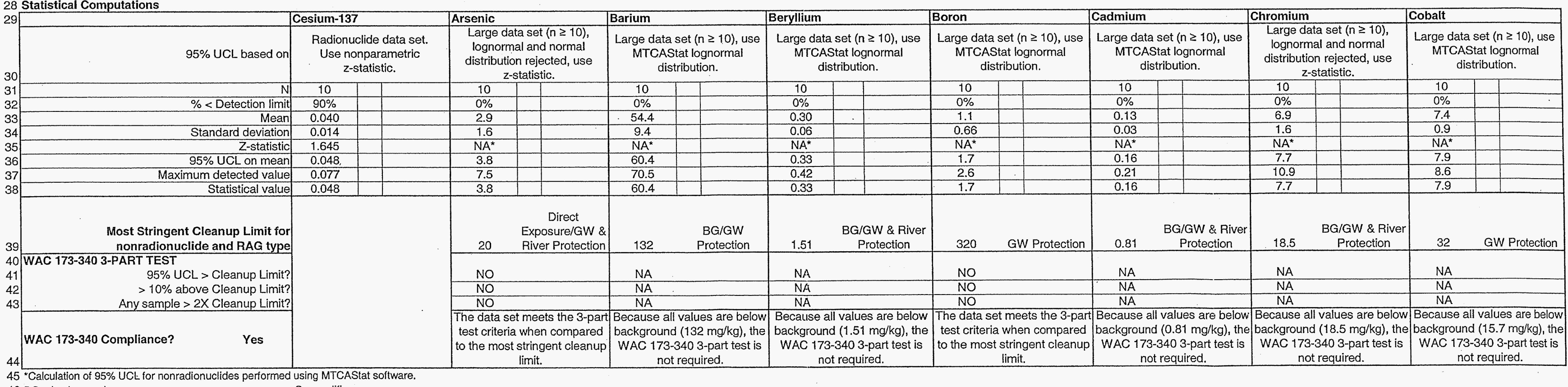

using MTCAStat sofitware.

$\begin{array}{ll}46 \mathrm{BG}=\text { background } & Q=\text { quallifer } \\ 47 \mathrm{GW}=\text { groundwater } & \mathrm{RA}=\mathrm{G}=\text { reme }\end{array}$

$48 \mathrm{MDA}=$ minimum detectable activity

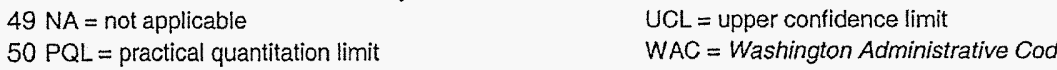


Originator J. M. Capron Q $\operatorname{me}$ Proct

\section{cactions}

) Waste Site Cleanup Venicalion 95\% UCL Calculations _-

1100-B-14:2 Area 5 Remediation Footprint Verification Data (continued)

\begin{tabular}{|c|c|c|c|c|c|c|c|c|c|c|c|c|c|c|c|c|c|c|c|c|c|c|c|c|c|c|}
\hline \multirow{2}{*}{\begin{tabular}{|c|} 
Sampling \\
Area \\
4
\end{tabular}} & \multirow{2}{*}{$\begin{array}{l}\text { Sample } \\
\text { Number } \\
\text { J11KB6 } \\
\end{array}$} & \multirow{2}{*}{$\begin{array}{c}\begin{array}{c}\text { Sample } \\
\text { Date }\end{array} \\
4 / 4 / 2006 \\
\end{array}$} & \multirow{2}{*}{\multicolumn{3}{|c|}{ 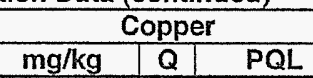 }} & \multicolumn{3}{|c|}{ Lead } & \multicolumn{3}{|c|}{ Lithium } & \multicolumn{3}{|c|}{ Manganese } & \multicolumn{3}{|c|}{$\begin{array}{c}\text { Molybdenum } \\
\end{array}$} & \multicolumn{3}{|c|}{$\begin{array}{l}\text { Nickel } \\
\end{array}$} & \multicolumn{3}{|c|}{ Strontium } & \multicolumn{3}{|c|}{ Tin } \\
\hline & & & $\frac{\mathrm{mg} / \mathrm{kg}}{16.4}$ & & $\frac{P Q L}{0.12}$ & $\frac{\mathrm{mg} / \mathrm{kg}}{5.8}$ & $Q$ & $\begin{array}{l}\mathrm{PQL} \\
0.31\end{array}$ & $\frac{\mathrm{mg} / \mathrm{kg}}{6.4}$ & \begin{tabular}{|l|l|}
$a$ \\
$C$ \\
\end{tabular} & $\begin{array}{l}\mathrm{PQL} \\
0.03 \\
\end{array}$ & $\frac{\mathrm{mg} / \mathrm{kg}}{315}$ & Q & $\begin{array}{l}\mathrm{PQL} \\
0.03 \\
\end{array}$ & $\frac{\mathrm{mg} / \mathrm{kg}}{0.43}$ & $Q$ & $\begin{array}{l}\mathrm{PQL} \\
0.29 \\
\end{array}$ & $\frac{\mathrm{mg} / \mathrm{kg}}{10.0}$ & Q & $\frac{P Q L}{0.24}$ & $\frac{\mathrm{mg} / \mathrm{kg}}{24.3}$ & \begin{tabular}{|l|l}
$Q$ \\
$C$
\end{tabular} & $\frac{P Q L}{0.01}$ & $\frac{\mathrm{mg} / \mathrm{kg}}{1.4}$ & Q & $\frac{P Q L}{1.1}$ \\
\hline $\begin{array}{l}\text { Duplicate of } \\
\text { J11KB6 }\end{array}$ & J11KC3 & $4 / 4 / 2006$ & 15.7 & & 0.12 & 5.8 & & 0.30 & 6.4 & c & 0.03 & ззз & & 0.03 & 0.52 & & 0.29 & 10.0 & & 0.24 & 23.7 & c & 0.01 & 1.3 & & 1.1 \\
\hline & & & 14.8 & & 0.12 & 5.1 & & & 5.6 & $\mathrm{C}$ & 0.03 & 289 & & & & & & & & & 21.6 & $\mathrm{C}$ & & 1.1 & & 1.1 \\
\hline 2 & & & 16.3 & & 0.12 & 4.8 & & & 8.4 & $C$ & & 306 & & & & & & & & & & & & 9.4 & & 1.0 \\
\hline 3 & & & 16.0 & & 0.12 & 6.5 & & 0.30 & 5.2 & $\mathrm{C}$ & 0.03 & 259 & & 0.03 & 0.41 & & 0.28 & 8.7 & & 0.23 & 24.2 & $\mathrm{C}$ & 0.01 & 1.2 & & 1.0 \\
\hline 5 & & & 17.5 & & 0.12 & 3.9 & & 0.30 & 5.7 & $c$ & 0.03 & 312. & & 0.03 & 0.50 & & 0.28 & 10.6 & & 0.23 & 25.7 & C & 0.01 & 1.5 & & 1.0 \\
\hline$\frac{6}{7}$ & & & $\frac{15.0}{15.4}$ & & 0.12 & 5.7 & & 0.31 & 5.5 & $\mathrm{C}$ & 0.03 & 308 & & 0.03 & 0.49 & & 0.29 & 9.7 & & 0.24 & 19.8 & $\mathrm{C}$ & 0.01 & 1.2 & & 1.1 \\
\hline$\frac{7}{8}$ & $\frac{\sqrt{111 K B 9}}{J 11 K C O}$ & & $\frac{15.4}{1148}$ & & 0.12 & $\frac{5.0}{32}$ & & 0.31 & $\frac{5.4}{3.9}$ & $\frac{c}{c}$ & 0.03 & 302 & & 0.03 & 0.49 & & 0.29 & 8.6 & & 0.24 & 25.6 & $\frac{\mathrm{C}}{\mathrm{C}}$ & 0.01 & $\frac{1.3}{12}$ & & 1.1 \\
\hline 8 & $\frac{\sqrt{11 K C O}}{\sqrt{111 K C 1}}$ & $\begin{array}{ll}4 / 4 / 2006 \\
4 / 2006\end{array}$ & $\frac{14.8}{18.4}$ & & 0.11 & $\frac{3.2}{56}$ & & 0.30 & $\begin{array}{l}3.9 \\
5.9\end{array}$ & $\frac{C}{c}$ & & $\frac{232}{362}$ & & 0.03 & 0.40 & & 0.28 & $\frac{8.3}{117}$ & & 0.23 & $\frac{20.2}{275}$ & $\mathrm{C}$ & 0.01 & $\frac{1.2}{13}$ & & $\frac{1.0}{10}$ \\
\hline$\frac{9}{10}$ & $\begin{array}{l}\mathrm{J} 1 \mathrm{KCC} \\
\mathrm{J} 1 \mathrm{KC} 2\end{array}$ & $\begin{array}{ll}44 / 2 / 2006 \\
4 / 4 / 2006\end{array}$ & $\frac{18.4}{21.2}$ & & 0.11 & $\frac{5.6}{10.6}$ & & 0.30 & $\begin{array}{r}5.9 \\
11.9\end{array}$ & $\mid \begin{array}{l} \\
c \\
\end{array}$ & 0.03 & $\frac{362}{388}$. & & 0.03 & $\begin{array}{l}0.53 \\
0.56 \\
\end{array}$ & & 0.28 & $\frac{11.7}{14.1}$ & & $\begin{array}{l}0.23 \\
0.27\end{array}$ & $\frac{27.5}{59.2}$ & $\begin{array}{l}\mathrm{C} \\
\mathrm{C}\end{array}$ & 0.01 & $\begin{array}{l}1.3 \\
1.2\end{array}$ & & $\frac{1.0}{1.2}$ \\
\hline
\end{tabular}

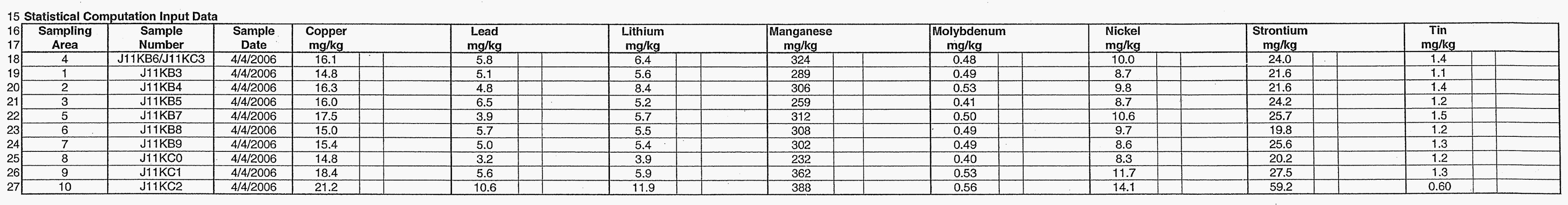

28 Statistical Computations

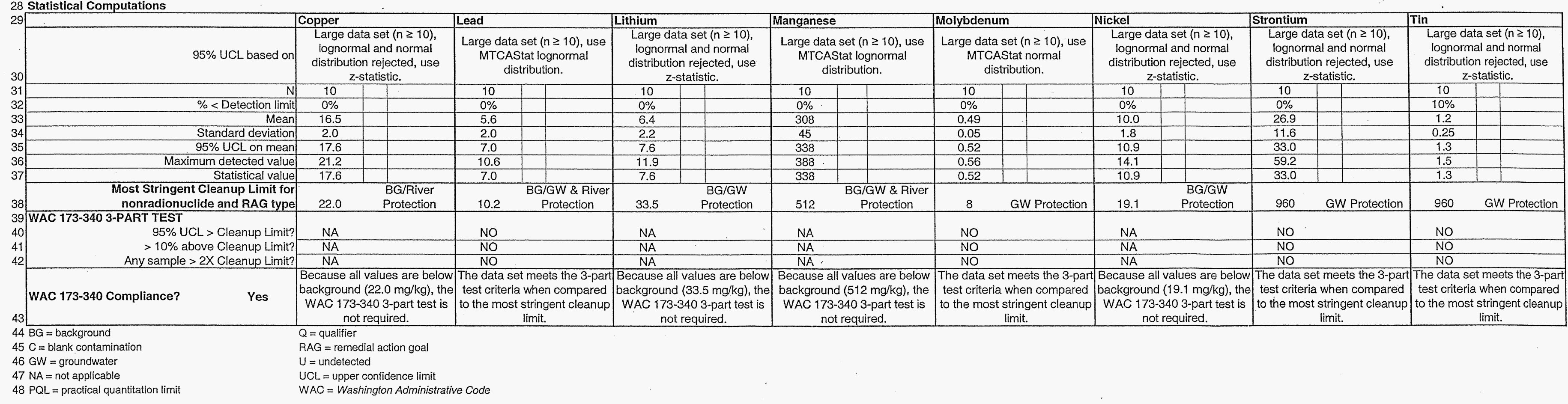


1 100-B-14:2 Area 5 Remediation Footprint Verification Data (continued)

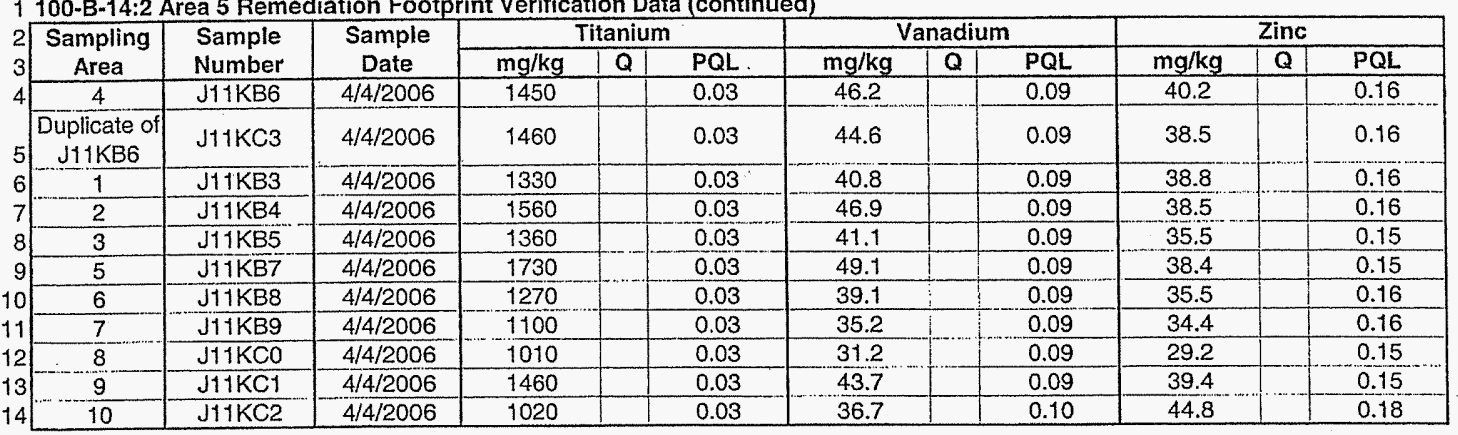

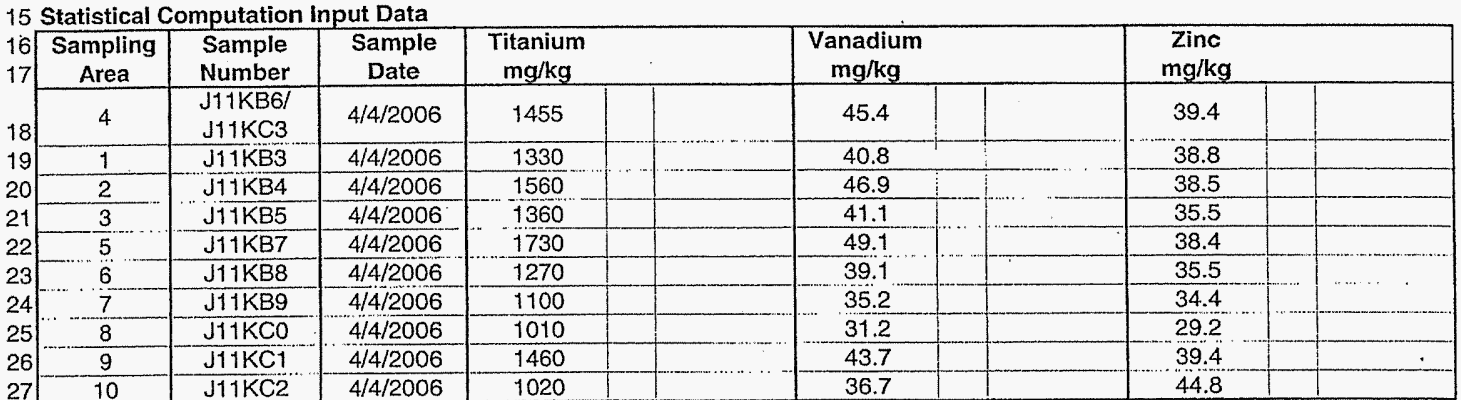

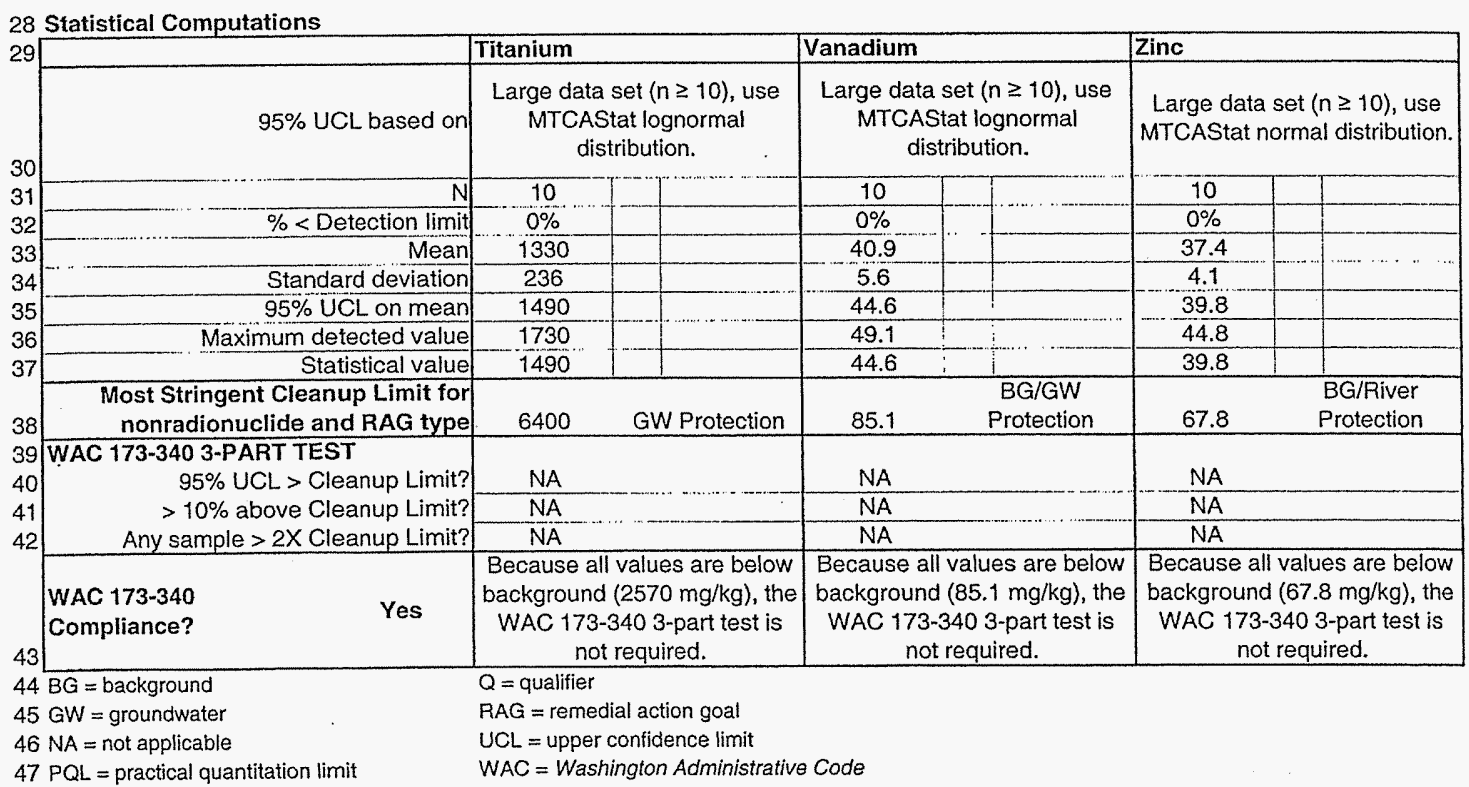


Originator J.M. Capron \&m C

1. 100-B-14:2 Area 5 Duplicate Analysis

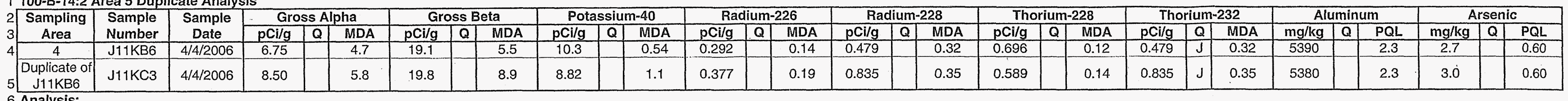

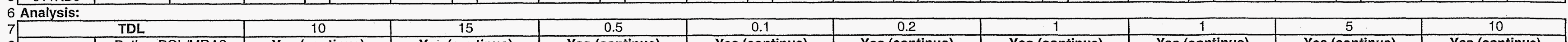

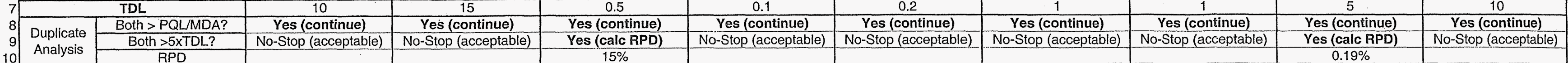

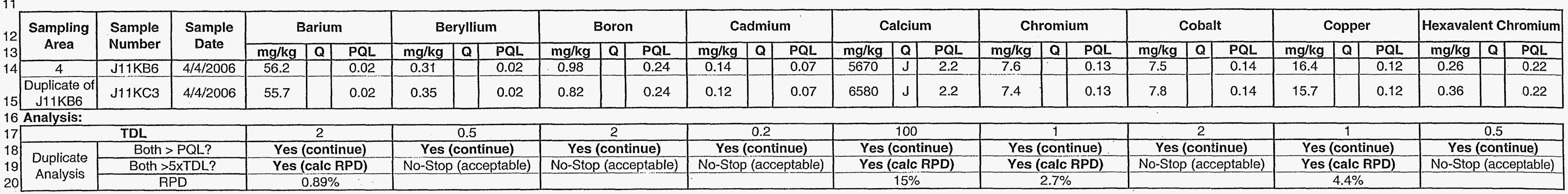

21 (1)

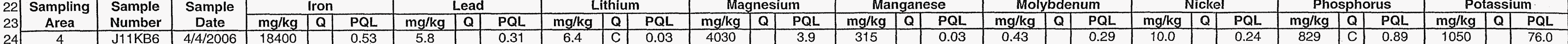

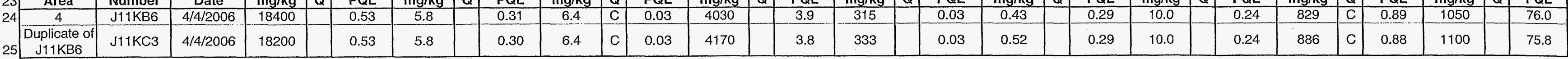

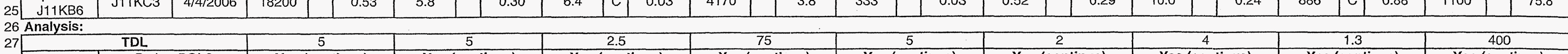

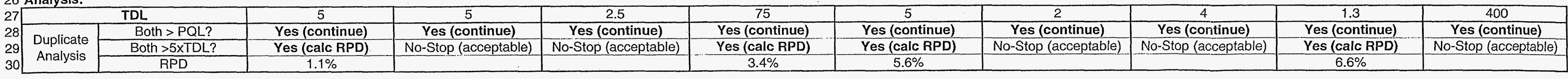

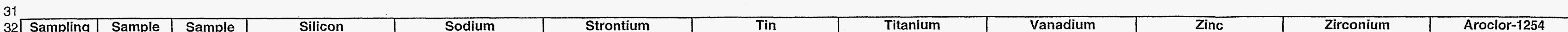

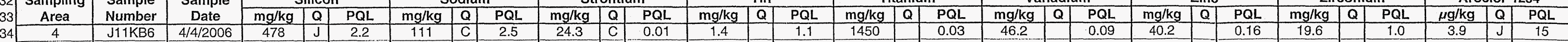

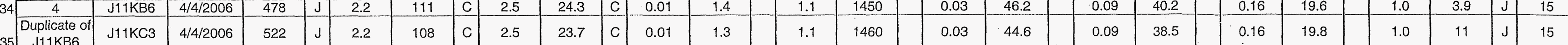

\begin{tabular}{|c|c|c|c|c|c|c|c|c|c|c|c|}
\hline & & TDL & 2 & 50 & 1 & 10 & 0.5 & 2.5 & 1 & 2.5 & 16.5 \\
\hline & & Both > PQL? & Yes (continue) & Yes (continue) & Yes (continue) & Yes (continue) & Yes (continue) & Yes (continue) & Yes (continue) & Yes (continue) & No-Stop (acceptable) \\
\hline & Analysis & $\begin{array}{l}\text { Both }>5 \times T D L ? \\
\text { RPD }\end{array}$ & $\begin{array}{l}\text { Yes (calc RPD) } \\
8.8 \%\end{array}$ & No-Stop (acceptable) & $\frac{\text { Yes (calc RPD) }}{2.5 \%}$ & No-Stop (acceptable) & $\begin{array}{l}\text { Yes (calc RPD) } \\
0.69 \%\end{array}$ & $\begin{array}{c}\text { Yes (calc RPD) } \\
3.5 \%\end{array}$ & $\frac{\text { Yes (calc RPD) }}{4.3 \%}$ & $\frac{\text { Yes (calc RPD) }}{1.0 \%}$ & \\
\hline
\end{tabular}
$41 \mathrm{C}=$ blank contamination $\quad Q=$ qualifier

$\begin{array}{ll}42 J=\text { estimated } & R P D=\text { relative percent difference } \\ 43 \mathrm{MDA}=\text { minimum detectable activity } & \mathrm{TDL}=\text { target detection limit }\end{array}$

$44 \mathrm{PQL}=$ practical quantitation limit 
Washington Closure Hanford

CALCULATION SHEET

Originator J. M. Capron $f$ he

Date $07 / 31 / 06$

Project
Subject
$100-B / C-14: 2$ (Areas 2 \& 5) Waste Site Cleanup Verification 95\% UCL Calculations

No. 0100B-CA-V0281

Subject 100-B-14:2 (Areas 2 \& 5) Waste Site Cleanup Verification 95\% UCL Calculations

No. $\frac{0}{8 / 06}$

Date $8 / 106$

Ecology Software (MTCAStat) Results (Area 5)

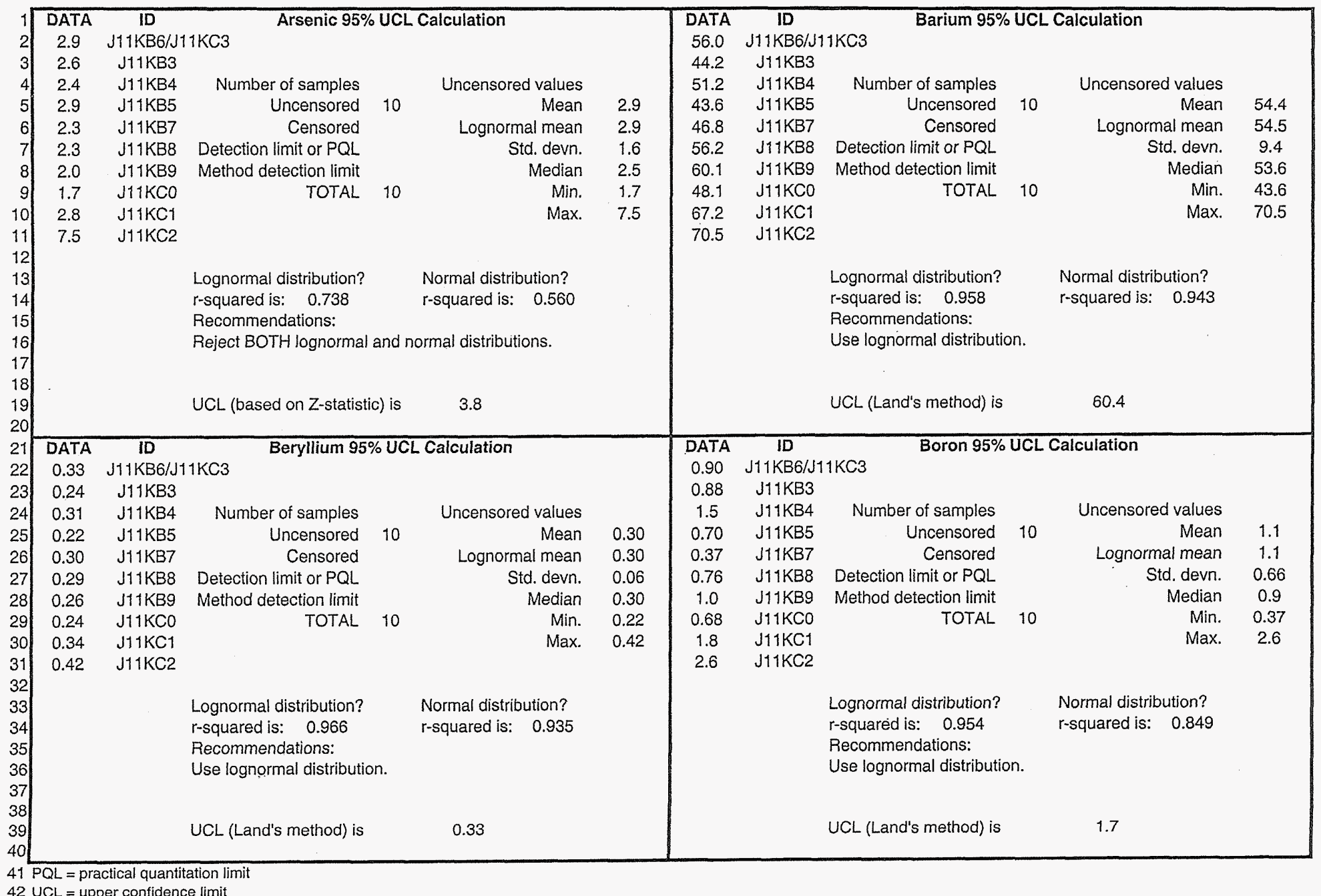


CALCULATION SHEET

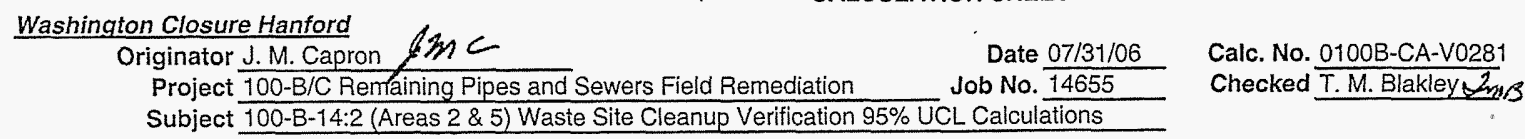

Calc. No. $0100 \mathrm{~B}-\mathrm{CA}-\mathrm{V} 0281$

Ecology Software (MTCAStat) Results (Area 5)

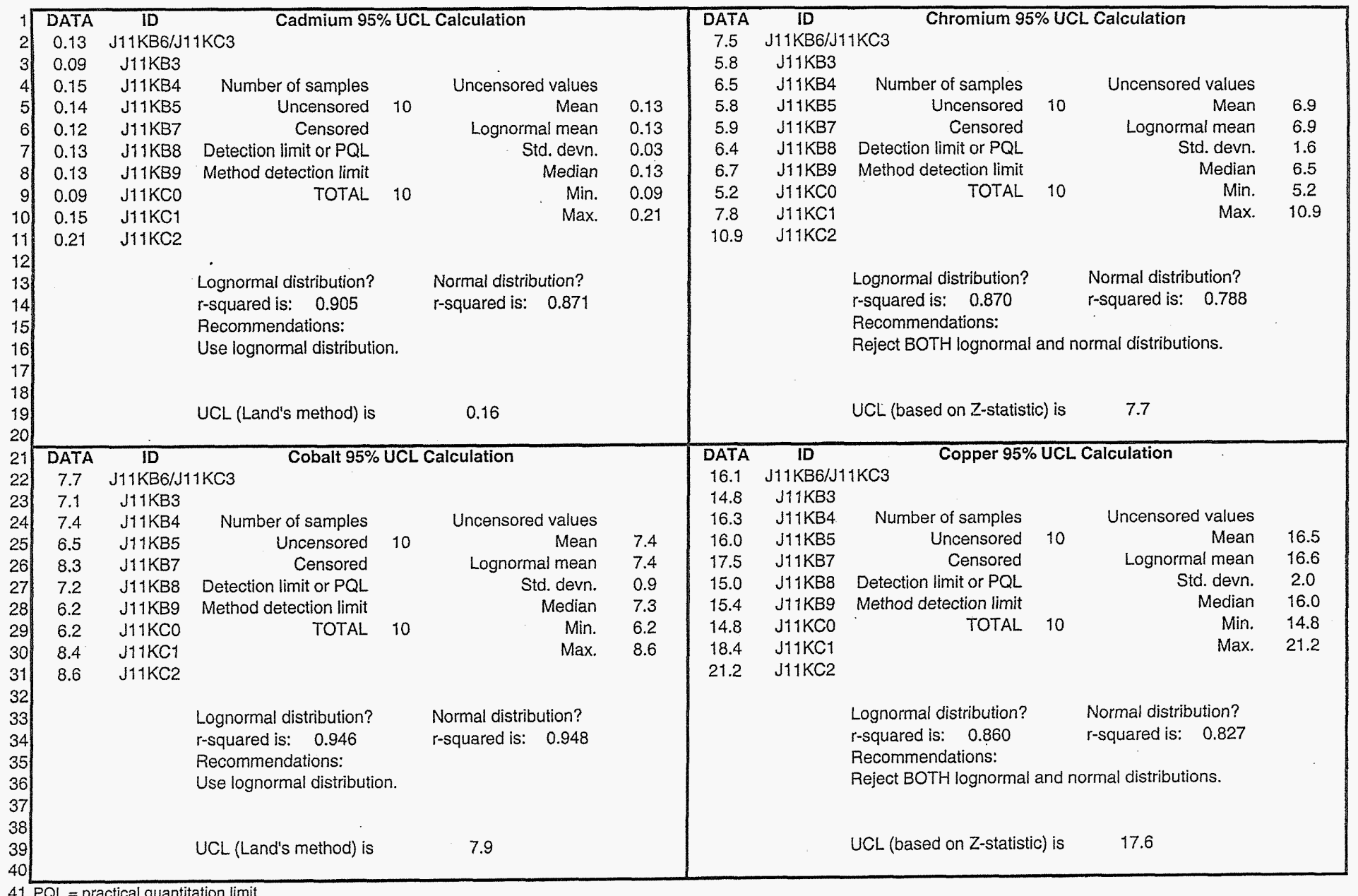




\section{CALCULATION SHEET}

Washington Closure Hanford

Ginc

Date $07 / 31 / 06$

Originator J.M. Capron G.MC Project $100-\mathrm{B} / \mathrm{C}$ Remaining Pipes and Sewers Field Remediation Job No. $\overline{14655}$

Calc. No. 0100B-CA-V028

Ecology Software (MTCAStat) Results (Area 5)

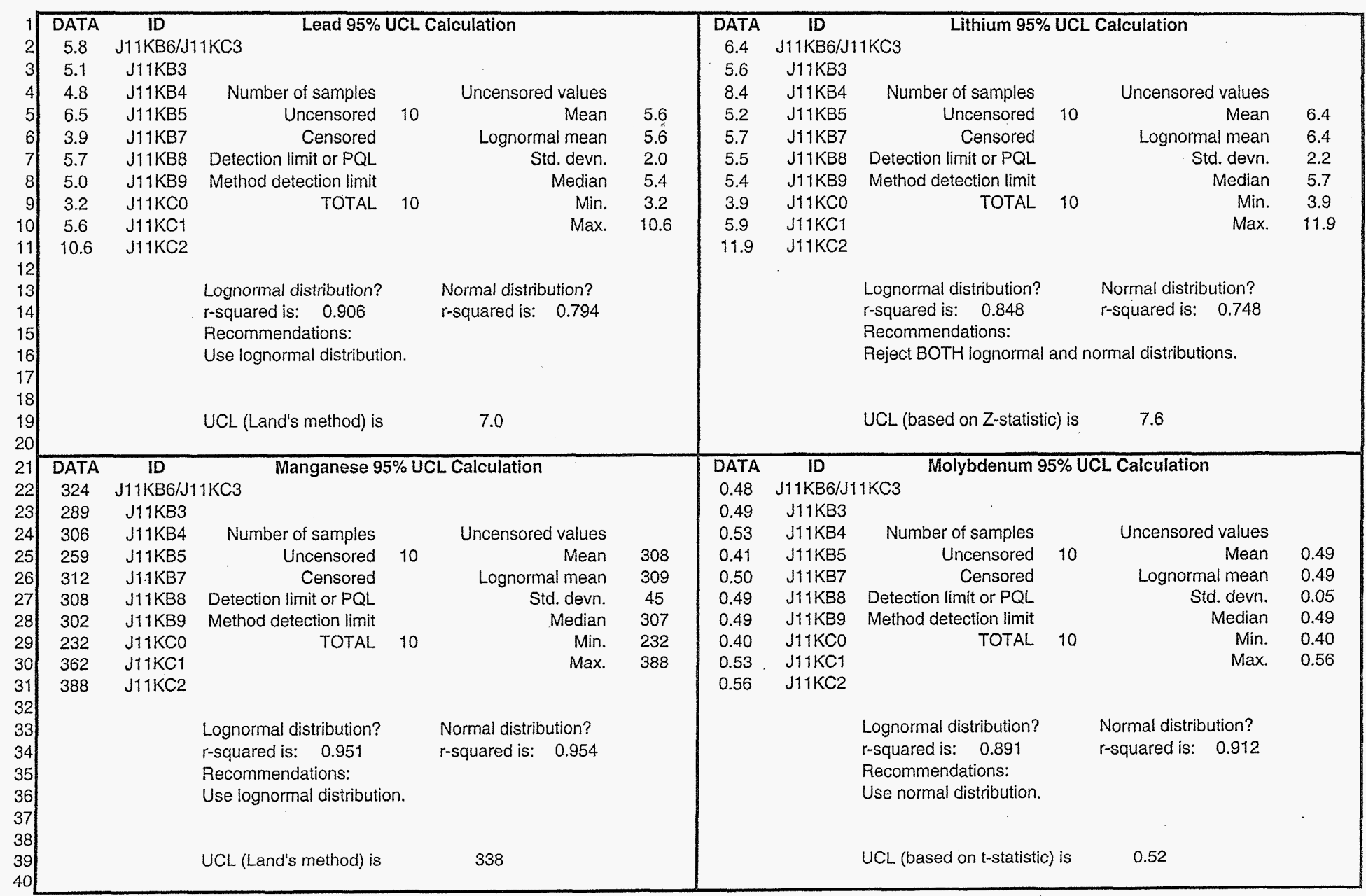


CALCULATION SHEET

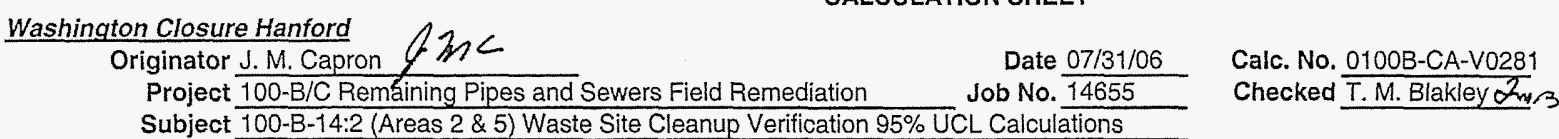
Subject 100-B-14:2 (Areas 2 \& 5) Waste Site Cleanup Verification 95\% UCL Calculation

Ecology Software (MTCAStat) Results (Area 5)

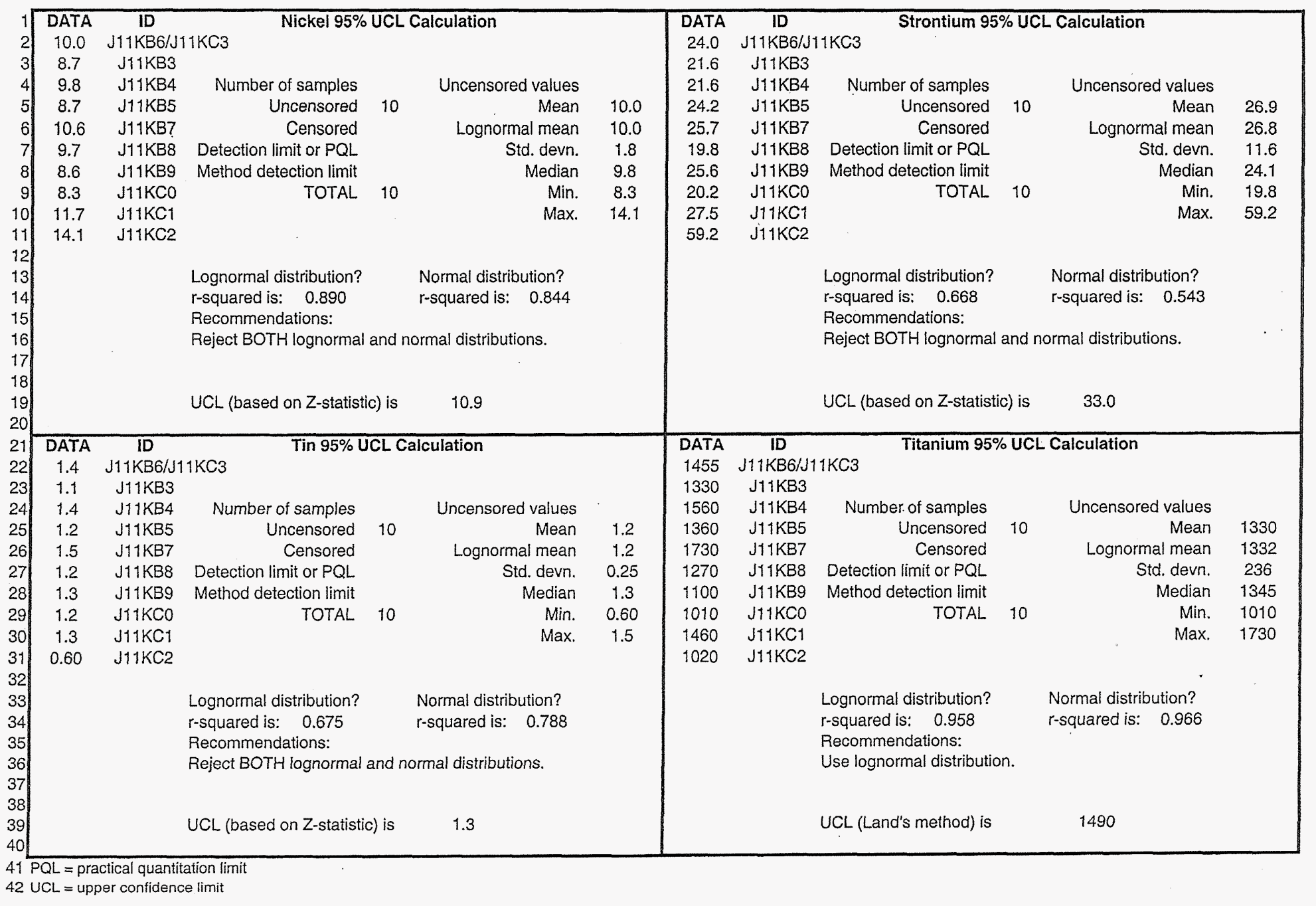




\section{CALCULATION SHEET}

Washington Closure Hanford

Originator JM Capron $4 \mathrm{MC}$

Project $100-\mathrm{B} / \mathrm{C}$ Rentaining Pipes and Sewers Field Remediation

Pubject 100-B-14.2 (Areas $2 \& 5$ ) Waste Site Cleanup Verification $95 \%$ UL
Calc. No. 0100B-CA-V0281

Checked T.M. Blakleyo2nB
Rev. No.

Date 81106

Ecology Software (MTCAStat) Results (Area 5)

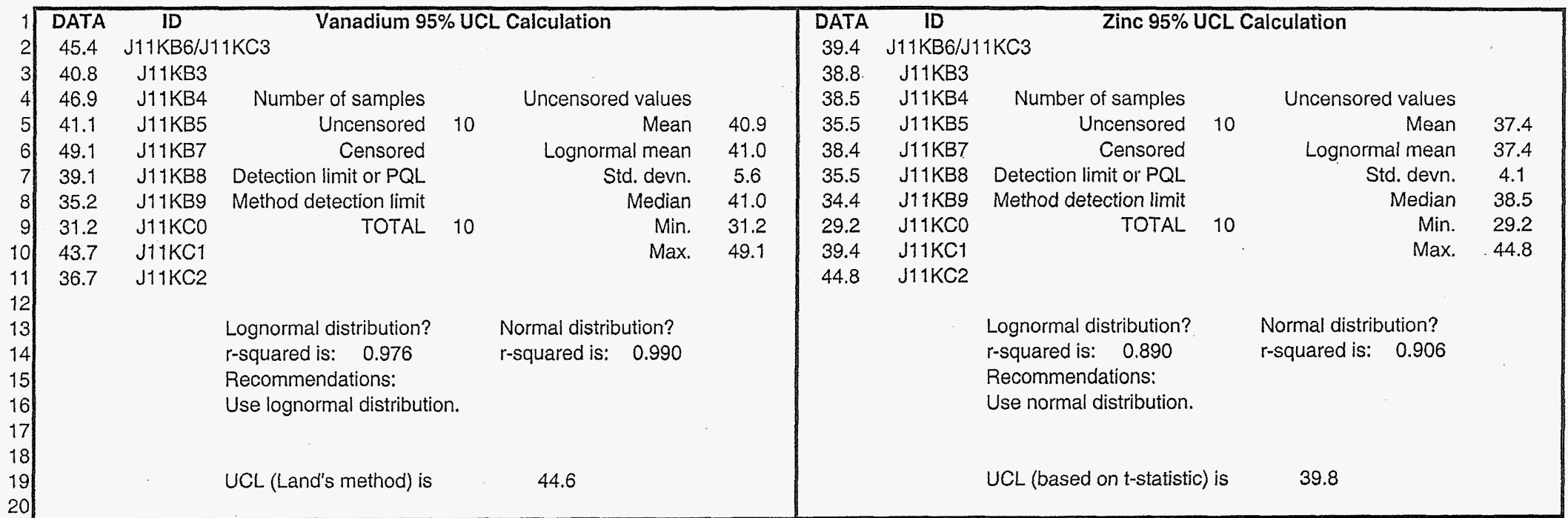

$21 \mathrm{PQL}=$ practical quantitation limit

$22 \mathrm{UCL}=$ upper confidence limit 
Attachment 1. 100-B-14:2 Area 2 Verification Sampling Results.

\begin{tabular}{|c|c|c|c|c|c|c|c|c|c|c|c|c|c|c|c|c|c|c|c|c|}
\hline \multirow{2}{*}{$\begin{array}{l}\text { Sample } \\
\text { Location }\end{array}$} & \multirow{2}{*}{$\begin{array}{l}\text { Sample } \\
\text { Number }\end{array}$} & \multirow{2}{*}{$\begin{array}{c}\text { Sample } \\
\text { Date }\end{array}$} & \multicolumn{3}{|c|}{ Americium-241 } & \multicolumn{3}{|c|}{ Antimony-125 } & \multicolumn{3}{|c|}{ Cesium-134 } & \multicolumn{3}{|c|}{ Cesium-137 } & \multicolumn{3}{|c|}{ Cobalt- 60} & \multicolumn{3}{|c|}{ Europium-152 } \\
\hline & & & $\mathrm{pCi} / \mathrm{g}$ & $Q$ & MDA & $\mathrm{pCi} / \mathrm{g}$ & $Q$ & $\mathrm{MDA}$ & $\mathrm{pCi} / \mathrm{g}$ & $Q$ & $\mathrm{MDA}$ & $\mathrm{pCi} / \mathrm{g}$ & Q & $\mathrm{MDA}$ & $\mathrm{pCi} / \mathrm{g}$ & $Q$ & $\mathrm{MDA}$ & $\mathrm{pCi} / \mathrm{g}$ & $Q$ & $\mathrm{MOA}$ \\
\hline 1 & J11K85 & $4 / 3 / 06$ & 0.26 & $\bar{U}$ & 0.26 & 0.15 & $\mathrm{U}$ & 0.15 & 0.12 & $\mathrm{U}$ & 0.12 & 0.072 & $U$ & 0.072 & 0.080 & $\overrightarrow{\mathrm{U}}$ & 0.080 & 0.18 & $\mathrm{U}$ & 0.18 \\
\hline 2 & $\mathrm{~J} 11 \mathrm{~K} 86$ & $4 / 3 / 06$ & 0.32 & $\mathrm{U}$ & 0.32 & 0.19 & $\bar{U}$ & 0.19 & 0.11 & $\bar{U}$ & 0.11 & 0.085 & $U$ & 0.085 & 0.089 & $\mathrm{U}$ & 0.089 & 0.18 & $\overline{\mathrm{U}}$ & 0.18 \\
\hline $\begin{array}{l}\text { Duplicate of } \\
\text { J11K } 86\end{array}$ & J11K95 & $4 / 3 / 06$ & 0.28 & U & 0.28 & 0.16 & U & 0.16 & 0.086 & $\mathrm{U}$ & 0.086 & 0.070 & $U$ & 0.070 & 0.069 & $U$ & 0.069 & 0.17 & U & 0.17 \\
\hline 3 & J11K87 & $4 / 3 / 06$ & 0.26 & $\mathrm{U}$ & 0.26 & 0.15 & $\mathrm{U}$ & 0.15 & 0.091 & $\mathrm{U}$ & 0.091 & 0.068 & $\mathrm{U}$ & 0.068 & 0.085 & $\mathrm{U}$ & 0.085 & 0.16 & $\mathrm{U}$ & 0.16 \\
\hline 4 & $\mathrm{J11K88}$ & $4 / 3 / 06$ & 0.20 & $\mathrm{U}$ & 0.20 & 0.19 & $\bar{U}$ & 0.19 & 0.098 & $U$ & 0.098 & 0.082 & $\mathrm{U}$ & 0.082 & 0.091 & $\mathrm{U}$ & 0.091 & 0.20 & $\mathrm{U}$ & 0.20 \\
\hline 5 & J11K89 & $4 / 3 / 06$ & 0.26 & U & 0.26 & 0.14 & $\mathrm{U}$ & 0.14 & 0.080 & $\mathrm{U}$ & 0.080 & 0.066 & $\mathrm{U}$ & 0.066 & 0.065 & $\mathrm{U}$ & 0.065 & 0.14 & $\mathrm{U}$ & 0.14 \\
\hline 6 & $\mathrm{~J} 11 \mathrm{~K} 90$ & $4 / 3 / 06$ & 0.19 & $\mathrm{U}$ & 0.19 & 0.11 & $\bar{U}$ & 0.11 & 0.079 & $\mathrm{U}$ & 0.079 & 0.060 & $\mathrm{U}$ & 0.060 & 0.069 & $\mathrm{U}$ & 0.069 & 0.13 & $\mathrm{U}$ & 0.13 \\
\hline 7 & $311 \mathrm{~K} 91$ & $4 / 3 / 06$ & 0.26 & $\mathrm{U}$ & 0.26 & 0.14 & $\mathrm{U}$ & 0.14 & 0.097 & $\mathrm{U}$ & 0.097 & 0.084 & $\mathrm{U}$ & 0.084 & 0.089 & $\overline{\mathrm{U}}$ & 0.089 & 0.18 & $\bar{U}$ & 0.18 \\
\hline 8 & $\mathrm{~J} 11 \mathrm{~K} 92$ & $4 / 3 / 06$ & 0.18 & $\mathrm{U}$ & 0.18 & 0.16 & $U$ & 0.16 & 0.089 & $\mathrm{U}$ & 0.089 & 0.071 & $\mathrm{U}$ & 0.071 & 0.069 & $\mathrm{U}$ & 0.069 & 0.16 & $\mathrm{U}$ & 0.16 \\
\hline 9 & J11K93 & $4 / 3 / 06$ & 0.21 & $\mathrm{U}$ & 0.21 & 0.21 & $\mathrm{U}$ & 0.21 & 0.12 & $U$ & 0.12 & 0.084 & $\mathrm{U}$ & 0.084 & 0.081 & $\mathrm{U}$ & 0.081 & 0.18 & $U$ & 0.18 \\
\hline 10 & $\mathrm{~J} 11 \mathrm{~K} 94$ & $4 / 3 / 06$ & 0.28 & $\overline{\mathrm{U}}$ & 0.28 & 0.18 & $\mathrm{U}$ & 0.18 & 0.10 & $\mathrm{U}$ & 0.10 & 0.080 & $\mathrm{U}$ & 0.080 & 0.088 & $\bar{U}$ & 0.088 & 0.20 & $\mathrm{U}$ & 0.20 \\
\hline $\begin{array}{l}\text { East BCL } \\
\text { stockpile }\end{array}$ & J11KD1 & $4 / 3 / 06$ & 0.21 & $\mathrm{U}$ & 0.21 & 0.14 & $\mathrm{U}$ & 0.14 & 0.081 & $\mathrm{U}$ & 0.081 & 0.071 & $\mathrm{U}$ & 0.071 & 0.079 & $\mathrm{U}$ & 0.079 & 0.15 & $U$ & 0.15 \\
\hline $\begin{array}{l}\text { West BCL } \\
\text { stockpile }\end{array}$ & J11KD2 & $4 / 3 / 06$ & 0.27 & U & 0.27 & 0.16 & $U$ & 0.16 & 0.098 & U & 0.098 & 0.077 & $\mathrm{U}$ & 0.077 & 0.085 & $\mathrm{U}$ & 0.085 & 0.17 & U & 0.17 \\
\hline
\end{tabular}

Note: The following abbreviations apply to all Attachment 1 and 2 tables.

Note: Data qualified with $C, D$ and/or J are considered acceptable values.

$\mathrm{BCL}=$ below cleanup levels

$\mathrm{D}=$ diluted

$\mathrm{PQL}=$ practical quantitation limit

$\mathrm{J}=$ estimated

$\mathrm{Q}=$ qualifier

$\mathrm{U}=$ undetected

\begin{tabular}{|c|c|c|c|}
\hline Attachment & 1 & Sheet No. & 1 of 13 \\
\hline Originator & J.M. Capron \&asc & Date & $07 / 31 / 06$ \\
\hline Checked & T.M. Blakle $2 \mathrm{mB}$ & Date & $8 / 1 / 06$ \\
\hline Calc. No. & $0100 \mathrm{~B}-\mathrm{CA}-\mathrm{V} 0281$ & Rev. No. & 0 \\
\hline
\end{tabular}


Attachment 1. 100-B-14:2 Area 2 Verification Sampling Results.

\begin{tabular}{|c|c|c|c|c|c|c|c|c|c|c|c|c|c|c|c|c|c|c|c|c|}
\hline \multirow{2}{*}{$\begin{array}{c}\text { Sample } \\
\text { Location }\end{array}$} & \multirow{2}{*}{$\begin{array}{c}\text { Sample } \\
\text { Number }\end{array}$} & \multirow{2}{*}{$\begin{array}{c}\text { Sample } \\
\text { Date }\end{array}$} & \multicolumn{3}{|c|}{ Europium-154 } & \multicolumn{3}{|c|}{ Europium-155 } & \multicolumn{3}{|c|}{ Gross Alpha } & \multicolumn{3}{|c|}{ Gross Beta } & \multicolumn{3}{|c|}{ Potassium-40 } & \multicolumn{3}{|c|}{ Radium-226 } \\
\hline & & & $\mathrm{pCi} / \mathrm{g}$ & $Q$ & MDA & $\mathrm{pCi} / \mathrm{g}$ & $Q$ & $\mathrm{MDA}$ & $\mathrm{pCi} / \mathrm{g}$ & $Q$ & $\mathrm{MDA}$ & $\mathrm{pCi} / \mathrm{g}$ & Q & MDA & $\mathrm{pCi} / \mathrm{g}$ & $Q$ & MDA & $\mathrm{pCi} / \mathrm{g}$ & $Q$ & $\overline{M D A}$ \\
\hline 1 & J11K85 & $4 / 3 / 06$ & 0.25 & $\mathrm{U}$ & 0.25 & 0.19 & $\mathrm{U}$ & 0.19 & -0.656 & $\mathrm{U}$ & 9.8 & 15.5 & & 6.2 & 6.66 & & 0.95 & 0.328 & $\mathrm{~J}$ & 0.13 \\
\hline 2 & $511 \mathrm{~K} 86$ & $4 / 3 / 06$ & 0.27 & $\bar{U}$ & 0.27 & 0.20 & $\bar{U}$ & 0.20 & 1.74 & $\mathrm{U}$ & 4.1 & 13.4 & & 4.0 & 10.2 & & 0.73 & 0.470 & $\mathrm{~J}$ & 0.16 \\
\hline $\begin{array}{c}\text { Duplicate of } \\
\text { J11K86 }\end{array}$ & J11K95 & $4 / 3 / 06$ & 0.23 & $\mathrm{U}$ & 0.23 & 0.18 & U & 0.18 & 8.98 & & 7.8 & 17.6 & & 4.6 & 9.92 & & 0.67 & 0.439 & $\mathrm{~J}$ & 0.13 \\
\hline 3 & J11K87 & $4 / 3 / 06$ & 0.24 & $\mathrm{U}$ & 0.24 & 0.20 & $\mathrm{U}$ & 0.20 & 11.2 & & 7.2 & 17.1 & & 10 & 9.24 & & 0.67 & 0.325 & $\mathrm{~J}$ & 0.13 \\
\hline 4 & J11K88 & $4 / 3 / 06$ & 0.27 & $\mathrm{U}$ & 0.27 & 0.19 & $\mathrm{U}$ & 0.19 & 3.31 & U & 5.3 & 12.2 & & 4.5 & 10.5 & & 0.74 & 0.399 & $\mathrm{~J}$ & 0.15 \\
\hline 5 & J11K89 & $4 / 3 / 06$ & 0.22 & $\mathrm{U}$ & 0.22 & 0.16 & $\mathrm{U}$ & 0.16 & 5.62 & $\mathrm{U}$ & 5.9 & 14.1 & & 5.6 & 9.36 & & 0.61 & 0.345 & $\mathrm{~J}$ & 0.12 \\
\hline 6 & $\mathrm{~J} 11 \mathrm{~K} 90$ & $4 / 3 / 06$ & 0.19 & $\mathrm{U}$ & 0.19 & 0.15 & $\mathrm{U}$ & 0.15 & 10.8 & & 5.7 & 16.9 & & 3.7 & 6.12 & & 0.72 & 0.185 & $\mathrm{~d}$ & 0.13 \\
\hline 7 & $\mathrm{~J} 11 \mathrm{~K} 91$ & $4 / 3 / 06$ & 0.26 & $\mathrm{U}$ & 0.26 & 0.20 & $\mathrm{U}$ & 0.20 & 2.50 & $\mathrm{U}$ & 5.4 & 28.8 & & 4.5 & 6.94 & & 0.95 & 0.443 & $\mathrm{~J}$ & 0.13 \\
\hline 8 & $\mathrm{~J} 11 \mathrm{~K} 92$ & $4 / 3 / 06$ & 0.21 & $\mathrm{U}$ & 0.21 & 0.17 & $\mathrm{U}$ & 0.17 & 1.21 & $\mathrm{U}$ & 11 & 17.3 & & 5.1 & 8.39 & & 0.93 & 0.354 & $\mathrm{~J}$ & 0.13 \\
\hline 9 & J11K93 & $4 / 3 / 06$ & 0.27 & $\mathrm{U}$ & 0.27 & 0.20 & $\mathrm{U}$ & 0.20 & 7.38 & & 6.7 & 14.1 & & 3.9 & 8.73 & & 0.84 & 0.508 & $J$ & 0.19 \\
\hline 10 & $\mathrm{~J} 11 \mathrm{~K} 94$ & $4 / 3 / 06$ & 0.27 & $\mathrm{U}$ & 0.27 & 0.22 & $\mathrm{U}$ & 0.22 & -2.10 & $U$ & 11 & 19.4 & & 5.1 & 9.27 & & 0.90 & 0.361 & $\mathrm{~J}$ & 0.15 \\
\hline $\begin{array}{l}\text { East } \mathrm{BCL} \\
\text { stockpile }\end{array}$ & $\mathrm{J} 11 \mathrm{KD} 1$ & $4 / 3 / 06$ & 0.20 & $\mathrm{U}$ & 0.20 & 0.16 & $\mathrm{U}$ & 0.16 & 0.744 & U & 9.5 & 16.4 & & 6.9 & 6.54 & & 0.59 & 0.386 & $\mathrm{~J}$ & 0.11 \\
\hline $\begin{array}{l}\text { West BCL } \\
\text { stockpile }\end{array}$ & $\mathrm{J} 11 \mathrm{KD} 2$ & $4 / 3 / 06$ & 0.27 & $\mathrm{U}$ & 0.27 & 0.21 & $\mathrm{U}$ & 0.21 & 2.52 & $\mathrm{U}$ & 7.9 & 17.9 & & 6.6 & 9.54 & & 0.66 & 0.400 & $\mathrm{~J}$ & 0.14 \\
\hline
\end{tabular}

\begin{tabular}{|c|c|c|c|c|c|c|c|c|c|c|c|c|c|c|c|c|c|c|c|c|}
\hline \multirow{2}{*}{$\begin{array}{l}\text { Sample } \\
\text { Location }\end{array}$} & \multirow{2}{*}{$\begin{array}{l}\text { Sample } \\
\text { Number }\end{array}$} & \multirow{2}{*}{$\begin{array}{c}\text { Sample } \\
\text { Date }\end{array}$} & \multicolumn{3}{|c|}{ Radium-228 } & \multicolumn{3}{|c|}{ Thorium-228 } & \multicolumn{3}{|c|}{ Thorium-232 } & \multicolumn{3}{|c|}{$\begin{array}{c}\text { Total Beta } \\
\text { Radiostrontium }\end{array}$} & \multicolumn{3}{|c|}{ Uranium-235 } & \multicolumn{3}{|c|}{ Uranium-238 } \\
\hline & & & $\mathrm{pCi} / \mathrm{g}$ & $Q$ & $\mathrm{MDA}$ & $\mathrm{pCi} / \mathrm{g}$ & $Q$ & $\overline{\mathrm{MDA}}$ & $\mathrm{pCi} / \mathrm{g}$ & $Q$ & $\mathrm{MDA}$ & $\mathrm{pCi} / \mathrm{g}$ & Q & MDA & $\mathrm{pCi} / \mathrm{g}$ & $Q$ & MDA & $\mathrm{pCi} / \mathrm{g}$ & $Q$ & MDA \\
\hline 1 & $\mathrm{J11K} \times 5$ & $4 / 3 / 06$ & 0.663 & & 0.29 & 0.423 & & 0.079 & 0.663 & & 0.29 & & & & 0.25 & $\mathrm{U}$ & 0.25 & 8.3 & $\bar{U}$ & 8.3 \\
\hline 2 & J11K86 & $4 / 3 / 06$ & 0.576 & & 0.38 & 0.595 & & 0.094 & 0.576 & & 0.38 & & & & 0.30 & $\bar{U}$ & 0.30 & 8.8 & $\mathrm{U}$ & 8.8 \\
\hline $\begin{array}{c}\text { Duplicate of } \\
\text { J11K86 }\end{array}$ & J11 K95 & $4 / 3 / 06$ & 0.860 & & 0.25 & 0.597 & & 0.082 & 0.860 & & 0.25 & & & & 0.27 & U & 0.27 & 8.6 & U & 8.6 \\
\hline 3 & J11K87 & $4 / 3 / 06$ & 0.766 & & 0.36 & 0.552 & & 0.13 & 0.766 & & 0.36 & & & & 0.25 & $\mathrm{U}$ & 0.25 & 8.4 & $\mathrm{U}$ & 8.4 \\
\hline 4 & J11K88 & $4 / 3 / 06$ & 0.493 & & 0.27 & 0.489 & & 0.081 & 0.493 & & 0.27 & & & & 0.27 & $\mathrm{U}$ & 0.27 & 11 & $\mathrm{U}$ & 11 \\
\hline 5 & J11K89 & $4 / 3 / 06$ & 0.463 & & 0.22 & 0.642 & & 0.11 & 0.463 & & 0.22 & & & & 0.24 & $\mathrm{U}$ & 0.24 & 7.8 & $\mathrm{U}$ & 7.8 \\
\hline 6 & $\mathrm{J11K} \mathrm{K}$ & $4 / 3 / 06$ & 0.497 & & 0.26 & 0.355 & & 0.065 & 0.497 & & 0.26 & & & & 0.18 & $\mathrm{U}$ & 0.18 & 7.1 & $\mathrm{U}$ & 7.1 \\
\hline 7 & J11K91 & $4 / 3 / 06$ & 0.565 & & 0.29 & 0.506 & & 0.078 & 0.565 & & 0.29 & 0.311 & & 0.19 & 0.25 & $\mathrm{U}$ & 0.25 & 8.6 & $\mathrm{U}$ & 8.6 \\
\hline 8 & $\mathrm{~J} 11 \mathrm{~K} 92$ & $4 / 3 / 06$ & 0.522 & & 0.25 & 0.556 & & 0.12 & 0.522 & & 0.25 & & & & 0.25 & $\mathrm{U}$ & 0.25 & 9.9 & $\mathrm{U}$ & 9.9 \\
\hline 9 & J11K93 & $4 / 3 / 06$ & 0.721 & & 0.40 & 0.751 & & 0.14 & 0.721 & & 0.40 & & & & 0.31 & $\mathrm{U}$ & 0.31 & 10 & $\mathrm{U}$ & 10 \\
\hline 10 & $\mathrm{J11K94}$ & $4 / 3 / 06$ & 0.832 & & 0.36 & 0.415 & & 0.10 & 0.832 & & 0.36 & & & & 0.29 & $\mathrm{U}$ & 0.29 & 9.9 & U & 9.9 \\
\hline $\begin{array}{l}\text { East } \mathrm{BCL} \\
\text { stockpile }\end{array}$ & $\mathrm{J} 11 \mathrm{KD} 1$ & $4 / 3 / 06$ & 0.616 & & 0.25 & 0.438 & & 0.11 & 0.616 & & 0.25 & & & & 0.20 & U & 0.20 & 7.3 & u & 7.3 \\
\hline $\begin{array}{l}\text { West BCL } \\
\text { stockpile }\end{array}$ & $\mathrm{J} 11 \mathrm{KD} 2$ & $4 / 3 / 06$ & 0.669 & & 0.32 & 0.536 & & 0.12 & 0.669 & & 0.32 & & & & 0.27 & U & 0.27 & 9.3 & U & 9.3 \\
\hline & & & & & & & & & & & & \multirow{2}{*}{\multicolumn{3}{|c|}{$\begin{array}{l}\text { Attachment } \\
\text { Originator }\end{array}$}} & \multicolumn{3}{|c|}{1} & \multicolumn{3}{|c|}{2 of 13} \\
\hline & & & & & & & & & & & & & & & \multicolumn{3}{|c|}{ J. M. Capron } & \multirow{3}{*}{$\begin{array}{l}\text { Date } \\
\text { Date } \\
\text { Rev. No. }\end{array}$} & \multicolumn{2}{|c|}{$07 / 31 / 06$} \\
\hline & & & & & & & & & & & & & \multirow{2}{*}{\multicolumn{2}{|c|}{$\begin{array}{l}\text { Checked } \\
\text { Calc. No. }\end{array}$}} & T.M. B1 & dey & & & & \\
\hline & & & & & & & & & & & & & & & $0100 \mathrm{~B}-\mathrm{C}$ & $-\mathrm{V} 0$ & & & & 0 \\
\hline
\end{tabular}


Attachment 1. 100-B-14:2 Area 2 Verification Sampling Results.

\begin{tabular}{|c|c|c|c|c|c|c|c|c|c|c|c|c|c|c|c|c|c|c|c|}
\hline \multirow{2}{*}{$\begin{array}{c}\text { Sample } \\
\text { Location }\end{array}$} & \multirow{2}{*}{$\begin{array}{l}\text { Sample } \\
\text { Number }\end{array}$} & \multirow{2}{*}{$\begin{array}{c}\text { Sample } \\
\text { Date }\end{array}$} & \multicolumn{3}{|c|}{ Aluminum } & \multicolumn{3}{|c|}{ Antimony } & \multicolumn{3}{|c|}{ Arsenic } & \multicolumn{2}{|c|}{ Barium } & \multicolumn{3}{|c|}{ Beryllium } & \multicolumn{3}{|c|}{ Boron } \\
\hline & & & $\mathrm{mg} / \mathrm{kg}$ & $Q$ & PQL & $\mathrm{mg} / \mathrm{kg}$ & $Q$ & $\mathrm{PQL}$ & $\mathrm{mg} / \mathrm{kg}$ & $Q$ & $\mathrm{PQL}$ & $\mathrm{mg} / \mathrm{kg}$ & \begin{tabular}{l|l}
$\mathrm{Q}$ & $\mathrm{PQL}$ \\
\end{tabular} & $\mathrm{mg} / \mathrm{kg}$ & $Q$ & $\mathrm{PQL}$ & $\mathrm{mg} / \mathrm{kg}$ & $Q$ & PQL \\
\hline 1 & J11K85 & $4 / 3 / 06$ & 6040 & C & 2.4 & 0.44 & U3 & 0.44 & 2.9 & & 0.61 & 61.3 & 0.02 & 0.36 & & 0.02 & 1.4 & C & 0.24 \\
\hline 2 & J11K86 & $4 / 3 / 06$ & 5210 & $\mathrm{C}$ & 2.3 & 0.44 & UJ & 0.44 & 1.8 & & 0.60 & 62.4 & 0.02 & 0.25 & & 0.02 & 1.1 & UJC & 0.24 \\
\hline $\begin{array}{c}\text { Duplicate of } \\
\text { J11K86 }\end{array}$ & $\mathrm{J11K95}$ & 4/3/06 & 4950 & c & 2.3 & 0.43 & UJ & 0.43 & 2.3 & & 0.60 & 64.3 & 0.02 & 0.29 & & 0.02 & 0.64 & UJC & 0.24 \\
\hline 3 & $\mathrm{J11K} 87$ & $4 / 3 / 06$ & 5840 & $\mathrm{C}$ & 2.4 & 0.44 & UJ & 0.44 & 3.2 & & 0.61 & 72.7 & 0.02 & 0.38 & & 0.02 & 1.6 & $\mathrm{C}$ & 0.24 \\
\hline 4 & $\mathrm{~J} 11 \mathrm{~K} 88$ & $4 / 3 / 06$ & 5450 & C & 2.4 & 0.44 & US & 0.44 & 2.9 & & 0.61 & 63.2 & 0.02 & 0.30 & & 0.02 & 1.1 & UJC & 0.24 \\
\hline 5 & $\mathrm{~J} 11 \mathrm{~K} 89$ & $4 / 3 / 06$ & 5470 & $\mathrm{C}$ & 2.4 & 0.44 & UI & 0.44 & 3.0 & & 0.61 & 55.8 & 0.02 & 0.35 & & 0.02 & 0.47 & UJC & 0.24 \\
\hline 6 & $\mathrm{J11K90}$ & $4 / 3 / 06$ & 4160 & $\mathrm{C}$ & 2.3 & 0.43 & UJ & 0.43 & 2.7 & & 0.60 & 49.6 & 0.02 & 0.33 & & 0.02 & 0.54 & USC & 0.24 \\
\hline 7 & $\mathrm{J11K91}$ & $4 / 3 / 06$ & 6390 & $\mathrm{C}$ & 2.3 & 0.43 & $\mathrm{UJ}$ & 0.43 & 3.4 & & 0.60 & 409 & 0.02 & 0.41 & & 0.02 & 5.0 & $\mathrm{C}$ & 0.24 \\
\hline 8 & $\mathrm{~J} 11 \mathrm{~K} 92$ & $4 / 3 / 06$ & 5020 & C & 2.3 & 0.43 & UJ & 0.43 & 3.5 & & 0.59 & 63.6 & 0.02 & 0.42 & & 0.02 & 0.83 & UJC & 0.23 \\
\hline 9 & J11K93 & $4 / 3 / 06$ & 5700 & C & 2.3 & 0.44 & UI & 0.44 & 3.3 & & 0.60 & 131 & 0.02 & 0.37 & & 0.02 & 2.7 & $\mathrm{C}$ & 0.24 \\
\hline 10 & J11K94 & $4 / 3 / 06$ & 6210 & C & 2.4 & 0.45 & UJ & 0.45 & 3.8 & & 0.63 & 95.6 & 0.02 & 0.32 & & 0.02 & 1.6 & C & 0.25 \\
\hline $\begin{array}{l}\text { East BCL } \\
\text { stockpile }\end{array}$ & $\mathrm{J} 11 \mathrm{KD} 1$ & $4 / 3 / 06$ & 5330 & c & 2.3 & 0.44 & UJ & 0.44 & 2.7 & & 0.60 & 141 & 0.02 & 0.34 & & 0.02 & 3.6 & c & 0.24 \\
\hline $\begin{array}{l}\text { West BCL } \\
\text { stockpile }\end{array}$ & J1/KD2 & $4 / 3 / 06$ & 6040 & c & 2.4 & 0.44 & UJ & 0.44 & 2.9 & & 0.61 & 86.6 & 0.02 & 0.31 & & 0.02 & 3.5 & c & 0.24 \\
\hline $\begin{array}{c}\text { Equipment } \\
\text { blank }\end{array}$ & J11K96 & $4 / 3 / 06$ & 50.7 & UIC & 2.2 & 0.41 & UJ & 0.41 & 0.56 & $\mathrm{U}$ & 0.56 & 1.4 & 0.02 & 0.02 & $\mathrm{U}$ & 0.02 & 0.61 & UJC & 0.22 \\
\hline
\end{tabular}

\begin{tabular}{|c|c|c|c|c|c|c|c|c|c|}
\hline Sample & Sample & Sample & Cadmium & Calcium & Chromium & Cobalt & Copper & Hexavalent Chromium \\
\hline
\end{tabular}

\begin{tabular}{|c|c|c|c|c|c|c|c|c|c|c|c|c|c|c|c|c|c|c|c|c|}
\hline \multirow{2}{*}{$\begin{array}{l}\text { Sample } \\
\text { Location }\end{array}$} & \multirow{2}{*}{$\begin{array}{l}\text { Sample } \\
\text { Number }\end{array}$} & \multirow{2}{*}{$\begin{array}{c}\text { Sampie } \\
\text { Date }\end{array}$} & \multicolumn{3}{|c|}{ Cauminin } & \multicolumn{3}{|c|}{ Cácium } & \multicolumn{3}{|c|}{ CITomimim } & \multicolumn{3}{|c|}{ Conall } & \multicolumn{3}{|c|}{ Coppex } & \multicolumn{3}{|c|}{ Hexavalent Cnromium } \\
\hline & & & $\mathrm{mg} / \mathrm{kg}$ & $Q$ & $\overline{\mathrm{PQL}}$ & $\mathrm{mg} / \mathrm{kg}$ & $\mathrm{Q}$ & $\mathrm{PQL}$ & $\mathrm{mg} / \mathrm{kg}$ & $Q$ & $\mathrm{PQL}$ & $\mathrm{mg} / \mathrm{kg}$ & Q & PQL & $\mathrm{mg} / \mathrm{kg}$ & $Q$ & $\mathrm{PQL}$ & $\mathrm{mg} / \mathrm{kg}$ & $\bar{Q}$ & $\overline{\mathrm{PQL}}$ \\
\hline 1 & J11K85 & $4 / 3 / 06$ & 0.17 & & 0.07 & 4880 & $\mathrm{~J}$ & 2.2 & 8.5 & & 0.133 & 8.7 & & 0.14 & 17.7 & & 0.12 & 0.25 & & 0.22 \\
\hline 2 & J11K86 & $4 / 3 / 06$ & 0.12 & & 0.07 & 4080 & $\mathrm{~J}$ & 2.2 & 7.7 & & 0.13 & 7.1 & & 0.14 & 12.9 & & 0.12 & 0.22 & & 0.21 \\
\hline $\begin{array}{l}\text { Duplicate of } \\
\text { J11K86 }\end{array}$ & J11K95 & $4 / 3 / 06$ & 0.07 & $\mathrm{U}$ & 0.07 & 3600 & $\mathrm{~J}$ & 2.2 & 7.5 & & 0.13 & 7.3 & & 0.14 & 12.6 & & 0.12 & 0.34 & & 0.21 \\
\hline 3 & $\mathrm{J11K87}$ & $4 / 3 / 06$ & 0.11 & & 0.07 & 8070 & $\mathrm{~J}$ & 2.2 & 8.6 & & 0.13 & 8.3 & & 0.14 & 17.1 & & 0.12 & 0.21 & U & 0.21 \\
\hline 4 & $\mathrm{~J} 11 \mathrm{~K} 88$ & $4 / 3 / 06$ & 0.11 & & 0.07 & 6710 & $\mathrm{~J}$ & 2.2 & 6.5 & & 0.13 & 7.8 & & 0.14 & 16.3 & & 0.12 & 0.26 & & 0.21 \\
\hline 5 & J11K89 & $4 / 3 / 06$ & 0.15 & & 0.07 & 6000 & $\mathrm{~J}$ & 2.2 & 7.6 & & 0.13 & 8.0 & & 0.14 & 17.2 & & 0.12 & 0.21 & $\mathrm{U}$ & 0.21 \\
\hline 6 & $\mathrm{J11K90}$ & $4 / 3 / 06$ & 0.12 & & 0.07 & 6290 & $\mathrm{~J}$ & 2.1 & 5.9 & & 0.13 & 8.0 & & 0.14 & 18.2 & & 0.12 & 0.22 & & 0.21 \\
\hline 7 & $\mathrm{J11K9I}$ & $4 / 3 / 06$ & 0.14 & & 0.07 & 7950 & $\mathrm{~J}$ & 2.2 & 10.1 & & 0.13 & 6.5 & & 0.14 & 15.9 & & 0.12 & 0.28 & & 0.21 \\
\hline 8 & $\mathrm{~J} 11 \mathrm{~K} 92$ & $4 / 3 / 06$ & 0.09 & & 0.07 & 8840 & $J$ & 2.1 & 6.1 & & 0.13 & 8.2 & & 0.14 & 18.3 & & 0.12 & 0.24 & & 0.21 \\
\hline 9 & $\mathrm{J11K93}$ & $4 / 3 / 06$ & 0.07 & & 0.07 & 8290 & $\mathrm{~J}$ & 2.2 & 7.9 & & 0.13 & 8.8 & & 0.14 & 17.4 & & 0.12 & 0.25 & & 0.21 \\
\hline 10 & J11K94 & $4 / 3 / 06$ & 0.09 & & 0.07 & 9770 & $\mathrm{~J}$ & 2.3 & 10.4 & & 0.13 & 7.5 & & 0.14 & 17.4 & & 0.12 & 0.27 & & 0.22 \\
\hline $\begin{array}{l}\text { East BCL } \\
\text { stockpile }\end{array}$ & $\mathrm{J} 11 \mathrm{KD} 1$ & $4 / 3 / 06$ & 0.21 & & 0.07 & 7610 & $\mathrm{~J}$ & 2.2 & 7.6 & & 0.13 & 9.2 & & 0.14 & 17.6 & & 0.12 & 0.21 & U & 0.21 \\
\hline $\begin{array}{l}\text { West BCL } \\
\text { stockpile }\end{array}$ & $\mathrm{J} 11 \mathrm{KD} 2$ & $4 / 3 / 06$ & 0.20 & & 0.07 & 5450 & J & 2.2 & 8.9 & & 0.13 & 8.4 & & 0.14 & 16.3 & & 0.12 & 0.28 & & 0.21 \\
\hline $\begin{array}{l}\text { Equipment } \\
\text { blank }\end{array}$ & J11K96 & $4 / 3 / 06$ & 0.06 & U & 0.06 & 22.9 & $J$ & 2.0 & 0.20 & & 0.12 & 0.13 & $\mathrm{U}$ & 0.13 & 0.11 & U & 0.11 & & & \\
\hline
\end{tabular}

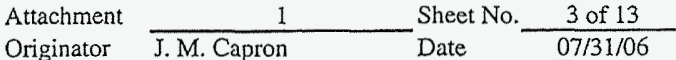

Checked T.M.Blakley Date

\begin{tabular}{lll} 
Checked & T. M. Blakley \\
Calc. No. & Rev. No. & \\
\hline
\end{tabular} 
Attachment 1. 100-B-14:2 Area 2 Verification Sampling Results.

\begin{tabular}{|c|c|c|c|c|c|c|c|c|c|c|c|c|c|c|c|c|c|c|c|c|}
\hline \multirow{2}{*}{$\begin{array}{c}\text { Sample } \\
\text { Location }\end{array}$} & \multirow{2}{*}{$\begin{array}{l}\text { Sample } \\
\text { Number }\end{array}$} & \multirow{2}{*}{$\begin{array}{c}\text { Sample } \\
\text { Date }\end{array}$} & \multicolumn{3}{|c|}{ Iron } & \multicolumn{3}{|c|}{ Lead } & \multicolumn{3}{|c|}{ Lithium } & \multicolumn{3}{|c|}{ Magnesium } & \multicolumn{3}{|c|}{ Manganese } & \multicolumn{3}{|c|}{ Mercury } \\
\hline & & & $\mathrm{mg} / \mathrm{kg}$ & $Q$ & $\mathrm{PQL}$ & $\mathrm{mg} / \mathrm{kg}$ & $Q$ & $\mathrm{PQL}$ & $\mathrm{mg} / \mathrm{kg}$ & $Q$ & $\mathrm{PQL}$ & $\mathrm{mg} / \mathrm{kg}$ & Q & PQL & $\mathrm{mg} / \mathrm{kg}$ & $Q$ & PQL & $\mathrm{mg} / \mathrm{kg}$ & $Q$ & $\mathrm{PQL}$ \\
\hline 1 & J11K85 & $4 / 3 / 06$ & 22700 & $\mathrm{C}$ & 3.5 & 6.1 & & 0.31 & 7.1 & $\mathrm{C}$ & 0.03 & 4480 & & 3.9 & 380 & & 0.03 & 0.02 & U & 0.02 \\
\hline 2 & J11K86 & $4 / 3 / 06$ & 17000 & $\mathrm{C}$ & 3.5 & 4.2 & & 0.31 & 5.1 & C & 0.03 & 3330 & & 3.9 & 334 & & 0.03 & 0.02 & $\mathrm{U}$ & 0.02 \\
\hline $\begin{array}{c}\text { Duplicate of } \\
\text { J11K86 }\end{array}$ & J11K95 & $4 / 3 / 06$ & 17300 & C & 3.4 & 4.4 & & 0.31 & 4.6 & C & 0.03 & 3300 & & 3.9 & 345 & & 0.03 & 0.01 & U & 0.01 \\
\hline 3 & J11K87 & $4 / 3 / 06$ & 20500 & $\mathrm{C}$ & 3.5 & 5.5 & & 0.31 & 7.0 & C & 0.03 & 4640 & & 3.9 & 355 & & 0.03 & 0.02 & U & 0.02 \\
\hline 4 & J11K88 & $4 / 3 / 06$ & 17900 & $\mathrm{C}$ & 3.5 & 4.7 & & 0.31 & 6.6 & $C$ & 0.03 & 4310 & & 3.9 & 315 & & 0.03 & 0.02 & $\mathrm{U}$ & 0.02 \\
\hline 5 & J11K89 & $4 / 3 / 06$ & 19600 & $\mathrm{C}$ & 3.5 & 4.6 & & 0.31 & 6.3 & $\bar{C}$ & 0.03 & 4120 & & 3.9 & 317 & & 0.03 & 0.02 & & 0.01 \\
\hline 16 & J11K90 & $4 / 3 / 06$ & 19100 & $\mathrm{C}$ & 3.4 & 4.4 & & 0.30 & 4.6 & $\bar{C}$ & 0.03 & 3740 & & 3.8 & 293 & & 0.03 & 0.05 & & 0.02 \\
\hline 7 & $511 \mathrm{~K} 91$ & $4 / 3 / 06$ & 13300 & $\mathrm{C}$ & 3.4 & 5.6 & & 0.31 & 8.6 & C & 0.03 & 3820 & & 3.9 & 279 & & 0.03 & 0.03 & & 0.02 \\
\hline 8 & $511 \mathrm{~K} 92$ & $4 / 3 / 06$ & 22000 & $\mathrm{C}$ & 3.4 & 5.5 & & 0.30 & 5.9 & $\bar{C}$ & 0.03 & 4610 & & 3.8 & 348 & & 0.03 & 0.01 & U & 0.01 \\
\hline 9 & $\mathrm{J11K93}$ & $4 / 3 / 06$ & 21200 & $\mathrm{C}$ & 3.5 & 5.7 & & 0.31 & 6.3 & $\mathrm{C}$ & 0.03 & 4210 & & 3.9 & 352 & & 0.03 & 0.03 & & 0.01 \\
\hline 10 & J11K94 & $4 / 3 / 06$ & 18700 & $\mathrm{C}$ & 3.6 & 6.5 & & 0.32 & 7.9 & C & 0.03 & 4590 & & 4.0 & 318 & & 0.03 & 0.02 & $\mathrm{U}$ & 0.02 \\
\hline $\begin{array}{l}\text { East BCL } \\
\text { stockpile }\end{array}$ & $311 \mathrm{KD} 1$ & $4 / 3 / 06$ & 19400 & C & 3.5 & 6.0 & & 0.31 & 6.3 & C & 0.03 & 3920 & & 3.9 & 322 & & 0.03 & 0.08 & & 0.01 \\
\hline $\begin{array}{l}\text { West BCL } \\
\text { stockpile }\end{array}$ & $311 \mathrm{KD} 2$ & $4 / 3 / 06$ & 20300 & C & 3.5 & 5.7 & & 0.31 & 6.7 & C & 0.03 & 4160 & & 3.9 & 351 & & 0.03 & 0.02 & $\mathrm{U}$ & 0.02 \\
\hline $\begin{array}{l}\text { Equipment } \\
\text { blank }\end{array}$ & $\mathrm{J} 11 \mathrm{~K} 96$ & $4 / 3 / 06$ & 283 & C & 3.2 & 0.34 & & 0.29 & 0.09 & UJC & 0.03 & 7.3 & & 3.6 & 4.1 & & 0.03 & 0.02 & U & 0.02 \\
\hline
\end{tabular}

\begin{tabular}{|c|c|c|c|c|c|c|c|c|c|c|}
\hline Sample & Sample & Sample & Molybdenum & Nickel & Phosphorus & Potassium & Selenium & Silicon \\
\hline
\end{tabular}

\begin{tabular}{|c|c|c|c|c|c|c|c|c|c|c|c|c|c|c|c|c|c|c|c|c|}
\hline \multirow{2}{*}{ Location } & \multirow{2}{*}{$\begin{array}{l}\text { Sampie } \\
\text { Number }\end{array}$} & \multirow{2}{*}{ Date } & \multicolumn{3}{|c|}{ - } & & & & & \\
\hline & & & $\mathrm{mg} / \mathrm{kg}$ & $Q$ & $\mathrm{PQL}$ & $\mathrm{mg} / \mathrm{kg}$ & $Q$ & $\mathrm{PQL}$ & $\mathrm{mg} / \mathrm{kg}$ & $Q$ & $\mathrm{PQL}$ & $\mathrm{mg} / \mathrm{kg}$ & $Q$ & PQL & $\mathrm{mg} / \mathrm{kg}$ & $Q$ & PQL & $\mathrm{mg} / \mathrm{kg}$ & $Q$ & $\mathrm{PQL}$ \\
\hline 1 & J11K85 & $4 / 3 / 06$ & 0.54 & & 0.29 & 11.1 & & 0.24 & 954 & $\mathrm{~J}$ & 0.91 & 1310 & & 77.6 & 0.47 & $\mathrm{U}$ & 0.47 & 468 & $J$ & 2.3 \\
\hline 2 & J11K86 & $4 / 3 / 06$ & 0.38 & & 0.29 & 9.3 & & 0.24 & 987 & $\mathrm{~J}$ & 0.89 & 1210 & & 76.4 & 0.47 & $\mathrm{U}$ & 0.47 & 502 & $\mathrm{~J}$ & 2.2 \\
\hline $\begin{array}{c}\text { Duplicate of } \\
\text { J11K86 }\end{array}$ & J11K95 & $4 / 3 / 06$ & 0.47 & & 0.29 & 9.7 & & 0.24 & 887 & $\mathrm{~J}$ & 0.89 & 1220 & & 76.1 & 0.46 & $\mathrm{U}$ & 0.46 & 452 & J & 2.2 \\
\hline 3 & J11K.87 & $4 / 3 / 06$ & 0.50 & & 0.29 & 11.6 & & 0.24 & 1030 & $\mathrm{~J}$ & 0.90 & 1130 & & 77.3 & 0.47 & $\mathrm{U}$ & 0.47 & 418 & $J$ & 2.3 \\
\hline 4 & J11K88 & $4 / 3 / 06$ & 0.29 & & 0.29 & 9.9 & & 0.24 & 975 & $\mathrm{~J}$ & 0.90 & 1060 & & 77.5 & 0.47 & $\mathrm{U}$ & 0.47 & 514 & 3 & 2.3 \\
\hline 5 & J11K89 & $4 / 3 / 06$ & 0.44 & & 0.29 & 10.2 & & 0.24 & 984 & $\mathrm{~J}$ & 0.90 & 1070 & & 76.9 & 0.47 & $U$ & 0.47 & 510 & J & 2.3 \\
\hline 6 & J11K90 & $4 / 3 / 06$ & 1.1 & & 0.28 & 9.9 & & 0.24 & 1150 & $\mathrm{~J}$ & 0.88 & 863 & & 75.6 & 0.46 & $\mathrm{U}$ & 0.46 & 417 & $\mathrm{~J}$ & 2.2 \\
\hline 7 & J11K91 & $4 / 3 / 06$ & 0.60 & & 0.29 & 12.1 & & 0.24 & 780 & $\mathrm{~J}$ & 0.89 & 1080 & & 76.1 & 0.46 & $\mathrm{U}$ & 0.46 & 600 & $J$ & 2.2 \\
\hline 8 & J11K92 & $4 / 3 / 06$ & 0.50 & & 0.28 & 10.6 & & 0.23 & 1200 & $\mathrm{~J}$ & 0.87 & 1030 & & 74.5 & 0.45 & $\mathrm{U}$ & 0.45 & 405 & $\mathrm{~J}$ & 2.2 \\
\hline 9 & J11K93 & $4 / 3 / 06$ & 0.58 & & 0.29 & 11.2 & & 0.24 & 1080 & $\mathrm{~J}$ & 0.89 & 1070 & & 76.3 & 0.47 & $\mathrm{U}$ & 0.47 & 541 & $\mathrm{~J}$ & 2.2 \\
\hline 10 & J11K94 & $4 / 3 / 06$ & 0.53 & & 0.30 & 12.5 & & 0.25 & 989 & $\mathrm{~J}$ & 0.93 & 1170 & & 79.5 & 0.48 & $\mathrm{U}$ & 0.48 & 560 & $\mathrm{~J}$ & 2.3 \\
\hline $\begin{array}{l}\text { East BCL } \\
\text { stockpile }\end{array}$ & J11KDI & $4 / 3 / 06$ & 0.51 & & 0.29 & 11.1 & & 0.24 & $1090^{\circ}$ & $\mathrm{J}$ & 0.89 & 1040 & & 76.3 & 0.46 & U & 0.46 & 517 & J & 2.2 \\
\hline $\begin{array}{l}\text { West BCL } \\
\text { stockpile }\end{array}$ & J11KD2 & $4 / 3 / 06$ & 0.51 & & 0.29 & 11.0 & & 0.24 & 923 & $\mathrm{~J}$ & 0.90 & 1150 & & 76.9 & 0.47 & U & 0.47 & 524 & $J$ & 2.3 \\
\hline $\begin{array}{c}\text { Equipment } \\
\text { blank }\end{array}$ & J11K96 & $4 / 3 / 06$ & 0.29 & & 0.27 & 0.22 & U & 0.22 & 5.4 & $\mathrm{~J}$ & 0.83 & 71.4 & U & 71.4 & 0.44 & U & 0.44 & 38.9 & J & 2.1 \\
\hline
\end{tabular}

Attachment

1 Sheet No. $\frac{4 \text { of } 13}{07 / 31 / 06}$

Originator J.M. Capron

Checked $\begin{array}{ll}\text { Date } & \\ \text { Rev. No. No0B-CA-V0281 } & \end{array}$ 
Attachment 1. 100-B-14:2 Area 2 Verification Sampling Results.

\begin{tabular}{|c|c|c|c|c|c|c|c|c|c|c|c|c|c|c|c|c|c|c|c|c|}
\hline \multirow{2}{*}{$\begin{array}{l}\text { Sample } \\
\text { Location }\end{array}$} & \multirow{2}{*}{$\begin{array}{l}\text { Sample } \\
\text { Number }\end{array}$} & \multirow{2}{*}{$\begin{array}{c}\text { Sample } \\
\text { Date }\end{array}$} & \multicolumn{3}{|c|}{ Silver } & \multicolumn{3}{|c|}{ Sodium } & \multicolumn{3}{|c|}{ Strontium } & \multicolumn{3}{|c|}{ Thallium } & \multicolumn{3}{|c|}{ Tin } & \multicolumn{3}{|c|}{ Titanium } \\
\hline & & & $\mathrm{mg} / \mathrm{kg}$ & $Q$ & $\mathrm{PQL}$ & $\mathrm{mg} / \mathrm{kg}$ & Q & $\mathrm{PQL}$ & $\mathrm{mg} / \mathrm{kg}$ & $\mathrm{Q}$ & PQL & $\mathrm{mg} / \mathrm{kg}$ & $Q$ & $\mathrm{PQL}$ & $\mathrm{mg} / \mathrm{kg}$ & $Q$ & PQL & $\mathrm{mg} / \mathrm{kg}$ & $Q$ & PQL \\
\hline 1 & J11K85 & $4 / 3 / 06$ & 0.07 & $\mathrm{U}$ & 0.07 & 139 & & 2.5 & $\frac{1}{23.8}$ & & 0.01 & 0.70 & $\frac{\vec{u}}{\mathrm{U}}$ & 0.70 & 1.2 & USC & 1.1 & 1860 & & 0.03 \\
\hline 2 & J11K86 & $4 / 3 / 06$ & 0.07 & $\mathrm{U}$ & 0.07 & 118 & & 2.5 & 21.5 & & 0.01 & 0.69 & $\mathrm{U}$ & 0.69 & 1.1 & UC & 1.1 & 1170 & & 0.03 \\
\hline $\begin{array}{l}\text { Duplicate of } \\
\text { J11K86 }\end{array}$ & J11K95 & $4 / 3 / 06$ & 0.07 & U & 0.07 & 109 & & 2.5 & 20.0 & & 0.01 & 0.69 & U & 0.69 & 1.1 & UC & 1.1 & 1260 & & 0.03 \\
\hline 3 & JIIK87 & $4 / 3 / 06$ & 0.07 & $U$ & 0.07 & 153 & & 2.5 & 36.1 & & 0.01 & 0.70 & $\mathrm{U}$ & 0.70 & 1.1 & UC & 1.1 & 1520 & & 0.03 \\
\hline 4 & J11K88 & $4 / 3 / 06$ & 0.07 & $\mathrm{U}$ & 0.07 & 154 & & 2.5 & 30.3 & & 0.01 & 0.70 & $\mathrm{U}$ & 0.70 & 1.1 & $U C$ & 1.1 & 1340 & & 0.03 \\
\hline 5 & J11K 89 & $4 / 3 / 06$ & 0.07 & $\mathrm{U}$ & 0.07 & 208 & & 2.5 & 23.4 & & 0.01 & 0.70 & $\mathrm{U}$ & 0.70 & 1.1 & UC & 1.1 & 1580 & & 0.03 \\
\hline 6 & J11K90 & $4 / 3 / 06$ & 0.07 & $\mathrm{U}$ & 0.07 & 142 & & 2.5 & 24.1 & & 0.01 & 0.69 & $\mathrm{U}$ & 0.69 & 1.0 & $\mathrm{UC}$ & 1.0 & 1580 & & 0.03 \\
\hline 7 & J11K91 & $4 / 3 / 06$ & 0.07 & $\mathrm{U}$ & 0.07 & 182 & & 2.5 & 118 & & 0.01 & 0.69 & $\mathrm{U}$ & 0.69 & 1.1 & $\mathrm{UC}$ & 1.1 & 727 & & 0.03 \\
\hline 8 & $\mathrm{~J} 11 \mathrm{~K} 92$ & $4 / 3 / 06$ & 0.07 & $\mathrm{U}$ & 0.07 & 156 & & 2.4 & 28.0 & & 0.01 & 0.68 & $\mathrm{U}$ & 0.68 & 1.0 & UC & 1.0 & 1820 & & 0.03 \\
\hline 9 & J11K93 & $4 / 3 / 06$ & 0.07 & $\mathrm{U}$ & 0.07 & 160 & & 2.5 & 64.2 & & 0.01 & 0.69 & $\mathrm{U}$ & 0.69 & 1.1 & UsC & 1.1 & 1590 & & 0.03 \\
\hline 10 & J11K94 & $4 / 3 / 06$ & 0.07 & $U$ & 0.07 & 163 & & 2.6 & 42.8 & & 0.01 & 0.72 & $\mathrm{U}$ & 0.72 & 3.1 & UC & 1.1 & 1190 & & 0.03 \\
\hline $\begin{array}{l}\text { East BCL } \\
\text { stockpile }\end{array}$ & J11KD1 & $4 / 3 / 06$ & 0.07 & $U$ & 0.07 & 160 & & 2.5 & 57.1 & & 0.01 & 0.69 & U & 0.69 & 1.3 & usc & 1.1 & 1250 & & 0.03 \\
\hline $\begin{array}{l}\text { West BCL } \\
\text { stockpile }\end{array}$ & $\mathrm{J} 11 \mathrm{KD} 2$ & $4 / 3 / 06$ & 0.07 & $\mathrm{U}$ & 0.07 & 146 & & 2.5 & 30.8 & & 0.01 & 0.70 & U & 0.70 & 1.1 & UC & 1.1 & 1490 & & 0.03 \\
\hline $\begin{array}{l}\text { Equipment } \\
\text { blank }\end{array}$ & J11K96 & $4 / 3 / 06$ & 0.06 & U & 0.06 & 7.6 & & 2.3 & 0.30 & & 0.009 & 0.65 & U & 0.65 & 0.99 & UC & 0.99 & 2.0 & & 0.03 \\
\hline
\end{tabular}

\begin{tabular}{|c|c|c|c|c|c|c|c|c|c|c|c|c|c|}
\hline \multirow{2}{*}{$\begin{array}{c}\text { Sample } \\
\text { Location }\end{array}$} & \multirow{2}{*}{$\begin{array}{l}\text { Sample } \\
\text { Number }\end{array}$} & \multirow{2}{*}{$\begin{array}{c}\text { Sample } \\
\text { Date }\end{array}$} & \multicolumn{3}{|c|}{ Uranium } & \multicolumn{3}{|c|}{ Vanadium } & \multicolumn{3}{|c|}{ Zinc } & \multicolumn{2}{|c|}{ Zirconium } \\
\hline & & & $\mathrm{mg} / \mathrm{kg}$ & $Q$ & PQL & $\mathrm{mg} / \mathrm{kg}$ & $Q$ & $\mathrm{PQL}$ & $\mathrm{mg} / \mathrm{kg}$ & $Q$ & PQL & $\mathrm{mg} / \mathrm{kg}$ & \begin{tabular}{l|l} 
Q & PQL
\end{tabular} \\
\hline 1 & J11K85 & $4 / 3 / 06$ & 0.89 & $\mathrm{UJ}$ & 0.89 & 53.6 & & 0.09 & 46.1 & & 0.16 & 24.8 & 1.1 \\
\hline 2 & $\mathrm{~J} 11 \mathrm{~K} 86$ & $4 / 3 / 06$ & 0.87 & $\overline{\mathrm{UI}}$ & 0.87 & 38.8 & & 0.09 & 36.9 & & 0.16 & 19.2 & 1.1 \\
\hline $\begin{array}{c}\text { Duplicate of } \\
\text { J11K86 }\end{array}$ & J11K95 & $4 / 3 / 06$ & 0.87 & UJ & 0.87 & 40.6 & & 0.09 & 37.3 & & 0.16 & 19.7 & 1.0 \\
\hline 3 & J11K87 & $4 / 3 / 06$ & 0.88 & UJ & 0.88 & 47.1 & & 0.09 & 44.6 & & 0.16 & 21.0 & 1.1 \\
\hline 4 & J11K88 & $4 / 3 / 06$ & 0.88 & UJ & 0.88 & 37.8 & & 0.09 & 39.5 & & 0.16 & 18.6 & 1.1 \\
\hline 5 & J11K89 & $4 / 3 / 06$ & 0.88 & UJ & 0.88 & 49.2 & & 0.09 & 42.8 & & 0.16 & 20.8 & 1.1 \\
\hline 6 & $\mathrm{~J} 11 \mathrm{~K} 90$ & $4 / 3 / 06$ & 0.86 & UJ & 0.86 & 43.8 & & 0.09 & 39.1 & & 0.16 & 20.9 & 1.0 \\
\hline 7 & $\mathrm{~J} 11 \mathrm{~K} 91$ & $4 / 3 / 06$ & 0.87 & UJ & 0.87 & 28.1 & & 0.09 & 38.5 & & 0.16 & 10.2 & 1.0 \\
\hline 8 & $\mathrm{~J} 11 \mathrm{~K} 92$ & $4 / 3 / 06$ & 0.85 & UJ & 0.85 & 51.4 & & 0.09 & 44.9 & & 0.15 & 22.7 & 1.0 \\
\hline 9 & J11K93 & $4 / 3 / 06$ & 0.87 & UJ & 0.87 & 49.8 & & 0.09 & 44.5 & & 0.16 & $21: 9$ & 1.0 \\
\hline 10 & J11K94 & $4 / 3 / 06$ & 0.91 & UJ & 0.91 & 40.7 & & 0.09 & 48.0 & & 0.16 & 14.7 & 1.1 \\
\hline $\begin{array}{l}\text { East BCL } \\
\text { stockpile }\end{array}$ & $\mathrm{J}_{11 \mathrm{KDI}}$ & $4 / 3 / 06$ & 2.2 & I & 0.87 & 39.8 & & 0.09 & 40.6 & & 0.16 & 19.6 & 1.0 \\
\hline $\begin{array}{l}\text { West BCL } \\
\text { stockpile }\end{array}$ & $\mathrm{J} 11 \mathrm{KD} 2$ & $4 / 3 / 06$ & 1.2 & $\mathrm{~J}$ & 0.88 & 47.2 & & 0.09 & 43.6 & & 0.16 & 20.4 & 1.1 \\
\hline Equipment & J11K96 & $4 / 3 / 06$ & 0.81 & UJ & 0.81 & 0.12 & & 0.08 & 1.4 & & 0.15 & 3.0 & 0.98 \\
\hline
\end{tabular}
$\begin{array}{lll}\text { Attachment } & 1 & \text { Sheet No. } \frac{5 \text { of } 13}{07 / 31 / 06} \\ \text { Originator } & \text { J.M. Capron } & \text { Date }\end{array}$ Checked T.M.Blakley Date 07/31/06 
Attachment 1. 100-B-14:2 Area 2 Verification Sampling Results.

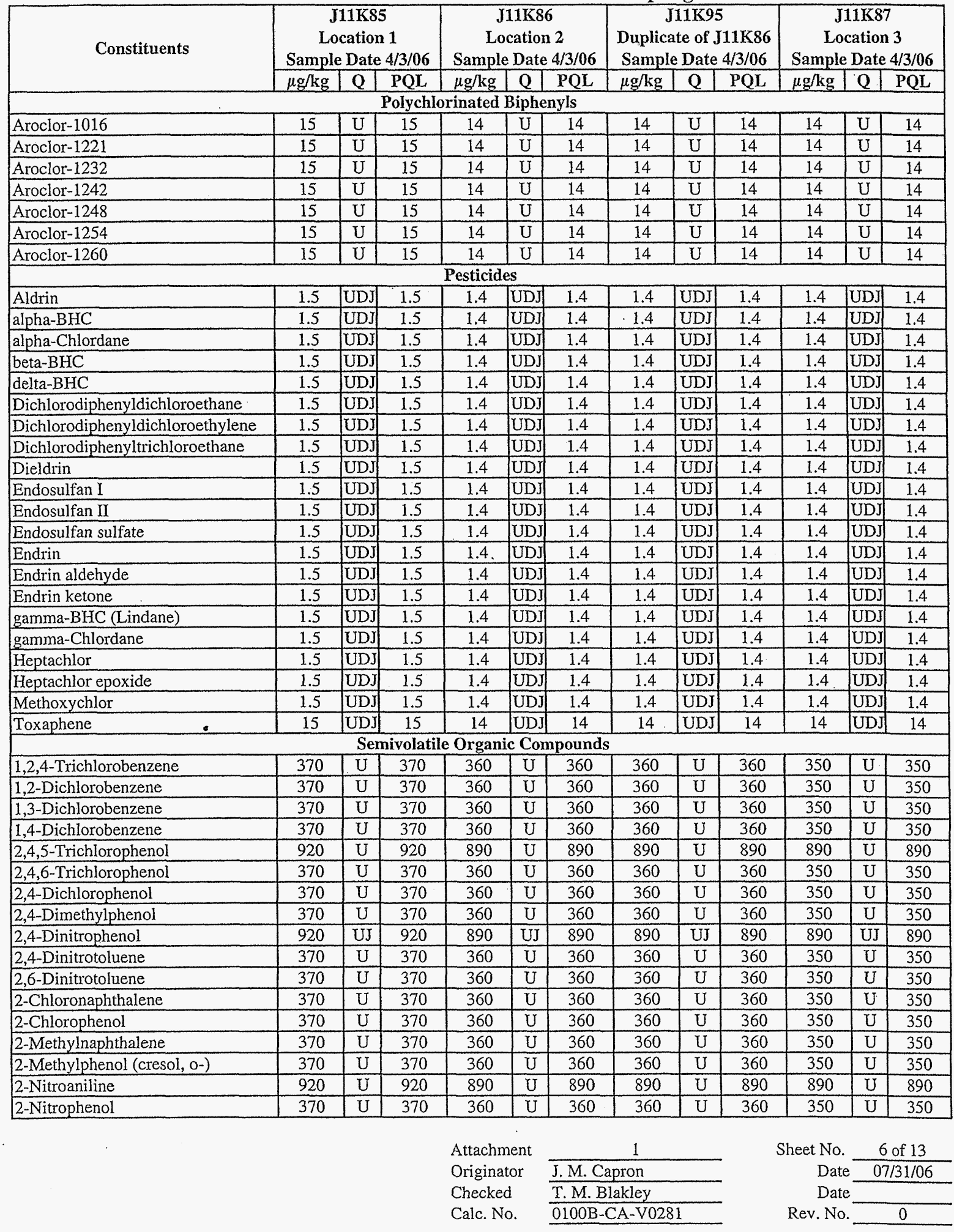


Attachment 1. 100-B-14:2 Area 2 Verification Sampling Results.

\begin{tabular}{|c|c|c|c|c|c|c|c|c|c|c|c|c|}
\hline \multirow[t]{2}{*}{ Constituents } & \multicolumn{3}{|c|}{$\begin{array}{c}\text { J11K85 } \\
\text { Location } 1 \\
\text { Sample Date } 4 / 3 / 06 \\
\end{array}$} & \multicolumn{3}{|c|}{$\begin{array}{c}\text { J11K86 } \\
\text { Location } 2 \\
\text { Sample Date 4/3/06 } \\
\end{array}$} & \multicolumn{3}{|c|}{$\begin{array}{c}\text { J11K95 } \\
\text { Duplicate of J11K86 } \\
\text { Sample Date } 4 / 3 / 06\end{array}$} & \multicolumn{3}{|c|}{$\begin{array}{c}\text { J11K87 } \\
\text { Location } 3 \\
\text { Sample Date 4/3/06 }\end{array}$} \\
\hline & $\mu \mathrm{g} / \mathrm{kg}$ & Q & PQL & $\mu \mathrm{g} / \mathrm{kg}$ & $Q$ & $\mathrm{PQL}$ & $\mu \mathrm{g} / \mathrm{kg}$ & $\mathbf{Q}$ & PQL & $\mu \mathrm{g} / \mathrm{kg}$ & Q & $\mathrm{PQL}$ \\
\hline \multicolumn{13}{|c|}{ Semivolatile Organic Compounds (continued) } \\
\hline 3,3'-Dichlorobenzidine & 370 & $\mathrm{U}$ & 370 & 360 & $\mathrm{U}$ & 360 & 360 & $\mathrm{U}$ & 360 & 350 & $\mathrm{U}$ & 350 \\
\hline 3-Nitroaniline & 920 & $\mathrm{U}$ & 920 & 890 & $\mathrm{U}$ & 890 & 890 & $\mathrm{U}$ & 890 & 890 & $\mathrm{U}$ & 890 \\
\hline 4,6-Dinitro-2-methylphenol & 920 & $\mathrm{U}$ & 920 & 890 & $\mathrm{U}$ & 890 & 890 & $\mathrm{U}$ & 890 & 890 & $\mathrm{U}$ & 890 \\
\hline 4-Bromophenyl-phenylether & 370 & $\mathrm{U}$ & 370 & 360 & $\mathrm{U}$ & 360 & 360 & $\mathrm{U}$ & 360 & 350 & $\bar{U}$ & 350 \\
\hline 4-Chloro-3-methylphenol & 370 & $\mathrm{U}$ & 370 & 360 & $\mathrm{U}$ & 360 & 360 & $\mathrm{U}$ & 360 & 350 & U & 350 \\
\hline 4-Chloroaniline & 370 & $\mathrm{U}$ & 370 & 360 & $\bar{U}$ & 360 & 360 & $\mathrm{U}$ & 360 & 350 & $\mathrm{U}$ & 350 \\
\hline 4-Chlorophenyl-phenylether & 370 & $\mathrm{U}$ & 370 & 360 & $\mathrm{U}$ & 360 & 360 & $\mathrm{U}$ & 360 & 350 & $\mathrm{U}$ & 350 \\
\hline 4-Methylphenol (p-cresol) & 370 & $\mathrm{U}$ & 370 & 360 & $\mathrm{U}$ & 360 & 360 & $\mathrm{U}$ & 360 & 350 & $\mathrm{U}$ & 350 \\
\hline 4-Nitroaniline & 920 & $\mathrm{U}$ & 920 & 890 & $\mathrm{U}$ & 890 & 890 & $\bar{U}$ & 890 & 890 & $\mathrm{U}$ & 890 \\
\hline 4-Nitrophenol & 920 & $\bar{U}$ & 920 & 890 & $\mathrm{U}$ & 890 & 890 & $\bar{U}$ & 890 & 890 & $\mathrm{U}$ & 890 \\
\hline \begin{tabular}{|l|} 
Acenaphthene \\
\end{tabular} & 370 & $\mathrm{U}$ & 370 & 360 & U & 360 & 360 & $\mathrm{U}$ & 360 & 350 & $\mathrm{U}$ & 350 \\
\hline \begin{tabular}{|l|} 
Acenaphthylene \\
\end{tabular} & 370 & $\mathrm{U}$ & 370 & 360 & $\mathrm{U}$ & 360 & 360 & $\mathrm{U}$ & 360 & 350 & $\mathrm{U}$ & 350 \\
\hline \begin{tabular}{|l|} 
Anthracene \\
\end{tabular} & 370 & $\mathrm{U}$ & 370 & 360 & $\mathrm{U}$ & 360 & 360 & $\mathrm{U}$ & 360 & 350 & $\mathrm{U}$ & 350 \\
\hline Benzo(a)anthracene & 370 & $\mathrm{U}$ & 370 & 360 & $\bar{U}$ & 360 & 360 & $\mathrm{U}$ & 360 & 350 & $\bar{U}$ & 350 \\
\hline Benzo(a)pyrene & 370 & $\mathrm{U}$ & 370 & 360 & $\bar{U}$ & 360 & 360 & $\overline{\mathrm{U}}$ & 360 & 350 & $\bar{U}$ & 350 \\
\hline Benzo(b)fluoranthene & 370 & $\overline{\mathrm{U}}$ & 370 & 360 & $\bar{U}$ & 360 & 360 & $\mathrm{U}$ & 360 & 350 & $\mathrm{U}$ & 350 \\
\hline Benzo $(g, h, i)$ perylene & 370 & $\bar{U}$ & 370 & 360 & $\mathrm{U}$ & 360 & 360 & $\mathrm{U}$ & 360 & 350 & $\bar{U}$ & 350 \\
\hline Benzo(k)fluoranthene & 370 & $\mathrm{U}$ & 370 & 360 & $\bar{U}$ & 360 & 360 & $\mathrm{U}$ & 360 & 350 & $\bar{U}$ & 350 \\
\hline bis(2-Chloro-1-methylethyl)ether & 370 & $\mathrm{U}$ & 370 & 360 & U & 360 & 360 & $\mathrm{U}$ & 360 & 350 & $\overline{\mathrm{U}}$ & 350 \\
\hline bis(2-Chloroethoxy)methane & 370 & $U$ & 370 & 360 & $\mathrm{U}$ & 360 & 360 & $\mathrm{U}$ & 360 & 350 & U & 350 \\
\hline bis(2-Chloroethyl)ether & 370 & $\mathrm{U}$ & 370 & 360 & $\mathrm{U}$ & 360 & 360 & $\mathrm{U}$ & 360 & 350 & $\mathrm{U}$ & 350 \\
\hline bis(2-Ethylhexyl)phthalate & 660 & $\mathrm{U}$ & 660 & 660 & $\mathrm{U}$ & 660 & 660 & $\mathrm{U}$ & 660 & 660 & $\mathrm{U}$ & 660 \\
\hline Butylbenzylphthalate & 370 & $\mathrm{U}$ & 370 & 360 & $U$ & 360 & 360 & $\mathrm{U}$ & 360 & 350 & $\mathrm{U}$ & 350 \\
\hline Carbazole & 370 & $\mathrm{U}$ & 370 & 360 & $\mathrm{U}$ & 360 & 360 & $\mathrm{U}$ & 360 & 350 & U & 350 \\
\hline \begin{tabular}{|l|} 
Chrysene \\
\end{tabular} & 370 & U & 370 & 360 & U & 360 & 360 & $\mathrm{U}$ & 360 & 350 & $\mathrm{U}$ & 350 \\
\hline Di-n-butylphthalate & 660 & $\mathrm{U}$ & 660 & 660 & $\mathrm{U}$ & 660 & 660 & $\mathrm{U}$ & 660 & 660 & $\mathrm{U}$ & 660 \\
\hline Di-n-octylphthalate & 370 & $\mathrm{U}$ & 370 & 360 & $\mathrm{U}$ & 360 & 360 & U & 360 & 350 & $\mathrm{U}$ & 350 \\
\hline Dibenz $(a, h)$ anthracene & 370 & $\mathrm{U}$ & 370 & 360 & $\mathrm{U}$ & 360 & 360 & $\mathrm{U}$ & 360 & 350 & $\mathrm{U}$ & 350 \\
\hline Dibenzofuran & 370 & $\mathrm{U}$ & 370 & 360 & $\mathrm{U}$ & 360 & 360 & $\mathrm{U}$ & 360 & 350 & $\mathrm{U}$ & 350 \\
\hline Diethylphthalate & 370 & $\mathrm{U}$ & 370 & $360^{\circ}$ & $\mathrm{U}$ & 360 & 360 & $\mathrm{U}$ & 360 & 350 & $\mathrm{U}$ & 350 \\
\hline Dimethylphthalate & 370 & U & 370 & 360 & $\mathrm{U}$ & 360 & 360 & $\mathrm{U}$ & 360 & 350 & U & 350 \\
\hline Fluoranthene & 370 & $\mathrm{U}$ & 370 & 360 & $\mathrm{U}$ & 360 & 360 & $\bar{U}$ & 360 & 350 & $\mathrm{U}$ & 350 \\
\hline Fluorene & 370 & $\mathrm{U}$ & 370 & 360 & $\mathrm{U}$ & 360 & 360 & $\mathrm{U}$ & 360 & 350 & $\mathrm{U}$ & 350 \\
\hline $\begin{array}{l}\text { Hexachlorobenzene } \\
\end{array}$ & 370 & $\mathrm{U}$ & 370 & 360 & $\mathrm{U}$ & 360 & 360 & $\overline{\mathrm{U}}$ & 360 & 350 & $\mathrm{U}$ & 350 \\
\hline \begin{tabular}{|l|} 
Hexachlorobutadiene \\
\end{tabular} & 370 & $\mathrm{U}$ & 370 & 360 & $\mathrm{U}$ & 360 & 360 & $\mathrm{U}$ & 360 & 350 & $\mathrm{U}$ & 350 \\
\hline Hexachlorocyclopentadiene & 370 & $\mathrm{U}$ & 370 & 360 & $\mathrm{U}$ & 360 & 360 & $\mathrm{U}$ & 360 & 350 & $\bar{U}$ & 350 \\
\hline $\begin{array}{l}\text { Hexachloroethane } \\
\end{array}$ & 370 & $\mathrm{U}$ & 370 & 360 & $\mathrm{U}$ & 360 & 360 & $\mathrm{U}$ & 360 & 350 & $\mathrm{U}$ & 350 \\
\hline Indeno $(1,2,3$-cd)pyrene & 370 & $\mathrm{U}$ & 370 & 360 & $\mathrm{U}$ & 360 & 360 & $U$ & 360 & 350 & $U$ & 350 \\
\hline $\begin{array}{l}\text { Isophorone } \\
\end{array}$ & 370 & $\mathrm{U}$ & 370 & 360 & $\mathrm{U}$ & 360 & 360 & U & 360 & 350 & $\mathrm{U}$ & 350 \\
\hline N-Nitroso-di-n-dipropylamine & 370 & $\bar{U}$ & 370 & 360 & $\mathrm{U}$ & 360 & 360 & $\bar{U}$ & 360 & 350 & $\mathrm{U}$ & 350 \\
\hline N-Nitrosodiphenylamine & 370 & $\mathrm{U}$ & 370 & 360 & $\mathrm{U}$ & 360 & 360 & $\mathrm{U}$ & 360 & 350 & $\mathrm{U}$ & 350 \\
\hline Naphthalene & 370 & $\bar{U}$ & 370 & 360 & $\mathrm{U}$ & 360 & 360 & $\mathrm{U}$ & 360 & 350 & $\mathrm{U}$ & 350 \\
\hline Nitrobenzene & 370 & $\mathrm{U}$ & 370 & 360 & $\mathrm{U}$ & 360 & 360 & $\mathrm{U}$ & 360 & 350 & $\mathrm{U}$ & 350 \\
\hline Pentachlorophenol & 920 & U & 920 & 890 & $\mathrm{U}$ & 890 & 890 & U & 890 & 890 & $\mathrm{U}$ & 890 \\
\hline \begin{tabular}{|l|} 
Phenanthrene \\
\end{tabular} & 370 & $\mathrm{U}$ & 370 & 360 & $\mathrm{U}$ & 360 & 360 & $\mathrm{U}$ & 360 & 350 & $\mathrm{U}$ & 350 \\
\hline \begin{tabular}{|l} 
Phenol \\
\end{tabular} & 370 & $\mathrm{U}$ & 370 & 360 & U & 360 & 360 & $\mathrm{U}$ & 360 & 350 & U & 350 \\
\hline \begin{tabular}{|l} 
Pyrene \\
\end{tabular} & 370 & $\mathrm{U}$ & 370 & 360 & $\mathrm{U}$ & 360 & 360 & $\mathrm{U}$ & 360 & 350 & $\mathrm{U}$ & 350 \\
\hline
\end{tabular}

\begin{tabular}{|c|c|c|c|}
\hline Attachment & 1 & Sheet No. & 7 of 13 \\
\hline Originator & J. M. Capron & Date & $07 / 31 / 06$ \\
\hline Checked & T.M. Blakley & Date & \\
\hline Calc. No. & $0100 \mathrm{~B}-\mathrm{CA}-\mathrm{V} 0281$ & Rev. No. & 0 \\
\hline
\end{tabular}


Attachment 1. 100-B-14:2 Area 2 Verification Sampling Results.

\begin{tabular}{|c|c|c|c|c|c|c|c|c|c|c|c|c|}
\hline \multirow[t]{2}{*}{ Constituents } & \multicolumn{3}{|c|}{$\begin{array}{c}\text { J11K88 } \\
\text { Location } 4 \\
\text { Sample Date } 4 / 3 / 06\end{array}$} & \multicolumn{3}{|c|}{$\begin{array}{c}\text { J11K89 } \\
\text { Location } 5 \\
\text { Sample Date 4/3/06 }\end{array}$} & \multicolumn{3}{|c|}{$\begin{array}{c}\text { J11K90 } \\
\text { Location } 6 \\
\text { Sample Date 4/3/06 }\end{array}$} & \multicolumn{3}{|c|}{$\begin{array}{c}\text { J11K91 } \\
\text { Location } 7 \\
\text { Sample Date 4/3/06 } \\
\end{array}$} \\
\hline & $\mu \mathrm{g} / \mathrm{kg}$ & Q & $\mathrm{PQL}$ & $\mu \mathrm{g} / \mathrm{kg}$ & Q & PQL & $\mu \mathrm{g} / \mathrm{kg}$ & Q & $\mathrm{PQL}$ & $\mu \mathrm{g} / \mathrm{kg}$ & Q & $\mathrm{PQL}$ \\
\hline \multicolumn{13}{|c|}{ Polychlorinated Biphenyls } \\
\hline Aroclor-1016 & 14 & $\mathrm{U}$ & 14 & 14 & $\mathrm{U}$ & 14 & 14 & $\mathrm{U}$ & 14 & 14 & $\mathrm{U}$ & 14 \\
\hline Aroclor-1221 & 14 & $\mathrm{U}$ & 14 & 14 & $\mathrm{U}$ & 14 & 14 & $\mathrm{U}$ & 14 & 14 & $\mathrm{U}$ & 14 \\
\hline Aroclor-1232 & 14 & $\mathrm{U}$ & 14 & 14 & $\mathrm{U}$ & 14 & 14 & $\mathrm{U}$ & 14 & 14 & $\mathrm{U}$ & 14 \\
\hline Aroclor-1242 & 14 & $\mathrm{U}$ & 14 & 14 & $\mathrm{U}$ & 14 & 14 & $\mathrm{U}$ & 14 & 14 & $\mathrm{U}$ & 14 \\
\hline Aroclor-1248 & 14 & $\mathrm{U}$ & 14 & 14 & $\mathrm{U}$ & 14 & 14 & $\mathrm{U}$ & 14 & 14 & $\mathrm{U}$ & 14 \\
\hline Aroclor-1254 & 14 & $\mathrm{U}$ & 14 & 14. & U & 14 & 14 & U & 14 & 14 & $\mathrm{U}$ & 14 \\
\hline Aroclor-1260 & 14 & $\mathrm{U}$ & 14 & 14 & $\mathrm{U}$ & 14 & 14 & $\mathrm{U}$ & 14 & 14 & $\mathrm{U}$ & 14 \\
\hline \multicolumn{13}{|c|}{ Pesticides } \\
\hline Aldrin & 1.4 & UDJ & 1.4 & \begin{tabular}{|l|}
1.4 \\
\end{tabular} & UDJ & 1.4 & 1.4 & UDJ & 1.4 & 1.4 & UDJ & 1.4 \\
\hline alpha-BHC & 1.4 & UDJ & 1.4 & 1.4 & UDJ & 1.4 & 1.4 & UDJ & 1.4 & 1.4 & UDJ & 1.4 \\
\hline alpha-Chlordane & 1.4 & UDJ & 1.4 & 1.4 & UDJ & 1.4 & 1.4 & UDJ & 1.4 & 1.4 & UDJ & 1.4 \\
\hline beta-BHC & 1.4 & UDJ & 1.4 & 1.4 & UDJ & 1.4 & 1.4 & UDJ & 1.4 & 1.4 & UDJ & 1.4 \\
\hline delta-BHC & 1.4 & UDJ & 1.4 & 1.4 & UDJ & 1.4 & 1.4 & UDJ & 1.4 & 1.4 & UDJ & 1.4 \\
\hline Dichlorodiphenyldichloroethane & 1.4 & UDJ & 1.4 & 1.4 & UDJ & 1.4 & 1.4 & UDJ & 1.4 & 1.4 & UDJ & 1.4 \\
\hline Dichlorodiphenyldichloroethylene & 1.4 & UDJ & 1.4 & 1.4 & UDJ & 1.4 & 1.4 & UDJ & 1.4 & 1.4 & UDJ & 1.4 \\
\hline Dichlorodiphenyltrichloroethane & 1.4 & UDJ & 1.4 & 1.4 & UDJ & 1.4 & 1.4 & UDJ & 1.4 & 1.4 & UDJ & 1.4 \\
\hline Dieldrin & 1.4 & UDJ & 1.4 & 1.4 & UDJ & 1.4 & 1.4 & UDJ & 1.4 & 1.4 & UDJ & 1.4 \\
\hline Endosulfan I & 1.4 & UDJ & 1.4 & 1.4 & UDJ & 1.4 & 1.4 & UDJ & 1.4 & 1.4 & UDJ & 1.4 \\
\hline Endosulfan II & 1.4 & UDJ & 1.4 & 1.4 & UDJ & 1.4 & 1.4 & UDJ & 1.4 & 1.4 & UDJ & 1.4 \\
\hline Endosulfan sulfate & 1.4 & UDI & 1.4 & 1.4 & UDJ & 1.4 & 1.4 & UDJ & 1.4 & 0.53 & JD & 1.4 \\
\hline Endrin & 1.4 & UDJ & 1.4 & 1.4 & UDJ & 1.4 & 1.4 & UDJ & 1.4 & 1.4 & UDJ & 1.4 \\
\hline Endrin aldehyde & 1.4 & UDJ & 1.4 & 1.4 & UDJ & 1.4 & 1.4 & UDJ & 1.4 & 1.4 & UDJ & 1.4 \\
\hline Endrin ketone & 1.4 & UDJ & 1.4 & 1.4 & UDJ & 1.4 & 1.4 & UDJ & 1.4 & 1.4 & UDJ & 1.4 \\
\hline gamma-BHC (Lindane) & 1.4 & UDJ & 1.4 & 1.4 & UDJ & 1.4 & 1.4 & UDJ & 1.4 & 1.4 & UDJ & 1.4 \\
\hline gamma-Chlordane & 1.4 & UDJ & 1.4 & 1.4 & UDJ & 1.4 & 1.4 & UDJ & 1.4 & 1.4 & UDJ & 1.4 \\
\hline Heptachlor & 1.4 & UDJ & 1.4 & 1.4 & UDJ & 1.4 & 1.4 & UDJ & 1.4 & 1.4 & UDJ & 1.4 \\
\hline Heptachlor epoxide & 1.4 & UDJ & 1.4 & 1.4 & UDJ & 1.4 & 1.4 & UDJ & 1.4 & 1.4 & UDJ & 1.4 \\
\hline Methoxychlor & 1.4 & UDJ & 1.4 & 1.4 & UDJ & 1.4 & 1.4 & UDJ & 1.4 & 1.4 & UDJ & 1.4 \\
\hline Toxaphene & 14 & UDJ & 14 & 14 & UDJ & 14 & 14 & UDJ & 14 & 14 & UDJ & 14 \\
\hline \multicolumn{13}{|c|}{ Semivolatile Organic Compounds } \\
\hline 1,2,4-Trichlorobenzene & 360 & U & 360 & 350 & $\mathrm{U}$ & 350 & 350 & $\mathrm{U}$ & 350 & 360 & $\bar{U}$ & 360 \\
\hline 1,2-Dichlorobenzene & 360 & $\mathrm{U}$ & 360 & 350 & $\mathrm{U}$ & 350 & 350 & $\mathrm{U}$ & 350 & 360 & $\mathrm{U}$ & 360 \\
\hline 1,3-Dichlorobenzene & 360 & $\mathrm{U}$ & 360 & 350 & $\mathrm{U}$ & 350 & 350 & $\mathrm{U}$ & 350 & 360 & $\mathrm{U}$ & 360 \\
\hline 1,4-Dichlorobenzene & 360 & $\mathrm{U}$ & 360 & 350 & $\mathrm{U}$ & 350 & 350 & $\mathrm{U}$ & 350 & 360 & $\mathrm{U}$ & 360 \\
\hline $2,4,5$-Trichlorophenol & 890 & $\mathrm{U}$ & 890 & 880 & $\mathrm{U}$ & 880 & 880 & $\mathrm{U}$ & 880 & 890 & $\mathrm{U}$ & 890 \\
\hline 2,4,6-Trichlorophenol & 360 & U & 360 & 350 & $\mathrm{U}$ & 350 & 350 & $\mathrm{U}$ & 350 & 360 & $\mathrm{U}$ & 360 \\
\hline 2,4-Dichlorophenol & 360 & $\mathrm{U}$ & 360 & 350 & $\mathrm{U}$ & 350 & 350 & $\mathrm{U}$ & 350 & 360 & $\mathrm{U}$ & 360 \\
\hline 2,4-Dimethylphenol & 360 & $\mathrm{U}$ & 360 & 350 & $\mathrm{U}$ & 350 & 350 & $\mathrm{U}$ & 350. & 360 & $\mathrm{U}$ & 360 \\
\hline 2,4-Dinitrophenol & 890 & UJ & 890 & 880 & UJ & 880 & 880 & UJ & 880 & 890 & UJ & 890 \\
\hline 2,4-Dinitrotoluene & 360 & $\mathrm{U}$ & 360 & 350 & $\mathrm{U}$ & 350 & 350 & $\mathrm{U}$ & 350 & 360 & $\mathrm{U}$ & 360 \\
\hline 2,6-Dinitrotoluene & 360 & $U$ & 360 & 350 & $\mathrm{U}$ & 350 & 350 & $\mathrm{U}$ & 350 & 360 & $\mathrm{U}$ & 360 \\
\hline 2-Chloronaphthalene & 360 & $\mathrm{U}$ & 360 & 350 & $\mathrm{U}$ & 350 & 350 & $\mathrm{U}$ & 350 & 360 & $\mathrm{U}$ & 360 \\
\hline 2-Chlorophenol & 360 & $\mathrm{U}$ & 360 & 350 & U & 350 & 350 & $\mathrm{U}$ & 350 & 360 & $\mathrm{U}$ & 360 \\
\hline 2-Methylnaphthalene & 360 & $\mathrm{U}$ & 360 & 350 & $\mathrm{U}$ & 350 & 350 & $\mathrm{U}$ & 350 & 34 & $\mathrm{~J}$ & 360 \\
\hline 2-Methylphenol (cresol, o-) & 360 & $\mathrm{U}$ & 360 & 350 & $\mathrm{U}$ & 350 & 350 & $\mathrm{U}$ & 350 & 360 & $\mathrm{U}$ & 360 \\
\hline 2-Nitroaniline & 890 & $\mathrm{U}$ & 890 & 880 & $\mathrm{U}$ & 880 & 880 & $\mathrm{U}$ & 880 & 890 & $\mathrm{U}$ & 890 \\
\hline 2-Nitrophenol & 360 & $\mathrm{U}$ & 360 & 350 & $\mathrm{U}$ & 350 & 350 & $\mathrm{U}$ & 350 & 360 & $\mathrm{U}$ & 360 \\
\hline & & & & Attachn & ient & & 1 & & & Sheet No. & & of 13 \\
\hline & & & & Origina & & J.M. Ce & pron & & & Date & & $131 / 06$ \\
\hline & & & & Checke & & T.M.B & lakley & & & Date & & \\
\hline & & & & Calc. $\mathrm{N}$ & & $0100 \mathrm{~B}-$ & A-V028 & & & Rev. No & & 0 \\
\hline
\end{tabular}


Attachment 1. 100-B-14:2 Area 2 Verification Sampling Results.

\begin{tabular}{|c|c|c|c|c|c|c|c|c|c|c|c|c|}
\hline \multirow[t]{2}{*}{ Constituents } & \multicolumn{3}{|c|}{$\begin{array}{c}\text { J11K88 } \\
\text { Location } 4 \\
\text { Sample Date } 4 / 3 / 06\end{array}$} & \multicolumn{3}{|c|}{$\begin{array}{c}\text { J11K89 } \\
\text { Location } 5 \\
\text { Sample Date 4/3/06 }\end{array}$} & \multicolumn{3}{|c|}{$\begin{array}{c}\text { J11K90 } \\
\text { Location } 6 \\
\text { Sample Date } 4 / 3 / 06\end{array}$} & \multicolumn{3}{|c|}{$\begin{array}{c}\text { J11K91 } \\
\text { Location } 7 \\
\text { Sample Date } 4 / 3 / 06\end{array}$} \\
\hline & $\mu \mathrm{g} / \mathrm{kg}$ & Q & PQL & $\mu \mathrm{g} / \mathrm{kg}$ & Q & PQL & $\mu \mathrm{g} / \mathrm{kg}$ & $Q$ & PQL & $\mu \mathrm{g} / \mathrm{kg}$ & Q & $\mathrm{PQL}$ \\
\hline \multicolumn{13}{|c|}{ Semivolatile Organic Compounds (continued) } \\
\hline 3,3'-Dichlorobenzidine & 360 & $\mathrm{U}$ & 360 & 350 & $\mathrm{U}$ & 350 & 350 & $\mathrm{U}$ & 350 & 360 & $\bar{U}$ & 360 \\
\hline 3-Nitroaniline & 890 & $\mathrm{U}$ & 890 & 880 & $\overline{\mathrm{U}}$ & 880 & 880 & $\mathrm{U}$ & 880 & 890 & $\bar{U}$ & 890 \\
\hline 4,6-Dinitro-2-methylphenol & 890 & $\mathrm{U}$ & 890 & 880 & $\mathrm{U}$ & 880 & 880 & U & 880 & 890 & $\bar{U}$ & 890 \\
\hline 4-Bromophenyl-phenylether & 360 & $\mathrm{U}$ & 360 & 350 & $\mathrm{U}$ & 350 & 350 & $\mathrm{U}$ & 350 & 360 & $\mathrm{U}$ & 360 \\
\hline 4-Chloro-3-methylphenol & 360 & $\mathrm{U}$ & 360 & 350 & $\mathrm{U}$ & 350 & 350 & $\mathrm{U}$ & 350 & 360 & $\bar{U}$ & 360 \\
\hline 4-Chloroaniline & 360 & $\mathrm{U}$ & 360 & 350 & $\mathrm{U}$ & 350 & 350 & $\mathrm{U}$ & 350 & 360 & $\mathrm{U}$ & 360 \\
\hline 4-Chlorophenyl-phenylether & 360 & $\mathrm{U}$ & 360 & 350 & $\mathrm{U}$ & 350 & 350 & U & 350 & 360 & U & 360 \\
\hline 4-Methylphenol (p-cresol) & 360 & $\mathrm{U}$ & 360 & 350 & $\mathrm{U}$ & 350 & 350 & U & 350 & 360 & $\mathrm{U}$ & 360 \\
\hline 4-Nitroaniline & 890 & $\mathrm{U}$ & 890 & 880 & $\mathrm{U}$ & 880 & 880 & $\mathrm{U}$ & 880 & 890 & $\bar{U}$ & 890 \\
\hline 4-Nitrophenol & 890 & $\mathrm{U}$ & 890 & 880 & $\mathrm{U}$ & 880 & 880 & $\mathrm{U}$ & 880 & 890 & $\mathrm{U}$ & 890 \\
\hline Acenaphthene & 360 & $\mathrm{U}$ & 360 & 350 & $\mathrm{U}$ & 350 & 350 & $\mathrm{U}$ & 350 & 360 & $\bar{U}$ & 360 \\
\hline Acenaphthylene & 360 & $\mathrm{U}$ & 360 & 350 & $\mathrm{U}$ & 350 & 350 & $\mathrm{U}$ & 350 & 360 & $\mathrm{U}$ & 360 \\
\hline Anthracene & 360 & $\mathrm{U}$ & 360 & 350 & $\mathrm{U}$ & 350 & 350 & $\mathrm{U}$ & 350 & 360 & $\mathrm{U}$ & 360 \\
\hline Benzo(a)anthracene & 360 & $\mathrm{U}$ & 360 & 350 & $\bar{U}$ & 350 & 350 & $\overline{\mathrm{U}}$ & 350 & 360 & $\bar{U}$ & 360 \\
\hline Benzo(a)pyrene & 360 & $\mathrm{U}$ & 360 & 350 & $\mathrm{U}$ & 350 & 350 & $\mathrm{U}$ & 350 & 360 & $\mathrm{U}$ & 360 \\
\hline Benzo(b)fluoranthene & 360 & $\mathrm{U}$ & 360 & 350 & $\mathrm{U}$ & 350 & 350 & $\mathrm{U}$ & 350 & 360 & $\bar{U}$ & 360 \\
\hline Benzo(g,h,i)perylene & 360 & $U$ & 360 & 350 & $\mathrm{U}$ & 350 & 350 & $\mathrm{U}$ & 350 & 360 & $\bar{U}$ & 360 \\
\hline Benzo(k)fluoranthene & 360 & $\mathrm{U}$ & 360 & 350 & $\mathrm{U}$ & 350 & 350 & U & 350 & 360 & $\mathrm{U}$ & 360 \\
\hline bis(2-Chloro-1-methylethyl)ether & 360 & $\mathrm{U}$ & 360 & 350 & $\mathrm{U}$ & 350 & 350 & $\mathrm{U}$ & 350 & 360 & $\mathrm{U}$ & 360 \\
\hline bis(2-Chloroethoxy)methane & 360 & $\mathrm{U}$ & 360 & 350 & $\mathrm{U}$ & 350 & 350 & $\mathrm{U}$ & 350 & 360 & $\overline{\mathrm{U}}$ & 360 \\
\hline bis(2-Chloroethyl)ether & 360 & $\mathrm{U}$ & 360 & 350 & $\mathrm{U}$ & 350 & 350 & $\mathrm{U}$ & 350 & 360 & $U$ & 360 \\
\hline bis(2-Ethylhexyl)phthalate & 660 & $\mathrm{U}$ & 660 & 660 & $\mathrm{U}$ & 660 & 660 & $\mathrm{U}$ & 660 & 660 & $U$ & 660 \\
\hline Butylbenzylphthalate & 360 & $\mathrm{U}$ & 360 & 350 & $\mathrm{U}$ & 350 & 350 & $\mathrm{U}$ & 350 & 360 & U & 360 \\
\hline Carbazole & 360 & $\mathrm{U}$ & 360 & 350 & $\mathrm{U}$ & 350 & 350 & $\mathrm{U}$ & 350 & 360 & $\mathrm{U}$ & 360 \\
\hline Chrysene & 360 & $\mathrm{U}$ & 360 & 350 & $\mathrm{U}$ & 350 & 350 & $\mathrm{U}$ & 350 & 360 & $\mathrm{U}$ & 360 \\
\hline Di-n-butylphthalate & 360 & $\mathrm{U}$ & 360 & 660 & U & 660 & 660 & U & 660 & 660 & $\bar{U}$ & 660 \\
\hline Di-n-octylphthalate & 360 & $\mathrm{U}$ & 360 & 350 & $\mathrm{U}$ & 350 & 350 & $\mathrm{U}$ & 350 & 360 & $\bar{U}$ & 360 \\
\hline Dibenz(a,h)anthracene & 360 & $\mathrm{U}$ & 360 & 350 & $\mathrm{U}$ & 350 & 350 & $\mathrm{U}$ & 350 & 360 & $\mathrm{U}$ & 360 \\
\hline Dibenzofuran & 360 & $\mathrm{U}$ & 360 & 350 & $\mathrm{U}$ & 350 & 350 & U & 350 & 360 & $\mathrm{U}$ & 360 \\
\hline Diethylphthalate & 360 & $\mathrm{U}$ & 360 & 350 & $\mathrm{U}$ & 350 & 350 & $\mathrm{U}$ & 350 & 360 & $\mathrm{U}$ & 360 \\
\hline Dimethylphthalate & 360 & U & 360 & 350 & $\mathrm{U}$ & 350 & 350 & $\mathrm{U}$ & 350 & 360 & $\overline{\mathrm{U}}$ & 360 \\
\hline Fluoranthene & 360 & $\mathrm{U}$ & 360 & 350 & $\mathrm{U}$ & 350 & 350 & $\mathrm{U}$ & 350 & 360 & $\mathrm{U}$ & 360 \\
\hline Fluorene & 360 & $\mathrm{U}$ & 360 & 350 & $\mathrm{U}$ & 350 & 350 & $\mathrm{U}$ & 350 & 360 & $\mathrm{U}$ & 360 \\
\hline Hexachlorobenzene & 360 & $\mathrm{U}$ & 360 & 350 & U & 350 & 350 & $\mathrm{U}$ & 350 & 360 & $\mathrm{U}$ & 360 \\
\hline Hexachlorobutadiene & 360 & $\mathrm{U}$ & 360 & 350 & $\mathrm{U}$ & 350 & 350 & $\mathrm{U}$ & 350 & 360 & $\mathrm{U}$ & 360 \\
\hline Hexachlorocyclopentadiene & 360 & $\mathrm{U}$ & 360 & 350 & $\mathrm{U}$ & 350 & 350 & $\mathrm{U}$ & 350 & 360 & $\mathrm{U}$ & 360 \\
\hline Hexachloroethane & 360 & $\mathrm{U}$ & 360 & 350 & $\mathrm{U}$ & 350 & 350 & $\mathrm{U}$ & 350 & 360 & $\mathrm{U}$ & 360 \\
\hline Indeno(1,2,3-cd)pyrene & 360 & $\mathrm{U}$ & 360 & 350 & $\mathrm{U}$ & 350 & 350 & $\mathrm{U}$ & 350 & 360 & $\mathrm{U}$ & 360 \\
\hline Isophorone & 360 & $\mathrm{U}$ & 360 & 350 & $\mathrm{U}$ & 350 & 350 & $\mathrm{U}$ & 350 & 360 & $\mathrm{U}$ & 360 \\
\hline N-Nitroso-di-n-dipropylamine & 360 & $\mathrm{U}$ & 360 & 350 & $\mathrm{U}$ & 350 & 350 & $\mathrm{U}$ & 350 & 360 & $\mathrm{U}$ & 360 \\
\hline N-Nitrosodiphenylamine & 360 & $\mathrm{U}$ & 360 & 350 & $\mathrm{U}$ & 350 & 350 & $\mathrm{U}$ & 350 & 360 & $\mathrm{U}$ & 360 \\
\hline Naphthalene & 360 & $\mathrm{U}$ & 360 & 350 & $\mathrm{U}$ & 350 & 350 & $\mathrm{U}$ & 350 & 24 & $\mathrm{~J}$ & 360 \\
\hline Nitrobenzene & 360 & $\mathrm{U}$ & 360 & 350 & $\mathrm{U}$ & 350 & 350 & $\mathrm{U}$ & 350 & 360 & $\mathrm{U}$ & 360 \\
\hline Pentachlorophenol & 890 & $\mathrm{U}$ & 890 & 880 & $\mathrm{U}$ & 880 & 880 & $\mathrm{U}$ & 880 & 890 & $\mathrm{U}$ & 890 \\
\hline Phenanthrene & 360 & $\mathrm{U}$ & 360 & 350 & $\mathrm{U}$ & 350 & 350 & $\mathrm{U}$ & 350 & 24 & $\mathrm{~J}$ & 360 \\
\hline \begin{tabular}{|l} 
Phenol \\
\end{tabular} & 360 & $\mathrm{U}$ & 360 & 350 & U & 350 & 350 & $\mathrm{U}$ & 350 & 360 & $\mathrm{U}$ & 360 \\
\hline Pyrene & 360 & $\mathrm{U}$ & 360 & 350 & $\mathrm{U}$ & 350 & 350 & U & 350 & 360 & $\mathrm{U}$ & 360 \\
\hline & & & & Attachm & & & 1 & & & Sheet No. & & of 13 \\
\hline & & & & Origina & & J.M.C & pron & & & Date & & /131/06 \\
\hline & & & & Checke & & T.M. B & akley & & & Date & & \\
\hline & & & & Calc. $\mathrm{N}$ & & $0100 \mathrm{~B}$ & $\mathrm{AA}-\mathrm{V} 028$ & & & Rev. No. & & 0 \\
\hline
\end{tabular}


Attachment 1. 100-B-14:2 Area 2 Verification Sampling Results.

\begin{tabular}{|c|c|c|c|c|c|c|c|c|c|c|c|c|}
\hline \multirow[t]{2}{*}{ Constituents } & \multicolumn{3}{|c|}{$\begin{array}{c}\text { J11K92 } \\
\text { Location } 8 \\
\text { Sample Date 4/3/06 }\end{array}$} & \multicolumn{3}{|c|}{$\begin{array}{c}\text { J11K93 } \\
\text { Location 9 } \\
\text { Sample Date 4/3/06 }\end{array}$} & \multicolumn{3}{|c|}{$\begin{array}{c}\text { J11K94 } \\
\text { Location } 10 \\
\text { Sample Date } 4 / 3 / 06\end{array}$} & \multicolumn{3}{|c|}{$\begin{array}{c}\text { J11KD1 } \\
\text { East BCL Stockpile } \\
\text { Sample Date } 4 / 3 / 06\end{array}$} \\
\hline & $\mu \mathrm{g} / \mathrm{kg}$ & $Q$ & PQL & $\mu \mathrm{g} / \mathrm{kg}$ & $Q$ & PQL & $\mu \mathrm{g} / \mathrm{kg}$ & $Q$ & PQL & $\mu \mathrm{g} / \mathrm{kg}$ & Q & PQL \\
\hline \multicolumn{13}{|c|}{ Polychlorinated Biphenyls } \\
\hline Aroclor-1016 & 14 & $\mathrm{U}$ & 14 & 14 & U & 14 & 15 & $\mathrm{U}$ & 15 & 14 & $\mathrm{U}$ & 14 \\
\hline Aroclor-1221 & 14 & $\mathrm{U}$ & 14 & 14 & $\mathrm{U}$ & 14 & 15 & $\mathrm{U}$ & 15 & 14 & $\bar{U}$ & 14 \\
\hline Aroclor -1232 & 14 & $\mathrm{U}$ & 14 & 14 & $\mathrm{U}$ & 14 & 15 & $\mathrm{U}$ & 15 & 14 & $\mathrm{U}$ & 14 \\
\hline Aroclor- 1242 & 14 & $\mathrm{U}$ & 14 & 14 & $\mathrm{U}$ & 14 & 15 & $\mathrm{U}$ & 15 & 14 & $U$ & 14 \\
\hline Aroclor- 1248 & 14 & $\mathrm{U}$ & 14 & 14 & $\mathrm{U}$ & 14 & 15 & $\mathrm{U}$ & 15 & 14 & U & 14 \\
\hline Aroclor- 1254 & 14 & $\mathrm{U}$ & 14 & 14 & $\mathrm{U}$ & 14 & 15 & $\mathrm{U}$ & 15 & 14 & $\mathrm{U}$ & 14 \\
\hline Aroclor-1260 & 14 & $\mathrm{U}$ & 14 & 14 & U & 14 & 15 & U & 15 & 14 & U & 14 \\
\hline \multicolumn{13}{|c|}{ Pesticides } \\
\hline Aldrin & 1.4 & UDJ & 1.4 & 1.4 & UDJ & 1.4 & 1.5 & UDJ & 1.5 & 1.4 & UDJ & 1.4 \\
\hline alpha-BHC & 1.4 & UDJ & 1.4 & 1.4 & UDJ & 1.4 & 1.5 & UDJ & 1.5 & 1.4 & UDJ & 1.4 \\
\hline alpha-Chlordane & 1.4 & UDI & 1.4 & 1.4 & UDJ & 1.4 & 1.5 & UDJ & 1.5 & 1.4 & UDJ & 1.4 \\
\hline beta-BHC & 1.4 & UDJ & 1.4 & 0.60 & $\mathrm{JD}$ & 0.60 & 1.5 & UDJ & 1.5 & 4.3 & JD & 4.3 \\
\hline delta-BHC & 1.4 & UDJ & 1.4 & 1.4 & UDJ & 1.4 & 1.5 & UDJ & 1.5 & 1.4 & UDJ & 1.4 \\
\hline Dichlorodiphenyldichloroethane & 1.4 & UDJ & 1.4 & 1.4 & UDJ & 1.4 & 1.5 & UDJ & 1.5 & 1.4 & UDJ & 1.4 \\
\hline Dichlorodiphenyldichloroethylene & 1.4 & UDJ & 1.4 & 1.4 & UDJ & 1.4 & 1.5 & UDJ & 1.5 & $1: 4$ & UDJ & 1.4 \\
\hline Dichlorodiphenyltrichloroethane & 1.4 & UDJ & 1.4 & 1.4 & UDJ & 1.4 & 1.5 & UDJ & 1.5 & 1.4 & UDJ & 1.4 \\
\hline \begin{tabular}{|l|} 
Dieldrin \\
\end{tabular} & 1.4 & UDJ & 1.4 & 1.4 & UDJ & 1.4 & 1.5 & UDJ & 1.5 & 1.4 & UDJ & 1.4 \\
\hline Endosulfan I & 1.4 & UDJ & 1.4 & 1.4 & UDJ & 1.4 & 1.5 & UDJ & 1.5 & 1.4 & UDJ & 1.4 \\
\hline Endosulfan II & 1.4 & UDJ & 1.4 & 1.4 & UDJ & 1.4 & 1.5 & UDJ & 1.5 & 1.4 & UDJ & 1.4 \\
\hline Endosulfan sulfate & 1.4 & UDJ & 1.4 & 1.4 & UDJ & 1.4 & 1.5 & UDJ & 1.5 & 1.4 & UDJ & 1.4 \\
\hline \begin{tabular}{|l|} 
Endrin \\
\end{tabular} & 1.4 & UDJ & 1.4 & 1.4 & UDJ & 1.4 & 1.5 & UDJ & 1.5 & 1.4 & UDJ & 1.4 \\
\hline Endrin aldehyde & 1.4 & UDJ & 1.4 & 1.4 & UDJ & 1.4 & 1.5 & UDJ & 1.5 & 1.4 & UDJ & 1.4 \\
\hline $\begin{array}{l}\text { Endrin ketone } \\
\end{array}$ & 1.4 & UDJ & 1.4 & 1.4 & UDJ & 1.4 & 1.5 & UDJ & 1.5 & 1.4 & UDJ & 1.4 \\
\hline gamma-BHC (Lindane) & 1.4 & UDJ & 1.4 & 1.4 & UDJ & 1.4 & 1.5 & UDJ & 1.5 & 1.4 & UDJ & 1.4 \\
\hline gamma-Chlordane & 1.4 & UDJ & 1.4 & 1.4 & UDJ & 1.4 & 1.5 & UDJ & 1.5 & 1.4 & UDJ & 1.4 \\
\hline Heptachlor & 1.4 & UDJ & 1.4 & 1.4 & UDJ & 1.4 & 1.5 & UDJ & 1.5 & 1.4 & UDJ & 1.4 \\
\hline Heptachlor epoxide & 1.4 & UDJ & 1.4 & 1.4 & UDJ & 1.4 & 1.5 & UDJ & 1.5 & 1.4 & UDJ & 1.4 \\
\hline \begin{tabular}{|l} 
Methoxychlor \\
\end{tabular} & 1.4 & UDJ & 1.4 & 1.4 & UDJ & 1.4 & 1.5 & UDJ & 1.5 & 1.4 & UDJ & 1.4 \\
\hline Toxaphene & 14 & UDJ & 14 & 14 & UDJ & 14 & 15 & UDJ & 15 & 14 & UDJ & 14 \\
\hline \multicolumn{13}{|c|}{ Semivolatile Organic Compounds } \\
\hline 1,2,4-Trichlorobenzene & 350 & U & 350 & 350 & $\mathrm{U}$ & 350 & 370 & U & 370 & 350 & $\overline{\mathrm{U}}$ & 350 \\
\hline 1,2-Dichlorobenzene & 350 & $\mathrm{U}$ & 350 & 350 & $\mathrm{U}$ & 350 & 370 & U & 370 & 350 & $\mathrm{U}$ & 350 \\
\hline 1,3-Dichlorobenzene & 350 & $\mathrm{U}$ & 350 & 350 & $\mathrm{U}$ & 350 & 370 & $\mathrm{U}$ & 370 & 350 & $\mathrm{U}$ & 350 \\
\hline 1,4-Dichlorobenzene & 350 & $\mathrm{U}$ & 350 & 350 & $\mathrm{U}$ & 350 & 370 & U & 370 & 350 & $\mathrm{U}$ & 350 \\
\hline 2,4,5-Trichlorophenol & 880 & $\mathrm{U}$ & 880 & 880 & $\mathrm{U}$ & 880 & 930 & $\mathrm{U}$ & 930 & 870 & $\mathrm{U}$ & 870 \\
\hline 2,4,6-Trichlorophenol & 350 & $\mathrm{U}$ & 350 & 350 & $\mathrm{U}$ & 350 & 370 & $\mathrm{U}$ & 370 & 350 & $\mathrm{U}$ & 350 \\
\hline 2,4-Dichlorophenol & 350 & $\mathrm{U}$ & 350 & 350 & $U$ & 350 & 370 & $\mathrm{U}$ & 370 & 350 & $\mathrm{U}$ & 350 \\
\hline 2,4-Dimethylphenol & 350 & $U$ & 350 & 350 & $\mathrm{U}$ & 350 & 370 & $\mathrm{U}$ & 370 & 350 & $\mathrm{U}$ & 350 \\
\hline 2,4-Dinitrophenol & 880 & UJ & 880 & 880 & $\overline{\mathrm{UJ}}$ & 880 & 930 & UJ & 930 & 870 & UJ & 870 \\
\hline 2,4-Dinitrotoluene & 350 & U & 350 & 350 & $\bar{U}$ & 350 & 370 & $\mathrm{U}$ & 370 & 350 & $\mathrm{U}$ & 350 \\
\hline 2,6-Dinitrotoluene & 350 & $\mathrm{U}$ & 350 & 350 & $U$ & 350 & 370 & $\mathrm{U}$ & 370 & 350 & $\mathrm{U}$ & 350 \\
\hline \begin{tabular}{|l|} 
2-Chloronaphthalene \\
\end{tabular} & 350 & $\mathrm{U}$ & 350 & 350 & $\mathrm{U}$ & 350 & 370 & $\mathrm{U}$ & 370 & 350 & U & 350 \\
\hline 2-Chlorophenol & 350 & $\mathrm{U}$ & 350 & 350 & $\mathrm{U}$ & 350 & 370 & U & 370 & 350 & $\mathrm{U}$ & 350 \\
\hline 2-Methylnaphthalene & 350 & $\mathrm{U}$ & 350 & 350 & $\mathrm{U}$ & 350 & 370 & $\mathrm{U}$ & 370 & 350 & $\mathrm{U}$ & 350 \\
\hline 2-Methylphenol (cresol, o-) & 350 & $\mathrm{U}$ & 350 & 350 & $\mathrm{U}$ & 350 & 370 & $\mathrm{U}$ & 370 & 350 & $\mathrm{U}$ & 350 \\
\hline \begin{tabular}{|l} 
2-Nitroaniline \\
\end{tabular} & 880 & $\mathrm{U}$ & 880 & 880 & U & 880 & 930 & $\mathrm{U}$ & 930 & 870 & $\mathrm{U}$ & 870 \\
\hline 2-Nitrophenol & 350 & $\mathrm{U}$ & 350 & 350 & $\mathrm{U}$ & 350 & 370 & $\mathrm{U}$ & 370 & 350 & $\mathrm{U}$ & 350 \\
\hline
\end{tabular}

Attachment

Originator

Checked

Calc. No.

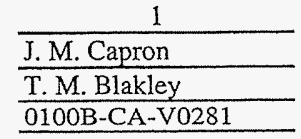

Sheet No. 10 of 13

Date $07 / 31 / 06$

Date

Rev. No. $\overline{0}$ 
Attachment 1. 100-B-14:2 Area 2 Verification Sampling Results.

\begin{tabular}{|c|c|c|c|c|c|c|c|c|c|c|c|c|}
\hline \multirow[t]{2}{*}{ Constituents } & \multicolumn{3}{|c|}{$\begin{array}{c}\text { J11K92 } \\
\text { Location } 8 \\
\text { Sample Date } 4 / 3 / 06\end{array}$} & \multicolumn{3}{|c|}{$\begin{array}{c}\text { J11K93 } \\
\text { Location } 9 \\
\text { Sample Date 4/3/06 }\end{array}$} & \multicolumn{3}{|c|}{$\begin{array}{c}\text { J11K94 } \\
\text { Location } 10 \\
\text { Sample Date } 4 / 3 / 06\end{array}$} & \multicolumn{3}{|c|}{$\begin{array}{c}\text { J11KD1 } \\
\text { East BCL Stockpile } \\
\text { Sample Date } 4 / 3 / 06\end{array}$} \\
\hline & $\mu \mathrm{g} / \mathrm{kg}$ & $\mathbf{Q}$ & PQL & $\mu \mathrm{g} / \mathrm{kg}$ & Q & PQL & $\mu \mathrm{g} / \mathrm{kg}$ & $Q$ & $\mathrm{PQL}$ & $\mu \mathrm{g} / \mathrm{kg}$ & $Q$ & PQL \\
\hline \multicolumn{13}{|c|}{ Semivolatile Organic Compounds (continued) } \\
\hline 3,3'-Dichlorobenzidine & 350 & $\mathrm{U}$ & 350 & 350 & $\mathrm{U}$ & 350 & 370 & $\bar{U}$ & 370 & 350 & $\mathrm{U}$ & 350 \\
\hline 3-Nitroaniline & 880 & $\mathrm{U}$ & 880 & 880 & U & 880 & 930 & $\mathrm{U}$ & 930 & 870 & U & 870 \\
\hline 4,6-Dinitro-2-methylphenol & 880 & $\mathrm{U}$ & 880 & 880 & $\mathrm{U}$ & 880 & 930 & $\mathrm{U}$ & 930 & 870 & $\mathrm{U}$ & 870 \\
\hline 4-Bromophenyl-phenylether & 350 & $\mathrm{U}$ & 350 & 350 & U & 350 & 370 & U & 370 & 350 & $\mathrm{U}$ & 350 \\
\hline 4-Chloro-3-methylphenol & 350 & $\mathrm{U}$ & 350 & 350 & U & 350 & 370 & $\overline{\mathrm{U}}$ & 370 & 350 & $\mathrm{U}$ & 350 \\
\hline 4-Chloroaniline & 350 & $\mathrm{U}$ & 350 & 350 & $\mathrm{U}$ & 350 & 370 & $\mathrm{U}$ & 370 & 350 & U & 350 \\
\hline 4-Chlorophenyl-phenylether & 350 & $\mathrm{U}$ & 350 & 350 & $\mathrm{U}$ & 350 & 370 & $\mathrm{U}$ & 370 & 350 & $\mathrm{U}$ & 350 \\
\hline 4-Methylphenol (p-cresol) & 350 & $\mathrm{U}$ & 350 & 350 & U & 350 & 370 & $\mathrm{U}$ & 370 & 350 & $\mathrm{U}$ & 350 \\
\hline 4-Nitroaniline & 880 & $\mathrm{U}$ & 880 & 880 & $\mathrm{U}$ & 880 & 930 & $\mathrm{U}$ & 930 & 870 & $\mathrm{U}$ & 870 \\
\hline 4-Nitrophenol & 880 & $\mathrm{U}$ & 880 & 880 & $\mathrm{U}$ & 880 & 930 & $\mathrm{U}$ & 930 & 870 & $\mathrm{U}$ & 870 \\
\hline Acenaphthene & 350 & $\mathrm{U}$ & 350 & 350 & $\mathrm{U}$ & 350 & 370 & $\mathrm{U}$ & 370 & 350 & $\mathrm{U}$ & 350 \\
\hline Acenaphthylene & 350 & $\mathrm{U}$ & 350 & 350 & $\mathrm{U}$ & 350 & 370 & $\mathrm{U}$ & 370 & 350 & $\mathrm{U}$ & 350 \\
\hline Anthracene & 350 & $\mathrm{U}$ & 350 & 350 & $\mathrm{U}$ & 350 & 370 & $\mathrm{U}$ & 370 & 350 & $\overline{\mathrm{U}}$ & 350 \\
\hline Benzo(a)anthracene & 350 & $\mathrm{U}$ & 350 & 350 & U & 350 & 370 & $\mathrm{U}$ & 370 & 350 & $\mathrm{U}$ & 350 \\
\hline Benzo(a)pyrene & 350 & $\mathrm{U}$ & 350 & 350 & U & 350 & 21 & $\mathrm{~J}$ & 370 & 350 & $\mathrm{U}$ & 350 \\
\hline Benzo(b)fluoranthene & 350 & $\mathrm{U}$ & 350 & 350 & U & 350 & 23 & $\mathrm{~J}$ & 370 & 350 & U & 350 \\
\hline Benzo $(\mathrm{g}, \mathrm{h}, \mathrm{i})$ perylene & 350 & $\mathrm{U}$ & 350 & 350 & $\mathrm{U}$ & 350 & 370 & $\mathrm{U}$ & 370 & 350 & $\mathrm{U}$ & 350 \\
\hline Benzo(k)fluoranthene & 350 & $\mathrm{U}$ & 350 & 350 & U & 350 & 22 & $\mathrm{~J}$ & 370 & 350 & $\mathrm{U}$ & 350 \\
\hline bis(2-Chloro-1-methylethyl)ether & 350 & $\mathrm{U}$ & 350 & 350 & $\mathrm{U}$ & 350 & 370 & $\mathrm{U}$ & 370 & 350 & $\mathrm{U}$ & 350 \\
\hline bis(2-Chloroethoxy)methane & 350 & $\mathrm{U}$ & 350 & 350 & U & 350 & 370 & $\mathrm{U}$ & 370 & 350 & $\mathrm{U}$ & 350 \\
\hline bis(2-Chloroethyl)ether & 350 & $\mathrm{U}$ & 350 & 350 & $\mathrm{U}$ & 350 & 370 & $\mathrm{U}$ & 370 & 350 & $\mathrm{U}$ & 350 \\
\hline bis(2-Ethylhexyl)phthalate & 660 & $\mathrm{U}$ & 660 & 660 & $\mathrm{U}$ & 660 & 660 & $\mathrm{U}$ & 660 & 660 & $\mathrm{U}$ & 660 \\
\hline Butylbenzylphthalate & 350 & $\mathrm{U}$ & 350 & 350 & $\mathrm{U}$ & 350 & 370 & $\mathrm{U}$ & 370 & 350 & $\mathrm{U}$ & 350 \\
\hline Carbazole & 350 & $\mathrm{U}$ & 350 & 350 & U & 350 & 370 & $\mathrm{U}$ & 370 & 350 & $\mathrm{U}$ & 350 \\
\hline Chrysene & 350 & $\mathrm{U}$ & 350 & 350 & $\mathrm{U}$ & 350 & 22 & $\mathrm{~J}$ & 370 & 350 & $\mathrm{U}$ & 350 \\
\hline Di-n-butylphthalate & 660 & $\mathrm{U}$ & 660 & 660 & $\mathrm{U}$ & 660 & 660 & $\mathrm{U}$ & 660 & 660 & $\mathrm{U}$ & 660 \\
\hline \begin{tabular}{|l|} 
Di-n-octylphthalate \\
\end{tabular} & 350 & $\mathrm{U}$ & 350 & 350 & $\mathrm{U}$ & 350 & 370 & $\mathrm{U}$ & 370 & 350 & $\bar{U}$ & 350 \\
\hline Dibenz $(\mathrm{a}, \mathrm{h})$ anthracene & 350 & $\mathrm{U}$ & 350 & 350 & $\mathrm{U}$ & 350 & 370 & $\mathrm{U}$ & 370 & 350 & $\mathrm{U}$ & 350 \\
\hline \begin{tabular}{|l|} 
Dibenzofuran \\
\end{tabular} & 350 & $\mathrm{U}$ & 350 & 350 & $\mathrm{U}$ & 350 & 370 & $\mathrm{U}$ & 370 & 350 & $\mathrm{U}$ & 350 \\
\hline Diethylphthalate & 350 & $\mathrm{U}$ & 350 & 350 & $\mathrm{U}$ & 350 & 370 & $\mathrm{U}$ & 370 & 350 & $\mathrm{U}$ & 350 \\
\hline \begin{tabular}{|l|} 
Dimethylphthalate \\
\end{tabular} & 350 & $\mathrm{U}$ & 350 & 350 & $\mathrm{U}$ & 350 & 370 & $\mathrm{U}$ & 370 & 350 & $\mathrm{U}$ & 350 \\
\hline Fluoranthene & 350 & $\mathrm{U}$ & 350 & 350 & $\mathrm{U}$ & 350 & 370 & $\mathrm{U}$ & 370 & 350 & $U$ & 350 \\
\hline Fluorene & 350 & $\mathrm{U}$ & 350 & 350 & $\mathrm{U}$ & 350 & 370 & U & 370 & 350 & U & 350 \\
\hline Hexachlorobenzene & 350 & $\mathrm{U}$ & 350 & 350 & $\mathrm{U}$ & 350 & 370 & U & 370 . & 350 & $U$ & 350 \\
\hline Hexachlorobutadiene & 350 & $\mathrm{U}$ & 350 & 350 & $\mathrm{U}$ & 350 & 370 & $\mathrm{U}$ & 370 & 350 & $\mathrm{U}$ & 350 \\
\hline Hexachlorocyclopentadiene & 350 & $\mathrm{U}$ & 350 & 350 & $\mathrm{U}$ & 350 & 370 & $\mathrm{U}$ & 370 & 350 & $\mathrm{U}$ & 350 \\
\hline Hexachloroethane & 350 & $\mathrm{U}$ & 350 & 350 & $\mathrm{U}$ & 350 & 370 & $\mathrm{U}$ & 370 & 350 & $U$ & 350 \\
\hline Indeno(1,2,3-cd)pyrene & 350 & $\mathrm{U}$ & 350 & 350 & $\mathrm{U}$ & 350 & 370 & $\mathrm{U}$ & 370 & 350 & U & 350 \\
\hline Isophorone & 350 & $\mathrm{U}$ & 350 & 350 & $\mathrm{U}$ & 350 & 370 & $\mathrm{U}$ & 370 & 350 & $\mathrm{U}$ & 350 \\
\hline N-Nitroso-di-n-dipropylamine & 350 & $\mathrm{U}$ & 350 & 350 & $\mathrm{U}$ & 350 & 370 & $\mathrm{U}$ & 370 & 350 & $\mathrm{U}$ & 350 \\
\hline N-Nitrosodiphenylamine & 350 & $\mathrm{U}$ & 350 & 350 & $\mathrm{U}$ & 350 & 370 & $\mathrm{U}$ & 370 & 350 & $\mathrm{U}$ & 350 \\
\hline \begin{tabular}{|l|} 
Naphthalene \\
\end{tabular} & 350 & $\mathrm{U}$ & 350 & 350 & $\mathrm{U}$ & 350 & 370 & $\mathrm{U}$ & 370 & 350 & U & 350 \\
\hline \begin{tabular}{|l} 
Nitrobenzene \\
\end{tabular} & 350 & $\mathrm{U}$ & 350 & 350 & $\mathrm{U}$ & 350 & 370 & $\mathrm{U}$ & 370 & 350 & $\mathrm{U}$ & 350 \\
\hline Pentachlorophenol & 880 & $\mathrm{U}$ & 880 & 880 & $\mathrm{U}$ & 880 & 930 & $\mathrm{U}$ & 930 & 870 & $\mathrm{U}$ & 870 \\
\hline Phenanthrene & 350 & $\mathrm{U}$ & 350 & 350 & $\mathrm{U}$ & 350 & 370 & $\mathrm{U}$ & 370 & 350 & $\mathrm{U}$ & 350 \\
\hline Phenol & 350 & $\mathrm{U}$ & 350 & 350 & U & 350 & 370 & $\mathrm{U}$ & 370 & 350 & $\mathrm{U}$ & 350 \\
\hline Pyrene & 350 & $\mathrm{U}$ & 350 & 350 & $U$ & 350 & 370 & $\mathrm{U}$ & 370 & 350 & U & 350 \\
\hline & & & & Attachn & & & 1 & & & Sheet No. & & 1 of 13 \\
\hline & & & & Origina & & J.M. C & pron & & & Date & & $7 / 31 / 06$ \\
\hline & & & & Checke & & T.M. B & akley & & & Dat & & \\
\hline & & & & Calc. $\mathrm{N}$ & & $0100 \mathrm{~B}$ & $\mathrm{~A}-\mathrm{V} 028$ & & & Rev. No & & 0 \\
\hline
\end{tabular}


Attachment 1. 100-B-14:2 Area 2 Verification Sampling Results.

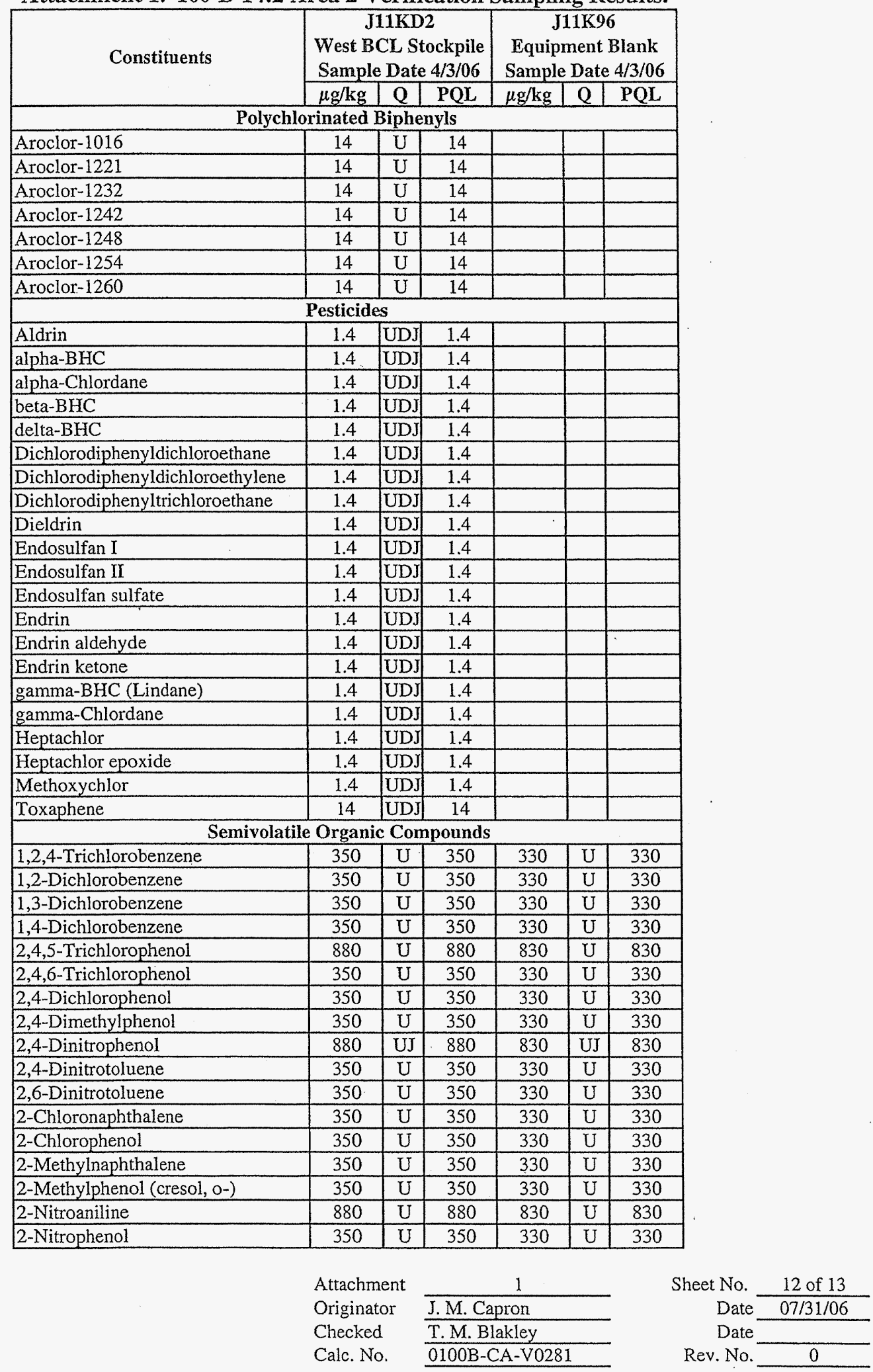


Attachment 1. 100-B-14:2 Area 2 Verification Sampling Results.

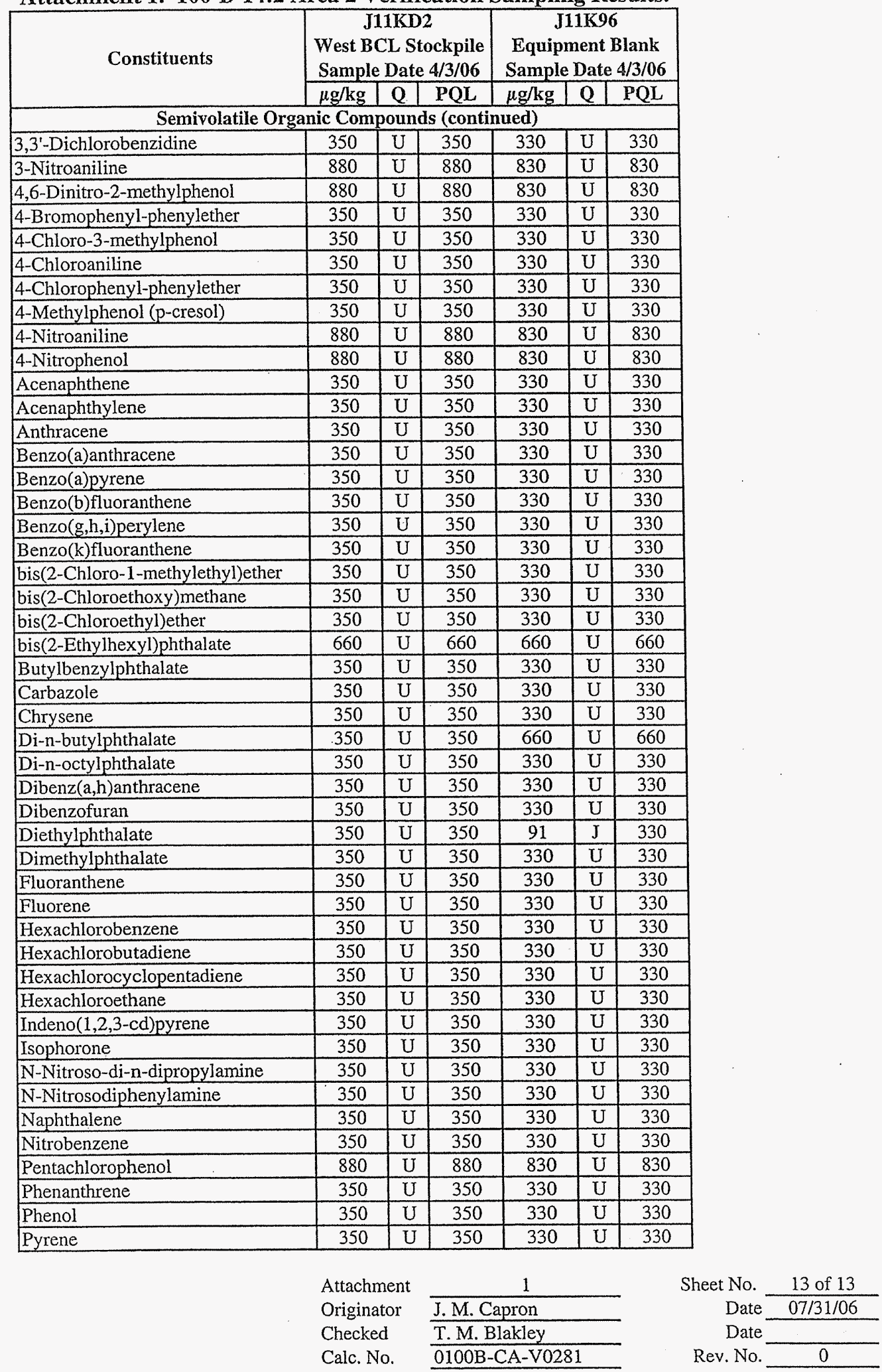


Attachment 2. 100-B-14:2 Area 5 Verification Sampling Results.

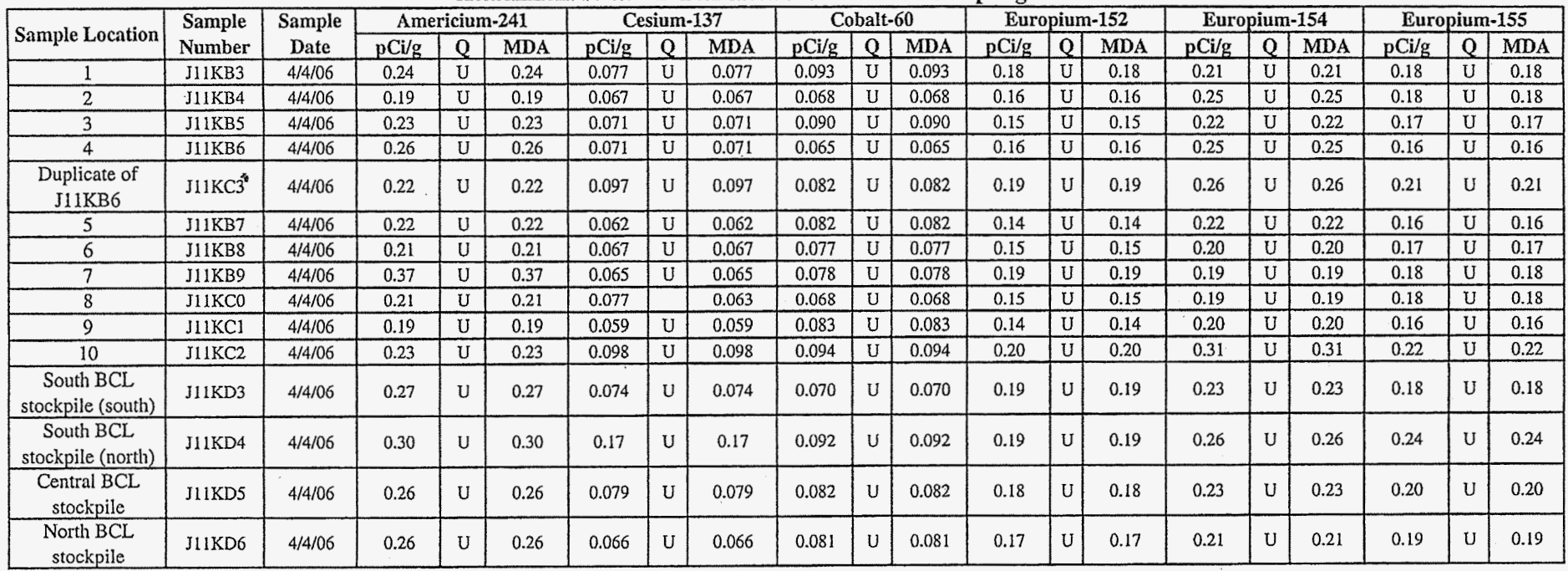

\begin{tabular}{|l|l|l|l|l|l|l|l|l|l|}
\hline & Radium-228 & Thorium-228 \\
\hline
\end{tabular} Sample Location $\begin{gathered}\text { Sumber } \\ \text { Nute }\end{gathered}$

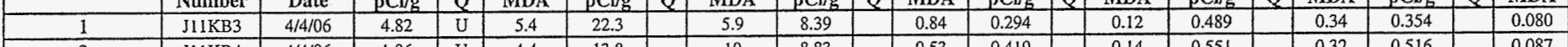

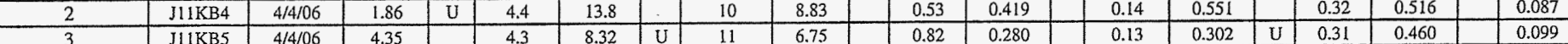

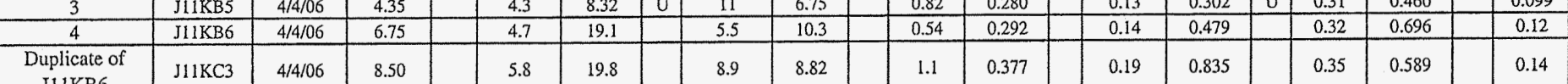

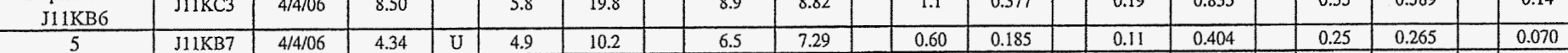

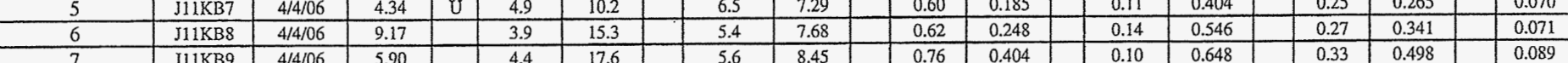

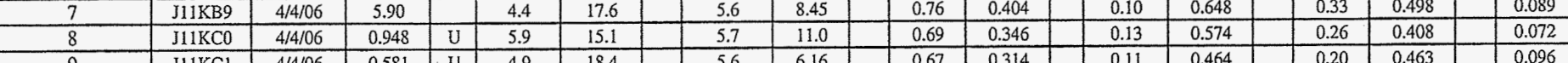

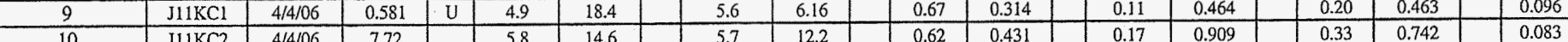

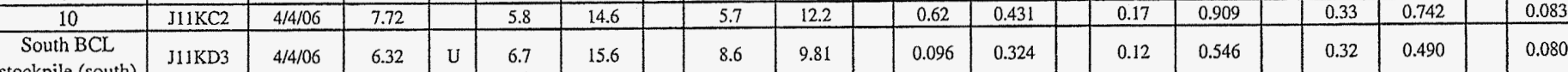

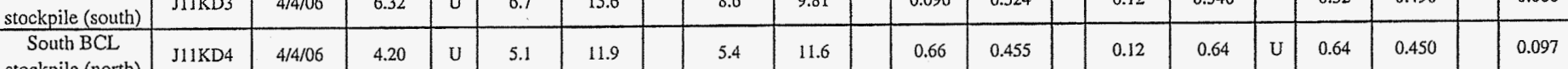
Central BCL

stockpile

North BCL \begin{tabular}{|l|l}
\hline J11KDS & $4 / 4 / 06$ \\
\hline
\end{tabular}

stockpile

\begin{tabular}{l|l|}
\hline 4.5 & 19.8 \\
\hline
\end{tabular}

\begin{tabular}{l|l}
10 & 9.42 \\
\hline
\end{tabular}

\begin{tabular}{l|l|}
\hline 0.81 & 0.349 \\
\hline
\end{tabular}

\begin{tabular}{l|l}
0.14 & 0.501 \\
\hline
\end{tabular}
Attachment $\frac{2}{2}$ Sheet No. $\frac{1 \text { of } 13}{07 / 31 / 06}$ Checked T.M.Blakley om Date $8 / 1 / 06$ 
Attachment 2. 100-B-14:2 Area 5 Verification Sampling Results.

\begin{tabular}{|c|c|c|c|c|c|c|c|c|c|c|c|c|c|c|c|c|c|}
\hline \multirow[t]{2}{*}{ Sample Location } & \multirow{2}{*}{$\begin{array}{l}\text { Sample } \\
\text { Number }\end{array}$} & \multirow{2}{*}{$\begin{array}{l}\text { Sample } \\
\text { Date }\end{array}$} & \multicolumn{3}{|c|}{ Thorium-232 } & \multicolumn{3}{|c|}{$\begin{array}{c}\text { Total Beta } \\
\text { Radiostrontium } \\
\end{array}$} & \multicolumn{3}{|c|}{ Tritium } & \multicolumn{3}{|c|}{ Uranium-235 } & \multicolumn{3}{|c|}{ Uranium-238 } \\
\hline & & & $\mathrm{pCi} / \mathrm{g}$ & $Q$ & MDA & $\mathrm{pCi} / \mathrm{g}$ & $Q$ & MDA & $\mathrm{pCi} / \mathrm{g}$ & Q & $\mathrm{MDA}$ & $\mathrm{pCi} / \mathrm{g}$ & Q & $\mathrm{MDA}$ & $\mathrm{pCi} / \mathrm{g}$ & $Q$ & $\mathrm{MDA}$ \\
\hline 1 & $\mathrm{~J} 11 \mathrm{~KB} 3$ & $4 / 4 / 06$ & 0.489 & $\mathrm{~J}$ & 0.34 & -0.051 & $\overline{\mathrm{U}}$ & 0.32 & -0.076 & UI & 0.37 & 0.23 & $\mathrm{U}$ & 0.23 & 9.4 & $\mathrm{U}$ & 9.4 \\
\hline 2 & J11KB4 & $4 / 4 / 06$ & 0.551 & $\mathrm{~J}$ & 0.32 & -0.052 & $\mathrm{U}$ & 0.31 & -0.130 & $\mathrm{UJ}$ & 0.36 & 0.26 & $\bar{U}$ & 0.26 & 10 & $\mathrm{U}$ & 10 \\
\hline 3 & J11KB5 & $4 / 4 / 06$ & 0.302 & UJ & 0.31 & -0.135 & $\mathrm{U}$ & 0.37 & -0.140 & UI & 0.36 & 0.22 & $\mathrm{U}$ & 0.22 & 8.2 & $\mathrm{U}$ & 8.2 \\
\hline 4 & J11KB6 & $4 / 4 / 06$ & 0.479 & $\mathrm{~J}$ & 0.32 & -0.039 & $\mathrm{U}$ & 0.28 & -0.028 & UJ & 0.36 & 0.26 & $\mathrm{U}$ & 0.26 & 7.9 & $\bar{U}$ & 7.9 \\
\hline $\begin{array}{c}\text { Duplicate of } \\
\text { J11KB6 }\end{array}$ & $\mathrm{J} 11 \mathrm{KC} 3$ & $4 / 4 / 06$ & 0.835 & $\mathrm{~J}$ & 0.35 & -0.045 & $\mathrm{U}$ & 0.25 & 0.151 & $\mathrm{u}$ & 0.27 & 0.31 & $\mathrm{U}$ & 0.31 & 11 & $\mathrm{U}$ & 11 \\
\hline 5 & J11KB7 & $4 / 4 / 06$ & 0.404 & $\mathrm{~J}$ & 0.25 & 0.090 & $\mathrm{U}$ & 0.29 & -0.024 & UJ & 0.37 & 0.21 & $\mathrm{U}$ & 0.21 & 7.1 & $\mathrm{U}$ & 7.1 \\
\hline 6 & J11KB8 & $4 / 4 / 06$ & 0.546 & $\mathrm{~J}$ & 0.27 & 0.085 & $\overline{\mathrm{U}}$ & 0.29 & -0.085 & $\mathrm{U}$ & 0.37 & 0.22 & $\bar{U}$ & 0.22 & 7.8 & $\mathrm{U}$ & 7.8 \\
\hline 7 & J11KB9 & $4 / 4 / 06$ & 0.648 & $\mathrm{~J}$ & 0.33 & 0.018 & $\mathrm{U}$ & 0.31 & 0.110 & $\bar{U}$ & 0.36 & 0.29 & $\mathrm{U}$ & 0.29 & 8.2 & $U$ & 8.2 \\
\hline 8 & $\mathrm{~J} 11 \mathrm{KCO}$ & $4 / 4 / 06$ & 0.574 & $\mathrm{~J}$ & 0.26 & -0.033 & $\mathrm{U}$ & 0.31 & 0.094 & $\mathrm{U}$ & 0.35 & 0.22 & $\mathrm{U}$ & 0.22 & 6.7 & $\mathrm{U}$ & 6.7 \\
\hline 9 & J11KCl & $4 / 4 / 06$ & 0.464 & $\mathrm{~J}$ & 0.20 & -0.041 & $\mathrm{U}$ & 0.26 & -0.005 & $\mathrm{U}$ & 0.38 & 0.19 & $\vec{U}$ & 0.19 & 7.8 & $\overline{\mathrm{U}}$ & 7.8 \\
\hline 10 & J11KC2 & $4 / 4 / 06$ & 0.909 & $\mathrm{~J}$ & 0.33 & 0.114 & $\mathrm{U}$ & 0.33 & 0.239 & $\mathrm{U}$ & 0.40 & 0.33 & $\mathrm{U}$ & 0.33 & 12 & $\bar{U}$ & 12 \\
\hline $\begin{array}{c}\text { South BCL } \\
\text { stockpile (south) }\end{array}$ & J11KD3 & $4 / 4 / 06$ & 0.546 & $\mathrm{~J}$ & 0.32 & & & & -0.105 & $\mathrm{U}$ & 0.25 & 0.27 & $\mathrm{U}$ & 0.27 & 9.4 & $\mathrm{U}$ & 9.4 \\
\hline $\begin{array}{c}\text { South BCL } \\
\text { stockpile (north) }\end{array}$ & $\mathrm{J} 1 \mathrm{IKD} 4$ & $4 / 4 / 06$ & 0.64 & UJ & 0.64 & & & & 0.296 & & 0.27 & 0.34 & $\mathrm{U}$ & 0.34 & 9.0 & U & 9.0 \\
\hline $\begin{array}{c}\text { Central BCL } \\
\text { stockpile }\end{array}$ & J11KD5 & $4 / 4 / 06$ & 0.575 & J & 0.33 & & & & 0.045 & U & 0.26 & 0.24 & $\mathrm{U}$ & 0.24 & 9.0 & $\mathrm{U}$ & 9.0 \\
\hline $\begin{array}{l}\text { North BCL } \\
\text { stockpile }\end{array}$ & J11KD6 & $4 / 4 / 06$ & 0.501 & $\mathrm{~J}$ & 0.31 & & & & -0.114 & $\mathrm{U}$ & 0.25 & 0.28 & $U$ & 0.28 & 8.3 & $\mathrm{U}$ & 8.3 \\
\hline
\end{tabular}

\begin{tabular}{|c|c|c|c|}
\hline Attachment & 2 & Sheet No. & 2 of 13 \\
\hline Originator & J.M. Capron & Date & $07 / 31 / 06$ \\
\hline Checked & T.M. Blakley & Date & \\
\hline Calc. No. & $0100 \mathrm{~B}-\mathrm{CA}-\mathrm{V} 0281$ & Rev. No. & 0 \\
\hline
\end{tabular}


Attachment 2, 100-B-14:2 Area 5 Verification Sampling Results.

\begin{tabular}{|c|c|c|c|c|c|c|c|c|c|c|c|c|c|c|c|c|c|c|c|c|}
\hline mole Location & Sample & Sample & & minum & & & ntimo & ay & Z & rsenic & & arium & & rylliwi & & & & & thiur & \\
\hline & Number & Date & $\mathrm{m} / \mathrm{kg}$ & \begin{tabular}{l|l} 
& 1 \\
\end{tabular} & PQL & $\mathrm{mg} / \mathrm{kg}$ & & $\mathrm{PQL}$ & $\mathrm{mg} / \mathrm{kg}$ & \begin{tabular}{l|l|}
$Q$ & $P Q$ \\
\end{tabular} & $\mathrm{mg} / \mathrm{kg}$ & \begin{tabular}{|l|l|}
$\mathrm{PQL}$ \\
\end{tabular} & $\mathrm{m} / \mathrm{kg}$ & Q & $\mathrm{POL}$ & $\mathrm{mg} / \mathrm{kg}$ & \begin{tabular}{l|l} 
Q & $\mathrm{PQL}$ \\
\end{tabular} & $\mathrm{mg} / \mathrm{kg}$ & $Q$ & \\
\hline$\frac{1}{2}$ & $511 K 3_{3}$ & 4/44106 & 4250 & & 2.4 & 0.44 & \begin{tabular}{|l|}
$\mathrm{UI}$ \\
\end{tabular} & 0.44 & 2.6 & 0.6 & $\frac{44.2}{5.2}$ & 0.02 & 0.24 & & 0.02 & $\begin{array}{l}0.88 \\
.5\end{array}$ & 0.24 & 0.09 & & 0.07 \\
\hline$\frac{2}{3}$ & J11KB4 & $\frac{44 / 106}{44 / 106}$ & $\frac{4720}{4002}$ & & $\frac{2.3}{23}$ & $\frac{0.43}{0.43}$ & \begin{tabular}{|l|}
$U_{3}$ \\
$\mathrm{u}_{1}$
\end{tabular} & 0.43 & 2.4 & $\frac{0.5}{0.5}$ & $\frac{51.2}{436}$ & 0.02 & 0.31 & & 0.02 & $\frac{1.5}{070}$ & 0.23 & 0.15 & & 0.07 \\
\hline$\frac{3}{4}$ & IKB6 & $4 / 41 / 106$ & 5390 & & 2.3 & 0.43 & \begin{tabular}{|l|} 
UJ \\
\end{tabular} & 0.43 & $\frac{2.7}{2.7}$ & 0.6 & $\frac{4.0}{56.2}$ & 0.02 & 0.02 & & $\frac{0.06}{0.02}$ & 0.10 & $\frac{0.23}{0.24}$ & $\frac{0.14}{0.14}$ & & $\frac{0.07}{0.07}$ \\
\hline Duplicate of & ЛИКСЗ & 4/4/106 & 5380 & & 2.3 & 0.43 & UJ & 0.43 & 3.0 & 0.6 & 55.7 & 0.02 & 0.35 & & 0.02 & 0.82 & 0.24 & 0.12 & & 0.07 \\
\hline$\frac{5}{5}$ & J11KB7 & 4/4/106 & 4310 & & 2.3 & 0.43 & Uu & 0.43 & $\overline{2.3}$ & 0.5 & 46.8 & $0.02^{\prime}$ & 0.30 & & 0.02 & 0.37 & 0.23 & 0.12 & & 0.07 \\
\hline & & & & & 2.3 & 0.43 & UI & 0.43 & 2.3 & 0.6 & 56.2 & & 070 & & 0.02 & 0.76 & & & & \\
\hline & $\mathrm{J11}$ & & 45 & & 2.3 & 0.43 & UJ & 0.43 & 2. & 0.6 & & & $\sqrt{2.26}$ & & 0.02 & 1.0 & & & & 0.07 \\
\hline 8 & J11KC & & & & 2.3 & 0.42 & UJ & 42 & 1.7 & & & & .24 & & 0.02 & 0.68 & & & & 0.07 \\
\hline 9 & J11KCI & & 5780 & & 2.33 & 0.42 & Us & 0.42 & 2.8. & & & & 0.34 & & 0.02 & & & & & 0.07 \\
\hline 10 & J11KC2 & 4/44106 & 7870 & & 2.7 & 0.50 & US & 0.50 & 7.5 & 0.6 & 70.5 & 0.02 & 0.42 & & 0.02 & 2.6 & 0.27 & 0.21 & & \\
\hline $\begin{array}{l}\text { South in } \\
\text { stockpile }\end{array}$ & J11KD3 & 4/4/106 & 3810 & & 2.3 & 0.43 & us & 0.43 & 2.0 & 0.5 & 50.6 & 0.02 & 0.24 & & 0.02 & 0.59 & 0.23 & 0.14 & & 0.07 \\
\hline $\begin{array}{c}\text { South } \\
\text { stockpile }\end{array}$ & J1KD4 & 4/4/106 & 5170 & & 2.3 & 0.42 & us & 0.42 & 2.4 & 0.5 & 58.2 & 0.02 & 0.32 & & 0.02 & 0.95 & 0.23 & 0.09 & & 0.07 \\
\hline Central BCL & J11KDS & $4 / 4 / 06$ & 4790 & & 2.4 & 0.44 & us & 0.44 & 2.3 & 0.6 & 65.5 & 0.02 & 0.32 & & 0.02 & 0.97 & 0.24 & 0.1 & & 0.07 \\
\hline & Л1КD 6 & 4/4406 & 4090 & & 2.2 & 0.42 & บJ & 0.42 & 2.6 & 0.5 & 75.0 & 0.02 & 0.26 & & 0.02 & 3.3 & 0.23 & 0.1 & & 0.07 \\
\hline & & 44100 & 40.8 & & & 030 & & 030 & & & & & & & & & & & & \\
\hline
\end{tabular}

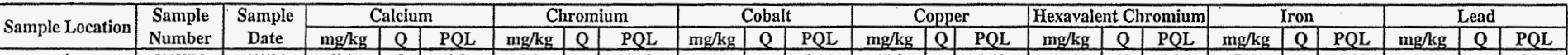

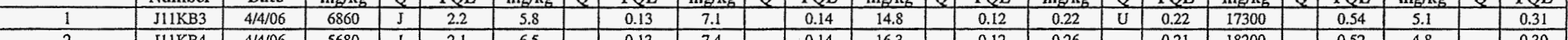

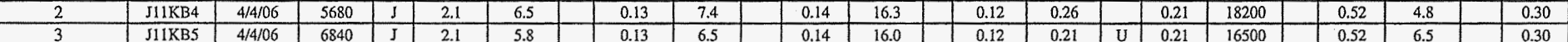

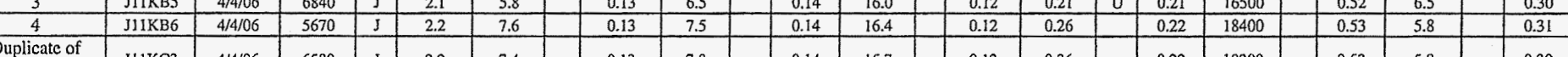

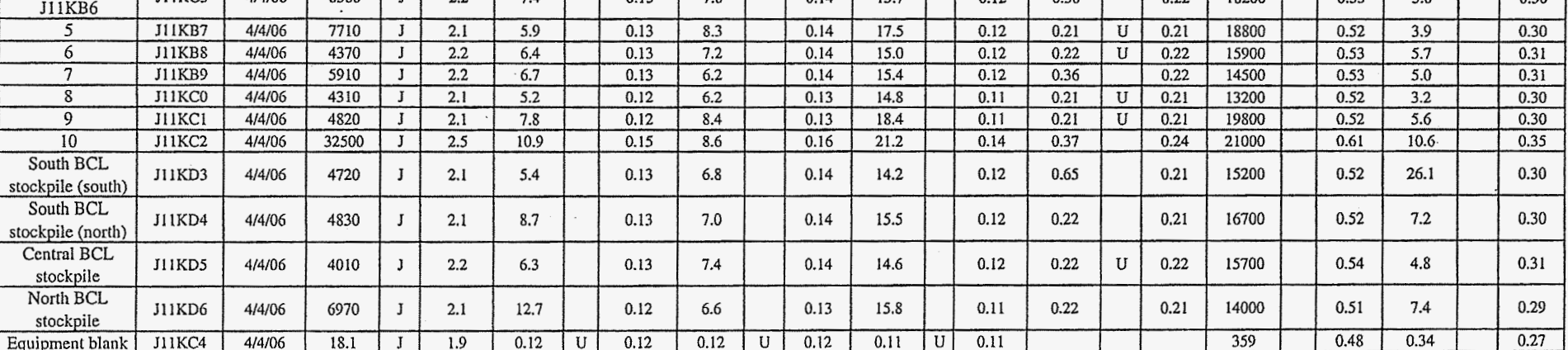


Attachment 2. 100-B-14:2 Area 5 Verification Sampling Results.

\begin{tabular}{|c|c|c|c|c|c|c|c|c|c|c|c|c|c|c|c|c|c|c|c|c|c|c|c|}
\hline \multirow{2}{*}{ Sample Location } & \multirow{2}{*}{$\begin{array}{l}\text { Sample } \\
\text { Number }\end{array}$} & \multirow{2}{*}{$\begin{array}{c}\text { Sample } \\
\text { Date }\end{array}$} & \multicolumn{3}{|c|}{ Lithium } & \multicolumn{3}{|c|}{ Magnesium } & \multicolumn{3}{|c|}{ Manganese } & \multicolumn{3}{|c|}{ Mercury } & \multicolumn{3}{|c|}{ Molybdenum } & \multicolumn{3}{|c|}{ Nickel } & \multicolumn{3}{|c|}{ Phosphorus } \\
\hline & & & $\mathrm{mg} / \mathrm{kg}$ & 0 & $\mathrm{PQL}$ & $\mathrm{mg} / \mathrm{kg}$ & Q & PQL & $\mathrm{mg} / \mathrm{kg}$ & $Q$ & $\mathrm{PQL}$ & $\mathrm{mg} / \mathrm{kg}$ & Q & $\mathrm{PQL}$ & $\mathrm{mg} / \mathrm{kg}$ & $Q$ & $\mathrm{PQL}$ & $\mathrm{mg} / \mathrm{kg}$ & 0 & POL & $\mathrm{mg} / \mathrm{kg}$ & 0 & PQL \\
\hline 1 & $\mathrm{J11 \textrm {KB } 3}$ & $4 / 4 / 06$ & 5.6 & C & 0.03 & 3680 & & 3.9 & 289 & & 0.03 & 0.02 & $\mathrm{U}$ & 0.02 & 0.49 & & 0.29 & 8.7 & & 0.24 & 939 & $\mathrm{C}$ & 0.90 \\
\hline 2 & J11KB4 & $4 / 4 / 06$ & 8.4 & C & 0.03 & 3690 & & 3.8 & 306 & & 0.03 & 0.04 & & 0.02 & 0.53 & & 0.28 & 9.8 & & 0.23 & 887 & C & 0.87 \\
\hline 3 & J11KB5 & $4 / 4 / 06$ & 5.2 & $\bar{c}$ & 0.03 & 3650 & & 3.8 & 259 & & 0.03 & 0.02 & $\mathrm{U}$ & 0.02 & 0.41 & & 0.28 & 8.7 & & 0.23 & 874 & $\frac{\mathrm{C}}{\mathrm{c}}$ & 0.87 \\
\hline 4 & J11KBO & $4 / 4 / 06$ & 6.4 & C & 0.03 & 4030 & & 3.9 & 315 & & 0.03 & 0.02 & $\mathrm{u}$ & 0.02 & 0.43 & & 0.29 & 10.0 & & 0.24 & 829 & C & 0.89 \\
\hline $\begin{array}{c}\text { Duplicate of } \\
\text { J11KB6 }\end{array}$ & $\mathrm{J11KC3}$ & $4 / 4 / 06$ & 6.4 & C & 0.03 & 4170 & & 3.8 & 333 & & 0.03 & 0.02 & $u$ & 0.02 & 0.52 & & 0.29 & 10.0 & & 0.24 & 886 & c & 0.88 \\
\hline 5 & J11KB7 & $4 / 4 / 06$ & 5.7 & $C$ & 0.03 & 4100 & & 3.8 & 312 & & 0.03 & 0.02 & U & 0.02 & 0.50 & & 0.28 & 10.6 & & 0.23 & 1030 & C & 0.87 \\
\hline 6 & J11KB8 & $4 / 4 / 06$ & 5.5 & $\mathrm{C}$ & 0.03 & 3550 & & 3.8 & 308 & & 0.03 & 0.02 & $\mathrm{u}$ & 0.02 & 0.49 & & 0.29 & 9.7 & & 0.24 & 808 & $\mathrm{C}$ & 0.89 \\
\hline 7 & J11KB9 & $4 / 4 / 06$ & 5.4 & $\mathrm{C}$ & 0.03 & 3360 & & 3.9 & 302 & & 0.03 & 0.03 & & 0.02 & 0.49 & & 0.29 & 8.6 & & 0.24 & 717 & $\mathrm{C}$ & 0.89 \\
\hline 8 & JIKKCO & $4 / 4106$ & 3.9 & C & 0.03 & 2930 & & 3.7 & 232 & & 0.03 & 0.02 & $\mathrm{U}$ & 0.02 & 0.40 & & 0.28 & 8.3 & & 0.23 & 801 & C & 0.86 \\
\hline 9 & J11KC1 & $4 / 4 / 106$ & 5.9 & $C$ & 0.03 & 4090 & & 3.7 & 362 & & 0.03 & 0.02 & & 0.01 & 0.53 & & 0.28 & 11.7 & & 0.23 & 944 & $\mathrm{C}$ & 0.86 \\
\hline 10 & J11KC2 & $4 / 4 / 06$ & 11.9 & $\mathrm{C}$ & 0.03 & 6020 & & 4.4 & 388 & & 0.03 & 0.02 & $\mathrm{U}$ & 0.02 & 0.56 & & 0.33 & 14.1 & & 0.27 & 802 & C & 1.0 \\
\hline $\begin{array}{c}\text { South BCL } \\
\text { stockpile (south) }\end{array}$ & J1KD3 & $4 / 4 / 06$ & 4.9 & c & 0.03 & 3060 & & 3.8 & 276 & & 0.03 & .0 .04 & & 0.02 & 0.45 & & 0.28 & 8.0 & & 0.23 & 889 & c & 0.87 \\
\hline $\begin{array}{c}\text { South BCL } \\
\text { stockpile (north) }\end{array}$ & J11KD4 & 4/4/06 & 5.9 & $c$ & 0.03 & 3630 & & 3.8 & 306 & & 0.03 & 0.02 & $\mathrm{u}$ & 0.02 & 0.50 & & 0.28 & 9.7 & & 0.23 & 801 & C & 0.87 \\
\hline $\begin{array}{c}\text { Central BCL } \\
\text { stockpile }\end{array}$ & J11KDS & $4 / 4 / 06$ & 5.0 & c & 0.03 & 3470 & & 3.9 & 324 & & 0.03 & 0.02 & $\mathrm{u}$ & 0.02 & 0.39 & & 0.29 & 9.0 & & 0.24 & 859 & C & 0.90 \\
\hline $\begin{array}{c}\text { North BCL } \\
\text { stockpile }\end{array}$ & J11KD6 & $4 / 4 / 06$ & 4.9 & C & 0.03 & 3120 & & 3.7 & 264 & & 0.03 & 0.1 & & 0.02 & 0.46 & & 0.28 & 8.1 & & 0.23 & 896 & C & 0.86 \\
\hline Equipment blank & J11KC4 & $4 / 4 / 06$ & 0.07 & UIC & 0.03 & 6.7 & & 3.5 & 5.4 & & 0.03 & 0.02 & $u$ & 0.02 & 0.26 & $\mathrm{U}$ & 0.26 & 0.21 & $\mathrm{U}$ & 0.21 & 3.3 & UJC & 0.80 \\
\hline
\end{tabular}

\begin{tabular}{|c|c|c|c|c|c|c|c|c|c|c|c|c|c|c|c|c|c|c|c|c|c|c|c|}
\hline \multirow{2}{*}{ Sample Location } & \multirow{2}{*}{$\begin{array}{l}\text { Sample } \\
\text { Number }\end{array}$} & \multirow{2}{*}{$\begin{array}{c}\text { Sample } \\
\text { Date }\end{array}$} & \multicolumn{3}{|c|}{ Potassium } & \multicolumn{3}{|c|}{ Selenium } & \multicolumn{3}{|c|}{ Silicon } & \multicolumn{3}{|c|}{ Silver } & \multicolumn{3}{|c|}{ Sodium } & \multicolumn{3}{|c|}{ Strontium } & \multicolumn{3}{|c|}{ Thallium } \\
\hline & & & $\mathrm{mg} / \mathrm{kg}$ & $Q$ & $\mathrm{PQL}$ & $\mathrm{mg} / \mathrm{kg}$ & $Q$ & $\mathrm{PQL}$ & $\mathrm{mg} / \mathrm{kg}$ & $Q$ & $\mathrm{PQL}$ & $\mathrm{mg} / \mathrm{kg}$ & Q & $\mathrm{PQL}$ & $\mathrm{mg} / \mathrm{kg}$ & $Q$ & $\mathrm{PQL}$ & $\mathrm{mg} / \mathrm{kg}$ & Q & $\mathrm{PQL}$ & $\mathrm{mg} / \mathrm{kg}$ & 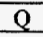 & $\mathrm{PQL}$ \\
\hline 1 & J11KB3 & $4 / 4 / 06$ & 951 & & 77.5 & 0.47 & $\mathrm{U}$ & 0.47 & 522 & $\mathrm{~J}$ & 2.3 & 0.07 & u & 0.07 & 119 & $\mathrm{C}$ & 2.5 & 21.6 & $\mathrm{C}$ & 0.01 & 0.70 & $\mathrm{U}$ & 0.70 \\
\hline 2 & J11KB4 & $4 / 4 / 06$ & 925 & & 74.9 & 0.46 & $\mathrm{U}$ & 0.46 & 441 & $J$ & 2.2 & 0.07 & $\mathrm{u}$ & 0.07 & 135 & C & 2.4 & 21.6 & $\mathrm{C}$ & 0.01 & 0.68 & $\mathrm{U}$ & 0.68 \\
\hline 3 & J11KB5 & $4 / 4 / 06$ & 685 & & 74.5 & 0.45 & $\mathrm{U}$ & 0.45 & 416 & $J$ & 2.2 & 0.07 & $\mathrm{U}$ & 0.07 & 98.4 & C & 2.4 & 24.2 & $C$ & 0.01 & 0.68 & $\mathrm{U}$ & 0.68 \\
\hline 4 & J11KB6 & $4 / 4 / 06$ & 1050 & & 76.0 & 0.46 & $\mathrm{U}$ & 0.46 & 478 & $\mathrm{~J}$ & 2.2 & 0.07 & $\mathrm{u}$ & 0.07 & 111 & $\mathrm{C}$ & 2.5 & 24.3 & $\mathrm{C}$ & 0.01 & 0.69 & $\mathrm{U}$ & 0.69 \\
\hline $\begin{array}{l}\text { Duplicate of } \\
\text { J11KB6 }\end{array}$ & J11KC3 & $4 / 4 / 06$ & 1100 & & 75.8 & 0.46 & U & 0.46 & 522 & $\mathrm{~J}$ & 2.2 & 0.07 & $\mathrm{u}$ & 0.07 & 108 & $\mathrm{C}$ & 2.5 & 23.7 & $\mathrm{C}$ & 0.01 & 0.69 & $\mathrm{u}$ & 0.69 \\
\hline 5 & J11KB7 & $4 / 4 / 06$ & 757 & & 74.5 & 0.45 & $\mathrm{U}$ & 0.45 & 466 & $\mathrm{~J}$ & 2.2 & 0.07 & U & 0.07 & 105 & $\mathrm{C}$ & 2.4 & 25.7 & $\mathrm{C}$ & 0.01 & 0.68 & $\mathrm{U}$ & 0.68 \\
\hline 6 & $\mathrm{J11KB8}$ & $4 / 4 / 06$ & 866 & & 74.9 & 0.46 & $\mathrm{U}$ & 0.46 & 587 & $\mathrm{~J}$ & 2.2 & 0.07 & $\mathrm{U}$ & 0.07 & 89.8 & C & 2.5 & 19.8 & $C$ & 0.01 & 0.69 & $\mathrm{u}$ & 0.69 \\
\hline 7 & J11KB9 & $4 / 4 / 06$ & 825 & & 76.2 & 0.46 & $\mathrm{U}$ & 0.46 & 491 & $\mathrm{~J}$ & 2.2 & 0.07 & $\mathrm{U}$ & 0.07 & 110 & C & 2.5 & 25.6 & C & 0.01 & 0.69 & 0 & 0.69 \\
\hline 8 & J11KCO & $4 / 4 / 06$ & 660 & & 73.9 & 0.45 & $\mathrm{U}$ & 0.45 & 387 & $\mathrm{~J}$ & 2.2 & 0.07 & $\mathrm{u}$ & 0.07 & 101 & C & 2.4 & 20.2 & $\mathrm{C}$ & 0.01 & 0.67 & U & 0.67 \\
\hline 9 & $\mathrm{IKCl}$ & & 1150 & & 73.7 & 0.45 & $\mathrm{U}$ & 0.45 & 483 & $\mathrm{~J}$ & 2.2 & 0.07 & $\mathrm{U}$ & 0.07 & 129 & $C$ & 2.4 & 27.5 & C & 0.01 & 0.67 & $\mathrm{U}$ & 0.67 \\
\hline 10 & J11KC2 & $4 / 4 / 06$ & 1290 & & 86.8 & 0.53 & U & 0.53 & 526 & $\mathrm{~J}$ & 2.6 & 0.08 & $\mathrm{u}$ & 0.08 & 218 & $\mathrm{C}$ & 2.8 & 59.2 & C & 0.01 & 0.79 & $\mathrm{U}$ & 0.79 \\
\hline $\begin{array}{c}\text { South BCL } \\
\text { stockpile (south) }\end{array}$ & J11KD3 & 4/4/06 & 772 & & 74.5 & 0.45 & $\mathrm{U}$ & 0.45 & 482 & J & 2.2 & 0.07 & $\mathrm{u}$ & 0.07 & 101 & $\mathrm{C}$ & 2.4 & 23.9 & c & 0.01 & 0.68 & $\mathrm{U}$ & 0.68 \\
\hline $\begin{array}{c}\text { South BCL } \\
\text { stockpile (north) }\end{array}$ & J11KD4 & $4 / 4 / 06$ & 1050 & & 74.4 & 0.45 & $\mathrm{U}$ & 0.45 & 486 & J & 2.2 & 0.07 & 0 & 0.07 & 103 & C & 2.4 & 21.6 & C & 0.01 & 0.68 & $\mathrm{U}$ & 0.68 \\
\hline $\begin{array}{c}\text { Central BCL } \\
\text { stockpile }\end{array}$ & J11KD5 & $4 / 4 / 06$ & 1130 & & 77.3 & 0.47 & $\mathrm{u}$ & 0.47 & 519 & $\mathrm{~J}$ & 2.3 & 0.07 & $u$ & 0.07 & 91.6 & C & 2.5 & 20.0 & C & 0.01 & 0.70 & $\mathrm{U}$ & 0.70 \\
\hline $\begin{array}{l}\text { North BCL } \\
\text { stockpile }\end{array}$ & J1IKDG & $4 / 4 / 06$ & 742 & & 73.2 & 0.45 & $\mathrm{U}$ & 0.45 & 517 & J & 2.2 & 0.07 & $u$ & 0.07 & 154 & $\mathrm{C}$ & 2.4 & 33.1 & $C$ & 0.01 & 0.66 & u & 0.66 \\
\hline Equipment blank & J11KC4 & $4 / 4 / 06$ & 68.3 & $\mathrm{U}$ & 68.3 & 0.42 & $\mathrm{U}$ & 0.42 & 42.2 & $\mathrm{~J}$ & 2.0 & 0.06 & U & 0.06 & 6.9 & UIC & 2.2 & 0.22 & C & 0.009 & 0.62 & $\mathrm{U}$ & 0.62 \\
\hline
\end{tabular}

Attachment 
Attachment 2. 100-B-14:2 Area 5 Verification Sampling Results.

\begin{tabular}{|c|c|c|c|c|c|c|c|c|c|c|c|c|c|c|c|c|c|c|c|c|}
\hline \multirow{2}{*}{ Sample Location } & \multirow{2}{*}{$\begin{array}{l}\text { Sample } \\
\text { Number } \\
\end{array}$} & \multirow{2}{*}{$\begin{array}{c}\text { Sample } \\
\text { Date }\end{array}$} & \multicolumn{3}{|c|}{ Tin } & \multicolumn{3}{|c|}{ Titanium } & \multicolumn{3}{|c|}{ Uranium } & \multicolumn{3}{|c|}{ Vanadium } & \multicolumn{3}{|c|}{ Zinc } & \multicolumn{3}{|c|}{ Zirconium } \\
\hline & & & $\mathrm{mg} / \mathrm{kg}$ & $Q$ & PQL & $\mathrm{mg} / \mathrm{kg}$ & $Q$ & $\mathbf{P Q L}$ & $\mathrm{mg} / \mathrm{kg}$ & Q & $\mathrm{PQL}$ & $\mathrm{mg} / \mathrm{kg}$ & Q & $\mathrm{PQL}$ & $\mathrm{mg} / \mathrm{kg}$ & $Q$ & PQL & $\mathrm{mg} / \mathrm{kg}$ & $Q$ & $\mathrm{PQL}$ \\
\hline 1 & J11KB3 & $4 / 4 / 06$ & 1.1 & & 1.1 & 1330 & & 0.03 & 0.88 & UC & 0.88 & 40.8 & & 0.09 & 38.8 & & 0.16 & 16.1 & & 1.1 \\
\hline 2 & J11KB4 & $4 / 4 / 06$ & 1.4 & & 1.0 & 1560 & & 0.03 & 0.85 & $\mathrm{UC}$ & 0.85 & 46.9 & & 0.09 & 38.5 & & 0.16 & 19.8 & & 1.0 \\
\hline 3 & J11KB5 & $4 / 4 / 06$ & 1.2 & & 1.0 & 1360 & & 0.03 & 0.85 & $\mathrm{UC}$ & 0.85 & 41.1 & & 0.09 & 35.5 & & 0.15 & 17.0 & & 1.0 \\
\hline 4 & J11KB6 & $4 / 4 / 06$ & 1.4 & & 1.1 & 1450 & & 0.03 & 0.87 & $\mathrm{UC}$ & 0.87 & 46.2 & & 0.09 & 40.2 & & 0.16 & 19.6 & & 1.0 \\
\hline $\begin{array}{c}\text { Duplicate of } \\
\mathrm{J} 11 \mathrm{~KB} 6\end{array}$ & J11KC3 & $4 / 4 / 06$ & 1.3 & & 1.1 & 1460 & & 0.03 & 2.2 & UJC & 0.87 & 44.6 & & 0.09 & 38.5 & & 0.16 & 19.8 & & 1.0 \\
\hline 5 & J11KB7 & $4 / 4 / 06$ & 1.5 & & 1.0 & 1730 & & 0.03 & 0.85 & $\mathrm{UC}$ & 0.85 & 49.1 & & 0.09 & 38.4 & & 0.15 & 18.3 & & 1.0 \\
\hline 6 & J11KB8 & $4 / 4 / 06$ & 1.2 & & 1.1 & 1270 & & 0.03 & 1.1 & UJC & 0.87 & 39.1 & & 0.09 & 35.5 & & 0.16 & 17.9 & & 1.0 \\
\hline 7 & J11KB9 & $4 / 4 / 06$ & 1.3 & & 1.1 & 1100 & & 0.03 & 1.3 & UJC & 0.87 & 35.2 & & 0.09 & 34.4 & & 0.16 & 14.5 & & 1.0 \\
\hline 8 & $\mathrm{~J} 11 \mathrm{KCO}$ & $4 / 4 / 06$ & 1.2 & & 1.0 & 1010 & & 0.03 & 0.84 & $\mathrm{UC}$ & 0.84 & 31.2 & & 0.09 & 29.2 & & 0.15 & 14.4 & & 1.0 \\
\hline 9 & $\mathrm{~J} 11 \mathrm{KCl}$ & $4 / 4 / 06$ & 1.3 & & 1.0 & 1460 & & 0.03 & 1.6 & UJC & 0.84 & 43.7 & & 0.09 & 39.4 & & 0.15 & 23.1 & & 1.0 \\
\hline 10 & J11KC2 & $4 / 4 / 06$ & 1.2 & $\mathrm{U}$ & 1.2 & 1020 & & 0.03 & 3.1 & UJC & 0.99 & 36.7 & & 0.10 & 44.8 & & 0.18 & 11.2 & & 1.2 \\
\hline $\begin{array}{c}\text { South BCL } \\
\text { stockpile (south) }\end{array}$ & $\mathrm{J} 11 \mathrm{KD} 3$ & $4 / 4 / 06$ & 1.1 & & 1.0 & 1020 & & 0.03 & 1.9 & USC & 0.85 & 33.6 & & 0.09 & 33.2 & & 0.15 & 14.6 & & 1.0 \\
\hline $\begin{array}{c}\text { South BCL } \\
\text { stockpile (north) }\end{array}$ & $\mathrm{J}_{11 \mathrm{KD} 4}$ & $4 / 4 / 06$ & 1.1 & & 1.0 & 1250 & & 0.03 & 1.8 & UsC & 0.85 & 42.0 & & 0.09 & 38.2 & & 0.15 & 17.4 & & 1.0 \\
\hline $\begin{array}{c}\text { Central BCL } \\
\text { stockpile }\end{array}$ & J11KDS & $4 / 4 / 06$ & 1.3 & & 1.1 & 1140 & & 0.03 & 1.8 & UJC & 0.88 & 35.7 & & 0.09 & 41.3 & & 0.16 & 19.4 & & 1.1 \\
\hline $\begin{array}{c}\text { North BCL } \\
\text { stockpile }\end{array}$ & J11KD6 & $4 / 4 / 06$ & 1.3 & & 1.0 & 1090 & & 0.03 & 2.2 & UJC & 0.84 & 33.9 & & 0.09 & 39.0 & & 0.15 & 14.5 & & 1.0 \\
\hline Equipment blank & J11KC4 & $4 / 4 / 06$ & 0.95 & $\mathrm{U}$ & 0.95 & 1.9 & & 0.03 & 0.78 & $\mathrm{UC}$ & 0.78 & 0.12 & & 0.08 & 1.2 & & 0.14 & 0.94 & $\mathrm{U}$ & 0.94 \\
\hline
\end{tabular}

\begin{tabular}{|c|c|c|c|}
\hline Attachment & 2 & Sheet No. & 5 of 13 \\
\hline Originator & J.M. Capron & Date & $07 / 31 / 06$ \\
\hline Checked & T.M. Blakley & Date & \\
\hline Calc. No. & $0100 \mathrm{~B}-\mathrm{CA}-\mathrm{V} 0281$ & Rev. No. & 0 \\
\hline
\end{tabular}


Attachment 2. 100-B-14:2 Area 5 Verification Sampling Results.

\begin{tabular}{|c|c|c|c|c|c|c|c|c|c|c|c|c|}
\hline \multirow[t]{2}{*}{ Constituents } & \multicolumn{3}{|c|}{$\begin{array}{c}\text { J11KB3 } \\
\text { Location } 1 \\
\text { Sample Date 4/4/06 }\end{array}$} & \multicolumn{3}{|c|}{$\begin{array}{c}\text { J11KB4 } \\
\text { Location } 2 \\
\text { Sample Date } 4 / 4 / 06\end{array}$} & \multicolumn{3}{|c|}{$\begin{array}{c}\text { J11KB5 } \\
\text { Location } 3 \\
\text { Sample Date } 4 / 4 / 06\end{array}$} & \multicolumn{3}{|c|}{$\begin{array}{c}\text { J11KB6 } \\
\text { Location } 4 \\
\text { Sample Date } 4 / 4 / 06\end{array}$} \\
\hline & $\mu \mathrm{g} / \mathrm{kg}$ & Q & $\mathrm{PQL}$ & $\mu \mathrm{g} / \mathrm{kg}$ & Q & PQL & $\mu \mathrm{g} / \mathrm{kg}$ & Q & PQL & $\mu \mathrm{g} / \mathrm{kg}$ & Q & PQL \\
\hline \multicolumn{13}{|c|}{ Polychlorinated Biphenyls } \\
\hline Aroclor -1016 & 15 & $\mathrm{U}$ & 15 & 14 & $\mathrm{U}$ & 14 & 14 & $\mathrm{U}$ & 14 & 15 & $\mathrm{U}$ & 15 \\
\hline Aroclor-1221 & 15 & $\mathrm{U}$ & 15 & 14 & $\mathrm{U}$ & 14 & 14 & $\mathrm{U}$ & 14 & 15 & $\mathrm{U}$ & 15 \\
\hline Aroclor-1232 & 15 & $\mathrm{U}$ & 15 & 14 & $\mathrm{U}$ & 14 & 14 & $\mathrm{U}$ & 14 & 15 & $\mathrm{U}$ & 15 \\
\hline Aroclor-1242 & 15 & $\mathrm{U}$ & 15 & 14 & $\mathrm{U}$ & 14 & 14 & $\mathrm{U}$ & 14 & 15 & $\mathrm{U}$ & 15 \\
\hline Aroclor-1248 & 15 & $\mathrm{U}$ & 15 & 14 & U & 14 & 14 & U & 14 & 15 & $\mathrm{U}$ & 15 \\
\hline Aroclor- 1254 & 15 & $\mathrm{U}$ & 15 & 14 & $\mathrm{U}$ & 14 & 3.5 & $\mathrm{~J}$ & 14 & 3.9 & $\mathrm{~J}$ & 15 \\
\hline Aroclor- 1260 & 15 & $\mathrm{U}$ & 15 & 14 & $\mathrm{U}$ & 14 & 14 & $\mathrm{U}$ & 14 & 15 & $\mathrm{U}$ & 15 \\
\hline \multicolumn{13}{|c|}{ Pesticides } \\
\hline Aldrin & 1.5 & UJD & 1.5 & 1.4 & UJD & 1.4 & 1.4 & UJD & 1.4 & 1.5 & UDD & 1.5 \\
\hline alpha-BHC & 1.5 & USD & 1.5 & 1.4 & UJD & 1.4 & 1.4 & UJD & 1.4 & 1.5 & UJD & 1.5 \\
\hline alpha-Chlordane & 1.5 & UJD & 1.5 & 1.4 & UJD & 1.4 & 1.4 & UJD & 1.4 & 1.5 & UJD & 1.5 \\
\hline beta-BHC & 0.62 & JD & 1.5 & 1.4 & UJD & 1.4 & 1.4 & UJD & 1.4 & 1.5 & UJD & 1.5 \\
\hline delta-BHC & 1.5 & UJD & 1.5 & 1.4 & UJD & 1.4 & 1.4 & UJD & 1.4 & 1.5 & UJD & 1.5 \\
\hline Dichlorodiphenyldichloroethane & 1.5 & UJD & 1.5 & 1.4 & UJD & 1.4 & 1.4 & UJD & 1.4 & 1.5 & UJD & 1.5 \\
\hline Dichlorodiphenyldichloroethylene & 1.5 & UJD & 1.5 & 1.4 & UJD & 1.4 & 1.4 & UJD & 1.4 & 1.5 & UJD & 1.5 \\
\hline Dichlorodiphenyltrichloroethane & 1.5 & UJD & 1.5 & 1.4 & UJD & 1.4 & 1.4 & UJD & 1.4 & 1.5 & UJD & 1.5 \\
\hline Dieldrin & 1.5 & UJD & 1.5 & 1.4 & UJD & 1.4 & 1.4 & UJD & 1.4 & 1.5 & UJD & 1.5 \\
\hline Endosulfan I & 1.5 & UJD & 1.5 & 1.4 & UJD & 1.4 & 1.4 & UJD & 1.4 & 1.5 & UJD & 1.5 \\
\hline Endosulfan II & 1.5 & UJD & 1.5 & 1.4 & UID & 1.4 & 1.4 & UJD & 1.4 & 1.5 & UID & 1.5 \\
\hline Endosulfan sulfate & 1.5 & UJD & 1.5 & 1.4 & UJD & 1.4 & 1.4 & UJD & 1.4 & 1.5 & UJD & 1.5 \\
\hline Endrin & 1.5 & UJD & 1.5 & 1.4 & UJD & 1.4 & 1.3 & JD & 1.3 & 1.5 & UJD & 1.5 \\
\hline Endrin aldehyde & 1.5 & UJD & 1.5 & 1.4 & UJD & 1.4 & 1.4 & UJD & 1.4 & 1.5 & UJD & 1.5 \\
\hline Endrin ketone & 1.5 & UJD & 1.5 & 1.4 & UJD & 1.4 & 1.4 & UJD & 1.4 & 1.5 & UJD & 1.5 \\
\hline gamma-BHC (Lindane) & 1.5 & UJD & 1.5 & 1.4 & UJD & 1.4 & 1.4 & UJD & 1.4 & 1.5 & UJD & 1.5 \\
\hline gamma-Chlordane & 1.5 & UJD & 1.5 & 1.4 & UJD & 1.4 & 1.4 & UJD & 1.4 & 1.5 & UJD & 1.5 \\
\hline Heptachlor & 1.5 & UJD & 1.5 & 1.4 & UJD & 1.4 & 1.4 & UJD & 1.4 & 1.5 & UJD & 1.5 \\
\hline Heptachlor epoxide & 1.5 & UJD & 1.5 & 1.4 & UJD & 1.4 & 1.4 & UJD & 1.4 & 1.5 & UJD & 1.5 \\
\hline $\begin{array}{l}\text { Methoxychlor } \\
\end{array}$ & 1.5 & UJD & 1.5 & 49 & D & 1.4 & 1.4 & UJD & 1.4 & 1.5 & UJD & 1.5 \\
\hline Toxaphene & 15 & UJD & 15 & 14 & UJD & 14 & 14 & UJD & 14 & 15 & UJD & 15 \\
\hline \multicolumn{13}{|c|}{ Semivolatile Organic Compounds } \\
\hline 1,2,4-Trichlorobenzene & 370 & UJ & 370 & 360 & UJ & 360 & 350 & UJ & 350 & 360 & $\mathrm{UJ}$ & 360 \\
\hline 1,2-Dichlorobenzene & 370 & $\mathrm{UJ}$ & 370 & 360 & UJ & 360 & 350 & UJ & 350 & 360 & UJ & 360 \\
\hline 1,3-Dichlorobenzene & 370 & $\mathrm{U}$ & 370 & 360 & $\mathrm{U}$ & 360 & 350 & $\mathrm{U}$ & 350 & 360 & $\mathrm{U}$ & 360 \\
\hline 1,4-Dichlorobenzene & 370 & $\mathrm{U}$ & 370 & 360 & $\mathrm{U}$ & 360 & 350 & $\mathrm{U}$ & 350 & 360 & $\mathrm{U}$ & 360 \\
\hline 2,4,5-Trichlorophenol & 910 & $\mathrm{U}$ & 910 & 890 & $\mathrm{U}$ & 890 & 890 & $U$ & 890 & 910 & $\mathrm{U}$ & 910 \\
\hline 2,4,6-Trichlorophenol & 370 & $\mathrm{U}$ & 370 & 360 & $\mathrm{U}$ & 360 & 350 & $\mathrm{U}$ & 350 & 360 & $\mathrm{U}$ & 360 \\
\hline 2,4-Dichlorophenol & 370 & UJ & 370 & 360 & UJ & 360 & 350 & UJ & 350 & 360 & UJ & 360 \\
\hline 2,4-Dimethylphenol & 370 & $\mathrm{UJ}$ & 370 & 360 & UJ & 360 & 350 & UJ & 350 & 360 & UJ & 360 \\
\hline 2,4-Dinitrophenol & 910 & UJ & 910 & 890 & UJ & 890 & 890 & UJ & 890 & 910 & UJ & 910 \\
\hline 2,4-Dinitrotoluene & 370 & $\mathrm{U}$ & 370 & 360 & U & 360 & 350 & $\mathrm{U}$ & 350 & 360 & $\mathrm{U}$ & 360 \\
\hline 2,6-Dinitrotoluene & 370 & $\mathrm{U}$ & 370 & 360 & $\mathrm{U}$ & 360 & 350 & U & 350 & 360 & U & 360 \\
\hline 2-Chloronaphthalene & 370 & $\mathrm{U}$ & 370 & 360 & $\mathrm{U}$ & 360 & 350 & U & 350 & 360 & U & 360 \\
\hline 2-Chlorophenol & 370 & $\mathrm{U}$ & 370 & 360 & $\mathrm{U}$ & 360 & 350 & $\mathrm{U}$ & 350 & 360 & $\mathrm{U}$ & 360 \\
\hline 2-Methylnaphthalene & 370 & $\mathrm{U}$ & 370 & 360 & $\mathrm{U}$ & 360 & 350 & $\mathrm{U}$ & 350 & 360 & $\mathrm{U}$ & 360 \\
\hline 2-Methylphenol (cresol, o-) & 370 & UJ & 370 & 360 & UJ & 360 & 350 & UJ & 350 & 360 & UJ & 360 \\
\hline $\begin{array}{l}\text {-Nitroaniline } \\
\end{array}$ & 910 & $\mathrm{U}$ & 910 & 890 & U & 890 & 890 & $\mathrm{U}$ & 890 & 910 & $\mathrm{U}$ & 910 \\
\hline 2-Nitrophenol & 370 & UJ & 370 & 360 & UJ & 360 & 350 & UJ & 350 & 360 & UJ & 360 \\
\hline & & & & \multicolumn{2}{|c|}{ Attachment } & \multicolumn{3}{|c|}{2} & & \multirow{4}{*}{$\begin{array}{r}\text { Sheet No. } \\
\text { Date } \\
\text { Date } \\
\text { Rev. No. }\end{array}$} & \multicolumn{2}{|c|}{6 of 13} \\
\hline & & & & \multicolumn{2}{|c|}{ Originator } & \multicolumn{4}{|c|}{ J. M. Capron } & & \multicolumn{2}{|c|}{$07 / 31 / 06$} \\
\hline & & & & \multirow{2}{*}{\multicolumn{2}{|c|}{$\begin{array}{l}\text { Checked } \\
\text { Calc. No. }\end{array}$}} & T.M. B & akley & & & & & \\
\hline & & & & & & $0100 \mathrm{~B}-$ & A-V028 & & & & & 0 \\
\hline
\end{tabular}


Attachment 2. 100-B-14:2 Area 5 Verification Sampling Results.

\begin{tabular}{|c|c|c|c|c|c|c|c|c|c|c|c|c|}
\hline \multirow[t]{2}{*}{ Constituents } & \multicolumn{3}{|c|}{$\begin{array}{c}\text { J11KB3 } \\
\text { Location } 1 \\
\text { Sample Date 4/4/06 }\end{array}$} & \multicolumn{3}{|c|}{$\begin{array}{c}\text { J11KB } 4 \\
\text { Location } 2 \\
\text { Sample Date } 4 / 4 / 06\end{array}$} & \multicolumn{3}{|c|}{$\begin{array}{c}\text { J11KB5 } \\
\text { Location } 3 \\
\text { Sample Date 4/4/06 }\end{array}$} & \multicolumn{3}{|c|}{$\begin{array}{c}\text { J11KB6 } \\
\text { Location } 4 \\
\text { Sample Date 4/4/06 } \\
\end{array}$} \\
\hline & $\mu \mathrm{g} / \mathrm{kg}$ & Q & PQL & $\mu \mathrm{g} / \mathrm{kg}$ & Q & PQL & $\mu \mathrm{g} / \mathrm{kg}$ & Q & $\mathrm{PQL}$ & $\mu \mathrm{g} / \mathrm{kg}$ & $\mathbf{Q}$ & $\mathrm{PQL}$ \\
\hline \multicolumn{13}{|c|}{ Semivolatile Organic Compounds (continued) } \\
\hline 3,3'-Dichlorobenzidine & 370 & $\mathrm{U}$ & 370 & 360 & $\mathrm{U}$ & 360 & 350 & $\mathrm{U}$ & 350 & 360 & $\mathrm{U}$ & 360 \\
\hline 3-Nitroaniline & 910 & $\mathrm{U}$ & 910 & 890 & $\mathrm{U}$ & 890 & 890 & $\mathrm{U}$ & 890 & 910 & $\mathrm{U}$ & 910 \\
\hline 4,6-Dinitro-2-methylphenol & 910 & UJ & 910 & 890 & UJ & 890 & 890 & UJ & 890 & 910 & $\overline{\mathrm{UJ}}$ & 910 \\
\hline 4-Bromophenyl-phenylether & 370 & $\mathrm{U}$ & 370 & 360 & $\mathrm{U}$ & 360 & 350 & $\mathrm{U}$ & 350 & 360 & $\mathrm{U}$ & 360 \\
\hline 4-Chloro-3-methylphenol & 370 & $\mathrm{U}$ & 370 & 360 & $\mathrm{U}$ & 360 & 350 & $\mathrm{U}$ & 350 & 360 & $\mathrm{U}$ & 360 \\
\hline 4-Chloroaniline & 370 & $\mathrm{U}$ & 370 & 360 & $\mathrm{U}$ & 360 & 350 & $\mathrm{U}$ & 350 & 360 & $\mathrm{U}$ & 360 \\
\hline 4-Chlorophenyl-phenylether & 370 & $\mathrm{U}$ & 370 & 360 & $\mathrm{U}$ & 360 & 350 & $\mathrm{U}$ & 350 & 360 & $\mathrm{U}$ & 360 \\
\hline 4-Methylphenol (p-cresol) & 370 & $\mathrm{UJ}$ & 370 & 360 & $\mathrm{UJ}$ & 360 & 350 & $\overline{\mathrm{UJ}}$ & 350 & 360 & UJ & 360 \\
\hline 4-Nitroaniline & 910 & $\mathrm{U}$ & 910 & 890 & $\mathrm{U}$ & 890 & 890 & $\mathrm{U}$ & 890 & 910 & $\mathrm{U}$ & 910 \\
\hline 4-Nitrophenol & 910 & $\mathrm{U}$ & 910 & 890 & $\mathrm{U}$ & 890 & 890 & $\mathrm{U}$ & 890 & 910 & $\mathrm{U}$ & 910 \\
\hline Acenaphthene & 370 & $\mathrm{U}$ & 370 & 360 & $\mathrm{U}$ & 360 & 350 & $\mathrm{U}$ & 350 & 360 & $\mathrm{U}$ & 360 \\
\hline Acenaphthylene & 370 & $\mathrm{U}$ & 370 & 360 & $\mathrm{U}$ & 360 & 350 & $\mathrm{U}$ & 350 & 360 & U & 360 \\
\hline Anthracene & 370 & $\mathrm{U}$ & 370 & 360 & $\mathrm{U}$ & 360 & 350 & $\mathrm{U}$ & 350 & 360 & $\mathrm{U}$ & 360 \\
\hline Benzo(a)anthracene & 370 & $U$ & 370 & 360 & $\bar{U}$ & 360 & 350 & $\mathrm{U}$ & 350 & 360 & $\mathrm{U}$ & 360 \\
\hline Benzo(a)pyrene & 370 & $\mathrm{U}$ & 370 & 360 & $\mathrm{U}$ & 360 & 350 & $\mathrm{U}$ & 350 & 360 & $\mathrm{U}$ & 360 \\
\hline Benzo(b)fluoranthene & 370 & $\mathrm{U}$ & 370 & 360 & $\mathrm{U}$ & 360 & 350 & $\mathrm{U}$ & 350 & 360 & $\bar{U}$ & 360 \\
\hline Benzo $(g, h, i)$ perylene & 370 & $\mathrm{U}$ & 370 & 360 & $\mathrm{U}$ & 360 & 350 & $\mathrm{U}$ & 350 & 360 & $\bar{U}$ & 360 \\
\hline Benzo $(k)$ fluoranthene & 370 & $\mathrm{U}$ & 370 & 360 & $\mathrm{U}$ & 360 & 350 & $\mathrm{U}$ & 350 & 360 & $\mathrm{U}$ & 360 \\
\hline bis(2-Chloro-1-methylethyl)ether & 370 & UJ & 370 & 360 & UJ & 360 & 350 & UJ & 350 & 360 & UJ & 360 \\
\hline bis(2-Chloroethoxy)methane & 370 & $\mathrm{U}$ & 370 & 360 & $\mathrm{U}$ & 360 & 350 & $\mathrm{U}$ & 350 & 360 & $\mathrm{U}$ & 360 \\
\hline bis(2-Chloroethyl)ether & 370 & $\mathrm{U}$ & 370 & 360 & $\mathrm{U}$ & 360 & 350 & $\mathrm{U}$ & 350 & 360 & $\mathrm{U}$ & 360 \\
\hline bis(2-Ethylhexyl)phthalate & 370 & $\mathrm{U}$ & 370 & 36 & $\mathrm{~J}$ & 360 & 33 & $\mathrm{~J}$ & 350 & 19 & $\mathrm{~J}$ & 360 \\
\hline Butylbenzylphthalate & 370 & $\mathrm{U}$ & 370 & 360 & $\mathrm{U}$ & 360 & 350 & $\mathrm{U}$ & 350 & 360 & $\mathrm{U}$ & 360 \\
\hline Carbazole & 370 & $\mathrm{U}$ & 370 & 360 & U & 360 & 350 & $\mathrm{U}$ & 350 & 360 & $\mathrm{U}$ & 360 \\
\hline Chrysene & 370 & $\mathrm{U}$ & 370 & 360 & $\mathrm{U}$ & 360 & 350 & $\mathrm{U}$ & 350 & 360 & $\mathrm{U}$ & 360 \\
\hline Di-n-butylphthalate & 370 & $\mathrm{U}$ & 370 & 360 & $\mathrm{U}$ & 360 & 21 & $\mathrm{~J}$ & 350 & 360 & $\mathrm{U}$ & 360 \\
\hline Di-n-octylphthalate & 370 & $\mathrm{U}$ & 370 & 360 & $U$ & 360 & 350 & $\mathrm{U}$ & 350 & 360 & $\mathrm{U}$ & 360 \\
\hline Dibenz $(\mathrm{a}, \mathrm{h})$ anthracene & 370 & $\mathrm{U}$ & 370 & 360 & $\mathrm{U}$ & 360 & 350 & $\mathrm{U}$ & 350 & 360 & $\mathrm{U}$ & 360 \\
\hline $\begin{array}{l}\text { Dibenzofuran } \\
\end{array}$ & 370 & $\mathrm{U}$ & 370 & 360 & $\mathrm{U}$ & 360 & 350 & U & 350 & 360 & $\bar{U}$ & 360 \\
\hline Diethylphthalate & 370 & $\mathrm{U}$ & 370 & 360 & $\mathrm{U}$ & 360 & 350 & $\mathrm{U}$ & 350 & 360 & $\mathrm{U}$ & 360 \\
\hline Dimethylphthalate & 370 & $\mathrm{U}$ & 370 & 360 & $\mathrm{U}$ & 360 & 350 & $\mathrm{U}$ & 350 & 360 & $\mathrm{U}$ & 360 \\
\hline Fluoranthene & 370 & $\mathrm{U}$ & 370 & 360 & $\mathrm{U}$ & 360 & 350 & $\mathrm{U}$ & 350 & 360 & $\mathrm{U}$ & 360 \\
\hline Fluorene & 370 & $\mathrm{U}$ & 370 & 360 & $\mathrm{U}$ & 360 & 350 & $\mathrm{U}$ & 350 & 360 & $\mathrm{U}$ & 360 \\
\hline Hexachlorobenzene & 370 & $\mathrm{U}$ & 370 & 360 & $\mathrm{U}$ & 360 & 350 & $\mathrm{U}$ & 350 & 360 & $\mathrm{U}$ & 360 \\
\hline Hexachlorobutadiene & 370 & $\bar{U}$ & 370 & 360 & $\mathrm{U}$ & 360 & 350 & $\mathrm{U}$ & 350 & 360 & $\mathrm{U}$ & 360 \\
\hline Hexachlorocyclopentadiene & 370 & $\mathrm{U}$ & 370 & 360 & $\mathrm{U}$ & 360 & 350 & $\mathrm{U}$ & 350 & 360 & $\mathrm{U}$ & 360 \\
\hline Hexachloroethane & 370 & $\mathrm{U}$ & 370 & 360 & $\mathrm{U}$ & 360 & 350 & $\mathrm{U}$ & 350 & 360 & $\mathrm{U}$ & 360 \\
\hline Indeno(1,2,3-cd)pyrene & 370 & $\mathrm{U}$ & 370 & 360 & U & 360 & 350 & $\mathrm{U}$ & 350 & 360 & $\mathrm{U}$ & 360 \\
\hline Isophorone & 370 & U & 370 & 360 & $\mathrm{U}$ & 360 & 350 & $\mathrm{U}$ & 350 & 360 & $\mathrm{U}$ & 360 \\
\hline N-Nitroso-di-n-dipropylamine & 370 & $\overline{U S}$ & 370 & 360 & UJ & 360 & 350 & UJ & 350 & 360 & UJ & 360 \\
\hline N-Nitrosodiphenylamine & 370 & $U$ & 370 & 360 & U & 360 & 350 & $\mathrm{U}$ & 350 & 360 & $\mathrm{U}$ & 360 \\
\hline Naphthalene & 370 & $\mathrm{U}$ & 370 & 360 & $\mathrm{U}$ & 360 & 350 & $\mathrm{U}$ & 350 & 360 & $\mathrm{U}$ & 360 \\
\hline \begin{tabular}{|l|} 
Nitrobenzene \\
\end{tabular} & 370 & $\mathrm{U}$ & 370 & 360 & $\mathrm{U}$ & 360 & 350 & $\mathrm{U}$ & 350 & 360 & $\mathrm{U}$ & 360 \\
\hline Pentachlorophenol & 910 & $\mathrm{U}$ & 910 & 890 & $\mathrm{U}$ & 890 & 890 & $\mathrm{U}$ & 890 & 910 & $\mathrm{U}$ & 910 \\
\hline Phenanthrene & 370 & $\mathrm{U}$ & 370 & 360 & $\mathrm{U}$ & 360 & 350 & $\mathrm{U}$ & 350 & 360 & $\mathrm{U}$ & 360 \\
\hline Phenol & 370 & UJ & 370 & 360 & UJ & 360 & 350 & UJ & 350 & 360 & UJ & 360 \\
\hline Pyrene & 370 & $\mathrm{U}$ & 370 & 360 & $\mathrm{U}$ & 360 & 350 & U & 350 & 360 & $\mathrm{U}$ & 360 \\
\hline & & & & Attachn & & & 2 & & & heet No. & & 7 of 13 \\
\hline & & & & Origina & & J.M. C & & & & Date & & $7 / 31 / 06$ \\
\hline & & & & Checke & & T.M. B & kley & & & & & \\
\hline & & & & Calc. $N$ & & $0100 \mathrm{~B}-$ & $\mathrm{A}-\mathrm{V} 028$ & & & Rev. No & & 0 \\
\hline
\end{tabular}


Attachment 2. 100-B-14:2 Area 5 Verification Sampling Results.

\begin{tabular}{|c|c|c|c|c|c|c|c|c|c|c|c|c|}
\hline \multirow[t]{2}{*}{ Constituents } & \multicolumn{3}{|c|}{$\begin{array}{c}\text { J11KC3 } \\
\text { Duplicate of J11KB6 } \\
\text { Sample Date } 4 / 4 / 06\end{array}$} & \multicolumn{3}{|c|}{$\begin{array}{c}\text { J11KB7 } \\
\text { Location } 5 \\
\text { Sample Date 4/4/06 }\end{array}$} & \multicolumn{3}{|c|}{$\begin{array}{c}\text { J11KB8 } \\
\text { Location } 6 \\
\text { Sample Date } 4 / 4 / 06\end{array}$} & \multicolumn{3}{|c|}{$\begin{array}{c}\text { J11KB9 } \\
\text { Location } 7 \\
\text { Sample Date 4/4/06 } \\
\end{array}$} \\
\hline & $\mu \mathrm{g} / \mathrm{kg}$ & $\mathbf{Q}$ & PQL & $\mu \mathrm{g} / \mathrm{kg}$ & Q & $\mathrm{PQL}$ & $\mu \mathrm{g} / \mathrm{kg}$ & $\mathbf{Q}$ & $\mathrm{PQL}$ & $\mu \mathrm{g} / \mathrm{kg}$ & Q & PQL \\
\hline \multicolumn{13}{|c|}{ Polychlorinated Biphenyls } \\
\hline Aroclor-1016 & 15 & $\mathrm{U}$ & 15 & 14 & $\mathrm{U}$ & 14 & 14 & $\mathrm{U}$ & 14 & 15 & $\mathrm{U}$ & 15 \\
\hline Aroclor-1221 & 15 & $\mathrm{U}$ & 15 & 14 & $\mathrm{U}$ & 14 & 14 & U & 14 & 15 & $\mathrm{U}$ & 15 \\
\hline Aroclor-1232 & 15 & $\mathrm{U}$ & 15 & 14 & $\mathrm{U}$ & 14 & 14 & $\mathrm{U}$ & 14 & 15 & $\mathrm{U}$ & 15 \\
\hline Aroclor-1242 & 15 & $\mathrm{U}$ & 15 & 14 & $\mathrm{U}$ & 14 & 14 & $\mathrm{U}$ & 14 & 15 & $\mathrm{U}$ & 15 \\
\hline Aroclor-1248 & 15 & $\mathrm{U}$ & 15 & 14 & $\mathrm{U}$ & 14 & 14 & $\mathrm{U}$ & 14 & 15 & $\mathrm{U}$ & 15 \\
\hline Aroclor-1254 & 11 & $\mathrm{~J}$ & 15 & 14 & $\mathrm{U}$ & 14 & 14 & $\mathrm{U}$ & 14 & 2.9 & $\mathrm{~J}$ & 15 \\
\hline Aroclor- 1260 & 15 & $\mathrm{U}$ & 15 & 14 & $\mathrm{U}$ & 14 & 14 & $\mathrm{U}$ & 14 & 15 & $\mathrm{U}$ & 15 \\
\hline \multicolumn{13}{|c|}{ Pesticides } \\
\hline Aldrin & 1.5 & UJD & 1.5 & 1.4 & UJD & 1.4 & 1.4 & UJD & 1.4 & 1.5 & UJD & 1.5 \\
\hline alpha-BHC & 1.5 & UJD & 1.5 & 1.4 & UJD & 1.4 & 1.4 & UJD & 1.4 & 1.5 & UJD & 1.5 \\
\hline alpha-Chlordane & 1.5 & UJD & 1.5 & 1.4 & UJD & 1.4 & 1.4 & UJD & 1.4 & 1.5 & UJD & 1.5 \\
\hline beta-BHC & 0.62 & $\mathrm{JD}$ & 1.5 & 1.4 & UJD & 1.4 & 1.4 & UJD & 1.4 & 1.5 & UJD & 1.5 \\
\hline delta-BHC & 1.5 & UJD & 1.5 & 1.4 & UID & 1.4 & 1.4 & UJD & 1.4 & 1.5 & UJD & 1.5 \\
\hline Dichlorodiphenyldichloroethane & 1.5 & UJD & 1.5 & 1.4 & UJD & 1.4 & 1.4 & UJD & 1.4 & 1.5 & USD & 1.5 \\
\hline Dichlorodiphenyldichloroethylene & 1.5 & UMD & 1.5 & 1.4 & USD & 1.4 & 1.4 & UJD & 1.4 & 1.5 & UJD & 1.5 \\
\hline Dichlorodiphenyltrichloroethane & 1.5 & UJD & 1.5 & 1.4 & UJD & 1.4 & 1.4 & UJD & 1.4 & 1.5 & UJD & 1.5 \\
\hline Dieldrin & 1.5 & UJD & 1.5 & 1.4 & UJD & 1.4 & 1.4 & UJD & 1.4 & 1.5 & UJD & 1.5 \\
\hline Endosulfan I & 1.5 & UJD & 1.5 & 1.4 & UJD & 1.4 & 1.4 & UID & 1.4 & 1.5 & UJD & 1.5 \\
\hline Endosulfan II & 1.5 & UJD & 1.5 & 1.4 & UJD & 1.4 & 1.4 & UJD & 1.4 & 1.5 & UJD & 1.5 \\
\hline Endosulfan sulfate & 1.5 & UJD & 1.5 & 1.4 & UJD & 1.4 & 1.4 & UJD & 1.4 & 1.5 & UJD & 1.5 \\
\hline Endrin & 1.5 & USD & 1.5 & 1.4 & UJD & 1.4 & 1.4 & UJD & 1.4 & 1.5 & UJD & 1.5 \\
\hline Endrin aldehyde & 1.5 & UJD & 1.5 & 1.4 & UJD & 1.4 & 1.4 & UJD & 1.4 & 1.5 & UJD & 1.5 \\
\hline Endrin ketone & 1.5 & UJD & 1.5 & 1.4 & UJD & 1.4 & 1.4 & UJD & 1.4 & 1.5 & UJD & 1.5 \\
\hline gamma-BHC (Lindane) & 1.5 & UJD & 1.5 & 1.4 & UJD & 1.4 & 1.4 & UJD & 1.4 & 1.5 & UJD & 1.5 \\
\hline gamma-Chlordane & 1.5 & UND & 1.5 & 1.4 & UJD & 1.4 & 1.4 & UJD & 1.4 & 1.5 & UJD & 1.5 \\
\hline Heptachlor & 1.5 & UJD & 1.5 & 1.4 & UJD & 1.4 & 1.4 & UJD & 1.4 & 1.5 & UJD & 1.5 \\
\hline Heptachlor epoxide & 1.5 & UJD & 1.5 & 1.4 & UJD & 1.4 & 1.4 & UJD & 1.4 & 1.5 & UJD & 1.5 \\
\hline Methoxychlor & 1.5 & UJD & 1.5 & 1.4 & UJD & 1.4 & 1.4 & UJD & 1.4 & 1.5 & UJD & 1.5 \\
\hline Toxaphene & 15 & UJD & 15 & 14 & UJD & 14 & 14 & UJD & 14 & 15 & UJD & 15 \\
\hline \multicolumn{13}{|c|}{ Semivolatile Organic Compounds } \\
\hline 1,2,4-Trichlorobenzene & 360 & UJ & 360 & 350 & \begin{tabular}{|l|l|}
$\mathrm{UJ}$ \\
\end{tabular} & 350 & 360 & UJ & 360 & 370 & UJ & 370 \\
\hline 1,2-Dichlorobenzene & 360 & UJ & 360 & 350 & $\mathrm{UJ}$ & 350 & 360 & UJ & 360 & 370 & UJ & 370 \\
\hline 1,3-Dichlorobenzene & 360 & $\mathrm{U}$ & 360 & 350 & $\mathrm{U}$ & 350 & 360 & $\mathrm{U}$ & 360 & 370 & $\mathrm{U}$ & 370 \\
\hline 1,4-Dichlorobenzene & 360 & $\mathrm{U}$ & 360 & 350 & $\mathrm{U}$ & 350 & 360 & $\mathrm{U}$ & 360 & 370 & U & 370 \\
\hline 2,4,5-Trichlorophenol & 910 & $\mathrm{U}$ & 910 & 890 & $\mathrm{U}$ & 890 & 900 & $\mathrm{U}$ & 900 & 910 & $\mathrm{U}$ & 910 \\
\hline 2,4,6-Trichlorophenol & 360 & $\mathrm{U}$ & 360 & 350 & $\mathrm{U}$ & 350 & 360 & $\mathrm{U}$ & 360 & 370 & $\mathrm{U}$ & 370 \\
\hline 2,4-Dichlorophenol & 360 & $\mathrm{UJ}$ & 360 & 350 & UJ & $350^{\circ}$ & 360 & $\mathrm{UJ}$ & 360 & 370 & UJ & 370 \\
\hline 2,4-Dimethylphenol & 360 & $\mathrm{UJ}$ & 360 & 350 & $\mathrm{UJ}$ & 350 & 360 & UJ & 360 & 370 & UJ & 370 \\
\hline 2,4-Dinitrophenol & 910 & UJ & 910 & 890 & UJ & 890 & 900 & UJ & 900 & 910 & UJ & 910 \\
\hline 2,4-Dinitrotoluene & 360 & $\mathrm{U}$ & 360 & 350 & $\mathrm{U}$ & 350 & 360 & $\mathrm{U}$ & 360 & 370 & U & 370 \\
\hline 2,6-Dinitrotoluene & 360 & $\mathrm{U}$ & 360 & 350 & $\mathrm{U}$ & 350 & 360 & $\mathrm{U}$ & 360 & 370 & $U$ & 370 \\
\hline 2-Chloronaphthalene & 360 & $\mathrm{U}$ & 360 & 350 & $\mathrm{U}$ & 350 & 360 & $\mathrm{U}$ & 360 & 370 & $\mathrm{U}$ & 370 \\
\hline 2-Chlorophenol & 360 & $\mathrm{U}$ & 360 & 350 & $\mathrm{U}$ & 350 & 360 & $\mathrm{U}$ & 360 & 370 & $\mathrm{U}$ & 370 \\
\hline 2-Methylnaphthalene & 360 & $\mathrm{U}$ & 360 & 350 & $\mathrm{U}$ & 350 & 360 & $\mathrm{U}$ & 360 & 370 & $\mathrm{U}$ & 370 \\
\hline 2-Methylphenol (cresol, o-) & 360 & UJ & 360 & 350 & $\mathrm{UJ}$ & 350 & 360 & UJ & 360 & 370 & $\mathrm{UJ}$ & 370 \\
\hline 2-Nitroaniline & 910 & $\mathrm{U}$ & 910 & 890 & $\mathrm{U}$ & 890 & 900 & $\mathrm{U}$ & 900 & 910 & $\mathrm{U}$ & 910 \\
\hline 2-Nitrophenol & 360 & UJ & 360 & 350 & $\mathrm{UJ}$ & 350 & 360 & UJ & 360 & 370 & $\mathrm{UJ}$ & 370 \\
\hline & & & & \multicolumn{2}{|c|}{ Attachment } & \multicolumn{3}{|c|}{2} & & Sheet No. & \multicolumn{2}{|c|}{8 of 13} \\
\hline & & & & \multicolumn{2}{|c|}{ Originator } & \multicolumn{3}{|c|}{ J.M. Capron } & & Date & \multicolumn{2}{|c|}{$07 / 31 / 06$} \\
\hline & & & & \multirow{2}{*}{\multicolumn{2}{|c|}{$\begin{array}{l}\text { Checked } \\
\text { Calc. No. }\end{array}$}} & T.M.E & & & & & & \\
\hline & & & & & & $0100 \mathrm{~B}$ & $\mathrm{~A}-\mathrm{V} 028$ & & & Rev. No. & & 0 \\
\hline
\end{tabular}


Attachment 2. 100-B-14:2 Area 5 Verification Sampling Results.

\begin{tabular}{|c|c|c|c|c|c|c|c|c|c|c|c|c|}
\hline \multirow[t]{2}{*}{ Constituents } & \multicolumn{3}{|c|}{\begin{tabular}{c|c|} 
J11KC3 \\
Duplicate of J11KB6 \\
Sample Date $4 / 4 / 06$ \\
\end{tabular}} & \multicolumn{3}{|c|}{$\begin{array}{c}\text { J11KB7 } \\
\text { Location } 5 \\
\text { Sample Date 4/4/06 } \\
\end{array}$} & \multicolumn{3}{|c|}{$\begin{array}{c}\text { J11KB8 } \\
\text { Location } 6 \\
\text { Sample Date 4/4/06 } \\
\end{array}$} & \multicolumn{3}{|c|}{$\begin{array}{c}\text { J11KB9 } \\
\text { Location } 7 \\
\text { Sample Date } 4 / 4 / 06 \\
\end{array}$} \\
\hline & $\mu \mathrm{g} / \mathrm{kg}$ & Q & PQL & $\mu \mathrm{g} / \mathrm{kg}$ & $Q$ & PQL & $\mu \mathrm{g} / \mathrm{kg}$ & $Q$ & PQL & $\mu \mathrm{g} / \mathrm{kg}$ & $\mathbf{Q}$ & PQL \\
\hline \multicolumn{13}{|c|}{ Semivolatile Organic Compounds (continued) } \\
\hline $3,3^{2}$-Dichlorobenzidine & 360 & $\mathrm{U}$ & 360 & 350 & $\mathrm{U}$ & 350 & 360 & $\mathrm{U}$ & 360 & 370 & $\mathrm{U}$ & 370 \\
\hline 3-Nitroaniline & 910 & $\mathrm{U}$ & 910 & 890 & $\mathrm{U}$ & 890 & 900 & $\mathrm{U}$ & 900 & 910 & $\mathrm{U}$ & 910 \\
\hline 4,6-Dinitro-2-methylphenol & 910 & UJ & 910 & 890 & UJ & 890 & 900 & UJ & 900 & 910 & $\mathrm{UJ}$ & 910 \\
\hline 4-Bromophenyl-phenylether & 360 & $\mathrm{U}$ & 360 & 350 & $\mathrm{U}$ & 350 & 360 & $\mathrm{U}$ & 360 & 370 & $\mathrm{U}$ & 370 \\
\hline 4-Chloro-3-methylphenol & 360 & $\mathrm{U}$ & 360 & 350 & $\mathrm{U}$ & 350 & 360 & $\mathrm{U}$ & 360 & 370 & $\bar{U}$ & 370 \\
\hline 4-Chloroaniline & 360 & $\mathrm{U}$ & 360 & 350 & $\mathrm{U}$ & 350 & 360 & $\mathrm{U}$ & 360 & 370 & $\mathrm{U}$ & 370 \\
\hline 4-Chlorophenyl-phenylether & 360 & $\mathrm{U}$ & 360 & 350 & $\mathrm{U}$ & 350 & 360 & $\mathrm{U}$ & 360 & 370 & $\mathrm{U}$ & 370 \\
\hline 4-Methylphenol (p-cresol) & 360 & UJ & 360 & 350 & UY & 350 & 360 & UJ & 360 & 370 & UJ & 370 \\
\hline 4-Nitroaniline & 910 & $\mathrm{U}$ & 910 & 890 & $\mathrm{U}$ & 890 & 900 & $\mathrm{U}$ & 900 & 910 & $\mathrm{U}$ & 910 \\
\hline 4-Nitrophenol & 910 & $\mathrm{U}$ & 910 & 890 & $\mathrm{U}$ & 890 & 900 & $\mathrm{U}$ & 900 & 910 & $\mathrm{U}$ & 910 \\
\hline Acenaphthene & 360 & $\mathrm{U}$ & 360 & 350 & $\mathrm{U}$ & 350 & 360 & $\mathrm{U}$ & 360 & 370 & $\mathrm{U}$ & 370 \\
\hline Acenaphthylene & 360 & $\mathrm{U}$ & 360 & 350 & $\mathrm{U}$ & 350 & 360 & $\mathrm{U}$ & 360 & 370 & $\mathrm{U}$ & 370 \\
\hline Anthracene & 360 & $\mathrm{U}$ & 360 & 350 & $\mathrm{U}$ & 350 & 360 & $\mathrm{U}$ & 360 & 370 & $\mathrm{U}$ & 370 \\
\hline Benzo(a)anthracene & 360 & $\mathrm{U}$ & 360 & 350 & $\mathrm{U}$ & 350 & 360 & $\mathrm{U}$ & 360 & 370 & $\mathrm{U}$ & 370 \\
\hline Benzo(a)pyrene & 360 & $\mathrm{U}$ & 360 & 350 & $\mathrm{U}$ & 350 & 360 & $\mathrm{U}$ & 360 & 370 & $\mathrm{U}$ & 370 \\
\hline Benzo(b)fluoranthene & 360 & $\mathrm{U}$ & 360 & 350 & $\mathrm{U}$ & 350 & 360 & U & 360 & 370 & $\mathrm{U}$ & 370 \\
\hline Benzo $(g, h, i)$ perylene & 360 & $\mathrm{U}$ & 360 & 350 & $\mathrm{U}$ & 350 & 360 & U & 360 & 370 & $\mathrm{U}$ & 370 \\
\hline Benzo(k)fluoranthene & 360 & $\mathrm{U}$ & 360 & 350 & $\mathrm{U}$ & 350 & 360 & $\mathrm{U}$ & 360 & 370 & $\mathrm{U}$ & 370 \\
\hline bis(2-Chloro-1-methylethyl)ether & 360 & UJ & 360 & 350 & $\mathrm{UJ}$ & 350 & 360 & UJ & 360 & 370 & UJ & 370 \\
\hline bis(2-Chloroethoxy)methane & 360 & $\mathrm{U}$ & 360 & 350 & $\mathrm{U}$ & 350 & 360 & $U$ & 360 & 370 & $\mathrm{U}$ & 370 \\
\hline bis(2-Chloroethyl)ether & 360 & $\mathrm{U}$ & 360 & 350 & $\mathrm{U}$ & 350 & 360 & $\mathrm{U}$ & 360 & 370 & $\mathrm{U}$ & 370 \\
\hline bis(2-Ethylhexyl)phthalate & 24 & $\mathrm{~J}$ & 360 & 350 & $\mathrm{U}$ & 350 & 360 & $\mathrm{U}$ & 360 & 370 & $\mathrm{U}$ & 370 \\
\hline Butylbenzylphthalate & 360 & $\mathrm{U}$ & 360 & 350 & $\mathrm{U}$ & 350 & 360 & $\mathrm{U}$ & 360 & 370 & $\mathrm{U}$ & 370 \\
\hline Carbazole & 360 & $\mathrm{U}$ & 360 & 350 & $\mathrm{U}$ & 350 & 360 & $\mathrm{U}$ & 360 & 370 & $\mathrm{U}$ & 370 \\
\hline Chrysene & 360 & $\mathrm{U}$ & 360 & 350 & $\mathrm{U}$ & 350 & 360 & $\mathrm{U}$ & 360 & 370 & $\mathrm{U}$ & 370 \\
\hline Di-n-butylphthalate & 360 & $\mathrm{U}$ & 360 & 350 & $\mathrm{U}$ & 350 & 360 & $\mathrm{U}$ & 360 & 370 & $\mathrm{U}$ & 370 \\
\hline Di-n-octylphthalate & 360 & $\mathrm{U}$ & 360 & 350 & $\mathrm{U}$ & 350 & 360 & $\mathrm{U}$ & 360 & 370 & $\mathrm{U}$ & 370 \\
\hline Dibenz $(a, h)$ anthracene & 360 & $\mathrm{U}$ & 360 & 350 & $\mathrm{U}$ & 350 & 360 & $\mathrm{U}$ & 360 & 370 & $\mathrm{U}$ & 370 \\
\hline Dibenzofuran & 360 & $\mathrm{U}$ & 360 & 350 & $U$ & 350 & 360 & $\mathrm{U}$ & 360 & 370 & $\mathrm{U}$ & 370 \\
\hline Diethylphthalate & 360 & $\mathrm{U}$ & 360 & 350 & $\mathrm{U}$ & 350 & 360 & $\mathrm{U}$ & 360 & 370 & $\mathrm{U}$ & 370 \\
\hline Dimethylphthalate & 360 & $\mathrm{U}$ & 360 & 350 & $U$ & 350 & 360 & $\mathrm{U}$ & 360 & 370 & $\mathrm{U}$ & 370 \\
\hline Fluoranthene & 360 & $\mathrm{U}$ & 360 & 350 & $\mathrm{U}$ & 350 & 360 & $\mathrm{U}$ & 360 & 370 & $\mathrm{U}$ & 370 \\
\hline Fluorene & 360 & $\mathrm{U}$ & 360 & 350 & $\mathrm{U}$ & 350 & 360 & $\mathrm{U}$ & 360 & 370 & $\mathrm{U}$ & 370 \\
\hline Hexachlorobenzene & 360 & $\mathrm{U}$ & 360 & 350 & $\mathrm{U}$ & 350 & 360 & $\mathrm{U}$ & 360 & 370 & $\mathrm{U}$ & 370 \\
\hline Hexachlorobutadiene & 360 & $\mathrm{U}$ & 360 & 350 & $\mathrm{U}$ & 350 & 360 & $\mathrm{U}$ & 360 & 370 & $\mathrm{U}$ & 370 \\
\hline Hexachlorocyclopentadiene & 360 & $\mathrm{U}$ & 360 & 350 & $\mathrm{U}$ & 350 & 360 & $\mathrm{U}$ & 360 & 370 & $U$ & 370 \\
\hline Hexachloroethane & 360 & $\mathrm{U}$ & 360 & 350 & $\mathrm{U}$ & 350 & 360 & $\mathrm{U}$ & 360 & 370 & $\mathrm{U}$ & 370 \\
\hline Indeno $(1,2,3-\mathrm{cd})$ pyrene & 360 & $\mathrm{U}$ & 360 & 350 & $\mathrm{U}$ & 350 & 360 & $\mathrm{U}$ & 360 & 370 & $\mathrm{U}$ & 370 \\
\hline Isophorone & 360 & $\mathrm{U}$ & 360 & 350 & $\mathrm{U}$ & 350 & 360 & $\mathrm{U}$ & 360 & 370 & $\mathrm{U}$ & 370 \\
\hline $\mathrm{N}$-Nitroso-di-n-dipropylamine & 360 & UJ & 360 & 350 & UJ & 350 & 360 & UJ & 360 & 370 & UJ & 370 \\
\hline N-Nitrosodiphenylamine & 360 & $\mathrm{U}$ & 360 & 350 & $\mathrm{U}$ & 350 & 360 & $\mathrm{U}$ & 360 & 370 & $\mathrm{U}$ & 370 \\
\hline Naphthalene & 360 & $\mathrm{U}$ & 360 & 350 & $\mathrm{U}$ & 350 & 360 & $\mathrm{U}$ & 360 & 370 & $\mathrm{U}$ & 370 \\
\hline Nitrobenzene & 360 & $\mathrm{U}$ & 360 & 350 & $\mathrm{U}$ & 350 & 360 & $\mathrm{U}$ & 360 & 370 & $\mathrm{U}$ & 370 \\
\hline Pentachlorophenol & 910 & $\mathrm{U}$ & 910 & 890 & $\mathrm{U}$ & 890 & 900 & $\mathrm{U}$ & 900 & 910 & $\mathrm{U}$ & 910 \\
\hline Phenanthrene & 360 & $\mathrm{U}$ & 360 & 350 & $\mathrm{U}$ & 350 & 360 & $\mathrm{U}$ & 360 & 370 & $\mathrm{U}$ & 370 \\
\hline Phenol & 360 & $\mathrm{UJ}$ & 360 & 350 & UJ & 350 & 360 & UJ & 360 & 370 & UJ & 370 \\
\hline Pyrene & 360 & $\mathrm{U}$ & 360 & 350 & $\mathrm{U}$ & 350 & 360 & $\mathrm{U}$ & 360 & 370 & $\mathrm{U}$ & 370 \\
\hline
\end{tabular}

Attachment

Originator

Calc. No.

\begin{tabular}{|c|}
\hline$\frac{2}{\text { J.M. Capron }}$ \\
\hline T. M. Blakley \\
\hline 0100B-CA-V0281 \\
\hline
\end{tabular}

Sheet No. 9 of 13

Date $07 / 31 / 06$

Date

Rev. No. $\overline{0}$ 
Attachment 2. 100-B-14:2 Area 5 Verification Sampling Results.

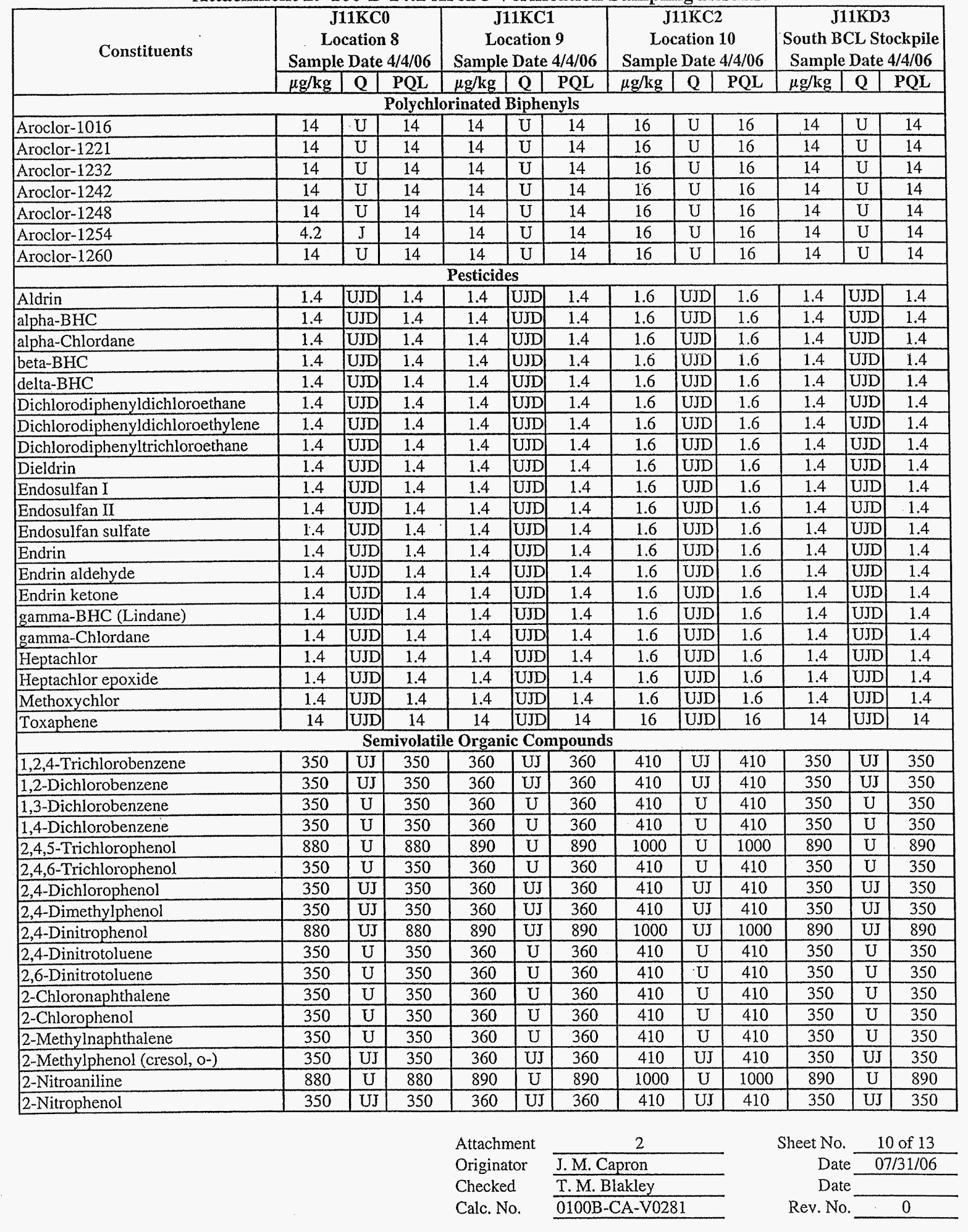


Attachment 2. 100-B-14:2 Area 5 Verification Sampling Results.

\begin{tabular}{|c|c|c|c|c|c|c|c|c|c|c|c|c|}
\hline \multirow[t]{2}{*}{ Constituents } & \multicolumn{3}{|c|}{$\begin{array}{c}\text { J11KC0 } \\
\text { Location } 8 \\
\text { Sample Date 4/4/06 }\end{array}$} & \multicolumn{3}{|c|}{$\begin{array}{c}\text { J11KC1 } \\
\text { Location } 9 \\
\text { Sample Date } 4 / 4 / 06 \\
\end{array}$} & \multicolumn{3}{|c|}{$\begin{array}{c}\text { J11KC2 } \\
\text { Location 10 } \\
\text { Sample Date } 4 / 4 / 06\end{array}$} & \multicolumn{3}{|c|}{$\begin{array}{c}\text { J11KD3 } \\
\text { South BCL Stockpile } \\
\text { Sample Date } 4 / 4 / 06 \\
\end{array}$} \\
\hline & $\mu \mathrm{g} / \mathrm{kg}$ & Q & PQL & $\mu \mathrm{g} / \mathrm{kg}$ & Q & PQL & $\mu \mathrm{g} / \mathrm{kg}$ & Q & PQL & $\mu \mathrm{g} / \mathrm{kg}$ & Q & PQL \\
\hline \multicolumn{13}{|c|}{ Semivolatile Organic Compounds (continued) } \\
\hline 3,3'-Dichlorobenzidine & 350 & $\mathrm{U}$ & 350 & 360 & U & 360 & 410 & $\mathrm{U}$ & 410 & 350 & $\mathrm{U}$ & 350 \\
\hline 3-Nitroaniline & 880 & $\mathrm{U}$ & 880 & 890 & $\bar{U}$ & 890 & 1000 & $\mathrm{U}$ & 1000 & 890 & $\mathrm{U}$ & 890 \\
\hline 4,6-Dinitro-2-methylphenol & 880 & UJ & 880 & 890 & $\mathrm{UJ}$ & 890 & 1000 & UJ & 1000 & 890 & UJ & 890 \\
\hline 4-Bromophenyl-phenylether & 350 & $\bar{U}$ & 350 & 360 & $\mathrm{U}$ & 360 & 410 & $U$ & 410 & 350 & $\mathrm{U}$ & 350 \\
\hline 4-Chloro-3-methylphenol & 350 & $\mathrm{U}$ & 350 & 360 & $\mathrm{U}$ & 360 & 410 & $\mathrm{U}$ & 410 & 350 & $\mathrm{U}$ & 350 \\
\hline 4-Chloroaniline & 350 & $\mathrm{U}$ & 350 & 360 & $\mathrm{U}$ & 360 & 410 & $\mathrm{U}$ & 410 & 350 & $\mathrm{U}$ & 350 \\
\hline 4-Chlorophenyl-phenylether & 350 & $\bar{U}$ & 350 & 360 & $\mathrm{U}$ & 360 & 410 & $\mathrm{U}$ & 410 & 350 & $\mathrm{U}$ & 350 \\
\hline 4-Methylphenol (p-cresol) & 350 & UJ & 350 & 360 & UJ & 360 & 410 & UJ & 410 & 350 & $\overline{\mathrm{UJ}}$ & 350 \\
\hline 4-Nitroaniline & 880 & $\mathrm{U}$ & 880 & 890 & $\mathrm{U}$ & 890 & 1000 & $\mathrm{U}$ & 1000 & 890 & $\mathrm{U}$ & 890 \\
\hline 4-Nitrophenol & 880 & $\mathrm{U}$ & 880 & 890 & $\mathrm{U}$ & 890 & 1000 & $\mathrm{U}$ & 1000 & 890 & $\mathrm{U}$ & 890 \\
\hline Acenaphthene & 350 & $\mathrm{U}$ & 350 & 360 & $\overline{\mathrm{U}}$ & 360 & 410 & $\mathrm{U}$ & 410 & 350 & $\mathrm{U}$ & 350 \\
\hline Acenaphthylene & 350 & $\bar{U}$ & 350 & 360 & $\mathrm{U}$ & 360 & 410 & $\mathrm{U}$ & 410 & 350 & $\mathrm{U}$ & 350 \\
\hline Anthracene & 350 & $\mathrm{U}$ & 350 & 360 & $\bar{U}$ & 360 & 410 & $\mathrm{U}$ & 410 & 350 & $U$ & 350 \\
\hline Benzo(a)anthracene & 350 & $\mathrm{U}$ & 350 & 360 & $\mathrm{U}$ & 360 & 410 & $\bar{U}$ & 410 & 350 & $\mathrm{U}$ & 350 \\
\hline Benzo(a)pyrene & 350 & $\mathrm{U}$ & 350 & 360 & $\mathrm{U}$ & 360 & 410 & $\mathrm{U}$ & 410 & 350 & $\bar{U}$ & 350 \\
\hline Benzo(b)fluoranthene & 350 & $\mathrm{U}$ & 350 & 360 & $\mathrm{U}$ & 360 & 410 & $\mathrm{U}$ & 410 & 350 & $\mathrm{U}$ & 350 \\
\hline Benzo $(\mathrm{g}, \mathrm{h}, \mathrm{i})$ perylene & 350 & $\mathrm{U}$ & 350 & 360 & $\mathrm{U}$ & 360 & 410 & $\mathrm{U}$ & 410 & 350 & $\mathrm{U}$ & 350 \\
\hline Benzo(k)fluoranthene & 350 & $\mathrm{U}$ & 350 & 360 & $U$ & 360 & 410 & $U$ & 410 & 350 & $\mathrm{U}$ & 350 \\
\hline bis(2-Chloro-1-methylethyl)ether & 350 & UI & 350 & 360 & UJ & 360 & 410 & UJ & 410 & 350 & UJ & 350 \\
\hline bis(2-Chloroethoxy)methane & 350 & $\mathrm{U}$ & 350 & 360 & $\mathrm{U}$ & 360 & 410 & $\mathrm{U}$ & 410 & 350 & $U$ & 350 \\
\hline bis (2-Chloroethyl)ether & 350 & $\mathrm{U}$ & 350 & 360 & $\mathrm{U}$ & 360 & 410 & $\mathrm{U}$ & 410 & 350 & $\mathrm{U}$ & 350 \\
\hline bis(2-Ethylhexyl)phthalate & 22 & $\mathrm{~J}$ & 350 & 360 & $\mathrm{U}$ & 360 & 410 & $\mathrm{U}$ & 410 & 25 & $\mathrm{~J}$ & 350 \\
\hline Butylbenzylphthalate & 350 & $\mathrm{U}$ & 350 & 360 & $\mathrm{U}$ & 360 & 410 & $\mathrm{U}$ & 410 & 350 & $\mathrm{U}$ & 350 \\
\hline Carbazole & 350 & $\mathrm{U}$ & 350 & 360 & $\mathrm{U}$ & 360 & 410 & $\mathrm{U}$ & 410 & 350 & $\mathrm{U}$ & 350 \\
\hline Chrysene & 350 & $\mathrm{U}$ & 350 & 360 & $\mathrm{U}$ & 360 & 410 & $\mathrm{U}$ & 410 & 21 & $\mathrm{~J}$ & 350 \\
\hline Di-n-butylphthalate & 350 & $\mathrm{U}$ & 350 & 360 & $\mathrm{U}$ & 360 & 410 & $\mathrm{U}$ & 410 & 350 & $\mathrm{U}$ & 350 \\
\hline Di-n-octylphthalate & 350 & $\mathrm{U}$ & 350 & 360 & U & 360 & 410 & $\mathrm{U}$ & 410 & 350 & $U$ & 350 \\
\hline Dibenz(a,h)anthracene & 350 & $\mathrm{U}$ & 350 & 360 & $\mathrm{U}$ & 360 & 410 & $\mathrm{U}$ & 410 & 350 & $\mathrm{U}$ & 350 \\
\hline Dibenzofuran & 350 & $\mathrm{U}$ & 350 & 360 & $\mathrm{U}$ & 3.60 & 410 & $\mathrm{U}$ & 410 & 350 & $\mathrm{U}$ & 350 \\
\hline Diethylphthalate & 350 & $\mathrm{U}$ & 350 & 360 & $\mathrm{U}$ & 360 & 410 & $\mathrm{U}$ & 410 & 350 & $\mathrm{U}$ & 350 \\
\hline Dimethylphthalate & 350 & $\mathrm{U}$ & 350 & 360 & $\mathrm{U}$ & 360 & 410 & $\mathrm{U}$ & 410 & 350 & $\mathrm{U}$ & 350 \\
\hline Fluoranthene & 350 & $\mathrm{U}$ & 350 & 360 & $\mathrm{U}$ & 360 & 410 & $\mathrm{U}$ & 410 & 19 & $\mathrm{~J}$ & 350 \\
\hline Fluorene & 350 & $\mathrm{U}$ & 350 & 360 & $\bar{U}$ & 360 & 410 & $\mathrm{U}$ & 410 & 350 & $\bar{U}$ & 350 \\
\hline Hexachlorobenzene & 350 & $\mathrm{U}$ & 350 & 360 & $\mathrm{U}$ & 360 & 410 & $\bar{U}$ & 410 & 350 & $\mathrm{U}$ & 350 \\
\hline Hexachlorobutadiene & 350 & $\bar{U}$ & 350 & 360 & $\bar{U}$ & 360 & 410 & $\mathrm{U}$ & 410 & 350 & $\mathrm{U}$ & 350 \\
\hline Hexachlorocyclopentadiene & 350 & $\mathrm{U}$ & 350 & 360 & $\mathrm{U}$ & 360 & 410 & $\mathrm{U}$ & 410 & 350 & $\mathrm{U}$ & 350 \\
\hline Hexachloroethane & 350 & $\mathrm{U}$ & 350 & 360 & $\mathrm{U}$ & 360 & 410 & $\mathrm{U}$ & 410 & 350 & $\overline{\mathrm{U}}$ & 350 \\
\hline Indeno( $1,2,3-c d)$ pyrene & 350 & $\mathrm{U}$ & 350 & 360 & $\mathrm{U}$ & 360 & 410 & $\mathrm{U}$ & 410 & 350 & $\mathrm{U}$ & 350 \\
\hline Isophorone & 350 & $\mathrm{U}$ & 350 & 360 & $\mathrm{U}$ & 360 & 410 & $\mathrm{U}$ & 410 & 350 & $\mathrm{U}$ & 350 \\
\hline N-Nitroso-di-n-dipropylamine & 350 & UJ & 350 & 360 & UJ & 360 & 410 & $\overline{\mathrm{UJ}}$ & 410 & 350 & $\mathrm{UJ}$ & 350 \\
\hline N-Nitrosodiphenylamine & 350 & $\mathrm{U}$ & 350 & 360 & $\mathrm{U}$ & 360 & 410 & $\mathrm{U}$ & 410 & 350 & $\mathrm{U}$ & 350 \\
\hline $\begin{array}{l}\text { Naphthalene } \\
\end{array}$ & 350 & $\mathrm{U}$ & 350 & 360 & $\mathrm{U}$ & 360 & 410 & $U$ & 410 & 350 & $\mathrm{U}$ & 350 \\
\hline Nitrobenzene & 350 & $\mathrm{U}$ & 350 & 360 & $\mathrm{U}$ & 360 & 410 & $\mathrm{U}$ & 410 & 350 & $\mathrm{U}$ & 350 \\
\hline Pentachlorophenol & 880 & $\mathrm{U}$ & 880 & 890 & $\mathrm{U}$ & 890 & 1000 & $\mathrm{U}$ & 1000 & 890 & $\mathrm{U}$ & 890 \\
\hline Phenanthrene & 350 & $\mathrm{U}$ & 350 & 360 & $\mathrm{U}$ & 360 & 410 & $\mathrm{U}$ & 410 & 350 & $\mathrm{U}$ & 350 \\
\hline Phenol & 350 & $\mathrm{UJ}$ & 350 & 360 & $\overline{\mathrm{UJ}}$ & 360 & 410 & UJ & 410 & 350 & UJ & 350 \\
\hline Pyrene & 20 & $\mathrm{~J}$ & 350 & 360 & $\mathrm{U}$ & 360 & 410 & $\mathrm{U}$ & 410 & 18 & $\mathrm{~J}$ & 350 \\
\hline & & & & Attachn & & & 2 & & & Sheet No. & & of 13 \\
\hline & & & & Origina & & J.M.C & pron & & & Date & & $31 / 06$ \\
\hline & & & & Checke & & T.M. B & akley & & & Date & & \\
\hline & & & & Calc. $\mathrm{N}$ & & $0100 \mathrm{~B}-$ & A-V028 & & & Rev. No. & & 0 \\
\hline
\end{tabular}


Attachment 2. 100-B-14:2 Area 5 Verification Sampling Results.

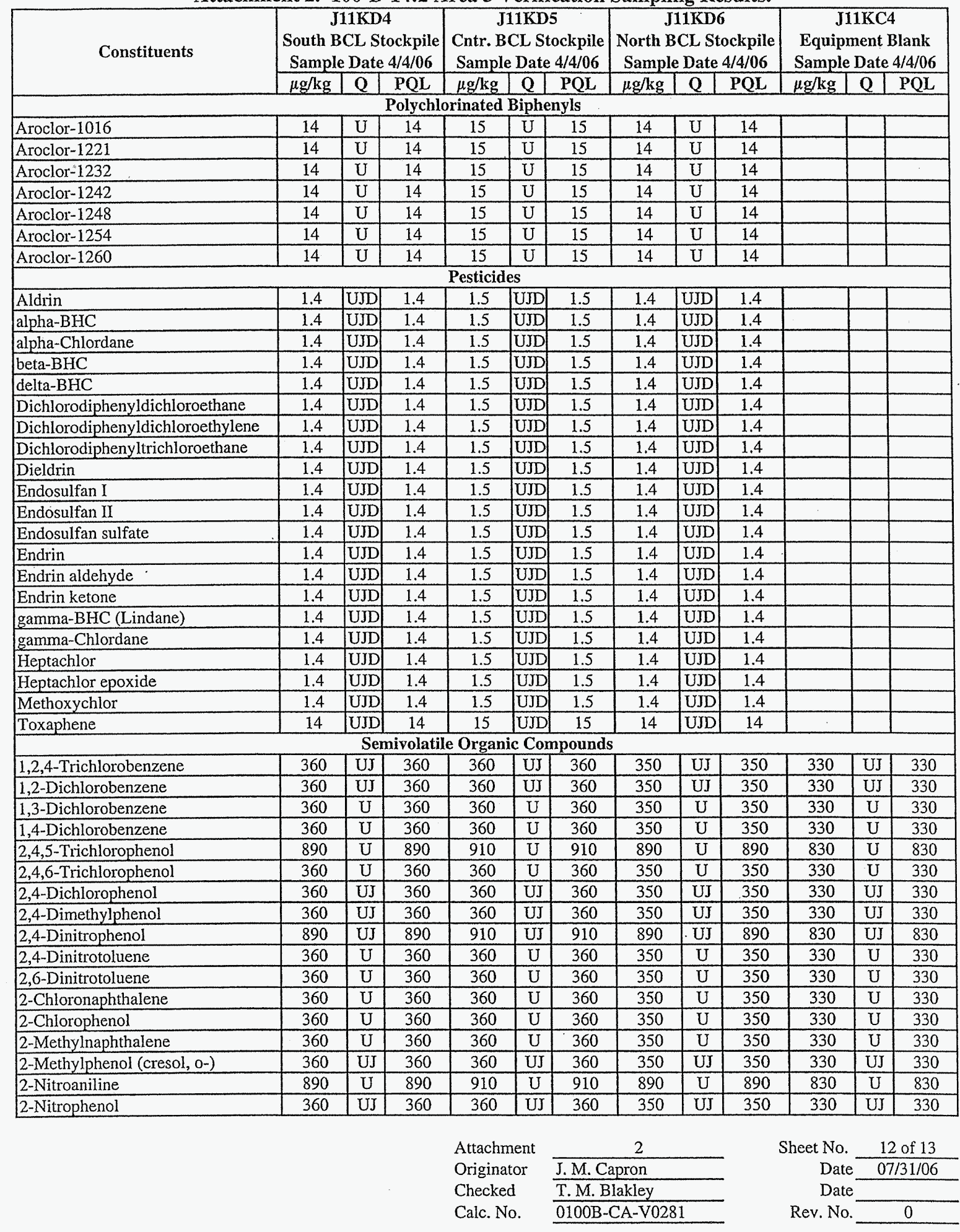


Attachment 2. 100-B-14:2 Area 5 Verification Sampling Results.

\begin{tabular}{|c|c|c|c|c|c|c|c|c|c|c|c|c|}
\hline \multirow[t]{2}{*}{ Constituents } & \multicolumn{3}{|c|}{\begin{tabular}{|c|} 
J11KD4 \\
South BCL Stockpile \\
Sample Date 4/4/06 \\
\end{tabular}} & \multicolumn{3}{|c|}{\begin{tabular}{|c|} 
J11KD5 \\
Cntr. BCL Stockpile \\
Sample Date $4 / 4 / 06$ \\
\end{tabular}} & \multicolumn{3}{|c|}{\begin{tabular}{|c|} 
J11KD6 \\
North BCL Stockpile \\
Sample Date 4/4/06 \\
\end{tabular}} & \multicolumn{3}{|c|}{$\begin{array}{c}\text { J11KC4 } \\
\text { Equipment Blank } \\
\text { Sample Date 4/4/06 }\end{array}$} \\
\hline & $\mu \mathrm{g} / \mathrm{kg}$ & $\mathrm{Q}$ & PQL & $\mu \mathrm{g} / \mathrm{kg}$ & $Q$ & PQL & $\mu \mathrm{g} / \mathrm{kg}$ & $\mathbf{Q}$ & PQL & $\mu \mathrm{g} / \mathrm{kg}$ & $Q$ & PQL \\
\hline \multicolumn{13}{|c|}{ Semivolatile Organic Compounds (continued) } \\
\hline 3,3'-Dichlorobenzidine & 360 & $\mathrm{U}$ & 360 & 360 & $U$ & 360 & 350 & $\mathrm{U}$ & 350 & 330 & $\mathrm{U}$ & 330 \\
\hline 3-Nitroaniline & 890 & $\mathrm{U}$ & 890 & 910 & $\mathrm{U}$ & 910 & 890 & $\mathrm{U}$ & 890 & 830 & $\mathrm{U}$ & 830 \\
\hline 4,6-Dinitro-2-methylphenol & 890 & UJ & 890 & 910 & UJ & 910 & 890 & UJ & 890 & 830 & UJ & 830 \\
\hline 4-Bromophenyl-phenylether & 360 & $\mathrm{U}$ & 360 & 360 & $\mathrm{U}$ & 360 & 350 & $\mathrm{U}$ & 350 & 330 & $\mathrm{U}$ & 330 \\
\hline 4-Chloro-3-methylphenol & 360 & $\mathrm{U}$ & 360 & 360 & $\mathrm{U}$ & 360 & 350 & $\mathrm{U}$ & 350 & 330 & $\mathrm{U}$ & 330 \\
\hline 4-Chloroaniline & 360 & $\mathrm{U}$ & 360 & 360 & $\mathrm{U}$ & 360 & 350 & $\mathrm{U}$ & 350 & 330 & $\mathrm{U}$ & 330 \\
\hline 4-Chlorophenyl-phenylether & 360 & $\mathrm{U}$ & 360 & 360 & $\mathrm{U}$ & 360 & 350 & $\mathrm{U}$ & 350 & 330 & $\mathrm{U}$ & 330 \\
\hline 4-Methylphenol (p-cresol) & 360 & UJ & 360 & 360 & UJ & 360 & 350 & UJ & 350 & 330 & UJ & 330 \\
\hline 4-Nitroaniline & 890 & $\mathrm{U}$ & 890 & 910 & $\mathrm{U}$ & 910 & 890 & $\mathrm{U}$ & 890 & 830 & $\mathrm{U}$ & 830 \\
\hline 4-Nitrophenol & 890 & $\mathrm{U}$ & 890 & 910 & $\mathrm{U}$ & 910 & 890 & $\mathrm{U}$ & 890 & 830 & $\mathrm{U}$ & 830 \\
\hline Acenaphthene & 360 & $\mathrm{U}$ & 360 & 360 & $\mathrm{U}$ & 360 & 350 & $\mathrm{U}$ & 350 & 330 & $\mathrm{U}$ & 330 \\
\hline Acenaphthylene & 360 & $\mathrm{U}$ & 360 & 360 & $\mathrm{U}$ & 360 & 350 & $\mathrm{U}$ & 350 & 330 & $\mathrm{U}$ & 330 \\
\hline Anthracene & 360 & $\mathrm{U}$ & 360 & 360 & $\mathrm{U}$ & 360 & 350 & $\mathrm{U}$ & 350 & 330 & $\bar{U}$ & 330 \\
\hline Benzo(a)anthracene & 360 & $\mathrm{U}$ & 360 & 360 & $\mathrm{U}$ & 360 & 350 & $\mathrm{U}$ & 350 & 330 & $\bar{U}$ & 330 \\
\hline Benzo(a)pyrene & 360 & $\mathrm{U}$ & 360 & 360 & $\mathrm{U}$ & 360 & 350 & $\mathrm{U}$ & 350 & 330 & $\mathrm{U}$ & 330 \\
\hline Benzo(b)fluoranthene & 360 & $\mathrm{U}$ & 360 & 360 & $\mathrm{U}$ & 360 & 350 & $\mathrm{U}$ & 350 & 330 & $\mathrm{U}$ & 330 \\
\hline Benzo $(\mathrm{g}, \mathrm{h}, \mathrm{i})$ perylene & 360 & $\mathrm{U}$ & 360 & 360 & $\mathrm{U}$ & 360 & 350 & $\mathrm{U}$ & 350 & 330 & $\bar{U}$ & 330 \\
\hline Benzo(k)fluoranthene & 360 & $\mathrm{U}$ & 360 & 360 & $\mathrm{U}$ & 360 & 350 & $\mathrm{U}$ & 350 & 330 & $\mathrm{U}$ & 330 \\
\hline bis(2-Chloro-1-methylethyl)ether & 360 & UJ & 360 & 360 & UJ & 360 & 350 & UJ & 350 & 330 & UJ & 330 \\
\hline bis(2-Chloroethoxy)methane & 360 & $\mathrm{U}$ & 360 & 360 & $\mathrm{U}$ & 360 & 350 & $\mathrm{U}$ & 350 & 330 & $\mathrm{U}$ & 330 \\
\hline bis(2-Chloroethyl)ether & 360 & $\mathrm{U}$ & 360 & 360 & $\mathrm{U}$ & 360 & 350 & $\mathrm{U}$ & 350 & 330 & $\mathrm{U}$ & 330 \\
\hline bis(2-Ethylhexyl)phthalate & 27 & $\mathrm{~J}$ & 360 & 360 & $\mathrm{U}$ & 360 & 27 & $\mathrm{~J}$ & 350 & 19 & $\mathrm{~J}$ & 330 \\
\hline Butylbenzylphthalate & 360 & $\mathrm{U}$ & 360 & 360 & $\mathrm{U}$ & 360 & 350 & $\mathrm{U}$ & 350 & 330 & $\mathrm{U}$ & 330 \\
\hline Carbazole & 360 & $U$ & 360 & 360 & $\mathrm{U}$ & 360 & 350 & $\mathrm{U}$ & 350 & 330 & $\mathrm{U}$ & 330 \\
\hline \begin{tabular}{|l} 
Chrysene \\
\end{tabular} & 360 & $\mathrm{U}$ & 360 & 360 & $\mathrm{U}$ & 360 & 350 & $\mathrm{U}$ & 350 & 330 & $\mathrm{U}$ & 330 \\
\hline Di-n-butylphthalate & 360 & $\mathrm{U}$ & 360 & 360 & $\mathrm{U}$ & 360 & 350 & $\mathrm{U}$ & 350 & 48 & $\mathrm{~J}$ & 330 \\
\hline Di-n-octylphthalate & 360 & $\mathrm{U}$ & 360 & 360 & $\mathrm{U}$ & 360 & 350 & $\mathrm{U}$ & 350 & 330 & $\mathrm{U}$ & 330 \\
\hline Dibenz $(a, h)$ anthracene & 360 & $\mathrm{U}$ & 360 & 360 & $\mathrm{U}$ & 360 & 350 & $\mathrm{U}$ & 350 & 330 & $\mathrm{U}$ & 330 \\
\hline Dibenzofuran & 360 & $\mathrm{U}$ & 360 & 360 & $\mathrm{U}$ & 360 & 350 & $\mathrm{U}$ & 350 & 330 & $\mathrm{U}$ & 330 \\
\hline Diethylphthalate & 360 & $\mathrm{U}$ & 360 & 360 & $\mathrm{U}$ & 360 & 350 & $\mathrm{U}$ & 350 & 330 & $\mathrm{U}$ & 330 \\
\hline Dimethylphthalate & 360 & $\mathrm{U}$ & 360 & 360 & $\mathrm{U}$ & 360 & 350 & $\mathrm{U}$ & 350 & 330 & $\mathrm{U}$ & 330 \\
\hline Fluoranthene & 360 & $\mathrm{U}$ & 360 & 360 & $\mathrm{U}$ & 360 & 350 & $\mathrm{U}$ & 350 & 330 & $\mathrm{U}$ & 330 \\
\hline Fluorene & 360 & $\mathrm{U}$ & 360 & 360 & $\mathrm{U}$ & 360 & 350 & $\mathrm{U}$ & 350 & 330 & $\mathrm{U}$ & 330 \\
\hline Hexachlorobenzene & 360 & $\mathrm{U}$ & 360 & 360 & $U$ & 360 & 350 & $\mathrm{U}$ & 350 & 330 & $\mathrm{U}$ & 330 \\
\hline \begin{tabular}{|l|} 
Hexachlorobutadiene \\
\end{tabular} & 360 & $\mathrm{U}$ & 360 & 360 & $\mathrm{U}$ & 360 & 350 & $\mathrm{U}$ & 350 & 330 & U & 330 \\
\hline Hexachlorocyclopentadiene & 360 & $\mathrm{U}$ & 360 & 360 & $\mathrm{U}$ & 360 & 350 & $\mathrm{U}$ & 350 & 330 & $\mathrm{U}$ & 330 \\
\hline Hexachloroethane & 360 & $\mathrm{U}$ & 360 & 360 & $\mathrm{U}$ & 360 & 350 & $\mathrm{U}$ & 350 & 330 & $\mathrm{U}$ & 330 \\
\hline Indeno(1,2,3-cd)pyrene & 360 & $\mathrm{U}$ & 360 & 360 & $\mathrm{U}$ & 360 & 350 & $\mathrm{U}$ & 350 & 330 & $\mathrm{U}$ & 330 \\
\hline Isophorone & 360 & $\mathrm{U}$ & 360 & 360 & $\mathrm{U}$ & 360 & 350 & $\mathrm{U}$ & 350 & 330 & $\mathrm{U}$ & 330 \\
\hline N-Nitroso-di-n-dipropylamine & 360 & UJ & 360 & 360 & UJ & 360 & 350 & UJ & 350 & 330 & $\mathrm{UJ}$ & 330 \\
\hline N-Nitrosodiphenylamine & 360 & $\mathrm{U}$ & 360 & 360 & $\mathrm{U}$ & 360 & 350 & $\mathrm{U}$ & 350 & 330 & $\mathrm{U}$ & 330 \\
\hline Naphthalene & 360 & $\mathrm{U}$ & 360 & 360 & $\mathrm{U}$ & 360 & 350 & $\mathrm{U}$ & 350 & 330 & $\mathrm{U}$ & 330 \\
\hline Nitrobenzene & 360 & $\mathrm{U}$ & 360 & 360 & $\mathrm{U}$ & 360 & 350 & $\mathrm{U}$ & 350 & 330 & $\mathrm{U}$ & 330 \\
\hline Pentachlorophenol & 890 & $\mathrm{U}$ & 890 & 910 & $\mathrm{U}$ & 910 & 890 & $\mathrm{U}$ & 890 & 830 & $\bar{U}$ & 830 \\
\hline Phenanthrene & 360 & $\mathrm{U}$ & 360 & 360 & $\mathrm{U}$ & 360 & 350 & $\mathrm{U}$ & 350 & 330 & $\mathrm{U}$ & 330 \\
\hline Phenol & 360 & UJ & 360 & 360 & UJ & 360 & 350 & $\mathrm{UJ}$ & 350 & 330 & UJ & 330 \\
\hline Pyrene & 19 & $\mathrm{~J}$ & 360 & 360 & $\mathrm{U}$ & 360 & 350 & $\mathrm{U}$ & 350 & 330 & $\mathrm{U}$ & 330 \\
\hline
\end{tabular}

Attachment

Originator

Checked

Calc. No.

$\frac{2}{\frac{2}{\text { J.M. Capron }}} \frac{}{\text { T.M. Blakley }}$

Sheet No. 13 of 13

Date $07 / 31 / 06$

Date

Rev. No

0 


\section{CALCULATION COVER SHEET}

Project Title 100-B/C Remaining Pipes and Sewers Field Remediation Job No. 14655

Area $100-\mathrm{B} / \mathrm{C}$

Discipline Environmental *Calc. No. 0100B-CA-V0282

Subject 100-B-14:2 (Areas 2 \& 5) Waste Site Hazard Quotient and Carcinogenic Risk Calculations Computer Program Excel Program No. Excel 2003

The attached calculations have been generated to document compliance with established cleanup levels. These calculations should be used in conjunction with other relevant documents in the administrative record.

\section{Committed Calculation $\otimes \quad$ Preliminary $\square \quad$ Superseded $\square \quad$ Voided}

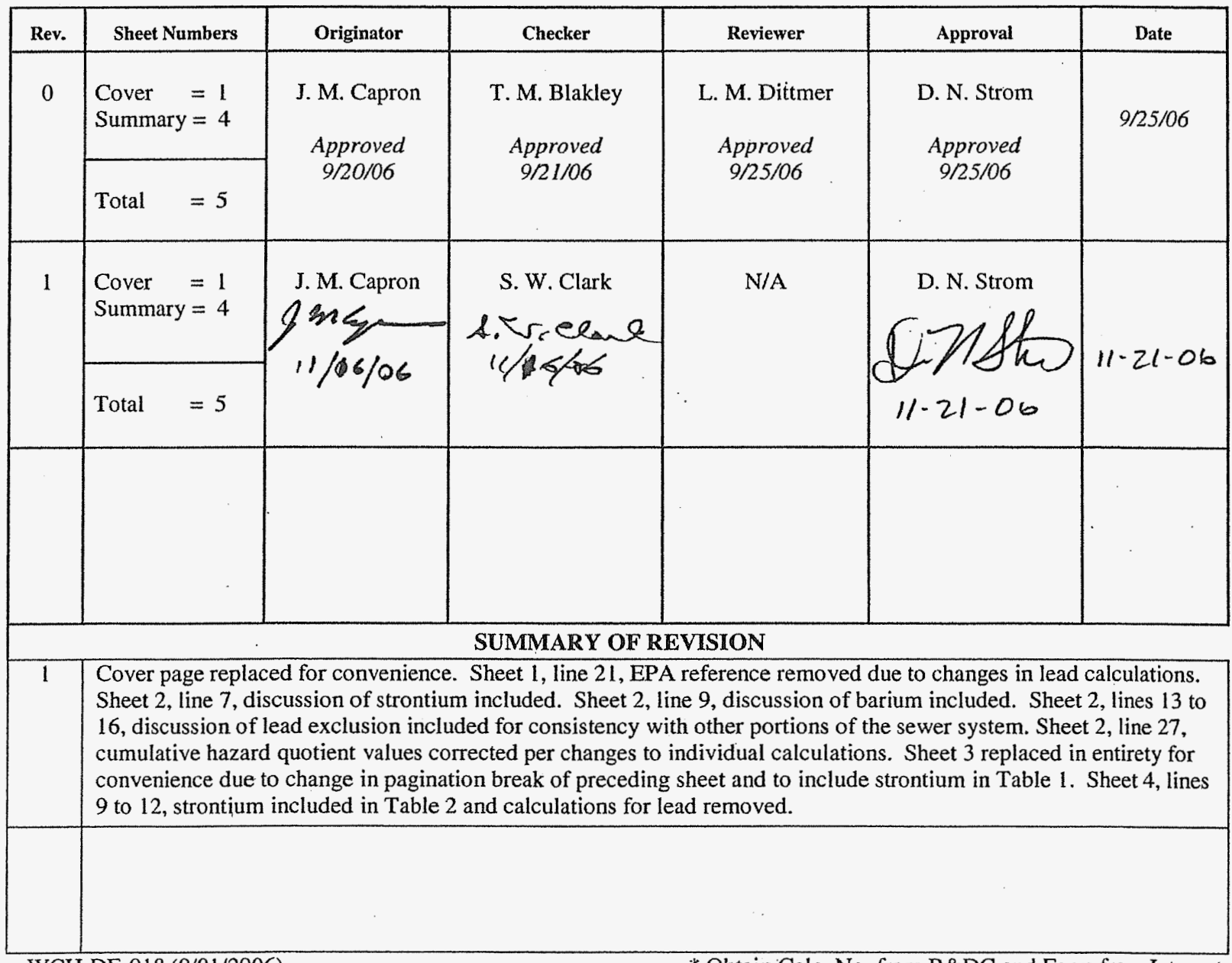


Washington Closure Hanford

CALCULATION SHEET

D. M. Capron ate: 11/16/06

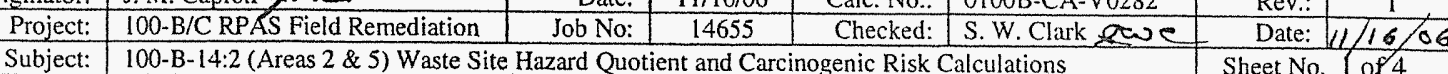

\section{PURPOSE:}

Provide documentation to support the calculation of the hazard quotient (HQ) and excess carcinogenic risk values for the 100-B-14:2 subsite, areas 2 and 5 remediation verification sampling results (area 5 was previously designated as the 100-B-14:9 subsite). In accordance with the remedial action goals (RAGs) in the remedial design report/remedial action work plan (RDR/RAWP) (DOE-RL 2005), the following criteria must be met:

1) An HQ of $<1.0$ for all individual noncarcinogens

2) A cumulative HQ of $<1.0$ for noncarcinogens

3) An excess carcinogenic risk of $<1 \times 10^{-6}$ for individual carcinogens

4) A cumulative excess carcinogenic risk of $<1 \times 10^{-5}$ for carcinogens.

\section{GIVEN/REFERENCES:}

1) DOE-RL, 2005, Remedial Design Report/Remedial Action Work Plan for the 100 Areas, DOE/RL-96-17, Rev. 5, U.S. Department of Energy, Richland Operations Office, Richland, Washington.

2) WAC 173-340, "Model Toxics Control Act - Cleanup," Washington Administrative Code, 1996.

3) WCH, 2006, 100-B-14:2 (Areas 2 \& 5) Waste Site Cleanup Verification 95\% UCL Calculations, Calculation No. 0100B-CA-V0281, Washington Closure Hanford, Richland, Washington.

\section{SOLUTION:}

1) Calculate an HQ for each noncarcinogenic constituent detected above background and compare to the individual HQ of $<1.0$ (DOE-RL 2005).

2) Sum the HQs and compare to the cumulative HQ criterion of $<1.0$.

3) Calculate an excess carcinogenic risk value for each carcinogenic constituent detected above background and compare to the individual excess carcinogenic risk criterion of $\angle 1 \times 10^{-6}$ (DOE-RL 2005).

4) Sum the excess carcinogenic risk values and compare to the cumulative excess carcinogenic risk criterion of $<1 \times 10^{-5}$. 
Washington Closure Hanford CALCULATION SHEET \begin{tabular}{l|l|l|l|}
\hline Originator: & J. M. Capron 0 & Date: & $1 / 16 / 06$
\end{tabular}

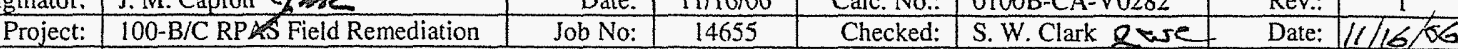

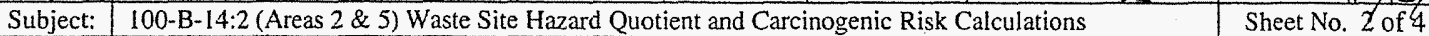

\section{METHODOLOGY:}

Hazard quotient and carcinogenic risk calculations were performed separately for area 2 and area 5 of the 100-B-14:2 subsite. Calculations for each area were performed using the higher of the remediation footprint statistical value and overburden/below cleanup levels (BCL) material maximum value for each analyte detected above background. Of the contaminants of concern (COCs) and contaminants of potential concern (COPCs) for the site, boron, molybdenum, strontium and tin require the HQ calculations for one or both areas because they were detected and Washington State or Hanford Site background values are not available. Barium was included for area 2 because it was quantified above the Hanford Site background value. Hexavalent chromium, aroclor-1254, and multiple chlorinated pesticides and semivolatile organic compounds (as identified in Tables 1 and 2) are included for one both areas because they were detected by laboratory analysis and cannot be attributed to natural occurrence. Lead does not have a reference dose for calculation of a hazard quotient because toxic effects of lead are correlated with blood-lead levels rather than exposure levels or daily intake. As a result, the maximum lead concentration for area 5 is reported but not included in the hazard quotient calculation. All other site nonradionuclide COCs and COPCs were not detected or were detected below background levels. An example of the HQ and risk calculations is presented below:

1) For example, the statistical value for boron in area 2 is $4.5 \mathrm{mg} / \mathrm{kg}$, divided by the noncarcinogenic $\mathrm{RAG}$ value of $16,000 \mathrm{mg} / \mathrm{kg}$ (calculated in accordance with the noncarcinogenic toxics effects formula in WAC 173-340-740[3]), is $2.8 \times 10^{-4}$. Comparing this value, and all other individual values, to the requirement of $<1.0$, this criterion is met.

2) After the HQ calculations are completed for the appropriate analytes, the cumulative HQ is obtained by summing the individual values. (To avoid errors due to intermediate rounding, the individual HQ values prior to rounding are used for this calculation.) The sums of the HQ values for areas 2 and 5 are $3.4 \times 10^{-2}$ and $1.2 \times 10^{-2}$, respectively. Comparing these values to the requirement of $<1.0$, this criterion is met.

3) To calculate the excess carcinogenic risk, the 95\% upper confidence limit or maximum value is divided by the carcinogenic RAG value, then multiplied by $1 \times 10^{-6}$. For example, the maximum value for hexavalent chromium in area 2 is $0.28 \mathrm{mg} / \mathrm{kg}$; divided by $2.1 \mathrm{mg} / \mathrm{kg}$ and multiplied as indicated is $1.3 \times 10^{-7}$. Comparing this value, and all other individual values, to the requirement of $<1 \times 10^{-6}$, this criterion is met.

4) After these calculations are completed for the carcinogenic analytes, the cumulative excess carcinogenic risk is obtained by summing the individual values. (To avoid errors due to intermediate rounding, the individual values prior to rounding are used for this calculation.) The sums of the excess carcinogenic risk values for areas 2 and 5 are $3.1 \times 10^{-7}$ and $3.3 \times 10^{-7}$, respectively. Comparing these values to the requirement of $<1 \times 10^{-5}$, this criterion is met.

\section{RESULTS:}

1) List individual noncarcinogens and corresponding $\mathrm{HQs}>1.0$ : None

2) List the cumulative noncarcinogenic HQ $>1.0$ : None

3) List individual carcinogens and corresponding excess cancer risk $>1 \times 10^{-6}$ : None

4) List the cumulative excess cancer risk for carcinogens $>1 \times 10^{-5}:$ None. 
Washington Closure Hanford CALCULATION SHEET

\begin{tabular}{|c|c|c|c|c|c|c|c|}
\hline Originator: & J. M. Capron gan & Date: & $11 / 16 / 06$ & Calc. No.: & 0100B-CA-V0282 & Rev.: & 1 \\
\hline Project: & 100-B/C RPAS Field Remediation & Job No: & 14655 & Checked: & S. W. Clark \&use & Date: & $11 / 16 / 06$ \\
\hline Subject: & \multicolumn{5}{|c|}{ 100-B-14:2 (Areas 2 \& 5) Waste Site Hazard Quotient and Carcinogenic Risk Calculations } & \multicolumn{2}{|c|}{ Sheet No. 3 of 4} \\
\hline
\end{tabular}

Table 1 shows the results of the calculations for the 100-B-14:2 subsite (area 2); results for area 5 are provided in Table 2.

Table 1. Hazard Quotient and Excess Cancer Risk Results for the 100-B-14:2 (Area 2) Subsite.

\begin{tabular}{|c|c|c|c|c|c|}
\hline $\begin{array}{c}\text { Contaminants of Concern/ } \\
\text { Contaminants of Potential Concern }\end{array}$ & $\begin{array}{c}\text { Maximum or } \\
\text { Statistical Value }^{\mathrm{a}} \\
(\mathrm{mg} / \mathrm{kg})\end{array}$ & $\begin{array}{c}\text { Noncarcinogen } \\
\mathbf{R A G}^{\mathbf{b}} \\
(\mathbf{m g} / \mathbf{k g})\end{array}$ & $\begin{array}{l}\text { Hazard } \\
\text { Quotient }\end{array}$ & $\begin{array}{c}\text { Carcinogen } \\
\mathbf{R A G}^{\mathbf{b}} \\
(\mathbf{m g} / \mathbf{k g})\end{array}$ & $\begin{array}{c}\text { Carcinogen } \\
\text { Risk }\end{array}$ \\
\hline \multicolumn{6}{|l|}{ Metals } \\
\hline Barium & 163 & 5,600 & $2.9 \mathrm{E}-02$ & - & -- \\
\hline Boron & 4.5 & 16,000 & $2.8 \mathrm{E}-04$ & - & -- \\
\hline Chromium, hexavalent ${ }^{\mathrm{c}}$ & 0.28 & 240 & $1.2 \mathrm{E}-03$ & 2.1 & $1.3 \mathrm{E}-07$ \\
\hline Molybdenum & 0.7 & 400 & $1.8 \mathrm{E}-03$ & -- & - \\
\hline Strontium & 57.1 & 48,000 & $1.2 \mathrm{E}-03$ & -- & -- \\
\hline \multicolumn{6}{|c|}{ Semivolatiles } \\
\hline Benzo(a)pyrene & 0.021 & - & -- & 0.137 & $1.5 \mathrm{E}-07$ \\
\hline Benzo(b)fluoranthene & 0.023 & 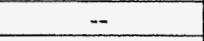 & -- & 1.37 & $1.7 \mathrm{E}-08$ \\
\hline Benzo(k)fluoranthene & 0.022 & - & $\sim$ & 13.7 & $1.6 \mathrm{E}-09$ \\
\hline Chrysene & 0.022 & -- & -- & 137 & $1.6 \mathrm{E}-10$ \\
\hline Methylnaphthalene; 2- & 0.034 & 320 & 1.1E-04 & -- & - \\
\hline Naphthalene & 0.024 & 1,600 & $1.5 \mathrm{E}-05$ & - & - \\
\hline Phenanthrene $^{\mathrm{d}}$ & 0.024 & 24,000 & $1.0 \mathrm{E}-06$ & - & - \\
\hline \multicolumn{6}{|c|}{ Pesticides } \\
\hline BHC, beta- & 0.00060 & -- & -- & 0.556 & $1.1 \mathrm{E}-09$ \\
\hline Endosulfan (I, II, sulfate) & 0.00053 & 480 & $1.1 \mathrm{E}-06$ & -- & -- \\
\hline \multirow{2}{*}{\multicolumn{6}{|c|}{$\begin{array}{l}\text { Totals } \\
\text { Cumulative Hazard Quotient: }\end{array}$}} \\
\hline & & & $3.4 \mathrm{E}-02$ & & \\
\hline \multicolumn{5}{|l|}{ Cumulative Excess Cancer Risk: } & $3.1 \mathrm{E}-07$ \\
\hline
\end{tabular}

Notes:

${ }^{a}=$ From WCH (2006).

" = Value obtained from Washington Administrative Code (WAC) 173-340-740(3), Method B, 1996, unless otherwise noted.

${ }^{c}=$ Value for the carcinogen RAG calculated based on the inhalation exposure pathway (WAC) 173-340-750(3), 1996.

$d=$ Toxicity data are not available for this constituent. RAGs for phenanthrene are based on the surrogate chemical anthracene.

-. = not applicable

$\mathrm{RAG}=$ remedial action goal 
Washington Closure Hanford CALCULATION SHEET

\begin{tabular}{|r|l|r|r|r|l|c|c|}
\hline Originator: & J. M. Capron & Dane: & $11 / 16 / 06$ & Calc. No.: & 0100 B-CA-V0282 & Rev.: & 1 \\
\hline Project: & $100-$ B/C RPAS Field Remediation & Job No: & 14655 & Checked: & S. W. Clark 2 se & Date: & $11 / 16 / 66$ \\
\hline Subject: & $100-$ B-14:2 (Areas 2 \& 5) Waste Site Hazard Quotient and Carcinogenic Risk Calculations & Sheet No. 4 of 4 \\
\hline
\end{tabular}

\section{Table 2. Hazard Quotient and Excess Cancer Risk Results for the 100-B-14:2 (Area 5) Subsite.}

\begin{tabular}{|c|c|c|c|c|c|}
\hline $\begin{array}{l}\text { Contaminants of Concern/ } \\
\text { Contaminants of Potential Concern }\end{array}$ & $\begin{array}{c}\text { Maximum or } \\
\text { Statistical Value } \\
(\mathrm{mg} / \mathrm{kg})\end{array}$ & $\begin{array}{c}\text { Noncarcinogen } \\
\mathbf{R A G}^{\mathrm{b}} \\
(\mathbf{m g} / \mathbf{k g})\end{array}$ & $\begin{array}{c}\text { Hazard } \\
\text { Quotient }\end{array}$ & $\begin{array}{c}\text { Carcinogen } \\
\mathbf{R A G}^{\mathbf{b}} \\
(\mathbf{m g} / \mathbf{k g})\end{array}$ & $\begin{array}{l}\text { Carcinogen } \\
\text { Risk }\end{array}$ \\
\hline \multirow{2}{*}{\multicolumn{6}{|c|}{ 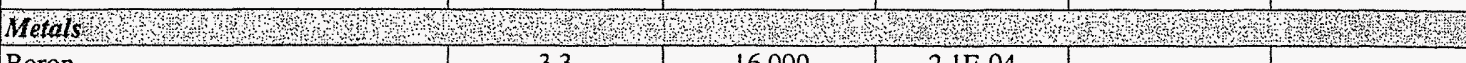 }} \\
\hline & & & & & \\
\hline Chromium, hexavalent ${ }^{c}$ & 0.65 & 240 & 2.7E-03 & 2.1 & 3.1E-07 \\
\hline Lead & 26.1 & $\cdots$ & $-\cdot$ & -- & -- \\
\hline Molybdenum & 0.52 & 400 & $1.3 \mathrm{E}-03$ & -. & -. \\
\hline Strontium & 33.1 & 48,000 & $6.9 \mathrm{E}-04$ & -. & -- \\
\hline Tin & 1.3 & 48,000 & $2.7 \mathrm{E}-05$ & - & - \\
\hline \multicolumn{6}{|c|}{ Semivolatites } \\
\hline Bis(2-ethylhexyl) phthalate & 0.036 & 1,600 & $2.3 \mathrm{E}-05$ & 71.4 & $5.0 \mathrm{E}-10$ \\
\hline Chrysene & 0.021 & $\ldots$ & -. & 137 & $1.5 \mathrm{E}-10$ \\
\hline Di-n-butylphthalate & 0.021 & 8,000 & $2.6 \mathrm{E}-06$ & $\ldots$ & -. \\
\hline Fluoranthene & 0.019 & 3,200 & $5.9 \mathrm{E}-06$ & -- & .. \\
\hline Pyrene & 0.020 & 2,400 & $8.3 \mathrm{E}-06$ & $\ldots$ & -. \\
\hline \multicolumn{6}{|c|}{ Pestictes } \\
\hline $\mathrm{BHC}$, beta- & 0.00062 & $-\ldots$ & -. & 0.556 & $1.1 \mathrm{E}-09$ \\
\hline Endrin (and ketone, aldehyde) & 0.0013 & 24 & 5.4E-05 & - & - \\
\hline Methoxychlor & 0.049 & 400 & $1.2 \mathrm{E}-04$ & -- & - \\
\hline \multicolumn{6}{|l|}{ Polychlorinoted Bipheruls } \\
\hline Aroclor- 1254 & 0.011 & 1.6 & $6.9 \mathrm{E}-03$ & 0.5 & $2.2 \mathrm{E}-08$ \\
\hline \multicolumn{6}{|l|}{ Totals } \\
\hline & $1.2 \mathrm{E}-02$ & & \\
\hline \multicolumn{5}{|l|}{ Cumulative Excess Cancer Risk: } & $3.3 \mathrm{E}-07$ \\
\hline \multicolumn{6}{|c|}{$\begin{array}{l}\text { Notes: } \\
a=\text { From WCH (2006). } \\
b=\text { Value obtained from Washington Administrative Code (WAC) 173-340-740(3), Method B, 1996, unless otherwise noted. } \\
c=\text { Value for the carcinogen RAG calculated based on the inhalation exposure pathway (WAC) 173-340-750(3), } 1996 . \\
-=\text { not applicable }\end{array}$} \\
\hline
\end{tabular}

\section{CONCLUSION:}

This calculation demonstrates that areas 2 and 5 of the 100-B-14:2 subsite meet the requirements for hazard quotient and excess carcinogenic risk as identified in the RDR/RAWP (DOE-RL 2005). 


\section{CALCULATION COVER SHEET}

Project Title:
Area
Discipline
Subject
Computer Program
100-B/C Remaining Pipes and Sewers Field Remediation $100-\mathrm{B} / \mathrm{C}$

Environmental

100-B-14:2 (Area 4) Waste Site Cleanup Veriffca

Excel
Job No.

*Calc. No.

0100B-CA-V0290

Program No. Excel 2003

The attached calculations have been generated to document compliance with established cleanup levels. These calculations should be used in conjunction with other relevant documents in the administrative record.

\section{Committed Calculation \\ 凶 \\ Preliminary \\ Superseded \\ Voided}

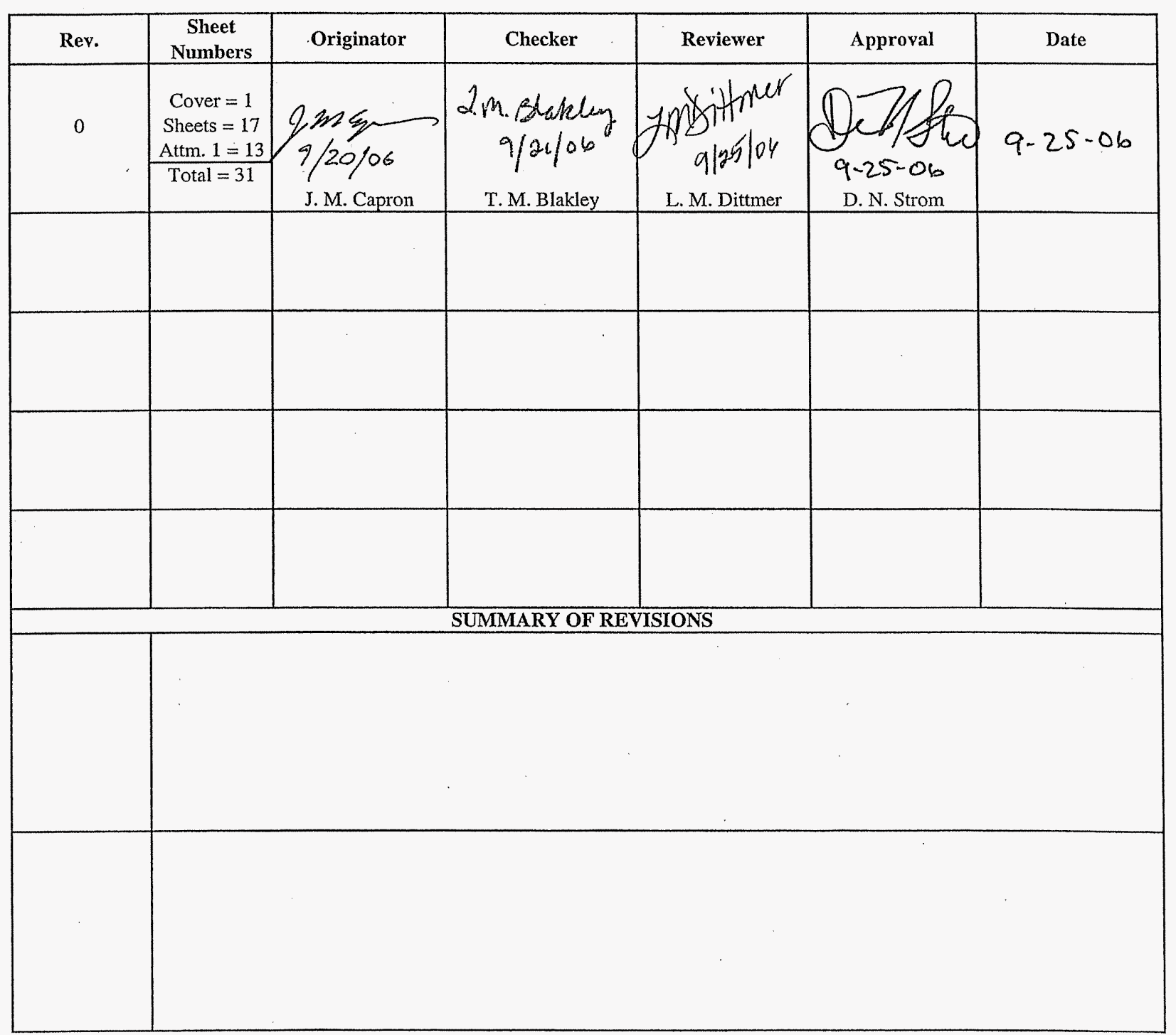


CALCULATION SHEET

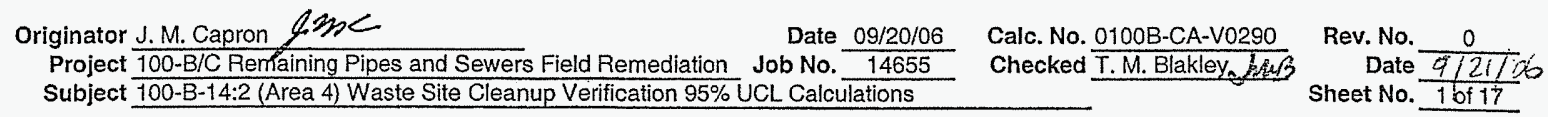

Summary

1 Purpose:

2 Calculate the $95 \%$ upper confidence limit (UCL) values to evaluate compliance with cleanup standards for the remediation footprint of the 100-B-14:2 subsite, area 4 (previously designated as the 100-B-14:8 subsite). Also, perform the Washington Administrative Code (WAC) 173-340-740(7)(e) 3-part test for each nonradioactive contaminant of concern (COC) and contaminant of potential concern (COPC) and calculate the relative percent difference (RPD) for primary-duplicate sample pairs, as necessary.

Table of Contents:

Sheets 1 to 4 - Calculation Sheet Summary

Sheets 5 to 8 - Calculation Sheet 100-B-14:2 Area 4 Remediation Footprint Verification Data

Sheet 9 - Calculation Sheet Duplicate Analysis

Sheets 10 to 17 - Ecology Software (MTCAStat) Results

Attachment 1 - 100-B-14:2 Area 4 Verification Sampling Results (13 sheets)

Given/References:

1) Sample Results (Attachment 1).

2) Background values and remedial action goals (RAGs) are taken from DOE-RL (2005b), DOE-RL (2001), Ecology (1994), and Ecology (2005).

3) DOE-RL, 2001, Hanford Site Background: Part 1, Soil Background for Nonradioactive Analytes, DOE/RL-92-24, Rev. 4, U.S. Department of Energy, Richland Operations Office, Richland, Washington.

4) DOE-RL, 2005a, 100 Area Remedial Action Sampling and Analysis Plan (SAP), DOE/RL-96-22, Rev. 4, U.S. Department of Energy, Richland Operations Office, Richland, Washington.

5) DOE-RL, 2005b, Remedial Design Report/Remedial Action Work Plan for the 100 Area (RDR/RAWP), DOE/RL-96-17, Rev. 5, U.S. Department of Energy, Richland Operations Office, Richland, Washington.

6) Ecology, 1992, Statistical Guidance for Ecology Site Managers, Publication \#92-54, Washington Department of Ecology, Olympia, Washington.

7) Ecology, 1993, Statistical Guidance for Ecology Site Managers, Supplement S-6, Analyzing Site or Background Data with Below-detection Limit or Below-PQL Values (Censored Data Sets). Publication \#92-54, Washington Department of Ecology, Olympia, Washington.

8) Ecology, 1994, Natural Background Soil Metals Concentrations in Washington State, Publication No. 94-115, Washington State Department of Ecology, Olympia, Washington.

9) Ecology, 2005, Cleanup Levels and Risk Calculations (CLARC) Database, Washington State Department of Ecology, Olympia, Washington, <https://fortress.wa.gov/ecy/clarc/CLARCHome.aspx>.

10) EPA, 1994, USEPA Contract Laboratory Program National Functional Guidelines for Inorganic Data Review, EPA 540/R-94/013. U.S. Environmental Protection Agency, Washington, D.C.

11) WAC 173-340, 1996, "Model Toxic Control Act - Cleanup," Washington Administrative Code.

Solution:

Calculation methodology is described in Ecology Pub. \#92-54 (Ecology 1992, 1993), below, and in the RDR/RAWP (DOE-RL $2005 \mathrm{~b})$. Use data from attached worksheets to perform the $95 \%$ UCL calculation for each analyte, the

WAC 173-340-740(7)(e) 3-part test for nonradionuclides, and the RPD calculations, as required. The hazard quotient and carcinogenic risk calculations are located in a separate calculation brief as an appendix to the Remaining Sites Verification Package (RSVP).

Calculation Description:

The subject calculations were performed on data from soil verification samples from the subject waste site. The data were entered into an EXCEL 2003 spreadsheet and calculations performed by using the built-in spreadsheet functions and/or creating formulae within the cells. The statistical evaluation of data for use in accordance with the RDR/RAWP (DOE-RL 2005b) is documented by this calculation. Duplicate RPD results are used in evaluation of data quality within the RSVP for this site.

\section{Methodology:}

For nonradioactive analytes with $\leq 50 \%$ of the data below detection limits and detected radionuclide analytes, the statistical value calculated to evaluate the effectiveness of cleanup is the $95 \%$ UCL. For nonradioactive analytes with $>50 \%$ of the data below detection limits, the maximum detected value for the data set is used instead of the $95 \%$ UCL. The $95 \%$ UCL is not calculated for data sets with no reported detections. The evaluation of the portion of each analyte's data set below detection limits was 1 performed by direct inspection of the attached sample results, and no further calculations were performed for those 62 nonradionuclide data sets where $>50 \%$ of the data was below detection limits or radionuclide data sets with no reported detections. 
Originator J. M. Capron $2 \approx<$

Date 09/20/06 Calc. No. 0100B-CA-V0290

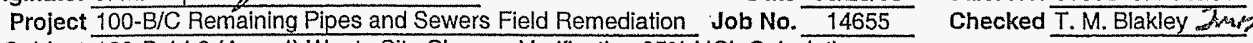
Subject 100-B-14:2 (Area 4) Waste Site Cleanup Verification 95\% UCL Calculations

Summary (continued)

not considered site COPCs (results for total phosphorus are attributed to phosphorus in phosphate). The $95 \%$ UCL values were also not calculated for potassium-40, radium-226, radium-228, thorium-228, and thorium-232, as these radionuclides are not related to the site history and are excluded from consideration as COCs/COPCs (DOE-RL 2005a).

All nonradionuclide data reported as being undetected are set to $1 / 2$ the detection limit value for calculation of the statistics (Ecology 1993). For radionuclide data, calculation of the statistics was done on the reported value. in cases where the laboratory does not report a value below the minimum detectable activity (MDA), half of the MDA is used in the calculation. For the statistical evaluation of duplicate sample pairs, the samples are averaged before being included in the data set, after adjustments for censored data as described above.

For nonradionuclides, the WAC 173-340 statistical guidance suggests that a test for distributional form be performed on the data and the $95 \%$ UCL calculated on the appropriate distribution using Ecology software. For nonradionuclide small data sets ( $\mathrm{n}<10$ ) and all radionuclide data sets, the calculations are performed assuming nonparametric distribution, so no tests for distribution are performed. For nonradionuclide data sets of ten or greater, as for the subject site, distributional testing and calculation of the $95 \%$ UCL is done using Ecology's MTCAStat software (Ecology 1993). Due to differences in addressing censored data between the RDR/RAWP (DOE-RL 2005b) and MTCAStat coding and due to a limitation in the MTCAStat coding (no direct capability to address variable quantitation limits within a data set), substitutions for censored data are performed before software input and the resulting input set treated as uncensored.

The WAC 173-340-740(7)(e) 3-part test is performed for nonradionuclide analytes only and determines if: 1) the $95 \%$ UCL exceeds the most stringent cleanup limit for each COPC/COC,

2) greater than $10 \%$ of the raw data exceed the most stringent cleanup limit for each $C O P C / C O C$,

3) the maximum value of the raw data set exceeds two times the most stringent cleanup limit for each COPC/COC.

The WAC 173-340-740(7)(e) 3-part test is not performed for COPCs/COCs where the statistical value defaults to the maximum value in the data set. Instead, direct comparison of the maximum value against site RAGs (within the RSVP) is used as the compliance basis.

The RPD is calculated when both the primary value and the duplicate value for a given analyte are above detection limits and are greater than 5 times the target detection limit (TDL). The TDL is a laboratory detection limit pre-determined for each analytical method, listed in Tabie II-1 of the SAP (DOE-RL 2005a). Where direct evaluation of the attached sample data showed that a given analyte was not detected in the primary and duplicate sample, further evaluation of the RPD value was not performed. The RPD calculations use the following formula:

$$
R P D=[|M-S| /((M+S) / 2)]^{*} 100
$$

where, $\quad M=$ main sample value $\quad S=$ split (or duplicate) sample value

For quality assurance/quality control (QA/QC) split and duplicate RPD calculations, a value less than $30 \%$ indicates the data compare favorably. For regulatory splits, a threshold of $35 \%$ is used (EPA 1994). If the RPD is greater than $30 \%$ (or $35 \%$ for regulatory split data), further investigation regarding the usability of the data is performed. No split samples were collected for cleanup verification of the subject site. As a matter of good practice, when an analyte is detected in the primary or duplicate sample, but was quantified at less than 5 times the TDL in one or both samples, an additional parameter is evaluated. In this case, if the difference between the primary and duplicate results exceeds a control limit of 2 times the TDL, further assessment regarding the usability of the data is performed. Additional discussion is provided in the data quality assessment section of the applicable RSVP, as necessary.

In addition to the statistical samples collected from the remediation footprint at the subject site, multi-aliquot samples were collected from stockpiles of overburden and other material assumed to be below cleanup levels. Statistical methodology is not applicable to non-statistical sampling, and direct evaluation of maximum detected values within these decision units will be used as the compliance basis. These maximum detected values are presented in the results summary for use in the RSVP. 
CALCULATION SHEET

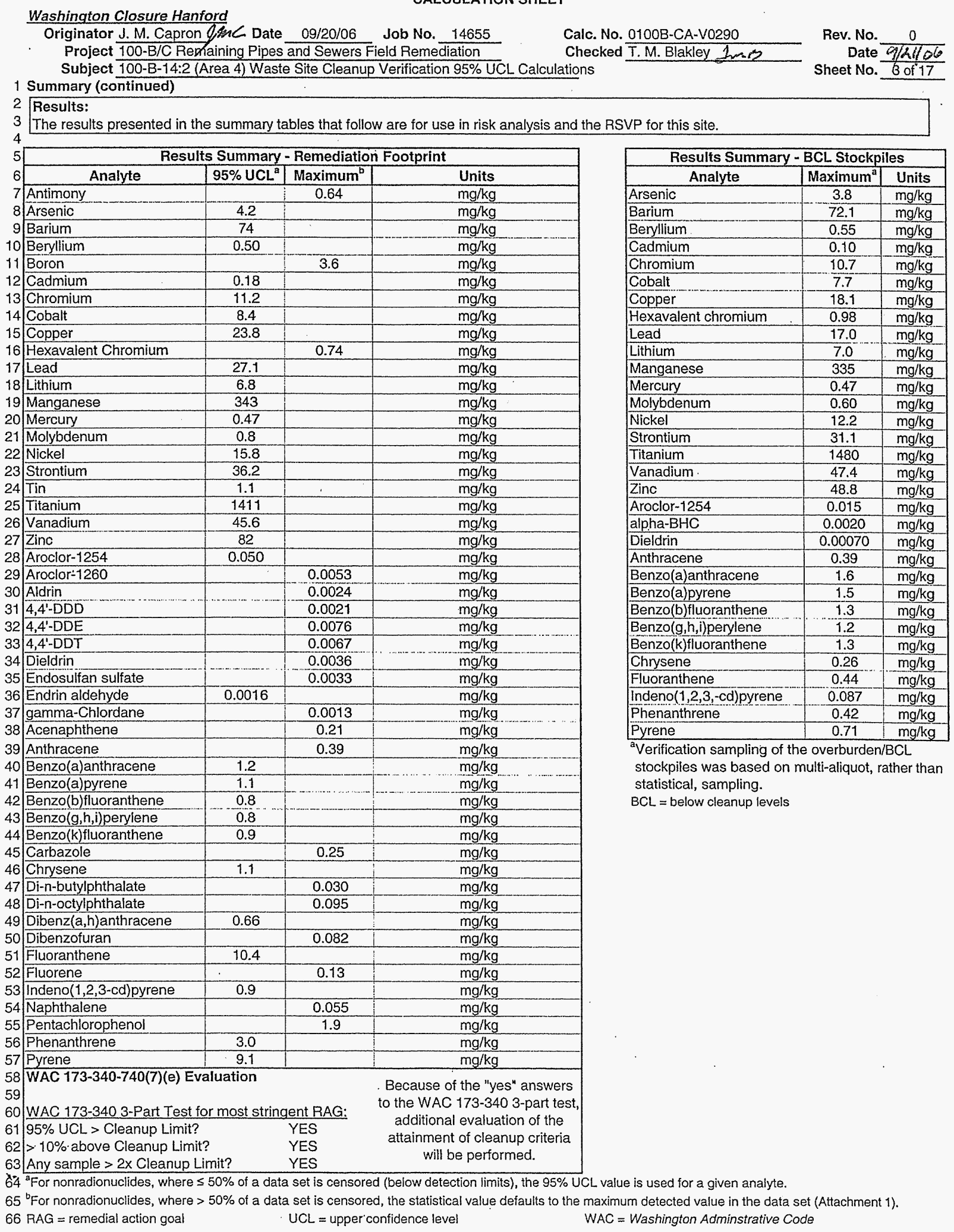


Washington Closure Hanford

\section{CALCULATION SHEET}

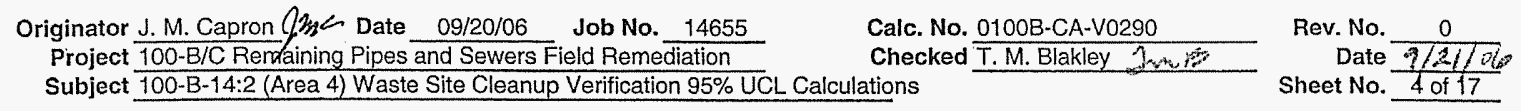

1 Summary (continued)

\begin{tabular}{|c|c|c|c|c|}
\hline \multicolumn{5}{|c|}{ Relative Percent Difference Results ${ }^{\mathrm{a}}$ - QAQC Analysis } \\
\hline & Analyte & $\begin{array}{l}\text { Duplicate } \\
\text { Analysis }\end{array}$ & Analyte & Duplicate Analysis ${ }^{b}$ \\
\hline & $4 \longdiv { \text { Potassium-40 } }$ & $25 \%$ & Manganese & $16 \%$ \\
\hline & 5 Aluminum & $9.6 \%$ & Phosphorus & $22 \%$ \\
\hline & Barium & $4.2 \%$ & Silicon & $5.0 \%$ \\
\hline & 7 Calcium & $8.7 \%$ & Strontium & $5.2 \%$ \\
\hline & 8 Chromium & $6.1 \%$ & Titanium & $12 \%$ \\
\hline & 9 Copper & $30 \%$ & Vanadium & $15 \%$ \\
\hline & 0 Iron & $17 \%$ & Zinc & $8.9 \%$ \\
\hline & 1 Magnesium & $9.6 \%$ & Zirconium & $4.1 \%$ \\
\hline
\end{tabular}

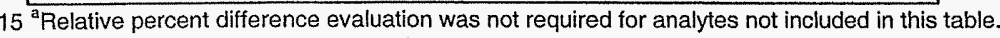

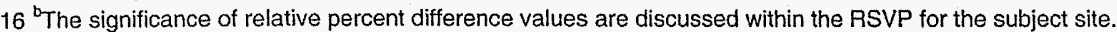

17 QAVQC = quality assurance/quality control

18 RSVP = remaining sites verification package

$19 \mathrm{TDL}=$ target detection limit 
Washington Closure Hanford

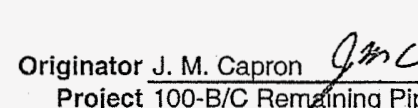

Project 100-B/C Remalining Pipes and Sewers Field Remediation

(1) Calculations
Date $\frac{09 / 20 / 06}{14655}$
Job No.

Calc. No. $\frac{0100 B-C A \cdot V 0290}{}$
Checked T. M. Blakley

Rev. No. $\frac{0}{\text { Date }}$
Sheet No. $\frac{9 / 2106}{15 \text { of } 17}$

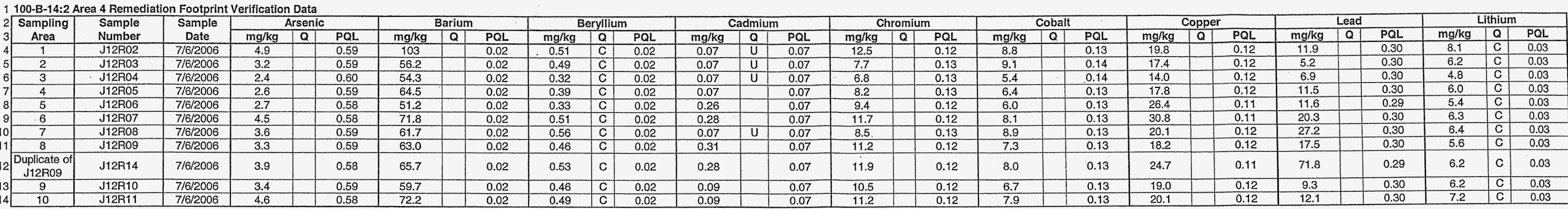

15 Statistical Computation Input Data

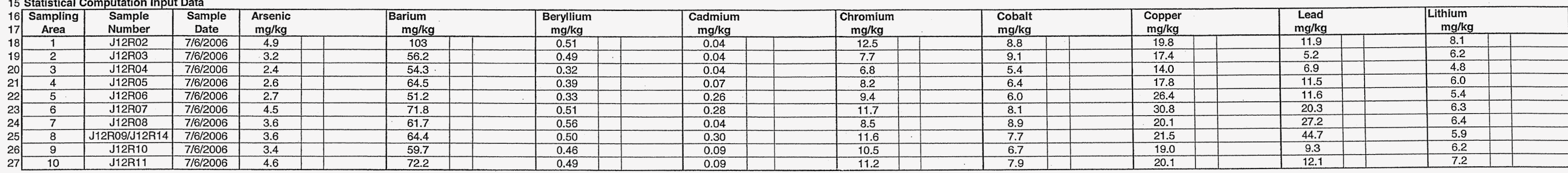

28 Statistical Computations

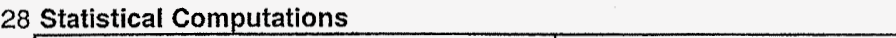

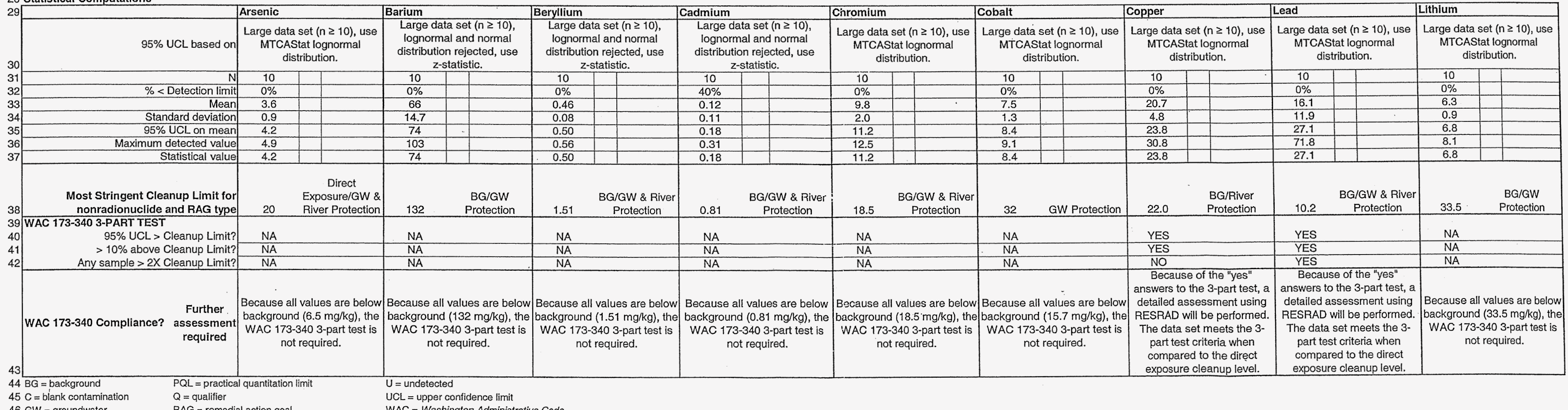

$46 \mathrm{GW}=$ groundwater
$47 \mathrm{NA}=$ not applicabl

$\mathrm{UCL}=$ upper confidence lilit
WAG = quallifier
$R A$ remedial action goal
RESRAD = RESidual RADioactivity (dose assessment model) 

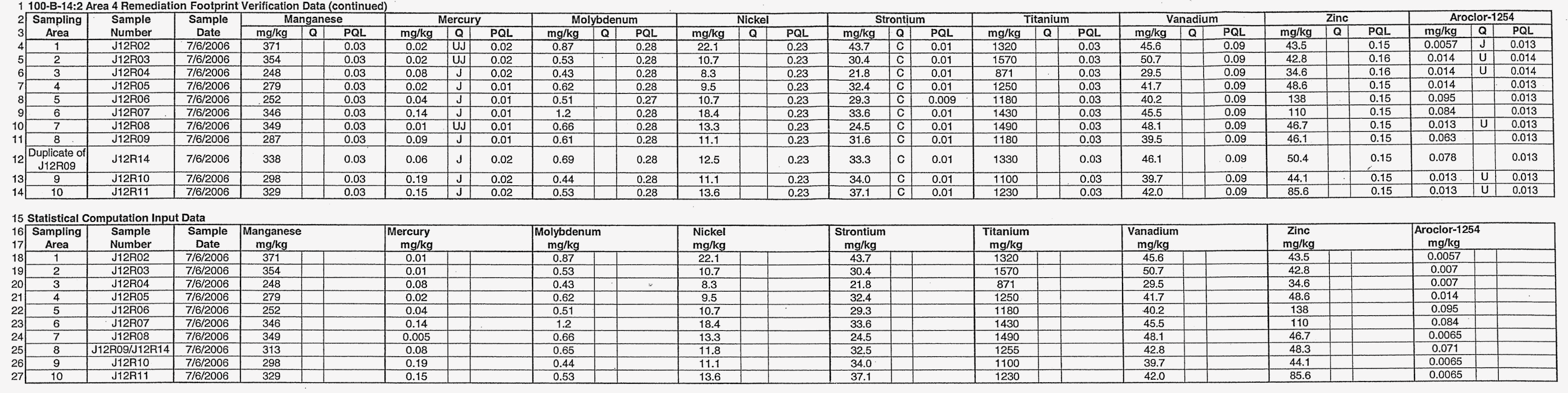

\section{Statistical Computations}

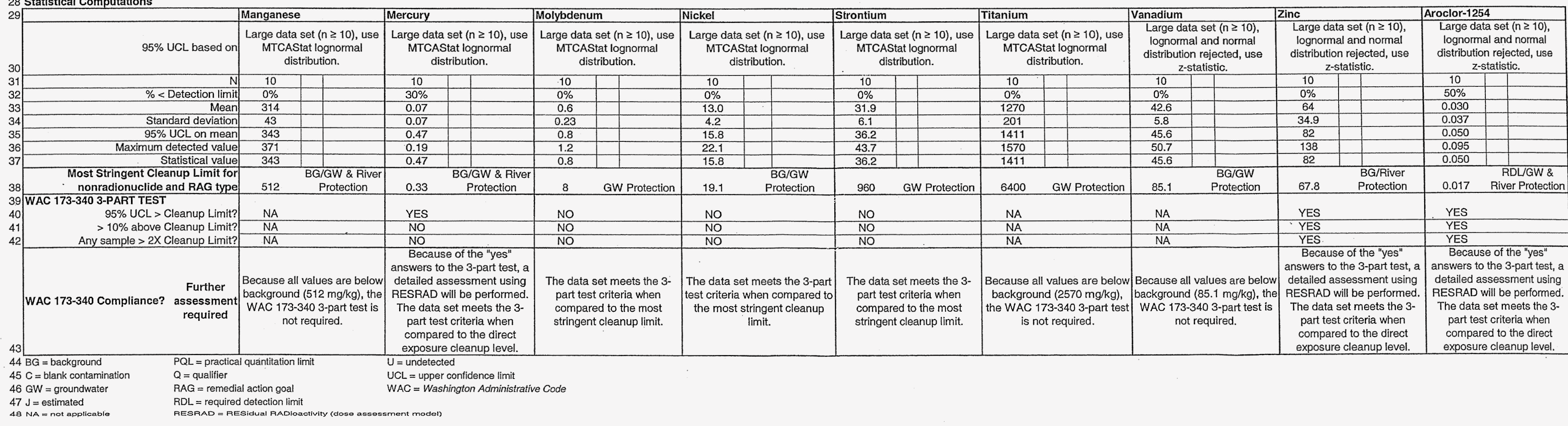




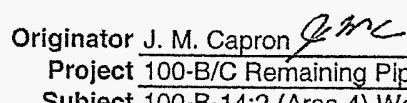

CALCULATION SHEET

Project $100-B / C$ Remaining Pipes and Sewers Field Remediation
Subject $100-B-14: 2$ (Area 4) Waste Site Cleanup Verification 95\% UCL Calculations

Job No. $\frac{09 / 20 / 06}{14655}$

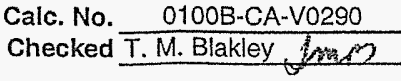

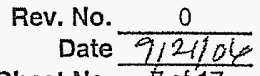

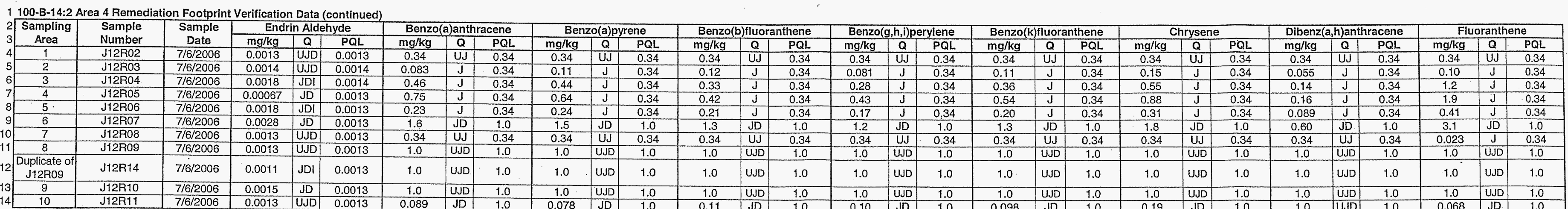

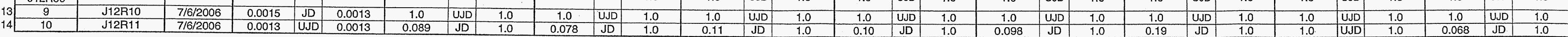

15 Statistical Computation Input Data
16 Sampling Sample

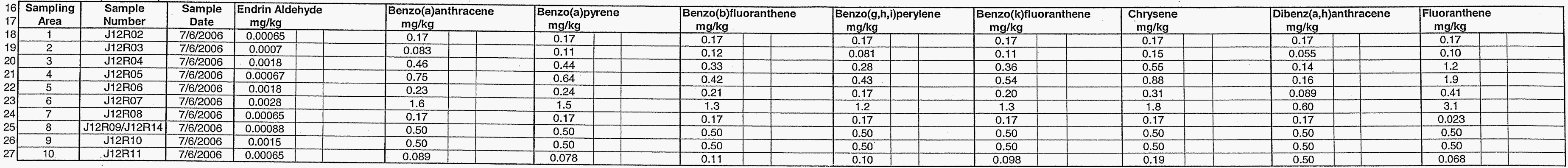

8 Statistical Computations

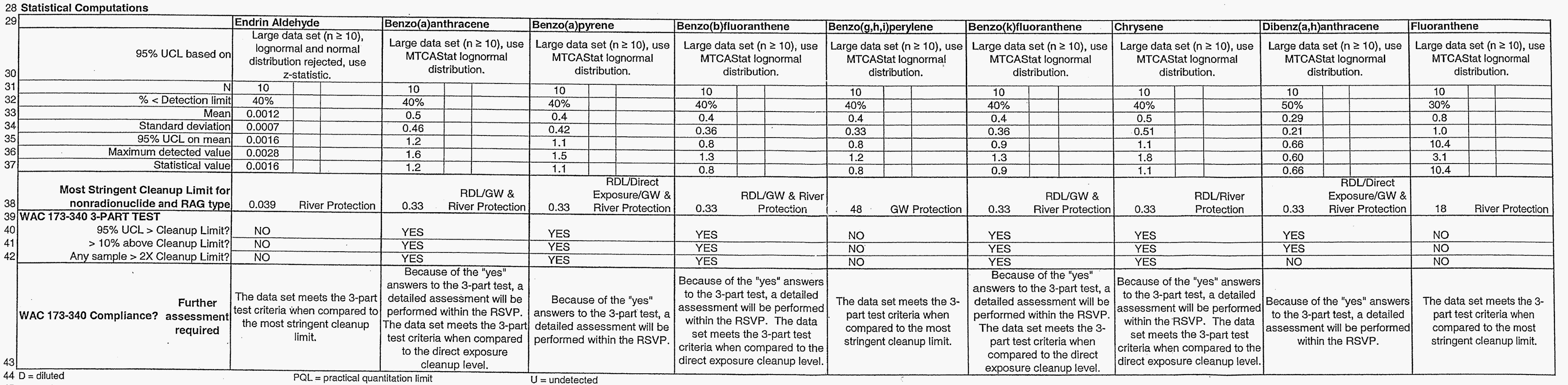

$44 \mathrm{D}=$ diluted
$45 \mathrm{I}$ intererence (on one analyical column)

$45 \mathrm{I}=$ interference
$46 \mathrm{GW}=$ groundwater
$47 \mathrm{~J}=$ estimated

$48 \mathrm{NA}=$ not applicable
$P Q L=$ practic
$Q=$ qualifitier

$\mathrm{RAG}=$ remedial action goal
$\mathrm{RDL}=$ required detection

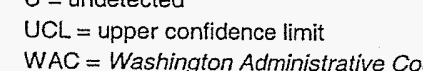




\section{CALCULATION SHEET}

\section{Washington Closure Hanford}

Originator J. M. Capron Q, $32 \mathrm{C}$ Checked T.M. Blakley

Project 100-B/C Remaining Pipes Date

Demers Field Remediation

Subject 100-B-14:2 (Area 4) Waste Site Cleanup Verification 95\% UCL Calculations
Calc. No. $0100 \mathrm{~B}-\mathrm{CA}-\mathrm{V} 0290$

Job No. 14655

1 100-B-14:2 Area 4 Remediation Footprint Verification Data (continued)

\begin{tabular}{|c|c|c|c|c|c|c|c|c|c|c|c|c|}
\hline \multirow{2}{*}{ 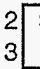 } & \multirow{2}{*}{$\begin{array}{c}\text { Sampling } \\
\text { Area }\end{array}$} & \multirow{2}{*}{$\begin{array}{l}\text { Sample } \\
\text { Number }\end{array}$} & \multirow{2}{*}{$\begin{array}{c}\text { Sample } \\
\text { Date }\end{array}$} & \multicolumn{3}{|c|}{ Indeno(1,2,3-cd)pyrene } & \multicolumn{3}{|c|}{ Phenanthrene } & \multicolumn{3}{|c|}{ Pyrene } \\
\hline & & & & $\mathrm{mg} / \mathrm{kg}$ & $Q$ & $P Q L$ & $\mathrm{mg} / \mathrm{kg}$ & $Q$ & PQL & $\mathrm{mg} / \mathrm{kg}$ & $Q$ & PQL \\
\hline 41 & 1 & J12R02 & $7 / 6 / 2006$ & 0.34 & UJ & 0.34 & 0.34 & UJ & 0.34 & 0.34 & UJ & 0.34 \\
\hline 5 & 2 & J12R03 & $7 / 6 / 2006$ & 0.072 & $\mathrm{~J}$ & 0.34 & 0.035 & $\mathrm{~J}$ & 0.34 & 0.13 & $\mathrm{~J}$ & 0.34 \\
\hline & 3 & $\mathrm{~J} 12 \mathrm{R} 04$ & $7 / 6 / 2006$ & 0.25 & $J$ & 0.34 & 0.74 & $\mathrm{~J}$ & 0.34 & 0.92 & $J$ & 0.34 \\
\hline & 4 & J12R05 & $7 / 6 / 2006$ & 0.42 & $J$ & 0.34 & 1.6 & $\mathrm{~J}$ & 0.34 & 1.5 & $J$ & 0.34 \\
\hline & 5 & J12R06 & $7 / 6 / 2006$ & 0.14 & $\mathrm{~J}$ & 0.34 & 0.27 & $J$ & 0.34 & 0.40 & $J$ & 0.34 \\
\hline & 6 & J12R07 & $7 / 6 / 2006$ & 1.1 & JD & 1.0 & 1.9 & $\mathrm{JD}$ & 1.0 & 3.4 & $\mathrm{JD}$ & 1.0 \\
\hline & 7 & J12R08 & $7 / 6 / 2006$ & 0.34 & UJ & 0.34 & 0.34 & UJ & 0.34 & 0.020 & $\mathrm{~J}$ & 0.34 \\
\hline & 8 & J12R09 & $7 / 6 / 2006$ & 1.0 & UJD & 1.0 & 1.0 & UJD & 1.0 & 1.0 & UJD & 1.0 \\
\hline & $\begin{array}{c}\text { Duplicate of } \\
\mathrm{J} 12 \mathrm{R} 09\end{array}$ & J12R14 & $7 / 6 / 2006$ & 1.0 & UJD & 1.0 & 1.0 & UJD & 1.0 & 1.0 & UJD & 1.0 \\
\hline & 9 & J12R10 & $7 / 6 / 2006$ & 1.0 & UJD & 1.0 & 1.0 & UJD & 1.0 & 1.0 & UJD & 1.0 \\
\hline & 10 & J12R11 & $7 / 6 / 2006$ & 0.054 & $\mathrm{JD}$ & 1.0 & 1.0 & UJD & 1.0 & 0.067 & JD & 1.0 \\
\hline
\end{tabular}

15 Statistical Computation Input Data

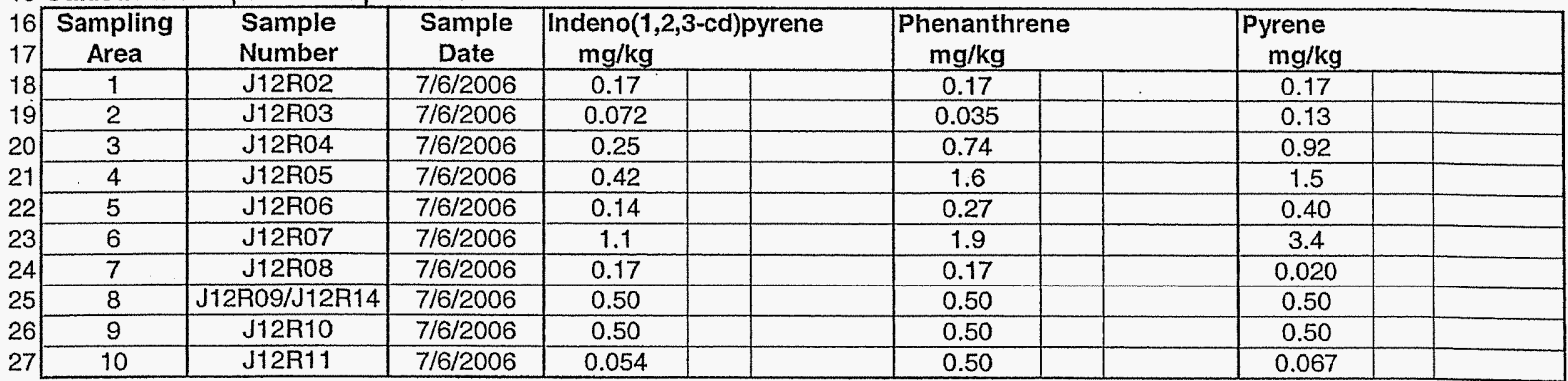

28 Statistical Computations

\begin{tabular}{|c|c|c|c|c|c|c|c|}
\hline \multirow[t]{2}{*}{29} & & \multicolumn{2}{|c|}{ Indeno(1,2,3-cd)pyrene } & \multicolumn{2}{|c|}{ Phenanthrene } & \multicolumn{2}{|l|}{ Pyrene } \\
\hline & $95 \%$ UCL based on & \multicolumn{2}{|c|}{$\begin{array}{l}\text { Large data set }(n \geq 10) \text {, use } \\
\text { MTCAStat lognormal } \\
\text { distribution. }\end{array}$} & \multicolumn{2}{|c|}{$\begin{array}{l}\text { Large data set }(n \geq 10) \text {, use } \\
\text { MTCAStat lognormal } \\
\text { distribution. }\end{array}$} & \multicolumn{2}{|c|}{$\begin{array}{l}\text { Large data set }(n \geq 10) \text {, use } \\
\text { MTCAStat lognormal } \\
\text { distribution. }\end{array}$} \\
\hline 31 & $\mathrm{~N}$ & 10 & & 10 & & 10 & \\
\hline 32 & $\%<$ Detection limit & $40 \%$ & & $50 \%$ & & $30 \%$ & \\
\hline 33 & Mean & 0.3 & & 0.6 & & 0.8 & \\
\hline 34 & Standard deviation & 0.32 & & 0.63 & & 1.0 & \\
\hline 35 & $95 \%$ UCL on mean & 0.9 & & 3.0 & & 9.1 & \\
\hline 36 & Maximum detected value & 1.1 & & 1.9 & & 3.4 & \\
\hline 37 & Statistical value & 0.9 & & 3.0 & & 9.1 & \\
\hline 38 & $\begin{array}{l}\text { Most Stringent Cleanup Limit for } \\
\text { nonradionuclide and RAG type }\end{array}$ & 0.33 & $\begin{array}{c}\text { RDL/GW \& River } \\
\text { Protection }\end{array}$ & 240 & GW Protection & 48 & GW Protection \\
\hline $\begin{array}{l}39 \\
40\end{array}$ & $\begin{array}{r}\text { WAC 173-340 3-PART TEST } \\
\text { 95\% UCL > Cleanup Limit? }\end{array}$ & YES & & NO & & NO & \\
\hline 41 & $>10 \%$ above Cleanup Limit? & YES & & NO & & NO & \\
\hline 42 & Any sample $>2 X$ Cleanup Limit? & YES & & NO & & No & \\
\hline 43 & WAC 173-340 Compliance? $\begin{array}{c}\begin{array}{c}\text { Further } \\
\text { assessment } \\
\text { required }\end{array} \\
\text { requat }\end{array}$ & $\begin{array}{l}\text { Because } \\
\text { to the } 3 \\
\text { assessm } \\
\text { within } t \\
\text { set } m \\
\text { criteria } \\
\text { direct ex }\end{array}$ & $\begin{array}{l}\text { the "yes" answers } \\
\text { tt test, a detailed } \\
\text { will be performed } \\
\text { RSVP. The data } \\
\text { the 3-part test } \\
\text { compared to the } \\
\text { ure cleanup level. }\end{array}$ & $\begin{array}{l}\text { The data } \\
\text { test crit } \\
\text { to the } m\end{array}$ & $\begin{array}{l}\text { meets the 3-part } \\
\text { when compared } \\
\text { stringent cleanup } \\
\text { limit. }\end{array}$ & $\begin{array}{l}\text { The da } \\
\text { part } \\
\text { comp } \\
\text { string }\end{array}$ & $\begin{array}{l}\text { et meets the 3- } \\
\text { criteria when } \\
\text { d to the most } \\
\text { cleanup limit. }\end{array}$ \\
\hline 44 & $\mathrm{PQL}=$ practica & 1 quantifati & & RSVP $=$ & ining sites & on packag & \\
\hline $\begin{array}{l}45 \\
46\end{array}$ & $\begin{array}{l}\text { GW = groundwater } \\
\mathrm{J}=\text { estimated }\end{array}$ & al action go & & $\begin{array}{l}U=\text { unde } \\
U C L=\text { up }\end{array}$ & $\begin{array}{l}\text { d } \\
\text { confidence limit }\end{array}$ & & \\
\hline 47 & $\mathrm{NA}=$ not applicable & detection & & $W A C=V$ & ington Administrativ & e Code & \\
\hline
\end{tabular}


Washington Closure Hanford

CALCULATION SHEET

Priginator J.M. Capron 1 Im Project 100-B/C Rehraining Pipes and Sewers Field Remediation
Subject 100-B-14:2 (Area 4) Waste Site Cleanup Verification 95\% UCL Calculations

Date $09 / 20 / 06$

Calc. No. $0100 B-$ CA-V0290
Checked T. M. Blakiey $\ln$ mo

Rev. No. $\frac{0}{9|2 i| 0 \mid}$
Date

Sheet No. $\frac{9 / 21 / 06}{90 t 17}$

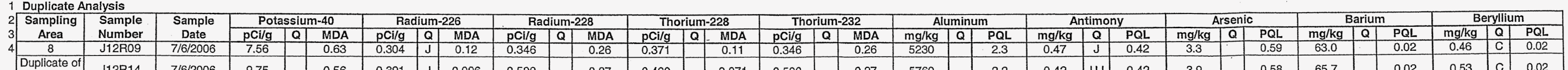

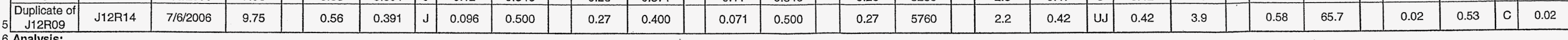

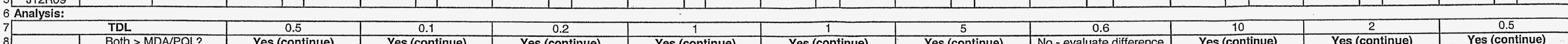

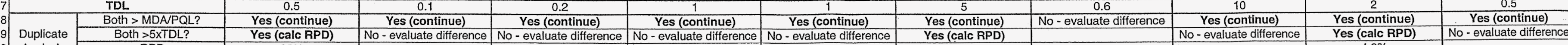

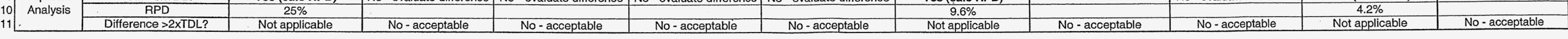

12

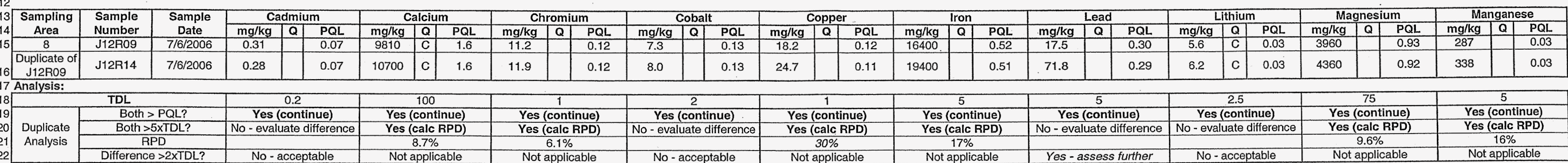

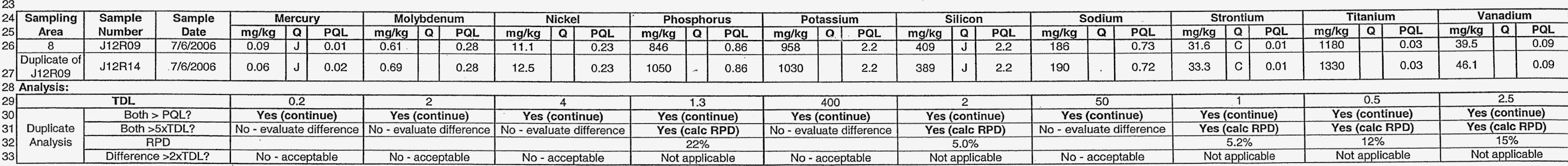

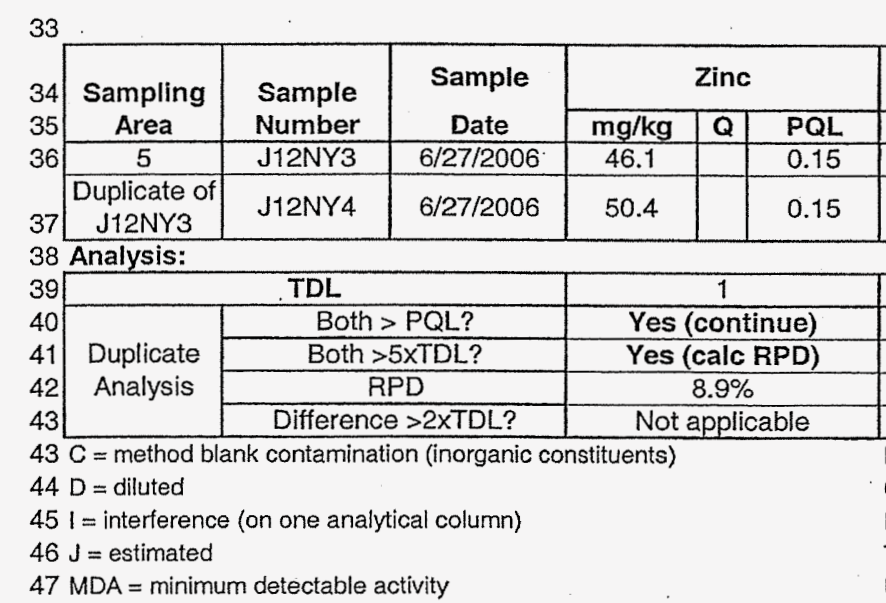

$46 J=$ estimated

47 MDA = minimum detectable activity

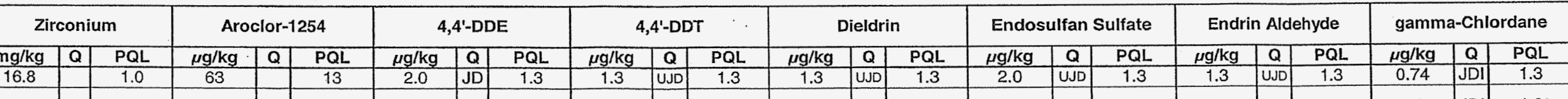
\begin{tabular}{|l|c|c|c|c|c|c|c|c|c|c|c|c|c|c|c|c|c|c|c|c|c|c|c|}
\hline 16.8 & 1.0 & 63 & 13 & 2.0 & $\mathrm{JD}$ & 1.3 & 1.3 & $\mathrm{UDD}$ & 1.3 & 1.3 & $\mathrm{UD}$ & 1.3 & 2.0 & $\mathrm{UD}$ & 1.3 & 1.3 & UDD & 1.3 & 0.74 & $\mathrm{JDI}$ & 1.3 \\
\hline 17.5 & & 1.0 & 78 & & 13 & 2.3 & $\mathrm{JD}$ & 1.3 & 6.7 & $\mathrm{JD}$ & 1.3 & 3.6 & $\mathrm{JD}$ & 1.3 & 3.3 & $\mathrm{JD}$ & 1.3 & 1.1 & $\mathrm{JDI}$ & 1.3 & 1.3 & $\mathrm{JDI}$ & 1.3 \\
\hline
\end{tabular} \begin{tabular}{|c|c|c|c|c|c|c|c|}
\hline 2.5 & 16.5 & 3.3 & 3.3 & 3.3 & 3.3 & 3.3 & 16.5 \\
\hline Yes (continue) & Yes (continue) & Yes (continue) & No-evaluate difference & No-evaluate difference & Yes (continue) & No- evaluate difference & No- evaluate difference \\
Yes (calc RPD) & No- evaluate difference & No- evaluate difference & & No- evaluate difference & &
\end{tabular} \begin{tabular}{|c|c|c|c|c|c|c|}
\hline $\begin{array}{c}4.1 \% \\
\text { Not aplicable }\end{array}$ No-acceptable & No-acceptable & No-acceptable & No-acceptable & No-acceptable & No-acceptable & No-acceptable \\
\hline
\end{tabular} $Q=$ qualifier $\mathrm{RPD}=$ relative percent difference
$\mathrm{TDL}=$ target detection limit 
Washington Closure Hanford

CALCULATION SHEET

Originator J.M. Capron

09/20/06 Calc. No. 0100B-CA-V0290 Project 100-B/C Renaining Pipes and Sewers Field Remediation Job No. $\frac{14655}{100}$ Checked T.M. Blakley $2 \times 1 / 3$ Subject 100-B-14:2 (Area 4) Waste Site Cleanup Verification 95\% UCL Calculation

Ecology Software (MTCAStat) Results

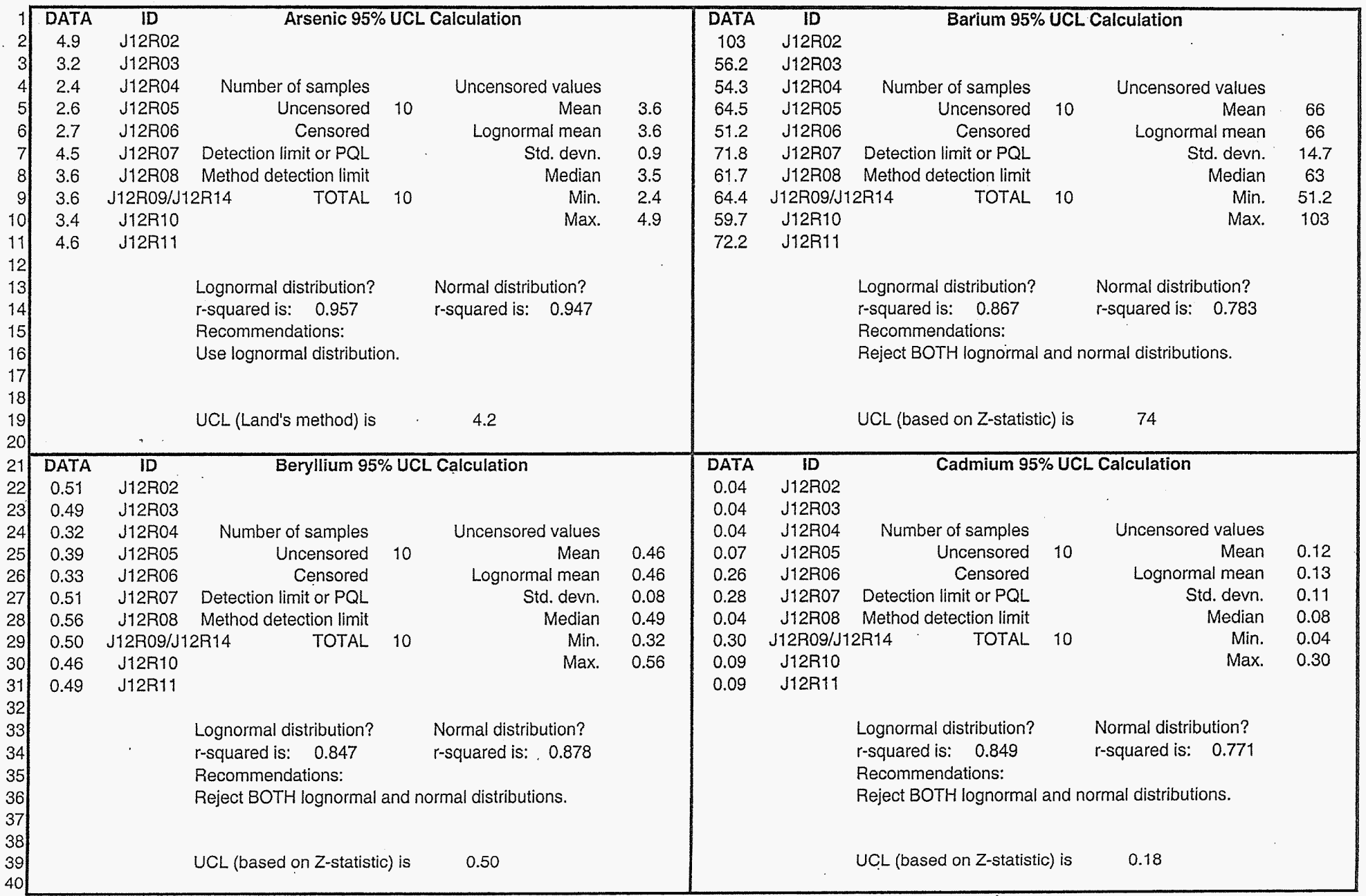

Rev. No. $\quad 0$

Date $\frac{0}{1 / 21 / 06}$

Sheet No. 10 of 17 
Washington Closure Hanford

CALCULATION SHEET

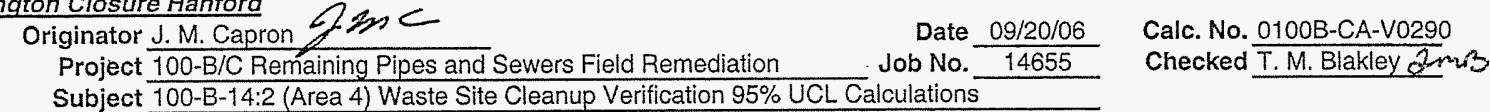

Rev. No. $\quad 0$

Date $2121 / 06$

Ecology Software (MTCAStat) Results

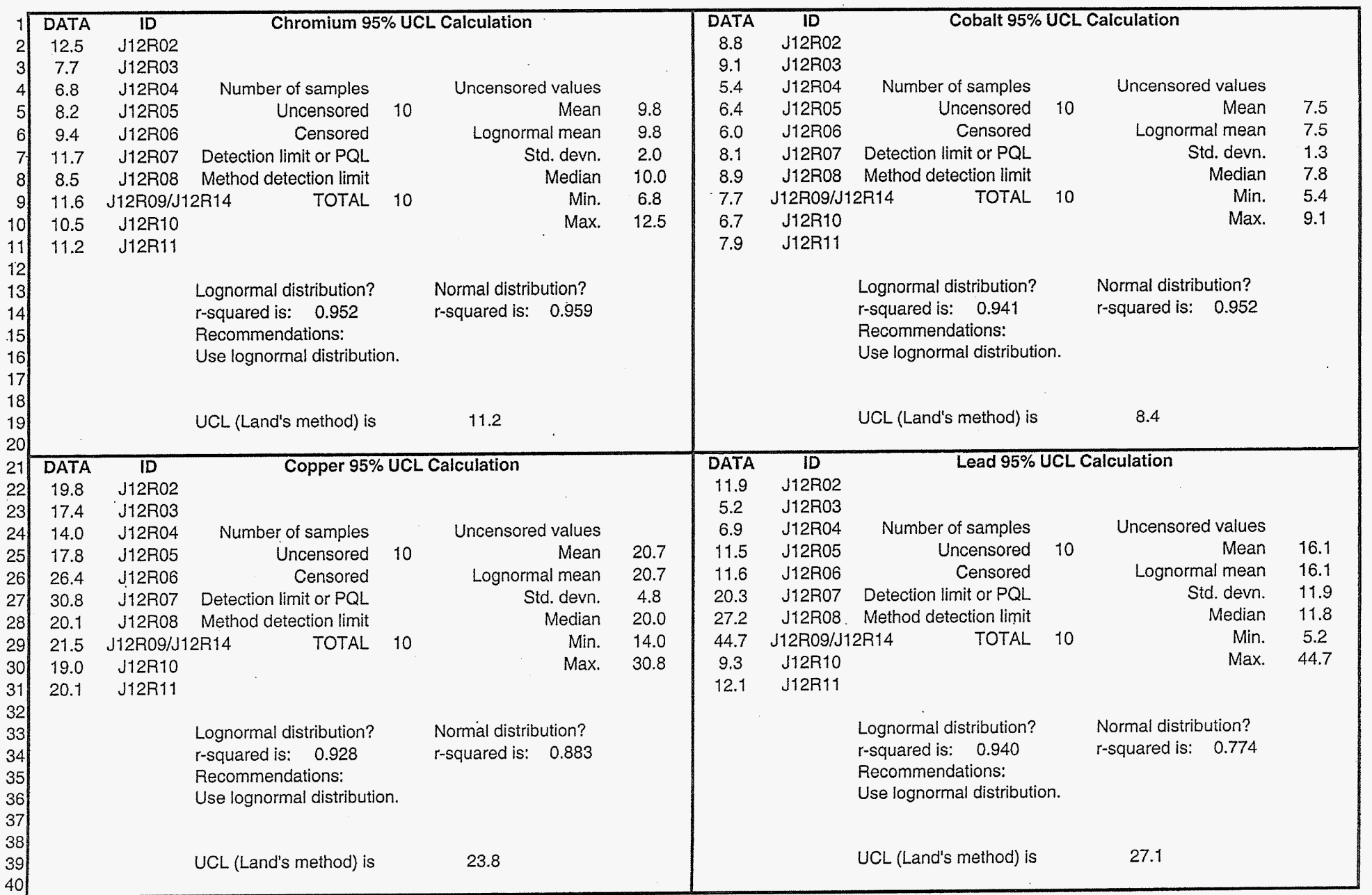


Originator $\mathrm{M}$ Capron libe

Date $09 / 20 / 06$ Job No. 14655

Project $100-B / C$ Remaining Pipes and Sewers Field Remediation Subject 100-B-14:2 (Area 4) Waste Site Cleanup Verification 95\% UCL Calculations

Calc. No. 0100B-CA-V0290 Checked T.M. Blakley In

Ecology Software (MTCAStat) Results

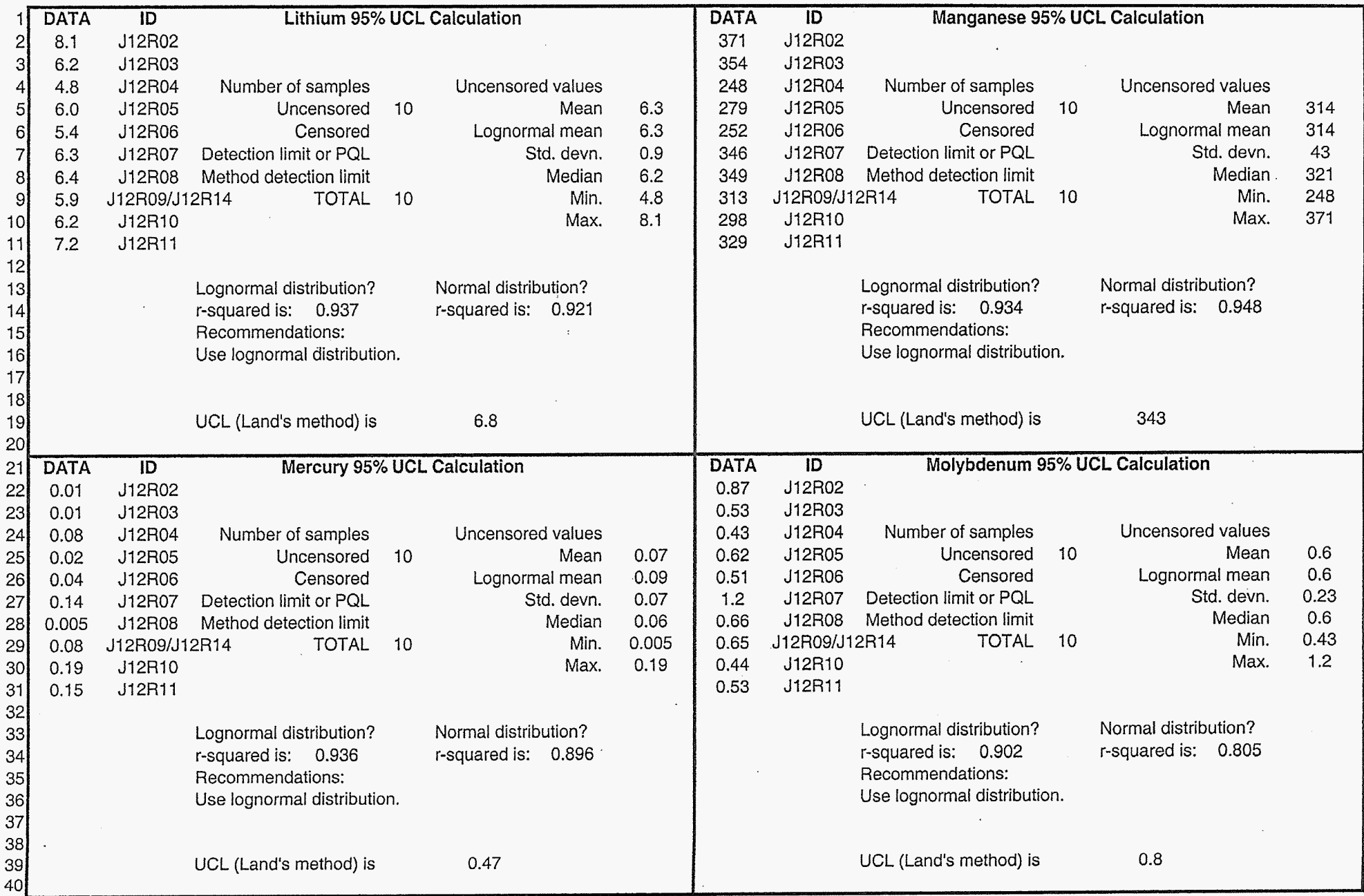

Rev. No. 0

Date $7 / 2 / 106$

Sheet No. 2 of 17 
Originator J. M. Capron $\varphi \notin$

Date $09 / 20 / 06$

Project 100-B/C Rentaining Pipes and Sewers Field Remediation

Job No. 14655

Calc. No. 0100B-CA-V0290

Subject 100-B-14:2 (Area 4) Waste Site Cleanup Verification 95\% UCL Calculation

Ecology Software (MTCAStat) Results

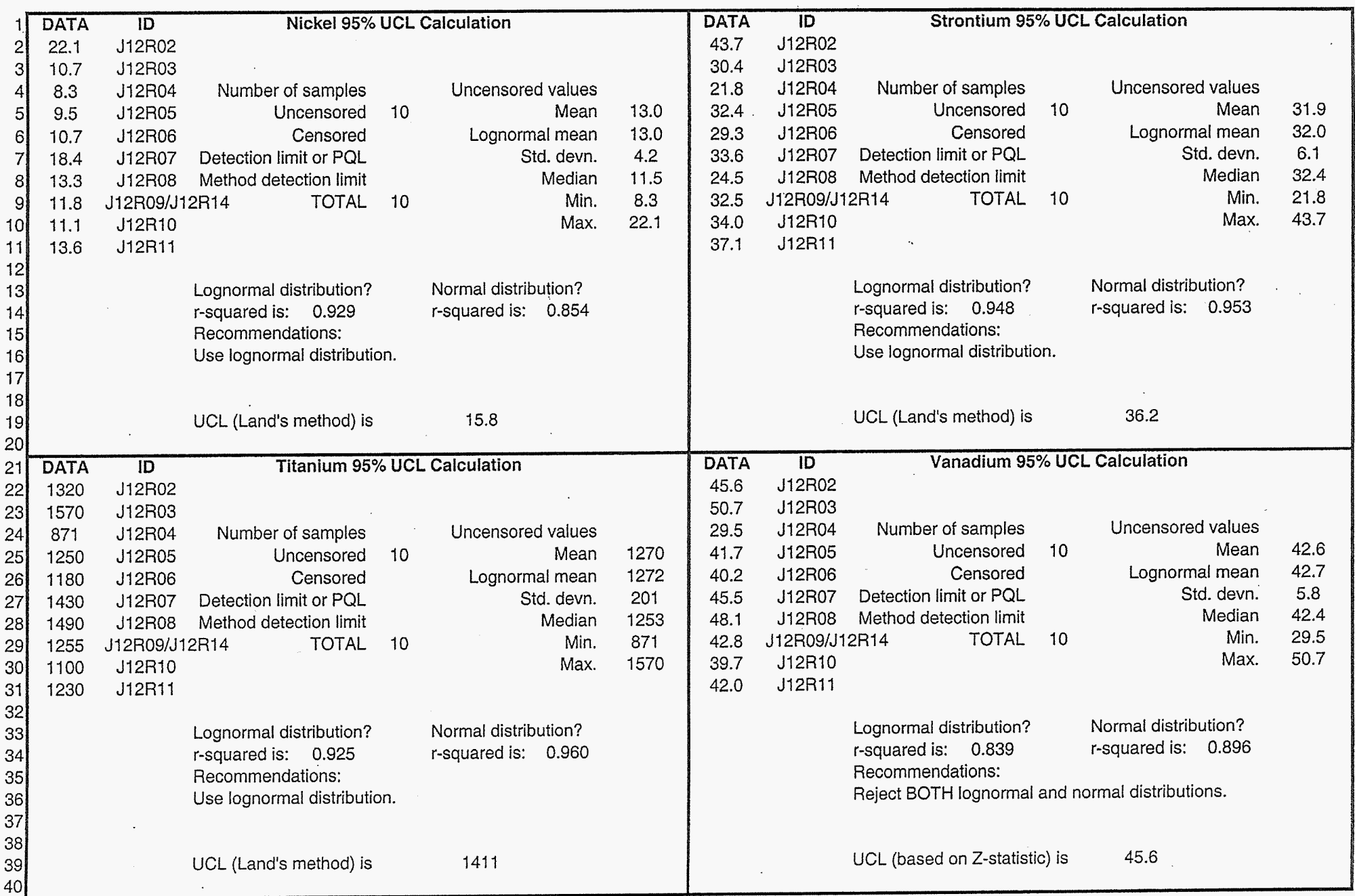


CALCULATION SHEET

Originator J.M. Capron

Project 100-B/C Remaining Pipes and Sewers Field $R$ enedalon

Date $\frac{09 / 20 / 06}{14655}$
Job No.

Calc. No. $0100 \mathrm{~B}-\mathrm{CA}-\mathrm{V} 0290$

Subject 100-B-14:2 (Area 4) Waste Site Cleanup Verification 95\% UCL Calculation

Rev. No.

Date $7 / 2 / 01$

Sheet No. $1 / 4$ of 17

Ecology Software (MTCAStat) Results

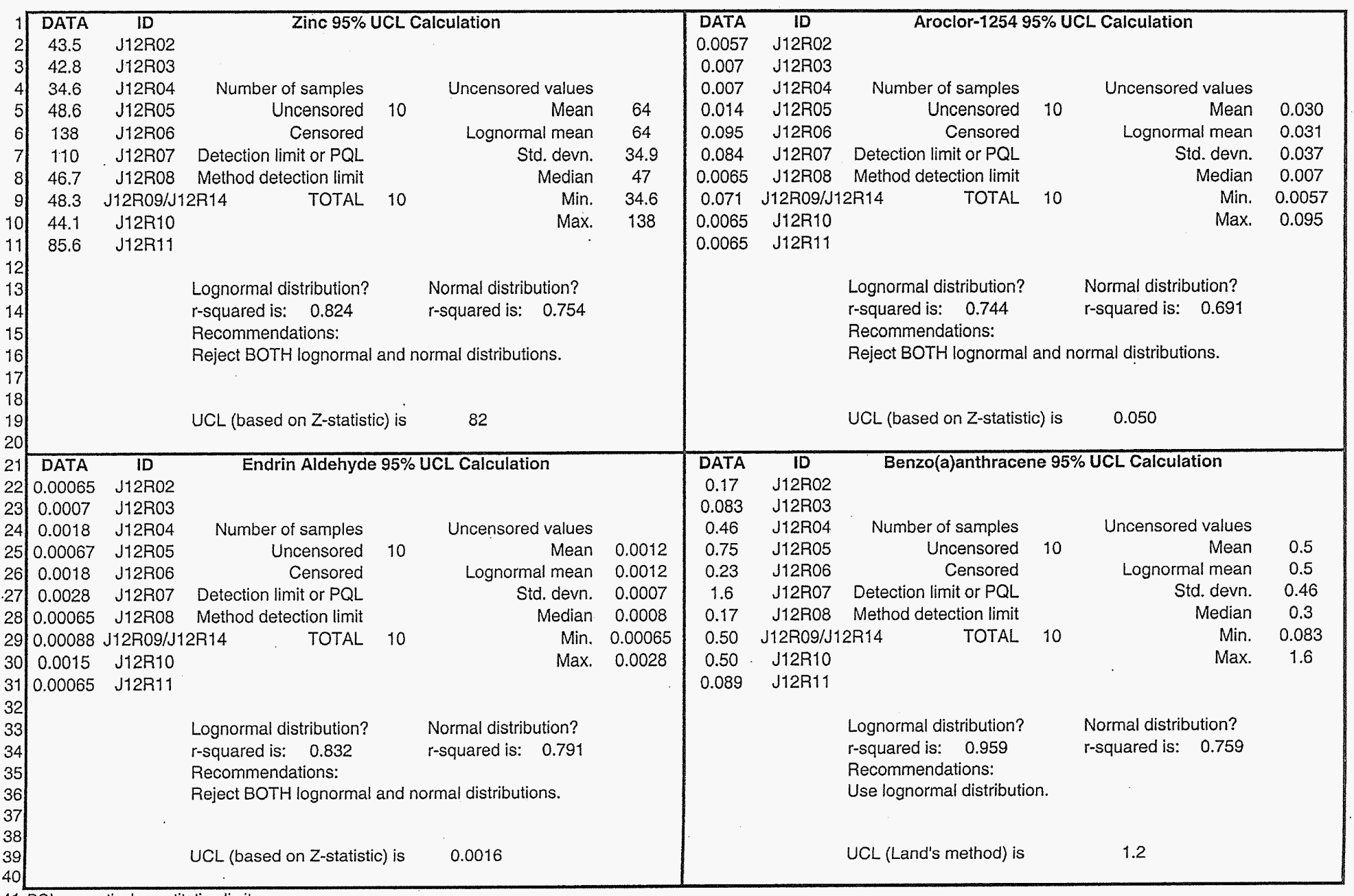



Originator J.M. Capron
Project 100 -B/C Renaining Pipes and Sewers Field Remediation $\begin{gathered}\text { Date } \\ \text { Job No. } \frac{09 / 20 / 06}{14655} \text { Calc. No. 0100B-CA-V0290 }\end{gathered}$

Subject 100-B-14:2 (Area 4) Waste Site Cleanup Verification 95\% UCL Calculation

Ecology Software (MTCAStat) Results

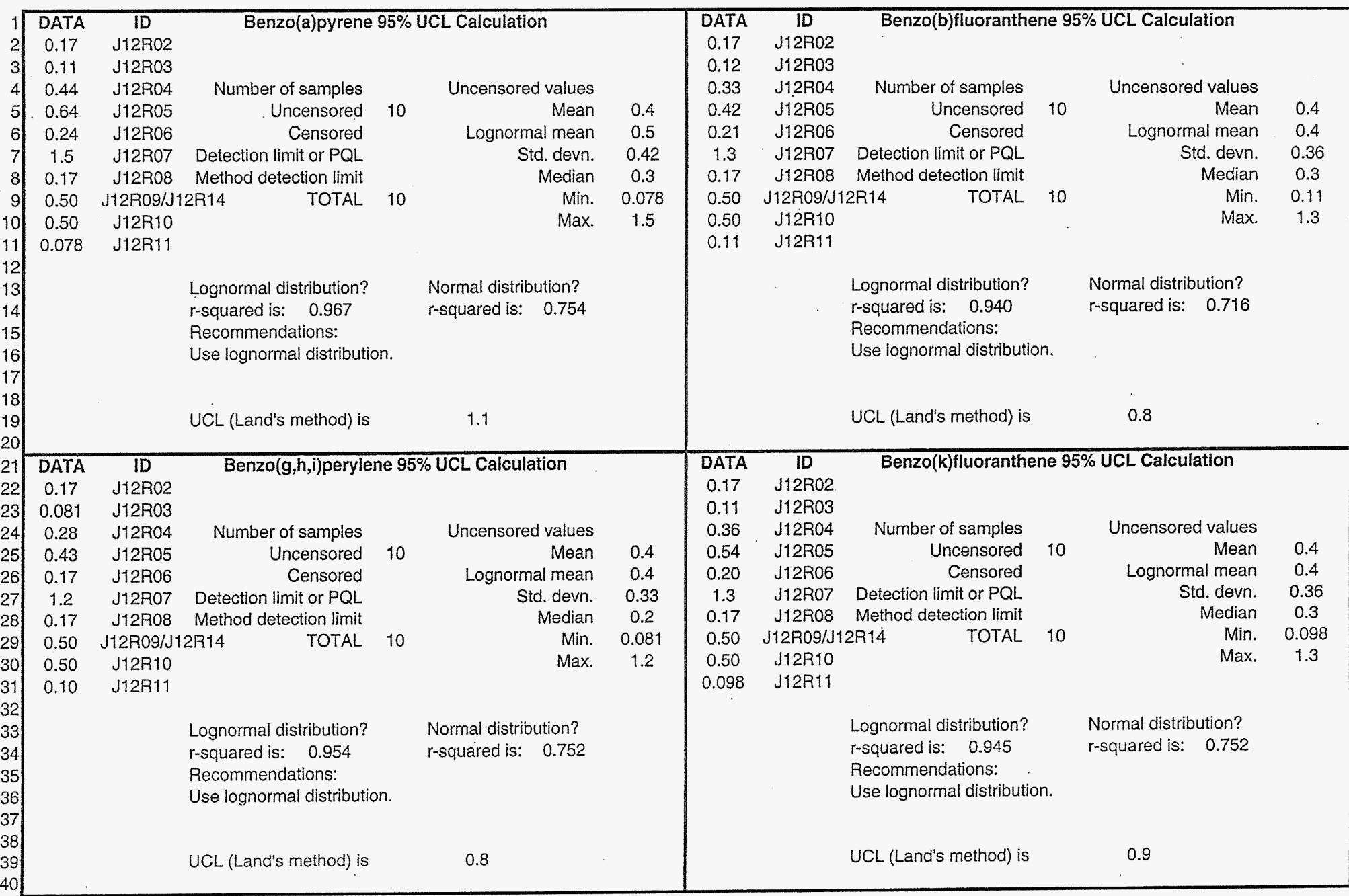


CALCULATION SHEET

Originator J.M. Capron 4 zac

Project $100-\mathrm{B} / \mathrm{C}$ Remaining Pipes and Sewers Field Remediation

Date $\frac{09 / 20 / 06}{14655}$
Job No.

146

Calc. No. 0100B-CA-V0290

Subject 100-B-14:2 (Area 4) Waste Site Cleanup Verification 95\% UCL Calculations

Ecology Software (MTCAStat) Results

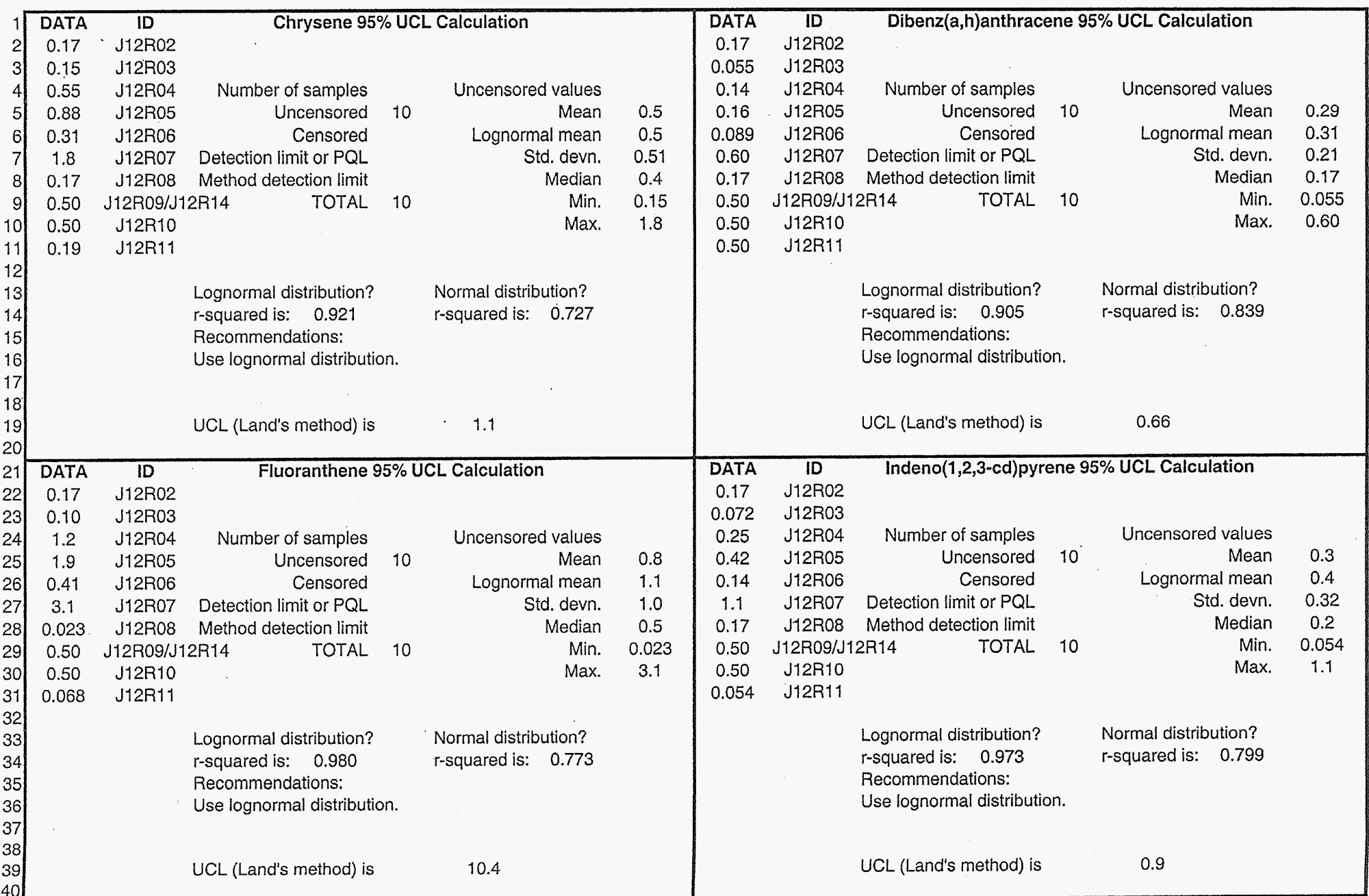


CALCULATION SHEET

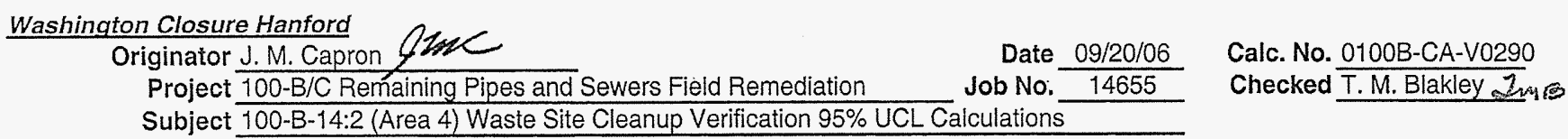
Subject 100-B-14:2 (Area 4) Waste Site Cleanup Verification 95\% UCL Calculations

Sheet No. 1t of 17

Ecology Software (MTCAStat) Results

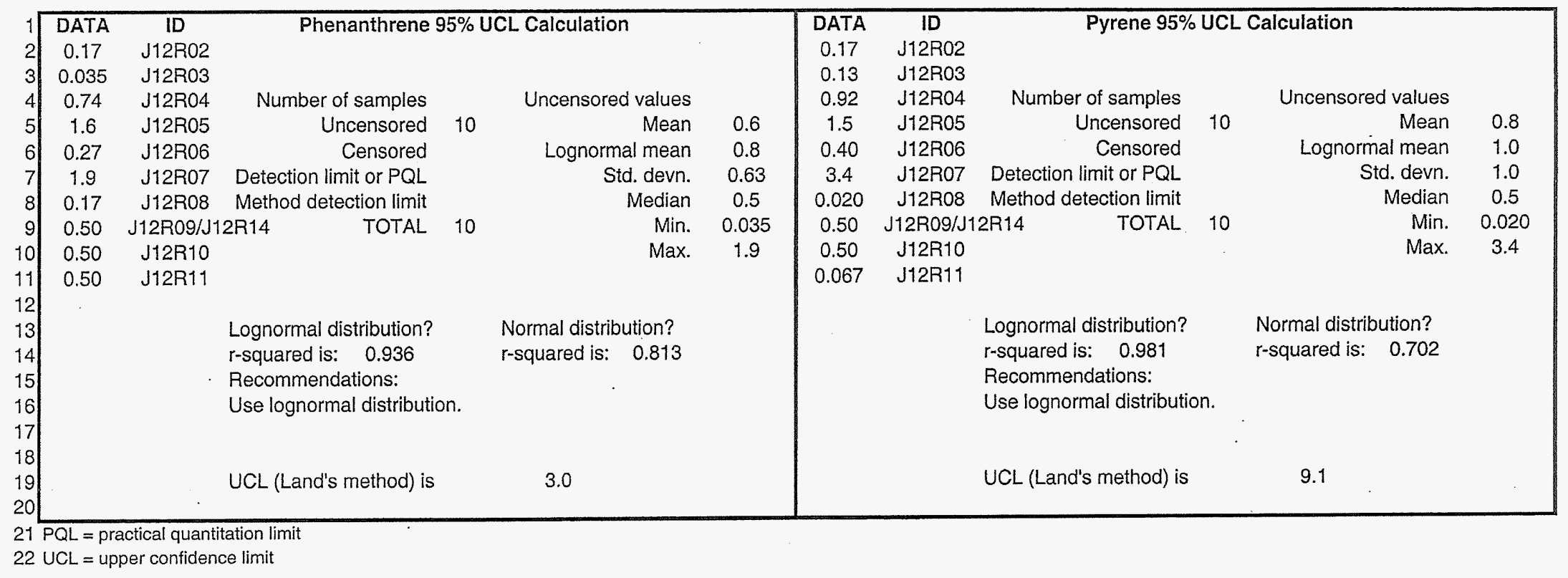


Attachment 1. 100-B-14:2 Area 4 Verification Sampling Results.

\begin{tabular}{|c|c|c|c|c|c|c|c|c|c|c|c|c|c|c|c|c|c|}
\hline \multirow{2}{*}{ Sample Location } & \multirow{2}{*}{$\begin{array}{l}\text { Sample } \\
\text { Number }\end{array}$} & \multirow{2}{*}{$\begin{array}{c}\text { Sample } \\
\text { Date }\end{array}$} & \multicolumn{3}{|c|}{ Americium-241 } & \multicolumn{3}{|c|}{ Cesium-137 } & \multicolumn{3}{|c|}{ Cobalt- 60} & \multicolumn{3}{|c|}{ Europium-152 } & \multicolumn{3}{|c|}{ Europium-154 } \\
\hline & & & $\mathrm{pCi} / \mathrm{g}$ & $Q$ & $\mathrm{MDA}$ & $\mathrm{pCi} / \mathrm{g}$ & $Q$ & MDA & $\mathrm{pCi} / \mathrm{g}$ & $Q$ & MDA & $\mathrm{pCi} / \mathrm{g}$ & $Q$ & $\mathrm{MDA}$ & $\mathrm{pCi} / \mathrm{g}$ & $Q$ & $\mathrm{MDA}$ \\
\hline 1 & $\mathrm{~J} 12 \mathrm{R} 02$ & $7 / 6 / 06$ & 0.28 & $\mathrm{U}$ & 0.28 & 0.078 & $\mathrm{U}$ & 0.078 & 0.085 & $\mathrm{U}$ & 0.085 & 0.20 & $\mathrm{U}$ & 0.20 & 0.26 & $\mathrm{U}$ & 0.26 \\
\hline 2 & J12R03 & $7 / 6 / 06$ & 0.23 & $\mathrm{U}$ & 0.23 & 0.063 & $\mathrm{U}$ & 0.063 & 0.072 & $\mathrm{U}$ & 0.072 & 0.16 & $\mathrm{U}$ & 0.16 & 0.21 & $\mathrm{U}$ & 0.21 \\
\hline 4 & J12R05 & $7 / 6 / 06$ & 0.042 & $\mathrm{U}$ & 0.042 & 0.047 & $\mathrm{U}$ & 0.047 & 0.053 & $\mathrm{U}$ & 0.053 & 0.11 & $\mathrm{U}$ & 0.11 & 0.15 & $\mathrm{U}$ & 0.15 \\
\hline 5 & J12R06 & $7 / 6 / 06$ & 0.045 & $U$ & 0.045 & 0.048 & $\mathrm{U}$ & 0.048 & 0.044 & $\mathrm{U}$ & 0.044 & 0.12 & $\mathrm{U}$ & 0.12 & 0.17 & $\mathrm{U}$ & 0.17 \\
\hline 6 & J12R07 & $7 / 6 / 06$ & 0.052 & U & 0.052 & 0.046 & $\mathrm{U}$ & 0.046 & 0.065 & $\mathrm{U}$ & 0.065 & 0.15 & $U$ & 0.15 & 0.17 & $U$ & 0.17 \\
\hline 7 & J12R08 & $7 / 6 / 06$ & 0.25 & $\mathrm{U}$ & 0.25 & 0.069 & $\mathrm{U}$ & 0.069 & 0.084 & $\mathrm{U}$ & 0.084 & 0.16 & $\mathrm{U}$ & 0.16 & 0.24 & $U$ & 0.24 \\
\hline 8 & J12R09 & $7 / 6 / 06$ & 0.22 & $\mathrm{U}$ & 0.22 & 0.068 & $\mathrm{U}$ & 0.068 & 0.079 & $\mathrm{U}$ & 0.079 & 0.16 & $U$ & 0.16 & 0.21 & $\mathrm{U}$ & 0.21 \\
\hline $\begin{array}{l}\text { Duplicate of } \\
\text { J12R09 }\end{array}$ & J12R14 & $7 / 6 / 06$ & 0.052 & U & 0.052 & 0.053 & U & 0.053 & 0.067 & $\mathrm{U}$ & 0.067 & 0.14 & $U$ & 0.14 & 0.19 & $U$ & 0.19 \\
\hline 9 & $\mathrm{~J} 12 \mathrm{R} 10$ & $7 / 6 / 06$ & 0.096 & $\mathrm{U}$ & 0.096 & 0.026 & $\mathrm{U}$ & 0.026 & 0.028 & $\mathrm{U}$ & 0.028 & 0.064 & $U$ & 0.064 & 0.087 & $\mathrm{U}$ & 0.087 \\
\hline 10 & J12R11 & $7 / 6 / 06$ & 0.091 & $\mathrm{U}$ & 0.091 & 0.099 & $\mathrm{U}$ & 0.099 & 0.15 & $\mathrm{U}$ & 0.15 & 0.15 & $\mathrm{U}$ & 0.15 & 0.39 & $\mathrm{U}$ & 0.39 \\
\hline East overburden & J12R12 & $7 / 6 / 06$ & 0.093 & $\bar{U}$ & 0.093 & 0.059 & $\mathrm{U}$ & 0.059 & 0.032 & $\mathrm{U}$ & 0.032 & 0.066 & U & 0.066 & 0.084 & U & 0.084 \\
\hline West overburden & J12R13 & $7 / 6 / 06$ & 0.019 & $\mathrm{U}$ & 0.019 & 0.017 & $\mathrm{U}$ & 0.017 & 0.023 & $\mathrm{U}$ & 0.023 & 0.051 & $\mathrm{U}$ & 0.051 & 0.066 & $\mathrm{U}$ & 0.066 \\
\hline
\end{tabular}

Note: The following abbreviations apply to all Attachment 1 tables.

Note: Data qualified with C, D, I and/or J are considered acceptable values.

$\mathrm{C}=$ method blank contamination (inorganic constituents)

$\mathrm{MDA}=$ minimum detectable activity

$\mathrm{D}=$ diluted

I = interference on one analytical column

$\mathrm{PQL}=$ practical quantitation limit

$\gamma=$ estimated

$\mathrm{Q}=$ qualifier

$\mathrm{U}=$ undetected

Attachment

Originator

Checked

Calc. No.

\begin{tabular}{|c|c|c|}
\hline J. M. Capron & Date & $\frac{10113}{09 / 20 / 06}$ \\
\hline T. M. Blakley JMB & Date & 9131106 \\
\hline 0100B-CA-V0290 & Rev. No. & \\
\hline
\end{tabular}


Attachment 1. 100-B-14:2 Area 4 Verification Sampling Results.

\begin{tabular}{|c|c|c|c|c|c|c|c|c|c|c|c|c|c|c|c|c|c|}
\hline \multirow{2}{*}{ Sample Location } & \multirow{2}{*}{$\begin{array}{l}\text { Sample } \\
\text { Number }\end{array}$} & \multirow{2}{*}{$\begin{array}{l}\text { Sample } \\
\text { Date }\end{array}$} & \multicolumn{3}{|c|}{ Europium-155 } & \multicolumn{3}{|c|}{ Potassium- 40} & \multicolumn{3}{|c|}{ Radium-226 } & \multicolumn{3}{|c|}{ Radium-228 } & \multicolumn{3}{|c|}{ Thorium-228 } \\
\hline & & & $\mathrm{pCi} / \mathrm{g}$ & $Q$ & $\mathrm{MDA}$ & $\mathrm{pCi} / \mathrm{g}$ & $Q$ & $\mathrm{MDA}$ & $\mathrm{pCi} / \mathrm{g}$ & $Q$ & $\mathrm{MDA}$ & $\mathrm{pCi} / \mathrm{g}$ & $Q$ & MDA & $\mathrm{pCi} / \mathrm{g}$ & $Q$ & $\mathrm{MDA}$ \\
\hline 1 & J12R02 & $7 / 6 / 06$ & 0.24 & $\mathrm{U}$ & 0.24 & 10.2 & & 0.75 & 0.364 & $\mathrm{~J}$ & 0.15 & 0.796 & & 0.31 & 0.470 & & 0.090 \\
\hline 2 & J12R03 & $7 / 6 / 06$ & 0.18 & $\bar{U}$ & 0.18 & 9.38 & & 0.46 & 0.316 & $\mathrm{~J}$ & 0.12 & 0.342 & & 0.28 & 0.398 & & 0.077 \\
\hline 3 & J12R04 & $7 / 6 / 06$ & 0.18 & $\mathrm{U}$ & 0.18 & 9.70 & & 0.63 & 0.300 & $\mathrm{~J}$ & 0.12 & 0.678 & & 0.29 & 0.393 & & 0.078 \\
\hline 4 & J12R05 & $7 / 6 / 06$ & 0.088 & $\mathrm{U}$ & 0.088 & 10.7 & & 0.37 & 0.294 & $\mathrm{~J}$ & 0.093 & 0.407 & & 0.19 & 0.438 & & 0.051 \\
\hline 5 & J12R06 & $7 / 6 / 06$ & 0.10 & $\bar{U}$ & 0.10 & 9.40 & & 0.46 & 0.320 & $\mathrm{~J}$ & 0.091 & 0.576 & & 0.20 & 0.377 & & 0.055 \\
\hline 6 & J12R07 & $7 / 6 / 06$ & 0.12 & $\mathrm{U}$ & 0.12 & 9.12 & & 0.64 & 0.337 & $\mathrm{~J}$ & 0.099 & 0.531 & & 0.23 & 0.529 & & 0.092 \\
\hline 7 & J12R08 & $7 / 6 / 06$ & 0.19 & $\bar{U}$ & 0.19 & 7.01 & & 0.65 & 0.277 & $\mathrm{~J}$ & 0.14 & 0.372 & & 0.29 & 0.344 & & 0.081 \\
\hline $\begin{array}{c}\text { Duplicate of } \\
\text { J12R09 }\end{array}$ & J12R14 & $7 / 6 / 06$ & 0.12 & U & 0.12 & 9.75 & & 0.56 & 0.391 & $\mathrm{~J}$ & 0.096 & 0.500 & & 0.27 & 0.400 & & 0.071 \\
\hline 9 & J12R10 & $7 / 6 / 06$ & 0.076 & $\mathrm{U}$ & 0.076 & 8.78 & & 0.29 & 0.320 & $\mathrm{~J}$ & 0.050 & 0.429 & & 0.11 & 0.360 & & 0.031 \\
\hline 10 & J12R11 & $7 / 6 / 06$ & 0.095 & $\bar{U}$ & 0.095 & 10.6 & & 1.3 & 0.725 & $\mathrm{~J}$ & 0.17 & 0.84 & $U$ & 0.84 & 0.263 & & 0.084 \\
\hline East overburden & J12R12 & $7 / 6 / 06$ & 0.073 & $\mathrm{U}$ & 0.073 & 8.27 & & 0.31 & 0.292 & $\mathrm{~J}$ & 0.046 & 0.485 & & 0.11 & 0.376 & & 0.032 \\
\hline West overburden & J12R13 & $7 / 6 / 06$ & 0.043 & $\bar{U}$ & 0.043 & 8.96 & & 0.20 & 0.390 & $\mathrm{~J}$ & 0.042 & 0.508 & & 0.081 & 0.396 & & 0.024 \\
\hline
\end{tabular}

\begin{tabular}{|c|c|c|c|c|c|c|c|c|c|c|c|}
\hline \multirow{2}{*}{ Sample Location } & \multirow{2}{*}{$\begin{array}{c}\text { Sample } \\
\text { Number }\end{array}$} & \multirow{2}{*}{\begin{tabular}{c} 
Dample \\
\cline { 5 - 12 }
\end{tabular}} & \multicolumn{3}{|c|}{ Thorium-232 } & \multicolumn{3}{|c|}{ Uranium-235 } & \multicolumn{3}{c|}{ Uranium-238 } \\
\hline 1 & $\mathrm{~J} 12 \mathrm{R} 02$ & $7 / 6 / 06$ & 0.796 & & 0.31 & 0.28 & $\mathrm{U}$ & 0.28 & 9.7 & $\mathrm{U}$ & 9.7 \\
\hline 2 & $\mathrm{~J} 12 \mathrm{R} 03$ & $7 / 6 / 06$ & 0.342 & & 0.28 & 0.22 & $\mathrm{U}$ & 0.22 & 7.4 & $\mathrm{U}$ & 7.4 \\
\hline 3 & $\mathrm{~J} 12 \mathrm{R} 04$ & $7 / 6 / 06$ & 0.678 & & 0.29 & 0.22 & $\mathrm{U}$ & 0.22 & 7.8 & $\mathrm{U}$ & 7.8 \\
\hline 4 & $\mathrm{~J} 12 \mathrm{R} 05$ & $7 / 6 / 06$ & 0.407 & & 0.19 & 0.14 & $\mathrm{U}$ & 0.14 & 5.7 & $\mathrm{U}$ & 5.7 \\
\hline 5 & $\mathrm{~J} 12 \mathrm{R} 06$ & $7 / 6 / 06$ & 0.576 & & 0.20 & 0.15 & $\mathrm{U}$ & 0.15 & 6.0 & $\mathrm{U}$ & 6.0 \\
\hline 6 & $\mathrm{~J} 12 \mathrm{R} 07$ & $7 / 6 / 06$ & 0.531 & & 0.23 & 0.17 & $\mathrm{U}$ & 0.17 & 7.5 & $\mathrm{U}$ & 7.5 \\
\hline 7 & $\mathrm{~J} 12 \mathrm{R} 08$ & $7 / 6 / 06$ & 0.372 & & 0.29 & 0.24 & $\mathrm{U}$ & 0.24 & 7.7 & $\mathrm{U}$ & 7.7 \\
\hline 8 & $\mathrm{~J} 12 \mathrm{R} 09$ & $7 / 6 / 06$ & 0.346 & & 0.26 & 0.22 & $\mathrm{U}$ & 0.22 & 8.2 & $\mathrm{U}$ & 8.2 \\
\hline $\begin{array}{c}\text { Duplicate of } \\
\text { J12R09 }\end{array}$ & $\mathrm{J} 12 \mathrm{R} 14$ & $7 / 6 / 06$ & 0.500 & & 0.27 & 0.17 & $\mathrm{U}$ & 0.17 & 7.8 & $\mathrm{U}$ & \multirow{2}{*}{7.8} \\
\hline 9 & $\mathrm{~J} 12 \mathrm{R} 10$ & $7 / 6 / 06$ & 0.429 & & 0.11 & 0.095 & $\mathrm{U}$ & 0.095 & 3.2 & $\mathrm{U}$ & 3.2 \\
\hline 10 & $\mathrm{~J} 12 \mathrm{R} 11$ & $7 / 6 / 06$ & 0.84 & $\mathrm{U}$ & 0.84 & 0.14 & $\mathrm{U}$ & 0.14 & 15 & $\mathrm{U}$ & 15 \\
\hline East overburden & $\mathrm{J} 12 \mathrm{R} 12$ & $7 / 6 / 06$ & 0.485 & & 0.11 & 0.093 & $\mathrm{U}$ & 0.093 & 3.3 & $\mathrm{U}$ & 3.3 \\
\hline West overburden & $\mathrm{J} 12 \mathrm{R} 13$ & $7 / 6 / 06$ & 0.508 & & 0.081 & 0.065 & $\mathrm{U}$ & 0.065 & 2.5 & $\mathrm{U}$ & 2.5 \\
\hline
\end{tabular}

Attachment

Originator

Checked

\begin{tabular}{|c|c|c|}
\hline 1 & Sheet No. & 2 of 13 \\
\hline J. M. Capron & Date & $09 / 20 / 06$ \\
\hline T. M. Blakley & Date & \\
\hline $0100 \mathrm{~B}-\mathrm{CA}-\mathrm{V} 0290$ & Rev. No. & 0 \\
\hline
\end{tabular}


Attachment 1. 100-B-14:2 Area 4 Verification Sampling Results.

\begin{tabular}{|c|c|c|c|c|c|c|c|c|c|c|c|c|c|c|c|c|c|c|c|c|}
\hline \multirow{2}{*}{ Sample Location } & \multirow{2}{*}{$\begin{array}{c}\text { Sample } \\
\text { Number }\end{array}$} & \multirow{2}{*}{$\begin{array}{c}\text { Sample } \\
\text { Date }\end{array}$} & \multicolumn{3}{|c|}{ Aluminum } & \multicolumn{3}{|c|}{ Antimony } & \multicolumn{3}{|c|}{ Arsenic } & \multicolumn{3}{|c|}{ Barium } & \multicolumn{3}{|c|}{ Beryllium } & \multicolumn{3}{|c|}{ Boron } \\
\hline & & & $\mathrm{mg} / \mathrm{kg}$ & $\mathrm{Q}$ & $\mathrm{PQL}$ & $\mathrm{mg} / \mathrm{kg}$ & $Q$ & $\mathrm{PQL}$ & $\mathrm{mg} / \mathrm{kg}$ & $Q$ & $\mathrm{PQL}$ & $\mathrm{mg} / \mathrm{kg}$ & Q & $\mathrm{PQL}$ & $\mathrm{mg} / \mathrm{kg}$ & $Q$ & PQL & $\mathrm{mg} / \mathrm{kg}$ & $Q$ & $\mathrm{PQL}$ \\
\hline 1 & J12R02 & $7 / 6 / 06$ & 6970 & & 2.3 & 0.42 & UJ & 0.42 & 4.9 & & 0.59 & 103 & & 0.02 & 0.51 & C & 0.02 & 3.6 & C & 0.23 \\
\hline 2 & $\mathrm{~J} 12 \mathrm{R} 03$ & $7 / 6 / 06$ & 5520 & & 2.3 & 0.43 & UJ & 0.43 & 3.2 & & 0.59 & 56.2 & & 0.02 & 0.49 & C & 0.02 & 2.1 & UJ & 0.23 \\
\hline 3 & $J 12 R 04$ & $7 / 6 / 06$ & 4290 & & 2.3 & 0.43 & UJ & 0.43 & 2.4 & & 0.60 & 54.3 & & 0.02 & 0.32 & C & 0.02 & 1.4 & UJ & 0.23 \\
\hline 5 & J12R06 & $7 / 6 / 06$ & 4450 & & 2.2 & 0.42 & UJ & 0.42 & 2.7 & & 0.58 & 51.2 & & 0.02 & 0.33 & $\mathrm{C}$ & 0.02 & 1.8 & UJ & 0.23 \\
\hline 6 & J12R07 & $7 / 6 / 06$ & 5650 & & 2.3 & 0.64 & $\mathrm{~J}$ & 0.42 & 4.5 & & 0.58 & 71.8 & & 0.02 & 0.51 & $\mathrm{C}$ & 0.02 & 3.3 & C & 0.23 \\
\hline 7 & $\mathrm{JI2R} 08$ & $7 / 6 / 06$ & 5630 & & 2.3 & 0.42 & UJ & 0.42 & 3.6 & & 0.59 & 61.7 & & 0.02 & 0.56 & C & 0.02 & 1.5 & UJ & 0.23 \\
\hline 8 & J12R09 & $7 / 6 / 06$ & 5230 & & 2.3 & 0.47 & $\mathrm{~J}$ & 0.42 & 3.3 & & 0.59 & 63.0 & & 0.02 & 0.46 & C & 0.02 & 2.1 & UJ & 0.23 \\
\hline 9 & $J 12 R 10$ & $7 / 6 / 06$ & 5610 & & 2.3 & 0.52 & $\mathrm{~J}$ & 0.42 & 3.4 & & 0.59 & 59.7 & & 0.02 & 0.46 & $\mathrm{C}$ & 0.02 & 2.1 & UJ & 0.23 \\
\hline 10 & $J 12 R 11$ & $7 / 6 / 06$ & 6320 & & 2.3 & 0.42 & UJ & 0.42 & 4.6 & & 0.58 & 72.2 & & 0.02 & 0.49 & C & 0.02 & 2.5 & C & 0.23 \\
\hline East overburden & $\mathrm{J} 12 \mathrm{R} 12$ & $7 / 6 / 06$ & 6190 & & 2.2 & 0.42 & UJ & 0.42 & 3.3 & & 0.58 & 72.1 & & 0.02 & 0.52 & $\mathrm{C}$ & 0.02 & 1.8 & UJ & 0.23 \\
\hline West overburden & $J 12 R 13$ & $7 / 6 / 06$ & 5460 & & 2.3 & 0.42 & $\mathrm{UJ}$ & 0.42 & 3.8 & & 0.58 & 68.6 & & 0.02 & 0.55 & C & 0.02 & 1.9 & UJ & 0.23 \\
\hline Equipment blank & $\mathrm{J} 12 \mathrm{R} 15$ & $7 / 6 / 06$ & 41.8 & & 2.2 & 0.42 & UJ & 0.42 & 0.58 & $\mathrm{U}$ & 0.58 & 1.1 & & 0.02 & 0.04 & UJ & 0.02 & 0.41 & $\mathrm{UJ}$ & 0.23 \\
\hline
\end{tabular}

\begin{tabular}{|c|c|c|c|c|c|c|c|c|c|c|c|c|c|c|c|c|c|c|c|c|}
\hline \multirow{2}{*}{ Sample Location } & \multirow{2}{*}{$\begin{array}{l}\text { Sample } \\
\text { Number }\end{array}$} & \multirow{2}{*}{$\begin{array}{c}\text { Sample } \\
\text { Date }\end{array}$} & \multicolumn{3}{|c|}{ Cadmium } & \multicolumn{3}{|c|}{ Calcium } & \multicolumn{3}{|c|}{ Chromium } & \multicolumn{3}{|c|}{ Cobalt } & \multicolumn{3}{|c|}{ Copper } & \multicolumn{3}{|c|}{ Hexavalent Chromium } \\
\hline & & & $\mathrm{mg} / \mathrm{kg}$ & $Q$ & PQL & $\mathrm{mg} / \mathrm{kg}$ & $Q$ & $\mathrm{PQL}$ & $\mathrm{mg} / \mathrm{kg}$ & $Q$ & $\mathrm{PQL}$ & $\mathrm{mg} / \mathrm{kg}$ & Q & PQL & $\mathrm{mg} / \mathrm{kg}$ & $Q$ & $\mathrm{PQL}$ & $\mathrm{mg} / \mathrm{kg}$ & $Q$ & PQL \\
\hline 1 & $\mathrm{~J} 12 \mathrm{R} 02$ & $7 / 6 / 06$ & 0.07 & $\mathrm{U}$ & 0.07 & 8420 & C & 1.6 & 12.5 & & 0.12 & 8.8 & & 0.13 & 19.8 & & 0.12 & 0.20 & $\mathrm{U}$ & 0.20 \\
\hline 2 & J12R03 & $7 / 6 / 06$ & 0.07 & $\mathrm{U}$ & 0.07 & 8640 & $\mathrm{C}$ & 1.6 & 7.7 & & 0.13 & 9.1 & & 0.14 & 17.4 & & 0.12 & 0.20 & $\mathrm{U}$ & 0.20 \\
\hline 3 & J12R04 & $7 / 6 / 06$ & 0.07 & $\mathrm{U}$ & 0.07 & 5110 & $\mathrm{C}$ & 1.6 & 6.8 & & 0.13 & 5.4 & & 0.14 & 14.0 & & 0.12 & 0.28 & & 0.20 \\
\hline 4 & J12R05 & $7 / 6 / 06$ & 0.07 & & 0.07 & 9200 & C & 1.6 & 8.2 & & 0.13 & 6.4 & & 0.13 & 17.8 & & 0.12 & 0.24 & & 0.20 \\
\hline 5 & J12R06 & $7 / 6 / 06$ & 0.26 & & 0.07 & 7690. & C & 1.6 & 9.4 & & 0.12 & 6.0 & & 0.13 & 26.4 & & 0.11 & 0.28 & & 0.20 \\
\hline 6 & J12R07 & $7 / 6 / 06$ & 0.28 & & 0.07 & 11500 & C & 1.6 & 11.7 & & 0.12 & 8.1 & & 0.13 & 30.8 & & 0.11 & 0.20 & $U$ & 0.20 \\
\hline 7 & J12R08 & $7 / 6 / 06$ & 0.07 & $\mathrm{U}$ & 0.07 & 6890 & C & 1.6 & 8.5 & & 0.13 & 8.9 & & 0.13 & 20.1 & & 0.12 & 0.20 & $U$ & 0.20 \\
\hline 8 & J12R09 & $7 / 6 / 06$ & 0.31 & & 0.07 & 9810 & C & 1.6 & 11.2 & & 0.12 & 7.3 & & 0.13 & 18.2 & & 0.12 & 0.20 & $\mathrm{U}$ & 0.20 \\
\hline $\begin{array}{c}\text { Duplicate of } \\
\text { J12R09 }\end{array}$ & $\mathrm{J} 12 \mathrm{R} 14$ & $7 / 6 / 06$ & 0.28 & & 0.07 & 10700 & C & 1.6 & 11.9 & & 0.12 & 8.0 & & 0.13 & 24.7 & & 0.11 & 0.20 & U & 0.20 \\
\hline 9 & J12R10 & $7 / 6 / 06$ & 0.09 & & 0.07 & 8380 & $\mathrm{C}$ & 1.6 & 10.5 & & 0.12 & 6.7 & & 0.13 & 19.0 & & 0.12 & 0.74 & & 0.20 \\
\hline 10 & J12R11 & $7 / 6 / 06$ & 0.09 & & 0.07 & 13700 & $\mathrm{C}$ & 1.6 & 11.2 & & 0.12 & 7.9 & & 0.13 & 20.1 & & 0.12 & 0.20 & $\mathrm{U}$ & 0.20 \\
\hline East overburden & J12R12 & $7 / 6 / 06$ & 0.07 & $\mathrm{U}$ & 0.07 & 7250 & C & 1.5 & 10.7 & & 0.12 & 7.7 & & 0.13 & 16.9 & & 0.11 & 0.98 & & 0.20 \\
\hline West overburden & J12R13 & $7 / 6 / 06$ & 0.10 & & 0.07 & 9200 & $\mathrm{C}$ & 1.6 & 9.1 & & 0.12 & 7.6 & & 0.13 & 18.1 & & 0.11 & 0.22 & & 0.20 \\
\hline Equipment blank & $\mathrm{J} 12 \mathrm{R} 15$ & $7 / 6 / 06$ & 0.07 & U & 0.07 & 22.8 & $\mathrm{C}$ & 1.6 & 0.17 & & 0.12 & 0.13 & $\mathrm{U}$ & 0.13 & 0.11 & $\mathrm{U}$ & 0.11 & & & \\
\hline
\end{tabular}

\begin{tabular}{|c|c|c|c|}
\hline Attachment & 1 & Sheet No & 3 of 13 \\
\hline Originator & J.M. Capron & Date & $09 / 20 / 06$ \\
\hline Checked & T.M. Blakley & Date & \\
\hline Calc. No. & $0100 \mathrm{~B}-\mathrm{CA}-\mathrm{V} 0290$ & Rev. No. & 0 \\
\hline
\end{tabular}


Attachment 1. 100-B-14:2 Area 4 Verification Sampling Results.

\begin{tabular}{|c|c|c|c|c|c|c|c|c|c|c|c|c|c|c|c|c|c|c|c|c|}
\hline \multirow{2}{*}{ Sample Location } & \multirow{2}{*}{$\begin{array}{l}\text { Sample } \\
\text { Number }\end{array}$} & \multirow{2}{*}{$\begin{array}{c}\text { Sample } \\
\text { Date }\end{array}$} & \multicolumn{3}{|c|}{ Iron } & \multicolumn{3}{|c|}{ Lead } & \multicolumn{3}{|c|}{ Lithium } & \multicolumn{3}{|c|}{ Magnesium } & \multicolumn{3}{|c|}{ Manganese } & \multicolumn{3}{|c|}{ Mercury } \\
\hline & & & $\mathrm{mg} / \mathrm{kg}$ & $Q$ & $\mathrm{PQL}$ & $\mathrm{mg} / \mathrm{kg}$ & $Q$ & PQL & $\mathrm{mg} / \mathrm{kg}$ & $Q$ & $\mathrm{PQL}$ & $\mathrm{mg} / \mathrm{kg}$ & Q & PQL & $\mathrm{mg} / \mathrm{kg}$ & Q & $\mathrm{PQL}$ & $\mathrm{mg} / \mathrm{kg}$ & $Q$ & $\mathrm{PQL}$ \\
\hline 1 & $\mathrm{~J} 12 \mathrm{R} 02$ & $7 / 6 / 06$ & 20900 & & 0.52 & 11.9 & & 0.30 & 8.1 & C & 0.03 & 4830 & & 0.93 & 371 & & 0.03 & 0.02 & UJ & 0.02 \\
\hline 2 & $\mathrm{~J} 12 \mathrm{R} 03$ & $7 / 6 / 06$ & 21400 & & 0.52 & 5.2 & & 0.30 & 6.2 & $\mathrm{C}$ & 0.03 & 4710 & & 0.94 & 354 & & 0.03 & 0.02 & UJ & 0.02 \\
\hline 3 & $\mathrm{~J} 12 \mathrm{R} 04$ & $7 / 6 / 06$ & 12400 & & 0.53 & 6.9 & & 0.30 & 4.8 & C & 0.03 & 3150 & & 0.95 & 248 & & 0.03 & 0.08 & $\mathrm{~J}$ & 0.02 \\
\hline 4 & J12R05 & $7 / 6 / 06$ & 16500 & & 0.52 & 11.5 & & 0.30 & 6.0 & C & 0.03 & 3890 & & 0.93 & 279 & & 0.03 & 0.02 & $\mathrm{~J}$ & 0.01 \\
\hline 5 & J12R06 & $7 / 6 / 06$ & 15300 & & 0.51 & 11.6 & & 0.29 & 5.4 & $\mathrm{C}$ & 0.03 & 3700 & & 0.92 & 252 & & 0.03 & 0.04 & $\mathrm{~J}$ & 0.01 \\
\hline 6 & J12R07 & $7 / 6 / 06$ & 25900 & & 0.52 & 20.3 & & 0.30 & 6.3 & C & 0.03 & 4140 & & 0.93 & 346 & & 0.03 & 0.14 & $\mathrm{~J}$ & 0.01 \\
\hline 7 & J12R08 & $7 / 6 / 06$ & 20800 & & 0.52 & 27.2 & & 0.30 & 6.4 & C & 0.03 & 4740 & & 0.93 & 349 & & 0.03 & 0.01 & UJ & 0.01 \\
\hline 8 & J12R09 & $7 / 6 / 06$ & 16400 & & 0.52 & 17.5 & & 0.30 & 5.6 & C & 0.03 & 3960 & & 0.93 & 287 & & 0.03 & 0.09 & $\mathrm{~J}$ & 0.01 \\
\hline $\begin{array}{c}\text { Duplicate of } \\
\text { J12R09 }\end{array}$ & J12R14 & $7 / 6 / 06$ & 19400 & & 0.51 & 71.8 & & 0.29 & 6.2 & C & 0.03 & 4360 & & 0.92 & 338 & & 0.03 & 0.06 & $J$ & 0.02 \\
\hline 9 & J12R10 & $7 / 6 / 06$ & 16700 & & 0.52 & 9.3 & & 0.30 & 6.2 & C & 0.03 & 4080 & & 0.93 & 298 & & 0.03 & 0.19 & $\mathrm{~J}$ & 0.02 \\
\hline 10 & J12R11 & $7 / 6 / 06$ & 17700 & & 0.52 & 12.1 & & 0.30 & 7.2 & C & 0.03 & 4740 & & 0.93 & 329 & & 0.03 & 0.15 & $\mathrm{~J}$ & 0.02 \\
\hline East overburden & $\mathrm{J} 12 \mathrm{R} 12$ & $7 / 6 / 06$ & 18300 & & 0.51 & 7.6 & & 0.29 & 7.0 & $\mathrm{C}$ & 0.03 & 4460 & & 0.92 & 329 & & 0.03 & 0.11 & $j$ & 0.01 \\
\hline West overburden & J12R13 & $7 / 6 / 06$ & 18100 & & 0.52 & 17.0 & & 0.30 & 5.6 & $\mathrm{C}$ & 0.03 & 3830 & & 0.93 & 335 & & 0.03 & 0.47 & $j$ & 0.02 \\
\hline Equipment blank & J12R15 & $7 / 6 / 06$ & 88.6 & & 0.51 & 0.30 & $\mathrm{U}$ & 0.30 & 0.06 & UJ & 0.03 & 6.2 & & 0.92 & 4.7 & & 0.03 & 0.01 & UJ & 0.01 \\
\hline
\end{tabular}

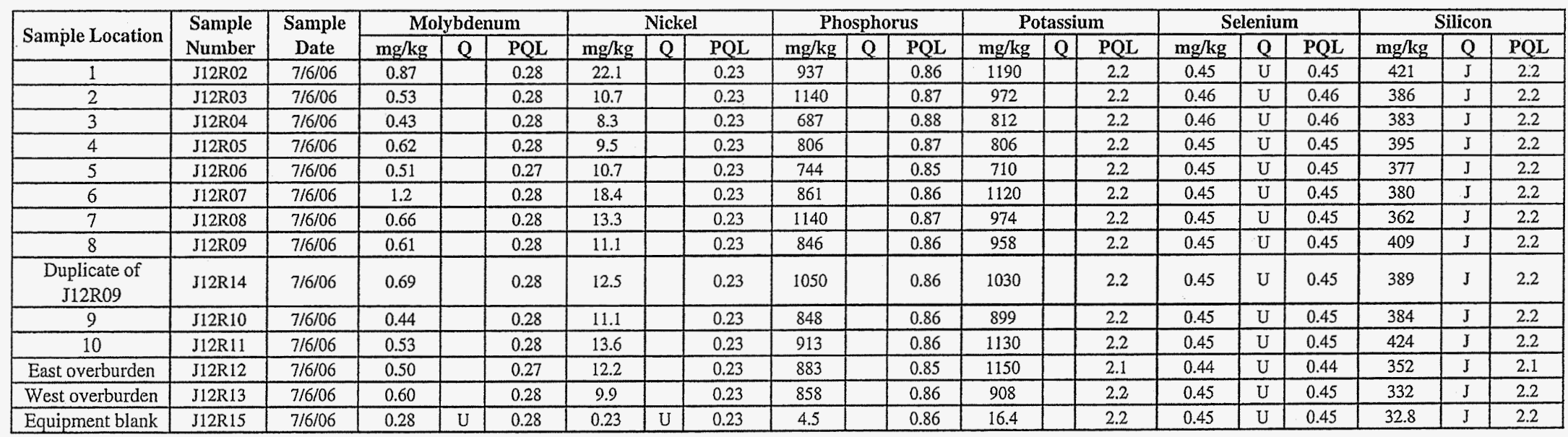

\begin{tabular}{|c|c|c|c|}
\hline Attachment & 1 & Sheet No. & 4 of 13 \\
\hline Originator & J.M. Capron & Date & $09 / 20 / 06$ \\
\hline Checked & T.M. Blakley & Date & \\
\hline Calc. No. & $0100 \mathrm{~B}-\mathrm{CA}-\mathrm{V} 0290$ & Rev. No. & 0 \\
\hline
\end{tabular}


Attachment 1. 100-B-14:2 Area 4 Verification Sampling Results.

\begin{tabular}{|c|c|c|c|c|c|c|c|c|c|c|c|c|c|c|c|c|c|c|c|c|}
\hline \multirow{2}{*}{ Sample Location } & \multirow{2}{*}{$\begin{array}{l}\text { Sample } \\
\text { Number }\end{array}$} & \multirow{2}{*}{$\begin{array}{c}\text { Sample } \\
\text { Date }\end{array}$} & \multicolumn{3}{|c|}{ Silver } & \multicolumn{3}{|c|}{ Sodium } & \multicolumn{3}{|c|}{ Strontium } & \multicolumn{3}{|c|}{ Thallium } & \multicolumn{3}{|c|}{ Tin } & \multicolumn{3}{|c|}{ Titanium } \\
\hline & & & $\mathrm{mg} / \mathrm{kg}$ & $Q$ & PQL & $\mathrm{mg} / \mathrm{kg}$ & $Q$ & PQL & $\mathrm{mg} / \mathrm{kg}$ & $Q$ & PQL & $\mathrm{mg} / \mathrm{kg}$ & $Q$ & PQL & $\mathrm{mg} / \mathrm{kg}$ & $Q$ & PQL & $\mathrm{mg} / \mathrm{kg}$ & $Q$ & $\mathrm{PQL}$ \\
\hline 1 & $\mathrm{~J} 12 \mathrm{R} 02$ & $7 / 6 / 06$ & 0.07 & $\mathrm{U}$ & 0.07 & 216 & & 0.73 & 43.7 & $\mathrm{C}$ & 0.01 & 0.67 & $\mathrm{U}$ & 0.67 & 1.0 & $\mathrm{U}$ & 1.0 & 1320 & & 0.03 \\
\hline 2 & $\mathrm{~J} 12 \mathrm{R} 03$ & $7 / 6 / 06$ & 0.07 & $\mathrm{U}$ & 0.07 & 164 & & 0.74 & 30.4 & $\mathrm{C}$ & 0.01 & 0.68 & $\bar{U}$ & 0.68 & 1.0 & $\mathrm{U}$ & 1.0 & 1570 & & 0.03 \\
\hline 3 & $\mathrm{J12R04}$ & $7 / 6 / 06$ & 0.07 & $\bar{U}$ & 0.07 & 110 & & 0.74 & 21.8 & $\mathrm{C}$ & 0.01 & 0.68 & $\mathrm{U}$ & 0.68 & 1.0 & $\mathrm{U}$ & 1.0 & 871 & & 0.03 \\
\hline 4 & J12R0S & $7 / 6 / 06$ & 0.07 & $\mathrm{U}$ & 0.07 & 159 & & 0.73 & 32.4 & $\mathrm{C}$ & 0.01 & 0.67 & $\mathrm{U}$ & 0.67 & 1.0 & $\mathrm{U}$ & 1.0 & 1250 & & 0.03 \\
\hline 5 & J12R06 & $7 / 6 / 06$ & 0.07 & $\mathrm{U}$ & 0.07 & 147 & & 0.72 & 29.3 & $\mathrm{C}$ & 0.009 & 0.66 & $\mathrm{U}$ & 0.66 & 1.0 & $\mathrm{U}$ & 1.0 & 1180 & & 0.03 \\
\hline 6 & J12R07 & $7 / 6 / 06$ & 0.07 & $\mathrm{U}$ & 0.07 & 202 & & 0.73 & 33.6 & $\mathrm{C}$ & 0.01 & 0.67 & $\mathrm{U}$ & 0.67 & 1.1 & & 1.0 & 1430 & & 0.03 \\
\hline 7 & J12R08 & $7 / 6 / 06$ & 0.07 & $\mathrm{U}$ & 0.07 & 243 & & 0.73 & 24.5 & $\mathrm{C}$ & 0.01 & 0.67 & $\mathrm{U}$ & 0.67 & 1.0 & $\mathrm{U}$ & 1.0 & 1490 & & 0.03 \\
\hline 8 & J12R09 & $7 / 6 / 06$ & 0.07 & $\bar{U}$ & 0.07 & 186 & & 0.73 & 31.6 & C & 0.01 & 0.67 & $\bar{U}$ & 0.67 & 1.0 & $\mathrm{U}$ & 1.0 & 1180 & & 0.03 \\
\hline $\begin{array}{c}\text { Duplicate of } \\
\text { J12R09 }\end{array}$ & $J 12 R 14$ & $7 / 6 / 06$ & 0.07 & U & 0.07 & 190 & & 0.72 & 33.3 & C & 0.01 & 0.67 & $U$ & 0.67 & 1.0 & $\mathrm{U}$ & 1.0 & 1330 & & 0.03 \\
\hline 9 & $\mathrm{~J} 12 \mathrm{R} 10$ & $7 / 6 / 06$ & 0.07 & U & 0.07 & 165 & & 0.73 & 34.0 & C & 0.01 & 0.67 & $\mathrm{U}$ & 0.67 & 1.0 & $\mathrm{U}$ & 1.0 & 1100 & & 0.03 \\
\hline 10 & $\mathrm{~J} 12 \mathrm{R} 11$ & $7 / 6 / 06$ & 0.07 & $\bar{U}$ & 0.07 & 210 & & 0.73 & 37.1 & $\mathrm{C}$ & 0.01 & 0.67 & $\mathrm{U}$ & 0.67 & 1.0 & $\mathrm{U}$ & 1.0 & 1230 & & 0.03 \\
\hline East overburden & J12R12 & $7 / 6 / 06$ & 0.07 & $\bar{U}$ & 0.07 & 166 & & 0.72 & 28.1 & $\mathrm{C}$ & 0.009 & 0.66 & $\dot{U}$ & 0.66 & 1.0 & $U$ & 1.0 & 1380 & & 0.03 \\
\hline West overburden & $\mathrm{J} 12 \mathrm{R} 13$ & $7 / 6 / 06$ & 0.07 & U & 0.07 & 182 & & 0.73 & 31.1 & $\bar{C}$ & 0.01 & 0.67 & $\bar{U}$ & 0.67 & 1.0 & $\bar{U}$ & 1.0 & 1480 & & 0.03 \\
\hline Equipment blank & J12R15 & $7 / 6 / 06$ & 0.07 & $\bar{U}$ & 0.07 & 7.7 & & 0.72 & 0.22 & $\bar{C}$ & 0.01 & 0.67 & $\bar{U}$ & 0.67 & 1.0 & $\bar{U}$ & 1.0 & 1.6 & & 0.03 \\
\hline
\end{tabular}

\begin{tabular}{|c|c|c|c|c|c|c|c|c|c|c|c|c|c|c|}
\hline \multirow{2}{*}{ Sample Location } & \multirow{2}{*}{$\begin{array}{l}\text { Sample } \\
\text { Number }\end{array}$} & \multirow{2}{*}{$\begin{array}{c}\text { Sample } \\
\text { Date }\end{array}$} & \multicolumn{3}{|c|}{ Uranium } & \multicolumn{3}{|c|}{ Vanadium } & \multicolumn{3}{|c|}{ Zinc } & \multicolumn{3}{|c|}{ Zirconium } \\
\hline & & & $\mathrm{mg} / \mathrm{kg}$ & $Q$ & PQL & $\mathrm{mg} / \mathrm{kg}$ & $\mathrm{Q}$ & PQL & $\mathrm{mg} / \mathrm{kg}$ & $\mathrm{Q}$ & PQL & $\mathrm{mg} / \mathrm{kg}$ & Q & PQL \\
\hline 1 & $\mathrm{~J} 12 \mathrm{R} 02$ & $7 / 6 / 06$ & 0.85 & $\vec{U}$ & 0.85 & 45.6 & & 0.09 & 43.5 & & 0.15 & 18.3 & & 1.0 \\
\hline 2 & J12R03 & $7 / 6 / 06$ & 0.85 & $\bar{U}$ & 0.85 & 50.7 & & 0.09 & 42.8 & & 0.16 & 21.2 & & 1.0 \\
\hline 3 & $\mathrm{~J} 12 \mathrm{R} 04$ & $7 / 6 / 06$ & 0.86 & $\bar{U}$ & 0.86 & 29.5 & & 0.09 & 34.6 & & 0.16 & 13.0 & & 1.0 \\
\hline 4 & J12R05 & $7 / 6 / 06$ & 0.85 & $\mathrm{U}$ & 0.85 & $41.7:$ & & 0.09 & 48.6 & & 0.15 & 14.2 & & 1.0 \\
\hline 5 & J12R06 & $7 / 6 / 06$ & 0.83 & $\mathrm{U}$ & 0.83 & 40.2 & & 0.09 & 138 & & 0.15 & 12.3 & & 1.0 \\
\hline 6 & J12R07 & $7 / 6 / 06$ & 0.84 & $\mathrm{U}$ & 0.84 & 45.5 & & 0.09 & 110 & & 0.15 & 19.5 & & 1.0 \\
\hline 7 & J12R08 & $7 / 6 / 06$ & 0.85 & $\mathrm{U}$ & 0.85 & 48.1 & & 0.09 & 46.7 & & 0.15 & 20.8 & & 1.0 \\
\hline 8 & J12R09 & $7 / 6 / 06$ & 0.84 & $\mathrm{U}$ & 0.84 & 39.5 & & 0.09 & 46.1 & & 0.15 & 16.8 & & 1.0 \\
\hline $\begin{array}{c}\text { Duplicate of } \\
\text { J12R09 }\end{array}$ & J12R14 & $7 / 6 / 06$ & 0.84 & $\mathrm{U}$ & 0.84 & 46.1 & & 0.09 & 50.4 & & 0.15 & 17.5 & & 1.0 \\
\hline 9 & J12R10 & $7 / 6 / 06$ & 0.84 & U & 0.84 & 39.7 & & 0.09 & 44.1 & & 0.15 & 13.6 & & 1.0 \\
\hline 10 & J12R11 & $7 / 6 / 06$ & 0.84 & $\mathrm{U}$ & 0.84 & 42.0 & & 0.09 & 85.6 & & 0.15 & 16.7 & & 1.0 \\
\hline East overburden & J12R12 & $7 / 6 / 06$ & 0.83 & $\mathrm{U}$ & 0.83 & 47.4 & & 0.09 & 47.6 & & 0.15 & 19.0 & & 1.0 \\
\hline West overburden & J12R13 & $7 / 6 / 06$ & 0.84 & $\mathrm{U}$ & 0.84 & 47.4 & & 0.09 & 48.8 & & 0.15 & 18.3 & & 1.0 \\
\hline Equipment blank & J12R15 & $7 / 6 / 06$ & 0.84 & $\mathrm{U}$ & 0.84 & 0.1 & & 0.09 & 0.58 & & 0.15 & 1.0 & $\mathrm{U}$ & 1.0 \\
\hline
\end{tabular}

Attachment

Originator

Checked

\begin{tabular}{|c|c|c|}
\hline 1 & Sheet No. & 5 of 13 \\
\hline J.M. Capron & Date & $09 / 20 / 06$ \\
\hline T.M. Blakley & Date & \\
\hline $0100 \mathrm{~B}-\mathrm{CA}-\mathrm{V} 0290$ & Rev. No. & 0 \\
\hline
\end{tabular}


Attachment 1. 100-B-14:2 Area 4 Verification Sampling Results.

\begin{tabular}{|c|c|c|c|c|c|c|c|c|c|c|c|c|}
\hline \multirow[t]{2}{*}{ Constituents } & \multicolumn{3}{|c|}{$\begin{array}{c}\text { J12R02 } \\
\text { Location } 1 \\
\text { Sample Date 7/6/06 }\end{array}$} & \multicolumn{3}{|c|}{$\begin{array}{c}\text { J12R03 } \\
\text { Location } 2 \\
\text { Sample Date } 7 / 6 / 06 \\
\end{array}$} & \multicolumn{3}{|c|}{$\begin{array}{c}\text { J12R04 } \\
\text { Location } 3 \\
\text { Sample Date } 7 / 6 / 06\end{array}$} & \multicolumn{3}{|c|}{$\begin{array}{c}\text { J12R05 } \\
\text { Location } 4 \\
\text { Sample Date } 7 / 6 / 06\end{array}$} \\
\hline & $\mu \mathrm{g} / \mathrm{kg}$ & $\mathbf{Q}$ & PQL & $\mu \mathrm{g} / \mathrm{kg}$ & $Q$ & $\mathrm{PQL}$ & $\mu \mathrm{g} / \mathrm{kg}$ & $Q$ & PQL & $\mu \mathrm{g} / \mathrm{kg}$ & $\mathbf{Q}$ & PQL \\
\hline \multicolumn{13}{|c|}{ Polychlorinated Biphenyls } \\
\hline Aroclor-1016 & 13 & $\mathrm{U}$ & 13 & 14 & U & 14 & 14 & U & 14 & 13 & U & 13 \\
\hline Aroclor -1221 & 13 & $U$ & 13 & 14 & $\mathrm{U}$ & 14 & 14 & $\mathrm{U}$ & 14 & 13 & $\mathrm{U}$ & 13 \\
\hline Aroclor-1232 & 13 & $\mathrm{U}$ & 13 & 14 & $\mathrm{U}$ & 14 & 14 & $\mathrm{U}$ & 14 & 13 & $\mathrm{U}$ & 13 \\
\hline Aroclor-1242 & 13 & $\mathrm{U}$ & 13 & 14 & $\mathrm{U}$ & 14 & 14 & $\mathrm{U}$ & 14 & 13 & U & 13 \\
\hline Aroclor-1248 & 13 & $\mathrm{U}$ & 13 & 14 & U & 14 & 14 & $\mathrm{U}$ & 14 & 13 & $\mathrm{U}$ & 13 \\
\hline Aroclor- 1254 & 5.7 & $\mathrm{~J}$ & 13 & 14 & $\mathrm{U}$ & 14 & 14 & $\mathrm{U}$ & 14 & 14 & & 13 \\
\hline Aroclor -1260 & 13 & $\mathrm{U}$ & 13 & 14 & $\mathrm{U}$ & 14 & 5.3 & $\mathrm{~J}$ & 14 & 13 & $\mathrm{U}$ & 13 \\
\hline \multicolumn{13}{|c|}{ Pesticides } \\
\hline Aldrin & 1.3 & UJD & 1.3 & 1.4 & UJD & 1.4 & 1.4 & UJD & 1.4 & 1.3 & UJD & 1.3 \\
\hline alpha-BHC & 1.3 & UJD & 1.3 & 1.4 & UJD & 1.4 & 1.4 & UJD & 1.4 & 1.3 & UJD & 1.3 \\
\hline alpha-Chlordane & 1.3 & UJD & 1.3 & 1.4 & UJD & 1.4 & 1.4 & UJD & 1.4 & 1.3 & UJD & 1.3 \\
\hline beta-BHC & 1.3 & UJD & 1.3 & 1.4 & UJD & 1.4 & 1.4 & UJD & 1.4 & 1.3 & UJD & 1.3 \\
\hline delta-BHC & 1.3 & UJD & 1.3 & 1.4 & UJD & 1.4 & 1.4 & UJD & 1.4 & 1.3 & UJD & 1.3 \\
\hline Dichlorodiphenyldichloroethane & 1.3 & UJD & 1.3 & 1.4 & UND & 1.4 & 2.1 & $\mathrm{JD}$ & 1.4 & 1.3 & UJD & 1.3 \\
\hline Dichlorodiphenyldichloroethylene & 1.3 & UJD & 1.3 & 1.4 & UJD & 1.4 & 1.4 & USD & 1.4 & 1.3 & UJD & 1.3 \\
\hline Dichlorodiphenyltrichloroethane & 1.3 & UID & 1.3 & 1.4 & UJD & 1.4 & 1.4 & UJD & 1.4 & 1.3 & UJD & 1.3 \\
\hline Dieldrin & $1: 3$ & UJD & 1.3 & 1.4 & UJD & 1.4 & 1.4 & UJD & 1.4 & 1.3 & UJD & 1.3 \\
\hline Endosulfan I & 1.3 & UJD & 1.3 & 1.4 & UJD & 1.4 & 1.4 & UJD & 1.4 & 1.3 & UJD & 1.3 \\
\hline Endosulfan II & 1.3 & UJD & 1.3 & 1.4 & UJD & 1.4 & 1.4 & UJD & 1.4 & 1.3 & UJD & 1.3 \\
\hline Endosulfan sulfate & 1.3 & UJD & 1.3 & 1.4 & UJD & 1.4 & 1.4 & UJD & 1.4 & 1.3 & UJD & 1.3 \\
\hline Endrin & 1.3 & UJD & 1.3 & 1.4 & UJD & 1.4 & 1.4 & UJD & 1.4 & 1.3 & UJD & 1.3 \\
\hline Endrin aldehyde & 1.3 & UJD & 1.3 & 1.4 & UJD & 1.4 & 1.8 & JDI & 1.4 & 0.67 & JD & 1.3 \\
\hline Endrin ketone & 1.3 & UJD & 1.3 & 1.4 & UJD & 1.4 & 1.4 & UJD & 1.4 & 1.3 & UJD & 1.3 \\
\hline gamma-BHC (Lindane) & 1.3 & UJD & 1.3 & 1.4 & UJD & 1.4 & 1.4 & UJD & 1.4 & 1.3 & UJD & 1.3 \\
\hline gamma-Chlordane & 1.3 & UJD & 1.3 & 1.4 & UJD & 1.4 & 1.4 & UJD & 1.4 & 1.3 & UJD & 1.3 \\
\hline Heptachlor & 1.3 & UJD & 1.3 & 1.4 & UJD & 1.4 & 1.4 & UJD & 1.4 & 1.3 & UJD & 1.3 \\
\hline Heptachlor epoxide & 1.3 & UJD & 1.3 & 1.4 & UJD & 1.4 & 1.4 & UJD & 1.4 & 1.3 & UJD & 1.3 \\
\hline Methoxychlor & 1.3 & UJD & 1.3 & 1.4 & UJD & 1.4 & 1.4 & UJD & 1.4 & 1.3 & UJD & 1.3 \\
\hline Toxaphene & 13 & UJD & 13 & 14 & UJD & 14 & 14 & UJD & 14 & 13 & UJD & 13 \\
\hline \multicolumn{13}{|c|}{ Semivolatile Organic Compounds } \\
\hline 1,2,4-Trichlorobenzene & 340 & UJ & 340 & 340 & \begin{tabular}{|l|} 
UJ \\
\end{tabular} & 340 & 340 & UJ & 340 & 340 & UJ & 340 \\
\hline 1,2-Dichlorobenzene & 340 & UJ & 340 & 340 & UJ & 340 & 340 & UJ & 340 & 340 & UJ & 340 \\
\hline 1,3-Dichlorobenzene & 340 & UJ & 340 & 340 & UJ & 340 & 340 & UJ & 340 & 340 & UJ & 340 \\
\hline 1,4-Dichlorobenzene & 340 & $\mathrm{UJ}$ & 340 & 340 & $\mathrm{UJ}$ & 340 & 340 & UJ & 340 & 340 & UI & 340 \\
\hline 2,4,5-Trichlorophenol & 840 & $\mathrm{UJ}$ & 840 & 850 & $\mathrm{UJ}$ & 850 & 850 & UJ & 850 & 840 & UJ & 840 \\
\hline 2,4,6-Trichlorophenol & 340 & $\mathrm{UJ}$ & 340 & 340 & UJ & 340 & 340 & UJ & 340 & 340 & UJ & 340 \\
\hline 2,4-Dichlorophenol & 340 & $\mathrm{UJ}$ & 340 & 340 & $\mathrm{UJ}$ & 340 & 340 & UJ & 340 & 340 & UJ & 340 \\
\hline 2,4-Dimethylphenol & 340 & UJ & 340 & 340 & $\mathrm{UJ}$ & 340 & 340 & UJ & 340 & 340 & US & 340 \\
\hline 2,4-Dinitrophenol & 840 & $\mathrm{UJ}$ & 840 & 850 & $\mathrm{UI}$ & 850 & 850 & UI & 850 & 840 & UJ & 840 \\
\hline 2,4-Dinitrotoluene & 340 & $\mathrm{UJ}$ & 340 & 340 & UJ & 340 & 340 & $\mathrm{UJ}$ & 340 & 340 & UJ & 340 \\
\hline 2,6-Dinitrotoluene & 340 & $\mathrm{UJ}$ & 340 & 340 & $\mathrm{UJ}$ & 340 & 340 & $\mathrm{UJ}$ & 340 & 340 & UJ & 340 \\
\hline 2-Chloronaphthalene & 340 & UJ & 340 & 340 & UJ & 340 & 340 & UJ & 340 & 340 & UJ & 340 \\
\hline 2-Chlorophenol & 340 & $\mathrm{UJ}$ & 340 & 340 & UJ & 340 & 340 & $\mathrm{UJ}$ & 340 & 340 & UJ & 340 \\
\hline 2-Methylnaphthalene & 340 & $\mathrm{UJ}$ & 340 & 340 & UJ & 340 & 340 & UJ & 340 & 340 & UJ & 340 \\
\hline 2-Methylphenol (cresol, o-) & 340 & UJ & 340 & 340 & UJ & 340 & 340 & UJ & 340 & 340 & UJ & 340 \\
\hline 2-Nitroaniline & 840 & $\mathrm{UI}$ & 840 & 850 & $\mathrm{UJ}$ & 850 & 850 & $\mathrm{UJ}$ & 850 & 840 & UJ & 840 \\
\hline 2-Nitrophenol & 340 & UY & 340 & 340 & UJ & 340 & 340 & UJ & 340 & 340 & UJ & 340 \\
\hline
\end{tabular}

Attachment

Originator

Checked

Calc. No.

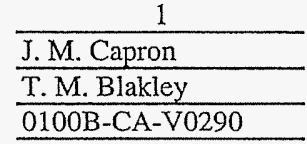

Sheet No. 6 of 13

Date $09 / 20 / 06$

Date

Rev. No. 
Attachment 1. 100-B-14:2 Area 4 Verification Sampling Results.

\begin{tabular}{|c|c|c|c|c|c|c|c|c|c|c|c|c|}
\hline \multirow[t]{2}{*}{ Constituents } & \multicolumn{3}{|c|}{$\begin{array}{c}\text { J12R02 } \\
\text { Location 1 } \\
\text { Sample Date 7/6/06 } \\
\end{array}$} & \multicolumn{3}{|c|}{$\begin{array}{c}\text { J12R03 } \\
\text { Location } 2 \\
\text { Sample Date } 7 / 6 / 06 \\
\end{array}$} & \multicolumn{3}{|c|}{$\begin{array}{c}\text { J12R04 } \\
\text { Location 3 } \\
\text { Sample Date 7/6/06 }\end{array}$} & \multicolumn{3}{|c|}{$\begin{array}{c}\text { J12R05 } \\
\text { Location } 4 \\
\text { Sample Date } 7 / 6 / 06\end{array}$} \\
\hline & $\mu \mathrm{g} / \mathrm{kg}$ & $\mathbf{Q}$ & PQL & $\mu \mathrm{g} / \mathrm{kg}$ & $\mathbf{Q}$ & PQL & $\mu \mathrm{g} / \mathrm{kg}$ & $\mathbf{Q}$ & PQL & $\mu \mathrm{g} / \mathrm{kg}$ & $\mathbf{Q}$ & $\mathrm{PQL}$ \\
\hline \multicolumn{13}{|c|}{ Semivolatile Organic Compounds (continued) } \\
\hline 3,3'-Dichlorobenzidine & 340 & $\mathrm{UJ}$ & 340 & 340 & UJ & 340 & 340 & UJ & 340 & 340 & UJ & 340 \\
\hline 3-Nitroaniline & 840 & UJ & 840 & 850 & UJ & 850 & 850 & UJ & 850 & 840 & UJ & 840 \\
\hline 4,6-Dinitro-2-methylphenol & 840 & UJ & 840 & 850 & UJ & 850 & 850 & UJ & 850 & 840 & $\mathrm{UJ}$ & 840 \\
\hline 4-Bromophenyl-phenylether & 340 & UJ & 340 & 340 & UJ & 340 & 340 & UJ & 340 & 340 & UI & 340 \\
\hline 4-Chloro-3-methylphenol & 340 & UJ & 340 & 340 & UJ & 340 & 340 & UJ & 340 & 340 & UJ & 340 \\
\hline 4-Chloroaniline. & 340 & UJ & 340 & 340 & $\mathrm{UJ}$ & 340 & 340 & UJ & 340 & 340 & $\mathrm{UJ}$ & 340 \\
\hline 4-Chlorophenyl-phenylether & 340 & $\mathrm{UJ}$ & 340 & 340 & UJ & 340 & 340 & UJ & 340 & 340 & $\mathrm{UJ}$ & 340 \\
\hline 4-Methylphenol (p-cresol) & 340 & UJ & 340 & 340 & UJ & 340 & 340 & UJ & 340 & 340 & UJ & 340 \\
\hline 4-Nitroaniline & 840 & UJ & 840 & 850 & UJ & 850 & 850 & UJ & 850 & 840 & UJ & 840 \\
\hline 4-Nitrophenol & 840 & UJ & 840 & 850 & UJ & 850 & 850 & UJ & 850 & 840 & UJ & 840 \\
\hline Acenaphthene & 340 & UJ & 340 & 340 & UJ & 340 & 68 & $\mathrm{~J}$ & 340 & 170 & $\mathrm{~J}$ & 340 \\
\hline Acenaphthylene & 340 & UJ & 340 & 340 & UJ & 340 & 340 & UJ & 340 & 340 & UJ & 340 \\
\hline Anthracene & 340 & UJ & 340 & 340 & UJ & 340 & 160 & $\mathrm{~J}$ & 340 & 330 & $\mathrm{~J}$ & 340 \\
\hline Benzo(a)anthracene & 340 & UJ & 340 & 83 & $\mathrm{~J}$ & 340 & 460 & $\mathrm{~J}$ & 340 & 750 & $\mathrm{~J}$ & 340 \\
\hline Benzo(a)pyrene & 340 & UJ & 340 & 110 & $\mathrm{~J}$ & 340 & 440 & $\mathrm{~J}$ & 340 & 640 & $\mathrm{~J}$ & 340 \\
\hline Benzo(b)fluoranthene & 340 & UJ & 340 & 120 & $\mathrm{~J}$ & 340 & 330 & $\mathrm{~J}$ & 340 & 420 & $\mathrm{~J}$ & 340 \\
\hline Benzo(g,h,i)perylene & 340 & UJ & 340 & 81 & $\mathrm{~J}$ & 340 & 280 & $\mathrm{~J}$ & 340 & 430 & $\mathrm{~J}$ & 340 \\
\hline Benzo(k)fluoranthene & 340 & UJ & 340 & 110 & $\mathrm{~J}$ & 340 & 360 & $\mathrm{~J}$ & 340 & 540 & $\mathrm{~J}$ & 340 \\
\hline bis(2-Chloro-1-methylethyl)ether & 340 & UJ & 340 & 340 & UJ & 340 & 340 & UJ & 340 & 340 & $\mathrm{UJ}$ & 340 \\
\hline bis(2-Chloroethoxy)methane & 340 & UJ & 340 & 340 & UJ & 340 & 340 & $\mathrm{UJ}$ & 340 & 340 & UJ & 340 \\
\hline bis(2-Chloroethyl)ether & 340 & UJ & 340 & 340 & UJ & 340 & 340 & UJ & 340 & 340 & UJ & 340 \\
\hline bis(2-Ethylhexyl)phthalate & 660 & UJ & 340 & 660 & UJ & 340 & 660 & UJ & 340 & 660 & UJ & 340 \\
\hline Butylbenzylphthalate & 340 & UJ & 340 & 340 & UJ & 340 & 340 & $\mathrm{UJ}$ & 340 & 340 & $\mathrm{UJ}$ & 340 \\
\hline Carbazole & 340 & UJ & 340 & 340 & UJ & 340 & 70 & $\mathrm{~J}$ & 340 & 150 & $\mathrm{~J}$ & 340 \\
\hline Chrysene & 340 & UJ & 340 & 150 & $\mathrm{~J}$ & 340 & 550 & $\mathrm{~J}$ & 340 & 880 & $\mathrm{~J}$ & 340 \\
\hline Di-n-butylphthalate & 340 & $\mathrm{UJ}$ & 340 & 30 & $\mathrm{~J}$ & 340 & 24 & $\mathrm{~J}$ & 340 & 340 & UJ & 340 \\
\hline Di-n-octylphthalate & 340 & $\mathrm{UJ}$ & 340 & 340 & UJ & 340 & 340 & UJ & 340 & 340 & UJ & 340 \\
\hline Dibenz $(\mathrm{a}, \mathrm{h})$ anthracene & 340 & UJ & 340 & 55 & $\mathrm{~J}$ & 340 & 140 & $\mathrm{~J}$ & 340 & 160 & $\mathrm{~J}$ & 340 \\
\hline Dibenzofuran & 340 & UJ & 340 & 340 & UJ & 340 & 20 & $\mathrm{~J}$ & 340 & .59 & $\mathrm{~J}$ & 340 \\
\hline Diethylphthalate & 340 & UJ & 340 & 340 & UJ & 340 & 340 & UJ & 340 & 340 & $\mathrm{UJ}$ & 340 \\
\hline Dimethylphthalate & 340 & $\mathrm{UJ}$ & 340 & 340 & UJ & 340 & 340 & UJ & 340 & 340 & $\mathrm{UJ}$ & 340 \\
\hline Fluoranthene & 340 & UJ & 340 & 100 & $\mathrm{~J}$ & 340 & 1200 & $\mathrm{~J}$ & 340 & 1900 & $\mathrm{~J}$ & 340 \\
\hline Fluorene & 340 & UJ & 340 & 340 & UJ & 340 & 45 & $\mathrm{~J}$ & 340 & 120 & $\mathrm{~J}$ & 340 \\
\hline Hexachlorobenzene & 340 & UJ & 340 & 340 & UJ & 340 & 340 & UJ & 340 & 340 & $\mathrm{UJ}$ & 340 \\
\hline Hexachlorobutadiene & 340 & UJ & 340 & 340 & UJ & 340 & 340 & UJ & 340 & 340 & $\mathrm{UJ}$ & 340 \\
\hline Hexachlorocyclopentadiene & 340 & UJ & 340 & 340 & UJ & 340 & 340 & UJ & 340 & 340 & UJ & 340 \\
\hline Hexachloroethane & 340 & UJ & 340 & 340 & UJ & 340 & 340 & UJ & 340 & 340 & UJ & 340 \\
\hline Indeno $(1,2,3$-cd)pyrene & 340 & $\mathrm{UJ}$ & 340 & 72 & $\mathrm{~J}$ & 340 & 250 & $\mathrm{~J}$ & 340 & 420 & $\mathrm{~J}$ & 340 \\
\hline Isophorone & 340 & UJ & 340 & 340 & UJ & 340 & 340 & UJ & 340 & 340 & UJ & 340 \\
\hline $\mathrm{N}$-Nitroso-di-n-dipropylamine & 340 & UJ & 340 & 340 & UJ & 340 & 340 & UJ & 340 & 340 & $\mathrm{UJ}$ & 340 \\
\hline N-Nitrosodiphenylamine & 340 & UJ & 340 & 340 & UJ & 340 & 340 & $\mathrm{UJ}$ & 340 & 340 & $\mathrm{UJ}$ & 340 \\
\hline Naphthalene & 340 & UJ & 340 & 340 & UJ & 340 & 340 & UJ & 340 & 27 & $\mathrm{~J}$ & 340 \\
\hline Nitrobenzene & 340 & UJ & 340 & 340 & UJ & 340 & 340 & UJ & 340 & 340 & UJ & 340 \\
\hline Pentachlorophenol & 840 & $\mathrm{UJ}$ & 840 & 850 & UJ & 850 & 850 & UJ & 850 & 840 & UJ & 840 \\
\hline Phenanthrene & 340 & UJ & 340 & 35 & $\mathrm{~J}$ & 340 & 740 & $\mathrm{~J}$ & 340 & 1600 & $\mathrm{~J}$ & 340 \\
\hline Phenol & 340 & UJ & 340 & 340 & UJ & 340 & 340 & US & 340 & 340 & UJ & 340 \\
\hline Pyrene. & 340 & UJ & 340 & 130 & $\mathrm{~J}$ & 340 & 920 & $\mathrm{~J}$ & 340 & 1500 & $\mathrm{~J}$ & 340 \\
\hline
\end{tabular}

Attachment

Originator

Checked

Calc. No.

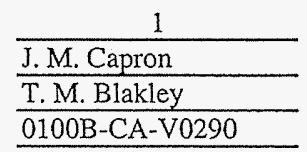

Sheet No. $\frac{7 \text { of } 13}{09 / 20 / 06}$
Date
Date
Rev. No. 0 
Attachment 1. 100-B-14:2 Area 4 Verification Sampling Results.

\begin{tabular}{|c|c|c|c|c|c|c|c|c|c|c|c|c|}
\hline \multirow[t]{2}{*}{ Constituents } & \multicolumn{3}{|c|}{$\begin{array}{c}\text { J12R06 } \\
\text { Location } 5 \\
\text { Sample Date } 7 / 6 / 06 \\
\end{array}$} & \multicolumn{3}{|c|}{$\begin{array}{c}\text { J12R07 } \\
\text { Location } 6 \\
\text { Sample Date } 7 / 6 / 06 \\
\end{array}$} & \multicolumn{3}{|c|}{$\begin{array}{c}\text { J12R08 } \\
\text { Location } 7 \\
\text { Sample Date } 7 / 6 / 06 \\
\end{array}$} & \multicolumn{3}{|c|}{$\begin{array}{c}\text { J12R09 } \\
\text { Location } 8 \\
\text { Sample Date 7/6/06 }\end{array}$} \\
\hline & $\mu \mathrm{g} / \mathrm{kg}$ & Q & PQL & $\mu \mathrm{g} / \mathrm{kg}$ & $Q$ & PQL & $\mu \mathrm{g} / \mathrm{kg}$ & Q & PQL & $\mu \mathrm{g} / \mathrm{kg}$ & \begin{tabular}{|l|l|} 
\\
\end{tabular} & PQL \\
\hline \multicolumn{13}{|c|}{ Polychlorinated Biphenyls } \\
\hline Aroclor-1016 & 13 & $\mathrm{U}$ & 13 & 13 & $\mathrm{U}$ & 13 & 13 & $\mathrm{U}$ & 13 & 13 & $\mathrm{U}$ & 13 \\
\hline Aroclor-1221 & 13 & $\mathrm{U}$ & 13 & 13 & $\mathrm{U}$ & 13 & 13 & $\mathrm{U}$ & 13 & 13 & $\mathrm{U}$ & 13 \\
\hline Aroclor-1232 & 13 & $\mathrm{U}$ & 13 & 13 & $\mathrm{U}$ & 13 & 13 & $\mathrm{U}$ & 13 & 13 & $\mathrm{U}$ & 13 \\
\hline Aroclor-1242 & 13 & $\mathrm{U}$ & 13 & 13 & $\mathrm{U}$ & 13 & 13 & $\mathrm{U}$ & 13 & 13 & $\mathrm{U}$ & 13 \\
\hline Aroclor-1248 & 13 & $\mathrm{U}$ & 13 & 13 & $\mathrm{U}$ & 13 & 13 & $\mathrm{U}$ & 13 & 13 & $\mathrm{U}$ & 13 \\
\hline Aroclor-1254 & 95 & & 13 & 84 & & 13 & 13 & $\mathrm{U}$ & 13 & 63 & & 13 \\
\hline Aroclor- 1260 & 13 & $\mathrm{U}$ & 13 & 13 & $\mathrm{U}$ & 13 & 13 & $\mathrm{U}$ & 13 & 13 & $\mathrm{U}$ & 13 \\
\hline \multicolumn{13}{|c|}{ Pesticides } \\
\hline Aldrin & 0.74 & $\mathrm{JD}$ & 1.3 & 2.4 & JD & 1.3 & 1.3 & UJD & 1.3 & 1.3 & UND & 1.3 \\
\hline alpha-BHC & 1.3 & UJD & 1.3 & 1.3 & UJD & 1.3 & 1.3 & USD & 1.3 & 1.3 & UJD & 1.3 \\
\hline alpha-Chlordane & 1.3 & UID & 1.3 & 1.3 & UJD & 1.3 & 1.3 & UJD & 1.3 & 1.3 & UJD & 1.3 \\
\hline beta-BHC & 1.3 & UJD & 1.3 & 1.3 & UMD & 1.3 & 1.3 & UJD & 1.3 & 1.3 & UJD & 1.3 \\
\hline delta-BHC & 1.3 & UJD & 1.3 & 1.3 & UMD & 1.3 & 1.3 & UID & 1.3 & 1.3 & UJD & 1.3 \\
\hline Dichlorodiphenyldichloroethane & 1.3 & UJD & 1.3 & 1.3 & UJD & 1.3 & 1.3 & UJD & 1.3 & 1.3 & UJD & 1.3 \\
\hline Dichlorodiphenyldichloroethylene & 3.9 & $\mathrm{JD}$ & 1.3 & 7.6 & $\mathrm{JD}$ & 1.3 & 1.3 & UJD & 1.3 & 2.0 & JD & 1.3 \\
\hline Dichlorodiphenyltrichloroethane & 1.3 & UJD & 1.3 & 1.3 & UJD & 1.3 & 1.3 & UJD & 1.3 & 1.3 & UJD & 1.3 \\
\hline Dieldrin & 1.3 & UND & 1.3 & 1.3 & UJD & 1.3 & 1.3 & UJD & 1.3 & 1.3 & UJD & 1.3 \\
\hline Endosulfan I & 1.3 & UJD & 1.3 & 1.3 & UJD & 1.3 & 1.3 & UJD & 1.3 & 1.3 & UJD & 1.3 \\
\hline Endosulfan II & 1.3 & UJD & 1.3 & 1.3 & UJD & 1.3 & 1.3 & UJD & 1.3 & 1.3 & UJD & 1.3 \\
\hline Endosulfan sulfate & 1.3 & UND & 1.3 & 1.3 & UJD & 1.3 & 1.3 & UJD & 1.3 & 2.0 & $\mathrm{JD}$ & 1.3 \\
\hline Endrin & 1.3 & UJD & 1.3 & 1.3 & UJD & 1.3 & 1.3 & UJD & 1.3 & 1.3 & UJD & 1.3 \\
\hline Endrin aldehyde & 1.8 & JDI & 1.3 & 2.8 & $\mathrm{JD}$ & 1.3 & 1.3 & UJD & 1.3 & 1.3 & UJD & 1.3 \\
\hline Endrin ketone & 1.3 & UJD & 1.3 & 1.3 & USD & 1.3 & 1.3 & UJD & 1.3 & 1.3 & UID & 1.3 \\
\hline gamma-BHC (Lindane) & 1.3 & UJD & 1.3 & 1.3 & USD & 1.3 & 1.3 & UJD & 1.3 & 1.3 & UJD & 1.3 \\
\hline gamma-Chlordane & 1.1 & JD & 1.3 & 1.3 & UJD & 1.3 & 1.3 & UJD & 1.3 & 0.74 & JDI & 1.3 \\
\hline Heptachlor & 1.3 & UJD & 1.3 & 1.3 & UJD & 1.3 & 1.3 & UJD & 1.3 & 1.3 & UID & 1.3 \\
\hline Heptachlor epoxide & 1.3 & UJD & 1.3 & 1.3 & UJD & 1.3 & 1.3 & UJD & 1.3 & 1.3 & UJD & 1.3 \\
\hline Methoxychlor & 1.3 & UJD & 1.3 & 1.3 & UJD & 1.3 & 1.3 & UJD & 1.3 & 1.3 & UJD & 1.3 \\
\hline Toxaphene & 13 & UND & 13 & 13 & UJD & 13 & 13 & UJD & 13 & 13 & UJD & 13 \\
\hline \multicolumn{13}{|c|}{ Semivolatile Organic Compounds } \\
\hline 1,2,4-Trichlorobenzene & 340 & UJ & 340 & 1000 & UJD & 1000 & 340 & UJ & 340 & 1000 & UID & 1000 \\
\hline 1,2-Dichlorobenzene & 340 & $\mathrm{UJ}$ & 340 & 1000 & UJD & 1000 & 340 & UJ & 340 & 1000 & UID & 1000 \\
\hline 1,3-Dichlorobenzene & 340 & $\mathrm{UJ}$ & 340 & 1000 & UJD & 1000 & 340 & UJ & 340 & 1000 & UJD & 1000 \\
\hline 1,4-Dichlorobenzene & 340 & UJ & 340 & 1000 & UJD & 1000 & 340 & UJ & 340 & 1000 & UTD & 1000 \\
\hline 2,4,5-Trichlorophenol & 840 & $\mathrm{UJ}$ & 840 & 2500 & UJD & 2500 & 840 & UJ & 840 & 2500 & UJD & 2500 \\
\hline 2,4,6-Trichlorophenol & 340 & $\mathrm{UJ}$ & 340 & 1000 & UID & 1000 & 340 & UJ & 340 & 1000 & UJD & 1000 \\
\hline 2,4-Dichlorophenol & 340 & UJ & 340 & 1000 & UJD & 1000 & 340 & UY & 340 & 1000 & UJD & 1000 \\
\hline 2,4-Dimethylphenol & 340 & UJ & 340 & 1000 & UJD & 1000 & 340 & UJ & 340 & 1000 & USD & 1000 \\
\hline 2,4-Dinitrophenol & 840 & UJ & 840 & 2500 & UJD & 2500 & 840 & UJ & 840 & 2500 & UJD & 2500 \\
\hline 2,4-Dinitrotoluene & 340 & UJ & 340 & 1000 & UJD & 1000 & 340 & UJ & 340 & 1000 & UJD & 1000 \\
\hline 2,6-Dinitrotoluene & 340 & UJ & 340 & 1000 & UJJ & 1000 & 340 & UJ & 340 & 1000 & UJD & 1000 \\
\hline 2-Chloronaphthalene & 340 & UJ & 340 & 1000 & UJD & 1000 & 340 & UJ & 340 & 1000 & UJD & 1000 \\
\hline 2-Chlorophenol & 340 & UJ & 340 & 1000 & UJD & 1000 & 340 & UJ & 340 & 1000 & UJD & 1000 \\
\hline 2-Methylnaphthalene & 340 & UJ & 340 & 1000 & UJD & 1000 & 340 & UJ & 340 & 1000 & UJD & 1000 \\
\hline 2-Methylphenol (cresol, o-) & 340 & UJ & 340 & 1000 & UJD & 1000 & 340 & UJ & 340 & 1000 & UJD & 1000 \\
\hline 2-Nitroaniline & 840 & UJ & 840 & 2500 & UJD & 2500 & 840 & $\mathrm{UJ}$ & 840 & 2500 & UJD & 2500 \\
\hline 2-Nitrophenol & 340 & UJ & 340 & 1000 & UJD & 1000 & 340 & $\mathrm{UJ}$ & 340 & 1000 & UJD & 1000 \\
\hline
\end{tabular}

\begin{tabular}{|c|c|c|c|}
\hline Attachment & 1 & Sheet No. & 8 of 13 \\
\hline Originator & J. M. Capron & Date & $09 / 20 / 06$ \\
\hline Checked & T.M. Blakley & Date & \\
\hline Calc. No. & $0100 \mathrm{~B}-\mathrm{CA}-\mathrm{V} 0290$ & Rev. No. & 0 \\
\hline
\end{tabular}


Attachment 1. 100-B-14:2 Area 4 Verification Sampling Results.

\begin{tabular}{|c|c|c|c|c|c|c|c|c|c|c|c|c|}
\hline \multirow[t]{2}{*}{ Constituents } & \multicolumn{3}{|c|}{$\begin{array}{c}\text { J12R06 } \\
\text { Location } 5 \\
\text { Sample Date } 7 / 6 / 06 \\
\end{array}$} & \multicolumn{3}{|c|}{$\begin{array}{c}\text { J12R07 } \\
\text { Location } 6 \\
\text { Sample Date 7/6/06 }\end{array}$} & \multicolumn{3}{|c|}{$\begin{array}{c}\text { J12R08 } \\
\text { Location } 7 \\
\text { Sample Date } 7 / 6 / 06\end{array}$} & \multicolumn{3}{|c|}{$\begin{array}{c}\text { J12R09 } \\
\text { Location } 8 \\
\text { Sample Date } 7 / 6 / 06\end{array}$} \\
\hline & $\mu \mathrm{g} / \mathrm{kg}$ & Q & PQL & $\mu \mathrm{g} / \mathrm{kg}$ & $Q$ & PQL & $\mu \mathrm{g} / \mathrm{kg}$ & $Q$ & $\mathrm{PQL}$ & $\mu \mathrm{g} / \mathrm{kg}$ & $Q$ & PQL \\
\hline \multicolumn{13}{|c|}{ Semivolatile Organic Compounds (continued) } \\
\hline 3,3'-Dichlorobenzidine & 340 & UJ & 340 & 1000 & UJD & 1000 & 340 & UJ & 340 & 1000 & UJD & 1000 \\
\hline 3-Nitroaniline & 840 & UJ & 840 & 2500 & UID & 2500 & 840 & UJ & 840 & 2500 & UJD & 2500 \\
\hline 4,6-Dinitro-2-methylphenol & 840 & UJ & 840 & 2500 & UJD & 2500 & 840 & UJ & 840 & 2500 & UJD & 2500 \\
\hline 4-Bromophenyl-phenylether & 340 & UJ & 340 & 1000 & UJD & 1000 & 340 & UJ & 340 & 1000 & UJD & 1000 \\
\hline 4-Chloro-3-methylphenol & 340 & UJ & 340 & 1000 & UJD & 1000 & 340 & UY & 340 & 1000 & UJD & 1000 \\
\hline 4-Chloroaniline & 340 & UJ & 340 & 1000 & USD & 1000 & 340 & $\mathrm{UJ}$ & 340 & 1000 & UJD & 1000 \\
\hline 4-Chlorophenyl-phenylether & 340 & UJ & 340 & 1000 & UID & 1000 & 340 & UJ & 340 & 1000 & UJD & 1000 \\
\hline 4-Methylphenol (p-cresol) & 340 & UJ & 340 & 1000 & UND & 1000 & 340 & UJ & 340 & 1000 & UJD & 1000 \\
\hline 4-Nitroaniline & 840 & $\mathrm{UJ}$ & 840 & 2500 & UID & 2500 & 840 & UJ & 840 & 2500 & UJD & 2500 \\
\hline 4-Nitrophenol & 840 & UJ & 840 & 2500 & UJD & 2500 & 840 & UJ & 840 & 2500 & UJD & 2500 \\
\hline Acenaphthene & 26 & $\mathrm{~J}$ & 340 & 210 & JD & 1000 & 340 & UJ & 340 & 1000 & UJD & 1000 \\
\hline Acenaphthylene & 340 & UJ & 340 & 1000 & UJD & 2500 & 340 & UJ & 340 & 1000 & UJD & 1000 \\
\hline Anthracene & 49 & $\mathrm{~J}$ & 340 & 390 & $\mathrm{JD}$ & 1000 & 340 & $\mathrm{UJ}$ & 340 & 1000 & UJD & 1000 \\
\hline Benzo(a)anthracene & 230 & $J$ & 340 & 1600 & JD & 1000 & 340 & $\overline{\mathrm{UJ}}$ & 340 & 1000 & UJD & 1000 \\
\hline Benzo(a)pyrene & 240 & $\mathrm{~J}$ & 340 & 1500 & $\mathrm{JD}$ & 1000 & 340 & UJ & 340 & 1000 & UJD & 1000 \\
\hline Benzo(b)fluoranthene & 210 & $\mathrm{~J}$ & 340 & 1300 & JD & 1000 & 340 & UJ & 340 & 1000 & UJD & 1000 \\
\hline Benzo(g,h,i)perylene & 170 & $\mathrm{~J}$ & 340 & 1200 & JD & 1000 & 340 & $\mathrm{UY}$ & 340 & 1000 & UJD & 1000 \\
\hline Benzo(k)fluoranthene & 200 & $\mathrm{~J}$ & 340 & 1300 & $\mathrm{SD}$ & 1000 & 340 & $\mathrm{UJ}$ & 340 & 1000 & UJD & 1000 \\
\hline bis(2-Chloro-1-methylethyl)ether & 340 & UJ & 340 & 1000 & UJD & 1000 & 340 & $\mathrm{UJ}$ & 340 & 1000 & UJD & 1000 \\
\hline bis(2-Chloroethoxy)methane & 340 & UJ & 340 & 1000 & UJD & 1000 & 340 & $\mathrm{UJ}$ & 340 & 1000 & UJD & 1000 \\
\hline bis(2-Chloroethyl)ether & 340 & UJ & 340 & 1000 & UJD & 1000 & 340 & UJ & 340 & 1000 & UMD & 1000 \\
\hline bis(2-Ethylhexyl)phthalate & 660 & UJ & 340 & 660 & UJD & 1000 & 660 & $\overline{\mathrm{UJ}}$ & 21 & 1000 & UJD & 1000 \\
\hline Butylbenzylphthalate & 340 & UJ & 340 & 1000 & UJD & 1000 & 340 & $\mathrm{UJ}$ & 340 & 1000 & UJD & 1000 \\
\hline Carbazole & 37 & $\mathrm{~J}$ & 340 & 250 & JD & 1000 & 340 & UJ & 340 & 1000 & UJD & 1000 \\
\hline Chrysene & 310 & $\mathrm{~J}$ & 340 & 1800 & JD & 1000 & 340 & UJ & 340 & 1000 & UJD & 1000 \\
\hline Di-n-butylphthalate & 24 & $\mathrm{~J}$ & 340 & 1000 & UJD & 1000 & 19 & $\mathrm{~J}$ & 340 & 1000 & UJD & 1000 \\
\hline Di-n-octylphthalate & 340 & UJ & 340 & 1000 & UJD & 1000 & 340 & UJ & 340 & 1000 & UJD & 1000 \\
\hline Dibenz $(\mathrm{a}, \mathrm{h})$ anthracene & 89 & $\mathrm{~J}$ & 340 & 600 & JD & 1000 & 340 & UJ & 340 & 1000 & UJD & 1000 \\
\hline Dibenzofuran & 340 & UJ & 340 & 82 & JD & 1000 & 340 & UJ & 340 & 1000 & UJD & 1000 \\
\hline Diethylphthalate & 340 & UJ & 340 & 1000 & UJD & 1000 & 340 & UJ & 340 & 1000 & UJD & 1000 \\
\hline Dimethylphthalate & 340 & $\mathrm{UJ}$ & 340 & 1000 & U:JD & 1000 & 340 & UJ & 340 & 1000 & UJD & 1000 \\
\hline Fluoranthene & 410 & $\mathrm{~J}$ & 340 & 3100 & JD & 1000 & 23 & $\mathrm{~J}$ & 340 & 1000 & UJD & 1000 \\
\hline Fluorene & 340 & UJ & 340 & 130 & JD & 1000 & 340 & UJ & 340 & 1000 & UJD & 1000 \\
\hline Hexachlorobenzene & 340 & UJ & 340 & 1000 & UJD & 1000 & 340 & UJ & 340 & 1000 & UJD & 1000 \\
\hline Hexachlorobutadiene & 340 & UJ & 340 & 1000 & UJD & 1000 & 340 & UJ & 340 & 1000 & UID & 1000 \\
\hline Hexachlorocyclopentadiene & 340 & UJ & 340 & 1000 & UJD & 1000 & 340 & UJ & 340 & 1000 & UJD & 1000 \\
\hline Hexachloroethane & 340 & $\mathrm{UJ}$ & 340 & 1000 & UJD & 1000 & 340 & UJ & 340 & 1000 & UJD & 1000 \\
\hline Indeno(1,2,3-cd)pyrene & 140 & $\mathrm{~J}$ & 340 & 1100 & JD & 1000 & 340 & UJ & 340 & 1000 & UJD & 1000 \\
\hline Isophorone & 340 & UJ & 340 & 1000 & UJD & 1000 & 340 & UJ & 340 & 1000 & UJD & 1000 \\
\hline N-Nitroso-di-n-dipropylamine & 340 & UJ & 340 & 1000 & UJD & 1000 & 340 & UJ & 340 & 1000 & UJD & 1000 \\
\hline N-Nitrosodiphenylamine & 340 & UJ & 340 & 1000 & UJD & 1000 & 340 & UJ & 340 & 1000 & UJD & 1000 \\
\hline Naphthalene & 340 & UJ & 340 & 55 & $\mathrm{JD}$ & 1000 & 340 & UJ & 340 & 1000 & UJD & 1000 \\
\hline Nitrobenzene & 340 & UJ & 340 & 1000 & UJD & 1000 & 340 & UJ & 340 & 1000 & UJD & 1000 \\
\hline Pentachlorophenol & 840 & UJ & 840 & 2500 & UJD & 2500 & 840 & UJ & 840 & 2500 & UJD & 2500 \\
\hline Phenanthrene & 270 & $\mathrm{~J}$ & 340 & 1900 & JD & 1000 & 340 & UJ & 340 & 1000 & UJD & 1000 \\
\hline Phenol & 340 & UJ & 340 & 1000 & UJD & 1000 & 340 & UJ & 340 & 1000 & UJD & 1000 \\
\hline Pyrene & 400 & $\mathrm{~J}$ & 340 & 3400 & $\mathrm{JD}$ & 1000 & 20 & $\mathrm{~J}$ & 340 & 1000 & UJD & 1000 \\
\hline & & & & Attachn & & & 1 & & & heet No. & & of 13 \\
\hline & & & & Origina & & J.M.C & ron & & & Date & & $120 / 06$ \\
\hline & & & & Checke & & T.M. & kley & & & & & \\
\hline & & & & Calc. N & & $0100 \mathrm{~B}$ & $\mathrm{~A}-\mathrm{V} 029$ & & & Rev. No & & 0 \\
\hline
\end{tabular}


Attachment 1. 100-B-14:2 Area 4 Verification Sampling Results.

\begin{tabular}{|c|c|c|c|c|c|c|c|c|c|c|c|c|}
\hline \multirow[t]{2}{*}{ Constituents } & \multicolumn{3}{|c|}{$\begin{array}{c}\text { J12R14 } \\
\text { Duplicate of J12R09 } \\
\text { Sample Date 7/6/06 } \\
\end{array}$} & \multicolumn{3}{|c|}{$\begin{array}{c}\text { J12R10 } \\
\text { Location } 9 \\
\text { Sample Date } 7 / 6 / 06 \\
\end{array}$} & \multicolumn{3}{|c|}{$\begin{array}{c}\text { J12R11 } \\
\text { Location } 10 \\
\text { Sample Date } 7 / 6 / 06\end{array}$} & \multicolumn{3}{|c|}{$\begin{array}{c}\text { J12R12 } \\
\text { East Overburden } \\
\text { Sample Date } 7 / 6 / 06\end{array}$} \\
\hline & $\mu \mathrm{g} / \mathrm{kg}$ & $\mathbf{Q}$ & PQL & $\mu \mathrm{g} / \mathrm{kg}$ & $\mathbf{Q}$ & PQL & $\mu \mathrm{g} / \mathrm{kg}$ & \begin{tabular}{|l|}
$\mathbf{Q}$ \\
\end{tabular} & PQL & $\mu \mathrm{g} / \mathrm{kg}$ & $Q$ & PQL \\
\hline \multicolumn{13}{|c|}{ Polychlorinated Biphenyls } \\
\hline Aroclor-1016 & 13 & $\mathrm{U}$ & 13 & 13 & $\mathrm{U}$ & 13 & 13 & $\mathrm{U}$ & 13 & 13 & $\mathrm{U}$ & 13 \\
\hline Aroclor-1221 & 13 & $\mathrm{U}$ & 13 & 13 & $\mathrm{U}$ & 13 & 13 & $\mathrm{U}$ & 13 & 13 & $\mathrm{U}$ & 13 \\
\hline Aroclor-1232 & 13 & $\mathrm{U}$ & 13 & 13 & $\mathrm{U}$ & 13 & 13 & $\mathrm{U}$ & 13 & 13 & $\mathrm{U}$ & 13 \\
\hline Aroclor-1242 & 13 & $\mathrm{U}$ & 13 & 13 & $\mathrm{U}$ & 13 & 13 & $\mathrm{U}$ & 13 & 13 & U & 13 \\
\hline Aroclor-1248 & 13 & $\mathrm{U}$ & 13 & 13 & $U$ & 13 & 13 & $\mathrm{U}$ & 13 & 13 & $\mathrm{U}$ & 13 \\
\hline Aroclor-1254 & 78 & & 13 & 13 & $\mathrm{U}$ & 13 & 13 & $\mathrm{U}$ & 13 & 13 & U & 13 \\
\hline Aroclor-1260 & 13 & $U$ & 13 & 13 & $\mathrm{U}$ & 13 & 13 & $\mathrm{U}$ & 13 & 13 & $\mathrm{U}$ & 13 \\
\hline \multicolumn{13}{|c|}{ Pesticides } \\
\hline Aldrin & 1.3 & UJD & 1.3 & 1.3 & UJD & 1.3 & 1.3 & UJD & 1.3 & 1.3 & UJD & 1.3 \\
\hline alpha-BHC & 1.3 & UJD & 1.3 & 1.3 & UJD & 1.3 & 1.3 & UJD & 1.3 & 1.3 & UJD & 1.3 \\
\hline alpha-Chlordane & 1.3 & UJD & 1.3 & 1.3 & UJD & 1.3 & 1.3 & UJD & 1.3 & 1.3 & UJD & 1.3 \\
\hline beta-BHC & 1.3 & UID & 1.3 & 1.3 & UJD & 1.3 & 1.3 & UJD & 1.3 & 1.3 & UJD & 1.3 \\
\hline delta-BHC & 1.3 & UJD & 1.3 & 1.3 & UJD & 1.3 & 1.3 & UJD & 1.3 & 1.3 & UJD & 1.3 \\
\hline Dichlorodiphenyldichloroethane & 1.3 & UJD & 1.3 & 1.3 & UJD & 1.3 & 1.3 & UJD & 1.3 & 1.3 & UJD & 1.3 \\
\hline Dichlorodiphenyldichloroethylene & 2.3 & $\mathrm{JD}$ & 1.3 & 1.3 & UJD & 1.3 & 1.3 & UJD & 1.3 & 1.3 & UJD & 1.3 \\
\hline Dichlorodiphenyltrichloroethane & 6.7 & $\mathrm{JD}$ & 1.3 & 1.3 & UJD & 1.3 & 1.3 & UJD & 1.3 & 1.3 & UJD & 1.3 \\
\hline Dieldrin & 3.6 & JD & 1.3 & 1.3 & UJD & 1.3 & 1.3 & UJD & 1.3 & 1.3 & UND & 1.3 \\
\hline Endosulfan I & 1.3 & UJD & 1.3 & 1.3 & UJD & 1.3 & 1.3 & UJD & 1.3 & 1.3 & UJD & 1.3 \\
\hline Endosulfan II & 1.3 & UJD & 1.3 & 1.3 & UND & 1.3 & 1.3 & UJD & 1.3 & 1.3 & UJD & 1.3 \\
\hline Endosulfan sulfate & 3.3 & JD & 1.3 & 1.3 & UJD & 1.3 & 1.3 & UJD & 1.3 & 1.3 & UJD & 1.3 \\
\hline Endrin & 1.3 & UJD & 1.3 & 1.3 & UJD & 1.3 & 1.3 & UJD & 1.3 & 1.3 & UJD & 1.3 \\
\hline Endrin aldehyde & 1.1 & JDI & 1.3 & 1.5 & JD & 1.3 & 1.3 & UJD & 1.3 & 1.3 & UJD & 1.3 \\
\hline Endrin ketone & 1.3 & UID & 1.3 & 1.3 & UJD & 1.3 & 1.3 & UJD & 1.3 & 1.3 & UJD & 1.3 \\
\hline gamma-BHC (Lindane) & 1.3 & UJD & 1.3 & 1.3 & UJD & 1.3 & 1.3 & UJD & 1.3 & 1.3 & UJD & 1.3 \\
\hline gamma-Chlordane & 1.3 & JDI & 1.3 & 1.3 & UJD & 1.3 & 1.3 & UJD & 1.3 & 1.3 & UJD & 1.3 \\
\hline Heptachlor & 1.3 & USD & 1.3 & 1.3 & UJD & 1.3 & 1.3 & UJD & 1.3 & 1.3 & UJD & 1.3 \\
\hline Heptachlor epoxide & 1.3 & USD & 1.3 & 1.3 & UJD & 1.3 & 1.3 & UJD & 1.3 & 1.3 & UJD & 1.3 \\
\hline Methoxychlor & 1.3 & UJD & 1.3 & 1.3 & UJD & 1.3 & 1.3 & UJD & 1.3 & 1.3 & UJD & 1.3 \\
\hline Toxaphene & 13 & UJD & 13 & 13 & UJD & 13 & 13 & UJD & 13 & 13 & UJD & 13 \\
\hline \multicolumn{13}{|c|}{ Semivolatile Organic Compounds } \\
\hline 1,2,4-Trichlorobenzene & 1000 & UJD & 1000 & 1000 & UJD & 1000 & 1000 & UND & 1000 & 1000 & UJD & 1000 \\
\hline 1,2-Dichlorobenzene & 1000 & UJD & 1000 & 1000 & UJD & 1000 & 1000 & UJD & 1000 & 1000 & UJD & 1000 \\
\hline 1,3-Dichlorobenzene & 1000 & USD & 1000 & 1000 & UJD & 1000 & 1000 & UJD & 1000 & 1000 & UJD & 1000 \\
\hline 1,4-Dichlorobenzene & 1000 & UJD & 1000 & 1000 & UND & 1000 & 1000 & UJD & 1000 & 1000 & UJD & 1000 \\
\hline 2,4,5-Trichlorophenol & 2500 & UJD & 2500 & 2500 & UJD & 2500 & 2500 & UJD & 2500 & 2500 & UTD & 2500 \\
\hline 2,4,6-Trichlorophenol & 1000 & UJD & 1000 & 1000 & UJD & 1000 & 1000 & UJD & 1000 & 1000 & UJD & 1000 \\
\hline 2,4-Dichlorophenol & 1000 & UJD & 1000 & 1000 & UJD & 1000 & 1000 & UJD & 1000 & 1000 & UJD & 1000 \\
\hline 2,4-Dimethylphenol & 1000 & UJD & 1000 & 1000 & UJD & 1000 & 1000 & UJD & 1000 & 1000 & UJD & 1000 \\
\hline 2,4-Dinitrophenol & 2500 & UJD & 2500 & 2500 & UJD & 2500 & 2500 & UJD & 2500 & 2500 & UJD & 2500 \\
\hline 2,4-Dinitrotoluene & 1000 & UID & 1000 & 1000 & UJD & 1000 & 1000 & UJD & 1000 & 1000 & UJD & 1000 \\
\hline 2,6-Dinitrotoluene & 1000 & UJD & 1000 & 1000 & UJD & 1000 & 1000 & UJD & 1000 & 1000 & Um & 1000 \\
\hline 2-Chloronaphthalene & 1000 & UJD & 1000 & 1000 & UJD & 1000 & 1000 & UJD & 1000 & 1000 & UJD & 1000 \\
\hline 2-Chlorophenol & 1000 & UJD & 1000 & 1000 & UJD & 1000 & 1000 & UJD & 1000 & 1000 & UJD & 1000 \\
\hline 2-Methylnaphthalene & 1000 & UJD & 1000 & 1000 & UJD & 1000 & 1000 & UJD & 1000 & 1000 & UJD & 1000 \\
\hline 2-Methylphenol (cresol, o-) & 1000 & UJD & 1000 & 1000 & UJD & 1000 & 1000 & UJD & 1000 & 1000 & USD & 1000 \\
\hline 2-Nitroaniline & 2500 & UJD & 2500 & 2500 & UJD & 2500 & 2500 & UND & 2500 & 2500 & USD & 2500 \\
\hline 2-Nitrophenol & 1000 & UJD & 1000 & 1000 & UJD & 1000 & 1000 & UJD & 1000 & 1000 & USD & 1000 \\
\hline
\end{tabular}

Attachment

Originator

Checked

Calc. No.

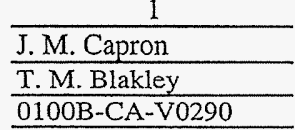

Sheet No. 10 of 13

Date 09/20/06

Date

Rev, No. 0 
Attachment 1. 100-B-14:2 Area 4 Verification Sampling Results.

\begin{tabular}{|c|c|c|c|c|c|c|c|c|c|c|c|c|}
\hline \multirow[t]{2}{*}{ Constituents } & \multicolumn{3}{|c|}{$\begin{array}{c}\text { J12R14 } \\
\text { Duplicate of J12R09 } \\
\text { Sample Date } 7 / 6 / 06 \\
\end{array}$} & \multicolumn{3}{|c|}{$\begin{array}{c}\text { J12R10 } \\
\text { Location } 9 \\
\text { Sample Date } 7 / 6 / 06 \\
\end{array}$} & \multicolumn{3}{|c|}{$\begin{array}{c}\text { J12R11 } \\
\text { Location } 10 \\
\text { Sample Date } 7 / 6 / 06\end{array}$} & \multicolumn{3}{|c|}{$\begin{array}{c}\text { J12R12 } \\
\text { East Overburden } \\
\text { Sample Date 7/6/06 }\end{array}$} \\
\hline & $\mu \mathrm{g} / \mathrm{kg}$ & $\mathbf{Q}$ & PQL & $\mu \mathrm{g} / \mathrm{kg}$ & $\mathbf{Q}$ & PQL & $\mu \mathrm{g} / \mathrm{kg}$ & $\mathrm{Q}$ & PQL & $\mu \mathrm{g} / \mathrm{kg}$ & $Q$ & $\mathrm{PQL}$ \\
\hline \multicolumn{13}{|c|}{ Semivolatile Organic Compounds (continued) } \\
\hline 3,3'-Dichlorobenzidine & 1000 & UUD & 1000 & 1000 & UJD & 1000 & 1000 & UJD| & 1000 & 1000 & UJD & 1000 \\
\hline 3-Nitroaniline. & 2500 & UJD & 2500 & 2500 & UND & 2500 & 2500 & UJD & 2500 & 2500 & UJD & 2500 \\
\hline 4,6-Dinitro-2-methylphenol & 2500 & UJD & 2500 & 2500 & UJD & 2500 & 2500 & UJD & 2500 & 2500 & UJD & 2500 \\
\hline 4-Bromophenyl-phenylether & 1000 & UJD & 1000 & 1000 & UJD & 1000 & 1000 & UJD & 1000 & 1000 & UJD & 1000 \\
\hline 4-Chloro-3-methylphenol & 1000 & UJD & 1000 & 1000 & UJD & 1000 & 1000 & UJD & 1000 & 1000 & UJD & 1000 \\
\hline 4-Chloroaniline & 1000 & UJD & 1000 & 1000 & UJD & 1000 & 1000 & UJD & 1000 & 1000 & UJD & 1000 \\
\hline 4-Chlorophenyl-phenylether & 1000 & UJD & 1000 & 1000 & UJD & 1000 & 1000 & UJD & 1000 & 1000 & UJD & 1000 \\
\hline 4-Methylphenol (p-cresol) & 1000 & UJD & 1000 & 1000 & UJD & 1000 & 1000 & UJD & 1000 & 1000 & UJD & 1000 \\
\hline 4-Nitroaniline & 2500 & UJD & 2500 & 2500 & UJD & 2500 & 2500 & UJD & 2500 & 2500 & UJD & 2500 \\
\hline 4-Nitrophenol & 2500 & UJD & 2500 & 2500 & UJD & 2500 & 2500 & UJD & 2500 & 2500 & UJD & 2500 \\
\hline Acenaphthene & 1000 & UJD & 1000 & 1000 & UJD & 1000 & 1000 & UJD & 1000 & 1000 & UJD & 1000 \\
\hline Acenaphthylene & 1000 & UJD & 1000 & 1000 & UJD & 1000 & 1000 & UJD & 1000 & 1000 & UJD & 1000 \\
\hline Anthracene & 1000 & UJD & 1000 & 1000 & UJD & 1000 & 1000 & UJD & 1000 & 86 & JD & 1000 \\
\hline Benzo(a)anthrace & 1000 & UJD & 1000 & 1000 & UJD & 1000 & 89 & $\mathrm{JD}$ & 1000 & 210 & YD & 1000 \\
\hline Benzo(a)pyrene & 1000 & UJD & 1000 & 1000 & UJD & 1000 & 78 & JD & 1000 & 170 & JD & 1000 \\
\hline Benzo(b)fluoranthene & 1000 & UJD & 1000 & 1000 & UJD & 1000 & 110 & $\mathrm{~J}$ & 1000 & 140 & $\mathrm{JD}$ & 1000 \\
\hline Benzo $(\mathrm{g}, \mathrm{h}, \mathrm{i})$ perylene & 1000 & UJD & 1000 & 1000 & UJD & 1000 & 100 & $\mathrm{JD}$ & 1000 & 96 & JD & 1000 \\
\hline Benzo(k)fluoranthene & 1000 & UJD & 1000 & 1000 & UJD & 1000 & 98 & JD & 1000 & 160 & JD & 1000 \\
\hline bis(2-Chloro-1-methylethyl)ether & 1000 & UJD & 1000 & 1000 & UJD & 1000 & 1000 & UJD & 1000 & 1000 & UJD & 1000 \\
\hline bis(2-Chloroethoxy)methane & 1000 & UJD & 1000 & 1000 & UJD & 1000 & 1000 & UJD & 1000 & 1000 & UJD & 1000 \\
\hline bis(2-Chloroethyl)ether & 1000 & UJD & 1000 & 1000 & UJD & 1000 & 1000 & UJD & 1000 & 1000 & UJD & 1000 \\
\hline bis $(2-\mathrm{B}$ & 1000 & UJD & 1000 & 1000 & UJD & 1000 & 660 & UJD & 1000 & 1000 & UJD & 1000 \\
\hline Butylbenzylphthalate & 1000 & UJD & 1000 & 1000 & UJD & 1000 & 1000 & UJD & 1000 & 1000 & UJD & 1000 \\
\hline Carbazole & 1000 & UJD & 1000 & 1000 & UJD & 1000 & 1000 & UJD & 1000 & 1000 & UID & 1000 \\
\hline Chrysene & 1000 & UJD & 1000 & 1000 & UJD & 1000 & 190 & $\mathrm{JD}$ & 1000 & 260 & JD & 1000 \\
\hline Di-n-butylphthalate & 1000 & UMD & 1000 & 1000 & UJD & 1000 & 1000 & UJD & 1000 & 1000 & UJD & 1000 \\
\hline Di-n-octylphthalate & 1000 & UJD & 1000 & 1000 & UJD & 1000 & 95 & $\mathrm{JD}$ & 1000 & 1000 & UJD & 1000 \\
\hline Dibenz $(\mathrm{a}, \mathrm{h})$ anthracene & 1000 & UJD & 1000 & 1000 & UJD & 1000 & 1000 & USD & 1000 & 1000 & UJD & 1000 \\
\hline Dibenzofuran & 1000 & UID & 1000 & 1000 & UJD & 1000 & 1000 & UJD & 1000 & 1000 & UJD & 1000 \\
\hline Diethylphthalate & 1000 & UID & 1000 & 1000 & UID & 1000 & 1000 & UJD & 1000 & 1000 & UJD & 1000 \\
\hline Dimethylphthalate & 1000 & UJD & 1000 & 1000 & UJD & 1000 & 1000 & UJD & 1000 & 1000 & UJD & 1000 \\
\hline Fluoranthene & 1000 & UID & 1000 & 1000 & UJD & 1000 & 68 & JD & 1000 & 440 & JD & 1000 \\
\hline Flüorene & 1000 & UJD & 1000 & 1000 & UJD & 1000 & 1000 & UJD & 1000 & 1000 & UJD & 1000 \\
\hline Hexachlorobenzene & 1000 & UJD & 1000 & 1000 & UJD & 1000 & 1000 & UJD & 1000 & 1000 & UJD & 1000 \\
\hline Hexachlorobutadiene & 1000 & UJD & 1000 & 1000 & UJD & 1000 & 1000 & UJD & 1000 & 1000 & UJD & 1000 \\
\hline Hexachlorocyclopentadiene & 1000 & UJD & 1000 & 1000 & UJD & 1000 & 1000 & UJD & 1000 & 1000 & UJD & 1000 \\
\hline Hexachloroethane & 1000 & UJD & 1000 & 1000 & UJD & 1000 & 1000 & UJD & 1000 & 1000 & UJD & 1000 \\
\hline Indeno(1,2,3-cd)pyrene & 1000 & UID & 1000 & 1000 & UJD & 1000 & 54 & $\mathrm{JD}$ & 1000 & 87 & JD & 1000 \\
\hline Isophorone & 1000 & UJD & 1000 & 1000 & UJD & 1000 & 1000 & UJD & 1000 & 1000 & UJD & 1000 \\
\hline N-Nitroso-di-n-dipropylamine & 1000 & UJD & 1000 & 1000 & UJD & 1000 & 1000 & UJD & 1000 & 1000 & UJD & 1000 \\
\hline N-Nitrosodiphenylamine & 1000 & UJD & 1000 & 1000 & UJD & 1000 & 1000 & UJD & 1000 & 1000 & UJD & 1000 \\
\hline Naphthalene & 1000 & UMD & 1000 & 1000 & UJD & 1000 & 1000 & UJD & 1000 & 1000 & USD & 1000 \\
\hline Nitrobenzene & 1000 & UJD & 1000 & 1000 & UJD & 1000 & 1000 & UJD & 1000 & 1000 & UJD & 1000 \\
\hline Pentachlorophenol & 2500 & UJD & 2500 & 2500 & UJD & 2500 & 1900 & JD & 2500 & 2500 & UJD & 2500 \\
\hline Phenanthrene & 1000 & UJD & 1000 & 1000 & UJD & 1000 & 1000 & UND & 1000 & 420 & JD & 1000 \\
\hline Phenol & 1000 & UJD & 1000 & 1000 & USD & 1000 & 1000 & UJD & 1000 & 1000 & UJD & 1000 \\
\hline Pyrene & 1000 & UJD & 1000 & 1000 & UJD & 1000 & 67 & $\mathrm{JD}$ & 1000 & 710 & JD. & 1000 \\
\hline
\end{tabular}

\begin{tabular}{|c|c|c|c|}
\hline Attachment & 1 & Sheet No. & 11 of 13 \\
\hline Originator & J.M. Capron & Date & $09 / 20 / 06$ \\
\hline Checked & T. M. Blakley & Date & \\
\hline Calc. No. & $0100 \mathrm{~B}-\mathrm{CA}-\mathrm{V} 0290$ & Rev. No. & 0 \\
\hline
\end{tabular}


Attachment 1. 100-B-14:2 Area 4 Verification Sampling Results.

\begin{tabular}{|c|c|c|c|c|c|c|}
\hline \multirow[t]{2}{*}{ Constituents } & \multicolumn{3}{|c|}{$\begin{array}{c}\text { J12R13 } \\
\text { West Overburden } \\
\text { Sample Date } 7 / 6 / 06\end{array}$} & \multicolumn{3}{|c|}{$\begin{array}{c}\text { J12R15 } \\
\text { Equipment Blank } \\
\text { Sample Date } 7 / 6 / 06\end{array}$} \\
\hline & $\mu \mathrm{g} / \mathrm{kg}$ & \begin{tabular}{l|l}
$\mathbf{Q}$ & \\
\end{tabular} & $\mathrm{PQL}$ & $\mu \mathrm{g} / \mathrm{kg}$ & $Q$ & PQL \\
\hline \multicolumn{7}{|c|}{ Polychlorinated Biphenyls } \\
\hline Aroclor-1016 & 13 & $\mathrm{U}$ & 13 & & & \\
\hline Aroclor-1221 & 13 & $\mathrm{U}$ & 13 & & & \\
\hline Aroclor- 1232 & 13 & $\mathrm{U}$ & 13 & & & \\
\hline Aroclor-1242 & 13 & $\mathrm{U}$ & 13 & & & \\
\hline Aroclor-1248 & 13 & $\mathrm{U}$ & 13 & & & \\
\hline Aroclor-1254 & 15 & & 13 & & & \\
\hline Aroclor- 1260 & 13 & $\mathrm{U}$ & 13 & & & \\
\hline \multicolumn{7}{|c|}{ Pesticides } \\
\hline Aldrin & 1.3 & UJD & 1.3 & & & \\
\hline alpha-BHC & 2.0 & $\mathrm{JD}$ & 1.3 & & & \\
\hline alpha-Chlordane & 1.3 & UJD & 1.3 & & & \\
\hline beta-BHC & 1.3 & UJD & 1.3 & & & \\
\hline delta-BHC & 1.3 & UJD & 1.3 & & & \\
\hline Dichlorodiphenyldichloroethane & 1.3 & UJD & 1.3 & & & \\
\hline Dichlorodiphenyldichloroethylene & 1.3 & UJD & 1.3 & & & \\
\hline Dichlorodiphenyltrichloroethane & 1.3 & UJD & 1.3 & & & \\
\hline Dieldrin & 0.70 & JD & 1.3 & & & \\
\hline Endosulfan I & 1.3 & UJD & 1.3 & & & \\
\hline Endosulfan II & 1.3 & UJD & 1.3 & & & \\
\hline Endosulfan sulfate & 1.3 & UJD & 1.3 & & & \\
\hline Endrin & 1.3 & UJD & 1.3 & & & \\
\hline Endrin aldehyde & 1.3 & UJD & 1.3 & & & \\
\hline Endrin ketone & 1.3 & UJD & 1.3 & & & \\
\hline gamma-BHC (Lindane) & 1.3 & UJD & 1.3 & & & \\
\hline gamma-Chlordane & 1.3 & UJD & 1.3 & & & \\
\hline Heptachlor & 1.3 & UJD & 1.3 & & & \\
\hline Heptachlor epoxide & 1.3 & UJD & 1.3 & & & \\
\hline Methoxychlor & 1.3 & UJD & 1.3 & & & \\
\hline Toxaphene & 13 & UJD & 13 & & & \\
\hline \multicolumn{7}{|c|}{ Semivolatile Organic Compounds } \\
\hline 1,2,4-Trichlorobenzene & 1000 & UJD & 1000 & 330 & UJ & 330 \\
\hline 1,2-Dichlorobenzene & 1000 & UJD & 1000 & 330 & $\mathrm{UJ}$ & 330 \\
\hline 1,3-Dichlorobenzene & 1000 & UJD & 1000 & 330 & UJ & 330 \\
\hline 1,4-Dichlorobenzene & 1000 & UJD & 1000 & 330 & $\mathrm{UJ}$ & 330 \\
\hline 2,4,5-Trichlorophenol & 2500 & UJD & 2500 & 830 & $\mathrm{UJ}$ & 830 \\
\hline 2,4,6-Trichlorophenol & 1000 & UJD & 1000 & 330 & UJ & 330 \\
\hline 2,4-Dichlorophenol & 1000 & UJD & 1000 & 330 & UJ & 330 \\
\hline 2,4-Dimethylphenol & 1000 & UJD & 1000 & 330 & UJ & 330 \\
\hline 2,4-Dinitrophenol & 2500 & UJD & 2500 & 830 & $\mathrm{UJ}$ & 830 \\
\hline 2,4-Dinitrotoluene & 1000 & UJD & 1000 & 330 & UJ & 330 \\
\hline 2,6-Dinitrotoluene & 1000 & UJD & 1000 & 330 & UJ & 330 \\
\hline 2-Chloronaphthalene & 1000 & UJD & 1000 & 330 & $\overline{\mathrm{UJ}}$ & 330 \\
\hline 2-Chlorophenol & 1000 & UID & 1000 & 330 & UJ & 330 \\
\hline 2-Methylnaphthalene & 1000 & UJD & 1000 & 330 & UJ & 330 \\
\hline 2-Methylphenol (cresol, o-) & 1000 & UJD & 1000 & 330 & $\mathrm{UJ}$ & 330 \\
\hline 2-Nitroaniline & 2500 & UJD & 2500 & 830 & UJ & 830 \\
\hline 2-Nitrophenol & 1000 & UJD & 1000 & 330 & UJ & 330 \\
\hline
\end{tabular}

$\begin{array}{ll}\text { Attachment } & \frac{1}{1} \\ \text { Originator } & \text { J.M. Capron } \\ \text { Checked } & \text { T.M. Blakley } \\ \text { Calc. No. } & \text { O100B-CA-V0290 }\end{array}$

Sheet No. 12 of 13

Date 09/20/06

Date

Rev. No. $\overline{0}$ 
Attachment 1. 100-B-14:2 Area 4 Verification Sampling Results.

\begin{tabular}{|c|c|c|c|c|c|c|}
\hline \multirow[t]{2}{*}{ Constituents } & \multicolumn{3}{|c|}{$\begin{array}{c}\text { J12R13 } \\
\text { West Overburden } \\
\text { Sample Date 7/6/06 }\end{array}$} & \multicolumn{3}{|c|}{$\begin{array}{c}\text { J12R15 } \\
\text { Equipment Blank } \\
\text { Sample Date 7/6/06 }\end{array}$} \\
\hline & $\mu \mathrm{g} / \mathrm{kg}$ & $\mathbf{Q}$ & PQL & $\mu \mathrm{g} / \mathrm{kg}$ & $Q$ & PQL \\
\hline \multicolumn{7}{|c|}{ Semivolatile Organic Compounds (continued) } \\
\hline 3,3'-Dichlorobenzidine & 1000 & UJD & 1000 & 330 & UJ & 330 \\
\hline 3-Nitroaniline & 2500 & UJD & 2500 & 830 & UJ & 830 \\
\hline 4,6-Dinitro-2-methylphenol & 2500 & UJD & 2500 & 830 & UJ & 830 \\
\hline 4-Bromophenyl-phenylether & 1000 & UJD & 1000 & 330 & UJ & 330 \\
\hline 4-Chloro-3-methylphenol & 1000 & UJD & 1000 & 330 & UJ & 330 \\
\hline 4-Chloroaniline & 1000 & UJD & 1000 & 330 & UJ & 330 \\
\hline 4-Chlorophenyl-phenylether & 1000 & UJD & 1000 & 330 & UJ & 330 \\
\hline 4-Methylphenol (p-cresol) & 1000 & UJD & 1000 & 330 & UJ & 330 \\
\hline 4-Nitroaniline & 2500 & UJD & 2500 & 830 & UJ & 830 \\
\hline 4-Nitrophenol & 2500 & UJD & 2500 & 830 & UJ & 830 \\
\hline Acenaphthene & 1000 & UJD & 1000 & 330 & UJ & 330 \\
\hline Acenaphthylene & 1000 & UJD & 1000 & 330 & UJ & 330 \\
\hline Anthracene & 1000 & UJD & 1000 & 330 & UJ & 330 \\
\hline Benzo(a)anthracene & 1000 & UID & 1000 & 330 & UJ & 330 \\
\hline Benzo(a)pyrene & 1000 & UJD & 1000 & 330 & UJ & 330 \\
\hline Benzo(b)fluoranthene & 1000 & UJD & 1000 & 330 & US. & 330 \\
\hline Benzo(g,h,i)perylene & 1000 & UJD & 1000 & 330 & UJ & 330 \\
\hline Benzo(k)fluoranthene & 1000 & UJD & 1000 & 330 & UJ & 330 \\
\hline bis(2-Chloro-1-methylethyl)ether & 1000 & UJD & 1000 & 330 & UJ & 330 \\
\hline bis(2-Chloroethoxy)methane & 1000 & UJD & 1000 & 330 & $\mathrm{UJ}$ & 330 \\
\hline bis(2-Chloroethyl)ether & 1000 & UJD & 1000 & 330 & UJ & 330 \\
\hline bis(2-Ethylhexyl)phthalate & 1000 & UJD & 1000 & 660 & UJ & 330 \\
\hline Butylbenzylphthalate & 1000 & UJD & 1000 & 330 & UJ & 330 \\
\hline Carbazole & 1000 & UJD & 1000 & 330 & $\mathrm{UJ}$ & 330 \\
\hline Chrysene & 1000 & UJD & 1000 & 330 & $\mathrm{UJ}$ & 330 \\
\hline Di-n-butylphthalate & 1000 & UJD & 1000 & 230 & $\mathrm{~J}$ & 330 \\
\hline Di-n-octylphthalate & 1000 & UJD & 1000 & 330 & UI & 330 \\
\hline Dibenz $(a, h)$ anthracene & 1000 & UJD & 1000 & 330 & UJ & 330 \\
\hline Dibenzofuran & 1000 & UJD & 1000 & 330 & UJ & 330 \\
\hline Diethylphthalate & 1000 & UJD & 1000 & 36 & $\mathrm{~J}$ & 330 \\
\hline Dimethylphthalate & 1000 & UJD & 1000 & 330 & UJ & 330 \\
\hline Fluoranthene & 1000 & UJD & 1000 & 330 & UJ & 330 \\
\hline Fluorene & 1000 & UJD & 1000 & 330 & UJ & 330 \\
\hline Hexachlorobenzene & 1000 & UJD & 1000 & 330 & UJ & 330 \\
\hline Hexachlorobutadiene & 1000 & UJD & 1000 & 330 & UJ & 330 \\
\hline Hexachlorocyclopentadiene & 1000 & UJD & 1000 & 330 & UJ & 330 \\
\hline Hexachloroethane & 1000 & UJD & 1000 & 330 & UJ & 330 \\
\hline Indeno( $1,2,3-c d)$ pyrene & 1000 & UJD & 1000 & 330 & UJ & 330 \\
\hline Isophorone & 1000 & UJD & 1000 & 330 & UJ & 330 \\
\hline N-Nitroso-di-n-dipropylamine & 1000 & UJD & 1000 & 330 & UJ & 330 \\
\hline N-Nitrosodiphenylamine & 1000 & UJD & 1000 & 330 & $\mathrm{UJ}$ & 330 \\
\hline Naphthalene & 1000 & UJD & 1000 & 330 & UJ & 330 \\
\hline Nitrobenzene & 1000 & UJD & 1000 & 330 . & UJ & 330 \\
\hline Pentachlorophenol & 2500 & UJD & 2500 & 830 & $\mathrm{UJ}$ & 830 \\
\hline Phenanthrene & 1000 & UJD & 1000 & 330 & UJ & 330 \\
\hline Phenol & 1000 & UJD & 1000 & 330 & UJ & 330 \\
\hline Pyrene & 59 & $\mathrm{JD}$ & 1000 & 330 & UJ & 330 \\
\hline
\end{tabular}

Attachment

Originator

Checked

Calc. No.

$\frac{\frac{1}{\text { J. M. Capron }}}{\frac{\text { T.M. Blakley }}{\text { 0100B-CA-V0290 }}}$

Sheet No. 13 of 13

Date $\frac{13 / 20 / 06}{0}$

Date

Rev. No. 


\section{CALCULATION COVER SHEET}

Project Title 100-B/C Remaining Pipes and Sewers Field Remediation Job No. 14655 Area $100-B / C$

Discipline Environmental *Calc. No. 0100B-CA-V0291

Subject 100-B-14:2 (Area 4) Waste Site Hazard Quotient and Carcinogenic Risk Calculations Computer Program Excel Program No. Excel 2003

The attached calculations have been generated to document compliance with established cleanup levels. These calculations should be used in conjunction with other relevant documents in the administrative record.

\section{Committed Calculation $\otimes \quad$ Preliminary $\square \quad$ Superseded $\square \quad$. Voided}

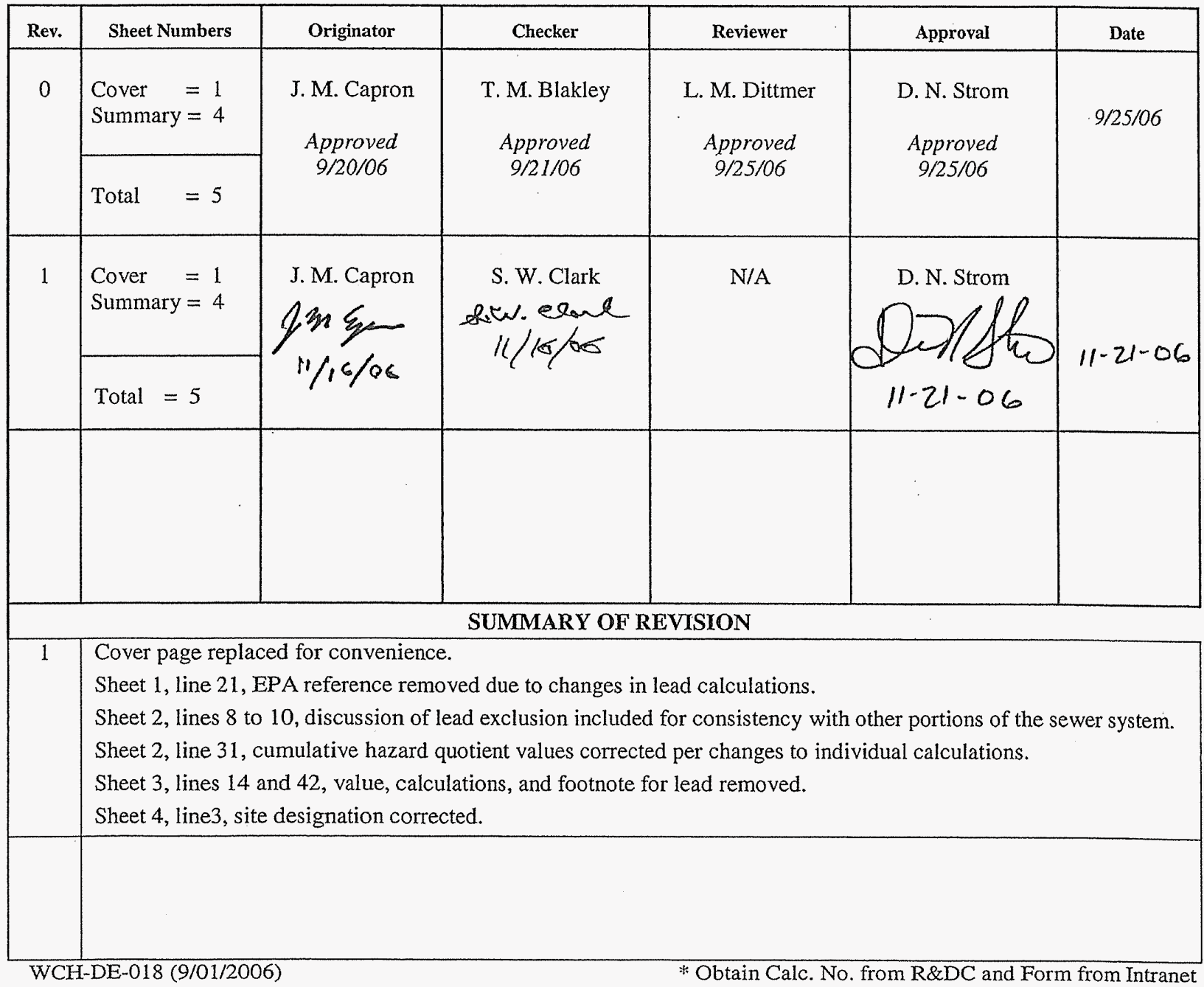


Washington Closure Hanford CALCULATION SHEET

\begin{tabular}{|c|c|c|c|c|c|c|c|}
\hline Originator: & J. M. Capron $2 \%<$ & Date: & $11 / 16 / 06$ & Calc. No.: & 0100B-CA-V0291 & Rev.: & 1 \\
\hline Project: & 100-B/C RPAS Field Remediation & Job No: & 14655 & Checked: & S. W. Clark \&so & Date: & 5 \\
\hline Subject: & 100-B-14:2 (Area 4) Waste Site Ha & Uuotient & Carcing & Risk Calcu & tions & Sheet No & 1 of 4 \\
\hline
\end{tabular}

\section{PURPOSE:}

Provide documentation to support the calculation of the hazard quotient (HQ) and excess carcinogenic risk values for the 100-B-14:2, area 4 (previously known as the 100-B-14:8 subsite) remediation verification sampling results. In accordance with the remedial action goals (RAGs) in the remedial design report/remedial action work plan (RDR/RAWP) (DOE-RL 2005), the following criteria must be met:

1) An HQ of $<1.0$ for all individual noncarcinogens

2) A cumulative $\mathrm{HQ}$ of $<1.0$ for noncarcinogens

3) An excess carcinogenic risk of $<1 \times 10^{-6}$ for individual carcinogens

4) A cumulative excess carcinogenic risk of $<1 \times 10^{-5}$ for carcinogens.

\section{GIVEN/REFERENCES:}

1) DOE-RL, 2005, Remedial Design Report/Remedial Action Work Plan for the 100 Areas, DOE/RL-96-17, Rev. 5, U.S. Department of Energy, Richland Operations Office, Richland, Washington.

2) WAC 173-303, "Dangerous Waste Regulations," Washington Administrative Code, 2004.

3) WAC 173-340, "Model Toxics Control Act - Cleanup," Washington Administrative Code, 1996.

4) WAC 173-350, "Solid Waste Handling Standards," Washington Administrative Code, 2005.

5) WCH, 2006, 100-B-14:2 (Area 4) Waste Site Cleanup Verification 95\% UCL Calculations, Calculation No. 0100B-CA-V0290, Washington Closure Hanford, Richland, Washington.

\section{SOLUTION:}

1) Calculate an HQ for each noncarcinogenic constituent detected above background and compare to the individual HQ of $<1.0$ (DOE-RL 2005).

2) Sum the HQs and compare to the cumulative HQ criterion of $<1.0$.

3) Calculate an excess carcinogenic risk value for each carcinogenic constituent detected above background and compare to the individual excess carcinogenic risk criterion of $<1 \times 10^{-6}$ (DOE-RL 2005).

4) Sum the excess carcinogenic risk values and compare to the cumulative excess carcinogenic risk criterion of $<1 \times 10^{-5}$. 
Washington Closure Hanford

Originator: I M Capron Q CALCULATION SHEET

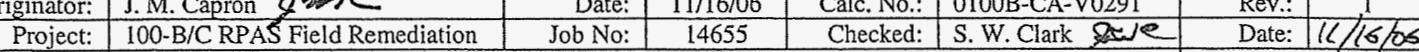

\begin{tabular}{l|l|l} 
Subject: & 100-B-14:2 (Area 4) Waste Site Hazard Quotient and Carcinogenic Risk Calculations & Sheet No. 2 of 4
\end{tabular}

\section{METHODOLOGY:}

Hazard quotient and carcinogenic risk calculations were performed for the 100-B-14:2 (area 4) subsite using the higher of the remediation footprint statistical value and overburden material maximum value for each analyte detected above background. Of the contaminants of concern (COCs) and contaminants of potential concern (COPCs) for the site, boron, molybdenum, strontium, and tin require the HQ calculations because they were detected and Washington State or Hanford Site background values are not available. Copper, lead, mercury, and zinc are included because they were quantified above their respective Hanford Site background values. Hexavalent chromium, aroclor-1254, aroclor-1260, and multiple chlorinated pesticides and semivolatile organic compounds (as identified in Table 1) are included because they were detected by laboratory analysis and cannot be attributed to natural occurrence. Lead does not have a reference dose for calculation of a hazard quotient because toxic effects of lead are correlated with blood-lead levels rather than exposure levels or daily intake. As a result, the maximum lead concentration is reported but not included in the hazard quotient calculation. Polycyclic aromatic hydrocarbons (PAHs) detected in the verification samples are not included because they are the result of asphalt cross-contamination in the sample matrix from a former overlying road and paved area. Asphalt that has been used for structural and construction purposes is excluded from consideration as a dangerous waste by WAC 173-303-071(3)(e), is listed as an inert waste in WAC 173350-990(2)(b), and its constituents are therefore not considered in attainment of soil RAGs (PAH concentrations in the soil matrix are assumed to be negligible). All other site nonradionuclide COCs and COPCs were not detected or were detected below background levels. An example of the HQ and risk calculations is presented below:

1) For example, the statistical value for boron is $3.6 \mathrm{mg} / \mathrm{kg}$, divided by the noncarcinogenic RAG value of $16,000 \mathrm{mg} / \mathrm{kg}$ (calculated in accordance with the noncarcinogenic toxics effects formula in WAC 173-340-740[3]), is $2.3 \times 10^{-4}$. Comparing this value, and all other individual values, to the requirement of $<1.0$, this criterion is met.

2) After the HQ calculations are completed for the appropriate analytes, the cumulative HQ is obtained by summing the individual values. (To avoid errors due to intermediate rounding, the individual HQ values prior to rounding are used for this calculation.) The sum of the HQ values is $7.3 \times 10^{-2}$. Comparing this value to the requirement of $<1.0$, this criterion is met.

3) To calculate the excess carcinogenic risk, the $95 \%$ upper confidence limit or maximum value is divided by the carcinogenic RAG value, then multiplied by $1 \times 10^{-6}$. For example, the maximum value for hexavalent chromium is $0.98 \mathrm{mg} / \mathrm{kg}$; divided by $2.1 \mathrm{mg} / \mathrm{kg}$ and multiplied as indicated is $4.7 \times 10^{-7}$. Comparing this value, and all other individual values, to the requirement of $<1 \times 10^{-6}$, this criterion is met.

4) After these calculations are completed for the carcinogenic analytes, the cumulative excess carcinogenic risk is obtained by summing the individual values. (To avoid errors due to intermediate rounding, the individual values prior to rounding are used for this calculation.) The sum of the excess carcinogenic risk values is $9.2 \times 10^{-7}$. Comparing this value to the requirement of $<1 \times 10^{-5}$, this criterion is met. 
Washington Closure Hanford CALCULATION SHEET

\begin{tabular}{|l|l|l|l|}
\hline Originator: & J. M. Capron $03<$ & Date: & $11 / 16 / 06$ \\
\hline
\end{tabular}

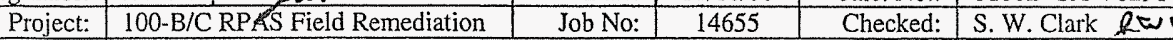

Subject: $100-B-14: 2$ (Area 4) Waste Site Hazard Quotient and Carcinogenic Risk Calculations

RESULTS:

1) List individual noncarcinogens and corresponding HQs $>1.0$ : None

2) List the cumulative noncarcinogenic $H Q>1.0$ : None

3) List individual carcinogens and corresponding excess cancer risk $>1 \times 10^{-6}$ : None

4) List the cumulative excess cancer risk for carcinogens $>1 \times 10^{-5}$ : None.

Table 1 shows the results of the calculations for the 100-B-14:2 (area 4) subsite.

Table 1. Hazard Quotient and Excess Cancer Risk Results for the 100-B-14:2 (Area 4) Subsite.

\begin{tabular}{|c|c|c|c|c|c|}
\hline $\begin{array}{l}\text { Contaminants of Concern/ } \\
\text { Contaminants of Potential Concern }\end{array}$ & $\begin{array}{c}\text { Maximum or } \\
\text { Statistical Value } \\
(\mathrm{mg} / \mathrm{kg})\end{array}$ & $\begin{array}{c}\text { Noncarcinogen } \\
\mathbf{R A G}^{\mathbf{b}} \\
(\mathrm{mg} / \mathbf{k g})\end{array}$ & $\begin{array}{l}\text { Hazard } \\
\text { Quotient }\end{array}$ & $\begin{array}{c}\text { Carcinogen } \\
\text { RAG }^{\mathbf{b}} \\
(\mathrm{mg} / \mathrm{kg})\end{array}$ & $\begin{array}{c}\text { Carcinogen } \\
\text { Risk }\end{array}$ \\
\hline \multicolumn{6}{|l|}{ Metals } \\
\hline Boron & 3.6 & 16,000 & $2.3 \mathrm{E}-04$ & - & -- \\
\hline Chromium, hexavalent ${ }^{c}$ & 0.98 & 240 & 4.1E-03 & 2.1 & $4.7 \mathrm{E}-07$ \\
\hline Copper & 23.8 & 2,960 & $8.0 \mathrm{E}-03$ & -- & $\ldots$ \\
\hline Lead & 27.1 & -- & -- & - & -- \\
\hline Mercury & 0.47 & 24 & $2.0 \mathrm{E}-02$ & -- & $\cdots$ \\
\hline Molybdenum & 0.8 & 400 & $2.0 \mathrm{E}-03$ & -- & $\ldots$ \\
\hline Strontium & 36.2 & 48,000 & $7.5 \mathrm{E}-04$ & - & $\ldots$ \\
\hline Tin & 1.1 & 48,000 & $2.3 \mathrm{E}-05$ & -- & -- \\
\hline Zinc & 82 & 24,000 & $3.4 \mathrm{E}-03$ & -. & - \\
\hline \multicolumn{6}{|c|}{ Semivolitiles } \\
\hline Dibenzofuran & 0.082 & 160 & $5.1 \mathrm{E}-04$ & -- & -- \\
\hline Di-n-butylphthalate & 0.030 & 8,000 & $3.8 \mathrm{E}-06$ & - & -- \\
\hline Di-n-octylphthalate & 0.095 & 1,600 & $5.9 \mathrm{E}-05$ & -- & - \\
\hline Pentachlorophenol & 1.9 & 2,400 & $7.9 \mathrm{E}-04$ & 8.33 & $2.3 \mathrm{E}-07$ \\
\hline Pesticides & The & 3.7. & 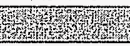 & 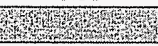 & - \\
\hline Aldrin & 0.0024 & 2.40 & $1.0 \mathrm{E}-03$ & 0.0588 & $4.1 \mathrm{E}-08$ \\
\hline BHC, alpha & 0.0020 & - & -- & 0.159 & $1.3 \mathrm{E}-08$ \\
\hline Chlordane (alpha and gamma) & 0.0013 & 40 & $3.3 \mathrm{E}-05$ & 2.86 & $4.5 \mathrm{E}-10$ \\
\hline $\mathrm{DDD}, 4,4^{\prime}-$ & 0.0021 & - & -- & 4.17 & $5.0 \mathrm{E}-10$ \\
\hline DDE, 4,4'- & 0.0076 & -- & -- & 2.94 & $2.6 \mathrm{E}-09$ \\
\hline DDT, $4,4^{\prime}-$ & 0.0067 & 40 & $1.7 \mathrm{E}-04$ & 2.94 & 2.3E-09 \\
\hline Dieldrin & 0.0036 & 4 & $9.0 \mathrm{E}-04$ & 0.0625 & $5.8 \mathrm{E}-08$ \\
\hline Endosulfan (I, II, sulfate) & 0.0033 & 480 & $6.9 \mathrm{E}-06$ & -- & -- \\
\hline Endrin (and ketone, aldehyde) & 0.0016 & 24 & $6.7 \mathrm{E}-05$ & - & -. \\
\hline \multicolumn{6}{|l|}{ 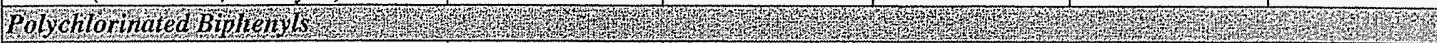 } \\
\hline Aroclor-1254 & 0.050 & 1.6 & $3.1 \mathrm{E}-02$ & 0.5 & $1.0 \mathrm{E}-07$ \\
\hline Aroclor- 1260 & 0.0053 & -- & -- & 0.5 & $1.1 \mathrm{E}-08$ \\
\hline \multicolumn{6}{|c|}{ potols } \\
\hline \multicolumn{3}{|c|}{ Cumulative Hazard Quotient: } & 7.3E-02 & & \\
\hline \multicolumn{5}{|l|}{ Cumulative Excess Cancer Risk: } & 9.2E-07 \\
\hline
\end{tabular}

Notes:

${ }^{a}=$ From WCH (2006).

${ }^{b}=$ Value obtained from Washington Administrative Code (WAC) 173-340-740(3), Method B, 1996.

${ }^{c}=$ Value for the carcinogen RAG calculated based on the inhalation exposure pathway (WAC) 173-340-750(3), 1996.

$\cdots=$ not applicable

$\mathrm{RAG}=$ remedial action goal 
Washington Closure Hanford CALCULATION SHEET

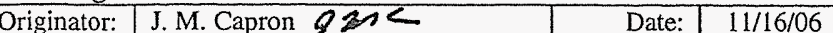

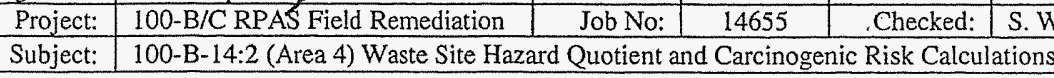

Calc. No.: $0100 \mathrm{~B}-\mathrm{CA}-\mathrm{V} 0291$

Rev.:

Rev.:

$\frac{1}{11 / 16 / 56}$

Sheet No. 4 of 4

1 CONCLUSION:

2

3 This calculation demonstrates that the 100-B-14:2 (area 4) subsite meets the requirements for hazard

4 quotient and excess carcinogenic risk as identified in the RDR/RAWP (DOE-RL 2005). 


\section{CALCULATION COVER SHEET}

Project Title:

Area

Discipline

Subject

Computer Program
100-B/C Area Field Remediation $100-\mathrm{B} / \mathrm{C}$

Environmental 1607-B2:1 Drain Excel
Job No.

*Calc. No. 0100B-CA-V0264

$\%$ UCL Calculations

Program No. Excel 2003

The attached calculations have been generated to document compliance with established cleanup levels. These documents should be used in conjunction with other relevant documents in the administrative record.

Committed Calculation $\quad \mathrm{X} \quad$ Preliminary $\square \quad$ Superseded $\square \quad$ Voided $\square$

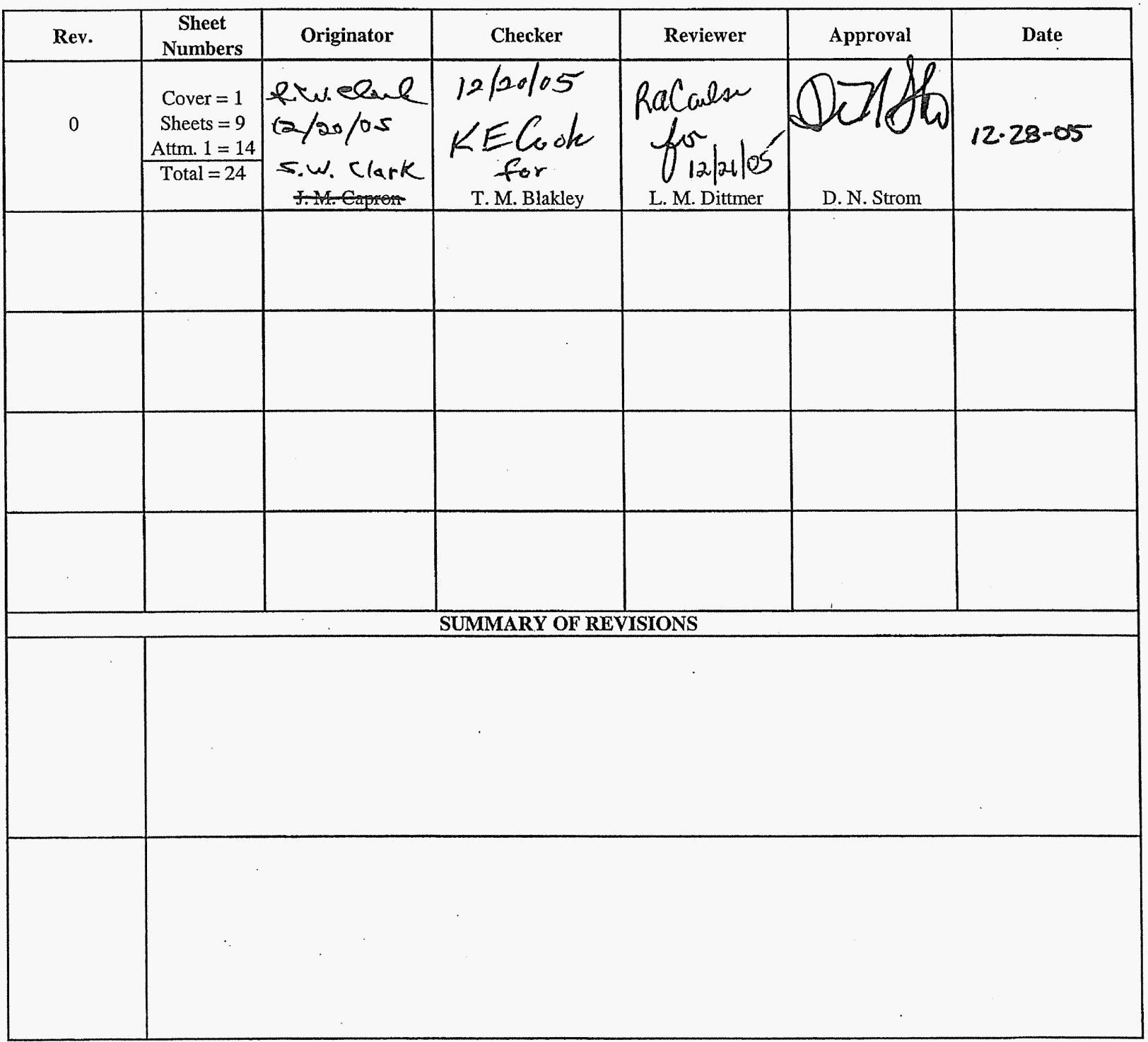

* Obtain calc no. from DIS

DE01437.03 (12/09/2004) 


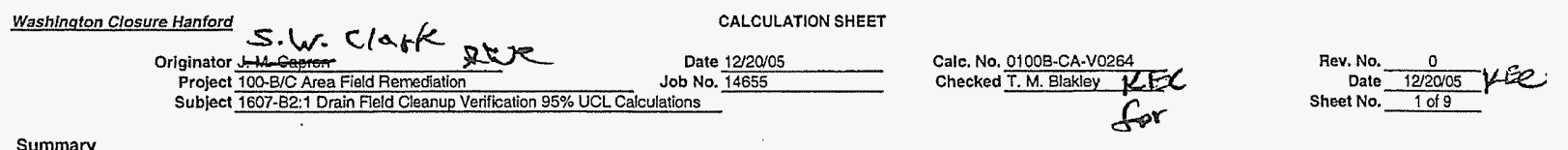

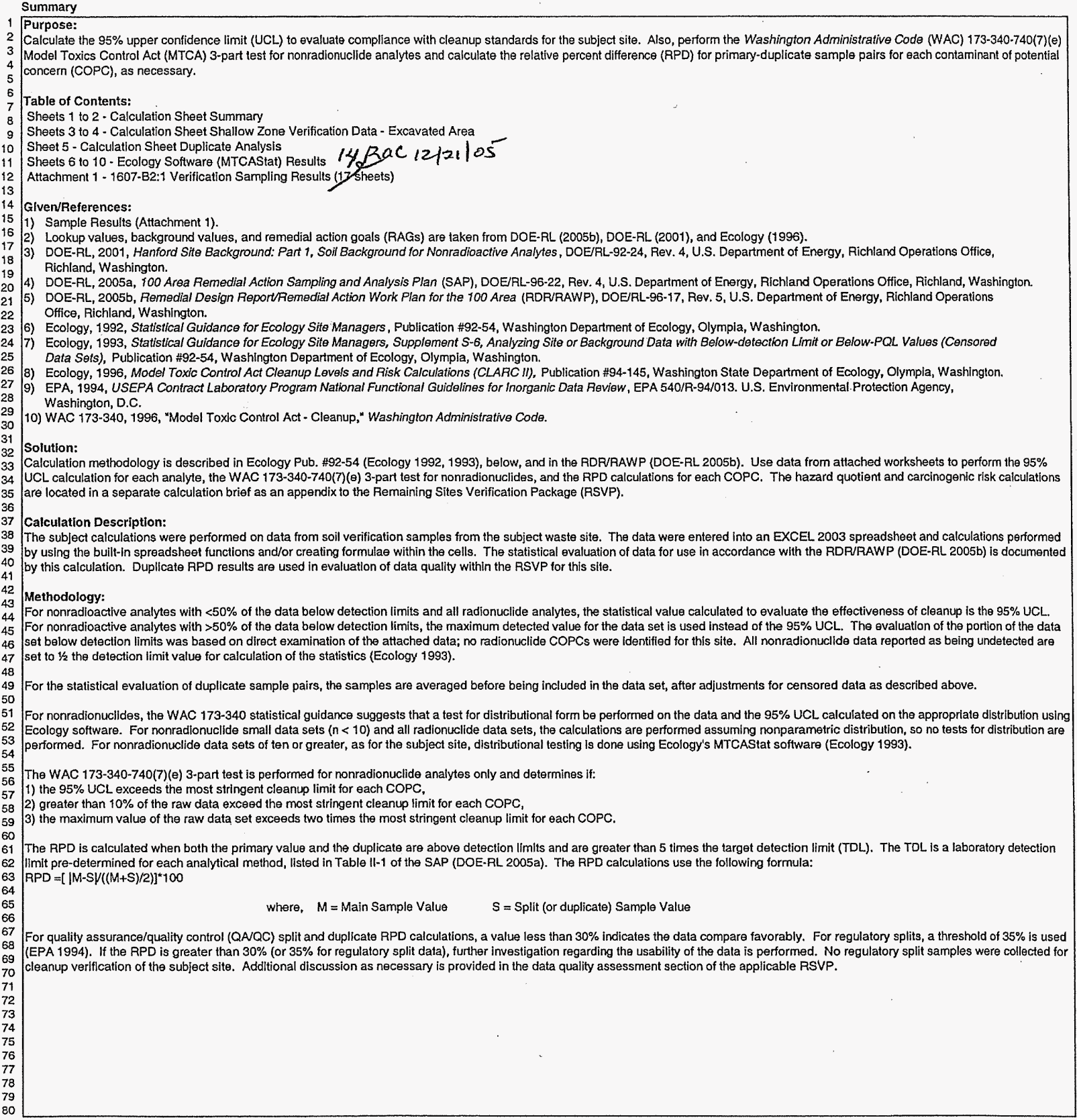




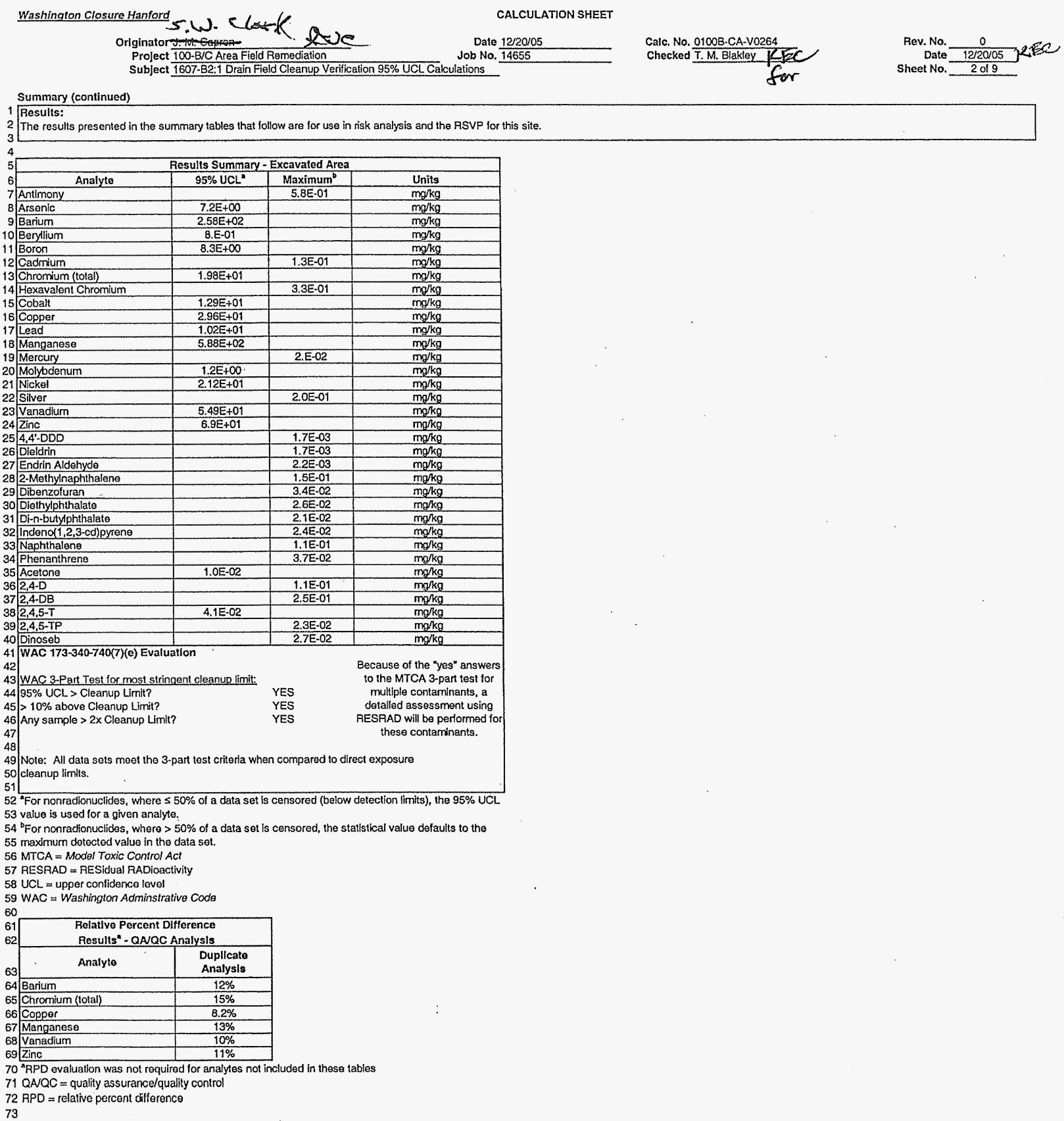


tark

CALCULATION SHEET

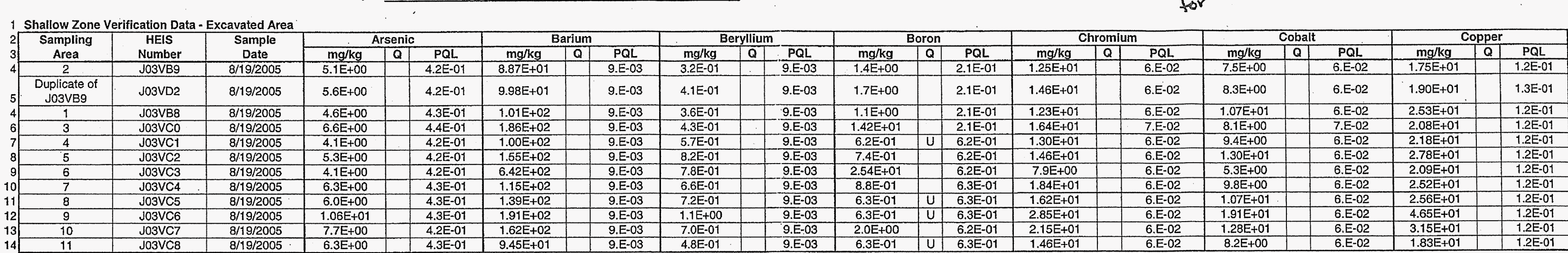
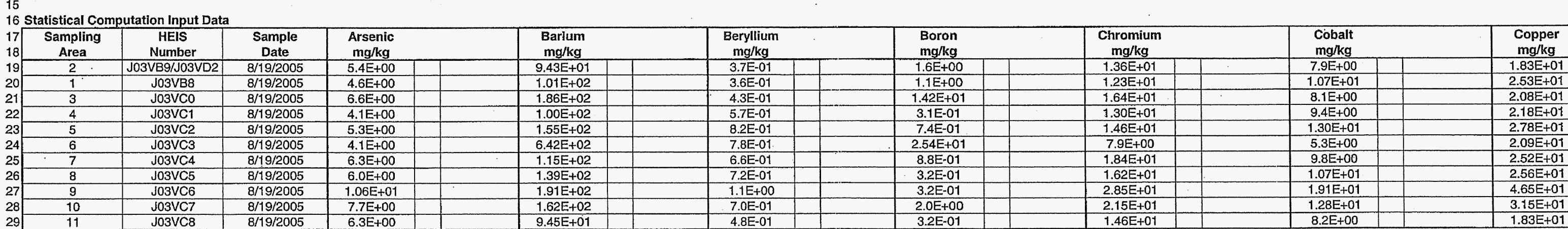

\section{Statistical Computatlons}

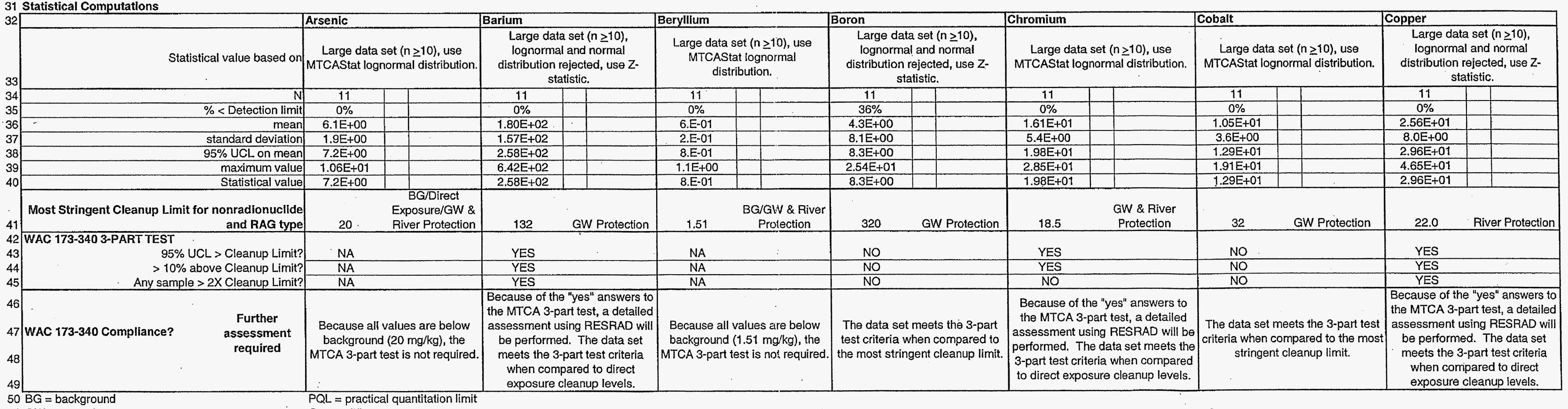

50 BG = background 
5. W. Clark

CALCULATION SHEET

s.w. Clark g.ve Project 100-B/C Area Field Remediatition
Subject 1607-B2:1 Drain Field Cleanup Verification 95\% UCL Calculations
Date $12 / 20 / 05$
Job No. $\frac{14655}{}$
Calc. No. $0100 \mathrm{O}-\mathrm{CA}-\mathrm{V} 0264$
Checked T. M. Blakley KLCC
Rev. No. $\frac{0}{120105}$
Date
Sheet No.

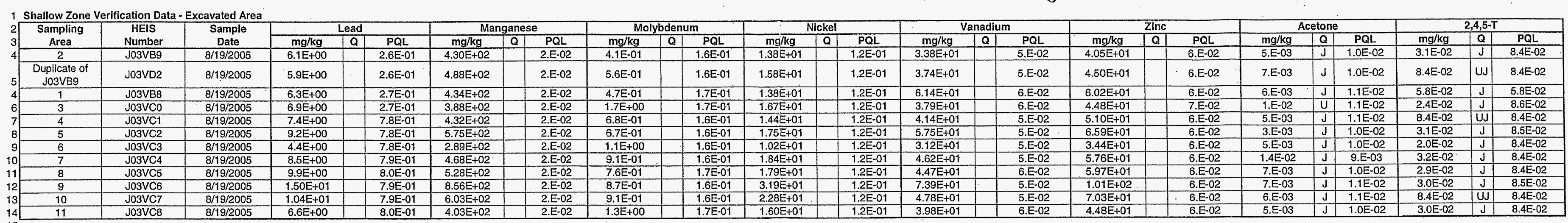

\section{Statistical Computation Input Data}
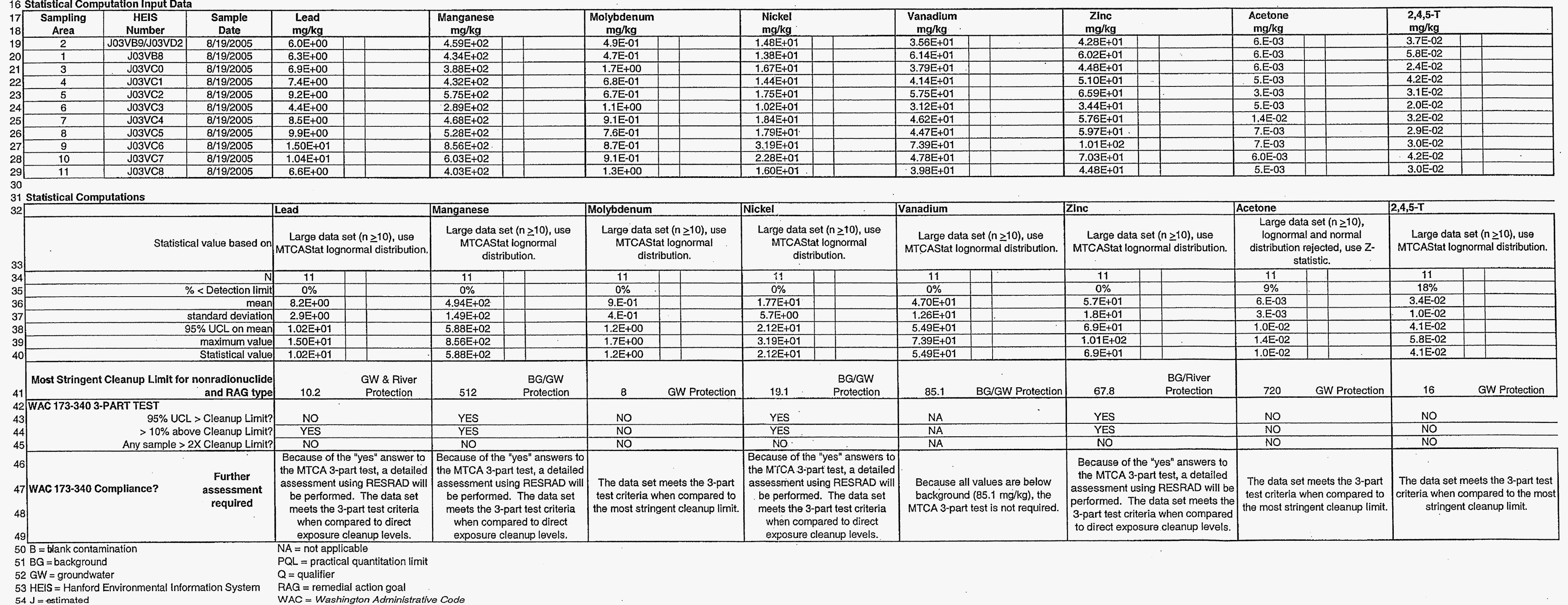

$54 J=$ estimated
55 MTCA $=$ Model Toxic Control Act

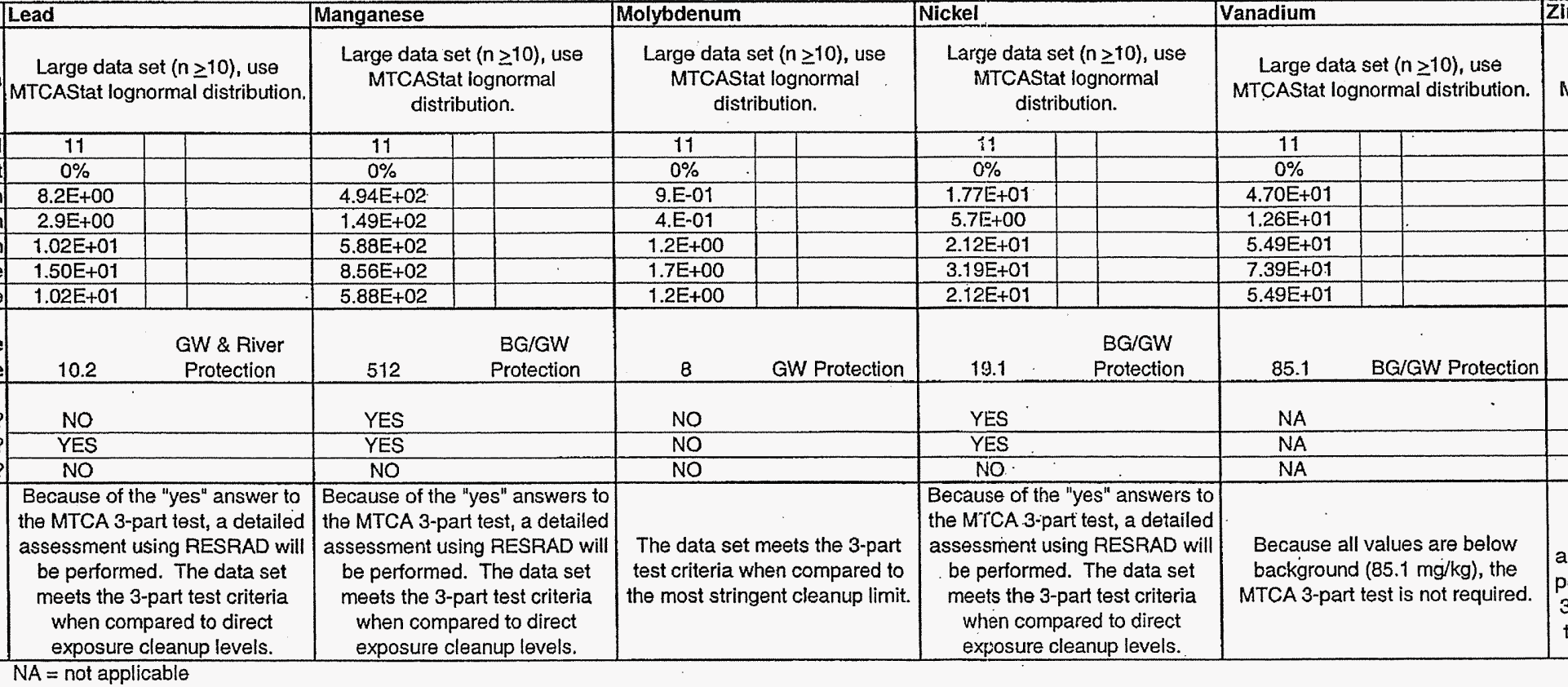

$\begin{aligned} & N A=\text { not applicable } \\ & P Q L\end{aligned}=$ practical quantitation limit

$P Q L=$ practical quantitation limit
$Q=$ qualifier
$R A G=$ remedial action goal
$W A C=$ Washington Administrative Codo 
Washington Closure Hanford

s.w. Clark

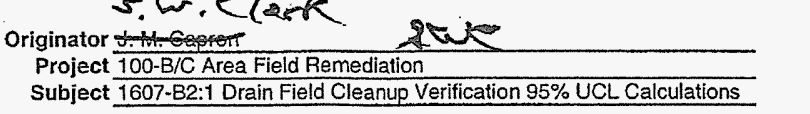

CALCULATION SHEET

Date 12200105

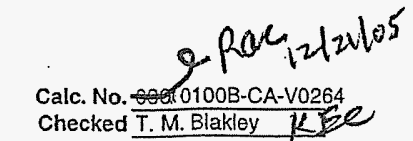

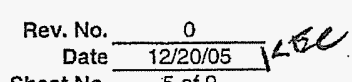

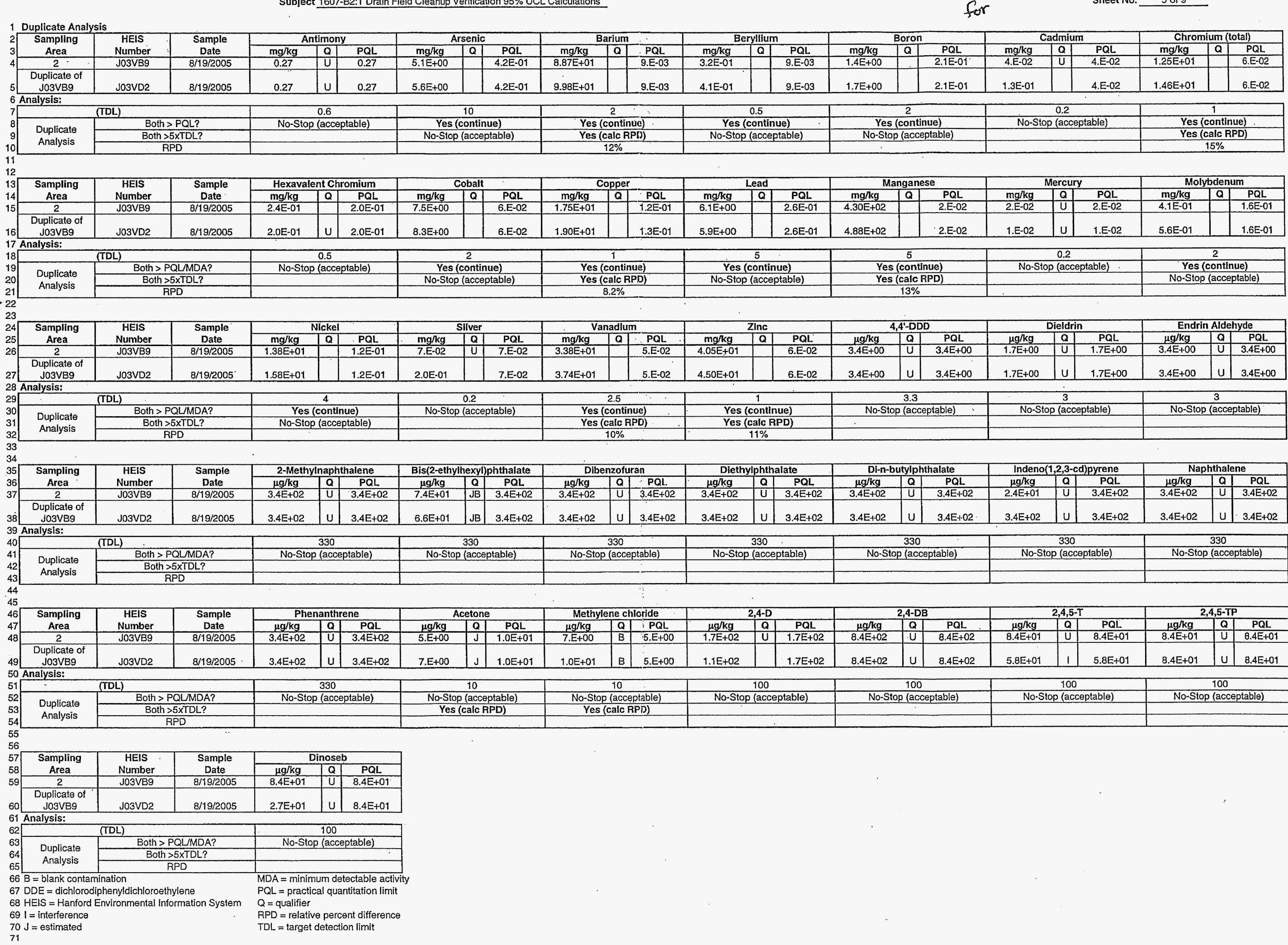


Washington Closure Hanford

Originator S.W. Clark Dwe

Project 100-B/C Area Field Remediation Subject 1607-B2-1 Drain Field Cleanup Verification 95\% UCL Calculations
CALCULATION SHEET

Date 12/20/05 Calc. No. 0100B-CA-V0264

Job No. 14655

Checked T.M. Blakley KEC
Rev. No. $\quad 0$

Date $12 / 20 / 05 \mathrm{~K}^{56}$

Sheet No. 6 of 9

Ecology Software (MTCAStat) Results

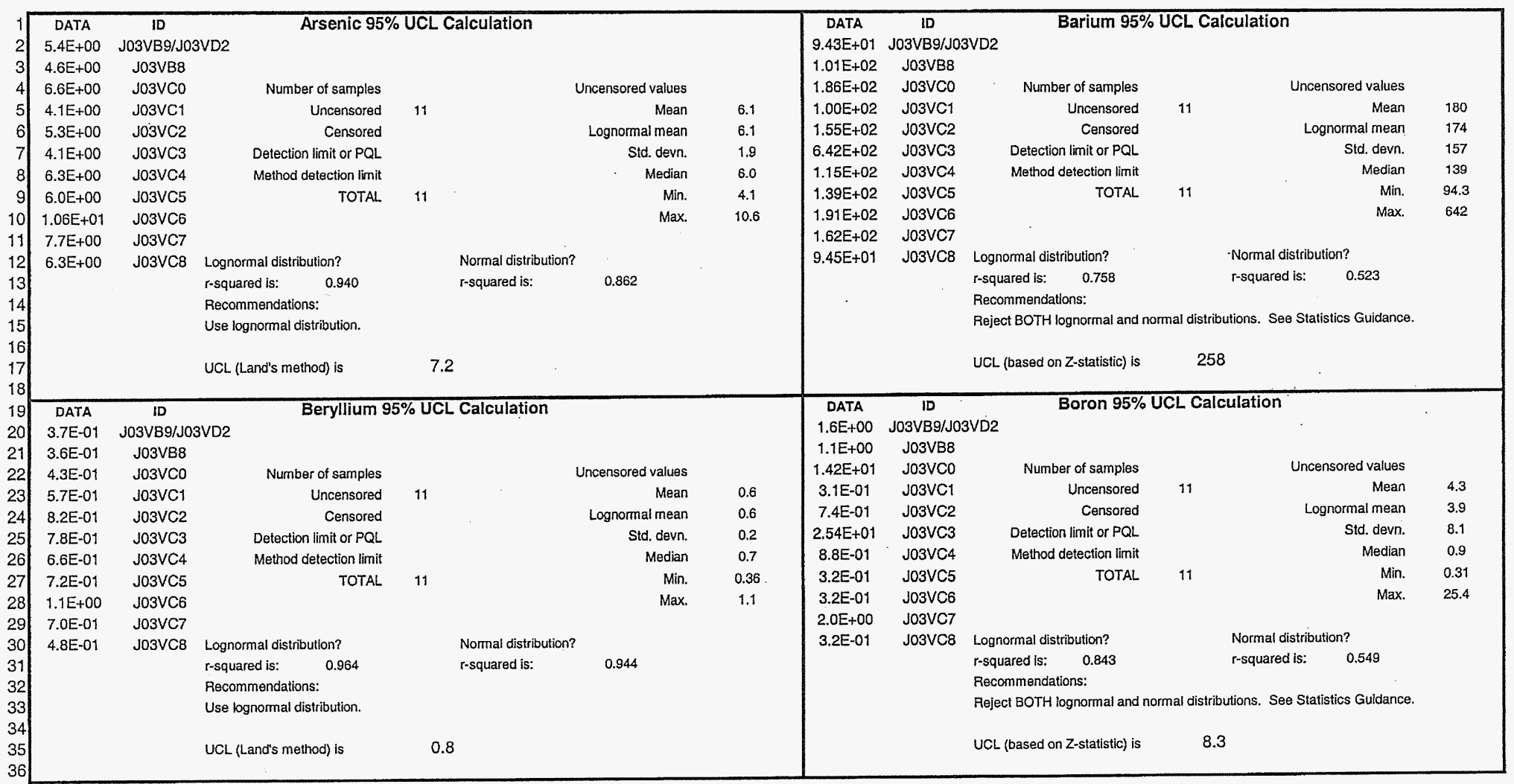


CALCULATION SHEET

Originator

$12 / 20 / 05$

Subject 1607-B2:1 Drain Field Cleanup Verification 95\% UCL Calculations

Date $\frac{12 / 20 / 05}{14655}$
Job No.

Calc. No. 0100B-CA-V0264

Checked T.M. Blakley KEZ

Rev. No. $\frac{0}{12 / 20 / 05<6 e}$

Sheet No.

. 7 of 9

Ecology Software (MTCAStat) Results

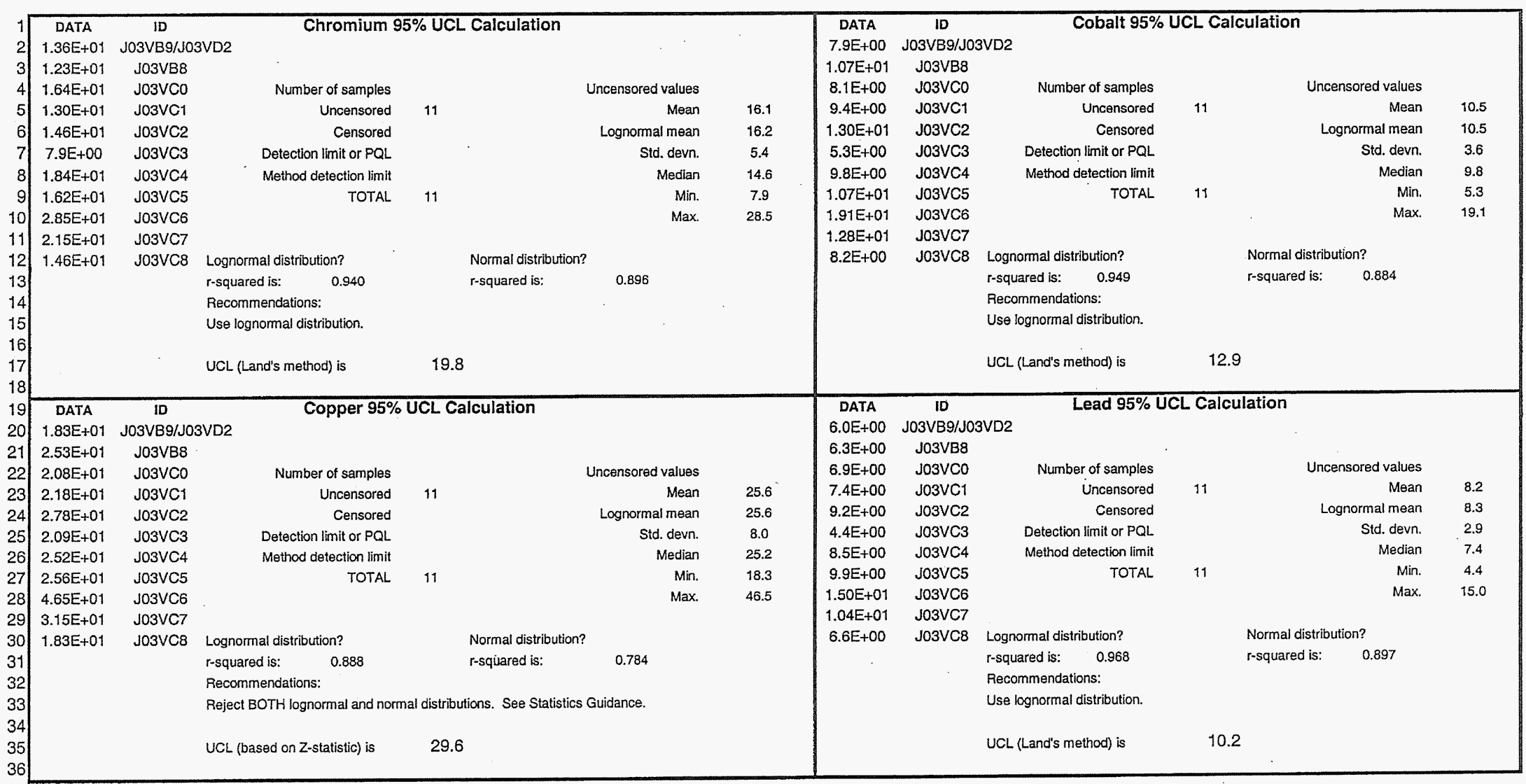


CALCULATION SHEET

Rev. No. 0

Date $\frac{0}{12 / 20 / 05 \mathrm{KEC}}$ Sheet No. 8 of 9 .

Ecology Software (MTCAStat) Results

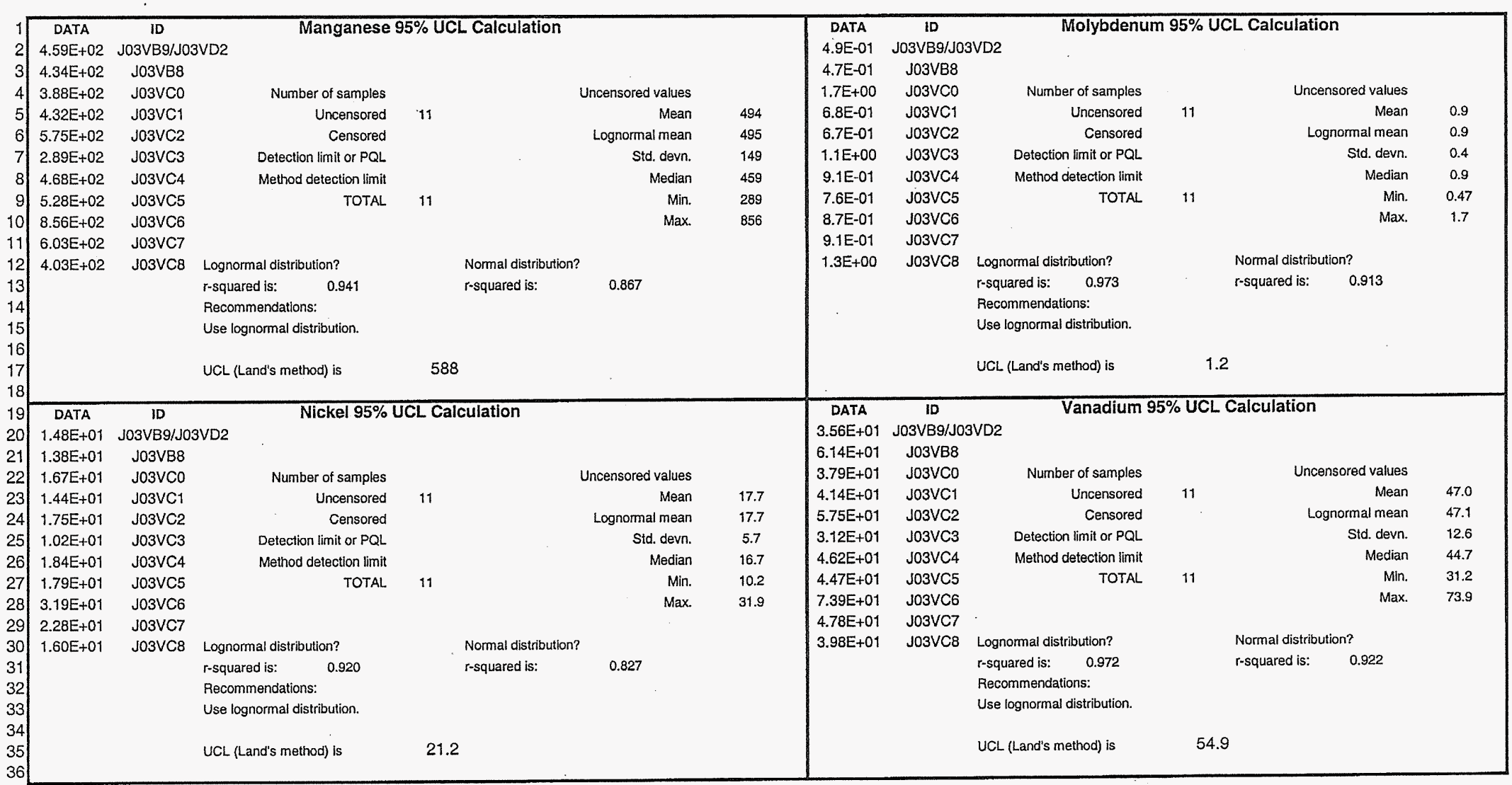




\section{Washington Closure Hanfor}

CALCULATION SHEET

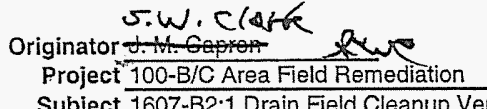

ate $12 / 20 / 05$

Job No. 14655

Calc. No. 0100B-CA-V0264

Project 100-B/C Area Field Remediation

Checked T.M. Blakley 1LEC

Rev. No.

Date $\frac{0}{12 / 20 / 05 \mathrm{~K}}$

Sheet No. $\frac{\text { of } 9}{9}$

Ecology Software (MTCAStat) Results

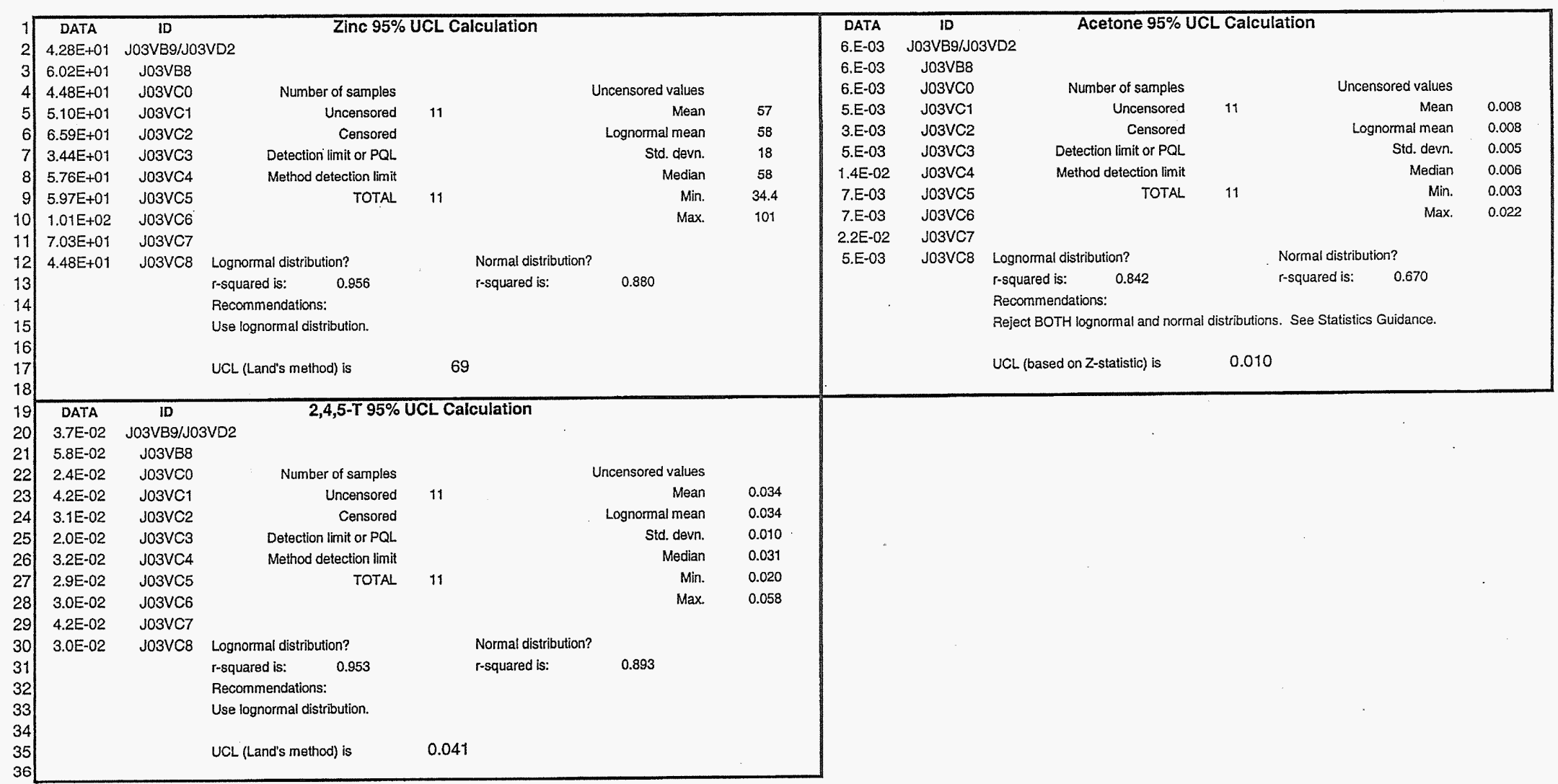


Attachment 1. 1607-B2 Verification Sampling Results.

\begin{tabular}{|c|c|c|c|c|c|c|c|c|c|c|c|c|c|c|c|c|c|c|c|c|}
\hline \multirow{2}{*}{$\begin{array}{c}\text { Sample } \\
\text { Location } \\
\end{array}$} & \multirow{2}{*}{$\begin{array}{c}\text { HEIS } \\
\text { Number }\end{array}$} & \multirow{2}{*}{$\begin{array}{c}\text { Sample } \\
\text { Date }\end{array}$} & \multicolumn{3}{|c|}{ Antimony } & \multicolumn{3}{|c|}{ Arsenic } & \multicolumn{3}{|c|}{ Barium } & \multicolumn{3}{|c|}{ Beryllium } & \multicolumn{3}{|c|}{ Boron } & \multicolumn{3}{|c|}{ Cadmium } \\
\hline & & & $\mathrm{mg} / \mathrm{kg}$ & $Q$ & PQL & $\mathrm{mg} / \mathrm{kg}$ & Q & $\mathrm{PQL}$ & $\mathrm{mg} / \mathrm{kg}$ & Q & PQL & $\mathrm{mg} / \mathrm{kg}$ & Q & $\mathrm{PQL}$ & $\mathrm{mg} / \mathrm{kg}$ & $Q$ & PQL & $\mathrm{mg} / \mathrm{kg}$ & Q & $\mathrm{PQL}$ \\
\hline 2 & J03VB9 & $8 / 19 / 05$ & 0.27 & UJ & 0.27 & 5.1 & & 0.42 & 88.7 & & 0.009 & 0.32 & & 0.009 & 1.4 & & 0.21 & 0.04 & $\mathrm{U}$ & 0.04 \\
\hline 3 & $\mathrm{~J} 03 \mathrm{VCO}$ & $8 / 19 / 05$ & 0.28 & $\mathrm{UJ}$ & 0.28 & 6.6 & & 0.44 & 186 & & 0.009 & 0.43 & & 0.009 & 14.2 & & 0.21 & 0.04 & $\mathrm{U}$ & 0.04 \\
\hline 4 & J03VC1 & $8 / 19 / 05$ & 0.27 & $\mathrm{UJ}$ & 0.27 & 4.1 & & 0.42 & 100 & & 0.009 & 0.57 & & 0.009 & 0.62 & $\mathrm{U}$ & 0.62 & 0.11 & $\mathrm{U}$ & 0.11 \\
\hline 5 & $\mathrm{~J} 03 \mathrm{VC2}$ & $8 / 19 / 05$ & 0.37 & $\mathrm{~d}$ & 0.27 & 5.3 & & 0.42 & 155 & & 0.009 & 0.82 & & 0.009 & 0.74 & & 0.62 & 0.11 & $\mathrm{U}$ & 0.11 \\
\hline 8 & J03VC5 & $8 / 19 / 05$ & 0.39 & $\mathrm{~J}$ & 0.28 & 6.0 & & 0.43 & 139 & & 0.009 & 0.72 & & 0.009 & 0.63 & $\mathrm{U}$ & 0.63 & 0.11 & U & 0.11 \\
\hline 9 & $\mathrm{~J} 03 \mathrm{VC6}$ & $8 / 19 / 05$ & 0.58 & $\mathrm{~J}$ & 0.27 & 10.6 & & 0.43 & 191 & & 0.009 & 1.1 & & 0.009 & 0.63 & $\mathrm{U}$ & 0.63 & 0.11 & $\mathrm{U}$ & 0.11 \\
\hline 10 & J03VC7 & $8 / 19 / 05$ & 0.43 & $\mathrm{~J}$ & 0.27 & 7.7 & & 0.42 & 162 & & 0.009 & 0.70 & & 0.009 & 2.0 & & 0.62 & 0.11 & $\mathrm{U}$ & 0.11 \\
\hline 11 & J03VC8 & $8 / 19 / 05$ & 0.28 & $\overline{\mathrm{UJ}}$ & 0.28 & 6.3 & & 0.43 & 94.5 & & 0.009 & 0.48 & & 0.009 & 0.63 & $\mathrm{U}$ & 0.63 & 0.11 & $\mathrm{U}$ & 0.11 \\
\hline Overburden & J03VC9 & $8 / 19 / 05$ & 0.42 & $\mathrm{~J}$ & 0.27 & 5.3 & & 0.43 & 101 & & 0.009 & 0.47 & & 0.009 & 2.3 & & 0.63 & 0.11 & $\mathrm{U}$ & 0.11 \\
\hline Overburden & J03VD0 & $8 / 19 / 05$ & 0.49 & $\mathrm{~J}$ & 0.27 & 4.4 & & 0.42 & 68.9 & & 0.009 & 0.36 & & 0.009 & 0.61 & $\mathrm{U}$ & 0.61 & 0.11 & $\mathrm{U}$ & 0.11 \\
\hline Overburden & J03VD1 & $8 / 19 / 05$ & 0.42 & $\mathrm{~J}$ & 0.27 & 4.9 & & 0.42 & 75.1 & & 0.009 & 0.33 & & 0.009 & 1.4 & & 0.21 & 0.13 & & 0.04 \\
\hline $\begin{array}{l}\text { Equipment } \\
\text { Blank }\end{array}$ & J03WW6 & $8 / 19 / 05$ & 0.27 & UJ & 0.27 & 0.42 & $\mathrm{U}$ & 0.42 & 0.93 & & 0.009 & 0.009 & $\mathrm{U}$ & 0.009 & 0.50 & & 0.21 & 0.04 & U & 0.04 \\
\hline
\end{tabular}

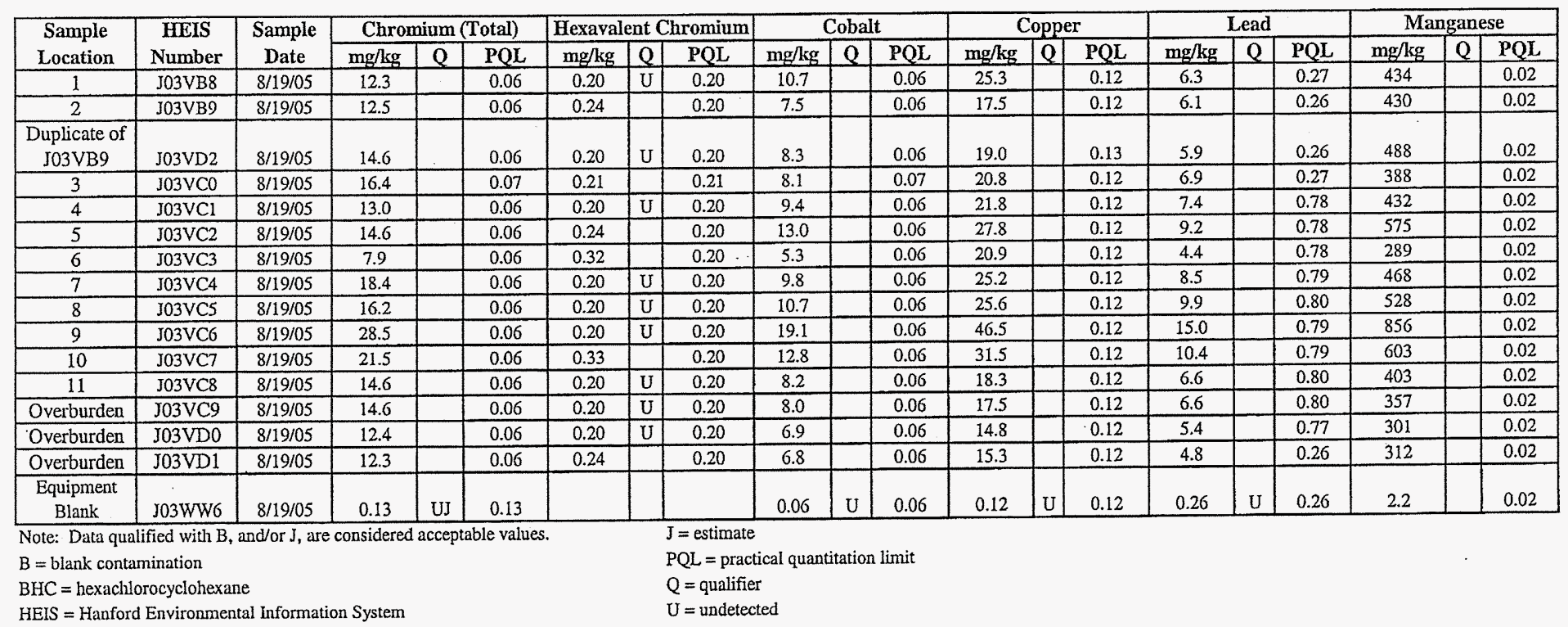


Attachment 1. 1607-B2 Verification Sampling Results.

\begin{tabular}{|c|c|c|c|c|c|c|c|c|c|c|c|c|c|c|c|c|c|c|c|c|}
\hline \multirow{2}{*}{$\begin{array}{l}\text { Sample } \\
\text { Location }\end{array}$} & \multirow{2}{*}{$\begin{array}{c}\text { HEIS } \\
\text { Number }\end{array}$} & \multirow{2}{*}{$\begin{array}{c}\text { Sample } \\
\text { Date }\end{array}$} & \multicolumn{3}{|c|}{ Mercury } & \multicolumn{3}{|c|}{ Molybdenum } & \multicolumn{3}{|c|}{ Nickel } & \multicolumn{3}{|c|}{ Selenium } & \multicolumn{3}{|c|}{ Silver } & \multicolumn{3}{|c|}{ Vanadium } \\
\hline & & & $\mathrm{mg} / \mathrm{kg}$ & Q & PQL & $\mathrm{mg} / \mathrm{kg}$ & Q & $\mathrm{PQL}$ & $\mathrm{mg} / \mathrm{kg}$ & $Q$ & PQL & $\mathrm{mg} / \mathrm{kg}$ & $Q$ & $\mathrm{PQL}$ & $\mathrm{mg} / \mathrm{kg}$ & $\mathrm{Q}$ & $\mathrm{PQL}$ & $\mathrm{mg} / \mathrm{kg}$ & $Q$ & $\mathrm{PQL}$ \\
\hline 1 & J03VB8 & $8 / 19 / 05$ & 0.02 & U & 0.02 & 0.47 & & 0.17 & 13.8 & & 0.12 & 0.39 & $\mathrm{U}$ & 0.39 & 0.07 & $\mathrm{U}$ & 0.07 & 61.4 & & 0.06 \\
\hline 2 & J03VB9 & $8 / 19 / 105$ & 0.02 & U & 0.02 & 0.41 & & 0.16 & 13.8 & & 0.12 & 0.39 & $\mathrm{U}$ & 0.39 & 0.07 & $\bar{U}$ & 0.07 & 33.8 & & 0.05 \\
\hline \multirow{2}{*}{\multicolumn{21}{|c|}{ Duplicate of }} \\
\hline & J03VD2 & $8 / 19 / 05$ & 0.01 & U & 0.01 & 0.56 & & 0.16 & 15.8 & & 0.12 & 0.39 & $u$ & 0.39 & 0.20 & & 0.07 & 37.4 & & 0.05 \\
\hline 3 & $\mathrm{~J} 03 \mathrm{VCO}$ & $8 / 19 / 05$ & 0.01 & $\bar{U}$ & 0.01 & 1.7 & & 0.17 & 16.7 & & 0.12 & 0.40 & $\mathrm{U}$ & 0.40 & 0.11 & & 0.07 & 37.9 & & 0.06 \\
\hline 4 & J03 VCl & $8 / 19 / 05$ & 0.02 & $\mathrm{U}$ & 0.02 & 0.68 & & 0.16 & 14.4 & & 0.12 & 0.39 & $\mathrm{U}$ & 0.39 & 0.07 & $U$ & 0.07 & $41: 4$ & & 0.05 \\
\hline 5 & J03VC2 & $8 / 19 / 05$ & 0.02 & $\bar{U}$ & 0.02 & 0.67 & & 0.16 & 17.5 & & 0.12 & 0.39 & $\mathrm{U}$ & 0.39 & 0.07 & $\mathrm{U}$ & 0.07 & 57.5 & & 0.05 \\
\hline 6 & J03VC3 & $8 / 19 / 05$ & 0.01 & $\mathrm{U}$ & 0.01 & 1.1 & & 0.16 & 10.2 & & 0.12 & 0.39 & $\mathrm{U}$ & 0.39 & 0.07 & $\mathrm{U}$ & 0.07 & 31.2 & & 0.05 \\
\hline 7 & J03VC4 & $8 / 19 / 05$ & 0.01 & $\bar{U}$ & 0.01 & 0.91 & & 0.16 & 18.4 & & 0.12 & 0.39 & $\mathrm{U}$ & 0.39 & 0.07 & U & 0.07 & 46.2 & & 0.05 \\
\hline 8 & J03VCS & $8 / 19 / 05$ & 0.01 & $\mathrm{U}$ & 0.01 & 0.76 & & 0.17 & 17.9 & & 0.12 & 0.39 & $\mathrm{U}$ & 0.39 & 0.07 & $\mathrm{U}$ & 0.07 & 44.7 & & 0.06 \\
\hline 9 & ग03VC6 & $8 / 19 / 05$ & 0.01 & $\mathrm{U}$ & 0.01 & 0.87 & & 0.16 & 31.9 & & 0.12 & 0.39 & $\mathrm{U}$ & 0.39 & 0.07 & U & 0.07 & 73.9 & & 0.05 \\
\hline 10 & $503 \mathrm{VC7}$ & $8 / 19105$ & 0.02 & & 0.01 & 0.91 & & 0.16 & 22.8 & & 0.12 & 0.39 & U & 0.39 & 0.07 & $\mathrm{U}$ & 0.07 & 47.8 & & 0.05 \\
\hline 11 & J03VC8 & $8 / 19 / 05$ & 0.01 & $\mathrm{U}$ & 0.01 & 1.3 & & 0.17 & 16.0 & & 0.12 & 0.39 & $\bar{U}$ & 0.39 & 0.07 & $\bar{U}$ & 0.07 & 39.8 & & 0.06 \\
\hline \multirow{2}{*}{\multicolumn{3}{|c|}{$\begin{array}{c}\%<\text { Detection limit } \\
\text { Maximum value }\end{array}$}} & \multirow{2}{*}{\multicolumn{3}{|c|}{$\frac{91 \%}{0.02}$}} & \multirow{2}{*}{\multicolumn{3}{|c|}{$0 \%$}} & \multicolumn{3}{|l|}{$0 \%$} & \multirow{2}{*}{\multicolumn{3}{|c|}{$100 \%$}} & \multirow{2}{*}{\multicolumn{3}{|c|}{$\frac{82 \%}{0.20}$}} & \multicolumn{3}{|l|}{$0 \%$} \\
\hline & & & & & & 1.7 & & & 31.9 & & & 0.4 & & & & & & 73.9 & & \\
\hline Overburden & J03VC9 & $8 / 19 / 05$ & 0.01 & $\bar{U}$ & 0.01 & 0.57 & & 0.16 & 14.4 & & 0.12 & 0.39 & $\mathrm{U}$ & 0.39 & 0.07 & $\mathrm{U}$ & 0.07 & 40.0 & & 0.05 \\
\hline Overburden & J03VD0 & $8 / 19 / 05$ & 0.02 & U & 0.02 & 0.60 & & 0.16 & 12.8 & & 0.12 & 0.38 & $\mathrm{U}$ & 0.38 & 0.07 & $\mathrm{U}$ & 0.07 & 34.9 & & 0.05 \\
\hline Overburden & J03VD1 & $8 / 19 / 05$ & 0.02 & $\mathrm{U}$ & 0.02 & 0.61 & & 0.16 & 13.1 & & 0.12 & 0.39 & $\mathrm{U}$ & 0.39 & 0.09 & & 0.07 & 31.7 & & 0.05 \\
\hline Equipment & $103 W W 6$ & $8 / 19105$ & 007 & II & 0.02 & 016 & $U$ & 0.16 & 0.12 & U & 0.12 & 0.39 & $U$ & 0.39 & 0.07 & U & 0.07 & 0.09 & & 0.05 \\
\hline
\end{tabular}

\begin{tabular}{|c|c|c|c|c|c|}
\hline \multirow{2}{*}{$\begin{array}{l}\text { Sample } \\
\text { Location }\end{array}$} & \multirow{2}{*}{$\begin{array}{l}\text { HEIS } \\
\text { Number }\end{array}$} & \multirow{2}{*}{$\begin{array}{c}\text { Sample } \\
\text { Date }\end{array}$} & \multicolumn{3}{|c|}{ Zinc } \\
\hline & & & $\mathrm{mg} / \mathrm{kg}$ & $Q$ & PQL \\
\hline 1 & J03VB8 & $8 / 19 / 05$ & 60.2 & & 0.06 \\
\hline 2 & J03VB9 & $8 / 19 / 05$ & 40.5 & & 0.06 \\
\hline $\begin{array}{l}\text { Duplicate of } \\
\text { J03VB9 }\end{array}$ & J03VD2 & $8 / 19 / 05$ & 45.0 & & 0.06 \\
\hline 3 & $\mathrm{~J} 03 \mathrm{VCO}$ & $8 / 19 / 05$ & 44.8 & & 0.07 \\
\hline 4 & $\mathrm{J03 \textrm {VCl }}$ & $8 / 19 / 05$ & 51.0 & & 0.06 \\
\hline 5 & $\mathrm{J03 \textrm {VC2 }}$ & $8 / 19 / 05$ & 65.9 & & 0.06 \\
\hline 6 & $\mathrm{~J} 03 \mathrm{VC} 3$ & $8 / 19 / 05$ & 34.4 & & 0.06 \\
\hline 7 & $\mathrm{~J} 03 \mathrm{VC} 4$ & $8 / 19 / 05$ & 57.6 & & 0.06 \\
\hline 8 & J03VC5 & $8 / 19 / 05$ & 59.7 & & 0.06 \\
\hline 9 & $\mathrm{~J} 03 \mathrm{VC} 6$ & $8 / 19 / 05$ & 101 & & 0.06 \\
\hline 10 & $503 \mathrm{VC7}$ & $8 / 19 / 05$ & 70.3 & & 0.06 \\
\hline 11 & J03VC8 & $8 / 19 / 05$ & 44.8 & & 0.06 \\
\hline \multirow{2}{*}{\multicolumn{3}{|c|}{$\%<$ Detection limit }} & \multicolumn{3}{|l|}{$095_{0}$} \\
\hline & & Maximum value & \multicolumn{3}{|l|}{101} \\
\hline Overburden & J03VC9 & $8 / 19 / 05$ & 42.5 & & 0.06 \\
\hline Overburden & J03VDO & $8 / 19 / 05$ & 36.1 & & 0.06 \\
\hline Overburden & J03VDI & $8 / 19105$ & 36.2 & & 0.06 \\
\hline $\begin{array}{l}\text { Equipment } \\
\text { Blank }\end{array}$ & J03WW6 & $8 / 19 / 05$ & 1.1 & & 0.06 \\
\hline
\end{tabular}

Attachment Originator Calc. No. 
Attachment 1. 1607-B2:1 Verification Sampling Results.

\begin{tabular}{|c|c|c|c|c|c|c|c|c|c|c|c|c|}
\hline \multirow[t]{2}{*}{ Constituent } & \multicolumn{3}{|c|}{$\begin{array}{c}\text { J03WW6 } \\
\text { Equipment Blank } \\
\text { Sample Date 8/19/05 }\end{array}$} & \multicolumn{3}{|c|}{$\begin{array}{c}\text { J03VB8 } \\
\text { Sample Location } 1 \\
\text { Sample Date 8/19/05 }\end{array}$} & \multicolumn{3}{|c|}{$\begin{array}{c}\text { J03VB9 } \\
\text { Sample Location } 2 \\
\text { Sample Date } 8 / 19 / 05\end{array}$} & \multicolumn{3}{|c|}{$\begin{array}{c}\text { J03VD2 } \\
\text { Duplicate of J03VB9 } \\
\text { Sample Date } 8 / 19 / 05\end{array}$} \\
\hline & $\mu \mathrm{g} / \mathrm{kg}$ & Q] & PQL & $\mu \mathrm{g} / \mathrm{kg}$ & Q & $\overline{\text { PQL }}$ & $\mu \mathrm{g} / \mathrm{kg}$ & Q & PQL & $\mu \mathrm{g} / \mathrm{kg}$ & $Q$ & PQL \\
\hline \multicolumn{13}{|c|}{ Polychlorinated Biphenyls } \\
\hline Aroclor -1016 & & & & 13 & U & 13 & 13 & $\mathrm{U}$ & 13 & 13 & $\mathrm{U}$ & 13 \\
\hline Aroclor-1221 & & & & 13 & $\mathrm{U}$ & 13 & 13 & $\mathrm{U}$ & 13 & 13 & $\bar{U}$ & 13 \\
\hline Aroclor-1232 & & & & 13 & U & 13 & 13 & $\mathrm{U}$ & 13 & 13 & $\mathrm{U}$ & 13 \\
\hline Aroclor-1242 & & & & 13 & $\mathrm{U}$ & 13 & 13 & $\mathrm{U}$ & 13 & 13 & $\mathrm{U}$ & 13 \\
\hline Aroclor -1248 & & & & 13 & $\mathrm{U}$ & 13 & 13 & $\mathrm{U}$ & 13 & 13 & U & 13 \\
\hline Aroclor-1254 & & & & 13 & $\mathrm{U}$ & 13 & 13 & $\mathrm{U}$ & 13 & 13 & $\mathrm{U}$ & 13 \\
\hline Aroclor- 1260 & & & & 13 & $\mathrm{U}$ & 13 & 13 & $\mathrm{U}$ & 13 & 13 & $\mathrm{U}$ & 13 \\
\hline \multicolumn{13}{|c|}{ Pesticides } \\
\hline Aldrin & & & & 1.7 & UJ & 1.7 & 1.7 & UJ & 1.7 & 1.7 & UJ & 1.7 \\
\hline Alpha-BHC & & & & 1.7 & UJ & 1.7 & 1.7 & UJ & 1.7 & 1.7 & UJ & 1.7 \\
\hline alpha-Chlordane & & & & 1.7 & UJ & 1.7 & 1.7 & UJ & 1.7 & 1.7 & UJ & 1.7 \\
\hline Beta-BHC & & & & 1.7 & $\mathrm{UJ}$ & 1.7 & 1.7 & UJ & 1.7 & 1.7 & UJ & 1.7 \\
\hline Delta-BHC & & & & 1.7 & UJ & 1.7 & 1.7 & UJ & 1.7 & 1.7 & UJ & 1.7 \\
\hline Dichlorodiphenyldichloroethane & & & & 3.4 & UJ & 3.4 & 3.4 & $\mathrm{UJ}$ & 3.4 & 3.4 & UJ & 3.4 \\
\hline Dichlorodiphenyldichloroethylene & & & & 3.4 & UJ & 3.4 & 3.4 & UJ & 3.4 & 3.4 & UJ & 3.4 \\
\hline Dichlorodiphenyltrichloroethane & & & & 3.4 & $\mathrm{UJ}$ & 3.4 & 3.4 & UJ & 3.4 & 3.4 & UJ & 3.4 \\
\hline Dieldrin & & & & 1.7 & UJ & 1.7 & 1.7 & UJ & 1.7 & 1.7 & $\mathrm{UJ}$ & 1.7 \\
\hline Endosulfan I & & & & 1.7 & UJ & 1.7 & 1.7 & UI & 1.7 & 1.7 & $\mathrm{UJ}$ & 1.7 \\
\hline Endosulfan II & & & & 3.4 & UI & 3.4 & 3.4 & UJ & 3.4 & 3.4 & UJ & 3.4 \\
\hline Endosulfan sulfate & & & & 3.4 & $\mathrm{UJ}$ & 3.4 & 3.4 & UJ & 3.4 & 3.4 & $\mathrm{UJ}$ & 3.4 \\
\hline Endrin & & & & 3.4 & $\mathrm{UJ}$ & 3.4 & 3.4 & UJ & 3.4 & 3.4 & UJ & 3.4 \\
\hline Endrin aldehyde & & & & 3.4 & UJ & 3.4 & 3.4 & UU & 3.4 & 3.4 & UJ & 3.4 \\
\hline Endrin ketone & & & & 3.4 & UJ & 3.4 & 3.4 & UJ & 3.4 & 3.4 & UJ & 3.4 \\
\hline Gamma-BHC (Lindane) & & & & 1.7 & UJ & 1.7 & 1.7 & UJ & 1.7 & 1.7 & UJ & 1.7 \\
\hline gamma-Chlordane & & & & 1.7 & UJ & 1.7 & 1.7 & UJ & 1.7 & 1.7 & UJ & 1.7 \\
\hline Heptachlor & & & & 1.7 & UJ & 1.7 & 1.7 & UJ & 1.7 & 1.7 & $\mathrm{UJ}$ & 1.7 \\
\hline Heptachlor epoxide & & & & 1.7 & UJ & 1.7 & 1.7 & UI & 1.7 & 1.7 & $\mathrm{UJ}$ & 1.7 \\
\hline Methoxychlor & & & & 17 & UT & 17 & 17 & $\mathrm{UJ}$ & 17 & 17 & UY & 17 \\
\hline Toxaphene & & & & 170 & UJ & 170 & 170 & $\mathrm{UJ}$ & 170 & 170 & UJ & 170 \\
\hline \multicolumn{13}{|c|}{ Semivolatile Organic Analytes } \\
\hline 1,2,4-Trichlorobenzene & 330 & $\mathrm{U}$ & 330 & 340 & $\mathrm{U}$ & 340 & 340 & $\overline{\mathrm{U}}$ & 340 & 340 & $\bar{U}$ & 340 \\
\hline 1,2-Dichlorobenzene & 330 & $\mathrm{U}$ & 330 & 340 & $\mathrm{U}$ & 340 & 340 & $\mathrm{U}$ & 340 & 340 & $\mathrm{U}$ & 340 \\
\hline 1,3-Dichlorobenzene & 330 & $\mathrm{U}$ & 330 & 340 & $\mathrm{U}$ & 340 & 340 & $\mathrm{U}$ & 340 & 340 & $\mathrm{U}$ & 340 \\
\hline 1,4-Dichlorobenzene & 330 & $\mathrm{U}$ & 330 & 340 & $\mathrm{U}$ & 340 & 340 & $\mathrm{U}$ & 340 & 340 & $\mathrm{U}$ & 340 \\
\hline 2,4,5-Trichlorophenol & 830 & $\mathrm{U}$ & 830 & 840 & U & 840 & 840 & $\mathrm{U}$ & 840 & 840 & $\mathrm{U}$ & 840 \\
\hline 2,4,6-Trichlorophenol & 330 & $\mathrm{U}$ & 330 & 340 & $\mathrm{U}$ & 340 & 340 & $\mathrm{U}$ & 340 & 340 & $\mathrm{U}$ & 340 \\
\hline 2,4-Dichlorophenol & 330 & $\mathrm{U}$ & 330 & 340 & U & 340 & 340 & $\mathrm{U}$ & 340 & 340 & $\mathrm{U}$ & 340 \\
\hline 2,4-Dimethylphenol & 330 & $\bar{U}$ & 330 & 340 & U & 340 & 340 & $U$ & 340 & 340 & $\mathrm{U}$ & 340 \\
\hline 2,4-Dinitrophenol & 830 & $\mathrm{U}$ & 830 & 840 & U & 840 & 840 & $\mathrm{U}$ & 840 & 840 & $\mathrm{U}$ & 840 \\
\hline 2,4-Dinitrotoluene & 330 & $\mathrm{U}$ & 330 & 340 & $\mathrm{U}$ & 340 & 340 & $\mathrm{U}$ & 340 & 340 & U & 340 \\
\hline 2,6-Dinitrotoluene & 330 & $\mathrm{U}$ & 330 & 340 & $\mathrm{U}$ & 340 & 340 & $\mathrm{U}$ & 340 & 340 & $\mathrm{U}$ & 340 \\
\hline 2-Chloronaphthalene & 330 & $\mathrm{U}$ & 330 & 340 & $\mathrm{U}$ & 340 & 340 & $\mathrm{U}$ & 340 & 340 & $\mathrm{U}$ & 340 \\
\hline 2-Chlorophenol & 330 & $\mathrm{U}$ & 330 & 340 & $\mathrm{U}$ & 340 & 340 & $\mathrm{U}$ & 340 & 340 & $\mathrm{U}$ & 340 \\
\hline 2-Methylnaphthalene & 330 & $\mathrm{U}$ & 330 & 340 & $\mathrm{U}$ & 340 & 340 & U & 340 & 340 & $\bar{U}$ & 340 \\
\hline 2-Methylphenol (cresol, o-) & 330 & $\mathrm{U}$ & 330 & 340 & $\mathrm{U}$ & 340 & 340 & $\mathrm{U}$ & 340 & 340 & $\mathrm{U}$ & 340 \\
\hline 2-Nitroaniline & 830 & $\mathrm{U}$ & 830 & 840 & $\mathrm{U}$ & 840 & 840 & $\mathrm{U}$ & 840 & 840 & $\mathrm{U}$ & 840 \\
\hline 2-Nitrophenol & 330 & $\mathrm{U}$ & 330 & 340 & $\mathrm{U}$ & 340 & 340 & $\mathrm{U}$ & 340 & 340 & $\mathrm{U}$ & 340 \\
\hline & & & & & \multirow{4}{*}{\multicolumn{2}{|c|}{$\begin{array}{l}\text { Attachment } \\
\text { Originator } \\
\text { Checked } \\
\text { Calc. No. }\end{array}$}} & \multicolumn{3}{|c|}{1} & \multirow[t]{4}{*}{ Sheet No. } & \multicolumn{2}{|r|}{3 of 14} \\
\hline & & & & & & & \multicolumn{3}{|c|}{ 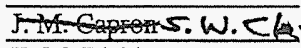 } & & \multicolumn{2}{|c|}{$<12 / 20 / 05$} \\
\hline & & & & & & & \multirow{2}{*}{\multicolumn{3}{|c|}{$\frac{\text { T. M. Blakley }}{\text { 0100B-CA-V0264 }}$}} & & \multicolumn{2}{|c|}{$12 / 20 / 05$} \\
\hline & & & & & & & & & & & & 0 \\
\hline
\end{tabular}


Attachment 1. 1607-B2:1 Verification Sampling Results.

\begin{tabular}{|c|c|c|c|c|c|c|c|c|c|c|c|c|}
\hline \multirow[t]{2}{*}{ Constituent } & \multicolumn{3}{|c|}{$\begin{array}{c}\text { J03WW6 } \\
\text { Equipment Blank } \\
\text { Sample Date 8/19/05 }\end{array}$} & \multicolumn{3}{|c|}{$\begin{array}{c}\text { J03VB8 } \\
\text { Sample Location } 1 \\
\text { Sample Date 8/19/05 } \\
\end{array}$} & \multicolumn{3}{|c|}{$\begin{array}{c}\text { J03VB9 } \\
\text { Sample Location } 2 \\
\text { Sample Date } 8 / 19 / 05 \\
\end{array}$} & \multicolumn{3}{|c|}{$\begin{array}{c}\text { J03VD2 } \\
\text { Duplicate of J03VB9 } \\
\text { Sample Date } 8 / 19 / 05\end{array}$} \\
\hline & $\mu \mathrm{g} / \mathrm{kg}$ & Q & PQL & $\mu \mathrm{g} / \mathrm{kg}$ & $\mathbf{Q}$ & PQL & $\mu \mathrm{g} / \mathrm{kg}$ & $\mathbf{Q}$ & PQL & $\mu \mathrm{g} / \mathrm{kg}$ & Q & PQL \\
\hline \multicolumn{13}{|c|}{ Semivolatile Organic Analytes (continued) } \\
\hline 3,3'-Dichlorobenzidine & 330 & $\mathrm{U}$ & 330 & 340 & $\mathrm{U}$ & 340 & 340 & $\mathrm{U}$ & 340 & 340 & $\mathrm{U}$ & 340 \\
\hline $3+4$ Methylphenol (cresol, $m+p)$ & 330 & $\mathrm{U}$ & 330 & 340 & $\mathrm{U}$ & 340 & 340 & $\mathrm{U}$ & 340 & 340 & $\mathrm{U}$ & 340 \\
\hline 3-Nitroaniline & 830 & UJ & 830 & 840 & UJ & 840 & 840 & UJ & 840 & 840 & UJ & 840 \\
\hline 4,6-Dinitro-2-methylphenol & 830 & $\mathrm{U}$ & 830 & 840 & $\mathrm{U}$ & 840 & 840 & $\mathrm{U}$ & 840 & 840 & $\mathrm{U}$ & 840 \\
\hline 4-Bromophenyl-phenylether & 330 & $\mathrm{U}$ & 330 & 340 & $\mathrm{U}$ & 340 & 340 & $\mathrm{U}$ & 340 & 340 & $\mathrm{U}$ & 340 \\
\hline 4-Chloro-3-methylphenol & 330 & $\mathrm{U}$ & 330 & 340 & $\mathrm{U}$ & 340 & 340 & $\mathrm{U}$ & 340 & 340 & $\mathrm{U}$ & 340 \\
\hline 4-Chloroaniline & 330 & $\mathrm{U}$ & 330 & 340 & $\mathrm{U}$ & 340 & 340 & $\mathrm{U}$ & 340 & 340 & $\mathrm{U}$ & 340 \\
\hline 4-Chlorophenyl-phenylether & 330 & $\mathrm{U}$ & 330 & 340 & $\mathrm{U}$ & 340 & 340 & $\mathrm{U}$ & 340 & 340 & $\mathrm{U}$ & 340 \\
\hline 4-Nitroaniline & 830 & UJ & 830 & 840 & UJ & 840 & 840 & $\mathrm{UJ}$ & 840 & 840 & $\mathrm{UJ}$ & 840 \\
\hline 4-Nitrophenol & 830 & UI & 830 & 840 & UI & 840 & 840 & UJ & 840 & 840 & UJ & 840 \\
\hline Acenaphthene & 330 & $\mathrm{U}$ & 330 & 340 & $\mathrm{U}$ & 340 & 340 & U & 340 & 340 & $\mathrm{U}$ & 340 \\
\hline Acenaphthylene & 330 & $\mathrm{U}$ & 330 & 340 & $\mathrm{U}$ & 340 & 340 & $\mathrm{U}$ & 340 & 340 & $\mathrm{U}$ & 340 \\
\hline Anthracene & 330 & $\mathrm{U}$ & 330 & 340 & $\mathrm{U}$ & 340 & 340 & $\mathrm{U}$ & 340 & 340 & $\mathrm{U}$ & 340 \\
\hline Benzo(a)anthracene & 330 & $\mathrm{U}$ & 330 & 340 & $\mathrm{U}$ & 340 & 340 & $U$ & 340 & 340 & $\mathrm{U}$ & 340 \\
\hline Benzo(a)pyrene & 330 & $\mathrm{U}$ & 330 & 340 & $\mathrm{U}$ & 340 & 340 & $\bar{U}$ & 340 & 340 & $\mathrm{U}$ & 340 \\
\hline Benzo(b)fluoranthene & 330 & $\mathrm{U}$ & 330 & 340 & $\mathrm{U}$ & 340 & 340 & $\mathrm{U}$ & 340 & 340 & $\mathrm{U}$ & 340 \\
\hline Benzo(ghi)perylene & 330 & $\mathrm{U}$ & 330 & 340 & $\mathrm{U}$ & 340 & 340 & $\mathrm{U}$ & 340 & 340 & $\mathrm{U}$ & 340 \\
\hline Benzo(k)fluoranthene & 330 & $\mathrm{U}$ & 330 & 340 & $\mathrm{U}$ & 340 & 340 & $\mathrm{U}$ & 340 & 340 & $\mathrm{U}$ & 340 \\
\hline Bis(2-chloro-1-methylethyl)ether & 330 & $\mathrm{U}$ & 330 & 340 & $\mathrm{U}$ & 340 & 340 & U & 340 & 340 & $\mathrm{U}$ & 340 \\
\hline Bis(2-chloroethoxy)methane & 330 & $\mathrm{U}$ & 330 & 340 & $\mathrm{U}$ & 340 & 340 & $\mathrm{U}$ & 340 & 340 & $\mathrm{U}$ & 340 \\
\hline Bis(2-chloroethyl) ether & 330 & $\mathrm{U}$ & 330 & 340 & $\mathrm{U}$ & 340 & 340 & $\mathrm{U}$ & 340 & 340 & $\mathrm{U}$ & 340 \\
\hline Bis(2-ethylhexyl) phthalate & 660 & $\mathrm{U}$ & 660 & 660 & $\mathrm{U}$ & 660 & 660 & $\mathrm{U}$ & 660 & 660 & $\mathrm{U}$ & 660 \\
\hline Butylbenzylphthalate & 330 & $\mathrm{U}$ & 330 & 340 & $\mathrm{U}$ & 340 & 340 & $\mathrm{U}$ & 340 & 340 & $\mathrm{U}$ & 340 \\
\hline Carbazole & 330 & $\mathrm{U}$ & 330 & 340 & $\mathrm{U}$ & 340 & 340 & $\mathrm{U}$ & 340 & 340 & $\mathrm{U}$ & 340 \\
\hline Chrysene & 330 & $\mathrm{U}$ & 330 & 340 & $\mathrm{U}$ & 340 & 340 & $\mathrm{U}$ & 340 & 340 & $\mathrm{U}$ & 340 \\
\hline Dibenz $(\mathrm{a}, \mathrm{h})$ anthracene & 330 & $\mathrm{U}$ & 330 & 340 & $\mathrm{U}$ & 340 & 340 & $\mathrm{U}$ & 340 & 340 & $\mathrm{U}$ & 340 \\
\hline Dibenzofuran & 330 & $\mathrm{U}$ & 330 & 340 & $\mathrm{U}$ & 340 & 340 & $\mathrm{U}$ & 340 & 340 & $\mathrm{U}$ & 340 \\
\hline Diethylphthalate & 330 & $\mathrm{U}$ & 330 & 340 & $\mathrm{U}$ & 340 & 340 & $\mathrm{U}$ & 340 & 340 & $\mathrm{U}$ & 340 \\
\hline Dimethylphthalate & 330 & $\mathrm{U}$ & 330 & 340 & $\mathrm{U}$ & 340 & 340 & $\mathrm{U}$ & 340 & 340 & $\mathrm{U}$ & 340 \\
\hline Di-n-butylphthalate & 72 & $\mathrm{~J}$ & 330 & 340 & $\mathrm{U}$ & 340 & 340 & $\mathrm{U}$ & 340 & 340 & $\mathrm{U}$ & 340 \\
\hline Di-n-octylphthalate & 330 & $\mathrm{U}$ & 330 & 340 & $\mathrm{U}$ & 340 & 340 & $\mathrm{U}$ & 340 & 340 & $\mathrm{U}$ & 340 \\
\hline Fluoranthene & 330 & $\mathrm{U}$ & 330 & 340 & $\mathrm{U}$ & 340 & 340 & $\mathrm{U}$ & 340 & 340 & $\mathrm{U}$ & 340 \\
\hline Fluorene & 330 & U & 330 & 340 & $\mathrm{U}$ & 340 & 340 & $\mathrm{U}$ & 340 & 340 & $\mathrm{U}$ & 340 \\
\hline Hexachlorobenzene & 330 & $\mathrm{U}$ & 330 & 340 & $\mathrm{U}$ & 340 & 340 & $\bar{U}$ & 340 & 340 & $\mathrm{U}$ & 340 \\
\hline Hexachlorobutadiene & 330 & $\mathrm{U}$ & 330 & 340 & $\mathrm{U}$ & 340 & 340 & $\mathrm{U}$ & 340 & 340 & $\mathrm{U}$ & 340 \\
\hline Hexachlorocyclopentadiene & 330 & $\mathrm{U}$ & 330 & 340 & $\mathrm{U}$ & 340 & 340 & $\mathrm{U}$ & 340 & 340 & $\mathrm{U}$ & 340 \\
\hline Hexachloroethane & 330 & $\mathrm{U}$ & 330 & 340 & $\mathrm{U}$ & 340 & 340 & $\mathrm{U}$ & 340 & 340 & $\mathrm{U}$ & 340 \\
\hline Indeno(1,2,3-cd)pyrene & 330 & $\mathrm{U}$ & 330 & 340 & $\bar{U}$ & 340 & 24 & $\mathrm{JB}$ & 340 & 340 & $\mathrm{U}$ & 340 \\
\hline Isophorone & 330 & $\mathrm{U}$ & 330 & 340 & $\mathrm{U}$ & 340 & 340 & $\mathrm{U}$ & 340 & 340 & $\mathrm{U}$ & 340 \\
\hline Naphthalene & 330 & $\mathrm{U}$ & 330 & 340 & $\mathrm{U}$ & 340 & 340 & $\mathrm{U}$ & 340 & 340 & $\mathrm{U}$ & 340 \\
\hline Nitrobenzene & 330 & $\underline{U}$ & 330 & 340 & $\mathrm{U}$ & 340 & 340 & $\mathrm{U}$ & 340 & 340 & $\mathrm{U}$ & 340 \\
\hline N-Nitroso-di-n-dipropylamine & 330 & $\mathrm{U}$ & 330 & 340 & $\mathrm{U}$ & 340 & 340 & $\mathrm{U}$ & 340 & 340 & $\mathrm{U}$ & 340 \\
\hline N-Nitrosodiphenylamine & 330 & $\mathrm{U}$ & 330 & 340 & $\mathbf{U}$ & 340 & 340 & $\mathrm{U}$ & 340 & 340 & $\mathrm{U}$ & 340 \\
\hline Pentachlorophenol & 830 & $\mathrm{U}$ & 830 & 840 & $\mathrm{U}$ & 840 & 840 & $\mathrm{U}$ & 840 & 840 & U & 840 \\
\hline Phenanthrene & 330 & $\mathrm{U}$ & 330 & 340 & $\mathrm{U}$ & 340 & 340 & $\mathrm{U}$ & 340 & 340 & $\mathrm{U}$ & 340 \\
\hline Phenol & 330 & $\mathrm{U}$ & 330 & 340 & $\mathrm{U}$ & 340 & 340 & $\mathrm{U}$ & 340 & 340 & $\mathrm{U}$ & 340 \\
\hline Pyrene & 330 & $\mathrm{U}$ & 330 & 340 & $\mathrm{U}$ & 340 & 340 & $\mathrm{U}$ & 340 & 340 & $U$ & 340 \\
\hline
\end{tabular}

Attachment Originator

Checked

Calc. No.

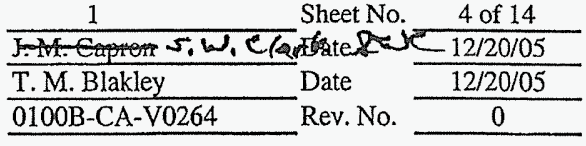


Attachment 1. 1607-B2:1 Verification Sampling Results.

\begin{tabular}{|c|c|c|c|c|c|c|c|c|c|c|c|c|}
\hline \multirow[t]{2}{*}{ Constituent } & \multicolumn{3}{|c|}{$\begin{array}{c}\text { J03WW6 } \\
\text { Equipment Blank } \\
\text { Sample Date 8/19/05 }\end{array}$} & \multicolumn{3}{|c|}{$\begin{array}{c}\text { J03VB8 } \\
\text { Sample Location } 1 \\
\text { Sample Date 8/19/05 }\end{array}$} & \multicolumn{3}{|c|}{$\begin{array}{c}\text { J03VB9 } \\
\text { Sample Location } 2 \\
\text { Sample Date 8/19/05 }\end{array}$} & \multicolumn{3}{|c|}{$\begin{array}{c}\text { J03VD2 } \\
\text { Duplicate of J03VB9 } \\
\text { Sample Date } 8 / 19 / 05\end{array}$} \\
\hline & $\mu \mathrm{g} / \mathrm{kg}$ & $\mathbf{Q}$ & $\mathrm{PQL}$ & $\mu \mathrm{g} / \mathrm{kg}$ & Q & $\mathrm{PQL}$ & $\mu \mathrm{g} / \mathrm{kg}$ & Q & PQL & $\mu \mathrm{g} / \mathrm{kg}$ & $\mathbf{Q}$ & $\mathrm{PQL}$ \\
\hline \multicolumn{13}{|c|}{ Volatile Organic Analytes } \\
\hline 1,1,1-Trichloroethane & & & & 6 & $\mathrm{UJ}$ & 6 & 5 & $\mathrm{U}$ & 5 & 5 & $\mathrm{U}$ & 5 \\
\hline 1,1,2,2-Tetrachloroethane & & & & 6 & UJ & 6 & 5 & $\mathrm{U}$ & 5 & 5 & $\mathrm{U}$ & 5 \\
\hline 1,1,2-Trichloroethane & & & & 6 & UJ & 6 & 5 & $\mathrm{U}$ & 5 & 5 & $\mathrm{U}$ & 5 \\
\hline 1,1-Dichloroethane & & & & 6 & UJ & 6 & 5 & $\mathrm{U}$ & 5 & 5 & $\overline{\mathrm{U}}$ & 5 \\
\hline 1,1-Dichloroethene & & & & 6 & UJ & 6 & 5 & $\mathrm{U}$ & 5 & 5 & $\mathrm{U}$ & 5 \\
\hline 1,2-Dichloroethane & & & & 6 & $\mathrm{UJ}$ & 6 & 5 & $\mathrm{U}$ & 5 & 5 & $\mathrm{U}$ & 5 \\
\hline 1,2-Dichloropropane & & & & 6 & UJ & 6 & 5 & $\bar{U}$ & 5 & 5 & $\mathrm{U}$ & 5 \\
\hline 2-Butanone & & & & 11 & $\mathrm{UJ}$ & 11 & 10 & $\mathrm{U}$ & 10 & 10 & U & 10 \\
\hline 2-Hexanone & & & & 11 & $\mathrm{UJ}$ & 11 & 10 & $\mathrm{U}$ & 10 & 10 & $\mathrm{U}$ & 10 \\
\hline 4-Methyl-2-Pentanone & & & & 11 & UJ & 11 & 10 & $\mathrm{U}$ & 10 & 10 & $\mathrm{U}$ & 10 \\
\hline Acetone & & & & 6 & $\mathrm{~J}$ & 11 & 5 & $\mathrm{~J}$ & 10 & 7 & $\mathrm{~J}$ & 10 \\
\hline Benzene & & & & 6 & UJ & 6 & 5 & $\mathrm{U}$ & 5 & 5 & $\mathrm{U}$ & 5 \\
\hline Bromodichloromethane & & & & 6 & UJ & 6 & 5 & $\mathrm{U}$ & 5 & 5 & $\mathrm{U}$ & 5 \\
\hline Bromoform & & & & 6 & UJ & 6 & 5 & $\mathrm{U}$ & 5 & 5 & $\mathrm{U}$ & 5 \\
\hline Bromomethane & & & & 11 & UJ & 11 & 10 & $\mathrm{U}$ & 10 & 10 & $\mathrm{U}$ & 10 \\
\hline Carbon disulfide & & & & 6 & $\mathrm{UJ}$ & 6 & 5 & $\mathrm{U}$ & 5 & 5 & $\mathrm{U}$ & 5 \\
\hline Carbon tetrachloride & & & & 6 & UJ & 6 & 5 & $\mathrm{U}$ & 5 & 5 &. $\mathrm{U}$ & 5 \\
\hline Chlorobenzene & & & & 6 & $\mathrm{UJ}$ & 6 & 5 & $\mathrm{U}$ & 5 & 5 & $\mathrm{U}$ & 5 \\
\hline Chloroethane & & & & 11 & $\mathrm{UJ}$ & 11 & 10 & $\mathrm{U}$ & 10 & 10 & $\mathrm{U}$ & 10 \\
\hline Chloroform & & & & 6 & UJ & 6 & 5 & $\mathrm{U}$ & 5 & 5 & $\mathrm{U}$ & 5 \\
\hline Chloromethane & & & & 11 & UJ & 11 & 10 & $\mathrm{U}$ & 10 & 10 & $\mathrm{U}$ & 10 \\
\hline cis-1,2-Dichloroethylene & & & & 6 & UJ & 6 & 5 & $\mathrm{U}$ & 5 & 5 & $\mathrm{U}$ & 5 \\
\hline cis-1,3-Dichloropropene & & & & 6 & UJ & 6 & 5 & $\mathrm{U}$ & 5 & 5 & $\mathrm{U}$ & 5 \\
\hline Dibromochloromethane & & & & 6 & UJ & 6 & 5 & $\mathrm{U}$ & 5 & 5 & $\mathrm{U}$ & 5 \\
\hline Ethylbenzene & & & & 6 & UJ & 6 & 5 & $\mathrm{U}$ & 5 & 5 & $\mathrm{U}$ & 5 \\
\hline Methylene chloride & & & & 10 & UJ & 10 & 10 & $U$ & 10 & 10 & $\mathrm{U}$ & 10 \\
\hline Styrene & & & & 6 & $\mathrm{UJ}$ & 6 & 5 & U & 5 & 5 & $\mathrm{U}$ & 5 \\
\hline Tetrachloroethene & & & & 6 & $\mathrm{UJ}$ & 6. & 5 & $\mathrm{U}$ & 5 & 5 & $\mathrm{U}$ & 5 \\
\hline Toluene & & & & 6 & UJ & 6 & 5 & $\mathrm{U}$ & 5 & 5 & $\mathrm{U}$ & 5 \\
\hline trans-1,2-Dichloroethylene & & & & 6 & $\mathrm{UJ}$ & 6 & 5 & $\mathrm{U}$ & 5 & 5 & $\mathrm{U}$ & 5 \\
\hline trans-1,3-Dichloropropene & & & & 6 & $\mathrm{UJ}$ & 6 & 5 & $\mathrm{U}$ & 5 & 5 & $\mathrm{U}$ & 5 \\
\hline Trichloroethene & & & & 6 & $\mathrm{UJ}$ & 6 & 5 & $\mathrm{U}$ & 5 & 5 & $\mathrm{U}$ & 5 \\
\hline Vinyl chloride & & & & 11 & UJ & 11 & 10 & $\mathrm{U}$ & 10 & 10 & $\mathrm{U}$ & 10 \\
\hline Xylenes (total) & & & & 6 & UJ & 6 & 5 & $\mathrm{U}$ & 5 & 5 & $\mathrm{U}$ & 5 \\
\hline \multicolumn{13}{|c|}{ Herbicides } \\
\hline $2,4-\mathrm{D}$ & & & & 170 & $\mathrm{U}$ & 170 & 170 & $\mathrm{U}$ & 170 & 110 & & 170 \\
\hline $2,4-\mathrm{DB}$ & & & & 840 & $\mathrm{U}$ & 840 & 840 & $\mathrm{U}$ & 840 & 840 & $\mathrm{U}$ & 840 \\
\hline $2,4,5-T$ & & & & 31 & $\mathrm{~J}$ & 84 & 84 & UI & 84 & 58 & $\mathrm{~J}$ & 84 \\
\hline 2,4,5-TP (Silvex) & & & & 84 & UJ & 84 & 84 & $\mathrm{UJ}$ & 84 & 84 & UJ & 84 \\
\hline Dalapon & & & & 840 & $\mathrm{U}$ & 840 & 840 & U & 840 & 840 & $\mathrm{U}$ & 840 \\
\hline Dicamba & & & & 340 & UJ & 340 & 340 & $\mathrm{UJ}$ & 340 & 340 & UJ & 340 \\
\hline Dichloroprop & & & & 840 & UJ & 840 & 840 & UJ & 840 & 840 & $\mathrm{UJ}$ & 840 \\
\hline Dinoseb & & & & 84 & $\mathrm{U}$ & 84 & 84 & $\mathrm{U}$ & 84 & 27 & & 84 \\
\hline
\end{tabular}

Attachment

Originator

Checked

Calc. No.

\begin{tabular}{|c|c|c|}
\hline 1 & Sheet No. & 5 of 14 \\
\hline 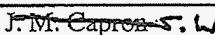 & Dete & $\longdiv { R _ { 1 2 / 2 0 / 0 5 } }$ \\
\hline T.M. Blakley & Date & $12 / 20 / 05$ \\
\hline $0100 \mathrm{~B}-\mathrm{CA}-\mathrm{V} 0264$ & Rev. No. & 0 \\
\hline
\end{tabular}


Attachment 1. 1607-B2:1 Verification Sampling Results.

\begin{tabular}{|c|c|c|c|c|c|c|c|c|c|c|c|c|}
\hline \multirow[t]{2}{*}{ Constituent } & \multicolumn{3}{|c|}{$\begin{array}{c}\text { J03VC0 } \\
\text { Sample Location } 3 \\
\text { Sample Date } 8 / 19 / 05\end{array}$} & \multicolumn{3}{|c|}{$\begin{array}{c}\text { J03VC1 } \\
\text { Sample Location } 4 \\
\text { Sample Date } 8 / 19 / 05\end{array}$} & \multicolumn{3}{|c|}{$\begin{array}{c}\text { J03VC2 } \\
\text { Sample Location } 5 \\
\text { Sample Date } 8 / 19 / 05\end{array}$} & \multicolumn{3}{|c|}{$\begin{array}{c}\text { J03VC3 } \\
\text { Sample Location } 6 \\
\text { Sample Date 8/19/05 }\end{array}$} \\
\hline & $\mu \mathrm{g} / \mathrm{kg}$ & $\mathbf{Q}$ & PQL & $\mu \mathrm{g} / \mathrm{kg}$ & Q & PQL & $\mu \mathrm{g} / \mathrm{kg}$ & $Q$ & PQL & $\mu \mathrm{g} / \mathrm{kg}$ & $Q$ & $\mathrm{PQL}$ \\
\hline \multicolumn{13}{|c|}{ Polychlorinated Biphenyls } \\
\hline Aroclor-1016 & 14 & $\mathrm{U}$ & 14 & 13 & $\mathrm{U}$ & 13 & 14 & $\mathrm{U}$ & 14 & 13 & $\bar{U}$ & 13 \\
\hline Aroclor-1221 & 14 & $\mathrm{U}$ & 14 & 13 & $\mathrm{U}$ & 13 & 14 & $\mathrm{U}$ & 14 & 13 & $\mathrm{U}$ & 13 \\
\hline Aroclor-1232 & 14 & $\mathrm{U}$ & 14 & 13 & $\mathrm{U}$ & 13 & 14 & $\mathrm{U}$ & 14 & 13 & $\mathrm{U}$ & 13 \\
\hline Aroclor-1242 & 14 & $\mathrm{U}$ & 14 & 13 & $\mathrm{U}$ & 13 & 14 & $\mathrm{U}$ & 14 & 13 & $\mathrm{U}$ & 13 \\
\hline Aroclor-1248 & 14 & $\mathrm{U}$ & 14 & 13 & $\mathrm{U}$ & 13 & 14 & $\mathrm{U}$ & 14 & 13 & $\mathrm{U}$ & 13 \\
\hline Aroclor-1254 & 14 & $\mathrm{U}$ & 14 & 13 & $\mathrm{U}$ & 13 & 14 & $\mathrm{U}$ & 14 & 13 & $\mathrm{U}$ & 13 \\
\hline Aroclor -1260 & 14 & $\mathrm{U}$ & 14 & 13 & $\mathrm{U}$ & 13 & 14 & $\mathrm{U}$ & 14 & 13 & $\mathrm{U}$ & 13 \\
\hline \multicolumn{13}{|c|}{ Pesticides } \\
\hline Aldrin & 1.7 & $\mathrm{UJ}$ & 1.7 & 1.7 & UJ & 1.7 & 1.7 & UY & 1.7 & 1.7 & UJ & 1.7 \\
\hline Alpha-BHC & 1.7 & $\mathrm{UJ}$ & 1.7 & 1.7 & $\mathrm{UJ}$ & 1.7 & 1.7 & UJ & 1.7 & 1.7 & UJ & 1.7 \\
\hline alpha-Chlordane & 1.7 & UJ & 1.7 & 1.7 & $\mathrm{UJ}$ & 1.7 & 1.7 & $\overline{U J}$ & 1.7 & 1.7 & $\mathrm{UJ}$ & 1.7 \\
\hline Beta-BHC & 1.7 & $\mathrm{UJ}$ & 1.7 & 1.7 & UJ & 1.7 & 1.7 & UJ & 1.7 & 1.7 & US & 1.7 \\
\hline Delta-BHC & 1.7 & $\mathrm{UJ}$ & 1.7 & 1.7 & UJ & 1.7 & 1.7 & $\mathrm{UJ}$ & 1.7 & 1.7 & UJ & 1.7 \\
\hline Dichlorodiphenyldichloroethane & 3.5 & $\mathrm{UJ}$ & 3.5 & 3.4 & $\mathrm{UJ}$ & 3.4 & 3.4 & $\mathrm{UJ}$ & 3.4 & 1.7 & $\mathrm{~J}$ & 3.4 \\
\hline Dichlorodiphenyldichloroethylene & 3.5 & UJ & 3.5 & 3.4 & UJ & 3.4 & 3.4 & UJ & 3.4 & 3.4 & $\overline{U J}$ & 3.4 \\
\hline Dichlorodiphenyltrichloroethane & 3.5 & $\mathrm{UJ}$ & 3.5 & 3.4 & UJ & 3.4 & 3.4 & UJ & 3.4 & 3.4 & UJ & 3.4 \\
\hline Dieldrin & 1.7 & $\mathrm{UJ}$ & 1.7 & 1.7 & $\mathrm{UJ}$ & 1.7 & 1.7 & UJ & 1.7 & 1.7 & $\mathrm{~J}$ & 1.7 \\
\hline Endosulfan I & 1.7 & UJ & 1.7 & 1.7 & UJ & 1.7 & 1.7 & UJ & 1.7 & 1.7 & UJ & 1.7 \\
\hline Endosulfan II & 3.5 & UJ & 3.5 & 3.4 & $\mathrm{UJ}$ & 3.4 & 3.4 & $\mathrm{UJ}$ & 3.4 & 3.4 & UJ & 3.4 \\
\hline Endosulfan sulfate & 3.5 & UJ & 3.5 & 3.4 & $\mathrm{UJ}$ & 3.4 & 3.4 & $\mathrm{UJ}$ & 3.4 & 3.4 & UJ & 3.4 \\
\hline Endrin & 3.5 & $\mathrm{UJ}$ & 3.5 & 3.4 & UJ & 3.4 & 3.4 & UJ & 3.4 & 3.4 & UJ & 3.4 \\
\hline Endrin aldehyde & 3.5 & UJ & 3.5 & 3.4 & $\mathrm{UJ}$ & 3.4 & 3.4 & $\mathrm{UJ}$ & 3.4 & 2.2 & $\mathbf{J}$ & 3.4 \\
\hline Endrin ketone & 3.5 & $\mathrm{UJ}$ & 3.5 & 3.4 & UJ & 3.4 & 3.4 & $\mathrm{UJ}$ & 3.4 & 3.4 & UJ & 3.4 \\
\hline Gamma-BHC (Lindane) & 1.7 & UJ & 1.7 & 1.7 & UJ & 1.7 & 1.7 & UJ & 1.7 & 1.7 & UJ & 1.7 \\
\hline gamma-Chlordane & 1.7 & $\mathrm{UJ}$ & 1.7 & 1.7 & $\mathrm{UJ}$ & 1.7 & 1.7 & $\mathrm{UJ}$ & 1.7 & 1.7 & $\mathrm{UJ}$ & 1.7 \\
\hline Heptachlor & 1.7 & UJ & 1.7 & 1.7 & UJ & 1.7 & 1.7 & UJ & 1.7 & 1.7 & $\mathrm{UJ}$ & 1.7 \\
\hline Heptachlor epoxide & 1.7 & UJ & 1.7 & 1.7 & UJ & 1.7 & 1.7 & UJ & 1.7 & 1.7 & $\mathrm{UJ}$ & 1.7 \\
\hline Methoxychlor & 17 & UJ & 17 & 17 & UJ & 17 & 17 & $\mathrm{UJ}$ & 17 & 17 & UJ & 17 \\
\hline Toxaphene & 170 & UJ & 170 & 170 & UJ & 170 & 170 & UJ & 170 & 170 & $\mathrm{UJ}$ & 170 \\
\hline \multicolumn{13}{|c|}{ Semivolatile Organic Analytes } \\
\hline 1,2,4-Trichlorobenzene & 350 & $\mathrm{U}$ & 350 & 340 & UJ & 340 & 340 & $\mathrm{U}$ & 340 & 340 & $\mathrm{U}$ & 340 \\
\hline 1,2-Dichlorobenzene & 350 & $\mathrm{U}$ & 350 & 340 & $\mathrm{UJ}$ & 340 & 340 & $\mathrm{U}$ & 340 & 340 & $\mathrm{U}$ & 340 \\
\hline 1,3-Dichlorobenzene & 350 & $\mathrm{U}$ & 350 & 340 & UJ & 340 & 340 & $\mathrm{U}$ & 340 & 340 & $\mathrm{U}$ & 340 \\
\hline 1,4-Dichlorobenzene & 350 & $\mathrm{U}$ & 350 & 340 & UJ & 340 & 340 & $\mathrm{U}$ & 340 & 340 & $\mathrm{U}$ & 340 \\
\hline $2,4,5$-Trichlorophenol & 860 & $\mathrm{U}$ & 860 & 840 & UJ & 840 & 850 & $\mathrm{U}$ & 850 & 840 & $\mathrm{U}$ & 840 \\
\hline 2,4,6-Trichlorophenol & 350 & $\mathrm{U}$ & 350 & 340 & $\mathrm{UJ}$ & 340 & 340 & U & 340 & 340 & $\mathrm{U}$ & 340 \\
\hline 2,4-Dichlorophenol & 350 & $\mathrm{U}$ & 350 & 340 & $\mathrm{UJ}$ & 340 & 340 & $\mathrm{U}$ & 340 & 340 & $\mathrm{U}$ & 340 \\
\hline 2,4-Dimethylphenol & 350 & $\mathrm{U}$ & 350 & 340 & UJ & 340 & 340 & $\mathrm{U}$ & 340 & 340 & $\mathrm{U}$ & 340 \\
\hline 2,4-Dinitrophenol & 860 & $\mathrm{U}$ & 860 & 840 & $\mathrm{UJ}$ & 840 & 850 & $\mathrm{U}$ & 850 & 840 & $\mathrm{U}$ & 840 \\
\hline 2,4-Dinitrotoluene & 350 & $\mathrm{U}$ & 350 & 340 & UJ & 340 & 340 & $\mathrm{U}$ & 340 & 340 & $\mathrm{U}$ & 340 \\
\hline 2,6-Dinitrotoluene & 350 & $\mathrm{U}$ & 350 & 340 & UJ & 340 & 340 & $\bar{U}$ & 340 & 340 & $\mathrm{U}$ & 340 \\
\hline 2-Chloronaphthalene & 350 & U & 350 & 340 & UJ & 340 & 340 & $\mathrm{U}$ & 340 & 340 & $\mathrm{U}$ & 340 \\
\hline 2-Chlorophenol & 350 & $\mathrm{U}$ & 350 & 340 & $\mathrm{UJ}$ & 340 & 340 & $\mathrm{U}$ & 340 & 340 & $\mathrm{U}$ & 340 \\
\hline 2-Methylnaphthalene & 350 & $\mathrm{U}$ & 350 & 340 & $\mathrm{UJ}$ & 340 & 340 & $\mathrm{U}$ & 340 & 150 & $\mathrm{~J}$ & 340 \\
\hline 2-Methylphenol (cresol, o-) & 350 & $\mathrm{U}$ & 350 & 340 & $\mathrm{UJ}$ & 340 & 340 & $\mathrm{U}$ & 340 & 340 & $\mathrm{U}$ & 340 \\
\hline 2-Nitroaniline & 860 & $\mathrm{U}$ & 860 & 840 & UJ & 840 & 850 & $\mathrm{U}$ & 850 & 840 & $\mathrm{U}$ & 840 \\
\hline 2-Nitrophenol & 350 & $\mathrm{U}$ & 350 & 340 & UJ & 340 & 340 & $\mathrm{U}$ & 340 & 340 & $\mathrm{U}$ & 340 \\
\hline & & & & & \multirow{4}{*}{\multicolumn{2}{|c|}{$\begin{array}{l}\text { Attachment } \\
\text { Originator } \\
\text { Checked } \\
\text { Calc. No. }\end{array}$}} & \multicolumn{3}{|c|}{1} & \multirow{4}{*}{$\begin{array}{l}\text { Sheet No. } \\
\text { Date } \\
\text { Rev. No. }\end{array}$} & \multicolumn{2}{|c|}{6 of 14} \\
\hline & & & & & & & \multicolumn{3}{|c|}{ 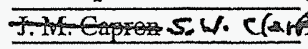 } & & \multicolumn{2}{|c|}{$-12 / 20 / 05$} \\
\hline & & & & & & & \multirow{2}{*}{\multicolumn{3}{|c|}{$\begin{array}{l}\text { T. M. Blakley } \\
0100 \text { B-CA-V0264 }\end{array}$}} & & \multicolumn{2}{|c|}{$12 / 20 / 05$} \\
\hline & & & & & & & & & & & & 0 \\
\hline
\end{tabular}


Attachment 1. 1607-B2:1 Verification Sampling Results.

\begin{tabular}{|c|c|c|c|c|c|c|c|c|c|c|c|c|}
\hline \multirow[t]{2}{*}{ Constituent } & \multicolumn{3}{|c|}{$\begin{array}{c}\text { J03VC0 } \\
\text { Sample Location } 3 \\
\text { Sample Date 8/19/05 }\end{array}$} & \multicolumn{3}{|c|}{$\begin{array}{c}\text { J03VC1 } \\
\text { Sample Location } 4 \\
\text { Sample Date 8/19/05 } \\
\end{array}$} & \multicolumn{3}{|c|}{$\begin{array}{c}\text { J03VC2 } \\
\text { Sample Location } 5 \\
\text { Sample Date 8/19/05 }\end{array}$} & \multicolumn{3}{|c|}{$\begin{array}{c}\text { J03VC3 } \\
\text { Sample Location } 6 \\
\text { Sample Date 8/19/05 }\end{array}$} \\
\hline & $\mu \mathrm{g} / \mathrm{kg}$ & Q & PQL & $\mu \mathrm{g} / \mathrm{kg}$ & $\mathbf{Q}$ & PQL & $\mu \mathrm{g} / \mathrm{kg}$ & $Q$ & PQL & $\mu \mathrm{g} / \mathrm{kg}$ & Q & PQL \\
\hline \multicolumn{13}{|c|}{ Semivolatile Organic Analytes (continued) } \\
\hline 3,3'-Dichlorobenzidine & 350 & $\mathrm{U}$ & 350 & 340 & UJ & 340 & 340 & $\mathrm{U}$ & 340 & 340 & $\mathrm{U}$ & 340 \\
\hline $3+4$ Methylphenol (cresol, $m+p)$ & 350 & U & 350 & 340 & UJ & 340 & 340 & $U$ & 340 & 340 & $\mathrm{U}$ & 340 \\
\hline 3-Nitroaniline & 860 & $\mathrm{UJ}$ & 860 & 840 & UJ & 840 & 850 & $\mathrm{UJ}$ & 850 & 840 & $\mathrm{UJ}$ & 840 \\
\hline 4,6-Dinitro-2-methylphenol & 860 & $\mathrm{U}$ & 860 & 840 & $\mathrm{UJ}$ & 840 & 850 & $\mathrm{U}$ & 850 & 840 & $\mathrm{U}$ & 840 \\
\hline 4-Bromophenyl-phenylether & 350 & $\mathrm{U}$ & 350 & 340 & $\mathrm{UJ}$ & 340 & 340 & $\mathrm{U}$ & 340 & 340 & $\mathrm{U}$ & 340 \\
\hline 4-Chloro-3-methylphenol & 350 & $\bar{U}$ & 350 & 340 & UJ & 340 & 340 & $\mathrm{U}$ & 340 & 340 & $\mathrm{U}$ & 340 \\
\hline 4-Chloroaniline & 350 & $\mathrm{U}$ & 350 & 340 & UJ & 340 & 340 & $\bar{U}$ & 340 & 340 & $\mathrm{U}$ & 340 \\
\hline 4-Chlorophenyl-phenylether & 350 & $\mathrm{U}$ & 350 & 340 & UJ & 340 & 340 & $\mathrm{U}$ & 340 & 340 & $\mathrm{U}$ & 340 \\
\hline 4-Nitroaniline & 860 & UJ & 860 & 840 & UJ & 840 & 850 & UJ & 850 & 840 & UJ & 840 \\
\hline 4-Nitrophenol & 860 & UJ & 860 & 840 & UJ & 840 & 850 & UJ & 850 & 840 & UJ & 840 \\
\hline Acenaphthene & 350 & $\mathrm{U}$ & 350 & 340 & UJ & 340 & 340 & $\mathrm{U}$ & 340 & 340 & $\mathrm{U}$ & 340 \\
\hline Acenaphthylene & 350 & $\mathrm{U}$ & 350 & 340 & UJ & 340 & 340 & $\mathrm{U}$ & 340 & 340 & $\mathrm{U}$ & 340 \\
\hline Anthracene & 350 & $\mathrm{U}$ & 350 & 340 & UJ & 340 & 340 & $\mathrm{U}$ & 340 & 340 & $\mathrm{U}$ & 340 \\
\hline Benzo(a)anthracene & 350 & $\mathrm{U}$ & 350 & 340 & UJ & 340 & 340 & $\mathrm{U}$ & 340 & 340 & $\mathrm{U}$ & 340 \\
\hline Benzo(a)pyrene & 350 & $\mathrm{U}$ & 350 & 340 & $\mathrm{UJ}$ & 340 & 340 & $\mathrm{U}$ & 340 & 340 & $\mathrm{U}$ & 340 \\
\hline Benzo(b)fluoranthene & 350 & $\mathrm{U}$ & 350 & 340 & UJ & 340 & 340 & $\mathrm{U}$ & 340 & 340 & $\mathrm{U}$ & 340 \\
\hline Benzo(ghi)perylene & 350 & $\mathrm{U}$ & 350 & 340 & UJ & 340 & 340 & $\mathrm{U}$ & 340 & 340 & $\mathrm{U}$ & 340 \\
\hline Benzo(k)fluoranthene & 350 & $\mathrm{U}$ & 350 & 340 & UI & 340 & 340 & $\mathrm{U}$ & 340 & 340 & $\mathrm{U}$ & 340 \\
\hline Bis(2-chloro-1-methylethyl)ether & 350 & $\mathrm{U}$ & 350 & 340 & UJ & 340 & 340 & $\mathrm{U}$ & 340 & 340 & $\mathrm{U}$ & 340 \\
\hline Bis(2-Chloroethoxy)methane & 350 & $\mathrm{U}$ & 350 & 340 & UJ & 340 & 340 & $\mathrm{U}$ & 340 & 340 & $\mathrm{U}$ & 340 \\
\hline Bis(2-chloroethyl) ether & 350 & $\mathrm{U}$ & 350 & 340 & UJ & 340 & 340 & $\mathrm{U}$ & 340 & 340 & $\mathrm{U}$ & 340 \\
\hline Bis(2-ethylhexyl) phthalate & 660 & $\mathrm{U}$ & 660 & 660 & UJ & 660 & 660 & $\mathrm{U}$ & 660 & 660 & $\mathrm{U}$ & 660 \\
\hline Butylbenzylphthalate & 350 & $\mathrm{U}$ & 350 & 340 & UJ & 340 & 340 & $\mathrm{U}$ & 340 & 340 & $\mathrm{U}$ & 340 \\
\hline Carbazole & 350 & $\mathrm{U}$ & 350 & 340 & UJ & 340 & 340 & $\mathrm{U}$ & 340 & 340 & $\mathrm{U}$ & 340 \\
\hline Chrysene & 350 & $\mathrm{U}$ & 350 & 340 & UJ & 340 & 340 & $\mathrm{U}$ & 340 & 340 & $\mathrm{U}$ & 340 \\
\hline Dibenz $(\mathrm{a}, \mathrm{h})$ anthracene & 350 & $\mathrm{U}$ & 350 & 340 & $\mathrm{UJ}$ & 340 & 340 & $\mathrm{U}$ & 340 & 340 & $\mathrm{U}$ & 340 \\
\hline Dibenzofuran & 350 & $\mathrm{U}$ & .350 & 340 & UJ & 340 & 340 & $\mathrm{U}$ & 340 & 34 & $\mathrm{~J}$ & 340 \\
\hline Diethylphthalate & 350 & $\mathrm{U}$ & 350 & 340 & $\mathrm{UJ}$ & 340 & 340 & $\mathrm{U}$ & 340 & 340 & $\mathrm{U}$ & 340 \\
\hline Dimethyl phthalate & 350 & $\mathrm{U}$ & 350 & 340 & UJ & 340 & 340 & $\mathrm{U}$ & 340 & 340 & $\mathrm{U}$ & 340 \\
\hline Di-n-butylphthalate & 350 & $\mathrm{U}$ & 350 & 340 & UJ & 340 & 340 & $\mathrm{U}$ & 340 & 21 & $\mathrm{~J}$ & 340 \\
\hline Di-n-octylphthalate & 350 & $\mathrm{U}$ & 350 & 340 & UJ & 340 & 340 & $\mathrm{U}$ & 340 & 340 & $\mathrm{U}$ & 340 \\
\hline Fluoranthene & 350 & $\mathrm{U}$ & 350 & 340 & UJ & 340 & 340 & $\mathrm{U}$ & 340 & 340 & $\mathrm{U}$ & 340 \\
\hline Fluorene & 350 & $\mathrm{U}$ & 350 & 340 & UJ & 340 & 340 & $\mathrm{U}$ & 340 & 340 & $\mathrm{U}$ & 340 \\
\hline Hexachlorobenzene & 350 & $\mathrm{U}$ & 350 & 340 & $\mathrm{UJ}$ & 340 & 340 & $\mathrm{U}$ & 340 & 340 & $\mathrm{U}$ & 340 \\
\hline Hexachlorobutadiene & 350 & $\mathrm{U}$ & 350 & 340 & UJ & 340 & 340 & $\mathrm{U}$ & 340 & 340 & $\mathrm{U}$ & 340 \\
\hline Hexachlorocyclopentadiene & 350 & $\mathrm{U}$ & 350 & 340 & US & 340 & 340 & $\mathrm{U}$ & 340 & 340 & $\mathrm{U}$ & 340 \\
\hline Hexachloroethane & 350 & $\mathrm{U}$ & 350 & 340 & UJ & 340 & 340 & $\mathrm{U}$ & 340 & 340 & $\mathrm{U}$ & 340 \\
\hline Indeno(1,2,3-cd)pyrene & 350 & $\mathrm{U}$ & 350 & 340 & UJ & 340 & 340 & $\mathrm{U}$ & 340 & 340 & $\mathrm{U}$ & 340 \\
\hline Isophorone & 350 & $\mathrm{U}$ & 350 & 340 & UI & 340 & 340 & $\mathrm{U}$ & 340 & 340 & $\mathrm{U}$ & 340 \\
\hline Naphthalene & 350 & $\mathrm{U}$ & 350 & 340 & UJ & 340 & 340 & $\mathrm{U}$ & 340 & 110 & $\mathrm{~J}$ & 340 \\
\hline Nitrobenzene & 350 & $\mathrm{U}$ & 350 & 340 & UJ & 340 & 340 & $\mathrm{U}$ & 340 & 340 & $\mathrm{U}$ & 340 \\
\hline N-Nitroso-di-n-dipropylamine & 350 & $\mathrm{U}$ & 350 & 340 & UJ & 340 & 340 & $\mathrm{U}$ & 340 & 340 & $\mathbf{U}$ & 340 \\
\hline N-Nitrosodiphenylamine & 350 & $\mathrm{U}$ & 350 & 340 & UJ & 340 & 340 & $\mathrm{U}$ & 340 & 340 & U & 340 \\
\hline Pentachlorophenol & 860 & $\mathrm{U}$ & 860 & 840 & UJ & 840 & 850 & $\mathrm{U}$ & 850 & 840 & U & 840 \\
\hline Phenanthrene & 350 & $\mathrm{U}$ & 350 & 340 & UJ & 340 & 340 & $\mathrm{U}$ & 340 & 37 & $\mathrm{~J}$ & 340 \\
\hline Phenol & 350 & $\mathrm{U}$ & 350 & 340 & UJ & 340 & 340 & $\mathrm{U}$ & 340 & 340 & $\mathrm{U}$ & 340 \\
\hline Pyrene & 350 & $\mathrm{U}$ & 350 & 340 & UJ & 340 & 340 & $\mathrm{U}$ & 340 & 340 & $\mathrm{U}$ & 340 \\
\hline
\end{tabular}

Attachment Originator

Checked

Calc. No.

$$
1
$$

Sheet No. 7 of 14

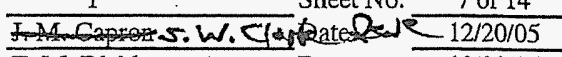
T.M. Blakley $0100 \mathrm{~B}-\mathrm{CA}-\mathrm{V} 0264$
Rev. No. 0 
Attachment 1. 1607-B2:1 Verification Sampling Results.

\begin{tabular}{|c|c|c|c|c|c|c|c|c|c|c|c|c|}
\hline \multirow[t]{2}{*}{ Constituent } & \multicolumn{3}{|c|}{$\begin{array}{c}\text { J03VC0 } \\
\text { Sample Location } 3 \\
\text { Sample Date } 8 / 19 / 05\end{array}$} & \multicolumn{3}{|c|}{$\begin{array}{c}\text { J03VC1 } \\
\text { Sample Location } 4 \\
\text { Sample Date 8/19/05 }\end{array}$} & \multicolumn{3}{|c|}{$\begin{array}{c}\text { J03VC2 } \\
\text { Sample Location } 5 \\
\text { Sample Date } 8 / 19 / 05\end{array}$} & \multicolumn{3}{|c|}{$\begin{array}{c}\text { J03VC3 } \\
\text { Sample Location } 6 \\
\text { Sample Date 8/19/05 }\end{array}$} \\
\hline & $\mu \mathrm{g} / \mathrm{kg}$ & Q & PQL & $\mu \mathrm{g} / \mathrm{kg}$ & $\mathbf{Q}$ & $\mathrm{PQL}$ & $\mu \mathrm{g} / \mathrm{kg}$ & Q & $\mathrm{PQL}$ & $\mu \mathrm{g} / \mathrm{kg}$ & Q & PQL \\
\hline \multicolumn{13}{|c|}{ Volatile Organic Analytes } \\
\hline 1,1,1-Trichloroethane & 6 & $\mathrm{U}$ & 6 & 6 & UJ & 6 & 5 & $\mathrm{U}$ & 5 & 5 & $\mathrm{U}$ & 5 \\
\hline 1,1,2,2-Tetrachloroethane & 6 & $\mathrm{U}$ & 6 & 6 & UY & 6 & 5 & $\mathrm{U}$ & 5 & 5 & $\mathrm{U}$ & 5 \\
\hline 1,1,2-Trichloroethane & 6 & $\mathrm{U}$ & 6 & 6 & UJ & 6 & 5 & $\mathrm{U}$ & 5 & 5 & $\mathrm{U}$ & 5 \\
\hline 1,1-Dichloroethane & 6 & $\mathrm{U}$ & 6 & 6 & UJ & 6 & 5 & $\mathrm{U}$ & 5 & 5 & $\mathrm{U}$ & 5 \\
\hline 1,1-Dichloroethene & 6 & $\bar{U}$ & 6 & 6 & $\mathrm{UJ}$ & 6 & 5 & $\mathrm{U}$ & 5 & 5 & $\mathrm{U}$ & 5 \\
\hline 1,2-Dichloroethane & 6 & $\mathrm{U}$ & 6 & 6 & UI & 6 & 5 & $\mathrm{U}$ & 5 & 5 & U & 5 \\
\hline 1,2-Dichloropropane & 6 & $\mathrm{U}$ & 6 & 6 & UJ & 6 & 5 & $\mathrm{U}$ & 5 & 5 & $\mathrm{U}$ & 5 \\
\hline 2-Butanone & 11 & $\mathrm{U}$ & 11 & 11 & UJ & 11 & 10 & $\mathrm{U}$ & 10 & 10 & $\mathrm{U}$ & 10 \\
\hline 2-Hexanone & 11 & $\mathrm{U}$ & 11 & 11 & UJ & 11 & 10 & $\mathrm{U}$ & 10 & 10 & $\mathrm{U}$ & 10 \\
\hline 4-Methyl-2-Pentanone & 11 & $\mathrm{U}$ & 11 & 11 & UJ & 11 & 10 & $\mathrm{U}$ & 10 & 10 & $\mathrm{U}$ & 10 \\
\hline Acetone & 11 & $\mathrm{U}$ & 11 & 5 & $\mathrm{~J}$ & 11 & 3 & $\mathrm{~J}$ & 10 & 5 & $\mathrm{~J}$ & 10 \\
\hline Benzene & 6 & $\mathrm{U}$ & 6 & 6 & UJ & 6 & 5 & $\mathrm{U}$ & 5 & 5 & $\mathrm{U}$ & 5 \\
\hline Bromodichloromethane & 6 & $\mathrm{U}$ & 6 & 6 & UJ & 6 & 5 & $\mathrm{U}$ & 5 & 5 & $\mathrm{U}$ & 5 \\
\hline Bromoform & 6 & $\mathrm{U}$ & 6 & 6 & UJ & 6 & 5 & $\mathrm{U}$ & 5 & 5 & $\mathrm{U}$ & 5 \\
\hline Bromomethane & 11 & $\mathrm{U}$ & 11 & 11 & $\mathrm{UJ}$ & 11 & 10 & $\mathrm{U}$ & 10 & 10 & $\mathrm{U}$ & 10 \\
\hline Carbon disulfide & 6 & $U$ & 6 & 6 & UJ & 6 & 5 & $\mathrm{U}$ & 5 & 5 & $\mathrm{U}$ & 5 \\
\hline Carbon tetrachloride & 6 & $\mathrm{U}$ & 6 & 6 & UJ & 6 & 5 & $\mathrm{U}$ & 5 & 5 & $\mathrm{U}$ & 5 \\
\hline Chlorobenzene & 6 & $\mathrm{U}$ & 6 & 6 & UJ & 6 & 5 & $\mathrm{U}$ & 5 & 5 & $\mathrm{U}$ & 5 \\
\hline Chloroethane & 11 & U & 11 & 11 & UJ & 11 & 10 & $\mathrm{U}$ & 10 & 10 & $\mathrm{U}$ & 10 \\
\hline Chloroform & 6 & $\mathrm{U}$ & 6 & 6 & UJ & 6 & 5 & $\mathrm{U}$ & 5 & 5 & $\mathrm{U}$ & 5 \\
\hline Chloromethane & 11 & $\bar{U}$ & 11 & 11 & UJ & 11 & 10 & $\mathrm{U}$ & 10 & 10 & $\mathrm{U}$ & 10 \\
\hline cis-1,2-Dichloroethylene & 6 & $\mathrm{U}$ & 6 & 6 & UJ & 6 & 5 & $\mathrm{U}$ & 5 & 5 & $\mathrm{U}$ & 5 \\
\hline cis-1,3-Dichloropropene & 6 & $\mathrm{U}$ & 6 & 6 & UJ & 6 & 5 & $\mathrm{U}$ & 5 & 5 & $\mathrm{U}$ & 5 \\
\hline Dibromochloromethane & 6 & $\mathrm{U}$ & 6 & 6 & UJ & 6 & 5 & $\mathrm{U}$ & 5 & 5 & $\mathrm{U}$ & 5 \\
\hline Ethylbenzene & 6 & $\mathrm{U}$ & 6 & 6 & UJ & 6 & 5 & $\mathrm{U}$ & 5 & 5 & $\bar{U}$ & 5 \\
\hline Methylene chloride & 10 & $\mathrm{U}$ & 10 & 10 & UI & 10 & 10 & $\mathrm{U}$ & 10 & 10 & $\mathrm{U}$ & 10 \\
\hline Styrene & 6 & U & 6 & 6 & UJ & 6 & 5 & $\mathrm{U}$ & 5 & 5 & $\mathrm{U}$ & 5 \\
\hline Tetrachloroethene & 6 & $\mathrm{U}$ & 6 & 6 & UJ & 6 & 5 & $\mathrm{U}$ & 5 & 5 & $\mathrm{U}$ & 5 \\
\hline Toluene & 6 & $\mathrm{U}$ & 6 & 6 & UJ & 6 & 5 & $\mathrm{U}$ & 5 & 5 & $\mathrm{U}$ & 5 \\
\hline trans-1,2-Dichloroethylene & 6 & $\mathrm{U}$ & 6 & 6 & UJ & 6 & 5 & $\mathrm{U}$ & 5 & 5 & $\mathrm{U}$ & 5 \\
\hline trans-1,3-Dichloropropene & 6 & $\mathrm{U}$ & 6 & 6 & UJ & 6 & 5 & $\mathrm{U}$ & 5 & 5 & $\mathrm{U}$ & 5 \\
\hline Trichloroethene & 6 & $\mathrm{U}$ & 6 & 6 & UJ & 6 & 5 & $\mathrm{U}$ & 5 & 5 & $\mathrm{U}$ & 5 \\
\hline Vinyl chloride & 11 & $\mathrm{U}$ & 11 & 11 & $\mathrm{UJ}$ & 11 & 10 & $\mathrm{U}$ & 10 & 10 & $\mathrm{U}$ & 10 \\
\hline Xylenes (total) & 6 & $\mathrm{U}$ & 6 & 6 & UJ & 6 & 5 & $\mathrm{U}$ & 5 & 5 & $\mathrm{U}$ & 5 \\
\hline \multicolumn{13}{|c|}{ Herbicides } \\
\hline $2,4-D$ & 170 & $\mathrm{U}$ & 170 & 170 & $\mathrm{U}$ & 170 & 170 & $\mathrm{U}$ & 170 & 170 & $\mathrm{U}$ & 170 \\
\hline $2,4-\mathrm{DB}$ & 860 & $\mathrm{U}$ & 860 & 840 & $\mathrm{U}$ & 840 & 850 & $\mathrm{U}$ & 850 & 840 & $\mathrm{U}$ & 840 \\
\hline $2,4,5-\mathrm{T}$ & 24 & $\mathrm{~J}$ & 86 & 84 & UJ & 84 & 31 & $\mathrm{~J}$ & 85 & 20 & $\mathrm{~J}$ & 84 \\
\hline $2,4,5-\mathrm{TP}$ (Silvex) & 86 & US & 86 & 84 & $\mathrm{UJ}$ & 84 & 85 & UJ & 85 & 84 & UJ & 84 \\
\hline Dalapon & 860 & $\mathrm{U}$ & 860 & 840 & $\mathrm{U}$ & 840 & 850 & $\mathrm{U}$ & 850 & 840 & $\mathrm{U}$ & 840 \\
\hline Dicamba & 350 & UJ & 350 & 340 & UJ & 340 & 340 & UJ & 340 & 340 & UJ] & 340 \\
\hline Dichloroprop & 860 & UJ & 860 & 840 & $\mathrm{UJ}$ & 840 & 850 & UJ & 850 & 840 & UJ & 840 \\
\hline Dinoseb & 86 & $\mathrm{U}$ & 86 & 84 & $\mathrm{U}$ & 84 & 85 & $\mathrm{U}$ & 85 & 84 & $U$ & 84 \\
\hline
\end{tabular}

\begin{tabular}{|c|c|c|c|}
\hline Attachment & 1 & Sheet No & 8 of 14 \\
\hline Originator & 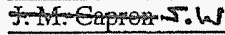 & ate & $-12 / 20 / 05$ \\
\hline Checked & T. M. Blakley & Date & $12 / 20 / 05$ \\
\hline Calc. No. & $0100 \mathrm{~B}-\mathrm{CA}-\mathrm{V} 0264$ & Rev. No. & 0 \\
\hline
\end{tabular}


Attachment 1. 1607-B2:1 Verification Sampling Results.

\begin{tabular}{|c|c|c|c|c|c|c|c|c|c|c|c|c|}
\hline \multirow[t]{2}{*}{ Constituent } & \multicolumn{3}{|c|}{$\begin{array}{c}\text { J03VC4 } \\
\text { Sample Location } 7 \\
\text { Sample Date 8/19/05 }\end{array}$} & \multicolumn{3}{|c|}{$\begin{array}{c}\text { J03VC5 } \\
\text { Sample Location } 8 \\
\text { Sample Date 8/19/05 }\end{array}$} & \multicolumn{3}{|c|}{$\begin{array}{c}\text { J03VC6 } \\
\text { Sample Location } 9 \\
\text { Sample Date } 8 / 19 / 05\end{array}$} & \multicolumn{3}{|c|}{$\begin{array}{c}\text { J03VC7 } \\
\text { Sample Location } 10 \\
\text { Sample Date } 8 / 19 / 05\end{array}$} \\
\hline & $\mu \mathrm{g} / \mathrm{kg}$ & Q & PQL & $\mu \mathrm{g} / \mathrm{kg}$ & Q & $\mathrm{PQL}$ & $\mu \mathrm{g} / \mathrm{kg}$ & $Q$ & $\mathrm{PQL}$ & $\mu \mathrm{g} / \mathrm{kg}$ & $\mathbf{Q}$ & $\mathrm{PQL}$ \\
\hline \multicolumn{13}{|c|}{ Polychlorinated Biphenyls } \\
\hline Aroclor-1016 & 13 & $\mathrm{U}$ & 13 & 13 & $\mathrm{U}$ & 13 & 14 & $\mathrm{U}$ & 14 & 13 & $\mathrm{U}$ & 13 \\
\hline Aroclor-1221 & 13 & $\mathrm{U}$ & 13 & 13 & $\mathrm{U}$ & 13 & 14 & $\mathrm{U}$ & 14 & 13 & $\mathrm{U}$ & 13 \\
\hline Aroclor-1232 & 13 & $\mathrm{U}$ & 13 & 13 & $\mathrm{U}$ & 13 & 14 & $U$ & 14 & 13 & $\mathrm{U}$ & 13 \\
\hline Aroclor-1242 & 13 & $\bar{U}$ & 13 & 13 & $\mathrm{U}$ & 13 & 14 & $U$ & 14 & 13 & $\mathrm{U}$ & 13 \\
\hline Aroclor-1248 & 13 & $\mathrm{U}$ & 13 & 13 & $\mathrm{U}$ & 13 & 14 & $\mathrm{U}$ & 14 & 13 & $\mathrm{U}$ & 13 \\
\hline Aroclor-1254 & 13 & $\mathrm{U}$ & 13 & 13 & $\mathrm{U}$ & 13 & 14 & $\mathrm{U}$ & 14 & 13 & $\mathrm{U}$ & 13 \\
\hline Aroclor-1260 & 13 & $\mathrm{U}$ & 13 & 13 & $\mathrm{U}$ & 13 & 14 & $\mathrm{U}$ & 14 & 13 & $\mathrm{U}$ & 13 \\
\hline \multicolumn{13}{|c|}{ Pesticides } \\
\hline Aldrin & 1.7 & UJ & 1.7 & 1.7 & $\mathrm{UJ}$ & 1.7 & 1.7 & UJ & 1.7 & 1.7 & UJ & 1.7 \\
\hline Alpha-BHC & 1.7 & UJ & 1.7 & 1.7 & UJ & 1.7 & 1.7 & UJ & 1.7 & 1.7 & $\mathrm{UJ}$ & 1.7 \\
\hline alpha-Chlordane & 1.7 & $\mathrm{UJ}$ & 1.7 & 1.7 & $\mathrm{UJ}$ & 1.7 & 1.7 & $\mathrm{UJ}$ & 1.7 & 1.7 & UJ & 1.7 \\
\hline Beta-BHC & 1.7 & $\mathrm{UJ}$ & 1.7 & 1.7 & UJ & 1.7 & 1.7 & UI & 1.7 & 1.7 & UJ & 1.7 \\
\hline Delta-BHC & 1.7 & UJ & 1.7 & 1.7 & UJ & 1.7 & 1.7 & UI & 1.7 & 1.7 & $\mathrm{UJ}$ & 1.7 \\
\hline Dichlorodiphenyldichloroethane & 3.4 & $\mathrm{UJ}$ & 3.4 & 3.4 & UJ & 3.4 & 3.4 & UJ & 3.4 & 3.4 & UJ & 3.4 \\
\hline Dichlorodiphenyldichloroethylene & 3.4 & UJ & 3.4 & 3.4 & UI & 3.4 & 3.4 & UJ & 3.4 & 3.4 & UJ & 3.4 \\
\hline Dichlorodiphenyltrichloroethane & 3.4 & UJ & 3.4 & 3.4 & UJ & 3.4 & 3.4 & $\mathrm{UJ}$ & 3.4 & 3.4 & UJ & 3.4 \\
\hline Dieldrin & 1.7 & $\mathrm{UJ}$ & 1.7 & 1.7 & UJ & 1.7 & 1.7 & $\mathrm{UJ}$ & 1.7 & 1.7 & UJ & 1.7 \\
\hline Endosulfan I & 1.7 & UJ & 1.7 & 1.7 & $\mathrm{UJ}$ & 1.7 & 1.7 & $\mathrm{UJ}$ & 1.7 & 1.7 & UJ & 1.7 \\
\hline Endosulfan II & 3.4 & UJ & 3.4 & 3.4 & UJ & 3.4 & 3.4 & $\mathrm{UJ}$ & 3.4 & 3.4 & UI & 3.4 \\
\hline Endosulfan sulfate & 3.4 & UJ & 3.4 & 3.4 & UJ & 3.4 & 3.4 & UJ & 3.4 & 3.4 & UI & 3.4 \\
\hline Endrin & 3.4 & UJ & 3.4 & 3.4 & UJ & 3.4 & 3.4 & $\mathrm{UJ}$ & 3.4 & 3.4 & UI & 3.4 \\
\hline Endrin aldehyde & 3.4 & UJ & 3.4 & 3.4 & UJ & 3.4 & 3.4 & UJ & 3.4 & 3.4 & UJ & 3.4 \\
\hline Endrin ketone & 3.4 & UJ & 3.4 & 3.4 & $\mathrm{UJ}$ & 3.4 & 3.4 & UJ & 3.4 & 3.4 & UJ & 3.4 \\
\hline Gamma-BHC (Lindane) & 1.7 & UJ & 1.7 & 1.7 & UJ & 1.7 & 1.7 & UJ & 1.7 & 1.7 & UJ & 1.7 \\
\hline gamma-Chlordane & 1.7 & UT & 1.7 & 1.7 & UJ & 1.7 & 1.7 & $\mathrm{UJ}$ & 1.7 & 1.7 & $\mathrm{UJ}$ & 1.7 \\
\hline Heptachlor & 1.7 & UJ & 1.7 & 1.7 & UJ & 1.7 & 1.7 & UJ & 1.7 & 1.7 & UJ & 1.7 \\
\hline Heptachlor epoxide & 1.7 & UJ & 1.7 & 1.7 & UJ & 1.7 & 1.7 & US & 1.7 & 1.7 & UY & 1.7 \\
\hline Methoxychlor & 17 & UJ & 17 & 17 & UJ & 17 & 17 & UJ & 17 & 17 & UI & 17 \\
\hline Toxaphene & 170 & UJ & 170 & 170 & UJ & 170 & 170 & UJ & 170 & 170 & $\mathrm{UJ}$ & 170 \\
\hline \multicolumn{13}{|c|}{ Semivolatile Organic Analytes } \\
\hline 1,2,4-Trichlorobenzene & 340 & $\mathrm{U}$ & 340 & 340 & $\mathrm{U}$ & 340 & 340 & $\mathrm{U}$ & 340 & 340 & $\mathrm{U}$ & 340 \\
\hline 1,2-Dichlorobenzene & 340 & $\mathrm{U}$ & 340 & 340 & $\mathrm{U}$ & 340 & 340 & $\mathrm{U}$ & 340 & 340 & $\mathrm{U}$ & 340 \\
\hline 1,3-Dichlorobenzene & 340 & $\mathrm{U}$ & 340 & 340 & $\mathrm{U}$ & 340 & 340 & $\mathrm{U}$ & 340 & 340 & $\mathrm{U}$ & 340 \\
\hline 1,4-Dichlorobenzene & 340 & $\mathrm{U}$ & 340 & 340 & $\mathrm{U}$ & 340 & 340 & $\mathrm{U}$ & 340 & 340 & $\mathrm{U}$ & 340 \\
\hline 2,4,5-Trichlorophenol & 840 & $\mathrm{U}$ & 840 & 840 & $\mathrm{U}$ & 840 & 850 & $\mathrm{U}$ & 850 & 840 & $\mathrm{U}$ & 840 \\
\hline 2,4,6-Trichlorophenol & 340 & $\mathrm{U}$ & 340 & 340 & $\mathrm{U}$ & 340 & 340 & $\mathrm{U}$ & 340 & 340 & $\mathrm{U}$ & 340 \\
\hline 2,4-Dichlorophenol & 340 & UJ & 340 & 340 & $\mathrm{U}$ & 340 & 340 & $\mathrm{U}$ & 340 & 340 & $\mathrm{U}$ & 340 \\
\hline 2,4-Dimethylphenol & 340 & $\mathrm{U}$ & 340 & 340 & $\mathrm{U}$ & 340 & 340 & $\mathrm{U}$ & 340 & 340 & $\mathrm{U}$ & 340 \\
\hline 2,4-Dinitrophenol & 840 & $\mathrm{U}$ & 840 & 840 & $\mathrm{U}$ & 840 & 850 & $\mathrm{U}$ & 850 & 840 & $\mathrm{U}$ & 840 \\
\hline 2,4-Dinitrotoluene & 340 & $\mathrm{U}$ & 340 & 340 & $\mathrm{U}$ & 340 & 340 & $\mathrm{U}$ & 340 & 340 & $\mathrm{U}$ & 340 \\
\hline 2,6-Dinitrotoluene & 340 & $\bar{U}$ & 340 & 340 & $\mathrm{U}$ & 340 & 340 & $\mathrm{U}$ & 340 & 340 & $\mathrm{U}$ & 340 \\
\hline 2-Chloronaphthalene & 340 & $\mathrm{U}$ & 340 & 340 & $\mathrm{U}$ & 340 & 340 & $\mathrm{U}$ & 340 & 340 & $\mathrm{U}$ & 340 \\
\hline 2-Chlorophenol & 340 & UJ & 340 & 340 & $\mathrm{U}$ & 340 & 340 & $\bar{U}$ & 340 & 340 & $\mathrm{U}$ & 340 \\
\hline 2-Methylnaphthalene & 340 & $\mathrm{U}$ & 340 & 340 & $\bar{U}$ & 340 & 340 & $\mathrm{U}$ & 340 & 340 & $\mathrm{U}$ & 340 \\
\hline 2-Methylphenol (cresol, o-) & 340 & $\mathrm{U}$ & 340 & 340 & $\mathrm{U}$ & 340 & 340 & $\mathrm{U}$ & 340 & 340 & $\mathrm{U}$ & 340 \\
\hline 2-Nitroaniline & 840 & $\mathrm{U}$ & 840 & 840 & U & 840 & 850 & $\mathrm{U}$ & 850 & 840 & $\mathrm{U}$ & 840 \\
\hline 2-Nitrophenol & 340 & $\mathrm{U}$ & 340 & 340 & $\mathrm{U}$ & 340 & 340 & $\mathrm{U}$ & 340 & 340 & $\mathrm{U}$ & 340 \\
\hline & & & & & Atta & hment & 1 & & & Sheet No. & & 9 of 14 \\
\hline & & & & & Ori & nator & thes & 22 & W.Ck & orbates & & $12 / 20 / 05$ \\
\hline & & & & & & ked & T.M. B & kley & & Date & & $12 / 20 / 05$ \\
\hline & & & & & $\mathrm{Cal}$ & No. & $\overline{0100 \mathrm{~B}-\mathrm{C}}$ & $\mathrm{A}-\mathrm{VO}$ & & Rev. No. & & 0 \\
\hline
\end{tabular}


Attachment 1. 1607-B2:1 Verification Sampling Results.

\begin{tabular}{|c|c|c|c|c|c|c|c|c|c|c|c|c|}
\hline \multirow[t]{2}{*}{ Constituent } & \multicolumn{3}{|c|}{$\begin{array}{c}\text { J03VC4 } \\
\text { Sample Location } 7 \\
\text { Sample Date } 8 / 19 / 05\end{array}$} & \multicolumn{3}{|c|}{$\begin{array}{c}\text { J03VC5 } \\
\text { Sample Location } 8 \\
\text { Sample Date 8/19/05 }\end{array}$} & \multicolumn{3}{|c|}{$\begin{array}{c}\text { J03VC6 } \\
\text { Sample Location } 9 \\
\text { Sample Date } 8 / 19 / 05\end{array}$} & \multicolumn{3}{|c|}{$\begin{array}{c}\text { J03VC7 } \\
\text { Sample Location } 10 \\
\text { Sample Date } 8 / 19 / 05\end{array}$} \\
\hline & $\mu \mathrm{g} / \mathrm{kg}$ & $Q$ & PQL & $\mu \mathrm{g} / \mathrm{kg}$ & $\mathbf{Q}$ & PQL & $\mu \mathrm{g} / \mathrm{kg}$ & $\mathbf{Q}$ & PQL & $\mu \mathrm{g} / \mathrm{kg}$ & $Q$ & PQL \\
\hline \multicolumn{13}{|c|}{ Semivolatile Organic Analytes (continued) } \\
\hline $3,3^{\prime}$-Dichlorobenzidine & 340 & $\mathrm{U}$ & 340 & 340 & U & 340 & 340 & $\mathrm{U}$ & 340 & 340 & $\mathrm{U}$ & 340 \\
\hline $3+4$ Methylphenol (cresol, $m+p)$ & 340 & $\mathrm{U}$ & 340 & 340 & $\mathrm{U}$ & 340 & 340 & $\mathrm{U}$ & 340 & 340 & $\bar{U}$ & 340 \\
\hline 3-Nitroaniline & 840 & UJ & 840 & 840 & UJ & 840 & 850 & UJ & 850 & 840 & UJ & 840 \\
\hline 4,6-Dinitro-2-methylphenol & 840 & $\mathrm{U}$ & 840 & 840 & $\mathrm{U}$ & 840 & 850 & $\mathrm{U}$ & 850 & 840 & $\mathrm{U}$ & 840 \\
\hline 4-Bromophenyl-phenylether & 340 & U & 340 & 340 & $\mathrm{U}$ & 340 & 340 & $\mathrm{U}$ & 340 & 340 & $\mathrm{U}$ & 340 \\
\hline 4-Chloro-3-methylphenol & 340 & $\mathrm{UJ}$ & 340 & 340 & $\mathrm{U}$ & 340 & 340 & $\mathrm{U}$ & 340 & 340 & $\mathrm{U}$ & 340 \\
\hline 4-Chloroaniline & 340 & $\mathrm{U}$ & 340 & 340 & $\mathrm{U}$ & 340 & 340 & $\mathrm{U}$ & 340 & 340 & $\mathrm{U}$ & 340 \\
\hline 4-Chlorophenyl-phenylether & 340 & $\mathrm{U}$ & 340 & 340 & $\mathrm{U}$ & 340 & 340 & $\mathrm{U}$ & 340 & 340 & $\mathrm{U}$ & 340 \\
\hline 4-Nitroaniline & 840 & UJ & 840 & 840 & $\mathrm{UJ}$ & 840 & 850 & UJ & 850 & 840 & UJ & 840 \\
\hline 4-Nitrophenol & 840 & UJ & 840 & 840 & UJ & 840 & 850 & UJ & 850 & 840 & $\mathrm{UJ}$ & 840 \\
\hline Acenaphthene & 340 & $\mathrm{U}$ & 340 & 340 & $\mathrm{U}$ & 340 & 340 & $\mathrm{U}$ & 340 & 340 & $\mathrm{U}$ & 340 \\
\hline Acenaphthylene & 340 & $\mathrm{U}$ & 340 & 340 & $\mathrm{U}$ & 340 & 340 & $\mathrm{U}$ & 340 & 340 & $\mathrm{U}$ & 340 \\
\hline Anthracene & 340 & $\mathrm{U}$ & 340 & 340 & $\mathrm{U}$ & 340 & 340 & $\mathrm{U}$ & 340 & 340 & $\mathrm{U}$ & 340 \\
\hline Benzo(a)anthracene & 340 & $\mathrm{U}$ & 340 & 340 & $\mathrm{U}$ & 340 & 340 & $\mathrm{U}$ & 340 & 340 & $\mathrm{U}$ & 340 \\
\hline Benzo(a)pyrene & 340 & $\mathrm{U}$ & 340 & 340 & $\bar{U}$ & 340 & 340 & $\mathrm{U}$ & 340 & 340 & $\mathrm{U}$ & 340 \\
\hline Benzo(b)fluoranthene & 340 & $\mathrm{U}$ & 340 & 340 & $\mathrm{U}$ & 340 & 340 & $\mathrm{U}$ & 340 & 340 & $\mathrm{U}$ & 340 \\
\hline Benzo(ghi)perylene & 340 & $\mathrm{U}$ & 340 & 340 & $\mathrm{U}$ & 340 & 340 & $\mathrm{U}$ & 340 & 340 & $\mathrm{U}$ & 340 \\
\hline Benzo $(\mathrm{k})$ fluoranthene & 340 & $\mathrm{U}$ & 340 & 340 & $\mathrm{U}$ & 340 & 340 & $\mathrm{U}$ & 340 & 340 & $\bar{U}$ & 340 \\
\hline Bis(2-chloro-1-methylethyl)ether & 340 & $\mathrm{U}$ & 340 & 340 & $\mathrm{U}$ & 340 & 340 & $\mathrm{U}$ & 340 & 340 & $\mathrm{U}$ & 340 \\
\hline Bis(2-Chloroethoxy)methane & 340 & $\mathrm{U}$ & 340 & 340 & $\mathrm{U}$ & 340 & 340 & $\mathrm{U}$ & 340 & 340 & $\mathrm{U}$ & 340 \\
\hline Bis(2-chloroethyl) ether & 340 & $\mathrm{U}$ & 340 & 340 & $\mathrm{U}$ & 340 & 340 & $\mathrm{U}$ & 340 & 340 & $\mathrm{U}$ & 340 \\
\hline Bis(2-ethylhexyl) phthalate & 660 & $\mathrm{U}$ & 660 & 660 & $\mathrm{U}$ & 660 & 660 & U & 660 & 660 & $\mathrm{U}$ & 660 \\
\hline Butylbenzylphthalate & 340 & $\bar{U}$ & 340 & 340 & $\mathrm{U}$ & 340 & 340 & $\mathrm{U}$ & 340 & 340 & $\mathrm{U}$ & 340 \\
\hline Carbazole & 340 & $\mathrm{U}$ & 340 & 340 & $\mathrm{U}$ & 340 & 340 & $\mathrm{U}$ & 340 & 340 & $\mathrm{U}$ & 340 \\
\hline Chrysene & 340 & $\mathrm{U}$ & 340 & 340 & $\mathrm{U}$ & 340 & 340 & $\mathrm{U}$ & 340 & 340 & $\mathrm{U}$ & 340 \\
\hline Dibenz $(\mathrm{a}, \mathrm{h})$ anthracene & 340 & $\mathrm{U}$ & 340 & 340 & $\mathrm{U}$ & 340 & 340 & $\mathrm{U}$ & 340 & 340 & $\mathrm{U}$ & 340 \\
\hline Dibenzofuran & 340 & $\mathrm{U}$ & 340 & 340 & $\mathrm{U}$ & 340 & 340 & $\mathrm{U}$ & 340 & 340 & U & 340 \\
\hline Diethylphthalate & 340 & $\mathrm{U}$ & 340 & 340 & $\mathrm{U}$ & 340 & 340 & $\mathrm{U}$ & 340 & 26 & $\mathrm{~J}$ & 340 \\
\hline Dimethyl phthalate & 340 & $\mathrm{U}$ & 340 & 340 & $\mathrm{U}$ & 340 & 340 & $\mathrm{U}$ & 340 & 340 & $\mathrm{U}$ & 340 \\
\hline Di-n-butylphthalate & 340 & $\mathrm{U}$ & 340 & 340 & $\mathrm{U}$ & 340 & 340 & $\mathrm{U}$ & 340 & 340 & $\mathrm{U}$ & 340 \\
\hline Di-n-octylphthalate & 340 & $\mathrm{U}$ & 340 & 340 & U & 340 & 340 & $\mathrm{U}$ & 340 & 340 & $\mathrm{U}$ & 340 \\
\hline \begin{tabular}{|l} 
Fluoranthene \\
\end{tabular} & 340 & $\mathrm{U}$ & 340 & 340 & $\mathrm{U}$ & 340 & 340 & $U$ & 340 & 340 & $\mathrm{U}$ & 340 \\
\hline Fluorene & 340 & $\mathrm{U}$ & 340 & 340 & $\mathrm{U}$ & 340 & 340 & $\mathrm{U}$ & 340 & 340 & $\mathrm{U}$ & 340 \\
\hline Hexachlorobenzene & 340 & $\mathrm{U}$ & 340 & 340 & $\mathrm{U}$ & 340 & 340 & $\mathrm{U}$ & 340 & 340 & $\mathrm{U}$ & 340 \\
\hline Hexachlorobutadiene & 340 & $\mathrm{U}$ & 340 & 340 & $\mathrm{U}$ & 340 & 340 & $\mathrm{U}$ & 340 & 340 & $\mathrm{U}$ & 340 \\
\hline Hexachlorocyclopentadiene & 340 & $\mathrm{U}$ & 340 & 340 & $\mathrm{U}$ & 340 & 340 & $\mathrm{U}$ & 340 & 340 & $\mathrm{U}$ & 340 \\
\hline Hexachloroethane & 340 & $\mathrm{U}$ & 340 & 340 & $\mathrm{U}$ & 340 & 340 & $\mathrm{U}$ & 340 & 340 & $\mathrm{U}$ & 340 \\
\hline Indeno(1,2,3-cd)pyrene & 340 & $\mathrm{U}$ & 340 & 340 & $\mathrm{U}$ & 340 & 340 & $\mathrm{U}$ & 340 & 340 & $\mathrm{U}$ & 340 \\
\hline Isophorone & 340 & $\mathrm{U}$ & 340 & 340 & $\mathrm{U}$ & 340 & 340 & $\mathrm{U}$ & 340 & 340 & $\mathrm{U}$ & 340 \\
\hline Naphthalene & 340 & $\mathrm{U}$ & 340 & 340 & $\mathrm{U}$ & 340 & 340 & $\mathrm{U}$ & 340 & 340 & $\bar{U}$ & 340 \\
\hline Nitrobenzene & 340 & $\mathrm{U}$ & 340 & 340 & $\mathrm{U}$ & 340 & 340 & $\mathrm{U}$ & 340 & 340 & $\mathrm{U}$ & 340 \\
\hline N-Nitroso-di-n-dipropylamine & 340 & $\mathrm{U}$ & 340 & 340 & $\mathrm{U}$ & 340 & 340 & $\mathrm{U}$ & 340 & 340 & $\mathrm{U}$ & 340 \\
\hline N-Nitrosodiphenylamine & 340 & $\mathrm{U}$ & 340 & 340 & $\mathrm{U}$ & 340 & 340 & $\mathrm{U}$ & 340 & 340 & $\mathrm{U}$ & 340 \\
\hline Pentachlorophenol & 840 & $\mathrm{U}$ & 840 & 840 & $\mathrm{U}$ & 840 & 850 & $\mathrm{U}$ & 850 & 840 & $\mathrm{U}$ & 840 \\
\hline Phenanthrene & 340 & $\mathrm{U}$ & 340 & 340 & $\mathrm{U}$ & 340 & 340 & $\mathrm{U}$ & 340 & 340 & U & 340 \\
\hline Phenol & 340 & $\mathrm{U}$ & 340 & 340 & $\mathrm{U}$ & 340 & 340 & $\mathrm{U}$ & 340 & 340 & $\mathrm{U}$ & 340 \\
\hline Pyrene & 340 & $\mathrm{U}$ & 340 & 340 & $\mathrm{U}$ & 340 & 340 & $\mathrm{U}$ & 340 & 340 & $\mathrm{U}$ & 340 \\
\hline
\end{tabular}

\begin{tabular}{|c|c|c|c|}
\hline Attachment & 1 & Sheet No. & 10 of 14 \\
\hline Originator & 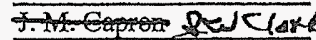 & (6ate $\mathrm{R}=\mathrm{J}$ & $-12 / 20 / 05$ \\
\hline Checked & T.M. Blakley & Date & $12 / 20 / 05$ \\
\hline Calc. No. & $0100 \mathrm{~B}-\mathrm{CA}-\mathrm{V} 0264$ & Rev. No. & 0 \\
\hline
\end{tabular}


Attachment 1. 1607-B2:1 Verification Sampling Results.

\begin{tabular}{|c|c|c|c|c|c|c|c|c|c|c|c|c|}
\hline \multirow[t]{2}{*}{ Constituent } & \multicolumn{3}{|c|}{$\begin{array}{c}\text { J03VC4 } \\
\text { Sample Location } 7 \\
\text { Sample Date 8/19/05 }\end{array}$} & \multicolumn{3}{|c|}{$\begin{array}{c}\text { J03VC5 } \\
\text { Sample Location } 8 \\
\text { Sample Date } 8 / 19 / 05\end{array}$} & \multicolumn{3}{|c|}{$\begin{array}{c}\text { J03VC6 } \\
\text { Sample Location } 9 \\
\text { Sample Date 8/19/05 }\end{array}$} & \multicolumn{3}{|c|}{$\begin{array}{c}\text { J03VC7 } \\
\text { Sample Location } 10 \\
\text { Sample Date 8/19/05 }\end{array}$} \\
\hline & $\mu \mathrm{g} / \mathrm{kg}$ & $Q$ & PQL & $\mu \mathrm{g} / \mathrm{kg}$ & Q & PQL & $\mu \mathrm{g} / \mathrm{kg}$ & $\mathbf{Q}$ & PQL & $\mu \mathrm{g} / \mathrm{kg}$ & $\mathbf{Q}$ & PQL \\
\hline \multicolumn{13}{|c|}{ Volatile Organic Analytes } \\
\hline 1,1,1-Trichloroethane & 4 & $\mathrm{UJ}$ & 4 & 5 & $\mathrm{U}$ & 5 & 6 & $\mathrm{U}$ & 6 & 6 & UJ & 6 \\
\hline 1,1,2,2-Tetrachloroethane & 4 & UJ & 4 & 5 & $\mathrm{U}$ & 5 & 6 & $\mathrm{U}$ & 6 & 6 & $\mathrm{UJ}$ & 6 \\
\hline 1,1,2-Trichloroethane & 4 & $\mathrm{UJ}$ & 4 & 5 & $\mathrm{U}$ & 5 & 6 & $\mathrm{U}$ & 6 & 6 & UJ & 6 \\
\hline 1,1-Dichloroethane & 4 & $\mathrm{UJ}$ & 4 & 5 & $\mathrm{U}$ & 5 & 6 & $\mathrm{U}$ & 6 & 6 & UJ & 6 \\
\hline 1,1-Dichloroethene & 4 & $\mathrm{UJ}$ & 4 & 5 & $\mathrm{U}$ & 5 & 6 & $\mathrm{U}$ & 6 & 6 & UI & 6 \\
\hline 1,2-Dichloroethane & 4 & $\mathrm{UJ}$ & 4 & 5 & $\mathrm{U}$ & 5 & 6 & $\mathrm{U}$ & 6 & 6 & $\mathrm{UJ}$ & 6 \\
\hline 1,2-Dichloropropane & 4 & $\mathrm{UJ}$ & 4 & 5 & $\mathrm{U}$ & 5 & 6 & $\mathrm{U}$ & 6 & 6 & UJ & 6 \\
\hline 2-Butanone & 9 & $\mathrm{UJ}$ & 9 & 10 & $\mathrm{U}$ & 10 & 11 & $\mathrm{U}$ & 11 & 11 & $\mathrm{UJ}$ & 11 \\
\hline 2-Hexanone & 9 & UJ & 9 & 10 & $\mathrm{U}$ & 10 & 11 & $\mathrm{U}$ & 11 & 11 & $\mathrm{UJ}$ & 11 \\
\hline 4-Methy1-2-Pentanone & 9 & $\mathrm{UJ}$ & 9 & 10 & $\mathrm{U}$ & 10 & 11 & $\mathrm{U}$ & 11 & 11 & UJ & 11 \\
\hline Acetone & 14 & $\mathbf{J}$ & 9 & 7 & $\mathrm{~J}$ & 10 & 7 & $\mathrm{~J}$ & 11 & 6 & $\mathrm{~J}$ & 11 \\
\hline Benzene & 4 & UJ & 4 & 5 & $\mathrm{U}$ & 5 & 6 & $\mathrm{U}$ & 6 & 6 & $\mathrm{UJ}$ & 6 \\
\hline Bromodichloromethane & 4 & $\mathrm{UJ}$ & 4 & 5 & $\mathrm{U}$ & 5 & 6 & $\mathrm{U}$ & 6 & 6 & US & 6 \\
\hline Bromoform & 4 & $\mathrm{UJ}$ & 4 & 5 & $\mathrm{U}$ & 5 & 6 & $\mathrm{U}$ & 6 & 6 & $\mathrm{UI}$ & 6 \\
\hline Bromomethane & 9 & $\mathrm{UJ}$ & 9 & 10 & $\mathrm{U}$ & 10 & 11 & $\mathrm{U}$ & 11 & 11 & UJ & 11 \\
\hline Carbon disulfide & 4 & $\mathrm{UJ}$ & 4 & 5 & $\mathrm{U}$ & 5 & 6 & $\mathrm{U}$ & 6 & 6 & $\mathrm{UJ}$ & 6 \\
\hline Carbon tetrachloride & 4 & $\mathrm{UJ}$ & 4 & 5 & $\mathrm{U}$ & 5 & 6 & $\mathrm{U}$ & 6 & 6 & $\mathrm{UJ}$ & 6 \\
\hline Chlorobenzene & 4 & UJ & 4 & 5 & $\mathrm{U}$ & 5 & 6 & $\mathrm{U}$ & 6 & 6 & $\mathrm{UJ}$ & 6 \\
\hline Chloroethane & 9 & $\mathrm{UJ}$ & 9 & 10 & $\mathrm{U}$ & 10 & 11 & $\mathrm{U}$ & 11 & 11 & $\mathrm{UJ}$ & 11 \\
\hline Chloroform & 4 & $\mathrm{UJ}$ & 4 & 5 & $\mathrm{U}$ & 5 & 6 & $\mathrm{U}$ & 6 & 6 & UJ & 6 \\
\hline Chloromethane & 9 & UJ & 9 & 10 & $\mathrm{U}$ & 10 & 11 & $\mathrm{U}$ & 11 & 11 & UJ & 11 \\
\hline cis-1,2-Dichloroethylene & 4 & $\mathrm{UJ}$ & 4 & 5 & $\mathrm{U}$ & 5 & 6 & $\mathrm{U}$ & 6 & 6 & $\mathrm{UJ}$ & 6 \\
\hline cis-1,3-Dichloropropene & 4 & US & 4 & 5 & $\mathrm{U}$ & 5 & 6 & $\mathrm{U}$ & 6 & 6 & UJ & 6 \\
\hline Dibromochloromethane & 4 & UJ & 4 & 5 & $\mathrm{U}$ & 5 & 6 & $\mathrm{U}$ & 6 & 6 & $\mathrm{UJ}$ & 6 \\
\hline Ethylbenzene & 4 & UJ & 4 & 5 & $\mathrm{U}$ & 5 & 6 & $\mathrm{U}$ & 6 & 6 & $\mathrm{UJ}$ & 6 \\
\hline Methylene chloride & 10 & $\mathrm{UJ}$ & 10 & 11 & $\mathrm{U}$ & 11 & 10 & $\mathrm{U}$ & 10 & 13 & UJ & 13 \\
\hline Styrene & 4 & UJ & 4 & 5 & $\mathrm{U}$ & 5 & 6 & $\mathrm{U}$ & 6 & 6 & UJ & 6 \\
\hline Tetrachloroethene & 4 & UJ & 4 & 5 & U & 5. & 6 & $\mathrm{U}$ & 6 & 6 & UJ & 6 \\
\hline Toluene & 4 & $\mathrm{UJ}$ & 4 & 5 & $\mathrm{U}$ & 5 & 6 & $\mathrm{U}$ & 6 & 6 & UJ & 6 \\
\hline trans-1,2-Dichloroethylene & 4 & UJ & 4 & 5 & $\mathrm{U}$ & 5 & 6 & $\mathrm{U}$ & 6 & 6 & UJ & 6 \\
\hline trans-1,3-Dichloropropene & 4 & UJ & 4 & 5 & $\mathrm{U}$ & 5 & 6 & $\mathrm{U}$ & 6 & 6 & UJ & 6 \\
\hline Trichloroethene & 4 & UJ & 4 & 5 & $\mathrm{U}$ & 5 & 6 & $\mathrm{U}$ & 6 & 6 & UJ & 6 \\
\hline \begin{tabular}{|l} 
Vinyl chloride \\
\end{tabular} & 9 & UJ & 9 & 10 & $\mathrm{U}$ & 10 & 11 & $\mathrm{U}$ & 11 & 11 & UJ & 11 \\
\hline Xylenes (total) & 4 & $\mathrm{UJ}$ & 4 & 5 & $\mathrm{U}$ & 5 & 6 & $\mathrm{U}$ & 6 & 6 & UJ & 6 \\
\hline \multicolumn{13}{|c|}{ Herbicides } \\
\hline 2,4-D & 170 & $\mathrm{U}$ & 170 & 170 & $\mathrm{U}$ & 170 & 170 & $\mathrm{U}$ & 170 & 170 & $\mathrm{U}$ & 170 \\
\hline 2,4-DB & 840 & $\mathrm{U}$ & 840 & 840 & $\mathrm{U}$ & 840 & 850 & $\mathrm{U}$ & 850 & 250 & & 840 \\
\hline $2,4,5-\mathrm{T}$ & 32 & $\mathrm{~J}$ & 84 & 29 & $\mathrm{~J}$ & 84 & 30 & $\mathrm{~J}$ & 85 & 84 & $\mathrm{UJ}$ & 84 \\
\hline 2,4,5-TP (Silvex) & 84 & UJ & 84 & 84 & UJ & 84 & 23 & $\mathrm{~J}$ & 85 & 84 & $\mathrm{UJ}$ & 84 \\
\hline Dalapon & 840 & $\mathrm{U}$ & 840 & 840 & $\mathrm{U}$ & 840 & 850 & $\mathrm{U}$ & 850 & 840 & $\mathrm{U}$ & 840 \\
\hline Dicamba & 340 & UJ & 340 & 340 & $\mathrm{UJ}$ & 340 & 340 & $\mathrm{UJ}$ & 340 & 340 & UJ & 340 \\
\hline Dichloroprop & 840 & UJ & 840 & 840 & $\mathrm{UJ}$ & 840 & 850 & $\mathrm{UI}$ & 850 & 840 & UI & 840 \\
\hline Dinoseb & 84 & $\mathrm{U}$ & 84 & 84 & $\mathrm{U}$ & 84 & 18 & & 85 & 21 & & 84 \\
\hline
\end{tabular}

Attachment

Originator

Checked

Calc. No.

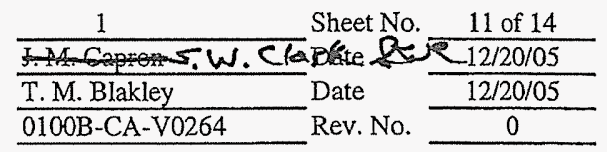


Attachment 1. 1607-B2:1 Verification Sampling Results.

\begin{tabular}{|c|c|c|c|c|c|c|c|c|c|c|c|c|}
\hline \multirow[t]{2}{*}{ Constituent } & \multicolumn{3}{|c|}{$\begin{array}{c}\text { J03VC8 } \\
\text { Sample Location } 11 \\
\text { Sample Date } 8 / 19 / 05\end{array}$} & \multicolumn{3}{|c|}{$\begin{array}{c}\text { J03VC9 } \\
\text { SW Overburden } \\
\text { Sample Date 8/19/05 }\end{array}$} & \multicolumn{3}{|c|}{$\begin{array}{c}\text { J03VD0 } \\
\text { NW Overburden } \\
\text { Sample Date } 8 / 19 / 05\end{array}$} & \multicolumn{3}{|c|}{$\begin{array}{c}\text { J03VD1 } \\
\text { NE Overburden } \\
\text { Sample Date 8/19/05 }\end{array}$} \\
\hline & $\mu \mathrm{g} / \mathrm{kg}$ & Q & PQL & $\mu \mathrm{g} / \mathrm{kg}$ & 01 & PQL & $\mu \mathrm{g} / \mathrm{kg}$ & Q & PQL & $\mu \mathrm{g} / \mathrm{kg}$ & Q & PQL \\
\hline \multicolumn{13}{|c|}{ Polychlorinated Biphenyls } \\
\hline Aroclor-1016 & 13 & $\mathrm{U}$ & 13 & 13 & U & 13 & 13 & $\mathrm{U}$ & 13 & 13 & $\mathrm{U}$ & 13 \\
\hline Aroclor-1221 & 13 & $\mathrm{U}$ & 13 & 13 & $\mathrm{U}$ & 13 & 13 & U & 13 & 13 & $\mathrm{U}$ & 13 \\
\hline Aroclor-1232 & 13 & $\mathrm{U}$ & 13 & 13 & $\mathrm{U}$ & 13 & 13 & $\mathrm{U}$ & 13 & 13 & U & 13 \\
\hline Aroclor-1242 & 13 & $\mathrm{U}$ & 13 & 13 & $\mathrm{U}$ & 13 & 13 & $\mathrm{U}$ & 13 & 13 & U & 13 \\
\hline Aroclor-1248 & 13 & $\mathrm{U}$ & 13 & 13 & $\mathrm{U}$ & 13 & 13 & $\mathrm{U}$ & 13 & 13 & $\mathrm{U}$ & 13 \\
\hline Aroclor-1254 & 13 & $\mathrm{U}$ & 13 & 13 & $\mathrm{U}$ & 13 & 13 & $\mathrm{U}$ & 13 & 13 & $\mathrm{U}$ & 13 \\
\hline Aroclor -1260 & 13 & $\mathrm{U}$ & 13 & 13 & $\mathrm{U}$ & 13 & 13 & $\mathrm{U}$ & 13 & 13 & $\mathrm{U}$ & 13 \\
\hline \multicolumn{13}{|c|}{ Pesticides } \\
\hline Aldrin & 1.7 & UJ & 1.7 & 1.7 & UJ & 1.7 & 1.7 & UJ & 1.7 & 1.7 & UJ & 1.7 \\
\hline Alpha-BHC & 1.7 & UJ & 1.7 & 1.7 & UJ & 1.7 & 1.7 & UJ & 1.7 & 1.7 & UJ & 1.7 \\
\hline alpha-Chlordane & 1.7 & UJ & 1.7 & 1.7 & UJ & 1.7 & 1.7 & $\overline{\mathrm{UJ}}$ & 1.7 & 1.7 & UI & 1.7 \\
\hline Beta-BHC & 1.7 & UJ & 1.7 & 1.7 & UI & 1.7 & 1.7 & UJ & 1.7 & 1.7 & UJ & 1.7 \\
\hline Delta-BHC & 1.7 & UJ & 1.7 & 1.7 & UJ & 1.7 & 1.7 & UJ & 1.7 & 1.7 & UJ & 1.7 \\
\hline Dichlorodiphenyldichloroethane & 3.4 & $\mathrm{UJ}$ & 3.4 & 3.4 & UJ & 3.4 & 3.3 & UJ & 3.3 & 3.3 & UJ & 3.3 \\
\hline Dichlorodiphenyldichloroethylene & 3.4 & $\mathrm{UJ}$ & 3.4 & 3.4 & UJ & 3.4 & 3.3 & UJ & 3.3 & 3.3 & UJ & 3.3 \\
\hline Dichlorodiphenyltrichloroethane & 3.4 & UJ & 3.4 & 3.4 & UJ & 3.4 & 3.3 & UJ & 3.3 & 3.3 & UJ & 3.3 \\
\hline Dieldrin & 1.7 & UJ & 1.7 & 1.7 & UJ & 1.7 & 1.7 & UJ & 1.7 & 1.7 & UJ & 1.7 \\
\hline Endosulfan I & 1.7 & UJ & 1.7 & 1.7 & UJ & 1.7 & 1.7 & UJ & 1.7 & 1.7 & UJ & 1.7 \\
\hline Endosulfan II & 3.4 & UJ & 3.4 & 3.4 & UJ & 3.4 & 3.3 & UJ & 3.3 & 3.3 & UJ & 3.3 \\
\hline Endosulfan sulfate & 3.4 & $\mathrm{UJ}$ & 3.4 & 3.4 & $\mathrm{UJ}$ & 3.4 & 3.3 & UJ & 3.3 & 3.3 & UJ & 3.3 \\
\hline Endrin & 3.4 & $\mathrm{UJ}$ & 3.4 & 3.4 & UJ & 3.4 & 3.3 & UJ & 3.3 & 3.3 & UJ & 3.3 \\
\hline Endrin aldehyde & 3.4 & $\mathrm{UJ}$ & 3.4 & 3.4 & $\mathrm{UJ}$ & 3.4 & 3.3 & UJ & 3.3 & 3.3 & UJ & 3.3 \\
\hline Endrin ketone & 3.4 & $\mathrm{UJ}$ & 3.4 & 3.4 & UI & 3.4 & 3.3 & $\mathrm{UJ}$ & 3.3 & 3.3 & UJ & 3.3 \\
\hline Gamma-BHC (Lindane) & 1.7 & $\mathrm{UJ}$ & 1.7 & 1.7 & UJ & 1.7 & 1.7 & UJ & 1.7 & 1.7 & UJ & 1.7 \\
\hline gamma-Chlordane & 1.7 & $\mathrm{UJ}$ & 1.7 & 1.7 & UJ & 1.7 & 1.7 & UJ & 1.7 & 1.7 & UJ & 1.7 \\
\hline Heptachlor & 1.7 & UJ & 1.7 & 1.7 & UJ & 1.7 & 1.7 & UI & 1.7 & 1.7 & UJ & 1.7 \\
\hline Heptachlor epoxide & 1.7 & $\overline{U I}$ & 1.7 & 1.7 & UJ & 1.7 & 1.7 & $\mathrm{UJ}$ & 1.7 & 1.7 & UJ & 1.7 \\
\hline Methoxychlor & 17 & UJ & 17 & 17 & UJ & 17 & 17 & UT & 17 & 17 & UJ & 17 \\
\hline Toxaphene & 170 & UJ & 170 & 170 & UJ & 170 & 170 & UJ & 170 & 170 & UJ & 170 \\
\hline \multicolumn{13}{|c|}{ Semivolatile Organic Analytes } \\
\hline 1,2,4-Trichlorobenzene & 340 & $\mathrm{U}$ & 340 & 340 & $\mathrm{U}$ & 340 & 340 & $\mathrm{U}$ & 340 & 340 & $\mathrm{U}$ & 340 \\
\hline 1,2-Dichlorobenzene & 340 & $\mathrm{U}$ & 340 & 340 & $\mathrm{U}$ & 340 & 340 & $\mathrm{U}$ & 340 & 340 & $\mathrm{U}$ & 340 \\
\hline 1,3-Dichlorobenzene & 340 & $\mathrm{U}$ & 340 & 340 & $\mathrm{U}$ & 340 & 340 & U & 340 & 340 & $\mathrm{U}$ & 340 \\
\hline 1,4-Dichlorobenzene & 340 & $\mathrm{U}$ & 340 & 340 & $\mathrm{U}$ & 340 & 340 & $\mathrm{U}$ & 340 & 340 & $\mathrm{U}$ & 340 \\
\hline 2,4,5-Trichlorophenol & 840 & $\bar{U}$ & 840 & 840 & $\mathrm{U}$ & 840 & 840 & U & 840 & 840 & U & 840 \\
\hline 2,4,6-Trichlorophenol & 340 & $\mathrm{U}$ & 340 & 340 & $\mathrm{U}$ & 340 & 340 & $\mathrm{U}$ & 340 & 340 & $U$ & 340 \\
\hline 2,4-Dichlorophenol & 340 & $\mathrm{U}$ & 340 & 340 & $\mathrm{U}$ & 340 & 340 & $\mathrm{U}$ & 340 & 340 & $\mathrm{U}$ & 340 \\
\hline 2,4-Dimethylphenol & 340 & $\mathrm{U}$ & 340 & 340 & $\mathrm{U}$ & 340 & 340 & $\mathrm{U}$ & 340 & 340 & $\mathrm{U}$ & 340 \\
\hline 2,4-Dinitrophenol & 840 & $\mathrm{U}$ & 840 & 840 & $\mathrm{U}$ & 840 & 840 & $\mathrm{U}$ & 840 & 840 & $\bar{U}$ & 840 \\
\hline 2,4-Dinitrotoluene & 340 & U & 340 & 340 & $\mathrm{U}$ & 340 & 340 & $\mathrm{U}$ & 340 & 340 & $\mathrm{U}$ & 340 \\
\hline 2,6-Dinitrotoluene & 340 & $\mathrm{U}$ & 340 & 340 & $\mathrm{U}$ & 340 & 340 & $\mathrm{U}$ & 340 & 340 & $\bar{U}$ & 340 \\
\hline 2-Chloronaphthalene & 340 & $\mathrm{U}$ & 340 & 340 & $\mathrm{U}$ & 340 & 340 & $\mathrm{U}$ & 340 & 340 & $\mathrm{U}$ & 340 \\
\hline 2-Chlorophenol & 340 & $\mathrm{U}$ & 340 & 340 & $\mathrm{U}$ & 340 & 340 & $\mathrm{U}$ & 340 & 340 & $\mathrm{U}$ & 340 \\
\hline 2-Methylnaphthalene & 340 & $\mathrm{U}$ & 340 & 340 & $\mathrm{U}$ & 340 & 340 & U & 340 & 340 & $\mathrm{U}$ & 340 \\
\hline 2-Methylphenol (cresol, o-) & 340 & $\mathrm{U}$ & 340 & 340 & $\mathrm{U}$ & 340 & 340 & $\mathrm{U}$ & 340 & 340 & $\mathrm{U}$ & 340 \\
\hline 2-Nitroaniline & 840 & $\mathrm{U}$ & 840 & 840 & $\mathrm{U}$ & 840 & 840 & $\mathrm{U}$ & 840 & 840 & $\mathrm{U}$ & 840 \\
\hline 2-Nitrophenol & 340 & $\mathrm{U}$ & 340 & 340 & $\mathrm{U}$ & 340 & 340 & U & 340 & 340 & $\mathrm{U}$ & 340 \\
\hline & & & & & \multirow{2}{*}{\multicolumn{2}{|c|}{$\begin{array}{l}\text { Attachment } \\
\text { Originator }\end{array}$}} & \multicolumn{3}{|c|}{1} & \multirow{2}{*}{ Sheet No. } & & 12 of 14 \\
\hline & & & & & & & \multicolumn{5}{|c|}{ 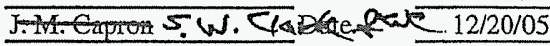 } & \\
\hline & & & & & \multirow{2}{*}{\multicolumn{2}{|c|}{$\begin{array}{l}\text { Checked } \\
\text { Calc. No. }\end{array}$}} & T.M.B & kley & & Date & & $12 / 20 / 05$ \\
\hline & & & & & & & $0100 \mathrm{~B}-1$ & $\frac{1}{1-V 0}$ & & Rev. No. & & 0 \\
\hline
\end{tabular}


Attachment 1. 1607-B2:1 Verification Sampling Results.

\begin{tabular}{|c|c|c|c|c|c|c|c|c|c|c|c|c|}
\hline \multirow[t]{2}{*}{ Constituent } & \multicolumn{3}{|c|}{$\begin{array}{c}\text { J03VC8 } \\
\text { Sample Location } 11 \\
\text { Sample Date } 8 / 19 / 05 \\
\end{array}$} & \multicolumn{3}{|c|}{$\begin{array}{c}\text { J03VC9 } \\
\text { SW Overburden } \\
\text { Sample Date } 8 / 19 / 05 \\
\end{array}$} & \multicolumn{3}{|c|}{$\begin{array}{c}\text { J03VD0 } \\
\text { NW Overburden } \\
\text { Sample Date 8/19/05 }\end{array}$} & \multicolumn{3}{|c|}{$\begin{array}{c}\text { J03VD1 } \\
\text { NE Overburden } \\
\text { Sample Date } 8 / 19 / 05\end{array}$} \\
\hline & $\mu \mathrm{g} / \mathrm{kg}$ & $Q$ & PQL & $\mu \mathrm{g} / \mathrm{kg}$ & $Q$ & PQL & $\mu \mathrm{g} / \mathrm{kg}$ & Q & PQL & $\mu \mathrm{g} / \mathrm{kg}$ & $Q$ & PQL \\
\hline \multicolumn{13}{|c|}{ Semivolatile Organic Analytes (continued) } \\
\hline 3,3'-Dichlorobenzidine & 340 & $\mathrm{U}$ & 340 & 340 & $U$ & 340 & 340 & $\mathrm{U}$ & 340 & 340 & $\mathrm{U}$ & 340 \\
\hline $3+4$ Methylphenol (cresol, $m+p)$ & 340 & $\mathrm{U}$ & 340 & 340 & $\mathrm{U}$ & 340 & 340 & $\mathrm{U}$ & 340 & 340 & $\mathrm{U}$ & 340 \\
\hline 3-Nitroaniline & 840 & UJ & 840 & 840 & UJ & 840 & 840 & UJ & 840 & 840 & UJ & 840 \\
\hline 4,6-Dinitro-2-methylphenol & 840 & $\mathrm{U}$ & 840 & 840 & $\mathrm{U}$ & 840 & 840 & $\mathrm{U}$ & 840 & 840 & $\mathrm{U}$ & 840 \\
\hline 4-Bromophenyl-phenylether & 340 & $\mathrm{U}$ & 340 & 340 & U & 340 & 340 & $\mathrm{U}$ & 340 & 340 & $\mathrm{U}$ & 340 \\
\hline 4-Chloro-3-methylphenol & 340 & $\mathrm{U}$ & 340 & 340 & $\mathrm{U}$ & 340 & 340 & $\mathrm{U}$ & 340 & 340 & U & 340 \\
\hline 4-Chloroaniline & 340 & U & 340 & 340 & $\mathrm{U}$ & 340 & 340 & U & 340 & 340 & $\mathrm{U}$ & 340 \\
\hline 4-Chlorophenyl-phenylether & 340 & $\mathrm{U}$ & 340 & 340 & $\mathrm{U}$ & 340 & 340 & $\mathrm{U}$ & 340 & 340 & $\mathrm{U}$ & 340 \\
\hline 4-Nitroaniline & 840 & $\mathrm{UJ}$ & 840 & 840 & $\mathrm{UJ}$ & 840 & 840 & UJ & 840 & 840 & $\mathrm{UJ}$ & 840 \\
\hline 4-Nitrophenol & 840 & UJ & 840 & 840 & $\mathrm{UJ}$ & 840 & 840 & UJ & 840 & 840 & UJ & 840 \\
\hline Acenaphthene & 340 & $\mathrm{U}$ & 340 & 340 & $\mathrm{U}$ & 340 & 340 & $\mathrm{U}$ & 340 & 340 & $\mathrm{U}$ & 340 \\
\hline Acenaphthylene & 340 & $\mathrm{U}$ & 340 & 340 & $\overline{\mathrm{U}}$ & 340 & 340 & $\overline{\mathrm{U}}$ & 340 & 340 & $\mathrm{U}$ & 340 \\
\hline Anthracene & 340 & $\mathrm{U}$ & 340 & 340 & $\bar{U}$ & 340 & 340 & $\bar{U}$ & 340 & 340 & $\mathrm{U}$ & 340 \\
\hline Benzo(a)anthracene & 340 & $\mathrm{U}$ & 340 & 340 & $\bar{U}$ & 340 & 340 & $\bar{U}$ & 340 & 340 & $\mathrm{U}$ & 340 \\
\hline Benzo(a)pyrene & 340 & $\mathrm{U}$ & 340 & 340 & $\bar{U}$ & 340 & 340 & $\bar{U}$ & 340 & 340 & $\mathrm{U}$ & 340 \\
\hline Benzo(b)fluoranthene & 340 & $\mathrm{U}$ & 340 & 340 & $\mathrm{U}$ & 340 & 340 & $\bar{U}$ & 340 & 340 & $\bar{U}$ & 340 \\
\hline Benzo(ghi)perylene & 340 & $\bar{U}$ & 340 & 340 & $\mathrm{U}$ & 340 & 340 & $\mathrm{U}$ & 340 & 340 & $\bar{U}$ & 340 \\
\hline Benzo(k)fluoranthene & 340 & $\mathrm{U}$ & 340 & 340 & $\mathrm{U}$ & 340 & 340 & $\bar{U}$ & 340 & 340 & $\bar{U}$ & 340 \\
\hline Bis(2-chloro-1-methylethyl)ether & 340 & $\mathrm{U}$ & 340 & 340 & $\overline{\mathrm{U}}$ & 340 & 340 & $\bar{U}$ & 340 & 340 & $\bar{U}$ & 340 \\
\hline Bis(2-Chloroethoxy)methane & 340 & $\mathrm{U}$ & 340 & 340 & $U$ & 340 & 340 & $\mathrm{U}$ & 340 & 340 & $\mathrm{U}$ & 340 \\
\hline Bis(2-chloroethyl) ether & 340 & $\bar{U}$ & 340 & 340 & $\mathrm{U}$ & 340 & 340 & $\mathrm{U}$ & 340 & 340 & $\mathrm{U}$ & 340 \\
\hline Bis(2-ethylhexyl) phthalate & 660 & $\mathrm{U}$ & 660 & 660 & $\mathrm{U}$ & 660 & 340 & $\mathrm{U}$ & 340 & 660 & $\mathrm{U}$ & 660 \\
\hline Butylbenzylphthalate & 340 & $\mathrm{U}$ & 340 & 340 & $\mathrm{U}$ & 340 & 340 & $\mathrm{U}$ & 340 & 340 & $\mathrm{U}$ & 340 \\
\hline Carbazole & 340 & $\mathrm{U}$ & 340 & 340 & $\mathrm{U}$ & 340 & 340 . & $\mathrm{U}$ & 340 & 340 & $\mathrm{U}$ & 340 \\
\hline Chrysene & 340 & $\mathrm{U}$ & 340 & 340 & $\mathrm{U}$ & 340 & 340 & $\mathrm{U}$ & 340 & 340 & $\bar{U}$ & 340 \\
\hline Dibenz(a,h)anthracene & 340 & $\mathrm{U}$ & 340 & 340 & $\mathrm{U}$ & 340 & 340 & $\mathrm{U}$ & 340 & 340 & $\mathrm{U}$ & 340 \\
\hline Dibenzofuran & 340 & $\mathrm{U}$ & 340 & 340 & $\mathrm{U}$ & 340 & 340 & $\mathrm{U}$ & 340 & 340 & $\mathrm{U}$ & 340 \\
\hline Diethylphthalate & 340 & $\mathrm{U}$ & 340 & 340 & $\mathrm{U}$ & 340 & 340 & $\mathrm{U}$ & 340 & 340 & $\mathrm{U}$ & 340 \\
\hline Dimethyl phthalate & 340 & $\mathrm{U}$ & 340 & 340 & $\mathrm{U}$ & 340 & 340 & $\mathrm{U}$ & 340 & 340 & $\mathrm{U}$ & 340 \\
\hline Di-n-butylphthalate & 340 & $\mathrm{U}$ & 340 & 340 & $\mathrm{U}$ & 340 & 340 & $\mathrm{U}$ & 340 & 340 & $\mathrm{U}$ & 340 \\
\hline Di-n-octylphthalate & 340 & $\mathrm{U}$ & 340 & 340 & $\mathrm{U}$ & 340 & 340 & $U$ & 340 & 340 & $\mathrm{U}$ & 340 \\
\hline Fluoranthene & 340 & $\mathrm{U}$ & 340 & 340 & $\mathrm{U}$ & 340 & 340 & $\mathrm{U}$ & 340 & 340 & $U$ & 340 \\
\hline Fluorene & 340 & $\mathrm{U}$ & 340 & 340 & $\mathrm{U}$ & 340 & 340 & $\mathrm{U}$ & 340 & 340 & $\mathrm{U}$ & 340 \\
\hline Hexachlorobenzene & 340 & $\mathrm{U}$ & 340 & 340 & $\mathrm{U}$ & 340 & 340 & $\mathrm{U}$ & 340 & 340 & $\mathrm{U}$ & 340 \\
\hline Hexachlorobutadiene & 340 & $\mathrm{U}$ & 340 & 340 & $\mathrm{U}$ & 340 & 340 & $\mathrm{U}$ & 340 & 340 & $\mathrm{U}$ & 340 \\
\hline Hexachlorocyclopentadiene & 340 & $\mathrm{U}$ & 340 & 340 & $\mathrm{U}$ & 340 & 340 & $\mathrm{U}$ & 340 & 340 & $\mathrm{U}$ & 340 \\
\hline Hexachloroethane & 340 & $\mathrm{U}$ & 340 & 340 & $\mathrm{U}$ & 340 & 340 & $\mathrm{U}$ & 340 & 340 & $\mathrm{U}$ & 340 \\
\hline Indeno $(1,2,3-c d)$ pyrene & 340 & $\mathrm{U}$ & 340 & 340 & $\mathrm{U}$ & 340 & 340 & $\mathrm{U}$ & 340 & 340 & $\mathrm{U}$ & 340 \\
\hline Isophorone & 340 & $\mathrm{U}$ & 340 & 340 & $\mathrm{U}$ & 340 & 340 & $\mathrm{U}$ & 340 & 340 & $\mathrm{U}$ & 340 \\
\hline Naphthalene & 340 & $\mathrm{U}$ & 340 & 340 & $\mathrm{U}$ & 340 & 340 & $\mathrm{U}$ & 340 & 340 & $\mathrm{U}$ & 340 \\
\hline Nitrobenzene & 340 & $\mathrm{U}$ & 340 & 340 & $\mathrm{U}$ & 340 & 340 & $\mathrm{U}$ & 340 & 340 & $\mathrm{U}$ & 340 \\
\hline N-Nitroso-di-n-dipropylamine & 340 & $\mathrm{U}$ & 340 & 340 & $\mathrm{U}$ & 340 & 340 & $\mathrm{U}$ & 340 & 340 & $\mathrm{U}$ & 340 \\
\hline N-Nitrosodiphenylamine & 340 & $\mathrm{U}$ & 340 & 340 & $\mathrm{U}$ & 340 & 340 & $\mathrm{U}$ & 340 & 340 & $\mathrm{U}$ & 340 \\
\hline Pentachlorophenol & 840 & $\mathrm{U}$ & 840 & 840 & $\mathrm{U}$ & 840 & 840 & $\mathrm{U}$ & 840 & 840 & $\mathrm{U}$ & 840 \\
\hline Phenanthrene & 340 & $\mathrm{U}$ & 340 & 340 & $\mathrm{U}$ & 340 & 340 & $\mathrm{U}$ & 340 & 340 & U & 340 \\
\hline Phenol & 340 & $\mathrm{U}$ & 340 & 340 & $\mathrm{U}$ & 340 & 340 & $\mathrm{U}$ & 340 & 340 & $\mathrm{U}$ & 340 \\
\hline Pyrene & 340 & $\mathrm{U}$ & 340 & 340 & $\bar{U}$ & 340 & 340 & $\mathrm{U}$ & 340 & 340 & $\mathrm{U}$ & 340 \\
\hline
\end{tabular}

Attachment Originator

Checked

Calc. No.

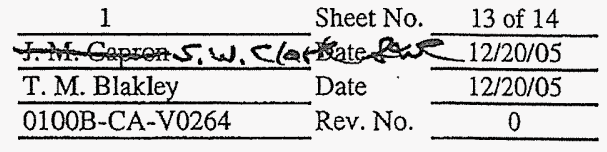


Attachment 1. 1607-B2:1 Verification Sampling Results.

\begin{tabular}{|c|c|c|c|c|c|c|c|c|c|c|c|c|}
\hline \multirow[t]{2}{*}{ Constituent } & \multicolumn{3}{|c|}{$\begin{array}{c}\text { J03VC8 } \\
\text { Sample Location } 11 \\
\text { Sample Date 8/19/05 }\end{array}$} & \multicolumn{3}{|c|}{$\begin{array}{c}\text { J03VC9 } \\
\text { SW Overburden } \\
\text { Sample Date } 8 / 19 / 05\end{array}$} & \multicolumn{3}{|c|}{$\begin{array}{c}\text { J03VD0 } \\
\text { NW Overburden } \\
\text { Sample Date 8/19/05 }\end{array}$} & \multicolumn{3}{|c|}{$\begin{array}{c}\text { J03VD1 } \\
\text { NE Overburden } \\
\text { Sample Date 8/19/05 }\end{array}$} \\
\hline & $\mu \mathrm{g} / \mathrm{kg}$ & $Q$ & PQL & $\mu \mathrm{g} / \mathrm{kg}$ & Q & PQL & $\mu \mathrm{g} / \mathrm{kg}$ & Q & $\mathrm{PQL}$ & $\mu \mathrm{g} / \mathrm{kg}$ & Q & PQL \\
\hline \multicolumn{13}{|c|}{ Volatile Organic Analytes } \\
\hline 1,1,1-Trichloroethane & 5 & UJ & 5 & 5 & $\mathrm{U}$ & 5 & 5 & $\mathrm{U}$ & 5 & 6 & $\mathrm{U}$ & 6 \\
\hline 1,1,2,2-Tetrachloroethane & 5 & UJ & 5 & 5 & $\mathrm{U}$ & 5 & 5 & $\mathrm{U}$ & 5 & 6 & $\mathrm{U}$ & 6 \\
\hline 1,1,2-Trichloroethane & 5 & UJ & 5 & 5 & $\mathrm{U}$ & 5 & 5 & $\mathrm{U}$ & 5 & 6 & $\mathrm{U}$ & 6 \\
\hline 1,1-Dichloroethane & 5 & UJ & 5 & 5 & $\mathrm{U}$ & 5 & 5 & $\mathrm{U}$ & 5 & 6 & $\mathrm{U}$ & 6 \\
\hline 1,1-Dichloroethene & 5 & UJ & 5 & 5 & $\bar{U}$ & 5 & 5 & $\mathrm{U}$ & 5 & 6 & $\mathrm{U}$ & 6 \\
\hline 1,2-Dichloroethane & 5 & \begin{tabular}{|l|} 
UJ \\
\end{tabular} & 5 & 5 & $\mathrm{U}$ & 5 & 5 & $\mathrm{U}$ & 5 & 6 & $\mathrm{U}$ & 6 \\
\hline 1,2-Dichloropropane & 5 & UJ & 5 & 5 & $\mathrm{U}$ & 5 & 5 & $\bar{U}$ & 5 & 6 & $\mathrm{U}$ & 6 \\
\hline 2-Butanone & 10 & \begin{tabular}{|l|l|}
$U J$ \\
\end{tabular} & 10 & 10 & $\mathrm{U}$ & 10 & 10 & $\mathrm{U}$ & 10 & 11 & $\mathrm{U}$ & 11 \\
\hline 2-Hexanone & 10 & UJ & 10 & 10 & $\mathrm{U}$ & 10 & 10 & $U$ & 10 & 11 & $\mathrm{U}$ & 11 \\
\hline 4-Methyl-2-Pentanone & 10 & UJ & 10 & 10 & $\mathrm{U}$ & 10 & 10 & $\bar{U}$ & 10 & 11 & $\mathrm{U}$ & 11 \\
\hline Acetone & 5 & $\mathrm{~J}$ & 10 & 9 & $\mathbf{J}$ & 10 & 6 & $\mathbf{J}$ & 10 & 6 & $\mathbf{J}$ & 11 \\
\hline Benzene & 5 & UJ & 5 & 5 & $\mathrm{U}$ & 5 & 5 & $\mathrm{U}$ & 5 & 6 & $\mathrm{U}$ & 6 \\
\hline Bromodichloromethane & 5 & UJ & 5 & 5 & $\mathrm{U}$ & 5 & 5 & $\mathrm{U}$ & 5 & 6 & $\bar{U}$ & 6 \\
\hline Bromoform & 5 & UJ & 5 & 5 & $\mathrm{U}$ & 5 & 5 & $\mathrm{U}$ & 5 & 6 & $\bar{U}$ & 6 \\
\hline Bromomethane & 10 & UI & 10 & 10 & $\mathrm{U}$ & 10 & 10 & $\mathrm{U}$ & 10 & 11 & $\mathrm{U}$ & 11 \\
\hline Carbon disulfide & 5 & UJ & 5 & 5 & $\mathrm{U}$ & 5 & 5 & $\mathrm{U}$ & 5 & 6 & $\mathrm{U}$ & 6 \\
\hline Carbon tetrachloride & 5 & UI & 5 & 5 & $\mathrm{U}$ & 5 & 5 & $\mathrm{U}$ & 5 & 6 & $\mathrm{U}$ & 6 \\
\hline Chlorobenzene & 5 & UJ & 5 & 5 & $\mathrm{U}$ & 5 & 5 & $U$ & 5 & 6 & $\mathrm{U}$ & 6 \\
\hline Chloroethane & 10 & UJ & 10 & 10 & $\mathrm{U}$ & 10 & 10 & $\mathrm{U}$ & 10 & 11 & $\mathrm{U}$ & 11 \\
\hline \begin{tabular}{|l} 
Chloroform \\
\end{tabular} & 5 & US & 5 & 5 & $\mathrm{U}$ & 5 & 5 & $\mathrm{U}$ & 5 & 6 & $\mathrm{U}$ & 6 \\
\hline Chloromethane & 10 & $\mathrm{UJ}$ & 10 & 10 & $\mathrm{U}$ & 10 & 10 & $\mathrm{U}$ & 10 & 11 & $\mathrm{U}$ & 11 \\
\hline cis-1,2-Dichloroethylene & 5 & UJ & 5 & 5 & $\mathrm{U}$ & 5 & 5 & $\mathrm{U}$ & 5 & 6 & $\mathrm{U}$ & 6 \\
\hline cis-1,3-Dichloropropene & 5 & UJ & 5 & 5 & $\mathrm{U}$ & 5 & 5 & $\mathrm{U}$ & 5 & 6 & $\mathrm{U}$ & 6 \\
\hline Dibromochloromethane & 5 & UJ & 5 & 5 & $\mathrm{U}$ & 5 & 5 & $\mathrm{U}$ & 5 & 6 & $\mathrm{U}$ & 6 \\
\hline Ethylbenzene & 5 & UJ & 5 & 5 & $\mathrm{U}$ & 5 & 5 & $\mathrm{U}$ & 5 & 6 & $\mathrm{U}$ & 6 \\
\hline Methylene chloride & 10 & US & 10 & 11 & $\mathrm{U}$ & 11 & 10 & $\mathrm{U}$ & 10 & 10 & $\mathrm{U}$ & 10 \\
\hline Styrene & 5 & UI & 5 & 5 & $\mathrm{U}$ & 5 & 5 & $\mathrm{U}$ & 5 & 6 & $\mathrm{U}$ & 6 \\
\hline Tetrachloroethene & 5 & UI & 5 & 5 & $\mathrm{U}$ & 5 & 5 & $\mathrm{U}$ & 5 & 6 & U & 6 \\
\hline Toluene & 5 & UJ & 5 & 5 & $\mathrm{U}$ & 5 & 5 & $\mathrm{U}$ & 5 & 6 & $\mathrm{U}$ & 6 \\
\hline trans-1,2-Dichloroethylene & 5 & $\mathrm{UJ}$ & 5 & 5 & $\mathrm{U}$ & 5 & 5 & $\mathrm{U}$ & 5 & 6 & $\mathrm{U}$ & 6 \\
\hline trans-1,3-Dichloropropene & 5 & UJ & 5 & 5 & U & 5 & 5 & $\mathrm{U}$ & 5 & 6 & $\mathrm{U}$ & 6 \\
\hline Trichloroethene & 5 & UJ & 5 & 5 & $\mathrm{U}$ & 5 & 5 & $\mathrm{U}$ & 5 & 6 & $\bar{U}$ & 6 \\
\hline Vinyl chloride & 10 & UJ & 10 & 10 & $\mathrm{U}$ & 10 & 10 & $\mathrm{U}$ & 10 & 11 & U & 11 \\
\hline Xylenes (total) & 5 & \begin{tabular}{|l|}
$\mathrm{UJ}$ \\
\end{tabular} & 5 & 5 & $\mathrm{U}$ & 5 & 5 & $\mathrm{U}$ & 5 & 6 & $\mathrm{U}$ & 6 \\
\hline \multicolumn{13}{|c|}{ Herbicides } \\
\hline $2,4-\mathrm{D}$ & 170 & $\mathrm{U}$ & 170 & 170 & $U$ & 170 & 170 & $\mathrm{U}$ & 170 & 170 & $\mathrm{U}$ & 170 \\
\hline $2,4-\mathrm{DB}$ & 840 & $\mathrm{U}$ & 840 & 840 & $\mathrm{U}$ & 840 & 840 & $\mathrm{U}$ & 840 & 840 & $\mathrm{U}$ & 840 \\
\hline $2,4,5-\mathrm{T}$ & 30 & $\mathrm{~J}$ & 84 & 49 & $\mathrm{~J}$ & 84 & 84 & $\mathrm{UJ}$ & 84 & 30 & $\mathrm{~J}$ & 84 \\
\hline 2,4,5-TP (Silvex) & 84 & UI & 84 & 84 & UJ & 84 & 84 & $\overline{\mathrm{UJ}}$ & 84 & 84 & UI & 84 \\
\hline Dalapon & 840 & $\mathrm{U}$ & 840 & 840 & $\mathrm{U}$ & 840 & 840 & $\mathrm{U}$ & 840 & 840 & $\mathrm{U}$ & 840 \\
\hline Dicamba & 340 & UI & 340 & 340 & UJ & 340 & 340 & $\mathrm{UJ}$ & 340 & 340 & UJ & 340 \\
\hline Dichloroprop & 840 & UJ & 840 & 840 & $\mathrm{UJ}$ & 840 & 840 & UJ & 840 & 840 & UJ & 840 \\
\hline Dinoseb & 84 & $\mathrm{U}$ & 84 & 84 & $\mathrm{U}$ & 84 & 84 & $\mathrm{U}$ & 84 & 84 & $\mathrm{U}$ & 84 \\
\hline
\end{tabular}

Attachment

Originator

Checked

Calc. No.
$15 \cdot \omega . c(\operatorname{ark} s$ Sheet No.

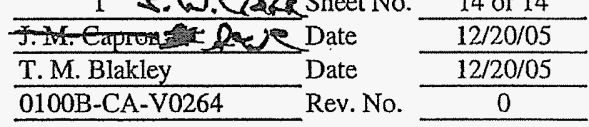




\section{CALCULATION COVER SHEET}

Project Title 100-B/C Field Remediation Project

Job No.

14655

Area $100-B / C$

Discipline Environmental $*$ Calc. No. 0100B-CA-V0265

Subject 1607-B2:1 Drain Field Hazard Quotient and Carcinogenic Risk Calculations

Computer Program Excel_Program No. Excel 2003

The attached calculations have been generated to document compliance with established cleanup levels. These documents should be used in conjunction with other relevant documents in the administrative record.

Committed Calculation $\otimes \quad$ Preliminary $\square \quad$ Superseded $\square \quad$ Voided $\square$

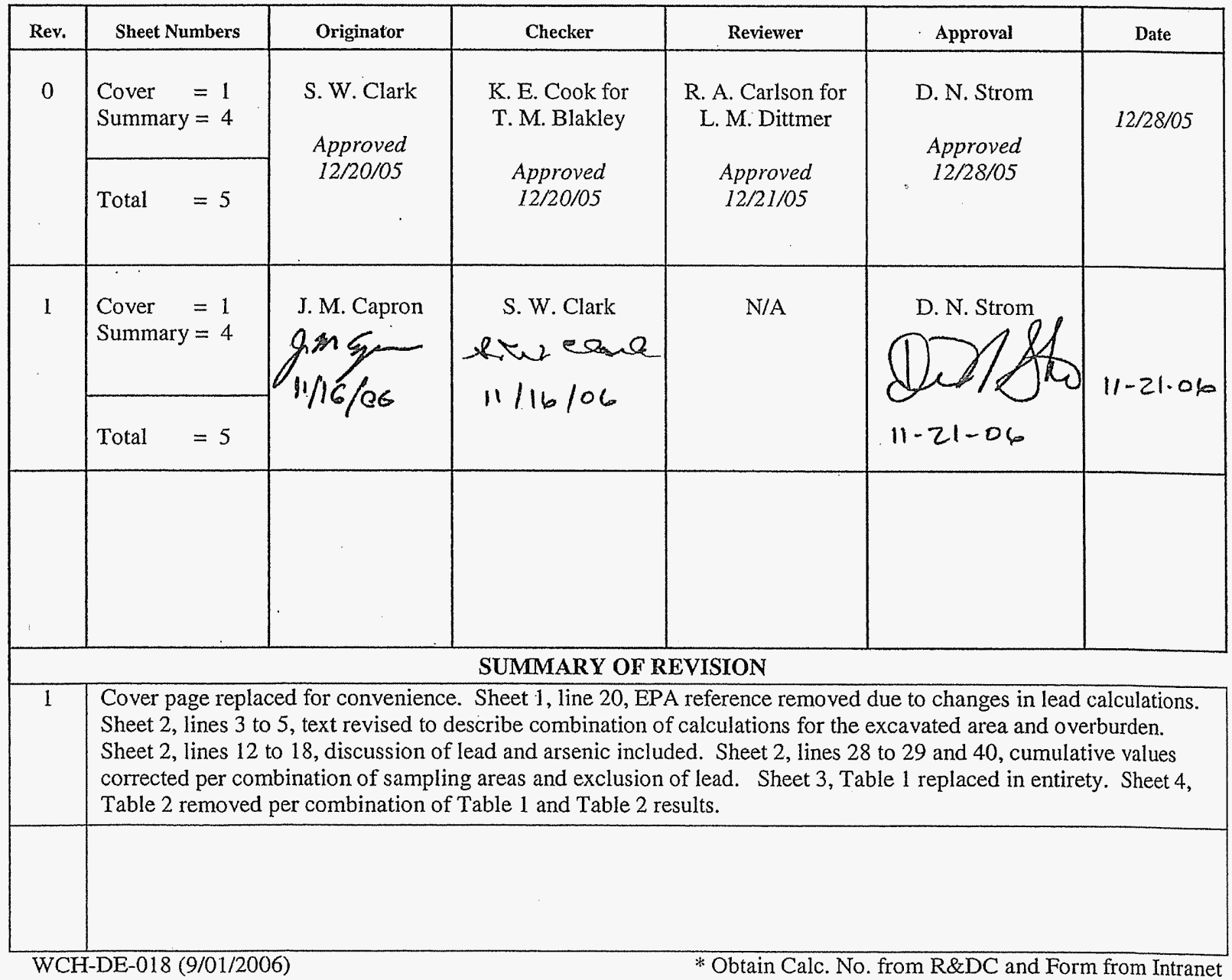


Washington Closure Hanford CALCULATION SHEET

\begin{tabular}{|c|c|c|c|c|c|c|c|}
\hline Originator: & J. M. Capron $Q 2$ & Date: & $11 / 16 / 06$ & Calc. No.: & 0100B-CA-V0265 & Rev.: & 1 \\
\hline Project: & 100-B/C Field Remediation & Job No: & 14655 & Checked: & S. W. Clark Rwke & Date: & $11 / 16 / 06$ \\
\hline
\end{tabular}

\section{PURPOSE:}

Provide documentation to support the calculation of the hazard quotient (HQ) and carcinogenic (excess cancer) risk values for the 1607-B2:1 drain field remedial action. In accordance with the remedial action goals (RAGs) in the remedial design report/remedial action work plan (RDR/RAWP) (DOE-RL 2005), the following criteria must be met:

1) An $\mathrm{HQ}$ of $<1.0$ for all individual noncarcinogens

2) A cumulative HQ of $<1.0$ for noncarcinogens

3) An excess cancer risk of $<1 \times 10^{-6}$ for individual carcinogens

4) A cumulative excess cancer risk of $<1 \times 10^{-5}$ for carcinogens.

\section{GIVEN/REFERENCES:}

1) DOE-RL, 2005, Remedial Design Report/Remedial Action Work Plan for the 100 Areas, DOE/RL-96-17, Rev. 5, U.S. Department of Energy, Richland Operations Office, Richland, Washington.

2) WAC 173-340, "Model Toxics Control Act - Cleanup," Washington Administrative Code, 1996.

3) WCH, 2005, 1607-B2:1 Drain Field Cleanup Verification 95\% UCL Calculations, 0100B-CA-V0264, Rev. 0, Washington Closure Hanford, Richland, Washington.

\section{SOLUTION:}

1) Calculate an HQ for each noncarcinogenic constituent detected above background or required detection limit/practical quantitation limit and compare it to the individual HQ of $<1.0$ (DOE-RL 2005).

2) Sum the HQs and compare to the cumulative HQ criterion of $<1.0$.

3) Calculate an excess cancer risk value for each carcinogenic constituent detected above background or required detection limit/practical quantitation limit and compare it to the individual excess cancer risk criterion of $<1 \times 10^{-6}$ (DOE-RL 2005).

4) Sum the excess cancer risk values and compare to the cumulative cancer risk criterion of $<1 \times 10^{-5}$. 
Washington Closure Hanford CALCULATION SHEET

\begin{tabular}{r|l|r|c|r|l|} 
Originator: & J. M. Capron q-zese & Date: & $11 / 16 / 06$ & Calc. No.: & $0100 B-C A-V 0265$ \\
\hline Project: & $100-B / C$ Field Remediation & Job No: & 14655 & Checked: & S. W. Clark \&we
\end{tabular}

Subject: $1607-B 2: 1$ Drain Field Hazard Quotient and Carcinogenic Risk Calculations

\section{METHODOLOGY:}

Hazard quotient and carcinogenic risk calculations were computed for the 1607-B2:1 site using the higher of the remediation footprint statistical value and overburden material maximum value for each analyte detected above background using the data from WCH (2005). Of the contaminants of potential concern for the site, boron and molybdenum require the HQ and risk calculations because these analytes were detected and a Washington State or Hanford Site background value is not available. Multiple additional metals are included for the excavated area (as shown in Table 1) because the statistical values for these constituents were above their respective Washington State or Hanford Site background values within those sampling areas. Hexavalent chromium, acetone, multiple pesticides, herbicides, and semivolatile organic analytes (as shown in Tables 1 and 2, below) are included because they were detected by laboratory analysis and cannot be attributed to natural occurrence. Lead does not have a reference dose for calculation of a hazard quotient because toxic effects of lead are correlated with blood-lead levels rather than exposure levels or daily intake. As a result, the maximum lead concentration is reported but not included in the hazard quotient calculation. Arsenic was detected above the Hanford Site Background value but below the WAC 173-340 Method A cleanup level. Due to the intent of Method A cleanup values and the allowance to use such values for arsenic (DOE-RL 2005), arsenic has been excluded from the Method B individual analyte and cumulative risk requirements. An example of the HQ and risk calculations is presented below:

1) For example, the statistical value for boron in the excavation sampling area is $8.3 \mathrm{mg} / \mathrm{kg}$, divided by the noncarcinogenic RAG value of $16,000 \mathrm{mg} / \mathrm{kg}$ (boron is identified as a noncarcinogen in WAC 173-340-740[3]), is $5.2 \times 10^{-4}$. Comparing this value, and all other individual values, to the requirement of $<1.0$, this criterion is met.

2) After the HQ calculations are completed for the appropriate analytes, the cumulative HQ is obtained by summing the individual values. (To avoid errors due to intermediate rounding, the individual HQ values prior to rounding are used for this calculation.) The sums of the HQ values is $1.3 \times 10^{-1}$. Comparing this value to the requirement of $<1.0$, this criterion is met.

3) To calculate the excess cancer risk, the statistical or maximum value is divided by the carcinogenic RAG value, then multiplied by $1 \times 10^{-6}$. For example, the maximum value for hexavalent chromium in the excavation sampling area is $0.33 \mathrm{mg} / \mathrm{kg}$; divided by $2.1 \mathrm{mg} / \mathrm{kg}$ and multiplied as indicated is $1.6 \times 10^{-7}$. Comparing this value, and all other individual values, to the requirement of $<1 \times 10^{-6}$, this criterion is met.

4) After these calculations are completed for the carcinogenic analytes, the cumulative excess cancer risk can be obtained by summing the individual values. (To avoid errors due to intermediate rounding, the individual excess carcinogenic risk values prior to rounding are used for this calculation.) The sum of the excess cancer risk values is $2.0 \times 10^{-7}$. Comparing this value to the requirement of $<1 \times 10^{-5}$, this criterion is met.

\section{RESULTS:}

1) List individual noncarcinogens and corresponding $\mathrm{HQs}>1.0$ : None

2) List the cumulative noncarcinogenic HQ $>1.0$ : None 
Washington Closure Hanford

CALCULATION SHEET

\begin{tabular}{l|c|c|c|} 
Date: & $11 / 16 / 06$ & Calc. No.: & 0100B-CA-V0265 \\
\hline
\end{tabular}

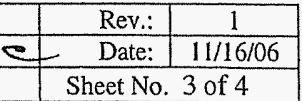

Subject: $1607-\mathrm{B} 2: 1$ Drain Field Hazard Quotient and Carcinogenic Risk Calculations

3) List individual carcinogens and corresponding excess cancer risk $>1 \times 10^{-6}$ : None

4) List the cumulative excess cancer risk for carcinogens $>1 \times 10^{-5}$ : None.

Table 1 shows the results of the calculations for the 1607-B2:1 subsite.

Table 1. Hazard Quotient and Excess Cancer Risk Results for the 1607-B2:1 Subsite.

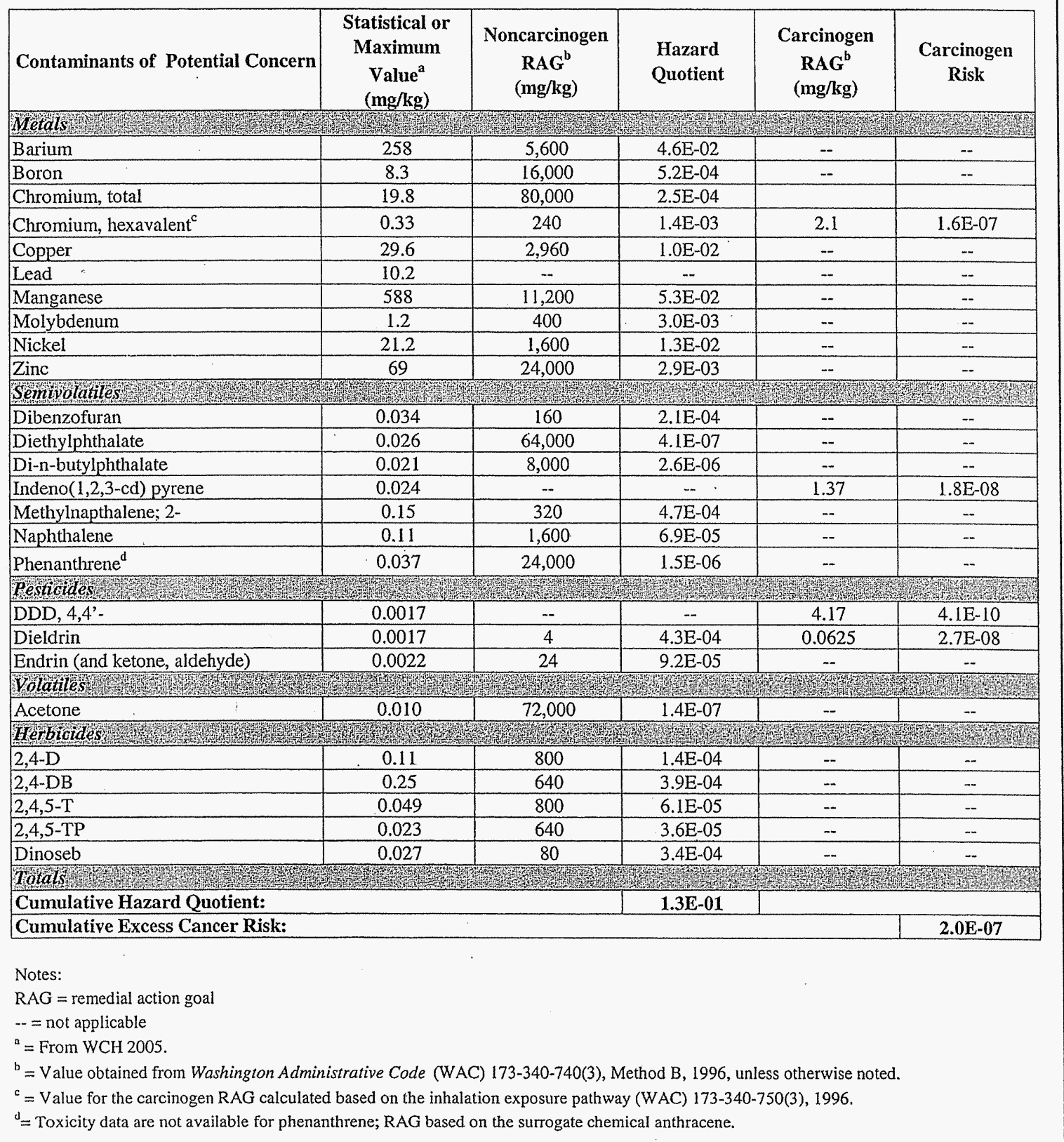


Washington Closure Hanford CALCULATION SHEET

Originator: J.M. Capron $2 \approx$ Date: $\mid 11 / 16 / 06$

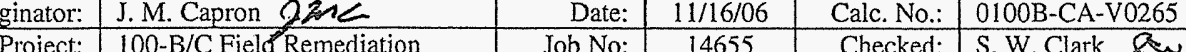

Subject: $1607-\mathrm{B} 2: 1$ Drain Field Hazard Quotient and Carcinogenic Risk Calculations

W. Clark \&ue

\begin{tabular}{c|c|} 
Rev.: & 1 \\
\hline Date: & $11 / 16 / 06$ \\
\hline
\end{tabular}

Sheet No. 4 of 4

\section{CONCLUSION:}

This calculation demonstrates that the 1607-B2:1 drain field waste site meets the requirements for the hazard quotients and carcinogenic (excess cancer) risk as identified in the RDR/RAWP (DOE-RL 2005). 


\section{CALCULATION COVER SHEET}

Project Title:

Area

Discipline

Subject

Computer Program
100-B/C Remaining Pipes and Sewers Field Remediation

$$
100-\mathrm{B} / \mathrm{C}
$$

Environmental

Calc. No. 0100B-CA-V0292

1607-B2:2 Waste Site Cleanup Verification 95\% UCL Calculations

Excel

Program No. Excel 2003

The attached calculations have been generated to document compliance with established cleanup levels. These calculations should be used in conjunction with other relevant documents in the administrative record.

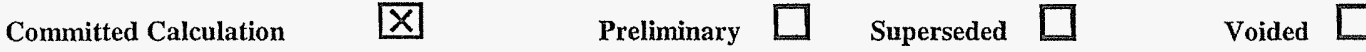

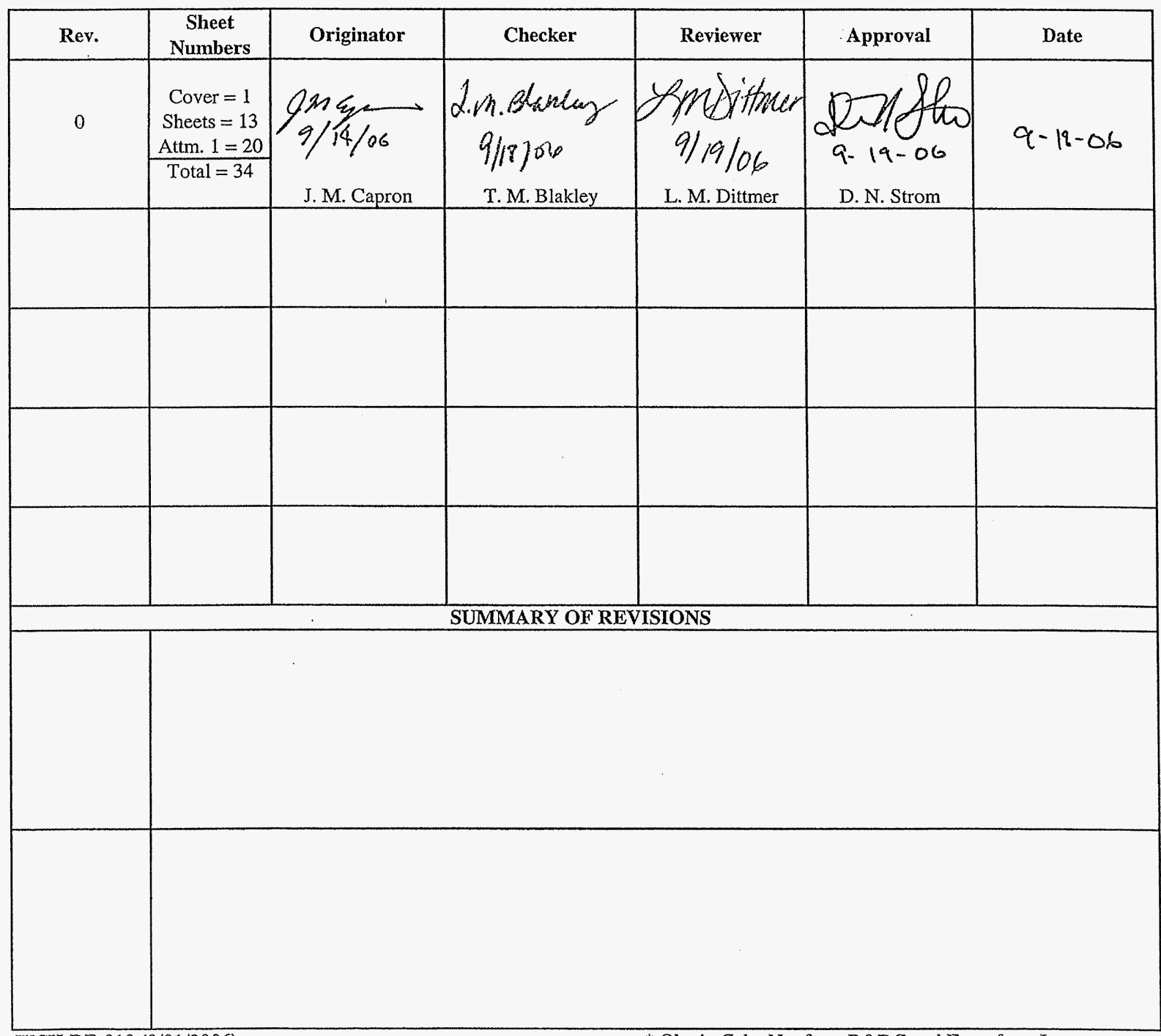




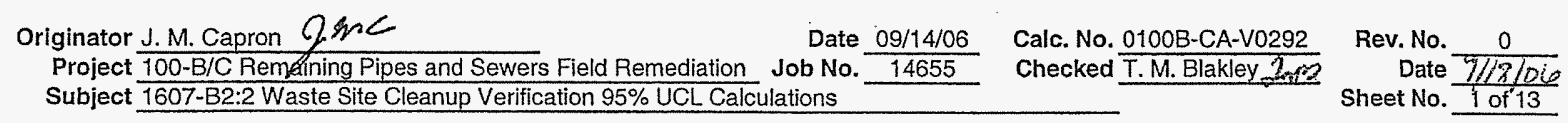

Summary

\section{Purpose:}

Calculate the $95 \%$ upper confidence limit (UCL) values to evaluate compliance with cleanup standards for the remediation footprint of the 1607-B2:2 subsite. Also, perform the Washington Administrative Code (WAC) 173-340-740(7)(e) 3-part test for each

nonradioactive contaminant of concern $(\mathrm{COC})$ and contaminant of potential concern (COPC) and calculate the relative percent

difference (RPD) for primary-duplicate sample pairs, as necessary.

\section{Table of Contents:}

Sheets 1 to 4 - Calculation Sheet Summary

Sheets 5 to 7 - Calculation Sheet 1607-B2:2 Remediation Footprint Verification Data

Sheet 8 - Calculation Sheet Duplicate Analysis

Sheets 9 to 13 - Ecology Software (MTCAStat) Results

Attachment 1 - 1607-B2:2 Verification Sampling Results (20 sheets)

Given/References:

1) Sample Results (Attachment 1).

2) Background values and remedial action goals (RAGs) are taken from DOE-RL (2005b), DOE-RL (2001), Ecology (1994), and Ecology (2005).

3) DOE-RL, 2001, Hanford Site Background: Part 1, Soil Background for Nonradioactive Analytes, DOE/RL-92-24, Rev. 4, U.S. Department of Energy, Richland Operations Office, Richland, Washington.

4) DOE-RL, 2005a, 100 Area Remedial Action Sampling and Analysis Plan (SAP), DOE/RL-96-22, Rev. 4, U.S. Department of Energy, Richland Operations Office, Richland, Washington.

5) DOE-RL, 2005b, Remedial Design Report/Remedial Action Work Plan for the 100 Area (RDR/RAWP), DOE/RL-96-17, Rev. 5, U.S. Department of Energy, Richland Operations Office, Richland, Washington.

6) Ecology, 1992, Statistical Guidance for Ecology Site Managers, Publication \#92-54, Washington Department of Ecology, Olympia, Washington.

7) Ecology, 1993, Statistical Guidance for Ecology Site Managers, Supplement S-6, Analyzing Site or Background Data with Below-detection Limit or Below-PQL Values (Censored Data Sets), Publication.\#92-54, Washington Department of Ecology, Olympia, Washington.

8) Ecology, 1994, Natural Background Soil Metals Concentrations in Washington State, Publication No. 94-115, Washington State Department of Ecology, Olympia, Washington.

9) Ecology, 2005, Cleanup Levels and Risk Calculations (CLARC) Database, Washington State Department of Ecology, Olympia, Washington, <https://fortress.wa.gov/ecy/clarc/CLARCHome.aspx>.

10) EPA, 1994, USEPA Contract Laboratory Program National Functional Guidelines for Inorganic Data Review, EPA 540/R-94/013. U.S. Environmental Protection Agency, Washington, D.C.

11) WAC 173-340, 1996, "Model Toxic Control Act - Cleanup," Washington Administrative Code.

Solution:

Calculation methodology is described in Ecology Pub. \#92-54 (Ecology 1992, 1993), below, and in the RDR/RAWP (DOE-RL

$2005 \mathrm{~b})$. Use data from attached worksheets to perform the $95 \%$ UCL calculation for each analyte, the

WAC 173-340-740(7)(e) 3-part test for nonradionuclides, and the RPD calculations, as required. The hazard quotient and
carcinogenic risk calculations are located in a separate calculation brief as an appendix to the Remaining Sites Verification

Package (RSVP).

\section{Calculation Description:}

The subject calculations were performed on data from soil verification samples from the subject waste site. The data were entered into an EXCEL 2003 spreadsheet and calculations performed by using the built-in spreadsheet functions and/or creating formulae within the cells. The statistical evaluation of data for use in accordance with the RDR/RAWP (DOE-RL 2005b) is documented by this calculation. Duplicate RPD results are used in evaluation of data quality within the RSVP for this site.

\section{Methodology:}

For nonradioactive analytes with $\leq 50 \%$ of the data below detection limits and detected radionuclide analytes, the statistical value calculated to evaluate the effectiveness of cleanup is the $95 \%$ UCL. For nonradioactive analytes with $>50 \%$ of the data below detection limits, the maximum detected value for the data set is used instead of the $95 \%$ UCL. The $95 \%$ UCL is not calculated for data sets with no reported detections. The evaluation of the portion of each analyte's data set below detection limits was performed by direct inspection of the attached sample results, and no further calculations were performed for those nonradionuclide data sets where $>50 \%$ of the data was below detection limits or radionuclide data sets with no reported detections. The $95 \%$ UCL values were not calculated for aluminum, calcium, iron, magnesium, phosphate, potassium, silicon, sodium, and zirconium, as no cleanup values are available in Ecology (2005) under WAC 173-340-740(3), and these constituents are thus 


\section{CALCULATION SHEET}

\section{Washington Closure Hanford}

Originator J. M. Capron 9 .

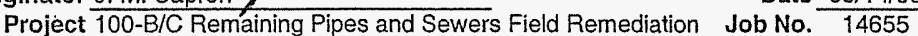

Subject 1607-B2:2 Waste Site Cleanup Verification 95\% UCL Calculations

alc. No. 0100B-CA-V0292 Checked T.M. Blakley 2 nom
Rev. No.

Date $\overline{9 / 18 / 06}$

Sheet No. 2 of 13

Summary (continued)

1 not considered site COPCs (results for total phosphorus are attributed to phosphorus in phosphate). The $95 \%$ UCL values were

also not calculated for potassium-40, radium-226, radium-228, thorium-228, and thorium-232, as these radionuclides are not

related to the site history and are excluded from consideration as COCs/COPCs (DOE-RL 2005a).

All nonradionuclide data reported as being undetected are set to $1 / 2$ the detection limit value for calculation of the statistics (Ecology 1993). For radionuclide data, calculation of the statistics was done on the reported value. In cases where the laboratory does not report a value below the minimum detectable activity (MDA), half of the MDA is used in the calculation. For the statistical evaluation of duplicate sample pairs, the samples are averaged before being included in the data set, after adjustments for censored data as described above.

For nonradionuclides, the WAC 173-340 statistical guidance suggests that a test for distributional form be performed on the data and the $95 \%$ UCL calculated on the appropriate distribution using Ecology software. For nonradionuclide small data sets ( $\mathrm{n}<10$ ) and all radionuclide data sets, the calculations are performed assuming nonparametric distribution, so no tests for distribution are performed. For nonradionuclide data sets of ten or greater, as for the subject site, distributional testing and calculation of the 95\% UCL is done using Ecology's MTCAStat software (Ecology 1993). Due to differences in addressing censored data between the RDR/RAWP (DOE-RL 2005b) and MTCAStat coding and due to a limitation in the MTCAStat coding (no direct capability to address variable quantitation limits within a data set), substitutions for censored data are performed before software input and the resulting input set treated as uncensored.

The WAC 173-340-740(7)(e) 3-part test is performed for nonradionuclide analytes only and determines if:

1) the $95 \%$ UCL exceeds the most stringent cleanup limit for each COPC/COC,

2) greater than $10 \%$ of the raw data exceed the most stringent cleanup limit for each COPC/COC,

3) the maximum value of the raw data set exceeds two times the most stringent cleanup limit for each COPC/COC.

The WAC 173-340-740(7)(e) 3-part test is not performed for COPCs/COCs where the statistical value defaults to the maximum value in the data set. Instead, direct comparison of the maximum value against site RAGs (within the RSVP) is used as the compliance basis.

The RPD is calculated when both the primary value and the duplicate value for a given analyte are above detection limits and are greater than 5 times the target detection limit (TDL). The TDL is a laboratory detection limit pre-determined for each analytical method, listed in Table II-1 of the SAP (DOE-RL 2005a). Where direct evaluation of the attached sample data showed that a given analyte was not detected in the primary and duplicate sample, further evaluation of the RPD value was not performed. The RPD calculations use the following formula:

$$
R P D=[|M-S| /((M+S) / 2)]^{*} 100
$$

$$
\text { where, } \quad M=\text { main sample value } \quad S=\text { split (or duplicate) sample value }
$$

For quality assurance/quality control (QAQC) split and duplicate RPD calculations, a value less than $30 \%$ indicates the data compare favorably. For regulatory splits, a threshold of $35 \%$ is used (EPA 1994). If the RPD is greater than $30 \%$ (or $35 \%$ for regulatory split data), further investigation regarding the usability of the data is performed. No split samples were collected for cleanup verification of the subject site. As a matter of good practice, when an analyte is detected in the primary or duplicate sample, but was quantified at less than 5 times the TDL in one or both samples, an additional parameter is evaluated. In this case, if the difference between the primary and duplicate results exceeds a control limit of 2 times the TDL, further assessment regarding the usability of the data is performed. No split samples were collected for cleanup verification of the subject site. Additional discussion is provided in the data quality assessment section of the applicable RSVP, as necessary.

In addition to the statistical samples collected from the remediation footprint at the subject site, multi-aliquot samples were collected from stockpiles of overburden and other material assumed to be below cleanup levels. Statistical methodology is not applicable to non-statistical sampling, and direct evaluation of maximum detected values within these decision units will be used as the compliance basis. These maximum detected values are presented in the results summary for use in the RSVP. 


\section{CALCULATION SHEET}

Washington Closure Hanford

Originator J.M. Capron g.me Date 09/14/06 Job No. 14655 Project 100-B/C Remaining Pipes and Sewers Field Remediation Subject 1607-B2:2 Waste Site Cleanup Verification 95\% UCL Calculations 1 Summary (continued)

2 Results:

3 The results presented in the summary tables that follow are for use in risk analysis and the RSVP for this site.

\section{Results Summary - Remediation Footprint .}

7 \begin{tabular}{r} 
An \\
\cline { 2 - 2 }
\end{tabular}

8 Strontium-90

9 Antimon

11 Barium

12 Beryllium

13 Boron

14 Cadmium

15 Chromium

16 Cobalt

17 Copper

18 Hexavalent Chromium

19 Lead

20 Lithium

21 Manganese

22 Mercury

23 Molybdenum

24 Nickel

25 Strontium

26 Titanium

27 Vanadium

28 Zinc

29 Aroclor-1254

30 Aroclor-1260

31 alpha-Chlordane

32 beta-BHC

33 4,4'-DDE

34 4,4'-DDT

35 Endosulfan

36 Endosulfan II

37 Endosulfan sulfat

38 Endrin aldehyde

39 Endrin ketone

40 gamma-Chlordane

41 Heptachlor epoxide

42 Methoxychlor

43 2-Methylnaphthalen

44 Benzo(a)anthracene

45 Benzo(a)pyrene

46 Benzo(b)fluoranthene

47 Benzo $(g, h, i)$ perylene

48 Benzo(k)fluoranthene

49 bis(2-Ethylhexyl)phthalate

50 Chrysene

51 Di-n-butylphthalate

52 Dibenz $(a, h)$ anthracene

53 Fluoranthene

54 Indeno(1,2,3-cd)pyrene

55 Naphthalene

56 Phenanthrene

57 Phenol

58 Pyrene

59 WAC 173-340-740(7)(e) Evaluation

61 WAC 173-340 3-Part Test for most stringent RAG:

62 95\% UCL > Cleanup Limit? YES

$63>10 \%$ above Cleanup Limit?

64 Any sample $>2 x$ Cleanup Limit?

NO

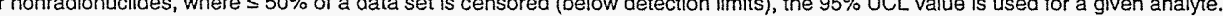

66 bor nonradionuclides, where $>50 \%$ of a data set is censored, the statistical value defaults to the maximum detected value in the

$67 \mathrm{RAG}=$ remedial action goal

Barium

Copper

Lead

Lithium

Manganese

Mercury

Molybdenum

Nickel

Strontium

Titanium

anadium

Aroclor-1254

Aroclor-1260

4,4'-DDE

4,4'-DDT

Endrin aldehyde

Benzo(b)fluoranthene

bis(2-Ethylhexyl)phthaiate

Di-n-butylphthalat

Diethylphthalate

Fluoranthene

Phenol

Pyrene

statistical, sampling.

$\mathrm{BCL}=$ below cleanup levels
Calc. No. 0100B-CA-V0292

Checked T.M. Blakley Ims Rev. No. $\frac{0}{\text { Date } \frac{9 / 6 / 66}{306}}$
Sheet No. 3 of 93

Results Summary - BCL Stockpiles

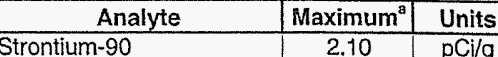

\begin{tabular}{|l|c|c|}
\hline Antimony & 2.10 & $\mathrm{pCi} / \mathrm{g}$ \\
\hline Arsenic & 0.47 & $\mathrm{mg} / \mathrm{kg}$ \\
\hline
\end{tabular}

\begin{tabular}{|c|c|c|}
\hline Arsenic & 0.47 & $\mathrm{mg} / \mathrm{kg}$ \\
\hline
\end{tabular}

Beryllium

\begin{tabular}{|c|c|c|}
\hline Boron & 0.39 & $\mathrm{mg} / \mathrm{kg}$ \\
\hline
\end{tabular}

\begin{tabular}{l|c|c|}
\hline Cadmium & 0.1 & $\mathrm{mg} / \mathrm{kg}$
\end{tabular}

\begin{tabular}{|l|c|c|}
\hline Chromium & 12.8 & $\mathrm{mg} / \mathrm{kg}$ \\
\hline
\end{tabular}

\begin{tabular}{|l|c|c|}
\hline Cobalt & 8.2 & $\mathrm{mg} / \mathrm{kg}$ \\
\hline
\end{tabular}

\begin{tabular}{|l|c|c|}
\hline Hexavalent chromium & 16.0 & $\mathrm{mg} / \mathrm{kg}$ \\
\hline
\end{tabular}

\begin{tabular}{l|l}
.28 & $\mathrm{mg} / \mathrm{kg}$ \\
\hline 8.9 & $\mathrm{mg} / \mathrm{kg}$ \\
\hline 7.7 & $\mathrm{mg} / \mathrm{kg}$
\end{tabular}

was based on multi-aliquot, rather than 
Washington Closure Hanford

CALCULATION SHEET

Originator J.M. Capron \& \& C Date 09/14/06 Job No. 14655 Project $100-\mathrm{B} / \mathrm{C}$ Rerhaining Pipes and Sewers Field Remediation Subject 1607-B2:2 Waste Site Cleanup Verification 95\% UCL Calculations
Calc. No. 0100B-CA-V0292

Checked T.M. Blakley $2 \mathrm{~m} / \mathrm{B}$
Rev. No.

Date $\frac{0}{5101}$

Sheet No. $\frac{1}{4 \text { of } 13}$

\section{Summary (continued)}

\begin{tabular}{|c|c|c|c|c|}
\hline 3 & Analyte & $\begin{array}{l}\text { Duplicate } \\
\text { Analysis }^{b}\end{array}$ & Analyte & Duplicate Analysis ${ }^{b}$ \\
\hline 4 & Potassium-40 & $58 \%$ & Manganese & $12 \%$ \\
\hline 5 & Aluminum & $0.75 \%$ & Phosphorus & $8.5 \%$ \\
\hline 6 & Barium & $14 \%$ & Silicon & $19 \%$ \\
\hline 7 & Calcium & $3.6 \%$ & Strontium & $10 \%$ \\
\hline 8 & Chromium & $4.1 \%$ & Titanium & $16 \%$ \\
\hline 9 & Copper & $3.6 \%$ & Vanadium & $14 \%$ \\
\hline 10 & Iron & $3.0 \%$ & Zinc & $2.6 \%$ \\
\hline & Magnesium & $5.2 \%$ & Zirconium & $2.7 \%$ \\
\hline
\end{tabular}

12 Quantitated results for radium-226 and radium-228 in both the primary and duplicate

13 field samples did not exceed the relative percent difference quality control threshold

14 of 5XTDL, but the difference between the results exceeded the 2XTDL control

15 threshold.

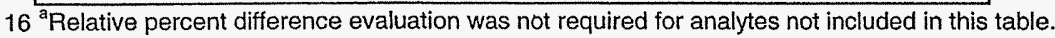

$17^{\mathrm{b}}$ The significance of relative percent difference values are discussed within the RSVP for the subject site.

$18 \mathrm{QAQC}=$ quality assurance/quality control

19 RSVP $=$ remaining sites verification package

$20 \mathrm{TDL}=$ target detection limit 
Originator J. M. Capron \me

CALCULATION SHEET

Project 100-B/C Renfaining Pipes and Sewers Field Remediation

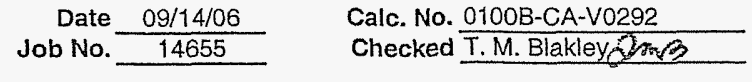

Rev. No. $\frac{0}{\text { Date }} \frac{9 / 18 / 06}{5 / 13}$

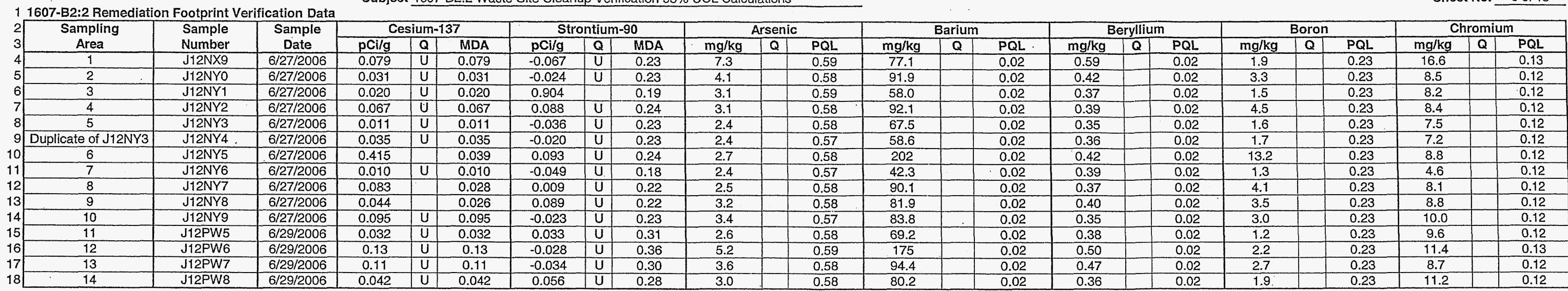
19 Statistical Computation Input Data
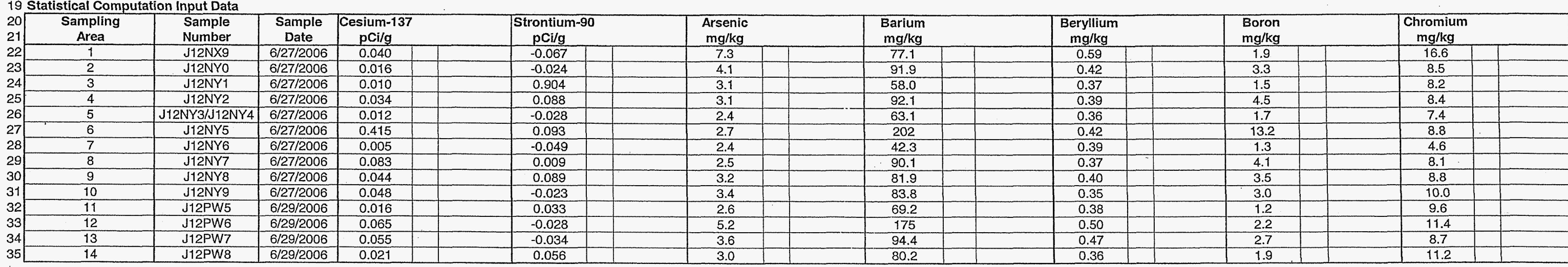

36 Statistical Compr

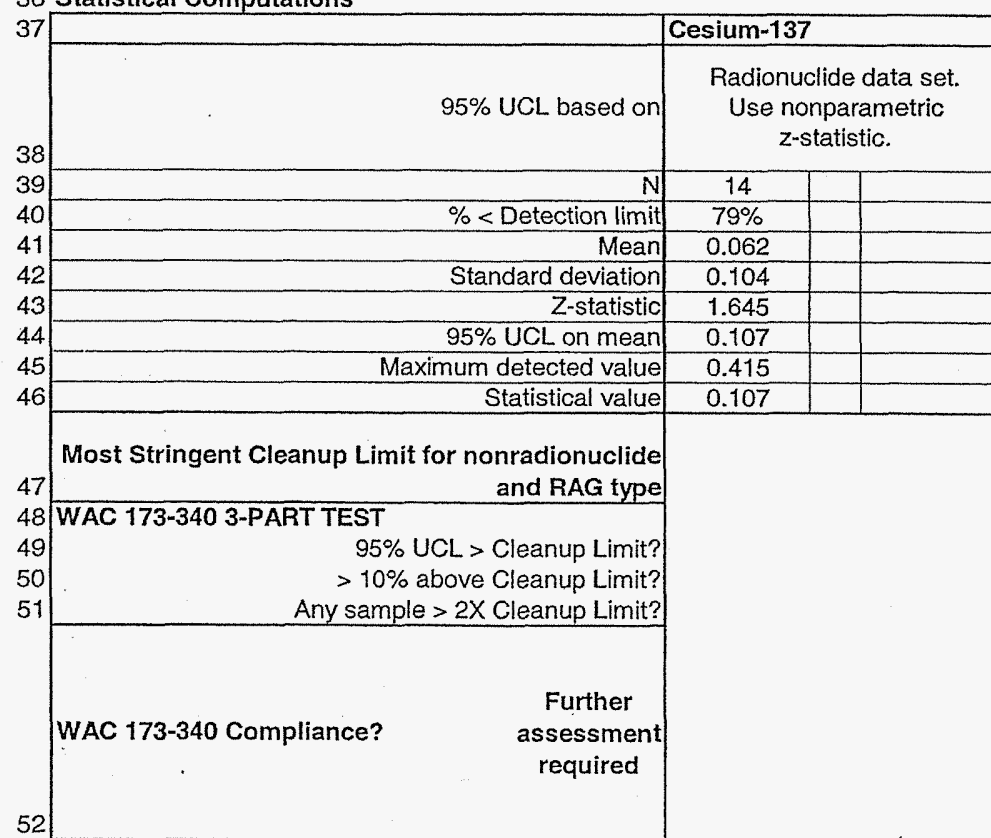

53 "Calculation of $95 \%$ UCL tor nonradionuclides pertormed using MTCAStat softwart.

$55 \mathrm{BG}=$ background
$55 \mathrm{GW}=$ groundwater

NA = not applicabl
POL

$=$ qualifier

$55 \mathrm{GW}=$ groundwate
$56 \mathrm{MDA}=$ minimum RAG $=$ remedial action goal
RESAD $=$ REESidual RADioad
$U=$ undetected
Strontium-90

Arsenic

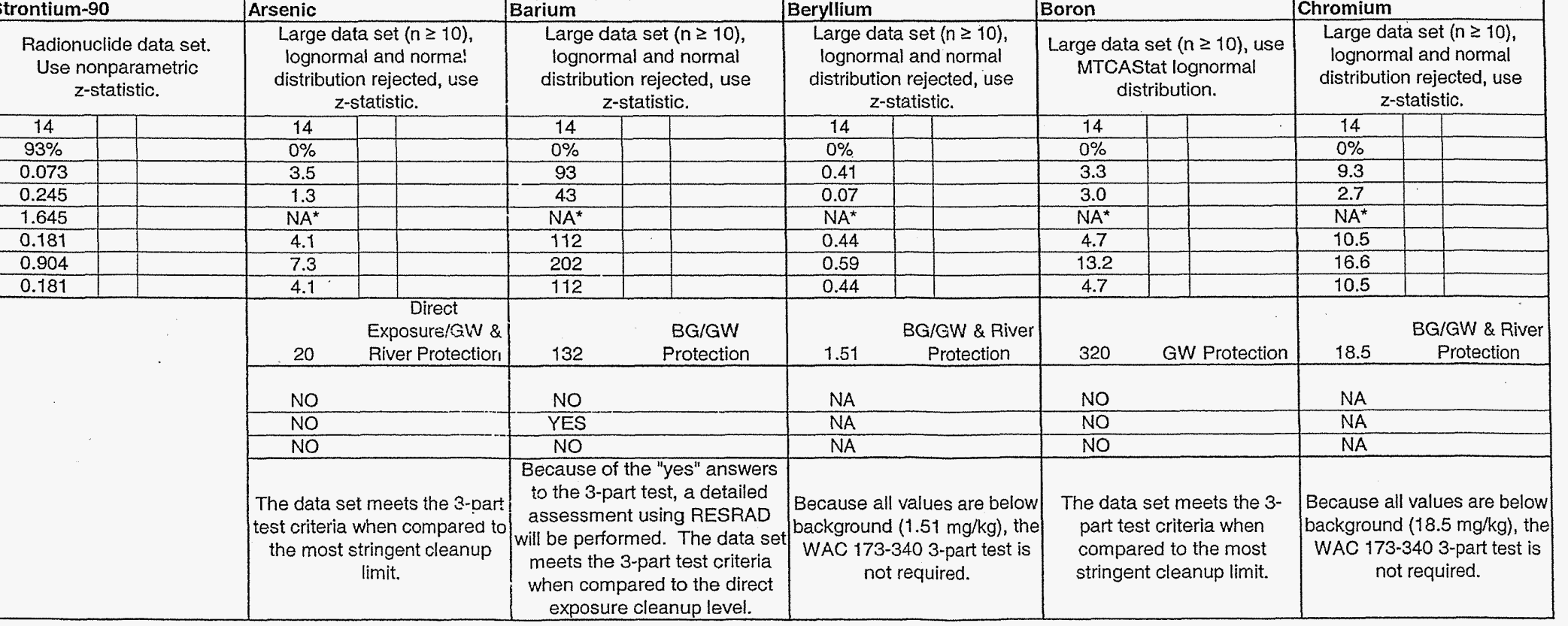

$$
\begin{aligned}
& \text { UCL = upper confidence limit } \\
& \text { WAC = Washington Administrative Code }
\end{aligned}
$$


CALCULATION SHEET

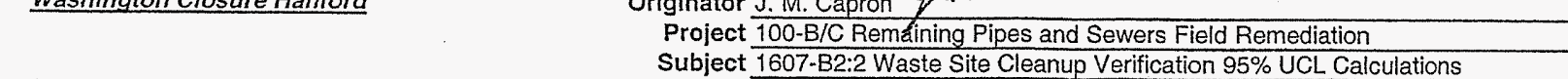

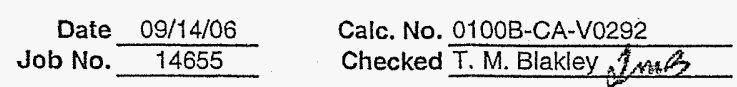

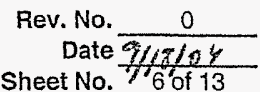

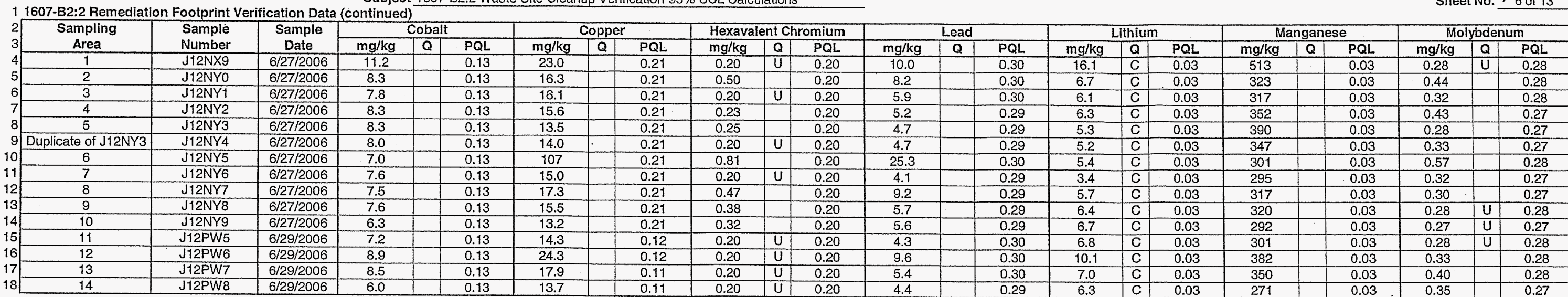

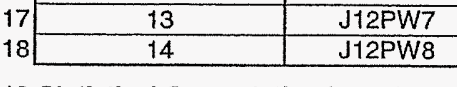

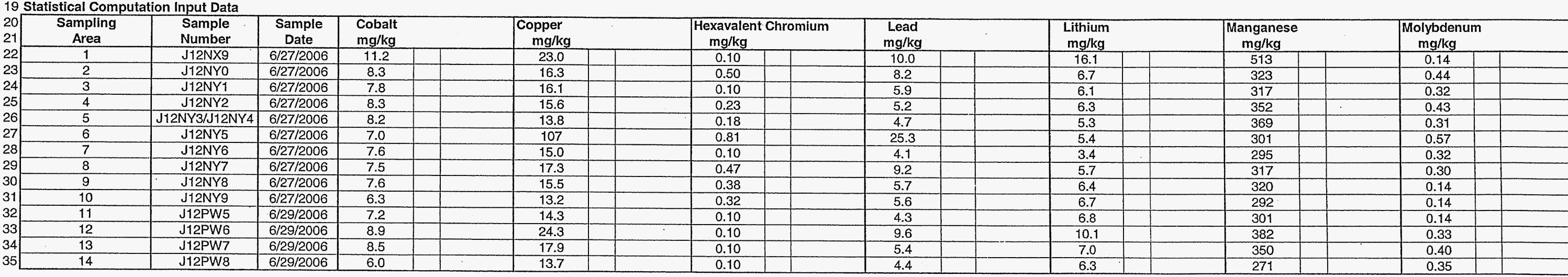

36 Statistical Computations

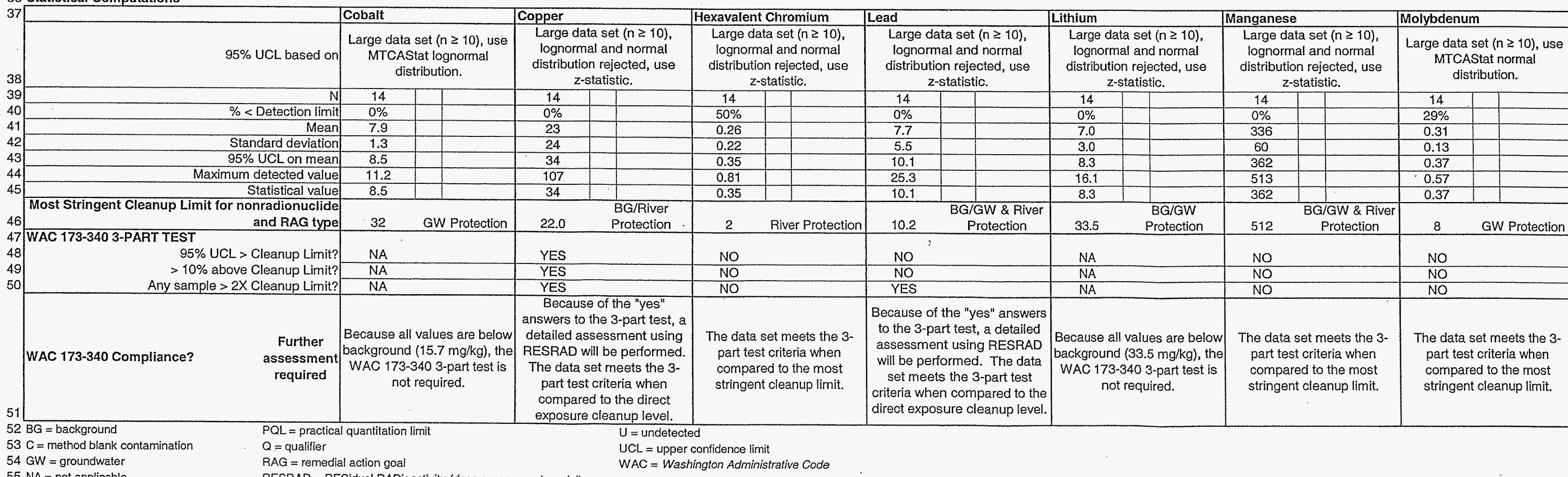


Washington Closure Hanford

originator J. M. Capron $\ell^{\text {menC }}$

CALCULATION SHEET

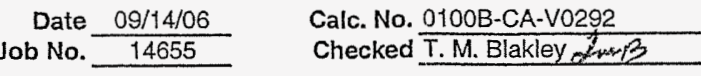

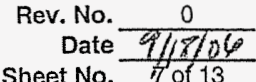
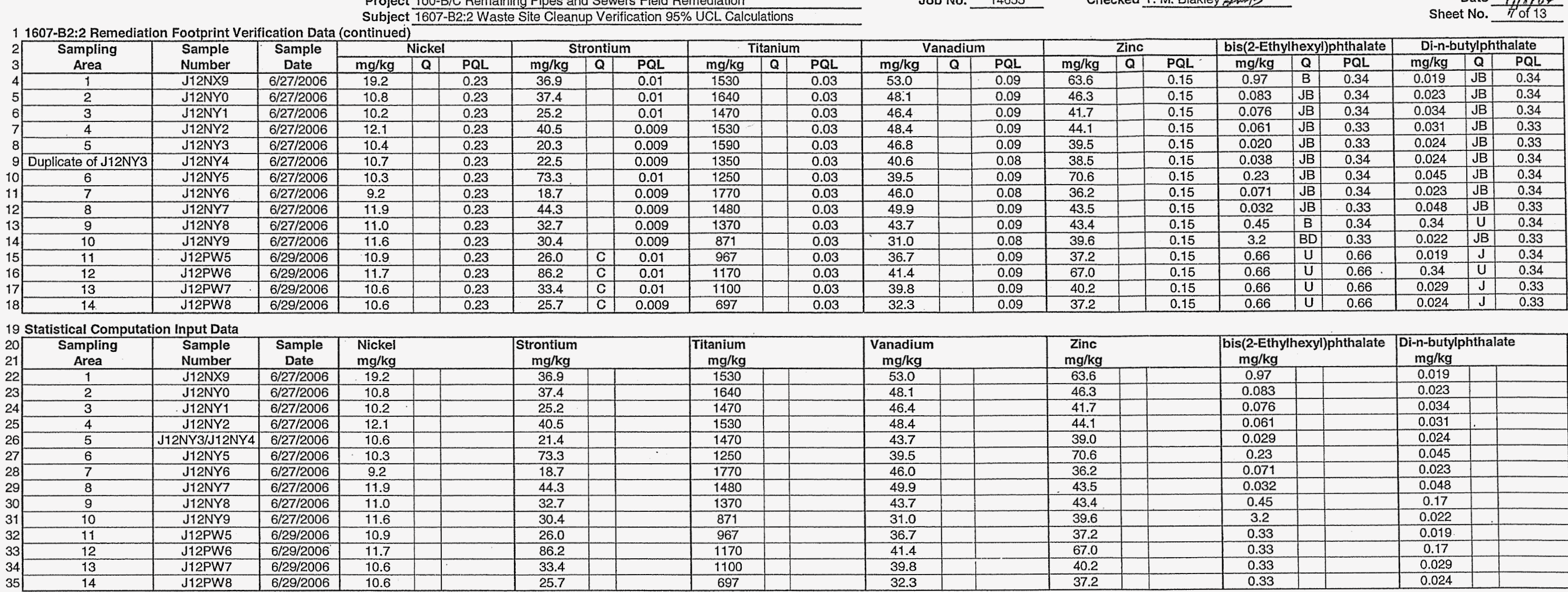

36 Statistical Computations

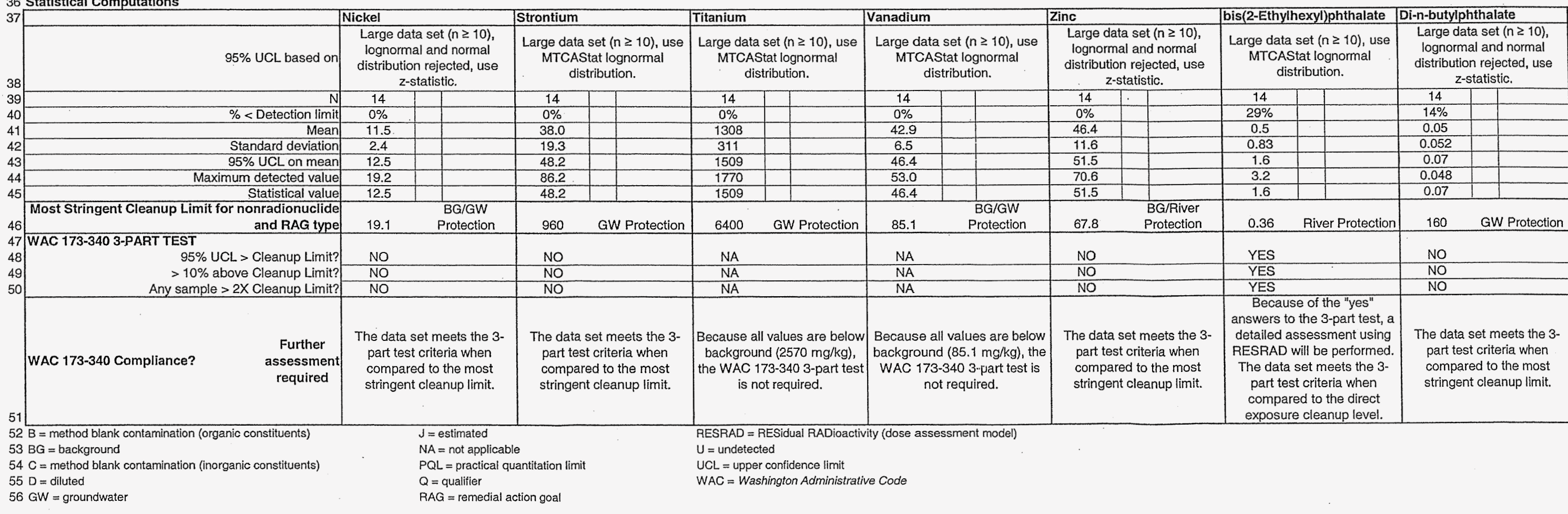


Washington Closure Hanford

CALCULATION SHEET

Jid Canron Ganc

Date $\quad 09 / 14 / 06$

Calc. No. 0100B-CA-V0292

Rev. No. $\frac{0}{\text { Date }}$ Project 100-B/C Remaining Pipes and Sewers Field Remediation

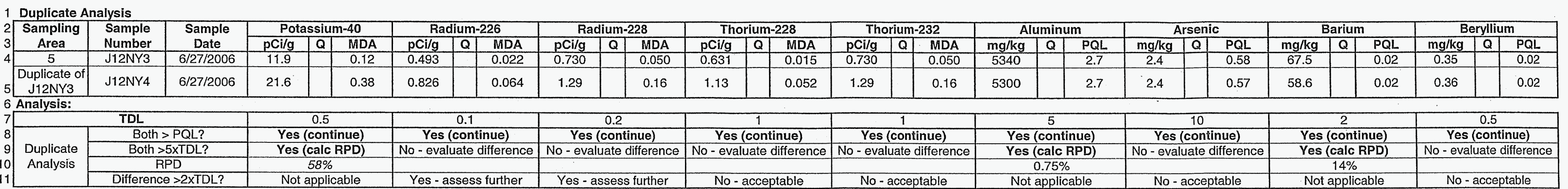

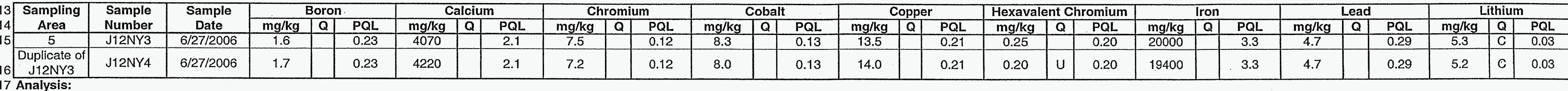

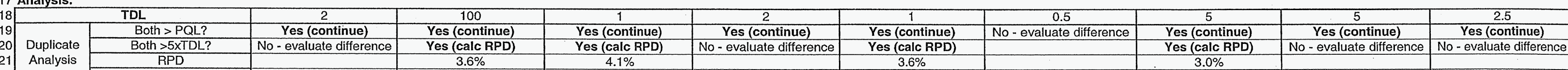

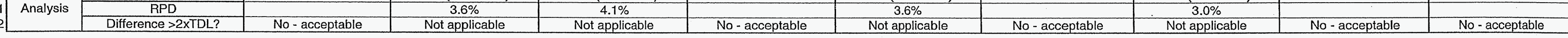

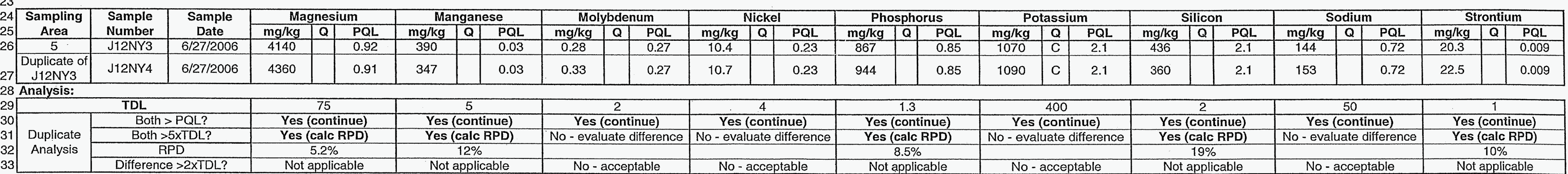

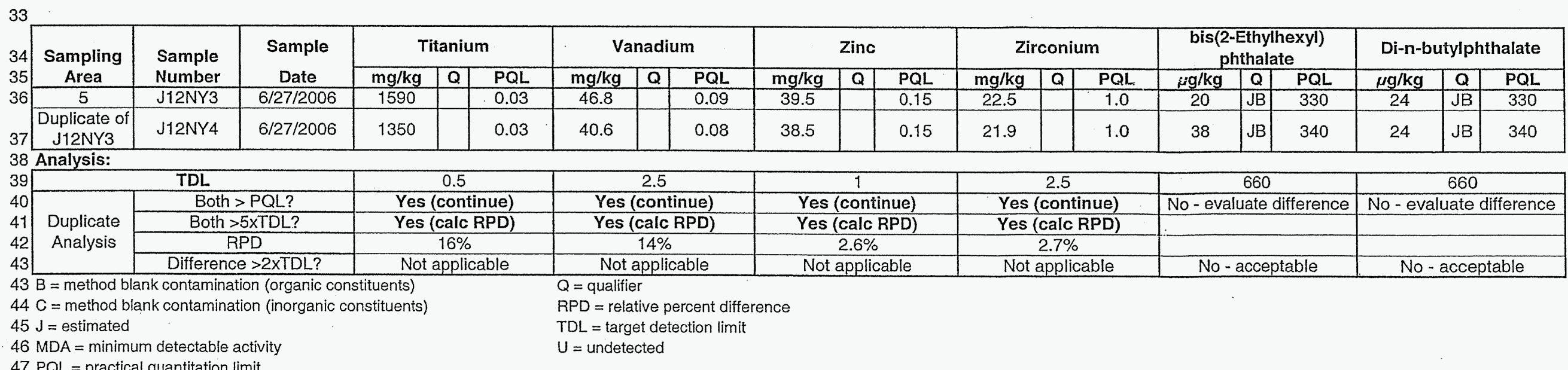

$46 \mathrm{MDA}=$ minimum detectable activity
$47 \mathrm{PQL}=$ practical quantitition limit

undetected 


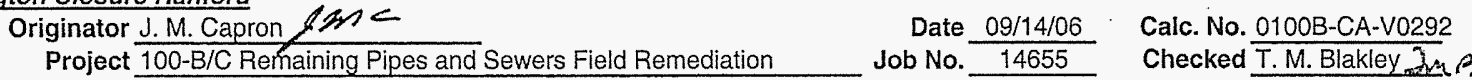
Subject 1607-B2:2 Waste Site Cleanup Verification 95\% UCL Calculations

Rev. No.

Date 211810

Sheet No. 19 of 13

Ecology Software (MTCAStat) Results

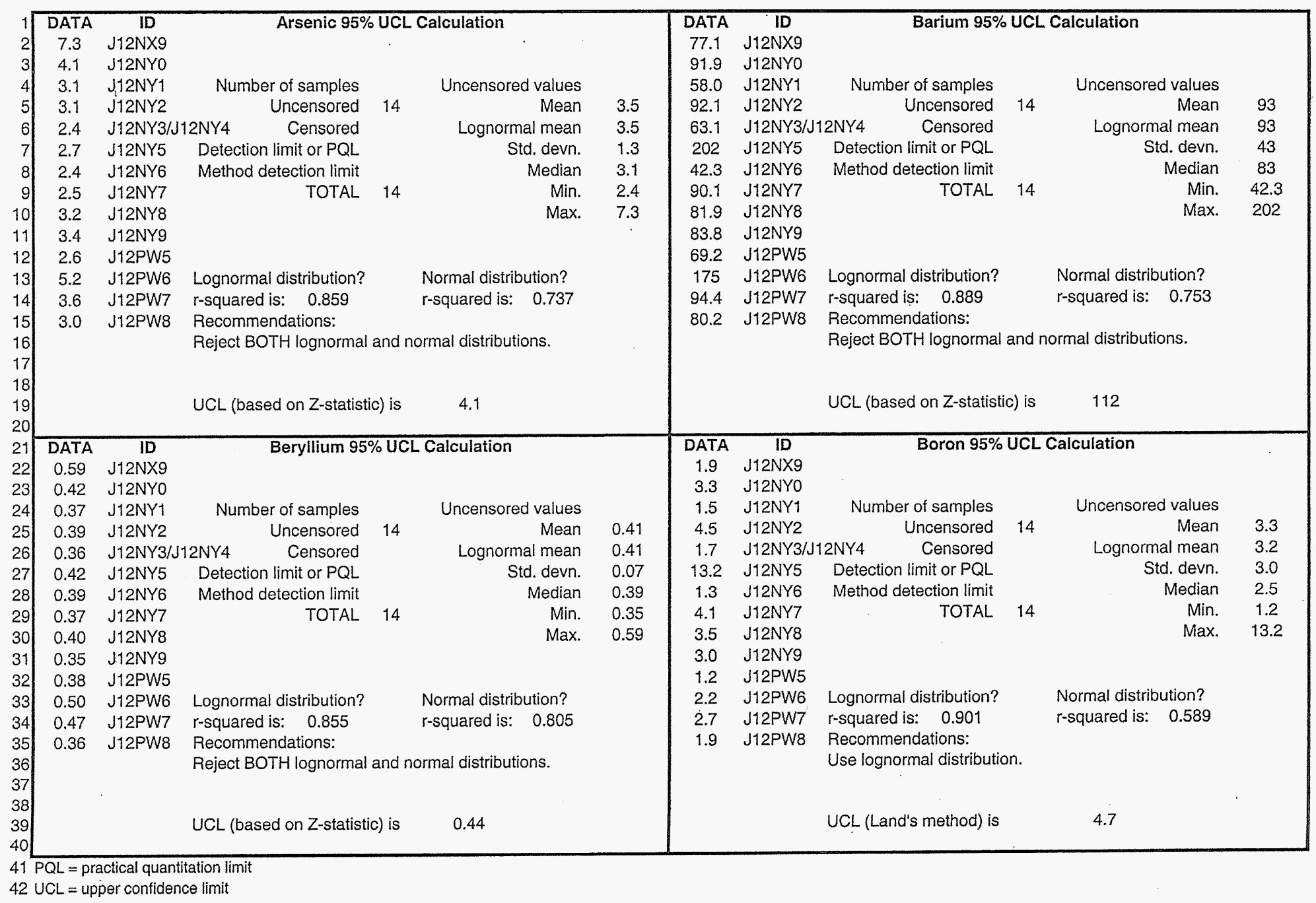




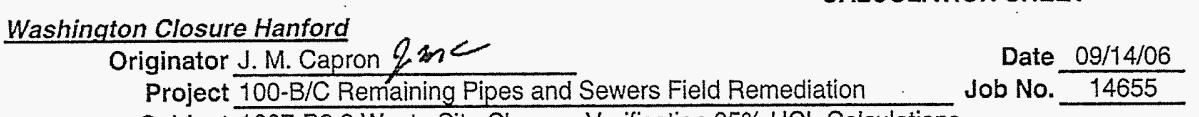

Rev. No.

Date 91810

Ecology Software (MTCAStat) Results

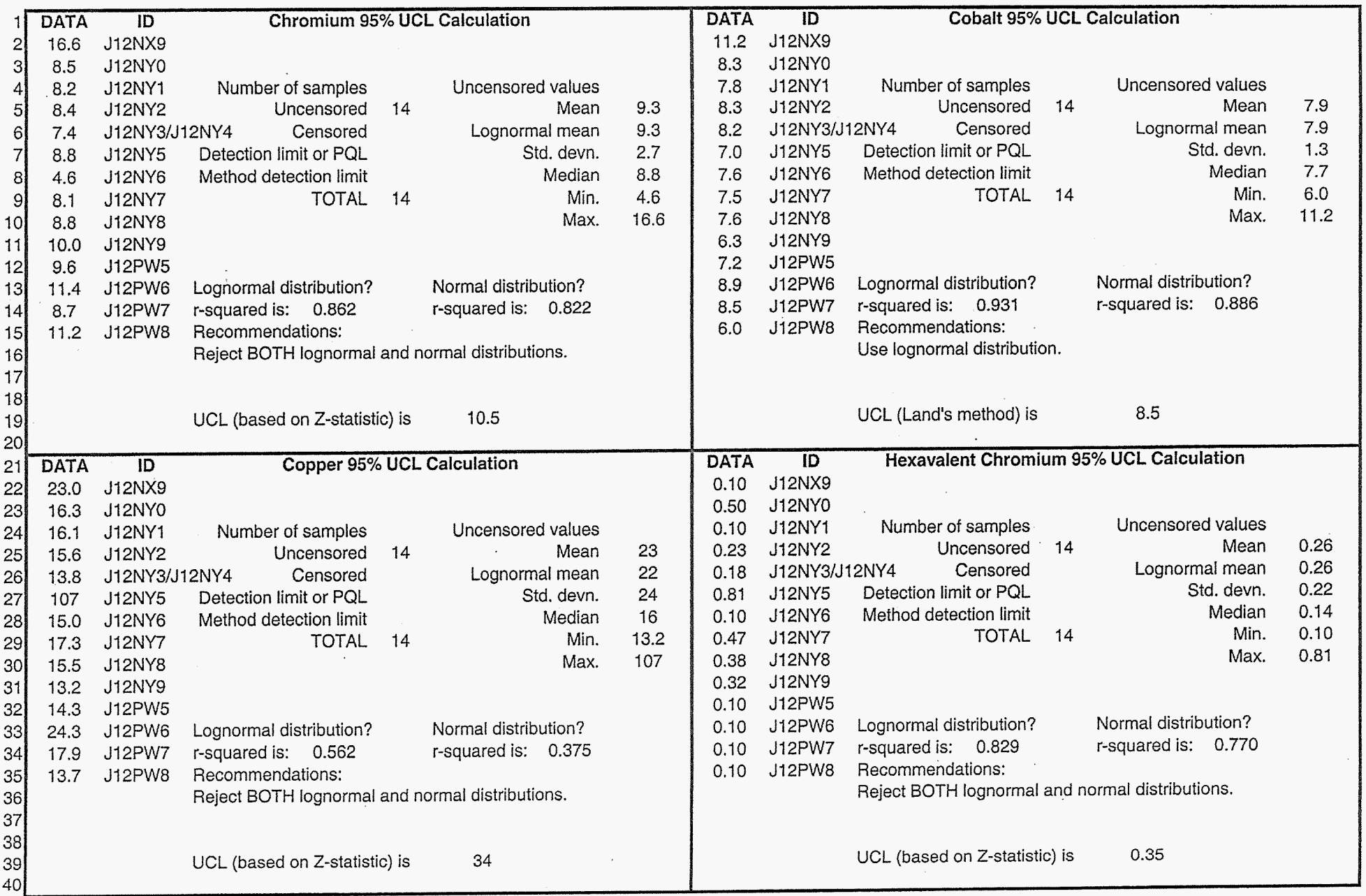




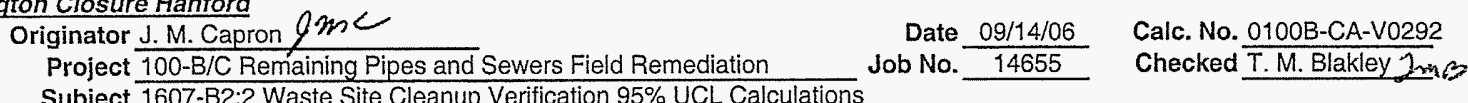

Calc. No. 0100B-CA-V0292 Checked T.M. Blakley 2 mas

Ecology Software (MTCAStat) Results

Rev. No. $\quad 0$

Date $91 / 7 / 0$

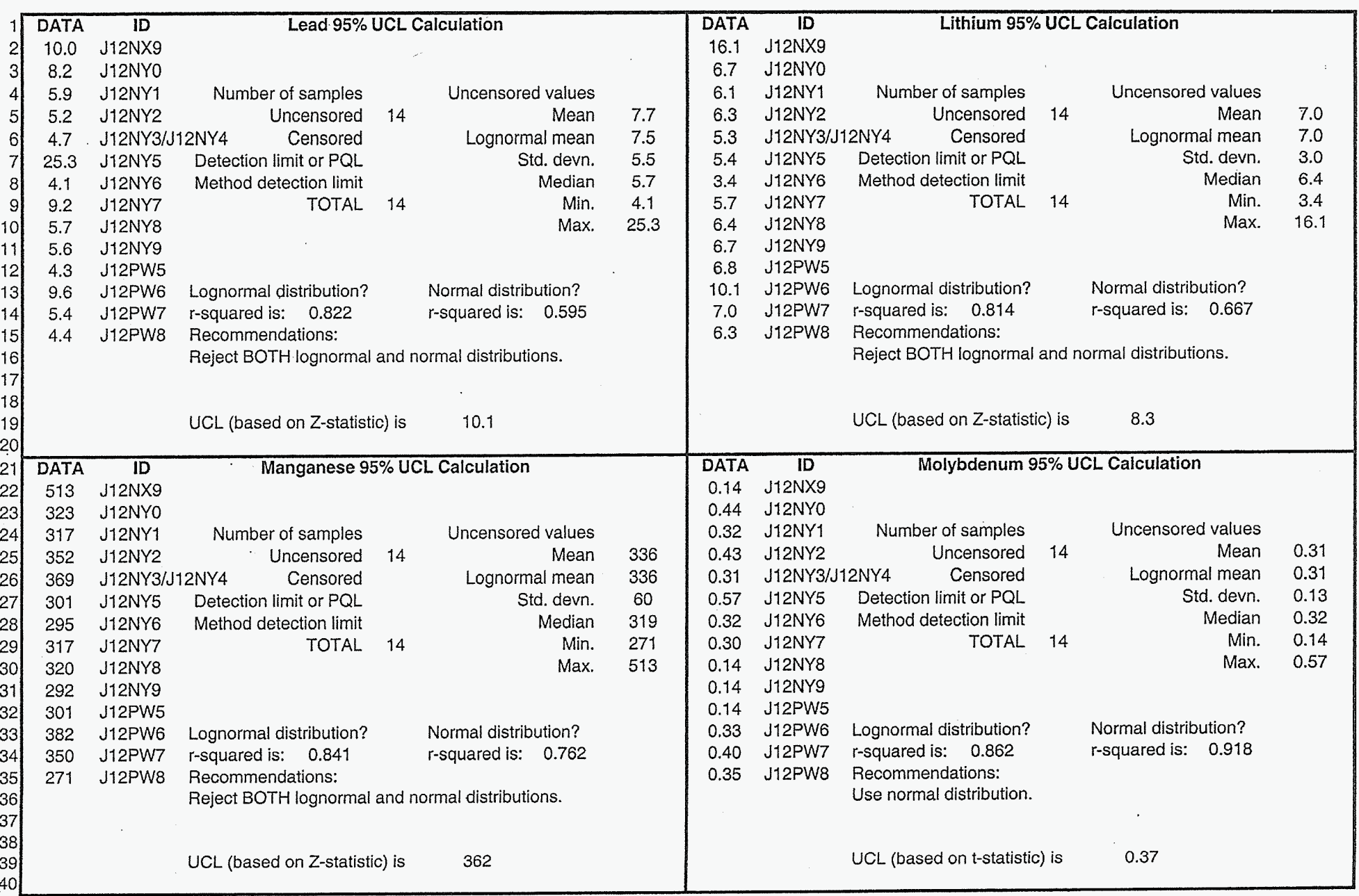


Washington Closure Hanford

CALCULATION SHEET

$$
\begin{aligned}
& \text { Originator J. M. Capron \&mC } \\
& \text { Project } 100 \text {-B/C Remaining Pipes and Sewers Field Remediation } \\
& \text { Subject 1607-B2:2 Waste Site Cleanup Verification 95\% UCL Calculation }
\end{aligned}
$$

Nev. No. Sheet No $\frac{9 / \% ? / 0 \%}{40 / 13}$

Ecology Software (MTCAStat) Results

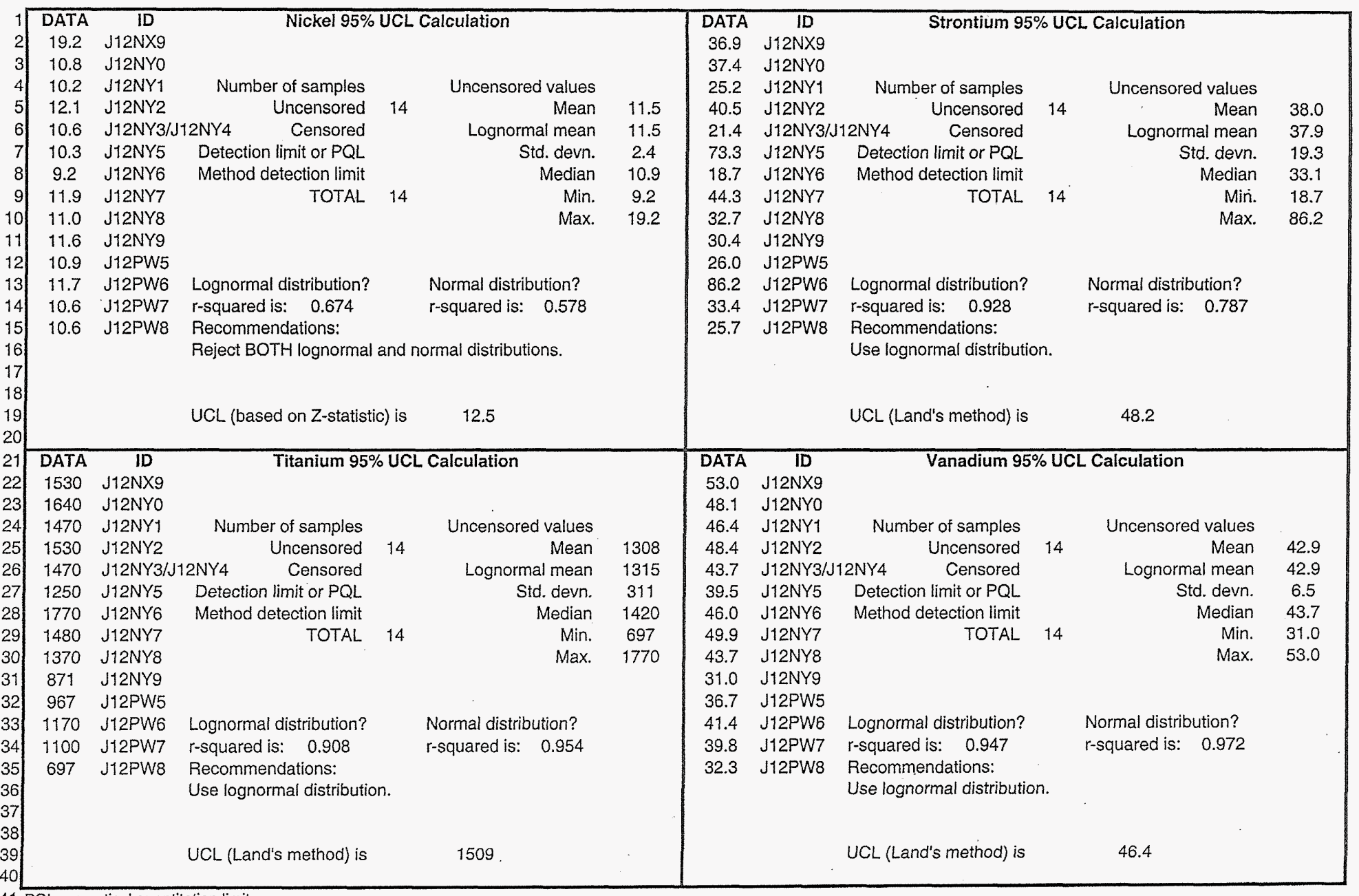


Washington Closure Hanford

CALCULATION SHEET

Originator J.M. Capron $g \mathrm{mc}$

Project 100-B/C Remaining Pipes and Sewers Field Remediation Job No. $\frac{14655}{1406}$

c. No. 0100B-CA-V0292 Subject 1607-B2:2 Waste Site Cleanup Verification 95\% UCL Calculation

Ecology Software (MTCAStat) Results

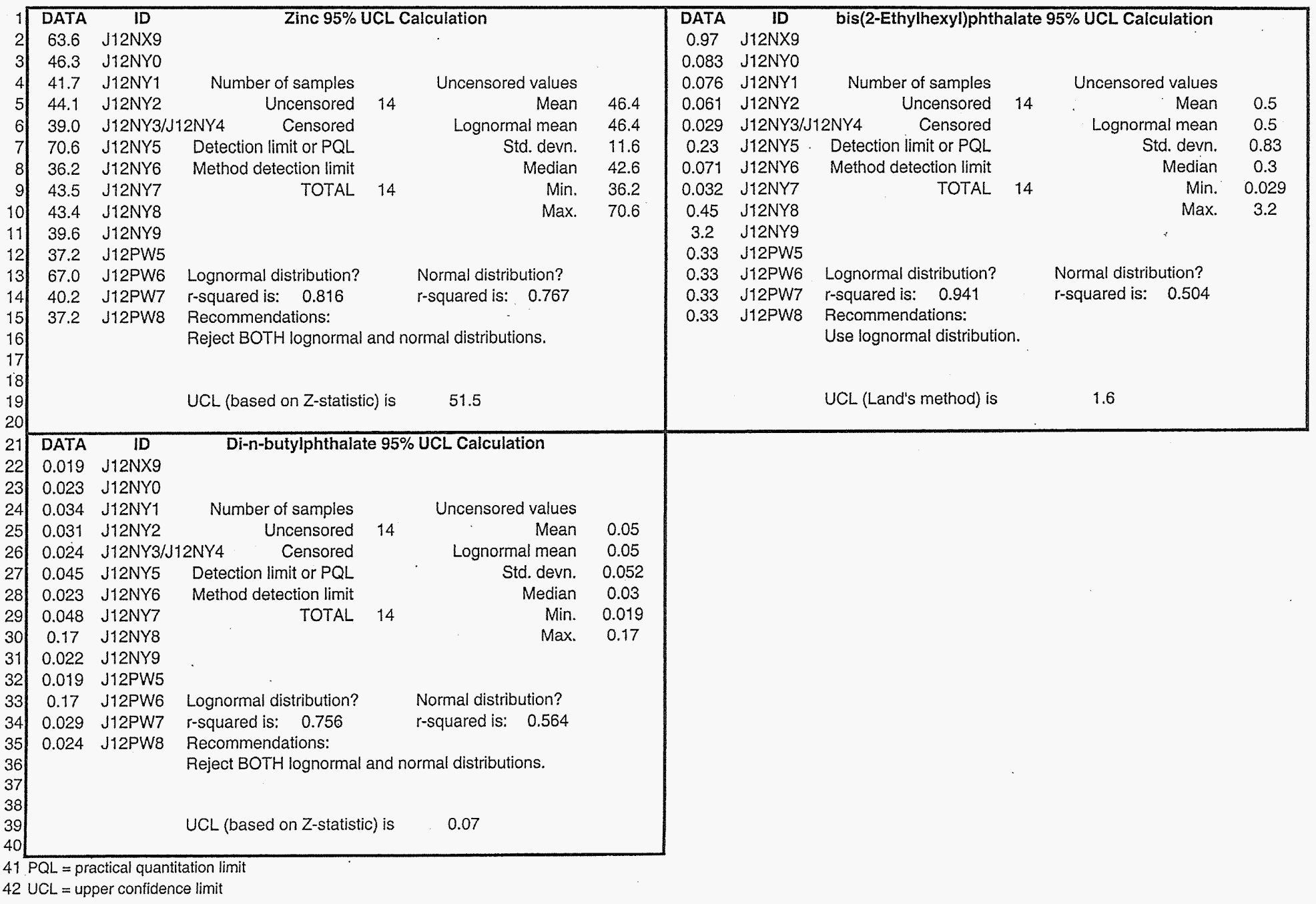


Attachment 1. 1607-B2:2 Verification Sampling Results.

\begin{tabular}{|c|c|c|c|c|c|c|c|c|c|c|c|c|c|c|c|c|c|}
\hline \multirow{2}{*}{ Sample Location } & \multirow{2}{*}{$\begin{array}{l}\text { Sample } \\
\text { Number }\end{array}$} & \multirow{2}{*}{$\begin{array}{c}\text { Sample } \\
\text { Date }\end{array}$} & \multicolumn{3}{|c|}{ Americium-241 } & \multicolumn{3}{|c|}{ Cesium-137 } & \multicolumn{3}{|c|}{ Cobalt- 60} & \multicolumn{3}{|c|}{ Europium-152 } & \multicolumn{3}{|c|}{ Europium-154 } \\
\hline & & & $\mathrm{pCi} / \mathrm{g}$ & $Q$ & $\mathrm{MDA}$ & $\mathrm{pCi} / \mathrm{g}$ & $Q$ & MDA & $\mathrm{pCi} / \mathrm{g}$ & $\mathbf{Q}$ & MDA & $\mathrm{pCi} / \mathrm{g}$ & $\mathbf{Q}$ & MDA & $\mathrm{pCi} / \mathrm{g}$ & $Q$ & MDA \\
\hline 1 & J12NX9 & $6 / 27 / 06$ & 0.29 & $\mathrm{U}$ & 0.29 & 0.079 & $\mathrm{U}$ & 0.079 & 0.093 & $\mathrm{U}$ & 0.093 & 0.19 & $\mathrm{U}$ & 0.19 & 0.25 & $\mathrm{U}$ & 0.25 \\
\hline 2 & J12NY0 & $6 / 27 / 06$ & 0.22 & $\mathrm{U}$ & 0.22 & 0.031 & $\mathrm{U}$ & 0.031 & 0.030 & $\bar{U}$ & 0.030 & 0.069 & $\bar{U}$ & 0.069 & 0.11 & $\mathrm{U}$ & 0.11 \\
\hline 3 & $\mathrm{~J} 12 \mathrm{NY} 1$ & $6 / 27 / 06$ & 0.11 & $\mathrm{U}$ & 0.11 & 0.020 & $U$ & 0.020 & 0.021 & $\mathrm{U}$ & 0.021 & 0.051 & $\mathrm{U}$ & 0.051 & 0.069 & $\mathrm{U}$ & 0.069 \\
\hline 4 & $\mathrm{~J} 12 \mathrm{NY} 2$ & $6 / 27 / 06$ & 0.30 & $\mathrm{U}$ & 0.30 & 0.067 & $\mathrm{U}$ & 0.067 & 0.074 & $\mathrm{U}$ & 0.074 & 0.16 & $\overline{\mathrm{U}}$ & 0.16 & 0.23 & $U$ & 0.23 \\
\hline 5 & J12NY3 & $6 / 27 / 06$ & 0.084 & $\mathrm{U}$ & 0.084 & 0.011 & $\mathrm{U}$ & 0.011 & 0.011 & $U$ & 0.011 & 0.027 & $\bar{U}$ & 0.027 & 0.040 & $\mathrm{U}$ & 0.040 \\
\hline $\begin{array}{l}\text { Duplicate of } \\
\text { J12NY3 }\end{array}$ & J12NY4 & $6 / 27 / 06$ & 0.37 & U & 0.37 & 0.035 & $\mathrm{U}$ & 0.035 & 0.041 & $\mathrm{U}$ & 0.041 & 0.10 & $\mathrm{U}$ & 0.10 & 0.12 & $\mathrm{U}$ & 0.12 \\
\hline 6 & J12NY5 & $6 / 27 / 06$ & 0.15 & $\mathrm{U}$ & 0.15 & 0.415 & & 0.039 & 0.033 & $\mathrm{U}$ & 0.033 & 0.079 & $\mathrm{U}$ & 0.079 & 0.11 & $\mathrm{U}$ & 0.11 \\
\hline 7 & J12NY6 & $6 / 27 / 106$ & 0.041 & $\mathrm{U}$ & 0.041 & 0.010 & $U$ & 0.010 & 0.008 & $\mathrm{U}$ & 0.008 & 0.018 & $\bar{U}$ & 0.018 & 0.024 & $\mathrm{U}$ & 0.024 \\
\hline 8 & $\mathrm{~J} 12 \mathrm{NY} 7$ & $6 / 27 / 06$ & 0.032 & U & 0.032 & 0.083 & & 0.028 & 0.033 & $\mathrm{U}$ & 0.033 & 0.066 & $\mathrm{U}$ & 0.066 & 0.094 & $\mathrm{U}$ & 0.094 \\
\hline 9 & J12NY8 & $6 / 27 / 06$ & 0.14 & $\mathrm{U}$ & 0.14 & 0.044 & & 0.026 & 0.026 & $\mathrm{U}$ & 0.026 & 0.063 & $\overline{\mathrm{U}}$ & 0.063 & 0.091 & $\mathrm{U}$ & 0.091 \\
\hline 10 & J12NY9 & $6 / 27 / 06$ & 0.41 & $\mathrm{U}$ & 0.41 & 0.095 & $\mathrm{U}$ & 0.095 & 0.097 & $\mathrm{U}$ & 0.097 & 0.21 & $\mathrm{U}$ & 0.21 & 0.33 & $U$ & 0.33 \\
\hline 11 & J12PW5 & $6 / 29 / 06$ & 0.029 & U & 0.029 & 0.032 & U & 0.032 & 0.038 & U & 0.038 & 0.048 & $\mathrm{U}$ & 0.048 & 0.12 & $\mathrm{U}$ & 0.12 \\
\hline 12 & J12PW6 & $6 / 29 / 06$ & 0.10 & $\mathrm{U}$ & 0.10 & 0.13 & $\mathrm{U}$ & 0.13 & 0.15 & $\mathrm{U}$ & 0.15 & 0.17 & $\bar{U}$ & 0.17 & 0.55 & $\mathrm{U}$ & 0.55 \\
\hline 13 & J12PW7 & $6 / 29 / 06$ & 0.095 & U & 0.095 & 0.11 & U & 0.11 & 0.13 & $\mathrm{U}$ & 0.13 & 0.14 & $\mathrm{U}$ & 0.14 & 0.39 & $\mathrm{U}$ & 0.39 \\
\hline 14 & J12PW8 & $6 / 29 / 06$ & 0.30 & U & 0.30 & 0.042 & $\mathrm{U}$ & 0.042 & 0.044 & U & 0.044 & 0.093 & $\mathrm{U}$ & 0.093 & 0.14 & $\mathrm{U}$ & 0.14 \\
\hline $\begin{array}{c}\text { North BCL } \\
\text { stockpile (north) }\end{array}$ & $J 12 \mathrm{NX} 4$ & $6 / 27 / 06$ & 0.48 & $\mathrm{U}$ & 0.48 & 0.048 & $U$ & 0.048 & 0.051 & $\mathrm{U}$ & 0.051 & 0.13 & U & 0.13 & 0.16 & $\mathrm{U}$ & 0.16 \\
\hline $\begin{array}{c}\text { North BCL } \\
\text { stockpile (middle) }\end{array}$ & J12NX5 & $6 / 27 / 06$ & 0.40 & $\mathrm{U}$ & 0.40 & 0.095 & $U$ & 0.095 & 0.094 & $U$ & 0.094 & 0.21 & U & 0.21 & 0.30 & $U$ & 0.30 \\
\hline $\begin{array}{c}\text { North BCL } \\
\text { stockpile (south) }\end{array}$ & J12NX6 & $6 / 27 / 06$ & 0.45 & $\mathrm{U}$ & 0.45 & 0.11 & $\mathrm{U}$ & 0.11 & 0.11 & U & 0.11 & 0.24 & U & 0.24 & 0.33 & $U$ & 0.33 \\
\hline $\begin{array}{c}\text { Southeastern } \mathrm{BCL} \\
\text { stockpile }\end{array}$ & $\mathrm{J} 12 \mathrm{NX7}$ & $6 / 27 / 06$ & 0.27 & U & 0.27 & 0.038 & $\mathrm{U}$ & 0.038 & 0.034 & U & 0.034 & 0.085 & U & 0.085 & 0.13 & U & 0.13 \\
\hline $\begin{array}{c}\begin{array}{c}\text { Small BCL } \\
\text { stockpiles }\end{array} \\
\end{array}$ & $\mathrm{J} 12 \mathrm{NX} 8$ & $6 / 27 / 06$ & 0.023 & $\mathrm{U}$ & 0.023 & 0.018 & $\mathrm{U}$ & 0.018 & 0.024 & $\mathrm{U}$ & 0.024 & 0.049 & $\mathrm{U}$ & 0.049 & 0.069 & $U$ & 0.069 \\
\hline
\end{tabular}

Note: The following abbreviations apply to all Attachment 1 tables.

Note: Data qualified with $B, C, D, I$ and/or J are considered acceptable values.

$\mathrm{B}=$ method blank contamination (organic constituents)

$\mathrm{J}=$ estimated

$\mathrm{BCL}=$ below cleanup levels

$\mathrm{MDA}=$ minimum detectable activity

$\mathrm{C}=$ method blank contamination (inorganic constituents)

$\mathrm{D}=$ diluted

$\mathrm{PQL}=$ practical quantitation limit

I = interference on one analytical column

$\mathrm{U}=$ undetected

Attachment

Calc No $\frac{\text { T.M. Blakley } 2 m \rho}{0100 \mathrm{~B}-\mathrm{CA}-\mathrm{V} 0292}$ Date $\frac{9418 / 06}{6}$ 
Attachment 1. 1607-B2:2 Verification Sampling Results.

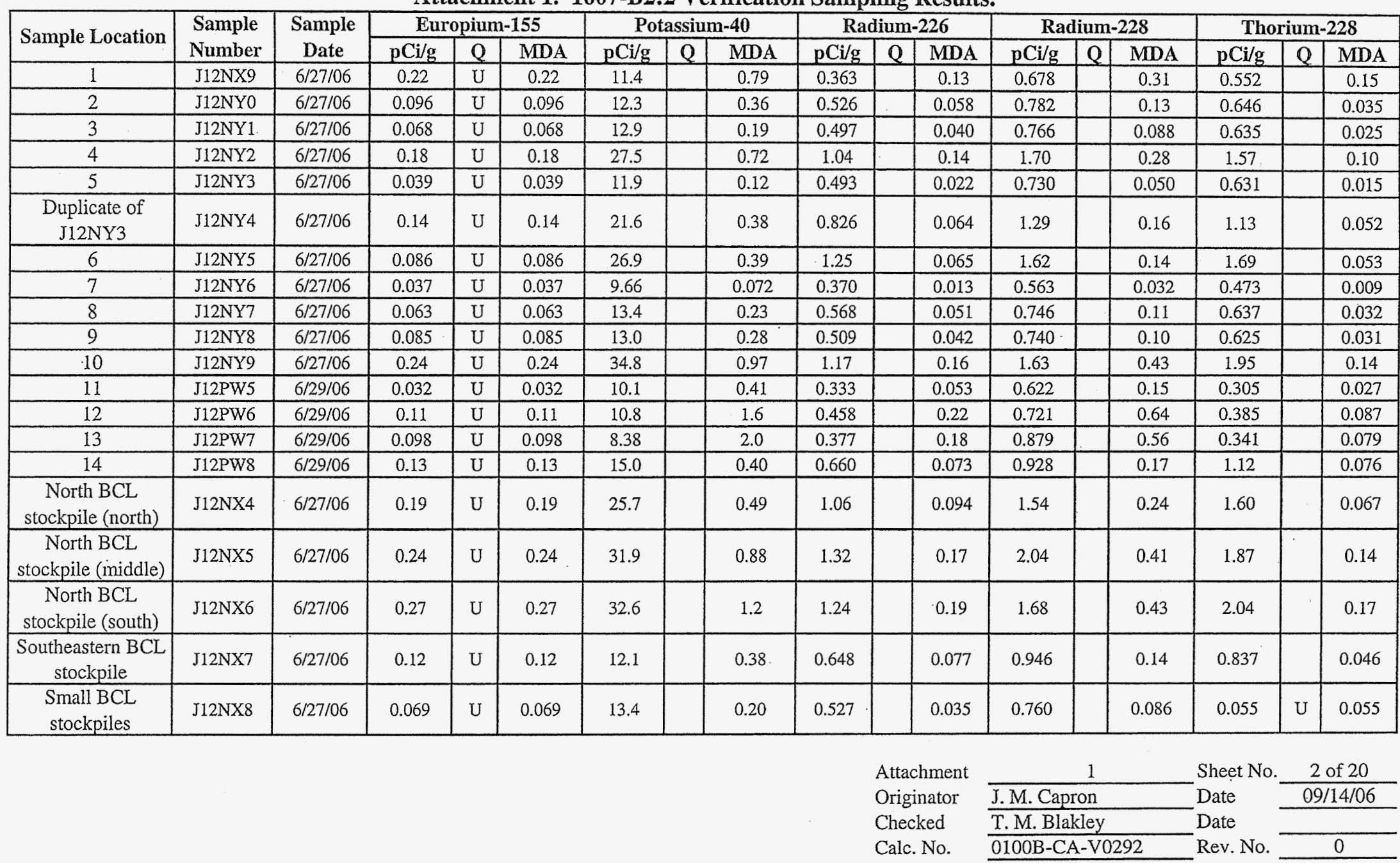


Attachment 1. 1607-B2:2 Verification Sampling Results.

\begin{tabular}{|c|c|c|c|c|c|c|c|c|c|c|c|c|c|c|c|c|c|}
\hline \multirow[t]{2}{*}{ Sample Location } & \multirow{2}{*}{$\begin{array}{l}\text { Sample } \\
\text { Number }\end{array}$} & \multirow{2}{*}{$\begin{array}{l}\text { Sample } \\
\text { Date }\end{array}$} & \multicolumn{3}{|c|}{ Thorium-232 } & \multicolumn{3}{|c|}{$\begin{array}{c}\text { Total Beta } \\
\text { Radiostrontium }\end{array}$} & \multicolumn{3}{|c|}{ Tritium } & \multicolumn{3}{|c|}{ Uranium-235 } & \multicolumn{3}{|c|}{ Uranium-238 } \\
\hline & & & $\mathrm{pCi} / \mathrm{g}$ & $Q$ & MDA & $\mathrm{pCi} / \mathrm{g}$ & $Q$ & MDA & $\mathrm{pCi} / \mathrm{g}$ & $Q$ & MDA & $\mathrm{pCi} / \mathrm{g}$ & $Q$ & MDA & $\mathrm{pCi} / \mathrm{g}$ & $Q$ & $\mathrm{MDA}$ \\
\hline 1 & J12NX9 & $6 / 27 / 06$ & 0.678 & & 0.31 & -0.067 & $\mathrm{U}$ & 0.23 & -2.36 & $U$ & 5.7 & 0.31 & $\mathrm{U}$ & 0.31 & 9.7 & $\mathrm{U}$ & 9.7 \\
\hline 2 & J12NYO & $6 / 27 / 06$ & 0.782 & & 0.13 & -0.024 & $\mathrm{U}$ & 0.23 & -1.13 & $\mathrm{U}$ & 5.0 & 0.14 & $\mathrm{U}$ & 0.14 & 3.9 & $\mathrm{U}$ & 3.9 \\
\hline 3 & $\mathrm{~J} 12 \mathrm{NY} 1$ & $6 / 27 / 06$ & 0.766 & & 0.088 & 0.904 & & 0.19 & -2.36 & $\mathrm{U}$ & 4.8 & 0.089 & $U$ & 0.089 & 2.4 & $\mathrm{U}$ & 2.4 \\
\hline 4 & $\mathrm{~J} 12 \mathrm{NY} 2$ & $6 / 27 / 06$ & 1.70 & & 0.28 & 0.088 & $\mathrm{U}$ & 0.24 & -1.92 & $\mathrm{U}$ & 4.9 & 0.25 & $\mathrm{U}$ & 0.25 & 8.5 & $\mathrm{U}$ & 8.5 \\
\hline 5 & J12NY3 & $6 / 27 / 06$ & 0.730 & & 0.050 & -0.036 & $\mathrm{U}$ & 0.23 & 1.50 & $\mathrm{U}$ & 5.0 & 0.053 & $\mathrm{U}$ & 0.053 & 1.5 & $\mathrm{U}$ & 1.5 \\
\hline $\begin{array}{c}\text { Duplicate of } \\
\text { J12NY3 }\end{array}$ & $\mathrm{J} 12 \mathrm{NY} 4$ & $6 / 27 / 06$ & 1.29 & & 0.16 & -0.020 & $U$ & 0.23 & -1.32 & $U$ & 5.8 & 0.19 & $\mathrm{U}$ & 0.19 & 4.4 & $\mathrm{U}$ & 4.4 \\
\hline 6 & J12NY5 & $6 / 27 / 06$ & 1.62 & & 0.14 & 0.093 & $\mathrm{U}$ & 0.24 & -2.44 & $\mathrm{U}$ & 5.7 & 0.12 & $\mathrm{U}$ & 0.12 & 4.0 & $\mathrm{U}$ & 4.0 \\
\hline 7 & J12NY6 & $6 / 27 / 06$ & 0.563 & & 0.032 & -0.049 & $\mathrm{U}$ & 0.18 & -2.81 & $\mathrm{U}$ & 4.6 & 0.032 & $\mathrm{U}$ & 0.032 & 1.8 & $\mathrm{U}$ & 1.8 \\
\hline 8 & $\mathrm{~J} 12 \mathrm{NY} 7$ & $6 / 27 / 06$ & 0.746 & & 0.11 & 0.009 & U & 0.22 & -1.16 & $\mathrm{U}$ & 5.4 & 0.090 & $\mathrm{U}$ & 0.090 & 3.3 & $\mathrm{U}$ & 3.3 \\
\hline 9 & $\mathrm{~J} 12 \mathrm{NY} 8$ & $6 / 27 / 06$ & 0.740 & & 0.10 & 0.089 & $\mathrm{U}$ & 0.22 & -1.58 & $\mathrm{U}$ & 5.0 & 0.11 & $\mathrm{U}$ & 0.11 & 2.9 & $\mathrm{U}$ & 2.9 \\
\hline 10 & J12NY9 & $6 / 27 / 06$ & 1.63 & & 0.43 & -0.023 & $\mathrm{U}$ & 0.23 & -1.69 & $\mathrm{U}$ & 5.2 & 0.34 & $\mathrm{U}$ & 0.34 & 11 & $\mathrm{U}$ & 11 \\
\hline 11 & J12PW5 & $6 / 29 / 06$ & 0.622 & & 0.15 & 0.033 & $\mathrm{U}$ & 0.31 & 0.902 & $\mathrm{U}$ & 3.2 & 0.049 & $\bar{U}$ & 0.049 & 4.7 & $\mathrm{U}$ & 4.7 \\
\hline 12 & J12PW6 & $6 / 29 / 06$ & 0.721 & & 0.64 & -0.028 & $\mathrm{U}$ & 0.36 & 2.03 & $\mathrm{U}$ & 3.8 & 0.17 & $\mathrm{U}$ & 0.17 & 15 & $\mathrm{U}$ & 15 \\
\hline 13 & J12PW7 & $6 / 29 / 06$ & 0.879 & & 0.56 & -0.034 & $\mathrm{U}$ & 0.30 & 0.379 & $\mathrm{U}$ & 3.0 & 0.15 & $\mathrm{U}$ & 0.15 & 16 & $\mathrm{U}$ & 16 \\
\hline 14 & J12PW8 & $6 / 29 / 06$ & 0.928 & & 0.17 & 0.056 & $\mathrm{U}$ & 0.28 & 1.30 & $\mathrm{U}$ & 3.1 & 0.16 & $\mathrm{U}$ & 0.16 & 5.4 & $\mathrm{U}$ & 5.4 \\
\hline $\begin{array}{c}\text { North BCL } \\
\text { stockpile (north) }\end{array}$ & $\mathrm{J} 12 \mathrm{NX} 4$ & $6 / 27 / 06$ & 1.54 & & 0.24 & 0.100 & $\mathrm{U}$ & 0.25 & -1.34 & $\mathrm{U}$ & 5.9 & 0.24 & $\mathrm{U}$ & 0.24 & 13 & $U$ & 13 \\
\hline $\begin{array}{c}\text { North BCL } \\
\text { stockpile (middle) }\end{array}$ & J12NX5 & $6 / 27 / 06$ & 2.04 & & 0.41 & 0.268 & $\mathrm{U}$ & 0.30 & -1.58 & U & 4.8 & 0.34 & $U$ & 0.34 & 12 & $\mathrm{U}$ & 12 \\
\hline $\begin{array}{c}\text { North BCL } \\
\text { stockpile (south) } \\
\end{array}$ & J12NX6 & $6 / 27 / 06$ & 1.68 & & 0.43 & -0.043 & $U$ & 0.25 & -1.78 & $U$ & 4.7 & 0.39 & $\mathrm{U}$ & 0.39 & 12 & $U$ & 12 \\
\hline $\begin{array}{c}\text { Southeastern BCL } \\
\text { stockpile }\end{array}$ & J12NX7 & $6 / 27 / 06$ & 0.946 & & 0.14 & 2.10 & & 0.23 & -1.67 & $U$ & 4.9 & 0.17 & $U$ & 0.17 & 4.6 & $\mathrm{U}$ & 4.6 \\
\hline $\begin{array}{c}\text { Small BCL } \\
\text { stockpiles }\end{array}$ & $\mathrm{J} 12 \mathrm{NX} 8$ & $6 / 27 / 06$ & 0.760 & & 0.086 & 0.294 & & 0.23 & -1.66 & $\mathrm{U}$ & 4.5 & 0.067 & $U$ & 0.067 & 2.4 & $\mathrm{U}$ & 2.4 \\
\hline & & & & & & & & & \multirow{4}{*}{\multicolumn{3}{|c|}{$\begin{array}{l}\text { Attachment } \\
\text { Originator } \\
\text { Checked } \\
\text { Calc. No. }\end{array}$}} & \multicolumn{3}{|c|}{1} & Sheet No. & \multicolumn{2}{|c|}{3 of 20} \\
\hline & & & & & & & & & & & & \multirow{2}{*}{\multicolumn{3}{|c|}{$\frac{\text { J.M. Capron }}{\text { T. M. Blakley }}$}} & Date & \multicolumn{2}{|c|}{$09 / 14 / 06$} \\
\hline & & & & & & & & & & & & & & & Date & & \\
\hline & & & & & & & & & & & & \multicolumn{3}{|c|}{ 0100B-CA-V0292 } & Rev. No. & & 0 \\
\hline
\end{tabular}


Attachment 1. 1607-B2:2 Verification Sampling Results.

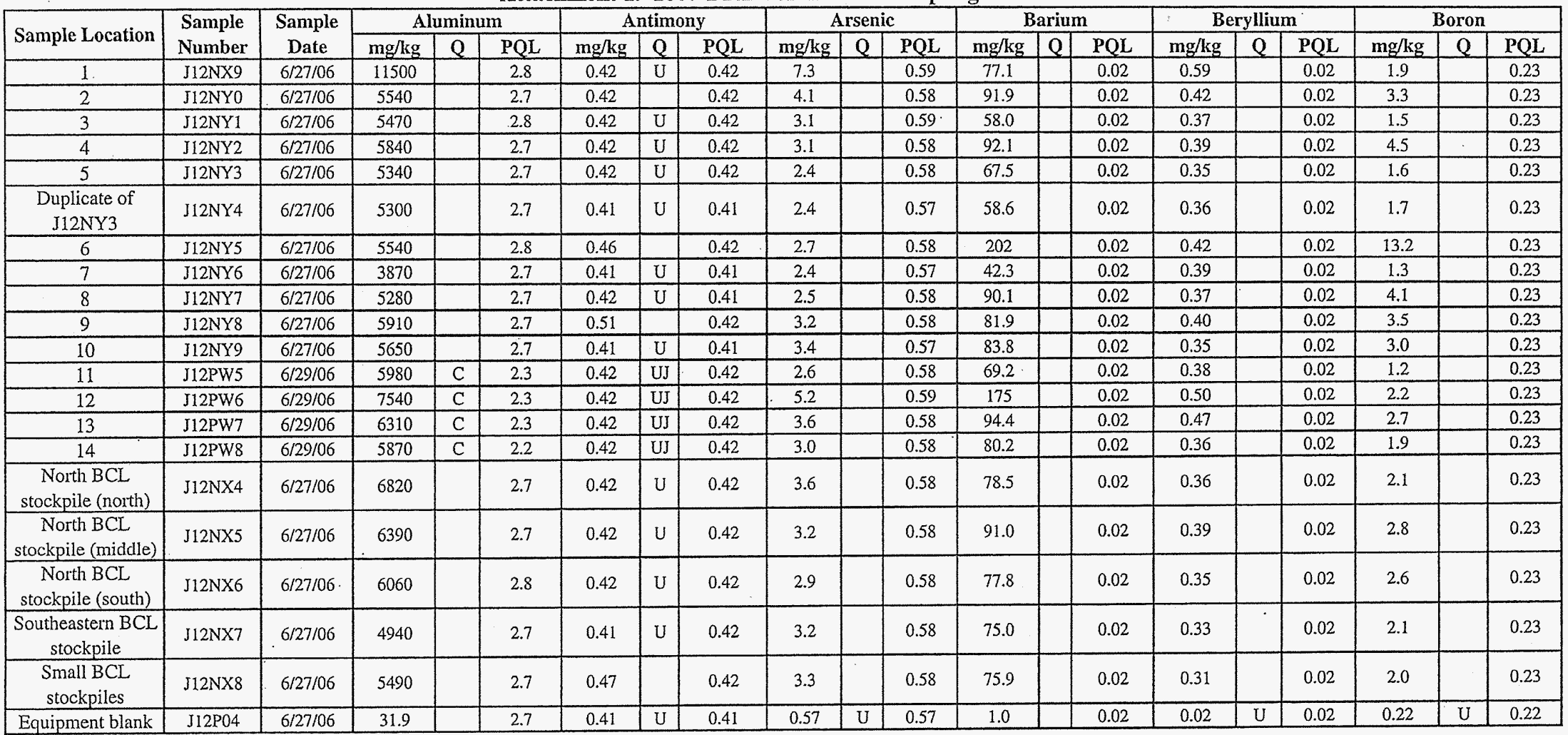

Attachment

Sheet No. $\quad 4$ of 20

Originator J.M. Capron Date 09/14/06

Checked T.M. Blakley

Calc. No.

0100B-CA-V0292

Rev. No.

0 
Attachment 1. 1607-B2:2 Verification Sampling Results.

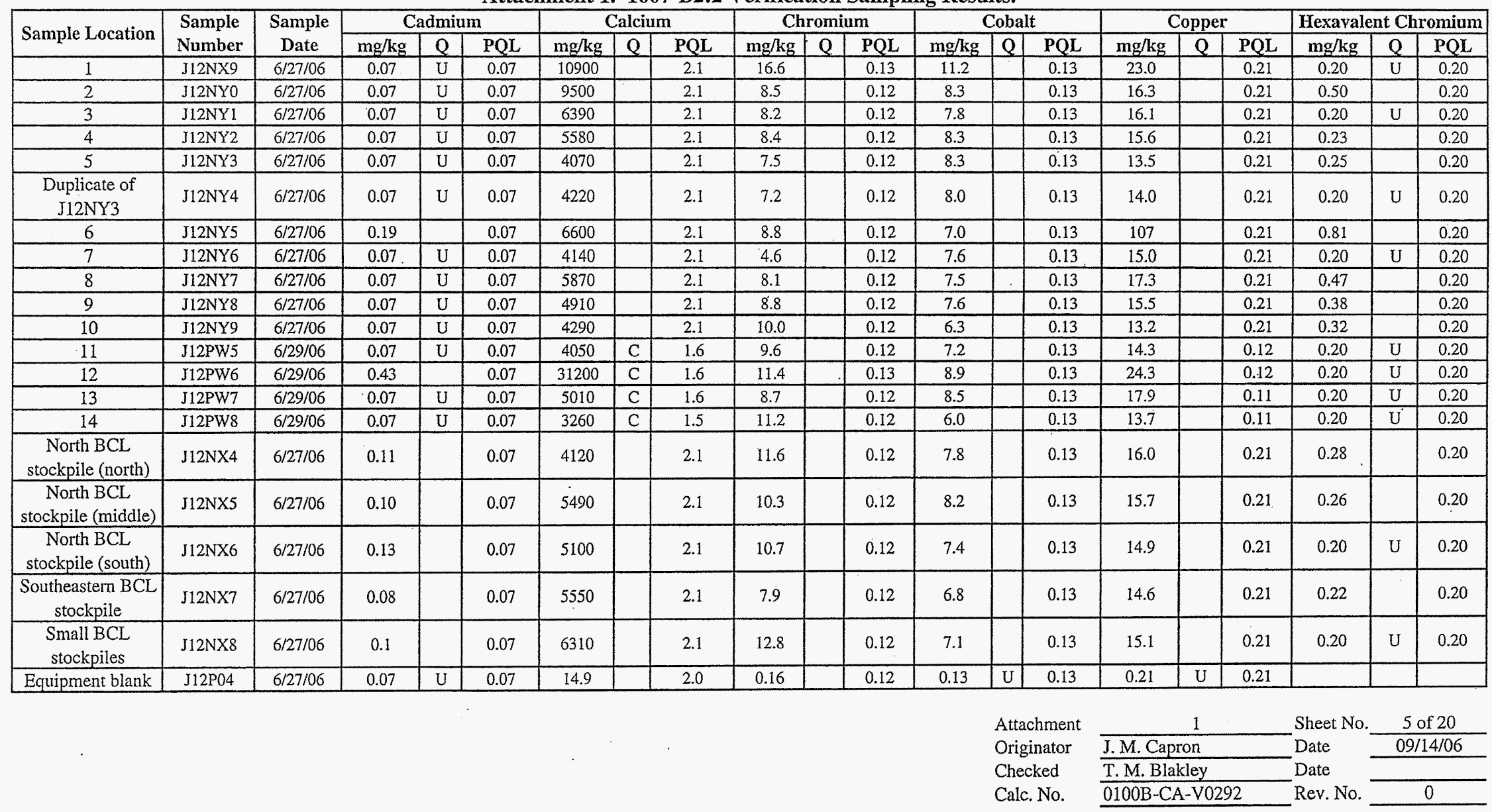


Attachment 1. 1607-B2:2 Verification Sampling Results.

\begin{tabular}{|c|c|c|c|c|c|c|c|c|c|c|c|c|c|c|c|c|c|c|c|c|}
\hline \multirow{2}{*}{ Sample Location } & \multirow{2}{*}{$\begin{array}{l}\text { Sample } \\
\text { Number }\end{array}$} & \multirow{2}{*}{$\begin{array}{c}\text { Sample } \\
\text { Date }\end{array}$} & \multicolumn{3}{|c|}{ Iron } & \multicolumn{3}{|c|}{ Lead } & \multicolumn{3}{|c|}{ Lithium } & \multicolumn{3}{|c|}{ Magnesium } & \multicolumn{3}{|c|}{ Manganese } & \multicolumn{3}{|c|}{ Mercury } \\
\hline & & & $\mathrm{mg} / \mathrm{kg}$ & $Q$ & PQL & $\mathrm{mg} / \mathrm{kg}$ & $Q$ & $\mathrm{PQL}$ & $\mathrm{mg} / \mathrm{kg}$ & $Q$ & $\mathrm{PQL}$ & $\mathrm{mg} / \mathrm{kg}$ & $Q$ & PQL & $\mathrm{mg} / \mathrm{kg}$ & $Q$ & $\overline{P Q L}$ & $\mathrm{mg} / \mathrm{kg}$ & $Q$ & $\mathrm{PQL}$ \\
\hline 1 & J12NX9 & $6 / 27 / 06$ & 26900 & & 3.4 & 10.0 & & 0.30 & 16.1 & C & 0.03 & 8580 & & 0.94 & 513 & & 0.03 & 0.02 & $\mathrm{U}$ & 0.02 \\
\hline 2 & J12NY0 & $6 / 27 / 06$ & 20400 & & 3.3 & 8.2 & & 0.30 & 6.7 & $\mathrm{C}$ & 0.03 & 4630 & & 0.92 & 323 & & 0.03 & 0.04 & & 0.01 \\
\hline 3 & J12NYI & $6 / 27 / 06$ & 20400 & & 3.4 & 5.9 & & 0.30 & 6.1 & $\mathrm{C}$ & 0.03 & 4330 & & 0.93 & 317 & & 0.03 & 0.01 & $U$ & 0.01 \\
\hline 4 & J12NY2 & $6 / 27 / 06$ & 21100 & & 3.3 & 5.2 & & 0.29 & 6.3 & $\mathrm{C}$ & 0.03 & 4700 & & 0.92 & 352 & & 0.03 & 0.02 & $\mathrm{U}$ & 0.02 \\
\hline 5 & J12NY3 & $6 / 27 / 06$ & 20000 & & 3.3 & 4.7 & & 0.29 & 5.3 & $\mathrm{C}$ & 0.03 & 4140 & & 0.92 & 390 & & 0.03 & 0.02 & $\mathrm{U}$ & 0.02 \\
\hline $\begin{array}{c}\text { Duplicate of } \\
\text { J12NY3 }\end{array}$ & $\mathrm{J} 12 \mathrm{NY} 4$ & $6 / 27 / 06$ & 19400 & & 3.3 & 4.7 & & 0.29 & 5.2 & $\mathrm{C}$ & 0.03 & 4360 & & 0.91 & 347 & & 0.03 & 0.02 & U & 0.02 \\
\hline 6 & J12NY5 & $6 / 27 / 06$ & 17400 & & 3.3 & 25.3 & & 0.30 & 5.4 & $\mathrm{C}$ & 0.03 & 4080 & & 0.93 & 301 & & 0.03 & 0.12 & & 0.02 \\
\hline 7 & J12NY6 & $6 / 27 / 06$ & 19900 & & 3.3 & 4.1 & & 0.29 & 3.4 & $C$ & 0.03 & 3800 & & 0.91 & 295 & & 0.03 & 0.02 & $\mathrm{U}$ & 0.02 \\
\hline 8 & J12NY7 & $6 / 27 / 06$ & 19800 & & 3.3 & 9.2 & & 0.29 & 5.7 & $\mathrm{C}$ & 0.03 & 4590 & & 0.92 & 317 & & 0.03 & 0.02 & U & 0.02 \\
\hline 9 & J12NY8 & $6 / 27 / 06$ & 18900 & & 3.3 & 5.7 & & 0.29 & 6.4 & $\mathrm{C}$ & 0.03 & 4520 & & 0.92 & 320 & & 0.03 & 0.01 & $\mathrm{U}$ & 0.01 \\
\hline 10 & J12NY9 & $6 / 27 / 06$ & 14600 & & 3.3 & 5.6 & & 0.29 & 6.7 & $\mathrm{C}$ & 0.03 & 4120 & & 0.91 & 292 & & 0.03 & 0.02 & $\mathrm{U}$ & 0.02 \\
\hline 11 & J12PW5 & $6 / 29 / 06$ & 15900 & $\mathrm{C}$ & 0.52 & 4.3 & & 0.30 & 6.8 & $\mathrm{C}$ & 0.03 & 4030 & & 0.93 & 301 & & 0.03 & 0.02 & $\mathrm{U}$ & 0.02 \\
\hline 12 & J12PW6 & $6 / 29 / 06$ & 18700 & C & 0.52 & 9.6 & & 0.30 & 10.1 & $\mathrm{C}$ & 0.03 & 5240 & & 0.94 & 382 & & 0.03 & 0.92 & & 0.02 \\
\hline 13 & J12PW7 & $6 / 29 / 06$ & 17400 & C & 0.52 & 5.4 & & 0.30 & 7.0 & $\mathrm{C}$ & 0.03 & 4190 & & 0.93 & 350 & & 0.03 & 0.01 & $\mathrm{U}$ & 0.01 . \\
\hline 14 & J12PW8 & $6 / 29 / 06$ & 13700 & $\mathrm{C}$ & 0.51 & 4.4 & & 0.29 & 6.3 & C & 0.03 & 3440 & & 0.92 & 271 & & 0.03 & 0.01 & $\mathrm{U}$ & 0.01 \\
\hline $\begin{array}{c}\text { North BCL } \\
\text { stockpile (north) }\end{array}$ & J12NX4 & $6 / 27 / 06$ & 20200 & & 3.3 & 8.0 & & 0.29 & 7.7 & C & 0.03 & 4390 & & 0.92 & 338 & & 0.03 & 0.01 & $\mathrm{U}$ & 0.01 \\
\hline $\begin{array}{c}\text { North BCL } \\
\text { stockpile (middle) }\end{array}$ & J12NX5 & $6 / 27 / 06$ & 20100 & & 3.3 & 7.0 & & 0.29 & 6.8 & $\mathrm{C}$ & 0.03 & 4610 & & 0.92 & 340 & & 0.03 & 0.01 & U & 0.01 \\
\hline $\begin{array}{c}\text { North BCL } \\
\text { stockpile (south) }\end{array}$ & J12NX6 & $6 / 27 / 06$ & 18200 & & 3.3 & $6: 0$ & & 0.30 & 6.7 & $\mathrm{C}$ & 0.03 & 4230 & & 0.93 & 336 & & 0.03 & 0.02 & U & 0.02 \\
\hline $\begin{array}{c}\text { Southeastern BCL } \\
\text { stockpile }\end{array}$ & $\mathrm{J} 12 \mathrm{NX7}$ & $6 / 27 / 06$ & 15700 & & 3.3 & 7.1 & & 0.29 & 5.7 & $\mathrm{C}$ & 0.03 & 3770 & & 0.91 & 294 & & 0.03 & 0.11 & & 0.02 \\
\hline $\begin{array}{c}\text { Small BCL } \\
\text { stockpiles }\end{array}$ & $\mathrm{J} 12 \mathrm{NX} 8$ & $6 / 27 / 06$ & 18900 & & 3.3 & 8.9 & & 0.30 & 6.2 & $\mathrm{C}$ & 0.03 & 4100 & & 0.92 & 338 & & 0.03 & 0.14 & & 0.01 \\
\hline Equipment blank & $\mathrm{J} 12 \mathrm{P} 04$ & $6 / 27 / 06$ & 85.1 & & 3.3. & 0.29 & $\mathrm{U}$ & 0.29 & 0.07 & $C$ & 0.03 & 4.9 & & 0.91 & 3.2 & & 0.03 & 0.02 & $U$ & 0.02 \\
\hline
\end{tabular}

Attachment 
Attachment 1. 1607-B2:2 Verification Sampling Results.

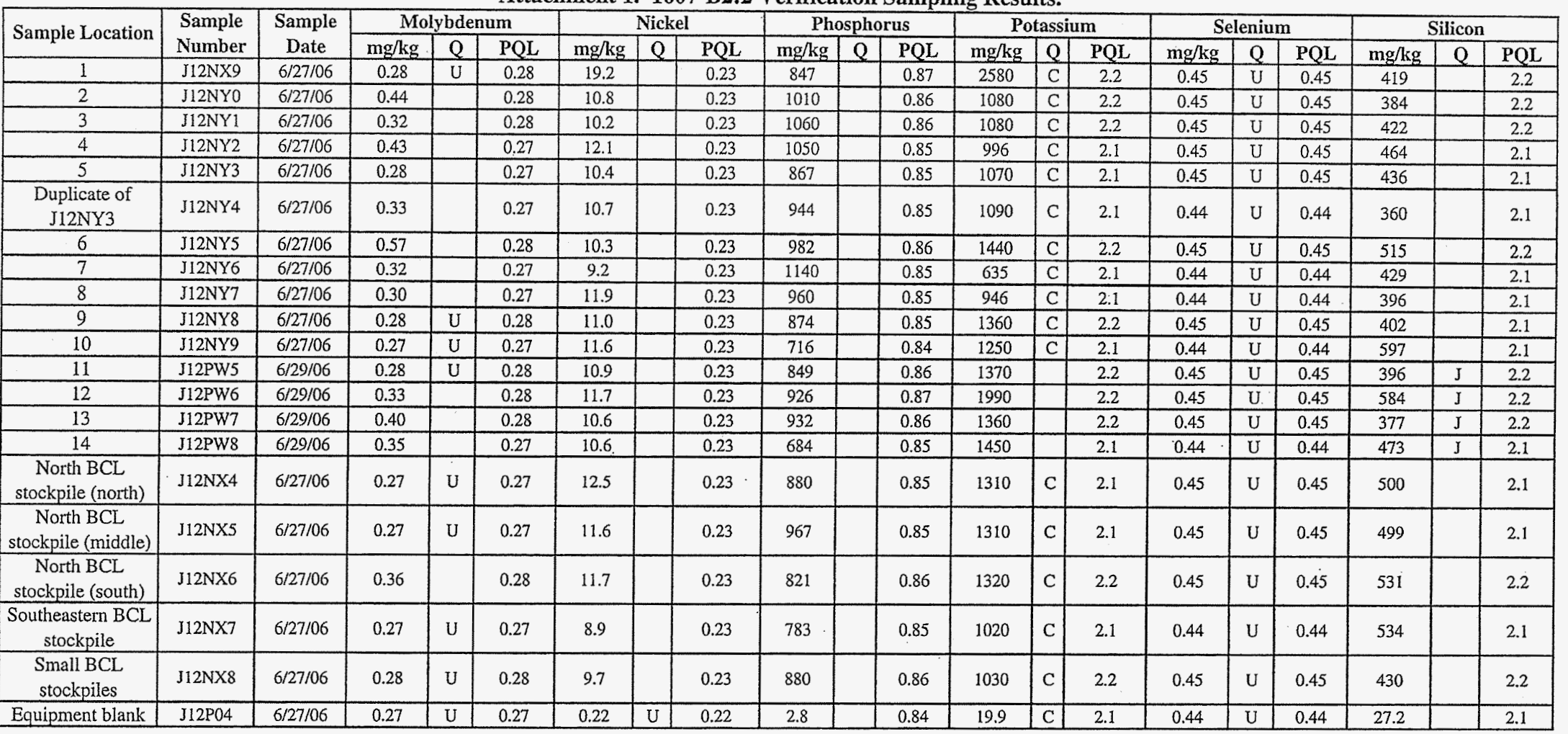

Attachment

Originator J.M. Capron Date Date

Date

Calc. No. $0100 \mathrm{~B}-\mathrm{CA}-\mathrm{V} 0292$ Rev. No. 
Attachment 1. 1607-B2:2 Verification Sampling Results.

\begin{tabular}{|c|c|c|c|c|c|c|c|c|c|c|c|c|c|c|c|c|c|c|c|c|}
\hline \multirow{2}{*}{ Sample Location } & \multirow{2}{*}{$\begin{array}{l}\text { Sample } \\
\text { Number }\end{array}$} & \multirow{2}{*}{$\begin{array}{c}\text { Sample } \\
\text { Date }\end{array}$} & \multicolumn{3}{|c|}{ Silver } & \multicolumn{3}{|c|}{ Sodium } & \multicolumn{3}{|c|}{ Strontium } & \multicolumn{3}{|c|}{ Thallium } & \multicolumn{3}{|c|}{ Tin } & \multicolumn{3}{|c|}{ Titanium } \\
\hline & & & $\mathrm{mg} / \mathrm{kg}$ & $\mathrm{Q}$ & PQL & $\mathrm{mg} / \mathrm{kg}$ & $\mathrm{Q}$ & PQL & $\mathrm{mg} / \mathrm{kg}$ & $Q$ & $\mathrm{PQL}$ & $\mathrm{mg} / \mathrm{kg}$ & Q & PQL & $\mathrm{mg} / \mathrm{kg}$ & $Q$ & $\overline{P Q L}$ & $\mathrm{mg} / \mathrm{kg}$ & $Q$ & PQL \\
\hline 1 & J12NX9 & $6 / 27 / 06$ & 0.07 & $\mathrm{U}$ & 0.07 & 578 & & 0.73 & 36.9 & & 0.01 & 0.67 & $\mathrm{U}$ & 0.67 & 1.0 & $\mathrm{U}$ & 1.0 & 1530 & & 0.03 \\
\hline 2 & $\mathrm{J12NY0}$ & $6 / 27 / 06$ & 0.07 & $\bar{U}$ & 0.07 & 205 & & 0.72 & 37.4 & & 0.01 & 0.67 & $\mathrm{U}$ & 0.67 & 1.0 & $\mathrm{U}$ & 1.0 & 1640 & & 0.03 \\
\hline 3 & J12NY1 & $6 / 27 / 06$ & 0.07 & $\mathrm{U}$ & 0.07 & 180 & & 0.73 & 25.2 & & 0.01 & 0.67 & $\overline{\mathrm{U}}$ & 0.67 & 1.0 & $\mathrm{U}$ & 1.0 & 1470 & & 0.03 \\
\hline 4 & J12NY2 & $6 / 27 / 06$ & 0.07 & $\mathrm{U}$ & 0.07 & 215 & & 0.72 & 40.5 & & 0.009 & 0.66 & $\mathrm{U}$ & 0.66 & 1.0 & $\mathrm{U}$ & 1.0 & 1530 & & 0.03 \\
\hline 5 & J12NY3 & $6 / 27 / 06$ & 0.07 & $\mathrm{U}$ & 0.07 & 144 & & 0.72 & 20.3 & & 0.009 & 0.66 & $U$ & 0.66 & 1.0 & $\mathrm{U}$ & 1.0 & 1590 & & 0.03 \\
\hline $\begin{array}{l}\text { Duplicate of } \\
\text { J12NY3 }\end{array}$ & J12NY4 & $6 / 27 / 06$ & 0.07 & $\mathrm{U}$ & 0.07 & 153 & & 0.72 & 22.5 & & 0.009 & 0.66 & U & 0.66 & 1.0 & U & 1.0 & 1350 & & 0.03 \\
\hline 6 & J12NY5 & $6 / 27 / 06$ & 0.07 & $\mathrm{U}$ & 0.07 & 254 & & 0.73 & 73.3 & & 0.01 & 0.67 & $\mathrm{U}$ & 0.67 & 1.0 & U & 1.0 & 1250 & & 0.03 \\
\hline 7 & J12NY6 & $6 / 27 / 06$ & 0.07 & $\mathrm{U}$ & 0.07 & 134 & & 0.71 & 18.7 & & 0.009 & 0.66 & $\mathrm{U}$ & 0.66 & 1.0 & $\bar{U}$ & 1.0 & 1770 & & 0.03 \\
\hline 8 & J12NY7 & $6 / 27 / 06$ & 0.07 & $\bar{U}$ & 0.07 & 172 & & 0.72 & 44.3 & & 0.009 & 0.66 & $\mathrm{U}$ & 0.66 & 1.0 & $\bar{U}$ & 1.0 & 1480 & & 0.03 \\
\hline 9 & J12NY8 & $6 / 27 / 06$ & 0.07 & $\mathrm{U}$ & 0.07 & 145 & & 0.72 & 32.7 & & 0.009 & 0.66 & $\mathrm{U}$ & 0.66 & 1.0 & $\mathrm{U}$ & 1.0 & 1370 & & 0.03 \\
\hline 10 & J12NY9 & $6 / 27 / 06$ & 0.07 & $\bar{U}$ & 0.07 & 125 & & 0.71 & 30.4 & & 0.009 & 0.66 & $\mathrm{U}$ & 0.66 & 1.0 & $\mathrm{U}$ & 1.0 & 871 & & 0.03 \\
\hline 11 & J12PW5 & $6 / 29 / 06$ & 0.07 & $\bar{U}$ & 0.07 & 151 & & 0.73 & 26.0 & C & 0.01 & 0.67 & $\mathrm{U}$ & 0.67 & 1.0 & $\mathrm{U}$ & 1.0 & 967 & & 0.03 \\
\hline 12 & J12PW6 & $6 / 29 / 06$ & 0.07 & $\bar{U}$ & 0.07 & 390 & & 0.73 & 86.2 & C & 0.01 & 0.67 & $\mathrm{U}$ & 0.67 & 1.0 & $\mathrm{U}$ & 1.0 & 1170 & & 0.03 \\
\hline 13 & J12PW7 & $6 / 29 / 06$ & 0.07 & $\mathrm{U}$ & 0.07 & 216 & & 0.73 & 33.4 & C & 0.01 & 0.67 & $\mathrm{U}$ & 0.67 & 1.0 & $\mathrm{U}$ & 1.0 & 1100 & & 0.03 \\
\hline 14 & $\mathrm{~J} 12 \mathrm{PW} 8$ & $6 / 29 / 06$ & 0.07 & U & 0.07 & 159 & & 0.72 & 25.7 & $\mathrm{C}$ & 0.009 & 0.66 & $\mathrm{U}$ & 0.66 & $1: 0$ & $\mathrm{U}$ & 1.0 & 697 & & 0.03 \\
\hline $\begin{array}{c}\text { North BCL } \\
\text { stockpile (north) }\end{array}$ & $\mathrm{J} 12 \mathrm{NX} 4$ & $6 / 27 / 06$ & 0.07 & U & 0.07 & 138 & & 0.72 & 27.6 & & 0.009 & 0.66 & $\mathrm{U}$ & 0.66 & 1.0 & U & 1.0 & 1330 & & 0.03 \\
\hline $\begin{array}{c}\text { North BCL } \\
\text { stockpile (middle) }\end{array}$ & J12NXS & $6 / 27 / 06$ & 0.07 & U & 0.07 & 150 & & 0.72 & 30.1 & & 0.009 & 0.66 & U & 0.66 & 1.0 & $\mathrm{U}$ & 1.0 & 1330 & & 0.03 \\
\hline $\begin{array}{c}\text { North BCL } \\
\text { stockpile (south) }\end{array}$ & $\mathrm{J} 12 \mathrm{NX} 6$ & $6 / 27 / 06$ & 0.07 & U & 0.07 & 154 & & 0.73 & 28.5 & & 0.01 & 0.67 & U & 0.67 & 1.0 & $\mathrm{U}$ & 1.0 & 1190 & & 0.03 \\
\hline $\begin{array}{c}\text { Southeastern } \mathrm{BCL} \\
\text { stockpile }\end{array}$ & $\mathrm{J} 12 \mathrm{NX7}$ & $6 / 27 / 06$ & 0.07 & U & 0.07 & 134 & & 0.72 & 31.0 & & 0.009 & 0.66 & U & 0.66 & 1.0 & U & 1.0 & 1020 & & 0.03 \\
\hline $\begin{array}{c}\text { Small BCL } \\
\text { stockpiles }\end{array}$ & $J 12 N \times 8$ & $6 / 27 / 06$ & 0.07 & U & 0.07 & 157 & & 0.72 & 28.9 & & 0.01 & 0.67 & $\mathrm{U}$ & 0.67 & 1.0 & U & 1.0 & 1330 & & 0.03 \\
\hline Equipment blank & $\mathrm{J} 12 \mathrm{PO} 4$ & $6 / 27 / 06$ & 0.07 & $\mathrm{U}$ & 0.07 & 7.5 & & 0.71 & 0.19 & & 0.009 & 0.66 & $\mathrm{U}$ & 0.66 & 1.0 & $\mathrm{U}$ & 1.0 & 1.4 & & 0.03 \\
\hline
\end{tabular}

Attachment

Originator J.M.Capron

Sheet No. $\quad 8$ of 20

Checked T.M. Blakley

Date $09 / 14 / 06$

0100B-CA-V0292

Date

0


Attachment 1. 1607-B2:2 Verification Sampling Results.

\begin{tabular}{|c|c|c|c|c|c|c|c|c|c|c|c|c|c|c|}
\hline \multirow{2}{*}{ Sample Location } & \multirow{2}{*}{$\begin{array}{l}\text { Sample } \\
\text { Number }\end{array}$} & \multirow{2}{*}{$\begin{array}{l}\text { Sample } \\
\text { Date }\end{array}$} & \multicolumn{3}{|c|}{ Uranium } & \multicolumn{3}{|c|}{ Vanadium } & \multicolumn{3}{|c|}{ Zinc } & \multicolumn{3}{|c|}{ Zirconium } \\
\hline & & & $\mathrm{mg} / \mathrm{kg}$ & $Q$ & $\mathrm{PQL}$ & $\mathrm{mg} / \mathrm{kg}$ & $Q$ & $\mathrm{PQL}$ & $\mathrm{mg} / \mathrm{kg}$ & $Q$ & PQL & $\mathrm{mg} / \mathrm{kg}$ & $Q$ & $\mathrm{PQL}$ \\
\hline 1 & $\mathrm{~J} 12 \mathrm{NX9}$ & $6 / 27 / 06$ & 0.85 & $\mathrm{U}$ & 0.85 & 53.0 & & 0.09 & 63.6 & & 0.15 & 22.8 & & 1.0 \\
\hline 2 & $\mathrm{~J} 12 \mathrm{NYO}$ & $6 / 27 / 06$ & 0.84 & U & 0.84 & 48.1 & & 0.09 & 46.3 & & 0.15 & 19.2 & & 1.0 \\
\hline 3 & $\mathrm{~J} 12 \mathrm{NY} 1$ & $6 / 27 / 06$ & 0.84 & $\mathrm{U}$ & 0.84 & 46.4 & & 0.09 & 41.7 & & 0.15 & 18.8 & & 1.0 \\
\hline 4 & J12NY2 & $6 / 27 / 06$ & 0.83 & $\bar{U}$ & 0.83 & 48.4 & & 0.09 & 44.1 & & 0.15 & 19.6 & & 1.0 \\
\hline 5 & $\mathrm{J12NY3}$ & $6 / 27 / 06$ & 0.83 & $\mathrm{U}$ & 0.83 & 46.8 & & 0.09 & 39.5 & & 0.15 & 22.5 & & 1.0 \\
\hline $\begin{array}{c}\text { Duplicate of } \\
\text { J12NY3 }\end{array}$ & $\mathrm{J} 12 \mathrm{NY} 4$ & $6 / 27 / 06$ & 0.83 & $U$ & 0.83 & 40.6 & & 0.08 & 38.5 & & 0.15 & 21.9 & & 1.0 \\
\hline 6 & J12NY5 & $6 / 27 / 06$ & 0.84 & $U$ & 0.84 & 39.5 & & 0.09 & 70.6 & & 0.15 & 17.0 & & 1.0 \\
\hline 7 & J12NY6 & $6 / 27 / 06$ & 0.83 & $U$ & 0.83 & 46.0 & & 0.08 & 36.2 & & 0.15 & 22.7 & & 1.0 \\
\hline 8 & J12NY7 & $6 / 27 / 06$ & 0.83 & $\mathrm{U}$ & 0.83 & 49.9 & & 0.09 & 43.5 & & 0.15 & 16.8 & & 1.0 \\
\hline 9 & J12NY8 & $6 / 27 / 06$ & 0.84 & $\mathrm{U}$ & 0.83 & 43.7 & & 0.09 & 43.4 & & 0.15 & 19.0 & & 1.0 \\
\hline 10 & J12NY9 & $6 / 27 / 06$ & 0.83 & U & 0.83 & 31.0 & & 0.08 & 39.6 & & 0.15 & 11.4 & & 0.99 \\
\hline 11 & J12PW5 & $6 / 29 / 06$ & 0.84 & $\mathrm{U}$ & 0.84 & 36.7 & & 0.09 & 37.2 & & 0.15 & 18.0 & & 1.0 \\
\hline 12 & J12PW6 & $6 / 29 / 06$ & 0.85 & $\mathrm{U}$ & 0.85 & 41.4 & & 0.09 & 67.0 & & 0.15 & 22.0 & & 1.0 \\
\hline 13 & J12PW7 & $6 / 29 / 06$ & 0.84 & $\mathrm{U}$ & 0.84 & 39.8 & & 0.09 & 40.2 & & 0.15 & 22.2 & & 1.0 \\
\hline 14 & J12PW8 & $6 / 29 / 06$ & 0.83 & U & 0.83 & 32.3 & & 0.09 & 37.2 & & 0.15 & 11.8 & & 1.0 \\
\hline $\begin{array}{c}\text { North BCL } \\
\text { stockpile (north) }\end{array}$ & $\mathrm{J} 12 \mathrm{NX} 4$ & $6 / 27 / 06$ & 0.83 & $\mathrm{U}$ & 0.83 & 46.6 & & 0.09 & 45.5 & & 0.15 & 16.7 & & 1.0 \\
\hline $\begin{array}{c}\text { North BCL. } \\
\text { stockpile (middle) }\end{array}$ & J12NX5 & $6 / 27 / 06$ & 0.83 & U & 0.83 & 44.7 & & 0.09 & 44.2 & & 0.15 & 19.0 & & 1.0 \\
\hline $\begin{array}{c}\text { North BCL } \\
\text { stockpile (south) }\end{array}$ & J12NX6 & $6 / 27 / 06$ & 0.84 & U & 0.84 & 42.9 & & 0.09 & 40.1 & & 0.15 & 17.0 & & 1.0 \\
\hline $\begin{array}{c}\text { Southeastern BCL } \\
\text { stockpile }\end{array}$ & $\mathrm{J} 12 \mathrm{NX} 7$ & $6 / 27 / 06$ & 0.83 & U & 0.83 & 32.3 & & 0.08 & 35.9 & & 0.15 & 14.7 & & 1.0 \\
\hline $\begin{array}{c}\text { Small BCL } \\
\text { stockpiles }\end{array}$ & $J 12 N X 8$ & $6 / 27 / 06$ & 0.84 & $U$ & 0.84 & 44.2 & & 0.09 & 49.6 & & 0.15 & 16.2 & & 1.0 \\
\hline Equipment blank & $\mathrm{J} 12 \mathrm{P} 04$ & $6 / 27 / 06$ & 0.82 & $\mathrm{U}$ & 0.82 & 0.08 & U & 0.08 & 0.70 & & 0.15 & 0.99 & $\mathrm{U}$ & 0.99 \\
\hline
\end{tabular}

\begin{tabular}{|c|c|c|c|}
\hline Attac & 1 & Sheet No. & 9 of 20 \\
\hline Origir & J.M. Capron & Date & $09 / 14 / 06$ \\
\hline Checked & T.M. Blakley & Date & \\
\hline Calc. No & $0100 \mathrm{~B}-\mathrm{CA}-\mathrm{V} 0292$ & Rev. No. & 0 \\
\hline
\end{tabular}


Attachment 1. 1607-B2:2 Verification Sampling Results.

\begin{tabular}{|c|c|c|c|c|c|c|c|c|c|c|c|c|}
\hline \multirow[t]{2}{*}{ Constituents } & \multicolumn{3}{|c|}{$\begin{array}{c}\text { J12NX9 } \\
\text { Location } 1 \\
\text { Sample Date } 6 / 27 / 06 \\
\end{array}$} & \multicolumn{3}{|c|}{$\begin{array}{c}\text { J12NY0 } \\
\text { Location } 2 \\
\text { Sample Date } 6 / 27 / 06 \\
\end{array}$} & \multicolumn{3}{|c|}{$\begin{array}{c}\text { J12NY1 } \\
\text { Location } 3 \\
\text { Sample Date } 6 / 27 / 06 \\
\end{array}$} & \multicolumn{3}{|c|}{$\begin{array}{c}\text { J12NY2 } \\
\text { Location } 4 \\
\text { Sample Date } 6 / 27 / 06\end{array}$} \\
\hline & $\mu \mathrm{g} / \mathrm{kg}$ & $Q$ & $\mathrm{PQL}$ & $\mu \mathrm{g} / \mathrm{kg}$ & Q & $\mathrm{PQL}$ & $\mu \mathrm{g} / \mathrm{kg}$ & $Q$ & PQL & $\mu \mathrm{g} / \mathrm{kg}$ & Q & PQL \\
\hline \multicolumn{13}{|c|}{ Polychlorinated Biphenyls } \\
\hline Aroclor -1016 & 14 & $\mathrm{U}$ & 14 & 13 & $\mathrm{U}$ & 13 & 13 & $\mathrm{U}$ & 13 & 13 & $\mathrm{U}$ & 13 \\
\hline Aroclor-1221 & 14 & $\mathrm{U}$ & 14 & 13 & $U$ & 13 & 13 & $\mathrm{U}$ & 13 & 13 & $\mathrm{U}$ & 13 \\
\hline Aroclor-1232 & 14 & $\mathrm{U}$ & 14 & 13 & $\mathrm{U}$ & 13 & 13 & $\mathrm{U}$ & 13 & 13 & $\mathrm{U}$ & 13 \\
\hline Aroclor -1242 & 14 & $\mathrm{U}$ & 14 & 13 & $\mathrm{U}$ & 13 & 13 & $\mathrm{U}$ & 13 & 13 & $\mathrm{U}$ & 13 \\
\hline Aroclor-1248 & 14 & $\mathrm{U}$ & 14 & 13 & $\mathrm{U}$ & 13 & 13 & $\mathrm{U}$ & 13 & 13 & $\mathrm{U}$ & 13 \\
\hline Aroclor-1254 & 14 & $\mathrm{U}$ & 14 & 13 & $\mathrm{U}$ & 13 & 13 & $\mathrm{U}$ & 13 & 13 & $\mathrm{U}$ & 13 \\
\hline Aroclor -1260 & 14 & $\mathrm{U}$ & 14 & 13 & $\mathrm{U}$ & 13 & 13 & $\mathrm{U}$ & 13 & 13 & $\mathrm{U}$ & 13 \\
\hline \multicolumn{13}{|c|}{ Pesticides } \\
\hline Aldrin & 1.4 & UD & 1.4 & 1.3 & UD & 1.3 & 1.3 & UD & 1.3 & 1.3 & UD & 1.3 \\
\hline alpha-BHC & 1.4 & UD & 1.4 & 1.3 & UD & 1.3 & 1.3 & UD & 1.3 & 1.3 & UD & 1.3 \\
\hline alpha-Chlordane & 1.4 & UD & 1.4 & 1.3 & UD & 1.3 & 1.3 & UD & 1.3 & 1.3 & UD & 1.3 \\
\hline beta-BHC & 1.4 & UD & 1.4 & 1.3 & UD & 1.3 & 1.3 & UD & 1.3 & 1.3 & UD & 1.3 \\
\hline delta-BHC & 1.4 & UD & 1.4 & 1.3 & UD & 1.3 & 1.3 & UD & 1.3 & 1.3 & UD & 1.3 \\
\hline Dichlorodiphenyldichloroethane & 1.4 & $\mathrm{UD}$ & 1.4 & 1.3 & UD & 1.3 & 1.3 & UD & 1.3 & 1.3 & UD & 1.3 \\
\hline Dichlorodiphenyldichloroethylene & 1.4 & UD & 1.4 & 1.3 & UD & 1.3 & 1.3 & UD & 1.3 & 1.3 & UD & 1.3 \\
\hline Dichlorodiphenyltrichloroethane & 1.4 & UD & 1.4 & 1.3 & UD & 1.3 & 1.3 & UD & 1.3 & 1.3 & UD & 1.3 \\
\hline Dieldrin & 1.4 & UD & 1.4 & 1.3 & UD & 1.3 & 1.3 & UD & 1.3 & 1.3 & UD & 1.3 \\
\hline Endosulfan I & 1.4 & UD & 1.4 & 1.3 & UD & 1.3 & 1.3 & UD & 1.3 & 1.3 & UD & 1.3 \\
\hline Endosulfan II & 1.4 & UD & 1.4 & 1.3 & UD & 1.3 & 1.3 & UD & 1.3 & 1.3 & UD & 1.3 \\
\hline Endosulfan sulfate & 1.4 & UD & 1.4 & 1.3 & UD & 1.3 & 1.3 & UD & 1.3 & 1.3 & UD & 1.3 \\
\hline Endrin & 1.4 & UD & 1.4 & 1.3 & UD & 1.3 & 1.3 & UD & 1.3 & 1.3 & UD & 1.3 \\
\hline Endrin aldehyde & 1.4 & UD & 1.4 & 1.3 & UD & 1.3 & 1.3 & UD & 1.3 & 1.3 & UD & 1.3 \\
\hline Endrin ketone & 1.4 & UD & 1.4 & 1.3 & UD & 1.3 & 1.3 & UD & 1.3 & 1.3 & UD & 1.3 \\
\hline gamma-BHC (Lindane) & 1.4 & UD & 1.4 & 1.3 & UD & 1.3 & 1.3 & UD & 1.3 & 1.3 & UD & 1.3 \\
\hline gamma-Chlordane & 1.4 & UD & 1.4 & 1.3 & UD & 1.3 & 1.3 & UD & 1.3 & 1.3 & UD & 1.3 \\
\hline Heptachlor & 1.4 & UD & 1.4 & 1.3 & UD & 1.3 & 1.3 & UD & 1.3 & 1.3 & UD & 1.3 \\
\hline Heptachlor epoxide & 1.4 & UD & 1.4 & 1.3 & UD & 1.3 & 1.3 & UD & 1.3 & 1.3 & UD & 1.3 \\
\hline Methoxychlor & 1.2 & JID & 1.4 & 1.6 & $\mathrm{IID}$ & 1.3 & 1.3 & UD & 1.3 & 1.3 & UD & 1.3 \\
\hline Toxaphene & 14 & UD & 14 & 13 & UD & 13 & 13 & UD & 13 & 13 & UD & 13 \\
\hline \multicolumn{13}{|c|}{ Semivolatile Organic Compounds } \\
\hline 1,2,4-Trichlorobenzene & 340 & $\mathrm{U}$ & 340 & 340 & $\mathrm{U}$ & 340 & 340 & $\mathrm{U}$ & 340 & 330 & $\mathrm{U}$ & 330 \\
\hline 1,2-Dichlorobenzene & 340 & $\mathrm{U}$ & 340 & 340 & U & 340 & 340 & $\mathrm{U}$ & 340 & 330 & $\mathrm{U}$ & 330 \\
\hline 1,3-Dichlorobenzene & 340 & $\mathrm{U}$ & 340 & 340 & $\mathrm{U}$ & 340 & 340 & $\mathrm{U}$ & 340 & 330 & $U$ & 330 \\
\hline 1,4-Dichlorobenzene & 340 & $\mathrm{U}$ & 340 & 340 & $\mathrm{U}$ & 340 & 340 & $\mathrm{U}$ & 340 & 330 & $\mathrm{U}$ & 330 \\
\hline 2,4,5-Trichlorophenol & 850 & $\mathrm{U}$ & 850 & 840 & $\mathrm{U}$ & 840 & 840 & $\mathrm{U}$ & 840 & 840 & $\mathrm{U}$ & 840 \\
\hline 2,4,6-Trichlorophenol & 340 & $\mathrm{U}$ & 340 & 340 & $\mathrm{U}$ & 340 & 340 & $\mathrm{U}$ & 340 & 330 & $\mathrm{U}$ & 330 \\
\hline 2,4-Dichlorophenol & 340 & $\mathrm{U}$ & 340 & 340 & $\mathrm{U}$ & 340 & 340 & $\mathrm{U}$ & 340 & 330 & $\mathrm{U}$ & 330 \\
\hline 2,4-Dimethylphenol & 340 & $\mathrm{U}$ & 340 & 340 & $\mathrm{U}$ & 340 & 340 & $\mathrm{U}$ & 340 & 330 & $\mathrm{U}$ & 330 \\
\hline 2,4-Dinitrophenol & 850 & $\mathrm{U}$ & 850 & 840 & $\mathrm{U}$ & 840 & 840 & $\mathrm{U}$ & 840 & 840 & $\mathrm{U}$ & 840 \\
\hline 2,4-Dinitrotoluene & 340 & $\mathrm{U}$ & 340 & 340 & $\mathrm{U}$ & 340 & 340 & $\mathrm{U}$ & 340 & 330 & $\mathrm{U}$ & 330 \\
\hline 2,6-Dinitrotoluene & 340 & $\mathrm{U}$ & 340 & 340 & $\mathrm{U}$ & 340 & 340 & $\bar{U}$ & 340 & 330 & $\mathrm{U}$ & 330 \\
\hline 2-Chloronaphtbalene & 340 & $\mathrm{U}$ & 340 & 340 & $\mathrm{U}$ & 340 & 340 & $\mathrm{U}$ & 340 & 330 & $\mathrm{U}$ & 330 \\
\hline 2-Chlorophenol & 340 & $\mathrm{U}$ & 340 & 340 & $\mathrm{U}$ & 340 & 340 & $\mathrm{U}$ & 340 & 330 & $\mathrm{U}$ & 330 \\
\hline 2-Methylnaphthalene & 340 & $\mathrm{U}$ & 340 & 340 & $\mathrm{U}$ & 340 & 340 & $\mathrm{U}$ & 340 & 330 & $\mathrm{U}$ & 330 \\
\hline 2-Methylphenol (cresol, o-) & 340 & $\mathrm{U}$ & 340 & 340 & $\mathrm{U}$ & 340 & 340 & $\mathrm{U}$ & 340 & 330 & $\mathrm{U}$ & 330 \\
\hline 2-Nitroaniline & 850 & $\mathrm{U}$ & 850 & 840 & $\mathrm{U}$ & 840 & 840 & $\mathrm{U}$ & 840 & 840 & $\mathrm{U}$ & 840 \\
\hline 2-Nitrophenol & 340 & $\mathrm{U}$ & 340 & 340 & $\mathrm{U}$ & 340 & 340 & $\mathrm{U}$ & 340 & 330 & $\mathrm{U}$ & 330 \\
\hline
\end{tabular}

Attachment

Originator

Checked

Calc. No.

\begin{tabular}{l}
$\frac{1}{\text { J.M. Capron }}$ \\
\hline T. M. Blakley \\
\hline 0100B-CA-V0292
\end{tabular}

Sheet No. $\quad 10$ of 20

Date $09 / 14 / 06$

Date

Rev. No. 0 
Attachment 1. 1607-B2:2 Verification Sampling Results.

\begin{tabular}{|c|c|c|c|c|c|c|c|c|c|c|c|c|}
\hline \multirow[t]{2}{*}{ Constituents } & \multicolumn{3}{|c|}{$\begin{array}{c}\text { J12NX9 } \\
\text { Location 1 } \\
\text { Sample Date 6/27/06 }\end{array}$} & \multicolumn{3}{|c|}{\begin{tabular}{c|} 
J12NY0 \\
Location 2 \\
Sample Date $6 / 27 / 06$ \\
\end{tabular}} & \multicolumn{3}{|c|}{$\begin{array}{c}\text { J12NY1 } \\
\text { Location } 3 \\
\text { Sample Date } 6 / 27 / 06\end{array}$} & \multicolumn{3}{|c|}{$\begin{array}{c}\text { J12NY2 } \\
\text { Location } 4 \\
\text { Sample Date } 6 / 27 / 06\end{array}$} \\
\hline & $\mu \mathrm{g} / \mathrm{kg}$ & Q & PQL & $\mu \mathrm{g} / \mathrm{kg}$ & Q & PQL & $\mu \mathrm{g} / \mathrm{kg}$ & $\mathbf{Q}$ & PQL & $\mu \mathrm{g} / \mathrm{kg}$ & $\mathbf{Q}$ & PQL \\
\hline \multicolumn{13}{|c|}{ Semivolatile Organic Compounds (continued) } \\
\hline 3,3'-Dichlorobenzidine & 340 & $\mathrm{U}$ & 340 & 340 & $\mathrm{U}$ & 340 & 340 & $U$ & 340 & 330 & $\mathrm{U}$ & 330 \\
\hline 3-Nitroaniline & 850 & $\mathrm{U}$ & 850 & 840 & $\mathrm{U}$ & 840 & 840 & $\mathrm{U}$ & 840 & 840 & $\bar{U}$ & 840 \\
\hline 4,6-Dinitro-2-methylphenol & 850 & $\mathrm{U}$ & 850 & 840 & $\mathrm{U}$ & 840 & 840 & $\mathrm{U}$ & 840 & 840 & $\mathrm{U}$ & 840 \\
\hline 4-Bromophenyl-phenylether & 340 & $\mathrm{U}$ & 340 & 340 & $\mathrm{U}$ & 340 & 340 & $\mathrm{U}$ & 340 & 330 & $\mathrm{U}$ & 330 \\
\hline 4-Chloro-3-methylphenol & 340 & $\mathrm{U}$ & 340 & 340 & $\mathrm{U}$ & 340 & 340 & $U$ & 340 & 330 & $\mathrm{U}$ & 330 \\
\hline 4-Chloroaniline & 340 & $\mathrm{U}$ & 340 & 340 & $\mathrm{U}$ & 340 & 340 & $\mathrm{U}$ & 340 & 330 & $\mathrm{U}$ & 330 \\
\hline 4-Chlorophenyl-phenylether & 340 & $\mathrm{U}$ & 340 & 340 & $\mathrm{U}$ & 340 & 340 & $\mathrm{U}$ & 340 & 330 & $\mathrm{U}$ & 330 \\
\hline 4-Methylphenol (p-cresol) & 340 & $\mathrm{U}$ & 340 & 340 & $\mathrm{U}$ & 340 & 340 & $\mathrm{U}$ & 340 & 330 & $\mathrm{U}$ & 330 \\
\hline 4-Nitroaniline & 850 & U & 850 & 840 & $U$ & 840 & 840 & $\mathrm{U}$ & 840 & 840 & $\mathrm{U}$ & 840 \\
\hline 4-Nitrophenol & 850 & $\mathrm{U}$ & 850 & 840 & $\mathrm{U}$ & 840 & 840 & $U$ & 840 & 840 & $\mathrm{U}$ & 840 \\
\hline Acenaphthene & 340 & $\mathrm{U}$ & 340 & 340 & $\mathrm{U}$ & 340 & 340 & $\mathrm{U}$ & 340 & 330 & $\mathrm{U}$ & 330 \\
\hline Acenaphthylene & 340 & $\mathrm{U}$ & 340 & 340 & $\mathrm{U}$ & 340 & 340 & $\mathrm{U}$ & 340 & 330 & $\mathrm{U}$ & 330 \\
\hline Anthracene & 340 & $\mathrm{U}$ & 340 & 340 & $\mathrm{U}$ & 340 & 340 & $\mathrm{U}$ & 340 & 330 & $\mathrm{U}$ & 330 \\
\hline Benzo(a)anthracene & 340 & $\mathrm{U}$ & 340 & 340 & $\mathrm{U}$ & 340 & 22 & $\mathrm{~J}$ & 340 & 330 & $\mathrm{U}$ & 330 \\
\hline Benzo(a)pyrene & 340 & $\mathrm{U}$ & 340 & 18 & $\mathrm{~J}$ & 340 & 340 & $\mathrm{U}$ & 340 & 330 & $\mathrm{U}$ & 330 \\
\hline Benzo(b)fluoranthene & 340 & $\mathrm{U}$ & 340 & 340 & $\mathrm{U}$ & 340 & 340 & $\mathrm{U}$ & 340 & 330 & $\mathrm{U}$ & 330 \\
\hline Benzo $(\mathrm{g}, \mathrm{h}, \mathrm{i})$ perylene & 340 & $\mathrm{U}$ & 340 & 23 & $\mathrm{~J}$ & 340 & 21 & $\mathrm{~J}$ & 340 & 330 & $\mathrm{U}$ & 330 \\
\hline Benzo(k)fluoranthene & 340 & $\mathrm{U}$ & 340 & 340 & $\mathrm{U}$ & 340 & 20 & $\mathrm{~J}$ & 340 & 330 & $\mathrm{U}$ & 330 \\
\hline bis(2-Chloro-1-methylethyl)ether & 340 & $\mathrm{U}$ & 340. & 340 & $\mathrm{U}$ & 340 & 340 & $\mathrm{U}$ & 340 & 330 & $\mathrm{U}$ & 330 \\
\hline bis(2-Chloroethoxy)methane & 340 & $\mathrm{U}$ & 340 & 340 & $\mathrm{U}$ & 340 & 340 & $\mathrm{U}$ & 340 & 330 & $\mathrm{U}$ & 330 \\
\hline bis(2-Chloroethyl)ether & 340 & $\mathrm{U}$ & 340 & 340 & $\mathrm{U}$ & 340 & 340 & $\bar{U}$ & 340 & 330 & $\mathrm{U}$ & 330 \\
\hline bis(2-Ethylhexyl)phthalate & 970 & B & 340 & 83 & JB & 340 & 76 & JB & 340 & 61 & $\mathrm{JB}$ & 330 \\
\hline Butylbenzylphthalate & 340 & $\mathrm{U}$ & 340 & 340 & $\mathrm{U}$ & 340 & 340 & $\mathrm{U}$ & 340 & 330 & $\mathrm{U}$ & 330 \\
\hline Carbazole & 340 & $\mathrm{U}$ & 340 & 340 & $\mathrm{U}$ & 340 & 340 & $\mathrm{U}$ & 340 & 330 & $\mathrm{U}$ & 330 \\
\hline Chrysene & 340 & $\mathrm{U}$ & 340 & 340 & $\mathrm{U}$ & 340 & 27 & $\mathrm{~J}$ & 340 & 330 & $\mathrm{U}$ & 330 \\
\hline Di-n-butylphthalate & 19 & $\mathrm{JB}$ & 340 & 23 & $\mathrm{JB}$ & 340 & 34 & $\mathrm{JB}$ & 340 & 31 & $\mathrm{JB}$ & 330 \\
\hline Di-n-octylphthalate & 340 & $\mathrm{U}$ & 340 & 340 & $\mathrm{U}$ & 340 & 340 & $\mathrm{U}$ & 340 & 330 & $\mathrm{U}$ & 330 \\
\hline Dibenz $(a, h)$ anthracene & 340 & $\mathrm{U}$ & 340 & 340 & $\mathrm{U}$ & 340 & 22 & $\mathrm{~J}$ & 340 & 330 & $\mathrm{U}$ & 330 \\
\hline Dibenzofuran & 340 & $\mathrm{U}$ & 340 & 340 & $\mathrm{U}$ & 340 & 340 & $\mathrm{U}$ & 340 & 330 & $\mathrm{U}$ & 330 \\
\hline Diethylphthalate & 340 & $\mathrm{U}$ & 340 & 340 & $\mathrm{U}$ & 340 & 340 & $\mathrm{U}$ & 340 & 330 & $\mathrm{U}$ & 330 \\
\hline Dimethylphthalate & 340 & $\mathrm{U}$ & 340 & 340 & $\bar{U}$ & 340 & 340 & $\mathrm{U}$ & 340 & 330 & $\mathrm{U}$ & 330 \\
\hline Fluoranthene & 340 & $\mathrm{U}$ & 340 & 340 & $\mathrm{U}$ & 340 & 30 & $\mathrm{~J}$ & 340 & 330 & $\mathrm{U}$ & 330 \\
\hline Fluorene & 340 & $\mathrm{U}$ & 340 & 340 & $\mathrm{U}$ & 340 & 340 & $\mathrm{U}$ & 340 & 330 & $\mathrm{U}$ & 330 \\
\hline Hexachlorobenzene & 340 & $\mathrm{U}$ & 340 & 340 & $\mathrm{U}$ & 340 & 340 & $\mathrm{U}$ & 340 & 330 & $\mathrm{U}$ & 330 \\
\hline Hexachlorobutadiene & 340 & $\mathrm{U}$ & 340 & 340 & $\mathrm{U}$ & 340 & 340 & $\mathrm{U}$ & 340 & 330 & $\mathrm{U}$ & 330 \\
\hline Hexachlorocyclopentadiene & 340 & $\mathrm{U}$ & 340 & 340 & $\mathrm{U}$ & 340 & 340 & $\mathrm{U}$ & 340 & 330 & $\mathrm{U}$ & 330 \\
\hline Hexachloroethane & 340 & $\mathrm{U}$ & 340 & 340 & $\mathrm{U}$ & 340 & 340 & $\mathrm{U}$ & 340 & 330 & $\mathrm{U}$ & 330 \\
\hline Indeno( $1,2,3$-cd)pyrene & 340 & $\mathrm{U}$ & 340 & 20 & $\mathrm{~J}$ & 340 & 19 & $J$ & 340 & 330 & $\mathrm{U}$ & 330 \\
\hline Isophorone & 340 & $\mathrm{U}$ & 340 & 340 & $\mathrm{U}$ & 340 & 340 & $\mathrm{U}$ & 340 & 330 & $\mathrm{U}$ & 330 \\
\hline N-Nitroso-di-n-dipropylamine & 340 & $\mathrm{U}$ & 340 & 340 & $\mathrm{U}$ & 340 & 340 & $\mathrm{U}$ & 340 & 330 & $\mathrm{U}$ & 330 \\
\hline N-Nitrosodiphenylamine & 340 & U & 340 & 340 & $\mathrm{U}$ & 340 & 340 & $\mathrm{U}$ & 340 & 330 & $\mathrm{U}$ & 330 \\
\hline Naphthalene & 340 & $\mathrm{U}$ & 340 & 340 & $\mathrm{U}$ & 340 & 340 & $\mathrm{U}$ & 340 & 330 & $\mathrm{U}$ & 330 \\
\hline Nitrobenzene & 340 & $\mathrm{U}$ & 340 & 340 & $\mathrm{U}$ & 340 & 340 & $\mathrm{U}$ & 340 & 330 & U & 330 \\
\hline Pentachlorophenol & 850 & $\mathrm{U}$ & 850 & 840 & $\mathrm{U}$ & 840 & 840 & $\mathrm{U}$ & 840 & 840 & $\mathrm{U}$ & 840 \\
\hline Phenanthrene & 340 & $U$ & 340 & 340 & $\mathrm{U}$ & .340 & 29 & $\mathrm{~J}$ & 340 & 330 & $\mathrm{U}$ & 330 \\
\hline Phenol & 340 & $\mathrm{U}$ & 340 & 340 & $\mathrm{U}$ & 340 & 340 & $\bar{U}$ & 340 & 330 & $\mathrm{U}$ & 330 \\
\hline Pyrene & 340 & $\mathrm{U}$ & 340 & 340 & $\mathrm{U}$ & 340 & 55 & $\mathrm{~J}$ & 340 & 330 & $\mathrm{U}$ & 330 \\
\hline
\end{tabular}

Attachment

Originator

Checked

Calc. No.

\begin{tabular}{|c|}
\hline J. M. Capron \\
\hline T. M. Blakley \\
\hline 0100B-CA-V0292 \\
\hline
\end{tabular}

Sheet No. 11 of 20

Date $09 / 14 / 06$

Date

Rev, No.

0 
Attachment 1. 1607-B2:2 Verification Sampling Results.

\begin{tabular}{|c|c|c|c|c|c|c|c|c|c|c|c|c|}
\hline \multirow[t]{2}{*}{ Constituents } & \multicolumn{3}{|c|}{$\begin{array}{c}\text { J12NY3 } \\
\text { Location 5 } \\
\text { Sample Date 6/27/06 }\end{array}$} & \multicolumn{3}{|c|}{\begin{tabular}{|c|}
$\mathrm{J12NY4}$ \\
Duplicate of J12NY3 \\
Sample Date $6 / 27 / 06$ \\
\end{tabular}} & \multicolumn{3}{|c|}{$\begin{array}{c}\text { J12NY5 } \\
\text { Location } 6 \\
\text { Sample Date } 6 / 27 / 06 \\
\end{array}$} & \multicolumn{3}{|c|}{$\begin{array}{c}\text { J12NY6 } \\
\text { Location } 7 \\
\text { Sample Date } 6 / 27 / 06 \\
\end{array}$} \\
\hline & $\mu \mathrm{g} / \mathrm{kg}$ & $Q$ & PQL & $\mu \mathrm{g} / \mathrm{kg}$ & $Q$ & PQL & $\mu \mathrm{g} / \mathrm{kg}$ & Q & PQL & $\mu \mathrm{g} / \mathrm{kg}$ & $Q$ & PQL \\
\hline \multicolumn{13}{|c|}{ Polychlorinated Biphenyls } \\
\hline Aroclor -1016 & 13 & $\mathrm{U}$ & 13 & 13 & $\mathrm{U}$ & 13 & 13 & $\mathrm{U}$ & 13 & 13 & $\mathrm{U}$ & 13 \\
\hline Aroclor- 1221 & 13 & $\mathrm{U}$ & 13 & 13 & $\mathrm{U}$ & 13 & 13 & $\mathrm{U}$ & 13 & 13 & $\mathrm{U}$ & 13 \\
\hline Aroclor-1232 & 13 & $\mathrm{U}$ & 13 & 13 & $\mathrm{U}$ & 13 & 13 & $\mathrm{U}$ & 13 & 13 & $\mathrm{U}$ & 13 \\
\hline Aroclor-1242 & 13 & $U$ & 13 & 13 & $\mathrm{U}$ & 13 & 13 & $\mathrm{U}$ & 13 & 13 & $\mathrm{U}$ & 13 \\
\hline Aroclor-1248 & 13 & $\mathrm{U}$ & 13 & 13 & $\mathrm{U}$ & 13 & 13 & $\mathrm{U}$ & 13 & 13 & $\mathrm{U}$ & 13 \\
\hline Aroclor- 1254 & 13 & $\mathrm{U}$ & 13 & 13 & $\mathrm{U}$ & 13 & 330 & & 13 & 13 & $\mathrm{U}$ & 13 \\
\hline Aroclor-1260 & 13 & $\mathrm{U}$ & 13 & 13 & $\mathrm{U}$ & 13 & 13 & $\mathrm{U}$ & 13 & 13 & $\mathrm{U}$ & 13 \\
\hline \multicolumn{13}{|c|}{ Pesticides } \\
\hline Aldrin & 1.3 & UD & 1.3 & 1.3 & UD & 1.3 & 1.3 & UD. & 1.3 & 1.3 & UD & 1.3 \\
\hline alpha-BHC & 1.3 & UD & 1.3 & 1.3 & UD & 1.3 & 1.3 & UD & 1.3 & 1.3 & UD & 1.3 \\
\hline alpha-Chlordane & 1.3 & UD & 1.3 & 1.3 & UD & 1.3 & 1.3 & UD & 1.3 & 1.3 & UD & 1.3 \\
\hline beta-BHC & 1.3 & UD & 1.3 & 1.3 & UD & 1.3 & 1.9 & $\mathrm{D}$ & 1.3 & 1.3 & UD & 1.3 \\
\hline delta-BHC & 1.3 & UD & 1.3 & 1.3 & UD & 1.3 & 1.3 & UD & 1.3 & 1.3 & UD & 1.3 \\
\hline Dichlorodiphenyldichloroethane & 1.3 & UD & 1.3 & 1.3 & UD & 1.3 & 1.3 & UD & 1.3 & 1.3 & UD & 1.3 \\
\hline Dichlorodiphenyldichloroethylene & 1.3 & UD & 1.3 & 1.3 & UD & 1.3 & 18 & D & 1.3 & 1.3 & UD & 1.3 \\
\hline Dichlorodiphenyltrichloroethane & 1.3 & UD & 1.3 & 1.3 & UD & 1.3 & 1.3 & UD & 1.3 & 1.3 & UD & 1.3 \\
\hline Dieldrin & 1.3 & UD & 1.3 & 1.3 & UD & 1.3 & 1.3 & UD & 1.3 & 1.3 & UD & 1.3 \\
\hline Endosulfan I & 1.3 & UD & 1.3 & 1.3 & UD & 1.3 & 6.9 & $\mathrm{D}$ & 1.3 & 1.3 & UD & 1.3 \\
\hline Endosulfan II & 1.3 & UD & 1.3 & 1.3 & UD & 1.3 & 3.4 & ID & 1.3 & 1.3 & UD & 1.3 \\
\hline Endosulfan sulfate & 1.3 & UD & 1.3 & 1.3 & UD & 1.3 & 1.3 & UD & 1.3 & 1.3 & UD & 1.3 \\
\hline Endrin & 1.3 & UD & 1.3 & 1.3 & UD & 1.3 & 1.3 & UD & 1.3 & 1.3 & UD & 1.3 \\
\hline Endrin aldehyde & 1.3 & UD & 1.3 & 1.3 & UD & 1.3 & 7.4 & D & 1.3 & 1.3 & UD & 1.3 \\
\hline Endrin ketone & 1.3 & UD & 1.3 & 1.3 & UD & 1.3 & 1.1 & JID & 1.3 & 1.3 & UD & 1.3 \\
\hline gamma-BHC (Lindane) & 1.3 & UD & 1.3 & 1.3 & UD & 1.3 & 1.3 & UD & 1.3 & 1.3 & UD & 1.3 \\
\hline gamma-Chlordane & 1.3 & UD & 1.3 & 1.3 & UD & 1.3 & 1.3 & UD & 1.3 & 1.3 & UD & 1.3 \\
\hline Heptachlor & 1.3 & UD & 1.3 & 1.3 & UD & 1.3 & 1.3 & UD & 1.3 & 1.3 & UD & 1.3 \\
\hline Heptachlor epoxide & 1.3 & UD & 1.3 & 1.3 & UD & 1.3 & 1.3 & UD & 1.3 & 1.3 & UD & 1.3 \\
\hline Methoxychlor & 1.3 & UD & 1.3 & 1.3 & UD & 1.3 & 1.3 & UD & 1.3 & 1.3 & UD & 1.3 \\
\hline Toxaphene & 13 & UD & 13 & 13 & UD & 13 & 13 & UD & 13 & 13 & UD & 13 \\
\hline \multicolumn{13}{|c|}{ Semivolatile Organic Compounds } \\
\hline 1,2,4-Trichlorobenzene & 330 & $\mathrm{U}$ & 330 & 340 & $\mathrm{U}$ & 340 & 340 & $\mathrm{U}$ & 340 & 340 & $\mathrm{U}$ & 340 \\
\hline 1,2-Dichlorobenzene & 330 & $U$ & 330 & 340 & U & 340 & 340 & $\mathrm{U}$ & 340 & 340 & $\mathrm{U}$ & 340 \\
\hline 1,3-Dichlorobenzene & 330 & $\mathrm{U}$ & 330 & 340 & $\mathrm{U}$ & 340 & 340 & $\mathrm{U}$ & 340 & 340 & $\mathrm{U}$ & 340 \\
\hline 1,4-Dichlorobenzene & 330 & U & 330 & 340 & $U$ & 340 & 340 & $\mathrm{U}$ & 340 & 340 & $\mathrm{U}$ & 340 \\
\hline 2,4,5-Trichlorophenol & 840 & $\mathrm{U}$ & 840 & 840 & $\mathrm{U}$ & 840 & 840 & $\mathrm{U}$ & 840 & 840 & $\mathrm{U}$ & 840 \\
\hline $2,4,6$-Trichlorophenol & 330 & $\mathrm{U}$ & 330 & 340 & $\mathrm{U}$ & 340 & 340 & $\mathrm{U}$ & 340 & 340 & $\mathrm{U}$ & 340 \\
\hline 2,4-Dichlorophenol & 330 & $\mathrm{U}$ & 330 & 340 & $\mathrm{U}$ & 340 & 340 & $\mathrm{U}$ & .340 & 340 & $\mathrm{U}$ & 340 \\
\hline 2,4-Dimethylphenol & 330 & $\mathrm{U}$ & 330 & 340 & $U$ & 340 & 340 & $\mathrm{U}$ & 340 & 340 & $\mathrm{U}$ & 340 \\
\hline 2,4-Dinitrophenol & 840 & $\mathrm{U}$ & 840 & 840 & U & 840 & 840 & $\mathrm{U}$ & 840 & 840 & $\mathrm{U}$ & 840 \\
\hline 2,4-Dinitrotoluene & 330 & $\mathrm{U}$ & 330 & 340 & U & 340 & 340 & $\mathrm{U}$ & 340 & 340 & $\mathrm{U}$ & 340 \\
\hline 2,6-Dinitrotoluene & 330 & $\mathrm{U}$ & 330 & 340 & $\mathrm{U}$ & 340 & 340 & $\mathrm{U}$ & 340 & 340 & $\mathrm{U}$ & 340 \\
\hline 2-Chloronaphthalene & 330 & $\mathrm{U}$ & 330 & 340 & $\mathrm{U}$ & 340 & 340 & $\mathrm{U}$ & 340 & 340 & $\mathrm{U}$ & 340 \\
\hline 2-Chlorophenol & 330 & $\mathrm{U}$ & 330 & 340 & $\mathrm{U}$ & 340 & 340 & $\mathrm{U}$ & 340 & 340 & $\bar{U}$ & 340 \\
\hline 2-Methylnaphthalene & 330 & $\mathrm{U}$ & 330 & 340 & $\mathrm{U}$ & 340 & 19 & $\mathrm{~J}$ & 340 & 340 & $\mathrm{U}$ & 340 \\
\hline 2-Methylphenol (cresol, o-) & 330 & $\mathrm{U}$ & 330 & 340 & $\mathrm{U}$ & 340 & 340 & $\mathrm{U}$ & 340 & 340 & U & 340 \\
\hline 2-Nitroaniline & 840 & $\mathrm{U}$ & 840 & 840 & $\mathrm{U}$ & 840 & 840 & $\mathrm{U}$ & 840 & 840 & $\mathrm{U}$ & 840 \\
\hline 2-Nitrophenol & 330 & $\mathrm{U}$ & 330 & 340 & $\mathrm{U}$ & 340 & 340 & $\mathrm{U}$ & 340 & 340 & U & 340 \\
\hline & & & & \multicolumn{2}{|c|}{ Attachment } & \multicolumn{3}{|c|}{1} & \multicolumn{2}{|c|}{ Sheet No. } & \multicolumn{2}{|c|}{12 of 20} \\
\hline & & & & \multirow{2}{*}{\multicolumn{2}{|c|}{ Originator }} & \multicolumn{4}{|c|}{ J.M. Capron } & Date & \multicolumn{2}{|c|}{$09 / 14 / 06$} \\
\hline & & & & \multirow{2}{*}{\multicolumn{2}{|c|}{$\begin{array}{l}\text { Checked } \\
\text { Calc. No. }\end{array}$}} & T.M. Bl & akley & & & Date & & \\
\hline & & & & & & $\overline{0100 \mathrm{~B}-\mathrm{C}}$ & A-V029 & & & Rev. No & & 0 \\
\hline
\end{tabular}


Attachment 1. 1607-B2:2 Verification Sampling Results.

\begin{tabular}{|c|c|c|c|c|c|c|c|c|c|c|c|c|}
\hline \multirow[t]{2}{*}{ Constituents } & \multicolumn{3}{|c|}{$\begin{array}{c}\text { J12NY3 } \\
\text { Location } 5 \\
\text { Sample Date 6/27/06 } \\
\end{array}$} & \multicolumn{3}{|c|}{$\begin{array}{c}\text { J12NY4 } \\
\text { Duplicate of J12NY3 } \\
\text { Sample Date } 6 / 27 / 06\end{array}$} & \multicolumn{3}{|c|}{$\begin{array}{c}\text { J12NY5 } \\
\text { Location } 6 \\
\text { Sample Date } 6 / 27 / 06 \\
\end{array}$} & \multicolumn{3}{|c|}{$\begin{array}{c}\text { J12NY6 } \\
\text { Location } 7 \\
\text { Sample Date } 6 / 27 / 06\end{array}$} \\
\hline & $\mu \mathrm{g} / \mathrm{kg}$ & Q & $\mathrm{PQL}$ & $\mu \mathrm{g} / \mathrm{kg}$ & Q & PQL & $\mu \mathrm{g} / \mathrm{kg}$ & Q & PQL & $\mu \mathrm{g} / \mathrm{kg}$ & $\mathbf{Q}$ & PQL \\
\hline \multicolumn{13}{|c|}{ Semivolatile Organic Compounds (continued) } \\
\hline 3,3'-Dichlorobenzidine & 330 & $\mathrm{U}$ & 330 & 340 & $\mathrm{U}$ & 340 & 340 & $\mathrm{U}$ & 340 & 340 & $\mathrm{U}$ & 340 \\
\hline 3-Nitroaniline & 840 & $\mathrm{U}$ & 840 & 840 & $\mathrm{U}$ & 840 & 840 & $\mathrm{U}$ & 840 & 840 & $\mathrm{U}$ & 840 \\
\hline 4,6-Dinitro-2-methylphenol & 840 & $\bar{U}$ & 840 & 840 & $\mathrm{U}$ & 840 & 840 & $\mathrm{U}$ & 840 & 840 & $\mathrm{U}$ & 840 \\
\hline 4-Bromophenyl-phenylether & 330 & $\mathrm{U}$ & 330 & 340 & $\mathrm{U}$ & 340 & 340 & $\mathrm{U}$ & 340 & 340 & $\mathrm{U}$ & 340 \\
\hline 4-Chloro-3-methylphenol & 330 & $\mathrm{U}$ & 330 & 340 & $\mathrm{U}$ & 340 & 340 & $\mathrm{U}$ & 340 & 340 & $\mathrm{U}$ & 340 \\
\hline 4-Chloroaniline & 330 & $\mathrm{U}$ & 330 & 340 & $\mathrm{U}$ & 340 & 340 & $\mathrm{U}$ & 340 & 340 & $\mathrm{U}$ & 340 \\
\hline 4-Chlorophenyl-phenylether & 330 & $\mathrm{U}$ & 330 & 340 & $U$ & 340 & 340 & $\mathrm{U}$ & 340 & 340 & $\mathrm{U}$ & 340 \\
\hline 4-Methylphenol (p-cresol) & 330 & $\mathrm{U}$ & 330 & 340 & $\mathrm{U}$ & 340 & 340 & $\mathrm{U}$ & 340 & 340 & $\mathrm{U}$ & 340 \\
\hline 4-Nitroaniline & 840 & $\mathrm{U}$ & 840 & 840 & $\mathrm{U}$ & 840 & 840 & $\mathrm{U}$ & 840 & 840 & $\mathrm{U}$ & 840 \\
\hline 4-Nitrophenol & 840 & $\mathrm{U}$ & 840 & 840 & $\mathrm{U}$ & 840 & 840 & $\mathrm{U}$ & 840 & 840 & $\mathrm{U}$ & 840 \\
\hline Acenaphthene & 330 & $\mathrm{U}$ & 330 & 340 & $\mathrm{U}$ & 340 & 340 & $\mathrm{U}$ & 340 & 340 & U & 340 \\
\hline Acenaphthylene & 330 & $\mathrm{U}$ & 330 & 340 & $\mathrm{U}$ & 340 & 340 & $\mathrm{U}$ & 340 & 340 & $\mathrm{U}$ & 340 \\
\hline Anthracene & 330 & $\mathrm{U}$ & 330 & 340 & $\mathrm{U}$ & 340 & 340 & $\mathrm{U}$ & 340 & 340 & $\mathrm{U}$ & 340 \\
\hline Benzo(a)anthracene & 330 & $\mathrm{U}$ & 330 & 340 & $\mathrm{U}$ & 340 & 41 & $\mathrm{~J}$ & 340 & 340 & $\mathrm{U}$ & 340 \\
\hline Benzo(a)pyrene & 330 & $\mathrm{U}$ & 330 & 340 & $\mathrm{U}$ & 340 & 33 & $\mathrm{~J}$ & 340 & 340 & $\mathrm{U}$ & 340 \\
\hline Benzo(b)fluoranthene & 330 & $\mathrm{U}$ & 330 & 340 & $\mathrm{U}$ & 340 & 41 & $\mathrm{~J}$ & 340 & 340 & $\mathrm{U}$ & 340 \\
\hline Benzo $(g, h, i)$ perylene & 330 & $\mathrm{U}$ & 330 & 340 & $\mathrm{U}$ & 340 & 30 & $\mathrm{~J}$ & 340 & 340 & $\mathrm{U}$ & 340 \\
\hline Benzo(k)fluoranthene & 330. & $\mathrm{U}$ & 330 & 340 & $\mathrm{U}$ & 340 & 35 & $\mathrm{~J}$ & 340 & 340 & $\mathrm{U}$ & 340 \\
\hline bis(2-Chloro-1-methylethyl)ether & 330 & $\mathrm{U}$ & 330 & 340 & $\mathrm{U}$ & 340 & 340 & $\mathrm{U}$ & 340 & 340 & $\mathrm{U}$ & 340 \\
\hline bis(2-Chloroethoxy)methane & 330 & $\mathrm{U}$ & 330 & 340 & $\mathrm{U}$ & 340 & 340 & $\mathrm{U}$ & 340 & 340 & U & 340 \\
\hline bis(2-Chloroethyl)ether & 330 & $\mathrm{U}$ & 330 & 340 & $\mathrm{U}$ & 340 & 340 & $\mathrm{U}$ & 340 & 340 & $\mathrm{U}$ & 340 \\
\hline bis(2-Ethylhexyl)phthalate & 20 & JB & 330 & 38 & JB & 340 & 230 & $\mathrm{JB}$ & 340 & 71 & $\mathrm{JB}$ & 340 \\
\hline Butylbenzylphthalate & 330 & $\mathrm{U}$ & 330 & 340 & $\mathrm{U}$ & 340 & 340 & $\mathrm{U}$ & 340 & 340 & $\mathrm{U}$ & 340 \\
\hline Carbazole & 330 & $\mathrm{U}$ & 330 & 340 & $\mathrm{U}$ & 340 & 340 & $\mathrm{U}$ & 340 & 340 & $\mathrm{U}$ & 340 \\
\hline Chrysene & 330 & $\mathrm{U}$ & 330 & 340 & $\mathrm{U}$ & 340 & 64 & $\mathrm{~J}$ & 340 & 340 & $\mathrm{U}$ & 340 \\
\hline Di-n-butylphthalate & 24 & $\mathrm{JB}$ & 330 & 24 & $\mathrm{JB}$ & 340 & 45 & $\mathrm{JB}$ & 340 & 23 & $\mathrm{JB}$ & 340 \\
\hline Di-n-octylphthalate & 330 & $\mathrm{U}$ & 330 & 340 & $\mathrm{U}$ & 340 & 340 & U. & 340 & 340 & $\mathrm{U}$ & 340 \\
\hline Dibenz $(a, h)$ anthracene & 330 & $\mathrm{U}$ & 330 & 340 & $\mathrm{U}$ & 340 & 340 & $\mathrm{U}$ & 340 & 340 & $\mathrm{U}$ & 340 \\
\hline Dibenzofuran & 330 & $\mathrm{U}$ & 330 & 340 & $\mathrm{U}$ & 340 & 340 & $\mathrm{U}$ & 340 & 340 & $\mathrm{U}$ & 340 \\
\hline Diethylphthalate & 330 & $\mathrm{U}$ & 330 & 340 & $\mathrm{U}$ & 340 & 340 & $\mathrm{U}$ & 340 & 340 & U & 340 \\
\hline Dimethylphthalate & 330 & $\mathrm{U}$ & 330 & 340 & $\mathrm{U}$ & 340 & 340 & $\mathrm{U}$ & 340 & 340 & U. & 340 \\
\hline Fluoranthene & 330 & U & 330 & 340 & $\mathrm{U}$ & 340 & 79 & $\mathrm{~J}$ & 340 & 340 & $\mathrm{U}$ & 340 \\
\hline Fluorene & 330 & $\mathrm{U}$ & 330 & 340 & $\mathrm{U}$ & 340 & 340 & $\mathrm{U}$ & 340 & 340 & $\mathrm{U}$ & 340 \\
\hline Hexachlorobenzene & 330 & $\mathrm{U}$ & 330 & 340 & $\mathrm{U}$ & 340 & 340 & $\mathrm{U}$ & 340 & 340 & $\mathrm{U}$ & 340 \\
\hline Hexachlorobutadiene & 330 & $\mathrm{U}$ & 330 & 340 & $\mathrm{U}$ & 340 & 340 & $\mathrm{U}$ & 340 & 340 & $\mathrm{U}$ & 340 \\
\hline Hexachlorocyclopentadiene & 330 & $\mathrm{U}$ & 330 & 340 & $\mathrm{U}$ & 340 & 340 & $\mathrm{U}$ & 340 & 340 & $\mathrm{U}$ & 340 \\
\hline Hexachloroethane & 330 & $\mathrm{U}$ & 330 & 340 & $\mathrm{U}$ & 340 & 340 & $\mathrm{U}$ & 340 & 340 & $\mathrm{U}$ & 340 \\
\hline Indeno( $1,2,3-\mathrm{cd})$ pyrene & 330 & U & 330 & 340 & $\mathrm{U}$ & 340 & 28 & $\mathrm{~J}$ & 340 & 340 & $\mathrm{U}$ & 340 \\
\hline Isophorone & 330 & U & 330 & 340 & $\mathrm{U}$ & 340 & 340 & $\mathrm{U}$ & 340 & 340 & $\mathrm{U}$ & 340 \\
\hline N-Nitroso-di-n-dipropylamine & 330 & $U$ & 330 & 340 & $\mathrm{U}$ & 340 & 340 & $\mathrm{U}$ & 340 & 340 & $\mathrm{U}$ & 340 \\
\hline N-Nitrosodiphenylamine & 330 & $\mathrm{U}$ & 330 & 340 & $\mathrm{U}$ & 340 & 340 & $\mathrm{U}$ & 340 & 340 & $\mathrm{U}$ & 340 \\
\hline Naphthalene & 330 & $\mathrm{U}$ & 330 & 340 & $\mathrm{U}$ & 340 & 17 & $\mathrm{~J}$ & 340 & 340 & $\mathrm{U}$ & 340 \\
\hline Nitrobenzene & 330 & $\mathrm{U}$ & 330 & 340 & $\mathrm{U}$ & 340 & 340 & $\mathrm{U}$ & 340 & 340 & $\mathrm{U}$ & 340 \\
\hline Pentachlorophenol & 840 & $\mathrm{U}$ & 840 & 840 & $\mathrm{U}$ & 840 & 840 & $\mathrm{U}$ & 840 & 840 & $\mathrm{U}$ & 840 \\
\hline Phenanthrene & 330 & $\mathrm{U}$ & 330 & 340 & $\mathrm{U}$ & 340 & 46 & $\mathrm{~J}$ & 340 & 340 & $\mathrm{U}$ & 340 \\
\hline Phenol & 330 & $\mathrm{U}$ & 330 & 340 & $\mathrm{U}$ & 340 & 17 & $\mathrm{~J}$ & 340 & 340 & $\mathrm{U}$ & 340 \\
\hline Pyrene & 330 & $\bar{U}$ & 330 & 340 & $\mathrm{U}$ & 340 & 66 & $\mathrm{~J}$ & 340 & 340 & $\mathrm{U}$ & 340 \\
\hline
\end{tabular}

Attachment

Originator

Checked

Calc. No.

$\frac{1}{\frac{1}{\text { J.M. Capron }}} \frac{}{\text { T.M. Blakley }}$

Sheet No. 13 of 20

Date $09 / 14 / 06$

Date

Rev. No. 0 
Attachment 1. 1607-B2:2 Verification Sampling Results.

\begin{tabular}{|c|c|c|c|c|c|c|c|c|c|c|c|c|}
\hline \multirow[t]{2}{*}{ Constituents } & \multicolumn{3}{|c|}{$\begin{array}{c}\text { J12NY7 } \\
\text { Location } 8 \\
\text { Sample Date } 6 / 27 / 06\end{array}$} & \multicolumn{3}{|c|}{$\begin{array}{c}\text { J12NY8 } \\
\text { Location } 9 \\
\text { Sample Date } 6 / 27 / 06\end{array}$} & \multicolumn{3}{|c|}{$\begin{array}{c}\text { J12NY9 } \\
\text { Location } 10 \\
\text { Sample Date } 6 / 27 / 06 \\
\end{array}$} & \multicolumn{3}{|c|}{\begin{tabular}{|c|} 
J12PW5 \\
Location 11 \\
Sample Date $6 / 29 / 06$ \\
\end{tabular}} \\
\hline & $\mu \mathrm{g} / \mathrm{kg}$ & $\mathbf{Q}$ & PQL & $\mu \mathrm{g} / \mathrm{kg}$ & $Q$ & PQL & $\mu \mathrm{g} / \mathrm{kg}$ & Q & PQL & $\mu g / \mathrm{kg}$ & $Q$ & PQL \\
\hline \multicolumn{13}{|c|}{ Polychlorinated Biphenyls } \\
\hline Aroclor-1016 & 13 & $\mathrm{U}$ & 13 & 13 & $\mathrm{U}$ & 13 & 13 & $\mathrm{U}$ & 13 & 13 & $\mathrm{U}$ & 13 \\
\hline Aroclor-1221 & 13 & $\mathrm{U}$ & 13 & 13 & $\mathrm{U}$ & 13 & 13 & $\mathrm{U}$ & 13 & 13 & $\mathrm{U}$ & 13 \\
\hline Aroclor -1232 & 13 & $U$ & 13 & 13 & $\bar{U}$ & 13 & 13 & $\mathrm{U}$ & 13 & 13 & $\mathrm{U}$ & 13 \\
\hline Aroclor-1242 & 13 & $\mathrm{U}$ & 13 & 13 & $\mathrm{U}$ & 13 & 13 & $\mathrm{U}$ & 13 & 13 & $\mathrm{U}$ & 13 \\
\hline Aroclor-1248 & 13 & $\mathrm{U}$ & 13 & 13 & $\mathrm{U}$ & 13 & 13 & $\mathrm{U}$ & 13 & 13 & $\mathrm{U}$ & 13 \\
\hline Aroclor-1254 & 38 & & 13 & 13 & $\mathrm{U}$ & 13 & 4.4 & & 13 & 13 & $\mathrm{U}$ & 13 \\
\hline Aroclor- 1260 & 6.7 & $\mathrm{~J}$ & 13 & 13 & $\mathrm{U}$ & 13 & 13 & $\mathrm{U}$ & 13 & 13 & $\mathrm{U}$ & 13 \\
\hline \multicolumn{13}{|c|}{ Pesticides } \\
\hline Aldrin & 1.3 & UD & 1.3 & 1.3 & UD & 1.3 & 1.3 & UD & 1.3 & 1.3 & UD & 1.3 \\
\hline alpha-BHC & 1.3 & UD & 1.3 & 1.3 & UD & 1.3 & 1.3 & UD & 1.3 & 1.3 & UD & 1.3 \\
\hline alpha-Chlordane & 1.3 & UD & 1.3 & 1.3 & UD & 1.3 & 1.3 & UD & 1.3 & 1.3 & UD & 1.3 \\
\hline beta-BHC & 1.3 & UD & 1.3 & 1.3 & UD & 1.3 & 1.3 & UD & 1.3 & 1.3 & UD & 1.3 \\
\hline delta-BHC & 1.3 & UD & 1.3 & 1.3 & UD & 1.3 & 1.3 & UD & 1.3 & 1.3 & UD & 1.3 \\
\hline Dichlorodiphenyldichloroethane & 1.3 & UD & 1.3 & 1.3 & UD & 1.3 & 1.3 & UD & 1.3 & 1.3 & UD & 1.3 \\
\hline Dichlorodiphenyldichloroethylene & 1.3 & UD & 1.3 & 1.3 & UD & 1.3 & 1.3 & UD & 1.3 & 1.3 & UD & 1.3 \\
\hline Dichlorodiphenyltrichloroethane & 17 & $\mathrm{D}$ & 1.3 & 1.3 & UD & 1.3 & 1.3 & UD & 1.3 & 1.3 & UD & 1.3 \\
\hline Dieldrin & 1.3 & UD & 1.3 & 1.3 & UD & 1.3 & 1.3 & UD & 1.3 & 1.3 & UD & 1.3 \\
\hline Endosulfan I & 1.3 & UD & 1.3 & 1.3 & UD & 1.3 & 1.3 & UD & 1.3 & 1.3 & UD & 1.3 \\
\hline Endosulfan II & 1.3 & UD & 1.3 & 1.3 & UD & 1.3 & 1.3 & UD & 1.3 & 1.3 & UD & 1.3 \\
\hline Endosulfan sulfate & 1.3 & UD & 1.3 & 1.3 & UD & 1.3 & 0.50 & $\mathrm{JD}$ & 1.3 & 1.3 & UD & 1.3 \\
\hline Endrin & 1.3 & UD & 1.3 & 1.3 & UD & 1.3 & 1.3 & UD & 1.3 & 1.3 & UD & 1.3 \\
\hline Endrin aldehyde & 1.3 & UD & 1.3 & 1.3 & UD & 1.3 & 0.77 & JID & 1.3 & 1.3 & UD & 1.3 \\
\hline Endrin ketone & 1.3 & UD & 1.3 & 1.3 & UD & 1.3 & 1.3 & UD & 1.3 & 1.3 & UD & 1.3 \\
\hline gamma-BHC (Lindane) & 1.3 & UD & 1.3 & 1.3 & UD & 1.3 & 1.3 & UD & 1.3 & 1.3 & UD & 1.3 \\
\hline gamma-Chlordane & 1.3 & UD & 1.3 & 1.3 & UD & 1.3 & 1.3 & UD & 1.3 & 1.3 & UD & 1.3 \\
\hline Heptachlor & 1.3 & UD & 1.3 & 1.3 & UD & 1.3 & 1.3 & UD & 1.3 & 1.3 & UD & 1.3 \\
\hline Heptachlor epoxide & 1.3 & UD & 1.3 & 1.3 & UD & 1.3 & 1.3 & UD & 1.3 & 1.3 & UD & 1.3 \\
\hline Methoxychlor & 1.3 & UD & 1.3 & 1.3 & UD & 1.3 & 1.3 & UD & 1.3 & 1.3 & UD & 1.3 \\
\hline Toxaphene & 13 & UD & 13 & 13 & UD & 13 & 13 & UD & 13 & 13 & UDJ & 13 \\
\hline \multicolumn{13}{|c|}{ Semivolatile Organic Compounds } \\
\hline 1,2,4-Trichlorobenzene & 330 & $\mathrm{U}$ & 330 & 340 & $\mathrm{U}$ & 340 & 330 & $\mathrm{U}$ & 330 & 340 & $\mathrm{U}$ & 340 \\
\hline 1,2-Dichlorobenzene & 330 & $\mathrm{U}$ & 330 & 340 & $\mathrm{U}$ & 340 & 330 & $\mathrm{U}$ & 330 & 340 & $\mathrm{U}$ & 340 \\
\hline 1,3-Dichlorobenzene & 330 & $\mathrm{U}$ & 330 & 340 & $\mathrm{U}$ & 340 & 330 & $U$ & 330 & 340 & $\mathrm{U}$ & 340 \\
\hline 1,4-Dichlorobenzene & 330 & $\mathrm{U}$ & 330 & 340 & $\mathrm{U}$ & 340 & 330 & $\mathrm{U}$ & 330 & 340 & $\mathrm{U}$ & 340 \\
\hline 2,4,5-Trichlorophenol & 840 & $\mathrm{U}$ & 840 & 840 & $\mathrm{U}$ & 840 & 840 & $\mathrm{U}$ & 840 & 840 & $\mathrm{U}$ & 840 \\
\hline 2,4,6-Trichlorophenol & 330 & $\mathrm{U}$ & 330 & 340 & $\mathrm{U}$ & 340 & 330 & $\mathrm{U}$ & 330 & 340 & $\mathrm{U}$ & 340 \\
\hline 2,4-Dichlorophenol & 330 & $\mathrm{U}$ & 330 & 340 & $\mathrm{U}$ & 340 & 330 & $\mathrm{U}$ & 330 & 340 & $\mathrm{U}$ & 340 \\
\hline 2,4-Dimethylphenol & 330 & $\mathrm{U}$ & 330 & 340 & $\mathrm{U}$ & 340 & 330 & $\mathrm{U}$ & 330 & 340 & $\mathrm{U}$ & 340 \\
\hline 2,4-Dinitrophenol & 840 & $\mathrm{U}$ & 840 & 840 & $\mathrm{U}$ & 840 & 840 & $\mathrm{U}$ & 840 & 840 & UI & 840 \\
\hline 2,4-Dinitrotoluene & 330 & $\mathrm{U}$ & 330 & 340 & $\mathrm{U}$ & 340 & 330 & U & 330 & 340 & $U$ & 340 \\
\hline 2,6-Dinitrotoluene & 330 & $\mathrm{U}$ & 330 & 340 & $\mathrm{U}$ & 340 & 330 & $\mathrm{U}$ & 330 & 340 & $\mathrm{U}$ & 340 \\
\hline 2-Chloronaphthalene & 330 & $\mathrm{U}$ & 330 & 340 & $\mathrm{U}$ & 340 & 330 & $\mathrm{U}$ & 330 & 340 & $\mathrm{U}$ & 340 \\
\hline 2-Chlorophenol & 330 & $\mathrm{U}$ & 330 & 340 & $\mathrm{U}$ & 340 & 330 & $\mathrm{U}$ & 330 & 340 & $\mathrm{U}$ & 340 \\
\hline 2-Methylnaphthalene & 330 & $\mathrm{U}$ & 330 & 340 & $\mathrm{U}$ & 340 & 330 & $\mathrm{U}$ & 330 & 340 & $U$ & 340 \\
\hline 2-Methylphenol (cresol, o-) & 330 & $\mathrm{U}$ & 330 & 340 & $\mathrm{U}$ & 340 & 330 & $\mathrm{U}$ & 330 & 340 & $\mathrm{U}$ & 340 \\
\hline 2-Nitroaniline & 840 & $\mathrm{U}$ & 840 & 840 & $\mathrm{U}$ & 840 & 840 & $\mathrm{U}$ & 840 & 840 & $U$ & 840 \\
\hline 2-Nitrophenol & 330 & $\mathrm{U}$ & 330 & 340 & $\mathrm{U}$ & 340 & 330 & $\mathrm{U}$ & 330 & 340 & $\mathrm{U}$ & 340 \\
\hline & & & & Attachn & & & 1 & & & Sheet No. & & of 20 \\
\hline & & & & Origina & & J.M. Ca & ron & & & Date & & $114 / 06$ \\
\hline & & & & Checke & & T.M. Bl & akley & & & Date & & \\
\hline & & & & Calc. N & & $0100 \mathrm{~B}-\mathrm{C}$ & $A-V 029$ & & & Rev. No. & & 0 \\
\hline
\end{tabular}


Attachment 1. 1607-B2:2 Verification Sampling Results.

\begin{tabular}{|c|c|c|c|c|c|c|c|c|c|c|c|c|}
\hline \multirow[t]{2}{*}{ Constituents } & \multicolumn{3}{|c|}{$\begin{array}{c}\text { J12NY7 } \\
\text { Location } 8 \\
\text { Sample Date } 6 / 27 / 06 \\
\end{array}$} & \multicolumn{3}{|c|}{\begin{tabular}{|c|} 
J12NY8 \\
Location 9 \\
Sample Date $6 / 27 / 06$ \\
\end{tabular}} & \multicolumn{3}{|c|}{$\begin{array}{c}\text { J12NY9 } \\
\text { Location } 10 \\
\text { Sample Date } 6 / 27 / 06\end{array}$} & \multicolumn{3}{|c|}{\begin{tabular}{|c|} 
J12PW5 \\
Location 11 \\
Sample Date 6/29/06 \\
\end{tabular}} \\
\hline & $\mu \mathrm{g} / \mathrm{kg}$ & Q & PQL & $\mu \mathrm{g} / \mathrm{kg}$ & Q & PQL & $\mu \mathrm{g} / \mathrm{kg}$ & Q & $\mathrm{PQL}$ & $\mu \mathrm{g} / \mathrm{kg}$ & Q & PQL \\
\hline \multicolumn{13}{|c|}{ Semivolatile Organic Compounds (continued) } \\
\hline 3,3'-Dichlorobenzidine & 330 & $\mathrm{U}$ & 330 & 340 & $\mathrm{U}$ & 340 & 330 & $\mathrm{U}$ & 330 & 340 & $\mathrm{U}$ & 340 \\
\hline 3-Nitroaniline & 840 & $\mathrm{U}$ & 840 & 840 & $\mathrm{U}$ & 840 & 840 & $U$ & 840 & 840 & $\mathrm{U}$ & 840 \\
\hline 4,6-Dinitro-2-methylphenol & 840 & $\mathrm{U}$ & 840 & 840 & $\mathrm{U}$ & 840 & 840 & $U$ & 840 & 840 & $\bar{U}$ & 840 \\
\hline 4-Bromophenyl-phenylether & 330 & $\mathrm{U}$ & 330 & 340 & $\mathrm{U}$ & 340 & 330 & $\mathrm{U}$ & 330 & 340 & $\mathrm{U}$ & 340 \\
\hline 4-Chloro-3-methylphenol & 330 & $\mathrm{U}$ & 330 & 340 & $\mathrm{U}$ & 340 & 330 & $\mathrm{U}$ & 330 & 340 & $\mathrm{U}$ & 340 \\
\hline 4-Chloroaniline & 330 & $\mathrm{U}$ & 330 & 340 & $\mathrm{U}$ & 340 & 330 & $\mathrm{U}$ & 330 & 340 & $\mathrm{U}$ & 340 \\
\hline 4-Chlorophenyl-phenylether & 330 & $\mathrm{U}$ & 330 & 340 & U & 340 & 330 & $\mathrm{U}$ & 330 & 340 & $\mathrm{U}$ & 340 \\
\hline 4-Methylphenol (p-cresol) & 330 & $\mathrm{U}$ & 330 & 340 & $\mathrm{U}$ & 340 & 330 & $\mathrm{U}$ & 330 & 340 & $\mathrm{U}$ & 340 \\
\hline 4-Nitroaniline & 840 & $\mathrm{U}$ & 840 & 840 & $\mathrm{U}$ & 840 & 840 & $\mathrm{U}$ & 840 & 840 & $\mathrm{U}$ & 840 \\
\hline 4-Nitrophenol & 840 & $\mathrm{U}$ & 840 & 840 & $\mathrm{U}$ & 840 & 840 & $\mathrm{U}$ & 840 & 840 & $\mathrm{U}$ & 840 \\
\hline Acenaphthene & 330 & $\bar{U}$ & 330 & 340 & $\mathrm{U}$ & 340 & 330 & $\mathrm{U}$ & 330 & 340 & $\mathrm{U}$ & 340 \\
\hline Acenaphthylene & 330 & $\mathrm{U}$ & 330 & 340 & $\mathrm{U}$ & 340 & 330 & $\mathrm{U}$ & 330 & 340 & $\mathrm{U}$ & 340 \\
\hline Anthracene & 330 & $\mathrm{U}$ & 330 & 340 & $\mathrm{U}$ & 340 & 330 & $\mathrm{U}$ & 330 & 340 & $\mathrm{U}$ & 340 \\
\hline Benzo(a)anthracene & 330 & $\mathrm{U}$ & 330 & 340 & $\mathrm{U}$ & 340 & 330 & $\mathrm{U}$ & 330 & 340 & $\mathrm{U}$ & 340 \\
\hline Benzo(a)pyrene & 330 & $\mathrm{U}$ & 330 & 340 & $\mathrm{U}$ & 340 & 330 & $\mathrm{U}$ & 330 & 340 & $\mathrm{U}$ & 340 \\
\hline Benzo(b)fluoranthene & 330 & $\mathrm{U}$ & 330 & 340 & $\mathrm{U}$ & 340 & 330 & $\mathrm{U}$ & 330 & 340 & $\mathrm{U}$ & 340 \\
\hline Benzo(g,h,i)perylene & 330 & $\mathrm{U}$ & 330 & 340 & U & 340 & 330 & $\mathrm{U}$ & 330 & 340 & $\mathrm{U}$ & 340 \\
\hline Benzo(k)fluoranthene & 330 & $\mathrm{U}$ & 330 & 340 & $\mathrm{U}$ & 340 & 330 & $\mathrm{U}$ & 330 & 340 & $\mathrm{U}$ & 340 \\
\hline bis(2-Chloro-1-methylethyl)ether & 330 & $\mathrm{U}$ & 330 & 340 & $\mathrm{U}$ & 340 & 330 & $\mathrm{U}$ & 330 & 340 & $\mathrm{U}$ & 340 \\
\hline bis(2-Chloroethoxy)methane & 330 & $\mathrm{U}$ & 330 & 340 & $\mathrm{U}$ & 340 & 330 & $\mathrm{U}$ & 330 & 340 & U & 340 \\
\hline bis(2-Chloroethyl)ether & 330 & $\mathrm{U}$ & 330 & 340 & $\mathrm{U}$ & 340 & 330 & $U$ & 330 & 340 & $\mathrm{U}$ & 340 \\
\hline bis(2-Ethylhexyl)phthalate & 32 & $\mathrm{JB}$ & 330 & 450 & $\mathrm{~B}$ & 340 & 3200 & $\mathrm{BD}$ & 330 & 660 & $\mathrm{U}$ & 660 \\
\hline Butylbenzylphthalate & 330 & $\mathrm{U}$ & 330 & 340 & $\mathrm{U}$ & 340 & 330 & $\mathrm{U}$ & 330 & 340 & U & 340 \\
\hline Carbazole & 330 & $\mathrm{U}$ & 330 & 340 & $\mathrm{U}$ & 340 & 330 & $\mathrm{U}$ & 330 & 340 & $U$ & 340 \\
\hline Chrysene & 330 & $\mathrm{U}$ & 330 & 340 & $\mathrm{U}$ & 340 & 330 & $\mathrm{U}$ & 330 & 340 & $\mathrm{U}$ & 340 \\
\hline Di-n-butylphthalate & 48 & $\mathrm{JB}$ & 330 & 340 & $\mathrm{U}$ & 340 & 22 & $\mathrm{JB}$ & 330 & 19 & $\mathrm{~J}$ & 340 \\
\hline Di-n-octylphthalate & 330 & $\mathrm{U}$ & 330 & 340 & $\mathrm{U}$ & 340 & 330 & $\mathrm{U}$ & 330 & 340 & $\mathrm{U}$ & 340 \\
\hline Dibenz $(a, h)$ anthracene & 330 & $\mathrm{U}$ & 330 & 340 & $\mathrm{U}$ & 340 & 330 & $\mathrm{U}$ & 330 & 340 & $\mathrm{U}$ & 340 \\
\hline Dibenzofuran & 330 & $\mathrm{U}$ & 330 & 340 & $\mathrm{U}$ & 340 & 330 & $\mathrm{U}$ & 330 & 340 & $\mathrm{U}$ & 340 \\
\hline Diethylphthalate & 330 & $\mathrm{U}$ & 330 & 340 & $\mathrm{U}$ & 340 & 330 & $U$ & 330 & 340 & $\mathrm{U}$ & 340 \\
\hline Dimethylphthalate & 330 & $\mathrm{U}$ & 330 & 340 & $\mathrm{U}$ & 340 & 330 & $\mathrm{U}$ & 330 & 340 & $\mathrm{U}$ & 340 \\
\hline Fluoranthene & 330 & $\mathrm{U}$ & 330 & 340 & $\mathrm{U}$ & 340 & 330 & U & 330 & 340 & $\mathrm{U}$ & 340 \\
\hline Fluorene & 330 & $\mathrm{U}$ & 330 & 340 & $\mathrm{U}$ & 340 & 330 & $\mathrm{U}$ & 330 & 340 & $\mathrm{U}$ & 340 \\
\hline Hexachlorobenzene & 330 & $\mathrm{U}$ & 330 & 340 & $\mathrm{U}$ & 340 & 330 & $\mathrm{U}$ & 330 & 340 & U & 340 \\
\hline Hexachlorobutadiene & 330 & $\mathrm{U}$ & 330 & 340 & $\mathrm{U}$ & 340 & 330 & $\mathrm{U}$ & 330 & 340 & $\mathrm{U}$ & 340 \\
\hline Hexachlorocyclopentadiene & 330 & $\mathrm{U}$ & 330 & 340 & $U$ & 340 & 330 & $\mathrm{U}$ & 330 & 340 & U & 340 \\
\hline Hexachloroethane & 330 & $\bar{U}$ & 330 & 340 & U & 340 & 330 & $\mathrm{U}$ & 330 & 340 & $\mathrm{U}$ & 340 \\
\hline Indeno(1,2,3-cd)pyrene & 330 & $\mathrm{U}$ & 330 & 340 & $\mathrm{U}$ & 340 & 330 & $\mathrm{U}$ & 330 & 340 & $\mathrm{U}$ & 340 \\
\hline Isophorone & 330 & $\mathrm{U}$ & 330 & 340 & $\mathrm{U}$ & 340 & 330 & $\mathrm{U}$ & 330 & 340 & U & 340 \\
\hline N-Nitroso-di-n-dipropylamine & 330 & $\mathrm{U}$ & 330 & 340 & $\mathrm{U}$ & 340 & 330 & $\mathrm{U}$ & 330 & 340 & $\mathrm{U}$ & 340 \\
\hline N-Nitrosodiphenylamine & 330 & $\mathrm{U}$ & 330 & 340 & $\mathrm{U}$ & 340 & 330 & $\mathrm{U}$ & 330 & 340 & $\mathrm{U}$ & 340 \\
\hline Naphthalene & 330 & $\mathrm{U}$ & 330 & 340 & $\mathrm{U}$ & 340 & 330 & $\mathrm{U}$ & 330 & 340 & $\mathrm{U}$ & 340 \\
\hline Nitrobenzene & 330 & $\mathrm{U}$ & 330 & 340 & $\mathrm{U}$ & 340 & 330 & $\mathrm{U}$ & 330 & 340 & U & 340 \\
\hline Pentachlorophenol & 840 & $\mathrm{U}$ & 840 & 840 & $\mathrm{U}$ & 840 & 840 & $\mathrm{U}$ & 840 & 840 & $\mathrm{U}$ & 840 \\
\hline Phenanthrene & 330 & $\mathrm{U}$ & 330 & 340 & $\mathrm{U}$ & 340 & 330 & $\mathrm{U}$ & 330 & 340 & $\mathrm{U}$ & 340 \\
\hline Phenol & 330 & $\mathrm{U}$ & 330 & 340 & $\mathrm{U}$ & 340 & 330 & $\mathrm{U}$ & 330 & 340 & $\mathrm{U}$ & 340 \\
\hline Pyrene & 330 & $\mathrm{U}$ & 330 & 340 & $\mathrm{U}$ & 340 & 330 & $\mathrm{U}$ & 330 & 340 & $\mathrm{U}$ & 340 \\
\hline & & & & Attachn & & & 1 & & & Sheet No. & & 5 of 20 \\
\hline & & & & Origina & & J.M.C & ron & & & Date & & $9 / 14 / 06$ \\
\hline & & & & Checke & & T.M. B & akley & & & Date & & \\
\hline & & & & Calc. $\mathrm{N}$ & & $0100 \mathrm{~B}-$ & A-V029 & & & Rev. No. & & 0 \\
\hline
\end{tabular}


Attachment 1. 1607-B2:2 Verification Sampling Results.

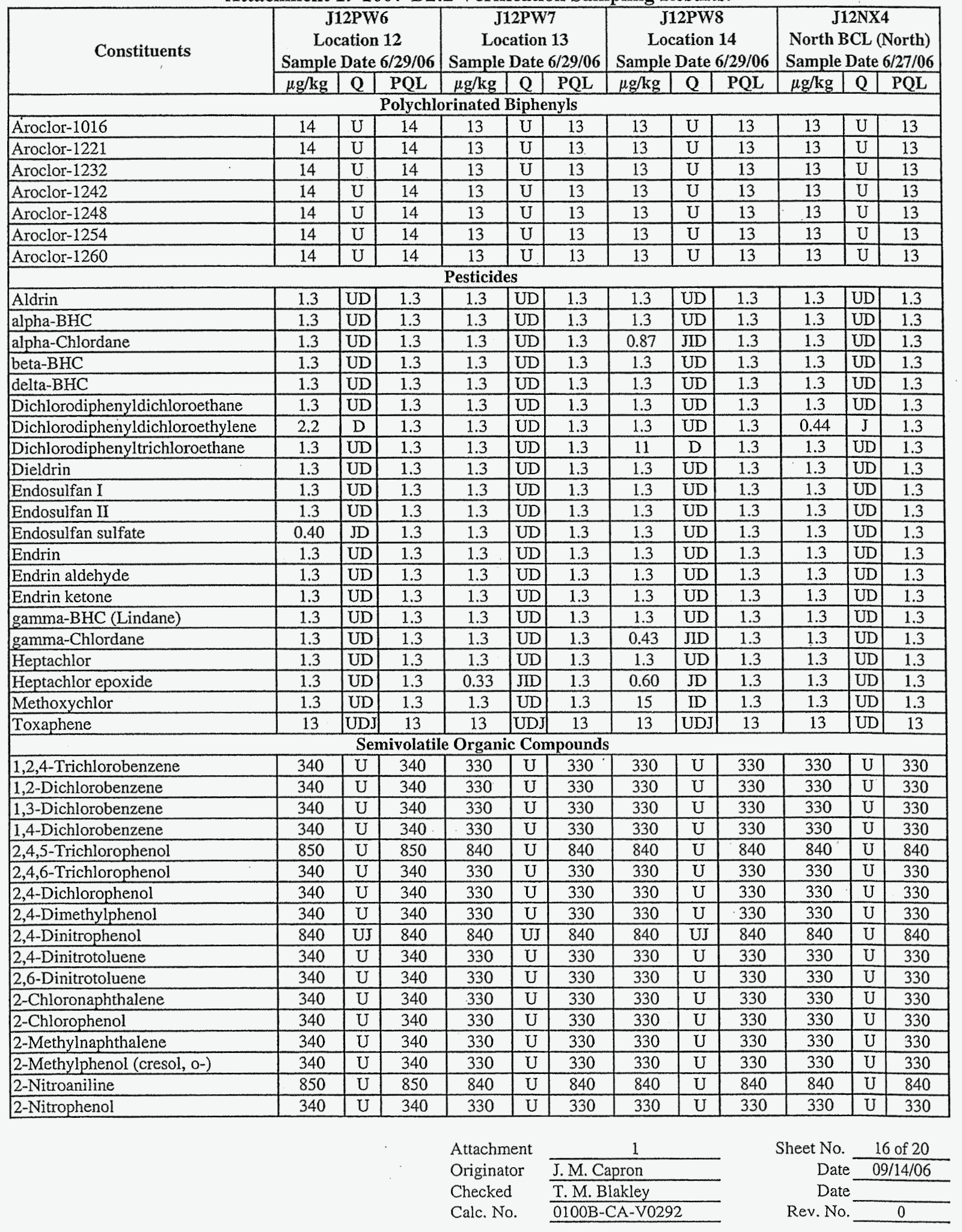


Attachment 1. 1607-B2:2 Verification Sampling Results.

\begin{tabular}{|c|c|c|c|c|c|c|c|c|c|c|c|c|}
\hline \multirow[t]{2}{*}{ Constituents } & \multicolumn{3}{|c|}{\begin{tabular}{|c|} 
J12PW6 \\
Location 12 \\
Sample Date $6 / 29 / 06$ \\
\end{tabular}} & \multicolumn{3}{|c|}{$\begin{array}{c}\text { J12PW7 } \\
\text { Location } 13 \\
\text { Sample Date 6/29/06 } \\
\end{array}$} & \multicolumn{3}{|c|}{$\begin{array}{c}\text { J12PW8 } \\
\text { Location } 14 \\
\text { Sample Date } 6 / 29 / 06 \\
\end{array}$} & \multicolumn{3}{|c|}{$\begin{array}{c}\text { J12NX4 } \\
\text { North BCL (North) } \\
\text { Sample Date } 6 / 27 / 06 \\
\end{array}$} \\
\hline & $\mu \mathrm{g} / \mathrm{kg}$ & $\mathbf{Q}$ & PQL & $\mu \mathrm{g} / \mathrm{kg}$ & $\mathbf{Q}$ & PQL & $\mu \mathrm{g} / \mathrm{kg}$ & Q & PQL & $\mu \mathrm{g} / \mathrm{kg}$ & $\mathbf{Q}$ & PQL \\
\hline \multicolumn{13}{|c|}{ Semivolatile Organic Compounds (continued) } \\
\hline 3,3'-Dichlorobenzidine & 340 & $\mathrm{U}$ & 340 & 330 & U & 330 & 330 & $\mathrm{U}$ & 330 & 330 & $\mathrm{U}$ & 330 \\
\hline 3-Nitroaniline & 850 & $\mathrm{U}$ & 850 & 840 & $\mathrm{U}$ & 840 & 840 & $\mathrm{U}$ & 840 & 840 & $\mathrm{U}$ & 840 \\
\hline 4,6-Dinitro-2-methylphenol & 850 & $\mathrm{U}$ & 850 & 840 & $\mathrm{U}$ & 840 & 840 & $\mathrm{U}$ & 840 & 840 & $\mathrm{U}$ & 840 \\
\hline 4-Bromophenyl-phenylether & 340 & $\mathrm{U}$ & 340 & 330 & $\mathrm{U}$ & 330 & 330 & $\mathrm{U}$ & 330 & 330 & $\mathrm{U}$ & 330 \\
\hline 4-Chloro-3-methylphenol & 340 & $\mathrm{U}$ & 340 & 330 & $\mathrm{U}$ & 330 & 330 & $\mathrm{U}$ & 330 & 330 & $\mathrm{U}$ & 330 \\
\hline 4-Chloroaniline & 340 & $\mathrm{U}$ & 340 & 330 & $\mathrm{U}$. & 330 & 330 & $\mathrm{U}$ & 330 & 330 & $\bar{U}$ & 330 \\
\hline 4-Chlorophenyl-phenylether & 340 & $\mathrm{U}$ & 340 & 330 & $\mathrm{U}$ & 330 & 330 & $\mathrm{U}$ & 330 & 330 & $\mathrm{U}$ & 330 \\
\hline 4-Methylphenol (p-cresol) & 340 & $\mathrm{U}$ & 340 & 330 & $\mathrm{U}$ & 330 & 330 & $\mathrm{U}$ & 330 & 330 & $\mathrm{U}$ & 330 \\
\hline 4-Nitroaniline & 850 & $\mathrm{U}$ & 850 & 840 & $\mathrm{U}$ & 840 & 840 & $\mathrm{U}$ & 840 & 840 & $\mathrm{U}$ & 840 \\
\hline 4-Nitrophenol & 850 & $\mathrm{U}$ & 850 & 840 & $\mathrm{U}$ & 840 & 840 & $\mathrm{U}$ & 840 & 840 & $\mathrm{U}$ & 840 \\
\hline Acenaphthene & 340 & $\mathrm{U}$ & 340 & 330 & $\mathrm{U}$ & 330 & 330 & $\mathrm{U}$ & 330 & 330 & $\mathrm{U}$ & 330 \\
\hline Acenaphthylene & 340 & $\mathrm{U}$ & 340 & 330 & $\mathrm{U}$ & 330 & 330 & $\mathrm{U}$ & 330 & 330 & $\mathrm{U}$ & 330 \\
\hline Anthracene & 340 & $\mathrm{U}$ & 340 & 330 & $\mathrm{U}$ & 330 & 330 & $\mathrm{U}$ & 330 & 330 & $\mathrm{U}$ & 330 \\
\hline Benzo(a)anthracene & 340 & $\mathrm{U}$ & 340 & 330 & $\mathrm{U}$ & 330 & 330 & $\mathrm{U}$ & 330 & 330 & $\mathrm{U}$ & 330 \\
\hline Benzo(a)pyrene & 340 & $\mathrm{U}$ & 340 & 330 & $\mathrm{U}$ & 330 & 330 & $\mathrm{U}$ & 330 & 330 & $\mathrm{U}$ & 330 \\
\hline Benzo(b)fluoranthene & 340 & $\mathrm{U}$ & 340 & 330 & $\mathrm{U}$ & 330 & 330 & $\mathrm{U}$ & 330 & 330 & $\mathrm{U}$ & 330 \\
\hline Benzo(g,h,i)perylene & 340 & $\mathrm{U}$ & 340 & 330 & $\mathrm{U}$ & 330 & 21 & $\mathrm{~J}$ & 330 & 330 & $\mathrm{U}$ & 330 \\
\hline Benzo(k)fluoranthene & 340 & $\mathrm{U}$ & 340 & 330 & $\mathrm{U}$ & 330 & 330 & $\mathrm{U}$ & 330 & 330 & $\mathrm{U}$ & 330 \\
\hline bis(2-Chloro-1-methylethyl)ether & 340 & $\mathrm{U}$ & 340 & 330 & $\mathrm{U}$ & 330 & 330 & $\mathrm{U}$ & 330 & 330 & $\mathrm{U}$ & 330 \\
\hline bis(2-Chloroethoxy)methane & 340 & $\mathrm{U}$ & 340 & 330 & $\mathrm{U}$ & 330 & 330 & $\mathrm{U}$ & 330 & 330 & $\mathrm{U}$ & 330 \\
\hline bis(2-Chloroethyl)ether & 340 & $\mathrm{U}$ & 340 & 330 & $\mathrm{U}$ & 330 & 330 & $\mathrm{U}$ & 330 & 330 & $\mathrm{U}$ & 330 \\
\hline bis(2-Ethylhexyl)phthalate & 660 & $\mathrm{U}$ & 660 & 660 & $\mathrm{U}$ & 660 & 660 & $\mathrm{U}$ & 660 & 1100 & $\mathrm{~B}$ & 330 \\
\hline Butylbenzylphthalate & 340 & $\mathrm{U}$ & 340 & 330 & $\mathrm{U}$ & 330 & 330 & $\mathrm{U}$ & 330 & 330 & $\mathrm{U}$ & 330 \\
\hline Carbazole & 340 & $\mathrm{U}$ & 340 & 330 & $\mathrm{U}$ & 330 & 330 & U & 330 & 330 & $\mathrm{U}$ & 330 \\
\hline Chrysene & 340 & U & 340 & 330 & $\mathrm{U}$ & 330 & 330 & $\mathrm{U}$ & 330 & 330 & $\mathrm{U}$ & 330 \\
\hline Di-n-butylphthalate & 340 & $\mathrm{U}$ & .340 & 29 & $\mathrm{~J}$ & 330 & 24 & $\mathrm{~J}$ & 330 & 80 & $\mathrm{JB}$ & 330 \\
\hline Di-n-octylphthalate & 340 & $\mathrm{U}$ & 340 & 330 & $\mathrm{U}$ & 330 & 330 & U & 330 & 330 & $\mathrm{U}$ & 330 \\
\hline Dibenz $(\mathrm{a}, \mathrm{h})$ anthracene & 340 & U & 340 & 330 & $\mathrm{U}$ & 330 & 18 & $\mathrm{~J}$ & 330 & 330 & $\mathrm{U}$ & 330 \\
\hline Dibenzofuran & 340 & $\mathrm{U}$ & 340 & 330 & $\mathrm{U}$ & 330 & 330 & $\mathrm{U}$ & 330 & 330 & $\mathrm{U}$ & 330 \\
\hline Diethylphthalate & 340 & $\mathrm{U}$ & 340 & 330 & $\mathrm{U}$ & 330 & 330 & $\mathrm{U}$ & 330 & 18 & $\mathrm{~J}$ & 330 \\
\hline Dimethylphthalate & 340 & $\mathrm{U}$ & 340 & 330 & $\mathrm{U}$ & 330 & 330 & $\mathrm{U}$ & 330 & 330 & $\mathrm{U}$ & 330 \\
\hline Fluoranthene & 340 & $\mathrm{U}$ & 340 & 330 & $\mathrm{U}$ & 330 & 330 & $\mathrm{U}$ & 330 & 330 & $\mathrm{U}$ & 330 \\
\hline Fluorene & 340 & $\mathrm{U}$ & 340 & 330 & $\mathrm{U}$ & 330 & 330 & $\mathrm{U}$ & 330 & 330 & $\mathrm{U}$ & 330 \\
\hline Hexachlorobenzene & 340 & $\mathrm{U}$ & 340 & 330 & $\mathrm{U}$ & 330 & 330 & $\mathrm{U}$ & 330 & 330 & $\mathrm{U}$ & 330 \\
\hline Hexachlorobutadiene & 340 & $\mathrm{U}$ & 340 & 330 & U & 330 & 330 & $\mathrm{U}$ & 330 & 330 & $\mathrm{U}$ & 330 \\
\hline Hexachlorocyclopentadiene & 340 & $\mathrm{U}$ & 340 & 330 & $\mathrm{U}$ & 330 & 330 & $\mathrm{U}$ & 330 & 330 & $\mathrm{U}$ & 330 \\
\hline Hexachloroethane & 340 & $\mathrm{U}$ & 340 & 330 & $\mathrm{U}$ & 330 & 330 & $\mathrm{U}$ & 330 & 330 & $\mathrm{U}$ & 330 \\
\hline Indeno $(1,2,3-\mathrm{cd})$ pyrene & 340 & $\mathrm{U}$ & 340 & 330 & $\mathrm{U}$ & 330 & 19 & $\mathrm{~J}$ & 330 & 330 & U & 330 \\
\hline Isophorone & 340 & $\mathrm{U}$ & 340 & 330 & $\mathrm{U}$ & 330 & 330 & $\mathrm{U}$ & 330 & 330 & $\mathrm{U}$ & 330 \\
\hline N-Nitroso-di-n-dipropylamine & 340 & $\mathrm{U}$ & 340 & 330 & $\mathrm{U}$ & 330 & 330 & $\mathrm{U}$ & 330 & 330 & $\mathrm{U}$ & 330 \\
\hline N-Nitrosodiphenylamine & 340 & $\mathrm{U}$ & 340 & 330 & $\mathrm{U}$ & 330 & 330 & $\mathrm{U}$ & 330 & 330 & $\mathrm{U}$ & 330 \\
\hline Naphthalene & 340 & $\mathrm{U}$ & 340 & 330 & $\mathrm{U}$ & 330 & 330 & $\mathrm{U}$ & 330 & 330 & $\mathrm{U}$ & 330 \\
\hline Nitrobenzene & 340 & $\mathrm{U}$ & 340 & 330 & $\mathrm{U}$ & 330 & 330 & $\mathrm{U}$ & 330 & 330 & U & 330 \\
\hline Pentachlorophenol & 850 & $\mathrm{U}$ & 850 & 840 & $\mathrm{U}$ & 840 & 840 & $\mathrm{U}$ & 840 & 840 & $\mathrm{U}$ & 840 \\
\hline Phenanthrene & 340 & $\mathrm{U}$ & 340 & 330 & $\mathrm{U}$ & 330 & 330 & $\mathrm{U}$ & 330 & 330 & $\mathrm{U}$ & 330 \\
\hline Phenol & 340 & $\mathrm{U}$ & 340 & 330 & $\mathrm{U}$ & 330 & 330 & $\mathrm{U}$ & 330 & 27 & $\mathrm{~J}$ & 330 \\
\hline Pyrene & 340 & $\mathrm{U}$ & 340 & 330 & $\mathrm{U}$ & 330 & 330 & $\mathrm{U}$ & 330 & 330 & $\mathrm{U}$ & 330 \\
\hline
\end{tabular}

Attachment

Originator

Checked

Calc. No.

$\frac{1}{\frac{1}{\text { J.M. Capron }}} \frac{\text { T.M. Blakley }}{\text { 0100B-CA-V0292 }}$

Sheet No. 17 of 20

Date $\underline{09 / 14 / 06}$

Date

Rev. No. 0 
Attachment 1. 1607-B2:2 Verification Sampling Results.

\begin{tabular}{|c|c|c|c|c|c|c|c|c|c|c|c|c|}
\hline \multirow[t]{2}{*}{ Constituents } & \multicolumn{3}{|c|}{$\begin{array}{c}\text { J12NX5 } \\
\text { North BCL (Middle) } \\
\text { Sample Date } 6 / 27 / 06 \\
\end{array}$} & \multicolumn{3}{|c|}{$\begin{array}{c}\text { J12NX6 } \\
\text { North BCL (South) } \\
\text { Sample Date } 6 / 27 / 06\end{array}$} & \multicolumn{3}{|c|}{$\begin{array}{c}\text { J12NX7 } \\
\text { Southeastern BCL } \\
\text { Sample Date } 6 / 27 / 06\end{array}$} & \multicolumn{3}{|c|}{$\begin{array}{c}\text { J12NX8 } \\
\text { Small BCL Piles } \\
\text { Sample Date } 6 / 27 / 06\end{array}$} \\
\hline & $\mu \mathrm{g} / \mathrm{kg}$ & $\mathrm{Q}$ & PQL & $\mu \mathrm{g} / \mathrm{kg}$ & $Q$ & PQL & $\mu \mathrm{g} / \mathrm{kg}$ & $\mathbf{Q}$ & PQL & $\mu \mathrm{g} / \mathrm{kg}$ & Q & PQL \\
\hline \multicolumn{13}{|c|}{ Polychlorinated Biphenyls } \\
\hline Aroclor-1016 & 13 & $\mathrm{U}$ & 13 & 13 & $\mathrm{U}$ & 13 & 13 & $\mathrm{U}$ & 13 & 13 & U & 13 \\
\hline Aroclor-1221 & 13 & $\mathrm{U}$ & 13 & 13 & $\mathrm{U}$ & 13 & 13 & $\mathrm{U}$ & 13 & 13 & $\mathrm{U}$ & 13 \\
\hline Aroclor-1232 & 13 & $\mathrm{U}$ & 13 & 13 & $\mathrm{U}$ & 13 & 13 & $\mathrm{U}$ & 13 & 13 & $\mathrm{U}$ & 13 \\
\hline Aroclor-1242 & 13 & $U$ & 13 & 13 & $\mathrm{U}$ & 13 & 13 & $\mathrm{U}$ & 13 & 13 & $\mathrm{U}$ & 13 \\
\hline Aroclor-1248 & 13 & $\mathrm{U}$ & 13 & 13 & $\mathrm{U}$ & 13 & 13 & $\mathrm{U}$ & 13 & 13 & $\mathrm{U}$ & 13 \\
\hline Aroclor-1254 & 6.2 & & 13 & 13 & $\mathrm{U}$ & 13 & 13 & $\mathrm{U}$ & 13 & 13 & $\mathrm{U}$ & 13 \\
\hline Aroclor- 1260 & 13 & $\mathrm{U}$ & 13 & 13 & $\mathrm{U}$ & 13 & 13 & $\mathrm{U}$ & 13 & 11 & & 13 \\
\hline \multicolumn{13}{|c|}{ Pesticides } \\
\hline Aldrin & 1.3 & UD & 1.3 & 1.3 & UD & 1.3 & 1.3 & UD & 1.3 & 1.3 & UD & 1.3 \\
\hline alpha-BHC & 1.3 & UD & 1.3 & 1.3 & UD & 1.3 & 1.3 & UD & 1.3 & 1.3 & UD & 1.3 \\
\hline alpha-Chlordane & 1.3 & UD & 1.3 & 1.3 & UD & 1.3 & 1.3 & UD & 1.3 & 1.3 & UD & 1.3 \\
\hline beta-BHC & 1.3 & UD & 1.3 & 1.3 & UD & 1.3 & 1.3 & UD & 1.3 & 1.3 & UD & 1.3 \\
\hline delta-BHC & 1.3 & UD & 1.3 & 1.3 & UD & 1.3 & 1.3 & UD & 1.3 & 1.3 & UD & 1.3 \\
\hline Dichlorodiphenyldichloroethane & 1.3 & UD & 1.3 & 1.3 & $\mathrm{UD}$ & 1.3 & 1.3 & UD & 1.3 & 1.3 & UD & 1.3 \\
\hline Dichlorodiphenyldichloroethylene & 1.3 & UD & 1.3 & 1.3 & UD & 1.3 & 1.3 & UD & 1.3 & 1.3 & UD & 1.3 \\
\hline Dichlorodiphenyltrichloroethane & 1.3 & UD & 1.3 & 1.3 & UD & 1.3 & 16 & $\mathrm{D}$ & 1.3 & 1.3 & UD & 1.3 \\
\hline Dieldrin & 1.3 & UD & 1.3 & 1.3 & UD & 1.3 & 1.3 & UD & 1.3 & 1.3 & UD & 1.3 \\
\hline Endosulfan I & 1.3 & UD & 1.3 & 1.3 & UD & 1.3 & 1.3 & UD & 1.3 & 1.3 & UD & 1.3 \\
\hline Endosulfan II & 1.3 & UD & 1.3 & 1.3 & UD & 1.3 & 1.3 & UD & 1.3 & 1.3 & UD & 1.3 \\
\hline Endosulfan sulfate & 1.3 & UD & 1.3 & 1.3 & UD & 1.3 & 1.3 & UD & 1.3 & 1.3 & UD & 1.3 \\
\hline Endrin & 1.3 & UD & 1.3 & 1.3 & UD & 1.3 & 1.3 & UD & 1.3 & 1.3 & UD & 1.3 \\
\hline Endrin aldehyde & 1.3 & UD & 1.3 & 2.2 & ID & 1.3 & 1.3 & UD & 1.3 & 1.3 & UD & 1.3 \\
\hline Endrin ketone & 1.3 & UD & 1.3 & 1.3 & UD & 1.3 & 1.3 & UD & 1.3 & 1.3 & UD & 1.3 \\
\hline gamma-BHC (Lindane) & 1.3 & UD & 1.3 & 1.3 & UD & 1.3 & 1.3 & UD & 1.3 & 1.3 & UD & 1.3 \\
\hline gamma-Chlordane & 1.3 & UD & 1.3 & 1.3 & $\mathrm{UD}$ & 1.3 & 1.3 & UD & 1.3 & 1.3 & UD & 1.3 \\
\hline Heptachlor & 1.3 & UD & 1.3 & 1.3 & UD & 1.3 & 1.3 & UD & 1.3 & 1.3 & UD & 1.3 \\
\hline Heptachlor epoxide & 1.3 & UD & 1.3 & 1.3 & UD & 1.3 & 1.3 & UD & 1.3 & 1.3 & UD & 1.3 \\
\hline Methoxychlor & 1.3 & UD & 1.3 & 1.3 & UD & 1.3 & 1.3 & UD & 1.3 & 1.3 & UD & 1.3 \\
\hline Toxaphene & 13 & UD & 13 & 13 & UD & 13 & 13 & UD & 13 & 13 & UD & 13 \\
\hline \multicolumn{13}{|c|}{ Semivolatile Organic Compounds } \\
\hline 1,2,4-Trichlorobenzene & 330 & $\mathrm{U}$ & 330 & 330 & $\mathrm{U}$ & 330 & 340 & $\mathrm{U}$ & 340 & 340 & $\mathrm{U}$ & 340 \\
\hline 1,2-Dichlorobenzene & 330 & $\mathrm{U}$ & 330 & 330 & $\mathrm{U}$ & 330 & 340 & $\mathrm{U}$ & 340 & 340 & $\mathrm{U}$ & 340 \\
\hline 1,3-Dichlorobenzene & 330 & $\mathrm{U}$ & 330 & 330 & $\mathrm{U}$ & 330 & 340 & $\mathrm{U}$ & 340 & 340 & $\mathrm{U}$ & 340 \\
\hline 1,4-Dichlorobenzene & 330 & $\mathrm{U}$ & 330 & 330 & $\mathrm{U}$ & 330 & 340 & $\mathrm{U}$ & 340 & 340 & $\mathrm{U}$ & 340 \\
\hline 2,4,5-Trichlorophenol & 840 & $\mathrm{U}$ & 840 & 840 & $\mathrm{U}$ & 840 & 840 & $\mathrm{U}$ & 840 & 840 & $\mathrm{U}$ & 840 \\
\hline 2,4,6-Trichlorophenol & 330 & $\mathrm{U}$ & 330 & 330 & $\mathrm{U}$ & 330 & 340 & $\mathrm{U}$ & 340 & 340 & $\mathrm{U}$ & 340 \\
\hline 2,4-Dichlorophenol & 330 & $\mathrm{U}$ & 330 & 330 & $\mathrm{U}$ & 330 & 340 & $\mathrm{U}$ & 340 & 340 & $\mathrm{U}$ & 340 \\
\hline 2,4-Dimethylphenol & 330 & $\mathrm{U}$ & 330 & 330 & $\mathrm{U}$ & 330 & 340 & $\mathrm{U}$ & 340 & 340 & $\mathrm{U}$ & 340 \\
\hline 2,4-Dinitrophenol & 840 & $\mathrm{U}$ & 840 & 840 & $\mathrm{U}$ & 840 & 840 & $\mathrm{U}$ & 840 & 840 & $\mathrm{U}$ & 840 \\
\hline 2,4-Dinitrotoluene & 330 & $\mathrm{U}$ & 330 & 330 & $\mathrm{U}$ & 330 & 340 & $\mathrm{U}$ & 340 & 340 & $U$ & 340 \\
\hline 2,6-Dinitrotoluene & 330 & $\mathrm{U}$ & 330 & 330 & $\mathrm{U}$ & 330 & 340 & $\mathrm{U}$ & 340 & 340 & $\mathrm{U}$ & 340 \\
\hline 2-Chloronaphthalene & 330 & $\mathrm{U}$ & 330 & 330 & $\mathrm{U}$ & 330 & 340 & $\mathrm{U}$ & 340 & 340 & $\mathrm{U}$ & 340 \\
\hline 2-Chlorophenol & 330 & $\mathrm{U}$ & 330 & 330 & $\mathrm{U}$ & 330 & 340 & $\mathrm{U}$ & 340 & 340 & $\bar{U}$ & 340 \\
\hline 2-Methylnaphthalene & 330 & $\mathrm{U}$ & 330 & 330 & $\mathrm{U}$ & 330 & 340 & $\mathrm{U}$ & 340 & 340 & $\mathrm{U}$ & 340 \\
\hline 2-Methylphenol (cresol, o-) & 330 & $\mathrm{U}$ & 330 & 330 & $\mathrm{U}$ & 330 & 340 & $\mathrm{U}$ & 340 & 340 & $\mathrm{U}$ & 340 \\
\hline 2-Nitroaniline & 840 & $\mathrm{U}$ & 840 & 840 & $\mathrm{U}$ & 840 & 840 & $\mathrm{U}$ & 840 & 840 & $\mathrm{U}$ & 840 \\
\hline 2-Nitrophenol & 330 & $\mathrm{U}$ & 330 & 330 & $\mathrm{U}$ & 330 & 340 & $\mathrm{U}$ & 340 & 340 & $\mathrm{U}$ & 340 \\
\hline
\end{tabular}

Attachment

Originator

Checked

Calc. No.

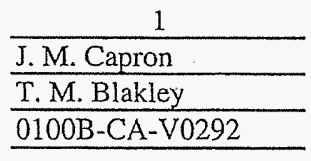

Sheet No. $\frac{18 \text { of } 20}{09 / 14 / 06}$
Date
Date
Rev. No. 
Attachment 1. 1607-B2:2 Verification Sampling Results.

\begin{tabular}{|c|c|c|c|c|c|c|c|c|c|c|c|c|}
\hline \multirow[t]{2}{*}{ Constituents } & \multicolumn{3}{|c|}{$\begin{array}{c}\text { J12NX5 } \\
\text { North BCL (Middle) } \\
\text { Sample Date } 6 / 27 / 06 \\
\end{array}$} & \multicolumn{3}{|c|}{\begin{tabular}{|c|} 
J12NX6 \\
North BCL (South) \\
Sample Date $6 / 27 / 06$ \\
\end{tabular}} & \multicolumn{3}{|c|}{$\begin{array}{c}\text { J12NX7 } \\
\text { Southeastern BCL } \\
\text { Sample Date } 6 / 27 / 06 \\
\end{array}$} & \multicolumn{3}{|c|}{$\begin{array}{c}\text { J12NX8 } \\
\text { Small BCL Piles } \\
\text { Sample Date } 6 / 27 / 06 \\
\end{array}$} \\
\hline & $\mu \mathrm{g} / \mathrm{kg}$ & $\mathbf{Q}$ & PQL & $\mu \mathrm{g} / \mathrm{kg}$ & $Q$ & PQL & $\mu \mathrm{g} / \mathrm{kg}$ & $\mathbf{Q}$ & PQL & $\mu \mathrm{g} / \mathrm{kg}$ & $Q$ & PQL \\
\hline \multicolumn{13}{|c|}{ Semivolatile Organic Compounds (continued) } \\
\hline 3,3'-Dichlorobenzidine & 330 & $\mathrm{U}$ & 330 & 330 & $\mathrm{U}$ & 330 & 340 & $\mathrm{U}$ & 340 & 340 & $\mathrm{U}$ & 340 \\
\hline 3-Nitroaniline & 840 & $\mathrm{U}$ & 840 & 840 & $\mathrm{U}$ & 840 & 840 & $\mathrm{U}$ & 840 & 840 & $\mathrm{U}$ & 840 \\
\hline 4,6-Dinitro-2-methylphenol & 840 & $\mathrm{U}$ & 840 & 840 & $\mathrm{U}$ & 840 & 840 & $\mathrm{U}$ & 840 & 840 & $\mathrm{U}$ & 840 \\
\hline 4-Bromophenyl-phenylether & 330 & $\mathrm{U}$ & 330 & 330 & $\bar{U}$ & 330 & 340 & $\mathrm{U}$ & 340 & 340 & $\mathrm{U}$ & 340 \\
\hline 4-Chloro-3-methylphenol & 330 & $\mathrm{U}$ & 330 & 330 & $\mathrm{U}$ & 330 & 340 & $\mathrm{U}$ & 340 & 340 & $\mathrm{U}$ & 340 \\
\hline 4-Chloroaniline & 330 & $\mathrm{U}$ & 330 & 330 & $\mathrm{U}$ & 330 & 340 & $\mathrm{U}$ & 340 & 340 & $\mathrm{U}$ & 340 \\
\hline 4-Chlorophenyl-phenylether & 330 & $\mathrm{U}$ & 330 & 330 & $\mathrm{U}$ & 330 & 340 & $\mathrm{U}$ & 340 & 340 & $\mathrm{U}$ & 340 \\
\hline 4-Methylphenol (p-cresol) & 330 & $\mathrm{U}$ & 330 & 330 & $\mathrm{U}$ & 330 & 340 & $\mathrm{U}$ & 340 & 340 & $\bar{U}$ & 340 \\
\hline 4-Nitroaniline & 840 & $\mathrm{U}$ & 840 & 840 & $\mathrm{U}$ & 840 & 840 & $\mathrm{U}$ & 840 & 840 & $\mathrm{U}$ & 840 \\
\hline 4-Nitrophenol & 840 & $\mathrm{U}$ & 840 & 840 & $\mathrm{U}$ & 840 & 840 & $\mathrm{U}$ & 840 & 840 & $\mathrm{U}$ & 840 \\
\hline Acenaphthene & 330 & $\mathrm{U}$ & 330 & 330 & $\mathrm{U}$ & 330 & 340 & $\mathrm{U}$ & 340 & 340 & U & 340 \\
\hline Acenaphthylene & 330 & $\mathrm{U}$ & 330 & 330 & $\mathrm{U}$ & 330 & 340 & $U$ & 340 & 340 & $\mathrm{U}$ & 340 \\
\hline Anthracene & 330 & $\mathrm{U}$ & 330 & 330 & $\mathrm{U}$ & 330 & 340 & $\mathrm{U}$ & 340 & 340 & $\mathrm{U}$ & 340 \\
\hline Benzo(a)anthracene & 330 & $\mathrm{U}$ & 330 & 330 & $\mathrm{U}$ & 330 & 340 & $U$ & 340 & 340 & $\mathrm{U}$ & 340 \\
\hline Benzo(a)pyrene & 330 & $\mathrm{U}$ & 330 & 330 & $\mathrm{U}$ & 330 & 340 & $\mathrm{U}$ & 340 & 340 & $\mathrm{U}$ & 340 \\
\hline Benzo(b)fluoranthene & 18 & $\mathrm{~J}$ & 330 & 330 & $\mathrm{U}$ & 330 & 340 & $\mathrm{U}$ & 340 & 340 & $\overline{\mathrm{U}}$ & 340 \\
\hline Benzo(g,h,i)perylene & 330 & $\mathrm{U}$ & 330 & 330 & $\mathrm{U}$ & 330 & 340 & $\mathrm{U}$ & 340 & 340 & $\mathrm{U}$ & 340 \\
\hline Benzo(k)fluoranthene & 330 & $\mathrm{U}$ & 330 & 330 & $\mathrm{U}$ & 330 & 340 & $\mathrm{U}$ & 340 & 340 & $\mathrm{U}$ & 340 \\
\hline bis(2-Chloro-1-methylethyl)ether & 330 & $\mathrm{U}$ & 330 & 330 & $\mathrm{U}$ & 330 & 340 & $\mathrm{U}$ & 340 & 340 & $\mathrm{U}$ & 340 \\
\hline bis(2-Chloroethoxy)methane & 330 & $\mathrm{U}$ & 330 & 330 & $\mathrm{U}$ & 330 & 340 & $\mathrm{U}$ & 340 & 340 & $\mathrm{U}$ & 340 \\
\hline bis(2-Chloroethyl)ether & 330 & $\mathrm{U}$ & 330 & 330 & $\mathrm{U}$ & 330 & 340 & U & 340 & 340 & U & 340 \\
\hline bis(2-Ethylhexyl)phthalate & 26 & $\mathrm{JB}$ & 330 & 25 & JB & 330 & 540 & $\mathrm{~B}$ & 340 & 160 & $\mathrm{JB}$ & 340 \\
\hline Butylbenzylphthalate & 330 & $\mathrm{U}$ & 330 & 330 & $\mathrm{U}$ & 330 & 340 & $\mathrm{U}$ & 340 & 340 & $\mathrm{U}$ & 340 \\
\hline Carbazole & 330 & $\mathrm{U}$ & 330 & 330 & $\bar{U}$ & 330 & 340 & $\mathrm{U}$ & 340 & 340 & $\bar{U}$ & 340 \\
\hline Chrysene & 330 & $U$ & 330 & 330 & $\mathrm{U}$ & 330 & 340 & $\mathrm{U}$ & 340 & 340 & $\mathrm{U}$ & 340 \\
\hline Di-n-butylphthalate & 23 & JB & 330 & 330 & $\mathrm{U}$ & 330 & 23 & $\mathrm{JB}$ & 340 & 36 & $\mathrm{JB}$ & 340 \\
\hline Di-n-octylphthalate & 330 & $\mathrm{U}$ & 330 & 330 & $\mathrm{U}$ & 330 & 340 & $\mathrm{U}$ & 340 & 340 & $\mathrm{U}$ & 340 \\
\hline Dibenz $(a, h)$ anthracene & 330 & $\mathrm{U}$ & 330 & 330 & $\mathrm{U}$ & 330 & 340 & $\mathrm{U}$ & 340 & 340 & $\mathrm{U}$ & 340 \\
\hline Dibenzofuran & 330 & $\mathrm{U}$ & 330 & 330 & $\mathrm{U}$ & 330 & 340 & $\mathrm{U}$ & 340 & 340 & $\mathrm{U}$ & 340 \\
\hline Diethylphthalate & 330 & $\mathrm{U}$ & 330 & 330 & $\mathrm{U}$ & 330 & 340 & U & 340 & 340 & $\mathrm{U}$ & 340 \\
\hline Dimethylphthalate & 330 & $\mathrm{U}$ & 330 & 330 & $\mathrm{U}$ & 330 & 340 & $\mathrm{U}$ & 340 & 340 & $\mathrm{U}$ & 340 \\
\hline Fluoranthene & 32 & $\mathrm{~J}$ & 330 & 330 & $\mathrm{U}$ & 330 & 340 & $\mathrm{U}$ & 340 & 340 & $\mathrm{U}$ & 340 \\
\hline Fluorene & 330 & $U$ & 330 & 330 & $\mathrm{U}$ & 330 & 340 & $\mathrm{U}$ & 340 & 340 & $\mathrm{U}$ & 340 \\
\hline Hexachlorobenzene & 330 & $\mathrm{U}$ & 330 & 330 & $\mathrm{U}$ & 330 & 340 & $\mathrm{U}$ & 340 & 340 & $\mathrm{U}$ & 340 \\
\hline Hexachlorobutadiene & 330 & $\mathrm{U}$ & 330 & 330 & $\mathrm{U}$ & 330 & 340 & $\mathrm{U}$ & 340 & 340 & $\mathrm{U}$ & 340 \\
\hline Hexachlorocyclopentadiene & 330 & $\mathrm{U}$ & 330 & 330 & $\mathrm{U}$ & 330 & 340 & $\mathrm{U}$ & 340 & 340 & $\mathrm{U}$ & 340 \\
\hline Hexachloroethane & 330 & $\mathrm{U}$ & 330 & 330 & $\mathrm{U}$ & 330 & 340 & $\mathrm{U}$ & 340 & 340 & $\bar{U}$ & 340 \\
\hline Indeno(1,2,3-cd)pyrene & 330 & $\mathrm{U}$ & 330 & 330 & $\mathrm{U}$ & 330 & 340 & $\mathrm{U}$ & 340 & 340 & $\mathrm{U}$ & 340 \\
\hline Isophorone & 330 & $\mathrm{U}$ & 330 & 330 & $\mathrm{U}$ & 330 & 340 & $\mathrm{U}$ & 340 & 340 & $\mathrm{U}$ & 340 \\
\hline N-Nitroso-di-n-dipropylamine & 330 & $\mathrm{U}$ & 330 & 330 & $\mathrm{U}$ & 330 & 340 & $\mathrm{U}$ & 340 & 340 & $\mathrm{U}$ & 340 \\
\hline N-Nitrosodiphenylamine & 330 & $\mathrm{U}$ & 330 & 330 & U & 330 & 340 & $\mathrm{U}$ & 340 & 340 & $\mathrm{U}$ & 340 \\
\hline Naphthalene & 330 & $\mathrm{U}$ & 330 & 330 & $\mathrm{U}$ & 330 & 340 & $\mathrm{U}$ & 340 & 340 & $\mathrm{U}$ & 340 \\
\hline Nitrobenzene & 330 & $\mathrm{U}$ & 330 & 330 & $\mathrm{U}$ & 330 & 340 & $\mathrm{U}$ & 340 & 340 & $\mathrm{U}$ & 340 \\
\hline Pentachlorophenol & 840 & $\mathrm{U}$ & 840 & 840 & $\mathrm{U}$ & 840 & 840 & $\mathrm{U}$ & 840 & 840 & $\mathrm{U}$ & 840 \\
\hline Phenanthrene & 330 & $\mathrm{U}$ & 330 & 330 & $\mathrm{U}$ & 330 & 340 & $\mathrm{U}$ & 340 & 340 & $\mathrm{U}$ & 340 \\
\hline Phenol & 330 & U & 330 & 330 & $\mathrm{U}$ & 330 & 340 & $\mathrm{U}$ & 340 & 340 & $\mathrm{U}$ & 340 \\
\hline Pyrene & 26 & $\mathrm{~J}$ & 330 & 330 & $\bar{U}$ & 330 & 17 & $\mathrm{~J}$ & 340 & 340 & $\mathrm{U}$ & 340 \\
\hline
\end{tabular}

Attachment

Originator

Checked

Calc. No.

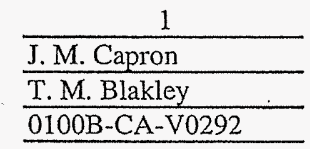

Sheet No. 19 of 20

Date $09 / 14 / 06$

Date

Rev. No. 0 
Attachment 1. 1607-B2:2 Verification Sampling Results.

\begin{tabular}{|c|c|c|c|c|c|c|c|}
\hline \multirow[t]{2}{*}{ Constituents } & \multicolumn{3}{|c|}{$\begin{array}{c}\text { J12P04 } \\
\text { Equipment Blank } \\
\text { Sample Date } 6 / 27 / 06\end{array}$} & \multirow[t]{2}{*}{ Constituents } & \multicolumn{3}{|c|}{$\begin{array}{c}\text { J12P04 } \\
\text { Equipment Blank } \\
\text { Sample Date 6/27/06 }\end{array}$} \\
\hline & $\mu \mathrm{g} / \mathrm{kg}$ & $\mathbf{Q}$ & PQL & & $\mu \mathrm{g} / \mathrm{kg}$ & Q & PQL \\
\hline \multicolumn{4}{|c|}{ Semivolatile Organic Compounds } & \multicolumn{4}{|c|}{ Semivolatile Organic Compounds (continued) } \\
\hline 1,2,4-Trichlorobenzene & 330 & $\mathrm{U}$ & 330 & Di-n-octylphthalate & 330 & $\mathrm{U}$ & 330 \\
\hline 1,2-Dichlorobenzene & 330 & $\mathrm{U}$ & 330 & Dibenz $(a, h)$ anthracene & 330 & $\mathrm{U}$ & 330 \\
\hline 1,3-Dichlorobenzene & 330 & $\mathrm{U}$ & 330 & Dibenzofuran & 330 & $\mathrm{U}$ & 330 \\
\hline 1,4-Dichlorobenzene & 330 & $\mathrm{U}$ & 330 & Diethylphthalate & 330 & $\mathrm{U}$ & 330 \\
\hline 2,4,5-Trichlorophenol & 830 & $\mathrm{U}$ & 830 & Dimethylphthalate & 330 & $\mathrm{U}$ & 330 \\
\hline 2,4,6-Trichlorophenol & 330 & $\mathrm{U}$ & 330 & Fluoranthene & 330 & $\mathrm{U}$ & 330 \\
\hline 2,4-Dichlorophenol & 330 & $\mathrm{U}$ & 330 & Fluorene & 330 & $\mathrm{U}$ & 330 \\
\hline 2,4-Dimethylphenol & 330 & U & 330 & Hexachlorobenzene & 330 & $\mathrm{U}$ & 330 \\
\hline 2,4-Dinitrophenol & 830 & $\mathrm{U}$ & 830 & Hexachlorobutadiene & 330 & $\mathrm{U}$ & 330 \\
\hline 2,4-Dinitrotoluene & 330 & $\mathrm{U}$ & 330 & Hexachlorocyclopentadiene & 330 & $\mathrm{U}$ & 330 \\
\hline 2,6-Dinitrotoluene & 330 & $\mathrm{U}$ & 330 & \begin{tabular}{|l} 
Hexachloroethane \\
\end{tabular} & 330 & U & 330 \\
\hline 2-Chloronaphthalene & 330 & U & 330 & Indeno(1,2,3-cd)pyrene & 330 & $\mathrm{U}$ & 330 \\
\hline 2-Chlorophenol & 330 & $\mathrm{U}$ & 330 & Isophorone & 330 & $\mathrm{U}$ & 330 \\
\hline 2-Methylnaphthalene & 330 & $\mathrm{U}$ & 330 & N-Nitroso-di-n-dipropylamine & 330 & $\mathrm{U}$ & 330 \\
\hline 2-Methylphenol (cresol, o-) & 330 & $\mathrm{U}$ & 330 & N-Nitrosodiphenylamine & 330 & U & 330 \\
\hline 2-Nitroaniline & 830 & $\mathrm{U}$ & 830 & \begin{tabular}{|l|} 
Naphthalene \\
\end{tabular} & 330 & $\mathrm{U}$ & 330 \\
\hline 2-Nitrophenol & 330 & $\mathrm{U}$ & 330 & \begin{tabular}{|l} 
Nitrobenzene \\
\end{tabular} & 330 & $\mathrm{U}$ & 330 \\
\hline 3,3'-Dichlorobenzidine & 330 & $\mathrm{U}$ & 330 & Pentachlorophenol & 830 & $\mathrm{U}$ & 830 \\
\hline 3-Nitroaniline & 830 & $\mathrm{U}$ & 830 & Phenanthrene & 330 & $\mathrm{U}$ & 330 \\
\hline 4,6-Dinitro-2-methylphenol & 830 & $\mathrm{U}$ & 830 & \begin{tabular}{|l|} 
Phenol \\
\end{tabular} & 330 & $\mathrm{U}$ & 330 \\
\hline 4-Bromophenyl-phenylether & 330 & $\mathrm{U}$ & 330 & Pyrene & 330 & $\mathrm{U}$ & 330 \\
\hline 4-Chloro-3-methylphenol & 330 & $\mathrm{U}$ & 330 & & & & \\
\hline
\end{tabular}

\begin{tabular}{|l|c|c|c|}
\hline 4-Chloro-3-methylphenol & 330 & $\mathrm{U}$ & 330 \\
\hline 4-Chloroaniline & 330 & $\mathrm{U}$ & 330 \\
\hline 4-Chlorophenyl-phenylether & 330 & $\mathrm{U}$ & 330 \\
\hline 4-Methylphenol (p-cresol) & 330 & $\mathrm{U}$ & 330 \\
\hline 4-Nitroaniline & 830 & $\mathrm{U}$ & 830 \\
\hline 4-Nitrophenol & 830 & $\mathrm{U}$ & 830 \\
\hline Acenaphthene & 330 & $\mathrm{U}$ & 330 \\
\hline Acenaphthylene & 330 & $\mathrm{U}$ & 330 \\
\hline Anthracene & 330 & $\mathrm{U}$ & 330 \\
\hline Benzo(a)anthracene & 330 & $\mathrm{U}$ & 330 \\
\hline Benzo(a)pyrene & 330 & $\mathrm{U}$ & 330 \\
\hline Benzo(b)fluoranthene & 330 & $\mathrm{U}$ & 330 \\
\hline Benzo(g,h,i)perylene & 330 & $\mathrm{U}$ & 330 \\
\hline Benzo(k)fluoranthene & 330 & $\mathrm{U}$ & 330 \\
\hline bis(2-Chloro-1-methylethyl)ether & 330 & $\mathrm{U}$ & 330 \\
\hline bis(2-Chloroethoxy)methane & 330 & $\mathrm{U}$ & 330 \\
\hline bis(2-Chloroethyl)ether & 330 & $\mathrm{U}$ & 330 \\
\hline bis(2-Ethylhexyl)phthalate & 4400 & $\mathrm{BD}$ & 330 \\
\hline Butylbenzylphthalate & 330 & $\mathrm{U}$ & 330 \\
\hline Carbazole & 330 & $\mathrm{U}$ & 330 \\
\hline Chrysene & 330 & $\mathrm{U}$ & 330 \\
\hline Di-n-butylphthalate & 45 & $\mathrm{JB}$ & 330 \\
\hline
\end{tabular}

Attachment $\frac{1}{\text { Originator J.M. Capron }}$
Checked T.M. Blakley
Calc. No. 0100 B-CA-V0292

Sheet No.

Date

Date

Rev. No.
20 of 20 $09 / 14 / 06$

0




\section{CALCULATION COVER SHEET}

Project Title 100-B/C Remaining Pipes and Sewers Field Remediation Job No. 14655 Area $100-\mathrm{B} / \mathrm{C}$

Discipline Environmental

*Calc. No. 0100B-CA-V0293

Subject 1607-B2:2 Waste Site Hazard Quotient and Carcinogenic Risk Calculations Computer Program Excel Program No. Excel 2003

The attached calculations have been generated to document compliance with established cleanup levels. These calculations should be used in conjunction with other relevant documents in the administrative record.

\section{Committed Calculation $\otimes \quad$ Preliminary $\square \quad$ Superseded $\square \quad$ Voided}

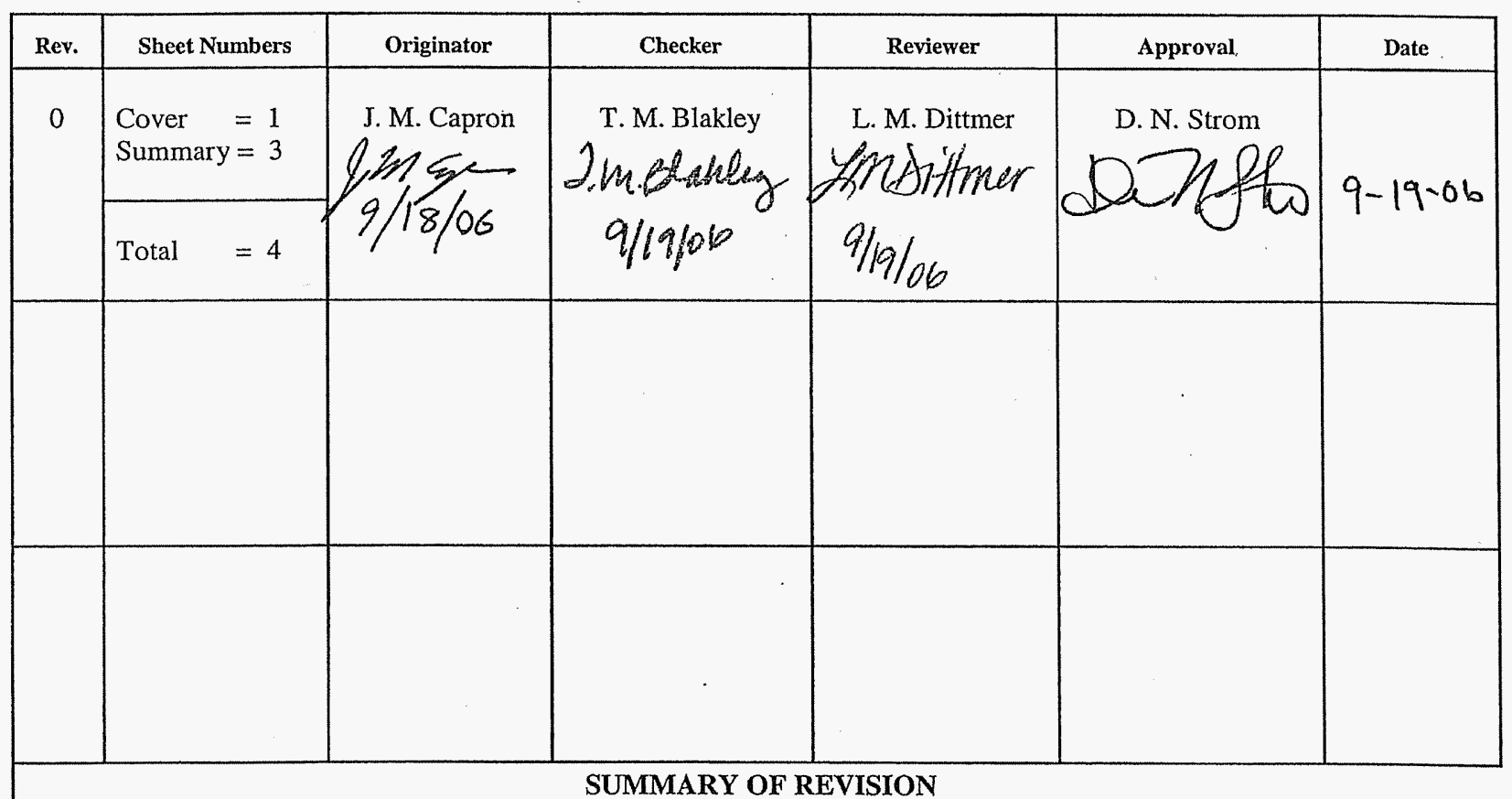

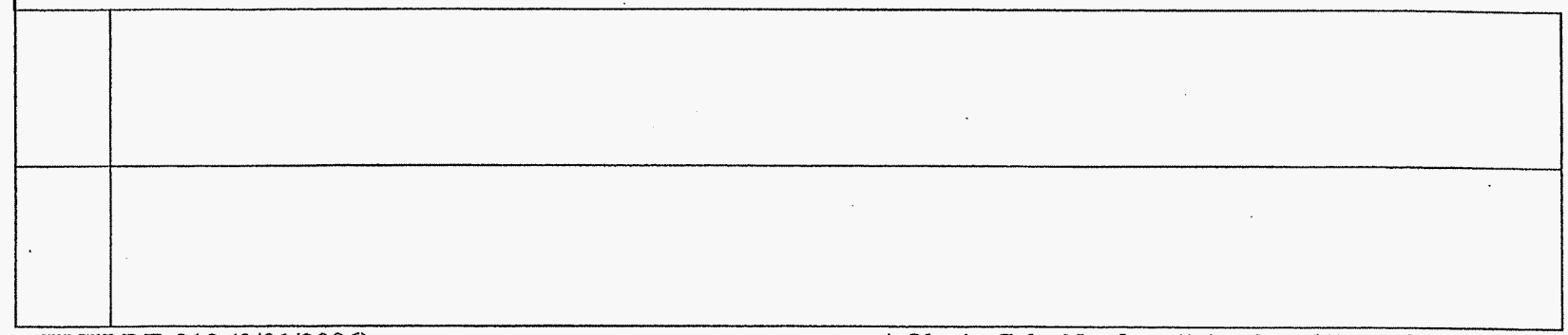


Washington Closure Hanford CALCULATION SHEET

\begin{tabular}{l|l|l|l|} 
Originator: & J. M. Capron Oanc & Date: & $09 / 18 / 06$ \\
\hline
\end{tabular}

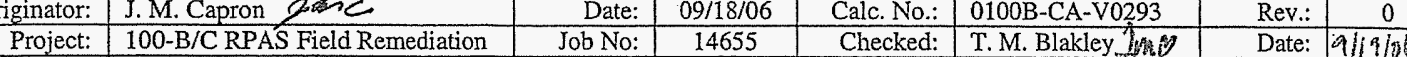

Subject: $1607-B 2: 2$ Waste Site Hazard Quotient and Carcinogenic Risk Calculations 1 Sheet No. 1 of 3

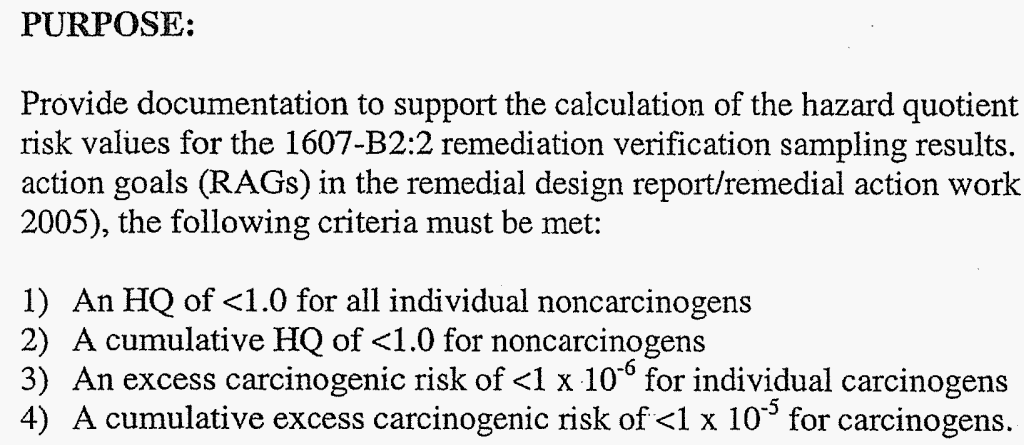
2005), the following criteria must be met:

1) An $\mathrm{HQ}$ of $<1.0$ for all individual noncarcinogens

2) A cumulative $\mathrm{HQ}$ of $<1.0$ for noncarcinogens

3) An excess carcinogenic risk of $<1 \times 10^{-6}$ for individual carcinogens

4) A cumulative excess carcinogenic risk of $<1 \times 10^{-5}$ for carcinogens.

Provide documentation to support the calculation of the hazard quotient (HQ) and excess carcinogenic risk values for the 1607-B2:2 remediation verification sampling results. In accordance with the remedial action goals (RAGs) in the remedial design report/remedial action work plan (RDR/RAWP) (DOE-RL

\section{GIVEN/REFERENCES:}

1) DOE-RL, 2005, Remedial Design Report/Remedial Action Work Plan for the 100 Areas, DOE/RL-96-17, Rev. 5, U.S. Department of Energy, Richland Operations Office, Richland, Washington.

2) WAC 173-340, "Model Toxics Control Act - Cleanup," Washington Administrative Code, 1996.

3) WCH, 2006, 1607-B2:2 Waste Site Cleanup Verification 95\% UCL Calculations, Calculation No. 0100B-CA-V0292, Washington Closure Hanford, Richland, Washington.

\section{SOLUTION:}

1) Calculate an HQ for each noncarcinogenic constituent detected above background and compare to the individual HQ of $<1.0$ (DOE-RL 2005).

2) Sum the HQs and compare to the cumulative HQ criterion of $<1.0$.

3) Calculate an excess carcinogenic risk value for each carcinogenic constituent detected above background and compare to the individual excess carcinogenic risk criterion of $<1 \times 10^{-6}$ (DOE-RL 2005).

4) Sum the excess carcinogenic risk values and compare to the cumulative excess carcinogenic risk criterion of $<1 \times 10^{-5}$.

\section{METHODOLOGY:}

Hazard quotient and carcinogenic risk calculations were performed for the $1607-\mathrm{B} 2: 2$ subsite using the higher of the remediation footprint statistical value and overburden material maximum value for each analyte detected above background. Of the contaminants of concern (COCs) and contaminants of potential concern (COPCs) for the site, boron, molybdenum, and strontium require the HQ calculations because they were detected and Washington State or Hanford Site background values are not available. 
Washington Closure Hanford

CALCULATTION SHEET

\begin{tabular}{|c|c|c|c|c|c|c|}
\hline Originator: & J.M. Capron gexc & Date: & $09 / 18 / 06$ & Calc. No.: & 0100B-CA-V0293 & Rev.: \\
\hline Project: & 100-B/C RPAS Field Remediation & Job No: & 14655 & Checked: & T. M. Blakley Ime & Date: 9119106 \\
\hline Subject: & \multicolumn{5}{|c|}{ 1607-B2:2 Waste Site Hazard Quotient and Carcinogenic Risk Calculations } & Sheet $N$ \\
\hline
\end{tabular}

Copper and mercury are included because they were quantified above their respective Hanford Site background values. Hexavalent chromium, aroclor-1254, aroclor-1260, and multiple chlorinated pesticides and semivolatile organic compounds (as identified in Table 1) are included because they were detected by laboratory analysis and cannot be attributed to natural occurrence. All other site nonradionuclide COCs and COPCs were not detected or were detected below background levels. An example of the HQ and risk calculations is presented below:

1) For example, the statistical value for boron is $4.7 \mathrm{mg} / \mathrm{kg}$, divided by the noncarcinogenic RAG value of $16,000 \mathrm{mg} / \mathrm{kg}$ (calculated in accordance with the noncarcinogenic toxics effects formula in WAC $173-340-740$ [3]), is $2.9 \times 10^{-4}$. Comparing this value, and all other individual values, to the requirement of $<1.0$, this criterion is met.

2) After the HQ calculations are completed for the appropriate analytes, the cumulative HQ is obtained by summing the individual values. (To avoid errors due to intermediate rounding, the individual HQ values prior to rounding are used for this calculation.) The sum of the HQ values is $2.6 \times 10^{-1}$. Comparing this value to the requirement of $<1.0$, this criterion is met.

3) To calculate the excess carcinogenic risk, the $95 \%$ upper confidence limit or maximum value is divided by the carcinogenic RAG value, then multiplied by $1 \times 10^{-6}$. For example, the $95 \%$ upper confidence limit value for hexavalent chromium is $0.35 \mathrm{mg} / \mathrm{kg}$; divided by $2.1 \mathrm{mg} / \mathrm{kg}$ and multiplied as indicated is $1.7 \times 10^{-7}$. Comparing this value, and all other individual values, to the requirement of $<1 \times 10^{-6}$, this criterion is met.

4) After these calculations are completed for the carcinogenic analytes, the cumulative excess carcinogenic risk is obtained by summing the individual values. (To avoid errors due to intermediate rounding, the individual values prior to rounding are used for this calculation.) The sum of the excess carcinogenic risk values is $1.4 \times 10^{-6}$. Comparing this value to the requirement of $<1 \times 10^{-5}$, this criterion is met.

\section{RESULTS:}

1) List individual noncarcinogens and corresponding HQs $>1.0$ : None

2) List the cumulative noncarcinogenic HQ $>1.0$ : None

3) List individual carcinogens and corresponding excess cancer risk $>1 \times 10^{-6}$ : None

4) List the cumulative excess cancer risk for carcinogens $>1 \times 10^{-5}$ : None.

Table 1 shows the results of the calculations for the 1607-B2:2 subsite.

\section{CONCLUSION:}

This calculation demonstrates that the 1607-B2:2 subsite meets the requirements for hazard quotient and excess carcinogenic risk as identified in the RDR/RAWP (DOE-RL 2005). 
Washington Closure Hanford

CALCULATION SHEET

Originator: J.M. Capron ganc Date: $109 / 18 / 06$

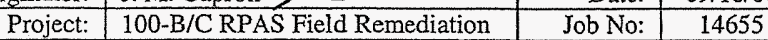

Subject: $1607-B 2: 2$ Waste Site Hazard Quotient and Carcinogenic Risk Calculations

\begin{tabular}{|c|c|c|}
\hline Rev.: & 0 \\
\hline Date: & $91 / 9106$ \\
\hline \multicolumn{2}{|c|}{ Sheet No. 3 of 3}
\end{tabular}

Table 1. Hazard Quotient and Excess Cancer Risk Results for the 1607-B2:2 Subsite.

\begin{tabular}{|c|c|c|c|c|c|}
\hline $\begin{array}{l}\text { Contaminants of Concern/ } \\
\text { Contaminants of Potential Concern }\end{array}$ & $\begin{array}{c}\text { Maximum or } \\
\text { Statistical Value } \mathrm{e}^{\mathrm{a}} \\
(\mathrm{mg} / \mathrm{kg})\end{array}$ & $\begin{array}{c}\text { Noncarcinogen } \\
\text { RAG }^{\mathbf{b}} \\
(\mathrm{mg} / \mathrm{kg})\end{array}$ & $\begin{array}{l}\text { Hazard } \\
\text { Quotient }\end{array}$ & $\begin{array}{c}\text { Carcinogen } \\
\mathbf{R A G}^{\mathbf{b}} \\
(\mathrm{mg} / \mathrm{kg})\end{array}$ & $\begin{array}{l}\text { Carcinogen } \\
\text { Risk }\end{array}$ \\
\hline \multicolumn{6}{|c|}{ 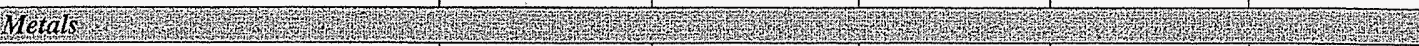 } \\
\hline Boron & 4.7 & 16,000 & $2.9 \mathrm{E}-04$ & -- & -- \\
\hline Chromium, hexavalent $^{\mathrm{c}}$ & 0.35 & 240 & $1.5 \mathrm{E}-03$ & 2.1 & $1.7 \mathrm{E}-07$ \\
\hline Copper & 34.0 & 2,960 & 1.1E-02 & - & $-\cdots$ \\
\hline Mercury & 0.92 & 24 & $3.8 \mathrm{E}-02$ & -- & -- \\
\hline Molybdenum & 0.37 & 400 & $9.3 \mathrm{E}-04$ & -- & -- \\
\hline Strontium & 48.2 & 48,000 & $1.0 \mathrm{E}-03$ & -- & -- \\
\hline \multicolumn{6}{|c|}{ Sentivoluifes } \\
\hline Benzo(a)anthracene & 0.041 & $\ldots$ & - & 1.37 & $3.0 \mathrm{E}-08$ \\
\hline Benzo(a)pyrene & 0.033 & -- & -- & 0.137 & $2.4 \mathrm{E}-07$ \\
\hline Benzo(b)fluoranthene & 0.041 & -- & -- & 1.37 & $3.0 \mathrm{E}-08$ \\
\hline Benzo(k)fluoranthene & 0.035 & -- & -- & 13.7 & $2.6 \mathrm{E}-09$ \\
\hline Benzo(g,h,i)perylene ${ }^{d}$ & 0.030 & 2,400 & $1.3 \mathrm{E}-05$ & - & -- \\
\hline Bis(2-ethylhexyl) phthalate & 1.6 & 1,600 & $1.0 \mathrm{E}-03$ & 71.4 & $2.2 \mathrm{E}-08$ \\
\hline Chrysene & 0.064 & -- & -- & 137 & $4.7 \mathrm{E}-10$ \\
\hline Dibenz $(\mathrm{a}, \mathrm{h})$ anthracene & 0.022 & -- & -- & 0.137 & $1.6 \mathrm{E}-07$ \\
\hline Diethylphthalate & 0.018 & 64,000 & $2.8 \mathrm{E}-07$ & -- & - \\
\hline Di-n-butylphthalate & 0.080 & 8,000 & $1.0 \mathrm{E}-05$ & - & -- \\
\hline Fluoranthene & 0.079 & 3,200 & $2.5 \mathrm{E}-05$ & -- & - \\
\hline Indeno $(1,2,3$-cd) pyrene & 0.028 & $-\cdots$ & -- & 1.37 & 2.0E-08 \\
\hline Methylnaphthalene; 2 - & 0.019 & 320 & $5.9 \mathrm{E}-05$ & -- & -- \\
\hline Naphthalene & 0.017 & 1,600 & $1.1 \mathrm{E}-05$ & - & - \\
\hline Phenanthrene $^{\mathrm{d}}$ & 0.046 & 24,000 & $1.9 \mathrm{E}-06$ & - & - \\
\hline Phenol & 0.027 & 24,000 & $1.1 \mathrm{E}-06$ & - & - \\
\hline Pyrene & 0.066 & 2,400 & $2.8 \mathrm{E}-05$ & - & - \\
\hline
\end{tabular}

Pesteides $\mathrm{BHC}$, beta-

Chlordane (alpha and gamma)

DDE, 4,4'-

DDT, 4,4'-

Endosulfan (I, II, sulfate)

Endrin (and ketone, aldehyde)

Heptachlor epoxide

Methoxychlor

0.0019

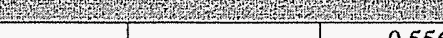

$0.556 \quad \mid-3.4 \mathrm{E}-09$

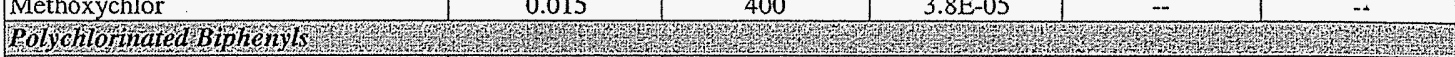

\begin{tabular}{|l|c|c|c|c|c|}
\hline Aroclor-1254 & 0.33 & 1.6 & $2.1 \mathrm{E}-01$ & 0.5 & $6.6 \mathrm{E}-07$ \\
\hline
\end{tabular}

\begin{tabular}{|l|l|l|l|l|l}
\hline Aroclor-1260 & 0.011 & - & - & 0.5 & $2.2 \mathrm{E}-08$
\end{tabular}

Totals s.

Cumulative Hazard Quotient:

Cumulative Excess Cancer Risk:

1.4E-06

Notes:

$\stackrel{a}{=}$ From WCH (2006).

${ }^{b}=$ Value obtained from Washington Administrative Code (WAC) 173-340-740(3), Method B, 1996.

${ }^{c}=$ Value for the carcinogen RAG calculated based on the inhalation exposure pathway (WAC) 173-340-750(3), 1996.

${ }^{d}=$ Toxicity data are not available for this constituent. RAGs for benzo(g,h,i)perylene and phenanthrene are based on the surrogate chemicals pyrene and anthracene, respectively.

-- = not applicable

$\mathrm{RAG}=$ remedial action goal 


\section{APPENDIX C}

\section{SITE PHOTOGRAPHY}




\section{Photograph C-1. Typical Concrete-Encased Vitrified Clay Pipe Segment.}

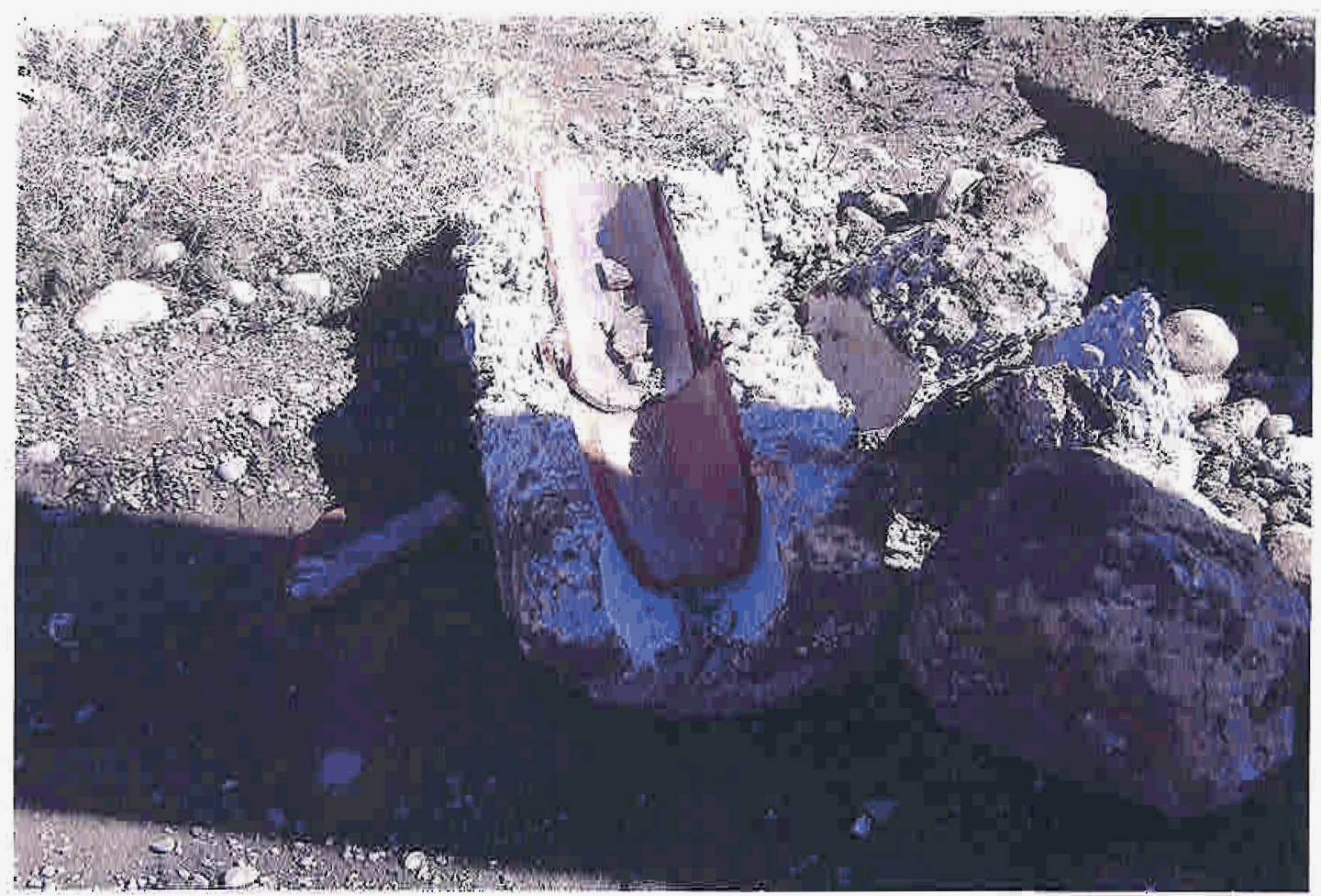

Photograph C-2. Excavation of the 100-B-14:2 (Area 4) Sewer Lines.

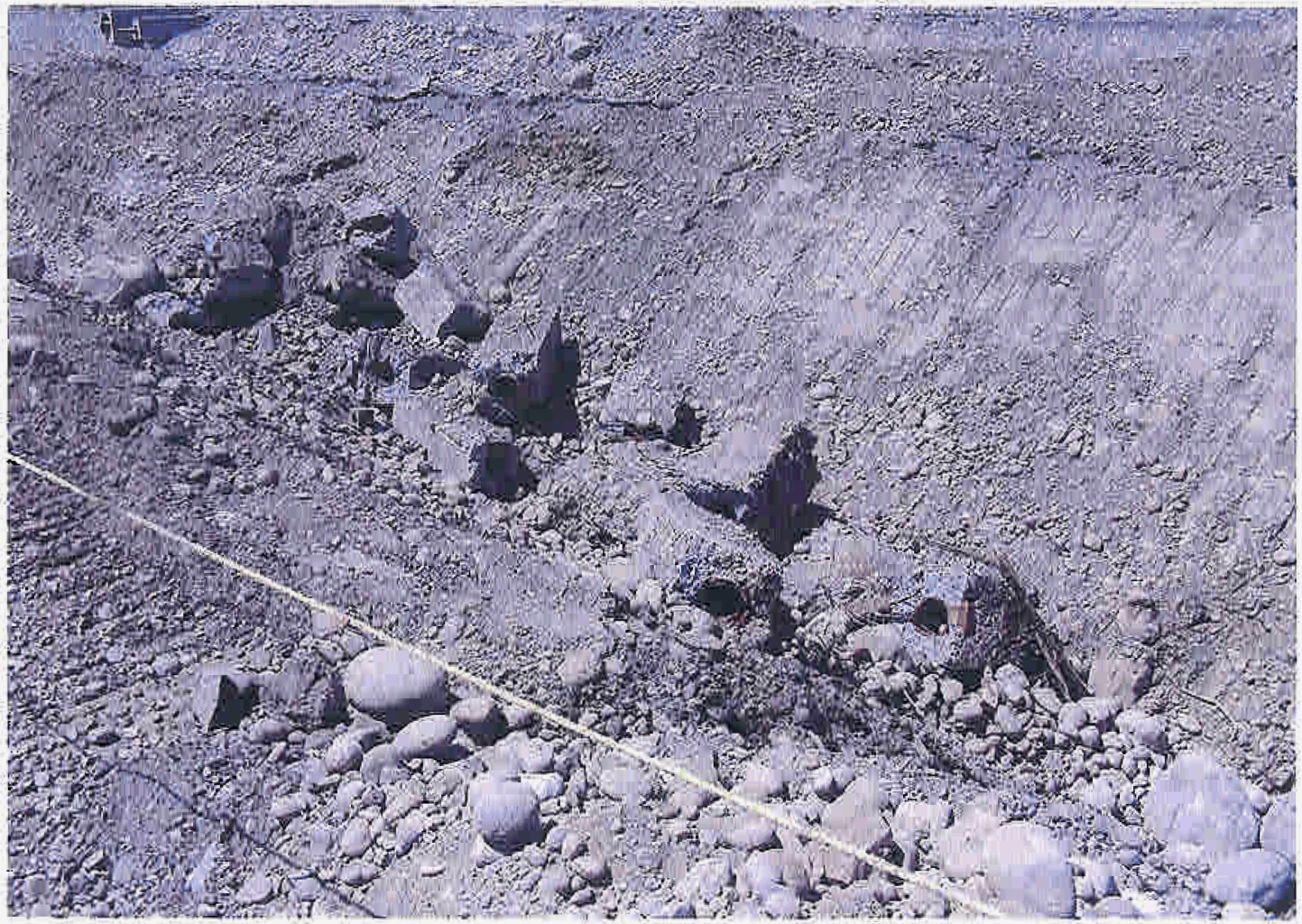


APPENDIX D

100 AREA ANALOGOUS SITES RESRAD CALCULATIONS 


\section{DISCLAIMER FOR CALCULATIONS}

The calculation provided in this appendix has been generated to document compliance with established cleanup levels. This calculation brief should be used in conjunction with other relevant documents in the administrative record. 


\section{CALCULATION COVER SHEET}

Project Title 100 Area Remedial Action

Job No. 22192

Area 100 Area

Discipline Environmental

*Calc. No. 0100X-CA-V0050

Subject 100 Area Analogous Sites RESRAD Calculations

Computer Program RESRAD

Program No.

Version 6.22

The attached calculations have been generated to document compliance with established cleanup levels.

These documents should be used in conjunction with other relevant documents in the administrative record.

\section{Committed Calculation $\otimes \quad$ Preliminary $\square \quad$ Superseded}

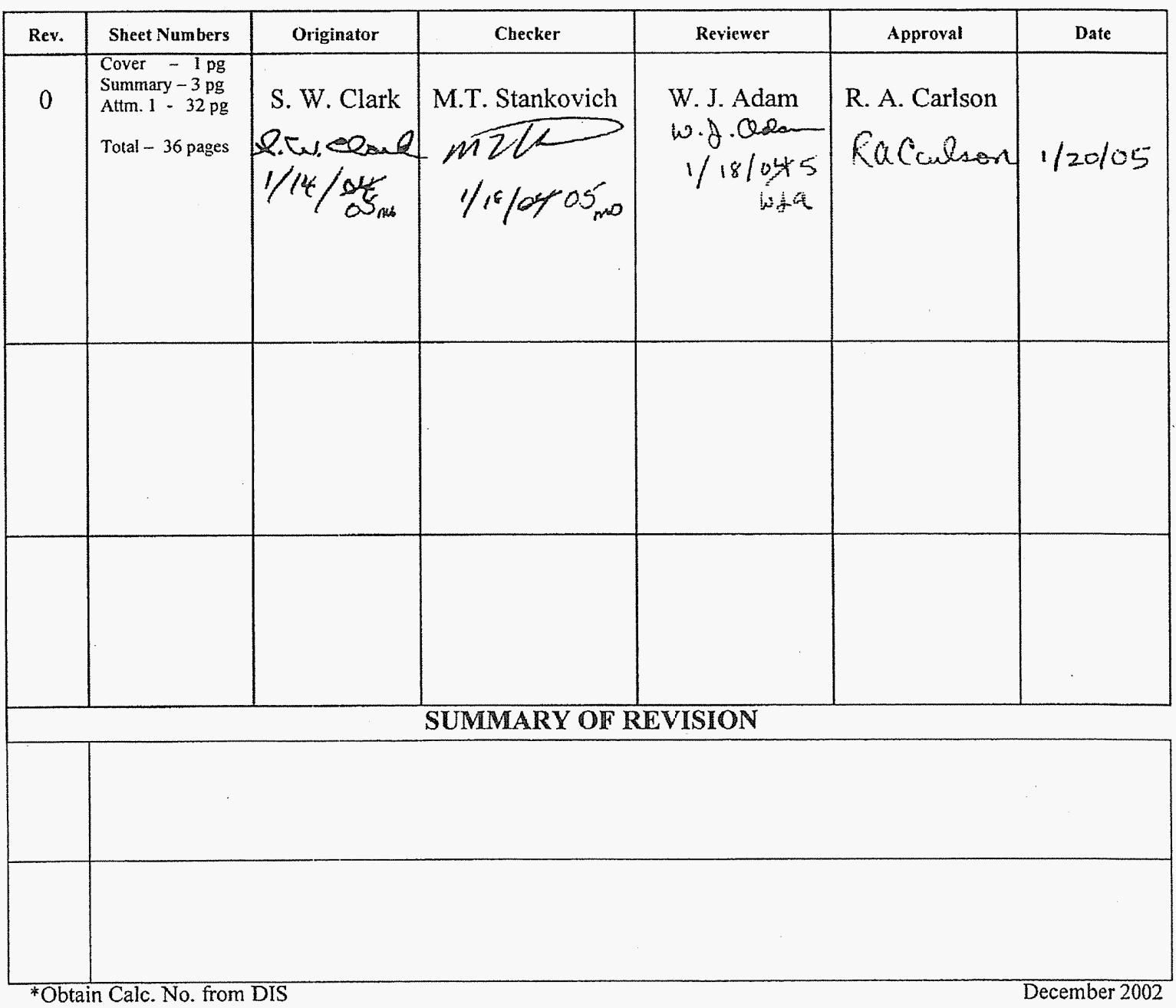

DE01-437.03 
Bechtel Hanford, Inc. CALCULATION SHEET

\begin{tabular}{|c|c|c|c|c|c|c|c|}
\hline ginator: & S.W. Clark X & Date: & $12 / 14704$ & Calc. No.: & $0100 \mathrm{X}-\mathrm{CA}-\mathrm{V} 0050$ & Rev.: & 0 \\
\hline Project: & 100 Area Remedial Action & Job No: & 22192 & Checked: & M. T. Stankovich nots & Date: & 118604 \\
\hline
\end{tabular}

\section{PURPOSE:}

Provide lookup values of contaminant distribution coefficients ( $\mathrm{Kd}$ values) that will be protective of groundwater as a function of uncontaminated vadose zone thickness for contaminants at 100 Area remaining sites. The RESRAD model was used with input parameters from the 100 Area RDR/RAWP to determine contaminant $\mathrm{Kd}$ values protective of groundwater as a function of the thickness of the unsaturated/ uncontaminated vadose zone over a period of 1,000 years.

\section{GIVEN/REFERENCES:}

1) RESidual RADioactivity (RESRAD) computer code, version 6.22, February 2004, to calculate compliance with residual radioactivity guidelines, developed for the U.S. Department of Energy by the Environmental Assessment Division of Argonne National Laboratory, Argonne, Illinois.

2) RESRAD input parameters from Table B-1 of the Remedial Design Report/Remedial Action Work Plan for the 100 Area (RDR/RAWP), DOE/RL-96-17, Rev. 4, U.S. Department of Energy, Richland Operations Office, Richland, Washington.

\section{SOLUTION:}

1) RESRAD modeling runs were performed to determine the minimum (lowest) contaminant $\mathrm{Kd}$ value that would be protective of groundwater at several unsaturated/uncontaminated vadose zone thicknesses. This was done by simultaneously running different $\mathrm{Kd}$ values for the long-lived surrogate radionuclides listed in Table 1 at each unsaturated/uncontaminated vadose zone thickness and determining the lowest $\mathrm{Kd}$ value that showed no concentration in the groundwater over a time period of 1,000 years.

2) Input parameters from Table B-1 of the 100 Area RDR/RAWP were used for RESRAD modeling. Thickness of the unsaturated/uncontaminated vadose zone was varied over a range of values from zero meters to 25 meters. Waste site area was varied from 10,000 square meters to 100 square meters to determine if waste site area affected the $\mathrm{Kd}$ value that would be calculated to be protective of groundwater (it did not). Surrogate radionuclide concentration was varied from 100 to $1,000 \mathrm{pCi} / \mathrm{g}$ to determine if soil concentration affected the Kd determined to be protective of groundwater (it did not). Typical input factors for modeling are shown in the "Summary" section of the RESRAD "Mixture Sums and Single Radionuclide Guidelines" printout in Attachment 1 to this Calculation Summary. 
HaAk Bard Bechtel Hanford, Inc. CALCULATION SHEET \begin{tabular}{r|l}
\hline Originator: & S. W. Clark Ru \\
\hline Project: & 100 Area Remedial Action
\end{tabular}

Subject: 100 Area Analogous Sites RESRAD Calculations Date: \begin{tabular}{|c|c|c|c} 
Job No: & 22192 & Checked: & M. T. Stankovich \\
\hline
\end{tabular}

\begin{tabular}{|c|c|c|}
\hline & Rev.: & 0 \\
\hline $\mathrm{nt}=$ & Date: & $1 / 18104$ \\
\hline
\end{tabular}

\begin{tabular}{|c|c|c|c|c|c|}
\hline \multicolumn{6}{|c|}{ Table 1. Long-Lived Surrogate Radionuclides for RESRAD Modeling } \\
\hline No. & Radionuclide & Half Life, (yr) & No. & Radionuclide & Half Life, (yr) \\
\hline 1 & Al-26 & $7.16 \mathrm{E}+05$ & 9 & Pu-242 & $3.76 \mathrm{E}+05$ \\
\hline 2 & $\mathrm{Cl}-36$ & $3.01 \mathrm{E}+05$ & 10 & $\mathrm{Pu}-244$ & $8.26 \mathrm{E}+08$ \\
\hline 3 & $\mathrm{Cm}-248$ & $3.39 \mathrm{E}+05$ & 11 & $\mathrm{Sm}-147$ & $1.06 \mathrm{E}+11$ \\
\hline 4 & $\mathrm{Cs}-135$ & $3.00 \mathrm{E}+06$ & 12 & Th-232 & $1.41 \mathrm{E}+10$ \\
\hline 5 & $\mathrm{Gd}-152$ & $1.08 \mathrm{E}+14$ & 13 & $\mathrm{U}-235$ & $7.04 \mathrm{E}+08$ \\
\hline 6 & $\mathrm{I}-129$ & $1.60 \mathrm{E}+07$ & 14 & $\mathrm{U}-236$ & $2.34 \mathrm{E}+06$ \\
\hline 7 & $\mathrm{~K}-40$ & $1.28 \mathrm{E}+09$ & 15 & $\mathrm{U}-238$ & $4.47 \mathrm{E}+09$ \\
\hline 8 & $\mathrm{~Np}-237$ & $2.14 \mathrm{E}+06$ & & & \\
\hline
\end{tabular}

\section{METHODOLOGY:}

1) Input parameters from Table B-1 of the 100 Area RDR/RAWP were entered into the RESRAD model with the all of the long-lived surrogate radionuclides from Table 1 at a concentration of $100 \mathrm{pCi} / \mathrm{g}$. The surrogate radionuclides were given a range of $\mathrm{Kd}$ values from $5 \mathrm{~mL} / \mathrm{g}$ to $100 \mathrm{~mL} / \mathrm{g}$. A thickness of zero meters for the unsaturated/uncontaminated vadose zone between the contaminated zone and groundwater was used for the first RESRAD modeling run.

2) The RESRAD model was run and the graphical output for drinking water concentration was examined for each of the surrogate radionuclides. The lowest $\mathrm{Kd}$ value at which the drinking water concentration was zero for 1,000 years was noted $(80 \mathrm{~mL} / \mathrm{g})$. The RESRAD model was rerun, this time using a range of $\mathrm{Kd}$ values for the surrogate radionuclides by increments of one from $70 \mathrm{~mL} / \mathrm{g}$ to $80 \mathrm{~mL} / \mathrm{g}$. The graphical output for drinking water concentration was examined for each of the surrogate radionuclides and the lowest $\mathrm{Kd}$ value at which the drinking water concentration was zero for 1,000 years was determined $(80 \mathrm{~mL} / \mathrm{g})$ and recorded in a table of $\mathrm{Kd}$ values versus unsaturated/uncontaminated vadose zone thickness.

3) This procedure was repeated for a series of uncontaminated/unsaturated vadose zone thicknesses from 1 meter to 25 meters. The lowest $\mathrm{Kd}$ values at which the drinking water concentration was zero for 1,000 years was determined at increments of $1 \mathrm{~mL} / \mathrm{g}$ for each unsaturated/ uncontaminated vadose zone thickness and recorded in Table 2.

\section{RESULTS:}

1) Table 2 and Figure 1 show the results of the series of determinations of the lowest $\mathrm{Kd}$ values at which the drinking water concentration was zero for 1,000 years at each unsaturated/ uncontaminated vadose zone thickness. It should be noted that the $\mathrm{Kd}$ values determined were not affected by the waste site area, the waste site length parallel to groundwater flow, or the concentration of the surrogate radionuclides. 


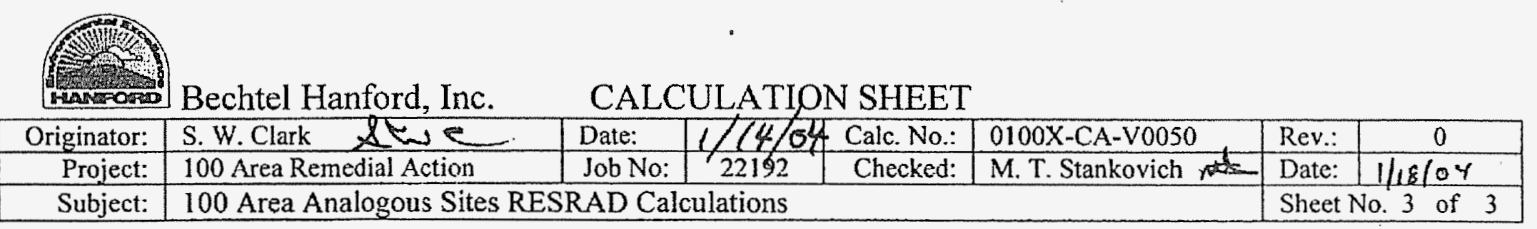

1

\section{Table 2:}

\begin{tabular}{|c|c|}
\hline $\begin{array}{c}\text { Unsaturated } \\
\text { Zone Thickness, } \\
\text { (meters) }\end{array}$ & $\begin{array}{c}\text { Distribution } \\
\text { Coefficient, } \\
(\mathrm{mL} / \mathrm{g})\end{array}$ \\
\hline 0 & $\mathrm{Kd}$ \\
\hline 1 & 80 \\
\hline 2 & 40 \\
\hline 3 & 27 \\
\hline 4 & 20 \\
\hline 5 & 16 \\
\hline 6 & 14 \\
\hline 7 & 12 \\
\hline 8 & 10 \\
\hline 9 & 9 \\
\hline 10 & 8 \\
\hline 11 & 8 \\
\hline 12 & 7 \\
\hline 16 & 7 \\
\hline 20 & 6 \\
\hline 25 & 4 \\
\hline
\end{tabular}

Figure 1:

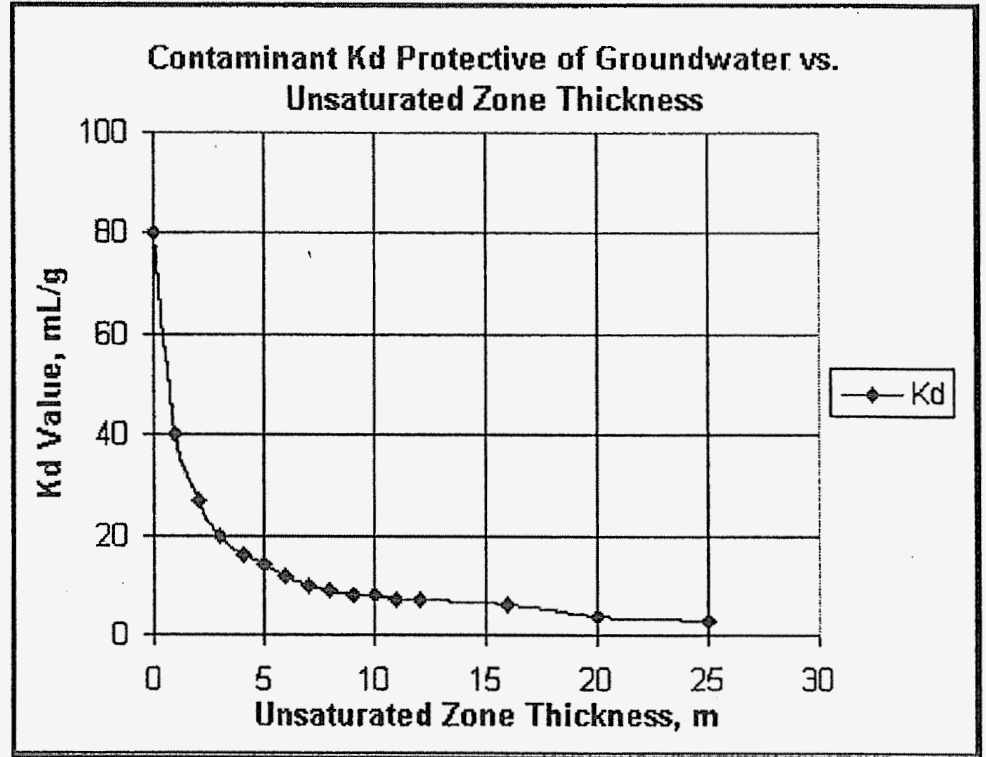

\section{CONCLUSIONS:}

- Table 2 and Figure 1 shows the results of the series of determinations of the lowest Kd values at which the drinking water concentration was zero for 1,000 years at each unsaturated/uncontaminated vadose zone thickness.

- The Kd values determined were not affected by:

- The area of the waste site,

- The waste site length parallel to groundwater flow, or

- The concentration of the surrogate radionuclides.

\section{ATTACHMENTS:}

1. RESRAD Output: Mixture Sums and Single Radionuclide Guidelines ( 32 pages) 


\section{ATTACHMENT 1}

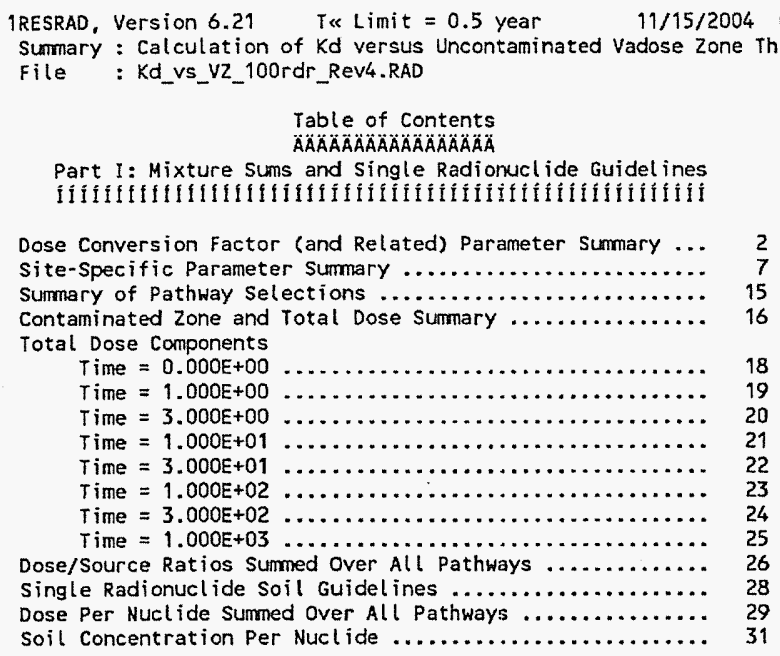




\section{ATTACHMENT 1}

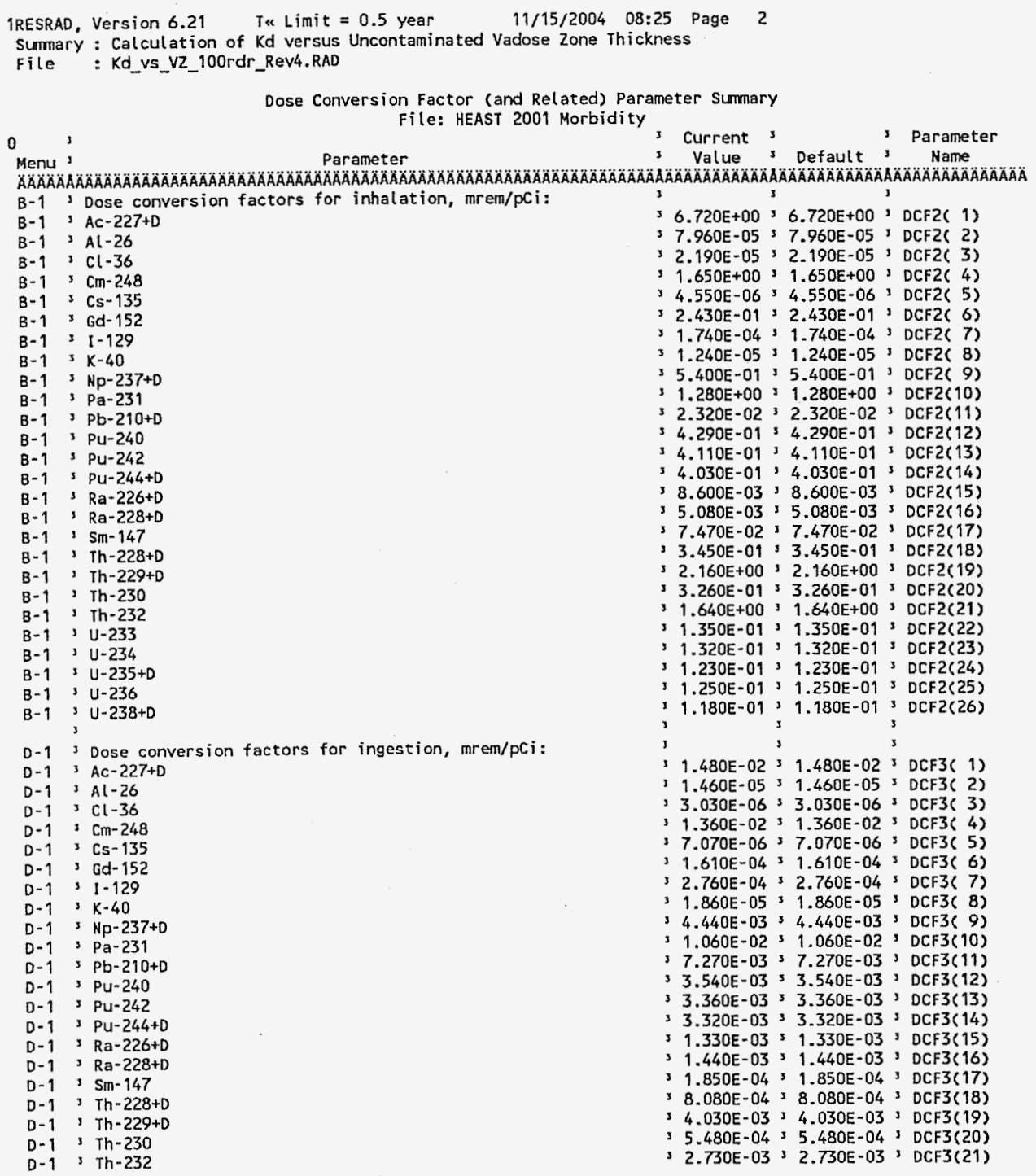

\begin{tabular}{ll} 
Attachment & $\frac{1}{\text { Sheet No. } 2 \text { of } 32}$ \\
Originator $\frac{32 . \text { W. Clark }}{\text { Sate }}$ & Date \\
Chk'd By M.T. Stankovich & Date \\
Calc. No. 0100X-CA-V0050 Rev. No. 0 \\
\hline
\end{tabular}




\section{ATTACHMENT 1}

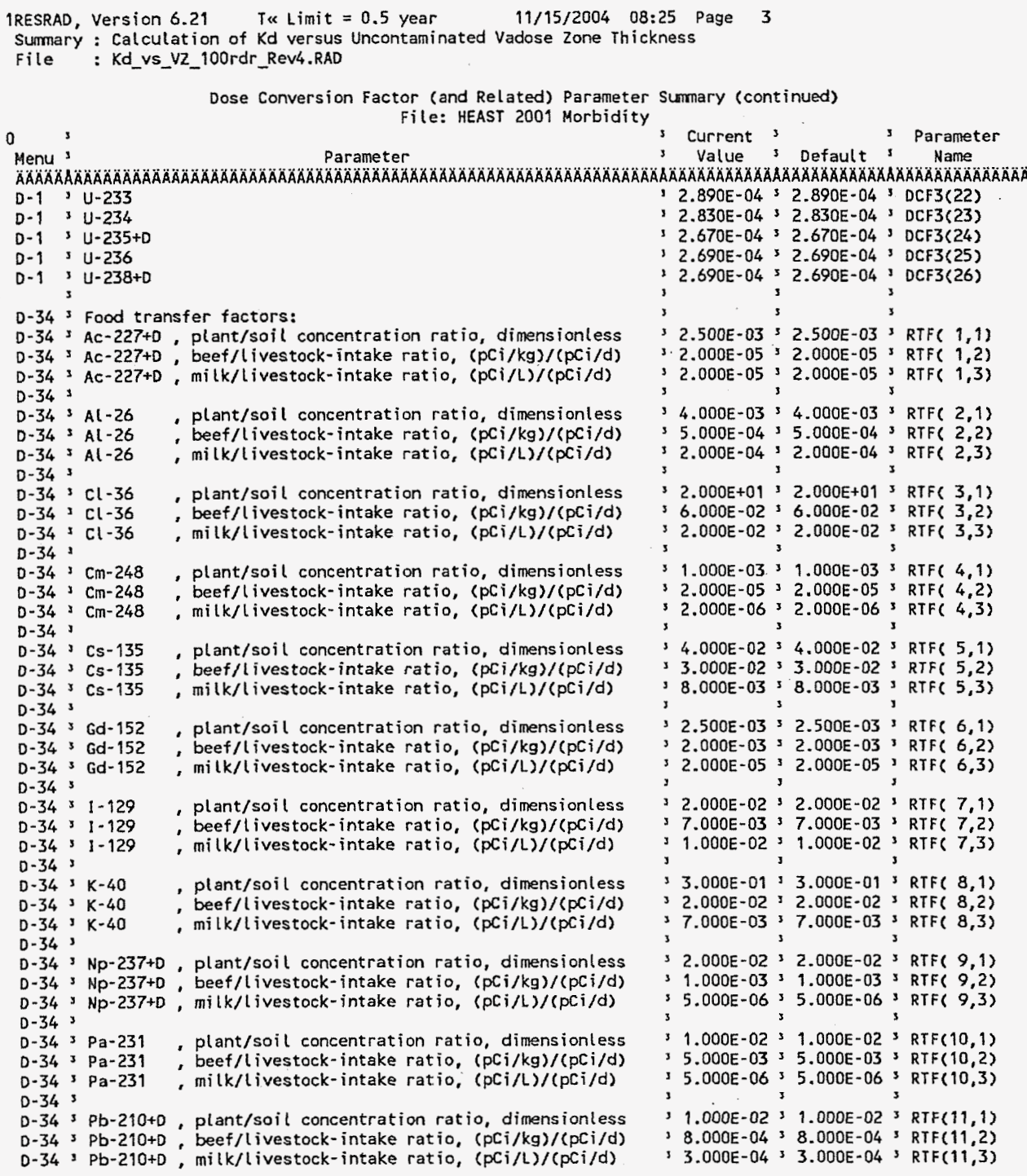

$0-343 \mathrm{pb}-210+0$. plant/soil concentration ratio, dimensionless

$\mathrm{D}-34$ s $\mathrm{Pb}-210+\mathrm{D}$; beef/livestock-intake ratio, $(\mathrm{pCi} / \mathrm{kg}) /(\mathrm{pCi} / \mathrm{d})$ D-34, $\mathrm{pb}-210+\mathrm{D}$, milk/livestock-intake ratio, $(\mathrm{pCi} / \mathrm{L}) /(\mathrm{pCi} / \mathrm{d})$ 


\section{ATTACHMENT 1}

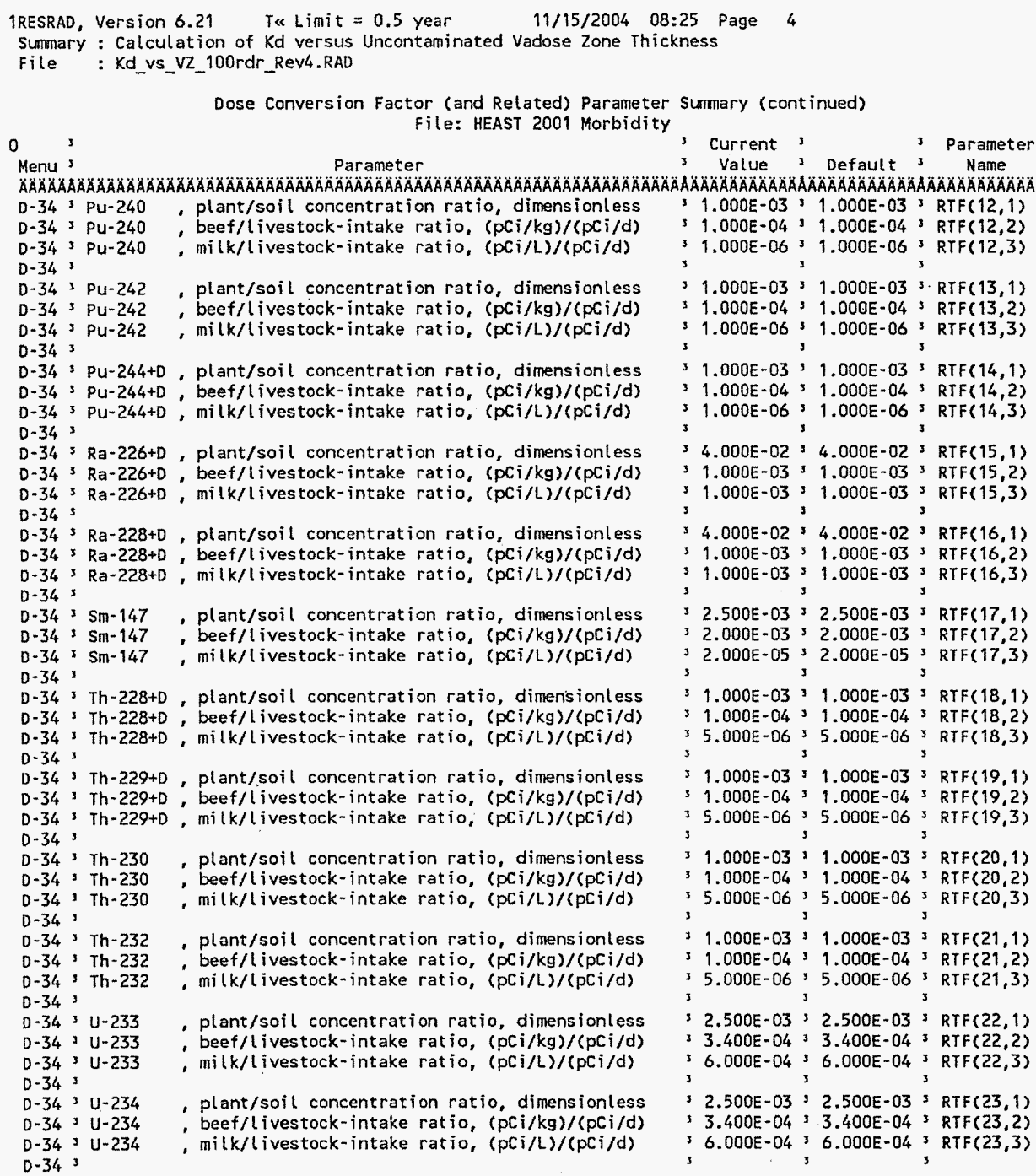




\section{ATTACHMENT 1}

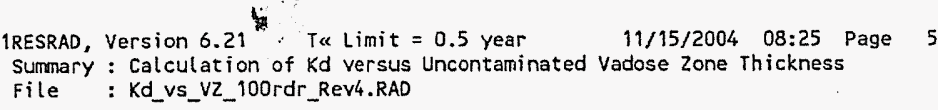




\section{ATTACHMENT 1}

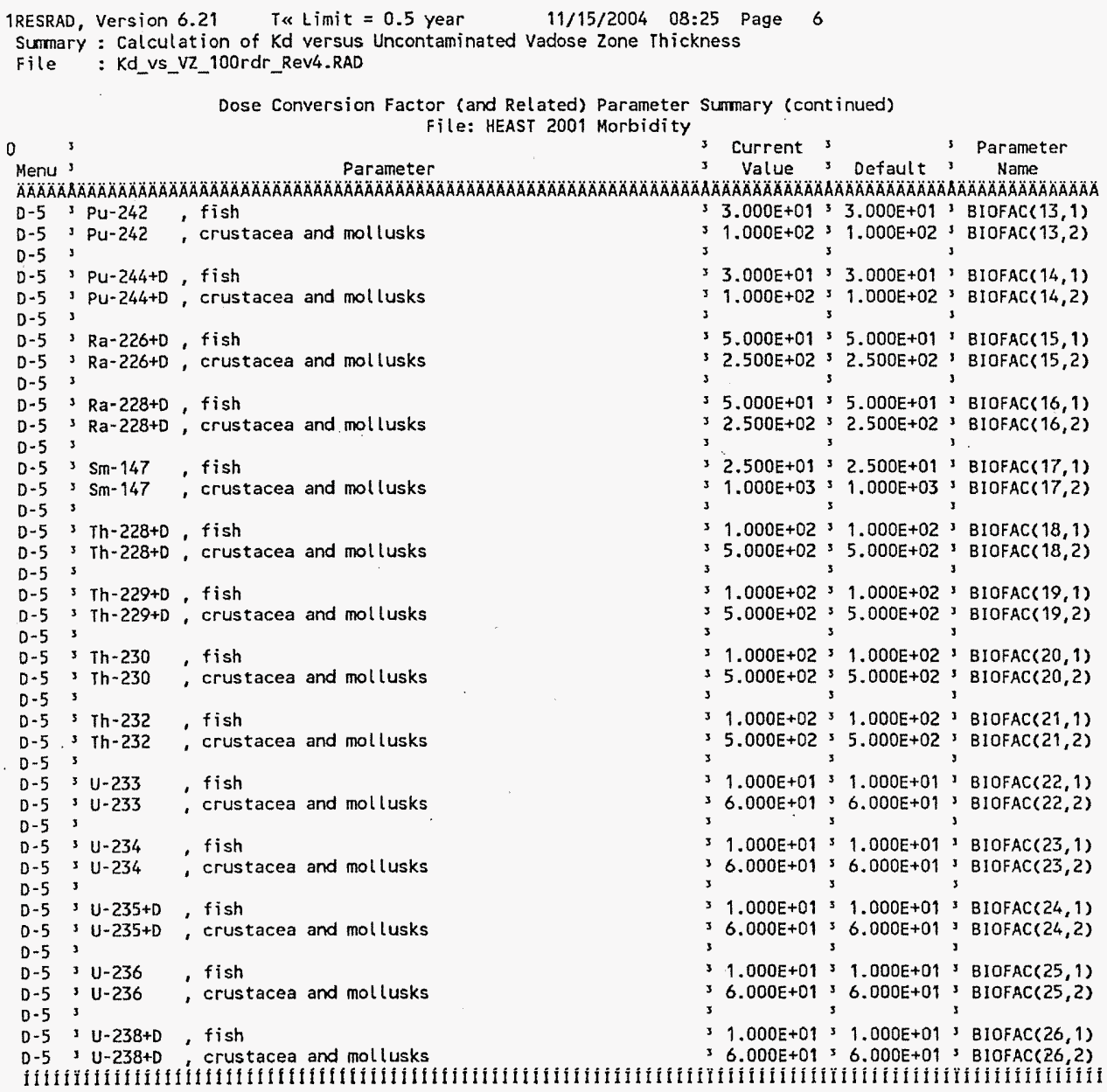

\begin{tabular}{l} 
Attachment $\frac{1}{1}$ Sheet No. $\underline{6}$ of 32 \\
Originator S.W. Clark \\
Chk'd By M.T. Stankovich \\
Date \\
Calc. No. 0100X-CA-V0050 Rev. No. 0 \\
\hline
\end{tabular}




\section{ATTACHMENT 1}

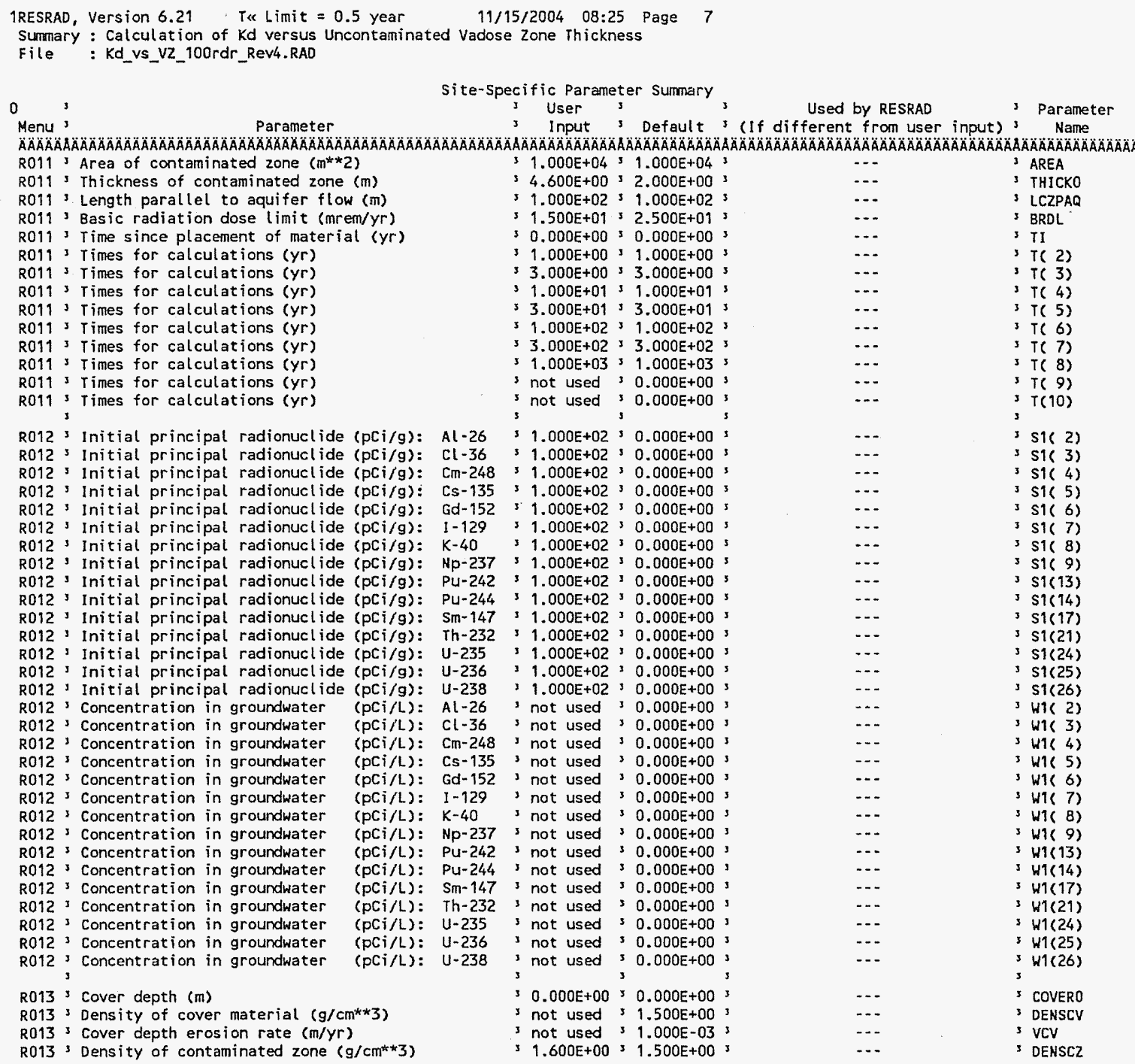

\begin{tabular}{ll} 
Attachment & \multicolumn{1}{c}{ Sheet No. 7 of 32} \\
Originator S.W. Clark & Date \\
Chk'd By M.T. Stankovich & Date \\
Calc. No. Ot00X-CA-V0050 Rev. No. 0
\end{tabular}




\section{ATTACHMENT 1}

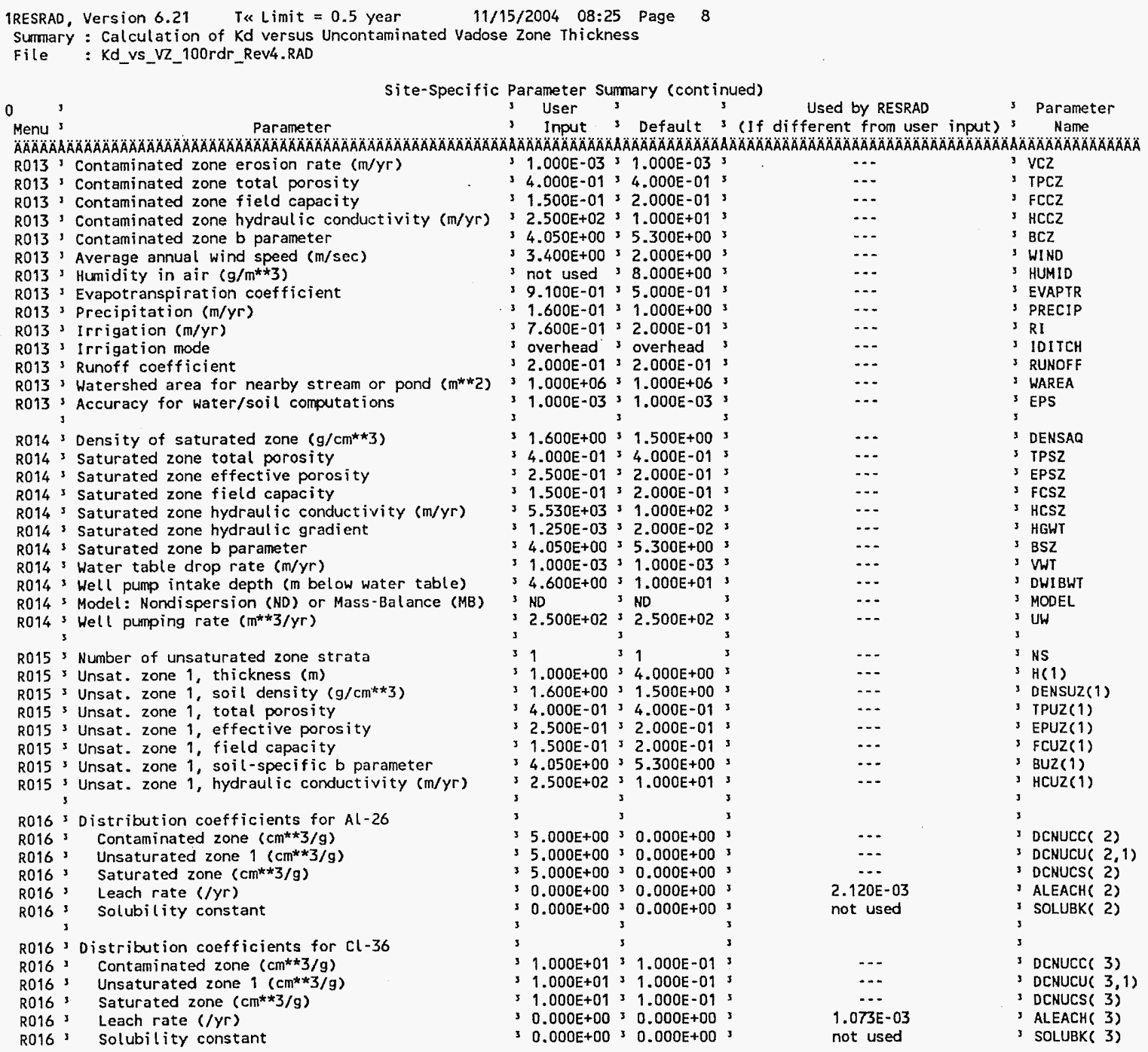

\begin{tabular}{|c|c|}
\hline Attachment $\quad 1$ & Sheet No. 8 of 32 \\
\hline Originator S.W. Clark & Date \\
\hline Chk'd By M.T. Stankovich & Date \\
\hline Calc. No. 0100X-CA-V0050 & Rev. No. \\
\hline
\end{tabular}




\section{ATTACHMENT 1}

1RESRAD, Version 6.21 T\& Limit $=0.5$ year $11 / 15 / 2004$ 08:25 Page 9 Sumary : Calculation of Kd versus Uncontaminated Vadose Zone Thickness file : Kd_vs_VZ_100rdr_Rev4.RAD

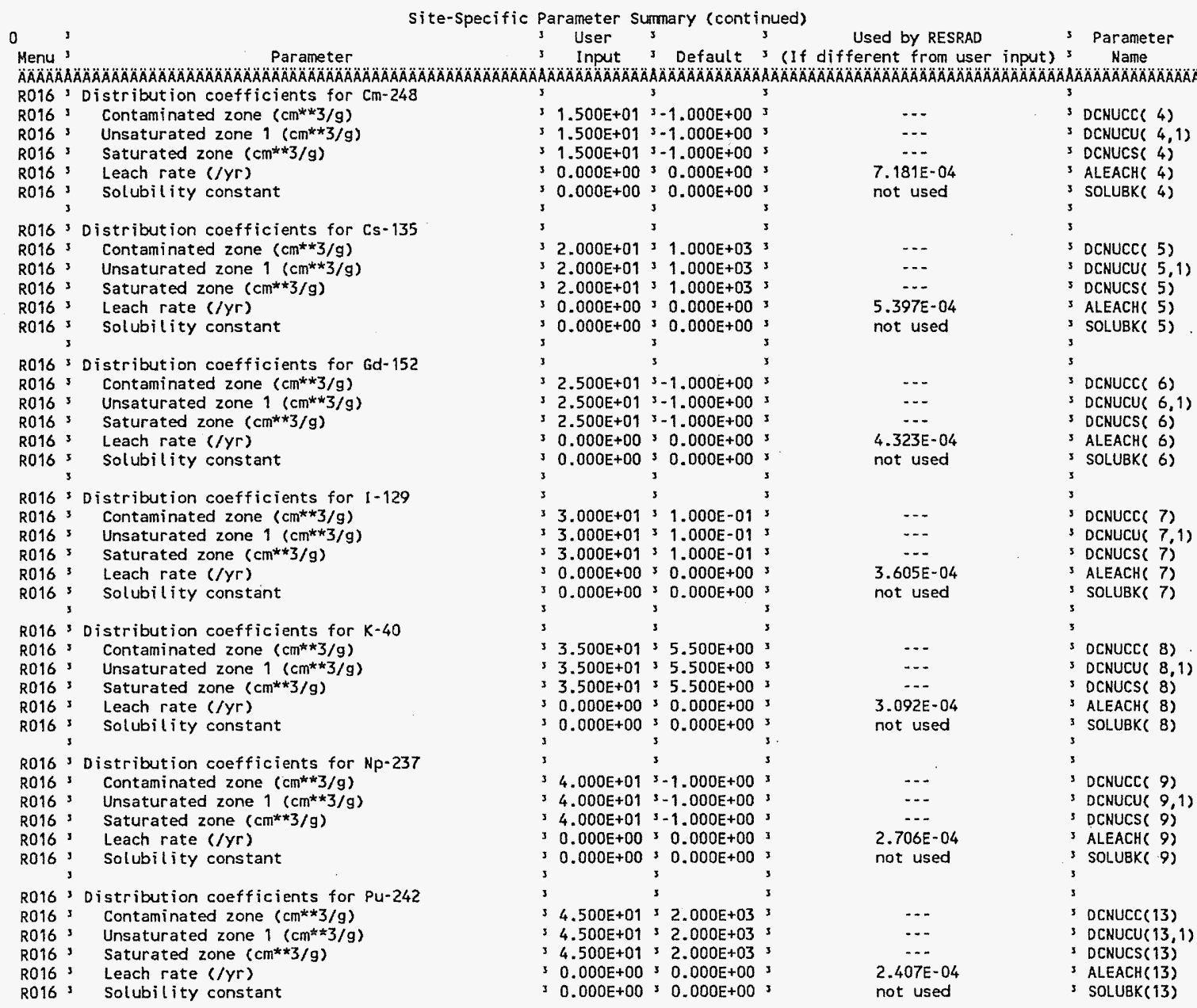

$\begin{array}{ll}\text { Attachment } \frac{1}{\text { Sta. Clark }} & \text { Sheet No. } \underline{9} \text { of } 32 \\ \text { Originator Sate } \\ \text { Chk'd By M.T. Stankovich } \\ \text { Calc. No. 0100X-CA-V0050 Rev. No. } 0\end{array}$




\section{ATTACHMENT 1}

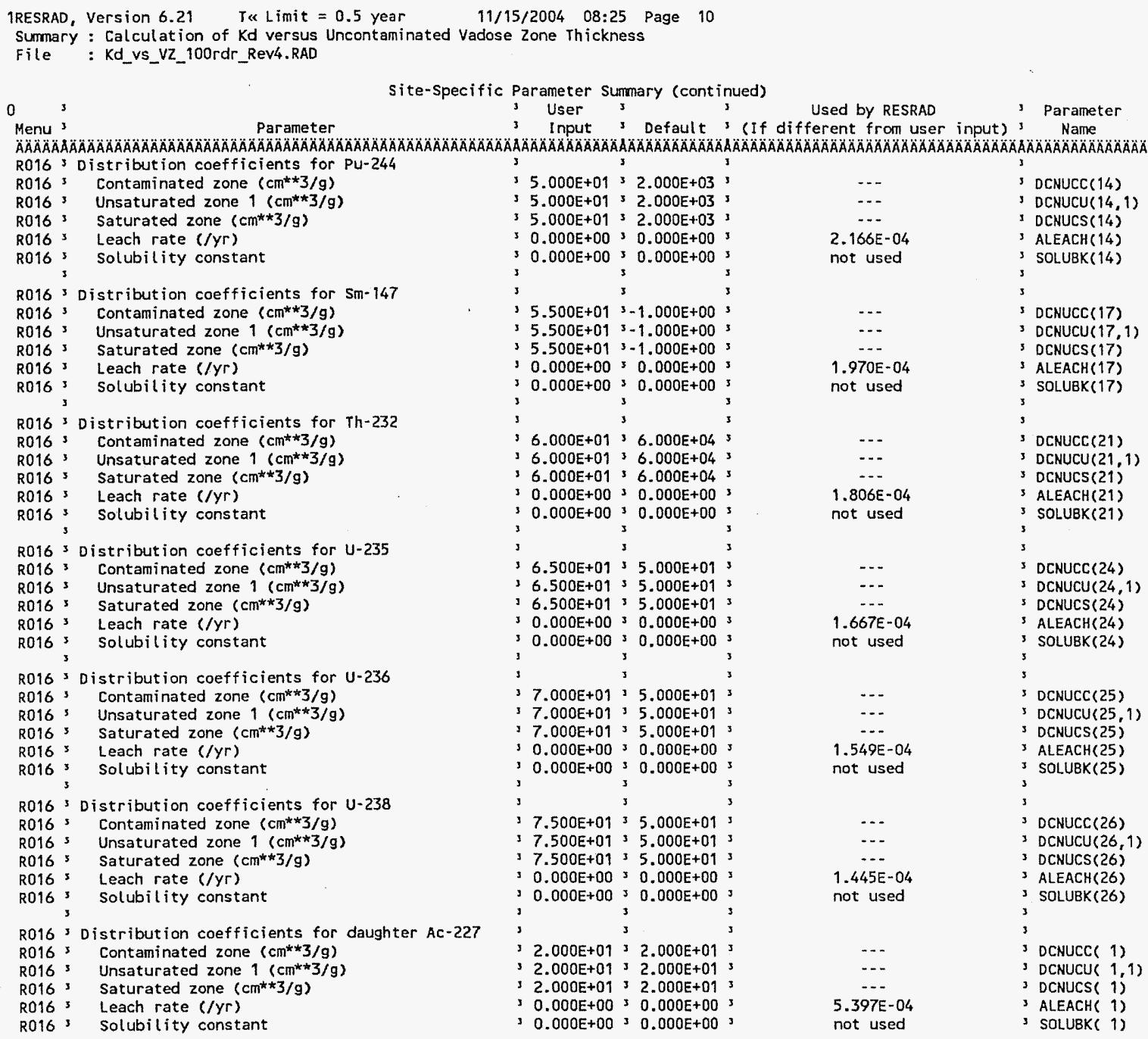

\begin{tabular}{|c|c|}
\hline Attachment & Sheet No. 10 of 32 \\
\hline Originator S.W. Clark & Date - - \\
\hline Chk'd By M.T. Stankovich & Date \\
\hline Calc. No. $0100 \mathrm{X}-\mathrm{CA}-\mathrm{V} 0050$ & Rev. No. \\
\hline
\end{tabular}




\section{ATTACHMENT 1}

1RESRAD, Version 6.21 T\& Limit $=0.5$ year $11 / 15 / 2004 \quad 08: 25$ Page 11 Sumary : Calculation of Kd versus Uncontaminated Vadose Zone Thickness

File : Kd_vs_vZ_100rdr_Rev4.RAD

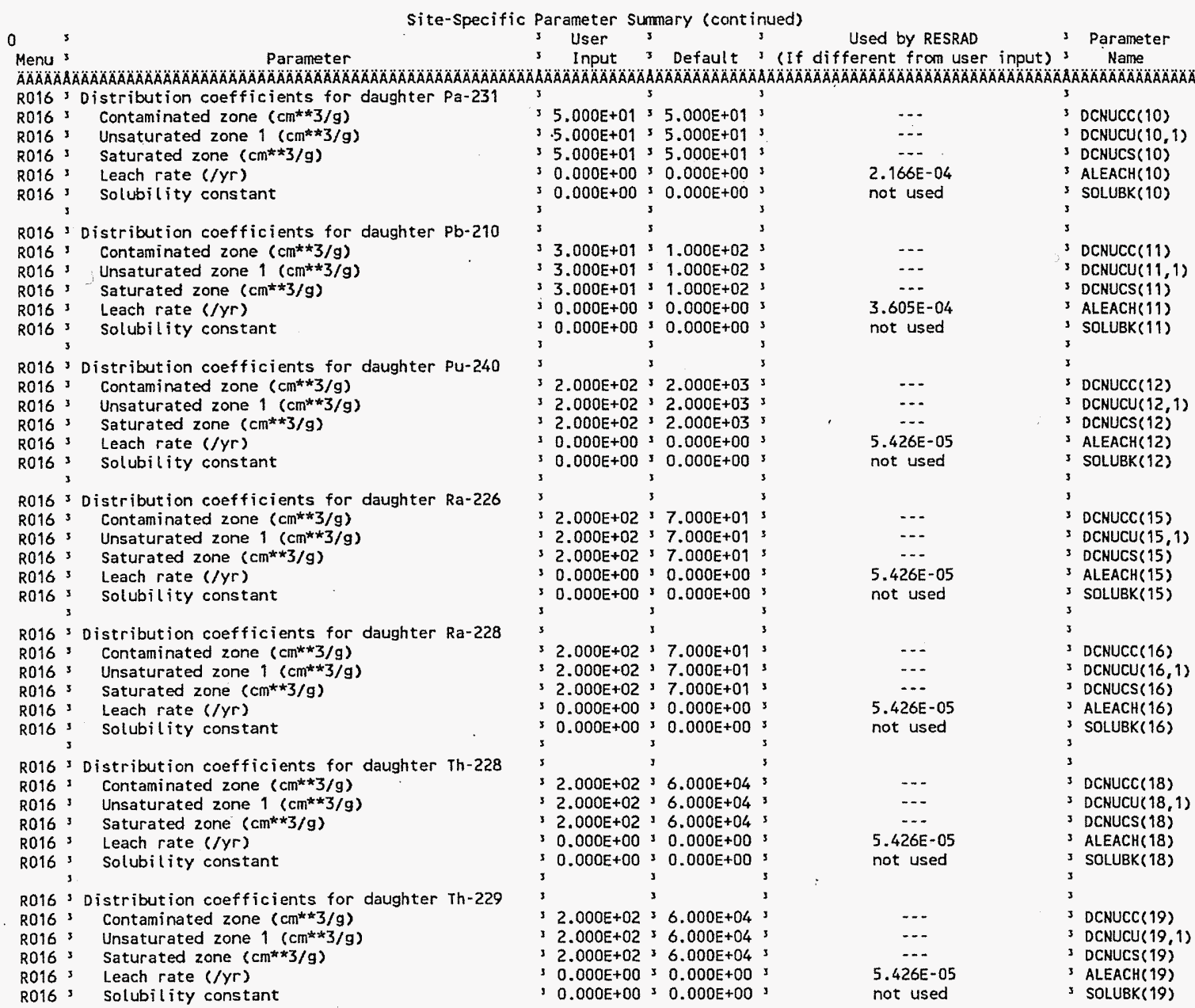

\begin{tabular}{ll} 
Attachment & \multicolumn{1}{c}{$\begin{array}{c}\text { Sheet No. } 11 \text { of } 32 \\
\text { Originator }\end{array}$} \\
S.W. Slark & Date \\
Chik'd By M.T. Stankovich & Date \\
Calc. No. 0100X-CA-V0050 & Rev. No. 0
\end{tabular}




\section{ATTACHMENT 1}

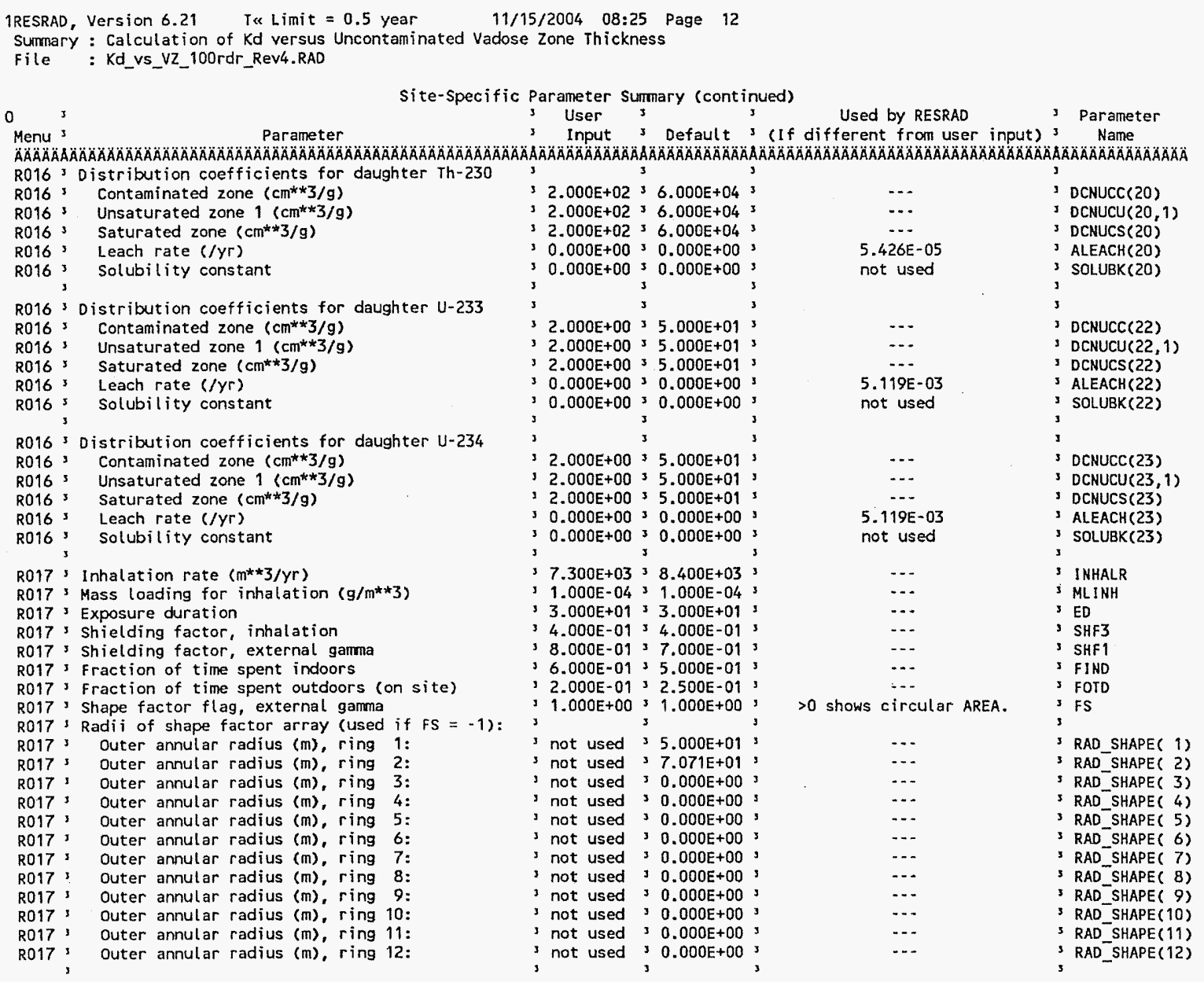

$\begin{array}{ll}\text { Attachment } \frac{1}{1} & \text { Sheet No. } 12 \text { of } 32 \\ \text { Originator S.W. Clark } & \text { Date } \\ \text { Chk'd By M.T. Stankovich } & \text { Date } \\ \text { Calc. No. 0100X-CA-V0050 Rev. No. } & \text { Re }\end{array}$




\section{ATTACHMENT 1}

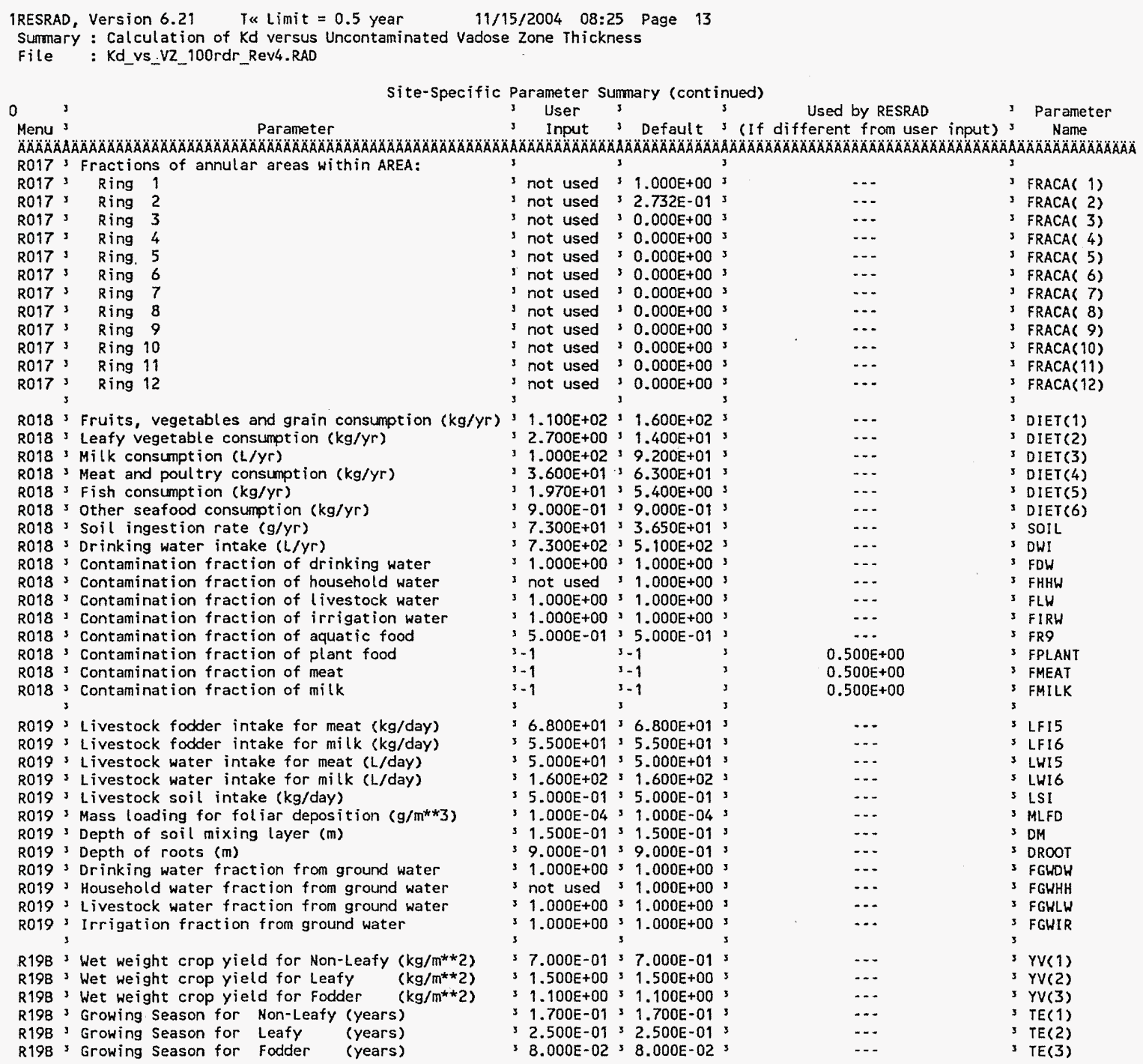

\begin{tabular}{|c|c|}
\hline Attachment $\quad 1$ & Sheet No. 13 of 32 \\
\hline Originator S.W. Clark & Date \\
\hline Chk'd By M.T. Stankovich & Date \\
\hline Calc. No. 0100X-CA-V0050 & Rev. No. \\
\hline
\end{tabular}




\section{ATTACHMENT 1}

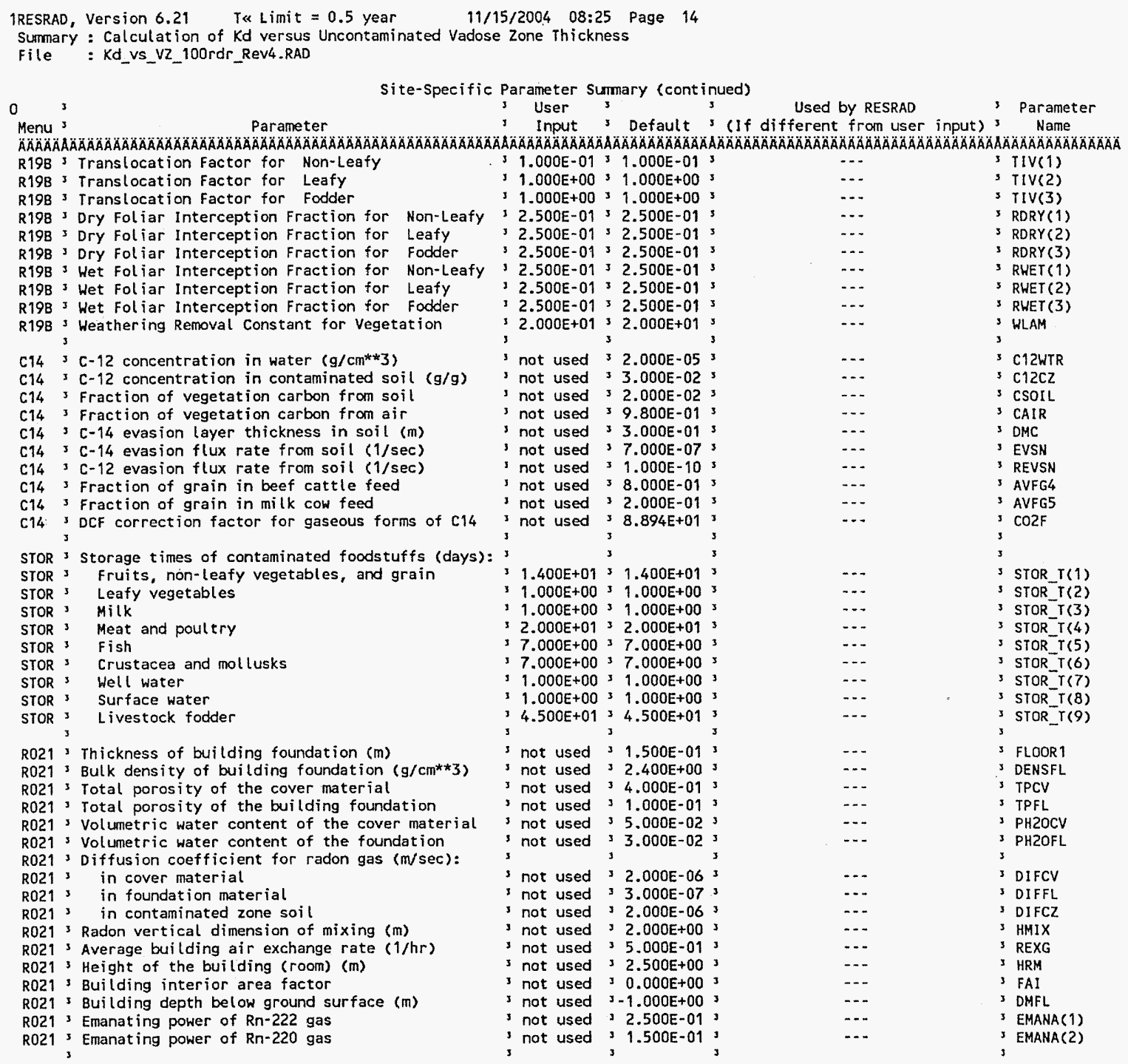

\begin{tabular}{|c|c|}
\hline Attachment & Sheet No. 14 of 32 \\
\hline Originator S.W. Clark & Date \\
\hline Chk'd By M.T. Stankovich & Date \\
\hline Calc. No. $0100 X-C A-V 0050$ & Rev. No. \\
\hline
\end{tabular}




\section{ATTACHMENT 1}

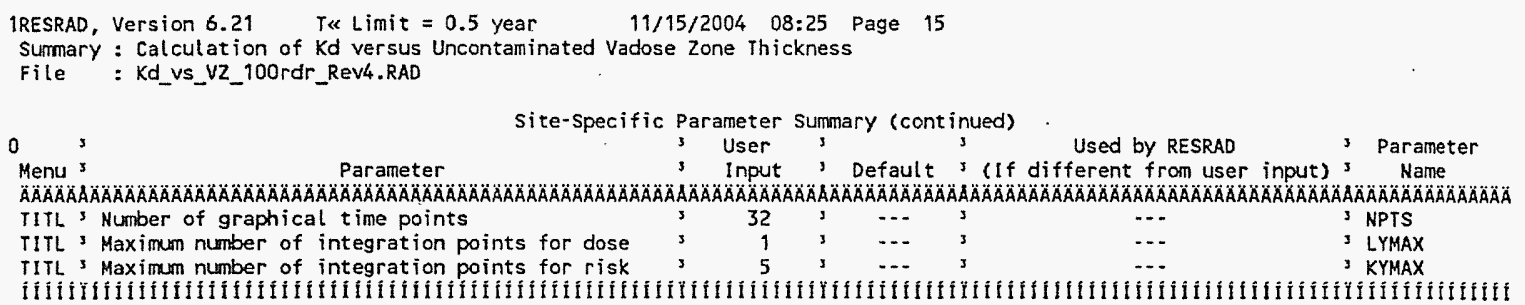




\section{ATTACHMENT 1}

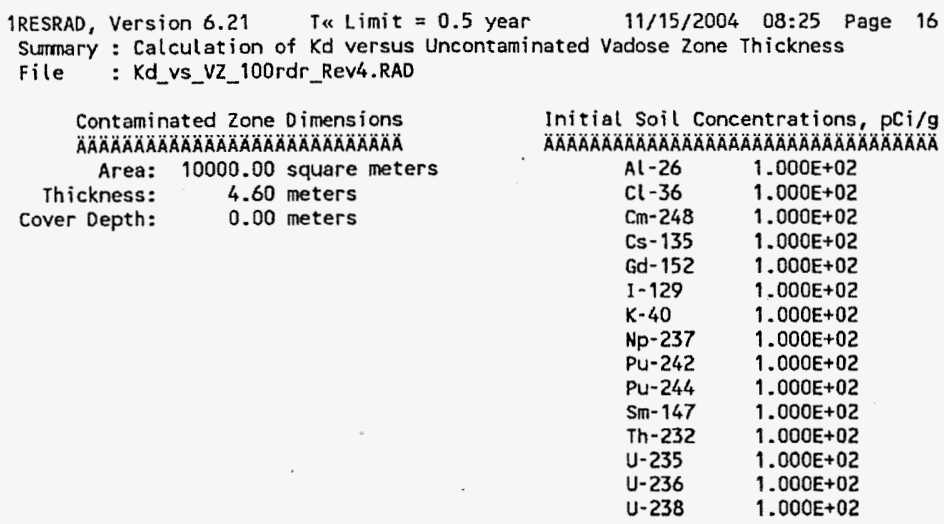

0

Total Dose TDOSE(t), mrem/yr

Basic Radiation Dose Limit $=1.500 \mathrm{E}+01 \mathrm{mrem} / \mathrm{yr}$

Total Mixture Sum $M(t)=$ Fraction of Basic Dose Limit Received at Time $(t)$

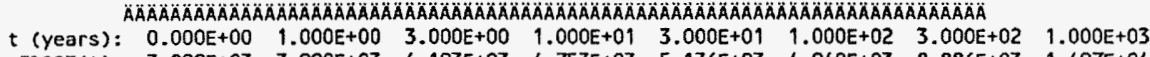

$\begin{array}{lllllllll}\operatorname{TDOSE}(t): & 3.898 E+03 & 3.990 E+03 & 4.193 E+03 & 4.753 E+03 & 5.136 E+03 & 4.940 E+03 & 8.886 E+03 & 1.497 E+04\end{array}$

$M(t)=2.599 E+02 \quad 2.660 E+02 \quad 2.796 E+02 \quad 3.169 E+02 \quad 3.424 E+02 \quad 3.293 E+02 \quad 5.924 E+02 \quad 9.977 E+02$ OMaximum $\operatorname{TDOSE}(t): 2.099 E+04 \mathrm{mrem} / \mathrm{yr}$ at $t=505 \overline{\mathrm{n}} 1$ years

0

Total Dose Contributions TOOSE $(i, p, t)$ for Individual Radionuclides (i) and Pathways ( $p$ )

As mrem/yr and Fraction of Total Dose At $t=5.054 E+02$ years

0
0 Water Independent Pathways (Inhalation excludes radon) Inhalation Radon Plant Meat Milk

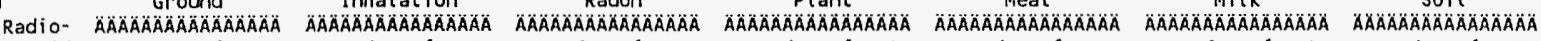
Nucl ide

A

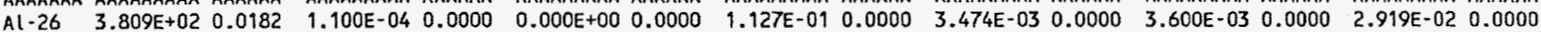

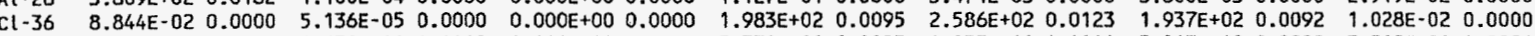

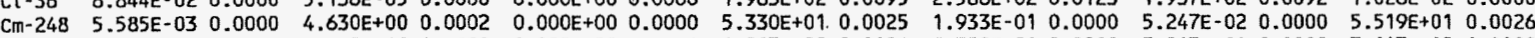
$\begin{array}{llllllllllllllll}C_{5}-135 & 1.913 E-03 & 0.0000 & 1.399 E-05 & 0.0000 & 0.000 E+00 & 0.0000 & 1.213 E+00 & 0.0001 & 9.359 E-01 & 0.0000 & 5.813 E-01 & 0.0000 & 3.143 E-02 & 0.0000\end{array}$

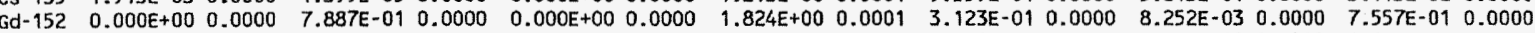
$\begin{array}{lllllllllllllll}1-129 & 7.260 \mathrm{E}-01 & 0.0000 & 5.856 \mathrm{E}-04 & 0.0000 & 0.000 E+00 & 0.0000 & 2.593 E+01 & 0.0012 & 5.398 E+00 & 0.0003 & 1.843 E+01 & 0.0009 & 1.343 E+00 & 0.0001\end{array}$ $\begin{array}{lllllllllllllll}K-40 & 5.692 E+01 & 0.0027 & 4.283 E-05 & 0.0000 & 0.000 E+00 & 0.0000 & 2.690 E+01 & 0.0013 & 1.197 E+01 & 0.0006 & 9.467 E+00 & 0.0005 & 9.291 E-02 & 0.0000\end{array}$ $4375.180+010.0029 \quad 1.902 E+000.0001 \quad 0.000 E+000.00004 .364 E+020.0208 \quad 1.297 E+01000061.553 E-010.00002 .261 E+010.0011$

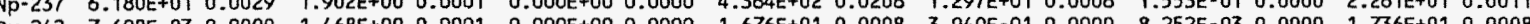
$\begin{array}{llllllll} & 0.0008\end{array}$

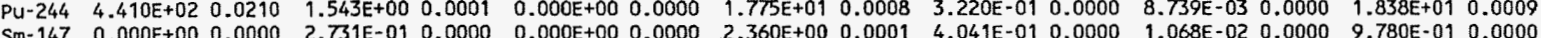
$\begin{array}{lllllllllllllll}\mathrm{Sm}-147 & 0.000 \mathrm{E}+00 & 0.0000 & 2.731 \mathrm{E}-01 & 0.0000 & 0.000 \mathrm{E}+00 & 0.0000 & 2.360 \mathrm{E}+00 & 0.0001 & 4.041 \mathrm{E}-01 & 0.0000 & 1.063 \mathrm{E}-02 & 0.0000 & 9.780 \mathrm{E}-01 & 0.0000 \\ \mathrm{Th}-232 & 9.475 \mathrm{E}+02 & 0.0452 & 7.337 \mathrm{E}+00 & 0.0003 & 0.000 \mathrm{E}+00 & 0.0000 & 3.157 \mathrm{E}+02 & 0.0150 & 7.912 \mathrm{E}+00 & 0.0004 & 1.761 \mathrm{E}+01 & 0.0008 & 2.655 \mathrm{E}+01 & 0.0013\end{array}$ $\begin{array}{lllllllllllllll}U-235 & 4.633 E+01 & 0.0022 & 7.497 E-01 & 0.0000 & 0.000 E+00 & 0.0000 & 1.110 E+01 & 0.0005 & 1.193 E+00 & 0.0001 & 4.807 E-01 & 0.0000 & 2.806 E+00 & 0.0001\end{array}$ $\begin{array}{llllllllllllllllll}U-236 & 1.319 E-02 & 0.0000 & 4.668 E-01 & 0.0000 & 0.000 E+00 & 0.0000 & 3.505 E+00 & 0.0002 & 1.020 E-01 & 0.0000 & 4.759 E-01 & 0.0000 & 1.453 E+00 & 0.0001\end{array}$

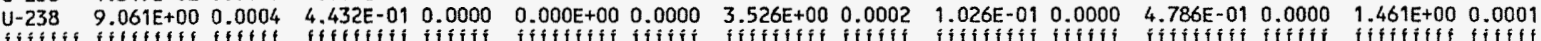

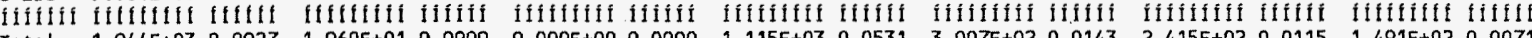

$\begin{array}{llllllllllllllllll}\text { Total } & 1.944 E+03 & 0.0927 & 1.960 E+01 & 0.0009 & 0.000 E+00 & 0.0000 & 1.115 E+03 & 0.0531 & 3.007 E+02 & 0.0143 & 2.415 E+02 & 0.0115 & 1.491 E+02 & 0.0071\end{array}$ 


\section{ATTACHMENT 1}

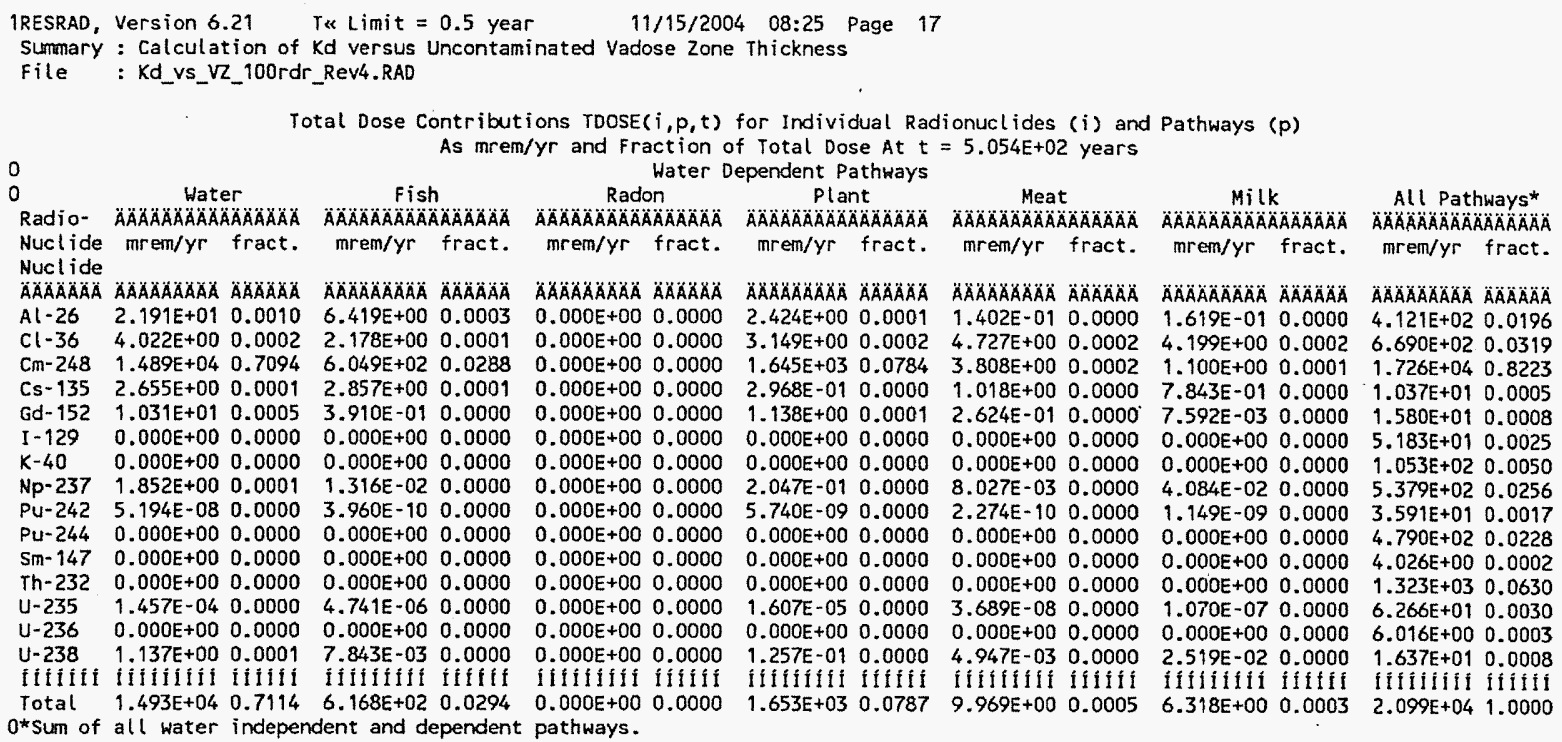




\section{ATTACHMENT 1}

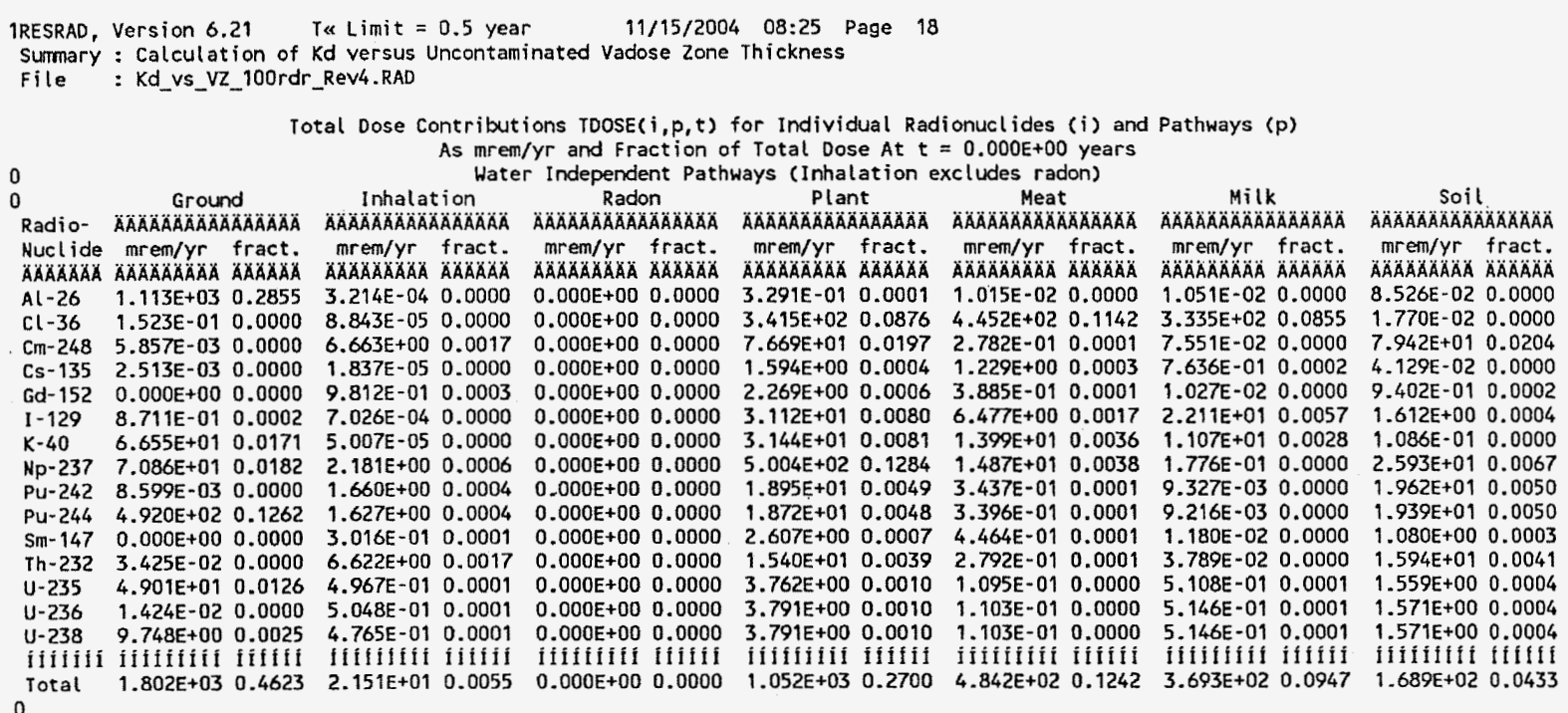

Total Dose Contributions TDOSE(i,p,t) for Individual Radionuclides (i) and Pathways (p) As $\mathrm{mrem} / \mathrm{yr}$ and Fraction of Total Dose At $t=0.000 \mathrm{E}+00$ years Hater Dependent Pathways

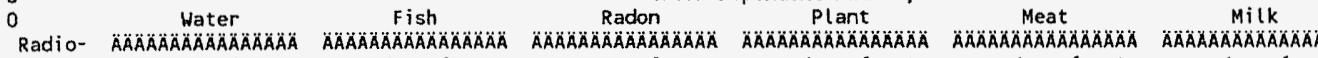
Nuclide mrem/yr fract. mrem/yr fract. mrem/yr fract. mrem/yr fract. mrem/yr fract. mrem/yr fract.

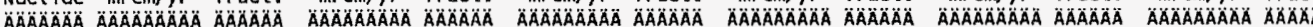
AL $\begin{array}{lllllllllllll}A & \text {-26 } & 0.000 E+00 & 0.0000 & 0.000 E+00 & 0.0000 & 0.000 E+00 & 0.0000 & 0.000 E+00 & 0.0000 & 0.000 E+00 & 0.0000\end{array}$ $\begin{array}{lllllllllll}\mathrm{Cl}-36 & 0.000 \mathrm{E}+00 & 0.0000 & 0.000 \mathrm{E}+00 & 0.0000 & 0.000 \mathrm{E}+00 & 0.0000 & 0.000 \mathrm{E}+00 & 0.0000 & 0.000 \mathrm{E}+00 & 0.0000\end{array}$ $\begin{array}{llllllllllll}\mathrm{C} m-248 & 0.000 E+00 & 0.0000 & 0.000 E+00 & 0.0000 & 0.000 E+00 & 0.0000 & 0.000 E+00 & 0.0000 & 0.000 E+00 & 0.0000\end{array}$

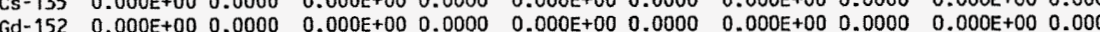

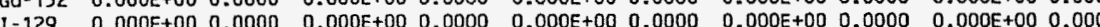

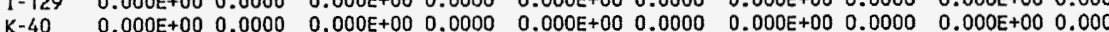
$\begin{array}{lllllllllll}K-40 & 0.000 E+00 & 0.0000 & 0.000 E+00 & 0.0000 & 0.000 E+00 & 0.0000 & 0.000 E+00 & 0.0000 & 0.000 E+00 & 0.0000\end{array}$ $\begin{array}{lllllllllll}\mathrm{NP}-237 & 0.000 E+00 & 0.0000 & 0.000 E+00 & 0.0000 & 0.000 E+00 & 0.0000 & 0.000 E+00 & 0.0000 & 0.000 E+00 & 0.0000\end{array}$ $\begin{array}{lllllllllll}\text { PU }-242 & 0.000 E+00 & 0.0000 & 0.000 E+00 & 0.0000 & 0.000 E+00 & 0.0000 & 0.000 E+00 & 0.0000 & 0.000 E+00 & 0.0000 \\ P U-244 & 0.000 E+00 & 0.0000 & 0.000 E+00 & 0.0000 & 0.000 E+00 & 0.0000 & 0.000 E+00 & 0.0000 & 0.000 E+00 & 0.0000\end{array}$ $\begin{array}{lllllllllll}\mathrm{PU}-244 & 0.000 \mathrm{E}+00 & 0.0000 & 0.000 \mathrm{E}+00 & 0.0000 & 0.000 E+00 & 0.0000 & 0.000 E+00 & 0.0000 & 0.000 E+00 & 0.0000 \\ \mathrm{~S}-147 & 0.000 \mathrm{E}+00 & 0.0000 & 0.000 \mathrm{E}+00 & 0.0000 & 0.000 \mathrm{E}+00 & 0.0000 & 0.000 \mathrm{E}+00 & 0.0000 & 0.000 \mathrm{E}+00 & 0.0000\end{array}$ $\begin{array}{lllllllllll}\mathrm{Sm}-147 & 0.000 \mathrm{E}+00 & 0.0000 & 0.000 \mathrm{E}+00 & 0.0000 & 0.000 \mathrm{E}+00 & 0.0000 & 0.000 E+00 & 0.0000 & 0.000 \mathrm{E}+00 & 0.0000 \\ \mathrm{Th}-232 & 0.000 \mathrm{E}+00 & 0.0000 & 0.000 \mathrm{E}+00 & 0.0000 & 0.000 \mathrm{E}+00 & 0.0000 & 0.000 \mathrm{E}+00 & 0.0000 & 0.000 \mathrm{E}+00 & 0.0000\end{array}$

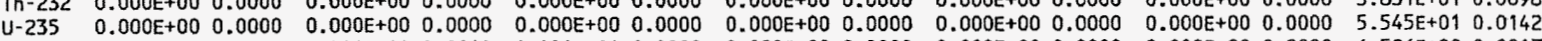
$\begin{array}{llllllllllllllll}U-236 & 0.000 E+00 & 0.0000 & 0.000 E+00 & 0.0000 & 0.000 E+00 & 0.0000 & 0.000 E+00 & 0.0000 & 0.000 E+00 & 0.0000 & 0.000 E+00 & 0.0000 & 6.506 E+00 & 0.0017\end{array}$

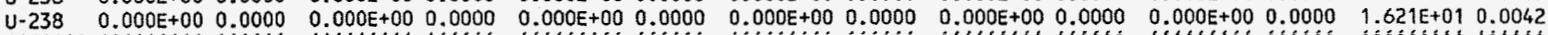

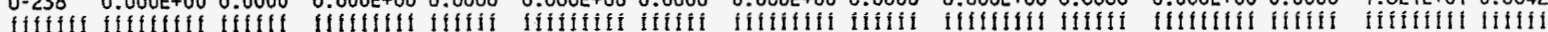
$\begin{array}{llllllllll} & \end{array}$ $0^{\star}$ Sum of all water independent and dependent pathways.

\begin{tabular}{|c|c|}
\hline Attachment & Sheet No. 18 of 32 \\
\hline Originator S.W. Clark & Date \\
\hline Chk'd By M.T.Stankovich & Date \\
\hline Calc. No. 0100X-CA-V0050 & Rev. No. \\
\hline
\end{tabular}




\section{ATTACHMENT 1}

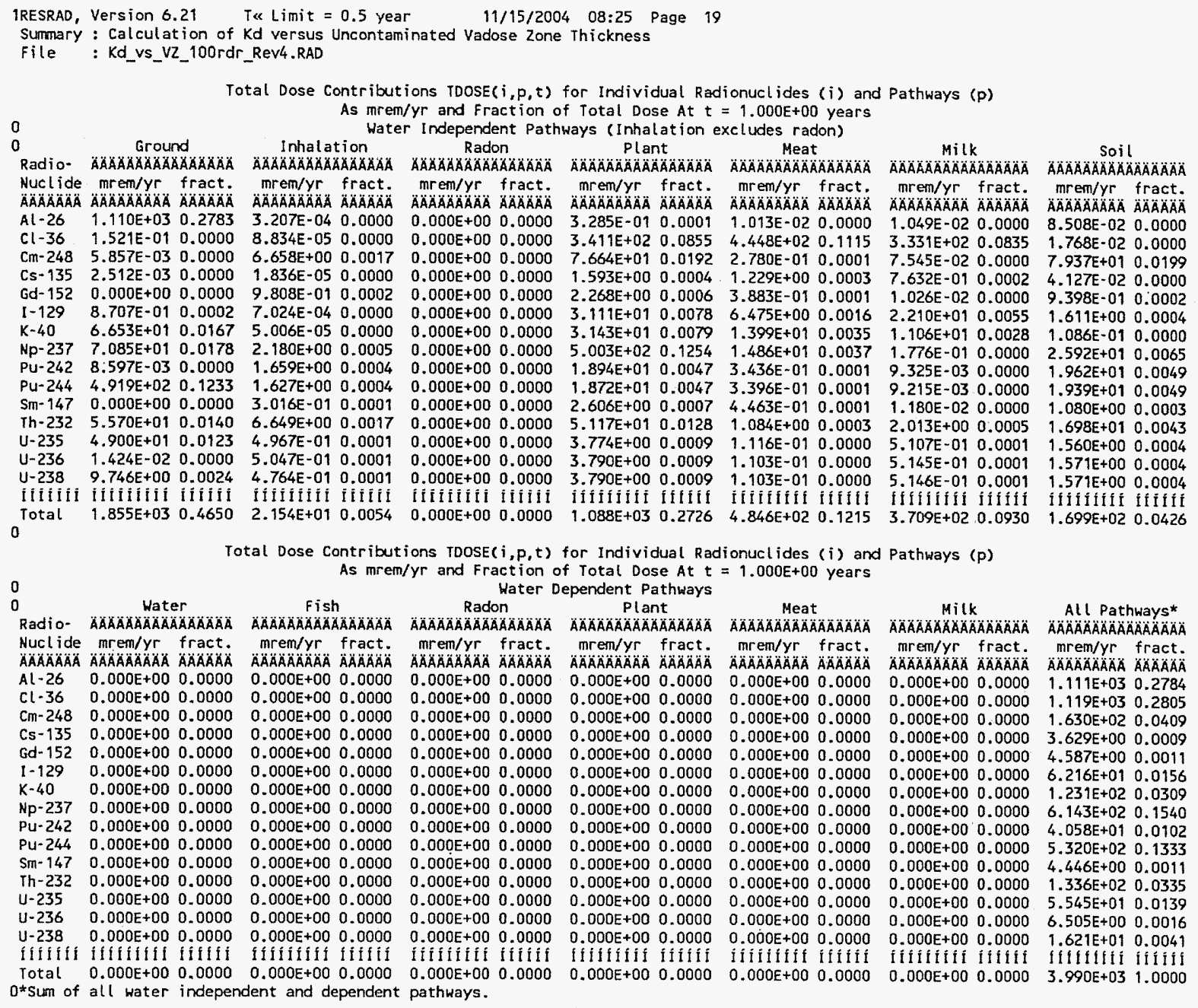

\begin{tabular}{|c|c|}
\hline \multirow{3}{*}{\multicolumn{2}{|c|}{$\begin{array}{ll}\text { Attachment } & \frac{1}{1} \text { Sheet No. } 19 \text { of } 32 \\
\text { Originator S.W. Clark } & \text { Date }\end{array}$}} \\
\hline & \\
\hline & \\
\hline $0100 X-C A-V 00$ & 0 \\
\hline
\end{tabular}




\section{ATTACHMENT 1}

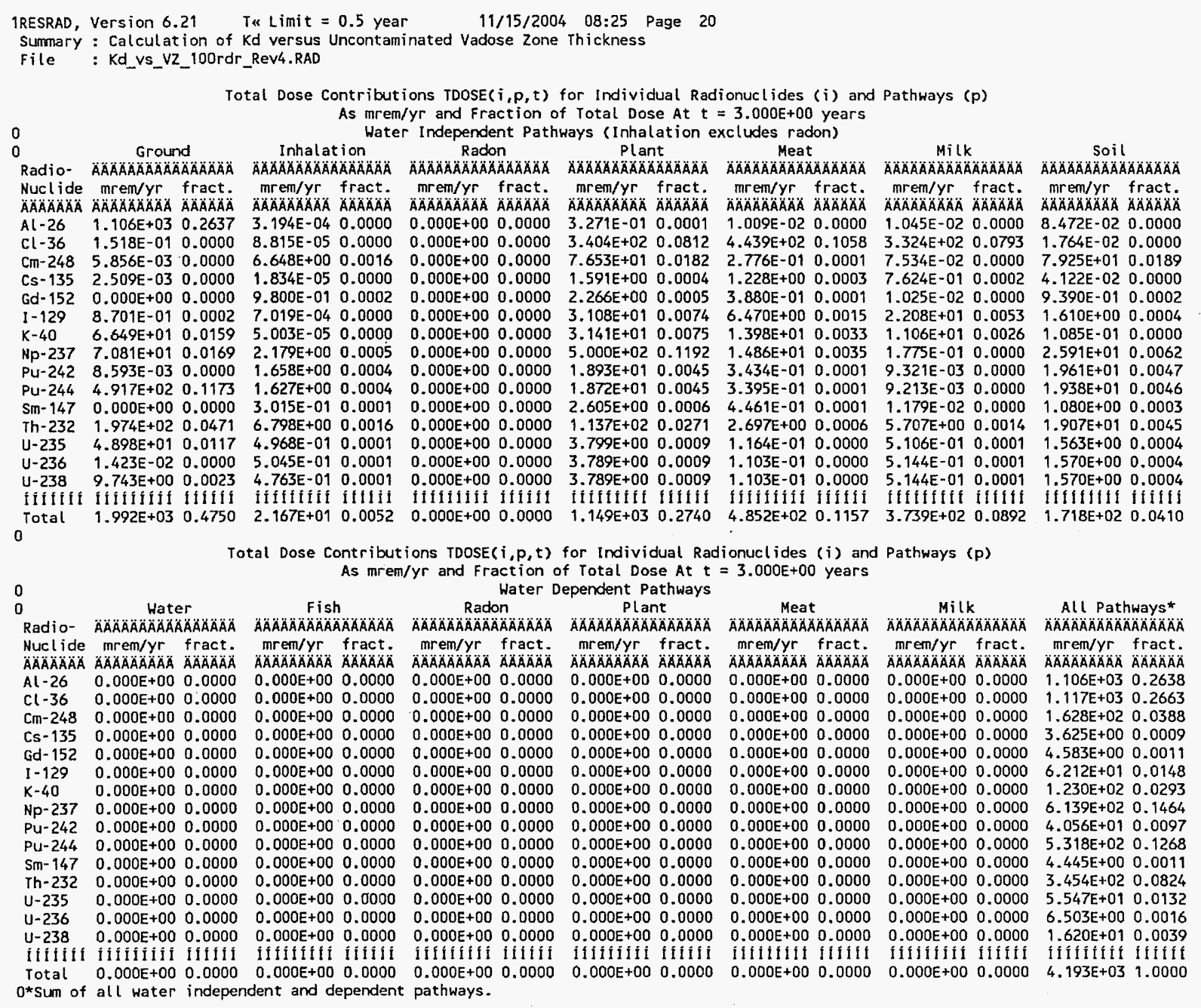

\begin{tabular}{ll} 
Attachment & \multicolumn{1}{c}{$\begin{array}{c}\text { Sheet No. } 20 \text { of } 32 \\
\text { Originator S.W. Clark }\end{array}$ Date } \\
Chk'd By M.T.Stankovich & Date \\
Calc. No. O100X-CA-V0050 Rev. No. 0
\end{tabular}




\section{ATTACHMENT 1}

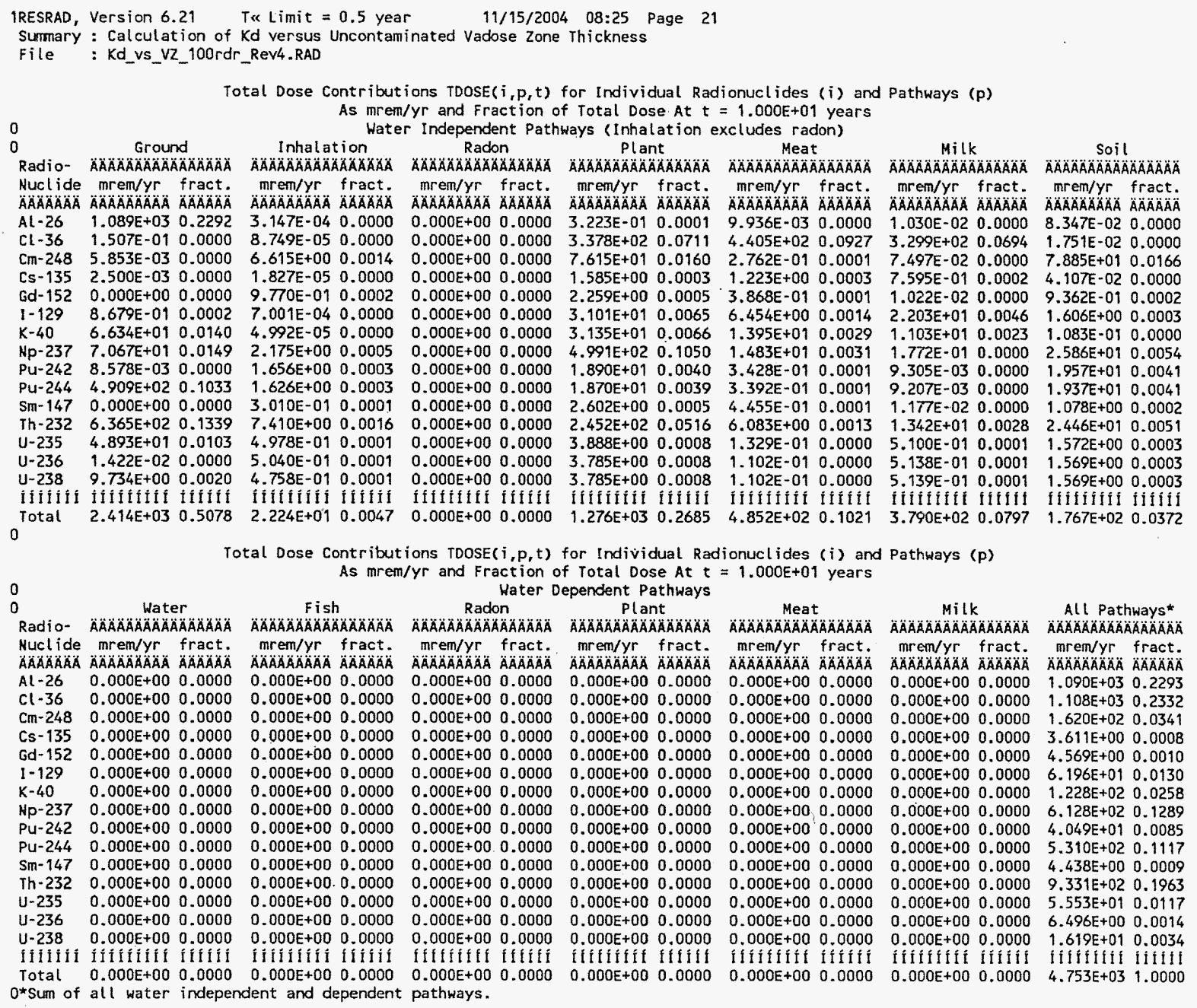

\begin{tabular}{|c|c|}
\hline Attachment & Sheet No. 21 of 32 \\
\hline Originator S.W. Clark & Date - \\
\hline Chk'd By M.T. Stankovich & Date \\
\hline Calc. No. $0100 X-C A-V 0050$ & Rev. No. \\
\hline
\end{tabular}




\section{ATTACHMENT 1}

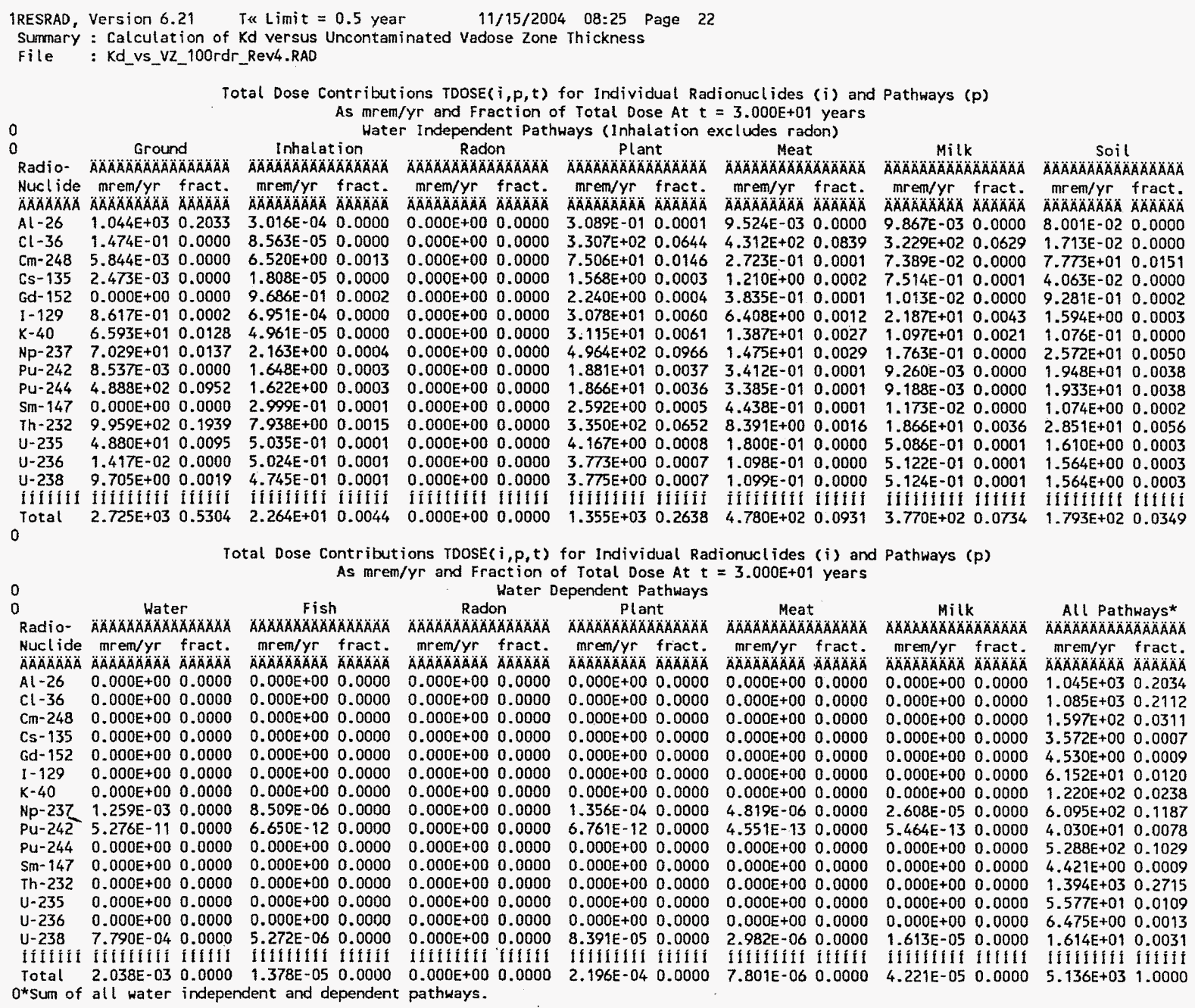

\begin{tabular}{ll} 
Attachment & \multicolumn{1}{c}{$\begin{array}{c}\text { Sheet No. } 22 \text { of } 32 \\
\text { Originator }\end{array}$} \\
Chk'W. Clark & Date \\
Chy M.T. Stankovich & Date \\
Calc. No. 0100X-CA-V0050 Rev. No. 0
\end{tabular}




\section{ATTACHMENT 1}

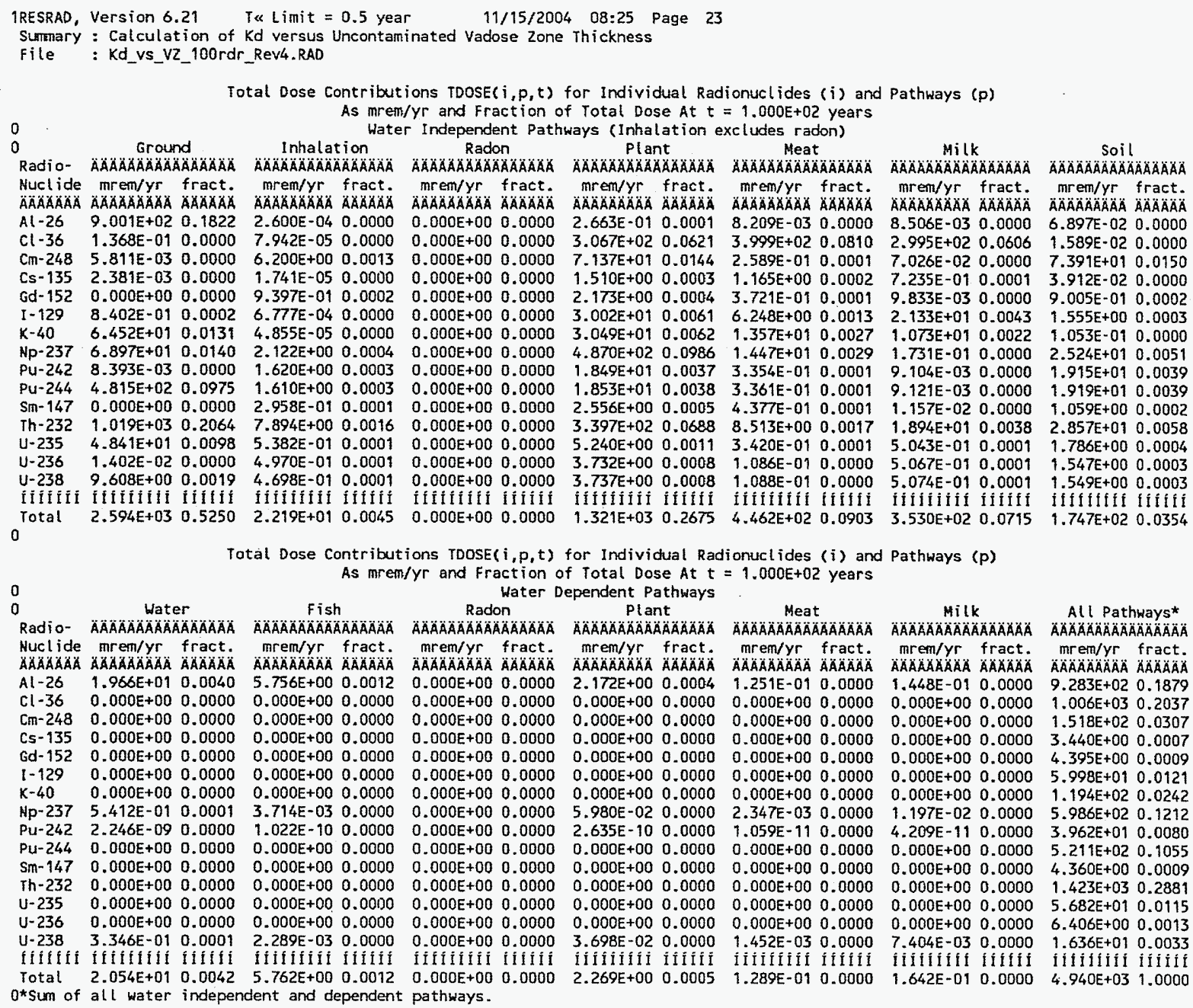

\begin{tabular}{ll} 
Attachment & \multicolumn{1}{c}{ Sheet No. 23 of 32} \\
Originator S.W. Clark & Date \\
Chk'd By M.T. Stankovich & Date \\
Calc. No. 0100X-CA-V0050 Rev. No. 0
\end{tabular}




\section{ATTACHMENT 1}

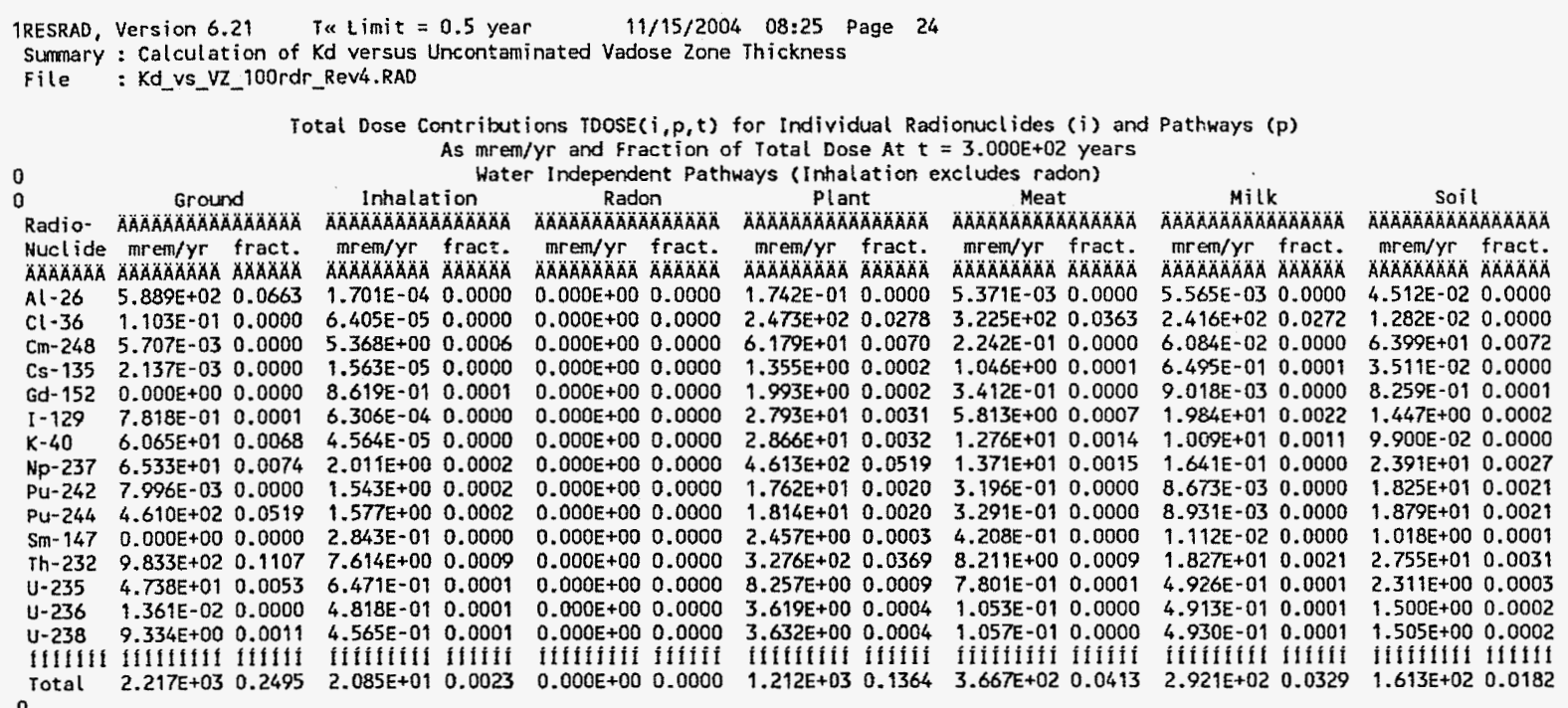

0

Total Dose Contributions TDOSE $(i, p, t)$ for Individual Radionuclides (i) and Pathways ( $p$ ) As mrem/yr and Fraction of Total Dose At $t=3.000 E+02$ years

Meat

Water Al-26 $3.444 \mathrm{E}+01 \quad 0.0039 \quad 1.009 \mathrm{E}+010.0011$ Cl-36 4.600E+00 $0.0005 \quad 2.491 E+00 \quad 0.0003$ $\begin{array}{cllll}\mathrm{Cm}-248 & 3.946 \mathrm{E}+03 \quad 0.4441 & 1.603 \mathrm{E}+02 & 0.0180\end{array}$ Cs $-135 \quad 0.000 \mathrm{E}+00 \quad 0.0000 \quad 0.000 \mathrm{E}+00 \quad 0.0000$ Gd-152 $0.000 \mathrm{E}+00 \quad 0.0000 \quad 0.000 \mathrm{E}+00 \quad 0.000$

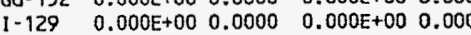

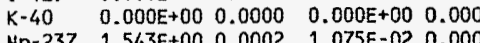
$\begin{array}{lllll}N p-237 & 1.543 E+00 & 0.0002 & 1.075 E-02 & 0.0000\end{array}$

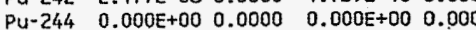

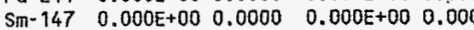

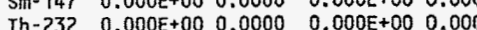
作 $\begin{array}{lllllllllll}U-236 & 0.000 E+00 & 0.0000 & 0.000 E+00 & 0.0000 & 0.000 E+00 & 0.0000 & 0.000 E+00 & 0.0000 & 0.000 E+00 & 0.0000\end{array}$

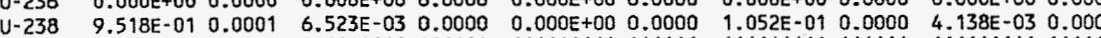

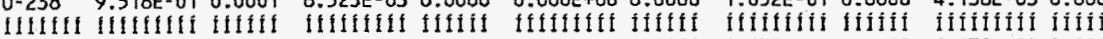
$\begin{array}{llllllllllllll}\text { Total } 3.988 E+03 & 0.4488 & 1.729 E+02 & 0.0195 & 0.000 E+00 & 0.0000 & 4.435 E+02 & 0.0499 & 6.638 E+00 & 0.0007\end{array}$ 0 * Sum of all water independent and dependent pathways.

$$
\text { Radon }
$$
AAAAAAA $2.204 E-010.0000$ $1.006 E+000.0001$ $\begin{array}{llllll}0.000 E+00 & 0.0000 & 4.358 E+02 & 0.0490 & 1.006 E+00 & 0.0001\end{array}$ $0.000 E+00 \quad 0.0000 \quad 0.000 E+00 \quad 0.0000 \quad 0.000 E+00 \quad 0.0000$

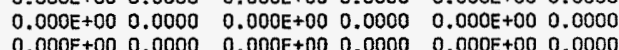

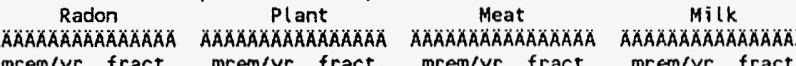
$2.544 E-01 \quad 0.0000$ $4.799 E+00 \quad 0.0005$ 2.910E-010.0000 $0.000 E+00 \quad 0.0000$ $0.000 E+00 \quad 0.0000 \quad 4.031 E+00 \quad 0.0005$ $\begin{array}{llll}0.000 E+00 & 0.0000 & 5.581 E+01 & 0.0063\end{array}$ $\begin{array}{llll}0.000 \mathrm{E}+00 & 0.0000 & 1.123 \mathrm{E}+02 & 0.0126\end{array}$ $\begin{array}{llll}0.010 E+00 & 0.0000 & 1.123 E+02 & 0.0126 \\ 0.411 E-02 & 0.0000 & 5.682 E+02 & 0.0639\end{array}$ $4.864 E-10 \quad 0.0000 \quad 3.774 E+01 \quad 0.0042$ $0.000 E+00 \quad 0.0000 \quad 4.999 E+02 \quad 0.0563$ $0.000 E+00 \quad 0.0000 \quad 4.192 E+00 \quad 0.0005$ $0.000 E+000.0000,1.373 E+03 \quad 0.1545$ $0.000 E+000.0000 \quad 5.986 E+010.0067$ 0.0 OOE $+00 \quad 0.0000 \quad 6.210 E+000.0007$

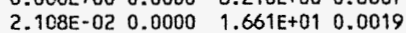

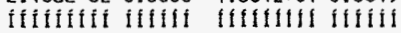
$5.399 E+00 \quad 0.0006 \quad 8.886 E+03 \quad 1.0000$
0

\begin{tabular}{|c|c|}
\hline Attachment & Sheet No. 24 of 32 \\
\hline Originator S.W. Clark & Date \\
\hline Chk'd By M.T. Stankovich & Date \\
\hline Calc. No. $0100 \times-C A-V 0050$ & Rev, No. \\
\hline
\end{tabular}




\section{ATTACHMENT 1}

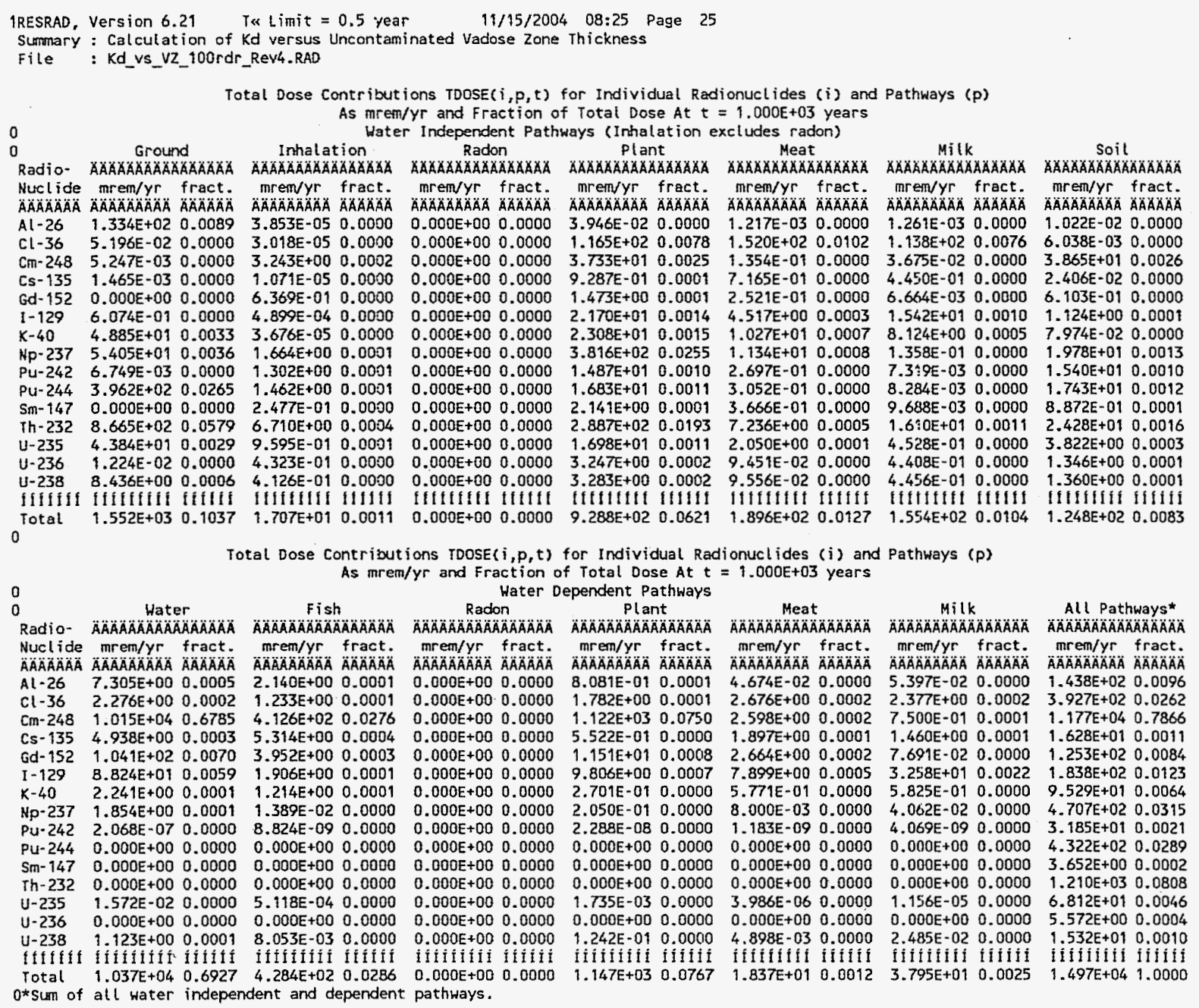

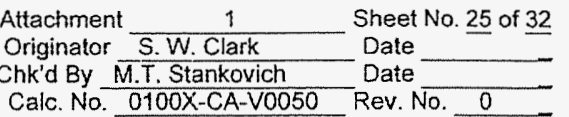




\section{ATTACHMENT 1}

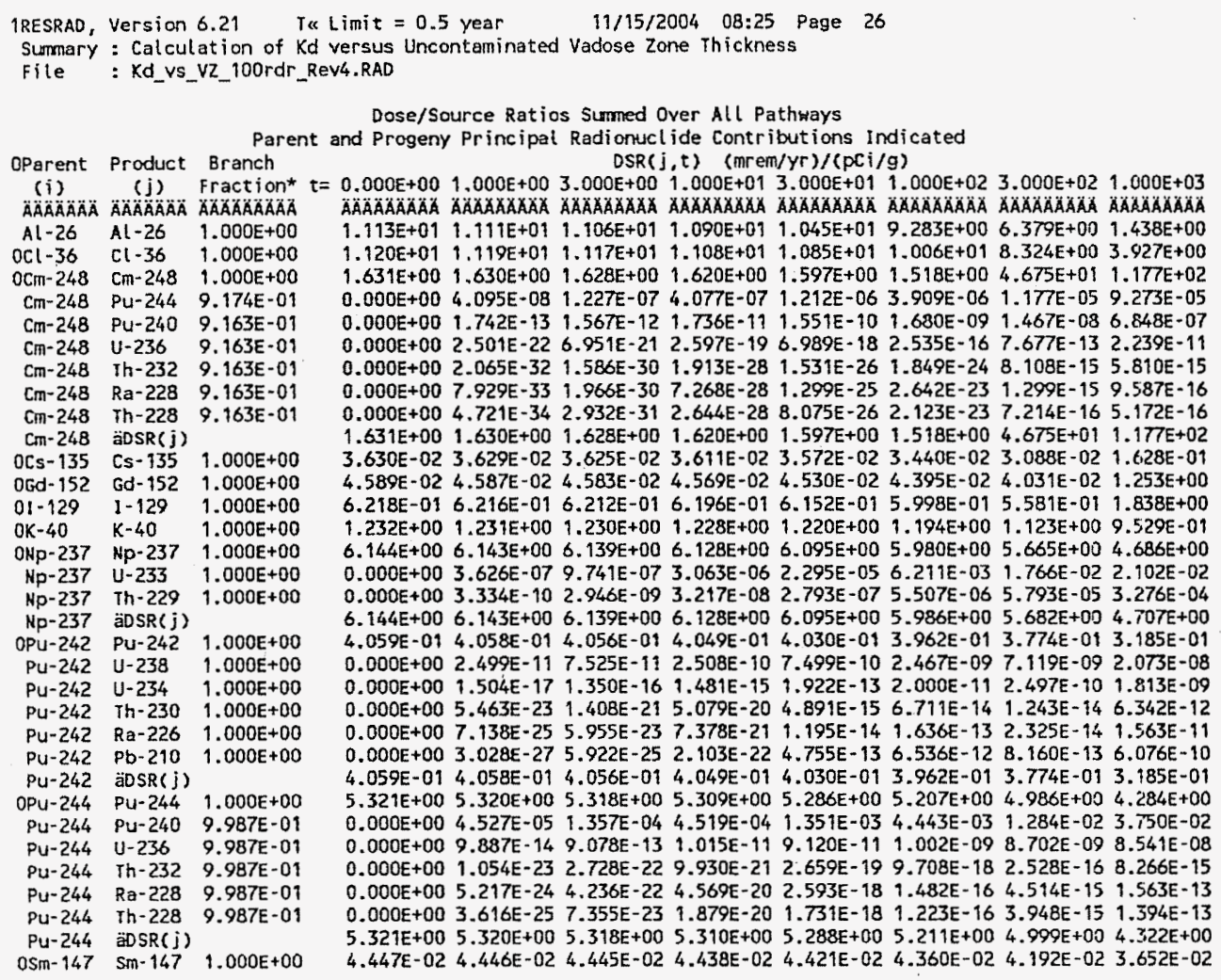

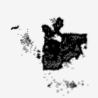

\begin{tabular}{|c|c|}
\hline Aftachment 1 & Sheet No. 26 of 32 \\
\hline Originator S.W. Clark & Date - - \\
\hline Chk'd By M.T. Stankovich & Date \\
\hline Calc. No. 0100入-CA-V0050 & Rev. No. \\
\hline
\end{tabular}




\section{ATTACHMENT 1}

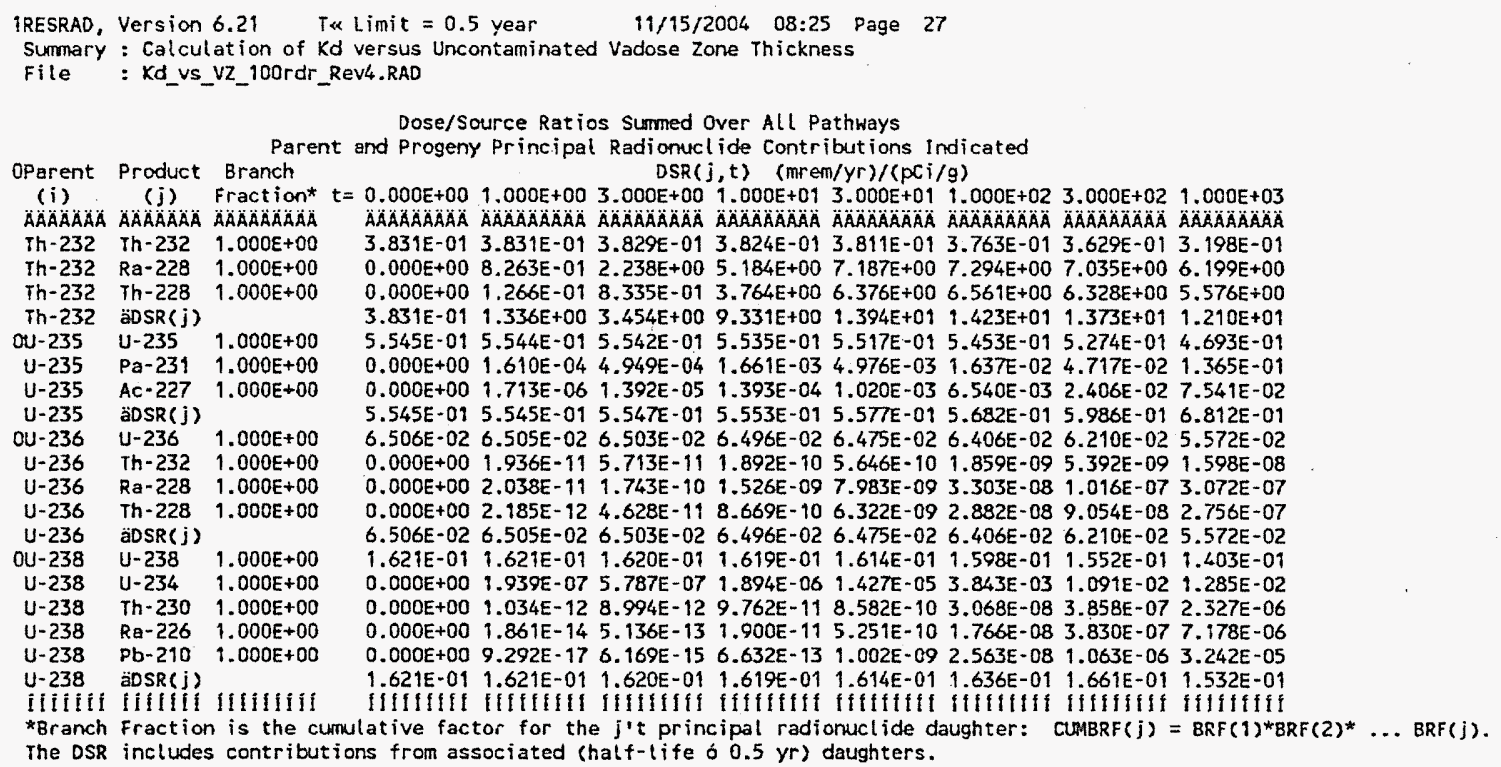

$\begin{array}{ll}\text { Attachment } & \frac{1}{\text { Sheet No. } 27 \text { of } 32} \\ \text { Originator S.W. Clark } & \text { Date } \\ \text { Chk'd By M.T. Stankovich } & \text { Date } \\ \text { Calc. No. 0100X-CA-V0050 Rev. No. } 0 & \end{array}$




\section{ATTACHMENT 1}

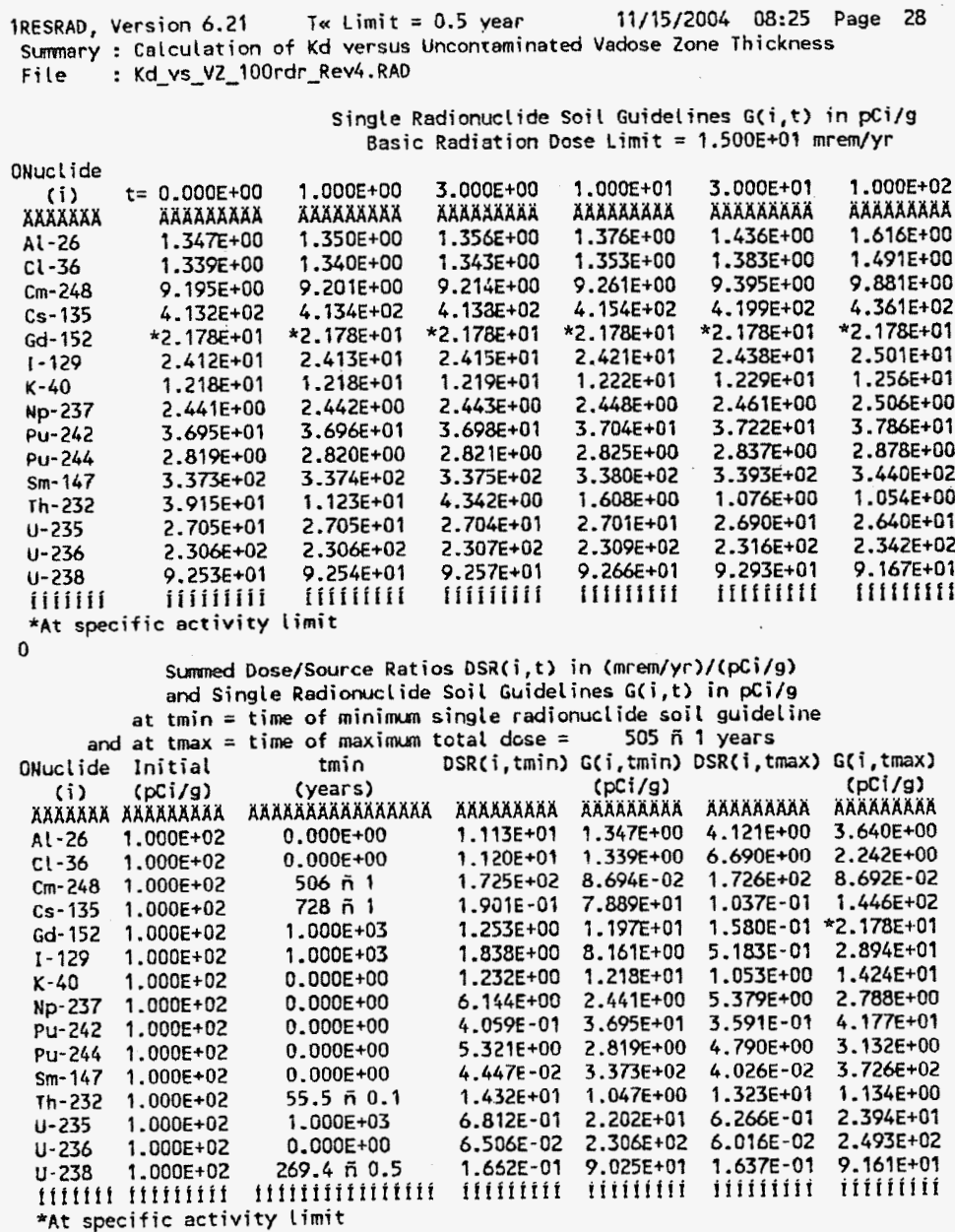

\begin{tabular}{|c|c|}
\hline $\begin{array}{l}3.000 E+02 \\
A A A A A B A A A \\
2.351 E+00 \\
1.802 E+00 \\
3.209 E-01 \\
4.858 E+02 \\
2.178 E+01 \\
2.688 E+01 \\
1.336 E+01 \\
2.640 E+00 \\
3.974 E+01 \\
3.001 E+00 \\
3.578 E+02 \\
1.093 E+00 \\
2.506 E+01 \\
2.415 E+02 \\
9.028 E+01 \\
111111111\end{array}$ & $\begin{array}{l}1.000 E+03 \\
A A A A A A A A A \\
1.043 E+01 \\
3.920 E+00 \\
1.274 E-01 \\
9.215 E+01 \\
1.197 E+01 \\
8.161 E+00 \\
1.574 E+01 \\
3.187 E+00 \\
4.709 E+01 \\
3.471 E+00 \\
4.107 E+02 \\
1.240 E+00 \\
2.202 E+01 \\
2.692 E+02 \\
9.792 E+01 \\
\text { if ff fff If }\end{array}$ \\
\hline
\end{tabular}




\section{ATTACHMENT 1}

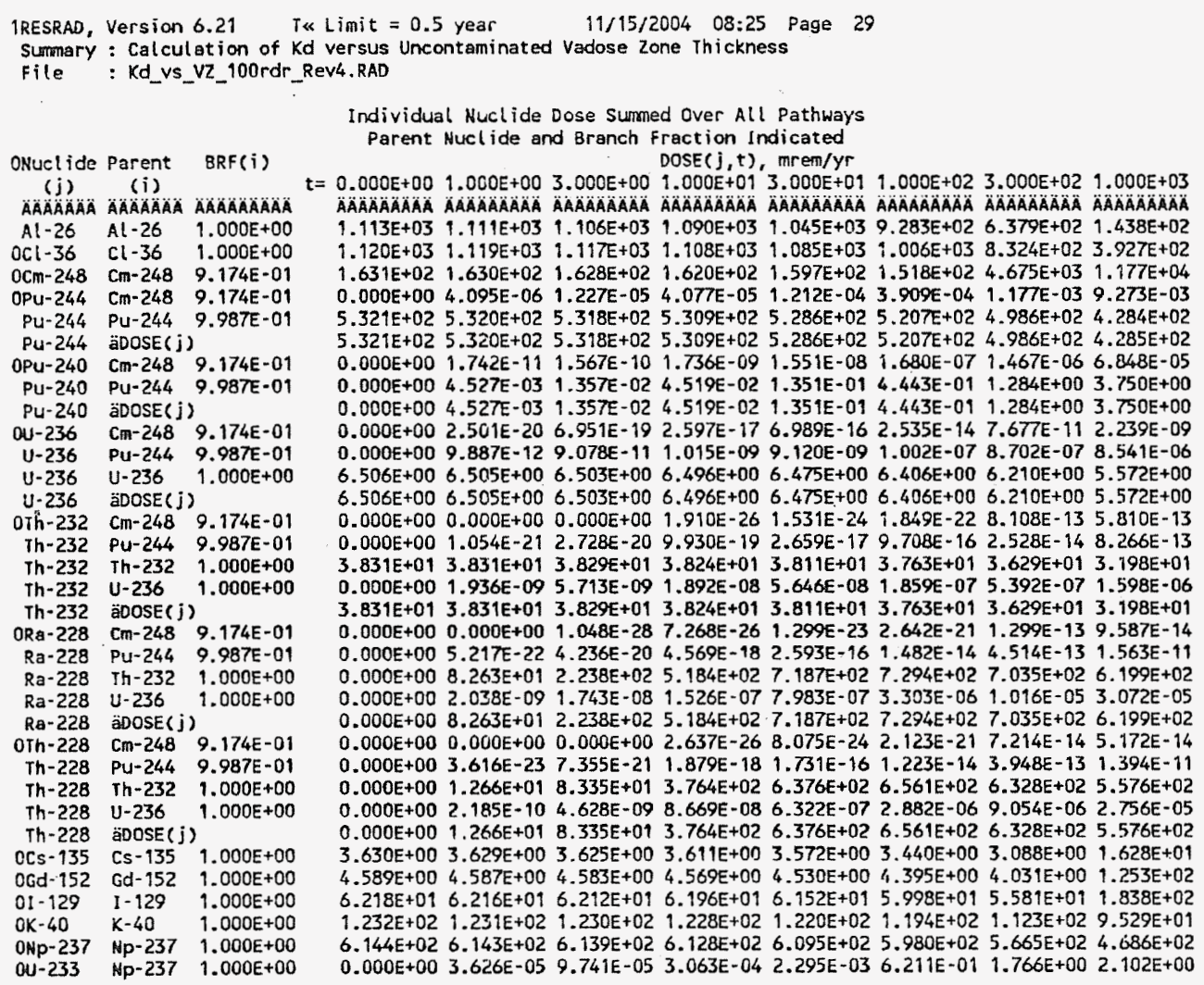

\begin{tabular}{|c|c|}
\hline Attachment $\quad 1$ & Sheet No. 29 of 32 \\
\hline Originator S.W. Clark & Date \\
\hline Chk'd By M.T. Stankovich & Date \\
\hline Calc. No. 0100X-CA-V0050 & Rev. No. \\
\hline
\end{tabular}




\section{ATTACHMENT 1}

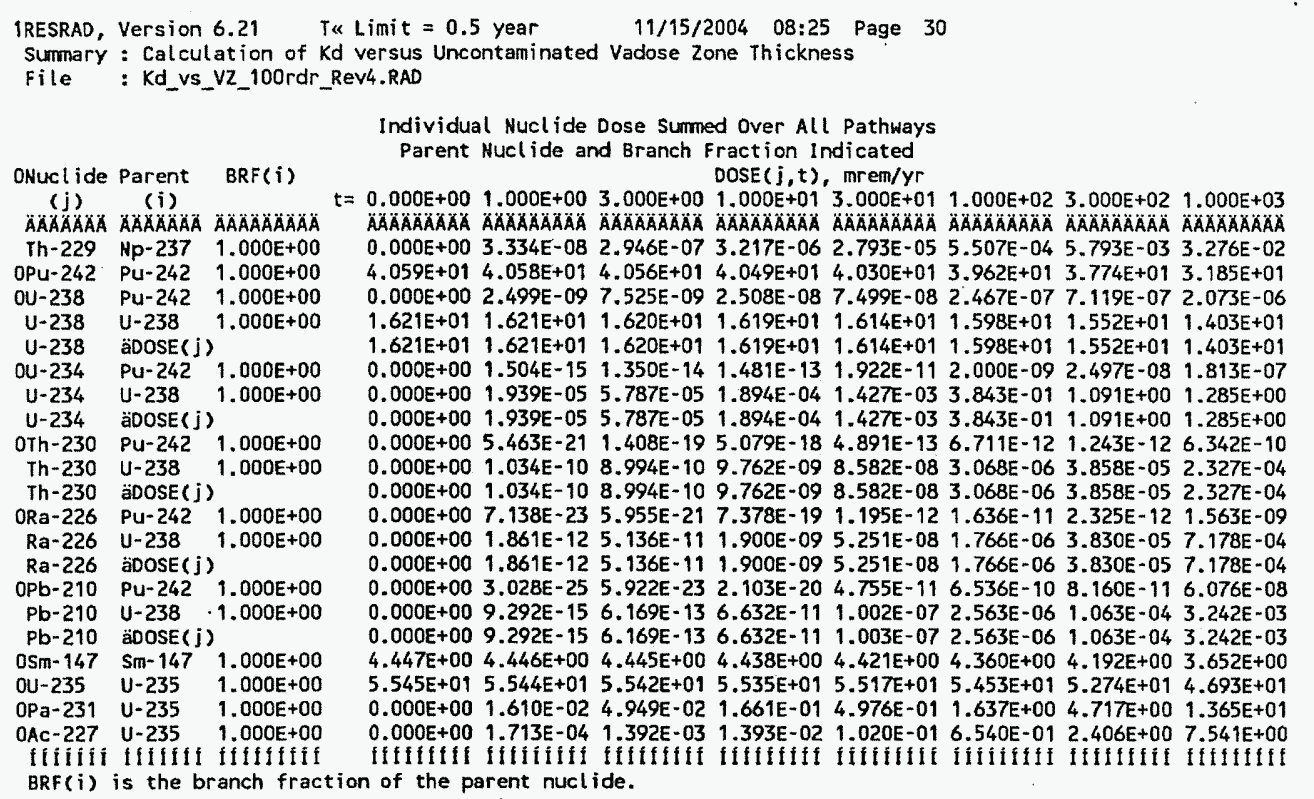

\begin{tabular}{|c|c|}
\hline Attachment & Sheet No. 30 of 32 \\
\hline S.W. Clark & Date \\
\hline Chk'd By M.T. Stankovich & Date \\
\hline Calc. No. 0100X-CA-V0050 & Rev. No. \\
\hline
\end{tabular}




\section{ATTACHMENT 1}

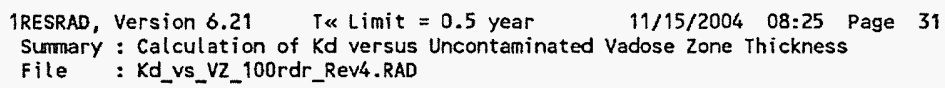

ONuclide Parent BRF(i) (j) (i)

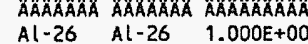
$\begin{array}{rrr}A l-26 & \mathrm{Al}-26 & 1.000 \mathrm{E}+00 \\ \text { OCl-36 } & \mathrm{Cl}-36 & 1.000 \mathrm{E}+00\end{array}$ $0 \mathrm{Cm}-248 \quad \mathrm{Cm}-248 \quad 9.174 \mathrm{E}-0$ OPu-244 Cm-248 $\quad 9.174 \mathrm{E}-01$ Pu-244 Pu-244 9.987E-01 Pu-244 äS(j):

$$
\begin{aligned}
& \text { Parent Nuclide and Branch Fraction Indicated } \\
& S(j, t), p C i / g
\end{aligned}
$$

$t=0.000 E+001.000 E+003.000 E+001.000 E+01, p C i / g$

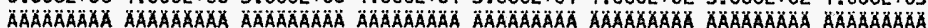

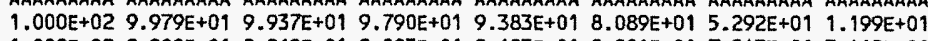

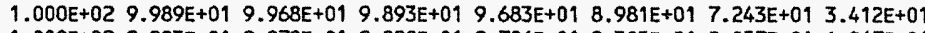

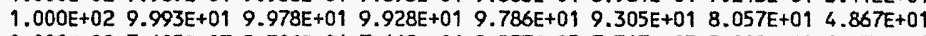
$0.000 E+00 \quad 7.695 E-07$ 2.306E-06 7.662E-06 2.277E-05 7.347E-05 2.009E-04 4.870E-04 $1.000 E+029.998 E+019.994 E+019.978 E+019.935 E+019.786 E+019.371 E+01 \quad 8.052 E+01$ $1.000 E+029.998 E+019.994 E+019.978 E+019.935 E+019.786 E+019.371 E+01 \quad 8.052 E+01$ $0.000 E+00 \quad 4.075 E-11 \quad 3.665 E-10 \quad 4.062 E-09 \quad 3.629 E-08 \quad 3.930 E-07 \quad 3.290 E-06 \quad 2.850 E-05$ $0.000 E+001.059 E-023.175 E-021.057 E-013.159 E-011.039 E+003.002 E+008.772 E+00$

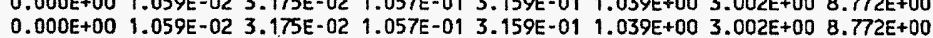

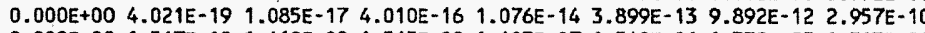
$0.000 E+00 \quad 1.567 E-10 \quad 1.410 E-09 \quad 1.565 E-08 \quad 1.403 E-07 \quad 1.540 E-06 \quad 1.338 E-05 \quad 1.313 E-04$ $1.000 E+029.998 E+019.995 E+019.985 E+019.954 E+019.846 E+019.546 E+018.565 E+01$ $1.000 E+029.998 E+019.995 E+019.985 E+019.954 E+019.846 E+019.546 E+018.565 E+01$ $0.000 E+00 \quad 4.960 E-30 \quad 4.015 E-28 \quad 4.947 E-26 \quad 3.984 E-24 \quad 4.821 E-22 \quad 3.689 E-20 \quad 3.739 E-18$ $0.000 E+00 \quad 2.577 E-21 \quad 6.956 E-20 \quad 2.573 E-18 \quad 6.923 E-17 \quad 2.532 E-15 \quad 6.598 E-14 \quad 2.157 E-12$ $1.000 E+02$ 9.998E+01 9.995E+01 9.982E+01 9.946E+019.821E+01 9.473E+01 B.348E+01 $0.000 E+00$ 4.933E-09 1.479E-0B 4.925E-08 1.473E-07 4.851E-07 $1.407 E-06$ 4. $0.000+00$ 4. $1.000 E+02$ 9.998E+01 9.995E+01 9.982E+01 9.946E+01 9.821E+01 9.473E+01 8.348E+01

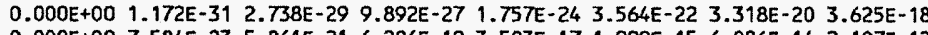
$0.000 E+00 \quad 7.584 E-23 \quad 5.861 E-21 \quad 6.206 E-19 \quad 3.503 E-17 \quad 1.999 E-15 \quad 6.086 E-14 \quad 2.107 E-12$ $0.000 E+001.136 E+01 \quad 3.034 E+01 \quad 6.995 E+01 \quad 9.688 E+01 \quad 9.831 E+01 \quad 9.483 E+01 \quad 8.356 E+01$ $0.000 E+00 \quad 2.857 E-10 \quad 2.380 E-09 \quad 2.064 E-08 \quad 1.077 E-07 \quad 4.453 E-07 \quad 1.370 E-06 \quad 4.141 E-06$ $0.000 E+001.136 E+01 \quad 3.034 E+01 \quad 6.995 E+01 \quad 9.688 E+01 \quad 9.831 E+01 \quad 9.483 E+01 \quad 8.356 E+01$

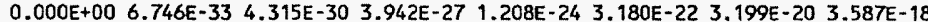
$0.000 E+00 \quad 5.200 E-24 \quad 1.086 E-212.804 E-192.591 E-171.832 E-15 \quad 5.918 E-142.090 E-12$ $0.000 E+001.864 \mathrm{E}+001.243 \mathrm{E}+015.638 \mathrm{E}+019.557 \mathrm{E}+019.835 \mathrm{E}+019.486 \mathrm{E}+01 \mathrm{~B} .359 \mathrm{E}+01$ $0.00 E+001.864 E+001.243 E+015.638 E+019.557 E+019.835 E+019.486 E+01$ 8.35 $0.000+00$ 3.189E-11 6. $0.000 E+001.864 E+001.243 E+015.638 E+019.557 E+01$ 9.835E+01 9.4B6E+01 8.359E+0

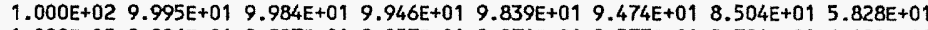

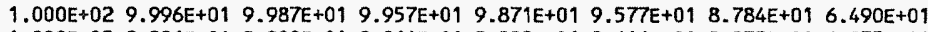
$1.000 E+029.996 E+019.989 E+019.964 E+019.892 E+019.646 E+01 \quad 8.975 E+01 \quad 6.973 E+01$ $1.000 E+029.997 E+019.991 E+019.969 E+019.908 E+019.696 E+019.114 E+01 \quad 7.340 E+01$ $1.000 E+029.997 E+019.992 E+019.973 E+019.919 E+019.733 E+019.219 E+01 \quad 7.626 E+01$

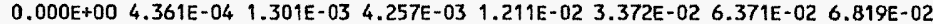

\begin{tabular}{ll} 
Attachment & \multicolumn{1}{c}{$\begin{array}{c}\text { Sheet No. } 31 \text { of } 32 \\
\text { Originator }\end{array}$} \\
Chk'd By S. Clark & Date \\
Calc. No. Stankovich & Date \\
O100X-CA-V0050 Rev. No. 0
\end{tabular}




\section{ATTACHMENT 1}

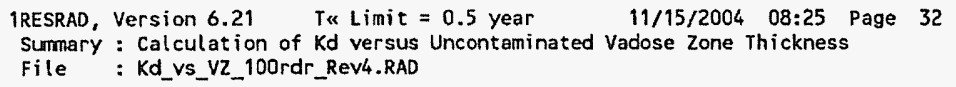

ONuclide Parent BRF(i) (j) (i) (i)

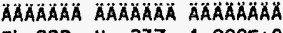
Th-229 Np-237 1.000E+00 OPU-242 PU-242 $1.000 \mathrm{E}+00$ OU-238 PU-242 1.000E +00 $\begin{array}{lll}\mathrm{U}-238 & \mathrm{U}-238 \quad 1.000 \mathrm{E}+00\end{array}$ U-238 äS(j):

OU-234 PU-242 $1.000 E+00$

$\mathrm{U}-234 \quad \mathrm{U}-238 \quad 1.000 \mathrm{E}+00$ $\mathrm{U}-234$ äs $(j)$

OTh-230 PU-242 1.000E+00 Th-230 U-238 1.000E +00 Th-230 äs(j):

Ra-226 PU-242 1.000E+00 Ra-226 U-238 1.000E+00 $R a-226$ äs $(j):$

OPb-210 PU-242 1.000E+00 $\mathrm{Pb}-210 \quad \mathrm{U}-238$ $\mathrm{Pb}-210$ äs(j) $\mathrm{Pb}-210$ as $(\mathrm{j}):$

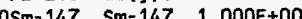
OU -235 (1.00E+00 OPa-231 U-235 $1.000+00$ OAC-227 U-235 $1.000 E+00$

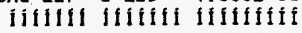
BRF( $i)$ is the branch fraction ORESCALC.EXE execution time =

$$
s(j, t), p C i / 9
$$

$=0.000 E+001.000 E+003.000 E+001.000 E+013.000 E+011.000 E+023.000 E+021.000 E+03$

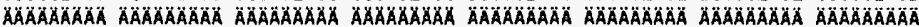

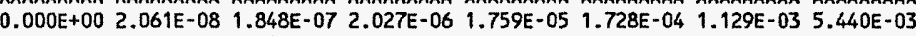

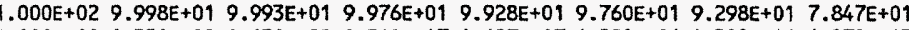
$0.000 E+00 \quad 1.551 E-08 \quad 4.651 E-08 \quad 1.548 E-07 \quad 4.627 E-07 \quad 1.522 E-06 \quad 4.392 E-06 \quad 1.279 E-05$ $1.000 E+029.999 E+019.996 E+019.986 E+019.957 E+019.856 E+019.576 E+01 \quad 8.654 E+01$ $1.000 E+029.999 E+019.996 E+019.986 E+019.957 E+019.856 E+019.576 E+01 \quad 8.654 E+01$ $0.000 E+002.195 E-14 \quad 1.968 E-13 \quad 2.159 E-12 \quad 1.874 E-11$ 1.842E-10 $1.207 E-09$ 5.874E-09 $0.000 E+002.828 E-04 \quad 8.438 E-04 \quad 2.762 E-03 \quad 7.866 E-03 \quad 2.201 E-02 \quad 4.229 E-02 \quad 4.895 E-02$ 0.000E+00 2.828E-04 8.438E-04 $2.762 E-037.866 E-03$ 2.201E-02 $4.220 E-024.895 E-02$ 0.00E+ $2.828 E-04$ 8.438E-04 2.762E-03 7.866E-03 2.201E-02 4.229E-02 4.895E-02 . $0.000 E+001.274 E-09$ 1.142E-08 1.254E-07 $1.089 E-06$ 1.076E-05 $7.145 E-05$ 3.677E-04 $0.000 E+00 \quad 1.274 E-09 \quad 1.142 E-08 \quad 1.254 E-07 \quad 1.089 \mathrm{E}-06 \quad 1.076 \mathrm{E}-05 \quad 7.145 \mathrm{E}-05 \quad 3.677 \mathrm{E}-04$ $0.000 E+00 \quad 7.138 E-24 \quad 5.768 E-22 \quad 7.061 E-20 \quad 5.583 E-18 \quad 6.350 E-16 \quad 4.132 E-142.718 E-12$ $0.000 E+00 \quad 1.840 E-13 \quad 4.953 E-12 \quad 1.816 E-10 \quad 4.765 E-09 \quad 1.600 E-07 \quad 3.338 E-06 \quad 6.062 E-05$ $0.000 E+00 \quad 1.840 E-13 \quad 4.953 E-12 \quad 1.816 E-10 \quad 4.765 E-09 \quad 1.600 E-07 \quad 3.338 E-06 \quad 6.062 E-05$

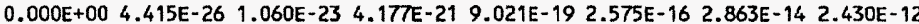
$0.000 E+001.421 E-151.134 E-13 \quad 1.331 E-119.373 E-107.545 E-08 \quad 2.521 E-06 \quad 5.597 E-05$ $0.000 E+001.421 E-151.134 E-131.331 E-119.373 E-107.545 E-082.521 E-06$ 5.597E 05 1.00E 00 1.421E-15 1.134E-13 1.331E 1.000E+02 $9.998 E+019.994 E+019.980 E+019.941 E+019.805 E+019.426 E+01$ 8.212E+0 .000E+02 $9.998 E+01$ 9.995E+01 $9.983 E+01$ 9.950E+01 $9.835 E+019.512 E+018.464 E+01$ $\begin{array}{llllllll}0.000 E+00 & 2.115 E-03 & 6.344 E-03 & 2.112 E-02 & 6.309 E-02 & 2.073 E-01 & 5.974 E-01 & 1.729 E+00\end{array}$

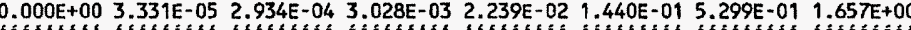

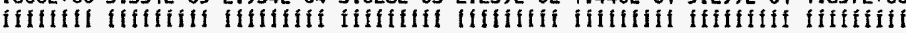
of the parent nuclide.

10.19 seconds 